\title{
Synthesis of indoles, biindoles, indole alkaloids, pyrroloindoles and benzimidazoles from aromatic nitro compounds and a study of the chemoselectivity in the Kosugi-Migita-Stille coupling
}

Nurul Hoda Ansari

Follow this and additional works at: https://researchrepository.wvu.edu/etd

\section{Recommended Citation}

Ansari, Nurul Hoda, "Synthesis of indoles, biindoles, indole alkaloids, pyrroloindoles and benzimidazoles from aromatic nitro compounds and a study of the chemoselectivity in the Kosugi-Migita-Stille coupling" (2017). Graduate Theses, Dissertations, and Problem Reports. 5114.

https://researchrepository.wvu.edu/etd/5114

This Dissertation is protected by copyright and/or related rights. It has been brought to you by the The Research Repository @ WVU with permission from the rights-holder(s). You are free to use this Dissertation in any way that is permitted by the copyright and related rights legislation that applies to your use. For other uses you must obtain permission from the rights-holder(s) directly, unless additional rights are indicated by a Creative Commons license in the record and/ or on the work itself. This Dissertation has been accepted for inclusion in WVU Graduate Theses, Dissertations, and Problem Reports collection by an authorized administrator of The Research Repository @ WVU.

For more information, please contact researchrepository@mail.wvu.edu. 


\title{
Synthesis of indoles, biindoles, indole alkaloids, pyrroloindoles and benzimidazoles from aromatic nitro compounds and a study of the chemoselectivity in the Kosugi-Migita-Stille coupling
}

\author{
Nurul Hoda Ansari \\ Dissertation submitted to \\ Eberly College of Arts and Sciences \\ West Virginia University \\ In partial fulfillment of the requirements \\ for the degree of \\ Doctor of Philosophy \\ in \\ Chemistry
}

\begin{abstract}
Björn Söderberg, Ph. D., Chair
Kung, K. Wang, Ph. D.

Brian V. Popp, Ph. D.

Jessica M. Hoover, Ph. D.

Mark McLaughlin, Ph. D.
\end{abstract}

Department of Chemistry

Morgantown, West Virginia

2017

Keywords: indoles, biindoles, indole alkaloids, pyrroloindoles, benzimidazoles, Kosugi-Migita-

Stille coupling

Copyright 2017 Nurul Hoda Ansari 


\section{Abstract \\ Synthesis of indoles, biindoles, indole alkaloids, pyrroloindoles and benzimidazoles from aromatic nitro compounds and a study of the chemoselectivity in the Kosugi-Migita-Stille coupling}

Nurul Hoda Ansari

Total syntheses of the three naturally occurring indole alkaloids alocasin A, scalaridine A and hyrtinadine A-B have been accomplished using three palladium catalyzed reactions, an alkyne hydrostannylation, a Kosugi-Migita-Stille coupling and a reductive $N$-heterocyclization as the key steps. A palladium catalyzed double reductive cyclization of 1,4-, 1,3-, and 2,3-bis(2-nitroaryl)-1,3-butadienes to afford 2,2', 2,3'-, and 3,3'-biindoles, respectively, has been developed. In an attempt to synthesize indolo[2,3- $b]$-and indolo[3,2-b]-indoles from the reductive cyclizations of 1,1'-, and 1,2'-bis(2-nitroaryl)ethenes respectively, nonselective product formation was observed giving rise to carbon monoxide insertion product as the sole or the major reaction product. The reductive cyclization strategy was extended to achieve the expedient synthesis of three naturally occurring polybrominated biindoles; 2,2',5,5'tetrabromo-3,3'-bi-1H-indole, 2,2',6,6'-tetrabromo-3,3'-bi-1H-indole and 2,2',5,5',6,6'-hexabromo-3,3'bi-1H-indole. In addition, palladium catalyzed, carbon monoxide mediated, double reductive $N$ heterocyclization of dialkenyldinitrobenzenes to the highly functionalized and highly elusive novel nonsymmetrical pyrroloindoles has been developed for the first time. The methodology presents the first common synthetic routes to all isomeric pyrroloindoles which are of interest in a number of applications in addition to their presence as a core moiety in bioactive natural products. The highly functionalized isomeric cyclization precursors were prepared through the use of Kosugi-Migita-Stille coupling. The generally mild reaction conditions offer significant improvements over the previously reported synthetic routes. A facile base-mediated cyclization of enamines derived from the condensation of 2-nitroanilines with $\alpha$-branched aldehydes, in the presence of a carbon-based electrophile, to give $N$-alkoxy-substituted benzimidazoles with or without an oxygenate side chain in the 2-position has also been developed. Finally, the chemoselectivity of Kosugi-Migita-Stille couplings of all isomeric permuatations of bromphenyl- and bromonitrophenyl trifluoromethanesulfonates was examined in order to compare and contrast the reactivity of $\mathrm{C}-\mathrm{Br}$ and $\mathrm{C}-\mathrm{OTf}$ bonds under three different reaction conditions. To the best of our knowledge, the study presented here represents the first of its kind. 
Dedicated to my parents and my wife

\author{
Shabana Tarannum
}




\section{Acknowledgements}

I would like to express my sincere gratitude to my research advisor professor Björn Söderberg for the continuous support, patience, motivation and immense knowledge. With his guidance, I learned organic chemistry and organic laboratory skills. I am very grateful to his guidance through all the difficulties that I encountered during my research work. His supervision and encouragement always helped me remain motivated in doing productive and meaningful research. This thesis which represents a milestone more than just a PhD requirement is a result of his insightful comments and discussions. In his lab, I gained the importance of honesty in the scientific world. I sincerely appreciate my committee members Dr. Kung Wang, Dr. Brian Popp, Dr. Jessica Hoover and Dr. Mark McLaughlin for their valuable involvement and suggestion in general throughout my graduate study at WVU. I truly appreciate Dr. Novruz Akhmedov's kindness and helpfulness that enabled me to learn various NMR techniques.

I appreciate the friendship and assistance of my present and past group members in the Söderberg research lab. Chapter 2 had been initiated by Dr. Christopher Dacko. Part of the work in chapter 4 had been carried out by Arica Jordan. Part of the work in chapter 6 was continued from Dr. Matthew Cummings. Similarly, the foundation stone for chapter 7 was laid by Dr. Matthew Cummings and Dr. Serge Banini. I am very grateful to their contributions. Partial work in chapter 3 was performed by Matthew Taylor, an undergraduate student in our lab. Similarly, I like to thank Dr. Yilin Zhang for being helpful running ${ }^{13} \mathrm{C}$ NMR spectra especially at night. I also like to thank my lab-mates Katharine Lambson, Ganesh Ghimire and SM Ashikur Rahman for their nice cooperation. I thank Katy for her impressive comments and suggestions during the organic divisional seminar talks. I appreciate Mr. Rahman's help during my thesis writing. Overall, these people will remain a lifelong memory for me.

I want to express my appreciation to my family members in Nepal and my brother Khursed Ansari at University of Toledo, for their endless spiritual support and encouragement. My wife's patience, bravery and encouragement served as an inspiration to make my graduate life productive. Through her patience was I able to complete seven research projects at WVU.

Last but not the least, I gratefully acknowledge the C. Eugene Bennett Department of Chemistry and funding from the National Institutes of Health (1 R15 GM122002-01) for support. The National Science Foundation-MRI program is also gratefully acknowledged for the funding of a $400 \mathrm{MHz}$ NMR system (CHE-1228366). I would like to thank Dr. Stephen Valentine for HRMS analyses. 


\section{Table of Contents}

Title page

i

Abstract

ii

Dedication

iii

Acknowledgements

iv

Table of Contents

$\mathrm{V}$

List of Tables

vii

List of Schemes

viii

List of Figures

$\mathrm{X}$

Chapter 1 Short syntheses of the indole alkaloids alocasin A, scalaridine A and hyrtinadine

A-B

1.A Introduction

1.B Results and Discussion

1.C Conclusion

Chapter 2 Double palladium catalyzed reductive cyclizations. Synthesis of 2,2'-, 2,3'-, and

2,2'-bi-1 $H$-indoles, indolo[3,2- $b$ ]indoles, and indolo[2,3- $b]$ indoles

2.A Introduction

2.B Results and Discussion

2.C Conclusion

Chapter 3 Syntheses of three naturally occurring polybrominated 3,3'-bi-1H-indoles

3.A Introduction

3.B Results and Discussion

3.C Conclusion

Chapter 4 A facile base-mediated synthesis of $N$-alkoxy-substituted benzimidazoles

4.A Introduction

4.B Results and Discussion

4.C Mechanistic Discussion

4.D Conclusion

Chapter 5 A facile base-mediated synthesis of $\mathrm{N}$-alkoxy-2H-benzimidazoles

5.A Introduction

5.B Results and Discussion

5.C Conclusion 
Chapter 6 Chemoselectivity in the Kosugi-Migita-Stille coupling of bromophenyl- and bromonitrophenyl-trifluoromethanesulfonates

6.A Introduction 45

6.B Results and Discussion $\quad 47$

6.C Conclusion 55

Chapter 7 Synthesis of pyrroloindoles via a double reductive $N$-heterocyclization 56

7.A Introduction $\quad 56$

$\begin{array}{lll}\text { 7.B Results and Discussion } & 60\end{array}$

7.C Synthesis of pyrroloindole (Type A-E) precursors 63

7.D Synthesis of 1H,8H-pyrrolo[3,2-g]indoles (Type A) 72

$\begin{array}{lll}\text { 7.E Conclusion } & 84\end{array}$

Experimental Section $\quad 85$

$\begin{array}{lr}\text { References and Footnotes } & 208\end{array}$

Appendix 


\section{List of Tables}

Table 2.1 Synthesis of 2,2'-bi-1H-indoles 13

Table 2.2 Synthesis of 3,3'-bi-1H-indoles 15

Table 2.3 Synthesis of 2,3'-bi-1H-indoles 17

Table 4.1 Condition screening

Table 4.2 Base-mediated synthesis of benzimidazoles under condition A 34

Table 4.3 Base-mediated synthesis of benzimidazoles under condition B 36

Table 4.4 Base-mediated synthesis of $N$-oxygenated benzimidazoles from enamine 127

Table 5.1 Formation of $N$-methoxybenzimidazoles from enamines 43

Table 5.2 Base-mediated synthesis of $N$-oxygenated benzimidazoles from enamine 12744

Table 6.1 Reactions of bromophenyl trifluoromethanesulfonates with ethenyltributyltin 48

Table 6.2 Cross-couplings of 213-222 with ethenyltributyltin 51

Table 7.1 Condition screening $\quad 61$

Table 7.2 Synthesis of cyclization precursors by double Kosugi-Migita-Stille coupling 64

Table 7.3 Selective cross coupling of bromo- and iodo-phenyl triflates 66

Table 7.4 Synthesis of unsymmetrical cyclization precursors $\quad 69$

Table 7.5 Synthesis of 1H,8H-pyrrolo[3,2-g]indoles (Type A) 73

Table 7.6 Attempted synthesis of pyrroloindoles (Type B-E) 76

Table 7.7 Synthesis of $N$-tosylated indoles $\quad 81$

Table 7.8 Synthesis of $N$-tosylated pyrroloindoles (Type B-E) $\quad 82$ 


\section{List of Schemes}

Scheme 1.1 Sperry's synthesis of alocasin A 2

Scheme 1.2 Sperry's synthesis of scalaridine A 2

Scheme 1.3 Tasch's synthesis of hyrtinadine A 3

Scheme 1.4 Mosquera's synthesis of scalaridien A 3

Scheme 1.5 Synthesis of alocasin A $\quad 5$

Scheme 1.6 Attempted cross coupling of 4 with 3,5-dibromopyridine (9) 6

$\begin{array}{ll}\text { Scheme 1.7 Synthesis of scalaridine A } & 7\end{array}$

Scheme 1.8 Attempted cross coupling of 4 with 2,5-dibromopyrimidine (15) 8

Scheme 1.9 Synthesis of hyrtinadine A 9

$\begin{array}{ll}\text { Scheme } 1.10 \text { Synthesis of hyrtinadine B } & 10\end{array}$

Scheme 2.1 Synthesis of 5-bromo-2-nitrocinnamaldehyde (32) 13

Scheme 2.2 Synthesis of 4,4'-diaza-3,3'-bi-1H-indole 15

Scheme 2.3 Synthesis of unsymmetrical 3,3'-bi-1H-indole 68

Scheme 2.4 Reductive cyclization of $79 \quad 18$

$\begin{array}{ll}\text { Scheme 2.5 Reductive Cyclization of } \mathbf{8 4} & 18\end{array}$

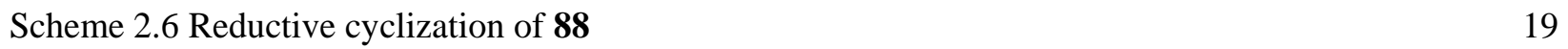

Scheme 2.7 Reductive cyclization of 91

Scheme 2.8 Synthesis of indolo[2,3-b]indole (96) and indolo[2,3-c]quinolinone (97) 20

Scheme 2.9 Reductive cyclization of $99 \quad 21$

Scheme 3.1 Palladium catalyzed double reductive cyclization to 3,3'-biindole 23

Scheme 3.2 Palladium catalyzed oxidative dimerization of 5-bromoindole 23

Scheme 3.3 Two common routes to $\mathbf{1 1 1} 24$

Scheme 3.4 Synthesis of precursor 111

Scheme 3.5 Bromination of 3-methylindole 25

Scheme 3.6 Synthesis of 2,2',5,5'-tetrabromo- and 2,2',5,5',6,6'-hexabromobiindoles 26

Scheme 3.7 Synthesis of 2,2',6,6'-tetrabromo-bi-1H-indole 27

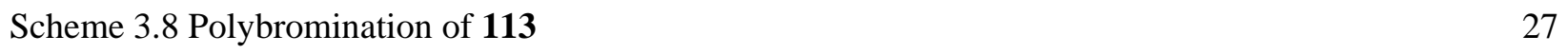

Scheme 3.9 Bromination of 3,3-bi-1H-indole $56 \quad 28$

Scheme 4.1 Formation of benzimidazoles 124-126 from enamine 122

Scheme 4.2 Formation of 2-ethenyl- $N$-propoxybenzimidazole 31

Scheme 4.3 Formation of $N$-methoxybenzimidazole $\mathbf{1 6 9}$ and benzophenone (170) 38

Scheme 4.4 Reaction of $\mathbf{1 2 7}$ with acetyl chloride 38 
Scheme 4.5 Proposed mechanism for the formation of $N$-oxygenated benzimidazoles 124-126 40

Scheme 5.1 Reaction of enamine $\mathbf{1 2 2}$ with methyl iodide 41

Scheme 5.2 Gardiner's synthesis of $N$-methoxybenzimidazole 42

Scheme 6.1 Preparation and Kosugi-Migita-Stille coupling of 204

Scheme 6.2 Kosugi-Migita-Stille coupling of $\mathbf{2 4 7}$ with ethenyltributyltin 54

Scheme 6.3 Kosugi-Migita-Stille coupling of $\mathbf{2 5 0}$

Scheme 6.4 Kosugi-Migita-Stille coupling of $\mathbf{2 5 3}$

Scheme 7.1 Vilsmeier formylation route to pyrroloindoles $\quad 57$

Scheme 7.2 Samsoniya et al synthesis of pyrroloindoles $\quad 57$

Scheme 7.3 Preparation of pyrroloindoles from aminoindoline 58

Scheme 7.4 Preparation of pyrroloindoles from diimines $\quad 58$

Scheme 7.5 Intramolecular hydroamination route to pyrroloindoles 58

Scheme 7.6 Possible routes to pyrroloindoles $\quad 59$

$\begin{array}{ll}\text { Scheme 7.7 Preparation of } \mathbf{2 5 8} & 61\end{array}$

Scheme 7.8 Synthesis of bromo- and iodo-phenyl trifluoromethanesulfonates 62

Scheme 7.9 Iodination of 2,5-dinitrophenol (267) 62

Scheme 7.10 Synthesis of bromo- and iodo-phenyl trifluoromethanesulfonates 63

Scheme 7.11 Synthesis of $\mathbf{2 8 1} \quad 63$

Scheme 7.12 Attempted selective Kosugi-Migita-Stille coupling of 258

Scheme 7.13 Problem in Kosugi-Migita-Stille coupling $\quad 68$

Scheme 7.14 Synthesis and attempted Kosugi-Migita-Stille coupling of $\mathbf{3 1 5} 68$

Scheme 7.15 Alternative route to $330 \quad 72$

Scheme 7.16 Attempted reductive cyclization of $\mathbf{3 6 8}$ and $\mathbf{3 6 9}$

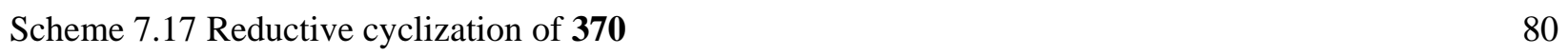




\section{List of Figures}

Figure 1.1 Structures of alocasin A, scalaridine A and hyrtinadine A-B 1

Figure 2.1 Possible cyclizations (A-E) of bis(2-nitrophenyl)butadienes and -ethenes 12

Figure 3.1 Naturally occurring polyhalogenated 3,3'-bi-1H-indoles 22

Figure 6.1 Isomeric bromonitrophenyl trifluoromethanesulfonates (213-222) 50

Figure 7.1 Isomeric pyrroloindoles $\quad 56$

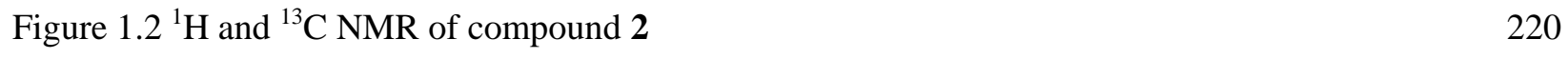

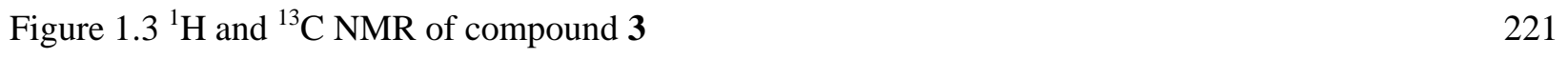

Figure $1.4{ }^{1} \mathrm{H}$ and ${ }^{13} \mathrm{C}$ NMR of compound 4

Figure $1.5{ }^{1} \mathrm{H}$ and ${ }^{13} \mathrm{C}$ NMR of compound 6

Figure $1.6{ }^{1} \mathrm{H}$ and ${ }^{13} \mathrm{C}$ NMR of compound 7

Figure $1.7{ }^{1} \mathrm{H}$ and ${ }^{13} \mathrm{C}$ NMR of compound 8

Figure $1.8{ }^{1} \mathrm{H}$ and ${ }^{13} \mathrm{C}$ NMR of alocasin A 226

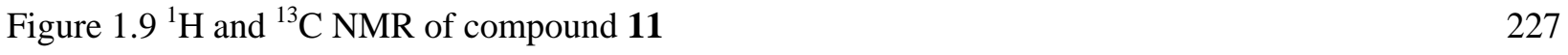

Figure $1.10{ }^{1} \mathrm{H}$ and ${ }^{13} \mathrm{C}$ NMR of compound $\mathbf{1 2} 228$

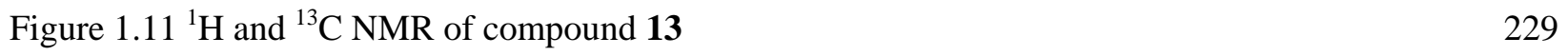

Figure $1.12{ }^{1} \mathrm{H}$ and ${ }^{13} \mathrm{C}$ NMR of compound $\mathbf{1 4}$

Figure $1.13{ }^{1} \mathrm{H}$ and ${ }^{13} \mathrm{C}$ NMR of scalaridine A 231

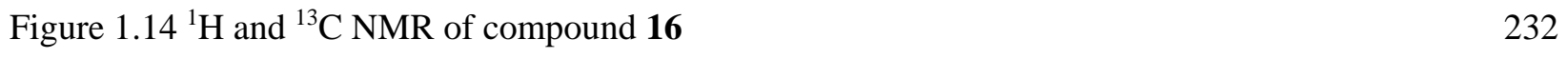

Figure $1.15{ }^{1} \mathrm{H}$ and ${ }^{13} \mathrm{C}$ NMR of compound $\mathbf{1 7}$

Figure $1.16{ }^{1} \mathrm{H}$ and ${ }^{13} \mathrm{C}$ NMR of compound $\mathbf{1 8}$

Figure $1.17{ }^{1} \mathrm{H}$ and ${ }^{13} \mathrm{C}$ NMR of compound 19

Figure $1.18{ }^{1} \mathrm{H}$ and ${ }^{13} \mathrm{C}$ NMR of compound 20 236

Figure $1.19^{1} \mathrm{H}$ and ${ }^{13} \mathrm{C}$ NMR of compound 21

Figure $1.20{ }^{1} \mathrm{H}$ and ${ }^{13} \mathrm{C}$ NMR of compound 22

Figure $1.21{ }^{1} \mathrm{H}$ and ${ }^{13} \mathrm{C}$ NMR of hyrtinadine A 239

Figure $1.22{ }^{1} \mathrm{H}$ and ${ }^{13} \mathrm{C}$ NMR of compound 24

Figure $1.23{ }^{1} \mathrm{H}$ and ${ }^{13} \mathrm{C}$ NMR of compound 25

Figure $1.24{ }^{1} \mathrm{H}$ and ${ }^{13} \mathrm{C}$ NMR of compound 26

Figure $1.25{ }^{1} \mathrm{H}$ and ${ }^{13} \mathrm{C}$ NMR of hyrtinadine B 243 
Figure $2.2{ }^{1} \mathrm{H}$ and ${ }^{13} \mathrm{C}$ NMR of compound 28

Figure $2.3{ }^{1} \mathrm{H}$ and ${ }^{13} \mathrm{C}$ NMR of compound 29

Figure $2.4{ }^{1} \mathrm{H}$ and ${ }^{13} \mathrm{C}$ NMR of compound 30

Figure $2.5{ }^{1} \mathrm{H}$ and ${ }^{13} \mathrm{C}$ NMR of compound 32

Figure $2.6{ }^{1} \mathrm{H}$ and ${ }^{13} \mathrm{C}$ NMR of compound 34 248

Figure $2.7{ }^{1} \mathrm{H}$ and ${ }^{13} \mathrm{C}$ NMR of compound $\mathbf{3 5}$ 249

Figure $2.8{ }^{1} \mathrm{H}$ and ${ }^{13} \mathrm{C}$ NMR of compound 36 250

Figure $2.9{ }^{1} \mathrm{H}$ and ${ }^{13} \mathrm{C}$ NMR of compound 37 251

Figure $2.10{ }^{1} \mathrm{H}$ and ${ }^{13} \mathrm{C}$ NMR of compound 38 252

Figure $2.11{ }^{1} \mathrm{H}$ and ${ }^{13} \mathrm{C}$ NMR of compound 39 253

Figure $2.12{ }^{1} \mathrm{H}$ and ${ }^{13} \mathrm{C}$ NMR of compound 40 254

Figure $2.13{ }^{1} \mathrm{H}$ and ${ }^{13} \mathrm{C}$ NMR of compound 41 255

Figure $2.14{ }^{1} \mathrm{H}$ and ${ }^{13} \mathrm{C}$ NMR of compound $\mathbf{4 2}$ 256

Figure $2.15{ }^{1} \mathrm{H}$ and ${ }^{13} \mathrm{C}$ NMR of compound 43 257

Figure $2.16{ }^{1} \mathrm{H}$ and ${ }^{13} \mathrm{C}$ NMR of compound 48 258

Figure $2.17{ }^{1} \mathrm{H}$ and ${ }^{13} \mathrm{C}$ NMR of compound 49 259

Figure $2.18{ }^{1} \mathrm{H}$ and ${ }^{13} \mathrm{C}$ NMR of compound 50 260

Figure $2.19{ }^{1} \mathrm{H}$ and ${ }^{13} \mathrm{C}$ NMR of compound $\mathbf{5 1}$ 261

Figure $2.20{ }^{1} \mathrm{H}$ and ${ }^{13} \mathrm{C}$ NMR of compound $\mathbf{5 2}$ 262

Figure $2.21{ }^{1} \mathrm{H}$ and ${ }^{13} \mathrm{C}$ NMR of compound 53 263

Figure $2.22{ }^{1} \mathrm{H}$ and ${ }^{13} \mathrm{C}$ NMR of compound $\mathbf{5 4}$ 264

Figure $2.23{ }^{1} \mathrm{H}$ and ${ }^{13} \mathrm{C}$ NMR of compound $\mathbf{5 5}$ 265

Figure $2.24{ }^{1} \mathrm{H}$ and ${ }^{13} \mathrm{C}$ NMR of compound 56 266

Figure $2.25{ }^{1} \mathrm{H}$ and ${ }^{13} \mathrm{C}$ NMR of compound 57 267

Figure $2.26{ }^{1} \mathrm{H}$ and ${ }^{13} \mathrm{C}$ NMR of compound $\mathbf{5 8}$ 268

Figure $2.27{ }^{1} \mathrm{H}$ and ${ }^{13} \mathrm{C}$ NMR of compound 59 269

Figure $2.28{ }^{1} \mathrm{H}$ and ${ }^{13} \mathrm{C}$ NMR of compound $\mathbf{6 0}$ 270

Figure $2.29{ }^{1} \mathrm{H}$ and ${ }^{13} \mathrm{C}$ NMR of compound 62 271

Figure $2.30{ }^{1} \mathrm{H}$ and ${ }^{13} \mathrm{C}$ NMR of compound 63 272 
Figure $2.31{ }^{1} \mathrm{H}$ and ${ }^{13} \mathrm{C}$ NMR of compound 64

Figure $2.32{ }^{1} \mathrm{H}$ and ${ }^{13} \mathrm{C}$ NMR of compound 66

Figure $2.33{ }^{1} \mathrm{H}$ and ${ }^{13} \mathrm{C}$ NMR of compound 67 275

Figure $2.34{ }^{1} \mathrm{H}$ and ${ }^{13} \mathrm{C}$ NMR of compound 68 276

Figure $2.35{ }^{1} \mathrm{H}$ and ${ }^{13} \mathrm{C}$ NMR of compound 69 277

Figure $2.36{ }^{1} \mathrm{H}$ and ${ }^{13} \mathrm{C}$ NMR of compound 71 278

Figure $2.37{ }^{1} \mathrm{H}$ and ${ }^{13} \mathrm{C}$ NMR of compound 72 279

Figure $2.38{ }^{1} \mathrm{H}$ and ${ }^{13} \mathrm{C}$ NMR of compound 73 280

Figure $2.39{ }^{1} \mathrm{H}$ and ${ }^{13} \mathrm{C}$ NMR of compound 74 281

Figure $2.40{ }^{1} \mathrm{H}$ and ${ }^{13} \mathrm{C}$ NMR of compound 75 282

Figure $2.41{ }^{1} \mathrm{H}$ and ${ }^{13} \mathrm{C}$ NMR of compound 76 283

Figure $2.42{ }^{1} \mathrm{H}$ and ${ }^{13} \mathrm{C}$ NMR of compound 77 284

Figure $2.43{ }^{1} \mathrm{H}$ and ${ }^{13} \mathrm{C}$ NMR of compound 78 285

Figure $2.44{ }^{1} \mathrm{H}$ and ${ }^{13} \mathrm{C}$ NMR of compound $\mathbf{8 1}$ 286

Figure $2.45{ }^{1} \mathrm{H}$ and ${ }^{13} \mathrm{C}$ NMR of compound $\mathbf{8 2}$ 287

Figure $2.46{ }^{1} \mathrm{H}$ and ${ }^{13} \mathrm{C}$ NMR of compound $\mathbf{8 8}$ 288

Figure $2.47{ }^{1} \mathrm{H}$ and ${ }^{13} \mathrm{C}$ NMR of compound 89 289

Figure $2.48{ }^{1} \mathrm{H}$ and ${ }^{13} \mathrm{C}$ NMR of compound 90 290

Figure $2.49{ }^{1} \mathrm{H}$ and ${ }^{13} \mathrm{C}$ NMR of compound 91 291

Figure $2.50{ }^{1} \mathrm{H}$ and ${ }^{13} \mathrm{C}$ NMR of compound 92 292

Figure $2.51{ }^{1} \mathrm{H}$ and ${ }^{13} \mathrm{C}$ NMR of compound 93 293

Figure $2.52{ }^{1} \mathrm{H}$ and ${ }^{13} \mathrm{C}$ NMR of compound 94 294

Figure $2.53{ }^{1} \mathrm{H}$ and ${ }^{13} \mathrm{C}$ NMR of compound 95 295

Figure $2.54{ }^{1} \mathrm{H}$ and ${ }^{13} \mathrm{C}$ NMR of compound 96 296

Figure $2.55{ }^{1} \mathrm{H}$ and ${ }^{13} \mathrm{C}$ NMR of compound 97 297

Figure $2.56{ }^{1} \mathrm{H}$ and ${ }^{13} \mathrm{C}$ NMR of compound 99 298

Figure $2.57{ }^{1} \mathrm{H}$ and ${ }^{13} \mathrm{C}$ NMR of compound $\mathbf{1 0 0}$ 299

Figure $2.58{ }^{1} \mathrm{H}$ and ${ }^{13} \mathrm{C}$ NMR of compound $\mathbf{1 0 1}$ 300

Figure $2.59{ }^{1} \mathrm{H}$ and ${ }^{13} \mathrm{C}$ NMR of compound $\mathbf{1 0 2}$ 301 
Figure $3.2{ }^{1} \mathrm{H}$ and ${ }^{13} \mathrm{C}$ NMR of compound 113

Figure $3.3{ }^{1} \mathrm{H}$ and ${ }^{13} \mathrm{C}$ NMR of compound $\mathbf{1 1 4}$

Figure $3.4{ }^{1} \mathrm{H}$ and ${ }^{13} \mathrm{C}$ NMR of compound $\mathbf{1 1 5}$

Figure $3.5{ }^{1} \mathrm{H}$ and ${ }^{13} \mathrm{C}$ NMR of compound $\mathbf{1 1 6}$ 305

Figure $3.6{ }^{1} \mathrm{H}$ and ${ }^{13} \mathrm{C}$ NMR of compound $\mathbf{1 1 1}$ 306

Figure $3.7{ }^{1} \mathrm{H}$ and ${ }^{13} \mathrm{C}$ NMR of compound $\mathbf{1 0 3}$

Figure $3.8{ }^{1} \mathrm{H}$ and ${ }^{13} \mathrm{C}$ NMR of compound $\mathbf{1 0 5}$ 308

Figure $3.9{ }^{1} \mathrm{H}$ and ${ }^{13} \mathrm{C}$ NMR of compound 118 309

Figure $3.10{ }^{1} \mathrm{H}$ and ${ }^{13} \mathrm{C}$ NMR of compound 119 310

Figure $3.11{ }^{1} \mathrm{H}$ and ${ }^{13} \mathrm{C}$ NMR of compound 120 311

Figure $3.12{ }^{1} \mathrm{H}$ and ${ }^{13} \mathrm{C}$ NMR of compound 104 312

Figure $3.13{ }^{1} \mathrm{H}$ and ${ }^{13} \mathrm{C}$ NMR of compound 121 
Figure $4.1{ }^{1} \mathrm{H}$ and ${ }^{13} \mathrm{C}$ NMR of compound $\mathbf{1 2 4}$

Figure $4.2{ }^{1} \mathrm{H}$ and ${ }^{13} \mathrm{C}$ NMR of compound $\mathbf{1 2 5}$

Figure $4.3{ }^{1} \mathrm{H}$ and ${ }^{13} \mathrm{C}$ NMR of compound $\mathbf{1 2 6}$

Figure $4.4{ }^{1} \mathrm{H}$ and ${ }^{13} \mathrm{C}$ NMR of compound $\mathbf{1 2 8}$

Figure $4.5{ }^{1} \mathrm{H}$ and ${ }^{13} \mathrm{C}$ NMR of compound 129

Figure $4.6{ }^{1} \mathrm{H}$ and ${ }^{13} \mathrm{C}$ NMR of compound $\mathbf{1 3 0}$

Figure $4.7{ }^{1} \mathrm{H}$ and ${ }^{13} \mathrm{C}$ NMR of compound 132

Figure $4.8{ }^{1} \mathrm{H}$ and ${ }^{13} \mathrm{C}$ NMR of compound $\mathbf{1 3 3}$

Figure $4.9{ }^{1} \mathrm{H}$ and ${ }^{13} \mathrm{C}$ NMR of compound $\mathbf{1 3 4}$

Figure $4.10{ }^{1} \mathrm{H}$ and ${ }^{13} \mathrm{C}$ NMR of compound $\mathbf{1 3 5}$

Figure $4.11{ }^{1} \mathrm{H}$ and ${ }^{13} \mathrm{C}$ NMR of compound $\mathbf{1 3 6}$

Figure $4.12{ }^{1} \mathrm{H}$ and ${ }^{13} \mathrm{C}$ NMR of compound 137

Figure $4.13{ }^{1} \mathrm{H}$ and ${ }^{13} \mathrm{C}$ NMR of compound 139

Figure $4.14{ }^{1} \mathrm{H}$ and ${ }^{13} \mathrm{C}$ NMR of compound 140

Figure $4.15{ }^{1} \mathrm{H}$ and ${ }^{13} \mathrm{C}$ NMR of compound 141

Figure $4.16{ }^{1} \mathrm{H}$ and ${ }^{13} \mathrm{C}$ NMR of compound $\mathbf{1 4 3}$

Figure $4.17{ }^{1} \mathrm{H}$ and ${ }^{13} \mathrm{C}$ NMR of compound 145

Figure $4.18{ }^{1} \mathrm{H}$ and ${ }^{13} \mathrm{C}$ NMR of compound 146

Figure $4.19{ }^{1} \mathrm{H}$ and ${ }^{13} \mathrm{C}$ NMR of compound 148 332

Figure $4.20{ }^{1} \mathrm{H}$ and ${ }^{13} \mathrm{C}$ NMR of compound 149 333

Figure $4.21{ }^{1} \mathrm{H}$ and ${ }^{13} \mathrm{C}$ NMR of compound $\mathbf{1 5 0}$ 334

Figure $4.22{ }^{1} \mathrm{H}$ and ${ }^{13} \mathrm{C}$ NMR of compound $\mathbf{1 5 1}$ 335

Figure $4.23{ }^{1} \mathrm{H}$ and ${ }^{13} \mathrm{C}$ NMR of compound $\mathbf{1 5 2}$ 336

Figure $4.24{ }^{1} \mathrm{H}$ and ${ }^{13} \mathrm{C}$ NMR of compound $\mathbf{1 5 3}$ 337

Figure $4.25{ }^{1} \mathrm{H}$ and ${ }^{13} \mathrm{C}$ NMR of compound $\mathbf{1 5 4}$ 338

Figure $4.26{ }^{1} \mathrm{H}$ and ${ }^{13} \mathrm{C}$ NMR of compound 155 339

Figure $4.27{ }^{1} \mathrm{H}$ and ${ }^{13} \mathrm{C}$ NMR of compound $\mathbf{1 5 6}$ 340

Figure $4.28{ }^{1} \mathrm{H}$ and ${ }^{13} \mathrm{C}$ NMR of compound $\mathbf{1 5 8}$ 341

Figure $4.29{ }^{1} \mathrm{H}$ and ${ }^{13} \mathrm{C}$ NMR of compound 159 342

Figure $4.30{ }^{1} \mathrm{H}$ and ${ }^{13} \mathrm{C}$ NMR of compound $\mathbf{1 6 0}$ 343

Figure $4.31{ }^{1} \mathrm{H}$ and ${ }^{13} \mathrm{C}$ NMR of compound $\mathbf{1 6 1}$ 344

Figure $4.32{ }^{1} \mathrm{H}$ and ${ }^{13} \mathrm{C}$ NMR of compound 162 345

Figure $4.33{ }^{1} \mathrm{H}$ and ${ }^{13} \mathrm{C}$ NMR of compound $\mathbf{1 6 3}$ 346

Figure $4.34{ }^{1} \mathrm{H}$ and ${ }^{13} \mathrm{C}$ NMR of compound 164 
Figure $4.35{ }^{1} \mathrm{H}$ and ${ }^{13} \mathrm{C}$ NMR of compound 165

Figure $4.36{ }^{1} \mathrm{H}$ and ${ }^{13} \mathrm{C}$ NMR of compound $\mathbf{1 6 6}$

Figure $4.37{ }^{1} \mathrm{H}$ and ${ }^{13} \mathrm{C}$ NMR of compound 167

Figure $4.38{ }^{1} \mathrm{H}$ and ${ }^{13} \mathrm{C}$ NMR of compound 169

Figure $4.39{ }^{1} \mathrm{H}$ and ${ }^{13} \mathrm{C}$ NMR of compound 171

Figure $4.40{ }^{1} \mathrm{H}$ and ${ }^{13} \mathrm{C}$ NMR of compound 172

Figure $4.41{ }^{1} \mathrm{H}$ and ${ }^{13} \mathrm{C}$ NMR of compound $\mathbf{1 7 3}$

Figure $4.42{ }^{1} \mathrm{H}$ and ${ }^{13} \mathrm{C}$ NMR of compound 174

Figure $4.43{ }^{1} \mathrm{H}$ and ${ }^{13} \mathrm{C}$ NMR of compound $\mathbf{1 7 5}$

Figure $4.44{ }^{1} \mathrm{H}$ and ${ }^{13} \mathrm{C}$ NMR of compound 176

Figure $4.45{ }^{1} \mathrm{H}$ and ${ }^{13} \mathrm{C}$ NMR of compound 177

Figure $4.46{ }^{1} \mathrm{H}$ and ${ }^{13} \mathrm{C}$ NMR of compound $\mathbf{1 7 8}$

Figure $4.47{ }^{1} \mathrm{H}$ and ${ }^{13} \mathrm{C}$ NMR of compound 179

Figure $4.48{ }^{1} \mathrm{H}$ and ${ }^{13} \mathrm{C}$ NMR of compound $\mathbf{1 8 0}$

Figure $4.49{ }^{1} \mathrm{H}$ and ${ }^{13} \mathrm{C}$ NMR of compound $\mathbf{1 8 1}$

Figure $4.50{ }^{1} \mathrm{H}$ and ${ }^{13} \mathrm{C}$ NMR of compound $\mathbf{1 8 2}$

Figure $4.51{ }^{1} \mathrm{H}$ and ${ }^{13} \mathrm{C}$ NMR of 4-carbomethoxy- $N$-methyl- $N$-(2-methyl-1-propen-1-yl)-2-

Figure 4.52 ${ }^{1} \mathrm{H}$ and ${ }^{13} \mathrm{C}$ NMR of 2,4-dinitro- $N$-methyl- $N$-(2-methyl-1-propen-1-yl)aniline

Figure $4.53{ }^{1} \mathrm{H}$ and ${ }^{13} \mathrm{C}$ NMR of 5-chloro- $N$-(2-methyl-1-propen-1-yl)-2-nitroaniline 
Figure $6.2{ }^{1} \mathrm{H}$ NMR of compound 204

Figure $6.3{ }^{13} \mathrm{C}$ and ${ }^{19} \mathrm{~F}$ NMR of compound 204

Figure $6.4{ }^{1} \mathrm{H}$ NMR of compound 205

Figure $6.5{ }^{13} \mathrm{C}$ and ${ }^{19} \mathrm{~F}$ NMR of compound 205

Figure $6.6{ }^{1} \mathrm{H}$ and ${ }^{13} \mathrm{C}$ NMR of compound 211

Figure $6.7{ }^{1} \mathrm{H}$ and ${ }^{13} \mathrm{C}$ NMR of compound 215

Figure $6.8{ }^{1} \mathrm{H}$ NMR of compound 216

Figure $6.9{ }^{13} \mathrm{C}$ and ${ }^{19} \mathrm{~F}$ NMR of compound 216

Figure $6.10{ }^{1} \mathrm{H}$ NMR of compound 217386

Figure $6.11{ }^{13} \mathrm{C}$ and ${ }^{19} \mathrm{~F}$ NMR of compound $\mathbf{2 1 7}$

Figure $6.12{ }^{1} \mathrm{H}$ and ${ }^{13} \mathrm{C}$ NMR of compound 219

Figure $6.13{ }^{1} \mathrm{H}$ and ${ }^{13} \mathrm{C}$ NMR of compound 222 389

Figure $6.14{ }^{1} \mathrm{H}$ and ${ }^{13} \mathrm{C}$ NMR of compound 224

Figure $6.15{ }^{1} \mathrm{H}$ and ${ }^{13} \mathrm{C}$ NMR of compound 225

Figure $6.16{ }^{1} \mathrm{H}$ and ${ }^{13} \mathrm{C}$ NMR of compound 226

Figure $6.17{ }^{1} \mathrm{H}$ and ${ }^{13} \mathrm{C}$ NMR of compound 227

Figure $6.18{ }^{1} \mathrm{H}$ NMR of a mixture of compounds $\mathbf{2 2 6}$ and $\mathbf{2 2 8} 394$

Figure $6.19^{1} \mathrm{H}$ and ${ }^{13} \mathrm{C}$ NMR of compound 229

Figure $6.20{ }^{1} \mathrm{H}$ and ${ }^{13} \mathrm{C}$ NMR of compound 232

Figure 6.21 ${ }^{1} \mathrm{H}$ and ${ }^{13} \mathrm{C}$ NMR of compound $\mathbf{2 3 3}$

Figure $6.22{ }^{1} \mathrm{H}$ and ${ }^{13} \mathrm{C}$ NMR of compound 234

Figure $6.23{ }^{1} \mathrm{H}$ and ${ }^{13} \mathrm{C}$ NMR of compound 235 
Figure $6.24{ }^{1} \mathrm{H}$ and ${ }^{13} \mathrm{C}$ NMR of compound 237

Figure $6.25{ }^{1} \mathrm{H}$ and ${ }^{13} \mathrm{C}$ NMR of compound 238

401

Figure $6.26{ }^{1} \mathrm{H}$ and ${ }^{13} \mathrm{C}$ NMR of compound 239

402

Figure $6.27{ }^{1} \mathrm{H}$ and ${ }^{13} \mathrm{C}$ NMR of compound 240

Figure $6.28{ }^{1} \mathrm{H}$ and ${ }^{13} \mathrm{C}$ NMR of compound 241

404

Figure $6.29{ }^{1} \mathrm{H}$ and ${ }^{13} \mathrm{C}$ NMR of compound 242

405

Figure $6.30{ }^{1} \mathrm{H}$ and ${ }^{13} \mathrm{C}$ NMR of compound 243

406

Figure $6.31{ }^{1} \mathrm{H}$ and ${ }^{13} \mathrm{C}$ NMR of compound 245

407

Figure $6.32{ }^{1} \mathrm{H}$ and ${ }^{13} \mathrm{C}$ NMR of compound 246

408

Figure $6.33{ }^{1} \mathrm{H}$ and ${ }^{13} \mathrm{C}$ NMR of compound 249

409

Figure $6.34{ }^{1} \mathrm{H}$ and ${ }^{13} \mathrm{C}$ NMR of compound 253

410

Figure $6.35{ }^{1} \mathrm{H}$ and ${ }^{13} \mathrm{C}$ NMR of compound 254

411

Figure $6.36{ }^{1} \mathrm{H}$ and ${ }^{13} \mathrm{C}$ NMR of compound $\mathbf{2 5 5}$ 
Figure $7.2{ }^{1} \mathrm{H}$ and ${ }^{13} \mathrm{C}$ NMR of compound 257

Figure $7.3{ }^{1} \mathrm{H}$ of compound 258

Figure $7.4{ }^{13} \mathrm{C}$ and ${ }^{19} \mathrm{~F}$ NMR of compound 258

Figure $7.5{ }^{1} \mathrm{H}$ and ${ }^{13} \mathrm{C}$ NMR of compound $\mathbf{2 6 1}$

Figure $7.6{ }^{1} \mathrm{H}$ and ${ }^{13} \mathrm{C}$ NMR of compound $\mathbf{2 6 2}$

Figure $7.7{ }^{1} \mathrm{H}$ NMR of compound 264

Figure $7.8{ }^{13} \mathrm{C}$ and ${ }^{19} \mathrm{~F}$ NMR of compound 264

Figure $7.9{ }^{1} \mathrm{H}$ NMR of compound 265

Figure $7.10{ }^{13} \mathrm{C}$ and ${ }^{19} \mathrm{~F}$ NMR of compound 265

Figure $7.11{ }^{1} \mathrm{H}$ NMR of compound 266

Figure $7.12{ }^{13} \mathrm{C}$ and ${ }^{19} \mathrm{~F}$ NMR of compound 266

Figure $7.13{ }^{1} \mathrm{H}$ NMR of a mixture of $\mathbf{2 7 0}$ and 271

Figure $7.14{ }^{1} \mathrm{H}$ NMR of compound 273

Figure $7.15{ }^{13} \mathrm{C}$ and ${ }^{19} \mathrm{~F}$ NMR of compound 273

Figure $7.16{ }^{1} \mathrm{H}$ and ${ }^{13} \mathrm{C}$ NMR of a mixture of 274 and 275

Figure $7.17{ }^{1} \mathrm{H}$ NMR of compound 276

Figure $7.18{ }^{13} \mathrm{C}$ and ${ }^{19} \mathrm{~F}$ NMR of compound 276

Figure $7.19{ }^{1} \mathrm{H}$ NMR of compound 277

Figure $7.20{ }^{13} \mathrm{C}$ and ${ }^{19} \mathrm{~F}$ NMR of compound 277

Figure $7.21{ }^{1} \mathrm{H}$ and ${ }^{13} \mathrm{C}$ NMR of compound 279

Figure $7.22{ }^{1} \mathrm{H}$ and ${ }^{13} \mathrm{C}$ NMR of compound $\mathbf{2 8 0}$

Figure $7.23{ }^{1} \mathrm{H}$ NMR of compound $\mathbf{2 8 1}$

Figure $7.24{ }^{13} \mathrm{C}$ and ${ }^{19} \mathrm{~F}$ NMR of compound 281

Figure $7.25{ }^{1} \mathrm{H}$ and ${ }^{13} \mathrm{C}$ NMR of compound 285

Figure $7.26{ }^{1} \mathrm{H}$ and ${ }^{13} \mathrm{C}$ NMR of compound 286

Figure $7.27{ }^{1} \mathrm{H}$ and ${ }^{13} \mathrm{C}$ NMR of compound 287

Figure $7.28{ }^{1} \mathrm{H}$ and ${ }^{13} \mathrm{C}$ NMR of compound 289

Figure $7.29{ }^{1} \mathrm{H}$ and ${ }^{13} \mathrm{C}$ NMR of compound 290

Figure $7.30{ }^{1} \mathrm{H}$ and ${ }^{13} \mathrm{C}$ NMR of compound 291

Figure $7.31{ }^{1} \mathrm{H}$ and ${ }^{13} \mathrm{C}$ NMR of compound 292

Figure $7.32{ }^{1} \mathrm{H}$ and ${ }^{13} \mathrm{C}$ NMR of compound 293

Figure $7.33{ }^{1} \mathrm{H}$ and ${ }^{13} \mathrm{C}$ NMR of compound 294

Figure $7.34{ }^{1} \mathrm{H}$ and ${ }^{13} \mathrm{C}$ NMR of compound 295

Figure $7.35{ }^{1} \mathrm{H}$ and ${ }^{13} \mathrm{C}$ NMR of compound 296 
Figure $7.36{ }^{1} \mathrm{H}$ and ${ }^{13} \mathrm{C}$ NMR of compound 297

Figure $7.37{ }^{1} \mathrm{H}$ and ${ }^{13} \mathrm{C}$ NMR of compound 298

Figure $7.38{ }^{1} \mathrm{H}$ NMR of compound $\mathbf{3 0 0}$

Figure $7.39{ }^{13} \mathrm{C}$ and ${ }^{19} \mathrm{~F}$ NMR of compound $\mathbf{3 0 0}$

Figure $7.40{ }^{1} \mathrm{H}$ NMR of compound $\mathbf{3 0 1}$

Figure $7.41{ }^{13} \mathrm{C}$ and ${ }^{19} \mathrm{~F}$ NMR of compound $\mathbf{3 0 0}$

Figure $7.42{ }^{1} \mathrm{H}$ and ${ }^{13} \mathrm{C}$ NMR of compound $\mathbf{3 0 2}$

Figure $7.43{ }^{1} \mathrm{H}$ NMR of compound $\mathbf{3 0 3}$

Figure $7.44{ }^{13} \mathrm{C}$ and ${ }^{19} \mathrm{~F}$ NMR of compound $\mathbf{3 0 3}$

Figure $7.45{ }^{1} \mathrm{H}$ NMR of compound $\mathbf{3 0 5}$

Figure $7.46{ }^{13} \mathrm{C}$ and ${ }^{19} \mathrm{~F}$ NMR of compound $\mathbf{3 0 5}$

Figure $7.47{ }^{1} \mathrm{H}$ NMR of compound 306

Figure $7.48{ }^{13} \mathrm{C}$ and ${ }^{19} \mathrm{~F}$ NMR of compound 306

Figure $7.49{ }^{1} \mathrm{H}$ NMR of compound $\mathbf{3 0 7}$

Figure $7.50{ }^{13} \mathrm{C}$ and ${ }^{19} \mathrm{~F}$ NMR of compound $\mathbf{3 0 7}$

Figure $7.51{ }^{1} \mathrm{HNMR}$ of compound $\mathbf{3 0 8}$

Figure $7.52{ }^{13} \mathrm{C}$ and ${ }^{19} \mathrm{~F}$ NMR of compound $\mathbf{3 0 8}$

Figure $7.53{ }^{1} \mathrm{H}$ NMR of compound 309

Figure $7.54{ }^{13} \mathrm{C}$ and ${ }^{19} \mathrm{~F}$ NMR of compound 309

Figure $7.55{ }^{1} \mathrm{H}$ NMR of compound $\mathbf{3 1 0}$

Figure $7.56{ }^{13} \mathrm{C}$ and ${ }^{19} \mathrm{~F}$ NMR of compound $\mathbf{3 1 0}$

Figure $7.57{ }^{1} \mathrm{H}$ NMR of compound $\mathbf{3 1 1}$

Figure $7.58{ }^{13} \mathrm{C}$ and ${ }^{19} \mathrm{~F}$ NMR of compound $\mathbf{3 1 1}$

Figure $7.59{ }^{1} \mathrm{H}$ NMR of compound $\mathbf{3 1 2}$

Figure $7.60{ }^{13} \mathrm{C}$ and ${ }^{19} \mathrm{~F}$ NMR of compound $\mathbf{3 1 2}$

Figure $7.61{ }^{1} \mathrm{H}$ NMR of compound $\mathbf{3 1 3}$

Figure $7.62{ }^{13} \mathrm{C}$ and ${ }^{19} \mathrm{~F}$ NMR of compound $\mathbf{3 1 3}$

Figure $7.63{ }^{1} \mathrm{H}$ and ${ }^{13} \mathrm{C}$ NMR of compound 314

Figure $7.64{ }^{1} \mathrm{H}$ and ${ }^{13} \mathrm{C}$ NMR of compound 315

Figure $7.65{ }^{1} \mathrm{H}$ and ${ }^{13} \mathrm{C}$ NMR of compound $\mathbf{3 1 6}$

Figure $7.66{ }^{1} \mathrm{H}$ and ${ }^{13} \mathrm{C}$ NMR of compound $\mathbf{3 1 7}$

Figure $7.67{ }^{1} \mathrm{H}$ and ${ }^{13} \mathrm{C}$ NMR of compound $\mathbf{3 1 8}$

Figure $7.68{ }^{1} \mathrm{H}$ and ${ }^{13} \mathrm{C}$ NMR of compound 319

Figure $7.69{ }^{1} \mathrm{H}$ and ${ }^{13} \mathrm{C}$ NMR of compound $\mathbf{3 2 0}$ 
Figure $7.70{ }^{1} \mathrm{H}$ and ${ }^{13} \mathrm{C}$ NMR of compound 321

Figure $7.71{ }^{1} \mathrm{H}$ and ${ }^{13} \mathrm{C}$ NMR of compound 322

Figure $7.72{ }^{1} \mathrm{H}$ and ${ }^{13} \mathrm{C}$ NMR of compound $\mathbf{3 2 3}$

Figure $7.73{ }^{1} \mathrm{H}$ and ${ }^{13} \mathrm{C}$ NMR of compound 324

Figure $7.74{ }^{1} \mathrm{H}$ and ${ }^{13} \mathrm{C}$ NMR of compound $\mathbf{3 2 5}$

Figure $7.75{ }^{1} \mathrm{H}$ and ${ }^{13} \mathrm{C}$ NMR of compound 326

Figure $7.76{ }^{1} \mathrm{H}$ and ${ }^{13} \mathrm{C}$ NMR of compound $\mathbf{3 2 7}$

Figure $7.77{ }^{1} \mathrm{H}$ and ${ }^{13} \mathrm{C}$ NMR of compound 328

Figure $7.78{ }^{1} \mathrm{H}$ and ${ }^{13} \mathrm{C}$ NMR of compound 329

Figure $7.79{ }^{1} \mathrm{H}$ and ${ }^{13} \mathrm{C}$ NMR of compound 330

Figure $7.80{ }^{1} \mathrm{H}$ and ${ }^{13} \mathrm{C}$ NMR of compound $\mathbf{3 3 1}$

Figure $7.81{ }^{1} \mathrm{H}$ and ${ }^{13} \mathrm{C}$ NMR of compound 336

Figure $7.82{ }^{1} \mathrm{H}$ and ${ }^{13} \mathrm{C}$ NMR of compound 337

Figure $7.83{ }^{1} \mathrm{H}$ and ${ }^{13} \mathrm{C}$ NMR of compound 338

Figure $7.84{ }^{1} \mathrm{H}$ and ${ }^{13} \mathrm{C}$ NMR of compound 339

Figure $7.85{ }^{1} \mathrm{H}$ and ${ }^{13} \mathrm{C}$ NMR of compound $\mathbf{3 4 0}$

Figure $7.86{ }^{1} \mathrm{H}$ and ${ }^{13} \mathrm{C}$ NMR of compound $\mathbf{3 4 1}$

Figure $7.87{ }^{1} \mathrm{H}$ and ${ }^{13} \mathrm{C}$ NMR of compound $\mathbf{3 4 2}$

Figure $7.88{ }^{1} \mathrm{H}$ and ${ }^{13} \mathrm{C}$ NMR of compound $\mathbf{3 4 3}$

Figure $7.89{ }^{1} \mathrm{H}$ and ${ }^{13} \mathrm{C}$ NMR of compound $\mathbf{3 4 4}$

Figure $7.90{ }^{1} \mathrm{H}$ and ${ }^{13} \mathrm{C}$ NMR of compound $\mathbf{3 4 5}$

Figure $7.91{ }^{1} \mathrm{H}$ and ${ }^{13} \mathrm{C}$ NMR of compound 346

Figure $7.92{ }^{1} \mathrm{H}$ and ${ }^{13} \mathrm{C}$ NMR of compound 347

Figure $7.93{ }^{1} \mathrm{H}$ and ${ }^{13} \mathrm{C}$ NMR of compound $\mathbf{3 4 8}$

Figure $7.94{ }^{1} \mathrm{H}$ and ${ }^{13} \mathrm{C}$ NMR of compound 349

Figure $7.95{ }^{1} \mathrm{H}$ and ${ }^{13} \mathrm{C}$ NMR of compound $\mathbf{3 5 0}$

Figure $7.96{ }^{1} \mathrm{H}$ and ${ }^{13} \mathrm{C}$ NMR of compound $\mathbf{3 5 1}$

Figure $7.97{ }^{1} \mathrm{H}$ and ${ }^{13} \mathrm{C}$ NMR of compound $\mathbf{3 5 2}$ 508

Figure $7.98{ }^{1} \mathrm{H}$ and ${ }^{13} \mathrm{C}$ NMR of compound $\mathbf{3 5 3}$

Figure $7.99{ }^{1} \mathrm{H}$ and ${ }^{13} \mathrm{C}$ NMR

Figure $7.100{ }^{1} \mathrm{H}$ and ${ }^{13} \mathrm{C}$ NMR of compound 354

Figure $7.101{ }^{1} \mathrm{H}$ and ${ }^{13} \mathrm{C}$ NMR of compound 355

Figure $7.102{ }^{1} \mathrm{H}$ and ${ }^{13} \mathrm{C}$ NMR of compound 356

Figure $7.103{ }^{1} \mathrm{H}$ and ${ }^{13} \mathrm{C}$ NMR of compound $\mathbf{3 6 3}$ 
Figure $7.104{ }^{1} \mathrm{H}$ and ${ }^{13} \mathrm{C}$ NMR of compound 364

Figure $7.105{ }^{1} \mathrm{H}$ and ${ }^{13} \mathrm{C}$ NMR of compound 365

Figure $7.106{ }^{1} \mathrm{H}$ and ${ }^{13} \mathrm{C}$ NMR of compound 366

Figure $7.107{ }^{1} \mathrm{H}$ and ${ }^{13} \mathrm{C}$ NMR of compound 368

Figure $7.108{ }^{1} \mathrm{H}$ and ${ }^{13} \mathrm{C}$ NMR of compound $\mathbf{3 6 9}$ 519

Figure $7.109{ }^{1} \mathrm{H}$ and ${ }^{13} \mathrm{C}$ NMR of compound 370 520

Figure $7.110{ }^{1} \mathrm{H}$ and ${ }^{13} \mathrm{C}$ NMR of compound 371 521

Figure $7.111{ }^{1} \mathrm{H}$ and ${ }^{13} \mathrm{C}$ NMR of compound 372 522

Figure $7.112{ }^{1} \mathrm{H}$ and ${ }^{13} \mathrm{C}$ NMR of compound 373 523

Figure $7.113{ }^{1} \mathrm{H}$ and ${ }^{13} \mathrm{C}$ NMR of compound 374 524

Figure $7.114{ }^{1} \mathrm{H}$ and ${ }^{13} \mathrm{C}$ NMR of compound 375 525

Figure $7.115{ }^{1} \mathrm{H}$ and ${ }^{13} \mathrm{C}$ NMR of compound 376 526

Figure $7.116{ }^{1} \mathrm{H}$ and ${ }^{13} \mathrm{C}$ NMR of compound 377 527

Figure $7.117{ }^{1} \mathrm{H}$ and ${ }^{13} \mathrm{C}$ NMR of compound 378 528

Figure $7.118{ }^{1} \mathrm{H}$ and ${ }^{13} \mathrm{C}$ NMR of compound 379 529

Figure $7.119{ }^{1} \mathrm{H}$ and ${ }^{13} \mathrm{C}$ NMR of compound 380 530

Figure $7.120{ }^{1} \mathrm{H}$ and ${ }^{13} \mathrm{C}$ NMR of compound 381 531

Figure $7.121{ }^{1} \mathrm{H}$ and ${ }^{13} \mathrm{C}$ NMR of compound $\mathbf{3 8 2}$ 532

Figure $7.122{ }^{1} \mathrm{H}$ and ${ }^{13} \mathrm{C}$ NMR of compound $\mathbf{3 8 3}$ 533

Figure $7.123{ }^{1} \mathrm{H}$ and ${ }^{13} \mathrm{C}$ NMR of compound 384 534

Figure $7.124{ }^{1} \mathrm{H}$ and ${ }^{13} \mathrm{C}$ NMR of compound 385 535

Figure $7.125{ }^{1} \mathrm{H}$ and ${ }^{13} \mathrm{C}$ NMR of compound 386 


\section{Chapter 1 Short syntheses of the indole alkaloids alocasin A, scalaridine A and hyrtinadine}

\section{A-B}

\section{A Introduction}

A number of bisindole alkaloids have been isolated from marine sponges and tunicates. Specific examples include hemacanthins, ${ }^{1}$ dragmacidins, ${ }^{2}$ topsentins (spongetins), ${ }^{1 \mathrm{c}-\mathrm{e}, 3}$ nortopsentins ${ }^{4}$ and rhopaladins. $^{5}$ These metabolites possess diverse bioactivities such as cytotoxic, ${ }^{1 \mathrm{f}, \mathrm{g}, 2 \mathrm{~d}, 3 \mathrm{a}, 4}{ }^{\text {antiviral, }}{ }^{1 \mathrm{~g}, 2 \mathrm{~b}, 4}$ antifungal, ${ }^{7}$ antibacterial $^{7}$ and antiplasmodial ${ }^{8}$ activities. The common feature of these alkaloids is the presence of a pyrazinone/imidazole or a reduced derivative linker either attached directly to the 3 positions of the two indoles or attached via a carbonyl carbon and/or methine. Hydroxylation in the 6 or 7 position can also be found in some of the metabolites.

Alocasin A was recently isolated from the dried rhizomes of the herbaceous plant Alocasia macrorrhiza, collected in the People Republic of China (Fig. 1.1). ${ }^{9}$ This is the only heterocycle-linked bisindole isolated from a terrestrial source. The presence of hydroxyl groups in the two 5-positions is also unusual for this type of bisindoles, but can be found in hyrtinadine A isolated from the marine sponge Hyrtios $s p .^{10}$ and scalaridine $\mathrm{A}$ and hyrtinadine $\mathrm{B}$ isolated from the marine sponge Scalarispongia $s p .{ }^{11}$ Hyrtinadine A and scalaridine A are the only examples of pyrimidine and pyridine linked bisindole alkaloids respectively. The structures of these natural products are depicted in Figure 1.1.

Figure 1.1 structures of alocasin A, scalaridine A, and hyrtinadine A-B

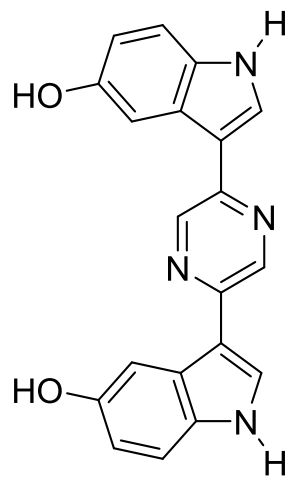

Alocasin A

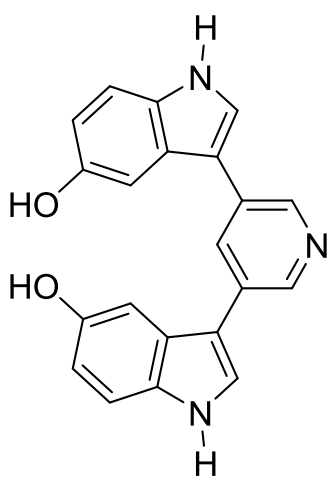

Scalaridine A<smiles>Oc1ccc2[nH]cc(-c3cnc(-c4c[nH]c5ccc(O)cc45)nc3)c2c1</smiles>

Hyrtinadine A<smiles>Oc1ccc2[nH]cc(-c3cncnc3)c2c1</smiles>

Hyrtinadine B

The structure elucidation of alocasin A, scalaridine A and hyrtinadine A-B was achieved using spectroscopic techniques. 
Total syntheses of alocasin A, scalaridine A and hyrtinadine A using cross-coupling strategy have recently been reported. In the syntheses of the pyrazine, pyridine and pyrimidine linked bisindoles, a one pot double Suzuki-Miyaura cross coupling of 1-t-butoxycarbonyl-5-methoxy-3-boryl indole with 2,5dibromopyrazine (alocasin A, Scheme 1.1), ${ }^{12}$ 3,5-dibromopyridine (scalaridine A, Scheme 1.2) ${ }^{13}$ and 2iodo-5-bromopyrimidine (hyrtinadine A, Scheme 1.3) ${ }^{14}$ were used as the key step. In another synthesis of hyrtinadine A, a palladium catalyzed double cross coupling of a triindolylindium reagent and 2-chloro-4bromopyrimidine was used (Scheme 1.4). ${ }^{15}$ Synthesis of hyrtinadine B has not been reported to date.

Scheme 1.1 Sperry's synthesis of alocasin A<smiles>COc1ccc2c(c1)c(Cc1ccccc1)cn2C(=O)OC(C)(C)OC(=O)c1cn(C(=O)OC(C)(C)C)c2ccc(OC)cc12</smiles>

Scheme 1.2 Sperry's synthesis of scalaridine A

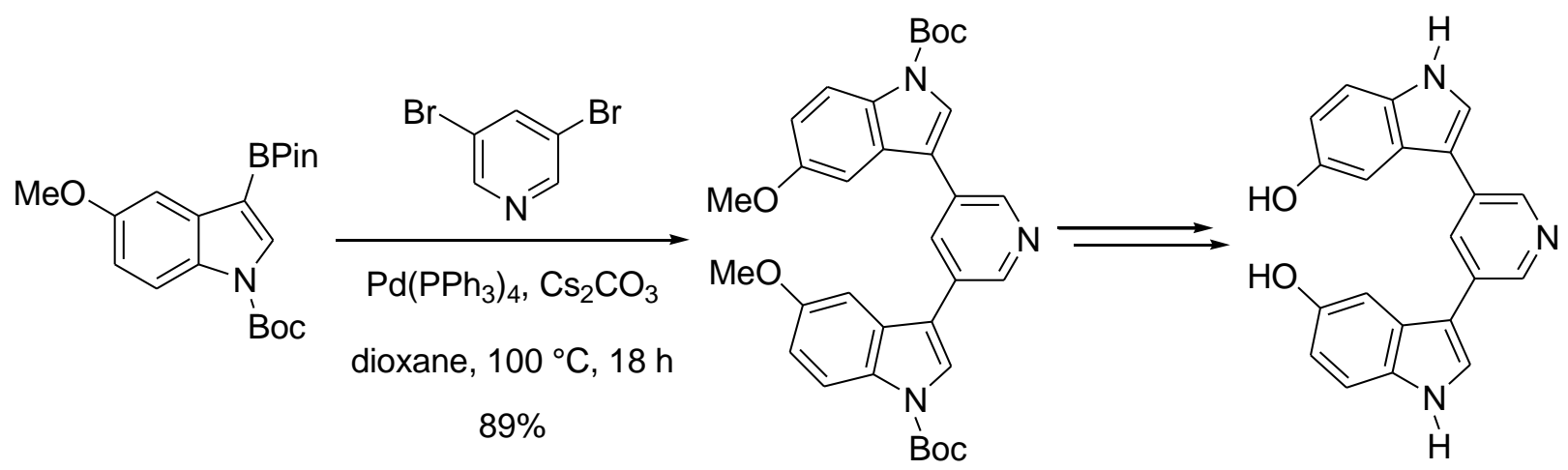


Scheme 1.3 Tasch's synthesis of hyrtinadine A

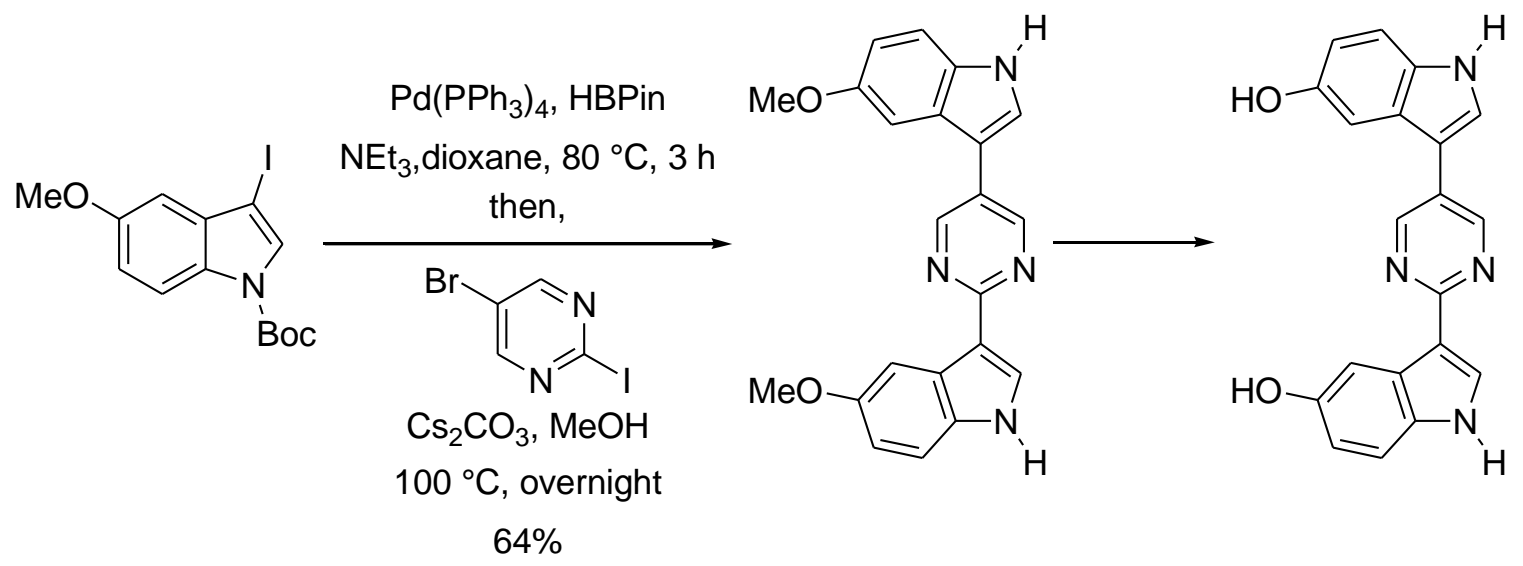

Scheme 1.4 Mosquera's synthesis of scalaridine A
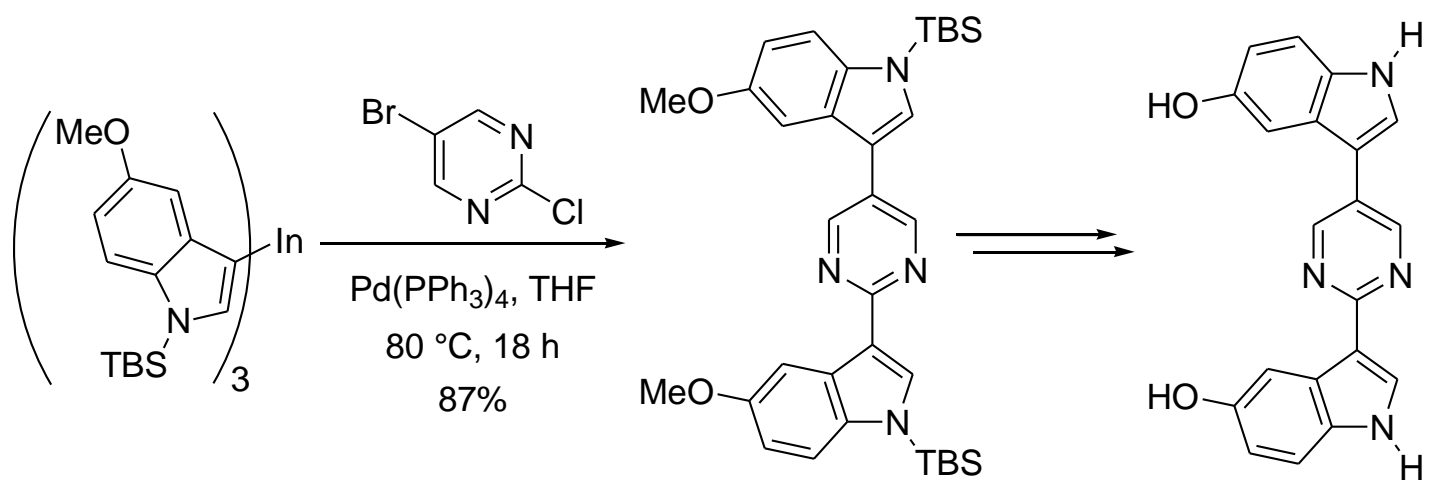

\section{B Results and Discussion}

The objective of this work was to develop short and efficient synthetic routes to all the four natural products namely alocasin A, scalaridine A, hyrtinadine A and hyrtinadine B using a late stage palladium catalyzed reductive $N$-heterocyclization as the key indole forming step. This methodology has been emerging as a powerful tool for the synthesis of a wide variety of substituted indoles. ${ }^{16}$ A number of naturally occurring indole alkaloids have been synthesized including tjipanazoles, ${ }^{17}$ murrayaquinone, ${ }^{18}$ bauerine $\mathrm{A},{ }^{19}$ carbazole alkaloids, ${ }^{20}$ mushroom metabolites, ${ }^{21}$ and the tetracyclic alkaloid ht-13-B ${ }^{22}$ using this reaction as the key indole forming step. The originally proposed plan was to use vinylstannane $\mathbf{4}$ as the common intermediate in the Kosugi-Migita-Stille cross coupling to assemble the reductive cyclization 
precursors. Although the plan worked well for alocasin A, it proved problematic for the remaining three natural products calling for a change in the synthetic strategy.

Alocasin A was synthesized in 6 steps from 4-benzyloxy-2-bromo-1-nitrobenzene (1) using a palladium catalyzed reaction in five of the steps (Scheme 1.5). The synthesis commenced with a Sonogashira coupling of 1 with trimethylsilylethyne followed by a tetrabutylammonium fluoride (TBAF) mediated deprotection affording products $\mathbf{2}$ and $\mathbf{3}$ respectively, in high isolated yields. Use of highly $\alpha$ regioselective palladium catalyzed hydrostannylation of ortho-substituted arylakynes reported by Alami et $\mathrm{al}^{23}$ gave rise to the expected vinylstannane $\mathbf{4}$ in excellent yield. ${ }^{24}$ Palladium catalyzed reactions of halopyrazines ${ }^{25}$ including Sonogashira, ${ }^{26}$ Suzuki-Miyaura, ${ }^{27}$ Negishi, ${ }^{28}$ and Kosugi-Migita-Stille ${ }^{29}$ cross couplings have been reported. A double Suzuki-Miyaura cross coupling of 2,5-dibromopyrazine (5) with 3-borylated indoles was used to prepare 2,5-bisindolopyrazines. ${ }^{14,30}$ A sequential Suzuki-Miyaura and Kosugi-Migita-Stille cross coupling of 2,5-dibromo-3-methoxypyrazine was used in the total synthesis of bisindolopyrazine alkaloid dragmacidin $\mathrm{D} .{ }^{31}$ In the present synthesis of alocasin A, the central pyrazine nucleus was installed by a one-pot double Kosugi-Migita-Stille cross coupling of vinylstannane $\mathbf{4}$ with $\mathbf{5}$ in a relatively low isolated yield along with the dimer 7 in $40 \%$ yield resulting from the dimerization of 4 . The two products were readily separated by chromatography on a silica gel. Cross coupling of related styryltin derivatives with aryl bromides and iodides have been reported. ${ }^{32}$

A double palladium catalyzed reductive $N$-heterocyclization of $\mathbf{6}$ using a bis(dibenzylideneactone)palladium-1,3-bis(diphenylphosphino)propane-1,10-phenanthroline catalyst system in the presence of carbon monoxide $\left(\mathrm{pCO}=6 \mathrm{~atm}, 120^{\circ} \mathrm{C}\right)$ in $N, N$-dimethylformamide, furnished protected dibenzyloxyalocasin A 8 in excellent isolated yield. The benzyl groups were smoothly reomoved by hydrogenolysis using $\mathrm{Pd} / \mathrm{C}-\mathrm{H}_{2}\left(10 \% \mathrm{Pd}, \mathrm{pH}_{2}=4 \mathrm{~atm}\right)$ in ethanol at $60{ }^{\circ} \mathrm{C}$ affording the naturally occurring bisindolopyrazine alocasin A. Thus the synthesis of this natural product was completed in six steps in $21 \%$ overall yield starting from 1 . The NMR, IR, HRMS, and melting point were compared with the literature reported data and all corroborated the originally proposed structure. It should be noted that all transformations from 6 to alocasin A involved reactions at two different centers making a total of nine connections/disconnections with an $84 \%$ average yield per step (Scheme 1.5). 
Scheme 1.5 Synthesis of alocasin A
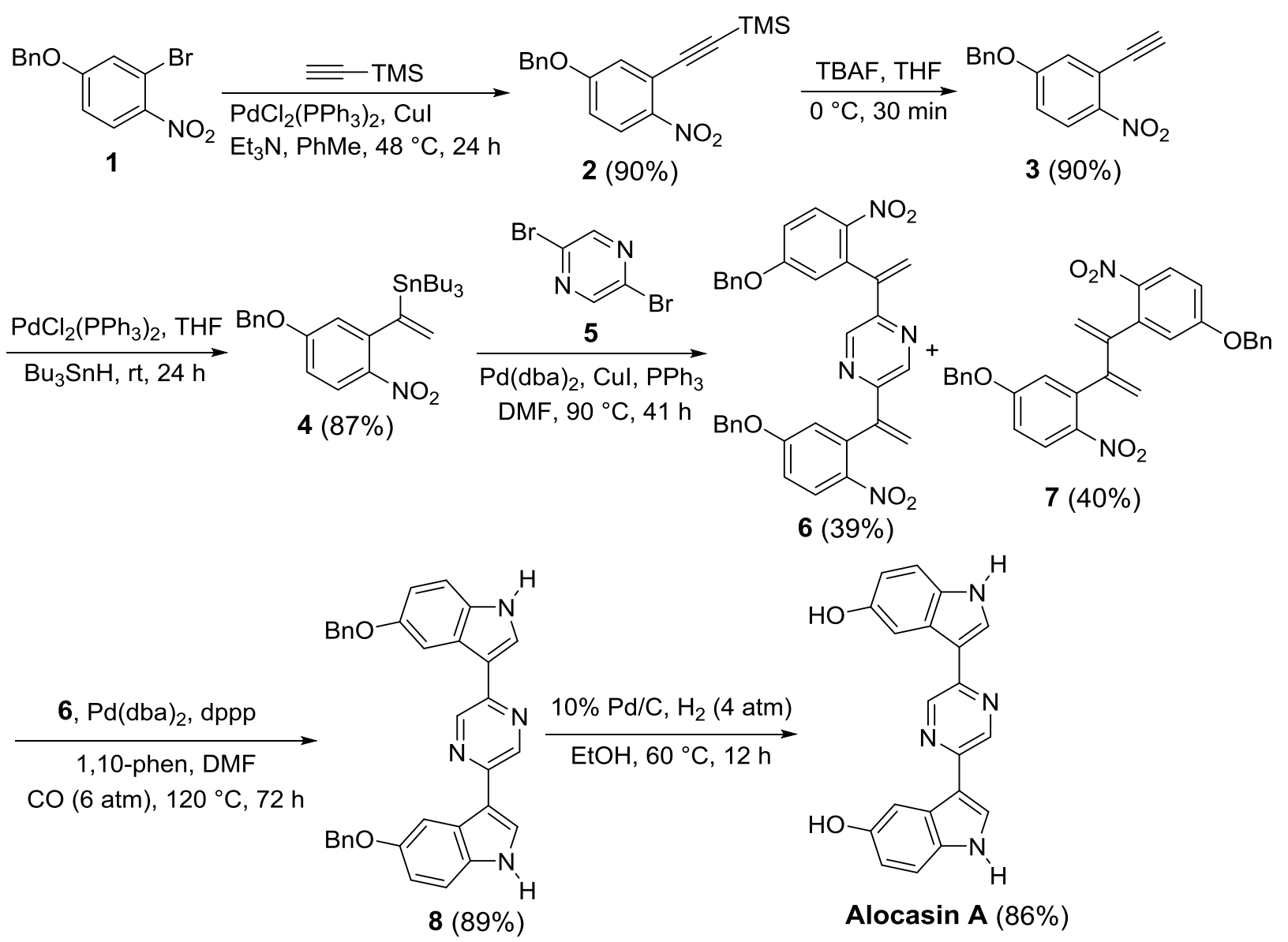

Alocasin A (86\%)

Excited by the successful synthesis of alocasin A, a similar sequence of Kosugi-Migita-Stille cross coupling of 3,5-dibromopyridine (9) was envisioned for the synthesis of scalaridine A. While 9 has been utilized in cross coupling reactions with aryltin reagents, ${ }^{33}$ the use of vinyl tin reagents has not been reported. Disappointingly, all attempted cross coupling reactions of 4 with 9 using a number of palladium-based catalysts failed to give the desired coupling product. The only product obtained from these reactions was the homocoupling product 7 derived from the dimerization of $\mathbf{4}$. Low yields in addition to a preference for cine-substitution ${ }^{34}$ have been observed in palladium catalyzed cross couplings using 1-trimethylstannyl-1-phenylethene and 2- and 3-bromopyridines (Scheme 1.6). ${ }^{35}$ 
Scheme 1.6 Attempted cross coupling of 4 with 3,5-dibromopyridine (9)

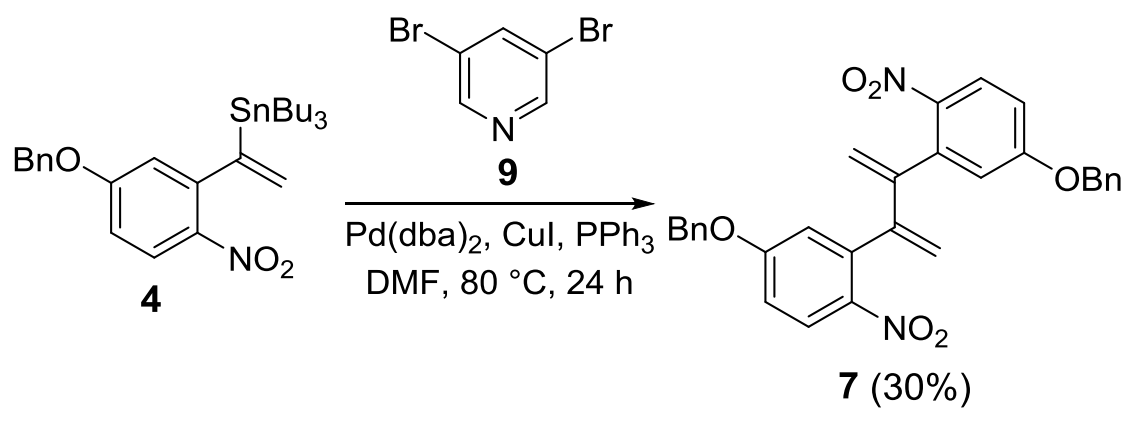

Thus, a slightly different strategy was pursued for the synthesis of scalaridine A where the polarity of the coupling partners was reversed, that is, a Kosugi-Migita-Stille coupling of heteroaryl vinylstannane with an aryl halide. Hydrostannation of ethyne-substituted heterocycles are relatively rare in the literature. A single example of a copper catalyzed, $\alpha$-regioselective hydrostannation, of 2-ethynylpyridine using hexamethylditin was reported by Yoshida et al. ${ }^{32}$ A palladium catalyzed hydrostannation of an $N$ protected 3-ethynylindole also gave exclusively $\alpha$-stannylated product. ${ }^{36}$ In contrast, palladium catalyzed hydrostannation of 2-ethynylpyridine with an ionic liquid supported stannane was shown to be relatively unselective having an $\alpha / \beta$-ratio of 38:62. ${ }^{37}$

In our case, palladium catalyzed hydrostannylation of 3,5-diethynylpyridine (10) gratifyingly furnished 11 with exclusive $\alpha$-selectivity. Subsequent Kosugi-Migita-Stille coupling of 11 with 4-benzyloxy-2iodo-1-nitrobenzene (12) gave the expected product 13. Reductive cyclization followed by debenzylation afforded scalaridine A. Synthetic scalaridine A was spectroscopically and thermally identical to sample obtained from natural sources $\left({ }^{1} \mathrm{H}\right.$ NMR, ${ }^{13} \mathrm{C}$ NMR, IR, HRMS, mp). Starting from 10, the overall yield was $13 \%$ in four synthetic transformations (Scheme 1.7). 
Scheme 1.7 Synthesis of scalaridine A

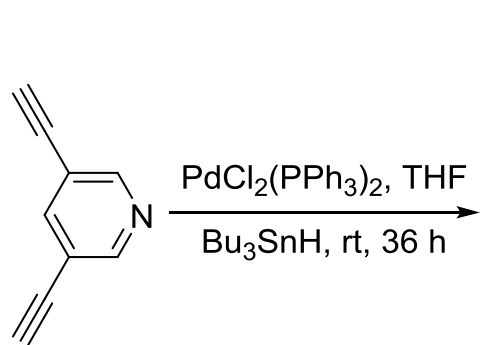

10
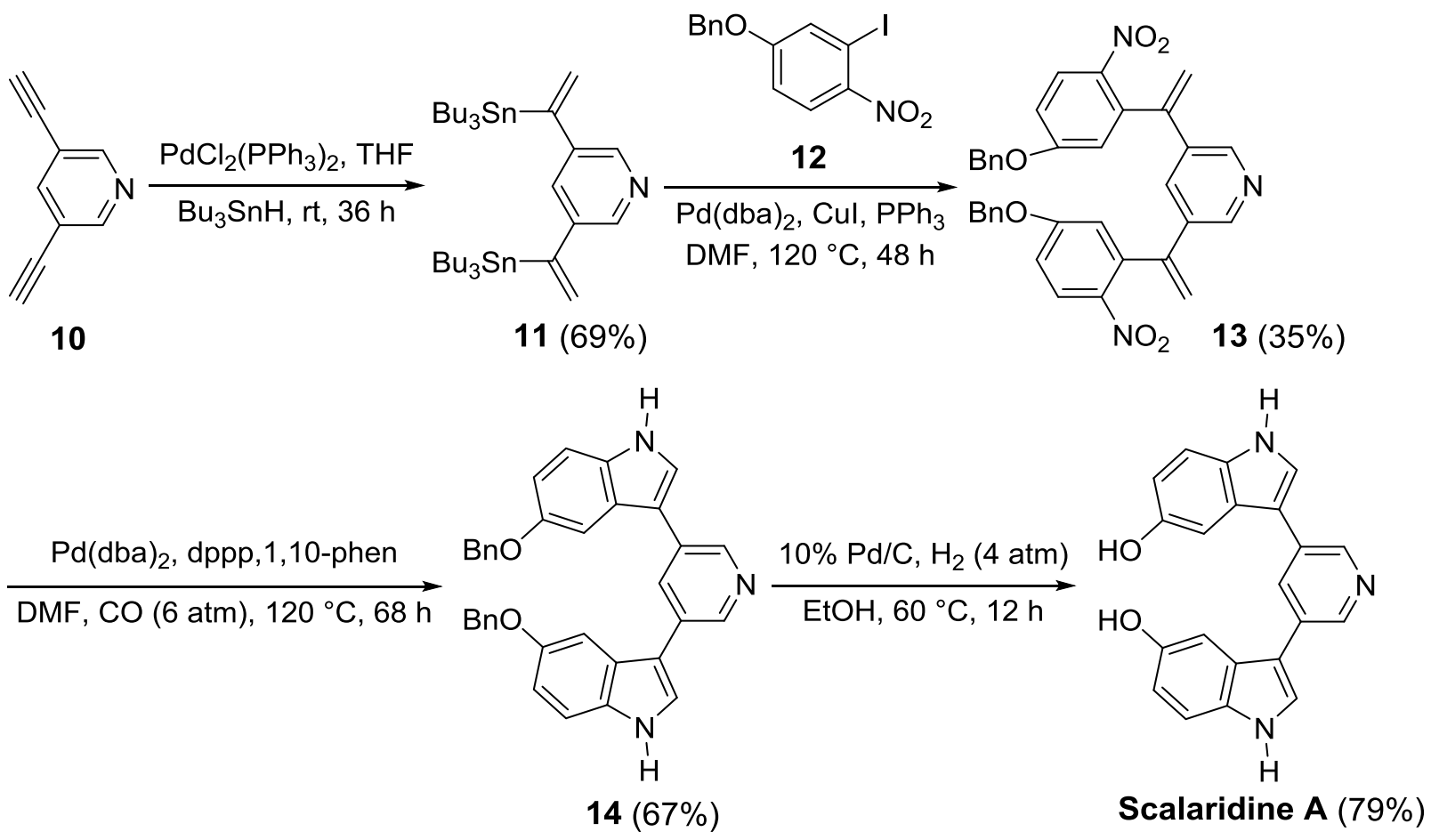

2,5-Dichloropyrimidine has been shown to participate in Kosugi-Migita-Stille cross coupling reactions with vinylstannanes, reacting first in the 2-position then in the 5-position. ${ }^{38}$ However, the related compound 2,5-dibromopyrimidine (15) proved to be problematic substrate in cross coupling reactions. Attempted coupling of $\mathbf{4}$ with $\mathbf{1 5}$ gave three identifiable products, destannylated product 16, an inseparable mixture of homocoupling product 7 and an unknown impurity, and the monocoupled, debrominated pyrimidine 17. The site of cross coupling was determined to be the 2-position based on ${ }^{1} \mathrm{H}$ NMR wherein a doublet resonance at $\delta 8.65 \mathrm{ppm}$ integrating to two protons and a triplet at $\delta 7.10 \mathrm{ppm}$ integrating to one proton with a coupling constant of $J=5.2 \mathrm{~Hz}$ was observed. Similar chemical shifts and coupling constants have been reported for related 2-substituted pyrimidines (Scheme 1.8). ${ }^{3}$ 
Scheme 1.8 Attemepted cross coupling of 4 with 2,5-dibromopyrimidine (15)

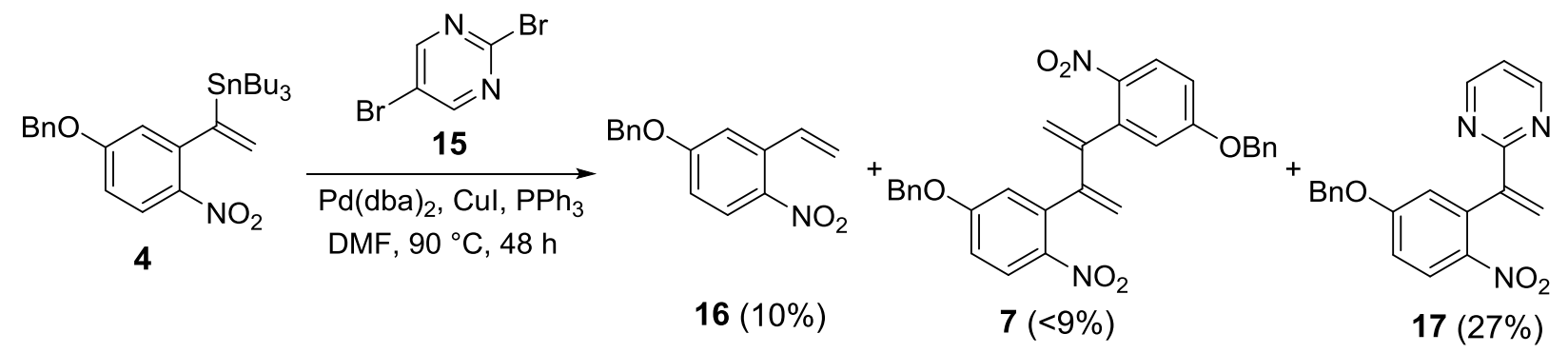

Here as well the reductive cyclization precursor was prepared by Kosugi-Migita-Stille coupling by reversing the polarity of the coupling partners. Double Sonogashira coupling ${ }^{40}$ of 2,5-dibromopyrimidine (15) with trimethylsilylethyne gave the expected product 18, which was used for desilylation using potassium carbonate in methanol/diethyl ether to give 2,5-diethynylpyrimidine (19). Palladium catalyzed hydrostannylation of 19 afforded the divinyltin-substituted pyrimidine 20, again with excellent $\alpha$ regioselectivity. The subsequent steps, Kosugi-Migita-Stille coupling of $\mathbf{2 0}$ with 12, reductive $\mathrm{N}$ heterocyclization, and debenzylation proceeded eventually to give hyrtinadine A. The spectroscopic data $\left({ }^{1} \mathrm{H}\right.$ and ${ }^{13} \mathrm{C}$ NMRs, HRMS) and melting point were fully consistent with those of the natural hyrtinadine A. The overall yield of hyrtinadine A starting from 15 was $12 \%$ in six steps (Scheme 1.9). 
Scheme 1.9 Synthesis of hyrtinadine A

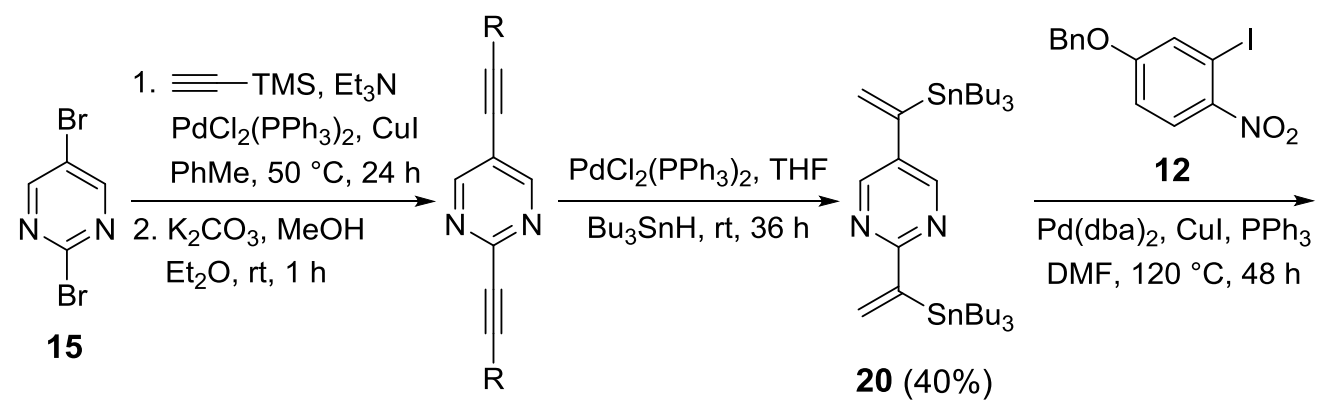

18 (R=TMS, $100 \%)$

$19(\mathrm{R}=\mathrm{H}, 93 \%)$<smiles>C=C(c1cnc(C(=C)c2cc(OCc3ccccc3)ccc2[N+](=O)[O-])nc1)c1cc(OCc2ccccc2)ccc1[N+](=O)[O-]</smiles>

$21(53 \%)$
$\frac{\mathrm{Pd}(\mathrm{dba})_{2}, \mathrm{dppp}, 1,10-\text { phen }}{\mathrm{DMF}, \mathrm{CO}(6 \mathrm{~atm}), 120^{\circ} \mathrm{C}, 60 \mathrm{~h}}$<smiles>CC(C)(C)Oc1ccc2[nH]ccc2c1</smiles>

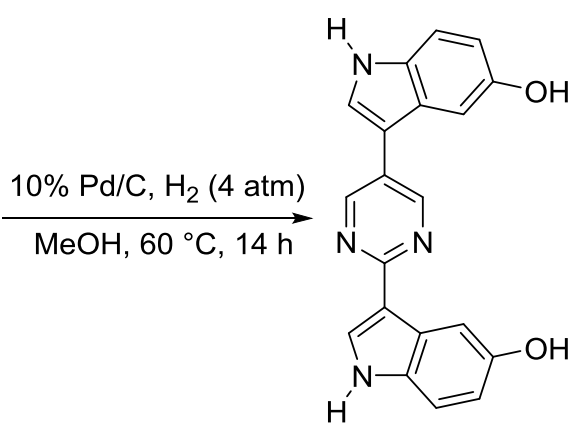

Hyrtinadine A (68\%)

Finally, 5-ethynylpyrimidine (23) served as the starting material for the synthesis of the fourth alkaloid hyrtinadine B. Following same sequence of reactions used in the synthesis of hyrtinadine A, (1) palladium catalyzed hydrostannylation, (2) cross coupling, (3) reductive cyclization, and (4) debenzylation gave hyrtinadine B in $28 \%$ overall yield in four steps. The spectroscopic data of the synthetic sample matched with those of the isolated compound (Scheme 1.10). 
Scheme 1.10 Synthesis of hyrtinadine B
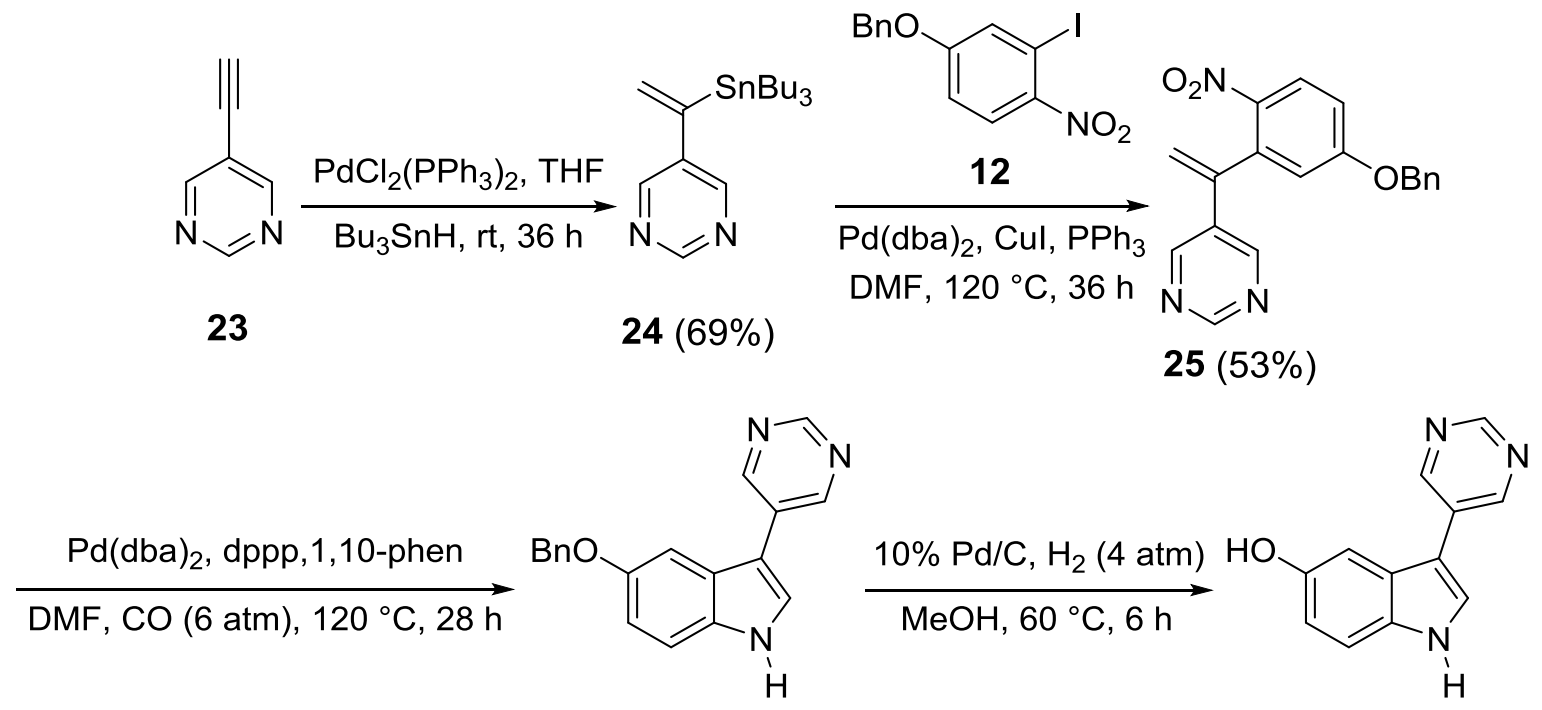

$26(90 \%)$

Hyrtinadine B (84\%)

\section{C Conclusion}

Expedient synthetic routes to four naturally occurring indole alkaloids, alocasin A, scalaridine A and hyrtinadine A-B have been developed. The overall yields were $21 \%$ (six steps), $13 \%$ (four steps), $12 \%$ (six steps), and $28 \%$ (four steps) respectively. Three palladium catalyzed reactions, namely, an alkyne hydrostannylation, a Kosugi-Migita-Stille cross coupling and a reductive $N$-heterocyclizations are the key steps in the syntheses. Results from the palladium catalyzed hydrostannylations of heteroarylethynes indicates that an ortho-substituent is not a requirement for high $\alpha$-regioselectivity when ethynyl substituted heterocycles are used. 
Chapter 2 Double palladium catalyzed reductive cyclizations. Synthesis of 2,2'-, 2,3'-, and 3,3'-bi-1H-indoles, indolo[3,2-b]indoles, and indolo[2,3-b]indoles

\section{A Introduction}

Indoles and carbazoles can be synthesized by the reductive cyclization of 2-nitrostyrenes and 2nitrobiphenyls using a number of reagents. Cadogan ${ }^{43}$ and Sundberg ${ }^{44}$ employed trialkyl phosphites or triphenylphsophine as reducing agents at elevated temperatures to carry out such transformations. More recently, however, reductive cyclizations using transition metal catalysts in the presence of carbon monoxide as the ultimate reducing agent have been developed. In fact, palladium complexes are the most commonly employed catalysts for effecting reductive cyclizations, and this facile method has been used in the total synthesis of a number of indole alkaloids. ${ }^{45}$ Therefore, we envisioned this methodology as an expedient pathway for the synthesis of a wide variety of compounds containing two indole units. Five different substrates in total, three bis(2-nitrophenyl)butadienes and two bis(2-nitrophenyl)ethenes were selected as biindole precursors (Figure 2.1). The formation of 2,2'-, 3,3'-, and 2,3'-biindoles from 1,4-, 2,3-, and 1,3-bis(2-nitroaryl)-1,3-butadienes, respectively, was anticipated via a cyclization onto each of the two carbon-carbon double bonds of the butadienes (A-C, Figure 2.1). In related reactions, successful reductive cyclyzations of 1,2-bis(2-nitroaryl)ethenes and 1,1-bis(2-nitroaryl)ethenes onto a shared olefin was hypothesized to furnish indolo[3,2-b]indoles and indolo[2,3-b]indoles respectively (D-E, Figure 2.1).

We disclose herein a short and efficient synthetic sequences to a variety of biindoles using a double reductive cyclization of 1,4-, 2,3-, and 1,3-bis(2-nitroaryl)-1,3-butadienes as the ultimate and key step leading to both the indole rings. Also disclosed are the nonselective reactions of 1,2-bis(2nitroaryl)ethenes and 1,1-bis(2-nitroaryl)ethenes forming a mixture of products. 
Figure 2.1 Possible cyclizations (A-E) of bis(2-nitrophenyl)butadienes and -ethenes.

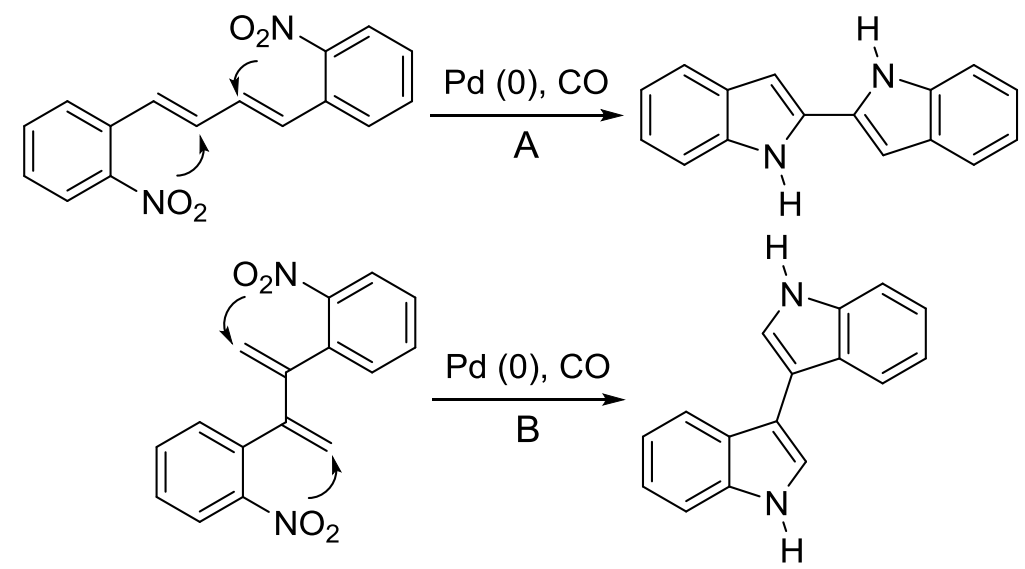<smiles>O=C(O)c1cccc2c1CCN2c1ccc2cc(-c3c[nH]c4ccccc34)[nH]c2c1</smiles><smiles>O=C(O)c1ccc2[nH]c3c4ccccc4[nH]c3c2c1</smiles><smiles>O=C(O)C(=O)c1cccc2c1[nH]c1[nH]c3ccccc3c12</smiles>

\section{B Results and Discussion}

The first biindole that was chosen for synthesis was 2,2 '-bi- $1 H$-indole (38). The cyclization precursor, 1,4-bis(2-nitrophenyl)-1,3-butadiene (33), was prepared following the procedure of Lowinger et $\mathrm{al}^{46}$ via a Wittig reaction of 2-nitrocinnamaldehyde (31) with 2-nitrobenzyl triphenylphosphonium bromide in the presence of a base. To our delight, subjecting compound $\mathbf{3 3}$ to our standard condition of reductive $N$ heterocyclization using a bis(dibenzylideneacetone)palladium-1,3-bis(diphenyl)propane-1,10phenanthroline catalyst symtem, in the presence of carbon monoxide (pCO=6 atm, $120{ }^{\circ} \mathrm{C}$ ) in $N, N^{\prime}-$ dimethylformamide, afforded compound $\mathbf{3 8}^{47}$ in good isolated yield.

A number of synthetic pathways to access 2,2'-biindoles have been reported in the literature. For instance, the parent compound $\mathbf{3 8}$ has been accessed by iridium, ${ }^{48}$ gold $^{49}$ and base ${ }^{50}$ catalyzed cyclization of the 
diamine corresponding to 33, Cadogan-Sunderberg type cyclizations using triphenylpshophine ${ }^{51}$ or tripheylphosphite, ${ }^{52}$ and double Madelung cyclization. ${ }^{53}$ Pertinent to the current methodology, Cenini et al. reported a $\mathrm{Pd}(\mathrm{TMB})_{2}-\mathrm{TMphenanthroline}{ }^{54}$ catalyzed carbon monoxide (pCO=40 atm, $140{ }^{\circ} \mathrm{C}$ ) mediated cyclization of $\mathbf{3 3}$ to give $\mathbf{3 8}$ (40\%) in addition to the monocyclized product 2-(2nitrophenyl)indole (26\%). ${ }^{55}$ A related reaction has also been described by Davies et al. using a palladium catalyzed route without any experimental details or yields. ${ }^{56}$

Excited by the above result, four additional substrates (34-37), in 55-75\% yields, were prepared by the treatment of the Wittig salts (28-30) with 31 or 5-bromo-2-nitrocinnamaldehyde (32) in the presence of a base. 5-Bromo-2-nitrocinnamaldehyde, in turn, was obtained by the reaction of 5-bromo-2nitrobenzaldehyde with (formylmethyl)triphenylphosphonium chloride in the presence of 4-(N,Ndimethylamino)pyridine (DMAP). Dienal 43 was also isolated resulting from two consecutive Wittig reactions in addition to 32 (Scheme 2.1). The dienes (34-37) were smoothly transformed to the corresponding 2,2'-bi-1H-indoles (39-42) under the same conditions. A good to excellent yield of product was observed for all substrates examined (Table 2.1).

Table 2.1 Synthesis of 2,2'-bi-1H-indoles

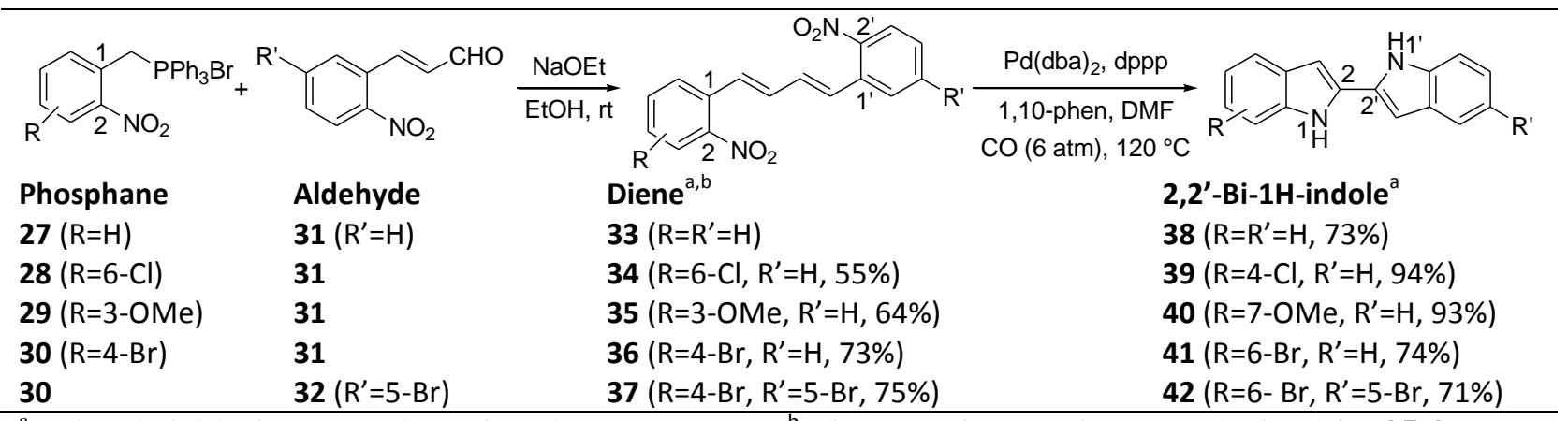

${ }^{\mathrm{a}}$ Isolated yield of pure product after chromatography. ${ }^{\mathrm{b}}$ Mixtures of $E E / Z Z$ isomers obtained for 35-37.

Scheme 2.1 Synthesis of 5-bromo-2-nitrocinnamaldehyde (32)<smiles>O=CC=CC=Cc1cc(Br)ccc1[N+](=O)[O-]</smiles> 
After the successful synthesis of 2,2'-bi-1H-indoles, double cyclization of 2,3-bis(2-nitrophenyl)-1,3butadienes to give 3,3'-bi-1H-indoles was examined next, and the results are summarized in Table 2.2, Scheme 2.2, and Scheme 2.3. The parent cyclization precursor 2,3-bis(2-nitrophenyl)-1,3-butadiene (52) has previously been prepared using a palladium catalyzed double cross coupling of 2-nitrophenylboronic acid with 1,4-bis(carbonyloxy)-2-butyne. ${ }^{57} \mathrm{We}$ have recently reported the formation of 7 , a substituted analogue of 52, as a side product in Kosugi-Migita-Stille reaction of $\mathbf{4}$ with an aryl bromide. ${ }^{58}$ It seemed plausible that homocoupling of $\mathbf{4}$, and related alkenyltin reagents, would be the major reaction path in the absence of a cross-coupling partner. In order to examine this idea, tributyl(1-(2-nitrophenyl)tin (48) was prepared from 1-ethynyl-2-nitrobenzene (44) in excellent isolated yield using the regioselective palladium catalyzed hydrostannation developed by Alami and coworkers. ${ }^{23 \mathrm{~b}}$ As expected, the $\beta$-isomer was not detected by ${ }^{1} \mathrm{H}$ NMR of the crude reaction mixture. To our delight, palladium catalyzed homocoupling of 48 proceeded smoothly in DMF to give dimer 52. Reductive cyclization of 52 resulted in the formation of 3,3'-bi- $1 H$-indole (56) ${ }^{59}$ as the sole product (Table 2.2).

3,3-Biindoles are relatively rare in nature, but a handful examples of halogenated and/or sulfur containing alkaloids have been isolated from algae. ${ }^{60-63}$ In addition, the unsubstituted 3,3'-biindole 56 was isolated from a terrestrial fungus. ${ }^{64}$ Recent synthesis of 3,3'-biindoles include, for example, a Masuda indole borylation-Suzuki arylation sequence, ${ }^{65}$ a tellurium tetrachloride ${ }^{66}$ mediated and palladium catalyzed oxidative dimerizations of indoles. ${ }^{67}$

To briefly examine the ease of synthesis of 3,3'-biindoles by reductive $N$-heterocyclization, four additional 1-(2-nitrophenyl)ethenyl)tin derivatives $(\mathbf{4}, \mathbf{4 9 - 5 1 )}$ were prepared in a similar fashion to 48 (Table 2.2). Although palladium catalyzed homocoupling of $\mathbf{4}$ gave the expected product $\mathbf{7}$ in good yield, the yield of chloro-substituted dimer $\mathbf{5 3}$ remained low and we were compelled to look for an alternative pathway. Homocoupling of organotin reagents have been reported using an excess of $\mathrm{CuCl}$ in $\mathrm{DMF},{ }^{68}$ an excess of $\mathrm{Cu}\left(\mathrm{NO}_{3}\right)_{2}$ in THF, ${ }^{69}$ or a catalytic amount of $\mathrm{CuCl}_{2}$ in the presence of 0.5 equivalent of iodine in DMF. ${ }^{70}$ After several trials for the preparation of reductive cyclization precursors using the literature conditions, a combination of 2.5 equivalent of $\mathrm{CuCl}$ in $\mathrm{DMF}$ at ambient temperature was found to work well for our substrates (49-51) giving excellent yields of porducts (Table 2.2). These precursors (7, 53-55) were successfully converted to expected 3,3'-biindoles (57-60) in 64-100\% isolated yields. The results are summarized in Table 2.2 below. 
Table 2.2 Synthesis of 3,3'-bi-1H-indoles

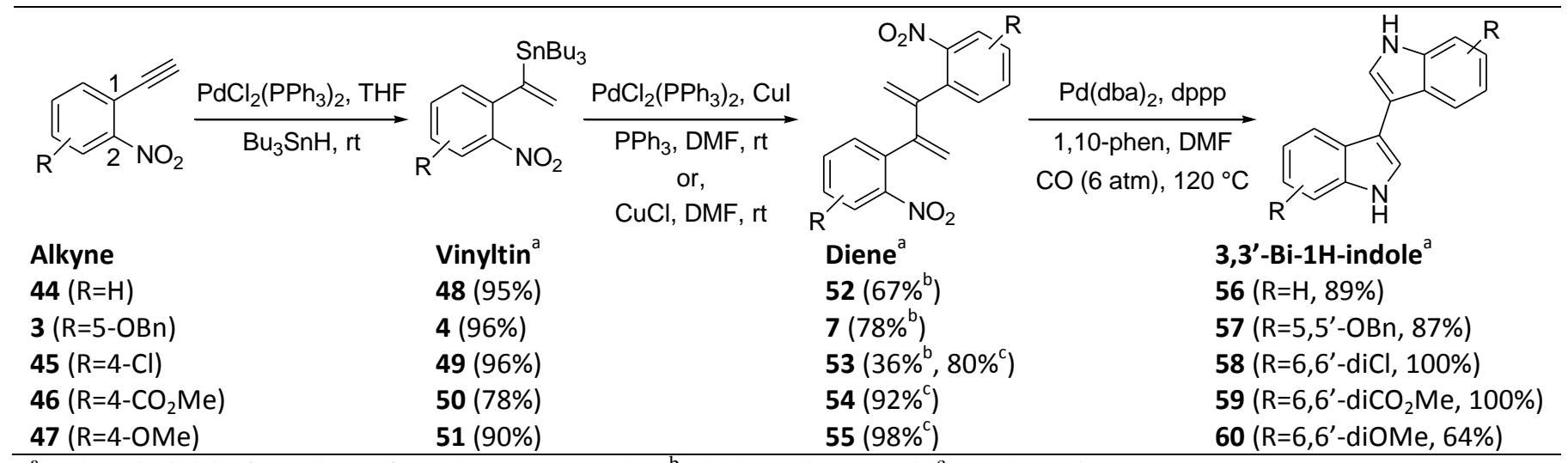

${ }^{\mathrm{a}}$ Isolated yield of product after chromatography. ${ }^{\mathrm{b}} \mathrm{PdCl}_{2}\left(\mathrm{PPh}_{3}\right)_{2}$ used. ${ }^{\mathrm{c}} \mathrm{CuCl}$ used.

As an example of pyridine-linked biindole, 4,4'-diaza-3,3'-bi-1H-indole (64) was synthesized using hydrostannation, dimerization, cyclization sequence discussed above. Thus, 64 was prepared in three steps from the previously reported 2-ethynyl-3-nitropyridine (Scheme 2.2).

Scheme 2.2 Synthesis of 4,4'-diaza-3,3'-bi-1H-indole (64)

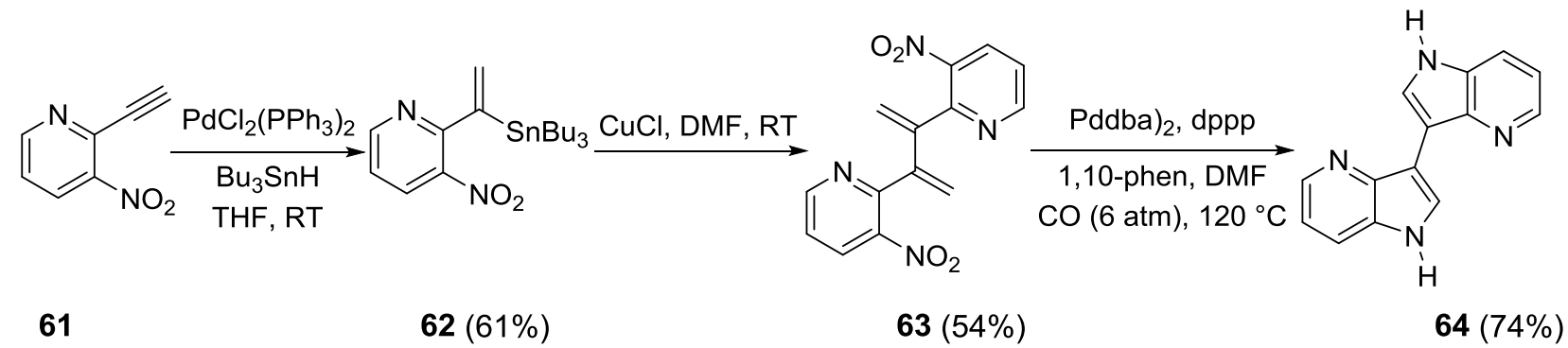

An unsymmetrical biindole $\mathbf{6 8}$ was also synthesized (Scheme 2.3). Treatment of $\mathbf{6 5}$ with $\mathrm{HBr}$ in 3pentanone as a solvent furnished vinyl bromide $\mathbf{6 6}^{71}$ in $57 \%$ yield. The vinyl bromide was then subjected to Kosugi-Migita-Stille coupling with ethenyltin 48 to give 2,3-bis(2-nitrophenyl)-1,3-butadiene (67). Tin impurities from the cross coupling were removed by chromatography using a 9:1 $\mathrm{SiO}_{2} / \mathrm{K}_{2} \mathrm{CO}_{3}$ stationary phase as described by Harrowven et al. ${ }^{72}$ Double reductive cyclization smoothly converted 67 to the desired 3,3'-biindole in quantitative yield (Scheme 2.3). ${ }^{73}$ 
Scheme 2.3 Synthesis of unsymmetrical 3,3'-bi-1H-indole 68
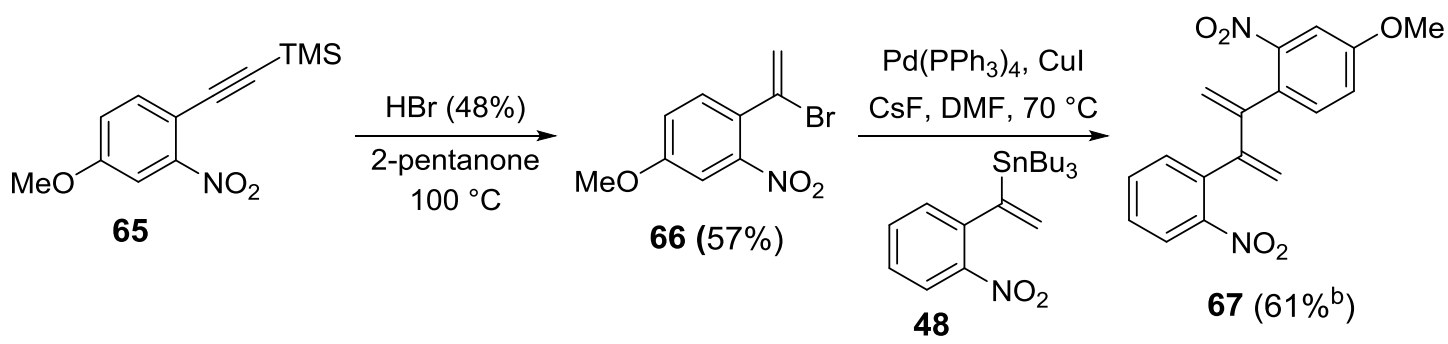

$+52(28 \%)$

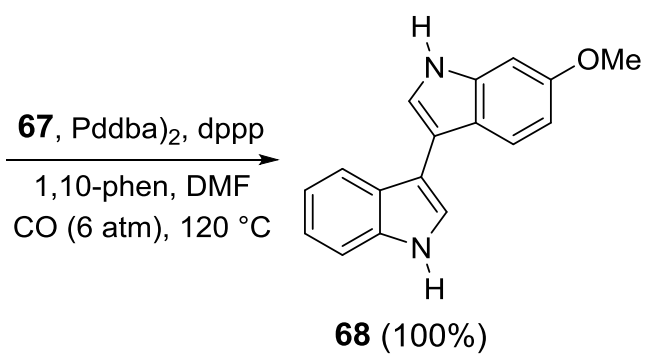

The third permutation of the double cyclization of substituted 1,3-butadienes is the synthesis of 2,3'-bi$1 H$-indoles from 1,3-bis(2-nitrophenyl)-1,3-butadienes (Table 2.3). Synthesis of 2,3-bi-1H-indoles have previously been reported via the Fischer indole, ${ }^{74}$ acid mediated reaction of 3-bromoindole with indole, ${ }^{75}$ and Lewis acid mediated dimerization to give 2,3-dihydro-2,3'-bisindole, followed by oxidation. ${ }^{76}$ To date, only four natural products containing a 2,3'-bisindole have been isolated. ${ }^{77}$ All four natural compounds are connected by an ethylene bridge between the 2 and 3' carbons of the respective indoles.

The parent cyclization precursor $\mathbf{7 1}$ was prepared in moderate yield by the treatment of the Wittig salt with 2-(2-nitrophenyl)-2-propenal (70) ${ }^{78}$ under basic conditions. Three additional substrates (72-74) were also synthesized in a similar fashion. Low to moderate yields of produtcs were isolated. Treatment of (7174) with carbon monoxide under palladium catalyzed conditions gave the anticipated 2,3'-bi-1H-indoles (75-78) in 61-72\% yields. The results are summarized in Table 2.3. 
Table 2.3 Synthesis of 2,3'-bi-1H-indoles

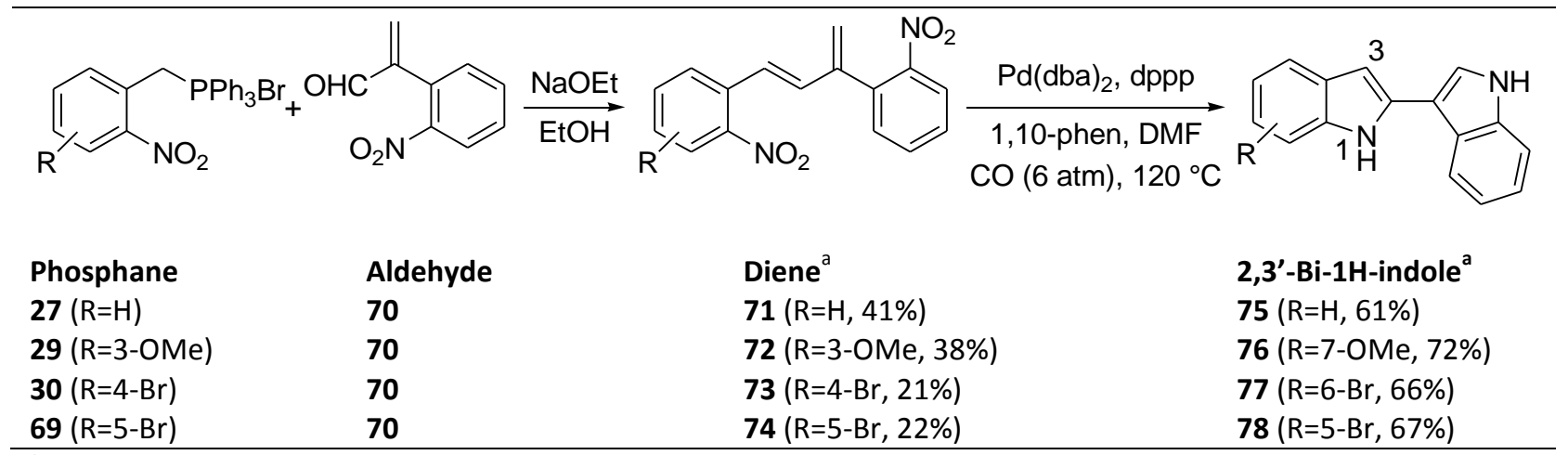

${ }^{\mathrm{a}}$ Isolated yield of pure product after chromatography

The fourth variation of the double cyclization is the cyclization of the two adjacent nitro-groups onto a shared olefin bond with the anticipated formation of indolo[3,2-b]indole. In this case, subjecting the known parent compound 1,2-bis(2-nitrophenyl)ethene $(\mathbf{7 9})^{79}$ to the palladium catalyzed reductive cyclization conditions for $56 \mathrm{~h}$ did not furnish the double cyclization product indolo[3,2-b]indole $(\mathbf{8 0})^{80}$ but gave two isomeric tetracyclic compounds indolo[1,2-c]quinazolin-6(5H)-one (81) and 5,11-dihyro$6 H$-indolo[3,2-c]quinoline-6-one (82). The products were analyzed by 2D NMR experiments and by comparison of analytical data with literature reported values. It should be noted that Nishiyama et al. have reported a reductive cyclization, related to the transition metal catalyzed reactions. For example, treatment of 79 with a catalytic amount of selenium (40 mol\%) under $30 \mathrm{~atm}$ of carbon monoxide at $100{ }^{\circ} \mathrm{C}$ gave 81 in $60 \%$ yield. ${ }^{81}$ In this catalytic process, carbon monoxide insertion to form an isocyanate was suggested as plausible intermediate that ultimately reacts with the indole $\mathrm{N}-\mathrm{H}$ giving rise to the insertion product. Isocyanate $\mathbf{8 3}$ has previously been reported as an intermediate in a Curtius rearrangement of the corresponding acid, affording a 1:1 mixture of $\mathbf{8 1}$ and $\mathbf{8 2} .{ }^{82}$ Compound $\mathbf{8 2}$ was not observed in the selenium catalyzed reactions (Scheme 2.4). Products derived from insertion of carbon monoxide have been isolated in a few additional cases of transition metal catalyzed reductive cyclizations aromatic nitro compounds. ${ }^{83}$ An example is the rhodium catalyzed reductive cyclization of $\mathbf{8 4}$ that gave, in addition to indole 85 as the major product, quinolinone $\mathbf{8 6}$ and amine $\mathbf{8 7}$ (Scheme 2.5). ${ }^{84}$ 
Scheme 2.4 Reductive cyclization of 79

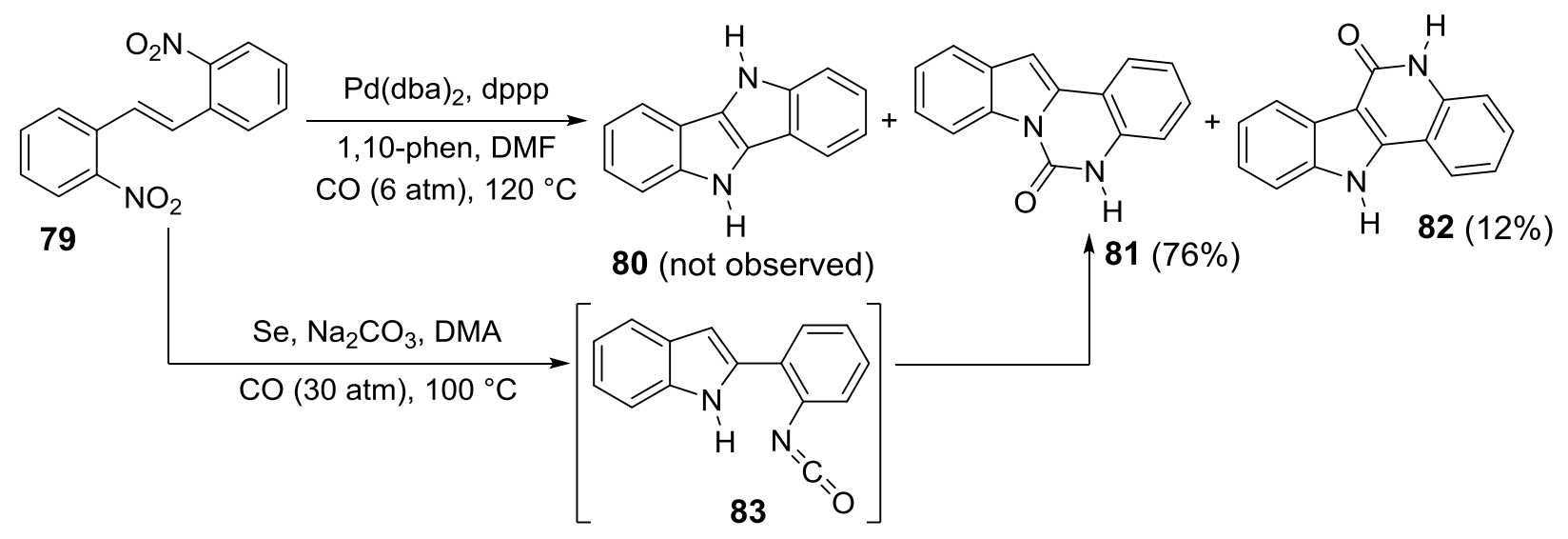

Scheme 2.5 Reductive cyclization of $\mathbf{8 4}{ }^{84}$

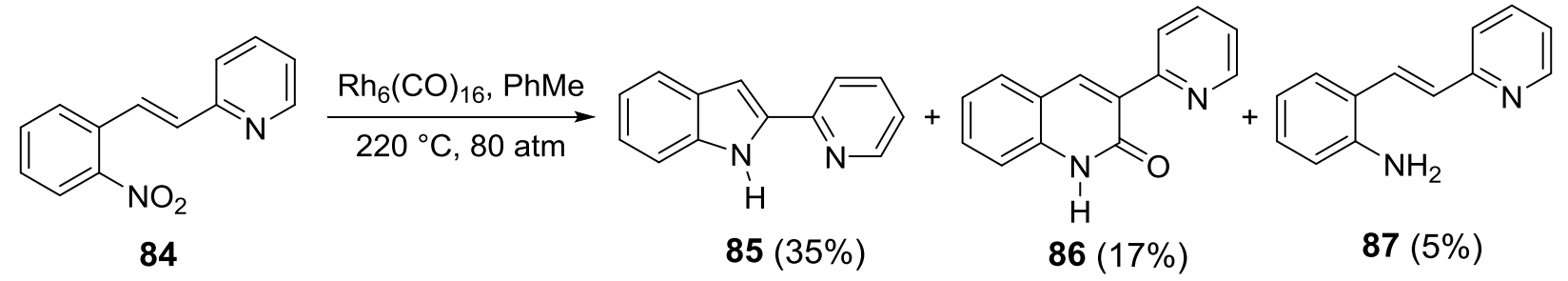

The formation of indolo[1,2-c]quinazolin- $6(5 H)$-one encouraged us to evaluate the palladium catalyzed pathway of 1,2-bis(2-nitrophenyl)ethenes as a general methodology toward these compounds. Two additional substrates $\mathbf{8 8}$ and $\mathbf{9 1}$ were prepared by Wittig reactions of $\mathbf{2 8}$ and $\mathbf{2 9}$ with 2-nitrobenzaldehyde. From the reductive cyclization of chloro-substituted alkene $\mathbf{8 8}$, both indolo[3,2-b]indole (90) and indolo[1,2-c]quinazolin-6(5H)-one (89) were isolated. The former compound $\mathbf{9 0}$ represents the first case of the formation of an indolo[3,2-b]indole from a transition metal catalyzed reductive cyclization, although as a minor product (Scheme 2.6). In contrast, the methoxy substituted substrate 91 did not participate in a double cyclization as readily as $\mathbf{7 9}$ and $\mathbf{8 8}$. In this case, two monocyclized products 92 and 93 were isolated in $10 \%$ and $74 \%$ respectively, together with a negligible amount of indolo[3,2c] quinolin-6-one (94) (Scheme 2.7). Monocyclized indole 93 was hypothesized to be intermediate precursor to the tetracyclic compound 94; however, only a miniscule amount of 94 (2\%) was isolated from an attempted cyclization of $\mathbf{9 3}$ under identical reaction conditions. Although all starting material was consumed in the reaction, no other side products were identified (Scheme 2.7). 
Scheme 2.6 Reductive cyclization of $\mathbf{8 8}$<smiles>CCON(OCc1ccccc1)c1ccccc1C=O</smiles>

28<smiles>O=[N+]([O-])c1ccccc1/C=C/c1c(Cl)cccc1[N+](=O)[O-]</smiles>

$88(28 \%)$

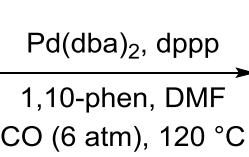

CO (6 atm), $120^{\circ} \mathrm{C}$

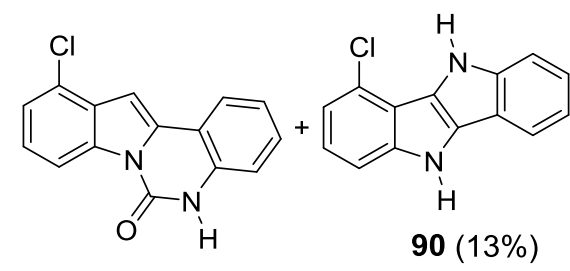

$89(69 \%)$

Scheme 2.7 Reductive cyclization of 91<smiles>CCON=C(Br)c1ccccc1C=O</smiles>

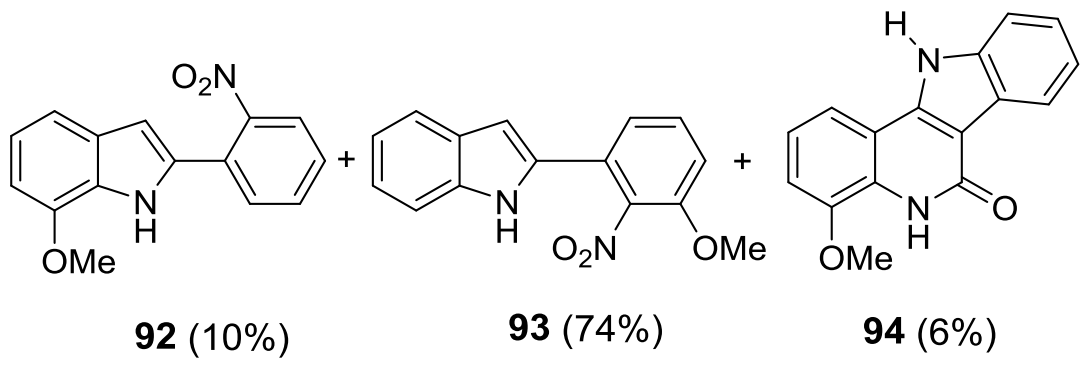

Finally, the feasibility of palladium catalyzed double reductive $N$-heterocyclization of 1,1-bis(2nitrophenyl)alkenes for the formation of indolo[2,3-b]indoles was also studied. The reductive cyclization precursor 95 was prepared by the Kosugi-Migita-Stille cross coupling of 1-iodo-2-nitrobenzene in good yield. ${ }^{85}$ As observed in the previous palladium catalyzed reactions involving tin compound $\mathbf{4 8}$, dimer $\mathbf{5 2}$ was also isolated in low yield (11\%). When subjected to palladium catalyzed reductive cyclization, compound 95 gave the anticipated product indolo[2,3-b]indole (96), although in a very low isolated yield together with the indolo[2,3-c]quinolinone (97) as the major product (Scheme 2.8). Cadogan-Sundberg type cyclizations of 3-(2-nitrophenyl)indoles using triphenylphosphine ${ }^{86}$ or triethyl phosphite ${ }^{87}$ to afford indolo[3,2-b]indoles have been reported. 
Scheme 2.8 Synthesis of indolo[2,3-b]indole (96) and indolo[2,3-c]quinlinone (97)
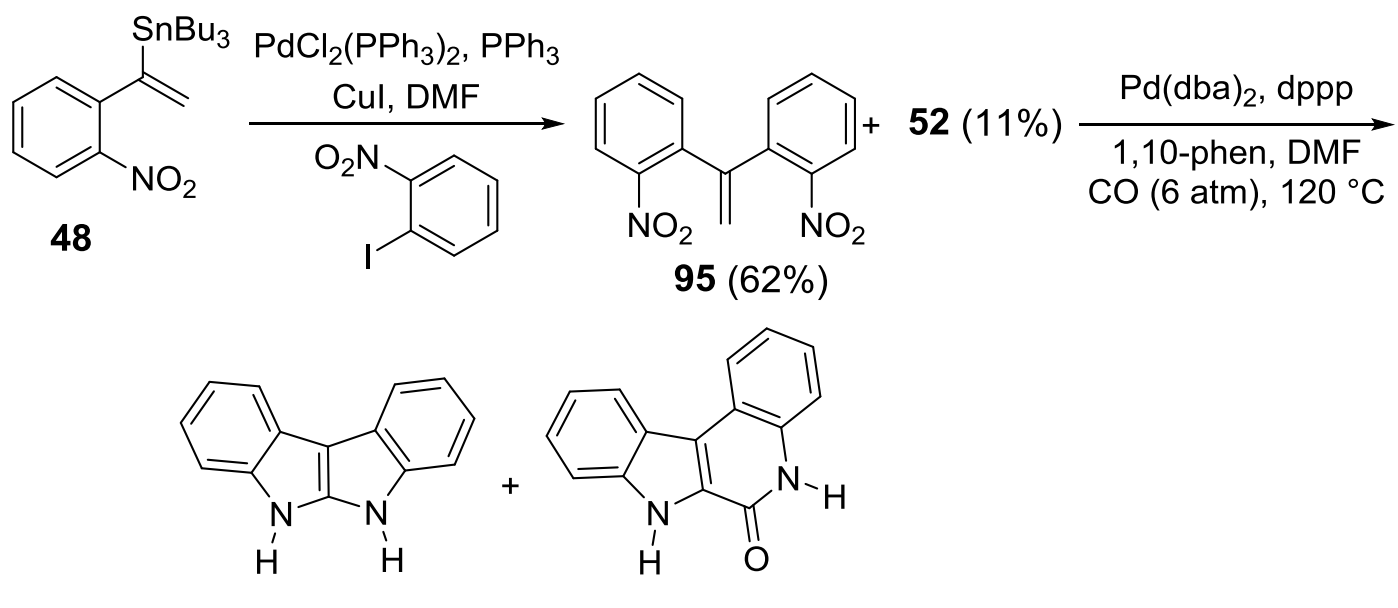

$96(14 \%) \quad 97(58 \%)$

The reaction pathway seen in Scheme 2.8 represents a very short and efficient route to access indolo[2,3c] quinolin-6-one starting from a symmetrical substrate like 95. A number of methodologies have been developed for the construction of the indoloquinoline-6-one skeleton. For example, cyclizations of $N$ arylindole-2-carboxamides utilizing a Heck reaction, ${ }^{88}$ photochemical cyclizations of indole-2-carboxylic acid arylamides, ${ }^{89}$ and 3-(2-azidophenyl)- $N$-phenylacrylamides, ${ }^{90}$ oxidative cyclizations of 3-arylindole-2carboxamides $^{91}$ and a platinum catalyzed reduction of ethyl 3-(2-nitrophenyl)-1H-indole-2carboxylates. $^{92}$

The unsymmetrical compound 99 containing a methoxy group meta to one of the nitro groups and a benzyloxy group para to the second nitro group was also synthesized by the Kosugi-Migita-Stille coupling of 4 with 98 (Scheme 2.9). Compound 99 was subjected to the cyclization conditions, and three different products were obtained after chromatography, an indolo[2,3-b]indole (100) and two isomeric indolo[2,3-c] quinolin-6-ones $\mathbf{1 0 1}$ and $\mathbf{1 0 2}$ in an approximately 1:1:1 ratio. The structures of the isomeric tetracycles (101-102) were elucidated using 2D NMR experiments (Scheme 2.9). It becomes clear that while the use of an unsymmetrical substrate will furnish two isomeric indolo[2,3-c]quinolin-6-ones, the methodology presented here may be of synthetic value employing symmetrical 1,1-bis(2-nitroaryl)alkenes as the starting materials. 
Scheme 2.9 Reductive cyclization of 99

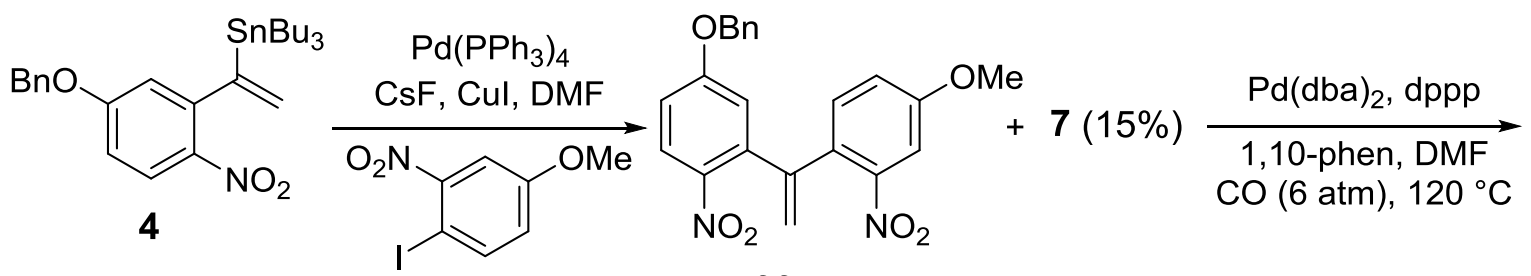

98

$99(69 \%)$

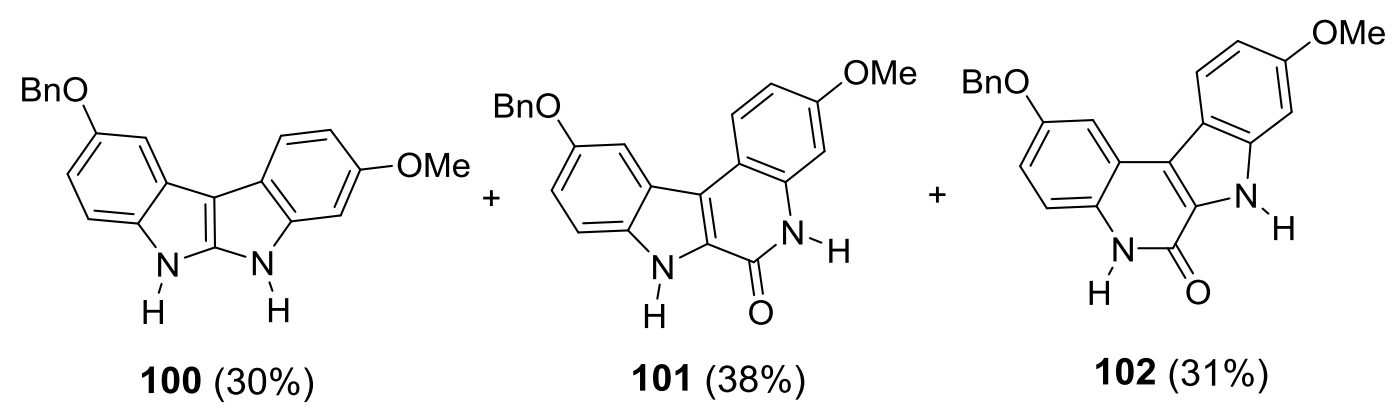

\section{C Conclusion}

A palladium catalyzed, carbon monoxide mediated, double reductive $N$-heterocyclization of 1,4-, 1,3-, and 2,3-bis(2-nitroaryl)-1,3-butadienes to afford 2,2'-, 2,3'-, and 3,3'-biindoles, respectively, have been developed. The methodology presented here clearly demonstrates potentially useful routes to access these ring systems. On the basis of the results in Schemes 2.4-2.8 and from the selenium catalyzed reactions reported in the literature, it appears that general syntheses of indolo[3,2- $b]$ indoles and indolo[2,3$b$ ]indoles via a palladium catalyzed carbon monoxide mediated reductive cyclizations of 1,2-bis(2nitroaryl)ethenes or 1,1-bis(2-nitroaryl)ethenes, respectively are not feasible. Products derived from carbon monoxide insertion and/or monocyclized products are the predominant products observed in these reactions. 


\section{Chapter 3 Syntheses of three naturally occurring polybrominated 3,3'-bi-1H-indoles}

\section{A Introduction}

Halogen and/ or sulfur containing 3,3'-bi-1H-indoles are relatively rare in nature. A small number of such indole alkaloids have been isolated mainly from marine algae and also from a terrestrial fungus. In 1982, Norton et al isolated 2,2',5,5'-tetrabromo-3,3'-bi-1H-indole (103) from Rivularia firma (Fig. 3.1). ${ }^{109}$ This was the first alkaloid of this family to be isolated and characterized. The related isomeric alkaloid 2,2',6,6'-tetrabromo-3,3'-bi-1H-indole (104) was later isolated from the same species. ${ }^{110}$ More recently was isolated 2,2',5,5',6,6'-hexabromo-3,3'-bi-1H-indole (105) from the red alga Laurentia similis. ${ }^{111}$ Compound $\mathbf{1 0 5}$ has been shown to be a protein tyrosine phospholipase 1B inhibitor. ${ }^{12}$

In addition to the polyhalogenated indoles $\mathbf{1 0 3 - 1 0 5}$, sulfur containing alkaloids $\mathbf{1 0 6},{ }^{60 \mathrm{a}} \mathbf{1 0 7 - 1 0 8},{ }^{60 \mathrm{~b}} \mathbf{1 0 9} \mathbf{9}^{60 \mathrm{c}}$ also exist in the nature and they are all produced by the red alga Laurencia brongniartii. The only example of a 3,3'-bi-1H-indole from a terrestrial source is the parent compound isolated from the fungus Gliocladium catenulatum. ${ }^{64}$ The polyfunctionalized biindole $\mathbf{1 1 0}$ was isolated from the green alga Chaetomorpha basiretorsa Setchell. ${ }^{63}$ To the best of our knowledge, the synthesis of none of these polyhalogenated alkaloids has been reported to date.

Figure 3.1 Naturally occurring polyhalogenated 3,3'-bi-1 $H$-indoles<smiles>[R]c1cc2[nH]c(Br)c(-c3c(Br)[nH]c4cc([R])c([R])cc34)c2cc1[R]</smiles>

$103\left(\mathrm{R}=\mathrm{Br}, \mathrm{R}^{\prime}=\mathrm{H}\right)$

$104\left(\mathrm{R}=\mathrm{H}, \mathrm{R}^{\prime}=\mathrm{Br}\right)$

$105\left(\mathrm{R}=\mathrm{R}^{\prime}=\mathrm{Br}\right)$<smiles>[R]c1[nH]c2cc(Br)cc(Br)c2c1-c1c(Br)[nH]c2cc(Br)cc(Br)c12</smiles>

$106\left(\mathrm{R}=\mathrm{R}^{\prime}=\mathrm{SMe}\right)$

$107\left(\mathrm{R}=\mathrm{SMe}, \mathrm{R}^{\prime}=\mathrm{SOMe}\right)$

$108\left(\mathrm{R}=\mathrm{R}^{\prime}=\mathrm{SOMe}\right)$<smiles>COc1cc(Br)c(Cl)c2c(-c3c[nH]c4c(OC)cc(Br)c(Cl)c34)c[nH]c12</smiles>

110

$109\left(\mathrm{R}=\mathrm{Br}, \mathrm{R}^{\prime}=\mathrm{SMe}\right)$ 
We have recently disclosed palladium catalyzed double reductive cyclization of 2,3-bis(2-nitrophenyl)1,3-butadienes for the synthesis of a variety of 3,3'-biindoles (Scheme 3.1). ${ }^{113}$ For instance, reductive cyclization of 2,3-bis(2-nitrophenyl)-1,3-butadiene (52) gave the parent 3,3'-bi-1H-indole (56) in excellent isolated yield. Herein are described synthetic routes to biindoles 103-105 based on this methodology.

Scheme 3.1 Palladium catalyzed double reductive cyclization to 3,3'-biindoles<smiles>C=C(C(=C)c1ccccc1[N+](=O)[O-])c1ccccc1[N+](=O)[O-]</smiles>

52
$\frac{\operatorname{Pd}(\mathrm{dba})_{2}, \mathrm{dppp}, 1,10-\text { phen }}{\mathrm{CO}(6 \mathrm{~atm}), 120^{\circ} \mathrm{C}, \mathrm{DMF}}$<smiles>c1ccc2c(-c3c[nH]c4ccccc34)c[nH]c2c1</smiles>

$56(89 \%)$

\section{B Results and Discussion}

Biindole 120 has previously been prepared by a palladium catalyzed oxidative dimerization of 5bromoindole (Scheme 3.2), ${ }^{67}$ reductive cyclization ${ }^{73}$ and a two-step procedure employing a nucleophilic addition of 5-bromoindole to 5-bromoisatine followed by borane reduction of the intermediate (Scheme $3.3) .^{114}$

Scheme 3.2 Palladium catalyzed oxidative dimerization of 5-bromoindole

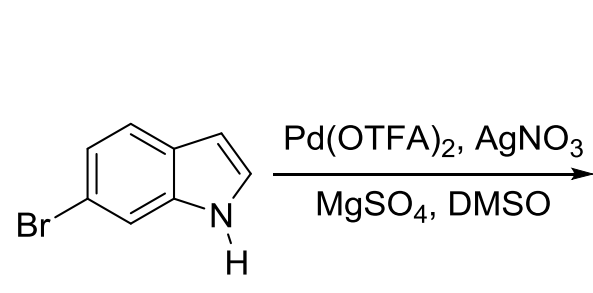<smiles>Brc1ccc2c(-c3c[nH]c4cc(Br)ccc34)c[nH]c2c1</smiles>

$120(69 \%)$ 


\section{Scheme 3.3 Two common routes to $\mathbf{1 6}$}

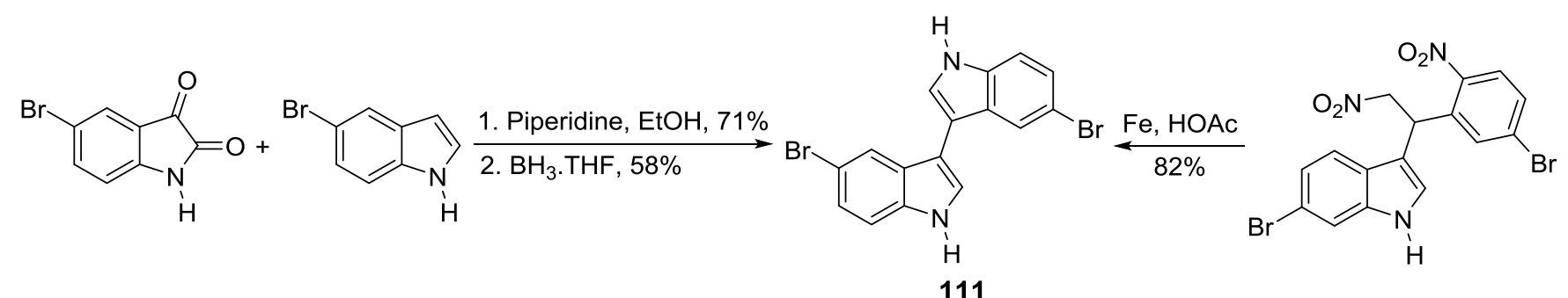

We hypothesized that comounds $\mathbf{1 0 3}$ and $\mathbf{1 0 4}$ can be derived from compound $\mathbf{1 1 1}$ by the introduction of extra bromine atoms. Accordingly, our synthesis commenced with the known compound 5-bromo-2nitro-1-iodobenzene (112) as the starting point for the synthesis of alkaloids 103 and 104. Sonogashira coupling of 112 with trimethylsilylethyne at ambient temperature gave 113. The silyl group was smoothly removed using potassium carbonate in a methanol-diethyl ether solvent system to afford 114. Treatment of $\mathbf{1 1 4}$ with tributyltin hydride in the presence of bis(triphenylphosphine)palladium dichloride in THF furnished the required vinyl tin derivative $\mathbf{1 1 5}$ with excellent $\alpha$-selectivity (Scheme 3.4). No trace of the $\beta$-isomer was observed by ${ }^{1} \mathrm{H}$ NMR of the crude reaction mixture. ${ }^{23 \mathrm{~b}}$ Tin compound $\mathbf{1 1 5}$ was smoothly transformed into the reductive cyclization precursor 116 using an excess of copper chloride. Once in hand, diene 116 was dissolved in $N, N$-dimethylformamide (DMF) and subjected to carbon monoxidemediated reductive cyclization ( $\mathrm{pCO}=6 \mathrm{~atm}, 120^{\circ} \mathrm{C}$ ) in the presence of a palladium catalyst system consisting of bis(dibenzylideneacetone)palladium - 1,3-bis(diphenyl)propane - 1,10-phenanthroline to give 5,5'-dibromo-3,3'-bi- $1 H$-indole 111. The ${ }^{1} \mathrm{H}$ NMR and ${ }^{13} \mathrm{C}$ NMR data recorded for 111 were in complete accordance with the reported values. 
Scheme 3.4 Synthesis of precursor 111

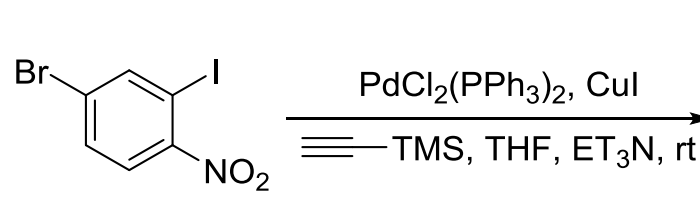

112<smiles>CC#Cc1cc(Br)ccc1[N+](=O)[O-]</smiles>

$113(92 \%)$

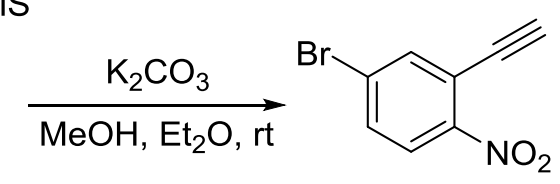

$114(91 \%)$<smiles>C=C(SCCC)c1cc(Br)ccc1[N+](=O)[O-]</smiles><smiles>Brc1ccc2[nH]cc(-c3c[nH]c4ccc(Br)cc34)c2c1</smiles>

$111(45 \%)$

3-Substituted indoles have been selectively brominated in the 2-position using NBS. For example, bromination of 3-methylindole using NBS in carbon tetrachloride $\left(\mathrm{CCl}_{4}\right)$ gave 2-bromo-3-methylindole in $97 \%$ yield. $^{115}$

Scheme 3.5 Bromination of 3-methylindole<smiles>Cc1c(N)[nH]c2cc([SeH])ccc12</smiles> 
Based on this result, biindole 111 was treated with 2.0 equivalents of NBS in $\mathrm{CCl}_{4}$ affording the naturally occurring tetrabrominated alkaloid $\mathbf{1 0 3}$ in 59\% yield after chromatographic purification. Reactions of 111 with 4-6 equivalents of NBS in $\mathrm{CCl}_{4}$ were not clean and afforded a number of inseparable products with low mass balance. However, replacing $\mathrm{CCl}_{4}$ with a 1:1 mixture of tetrahydrofuran and dichloromethane as the solvent system gave $\mathbf{1 0 5}$ in $46 \%$ isolated yield upon treatment with six equivalents of NBS. All analytical data for $\mathbf{1 0 3}{ }^{109}$ and $\mathbf{1 0 5}{ }^{111}$ were in complete agreement with the data reported for the isolated natural products.

Scheme 3.6 Synthesis of 2,2',5,5'-tetrabromo- and 2,2',5,5',6,6'-hexabromobiindoles

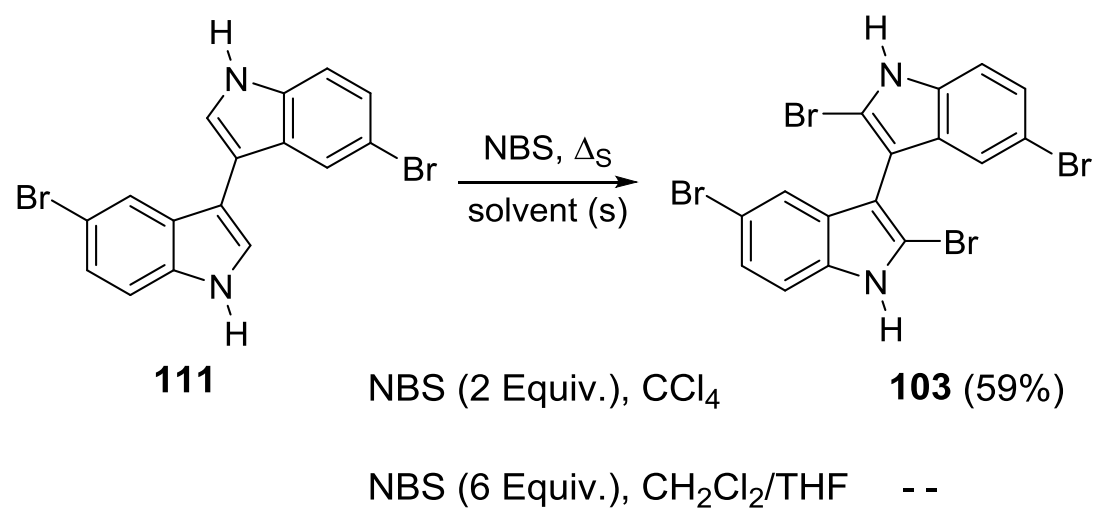<smiles>Brc1cc2[nH]c(Br)c(-c3c(Br)[nH]c4cc(Br)c(Br)cc34)c2cc1Br</smiles>

A synthetic sequence similar to the one depicted in Scheme 3.6 was used to prepare the remaining 2,2',6,6'-tetrabrominated alkaloid 104. 1-(4-Bromo-2-nitrophenyl)ethyne (117) served as the starting point for the synthesis of $\mathbf{1 0 4}$ (Scheme 3.7). Both the regioselective palladium catalyzed hydrostannylation of $\mathbf{1 1 7}$ furnishing vinyl tin $\mathbf{1 1 8}$ and the subsequent copper mediated coupling affording dimer 119 proceeded uneventfully. Reductive cyclization of 119 afforded 6,6'-dibromo-3,3'-biindole (120) in 64\% isolated yield. Compound $\mathbf{1 2 0}$ has previously been prepared via a palladium catalyzed oxidative dimerization of 6-bromoindole. ${ }^{67}$

Similar to the synthesis of $\mathbf{1 0 3}$, biindole $\mathbf{1 2 0}$ was treated with 2.0 equivalents of $\mathrm{NBS}$ in $\mathrm{CCl}_{4}$ affording the tetrabrominated biindole alkaloid $\mathbf{1 0 4}$ in 59\% yield after chromatographic purification. All analytical data were in complete agreement with the data reported for isolated $\mathbf{1 0 4} .^{109}$ 
Scheme 3.7 Synthesis of 2,2',6,6'-tetrabromo-bi- $1 H$-indole

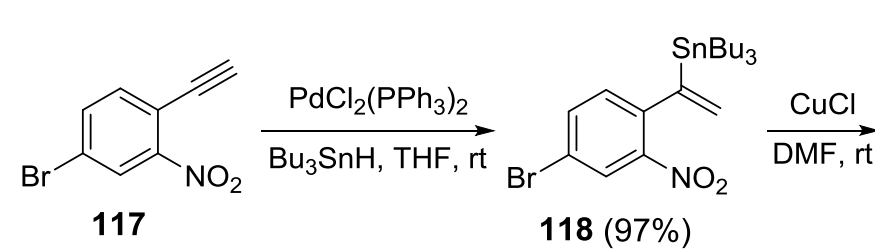<smiles>Brc1ccc2c(-c3c[nH]c4cc(Br)ccc34)c[nH]c2c1</smiles>

$120(64 \%)$

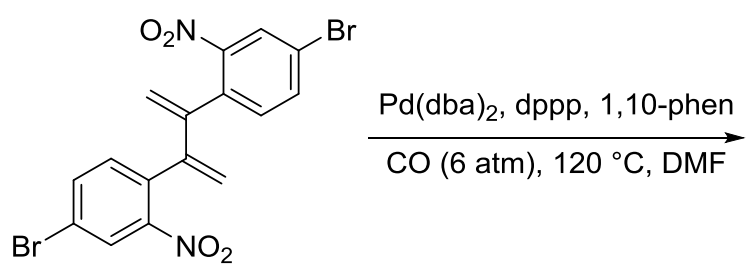

$119(82 \%)$

Biindole 120 was percieved to be a suitable precursor to the hexabromo substituted natural product 105 . In the event, treatment of $\mathbf{1 2 0}$ with 5 equivalents of NBS in a THF-dichloromethane solvent mixture gave a separable mixture of the expected product 105 along with a significant amount of 2,2',5,6,6'pentabromo-bi-1H-indole 121 (Scheme 3.8). Disappointingly, increasing the amount of NBS to eight equivalents gave roughly the same product ratio but in a much diminished yield (Scheme 3.8). In addition, an attempted bromination of the isolated pentabromobiindole $\mathbf{1 2 1}$ using three equivalents of NBS was unsuccessful. The starting material was recoved in high yield (94\%) after chromatography.

Scheme 3.8 Polybromination of $\mathbf{1 2 0}$<smiles>Brc1ccc2c(-c3c[nH]c4cc(Br)ccc34)c[nH]c2c1</smiles>

120

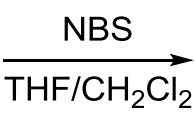

NBS (5 Equiv.), $\mathrm{CH}_{2} \mathrm{Cl}_{2} / \mathrm{THF}$

NBS (8 Equiv.), $\mathrm{CH}_{2} \mathrm{Cl}_{2} / \mathrm{THF}$<smiles>Brc1ccc2c(-c3c(Br)[nH]c4cc(Br)c(Br)cc34)c(Br)[nH]c2c1</smiles>

$121(59 \%)$

$121(11 \%)$<smiles>Brc1cc2[nH]c(Br)c(-c3c(Br)[nH]c4cc(Br)c(Br)cc34)c2cc1Br</smiles>

$105(47 \%)$

$105(13 \%)$ 
Reports for direct 2,6-dibromination and 2,5,6-tribromination of 3-substituted indoles can be found in the

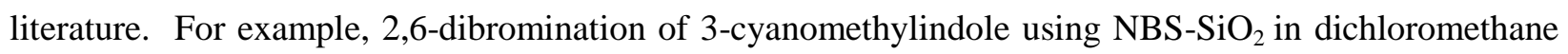
gave 2,6-dibromo-3-cyanomethylindole (81\%), 2,5-dibromo-3-cyanomethylindole (3\%), and 2,4dibromo-3-cyanomethylindole (5\%). ${ }^{116}$ 3-Methylindole afforded selectively 2,6-dibromo-3-methylindole using $\mathrm{NBS}_{-} \mathrm{SiO}_{2}$ in dichloromethane ${ }^{117}$ and 3-phenylindole using NBS in acetic acid furnished a 6:1 ratio of 2,6-dibromo-3-phenylindole and 2,5-dibromo-3-phenylindole. ${ }^{118}$ In contrast, a 3:4 ratio of 2,6: 2,5dibromination was observed from brominations of methyltryptophan employing NBS in AcOH/HCOOH. ${ }^{119}$ Tribromination of 3-methylindole with bromine in acetic anhydride gave 2,5,6tribromo-3-methyl- $N$-acetylindole. ${ }^{120}$ Based on these previously reported brominations, polybrominations of the parent 3,3'-biindole 56 to give either 104 or 105, depending on the stoichiometry of the reagents, were also pursued. Thus, 3,3'-biindole 56 was treated with 4.0, 5.1, and 8.0 equivalents of NBS in THF/dichloromethane (Scheme 3.9). The brominations proved to be substantially more difficult and only low yields of product(s) were obtained. Using 4.0 equivalents of NBS gave a mixture of $\mathbf{1 0 4}$ and 121 both in $8 \%$ isolated yield. Increasing the amount of NBS to 5.1 equivalents eliminated the formation of biindole 104 but gave instead pentabrominated and hexabrominated compounds 121 and 105, respectively. Finally, treatment of $\mathbf{5 6}$ with 8.0 equivalents of NBS gave $\mathbf{1 0 5}$ in $16 \%$ yield. No other products were isolated from the three reactions of $\mathbf{5 6}$.

Scheme 3.9 Bromination of 3,3-bi-1H-indole 56

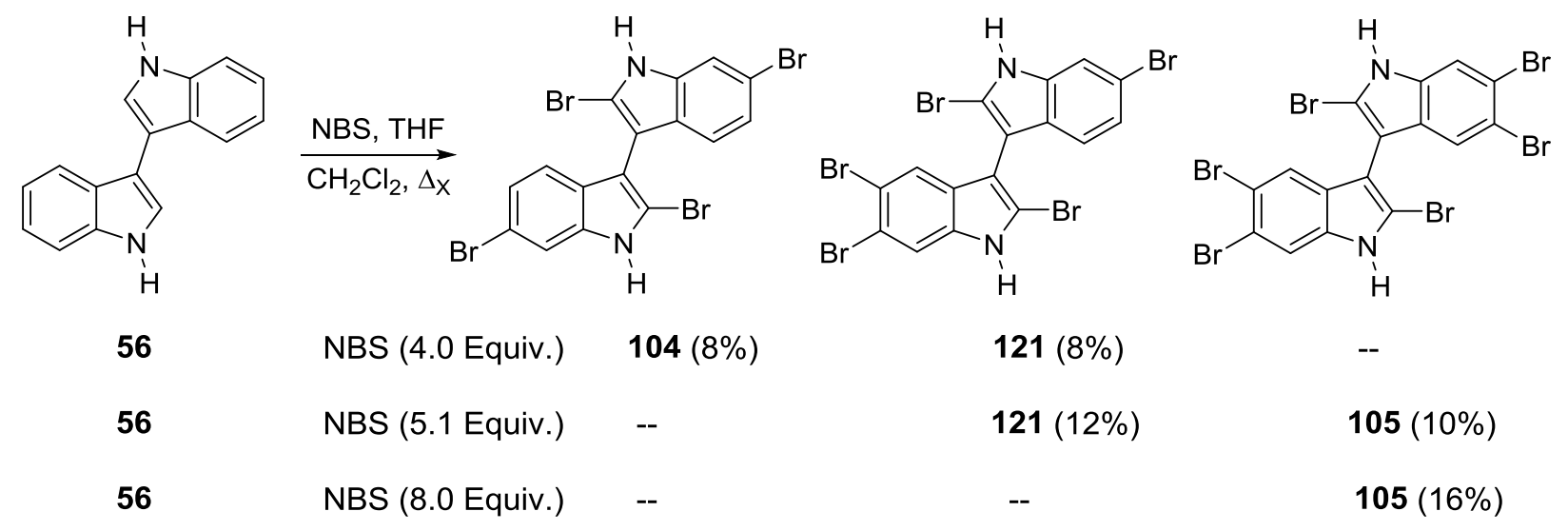




\section{C Conclusion}

The naturally occurring polybrominated indoles 2,2',5,5'-tetrabromo-3,3'-bi-1H-indole, 2,2',6,6'tetrabromo-3,3'-bi-1H-indole, and 2,2',5,5',6,6'-hexabromo-3,3'-bi-1H-indole were synthesized for the first time using a palladium catalyzed, carbon monoxide mediated, double $N$-heterocyclization of 2,3bis(2-nitro-4(or 5)-bromophenyl)-1,4-butadienes as the key indolization step. The synthetically derived compounds were in all aspects identical to the biindoles isolated from the natural sources. 


\section{Chapter 4 A facile base-mediated synthesis of $N$-alkoxy-substituted benzimidazoles}

\section{A Introduction}

Benzimidazoles are a large class of heterocyclic aromatic compounds consisting of a benzene ring fused to imidazole. The benzo derivative of imidazole is referred to as benzimidazole. The parent compound of the series is commonly called benzimidazole although other name such as 1,3-benzodiazole is often used.

Our group has reported the synthesis of a series of $N$-substituted 2-nitrobenzenamines. We were proposing to use compound $\mathbf{1 2 3}$ as a precursor to quinolines via the palladium catalyzed, carbon monoxide, mediated reductive $N$-heterocyclization. With this in mind, compound 122 was dissolved in dimethylsulfoxide (DMSO) and the solution was added to a suspension of sodium hydride (NaH) in DMSO at an ambient temperature. After stirring the mixture for $1 \mathrm{~h}$, the resulting solution was cooled to 0 ${ }^{\circ} \mathrm{C}$ and methyl iodide (MeI) was added. After slowly warming to ambient temperature over $1 \mathrm{~h}$, the reaction mixture was worked up and purified. To our surprise, the expected $N$-methylated product $\mathbf{1 2 3}$ was not observed in the crude; instead 1-methoxybenzimidazole (124), 1-methoxy-2-(2-methoxy-2propyl)benzimidazole (125), and 1-methoxy-2-(2-hydroxy-2-propyl)benzimidazole (126) were obtained in 5\%, 57\%, and 15\% yield, respectively (Scheme 4.1). Cyclization of $N$-phenacyl-2-nitroaniline ${ }^{121}$ and $N$ benzyl-2-nitroanilines to give $N$-oxygenated benzimidazoles ${ }^{122}$ has been reported.

Scheme 4.1 Formation of benzimidazoles 124-126 from enamine 122

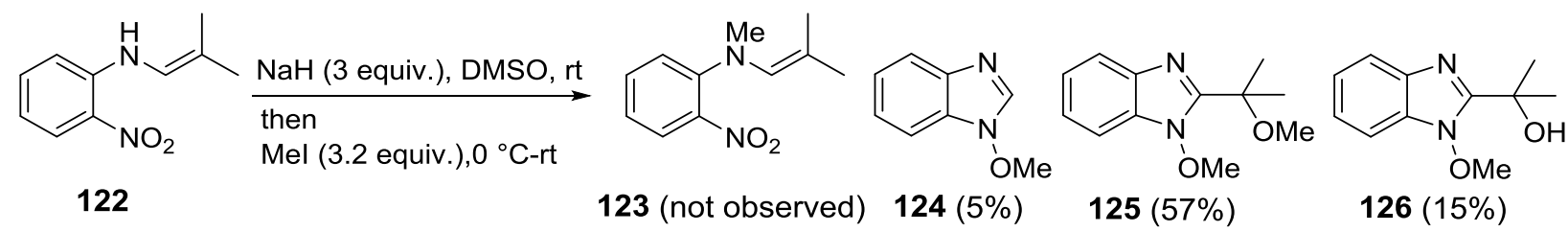

The formation of the observed products in the preceding reaction can be accounted for based on the methodologies developed by Gardiner et al for the formation of 2 -substituted $N$-alkoxybenzimidazoles from 2-nitrobenzeneamines and organic halides in the presence of a base and by Popov and Kryshtalyuk for the formation of 2-substituted $N$-hydroxybenzimidazoles from 2-nitrobenzeneamines (Scheme 4.2). ${ }^{123,124} \mathrm{~N}$-alkoxybenzimidazles can also be prepared from $N$-hydroxybenzimidazoles either via a Williamson type ether synthesis ${ }^{125}$ or by a Mitsunobu reaction ${ }^{126}$ with an alcohol. The reaction of 122 to give 124-126 differs in that either a loss of or oxygenation of the side chain is observed. The formation of compounds 125 and 126 was particularly interesting in the sense that no synthetic routes to $\mathrm{N}$-alkoxy-2(alkoxyalkyl)benzimidazoles or $\mathrm{N}$-alkoxy-2-(hydroxyalkyl)benzimidazoles have been reported to date. This chapter presents detailed studies of the scope and limitation of the base mediated cyclization of 
enamines to give $N$-oxygenated benzimidazoles. A plausible mechanism has also been included. $N$ Oxygenated benzimidazoles have been shown to exhibit anti-protozoa ${ }^{127}$ and anti-HIV activities. ${ }^{123 d}$

Scheme 4.2 Formation of 2-ethenyl- $N$-propoxybenzimidazole ${ }^{123 f}$

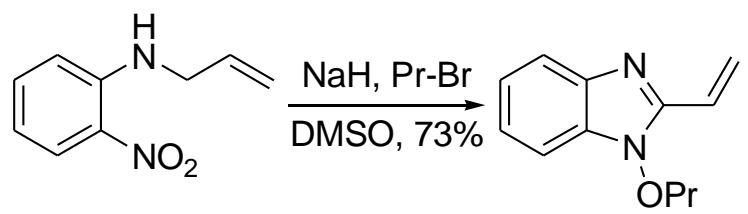

\section{B Results and Discussion}

In the reaction above, 3 equivalents of base and 3.2 equivalents of MeI were used to give rise to products 124-126. With the hope to control the selectivity of the reaction, we carried out a screening of the reaction conditions by varying the amounts of base and the electrophiles. We chose compound $\mathbf{1 2 7}$ for this purpose because it contains a methoxy group on the aromatic ring that may be useful to determine the product ratios in the crude reaction. As can be seen in table 4.1 (entries 1-3), increasing the amount of MeI while keeping the amount of base the same did not improve the reaction in favor of any of the products and the starting material was recovered in each case. Products 128 and 130 were inseparable by column chromatography; the approximate ratios of these products were determined by ${ }^{1} \mathrm{H}$ NMR. Gratifyingly, a significant improvement was observed when the amount of base was increased to 2.10 equivalents with 1.05 equivalents of MeI (table 4.1, entry 4). However, a mixture of products was observed upon increasing the amount of MeI while keeping the amount of base the same (table 4.1, entries 5-6). Finally, using 3.15 equivalents of both $\mathrm{NaH}$ and $\mathrm{MeI}$ resulted in a clean conversion of 127 to 129 with only trace amounts of the other two benzimidazoles observed in the ${ }^{1} \mathrm{H}$ NMR of the crude reaction mixture. Other possible solvents were also examined for the cyclization, however only a low yield of 129 was obtained. So, we concluded that DMF, NMP, and MeCN were not a good suit to this reaction. 
Table 4.1 Condition screening

\begin{tabular}{|c|c|c|c|c|c|c|}
\hline Entry & Eq. NaH & Eq. MeI & Solvent & \multicolumn{3}{|c|}{ Alkoxybenzimidazoles $^{a}$} \\
\hline $1^{b}$ & 1.05 & 1.05 & DMSO & $128^{\mathrm{c}}$ & -- & -- \\
\hline $2^{\mathrm{d}}$ & 1.05 & 2.10 & DMSO & $128(1 \%)$ & -- & $130(34 \%)$ \\
\hline $3^{\mathrm{e}}$ & 1.05 & 3.17 & DMSO & $128(3 \%)$ & -- & $130(30 \%)$ \\
\hline 4 & 2.10 & 1.05 & DMSO & $128(2 \%)$ & 129 tr. & $130(74 \%)$ \\
\hline 5 & 2.10 & 2.10 & DMSO & $128(5 \%)$ & $129(17 \%)$ & $130(56 \%)$ \\
\hline 6 & 2.10 & 3.16 & DMSO & $128(7 \%)$ & $129(18 \%)$ & $130(46 \%)$ \\
\hline 7 & 3.15 & 3.15 & DMSO & $128^{\mathrm{c}}$ & $129(69 \%)$ & $130^{c}$ \\
\hline 8 & 3.75 & 3.33 & DMSO & $128(5 \%)$ & $129(64 \%)$ & $130(2 \%)$ \\
\hline 9 & 5.0 & 5.0 & DMSO & $128^{c}$ & $129(65 \%)$ & -- \\
\hline 10 & 3.74 & 3.20 & DMF & $\mathbf{1 2 8}(21 \%)$ & $129(8 \%)$ & -- \\
\hline 11 & 3.75 & 3.32 & NMP & $128^{c}$ & $129(2(\%)$ & -- \\
\hline 12 & 3.74 & 3.20 & $\mathrm{MeCN}$ & $\mathbf{1 2 8}(65 \%)$ & $\mathbf{1 2 9}(13 \%)$ & -- \\
\hline
\end{tabular}

a) Isolated yields of products after chromatography unless stated as $\sim \%$. The $\sim \%$ are yields calculated from the ${ }^{1} \mathrm{H}$ NMR spectrum of an inseparable mixture. b) The starting material was recovered in $48 \%$ yield. c) Trace amount of the compound was observed in the ${ }^{1} \mathrm{H}$ NMR of the crude reaction mixture. d) The starting material was recovered in $40 \%$ yield. e) The starting material was recovered in $35 \%$ yield.

Based on the results from table 4.1, we devised two set of reaction conditions. Experiments using 3.15 equivalents of both $\mathrm{NaH}$ and $\mathrm{MeI}$ to afford dimethylated benzimidazoles are referred to as Conditions $\mathrm{A}$ (See Table 4.1, entry 7); reactions using 2.1 equivalents of $\mathrm{NaH}$ and 1.05 equivalents of MeI to give monomethylated benzimidazoles as the major product are referred to as Conditions B (See Table 4.1, entry 4). The scope and limitation of the cyclization to give $N$-oxygenated benzimidazoles were probed using fifteen different enamines in total and the results are summarized in Table 4.2. Ten of the enamines were previously reported and five new were prepared by condensation of the appropriately substituted 2nitroaniline with an aldehyde in dichloromethane or benzene in the presence of molecular sieves. Enamines derived from condensation of 2-nitroanilines bearing a substituent in the 4- or 5- position and 3-methylpropanal were selected in order to evaluate the effects of electronic properties and the relative position of the substituent on the benzene ring in a systematic fashion without a significant steric 
contribution (Table 4.2, entries 1-25). The enamines were tested under both Conditions A and Conditions $\mathrm{B}$ and the results are summarized in Table 4.2. Although we initially considered acetonitrile and ratio of the reagents (Table 4.1, entry 12) to be plausible pathways for the synthesis of $2 \mathrm{H}-\mathrm{N}$ alkoxybenzimidazoles, complex mixtures of products in inferior yields were obtained when applied to other enamines.

As can be seen in Table 4.1, all substrates except 4-nitro-substituted enamine 144 furnished $N$ methoxybenzimidazoles under Condition A. Enamines 127, 131, 133, and 136 containing methoxy-, chloro-, bromo- and fluoro-substituent respectively at the 4-position gave good to excellent yields (6994\%) of dimethylated benzimidazoles as the sole product isolated after chromatography (Table 4.2, entries 1-8). In each of these reactions, an immediate color change to deep purple was observed upon mixing the enamine with the base in DMSO. In most cases, the purple color rapidly changed to brown or orange-brown within two minutes. The appearance of brown color was an indicative of a resting state of the reaction and no further reaction would occur until MeI had been added. For example, addition of MeI either after 2 minutes or $9 \mathrm{~h}$ of stirring at ambient temperature gave almost identical isolated yield of product 129 (entries 2-3). Similar color changes were observed for all substrate types although longer times were required in some cases before the purple color would disappear.

Apart from the selectivity observed for the first four substrates in Table 4.2, the rest of enamines gave either $2 \mathrm{H}$-unsubstituted $\mathrm{N}$-methoxybenzimidazoles, mixtures of $\mathrm{N}$-methoxybenzimidazoles, or $\mathrm{N}$ methylation of the starting material. The parent substrates 122, 138 and 153 gave rise to mixtures of three benzimidazoles when MeI was added at $0{ }^{\circ} \mathrm{C}$ (entries 9, 11, 24). In contrast monoalkylated benzimidazoles were not observed when MeI was added at ambient temperature (entries 10, 12, 15). The reason for this product distribution is not clear.

Two substrates containing an electron withdrawing group at the 4-position of the aromatic ring were also examined (Table 4.2, entries 13-16). Reaction of ester functionalized substrate 142 gave $N$-methylation product in $62 \%$ isolated yield when MeI was added at ambient temperature. In this reaction no sign of benzimidazoles was observed in the crude. However, when MeI was added after mixing the reaction mixture for $24 \mathrm{~h}$ at ambient temperature, $N$-methoxybenzimidazole was isolated as the only product in good yield. No trace of a 2-substituted product was observed in the crude reaction mixture. Reaction of 144 in a similar fashion gave $N$-methylated product in $85 \%$ isolated yield as the sole product. Extending the reaction time for the nitro analog 144 prior to addition of MeI did give neither benzimidazole, or any other product nor was the starting material observed in the crude reaction mixture. $N$-methylation was not limited to substrates containing electron withdrawing groups on the aromatic ring, but it was observed 
with 5-methoxy- and 5-chloro-substituted enamines 145 and 147 when insufficient time was given before the mixing of MeI.

Two additional enamines 157 and 160 were also tested. The methoxy-substituted enamine 157 behaved similar to 127, affording the dialkylated produt 158. In addition, a minor amount of the monoalkylated product 159 was isolated upon addition of MeI at ambient temperature (entry 27). Enamine 160 proved to be remarkably sensitive to temperature. All three $N$-oxygenated benzimidazoles were obtained in low yield at $0{ }^{\circ} \mathrm{C}$. In contrast, only $\mathbf{1 3 4}$ was obtained when the reaction was carried out at ambient temperature.

Table 4.2 Base-mediated synthesis of benzimidazoles under Condition A

\section{Condition A Alkoxybenzimidazoles ${ }^{b}$}

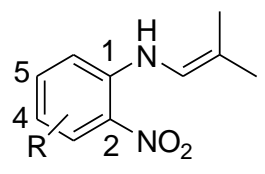

\section{Entry Enamine}

$1 \quad 127(\mathrm{R}=4-\mathrm{OMe})$

2

3

4 5

$$
6
$$$$
7
$$

$10 \quad \mathbf{1 3 8}$

$11 \quad 122(\mathrm{R}=\mathrm{H})$

$12 \quad \mathbf{1 2 2}$

$13142\left(\mathrm{R}=4-\mathrm{CO}_{2} \mathrm{Me}\right)$

$14 \quad 142$

$15 \quad 23\left(\mathrm{R}=4-\mathrm{NO}_{2}\right)$

$16 \quad 144$

17

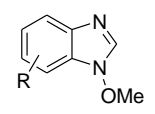

Time/Temp ${ }^{\mathrm{a}}$

$1 \mathrm{~h}-0{ }^{\circ} \mathrm{C}$

2 min-rt

9 h-rt

$1 \mathrm{~h}-0{ }^{\circ} \mathrm{C}$

1 h-rt

$1 \mathrm{~h}-0{ }^{\circ} \mathrm{C}$

4 min-rt

1 h-rt

$1 \mathrm{~h}-0{ }^{\circ} \mathrm{C}$

1 h-rt

$1 \mathrm{~h}-0{ }^{\circ} \mathrm{C}$

$1 \mathrm{~h}-\mathrm{rt}$

10 min-rt $^{\mathrm{f}}$

24 h-rt

$1 \mathrm{~h}-0{ }^{\circ} \mathrm{C}^{\mathrm{g}}$

24 h-rt

1 h-rt
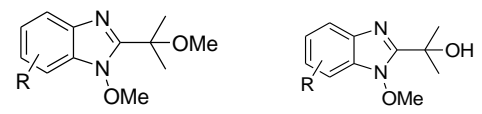

$\%$ Yield

$\%$ Yield

$\%$ Yield

$\sum(\%)^{\mathrm{c}}$

$128^{\mathrm{d}}$

129 (71)

71

129 (71)

71

$128^{\mathrm{d}}$

129 (69)

69

132 (86)

86

132 (94)

94

$134^{\mathrm{d}} \quad 135(78)$

78

$134^{\mathrm{d}} \quad 135(93)$

93

137 (75)

75

139 (28)

140 (52)

141 (5)

85

139 (27) 140 (68)

95

$124(5) \quad 125$ (57)

$126(15)$

77

124 (31) 125 (34)

65

143 (74)

74 


\begin{tabular}{|c|c|c|c|c|c|c|}
\hline 18 & $145^{j}$ & 24 h-rt & $146(51)$ & & & 51 \\
\hline 19 & $147(\mathrm{R}=5-\mathrm{Cl})$ & 6 min- $\mathrm{rt}^{\mathrm{k}}$ & $149(9)$ & & & 9 \\
\hline 20 & 147 & $1 \mathrm{~h}-0{ }^{\circ} \mathrm{C}$ & $148(56)$ & $149(27)$ & & 83 \\
\hline 21 & 147 & $1 \mathrm{~h}-\mathrm{rt}$ & $148(52)$ & $149(31)$ & & 83 \\
\hline 22 & $150(\mathrm{R}=5-\mathrm{Br})$ & 10 min-rt & $151(67)$ & $152(28)$ & & 95 \\
\hline 23 & 150 & $1 \mathrm{~h}-\mathrm{rt}$ & $151(55)$ & $152(29)$ & & 84 \\
\hline 24 & $153(\mathrm{R}=5-\mathrm{Me})$ & $1 \mathrm{~h}-0{ }^{\circ} \mathrm{C}$ & $154(21)$ & $155(22)$ & $156(20)$ & 63 \\
\hline 25 & 153 & 10 min-rt & $154(31)$ & $155(26)$ & & 57 \\
\hline & $157(\mathrm{R}=4-\mathrm{OMe})$ & & & 158 & 159 & \\
\hline 26 & 157 & $1 \mathrm{~h}-0{ }^{\circ} \mathrm{C}$ & $128^{d}$ & $158(78)$ & & 78 \\
\hline 27 & 157 & 2 min-rt & $128^{d}$ & $158(84)$ & $159(11)^{1}$ & 95 \\
\hline & $160(\mathrm{R}=\mathrm{Br})$ & & 134 & 161 & 162 & \\
\hline 28 & 160 & $1 \mathrm{~h}-0{ }^{\circ} \mathrm{C}$ & $134(22)$ & $161(22)$ & $162(13)$ & 57 \\
\hline 29 & 160 & 38 min-rt & $134(92)$ & & & 92 \\
\hline
\end{tabular}

a. 3.15 equivalents of $\mathrm{NaH}$ and $\mathrm{MeI}$ were used. The Time/Temp is the time elapsed before addition of MeI and the temperature of the reaction mixture for the addition.

b. Isolated yields of pure products after chromatography unless stated as $\sim \%$. The $\sim \%$ are yields calculated from ${ }^{1} \mathrm{H}$ NMR spectrum of an inseparable mixture.

c. Total yield of benzimidazoles isolated

d. Trace amount of the compound was observed in the ${ }^{1} \mathrm{H}$ NMR of the crude reaction mixture.

e. Trace amount of $\mathbf{1 3 8}$ was observed in the ${ }^{1} \mathrm{H}$ NMR spectrum.

f. 4-Carbomethoxy- $N$-methyl- $N$-(2-methyl-1-propen-1-yl)-2-nitroaniline, the $N$-methylation product of 142 was isolated in $62 \%$ yield.

g. 2,4-Dinitro- $N$-methyl- $N$-(2-methyl-1-propen-1-yl)aniline, the $N$-methylation product from 144, was isolated in $85 \%$ yield. 
h. No product or unreacted 144 was observed.

i. 5-Methoxy-2-nitro- $N$-methylaniline ${ }^{128}$ was isolated in $55 \%$ yield.

j. 5.0 Equivalents of $\mathrm{NaH}$ and 3.9 equivalents of MeI were used.

k. 5-Chloro- $N$-methyl- $N$-(2-methyl-1-propen-1-yl)-2-nitroaniline, the $N$-methylation product of 147, was isolated in $52 \%$ yield.

1. Trace amount of $\mathbf{1 2 8}$ was observed in the ${ }^{1} \mathrm{H}$ NMR spectrum.

Finally, feasibility of formation of mono-alkylated benzimidazoles was evaluated next using Conditions B. Under this condition as well all enamines but except 4-nitro-substituted enamine 144, gave the expected products. In general, reactions under this condition were not as selective as the reaction of the 4methoxy-substituted enamine 127 to give 130 (See Table 4.1, entry 4 and Table 4.3, entry 1). However, cyclization to produce benzimidazoles was observed in all cases. For all enamines except 4carbomethoxy- and the 5-methoxy-substituted compounds 142 and $\mathbf{1 4 5}$, the reaction went on smoothly to give monoalkylated benzimidazoles in $45-74 \%$ yields as the major product in addition to minor amounts of either $2 \mathrm{H}-\mathrm{N}$-methoxybenzimidazoles $(2-21 \%)$ or dimethylated benzimidazoles (15-22\%). Under conditions $\mathrm{B}$, the position and the electronic properties of the substituents on the aromatic ring had little effect on the product distribution. Similar to Condition A, enamines 142 and 145 gave rise to $2 H-N$ methoxybenzimidazoles.

Table 4.3 Base-mediated synthesis of bezimidazoles under Condition B

\section{Alkoxybenzimidazoles ${ }^{b}$}

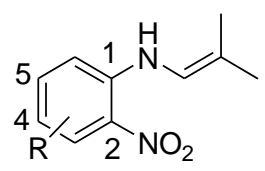

Entry $^{\mathrm{a}}$

Enamine

1

2

3

4

5

6

7
$122(\mathrm{R}=\mathrm{H})$

$123(\mathrm{R}=4-\mathrm{OMe})$

$131(\mathrm{R}=4-\mathrm{Cl})$

$133(\mathrm{R}=4-\mathrm{Br})$

$136(\mathrm{R}=4-\mathrm{F})$

$138(\mathrm{R}=4-\mathrm{Me})$

139 (11)

128 (2)

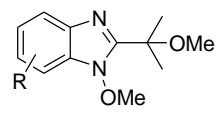

\% Yield

132 (20)

135 (22)

137 (15)
$\%$ Yield

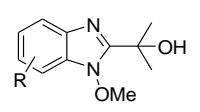

$\%$ Yield

$126(55)^{\mathrm{d}}$

130 (74)

163 (59)

164 (68)

165 (56)

141 (45) $\sum(\%)^{\mathrm{c}}$

55

76

79

90

72

55 


\begin{tabular}{|c|c|c|c|c|}
\hline 8 & $142^{\mathrm{f}}$ & $143(73)$ & & 73 \\
\hline 9 & $145(\mathrm{R}=5-\mathrm{OMe})^{\mathrm{g}}$ & & & - \\
\hline 10 & $145^{\mathrm{f}}$ & $146(64)$ & & 64 \\
\hline 11 & $147(\mathrm{R}=5-\mathrm{Cl})$ & 149 (15) & $166(55)$ & 70 \\
\hline 12 & $140(\mathrm{R}=5-\mathrm{Br})$ & $152(16)$ & $167(63)$ & 79 \\
\hline 13 & $153(\mathrm{R}=5-\mathrm{Me})$ & & $156(61)$ & 61 \\
\hline 14 & $157(\mathrm{R}=4-\mathrm{OMe})$ & 158 (19) & $159(51)$ & 70 \\
\hline 15 & $160(\mathrm{R}=4-\mathrm{Br})$ & $134(22)$ & $162(52)$ & 74 \\
\hline
\end{tabular}

a. Condition B: 2.1 equivalents of $\mathrm{NaH}$ and 1.05 equivalents of MeI. The mixture was stirred for $1 \mathrm{~h}$ at ambient temperature then cooled to $0{ }^{\circ} \mathrm{C}$ prior to addition of $\mathrm{MeI}$ unless otherwise stated.

b. Isolated yields of pure products after chromatography unless otherwise stated. The \% yields are calculated from the ${ }^{1} \mathrm{H}$ NMR spectrum of an inseparable mixture.

c. Total yield of benzimidazoles.

d. Trace amount of $\mathbf{1 2 4}$ was observed in the ${ }^{1} \mathrm{H}$ NMR spectrum.

e. A nearly 3:1 mixture of 4-carbomethoxy- $N$-methyl- $N$-(2-methyl-1-propen-1-yl)-2-nitroaniline and 142 was observed in the ${ }^{1} \mathrm{H}$ NMR spectrum of the crude reaction mixture.

f. The base substrate mixture was stirred for $24 \mathrm{~h}$ before addition of MeI.

g. Neither product nor starting material was observed.

The formation of $2 \mathrm{H}-\mathrm{N}$-alkoxybenzimidazoles in the above reactions involves a carbon-carbon bond fission during the reaction and a loss of three carbon unit probably in the form of acetone (see mechanism below). However, neither acetone nor acetophenone or cyclohexanone, the anticipated by-products from enamines 157 and 160 were recovered from the reactions. This is probably due to evaporative loss upon removal of solvents. With a hope to isolate, characterize and quantify any potential by-product derived from carbon-carbon bond cleavage, enamine $\mathbf{1 6 8}$ was prepared. The enamine was subjected to reaction 
under Conditions A and a small amount of benzophenone (170) was obtained along with the expected product 169. Gratifyingly, when the base and the solvent system described by Gardiner et al ${ }^{123 b}(t$ $\mathrm{BuOK} / t-\mathrm{BuOH}$ ) was used, an almost quantitative yield of benzophenone was isolated in addition to the product $\mathbf{1 2 8}$ in $\mathbf{7 8 \%}$ yield (Scheme 4.3 ).

Scheme 4.3 Formation of $N$-methoxybenzimidazole 169 and benzophenone 170<smiles>COc1ccc(NC=C(c2ccccc2)c2ccccc2)c([N+](=O)[O-])c1</smiles>

A small selection of other carbon-based electrophiles including benzyl bromide, allyl bromide, propargyl bromide, 3-bromo-1-butyne, diiodomethane, and acetyl chloride were also employed with enamine 127. Moderate yields of products were obtained. The result from acetyl chloride is presented in Scheme 4.4.

Scheme 4.4 Reaction of 127 with Acetyl Chloride<smiles>C=C(C)c1nc2ccc(OC)cc2n1OC(=O)OC(C)(C)C(=O)OC(C)(C)c1nc2ccc(OC)cc2n1OC(=O)O</smiles> 
Table 4.4 Base-mediated synthesis of $N$-oxygenated benzimidazoles from enamine 127

\begin{tabular}{llllll}
\hline Entry & Time/Temp $^{\mathrm{a}}$ & Electrophile (R-X) & Alkoxybenzimidazoles & \\
\hline \\
3
\end{tabular}

a. Condition A: 3.15 equivalents of $\mathrm{NaH}$ and $\mathrm{R}-\mathrm{X}$. The Time/Temp is the time before addition of electrophile $(\mathrm{R}-\mathrm{X})$ and addition temperature.

b. Isolated yields of pure products after chromatography unless otherwise stated. The yields are calculated from the ${ }^{1} \mathrm{H}$ NMR spectrum of an inseparable mixture.

c. Total yield of benzimidazole(s) isolated.

A limitation of this methodology is the use of enamines derived from condensation of alpha-branched aldehydes and 4- or 5-substituted-2-nitroanilines. Attempts to prepare enamines from simple and unbranched aliphatic aldehydes, for example propanal and hexanal, were unsuccessful. In addition, 3- and 6-substituted 2-nitrobenzenamines did not form any enamines using the conditions described. Only unreacted starting materials were obtained from the reactions.

\section{C Mechanistic Discussion}

The formation of $\mathrm{N}$-oxygenated benzimidazoles in the above transformations can be mechanistically understood as follows. Deprotonation of 122 forms anions of type $\mathbf{1 8 3}$ and 184. 1,7-Electrocyclization of 184 will give 185 which in turn may undergo ring opening to form nitroso-imine 186. 1,5Electrocyclization of $\mathbf{1 8 6}$ to $\mathbf{1 8 7}$ is plausible. Aromatization of intermediate 187 would furnish alkoxybenzimidazole 188 by the loss of acetone or dialkoxybenzimidazole 189 via deprotonation. It is 
unclear when the alkylation is taking place; it is possible that alkylation can occur both prior to and after aromatization. The proposed mechanism hints that two equivalents of a base will be required for the transformation of $\mathbf{1 2 2}$ to $\mathbf{1 2 5}$ and 126; one for the initial deprotonation to give 183/184 and another for deprotonation-aromatization of $\mathbf{1 8 7}$ to give 189. Although 2.10 equivalents of base proved optimal for the formation of monoalkylated products, it remained unclear why an addition equivalent of the base was required to maximize the yield of dialkylated products.

Scheme 4.5 Proposed mechanism for the $N$-oxygenated benzimidazoles 124-126

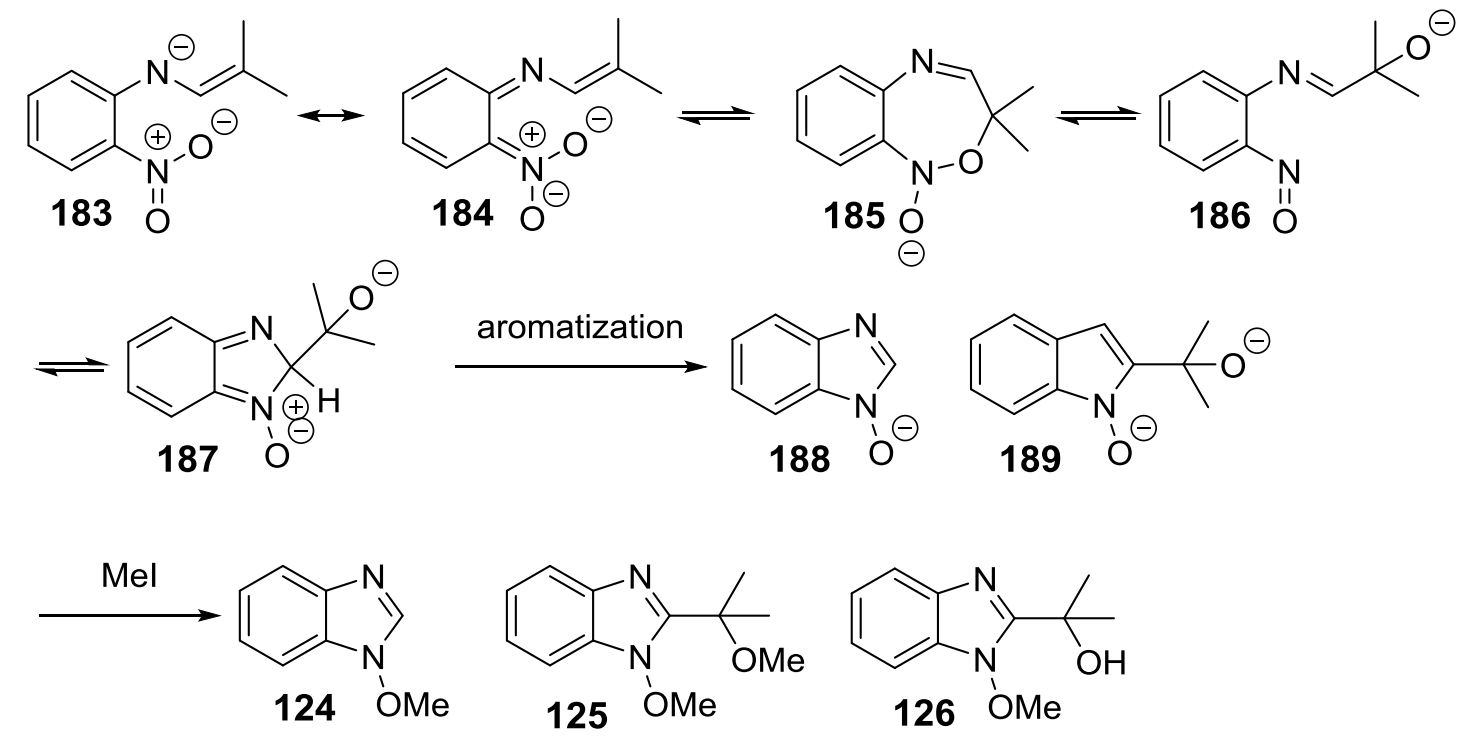

The mechanism outlined here is supported by previous studies in the literature. ${ }^{129}$ In the preparation of indazole- $N$-oxide from azomethine ylides, Nyerges et al proposed 1,7-electrocyclization. ${ }^{130,131}$ The authors suggested a ring-contraction with loss of formaldehyde followed by a nitroso-imine 1,5electrocyclization. We have reported a related palladium catalyzed reductive cyclization of 2nitrostyrenes in the presence of base to give oxygenated indoles. ${ }^{132}$

\section{D Conclusion}

We have developed a mild base mediated cyclization of enamines derived from the condensation of 2nitroanilines with $\alpha$-branched aldehydes to give $N$-alkoxy-substituted benzimidazoles with or without an oxygenated side chain in the second position. A large number of carbon-based electrophiles can be employed. 


\section{Chapter 5 A facile base-mediated synthesis of $\mathrm{N}$-alkoxy-2H-benzimidazoles}

\section{A Introduction}

The methodology presented here can be viewed as a modification of the synthesis of $\mathrm{N}$-alkoxy -2substituted benzimidazoles discussed in chapter 4. Reaction of 2-nitrobenzenamines with an electrophile in the presence of sodium hydride in dimethylsulfoxide or acetonitrile is not specific giving rise to a mixture of 2-substituted $\mathrm{N}$-alkoxybenzimidazoles. The product distribution was very much dependent on the amount of base used. For example, treatment of enamine 127 with MeI in dimethylsulfoxide (DMSO) in the presence of sodium hydride $(\mathrm{NaH})$ with varying amount of base gave the following products (Scheme 5.1). ${ }^{136}$

Scheme 5.1 Reaction of enamine 127 with methyl iodide

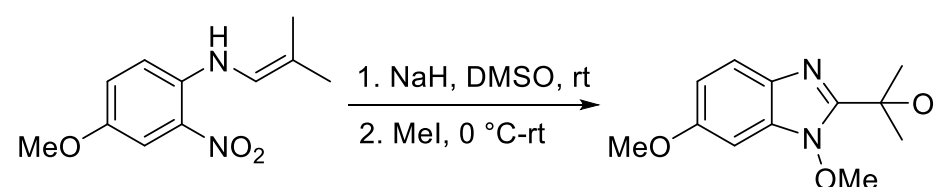

1272.10 equiv. $\mathrm{NaH} / 1.05$ equiv. Mel

1273.50 equiv. $\mathrm{NaH} / 3.50$ equiv. Mel
$130(74 \%)$

$130(-)$

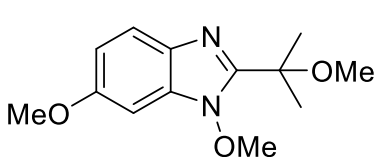

$129(-)$

$129(71 \%)$

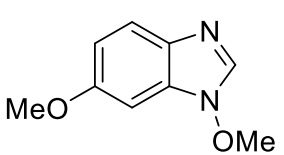

$128(2 \%)$

128 (trace)

In the course of our investigation for suitable reaction conditions, we found that the reaction can be tailored to give $\mathrm{N}$-alkoxy- $2 \mathrm{H}$-benzimidazole as the sole product when enamines are allowed to react with potassium $t$-butoxide $(t$-BuOK) for prolonged period of time followed by the trapping of the electrophile. Herein we report the scope and limitations of the cyclization to give $N$-oxygenated benzimidazoles from reactions of 2-nitrobenzenamines with an array of carbon-centered electrophiles.

\section{B Results and Discussion}

Very limited number of methods have been reported for the synthesis of $N$-alkoxybenzimidazoles. Direct alkylation of $\mathrm{N}$-hydroxybenzimidazole or its tautomer $1 \mathrm{H}$-benzimidazole-3-oxide has been used to prepare $N$-methoxy, $N$-ethoxy and $N$-allyloxybenzimidazoles ${ }^{137}$ Gardiner et al has previously reported the synthesis of $N$-alkoxybenzimidazoles from 2-nitroanilines and an alkyl halide. ${ }^{123}$ However, mixtures of $N$ alkylated anilines and $\mathrm{N}$-alkoxybenzimidazoles were observed in many cases. In addition, only one case of a $2 \mathrm{H}$ - $\mathrm{N}$-alkoxybenzimidazole was reported (Scheme 5.2). 
Scheme 5.2 Gardiner's synthesis of $N$-methoxybenzimidazole

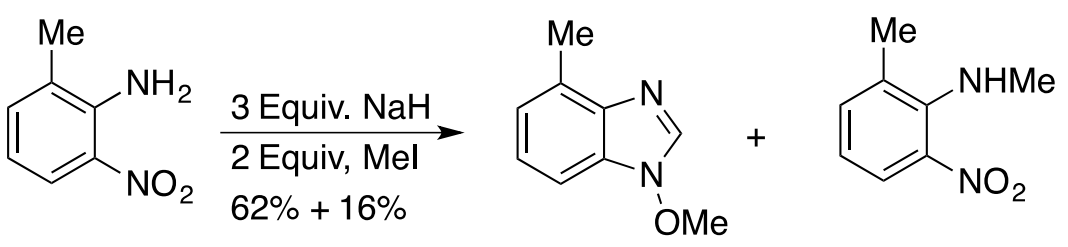

The objective of this work was to develop short and efficient synthetic routes to $2 \mathrm{H}-\mathrm{N}$ alkoxybenzimidazoles from easily accessible starting materials. A detailed study on the screening of reaction conditions and the suitable solvents can be found in chapter 4. Our group has disclosed the synthesis of a variety of $N$-(2-methyl-1-propen-1-yl)-2-nitrobenzenamines. ${ }^{134}$ Following the same procedure, we synthesized 2-nitrobenzenamines and subjected them to the reaction with $t$-BuOK for $24 \mathrm{~h}$ and then trapped the intermediates with the desired carbon-based electrophiles to afford $2 H-N$ alkoxybenzimidazoles in good to excellent yields (Table 5.1). Four equivalents of base and longer reaction time were required for the completion of the reaction. Reaction of 4-carbomethoxy- $N$-methyl- $N$ (2-methyl-1-propen-1-yl)-2-nitrobenzenamine and 2,4-dinitro- $N$-(2-methyl-1-propen-1-yl)benzenamine, however, did give neither the starting materials back nor any identifiable products for the similar reason discussed in the preceding chapter (chapter 4). 
Table 5.1 Formation of $N$-methoxybenzimidazoles from enamines

\begin{tabular}{|c|c|c|}
\hline Entry & Enamine $^{\mathrm{a}}$ & Methoxybenzimidazole ${ }^{b}$ \\
\hline 1 & $127(\mathrm{R}=4-\mathrm{OMe})$ & $128(76 \%)$ \\
\hline 2 & $122(\mathrm{R}=\mathrm{H})$ & $124(100 \%)$ \\
\hline 3 & $138(\mathrm{R}=4-\mathrm{Me})$ & $139(76 \%)$ \\
\hline 4 & $131(\mathrm{R}=4-\mathrm{Cl})$ & $191(92 \%)$ \\
\hline 5 & $133(\mathrm{R}=4-\mathrm{Br})$ & $134(85 \%)$ \\
\hline 6 & $136(R=4-F)$ & $192(67 \%)$ \\
\hline 7 & $142\left(\mathrm{R}=4-\mathrm{CO}_{2} \mathrm{Me}\right)$ & not observed \\
\hline 8 & $144\left(\mathrm{R}=4-\mathrm{NO}_{2}\right)$ & not observed \\
\hline 9 & $145(\mathrm{R}=5-\mathrm{OMe})$ & $146(85 \%)$ \\
\hline 10 & $153(\mathrm{R}=5-\mathrm{Me})$ & $154(71 \%)$ \\
\hline 11 & $147(R=5-C l)$ & $148(71 \%)$ \\
\hline 12 & $150(R=5-B r)$ & $151(73 \%)$ \\
\hline 13 & 190 ( $\mathrm{R}=\mathrm{OMe})$ & $128(94 \%)$ \\
\hline 14 & $160(\mathrm{R}=\mathrm{Br})$ & $134(94 \%)$ \\
\hline 15 & 157 & $128(76 \%)$ \\
\hline 16 & 168 & $128(78 \%)$ \\
\hline
\end{tabular}

a) A solution of the enamine in $t$ - $\mathrm{BuOH}$ was treated with $t$ - $\mathrm{BuOK}$ (4 equiv.) stirred for $24 \mathrm{~h}$ then treated with MeI (3 equiv.) b) Isolated yield of pure product after chromatography on silica gel.

Results from a number of other electrophiles are presented in Table 5.2 below. Of the various electrophiles tested, propargyl bromide and 3-bromo-1-butyne gave allenes instead of the normal products, albeit in low yield. Similarly, less reactive electrophiles, such as, 1-bromoheptane, 1-iodobutane and 2-iodopropane gave low isolated yield of the products. It should be born in mind that the pathway presented here represents the easiest and the cheapest route to access a wide variety of $2 \mathrm{H}-\mathrm{N}$ - 
alkoxybenzimidazoles to date. Clean reaction, easy work up and isolation of compounds will make this methodology useful.

Table 5.2 Base-mediated synthesis of $N$-oxygenated benzimidazoles from enamine $\mathbf{1 2 7}$

\begin{tabular}{|c|c|c|c|}
\hline Entry & Enamine $^{a}$ & Electrophile & Benzimidazole $^{b}$ \\
\hline 1 & & Benzyl bromide & $193(82 \%)$ \\
\hline 2 & & Allyl bromide & $194(100 \%)$ \\
\hline 3 & & 2-Methyl-3-bromopropene & $195(90 \%)$ \\
\hline 4 & & Propargyl bromide & $196(15 \%)$ \\
\hline 5 & $\mathrm{MeO}^{-}$ & 3-Bromo-1-butyne & $197(12 \%)$ \\
\hline 6 & 12 & Acetyl chloride & $198(54 \%)$ \\
\hline 7 & & 1-Bromoheptane & $199(16 \%)$ \\
\hline 8 & & 1-lodobutane & $200(15 \%)$ \\
\hline 9 & & 2-lodopropane & 201 (8\%) \\
\hline
\end{tabular}

a) A solution of the enamine in $t$ - $\mathrm{BuOH}$ was treated with $t$ - $\mathrm{BuOK}$ (4 equiv.) stirred for $24 \mathrm{~h}$ then treated with MeI (3 equiv.) b) Isolated yield of pure product after chromatography on silica gel.

The formation of product can be rationalized via 1,7 -electrocyclization ${ }^{130,131}$ of the azomethine ylide followed by ring opening. The second ring closing step leads to a relatively unstable intermediate that loses the side chain possibly in the form of acetone opening the door for aromatization. The $N$-alkoxy anion then abstracts the electrophile giving rise to $\mathrm{N}$-alkoxybenzimidazole. A detailed mechanistic viewpoint has been discussed in chapter 4 . We have isolated benzophenone from the reaction of $\mathbf{1 6 8}$ with $\mathrm{MeI}$ in the presence of $t$ - $\mathrm{BuOK} / t$-BuOH. ${ }^{136}$

\section{C Conclusion}

In summary, short and efficient synthetic route to a variety of $2 \mathrm{H}-\mathrm{N}$-alkoxybenzimidazoles from easily accessible $N$-(2-methyl-1-propen-1-yl)-2-nitrobenzenamines has been developed for the first time. This methodology presents a useful approach to the formation of NO-C bond. Mild reaction conditions, easy work up and isolation of compound are additional benefits. 


\section{Chapter 6 Chemoselectivity in the Kosugi-Migita-Stille coupling of bromonitrophenyl trifluoromethanesulfonates}

\section{A Introduction}

Palladium catalyzed cross coupling reactions of aromatic halides and trifluoromethanesulfonates have long history of use in the modern organic synthesis. The reactions have undergone extensive modifications over the last four decades. In this chapter will be discussed the shortcomings and recent development related to Stille and Echavarren's study on the relative chemoselectivity of carbon-bromine (C-Br) versus carbon-trifluoromethanesulfonate (C-OTf) in the palladium catalyzed cross couplings of bromophenyl- and bromonitrophenyl- trifluoromethanesulfonates with ethenyltributyltin. Stille and Echavarren disclosed that use of additives and varying reaction conditions can dramatically alter the product distribution. ${ }^{138}$ For example, treatment of 202 with vinyltin in the presence of $\mathrm{Pd}\left(\mathrm{PPh}_{3}\right)_{4}$ in dioxane at $98{ }^{\circ} \mathrm{C}$ gave product derived from highly selective oxidative addition to $\mathrm{C}-\mathrm{Br}$ bond whereas product derived from highly selective oxidative addition to C-OTf bond was obtained when the reaction was carried out using $\mathrm{PdCl}_{2}\left(\mathrm{PPh}_{3}\right)_{2}$ and $\mathrm{LiCl}$ in DMF at $24{ }^{\circ} \mathrm{C} .{ }^{139,140}$ In the same seminal work was reported the formation of products from both 4-iodophenyl trifluoromethanesulfonate and 1-bromo-4iodobenzene via the completely selective oxidative addition to C-I bond. After a careful consideration, they proposed the following reactivity order:

1. $\mathrm{I}>\mathrm{Br}>\mathrm{OTf}>\mathrm{Cl}$ in the presence of $\mathrm{LiCl}$

2. $\mathrm{I}>\mathrm{OTf}>\mathrm{Br}>\mathrm{Cl}$ in the absence of $\mathrm{LiCl}$

However, the proposed reactivity order should not be taken as an absolute scale. The presence of other functional groups in the substrate, catalyst design and reaction conditions may greatly change the reactivity order where product derived from much less selective coupling site predominates. For example, reaction of 4-chlorophenyl trifluoromethanesulfonate with $\mathrm{PhBu}_{3} \mathrm{Sn}$ in the presence of $\mathrm{Pd}_{2}(\mathrm{dba})_{3}-\mathrm{Pd}(t-$ $\left.\mathrm{Bu}_{3}\right)_{2}{ }^{141}$ and $\mathrm{CsF}$ in dioxane at ambient temperature gave exclusive $\mathrm{C}-\mathrm{Cl}$ coupling products. ${ }^{142,143}$ The effect of added $\mathrm{LiCl}$ has been attributed to Ar-Pd-OTf to Ar-Pd-Cl metathesis followed by rapid transmetallation and reductive elimination. Thus, use of $\mathrm{LiCl}$ certainly affects chemoselectivity but literature reports ${ }^{144-149}$ suggest that the magnitude of effect is related to more than one factor. An increase in the rate of oxidative addition cannot be the sole reason behind the change in selectivity for C-Br to COTf bond with the added LiCl. To the best of our knowledge, there are no Kosugi-Migita-Stille couplings reported to date wherein even a fraction of $\mathrm{C}-\mathrm{Br}$ or C-OTf coupling occurred on benzene ring also containing a C-I bond. ${ }^{150}$ 
We have reported the synthesis of a wide variety of indoles, biindoles, and the total synthesis of a number of indole alkaloids via the palladium catalyzed carbon monoxide mediated en route reductive $\mathrm{N}$ heteroannulation of orthonitrostyrene derivatives. ${ }^{45,113}$ We envisioned that chemoselective sequential introduction of two different alkenes to dinitrobenzenes may lead to dialkenyldinitrobenzenes that are the potential precursors to pyrroloindoles. We thought that the chemoselectivity reported by Stille and Echavarren would enable us to prepare a variety of dialkenyldinitrobenzenes that can be used for palladium catalyzed double reductive cyclization.

In order to test the hypothesis, 5-bromo-2,4-dinitrophenyl trifluoromethanesulfonate (204) was prepared in good yield by dinitration of 3-bromophenyl trifluoromethanesulfonate (203). Treatment of 204 with ethenyltributyltin in DMF under Stille-Echavarren conditions discussed above that should result in C-OTf coupling did not give any identifiable product. To simplify possible work up and the analysis of crude ${ }^{1} \mathrm{H}$ NMR, the solvent was changed to toluene and the reaction was carried out at ambient temperature. However, no appreciable reaction was observed. The reaction temperature was then raised to $80{ }^{\circ} \mathrm{C}$ whereby all starting material was consumed within $17 \mathrm{~h}$. The ${ }^{1} \mathrm{H}$ NMR of the crude reaction mixture revealed the presence of one site coupling product. The crude reaction mixture was purified by chromatography on a silica gel to afford $\mathbf{2 0 5}$ in good isolated yield. The product was identified and characterized by ${ }^{1} \mathrm{H},{ }^{19} \mathrm{~F}$ and ${ }^{13} \mathrm{C}$ NMRs. A very indicative quartet $(J=320 \mathrm{~Hz})$ in the ${ }^{13} \mathrm{C}$ NMR and a resonance at $-72.9 \mathrm{~Hz}$ in the ${ }^{19} \mathrm{~F}$ NMR confirmed the product to be $\mathbf{2 0 5}$; the expected C-OTf coupling product was not observed.

Scheme 6.1 Preparation and Kosugi-Migita-Stille coupling of 204

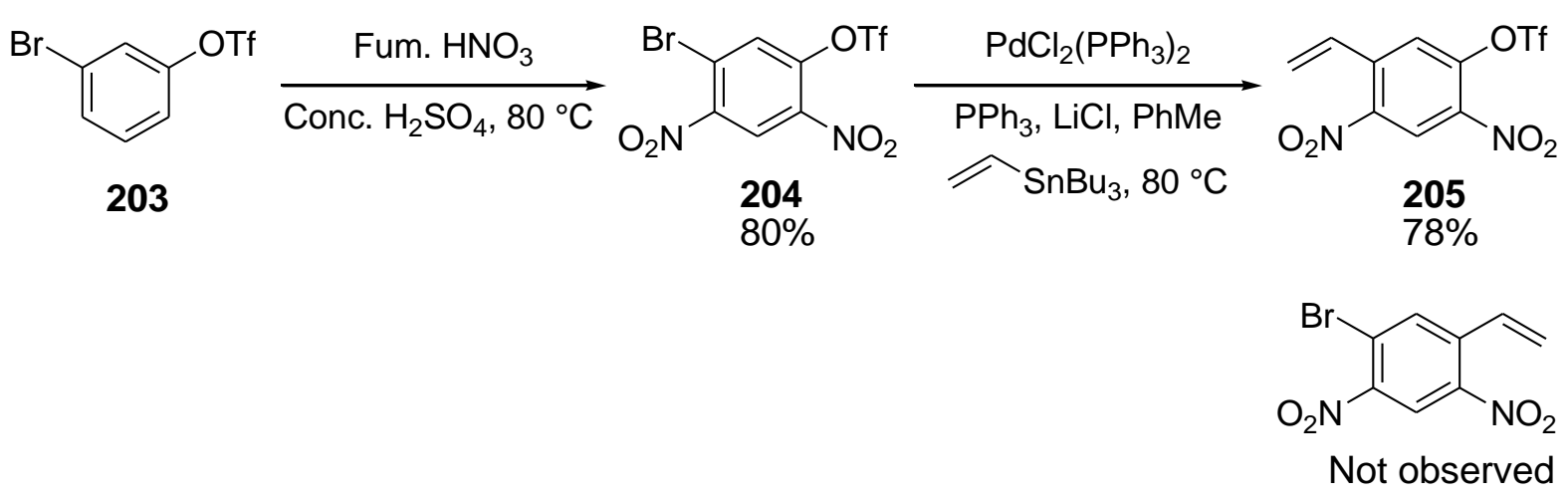

The absence of C-OTf coupling in the above reaction is in sharp contrast to what Stille and Echavarren reported. The structure of compound 204 suggests that both the coupling sites have to be activated by the adjacent nitro groups more or less to the same extent for the oxidative addition. In addition, the steric 
environments for both the sites seem to be more or less equal as both are followed by an ortho nitro group and separated by a free carbon. The absence of the C-OTf coupling under the reaction conditions known to be selective for C-OTf coupling became a driving force for us to consider a detailed systematic study of chemoselectivity in the palladium catalyzed Kosugi-Migita-Stille cross couplings of halophenyl triflates and halonitrophenyl triflates with ethenyltributyltin. Thus, all possible permutations of bromophenyl trifluoromethanesulfonates and bromonitrophenyl trifluoromethanesulfonates were synthesized although few additional substrate types were also pursued in order to evaluate the selectivity more accurately. To the best of our knowledge, the study presented in this chapter represents the first of its kind.

\section{B Results and Discussion}

All the bromophenyl trifluoromethanesulfonates and bromonitrophenyl trifluoromethanesulfonates were tested under the following three different conditions:

1. Conditions $\mathrm{A}: \mathrm{Pd}(\mathrm{dba})_{2}, \mathrm{PPh}_{3}, 1,4$-dioxane, ethenyltributyltin, reflux

2. Conditions B: $\mathrm{PdCl}_{2}\left(\mathrm{PPh}_{3}\right)_{2}, \mathrm{LiCl}$, ethenyltributyltin, $\mathrm{DMF}, 24{ }^{\circ} \mathrm{C}$

3. Conditions $\mathrm{C}: \mathrm{PdCl}_{2}\left(\mathrm{PPh}_{3}\right)_{2}$, ethenyltributyltin, 1,4-dioxane, reflux

It is worth mentioning that Conditions $\mathrm{A}$ are the reported conditions for $\mathrm{C}-\mathrm{Br}$ coupling, Conditions $\mathrm{B}$ are the reported conditions for C-OTf coupling. Under conditions A and B, efforts were focused on duplicating the results reported by Stille and Echavarren. Under C-Br selective conditions, compound 202 is reported to give a 33:1 ratio of $\mathrm{C}$-Br to C-OTf bond coupling based on ${ }^{1} \mathrm{H}$ NMR of the crude reaction mixture (Table 6.1). When the reaction was repeated, in our hands only product 206 was detected in the ${ }^{1} \mathrm{H}$ NMR of the crude reaction mixture (600 MHz, Table 6.1, entry 2). The palladium catalyst, $\left(\mathrm{Pd}\left(\mathrm{PPh}_{3}\right)_{4}\right)$, used by Stille and Echavarren is not very air stable; handling and storage of this catalyst often results into diminished catalytic activity. In our hands, a combination of $2 \mathrm{~mol} \%$ of $\mathrm{Pd}(\mathrm{dba})_{2}$ and 8 mol\% of $\mathrm{PPh}_{3}$ gave the same exclusive selectivity as $\mathrm{Pd}\left(\mathrm{PPh}_{3}\right)_{4}$ (entry 3). So, this catalyst system $\left(\mathrm{Pd}(\mathrm{dba})_{2}\right.$ and $\left.\mathrm{PPh}_{3}\right)$ was used as a substitute for $\mathrm{Pd}\left(\mathrm{PPh}_{3}\right)_{4}$ in order to minimize possible catalytic degredation under Conditions A. Under Conditions B, Stille and Echavarren reported exclusive C-OTf coupling (entry 4). In our hands, use of the same reaction conditions gave a much lower selectivity (1:6.7, entry 5). This product distribution is same as the one reported by Stille and Echavarren at higher temperature $\left(70{ }^{\circ} \mathrm{C}\right.$, entry 6). ${ }^{151}$ It should be noted that products (206-207) are susceptible to decomposition/polymerization while performing chromatography on silica gel.

In order to examine the effect of relative position of bromide and trifluoromethanesulfonate on chemoselectivity, isomeric compounds 2-bromophenyl- and 3-bromophenyl- trifluoromethanesulfonates (208 and 203) were prepared according to literature procedures. Reactions of both the isomers displayed 
the same high selectivity for $\mathrm{C}-\mathrm{Br}$ coupling under Conditions $\mathrm{A}$ and a similar selectivity for C-OTf coupling under Conditions B as was observed with compound 202. Reaction of 203 under Conditions B using $\mathrm{PdCl}_{2}$ (dppp) has been reported. ${ }^{152}$ Only C-OTf coupling was reported in $25 \%$ yield although it remains unclear if the reported selectivity is based on crude reaction mixture or after chromatographic purification.

Table 6.1 Reactions of bromophenyl trifluoromethanesulfonates with ethenytributyltin

\begin{tabular}{|c|c|c|c|c|}
\hline Entry & Conditions $^{\mathrm{a}}$ & & atio $^{b}$ & Yield $^{\mathrm{c}}$ \\
\hline & 202 & 206 & 207 & \\
\hline $1^{\mathrm{d}}$ & $\mathrm{Pd}\left(\mathrm{PPh}_{3}\right)_{4}$, 1,4-dioxane, reflux & 33 & 1 & $77 \%$ \\
\hline 2 & $\mathrm{Pd}\left(\mathrm{PPh}_{3}\right)_{4}$, 1,4-dioxane, reflux & $>30$ & 1 & \\
\hline 3 & $\mathrm{Pd}(\mathrm{dba})_{2}, 4 \mathrm{PPh}_{3}, 1,4$-dioxane, reflux & $>30$ & 1 & $30 \%$ \\
\hline 4 & $\mathrm{PdCl}_{2}\left(\mathrm{PPh}_{3}\right)_{2}$, 1,4-dioxane, reflux & $>30$ & 1 & $90 \%$ \\
\hline $5^{\mathrm{d}}$ & $\mathrm{PdCl}_{2}\left(\mathrm{PPh}_{3}\right)_{2}, \mathrm{LiCl}, \mathrm{DMF}, 24{ }^{\circ} \mathrm{C}$ & 0 & 100 & $77 \%$ \\
\hline 6 & $\mathrm{PdCl}_{2}\left(\mathrm{PPh}_{3}\right)_{2}, \mathrm{LiCl}, \mathrm{DMF}, 24{ }^{\circ} \mathrm{C}$ & 1 & 6.7 & \\
\hline $7^{\mathrm{d}}$ & $\mathrm{PdCl}_{2}\left(\mathrm{PPh}_{3}\right)_{2}, \mathrm{LiCl}, \mathrm{DMF}, 70{ }^{\circ} \mathrm{C}$ & 1 & 5 & $45 \%$ \\
\hline $8^{\mathrm{d}}$ & $\mathrm{PdCl}_{2}\left(\mathrm{PPh}_{3}\right)_{2}, \mathrm{LiCl}, 1,4$-dioxane, reflux & 1 & 6 & $75 \%$ \\
\hline & 208 & 209 & 210 & \\
\hline 9 & $\mathrm{Pd}(\mathrm{dba})_{2}, 4 \mathrm{PPh}_{3}, 1,4$-dioxane, reflux & $>30$ & 1 & $5 \%$ \\
\hline 10 & $\mathrm{PdCl}_{2}\left(\mathrm{PPh}_{3}\right)_{2}, \mathrm{LiCl}, \mathrm{DMF}, 24^{\circ} \mathrm{C}$ & 1 & 5.9 & \\
\hline & 203 & 211 & 212 & \\
\hline 11 & $\mathrm{Pd}(\mathrm{dba})_{2}, 4 \mathrm{PPh}_{3}, 1,4$-dioxane, reflux & 30 & $1^{\mathrm{f}}$ & $34 \%^{\mathrm{g}}$ \\
\hline 12 & $\mathrm{PdCl}_{2}\left(\mathrm{PPh}_{3}\right)_{2}$, 1,4-dioxane, reflux ${ }^{\mathrm{h}, \mathrm{i}}$ & 30 & 1 & $47 \%$ \\
\hline 13 & $\mathrm{PdCl}_{2}\left(\mathrm{PPh}_{3}\right)_{2}, \mathrm{LiCl}, \mathrm{DMF}, 24^{\circ} \mathrm{C}$ & 1 & $6.3^{\mathrm{j}}$ & K \\
\hline $14^{1}$ & $\mathrm{PdCl}$ (dppp), $\mathrm{LiCl}, \mathrm{DMF} 25^{\circ} \mathrm{C}^{\mathrm{m}}$ & 0 & 100 & $25 \%$ \\
\hline
\end{tabular}


a) See experimental section for details. b) Ratio of products in crude reaction mixture. c) Isolated yield of pure product after chromatography. d) See reference ${ }^{138}$. e) Less than nearly $10 \%$ of decoupling product was also observed. f) A 19:30:1:6 ratio of 203/211/212:ethenytributyltin was observed in the crude ${ }^{1} \mathrm{H}$ NMR spectrum. g) Isolated as a mixture with 203. Yield calculated from ${ }^{1} \mathrm{H}$ NMR spectrum. h) A significant amount of $\mathbf{2 0 3}$ remained unreacted even after $72 \mathrm{~h}$. i). The reaction time was $39 \mathrm{~h}$. j) A 5.6:1:6.3 ratio of 203/211/212 was observed in the crude ${ }^{1} \mathrm{H}$ NMR spectrum. K) Product 212 was not isolated. 1) See reference ${ }^{152}$. m) The reaction time was $39 \mathrm{~h}$.

Chemoselectivity of substrates containing both $\mathrm{C}-\mathrm{Br}$ and C-OTf bonds can be significantly affected by electronic effects. For example, exclusive coupling at C-OTf bond was observed in the Suzuki-Miyaura coupling of 202 with triethylboron. ${ }^{151}$ On the other hand, reaction of 202 with $4-\mathrm{MeC}_{6} \mathrm{H}_{4} \mathrm{SnBu}_{3}$ in the presence of $\mathrm{PdCl}_{2}\left(\mathrm{PPh}_{3}\right)_{2}, \mathrm{PPh}_{3}$, and $\mathrm{CuBr}$ in refluxing dioxane afforded $\mathrm{C}-\mathrm{Br}$ coupling product exclusively. ${ }^{139}$ For Suzuki-Miyaura couplings with aryl/alkenyboronic acids, the C-Br bond generally undergoes selective coupling regardless of electron density of the substrate and regardless of the palladium based catalyst used. ${ }^{143 a, 152-156}$ For substrates containing two trifluoromethanesulfonate groups in Suzuki-Miyaura reactions, the cross coupling may take place either at the more electron deficient site ${ }^{157}$ or at the least hindered but more electron rich site. ${ }^{158}$ Oh-e, Miyaura and Suzuki established the order of reactivity as I>Br>OTf for Suzuki-Miyaura couplings with 9-BBN reagent. ${ }^{159} \mathrm{Fu}$ and coworkers have shown that the chemoselectivity could be changed depending on the ligand system and the type of the reaction solvents. ${ }^{143}$ Similarly, Sonogashira coupling of $\mathbf{2 0 2}$ and $\mathbf{2 0 3}$ with phenylethyne gave exclusive coupling at $\mathrm{C}-\mathrm{Br}$ bond in the presence of $10 \mathrm{~mol} \%$ of $\mathrm{ZnCl}_{2}$ at ambient temperature ${ }^{160}$ whereas the same reaction gave only C-OTf coupling product when trimethylsilylethyne was used as a coupling partner in the presence of CuI. ${ }^{161}$ Kumada coupling of $\mathbf{2 0 2}$ and a variety of ortho bromo trifluoromethanesulfonates with phenylethynyl magnesium bromide or phenyl magnesium bromide afforded product derived from COTf coupling. ${ }^{152,162}$ Negishi coupling of $\mathbf{2 0 7}$ with organozinc reagents gave C-OTf coupling product in the presence of $\mathrm{PdCl}_{2}(\mathrm{dppp})^{152},\left[\mathrm{Pd}_{2}(\mathrm{dba})_{3}\right]-\mathrm{dppb}^{163}$. In contrast, coupling of 202 with $\mathrm{Me}_{2} \mathrm{Zn}$ gave C-Br coupling product using $\mathrm{Pd}(\mathrm{OAc})_{2}-\mathrm{AsPPh}_{3}$ and a 3:1 ratio of $\mathrm{C}-\mathrm{Br}$ to $\mathrm{C}-\mathrm{OTf}$ coupling products when $\mathrm{PdCl}_{2}(\text { dppe })_{2}$ was used. ${ }^{164}$

The effects of substituents on the coupling reactions were further examined with additional substrates. All the possible isomeric permutations of bromonitrophenyl trifluoromethanesulfonates (213-222) were prepared from the corresponding phenols using literature procedures. The phenols were treated with trifluoromethanesulfonic anhydride in the presence of either pyridine or triethylamine. 
Figure 6.1 Isomeric bromonitrophenyl trifluoromethanesulfonates (213-222)

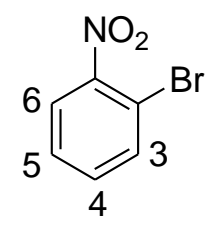

213 (3-OTf)

214 (4-OTf)

215 (5-OTf)

216 (6-OTf)<smiles>O=[N+]([O-])c1cccc(Br)c1</smiles>

217 (2-OTf)

218 (3-OTf)

219 (4-OTf)<smiles>O=[N+]([O-])C1=[C+]C(Br)=CC1</smiles>

221 (2-OTf)

222 (3-OTf)

All the above isomeric compounds were tested under the previously mentioned three reaction conditions for the cross coupling results with ethenyltributytin. Under Conditions A, a combination of relatively air stable $\mathrm{Pd}(\mathrm{dba})_{2}$ and $\mathrm{PPh}_{3}$, a substitute for $\mathrm{Pd}\left(\mathrm{PPh}_{3}\right)_{4}$ was used. The reactions were carried out in refluxing 1,4-dioxane. This condition is known to afford product derived from highly selective oxidative addition to C-Br bond. Conditions B comprise of a combination of $2 \mathrm{~mol} \%$ of $\mathrm{PdCl}_{2}\left(\mathrm{PPh}_{3}\right)_{2}$ and 3 equivalents of $\mathrm{LiCl}$. The reactions were carried out in DMF at ambient temperature. This condition as reported by Stille and Echavarren is known to give product derived from highly selective oxidative addition to C-OTf bond. Under conditions $\mathrm{C}$, the reactions were carried out in the presence of $2 \mathrm{~mol} \%$ of $\mathrm{PdCl}_{2}\left(\mathrm{PPh}_{3}\right)_{2}$ in refluxing 1,4-dioxane. Under conditions A and B, all the reactions were carried out for $24 \mathrm{~h}$ regardless of whether the starting material was consumed or left over within $24 \mathrm{~h}$. Starting materials were observed in the ${ }^{1} \mathrm{H}$ NMR of the crude reaction mixture in many cases after the allocated reaction time. Under conditions $\mathrm{C}$, a majority of reactions were run for approximately $24 \mathrm{~h}$. For details, visit the experimental section. For all the reactions, the ratio of products was analyzed by ${ }^{1} \mathrm{H}$ NMR of the crude reaction mixtures before chromatographic separation. Since the objective of this study was to examine the selectivity of the coupling reactions (C-Br vs $\mathrm{C}-\mathrm{OTf}$ ), no attempts were made to optimize the yield of a product for a particular substrate. High selectivity was observed under both conditions A and B for 216 and 219 wherein the bromo and the trifluromethanesulfonate groups are both ortho or meta to the nitro group. Similarly, enhanced selectivity for C-OTf couplings was observed for 217-220 under Condtions B when the bromide was meta to the nitro group. Under Conditions $\mathrm{C}$, a completely different selectivity pattern was observed for all the substrates tested regardless of positions of the groups on the aromatic rings. Under this condition, all substrates gave products derived from highly selective oxidative addition to $\mathrm{C}-\mathrm{Br}$ or C-I bond. The results are summarized in the table below. 
Table 6.2 Cross-couplings of 213-222 with ethenyltributyltin

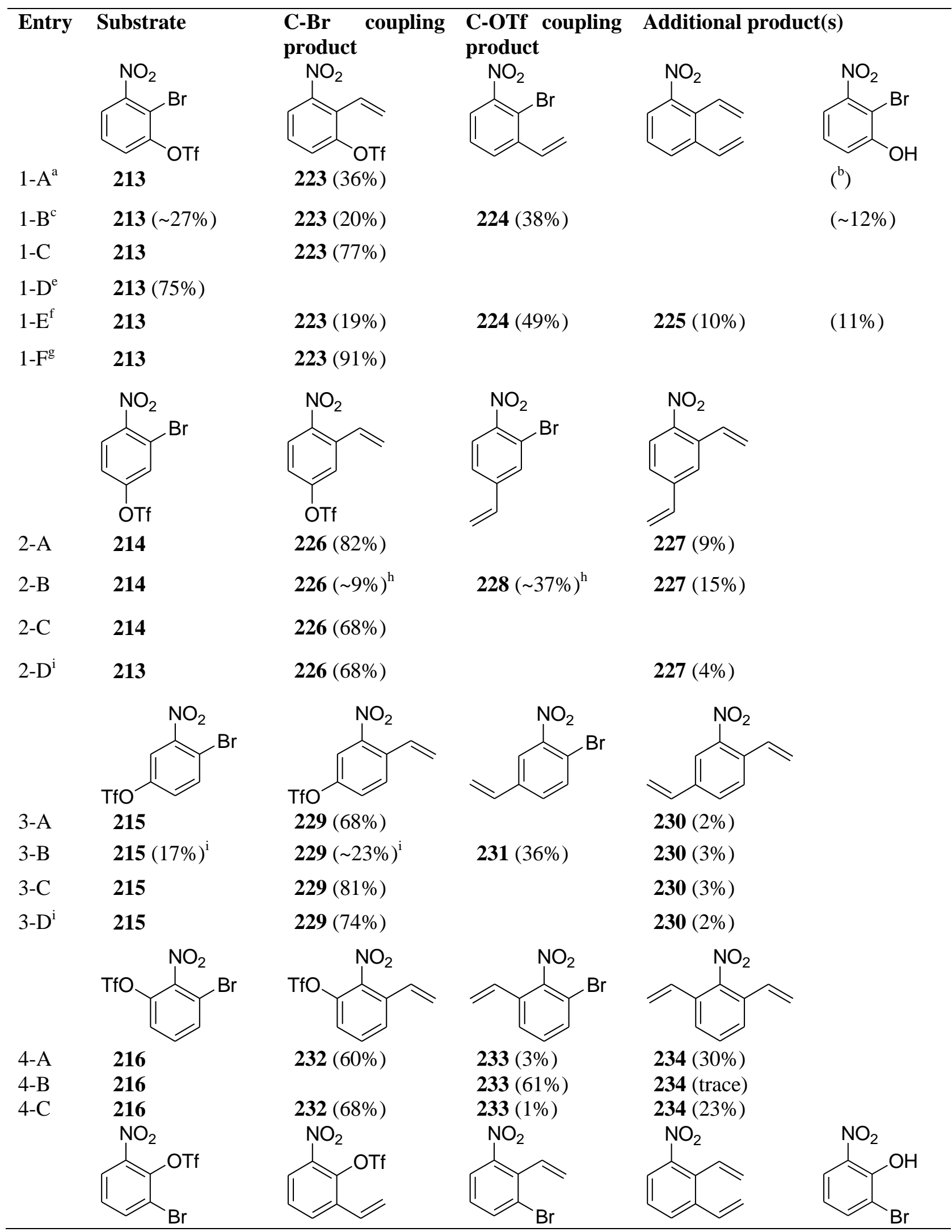




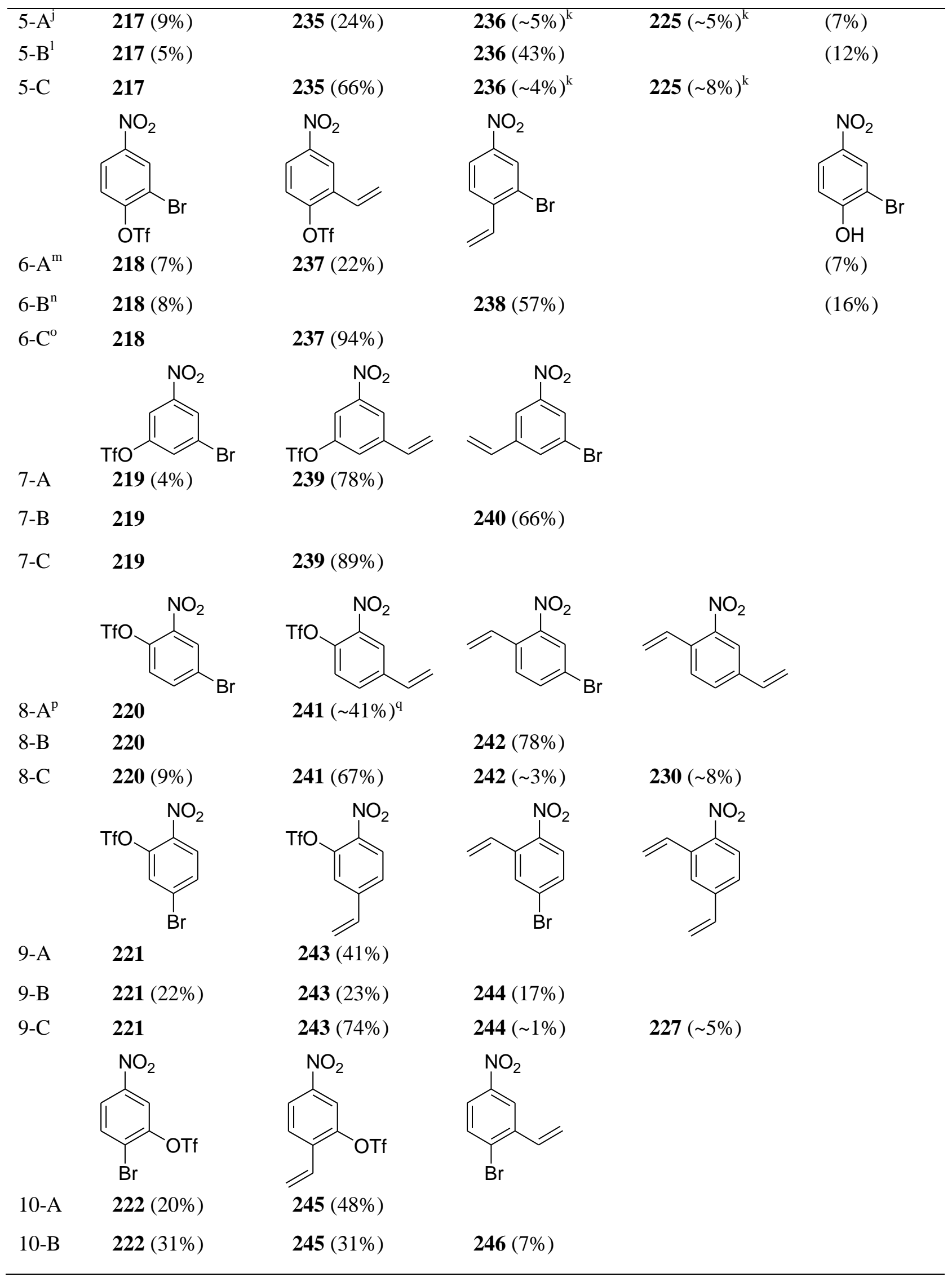




10-C 222

Details of the reaction Conditions A-C

A) $\mathrm{Pd}(\mathrm{dba})_{2}(2 \mathrm{~mol} \%), \mathrm{PPh}_{3}(8 \mathrm{~mol} \%), 14$,-dioxane, reflux

B) $\mathrm{PdCl}_{2}\left(\mathrm{PPh}_{3}\right)_{2}(2 \mathrm{~mol} \%), \mathrm{LiCl}(300 \mathrm{~mol} \%), \mathrm{DMF}$, ambient temperature

C) $\mathrm{PdCl}_{2}\left(\mathrm{PPh}_{3}\right)_{2}(2 \mathrm{~mol} \%)$, 1,4-dioxane, reflux

a) A ratio of 1:2 was observed for $\mathbf{2 1 3 / 2 2 3}$ in the ${ }^{1} \mathrm{H}$ NMR of the crude reaction mixture. b) Isolated as a mixture with unknown impurities. c) A ratio of 1.14:1:1.93 was observed for 213/223/224 in the ${ }^{1} \mathrm{H}$ NMR of the crude reaction mixture. d) A mixture of 213 and 2-bromo-3-nitrophenol was obtained from the ${ }^{1} \mathrm{H}$ NMR of the mixture. e) $\mathrm{PdCl}_{2}(\mathrm{dppp})_{2}(2 \mathrm{~mol} \%), \mathrm{LiCl}(300 \mathrm{~mol} \%), \mathrm{DMF}$, ambient temperature used for the reaction. f) $\mathrm{PdCl}_{2}(\mathrm{dppp})_{2}(2 \mathrm{~mol} \%), \mathrm{DMF}$, ambient temperature was used for the reaction. $\mathrm{g}$ ) $\mathrm{Pd}\left(\mathrm{PPh}_{3}\right)_{4}(2 \mathrm{~mol} \%)$, toluene, reflux condition used for the reaction. h) An inseparable mixture of 226 and 228 was obtained; yield calculated from ${ }^{1} \mathrm{H}$ NMR of the crude reaction mixture. i) An inseparable mixture of 215 and 229 was obtained; yield calculated from the ${ }^{1} \mathrm{H}$ NMR of the crude reaction mixture. j) A ratio of 3.22:3.55:1.10:1 was observed for $\mathbf{2 1 7 / 2 3 5 / 2 3 6 / 2 2 5}$ in the crude reaction mixture. $\mathrm{k}$ ) An inseparable mixture of $\mathbf{2 2 5}$ and $\mathbf{2 3 6}$ was obtained; yield calculated from ${ }^{1} \mathrm{H}$ NMR of the crude reaction mixture. 1) A ratio of 1:6.67 for compounds $\mathbf{2 1 7 / 2 3 6}$ was observed by ${ }^{1} \mathrm{H}$ NMR of the crude reaction mixture. m) A ratio of 1:2.58 for compounds $\mathbf{2 1 8} / \mathbf{2 3 7}$ was observed by ${ }^{1} \mathrm{H}$ NMR of the crude reaction mixture. $\mathrm{n}$ ) A ratio of 1:5 for compounds $\mathbf{2 1 8 / 2 3 9}$ was observed by ${ }^{1} \mathrm{H}$ NMR of the crude reaction mixture. o) Reaction time 36 h. p) A ratio of 1:2.2 was observed for mopounds $\mathbf{2 2 0 / 2 4 1}$ by the ${ }^{1} \mathrm{H}$ NMR of the crude reaction mixture. q) Calculated from a mixture of $\mathbf{2 4 1}$ and dibenzylideneacetone.

Hayashi and Kamikawa have demonstrated that chemoselectivity of oxidative addition to C-Br vs C-OTf bond can be modulated by proper choice of ligands. ${ }^{165}$ Jutand and Mosleh have shown that a faster oxidative addition to C-OTf bond generally predominates when the OTf group sits para to an electron withdrawing group such as, a nitro group. ${ }^{166}$ 4-Cyanobromobenzene has been reported to be 326 times more reactive than the bromobenzene. ${ }^{167}$ Stille couplings of usually unreactive aryl fluorides have also been reported. ${ }^{168,169}$

The chemoselectivity of C-I vs C-OTf bond was evaluated under all the three different conditions (A, B and $\mathrm{C}$ ). The trifluoromethanesulfonate group was placed at an activated site. The results are outlined in the scheme below. In general, iodides are known to undergo coupling much faster than the OTf group even in the presence of $\mathrm{LiCl}$. Reaction of 3-bromo-4-iodo-1-methoxybenzene with an alkenyltin reagent 
to give C-I coupling product had been reported although C-I is positioned at an electronically unfavorable site. ${ }^{170}$

Scheme 6.2 Kosugi-Migita-Stille coupling of 247 with ethenyltributyltin<smiles>C=C[Sb]CCCC</smiles><smiles>C=Cc1ccc(OCC)c([N+](=O)[O-])c1</smiles><smiles>C=Cc1ccc(O)c([N+](=O)[O-])c1</smiles><smiles>C=Cc1ccc(C=C)c([N+](=O)[O-])c1</smiles><smiles>C=Cc1ccc(I)cc1[N+](=O)[O-]</smiles><smiles>O=[N+]([O-])c1cc(I)ccc1O</smiles>

247 Conditions A

$241(38 \%)$

$248(14 \%)$

$230(5 \%)$

247 Conditions B

$249(4 \%)$

247 Conditions C 241 (87\%)

$230(8 \%)$

Reaction of 5-bromo-2-trifloxybenzaldehyde has been shown to give C-OTf coupling product when the reaction is carried out with $\mathrm{Pd}_{2}(\mathrm{dba})_{3}$ and chiral ferrocenyldiphosphine in DMF at $80{ }^{\circ} \mathrm{C}$ without added LiCl. Only a very limited number of palladium catalyzed coupling reactions of bromophenyl trifluoromethanesulfonates containing an electron donating group has been reported. ${ }^{139,171}$ Compound 250 was selected to minimize the possible steric interactions between the Br/OTf and the methoxy groups. Based on electronic considerations, the trifluoromethanesulfonate group may be expected to show less selectivity while the bromide in the less electron rich position should be more selective. This compound was also tested under all the three reaction conditions. The results are summarized in the scheme below. The reaction of $\mathbf{2 5 0}$ did not reach completion under Conditions $\mathrm{C}$ even with an excess of ethenyltributytin.

Scheme 6.3 Kosugi-Migita-Stille coupling of 250<smiles>C=C[SbH]=CCc1ccc(OC)cc1Br</smiles>

250

Conditions A

250<smiles>C=Cc1cc(OC)ccc1OCC</smiles>

$251(33 \%)$

$251(4 \%)$<smiles>C=Cc1ccc(OC)cc1Br</smiles>

$252(39 \%)$

As a final test substrate, compound 253 was chosen. The purpose here was to examine if the generalization of chemoselectivity may be extended to other electron withdrawing groups. Under all the 
three reaction conditions, only one cross coupling product was observed in the ${ }^{1} \mathrm{H}$ NMR spectrum of the crude reaction mixtures.

Scheme 6.4 Kosugi-Migita-Stille coupling of 253

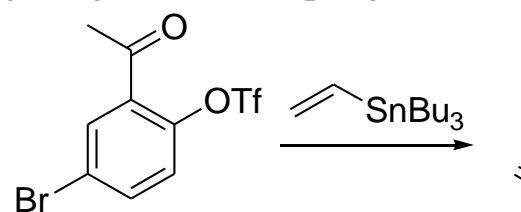

253 Conditions A

253 Conditions B

252 Conditions $\mathrm{C}$<smiles>C=Cc1ccc(Br)c(C(C)=O)c1</smiles>

$254(14 \%)$
$255(58 \%)$<smiles>C=Cc1ccc(Br)cc1C(C)=O</smiles>

$\mathrm{Br}$

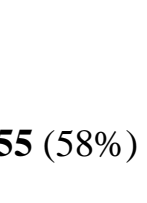

\section{C Conclusion}

The chemoselectivity of Kosugi-Migita-Stille couplings of all isomeric permuatations of bromphenyl trifluoromethanesulfonates and bromonitrophenyl trifluoromethanesulfonates was examined in order to compare and contrast the reactivity of $\mathrm{C}-\mathrm{Br}$ and $\mathrm{C}$-OTf bonds under three different reaction conditions. Under all conditions, discrepancies were observed from the literature reported results. High selectivity was observed only for few of the compounds under both the reaction conditions (A and B). Enhanced selectivity for carbon-triflate bond was observed under conditions B only for some of the compounds. All substrates tested under conditions $\mathrm{C}$ showed very high selectivity for carbon-bromine bond coupling regardless of the type of functional groups and their positions on the aromatic rings. Although some generalizations can be made for some of the isomeric and potentially useful compounds, it is worth not to extend them to compounds having additional functional groups and/or more sterically demanding functionalities. 


\section{Chapter 7 Synthesis of pyrroloindoles via a double reductive $N$-heterocyclization}

\section{A Introduction}

There are five isomeric pyrroloindoles. For simplicity, they have been grouped into five types (types A-E, Figure 7.1). The structures of these compounds remind of the notion that chemistry is indeed a creative science. Another interesting fact about these compounds is that they form a good basis for separate NMR studies. For example, the ${ }^{1} \mathrm{H}$ and ${ }^{13} \mathrm{C}$ NMRs of pyrroloindoles (of types $\mathrm{A}, \mathrm{C}$ and $\mathrm{D}$ ) look similar. Unless one knows the identity of the starting material, it is hard to tell what pyrroloindoles have been synthesized. In addition to their wide range of possible applications, the pyrroloindole skeleton can be found in natural products. Pyrroloindoles have been shown to possess bactericidal, antimicrobial, and antitumor activities. ${ }^{193}$ Among other representative examples of pyrroloindole containing natural products include terreusinone A, ${ }^{194,195}$ and indolo[3,2-b]carbazole. ${ }^{196-198}$ Terreusinone A possesses significant UVA protecting properties. ${ }^{195}$

Figure 7.1 Isomeric pyrroloindoles<smiles>c1cc2ccc3cc[nH]c3c2[nH]1</smiles>

\section{$1 H, 8 H$-Pyrrolo[3,2-g]indole}

(Type A)<smiles>c1cc2cc3cc[nH]c3cc2[nH]1</smiles>

1H,7H-Pyrrolo[3,2-f]indole

(Type B)<smiles>c1cc2c(ccc3[nH]ccc32)[nH]1</smiles>

\section{$1 H, 6 H$-Pyrrolo[3,2-e $]$ indole}

(Type D)<smiles>c1cc2cc3[nH]ccc3cc2[nH]1</smiles>

$1 H, 5 H$-Pyrrolo[2,3-f]indole (Type C)<smiles>c1cc2c(ccc3cc[nH]c32)[nH]1</smiles>

$1 H, 5 H$-Pyrrolo[2,3-g]indole

(Type E) 
There are several methods reported to access these isomeric compounds. They can be broadly classified into two categories. One route consists of fusing a pyrrole ring onto a preformed indole or indoline ring, and the other route is the direct double ring closure of the precursors without a preformed indole or indoline. Examples of the first methodology includes a Fischer indole synthesis ${ }^{199}$ intramolecular cyclization of a bis-( $\beta$-aminoethyl)-hydroquinone derivative in presence of water. ${ }^{200}$ The second general methodology includes a double Reissert indole synthesis ${ }^{201,202}$ and a double Batcho-Leimgruber ${ }^{203}$

Shannon et al. prepared pyrrolo[3,2-f]- and -[2,3-f]-indoles through the Montmorillonite K-10 clay catalyzed Vilsmeier formylation of pyrrole. ${ }^{204}$

Scheme 7.1 Vilsmeier formylation route to pyrroloindoles<smiles>CCOC(=O)c1[nH]c(Cc2cc(CCC(C)C(=O)OCC)c(C(=O)OCC)[nH]2)cc1C</smiles>

Samsoniya et al. reported numerous methods to prepare pyrroloindoles. ${ }^{205,206}$ The first method utilized a Fischer-type bicyclization of bishydrazone to form the two pyrrole rings (Scheme 7.2). An inherent drawback of this methodology was the formation of four isomers.

Scheme 7.2 Samsoniya et al synthesis of pyrroloindoles
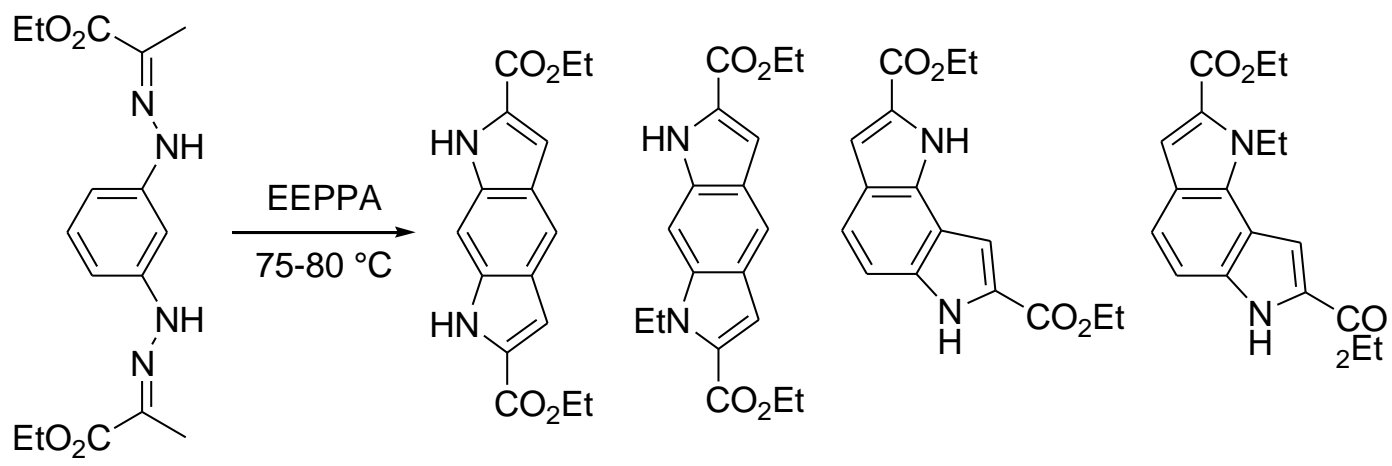

Although the second methodology by Samsoniya et al attempted to circumvent the previously reported issue of isomer formation by using a pre-formed aminoindoline, two non-substituted pyrroloindoles were obtained only at the expense of additional steps. 
Scheme 7.3 Preparation of pyrroloindoles from Aminoindoline<smiles>CCOC(C)=NNc1ccc2c(c1)N(C)CC2</smiles><smiles>CC#CCOC(=O)c1cc2cc3c(cc2[nH]1)N(C)CC3</smiles><smiles>c1cc2cc3cc[nH]c3cc2[nH]1</smiles><smiles>CCOC(=O)c1cc2c3c(ccc2[nH]1)CCN3C</smiles><smiles>C#CC#C</smiles>

While the palladium-catalyzed aerobic oxidative bicyclization route to the preparation of pyrroloindoles from $N$-aryl imines reported by Yoshikai et $\mathrm{al}^{207}$ seemed to have broader substrate scope, the authors did not attempt to prepare non-symmetrical compounds. The yields of products were modest at best and the regioselectivities observed in the cyclizations were also not explained.

Scheme 7.4 Preparation of pyrroloindoles from Diimines

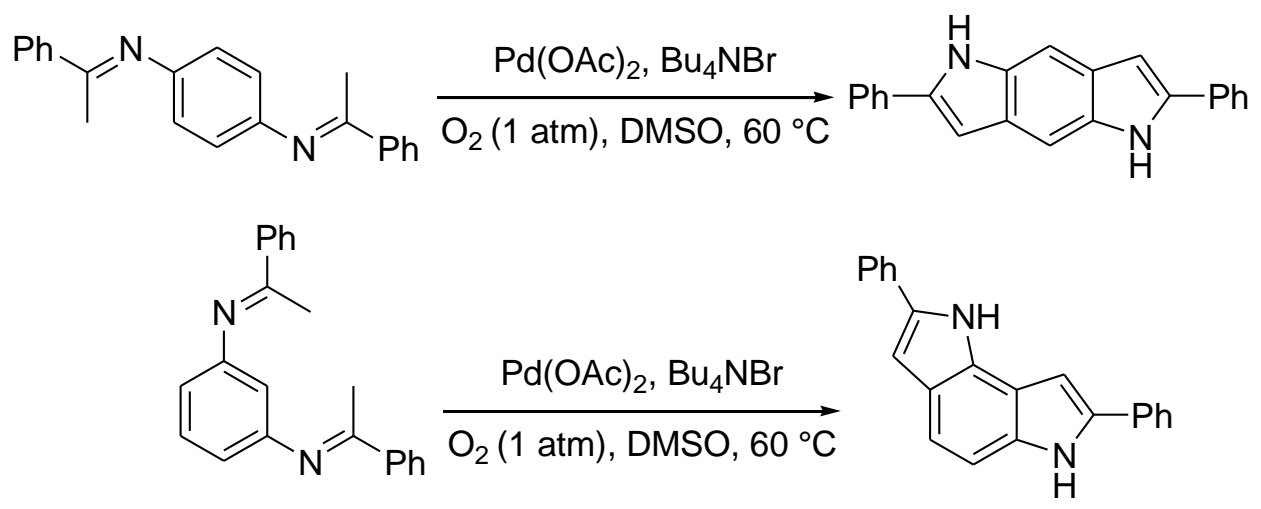

Fujii and Ohno reported a copper-catalyzed bis-cyclization of di-alkynyl-dimesylamide (Scheme 7.5). ${ }^{208}$ This approach was limited to terminal alkynes, affording only non-substituted pyrroloindoles.

A related rhodium catalyzed hydroamination of di-alkynyl-diamines and di-alkynyl-acetylamide have been used to prepare type $\mathrm{B}$-and $\mathrm{C}$ benzoindoles. ${ }^{209}$ 2-Substituted pyrroloindoles cannot be made by this methodology.

Scheme 7.5 Intramolecular Hydroamination route to pyrroloindoles<smiles>C#Cc1cc(C#C)c(NC(C)C)cc1NC(=O)c1cc2cc3ccn(S(C)(=O)=O)c3cc2n1C</smiles> 
One serious limitation of the previously reported synthetic routes is that they allow only for the preparation of symmetrical pyrroloindoles. This is in part due to limitations associated with the methods used to prepare the required starting materials, most of which also involve symmetrical compounds. Therefore, methods to prepare non-symmetrical substrates that can be converted to non-symmetrical pyrroloindoles are highly desirable.

Over the last twenty years, our group has extensively studied applications of palladium catalyzed carbon monoxide mediated reductive cyclizations. This versatile methodology has been used to prepare a wide variety of indoles, bi-1H-indoles and indole alkaloids. ${ }^{45,113} \mathrm{We}$ envisioned that this methodology could be applied in the synthesis of benzoindoles from dialkenyldinitrobenzenes. The relatively mild conditions used in such reactions could allow for broad functional group compatibility and offer significant improvements over the previously disclosed methods. With the goal of preparing non-symmetrical pyrroloindoles using the reductive cyclization methodology, efforts were made to establish routes to prepare the requisite dialkenyldinitrobenzenes. Specifically, efforts were focused on synthesizing nonsymmetrical cyclization precursors in a controlled, sequential manner. For organizational clarity, each isomeric substrate is presented separately, although numerous commonalities were encountered in the preparation of each substrate. The synthetic strategy is presented below.

Scheme 7.6 Possible routes to pyrroloindoles<smiles>[X]c1ccc([X])c([N+](=O)[O-])c1[N+](=O)[O-]</smiles><smiles>[X]c1cc([N+](=O)[O-])c([N+](=O)[O-])cc1[X]</smiles><smiles>[X]c1cc([N+](=O)[O-])c([X])cc1[N+](=O)[O-]</smiles><smiles>[X]c1c([N+](=O)[O-])ccc([N+](=O)[O-])c1[X]</smiles><smiles>[X]c1ccc([N+](=O)[O-])c([X])c1[N+](=O)[O-]</smiles> 
Accordingly, we hypothesized that pyrroloindole of type A could be obtained by reductive cyclization of 1,4-dialkenyl-2,3-dinitrobenzenes which, in turn, could be derived by Kosugi-Migita-Stille coupling with the appropriate tin reagents. A similar strategy was envisioned for the synthesis of the other pyrroloindole types.

\section{B Results and Discussion}

Compound 285 was chosen as a test substrate. Compound 285 was synthesized by a double KosugiMigita-Stille coupling of 1,4-dibromo-2,3-dinitrobenzene with vinyltin 282. Treatment of $\mathbf{2 8 5}$ under our standard conditions for reductive cyclization ( $\mathrm{Pd}(\mathrm{dba})_{2}$, dppp, 1,10-phen, CO (6 atm), DMF, $\left.120{ }^{\circ} \mathrm{C}\right)$ afforded the expected product 336 in $23 \%$ isolated yield. The monocyclized product 337 was not observed. The problem, however, was the low yield of product. We hypothesized that the low yield (Table 7.1, entry 1) might be a result of the catalyst inactivity after prolonged period of reaction and is probably a call for the reevaluation of the catalytic systems. This catalyst was replaced with a robust catalyst system $\left(\mathrm{Pd}(\mathrm{OAc})_{2}\right.$, dppp, 1,10-phen) which improved the yield, but this was again not very satisfactory (Table 7.1, entry 2). Although a combination of $\mathrm{Pd}(\mathrm{dba})_{2}$ and $\mathrm{PPh}_{3}$ did improve the yield tremendously (64\%, entry 3$)$, the best result (70\%, entry 6$)$, was obtained with the combination of the very simple catalyst $\left(\mathrm{Pd}(\mathrm{OAc})_{2}, 1,10\right.$-phen $)$. Notice that entries 4 and 5 are conditions leading to monocyclized product $\mathbf{3 3 7}$ although a minor amount of pyrroloindole was also isolated (9\%, entry 5). 
Table 7.1 Condition screening

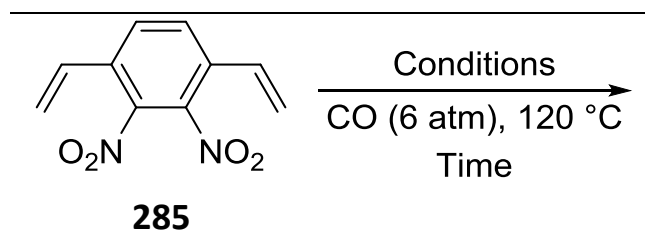

Conditions

Entry

1. $P d(d b a)_{2}, d p p p, 1,10$-phen

2. $\mathrm{Pd}(\mathrm{OAc})_{2}, \mathrm{dppp}, 1,10$-phen

3. $\quad \mathrm{Pd}(\mathrm{dba})_{2}, \mathrm{PPh}_{3}$

4. $\quad \mathrm{Pd}(\mathrm{OAc})_{2}, \mathrm{PPh}_{3}$

5. $\quad \operatorname{Pd}(\mathrm{OAc})_{2}, \mathrm{dppp}$

6. $P d(O A c)_{2}, 1,10$-phen

7. $\quad \mathrm{Pd}(\mathrm{OAc})_{2}, 1,10$-phen

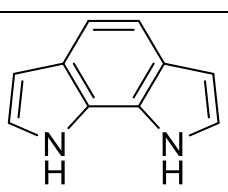

336

Time \% Yield

(h)

89

100

126

126

36

48

90

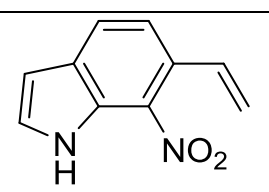

337

$\%$ Yield

$\Sigma(\%)$

23

35

64

54

58

48

70

In order to validate the result presented in Table 7.1, we commenced to synthesize symmetrical as well as unsymmetrical cyclization precursors. Most of the cross-coupling precursors were novel and they were prepared as described below. All other starting materials were commercially available or prepared according to literature procedures. For the $1 \mathrm{H,}, 8 \mathrm{H}$-pyrrolo[3,2-g]indole (Type A) precursors, 4-bromo-2,3dinitrophenol (257) and 4-bromo-2,3-dinitrophenyltriflate (258) were prepared from 1,4-dibromo-2,3dinitrobenzene $(\mathbf{2 5 6})^{210}$ by a sequential nucleophilic aromatic substitution using an excess of sodium hydroxide in a water-THF mixture and triflation (Scheme 7.7).

Scheme 7.7 Preparation of 258<smiles>O=[N+]([O-])c1c(Br)ccc(Br)c1[N+](=O)[O-]</smiles><smiles>O=[N+]([O-])c1c(O)ccc(Br)c1[N+](=O)[O-]</smiles>
$\mathrm{TF}_{2} \mathrm{O}$, Pyridine<smiles>CCCCCCC</smiles><smiles>CCCOc1ccc(Br)c([N+](=O)[O-])c1[N+](=O)[O-]</smiles>

4-Bromo-2,3-dinitrophenol (257) can alternatively be obtained from nitration of 4-bromo-3-nitrophenol (259). In addition to $\mathbf{2 5 7}$, the isomeric nitration product 4-bromo-2,5-dinitrophenol (262) was also obtained. The latter is a plausible precursor to Type $\mathrm{C}$ pyrroloindoles. The isomers were readily separated 
and transformed into the corresponding triflates $\mathbf{2 5 8}$, and $\mathbf{2 6 5}$, respectively (Scheme 7.8). In a similar fashion, nitration of 4-iodo-3-nitrophenol $(\mathbf{2 6 0})^{211}$ gave rise to two readily separable products, 4-iodo-2,5dinitrophenol (263) and 4-iodo-2,3-dinitrophenol (261), and after triflation the corresponding triflates 266 and 264 respectively.

Scheme 7.8 Synthesis of bromo- and iodo-phenyl trifluoromethanesulfonates

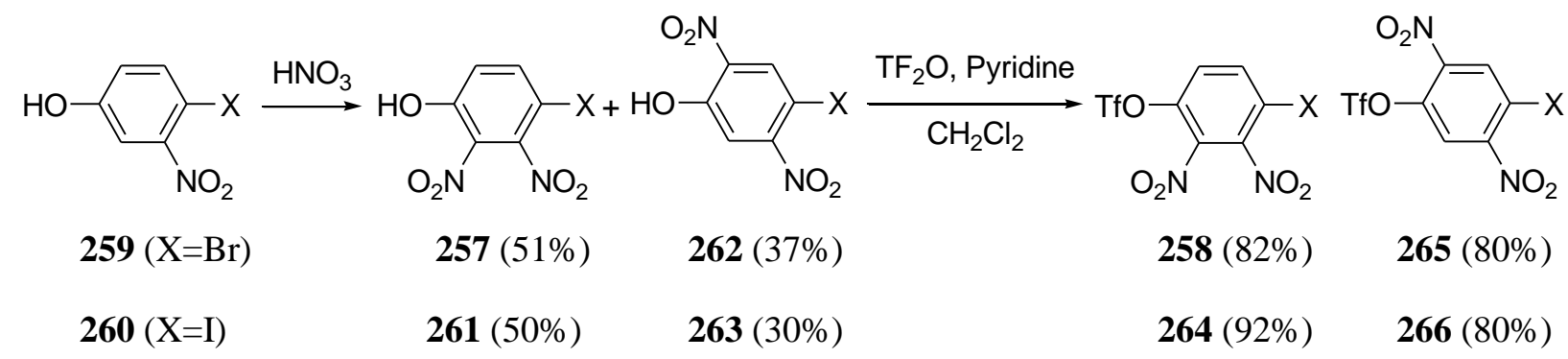

A selective oxidative iodination of 2,5-dinitrophenol $(\mathbf{2 6 7})^{212}$ using potassium iodide and benzyltriphenylphosphonium peroxymonosulfate to give 263 has been reported. However, we were unable to repeat the reported result using this procedure. Iodination of deactivated aromatic compounds using $N$-iodosuccinimide (NIS) in trifluoromethanesulfonic acid has been reported by Olah et al. ${ }^{213}$ In a slight modification of this procedure, treatment of $\mathbf{2 6 7}$ with NIS in sulfuric acid afforded the desired 4iodo-2,5-dinitrophenol (263) in moderate yield (Scheme 7.9).

Scheme 7.9 Iodination of 2,5-dinitrophenol (267).<smiles>O=[N+]([O-])c1ccc([N+](=O)[O-])c(O)c1</smiles>

267

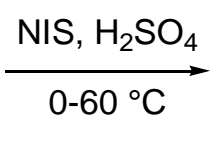<smiles>O=[N+]([O-])c1cc(I)c([N+](=O)[O-])cc1O</smiles>

$263(57 \%)$

Nitration of 5-iodo-2-nitrophenol (269) ${ }^{214}$ with sodium nitrate in sulfuric acid afforded 5-iodo-2,4dinitrophenol (274) $)^{215}$ as the major product along with the novel nitration product 3-iodo-2,4dinitrophenol (275) as an inseparable mixture by chromatography (Scheme 7.10). Since the products were inseparable, they were treated with triflic anhydride to give the readily separated triflates 276 and 277. The same results were obtained using 5-bromo-2-nitrophenol (268) affording an inseparable mixture of dinitrobromophenols 270 and 271 and the readily separable corresponding triflates 204 and 273 (Scheme 7.10). 
Scheme 7.10 Synthesis of bromo- and iodo-phenyl trifluoromethanesulfonates

$$
\begin{aligned}
& \text { O }{ }^{\circ} \mathrm{C} \\
& \mathbf{2 6 8}(\mathrm{X}=\mathrm{Br}) \quad \mathbf{2 7 0}+\mathbf{2 7 1}(99 \%) \quad \mathbf{2 0 4}(57 \%) \quad \mathbf{2 7 3}(16 \%) \\
& 269(\mathrm{X}=\mathrm{I}) \quad \mathbf{2 7 4}+\mathbf{2 7 5}(99 \%) \quad \mathbf{2 7 6}(62 \%) \quad \mathbf{2 7 7}(28 \%)
\end{aligned}
$$

Finally, the elusive Kosugi-Migita-Stille coupling precursor, 2-bromo-3,6-dintrophenyl trifluoromethanesulfonate (281) was prepared in the same fashion as discussed above for compounds 204 and 273. A minor amount of the undesired product $\mathbf{2 8 0}$ was also obtained from the nitration reaction (Scheme 7.11).

Scheme 7.11 Synthesis of 281

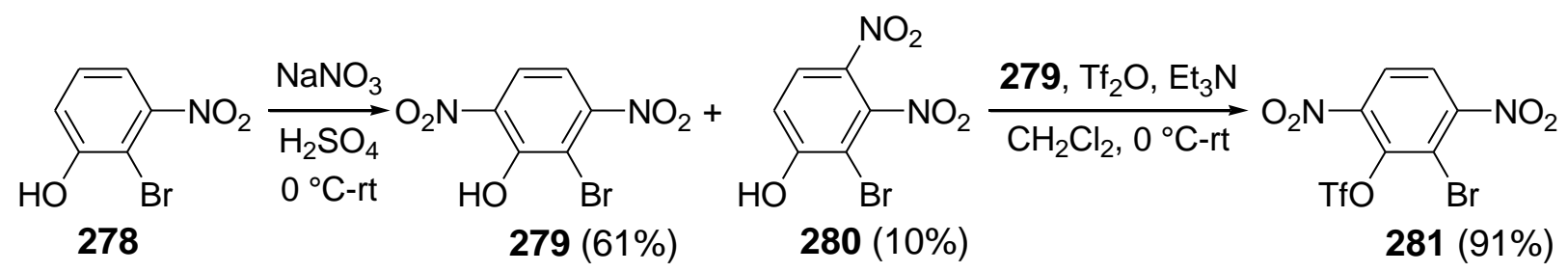

\section{C Synthesis of pyrroloindole (Type A-E) precursors}

The Kosugi-Migita-Stille cross coupling was used to introduce the required alkenes onto the benzene ring. Some of the advantages of this type of coupling reaction include the vast array of alkenyltins already reported in the literature, their ease of preparation either from Grignard reagents or hydrostannation of alkynes and their stability relative to alternative reagents. Variation of the alkenyl tin used in either coupling step would allow for preparation of a number of structurally diverse pyrroloindoles. A double Kosugi-Migita-Stille cross-coupling was used for the synthesis of symmetrical cyclization precursors using 1,4-dibromo-2,3-dinitrobenzene (256), 1,5-dibromo-2,4-dinitrobenzene $\quad(\mathbf{2 8 8}),{ }^{209}$ 4-iodo-2,5dinitrophenyl triflate (266) or 4-bromo-2,5-dinitrophenyl triflate (265), 5-bromo-2,4-dinitrophenyl triflate (204), 5-iodo-2,4-dinitrophenyl triflate (276), 3-bromo-2,6-dinitrophenyl triflate (273), and 2-bromo-3,6dinitrophenyl triflate (281).

Thus, ethenyl (282), 1-propen-1-yl (283), and 1-propen-2-yl (284) tributyltin were treated with the appropriate substrate in the presence of $\mathrm{PdCl}_{2}\left(\mathrm{PPh}_{3}\right)_{2}$ and/or $\mathrm{PPh}_{3}$ in 1,4-dioxane at $90-100{ }^{\circ} \mathrm{C}$ and the results are summarized in Table 7.2. 
Table 7.2 Synthesis of cyclization precursor by double Kosugi-Migita-Stille coupling

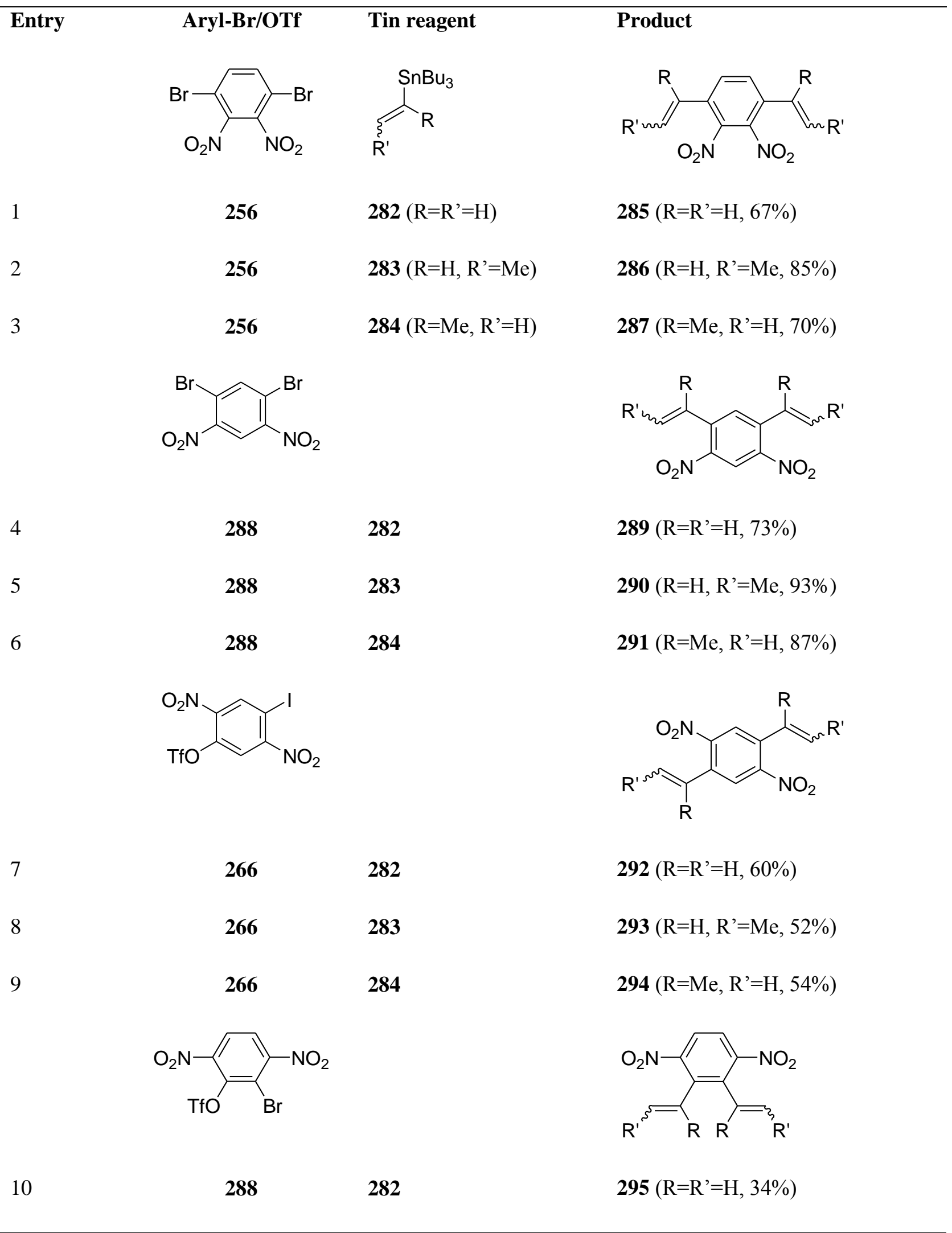




122894

It was anticipated that the chemoselectivity between bromides and triflates (discussed in chapter 6) could be used to our advantage for the introduction of two different alkenes onto an aromatic ring. However, cross coupling of $\mathbf{2 5 8}$ proved to be relatively unselective under the two conditions established by Echavarren and Stille and under some additional conditions examined (Scheme 7.12). Inseparable mixtures of di- and C-OTf coupled products and C-Br coupled product and starting material in addition to unknown impurities were obtained in each case. Compound $\mathbf{2 5 8}$ was not further examined as a possible starting material to unsymmetrically substituted precursors for pyrroloindole type A.

Scheme 7.12 Attempted selective Kosugi-Migita-Stille coupling of $\mathbf{2 5 8}$<smiles></smiles>

A selective introduction of one alkene was, however, feasible using iodo- and bromo-triflates and limited amounts of alkenyl tin reagent (Table 7.3). Attempted coupling at the triflate using an excess of lithium chloride resulted in an OTf-chloride exchange (Scheme 7.13, 7.14). Thus, all reactions were performed in the absence of $\mathrm{LiCl}$. 
Table 7.3 Selective cross coupling of bromo- and iodo-phenyl triflates.

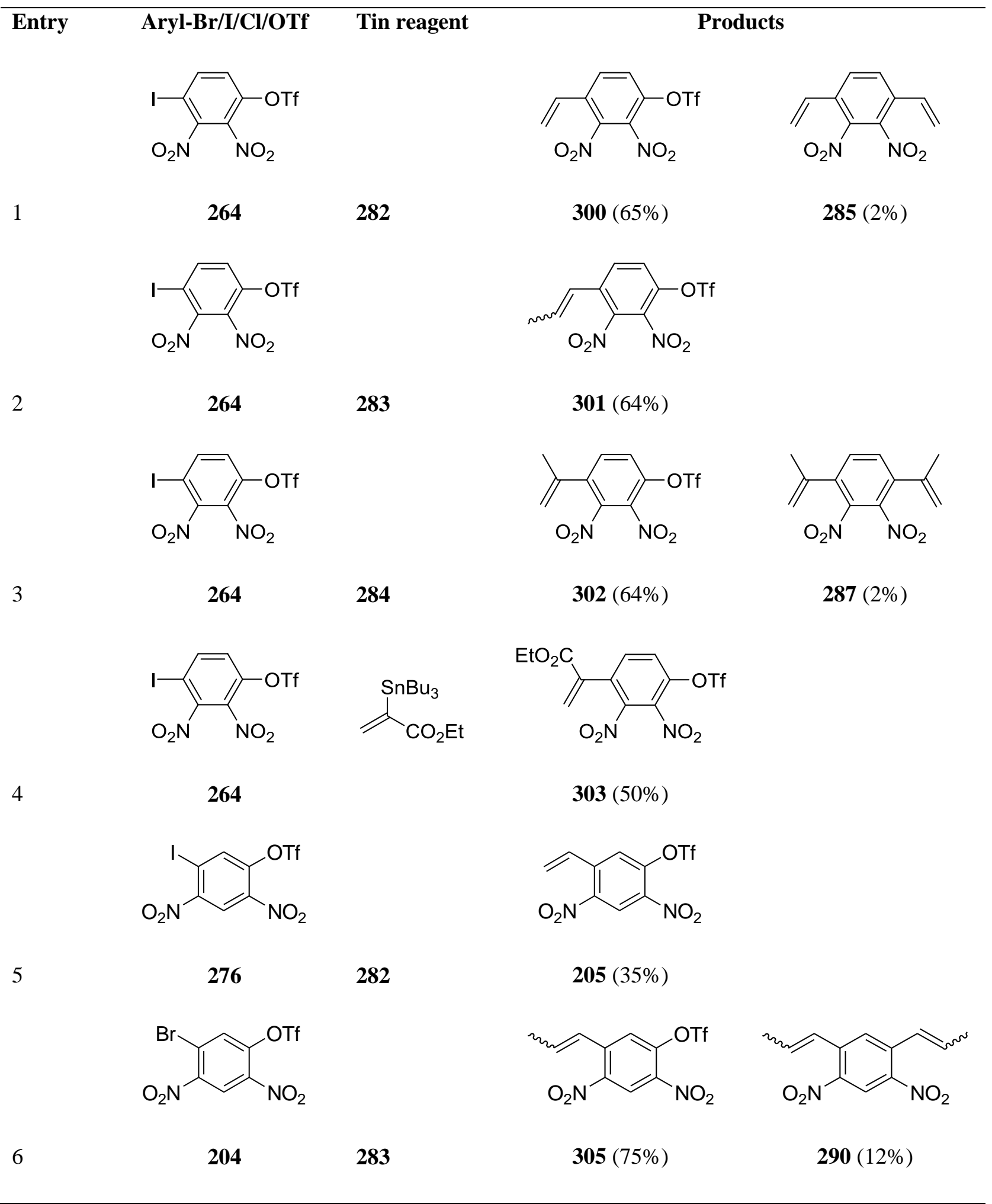




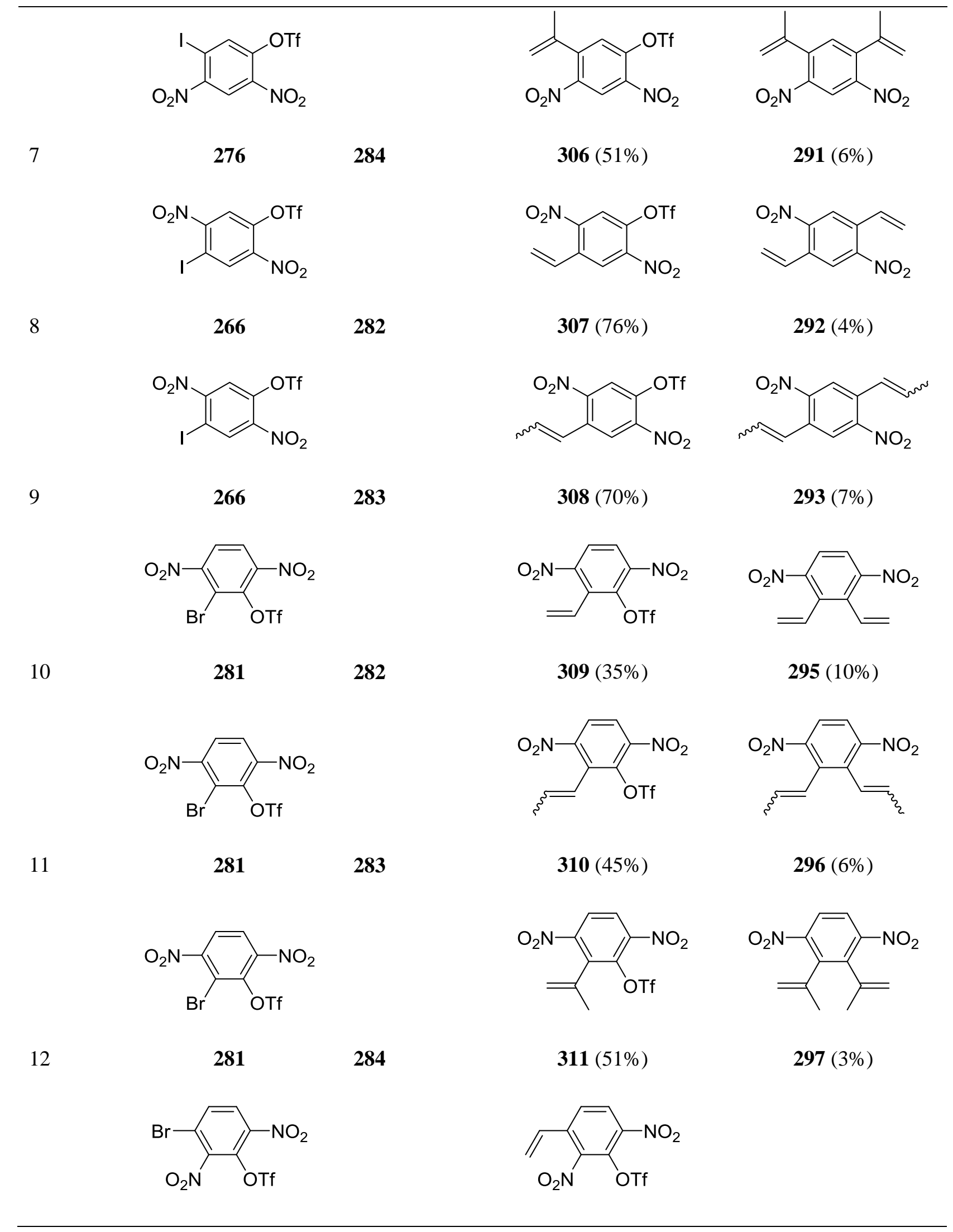


13 273<smiles>O=[N+]([O-])c1ccc(Br)c([N+](=O)[O-])c1[O+]</smiles>

273
282

$312(42 \%)$<smiles>C=C(C)c1ccc([N+](=O)[O-])c([O-])c1[N+](=O)[O-]</smiles>

$313(45 \%)$

The second alkene was thought to be readily introduced by an aryl triflate - organotin cross coupling using the conditions described by Echavarren and Stille $\left(\mathrm{PdCl}_{2}\left(\mathrm{PPh}_{3}\right)_{2}, \mathrm{LiCl}, \mathrm{DMF}, \mathrm{RT}\right)$. However attempted coupling of $\mathbf{3 0 0}$ and $\mathbf{3 0 7}$ resulted only in a replacement of the triflate with a chlorine to give 314 and 315, respectively (Schemes 7.13-7.14). Cross coupling of the chlorine was not feasible at ambient temperature but $\mathbf{3 1 7}$ underwent coupling at elevated temperature to give $\mathbf{3 1 6}$ in excellent isolated yield after chromatography. The triflate to chloride transformation could readily be avoided by either excluding lithium chloride at ambient temperatures or perform the reaction at elevated temperatures in the presence of lithium chloride. The results from the second Kosugi-Migita-Stille coupling of phenyl triflates are summarized in the following table (Table 7.13).

Scheme 7.13 Problem in Kosugi-Migita-Stille coupling

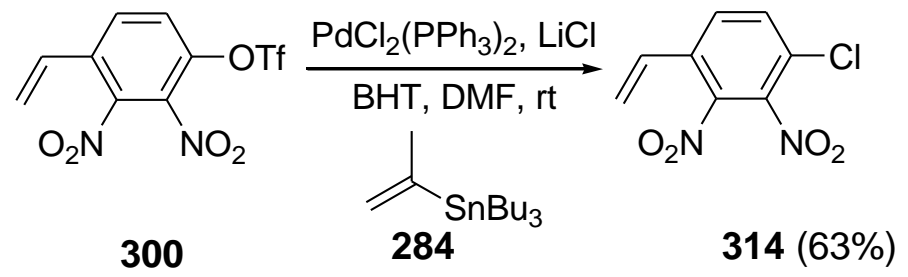

Scheme 7.14 Synthesis and attempted Kosugi-Migita-Stille coupling of $\mathbf{3 1 5}$<smiles>C=Cc1cc([N+](=O)[O-])c([N+](=O)[O-])cc1OC</smiles>

307

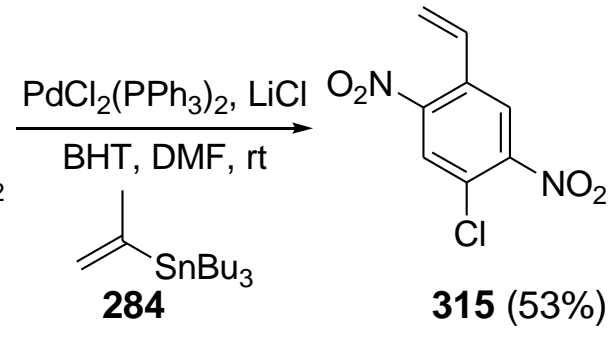

$315(53 \%)$

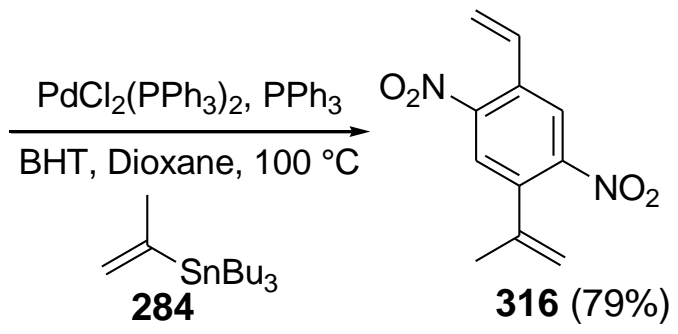


Table 7.4 Synthesis of unsymmetrical cyclization precursors

\section{Entry} Aryl-Br/I/Cl/OTf<smiles>C=Cc1ccc(OCC)c([N+](=O)[O-])c1[N+](=O)[O-]</smiles>

1

300

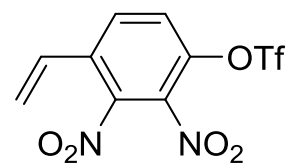

2<smiles>C=C(C)c1ccc(OCC)c([N+](=O)[O-])c1[N+](=O)[O-]</smiles>

3<smiles>C=Cc1ccc(OC)c([N+](=O)[O-])c1[N+](=O)[O-]</smiles>

4<smiles>CC=Cc1ccc(OCC)c([N+](=O)[O-])c1[N+](=O)[O-]</smiles>

5<smiles>C=C(C(=O)OCC)c1ccc(OCC)c([N+](=O)[O-])c1[N+](=O)[O-]</smiles>

Tin reagent

283

284

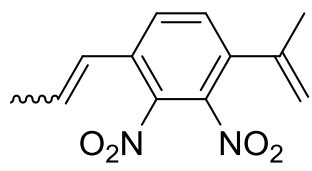

283<smiles>C=C(CCCC)C(=O)OCC</smiles><smiles>C=Cc1ccc(C(=C)C(=O)OCC)c([N+](=O)[O-])c1[N+](=O)[O-]</smiles>

$320(51 \%)$

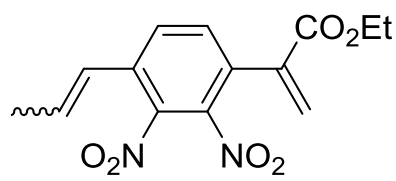

$\mathrm{Bu}_{3} \mathrm{Sn}_{\mathrm{CO}_{2} \mathrm{Et}}$

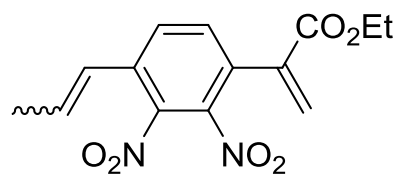

$321(43 \%)$ 


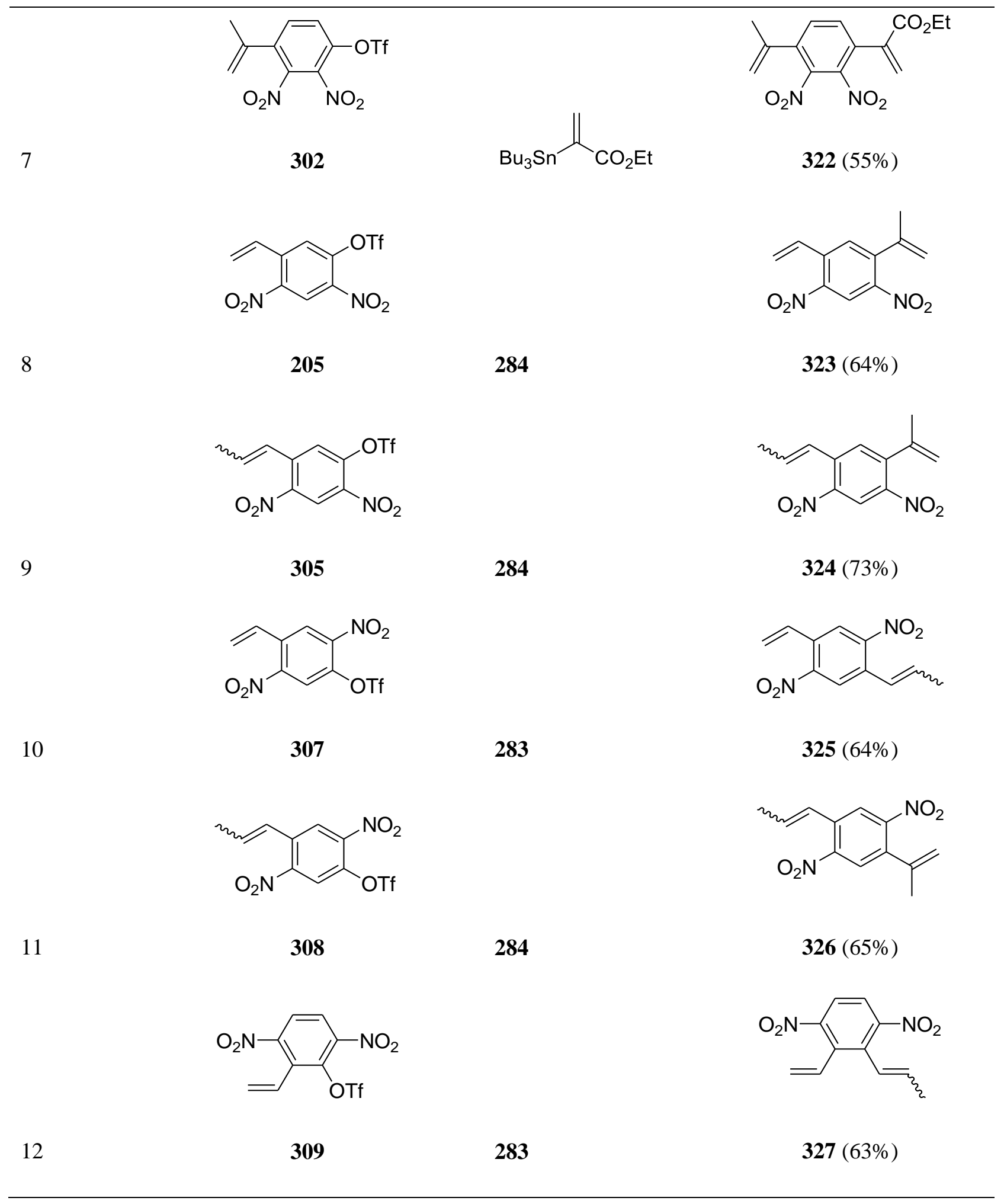




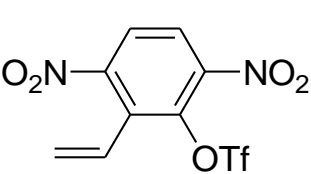

13

14

15

16

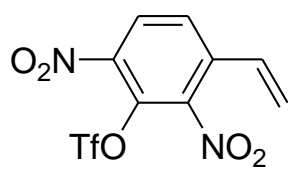

284

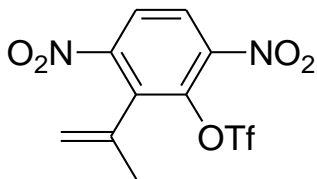

311

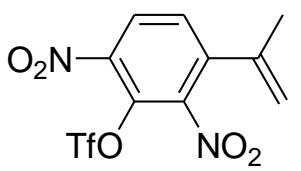

313

312

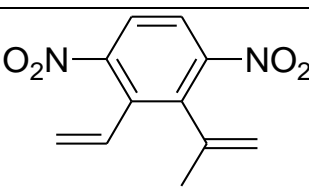

$328(64 \%)$

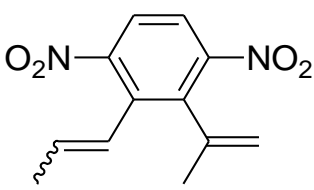

$329(50 \%)$

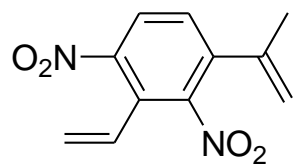

$330(45 \%)$

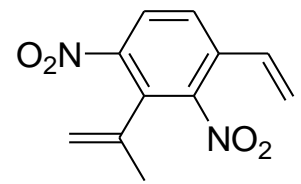

$331(27 \%)$

The route to Type E pyrroloindoles involved the separation of isomeric nitration product affording the precursor in moderate to low yield. Thus, a second route to the precursors was pursued. Aniline $\mathbf{3 3 2}^{216}$ was converted to aryl iodide $\mathbf{3 3 3}$ via reaction of the corresponding diazonium salt with potassium iodide (Scheme 7.15). Employing Mundla's methodology ${ }^{217}$ condensation of $\mathbf{3 3 3}$ with para-formaldehyde in the presence of a catalytic amount of potassium hydroxide afforded alcohol 334. Conversion of alcohol 334 to the corresponding mesylate, which was not isolated, followed by subsequent elimination afforded dinitrostyrene 330. Kosugi-Migita-Stille coupling between iodide 335 and 284 gave the expected substrate 330. Unfortunately, all attempts to broaden the scope of Mundla's methodology substituting para-formaldehyde with other aldehydes, for example hexanal, were unsuccessful. 
Scheme 7.15 Alternative route to $\mathbf{3 3 0}$
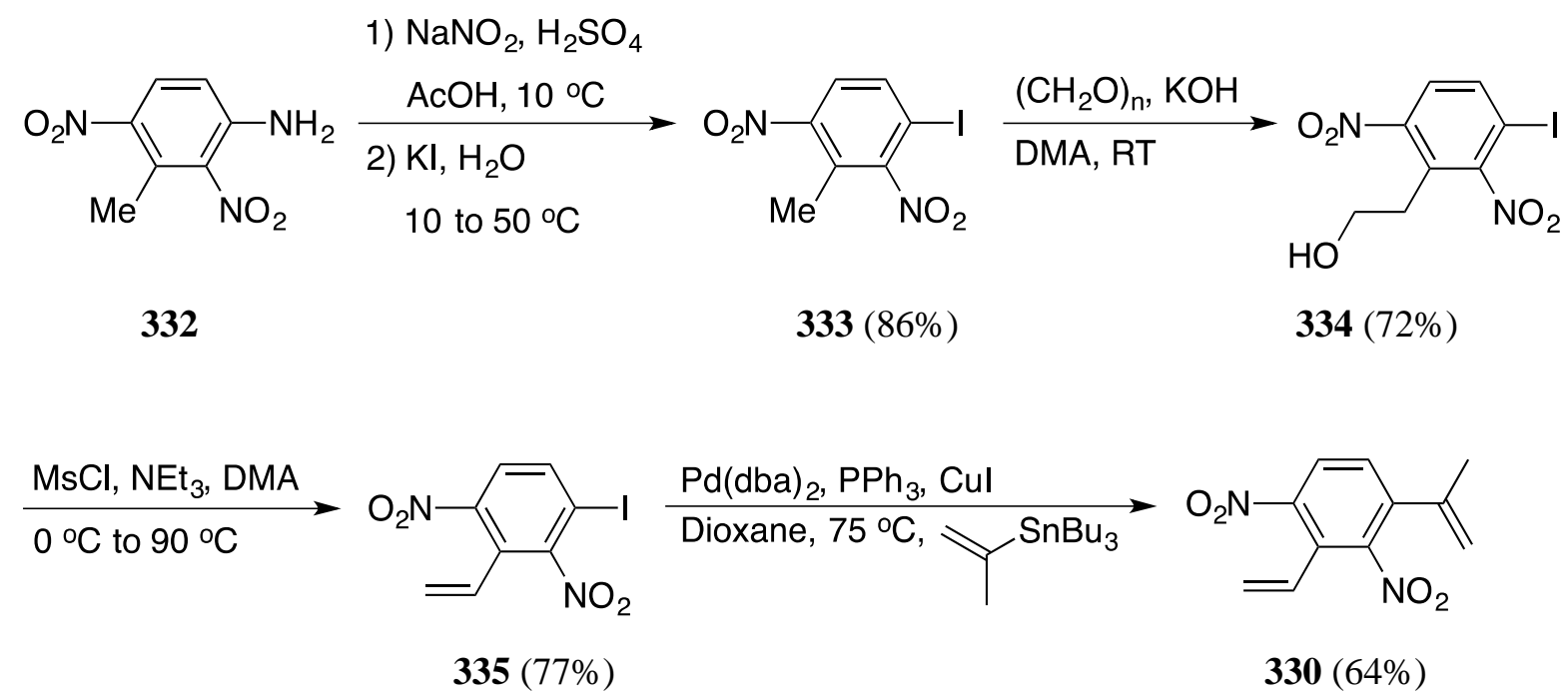

\section{D Synthesis of $1 \mathrm{H}, 8 \mathrm{H}$-pyrrolo[3,2-g]indoles (Type A)}

After successful synthesis of symmetrically as well as unsymmetrically substituted cyclization precursors, they were subjected to the reductive cyclization conditions as discussed in table 7.1. To our delight, all diakenyl-dinitrobenzenes smoothly participated in the reactions to give rise to symmetrical as well as unsymmetrical pyrroloindoles of type A (Table 7.5). The symmetrical substrates 286 and 287 also behaved as $\mathbf{2 8 5}$ to give rise to monocyclized indoles 339 and $\mathbf{3 4 1}$ along with pyrroloindoles $\mathbf{3 3 8}$ and 340 respectively. In order to compare the reactivity of substituted and the unsubstituted double bonds, compound 317 was subjected to the monocyclization conditions $\left(\mathrm{Pd}(\mathrm{OAc})_{2}, \mathrm{PPh}_{3}\right)$ as revealed in Table 7.1. Only double cyclization product 342 (53\%) was obtained; the expected monocyclization product was not detected. The formation of unsymmetrically substituted pyrroloindoles here represents the first synthesis of these compounds. All the results are summarized the table below (Table 7.5). 
Table 7.5 Synthesis of $1 H, 8 H$-pyrrolo[3,2-g]indoles (Type A)

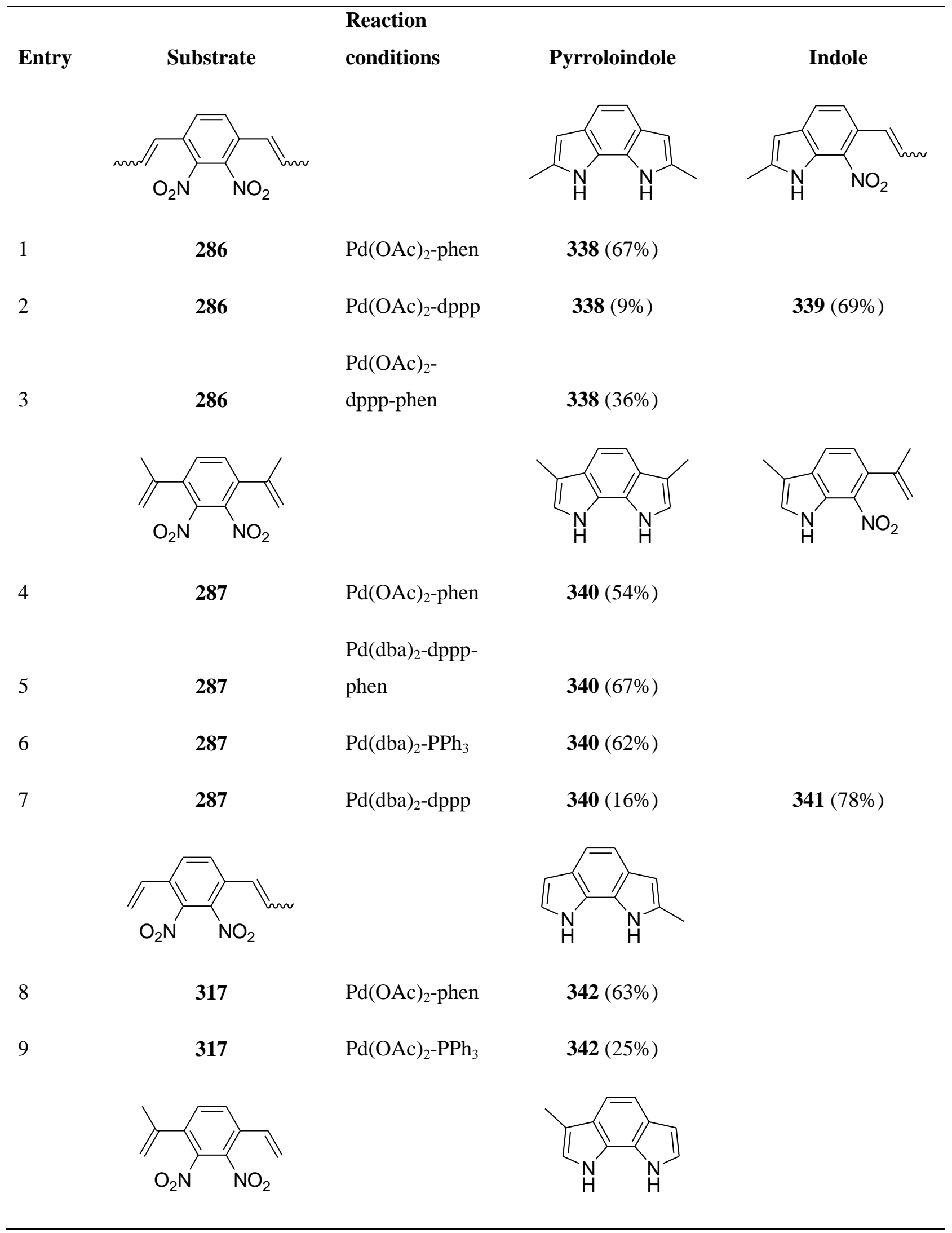




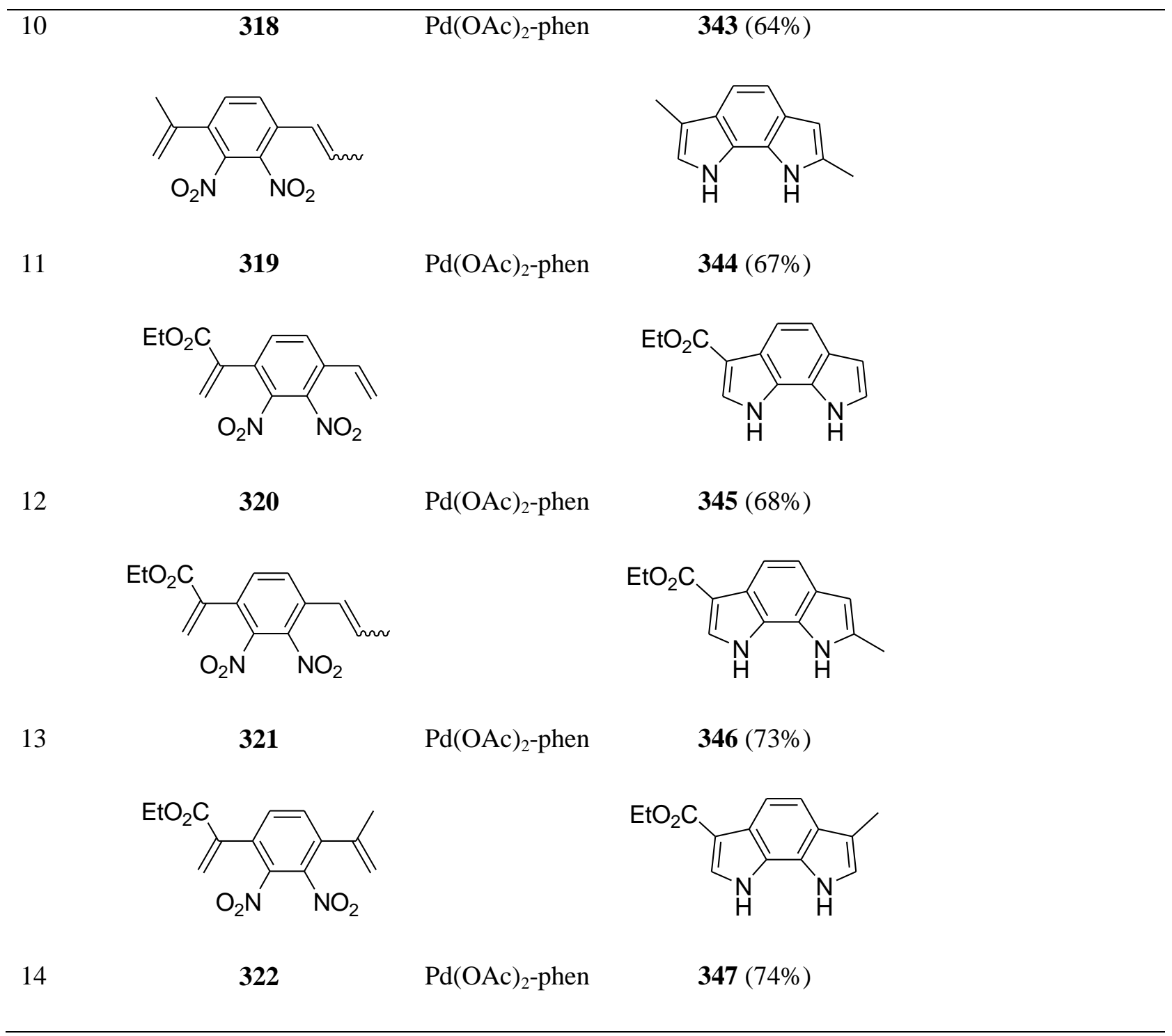

The results summarized in Table 7.5 are exciting in the sense that they open the door for the synthesis of unsymmetrically substituted pyrroloindoles of type A. Encouraged with these results, we were very sure that a combination of $\mathrm{Pd}(\mathrm{OAc})_{2}-1,10$-phen would be a good catalyst for the synthesis of rest of the pyrroloindole types. Problems then started arising. Although compound 291 gave pyrroloindole in moderate yield (53\%), the case of formation of pyrroloindole $\mathbf{3 5 0}$ was realized only with $\mathrm{Pd}(\mathrm{dba})_{2}$-dppp1,10-phen; all other conditions gave monocyclized indole (Table 7.6, entries 4). Compound 290 gave monocyclized indole even after prolonged reaction time (Table 7.6, entries 2, 3). On the other hand, the non-substituted 1,5-diethenyl-2,4-dinitrobenzene (289) did not afford any identifiable monocyclized or double cyclized product although all the starting material was consumed under any of the several conditions tested. A possible route is the polymerization but this possibility was also ruled out when similar result was obtained with $\mathrm{Pd}(\mathrm{dba})_{2}$-dppp-1,10-phen, BHT. The unsymmetrically substituted 1,5- 
dialkenyl-2,4-dinitrobenzenes of this series (compounds $\mathbf{3 2 3}$ and 324) afforded monocyclized products in very diminished yields along with an inseparable mixture of unknown impurities under all reaction conditions tried. We were unable to find a good condition for the cyclization of compounds $\mathbf{3 2 3}$ and $\mathbf{3 2 4}$.

Similar results were obtained when we attempted to synthesize pyrroloindoles of type $\mathrm{C}$. The nonsubstituted 1,4-dialkenyl-2,5-dinitrobenzene 292 participated in double cyclization to afford pyrroloindole $351(60 \%)$ uneventfully. The related cyclization precursor 294 gave pyrroloindole 387 only with $\mathrm{Pd}(\mathrm{dba})_{2}-\mathrm{PPh}_{3}$ albeit in low yield (26\%). Under all other conditions tried, compounds 293 and 294 gave monocyclized normal indoles $\mathbf{3 5 2}$ and $\mathbf{3 5 3}$ respectively. Here also the unsymmetrically substituted cyclization partners gave monocyclized indoles in diminished yield along with an inseparable mixture of unknow impurities. These compounds were not further explored for the unsymmetrically substituted pyrroloindoles of type $\mathrm{C}$.

The results from the attempted cyclizations of 298, $\mathbf{3 3 0}$ and $\mathbf{3 3 1}$ that are possible precursors to pyrroloindoles of type $\mathrm{E}$ are outlined in Table 7.6. As can been, compound $\mathbf{2 9 8}$ gave monocyclized and dicyclized products 364 (20\%) and $\mathbf{3 6 3}$ (50\%) respectively whereas the unsymmetrical partners 330 and 331 gave indoles. Compound 366 was obtained along with minor amount of pyrroloindole $\mathbf{3 6 7}$ as an inseparable mixture by chromography.

The cyclization precursors to type D were also tested for any possibility of formation of pyrroloindoles. Unsurprisingly, however, all the symmetrical dialkeynyldinitrobenzenes went on smoothly to give rise to indoles in high isolated yields after chromatography. Completely different observations were noticed when the unsymmetrically substituted dialkenyldinitrobenzenes were subjected to double cyclization; these compounds gave rise to two indoles resulting from each of the double bond cyclization as an iseparable mixture in nearly 1:1 ratio. The results are included in Table 7.6. 
Table 7.6 Attempted synthesis of pyrroloindoles (Type B-E)
Entr Substrate
$\mathbf{y}$<smiles>C=Cc1cc([N+](=O)[O-])cc([N+](=O)[O-])c1C=C</smiles>
1
289<smiles>C/C=C/c1cc(/C=C/C)c([N+](=O)[O-])cc1[N+](=O)[O-]</smiles>
2
3
290<smiles>C=C(C)c1cc(C(=C)C)c([N+](=O)[O-])cc1[N+](=O)[O-]</smiles>
4
5
6
7
291<smiles>C=Cc1cc([N+](=O)[O-])c(C=C)cc1[N+](=O)[O-]</smiles>
8
292

\section{Conditions}
Pyrroloindole
Indole
$\mathrm{Pd}(\mathrm{OAc})_{2}$, phen
$\mathrm{Pd}(\mathrm{OAc})_{2}$, phen
$\mathrm{Pd}(\mathrm{dba})_{2}, \mathrm{PPh}_{3}$<smiles>Cc1c[nH]c2cc3[nH]cc(C)c3cc12</smiles>
348 (64\%)<smiles>C=C(C)c1cc2c(C)c[nH]c2cc1[N+](=O)[O-]</smiles>
$\mathrm{Pd}(\mathrm{dba})_{2}, \mathrm{dppp}, \quad \mathbf{3 4 9}(56 \%)$ phen
$\operatorname{Pd}(\mathrm{dba})_{2}$, dppp $\mathbf{3 4 9}(16 \%)$
$350(78 \%)$
$\operatorname{Pd}(\mathrm{dba})_{2}$, phen
$350(20 \%)$
$\mathrm{Pd}(\mathrm{dba})_{2}, \mathrm{PPh}_{3}$
$350(85 \%)$ 


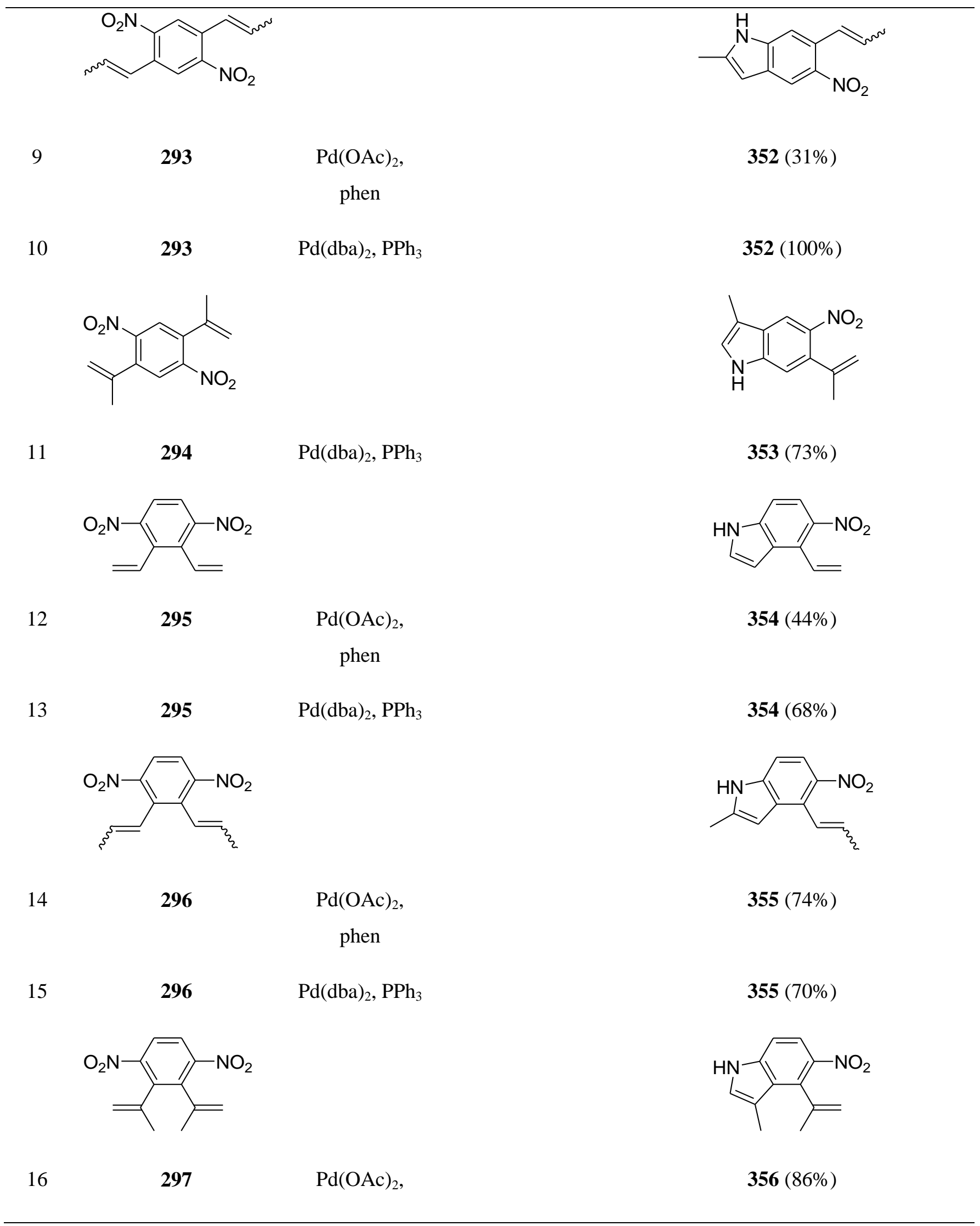




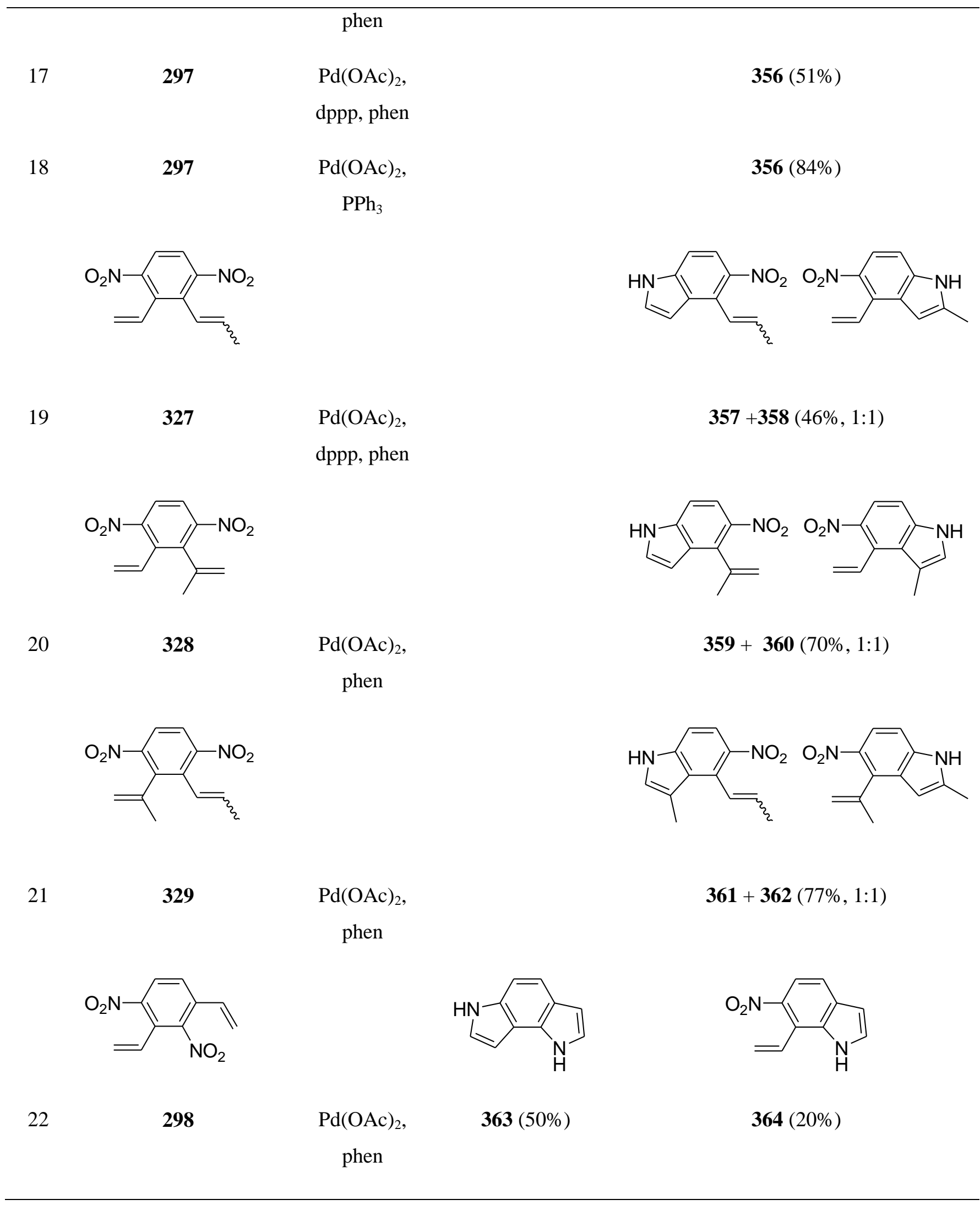




23

A second cyclization of the indoles $\mathbf{3 4 8}$ and $\mathbf{3 5 2}$ was also attempted. Submitting the mono-cyclized product from 290 to the reaction conditions $\mathrm{Pd}(\mathrm{OAc})_{2}-\mathrm{PPh}_{3}-30 \mathrm{~h}$ gave only $38 \%$ of recovered starting material whereas quantitative amount of starting material was recovered from the attempted second cyclization of 352 under the reaction conditions $\mathrm{Pd}(\mathrm{OAc})_{2}-\mathrm{PPh}_{3}-30 \mathrm{~h}$. A possible hindrance to the second cyclization may be the electronic factor associated with the indoles resulting from the first cyclization. Once the indole is formed, the ring becomes electron rich and this may be acting as an obstacle to the second cyclization. We then considered introducing electron withdrawing group to the NH group of the indole and subjecting the resulting indole to the second cyclization. The first electron withdrawing that came into our consideration was the acetyl group. Accordingly, we synthesized the acetylated indoles $\mathbf{3 6 8}$ and 369 and submitted them to our standard conditions of reductive cyclization. 
Scheme 7.16 Attempted reductive cyclization of $\mathbf{3 6 8}$ and $\mathbf{3 6 9}$
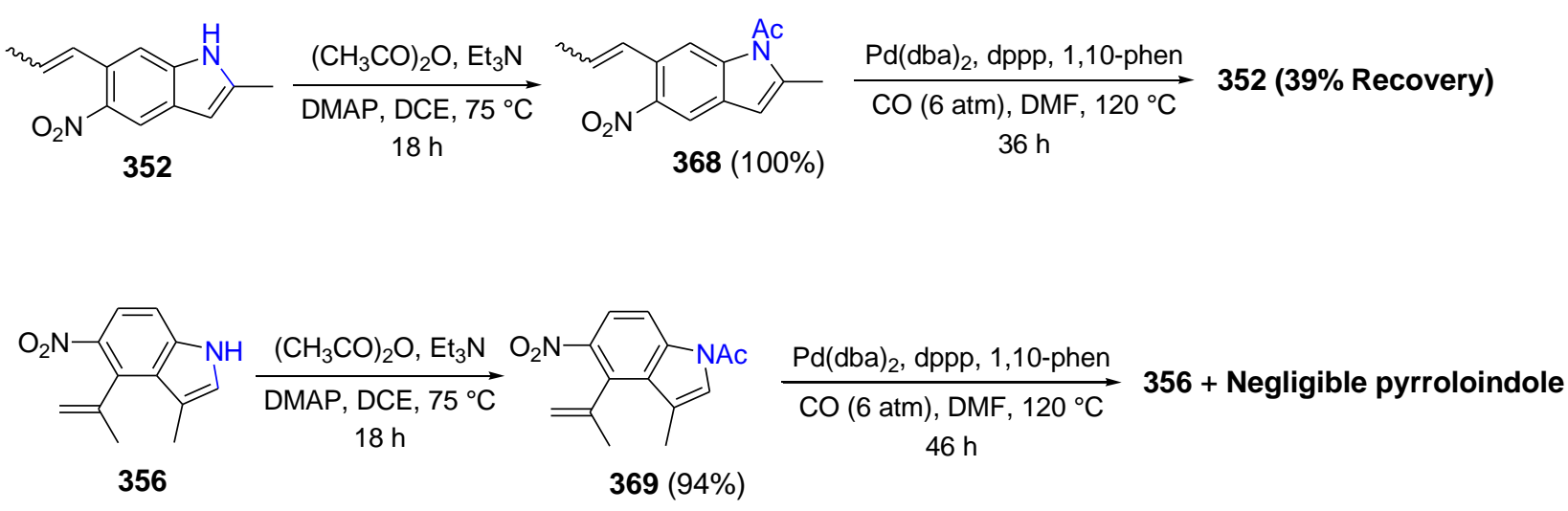

Although the result from the attempted cyclization of $\mathbf{3 6 8}$ was frustrating, the presence of a minor amount of pyrroloindole in the isolated starting material from the reductive cyclization of $\mathbf{3 6 9}$ was an indication that an electron withdrawing group may play a crucial role in the second cyclization. With this in mind, we synthesized the tosylated indole $\mathbf{3 7 0}$ by reacting $\mathbf{3 5 6}$ with $\mathrm{TsCl}$ in the presence of a base in DMF and subjected to reductive cyclization. To our delight, the tosylated pyrroloindole $\mathbf{3 7 1}$ was obtained in excellent yield (88\%) after chromatographic separation.

Scheme 7.17 Reductive cyclization of $\mathbf{3 7 0}$

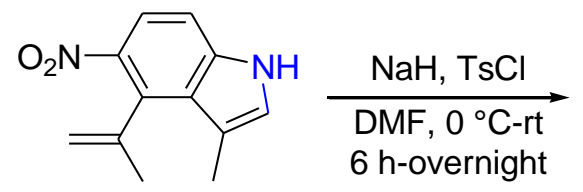

356

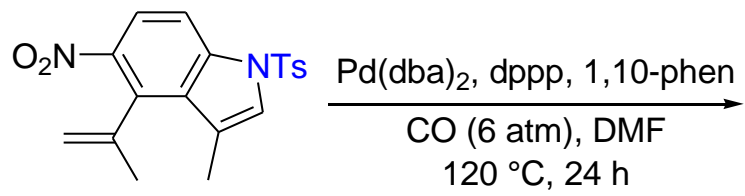

$370(78 \%)$

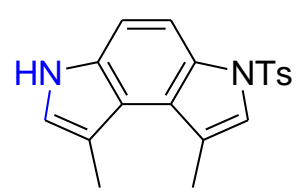

$371(88 \%)$

Encouraged with this result, the tosylated indoles 372-379 were prepared in a similar fashion and subjected to reductive cyclization. The results are summarized in the table below. 
Table 7.7 Synthesis of $N$-tosylated indoles

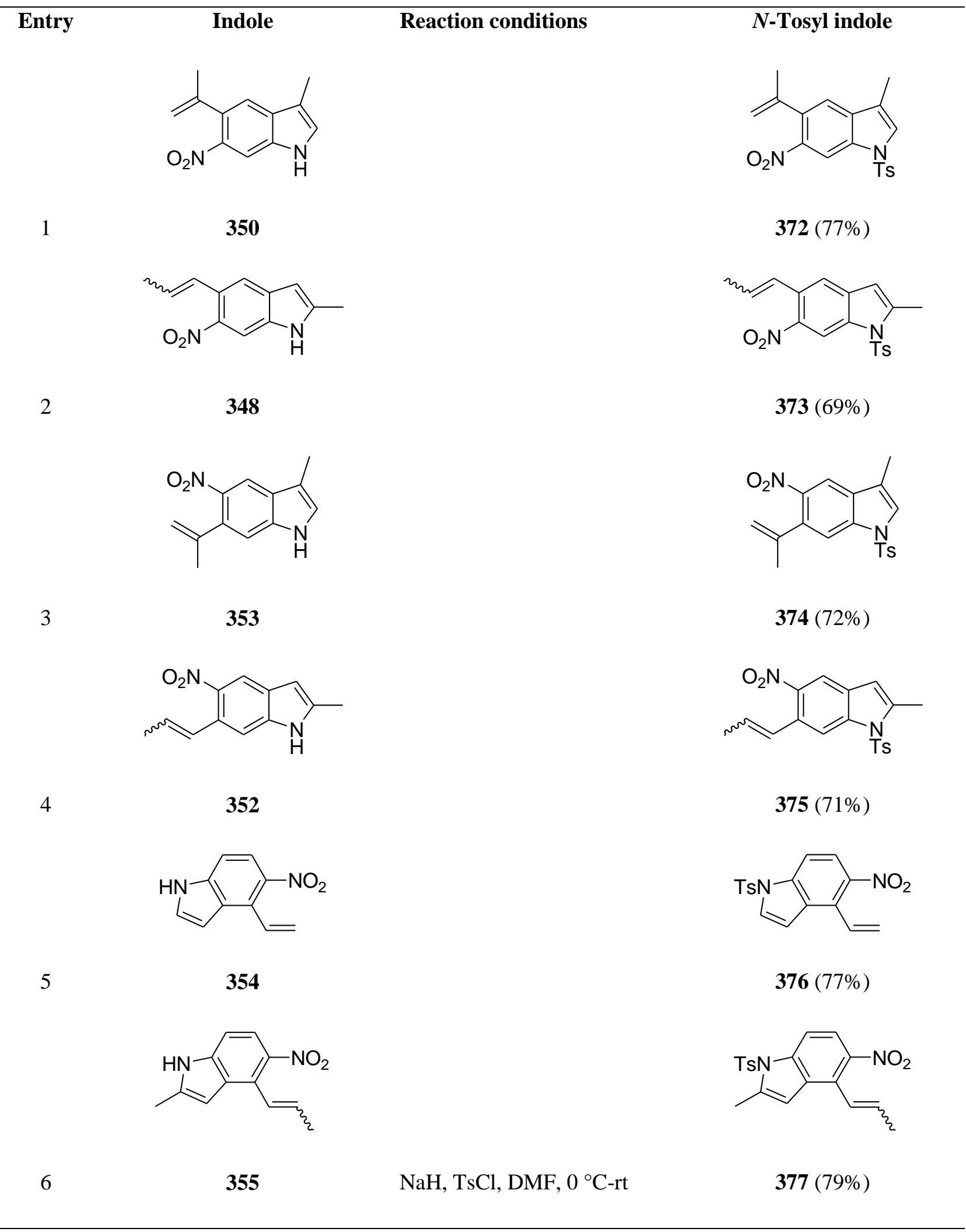




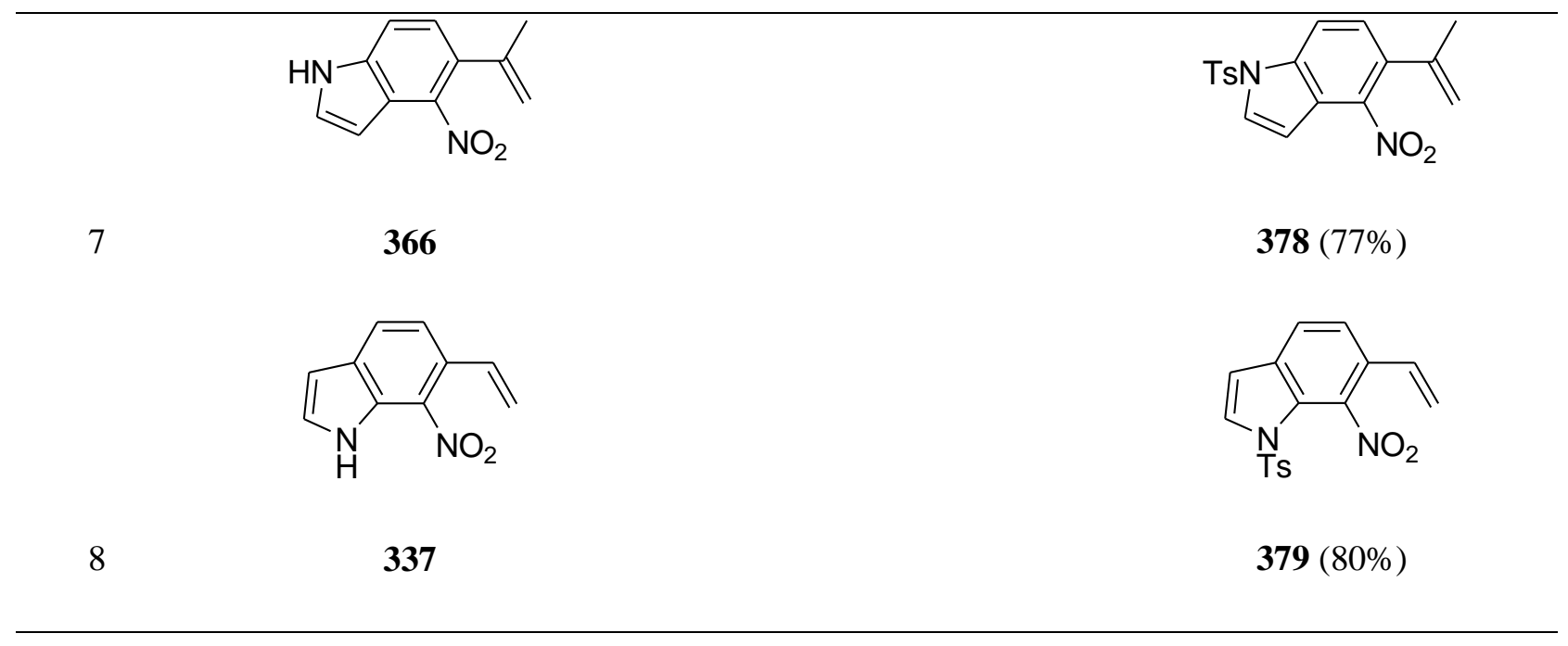

As is clear, all the problematic substrates but 379 smoothly participated in cyclization giving rise to pyrroloindoles in high to excellent isolated yields. Compound $\mathbf{3 7 9}$ was tested for cyclization under two different catalytic conditions (Table 7.8, entries 8,9). The use of tosyl group as an electron withdrawing group has several advantages, for example, it improves the yields and leads to enhanced stability of the pyrroloindoles and diminishes the overall reaction time. The non-tosylated indoles are relatively unstable in air. The results are summarized in Table 7.8.

Table 7.8 Synthesis of $N$-tosylated pyrroloindoles (Types B-E)

Entry Reaction conditions




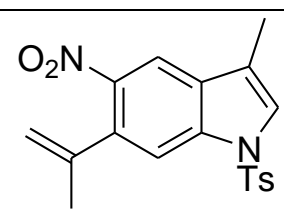

3

374<smiles>CC=Cc1cc2c(cc1C)cc(C)n2[135I]</smiles>

4<smiles></smiles>

5

376

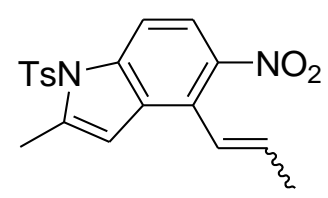

6

377<smiles>C=C(C)c1ccc2c(ccn2[Al])c1[N+](=O)[O-]</smiles>

7

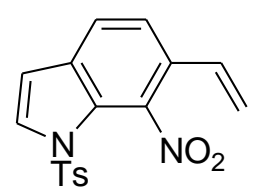

8

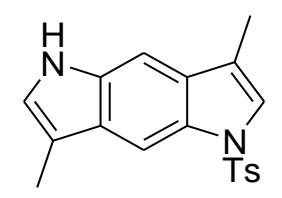

$382(88 \%)$

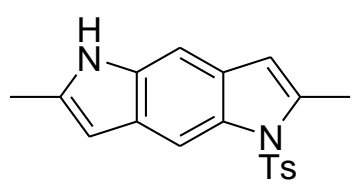

$383(76 \%)$

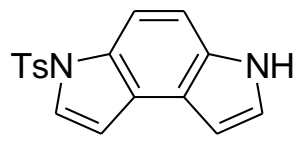

$384(89 \%)$

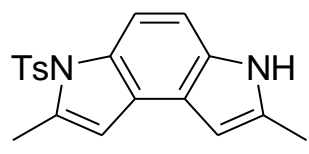

$385(77 \%)$

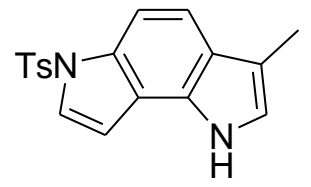

$386(87 \%)$

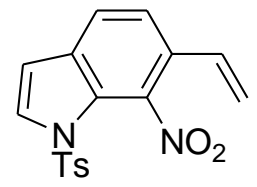

$379(70 \%)$ 


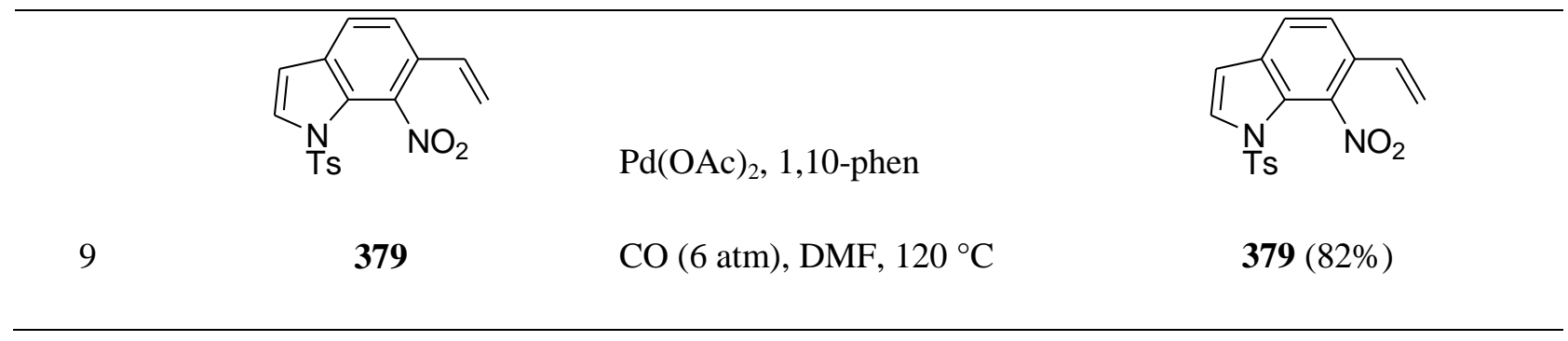

7.E Conclusion Palladium catalyzed, carbon monoxide mediated, double reductive $N$-heterocyclization of dialkenyldinitrobenzenes to the functionalized and novel non-symmetrical pyrroloindoles has been developed for the first time. This methodology represents the first common synthetic routes to all the isomeric pyrroloindoles which are of interest in a number of applications in addition to their presence as a core moiety in a wide range of bioactive natural products. The functionalized isomeric cyclization precursors were prepared through use of Kosugi-Migita-Stille coupling reaction. The generally mild catalyst conditions offer significant improvements over previously reported methods that utilize harsh conditions. This pathway opens the door for the synthesis of all the otherwise synthetically challenging unsymmetrically substituted pyrroloindoles in general. 


\section{Experimental Section}

General Procedures. All NMR spectra were recorded in $\mathrm{CDCl}_{3}$ at $600 \mathrm{MHz}\left({ }^{1} \mathrm{H} \mathrm{NMR}\right), 150 \mathrm{MHz}\left({ }^{13} \mathrm{C}\right.$ NMR, $1 \mathrm{H}$ broadband decoupled) and $376 \mathrm{MHz}{ }^{19} \mathrm{~F}$ NMR at ambient temperature unless otherwise stated. The chemical shifts are expressed in $\delta$ values relative to $\mathrm{SiMe}_{4}\left(0.0 \mathrm{ppm},{ }^{1} \mathrm{H}\right.$ and $\left.{ }^{13} \mathrm{C}\right)$ or $\mathrm{CDCl}_{3}(77.0$ ppm, ${ }^{13} \mathrm{C}$ ) internal standards. HRMS data were obtained via electrospray ionization (ESI) with an ion trap mass analyzer. THF, dichloromethane and toluene were purified and dried via a two consecutive columns composed of activated alumina and Q5 catalyst on a Glass Contours solvent purification system. Anhydrous $\mathrm{N}, \mathrm{N}$-dimethylformamide was used as received. Hexanes, ethyl acetate, and 1,4-dioxane were distilled from calcium hydride. Chemicals prepared according to literature procedures have been footnoted the first time used; all other reagents were obtained from commercial sources and used as received. All reactions were performed under a nitrogen atmosphere in oven-dried glassware. Solvents were removed from reaction mixtures and products on a rotary evaporator at water aspirator pressure unless otherwise stated. Melting points (uncorrected) were recorded from the pure products obtained by chromatography.

2-(5-Benzyloxy-2-nitrophenyl)-1-trimethylsilylethyne (2). 5-Benzyloxy-2-nitro-1-bromobenzene ${ }^{41}$ (3.91 g, $12.7 \mathrm{mmol})$ was dissolved in toluene $(10 \mathrm{~mL})$ and triethylamine $\left(\mathrm{NEt}_{3}, 40 \mathrm{~mL}\right)$ whereby bis(triphenylphosphinepalladium dichloride $\left(\mathrm{PdCl}_{2}\left(\mathrm{PPh}_{3}\right)_{2}, 0.62 \mathrm{~g}, 0.88 \mathrm{mmol}\right)$ and copper iodide (CuI, $0.17 \mathrm{~g}, 0.89 \mathrm{mmol}$ ) were added successively. The reaction mixture was stirred under a nitrogen atmosphere for five minutes. Trimethylsilylethyne $(2.17 \mathrm{~mL}, 15.2 \mathrm{mmol})$ was added drop wise and the resulting mixture was stirred at $48{ }^{\circ} \mathrm{C}$ for $24 \mathrm{~h}$. After cooling to ambient temperature, the mixture was filtered through celite, and the celite was washed with EtOAc. The solvents were removed from the filtrate under reduced pressure. The resulting residue was purified by chromatography (EtOAc/hexane, 1:9) affording 2 (3.71 g, $11.4 \mathrm{mmol}, 90 \%)$ as a faint yellow solid. $\mathrm{mp}=96{ }^{\circ} \mathrm{C}$; ${ }^{1} \mathrm{H} \mathrm{NMR}\left(400 \mathrm{MHz}, \mathrm{CDCl}_{3}\right)$ $\delta 8.07(\mathrm{~d}, J=9.2 \mathrm{~Hz}, 1 \mathrm{H}), 7.43-7.36(\mathrm{~m}, 5 \mathrm{H}), 7.18(\mathrm{~d}, J=2.8 \mathrm{~Hz}, 1 \mathrm{H}), 6.97$ (dd, $J=9.2,2.8 \mathrm{~Hz}, 1 \mathrm{H}), 5.14$ (s, 2H), 0.29 (s, 9H); ${ }^{13} \mathrm{C}$ NMR (100 MHz, $\left.\mathrm{CDCl}_{3}\right) \delta 161.8,143.2,135.3,128.8,128.5,127.5,127.0,120.7$, 120.1, 115.4, 103.7, 99.9, 70.7, 0.4; IR (APT) 1572, 1515, 1333. 1248, $836 \mathrm{~cm}^{-1}$; HRMS (ESI) calcd for $\mathrm{C}_{18} \mathrm{H}_{20} \mathrm{NO}_{3} \mathrm{Si}\left(\mathrm{M}+\mathrm{H}^{+}\right)$326.1212, found 326.1195.

1-(5-Benzyloxy-2-nitrophenyl)ethyne (3). A solution of tetrabutylammonium fluoride (1M in THF) was added drop wise to a $0{ }^{\circ} \mathrm{C}$ cold solution of $2(2.32 \mathrm{~g}, 7.13 \mathrm{mmol})$ in THF $(30 \mathrm{~mL})$. The resulting mixture was stirred at $0{ }^{\circ} \mathrm{C}$ for $30 \mathrm{~min}$. The solvent was removed under reduced pressure and the resulting residue was purified by chromatography (EtOAc/hexane, 2:8) to give $3(1.63 \mathrm{~g}, 6.44 \mathrm{mmol}, 90 \%)$ as a white solid. $\mathrm{mp}=122{ }^{\circ} \mathrm{C} ;{ }^{1} \mathrm{H}$ NMR $\left(400 \mathrm{MHz}, \mathrm{CDCl}_{3}\right) \delta 8.10(\mathrm{~d}, J=9.2 \mathrm{~Hz}, 1 \mathrm{H}), 7.45-7.35(\mathrm{~m}, 5 \mathrm{H}), 7.22(\mathrm{~d}$, 
$J=2.8 \mathrm{~Hz}, 1 \mathrm{H}), 7.02(\mathrm{dd}, J=9.2,2.8 \mathrm{~Hz}, 1 \mathrm{H}), 5.15(\mathrm{~s}, 2 \mathrm{H}), 3.52(\mathrm{~s}, 1 \mathrm{H}) ;{ }^{13} \mathrm{C} \mathrm{NMR}\left(100 \mathrm{MHz}, \mathrm{CDCl}_{3}\right) \delta$ 161.9, 143.2, 135.1, 128.8, 128.7, 127.5, 127.1, 120.8, 119.7, 115.7, 85.0, 79.0, 70.8; IR (APT) 3284, 1579, 1507, 1327, 1249, $1073 \mathrm{~cm}^{-1}$; HRMS (ESI) calcd for $\mathrm{C}_{15} \mathrm{H}_{12} \mathrm{NO}_{3}\left(\mathrm{M}+\mathrm{H}^{+}\right)$254.0817, found 254.0805 .

1-(5-Benzyloxy-2-nitrophenyl)-1-tributylstannylethene (4). To a solution of $\mathbf{3}$ (1.60 g, $6.32 \mathrm{mmol})$ in THF (30 mL) under $\mathrm{N}_{2}$ was added $\mathrm{PdCl}_{2}\left(\mathrm{PPh}_{3}\right)_{2}(0.44 \mathrm{~g}, 0.63 \mathrm{mmol})$ and tributyltin hydride $(2.76 \mathrm{~g}, 9.48$ mmol). The resulting dark brown mixture was stirred at ambient temperature under $\mathrm{N}_{2}$ for $24 \mathrm{~h}$. The mixture was diluted with EtOAc $(30 \mathrm{~mL})$ and washed with $\mathrm{H}_{2} \mathrm{O}(3 \times 30 \mathrm{~mL})$ and brine $(30 \mathrm{~mL})$, The organic layer was dried $\left(\mathrm{MgSO}_{4}\right)$, filtered, at the solvent was removed under reduced pressure. The residue was purified by chromatography (EtOAc/hexane, 1:19) affording 4 (2.99 g, $5.49 \mathrm{mmol}, 87 \%)$ as a brown viscous liquid. ${ }^{1} \mathrm{H}$ NMR $\left(400 \mathrm{MHz}, \mathrm{CDCl}_{3}\right) \delta 8.10(\mathrm{~d}, J=9.2 \mathrm{~Hz}, 1 \mathrm{H}), 7.45-7.33(\mathrm{~m}, 5 \mathrm{H}), 6.86$ (dd, $J=9.2,2.4 \mathrm{~Hz}, 1 \mathrm{H}), 6.63(\mathrm{~d}, J=2.0 \mathrm{~Hz}, 1 \mathrm{H}), 5.72$ (d, J=2.4 Hz, 1H), 5.41 (d, J=2.0 Hz, 1H), 5.14 (s, 2H), 1.56-1.37 (m, 6H), 1.27 (sext, $J=7.2 \mathrm{~Hz}, 6 \mathrm{H}), 0.95-0.84(\mathrm{~m}, 15 \mathrm{H}) ;{ }^{13} \mathrm{C} \mathrm{NMR}\left(100 \mathrm{MHz}, \mathrm{CDCl}_{3}\right) \delta 162.3$, 155.7, 147.6, 139.1, 135.6, 128.7, 128.4, 127.5, 127.1, 124.8, 114.6, 112.7,70.5, 28.8, 27.3, 13.6, 11.0; IR (ATR) 2954, 2922, 1567, 1329, 1283, 1243, 1228, $694 \mathrm{~cm}^{-1}$; HRMS (ESI) calcd for $\mathrm{C}_{27} \mathrm{H}_{40} \mathrm{NO}_{3} \mathrm{Sn}$ $\left(\mathrm{M}+\mathrm{H}^{+}\right)$546.2030, found 546.2025.

2,5-Di(1-(5-benzyloxy-2-nitrophenyl)-1-ethenyl)pyrazine (6) and 2,3-di(5-benzyloxy-2-nitrophenyl)1,3-butadiene (7). To a solution of 2,5-dibromopyrazine (5) (100 mg, $0.420 \mathrm{mmol})$ and 4 (458 mg, 0.841 $\mathrm{mmol})$ in dry DMF $(2 \mathrm{~mL})$ was added bis(dibenzylideneacetone)palladium ( $\operatorname{Pd}(\mathrm{dba})_{2}, 19.4 \mathrm{mg}, 0.034$ $\mathrm{mmol}), \mathrm{CuI}(160 \mathrm{mg}, 0.841 \mathrm{mmol})$, and $\mathrm{PPh}_{3}(35.3 \mathrm{mg}, 0.135 \mathrm{mmol})$ respectively. The resulting brown mixture was stirred under an atmosphere of $\mathrm{N}_{2}$ at $90{ }^{\circ} \mathrm{C}$ for $41 \mathrm{~h}$. EtOAc $(25 \mathrm{~mL})$ was added and the mixture was washed with $\mathrm{H}_{2} \mathrm{O}(3 \times 25 \mathrm{~mL})$ and brine $(3 \times 25 \mathrm{~mL})$. The organic layer was dried $\left(\mathrm{MgSO}_{4}\right)$, filtered, and the solvents were removed under reduced pressure. The residue was purified by chromatography on $\mathrm{SiO}_{2} / \mathrm{K}_{2} \mathrm{CO}_{3}\left(10 \% \mathrm{~K}_{2} \mathrm{CO}_{3}\right.$, EtOAc/hexane, 1:9, then 2:8) to give, in order of elution, 7 ( $85.5 \mathrm{mg}, 0.168 \mathrm{mmol}, 40 \%)$ followed by 6 (96 mg, $0.16 \mathrm{mmol}, 39 \%)$ both as white solids. Analytical data for 6: $\mathrm{mp}=186-187{ }^{\circ} \mathrm{C}$; ${ }^{1} \mathrm{H}$ NMR $\left(400 \mathrm{MHz}, \mathrm{CDCl}_{3}\right) \delta 8.51(\mathrm{~s}, 1 \mathrm{H}), 8.15(\mathrm{~d}, J=9.0 \mathrm{~Hz}, 1 \mathrm{H}), 7.45-7.33$ (m, 5H), $7.04(\mathrm{dd}, J=8.8,2.4 \mathrm{~Hz}, 1 \mathrm{H}), 7.01(\mathrm{~d}, J=2.4 \mathrm{~Hz}, 1 \mathrm{H}), 6.29(\mathrm{~s}, 1 \mathrm{H}), 5.57(\mathrm{~s}, 1 \mathrm{H}), 5.17(\mathrm{~s}, 2 \mathrm{H}) ;{ }^{13} \mathrm{C}$ NMR (100 MHz, $\left.\mathrm{CDCl}_{3}\right) \delta 162.7,149.9,144.7,141.2,140.5,137.8,135.4,128.8,128.5,127.6,127.3$, 118.8, 118.4, 114.6, 70.8; IR (APT) 1578, 1508, 1326, 1242, 1001, 919, $750 \mathrm{~cm}^{-1}$; HRMS (ESI) calcd for $\mathrm{C}_{34} \mathrm{H}_{27} \mathrm{~N}_{4} \mathrm{O}_{6}\left(\mathrm{M}+\mathrm{H}^{+}\right)$587.1931, found 587.1925. Analytical data for 7: $\mathrm{mp}=158-160{ }^{\circ} \mathrm{C}$; ${ }^{1} \mathrm{H}$ NMR (400 $\left.\mathrm{MHz}, \mathrm{CDCl}_{3}\right) \delta 8.08(\mathrm{~d}, J=9.2 \mathrm{~Hz}, 1 \mathrm{H}), 7.48-7.36(\mathrm{~m}, 5 \mathrm{H}), 7.17(\mathrm{~d}, J=2.8 \mathrm{~Hz}, 1 \mathrm{H}), 7.03$ (dd, J=9.2, 2.8 $\mathrm{Hz}, 1 \mathrm{H}), 5.20(\mathrm{~s}, 2 \mathrm{H}), 5.08(\mathrm{~s}, 1 \mathrm{H}), 4.88(\mathrm{~s}, 1 \mathrm{H}) ;{ }^{13} \mathrm{C} \mathrm{NMR}\left(100 \mathrm{MHz}, \mathrm{CDCl}_{3}\right) \delta 162.2,145.6,141.7$, 
138.3, 135.5, 128.7, 128.4, 127.8, 126.8, 117.5, 117.1, 114.9, 70.7; IR (ATR) 1573, 1513, 1343, 1230, $1007 \mathrm{~cm}^{-1}$; HRMS (ESI) calcd for $\mathrm{C}_{30} \mathrm{H}_{25} \mathrm{~N}_{2} \mathrm{O}_{6}\left(\mathrm{M}+\mathrm{H}^{+}\right)$509.1713, found 509.1715.

5,5'-Dibenzyloxy-3,3'-(2,5-pyrazindiyl)bis-1(H)-indole (8). To a solution of 5 (114 mg, $0.194 \mathrm{mmol})$ in dry DMF (2 mL), in a treaded ACE-Glass pressure tube, was added 1,3-bis(diphenylphosphino)propane (dppp, $6 \mathrm{mg}, 0.014 \mathrm{mmol}$ ), 1,10-phenanthroline (phen, $5 \mathrm{mg}, 0.027 \mathrm{mmol}$ ) and Pd(dba) 2 (8 mg, 0.014 mmol). The tube was fitted with a pressure head, and the solution was saturated with 3 cycles of CO (6 atm) and stirred at $120^{\circ} \mathrm{C}(6 \mathrm{~atm} \mathrm{CO}, 72 \mathrm{~h})$. The reaction mixture was cooled to ambient temperature, diluted with EtOAc $(30 \mathrm{~mL})$, and washed with $\mathrm{H}_{2} \mathrm{O}(3 \times 30 \mathrm{~mL})$ and brine $(3 \times 30 \mathrm{~mL})$. The organic layer was dried $\left(\mathrm{MgSO}_{4}\right)$, filtered, and the solvents were removed under reduced pressure. The residue was purified by chromatography (EtOAc/hexane, 3:7, then 6:4) to afford 8 (90 mg, $0.172 \mathrm{mmol}, 89 \%)$ as yellow powder. $\mathrm{mp}=264-265{ }^{\circ} \mathrm{C} ;{ }^{1} \mathrm{H}$ NMR $\left(400 \mathrm{MHz}\right.$, acetone-d $\left.\mathrm{d}_{6}\right) \delta 10.63(\mathrm{~s}, 1 \mathrm{H}), 9.09(\mathrm{~s}, 1 \mathrm{H}), 8.22(\mathrm{~d}$, $J=2.5 \mathrm{~Hz}, 1 \mathrm{H}), 8.14(\mathrm{~d}, J=2.8 \mathrm{~Hz}, 1 \mathrm{H}), 7.58$ (d, $J=7.6 \mathrm{~Hz}, 2 \mathrm{H}), 7.46-7.29$ (m, 5H), 6.98 (dd, J=8.8, 2.5 $\mathrm{Hz}, 1 \mathrm{H}), 5.22(\mathrm{~s}, 2 \mathrm{H}) ;{ }^{13} \mathrm{C}$ NMR (100 MHz, acetone-d $\left.{ }_{6}\right) \delta 155.0,148.0,141.0,139.2,133.6,129.2,128.6$, 128.5, 127.2, 126.3, 114.5, 113.8, 113.2, 106.3, 71.3; IR (APT) 3228, 2927, 1546, 1478, $1154 \mathrm{~cm}^{-1}$; HRMS (ESI) calcd for $\mathrm{C}_{34} \mathrm{H}_{26} \mathrm{~N}_{4} \mathrm{O}_{2}\left(\mathrm{M}+\mathrm{H}^{+}\right)$523.2134, found 523.2116.

Alocasin A. To a solution of $8(41 \mathrm{mg}, 0.078 \mathrm{mmol})$ in EtOH $(2 \mathrm{~mL})$, in a treaded pressure tube, was added $\mathrm{Pd} / \mathrm{C}(10 \%-\mathrm{Pd}, 18 \mathrm{mg}, 0.017 \mathrm{mmol})$. The tube was fitted with a pressure head and the mixture was pressurized with $\mathrm{H}_{2}(4 \mathrm{~atm})$ and stirred at $60{ }^{\circ} \mathrm{C}(12 \mathrm{~h})$. After cooling to ambient temperature, the mixture was filtered through a pad of celite and the celite was washed with warm ethanol ( $5 \mathrm{~mL})$. The solvent was removed under reduced pressure and residue was purified by chromatography (EtOAc/hexane, 7:3) to afford alocasin A (23 mg, $0.067 \mathrm{mmol}, 86 \%$ ) as faint yellow solid. $\mathrm{mp}=244-245{ }^{\circ} \mathrm{C}$ (lit. $\mathrm{mp}=243-244$ $\left.{ }^{\circ} \mathrm{C}\right) ;{ }^{9} \mathrm{H}$ NMR $\left(400 \mathrm{MHz}, \mathrm{CD}_{3} \mathrm{OD}\right) \delta 8.94(\mathrm{~s}, 2 \mathrm{H}), 7.90(\mathrm{~s}, 2 \mathrm{H}), 7.72(\mathrm{~d}, J=2.4 \mathrm{~Hz}, 2 \mathrm{H}), 7.30$ (d, J=8.4 Hz, 2H), $6.79(\mathrm{dd}, J=8.8,2.4 \mathrm{~Hz}, 2 \mathrm{H}) ;{ }^{13} \mathrm{C}$ NMR (100 MHz, $\left.\mathrm{CD}_{3} \mathrm{OD}\right) \delta 152.8,148.4,141.4,133.6,127.3$, 126.6, 113.8, 113.3, 113.2, 106.1; IR (ATR) 3298, 2927, 1551, 1466, $1154 \mathrm{~cm}^{-1}$; HRMS (ESI) calcd for $\mathrm{C}_{20} \mathrm{H}_{15} \mathrm{~N}_{4} \mathrm{O}_{2}\left(\mathrm{M}+\mathrm{H}^{+}\right)$343.1195, found 343.1188.

2,3-Di(5-benzyloxy-2-nitrophenyl)-1,3-butadiene (7). To a solution of 3,5-dibromopyridine (9) (217 $\mathrm{mg}, 0.919 \mathrm{mmol})$ and $4(1.00 \mathrm{~g}, 1.84 \mathrm{mmol})$ in dry DMF $(6 \mathrm{~mL})$ was added Pd(dba) $2(52.8 \mathrm{mg}, 0.092$ $\mathrm{mmol}), \mathrm{CuI}$ (350 mg, $1.84 \mathrm{mmol})$, and $\mathrm{PPh}_{3}(96.4 \mathrm{mg}, 0.367 \mathrm{mmol})$ respectively. The resulting brown mixture was stirred under an atmosphere of $\mathrm{N}_{2}$ at $80{ }^{\circ} \mathrm{C}$ for $24 \mathrm{~h}$. EtOAc $(25 \mathrm{~mL})$ was added and the mixture was washed with brine $(6 \times 50 \mathrm{~mL})$. The organic layer was dried $\left(\mathrm{MgSO}_{4}\right)$, filtered, and the solvents were removed under reduced pressure. The residue was purified by chromatography on $\mathrm{SiO}_{2} / \mathrm{K}_{2} \mathrm{CO}_{3}\left(10 \% \mathrm{~K}_{2} \mathrm{CO}_{3}\right.$, EtOAc/hexane, 1:9, then 2:8) to give 7 (139 mg, $\left.0.273 \mathrm{mmol}, 30 \%\right)$. 
3,5-(1-Tributylstannyl-1-ethenyl)pyridine (11). Treatment of 3,5-diethynylpyridine $(\mathbf{1 0})^{42}(150 \mathrm{mg}$, $1.18 \mathrm{mmol})$ with $\mathrm{Bu}_{3} \mathrm{SnH}(1.03 \mathrm{mg}, 3.59 \mathrm{mmol})$ in the presence of $\mathrm{PdCl}_{2}\left(\mathrm{PPh}_{3}\right)_{2}(100 \mathrm{mg}, 0.142 \mathrm{mmol})$ in THF (4 mL), as described for 4 (36 h), gave after work up and chromatography (EtOAc/hexanes, 2:98) 11 (510 mg, $0.719 \mathrm{mmol}, 61 \%$ ) as yellow oil. ${ }^{1} \mathrm{H}$ NMR (400 MHz, $\left.\mathrm{CDCl}_{3}\right) \delta 8.24$ (d, J=2.4 Hz, 1H), $7.23(\mathrm{t}$, $J=2.0 \mathrm{~Hz}, 1 \mathrm{H}), 6.05$ (d, $J=2.4 \mathrm{~Hz}, 1 \mathrm{H}), 5.51$ (d, $J=2.4 \mathrm{~Hz}, 1 \mathrm{H}), 1.57-1.24$ (m, 6H), 1.29 (sext, $J=7.2 \mathrm{~Hz}$, 6H), 1.07-0.79 (m, 15H); ${ }^{13} \mathrm{C}$ NMR (100 MHz, $\left.\mathrm{CDCl}_{3}\right) \delta 151.3,145.4,141.5,130.4,128.7,29.0,27.2$, 13.6, 10.3; IR (ATR) 2956, 2925, 1463, $920 \mathrm{~cm}^{-1}$; HRMS (ESI) calcd for $\mathrm{C}_{33} \mathrm{H}_{62} \mathrm{NSn}_{2}\left(\mathrm{M}+\mathrm{H}^{+}\right) 712.2926$, found 712.2929 .

2-Iodo-4-benzyloxy-1-nitrobenzene (12). A mixture of 3-iodo-4-nitrophenol (5.83 g, $22.0 \mathrm{mmol}$ ), benzyl bromide $(4.86 \mathrm{~g}, 35.2 \mathrm{mmol})$ and $\mathrm{K}_{2} \mathrm{CO}_{3}(5.64 \mathrm{~g}, 40.8 \mathrm{mmol})$ in absolute EtOH (50 mL) was heated ate reflux for $18 \mathrm{~h}$. The resulting mixture was concentrated under reduced pressure and EtOAc (50 $\mathrm{mL})$ was added to the resulting residue. The mixture was washed with water $(3 \times 40 \mathrm{~mL})$, dried $\left(\mathrm{MgSO}_{4}\right)$, and filtered. The filtrate was concentrated under reduced pressure and the resulting residue was purified by chromatography (EtOAc/hexanes, 2:8) to afford 12 (7.32 g, $20.6 \mathrm{mmol}, 94 \%)$ as an orange-yellow solid. $\mathrm{mp}=73-75^{\circ} \mathrm{C}$; ${ }^{1} \mathrm{H}$ NMR $\left(400 \mathrm{MHz}, \mathrm{CDCl}_{3}\right) \delta 7.97(\mathrm{~d}, J=9.2 \mathrm{~Hz}, 1 \mathrm{H}), 7.63(\mathrm{~d}, J=2.8 \mathrm{~Hz}, 1 \mathrm{H}), 7.45$ $7.36(\mathrm{~m}, 5 \mathrm{H}), 7.00(\mathrm{dd}, J=8.8,2.8 \mathrm{~Hz}, 1 \mathrm{H}), 5.12(\mathrm{~s}, 2 \mathrm{H}) ;{ }^{13} \mathrm{C} \mathrm{NMR}\left(100 \mathrm{MHz}, \mathrm{CDCl}_{3}\right) \delta 161.6,145.4$, 135.0, 128.8, 128.6, 128.0, 127.5, 127.5, 114.7, 88.1, 70.8; IR (ATR) 1575, 1507, 1332, 1241, $982 \mathrm{~cm}^{-1}$; HRMS (ESI) calcd for $\mathrm{C}_{13} \mathrm{H}_{11} \mathrm{INO}_{3}\left(\mathrm{M}+\mathrm{H}^{+}\right) 355.9784$, found 355.9785 .

3,5-Di(5-benzyloxy-2-nitrophenyl)pyridine (13). Reaction of 11 (750 mg, $1.06 \mathrm{mmol})$ with 12 (1.13 g, $3.17 \mathrm{mmol})$ in the presence of $\mathrm{Pd}(\mathrm{dba})_{2}\left(61 \mathrm{mg}, 0.11 \mathrm{mmol}, \mathrm{PPh}_{3}(111 \mathrm{mg}, 0.423 \mathrm{mmol})\right.$ and $\mathrm{CuI}(403$ $\mathrm{mg}, 2.11 \mathrm{mmol})$ in DMF $(6 \mathrm{~mL})$, as described for $5\left(120^{\circ} \mathrm{C}, 48 \mathrm{~h}\right)$ gave after work up and chromatography (EtOAc/hexanes, 2:8 then 3:7) 13 (216 mg, $0.369 \mathrm{mmol}, 35 \%)$ as viscous yellow oil. ${ }^{1} \mathrm{H}$ NMR (400 MHz, $\left.\mathrm{CDCl}_{3}\right) \delta 8.33(\mathrm{~d}, J=2.0 \mathrm{~Hz}, 2 \mathrm{H}), 8.07$ (d, J=8.8 Hz, 2H), $7.51(\mathrm{t}, J=2.0 \mathrm{~Hz}, 1 \mathrm{H}), 7.46-$ 7.37 (m, 10H), 7.04 (dd, J=9.2, $2.8 \mathrm{~Hz}, 2 \mathrm{H}), 6.98$ (d, J=2.8 Hz, 2H), 5.73 (s, 2H), 5.36 (s, 2H), 5.17 (s, $4 \mathrm{H}) ;{ }^{13} \mathrm{C}$ NMR $\left(100 \mathrm{MHz}, \mathrm{CDCl}_{3}\right) \delta 162.4,146.8,144.1,141.2,138.4,135.3,134.4,130.8,128.8,128.5$, 127.6, 127.4, 118.2, 116.8, 114.7, 70.8; IR (ATR) 1572, 1509, 1332, $1224 \mathrm{~cm}^{-1}$; HRMS (ESI) calcd for $\mathrm{C}_{35} \mathrm{H}_{28} \mathrm{~N}_{3} \mathrm{O}_{6}\left(\mathrm{M}+\mathrm{H}^{+}\right)$586.1978, found 586.1979.

3,5-Bis(5-benzyloxy-3-indolyl)pyridine (14). Treatment of a solution of $\mathbf{1 3}$ (176 $\mathrm{mg}, 0.300 \mathrm{mmol})$ in the presence of $\mathrm{Pd}_{2}(\mathrm{dba})_{3}(28 \mathrm{mg}, 0.030 \mathrm{mmol})$, dppp (12 mg, $\left.0.030 \mathrm{mmol}\right)$, and phen $(11 \mathrm{mg}, 0.060 \mathrm{mmol})$ in DMF (1.5 mL) with CO (6 atm), as described for $8\left(120{ }^{\circ} \mathrm{C}, 68 \mathrm{~h}\right)$, gave after work up and chromatography (EtOAc/hexanes, in order 7:3, 3:7) 14 (105 $\mathrm{mg}, 0.201 \mathrm{mmol}, 67 \%)$ as a viscous yellow oil. ${ }^{1} \mathrm{H}$ NMR (400 MHz, $\left.\mathrm{CDCl}_{3}\right) \delta 8.91(\mathrm{br}, \mathrm{s}, 2 \mathrm{H}), 8.84(\mathrm{~d}, J=2.0 \mathrm{~Hz}, 2 \mathrm{H}), 8.12(\mathrm{t}, J=2.0 \mathrm{~Hz}, 1 \mathrm{H}), 7.50$ 
(d, $J=2.4 \mathrm{~Hz}, 2 \mathrm{H}), 7.44-7.42(\mathrm{~m}, 4 \mathrm{H}), 7.35-7.24(\mathrm{~m}, 10 \mathrm{H}), 7.02(\mathrm{dd}, J=8.8,2.4 \mathrm{~Hz}, 2 \mathrm{H}), 5.07(\mathrm{~s}, 4 \mathrm{H}) ;{ }^{13} \mathrm{C}$ NMR $\left(100 \mathrm{MHz}, \mathrm{CDCl}_{3}\right) \delta 154.0,145,2,137.4,132.6,132.1,131.9,128.4,127.7,127.5,125.9,123.4$, 114.1, 113.5, 112.4, 102.9, 70.9; IR (ATR) 3427, 3287, 1594, 1477, $1196 \mathrm{~cm}^{-1}$; HRMS (ESI) calcd for $\mathrm{C}_{35} \mathrm{H}_{28} \mathrm{~N}_{3} \mathrm{O}_{2}\left(\mathrm{M}+\mathrm{H}^{+}\right)$522.2181, found 522.2182.

Scalaridine A. Treatment of a solution of $14(87 \mathrm{mg}, 0.168 \mathrm{mmol})$ with Pd/C (10\%, $21 \mathrm{mg}, 0.020 \mathrm{mmol})$ in $\mathrm{EtOH}(1.5 \mathrm{~mL})$, as decribed for alocasin $\mathrm{A}\left(4 \mathrm{~atm}, 60^{\circ} \mathrm{C}, 12 \mathrm{~h}\right)$ gave after work up and chromatography (EtOAc), scalaradine A (45 mg, $0.13 \mathrm{mmol}, 79 \%$ ) as yellow solid. $\mathrm{mp}=321^{\circ} \mathrm{C}$ dec. (lit. $\mathrm{mp}=319-329^{\circ} \mathrm{C}$ dec.); ${ }^{13}{ }^{1} \mathrm{H}$ NMR (400 MHz, DMSO-d 6 ) $\delta 11.26$ (br, s, 2H), 8.80 (s, 2H), 8.69 (br, s, 2H), 8.18 (s, 1H), $7.78(\mathrm{~d}, J=2.4 \mathrm{~Hz}, 2 \mathrm{H}), 7.29(\mathrm{~d}, J=8.8 \mathrm{~Hz}, 2 \mathrm{H}), 7.23(\mathrm{~d}, J=2.4 \mathrm{~Hz}, 2 \mathrm{H}), 6.70(\mathrm{dd}, J=8.4,2.0 \mathrm{~Hz}, 2 \mathrm{H}) ;{ }^{13} \mathrm{C}$ NMR (100 MHz,DMSO-d 6 ) $\delta$ 151.6, 143.7, 132.0, 131.3, 130.0, 125.6, 124.5, 112.6, 112.0, 111.5, 102.6 ; IR (ATR) 3427, 3286, 1594, 1475, $1195 \mathrm{~cm}^{-1}$; HRMS (ESI) calcd for $\mathrm{C}_{21} \mathrm{H}_{16} \mathrm{~N}_{3} \mathrm{O}_{2}\left(\mathrm{M}+\mathrm{H}^{+}\right) 342.1243$, found 342.1240 .

(5-Benzyloxy-2-nitrophenyl)ethene (16), 2,3-di(5-benzyloxy-2-nitrophenyl)-1,3-butadiene (7), 1-(5benzyloxy-2-nitrophenyl)-1-(2-pyrimidyl)ethene (17). Reaction of 2,5-dibromopyrimidine (15) (300 $\mathrm{mg}, 1.26 \mathrm{mmol})$ and $4(2.06 \mathrm{~g}, 3.78 \mathrm{mmol})$ in the presence of $\mathrm{Pd}(\mathrm{dba})_{2}(73 \mathrm{mg}, 0.13 \mathrm{mmol}), \mathrm{CuI}(720$ $\mathrm{mg}, 3.78 \mathrm{mmol}$ ), and $\mathrm{PPh}_{3}(132 \mathrm{mg}, 0.504 \mathrm{mmol})$ in dry DMF (5 mL) under argon as described for 5 (100 $\left.{ }^{\circ} \mathrm{C}, 48 \mathrm{~h}\right)$, gave after work up and chromatography on $\mathrm{SiO}_{2} / \mathrm{K}_{2} \mathrm{CO}_{3}\left(10 \% \mathrm{~K}_{2} \mathrm{CO}_{3}\right.$, EtOAc/hexane, 1:9, then 2:8), in order of elution, 16 (101 mg, $0.396 \mathrm{mmol}, 10 \%)$ as a white solid, an inseparable mixture of 7 and an unknown impurity $(90 \mathrm{mg},<8 \%)$ as a white solid, and $17(114 \mathrm{mg}, 0.342 \mathrm{mmol}, 27 \%)$ as a viscous greenish-brown oil. Analytical data for $16 \mathrm{mp}=58-59{ }^{\circ} \mathrm{C}$; ${ }^{1} \mathrm{H} \mathrm{NMR}\left(400 \mathrm{MHz}, \mathrm{CDCl}_{3}\right) \delta 8.05(\mathrm{~d}, J=9.2$ $\mathrm{Hz}, 1 \mathrm{H}), 7.44-7.32(\mathrm{~m}, 5 \mathrm{H}), 7.30$ (dd, $J=17.2,10.8 \mathrm{~Hz}, 1 \mathrm{H}), 7.11$ (d, $J=2.8 \mathrm{~Hz}, 1 \mathrm{H}), 6.94$ (dd, J=9.2, 2.8 $\mathrm{Hz}, 1 \mathrm{H}), 5.65$ (dd, $J=17.2,0.8 \mathrm{~Hz}, 1 \mathrm{H}), 5.46$ (dd, $J=11.2,0.8 \mathrm{~Hz}, 1 \mathrm{H}), 5.17$ (s, 2H); ${ }^{13} \mathrm{C} \mathrm{NMR}(100 \mathrm{MHz}$, $\left.\mathrm{CDCl}_{3}\right) \delta 162.3,140.9,136.7,135.5,133.7,128.8,128.5,127.5,127.3,118.6,114.4,114.0,70.6$; IR (ATR) $1581,1501,1238,1078,987,930 \mathrm{~cm}^{-1}$.

Analytical data for 17: ${ }^{1} \mathrm{H} \mathrm{NMR}\left(400 \mathrm{MHz}, \mathrm{CDCl}_{3}\right) \delta 8.65$ (d, J=5.2 Hz, 1H), 8.18 (d, J=8.8 Hz, 1H), 7.46-7.35 (m, 5H), 7.10 (t, J=5.2 Hz, 1H), 7.07 (d, $J=2.8 \mathrm{~Hz}, 1 \mathrm{H}), 7.04$ (dd, J=8.8, $2.8 \mathrm{~Hz}, 1 \mathrm{H}), 6.86$ (d, $J=1.2 \mathrm{~Hz}, 1 \mathrm{H}), 5.78(\mathrm{~d}, J=1.2 \mathrm{~Hz}, 1 \mathrm{H}), 5.17(\mathrm{~s}, 2 \mathrm{H}) ;{ }^{13} \mathrm{C} \mathrm{NMR}\left(100 \mathrm{MHz}, \mathrm{CDCl}_{3}\right) \delta 163.9,162.6,156.9$, 146.9, 141.6, 138.2, 135.5, 128.7, 128.5, 127.6, 127.0, 122.4, 119.2, 118.4, 113.9, 70.6; IR (ATR) 1583, 1567, 1554, 1506, 1336, 1249, 758, $698 \mathrm{~cm}^{-1}$; HRMS (ESI) calcd for $\mathrm{C}_{19} \mathrm{H}_{16} \mathrm{~N}_{3} \mathrm{O}_{3}\left(\mathrm{M}+\mathrm{H}^{+}\right) 334.1192$, found 334.1190 .

2,5-Di(2-trimethylsilyl-1-ethynyl)pyrimidine (18). A solution of 15 (2.25 g, 9.49 mmol), $\mathrm{PdCl}_{2}\left(\mathrm{PPh}_{3}\right)_{2}$ (666 mg, $0.949 \mathrm{mmol})$, and $\mathrm{CuI}(362 \mathrm{mg}, 1.90 \mathrm{mmol})$ in toluene $(9 \mathrm{~mL})$ was stirred at ambient 
temperature under an atmosphere of $\mathrm{N}_{2}$ for $5 \mathrm{~min}$. Triethylamine $(25 \mathrm{~mL}$ ) and a solution of trimethylsilylethyne $(2.52 \mathrm{~g}, 25.6 \mathrm{mmol})$ in $\mathrm{NEt}_{3}(4 \mathrm{~mL})$ was added. The resulting dark brown reaction mixture was stirred at $50{ }^{\circ} \mathrm{C}$ for $24 \mathrm{~h}$. After cooling to ambient temperature, the mixture was filtered through Celite and the bed was washed with $\mathrm{Et}_{2} \mathrm{O}(25 \mathrm{~mL})$. The filtrate was concentrated under reduced pressure and the resulting residue was purified by chromatography (EtOAc/hexanes, 5:95) to afford 18 (2.59 g, $0.949 \mathrm{mmol}, 100 \%)$ as pale green solid. mp=99-100 ${ }^{\circ} \mathrm{C} ;{ }^{1} \mathrm{H} \mathrm{NMR}\left(400 \mathrm{MHz}, \mathrm{CDCl}_{3}\right) \delta 8.72(\mathrm{~s}$, $2 \mathrm{H}), 0.29(\mathrm{~s}, 9 \mathrm{H}), 0.27(\mathrm{~s}, 9 \mathrm{H}) ;{ }^{13} \mathrm{C} \mathrm{NMR}\left(100 \mathrm{MHz}, \mathrm{CDCl}_{3}\right) \delta$ 159.2, 149.9, 118.2, 104.6, 102.1, 97.6, 96.8, -0.4, -0.6; IR (ATR) 2963, 2163,1413, 1245, $838 \mathrm{~cm}^{-1}$; HRMS (ESI) calcd for $\mathrm{C}_{14} \mathrm{H}_{21} \mathrm{~N}_{2} \mathrm{Si}_{2}\left(\mathrm{M}+\mathrm{H}^{+}\right)$ 273.1243 , found 273.1240 .

2,5-Diethynylpyrimidine (19). A heterogeneous mixture of 18 (744 mg, $2.94 \mathrm{mmol}), \mathrm{K}_{2} \mathrm{CO}_{3}(2.43 \mathrm{~g}$, $17.6 \mathrm{mmol})$ in $\mathrm{MeOH} / \mathrm{Et}_{2} \mathrm{O}(1: 2,15 \mathrm{~mL})$ was stirred at ambient temperature under an $\mathrm{N}_{2}$ atmosphere for 1 h. Water $(20 \mathrm{~mL})$ was added and the mixture was extracted in EtOAc $(3 \times 10 \mathrm{~mL})$. The combined organic layer was concentrated under reduced pressure and the resulting crude product was purified by chromatography (EtOAc/hexanes, 1:1) to afford $19(326 \mathrm{mg}, 2.54 \mathrm{mmol}, 93 \%)$ as a white solid. $\mathrm{mp}=97$ $98{ }^{\circ} \mathrm{C} ;{ }^{1} \mathrm{H}$ NMR $\left(400 \mathrm{MHz}, \mathrm{CDCl}_{3}\right) \delta 8.79(\mathrm{~s}, 2 \mathrm{H}), 3.49(\mathrm{~s}, 1 \mathrm{H}), 3.26(\mathrm{~s}, 1 \mathrm{H}) ;{ }^{13} \mathrm{C} \mathrm{NMR}(100 \mathrm{MHz}$, $\left.\mathrm{CDCl}_{3}\right) \delta 159.6,150.2,117.8,86.1,81.5,78.0,76.7$; IR (ATR) 3291, 3180, 2129, 2100, $1415 \mathrm{~cm}^{-1}$; HRMS (ESI) calcd for $\mathrm{C}_{8} \mathrm{H}_{5} \mathrm{~N}_{2}\left(\mathrm{M}+\mathrm{H}^{+}\right)$129.0452, found 129.0448 .

2,5-Di(1-tributylstannyl-1-ethenyl)pyrimidine (20). THF (4 mL) was added to a flask containing 19 (257 mg, $2.01 \mathrm{mmol})$ and $\mathrm{PdCl}_{2}\left(\mathrm{PPh}_{3}\right)_{2}(169 \mathrm{mg}, 0.241 \mathrm{mmol})$ under an atmosphere of $\mathrm{N}_{2}$. The mixture was stirred at ambient temperature for $5 \mathrm{~min}$ followed by slow addition of $\mathrm{Bu}_{3} \mathrm{SnH}(1.75 \mathrm{~g}, 6.03 \mathrm{mmol})$ dissolved in THF $(1 \mathrm{~mL})$. The resulting brown mixture was stirred at the ambient temperature for $34 \mathrm{~h}$. The mixture was then concentrated under reduced pressure and the residue was purified by chromatography (EtOAc/hexanes, 2:98) to give $20(566 \mathrm{mg}, 0.797 \mathrm{mmol}, 40 \%)$ as pale yellow oil. ${ }^{1} \mathrm{H}$ NMR $\left(400 \mathrm{MHz}, \mathrm{CDCl}_{3}\right) \delta 8.47(\mathrm{~s}, 2 \mathrm{H}), 7.10(\mathrm{~d}, J=2.8 \mathrm{~Hz}, 1 \mathrm{H}), 6.12(\mathrm{~d}, J=2.0 \mathrm{~Hz}, 1 \mathrm{H}), 5.86(\mathrm{~d}, J=2.8$ $\mathrm{Hz}, 1 \mathrm{H}), 5.56$ (d, J=2.4 Hz, 1H), 1.58-1.43 (m, 12H), 1.30 (dhex, J=7.2, 3.2 Hz, 12H), 1.04-0.84 (m, $30 \mathrm{H}) ;{ }^{13} \mathrm{C} \mathrm{NMR}\left(100 \mathrm{MHz}, \mathrm{CDCl}_{3}\right) \delta 166.7,154.4,153.9,147.8,136.3,132.4,129.7,29.1,28.9,27.3$, 27.2, 13.7, 13.6, 10.4, 10.3; IR (ATR) 2955, 2922, 2852, $1427 \mathrm{~cm}^{-1}$; HRMS (ESI) calcd for $\mathrm{C}_{32} \mathrm{H}_{61} \mathrm{~N}_{2} \mathrm{Sn}_{2}$ $\left(\mathrm{M}+\mathrm{H}^{+}\right)$713.2879, found 713.2883.

2,5-Di[1-(5-benzyloxy-2-nitrophenyl)-1-ethenyl]pyrimidine (21). Treatment of 20 (436 mg, 0.615 mmol) with $12(655 \mathrm{mg}, 1.84 \mathrm{mmol})$ in the presence of $\mathrm{Pd}_{2}(\mathrm{dba})_{3}(56 \mathrm{mg}, 0.061 \mathrm{mmol}), \mathrm{PPh}_{3}(65 \mathrm{mg}$, $0.25 \mathrm{mmol})$ and $\mathrm{CuI}(234 \mathrm{mg}, 1.23 \mathrm{mmol})$ in DMF $(5 \mathrm{~mL})$, as described for $5\left(120^{\circ} \mathrm{C}, 48 \mathrm{~h}\right)$, gave after work up and chromatography (EtOAc/hexanes, 1:9 then 8:2) 21 (189 mg, $0.323 \mathrm{mmol}, 53 \%$ ) as viscous 
yellow oil. ${ }^{1} \mathrm{H}$ NMR (400 MHz, $\left.\mathrm{CDCl}_{3}\right) \delta 8.52(\mathrm{~s}, 2 \mathrm{H}), 8.16(\mathrm{dd}, J=8.0,0.8 \mathrm{~Hz}, 1 \mathrm{H}), 8.12(\mathrm{~d}, J=8.8 \mathrm{~Hz}$, $1 \mathrm{H}), 7.44-7.35(\mathrm{~m}, 10 \mathrm{H}), 7.06-7.01(\mathrm{~m}, 3 \mathrm{H}), 6.96(\mathrm{~d}, J=2.4 \mathrm{~Hz}, 1 \mathrm{H}), 6.86(\mathrm{~d}, J=1.2 \mathrm{~Hz}, 1 \mathrm{H}), 5.81(\mathrm{~s}, 1 \mathrm{H})$, $5.76(\mathrm{~d}, J=1.2 \mathrm{~Hz}, 1 \mathrm{H}), 5.37(\mathrm{~s}, 1 \mathrm{H}), 5.17(\mathrm{~s}, 2 \mathrm{H}), 5.16(\mathrm{~s}, 2 \mathrm{H}) ;{ }^{13} \mathrm{C} \mathrm{NMR}\left(100 \mathrm{MHz}, \mathrm{CDCl}_{3}\right) \delta 162.7$, $162.5,162.5,154.0,146.4,141.5,141.5,140.7,138.1,137.5,135.5,135.1,130.1,128.8,128.7,128.5$, 128.4, 127.6, 127.6, 127.5, 126.9, 122.4, 118.2, 118.2, 116.9, 114.8, 114.0, 70.8, 70.6; IR (ATR) 1574, 1509, 1334, 1228, $1003 \mathrm{~cm}^{-1}$; HRMS (ESI) calcd for $\mathrm{C}_{34} \mathrm{H}_{27} \mathrm{~N}_{4} \mathrm{O}_{6}\left(\mathrm{M}+\mathrm{H}^{+}\right)$587.1931, found 587.1934.

3-[2-(5-benzyloxy-1H-indol-3-yl)-5-pyrimidinyl]-5-benzyloxy- $1 H$-indole (22). Treatment of a solution of 21 (141 mg, $0.240 \mathrm{mmol})$ in the presence of tris(dibenzylideneacetone)dipalladium $\left(\mathrm{Pd}_{2}(\mathrm{dba})_{3}, 22 \mathrm{mg}\right.$, $0.024 \mathrm{mmol})$, dppp (10 mg, $0.024 \mathrm{mmol})$, and phen $(9 \mathrm{mg}, 0.048 \mathrm{mmol})$ in DMF (1.5 mL) with CO (6 atm), as described for $8\left(120^{\circ} \mathrm{C}, 60 \mathrm{~h}\right)$, gave after work up and chromatography (hexanes/EtOAc, 3:7) 22 (85 mg, $0.163 \mathrm{mmol}, 68 \%$ ) as a yellow solid. $\mathrm{mp}=199-200{ }^{\circ} \mathrm{C} ;{ }^{1} \mathrm{H}$ NMR (400 MHz, DMSO-d 6 ) $\delta 11.54$ (br, s, 1H), 11.46 (br, s, 1H), 9.09 (s, 2H), 8.26 (s, 1H), 8.19 (s, 1H), 7.88 (s, 1H), 7.55-7.32 (m, 13H), $6.94(\mathrm{~d}, J=8.4 \mathrm{~Hz}, 2 \mathrm{H}), 5.20(\mathrm{~s}, 2 \mathrm{H}), 5.17$ (s, 2H); ${ }^{13} \mathrm{C}$ NMR (100 MHz, DMSO-d 6 ) $\delta 160.6,153.7,154.4$, 153.2, 137.8, 137.7, 132.3, 132.0, 129.0, 128.3, 128.3, 127.8, 127.7, 127.6, 127.6, 126.1, 125.0, 124.9, 124.6, 114.6, 112.8, 112.7, 112.5, 112.2, 109.3, 105.7, 102.3, 69.9, 69.9; IR (ATR) 3409, 2884, 1543, 1447, $1199 \mathrm{~cm}^{-1}$; HRMS (ESI) calcd for $\mathrm{C}_{34} \mathrm{H}_{27} \mathrm{~N}_{4} \mathrm{O}_{2}\left(\mathrm{M}+\mathrm{H}^{+}\right)$523.2135, found 523.2135.

Hyrtinandine A. Treatment of a solution of $22(56 \mathrm{mg}, 0.107 \mathrm{mmol})$ with Pd/C (10\%-Pd, $14 \mathrm{mg}, 0.013$ mmol) in $\mathrm{MeOH}(2 \mathrm{~mL})$, as described for alocasine $\mathrm{A}\left(4 \mathrm{~atm} \mathrm{H}_{2}, 60{ }^{\circ} \mathrm{C}, 14 \mathrm{~h}\right)$ gave after work up and chromatography (EtOAc/hexanes, 9:1) hyrtinadine A (32 $\mathrm{mg}, 0.093 \mathrm{mmol}, 87 \%)$ as a white solid. $\mathrm{mp}=290-291{ }^{\circ} \mathrm{C}$ (lit. $\left.\mathrm{mp}=296{ }^{\circ} \mathrm{C}\right) ;{ }^{14}{ }^{1} \mathrm{H}$ NMR (400 MHz, DMSO-d 6 ) $\delta 11.38$ (br s, 1H), 11.29 (br s, 1H), 8.99 (s, 2H), 8.84 (s, 2H), 8.11 (d, J=3.2 Hz, 1H), 7.97 (d, J=2.4 Hz, 1H), 7.80 (d, J=2.8 Hz, 1H), 7.30$7.25(\mathrm{~m}, 2 \mathrm{H}), 7.20(\mathrm{~d}, J=1.6 \mathrm{~Hz}, 1 \mathrm{H}), 6.73-6.68(\mathrm{~m}, 2 \mathrm{H}) ;{ }^{13} \mathrm{C}$ NMR (100 MHz, DMSO-d 6 ) $\delta 160.7,153.5$, 151.8, 151.6, 131.4, 131.3, 128.7, 126.4, 125.4, 125.0, 124.2, 114.1, 112.6, 112.2, 112.1, 112.0, 108.6, 106.2, 102.7. IR (ATR) 3295, 1542, 1448, 1364, $1229 \mathrm{~cm}^{-1}$; HRMS (ESI) calcd for $\mathrm{C}_{20} \mathrm{H}_{15} \mathrm{~N}_{4} \mathrm{O}_{2}\left(\mathrm{M}+\mathrm{H}^{+}\right)$ 343.1195, found 343.1194 .

1-(5-Pyrimidyl)-1-tributylstannylethene (24). Treatment of 5-ethynylpyrimidine 23 (388 $\mathrm{mg}, 3.73$ mmol) with tributyltin hydride $(1.63 \mathrm{~g}, 5.59 \mathrm{mmol})$ in the presence of $\mathrm{PdCl}_{2}\left(\mathrm{PPh}_{3}\right)_{2}(262 \mathrm{mg}, 0.373$ $\mathrm{mmol}$ ) in THF $(9 \mathrm{~mL}$ ), as described for 4 (ambient temperature, $36 \mathrm{~h}$ ), gave after work up and chromatography (EtOAc/hexanes, 5:95) 24 (1.02 g, $2.57 \mathrm{mmol}, 69 \%)$ as pale yellow oil. ${ }^{1} \mathrm{H}$ NMR (600 $\left.\mathrm{MHz}_{\mathrm{CDCl}}\right) \delta 9.04(\mathrm{~s}, 1 \mathrm{H}), 8.52(\mathrm{~s}, 2 \mathrm{H}), 6.10(\mathrm{~d}, J=1.8 \mathrm{~Hz}, 1 \mathrm{H}), 5.62(\mathrm{~d}, J=2.4 \mathrm{~Hz}, 1 \mathrm{H}), 1.52-1.42(\mathrm{~m}$, $6 \mathrm{H}), 1.29$ (sext, $J=7.8 \mathrm{~Hz}, 6 \mathrm{H}), 1.06-0.84(\mathrm{~m}, 15 \mathrm{H}) ;{ }^{13} \mathrm{C} \mathrm{NMR}\left(100 \mathrm{MHz}, \mathrm{CDCl}_{3}\right) \delta 156.4,153.9,147.5$, 
139.7, 130.9, 28.8, 27.1, 13.5, 10.2; IR (APT) 2956, 2925, 1544, $1407 \mathrm{~cm}^{-1}$; HRMS (ESI) calcd for $\mathrm{C}_{18} \mathrm{H}_{33} \mathrm{~N}_{2} \mathrm{Sn}\left(\mathrm{M}+\mathrm{H}^{+}\right)$397.1666, found 397.1666.

1-(5-Benzyloxy-2-nitrophenyl)-1-(4-pyrimidyl)ethene (25). To mixture of 12 (584 mg, $1.64 \mathrm{mmol}$ ), $\mathrm{PPh}_{3}(66 \mathrm{mg}, 0.25 \mathrm{mmol}), \mathrm{CuI}(241 \mathrm{mg}, 1.26 \mathrm{mmol})$ and $\mathrm{Pd}_{2} \mathrm{dba}_{3}(58 \mathrm{mg}, 0.063 \mathrm{mmol})$ in DMF (5 mL) at ambient under an atmosphere of $\mathrm{N}_{2}$, was added a solution of $\mathbf{2 4}(500 \mathrm{mg}, 1.26 \mathrm{mmol})$ in DMF (1 mL). The resulting brown reaction mixture was heated at $120^{\circ} \mathrm{C}$ for $36 \mathrm{~h}$. After cooling to ambient temperature, the mixture was diluted with EtOAc $(30 \mathrm{~mL})$ and the organic layer was washed with water $(4 \times 20 \mathrm{~mL})$ and brine $(20 \mathrm{~mL})$. The organic phase was dried over $\mathrm{MgSO}_{4}$, filtered, and the solvent was removed under reduced pressure. The crude product was purified by column (EtOAc/hexanes, 3:7, then 1:1) to afford 25 (223 mg, $0.669 \mathrm{mmol}, 53 \%$ ) as viscous pale brown oil that slowly solidified. $\mathrm{mp}=113{ }^{\circ} \mathrm{C} ;{ }^{1} \mathrm{H}$ NMR $(400$ $\left.\mathrm{MHz}, \mathrm{CDCl}_{3}\right) \delta 9.11(\mathrm{~s}, 1 \mathrm{H}), 8.62(\mathrm{~s}, 2 \mathrm{H}), 8.17(\mathrm{~d}, J=8.8,1 \mathrm{H}), 7.45-7.37(\mathrm{~m}, 5 \mathrm{H}), 7.09$ (dd, J=8.8, 2.4 Hz, 1H), $6.99(\mathrm{~d}, J=2.8 \mathrm{~Hz}, 1 \mathrm{H}), 5.82(\mathrm{~s}, 1 \mathrm{H}), 5.44(\mathrm{~s}, 1 \mathrm{H}), 5.19(\mathrm{~s}, 2 \mathrm{H}) ;{ }^{13} \mathrm{C} \mathrm{NMR}\left(100 \mathrm{MHz}, \mathrm{CDCl}_{3}\right) \delta$ 162.5, 157.7, 153.9, 141.5, 137.3, 135.0, 132.6, 128.7, 128.5, 127.7, 127.4, 118.4, 117.7, 114.6, 70.7; IR (APT) $1575,1510,1340,1223 \mathrm{~cm}^{-1}$; HRMS calcd for $\mathrm{C}_{19} \mathrm{H}_{16} \mathrm{~N}_{3} \mathrm{O}_{3}\left(\mathrm{M}+\mathrm{H}^{+}\right)$334.1192, found 334.1190.

5-Benzyloxy-3-(4-pyrimidyl)indole (26). Treatment of a solution of 25 (200 $\mathrm{mg}, 0.600 \mathrm{mmol})$ in the presence of $\mathrm{Pd}_{2}(\mathrm{dba})_{3}(33 \mathrm{mg}, 0.036 \mathrm{mmol})$, dppp (15 mg, $\left.0.036 \mathrm{mmol}\right)$, and phen $(13 \mathrm{mg}, 0.072 \mathrm{mmol})$ in DMF (2 mL) with CO (6 atm), as described for $8\left(120^{\circ} \mathrm{C}, 28 \mathrm{~h}\right)$, gave after work up and chromatography (EtOAc/hexanes, 7:3) 26 (163 mg, $0.540 \mathrm{mmol}, 90 \%)$ as a white solid. $\mathrm{mp}=189{ }^{\circ} \mathrm{C} ;{ }^{1} \mathrm{H}$ NMR (400 MHz, $\left.\mathrm{CDCl}_{3}\right) \delta 9.14(\mathrm{~s}, 1 \mathrm{H}), 9.00(\mathrm{~s}, 2 \mathrm{H}), 8.52$ (br, s, 1H), 7.48 (d, J=7.2 Hz, 2H), 7.45 (d, $J=2.8 \mathrm{~Hz}, 1 \mathrm{H}), 7.42-7.31(\mathrm{~m}, 5 \mathrm{H}), 7.05(\mathrm{dd}, J=8.4,2.0 \mathrm{~Hz}, 1 \mathrm{H}), 5.12(\mathrm{~s}, 2 \mathrm{H}) ;{ }^{13} \mathrm{C} \mathrm{NMR}(100 \mathrm{MHz}$, $\left.\mathrm{CDCl}_{3}\right) \delta 156.0,154.5,154.4,137.2,131.9,129.9,128.6,127.9,127.6,125.5,123.3,114.2,112.6,110.7$, 102.3, 70.9; IR (ATR) 3017,1533, 1486, $1157 \mathrm{~cm}^{-1}$; HRMS (ESI) calcd for $\mathrm{C}_{19} \mathrm{H}_{16} \mathrm{~N}_{3} \mathrm{O}\left(\mathrm{M}+\mathrm{H}^{+}\right) 302.1293$ , found 302.1291 .

Hyrtinadine B. Treatment of a solution of 21 (90 mg, $0.30 \mathrm{mmol})$ with $\mathrm{Pd} / \mathrm{C}(10 \%, 28 \mathrm{mg}, 0.026 \mathrm{mmol})$ in $\mathrm{MeOH}(1.5 \mathrm{~mL})$, as described for alocasin $\mathrm{A}\left(4\right.$ atm $\mathrm{H}_{2}, 60{ }^{\circ} \mathrm{C}, 6 \mathrm{~h}$ ) gave after work up and chromatography (EtOAc) hyrtinadine B (53 mg, $0.251 \mathrm{mmol}, 84 \%)$ as a white solid. $\mathrm{mp}=289-290{ }^{\circ} \mathrm{C} ;{ }^{1} \mathrm{H}$ NMR (400 MHz, CD 3 OD) $\delta 9.05(\mathrm{~s}, 2 \mathrm{H}), 8.89(\mathrm{~s}, 1 \mathrm{H}), 7.62(\mathrm{~s}, 1 \mathrm{H}), 7.24(\mathrm{~d}, J=8.4 \mathrm{~Hz}, 1 \mathrm{H}), 7.16(\mathrm{~d}$, $J=2.4 \mathrm{~Hz}, 1 \mathrm{H}), 6.72(\mathrm{dd}, J=8.8,2.0 \mathrm{~Hz}, 1 \mathrm{H}) ;{ }^{13} \mathrm{C} \mathrm{NMR}\left(100 \mathrm{MHz}, \mathrm{CD}_{3} \mathrm{OD}\right) \delta 155.5,155.0,153.3,133.7$, 133.1, 127.0, 126.1, 113.8, 113.8, 109.5, 103.8; IR (ATR) 3327, 2727, 1557, 1420, $1386 \mathrm{~cm}^{-1}$; HRMS (ESI) calcd for $\mathrm{C}_{12} \mathrm{H}_{10} \mathrm{~N}_{3} \mathrm{O}\left(\mathrm{M}+\mathrm{H}^{+}\right) 212.0824$, found 212.0821 . 
[(6-Chloro-2-nitrophenyl)methyl]triphenylphosphonium bromide (28). To a solution of 2-chloro-6nitrobenzylbromide $(3.03 \mathrm{~g}, 12.1 \mathrm{mmol})$ in toluene $(36 \mathrm{~mL})$ was added, in portions, triphenylphosphine $\left(\mathrm{PPh}_{3}, 3.49 \mathrm{~g}, 13.3 \mathrm{mmol}\right)$. The resulting solution was stirred under a nitrogen atmosphere, at $100{ }^{\circ} \mathrm{C}$, for $12 \mathrm{~h}$. A precipitate started to form after $15 \mathrm{~min}$. The mixture was cooled to ambient temperature, the precipitate was removed by filtration, the solid was washed with $\mathrm{Et}_{2} \mathrm{O}$ and the solvents were removed to afford 28 (6.21 g, $12.1 \mathrm{mmol}, 100 \%)$ as a white solid. mp=235-243 ${ }^{\circ} \mathrm{C} ;{ }^{1} \mathrm{H}$ NMR (600 MHz, DMSO-d 6 ) $\delta$ $7.96(\mathrm{~d}, J=8.4 \mathrm{~Hz}, 1 \mathrm{H}), 7.89-7.85(\mathrm{~m}, 4 \mathrm{H}), 7.73-7.66$ (m, 12H), 7.63 (td, J=7.8, $1.8 \mathrm{~Hz}, 1 \mathrm{H}), 5.34$ (d, $J=14.4 \mathrm{~Hz}, 2 \mathrm{H}) ;{ }^{13} \mathrm{C}$ NMR $\left(150 \mathrm{MHz}, \mathrm{DMSO}_{6}\right) \delta 149.7\left(\mathrm{~d}, J^{\mathrm{CP}}=4.5 \mathrm{~Hz}\right), 136.4\left(\mathrm{~d}, J^{\mathrm{CP}}=6.0 \mathrm{~Hz}\right), 135.4$ $\left(\mathrm{d}, J^{\mathrm{CP}}=3.0 \mathrm{~Hz}\right), 135.1\left(\mathrm{~d}, J^{\mathrm{CP}}=3.0 \mathrm{~Hz}\right), 133.8\left(\mathrm{~d}, J^{\mathrm{CP}}=10.5 \mathrm{~Hz}\right), 131.3\left(\mathrm{~d}, J^{\mathrm{CP}}=3.0 \mathrm{~Hz}\right), 130.0\left(\mathrm{~d}, J^{\mathrm{CP}}=13.5\right.$ $\mathrm{Hz}), 124.9\left(\mathrm{~d}, J^{\mathrm{CP}}=3.0 \mathrm{~Hz}\right), 123.5\left(\mathrm{~d}, J^{\mathrm{CP}}=9.0 \mathrm{~Hz}\right), 117.9\left(\mathrm{~d}, J^{\mathrm{CP}}=85.5 \mathrm{~Hz}\right), 25.9\left(\mathrm{~d}, J^{\mathrm{CP}}=51.0 \mathrm{~Hz}\right) ; \mathrm{IR}$ (ATR) 1533, 1434, 1355, 1107, $748 \mathrm{~cm}^{-1}$; HRMS (ESI, negative ion mode) calcd for $\mathrm{C}_{25} \mathrm{H}_{20} \mathrm{BrClNO}_{2} \mathrm{P}$ (M) 511.0104, found 511.0133.

[(3-Methoxy-2-nitrophenyl)methyl] triphenylphosphonium bromide (29). Treatment of 3-methoxy-2nitrobenzylbromide $(1.48 \mathrm{~g}, 6.05 \mathrm{mmol})$ with $\mathrm{PPh}_{3}(1.74 \mathrm{~g}, 6.64 \mathrm{mmol})$ in toluene $(15 \mathrm{~mL})$ as described for $28\left(100{ }^{\circ} \mathrm{C}, 12 \mathrm{~h}\right)$, gave after work up $29(2.67 \mathrm{~g}, 5.26 \mathrm{mmol}, 87 \%)$ as a white solid. $\mathrm{mp}=213-215^{\circ} \mathrm{C}$; ${ }^{1} \mathrm{H}$ NMR (600 MHz, DMSO-d 6 ) $\delta 7.92(\mathrm{t}, J=7.8 \mathrm{~Hz}, 3 \mathrm{H}), 7.74(\mathrm{dt}, J=7.8,3.6 \mathrm{~Hz}, 6 \mathrm{H}), 7.63(\mathrm{dd}, J=8.4$, $7.2 \mathrm{~Hz}, 6 \mathrm{H}), 7.42$ (t, J=8.4 Hz, 1H), 7.35 (d, J=9.0 Hz, 1H), 6.66 (dd, $J=8.4,2.4 \mathrm{~Hz}, 1 \mathrm{H}), 5.10$ (d, $J=15.0$ $\mathrm{Hz}, 2 \mathrm{H}), 3.84(\mathrm{~s}, 3 \mathrm{H}) ;{ }^{13} \mathrm{C} \mathrm{NMR}\left(150 \mathrm{MHz}, \mathrm{DMSO}_{6}\right) \delta 151.4\left(\mathrm{~d}, J^{\mathrm{CP}}=2.2 \mathrm{~Hz}\right), 140.9\left(\mathrm{~d}, J^{\mathrm{CP}}=6.9 \mathrm{~Hz}\right)$, $135.3\left(\mathrm{~d}, J^{\mathrm{CP}}=3.4 \mathrm{~Hz}\right), 133.9\left(\mathrm{~d}, J^{\mathrm{CP}}=10.2 \mathrm{~Hz}\right), 132.1\left(\mathrm{~d}, J^{\mathrm{CP}}=3.4 \mathrm{~Hz}\right), 130.2\left(\mathrm{~d}, J^{\mathrm{CP}}=12.6 \mathrm{~Hz}\right), 123.1(\mathrm{~d}$, $\left.J^{\mathrm{CP}}=4.6 \mathrm{~Hz}\right), 121.8\left(\mathrm{~d}, J^{\mathrm{CP}}=7.9 \mathrm{~Hz}\right), 117.1\left(\mathrm{~d}, J^{\mathrm{CP}}=84.6 \mathrm{~Hz}\right), 114.3\left(\mathrm{~d}, J^{\mathrm{CP}}=2.2 \mathrm{~Hz}\right), 57.0,25.3\left(\mathrm{~d}, J^{\mathrm{CP}}=49.2\right.$ $\mathrm{Hz}$ ); IR (ATR) 1536, 1433, 1281, 1108, $685 \mathrm{~cm}^{-1}$; HRMS (ESI, negative ion mode) calcd for $\mathrm{C}_{26} \mathrm{H}_{23} \mathrm{BrNO}_{3} \mathrm{P}\left(\mathrm{M}^{-}\right)$507.0599, found 507.0628.

[(4-Bromo-2-nitrophenyl)methyl] triphenylphosphonium bromide (30). Treatment of 4-bromo-2nitrobenzylbromide (208 mg, $0.705 \mathrm{mmol})$ with $\mathrm{PPh}_{3}(203 \mathrm{mg}, 0.774 \mathrm{mmol})$ in toluene $(8 \mathrm{~mL})$ as described for $28\left(100{ }^{\circ} \mathrm{C}, 8 \mathrm{~h}\right)$, gave after work up 30 (393 mg, $\left.0.705 \mathrm{mmol}, 100 \%\right)$ as a white solid. $\mathrm{mp}=242-244{ }^{\circ} \mathrm{C} ;{ }^{1} \mathrm{H}$ NMR $\left(400 \mathrm{MHz}, \mathrm{DMSO}_{6}\right) \delta 8.24(\mathrm{~d}, J=2.0 \mathrm{~Hz}, 1 \mathrm{H}), 7.97-7.90(\mathrm{~m}, 3 \mathrm{H}), 7.92(\mathrm{~d}$, $J=1.6 \mathrm{~Hz}, 1 \mathrm{H}), 7.77-7.72(\mathrm{~m}, 6 \mathrm{H}), 7.66-7.61(\mathrm{~m}, 6 \mathrm{H}), 7.31(\mathrm{dd}, J=8.0,2.4 \mathrm{~Hz}, 1 \mathrm{H}), 5.42(\mathrm{~d}, J=15.2 \mathrm{~Hz}$, $2 \mathrm{H}) ;{ }^{13} \mathrm{C}$ NMR $\left(150 \mathrm{MHz}, \mathrm{DMSO}-\mathrm{d}_{6}\right) \delta 148.8\left(\mathrm{~d}, J^{\mathrm{CP}}=6.1 \mathrm{~Hz}\right), 137.3\left(\mathrm{~d}, J^{\mathrm{CP}}=3.0 \mathrm{~Hz}\right), 135.3\left(\mathrm{~d}, J^{\mathrm{CP}}=3.0\right.$ $\mathrm{Hz}), 133.9\left(\mathrm{~d}, J^{\mathrm{CP}}=10.6 \mathrm{~Hz}\right), 130.3\left(\mathrm{~d}, J^{\mathrm{CP}}=12.1 \mathrm{~Hz}\right), 128.4\left(\mathrm{~d}, J^{\mathrm{CP}}=2.3 \mathrm{~Hz}\right), 123.1\left(\mathrm{~d}, J^{\mathrm{CP}}=8.4 \mathrm{~Hz}\right), 122.6$ $\left(\mathrm{d}, J^{\mathrm{CP}}=4.5 \mathrm{~Hz}\right), 117.4,116.6,26.4\left(\mathrm{~d}, J^{\mathrm{CP}}=49.3 \mathrm{~Hz}\right)$; IR (ATR) 1521, 1367, 1346, 1284, 1066, $852 \mathrm{~cm}^{-1}$; HRMS (ESI) calcd for $\mathrm{C}_{25} \mathrm{H}_{20} \mathrm{Br}_{2} \mathrm{NO}_{2} \mathrm{P}$ (M-Br) 476.0415, found 476.0416 . 
3-(5-Bromo-2-nitrophenyl)-2-propenal (32) and 5-(5-Bromo-2-nitrophenyl)-2,4-pentadienal (43). A mixture of 5-bromo-2-nitrobenzaldehyde $(600 \mathrm{mg}, 2.61 \mathrm{mmol})$, (formylmethyl)triphenylphosphonium chloride (1.78 mg, $5.22 \mathrm{mmol}$ ) and DMAP (956 mg, $7.82 \mathrm{mmol})$ in $\mathrm{CHCl}_{3}(36 \mathrm{~mL})$ was stirred at ambient temperature for $4 \mathrm{~h}$ followed by heating at reflux for $3 \mathrm{~h}$. After cooling to ambient temperature, the solvent was removed under reduced pressure and the resulting crude product was purified by chromatography (hexane/EtOAc, 95:5) to afford, in order of elution, 32 (337 mg, $1.32 \mathrm{mmol}, 51 \%$ ) and 43 (153 mg, $0.542 \mathrm{mmol}, 21 \%)$ both as pale yellow solids. Analytical data for 32: $\mathrm{mp}=125-130{ }^{\circ} \mathrm{C} ;{ }^{1} \mathrm{H}$ $\operatorname{NMR}\left(600 \mathrm{MHz}, \mathrm{CDCl}_{3}\right) \delta 9.78(\mathrm{~d}, J=7.2 \mathrm{~Hz}, 1 \mathrm{H}), 8.01$ (d, J=3.0 Hz, 1H), 7.99 (d, J=9.6 Hz, 1H), 7.80 $(\mathrm{d}, J=1.8 \mathrm{~Hz}, 1 \mathrm{H}), 7.73(\mathrm{dd}, J=9.0,1.8 \mathrm{~Hz}, 1 \mathrm{H}), 6.61(\mathrm{dd}, J=15.5,7.8 \mathrm{~Hz}, 1 \mathrm{H}) ;{ }^{13} \mathrm{C} \mathrm{NMR}(150 \mathrm{MHz}$, $\left.\mathrm{CDCl}_{3}\right) \delta 192.5,146.5,145.7,133.9,133.4,132.0,131.9,128.8,126.7$; IR (ATR) 1675, 1521, 1345, $1098,834 \mathrm{~cm}^{-1}$; HRMS (ESI, negative ion mode) calcd for $\mathrm{C}_{9} \mathrm{H}_{6} \mathrm{BrNO}_{3}\left(\mathrm{M}^{-}\right) 254.9531$, found 254.9530 .

Analytical data for 43: $\mathrm{mp}=110-119{ }^{\circ} \mathrm{C} ;{ }^{1} \mathrm{H}$ NMR $\left(600 \mathrm{MHz}, \mathrm{CDCl}_{3}\right) \delta 9.68(\mathrm{~d}, J=7.8 \mathrm{~Hz}, 1 \mathrm{H}), 7.92(\mathrm{~d}$, $J=8.4 \mathrm{~Hz}, 1 \mathrm{H}), 7.84(\mathrm{~d}, J=2.4 \mathrm{~Hz}, 1 \mathrm{H}), 7.62$ (dd, $J=9.0,1.8 \mathrm{~Hz}, 1 \mathrm{H}), 7.50(\mathrm{~d}, J=15.6 \mathrm{~Hz}, 1 \mathrm{H}), 7.30$ (dd, $J=15.6,10.8 \mathrm{~Hz}, 1 \mathrm{H}), 6.96(\mathrm{dd}, J=15.6,11.4 \mathrm{~Hz}, 1 \mathrm{H}), 6.36(\mathrm{dd}, J=15.0,7.8 \mathrm{~Hz}, 1 \mathrm{H}) ;{ }^{13} \mathrm{C} \mathrm{NMR}(150 \mathrm{MHz}$, $\left.\mathrm{CDCl}_{3}\right) \delta 193.2,149.8,146.6,135.0,134.1,133.1,132.5,131.8,131.4,128.4,126.6$;R (ATR) 3417, 1676, 1512, 1345, 1334, 1117, $983 \mathrm{~cm}^{-1}$; HRMS (ESI, negative ion mode) calcd for $\mathrm{C}_{11} \mathrm{H}_{8} \mathrm{BrNO}_{3}\left(\mathrm{M}^{-}\right)$ 280.9688 , found 280.9709 .

E,E-1-(2-Chloro-6-nitrophenyl)-4-(2-nitrophenyl)-1,3-butadiene (34). A solution of freshly prepared sodium ethoxide (NaOEt, $1.5 \mathrm{M}$ in EtOH, $6 \mathrm{~mL}, 9.0 \mathrm{mmol}$ ) was added drop wise to a solution of 31 (389 $\mathrm{mg}, 2.20 \mathrm{mmol})$ and $28(1.13 \mathrm{~g}, 2.20 \mathrm{mmol})$ in absolute EtOH $(9 \mathrm{~mL})$ at ambient temperature. The reaction mixture turned dark purple slowly changing to red. After $36 \mathrm{~h}$ at ambient temperature, water (30 $\mathrm{mL}$ ) was added, and the mixture was extracted with EtOAc $(3 \times 15 \mathrm{~mL})$. The combined organic phases were dried $\left(\mathrm{MgSO}_{4}\right)$, filtered, and the solvents were removed under reduced pressure. The residue was purified by chromatography (hexane/EtOAc, 9:1, then 8:2) to give 34 (399 $\mathrm{mg}, 1.21 \mathrm{mmol}, 55 \%$ ) as an orange solid. $\mathrm{mp}=153-155{ }^{\circ} \mathrm{C} ;{ }^{1} \mathrm{H}$ NMR $\left(400 \mathrm{MHz}, \mathrm{CDCl}_{3}\right) \delta 7.92(\mathrm{dd}, J=8.0,0.8 \mathrm{~Hz}, 1 \mathrm{H}), 7.90(\mathrm{dd}$, $J=8.4,1.2 \mathrm{~Hz}, 1 \mathrm{H}), 7.82(\mathrm{dd}, J=8.4,1.2 \mathrm{~Hz}, 1 \mathrm{H}), 7.73-7.63(\mathrm{~m}, 3 \mathrm{H}), 7.60(\mathrm{td}, J=7.2,0.4 \mathrm{~Hz}, 1 \mathrm{H}), 7.51(\mathrm{td}$, $J=8.0,0.8 \mathrm{~Hz}, 1 \mathrm{H}), 7.46-7.32(\mathrm{~m}, 6 \mathrm{H}), 7.19$ (d, J=15.2 Hz, 1H), 7.17 (d, J=15.2 Hz, 1H), 6.93 (dd, $J=13.4,10.0 \mathrm{~Hz}, 1 \mathrm{H}), 6.83(\mathrm{~d}, J=15.6 \mathrm{~Hz}, 1 \mathrm{H}), 6.73-6.59(\mathrm{~m}, 3 \mathrm{H}), 6.40(\mathrm{dd}, J=15.2,10.0 \mathrm{~Hz}, 1 \mathrm{H}) ;{ }^{13} \mathrm{C}$ NMR (100 MHz, DMSO-d $\left.{ }_{6}\right) \delta 150.7,150.3,147.9,147.8,136.6,135.7,135.1,133.7,133.6,133.2,133.0$, 133.0, 132.9, 132.2, 132.0, 130.8, 130.2, 130.2, 129.7, 128.9, 128.4, 128.4, 128.3, 128.3, 127.9, 127.7, 125.8, 124.7, 124.6, 124.3, 122.5, 122.4; IR (ATR) 1515, 1343, 986, 751, $725 \mathrm{~cm}^{-1}$; HRMS (ESI) calcd for $\mathrm{C}_{16} \mathrm{H}_{12} \mathrm{ClN}_{2} \mathrm{O}_{2}\left(\mathrm{M}+\mathrm{H}^{+}\right)$331.0485, found 331.0480. 
EE/ZE-1-(3-Methoxy-2-nitrophenyl)-4-(2-nitrophenyl)-1,3-butadiene (35). Treatment of a solution of 31 (300 mg, $1.69 \mathrm{mmol})$ and $29(861 \mathrm{mg}, 1.69 \mathrm{mmol})$ in EtOH $(18 \mathrm{~mL})$ in the presence of NaOEt $(1.7 \mathrm{M}$, $11 \mathrm{~mL}, 18.7 \mathrm{mmol})$, as described for $34(24 \mathrm{~h})$, gave after work up and chromatography (hexane/EtOAc, 8:2, then 7:3) 35 (354 mg, $1.08 \mathrm{mmol}, 64 \%, E E / Z E=1: 1)$ as an orange solid. mp=130-135 ${ }^{\circ} \mathrm{C} ;{ }^{1} \mathrm{H} \mathrm{NMR}$ (600 MHz, DMSO-d6) $\delta$ 7.97-7.95 (m, 3H), 7.76 (dd, $J=7.9,1.4 \mathrm{~Hz}, 1 \mathrm{H}), 7.71$ (dd, $J=7.8,1.3 \mathrm{~Hz}, 1 \mathrm{H})$, $7.68(\mathrm{dd}, J=7.9,1.3 \mathrm{~Hz}, 1 \mathrm{H}), 7.59(\mathrm{t}, J=8.3 \mathrm{~Hz}, 1 \mathrm{H}), 7.56-7.51(\mathrm{~m}, 4 \mathrm{H}), 7.41-7.30(\mathrm{~m}, 3 \mathrm{H}), 7.25$ (dd, $J=8.3,0.8 \mathrm{~Hz}, 1 \mathrm{H}), 7.17$ (d, $J=15.2 \mathrm{~Hz}, 1 \mathrm{H}), 7.16$ (d, J=7.9 Hz, 1H), 7.13 (d, J=15.2 Hz, 1H), 7.03 (dd, $J=15.2,11.5 \mathrm{~Hz}, 1 \mathrm{H}), 6.73$ (t, $J=11.4 \mathrm{~Hz}, 1 \mathrm{H}), 6.59$ (d, $J=15.5 \mathrm{~Hz}, 1 \mathrm{H}), 6.45$ (d, $J=11.3 \mathrm{~Hz}, 1 \mathrm{H}), 3.92$ (s, $3 \mathrm{H}), 3.90(\mathrm{~s}, 3 \mathrm{H}) ;{ }^{13} \mathrm{C} \mathrm{NMR}\left(150 \mathrm{MHz}, \mathrm{CDCl}_{3}\right.$, major and minor) $\delta 150.3,150.2,147.9,147.8,139.9$, $139.7,134.4,133.9,133.6,133.5,133.2,131.6,131.3,130.9,130.9,130.8,129.3,129.1,129.1,128.9$, 128.7, 128.3, 128.2, 127.7, 125.5, 124.4, 124.4, 124.1, 121.9, 117.4, 112.8, 112.5, 56.7, 56.7; IR (ATR) 1519, 1369, 1343, 1285, $1063 \mathrm{~cm}^{-1}$; HRMS (ESI) calcd for $\mathrm{C}_{17} \mathrm{H}_{15} \mathrm{~N}_{2} \mathrm{O}_{5}\left(\mathrm{M}+\mathrm{H}^{+}\right)$327.0981, found 327.0976.

EE/EZ-1-(4-Bromo-2-nitrophenyl)-4-(2-nitrophenyl)-1,3-butadiene (36). Treatment of a solution of $31(51 \mathrm{mg}, 0.29 \mathrm{mmol})$ and $\mathbf{3 0}(155 \mathrm{mg}, 0.278 \mathrm{mmol})$ in EtOH $(7 \mathrm{~mL})$ in the presence of NaOEt $(1.7 \mathrm{M}, 7$ $\mathrm{mL}, 11.9 \mathrm{mmol})$, as described for 34 (36 h), gave after work up and chromatography (hexane/EtOAc, 9:1) 36 (78.4 mg, $0.209 \mathrm{mmol}, 73 \%, E E / E Z=1: 2)$ as an orange solid. NMR data from the $E E / E Z=1: 2$ mixture of 36: $\mathrm{mp}=141-142{ }^{\circ} \mathrm{C} ;{ }^{1} \mathrm{H}$ NMR (600 MHz, DMSO-d6) $\delta 8.32(\mathrm{~d}, J=2.1 \mathrm{~Hz}, 1 \mathrm{H}), 8.19$ (d, J=2.1 Hz, 0.5H), 7.99-7.94 (m, 3H), 7.91 (dd, $J=8.6,2.0 \mathrm{~Hz}, 0.5 \mathrm{H}), 7.78(\mathrm{dd}, J=8.0,1.3 \mathrm{~Hz}, 1 \mathrm{H}), 7.72(\mathrm{t}, J=6.7 \mathrm{~Hz}$, $0.5 \mathrm{H}), 7.65(\mathrm{dd}, J=7.4,0.7 \mathrm{~Hz}, 1 \mathrm{H}), 7.54-7.50$ (m, 3H), 7.38 (dd, $J=15.1,10.5 \mathrm{~Hz}, 0.5 \mathrm{H}), 7.31$ (dd, $J=14.9,10.6 \mathrm{~Hz}, 0.5 \mathrm{H}), 7.17-7.14(\mathrm{~m}, 1.5 \mathrm{H}), 7.05-6.98(\mathrm{~m}, 1.5 \mathrm{H}), 6.79(\mathrm{~d}, J=11.4 \mathrm{~Hz}, 1 \mathrm{H}), 7.73(\mathrm{t}$, $J=11.2 \mathrm{~Hz}, 1 \mathrm{H}) ;{ }^{13} \mathrm{C}$ NMR (150 MHz, DMSO-d6) $\delta 148.3,148.2,147.9,147.8,136.3,135.8,134.6$, 134.0, 133.7, 133.3, 133.2, 131.8, 130.9, 130.7, 130.4, 130.2, 129.3, 129.0, 128.9, 128.9, 128.5, 128.2, 128.2, 127.7, 127.2, 127.1, 126.9, 122.4, 124.3, 120.7, 120.6; ${ }^{93}$ IR (ATR) 3027, 1520, 1347, 1217, 748 $\mathrm{cm}^{-1}$; HRMS (ESI) calcd for $\mathrm{C}_{16} \mathrm{H}_{12} \mathrm{BrN}_{2} \mathrm{O}_{4}\left(\mathrm{M}+\mathrm{H}^{+}\right)$374.9980, found 374.9975 .

EZ/EE-1-(4-Bromo-2-nitro-nitrophenyl)-4-(5-bromo-2-nitrophenyl)-1,3-butadiene (37). Treatment of a solution of $\mathbf{3 2}(320 \mathrm{mg}, 1.25 \mathrm{mmol})$ with $\mathbf{3 0}(696 \mathrm{mg}, 1.25 \mathrm{mmol})$ in the presence of NaOEt $(2.0 \mathrm{M}$ in EtOH, $2.81 \mathrm{~mL}, 5.62 \mathrm{mmol})$ in EtOH $(16 \mathrm{~mL})$, as described for $34(24 \mathrm{~h})$ gave after work up and chromatography (hexane/EtOAc, 9:1) 37 (424 mg, $0.934 \mathrm{mmol}, 75 \%, E Z / E E=7: 1)$ as an orange solid. $\mathrm{mp}=159-162{ }^{\circ} \mathrm{C} ;{ }^{1} \mathrm{H} \mathrm{NMR}$ data from the $E Z / E E=7: 1$ mixture of 37, major isomer: ${ }^{1} \mathrm{H}$ NMR $(600 \mathrm{MHz}$, $\left.\mathrm{CDCl}_{3}\right) \delta 8.29(\mathrm{~d}, J=1.8 \mathrm{~Hz}, 1 \mathrm{H}), 7.83(\mathrm{~d}, J=8.6 \mathrm{~Hz}, 1 \mathrm{H}), 7.78(\mathrm{dd}, J=8.3,1.8 \mathrm{~Hz}, 1 \mathrm{H}), 7.60(\mathrm{~d}, J=2.0 \mathrm{~Hz}$, $1 \mathrm{H}), 7.51(\mathrm{dd}, J=8.8,1.9 \mathrm{~Hz}, 1 \mathrm{H}), 7.35(\mathrm{~d}, J=8.1 \mathrm{~Hz}, 1 \mathrm{H}), 7.22(\mathrm{~d}, J=15.3 \mathrm{~Hz}, 1 \mathrm{H}), 6.90(\mathrm{~d}, J=11.2 \mathrm{~Hz}$, $1 \mathrm{H}), 6.78(\mathrm{dd}, J=15.2,11.2 \mathrm{~Hz}, 1 \mathrm{H}), 6.64(\mathrm{t}, J=11.4 \mathrm{~Hz}, 1 \mathrm{H})$; partial ${ }^{1} \mathrm{H}$ NMR data for minor isomer: ${ }^{1} \mathrm{H}$ 
$\operatorname{NMR}\left(600 \mathrm{MHz}, \mathrm{CDCl}_{3}\right) \delta 8.11(\mathrm{~d}, J=1.6 \mathrm{~Hz}, 1 \mathrm{H}), 7.87(\mathrm{~d}, J=2.5 \mathrm{~Hz}, 1 \mathrm{H}), 7.73(\mathrm{dd}, J=9.1,1.8 \mathrm{~Hz}, 1 \mathrm{H})$, 7.62 (d, $J=8.3 \mathrm{~Hz} .1 \mathrm{H}), 7.54$ (dd, $J=8.6,1.7 \mathrm{~Hz}, 1 \mathrm{H}), 7.02-6.96$ (m, 2H); ${ }^{13} \mathrm{C}$ NMR (150 MHz, DMSO-d6) $\delta 148.1,148.0,146.4,146.3,136.3,136.2,134.4,134.2$, 134.0, 133.7, 133.6, 131.5, 131.4, 131.0, 130.9, 130.8, 130.0, 129.5, 129.3, 129.2, 128.8, 128.6, 128.1, 128.1, 128.0, 127.8, 126.5, 126.4, 126.4, 126.3, 121.9, 121.7; IR (ATR) 3381, 1589, 1517, 1335, $748 \mathrm{~cm}^{-1}$; HRMS (ESI, negative ion mode) calcd for $\mathrm{C}_{16} \mathrm{H}_{10} \mathrm{Br}_{2} \mathrm{~N}_{2} \mathrm{O}_{4}\left(\mathrm{M}^{-}\right)$451.9007, found 451.9018 .

2,2'-Bi-1H-indole (38). 1,4-Di(2-nitrophenyl)-1,3-butadiene (33) ${ }^{94}(51 \mathrm{mg}, 0.17 \mathrm{mmol}), \mathrm{Pd}(\mathrm{dba})_{2}(6 \mathrm{mg}$, $0.01 \mathrm{mmol})$, dppp (4 mg, $0.01 \mathrm{mmol})$ and phen $(5 \mathrm{mg}, 0.02 \mathrm{mmol})$ were dissolved in anhydrous DMF (1 $\mathrm{mL}$ ) in a threaded ACE glass pressure tube. The tube was fitted with a pressure head, and the solution was saturated with carbon monoxide (four cycles of $6 \mathrm{~atm}$ of $\mathrm{CO}$ ). The reaction mixture was heated at $120{ }^{\circ} \mathrm{C}$ under CO (6 atm) until all starting material was consumed $(60 \mathrm{~h})$, as judged by TLC (hexanes/EtOAc, 9:1). Brine $(10 \mathrm{~mL})$ was added and the red-brown solution was extracted with EtOAc $(3 \times 20 \mathrm{~mL})$. The combined organic phases were dried $\left(\mathrm{MgSO}_{4}\right)$, filtered, and the solvent was removed under reduced pressure. The resulting crude product was purified by chromatography (hexane/EtOAc, 19:1) to afford 38 (29 mg, $0.13 \mathrm{mmol}, 73 \%$ ) as a white solid. mp 310-313 ${ }^{\circ} \mathrm{C}\left(\right.$ lit. $\left.{ }^{95} \mathrm{mp} 310-312{ }^{\circ} \mathrm{C}\right)$.

4-Chloro-2,2'-bi-1H-indole (39). Treatment of a solution of 34 (297 mg, $0.898 \mathrm{mmol})$ in DMF (2 mL) with $\mathrm{Pd}(\mathrm{dba})_{2}(36 \mathrm{mg}, 0.063 \mathrm{mmol})$, dppp $(26 \mathrm{mg}, 0.063 \mathrm{mmol})$ and phen $(23 \mathrm{mg}, 0.126 \mathrm{mmol})$, as described for 38 ( $6 \mathrm{~atm} \mathrm{CO}, 120{ }^{\circ} \mathrm{C}, 40 \mathrm{~h}$ ), gave after work up and chromatography (hexane/EtOAc, 9:1) 39 (226 mg, $0.846 \mathrm{mmol}, 94 \%)$ as a white solid. $\mathrm{mp}=186-187{ }^{\circ} \mathrm{C} ;{ }^{1} \mathrm{H}$ NMR (400 MHz, Acetone-d $\left.\mathrm{d}_{6}\right) \delta$ 11.03 (br, s, 1H), 10.81 (br, s, 1H), 7.60 (dd, J=7.6, $0.8 \mathrm{~Hz}, 1 \mathrm{H}), 7.46$ (dd, J=8.0, 0.8 Hz, 1H), 7.39 (ddd, $J=7.2,2.4,0.8 \mathrm{~Hz}, 1 \mathrm{H}), 7.18(\mathrm{td}, J=7.6,0.4 \mathrm{~Hz}, 1 \mathrm{H}), 7.16(\mathrm{t}, J=7.6 \mathrm{~Hz}, 1 \mathrm{H}), 7.14-7.05(\mathrm{~m}, 4 \mathrm{H}), 7.01$ (dd, $J=2.4,0.8 \mathrm{~Hz}, 1 \mathrm{H}) ;{ }^{13} \mathrm{C}$ NMR (100 MHz, Acetone-d $\left.{ }_{6}\right) \delta 138.9,138.3,133.3,131.5,129.8,128.6,125.7$, 123.5, 123.2, 121.2, 120.7, 120.1, 111.9, 110.8, 100.2, 97.6; IR (ATR) 3409, 3063, 1334, 1185, $747 \mathrm{~cm}^{-1}$; HRMS (ESI) calcd for $\mathrm{C}_{16} \mathrm{H}_{12} \mathrm{ClN}_{2}\left(\mathrm{M}+\mathrm{H}^{+}\right)$267.0689, found 267.0686 .

7-Methoxy-2,2'-bi-1H-indole (40). Treatment of a solution of 35 (86 mg, $0.26 \mathrm{mmol})$ in DMF (2 mL) with $\mathrm{Pd}(\mathrm{dba})_{2}(11 \mathrm{mg}, 0.018 \mathrm{mmol})$, dppp, $(8 \mathrm{mg}, 0.018 \mathrm{mmol})$ and phen $(23 \mathrm{mg}, 0.126 \mathrm{mmol})$, as described for 38 (CO $6 \mathrm{~atm}, 120{ }^{\circ} \mathrm{C}, 72 \mathrm{~h}$ ), gave after work up and chromatography (hexane/EtOAc, 9:1) 40 (64 mg, $0.24 \mathrm{mmol}, 93 \%$ ) as a white solid. mp=228-229 ${ }^{\circ} \mathrm{C} ;{ }^{1} \mathrm{H}$ NMR (400 MHz, Acetone-d $\left.\mathrm{d}_{6}\right) \delta 10.68$, (br, s, 1H), 10.58 (br, s, 1H), 7.58 (d, J=8.0 Hz, 1H), 7.42 (dd, J=8.0 Hz, 1.2 Hz, 1H), 7.17 (d, J=8.0 Hz, $1 \mathrm{H}), 7.13(\mathrm{td}, J=6.8,1.2 \mathrm{~Hz}, 1 \mathrm{H}), 7.11(\mathrm{~d}, J=1.2 \mathrm{~Hz}, 1 \mathrm{H}), 7.04(\mathrm{td}, J=6.8,1.2 \mathrm{~Hz}, 1 \mathrm{H}), 6.98$ (d, J=8.0 Hz, $1 \mathrm{H}), 6.94(\mathrm{~d}, J=2.4 \mathrm{~Hz}, 1 \mathrm{H}), 6.68(\mathrm{~d}, J=7.6 \mathrm{~Hz}, 1 \mathrm{H}), 3.94(\mathrm{~s}, 3 \mathrm{H}) ;{ }^{13} \mathrm{C}$ NMR (100 MHz, Acetone-d $\mathrm{d}_{6} \delta$ 147.2 , 138.1, 132.3, 132.1, 131.3, 130.0, 128.3, 122.7, 121.2, 121.0, 120.5, 113.8, 111.8, 103.7, 100.2, 
100.0, 55.6; IR (ATR) 3346, 1580, 1344, 1252, 1094, $729 \mathrm{~cm}^{-1}$; HRMS (ESI) calcd for $\mathrm{C}_{17} \mathrm{H}_{15} \mathrm{~N}_{2} \mathrm{O}$ $\left(\mathrm{M}+\mathrm{H}^{+}\right) 263.1184$, found 263.1184 .

6-Bromo-2,2'-bi-1H-indole (41). Treatment of a solution of $\mathbf{3 6}$ (72 $\mathrm{mg}, 0.19 \mathrm{mmol})$ in DMF (1.5 mL) with $\mathrm{Pd}(\mathrm{dba})_{2}(8 \mathrm{mg}, 0.013 \mathrm{mmol})$, dppp, $(6 \mathrm{mg}, 0.013 \mathrm{mmol})$ and phen $(5 \mathrm{mg}, 0.027 \mathrm{mmol})$, as described for $38\left(\mathrm{CO} 6 \mathrm{~atm}, 120^{\circ} \mathrm{C}, 49 \mathrm{~h}\right.$ ), gave after work up and chromatography (hexane/EtOAc, 1:9) 41 (44 mg, $0.142 \mathrm{mmol}, 74 \%)$ as a white solid. $\mathrm{mp}=251-252{ }^{\circ} \mathrm{C} ;{ }^{1} \mathrm{H}$ NMR $\left(400 \mathrm{MHz}\right.$, Acetone- $\left.\mathrm{d}_{6}\right) \delta 10.93(\mathrm{~s}, 1 \mathrm{H})$, 10.79 (s, 1H), $7.58(\mathrm{~s}, 1 \mathrm{H}), 7.57(\mathrm{~d}, J=8.8 \mathrm{~Hz}, 1 \mathrm{H}), 7.51(\mathrm{~d}, J=8.0 \mathrm{~Hz}, 1 \mathrm{H}), 7.41(\mathrm{dd}, J=7.6,0.8 \mathrm{~Hz}, 1 \mathrm{H})$, $7.17(\mathrm{dd}, J=8.8,2.0 \mathrm{~Hz}, 1 \mathrm{H}), 7.14(\mathrm{dd}, J=8.4,1.2 \mathrm{~Hz}, 1 \mathrm{H}), 7.04$ (t with further fine splittings, $J=7.2 \mathrm{~Hz}$, 1H), 6.96 (br s, 2H); ${ }^{13} \mathrm{C}$ NMR (100 MHz, Acetone-d $\left.{ }_{6}\right) \delta$ 138.9, 138.2, 133.4, 131.7, 129.8, 129.0, 123.6, 123.1, 122.5, 121.1, 120.6, 115.6, 114.5, 111.9, 100.0, 99.5; IR (ATR) 3425, 1386, 1338, 1218, $741 \mathrm{~cm}^{-1}$; HRMS (ESI) calcd for $\mathrm{C}_{16} \mathrm{H}_{12} \mathrm{BrN}_{2}\left(\mathrm{M}+\mathrm{H}^{+}\right)$311.0183, found 311.0176.

5,6'-Dibromo-2,2'-bi-1H-indole (42). Treatment of a solution of $37(100 \mathrm{mg}, 0.220 \mathrm{mmol})$ in the presence of $\mathrm{Pd}(\mathrm{dba})_{2}(8.9 \mathrm{mg}, 0.015 \mathrm{mmol})$, dppp $(6.4 \mathrm{mg}, 0.015 \mathrm{mmol})$ and phen $(5.6 \mathrm{mg}, 0.031 \mathrm{mmol})$ in DMF (1.5 mL) with CO (6 atm), as described for $38\left(120{ }^{\circ} \mathrm{C}, 58 \mathrm{~h}\right)$, gave after work up and chromatography (hexane/EtOAc, 9:1) 42 (61.3 mg, $0.157 \mathrm{mmol}, 71 \%$ ) as a white solid. $\mathrm{mp}=275-276{ }^{\circ} \mathrm{C}$; ${ }^{1} \mathrm{H}$ NMR (600 MHz, CDCl $3 / \mathrm{DMSO}_{6}$ ) $\delta 11.73$ (br, s, 1H), 11.69 (br, s, 1H), 7.74 (s, 1H), 7.53 (s, 1H), $7.50(\mathrm{~d}, J=8.4 \mathrm{~Hz}, 1 \mathrm{H}), 7.34$ (d, J=9.0 Hz, 1H), 7.19 (dd, J=8.4, 1.8 Hz, 1H), 7.12 (dd, $J=8.4,1.8 \mathrm{~Hz}, 1 \mathrm{H})$, $6.94(\mathrm{~s}, 1 \mathrm{H}), 6.90(\mathrm{~s}, 1 \mathrm{H}) ;{ }^{13} \mathrm{C} \mathrm{NMR}\left(150 \mathrm{MHz}, \mathrm{CDCl}_{3} / \mathrm{DMSO}_{-} \mathrm{d}_{6}\right) \delta 137.8,135.6,132.2,131.6,130.2$, 127.3, 124.1, 122.3, 122.1, 121.6, 114.4, 113.5, 112.8, 111.9, 99.0, 98.3; IR (ATR) 3416, 1599, 1437, 1332, $802 \mathrm{~cm}^{-1}$; HRMS (ESI) calcd for $\mathrm{C}_{16} \mathrm{H}_{11} \mathrm{Br}_{2} \mathrm{~N}_{2}\left(\mathrm{M}+\mathrm{H}^{+}\right)$388.9289, found 388.9286.

Tributyl(1-(2-nitrophenyl)ethenyl)tin (48). Tributyltin hydride (742 mg, $2.55 \mathrm{mmol})$ was added drop wise, at ambient temperature, to a solution of $\mathrm{PdCl}_{2}\left(\mathrm{PPh}_{3}\right)_{2}(119 \mathrm{mg}, 0.170 \mathrm{mmol})$ and 1-ethynyl-2nitrobenzene (44) ${ }^{96}(250 \mathrm{mg}, 1.70 \mathrm{mmol})$ in THF $(5 \mathrm{~mL})$. The dark brown reaction mixture was stirred for $34 \mathrm{~h}$ followed by removal of the solvent under reduced pressure. Purification by chromatography (hexane/EtOAc, 95:5) gave 48 (707 mg, $1.61 \mathrm{mmol}$, 95\%) as a pale green oil. ${ }^{1} \mathrm{H}$ NMR (600 MHz, $\left.\mathrm{CDCl}_{3}\right) \delta 7.99(\mathrm{dd}, J=8.4,1.2 \mathrm{~Hz}, 1 \mathrm{H}), 7.52(\mathrm{dt}, J=7.8,1.2 \mathrm{~Hz}, 1 \mathrm{H}), 7.31(\mathrm{dt}, J=7.2,1.2 \mathrm{~Hz}, 1 \mathrm{H}), 7.11$ $(\mathrm{dd}, J=7.8,1.2 \mathrm{~Hz}, 1 \mathrm{H}), 5.74(\mathrm{~d}, J=3.0 \mathrm{~Hz}, 1 \mathrm{H}), 5.45$ (d, $J=2.4 \mathrm{~Hz}, 1 \mathrm{H}), 1.43-1.48(\mathrm{~m}, 6 \mathrm{H}), 1.26$ (sext, $J=7.8 \mathrm{~Hz}, 6 \mathrm{H}), 0.91-0.94(\mathrm{~m}, 6 \mathrm{H}), 0.86(\mathrm{t}, J=7.2 \mathrm{~Hz}, 9 \mathrm{H}) ;{ }^{13} \mathrm{C} \mathrm{NMR}\left(150 \mathrm{MHz}, \mathrm{CDCl}_{3}\right) \delta 154.6,146.0$, 144.3, 133.2, 129.7, 126.3, 126.0, 124.3, 28.8, 27.3, 13.6, 10.9; IR (ATR) 2922, 1518, 1339, $1038 \mathrm{~cm}^{-1}$; HRMS (ESI) calcd for $\mathrm{C}_{20} \mathrm{H}_{34} \mathrm{NO}_{2}{ }^{120} \mathrm{Sn}\left(\mathrm{M}+\mathrm{H}^{+}\right) 440.1606$, found 440.1607 .

Tributyl(1-(5-benzyloxy-2-nitrophenyl)ethenyl)tin (4). Treatment of $\mathbf{3}^{58}$ (1.30 g, $\left.5.15 \mathrm{mmol}\right)$ in dry THF (15 mL) with tributyltin hydride $(2.30 \mathrm{~g}, 7.90 \mathrm{mmol})$ in the presence of $\mathrm{PdCl}_{2}\left(\mathrm{PPh}_{3}\right)_{2}(362 \mathrm{mg}, 0.515$ 
mmol), as described for 48 (24 h), gave after solvent removal and chromatography (hexane/EtOAc, 9:1) 4 (2.70 g, $4.96 \mathrm{mmol}, 96 \%)$ as a brown viscous oil. ${ }^{1} \mathrm{H}$ NMR $\left(400 \mathrm{MHz}, \mathrm{CDCl}_{3}\right) \delta 8.10(\mathrm{~d}, J=9.2 \mathrm{~Hz}, 1 \mathrm{H})$, 7.45-7.33 (m, 5H), $6.86(\mathrm{dd}, J=9.2,2.4 \mathrm{~Hz}, 1 \mathrm{H}), 6.63$ (d, J=2.0 Hz, 1H), 5.72 (d, J=2.4 Hz, 1H), 5.41 (d, $J=2.0 \mathrm{~Hz}, 1 \mathrm{H}), 5.14(\mathrm{~s}, 2 \mathrm{H}), 1.56-1.37(\mathrm{~m}, 6 \mathrm{H}), 1.27$ (sext, $J=7.2 \mathrm{~Hz}, 6 \mathrm{H}), 0.95-0.84(\mathrm{~m}, 15 \mathrm{H}) ;{ }^{13} \mathrm{C}$ NMR $\left(100 \mathrm{MHz}, \mathrm{CDCl}_{3}\right) \delta 162.3,155.7,147.6,139.1,135.6,128.7,128.4,127.5,127.1,124.8,114.6$, 112.7,70.5, 28.8, 27.3, 13.6, 11.0; IR (ATR) 2954, 2922, 1567, 1329, 1283, 1243, 1228, $694 \mathrm{~cm}^{-1}$; HRMS calcd for $\mathrm{C}_{27} \mathrm{H}_{40} \mathrm{NO}_{3} \mathrm{Sn}\left(\mathrm{M}+\mathrm{H}^{+}\right)$546.2030, found 546.2030.

Tributyl(1-(4-chloro-2-nitrophenyl)ethenyl)tin (49). Treatment of a solution of $\mathbf{4 5}^{97}$ (215 $\mathrm{mg}, 1.18$ mmol) in THF (3 mL) with tributyltin hydride $(577 \mathrm{mg}, 1.78 \mathrm{mmol})$ in the presence of $\mathrm{PdCl}_{2}\left(\mathrm{PPh}_{3}\right)_{2}(83.1$ $\mathrm{mg}, 0.118 \mathrm{mmol})$, as described for $48(24 \mathrm{~h})$, gave after solvent removal and chromatography (hexane/EtOAc, 19:1) 49 (537 mg, $1.13 \mathrm{mmol}, 96 \%)$ as a brown viscous oil. ${ }^{1} \mathrm{H} \mathrm{NMR}\left(400 \mathrm{MHz}, \mathrm{CDCl}_{3}\right.$ ) $\delta 7.99(\mathrm{~d}, J=2.0 \mathrm{~Hz}, 1 \mathrm{H}), 7.48$ (dd, $J=8.4,2.4 \mathrm{~Hz}, 1 \mathrm{H}), 7.05$ (d, J=8.0, Hz, 1H), 5.73 (d, J=2.4 Hz, 1H), $5.46(\mathrm{~d}, J=2.4 \mathrm{~Hz}, 1 \mathrm{H}), 1.51-1.43(\mathrm{~m}, 6 \mathrm{H}), 1.30$ (sext, $J=8.0 \mathrm{~Hz}, 6 \mathrm{H}), 0.99-0.82(\mathrm{~m}, 15 \mathrm{H}) ;{ }^{13} \mathrm{C}$ NMR $(100$ $\left.\mathrm{MHz}, \mathrm{CDCl}_{3}\right) \delta 153.6,146.1,142.8,133.2,131.8,130.8,126.8,124.2,28.8,27.2,13.6,10.9$; IR (ATR) 2954, 2922, 1567, 1329, 1283, 1243, 1228, $694 \mathrm{~cm}^{-1}$; HRMS (ESI, negative ion mode) calcd for $\mathrm{C}_{20} \mathrm{H}_{32} \mathrm{ClNO}_{2} \mathrm{Sn}\left(\mathrm{M}^{-}\right) 473.1144$, found 473.1188.

Tributyl(1-(4-carbomethoxy-2-nitrophenyl)ethenyl)tin (50). Treatment of a solution of $\mathbf{4 6}^{90}$ (100 mg, $0.487 \mathrm{mmol})$ in THF $(2 \mathrm{~mL})$ with tributyltin hydride $(213 \mathrm{mg}, 0.731 \mathrm{mmol})$ in the presence of $\mathrm{PdCl}_{2}\left(\mathrm{PPh}_{3}\right)_{2}(34 \mathrm{mg}, 0.049 \mathrm{mmol})$, as described for $48(35 \mathrm{~h})$ gave after solvent removal and chromatography (hexane/EtOAc, 19:1) $\mathbf{5 0}(189 \mathrm{mg}, 0.380 \mathrm{mmol}, 78 \%)$ as a brown viscous oil. ${ }^{1} \mathrm{H}$ NMR $\left(400 \mathrm{MHz}, \mathrm{CDCl}_{3}\right) \delta 8.63(\mathrm{~d}, J=1.6 \mathrm{~Hz}, 1 \mathrm{H}), 8.15(\mathrm{dd}, \mathrm{J}=7.6,1.6 \mathrm{~Hz}, 1 \mathrm{H}), 7.17(\mathrm{~d}, J=8.0 \mathrm{~Hz}, 1 \mathrm{H}), 5.75$ $(\mathrm{d}, J=2.8 \mathrm{~Hz}, 1 \mathrm{H}), 5.48(\mathrm{~d}, J=2.8 \mathrm{~Hz}, 1 \mathrm{H}), 3.96(\mathrm{~s}, 3 \mathrm{H}), 1.51-1.43(\mathrm{~m}, 6 \mathrm{H}), 1.25$ (sext, $J=7.2 \mathrm{~Hz}, 6 \mathrm{H})$, 0.94-0.90 (m, 6H), 0.85 (t, J=6.8 Hz, 9H); $\left.{ }^{13} \mathrm{C} \mathrm{NMR} \mathrm{(100} \mathrm{MHz,} \mathrm{CDCl}_{3}\right) \delta 165.0,154.1,148.7,145.8$, 133.5, 129.9, 128.6, 126.4, 125.6, 52.4, 28.7, 27.2, 13.6, 11.0; IR (ATR) 2954, 2922, 1567, 1329, 1283, 1243, 1228, $694 \mathrm{~cm}^{-1}$; HRMS (ESI, negative ion mode) calcd for $\mathrm{C}_{22} \mathrm{H}_{35} \mathrm{~N}_{2} \mathrm{O}_{4} \mathrm{Sn}\left(\mathrm{M}^{-}\right)$497.1588, found 497.1645.

Tributyl(1-(4-methoxy-2-nitrophenyl)ethenyl)tin (51). Treatment of a solution of $\mathbf{4 7}$ (1.10 g, 6.21 mmol) in THF $(130 \mathrm{~mL})$ with tributyltin hydride $(2.71 \mathrm{~g}, 9.31 \mathrm{mmol})$ in the presence of $\mathrm{PdCl}_{2}\left(\mathrm{PPh}_{3}\right)_{2}$ (436 mg, $0.621 \mathrm{mmol}$ ), as described for $48(32 \mathrm{~h}$ ), gave after solvent removal and chromatography (hexane/EtOAc, 9:1) 51 (2.62 g, $5.58 \mathrm{mmol}, 90 \%)$ as a brown viscous oil. ${ }^{1} \mathrm{H} \mathrm{NMR}\left(400 \mathrm{MHz}, \mathrm{CDCl}_{3}\right) \delta$ $7.52(\mathrm{~d}, J=2.8 \mathrm{~Hz}, 1 \mathrm{H}), 7.10(\mathrm{dd}, J=8.4,2.8 \mathrm{~Hz}, 1 \mathrm{H}), 7.01$ (d, J=8.0,Hz, 1H), 5.72 (d, J=2.4 Hz, 1H), 5.42 $(\mathrm{d}, J=2.8 \mathrm{~Hz}, 1 \mathrm{H}), 3.86(\mathrm{~s}, 3 \mathrm{H}), 1.51-1.32(\mathrm{~m}, 6 \mathrm{H}), 1.26$ (sext, $J=7.6 \mathrm{~Hz}, 6 \mathrm{H}), 0.98-0.82(\mathrm{~m}, 15 \mathrm{H}) ;{ }^{13} \mathrm{C}$ 
NMR (100 MHz, $\left.\mathrm{CDCl}_{3}\right) \delta 157.9,154.3,146.1,136.8,130.7,126.1,120.6,108.2,55.8,28.8,27.3,13.6$, 10.8; IR (ATR) 2924, 1521, 1341, 1303, $1034 \mathrm{~cm}^{-1}$; HRMS (ESI) calcd for $\mathrm{C}_{21} \mathrm{H}_{36} \mathrm{NO}_{3}{ }^{120} \mathrm{Sn}\left(\mathrm{M}+\mathrm{H}^{+}-\mathrm{C}_{4} \mathrm{H}_{8}\right)$ 412.0935, found 412.0931.

2,3-Di(2-nitrophenyl)-1,3-butadiene (52). ${ }^{58}$ To a solution of 48 (348 mg, $\left.0.794 \mathrm{mmol}\right)$ in DMF (10 mL) was added $\mathrm{PdCl}_{2}\left(\mathrm{PPh}_{3}\right)_{2}(28 \mathrm{mg}, 0.040 \mathrm{mmol}), \mathrm{PPh}_{3}(21 \mathrm{mg}, 0.080 \mathrm{mmol})$ and $\mathrm{CuI}(113 \mathrm{mg}, 0.595$ $\mathrm{mmol})$. The reaction was stirred at ambient temperature for $36 \mathrm{~h} . \mathrm{Et}_{2} \mathrm{O}(30 \mathrm{~mL})$ was added and the organic phase was washed with $\mathrm{NH}_{4} \mathrm{OH}(10 \%$, aqueous, $3 \mathrm{X} 30 \mathrm{~mL}), \mathrm{H}_{2} \mathrm{O}(30 \mathrm{~mL})$, and brine $(30 \mathrm{~mL})$. The organic phase was dried $\left(\mathrm{MgSO}_{4}\right)$, filtered, and the solvents were removed under reduced pressure. The resulting solid was purified by chromatography (hexane/EtOAc, 95:5) to afford 52 (78 $\mathrm{mg}, 0.263 \mathrm{mmol}$, $67 \%$ ) as a dark yellow solid. $\mathrm{mp}=122-124{ }^{\circ} \mathrm{C} ;{ }^{1} \mathrm{H} \mathrm{NMR}\left(600 \mathrm{MHz}, \mathrm{CDCl}_{3}\right) \delta 7.98(\mathrm{dd}, J=8.4,1.2 \mathrm{~Hz}, 2 \mathrm{H})$, $7.66(\mathrm{dt}, J=7.8,1.2 \mathrm{~Hz}, 2 \mathrm{H}), 7.61(\mathrm{dd}, J=7.8,1.2 \mathrm{~Hz}, 2 \mathrm{H}), 7.52$ (dt, J=7.2, $1.2 \mathrm{~Hz}, 2 \mathrm{H}), 5.15$ (s, 2H), 4.92 (s, 2H); ${ }^{13} \mathrm{C} \mathrm{NMR}\left(150 \mathrm{MHz}, \mathrm{CDCl}_{3}\right) \delta 149.0,145.2,135.3,132.9,132.4,128.7,124.0,118.3$; IR (ATR) 1514, 1332, 911, 790, 749, $697 \mathrm{~cm}^{-1}$; HRMS (ESI) calcd for $\mathrm{C}_{16} \mathrm{H}_{12} \mathrm{~N}_{2} \mathrm{NaO}_{4}\left(\mathrm{M}+\mathrm{Na}^{+}\right)$319.0695, found 319.0690 .

2,3-Di(5-benzyloxy-2-nitrophenyl)-1,3-butadiene (7). Treatment of 4 (103 mg, $0.189 \mathrm{mmol})$ in DMF (1.5 mL) with Pd(dba) 2 (7.2 mg, $0.013 \mathrm{mmol}), \mathrm{PPh}_{3}(14 \mathrm{mg}, 0.053 \mathrm{mmol})$, and CuI (36 mg, $\left.0.189 \mathrm{mmol}\right)$, as described for 52 (28 h), gave after work up and chromatography ${ }^{100}$ (hexane/EtOAc, 9:1) 7 (37.6 mg, $0.074 \mathrm{mmol}, 78 \%)$ as a white solid. $\mathrm{mp}=158-160{ }^{\circ} \mathrm{C} ;{ }^{1} \mathrm{H} \mathrm{NMR}\left(400 \mathrm{MHz}, \mathrm{CDCl}_{3}\right) \delta 8.08(\mathrm{~d}, J=9.2, \mathrm{~Hz}$, 1H), 7.48-7.36 (m, 5H), 7.17 (d, J=2.8 Hz, 1H), 7.02 (dd, J=9.6, 3.2 Hz, 1H), 5.19 (s, 2H), 5.08 (s, 1H), $4.88(\mathrm{~s}, 1 \mathrm{H}) ;{ }^{13} \mathrm{C} \mathrm{NMR}\left(100 \mathrm{MHz}, \mathrm{CDCl}_{3}\right) \delta 162.2,145.6,141.7,138.3,135.5,128.7,128.4,127.8,126.8$, 117.5, 117.1, 114.9, 70.7; IR (ATR) 1573, 1513, 1343, 1230, $1007 \mathrm{~cm}^{-1}$; HRMS (ESI) calcd for $\mathrm{C}_{30} \mathrm{H}_{25} \mathrm{~N}_{2} \mathrm{O}_{6}\left(\mathrm{M}+\mathrm{H}^{+}\right)$509.1713, found 509.1715.

2,3-Di(4-chloro-2-nitrophenyl)-1,3-butadiene (53). To a slurry of copper chloride (210 mg, $2.12 \mathrm{mmol}$ ) in DMF (2 $\mathrm{mL}$ ) in a round bottomed flask covered with aluminum foil to exclude light was added a solution of 49 (400 mg, $0.846 \mathrm{mmol})$ in DMF (2 mL). The resulting mixture was stirred at ambient temperature for $1 \mathrm{~h}$. A saturated solution of $\mathrm{NH}_{4} \mathrm{Cl}$ (aqueous, $4 \mathrm{~mL}$ ) was added and the mixture was allowed to stir for an additional hour. The mixture was diluted with EtOAc $(30 \mathrm{~mL})$, was washed with $\mathrm{H}_{2} \mathrm{O}(3 \times 30 \mathrm{~mL})$ and brine $(20 \mathrm{~mL})$. The organic phase was dried $\left(\mathrm{MgSO}_{4}\right)$, filtered, and the solvent was removed under reduced pressure. The residue was purified by chromatography ${ }^{100}$ (hexane/EtOAc, 9:1) affording 53 (124 mg, $0.340 \mathrm{mmol}, 80 \%)$ as a white solid. $\mathrm{mp}=182{ }^{\circ} \mathrm{C} ;{ }^{1} \mathrm{H}$ NMR $\left(400 \mathrm{MHz}, \mathrm{CDCl}_{3}\right) \delta$ $7.98(\mathrm{~d}, J=2.0, \mathrm{~Hz}, 1 \mathrm{H}), 7.64(\mathrm{dd}, J=8.0,1.6 \mathrm{~Hz} 1 \mathrm{H}), 7.54(\mathrm{~d}, J=8.4 \mathrm{~Hz}, 1 \mathrm{H}), 5.15(\mathrm{~s}, 1 \mathrm{H}), 4.92(\mathrm{~s}, 1 \mathrm{H})$; ${ }^{13}$ C NMR (100 MHz, DMSO-d $\left.{ }_{6}\right) \delta 149.2,143.4,133.5,133.3,133.2,132.6,124.1,119.4$; IR (ATR) 
3086, 1523, 1344, 914, $700 \mathrm{~cm}^{-1}$; HRMS (ESI, negative ion mode) calcd for $\mathrm{C}_{16} \mathrm{H}_{10} \mathrm{Cl}_{2} \mathrm{~N}_{2} \mathrm{O}_{4}\left(\mathrm{M}^{-}\right)$ 364.0018 , found 364.0048 .

2,3-Di(4-carbomethoxy-2-nitrophenyl)-1,3-butadiene (54). Treatment of 50 (400 mg, $0.806 \mathrm{mmol}$ ) with $\mathrm{CuCl}$ (207 mg, $2.10 \mathrm{mmol})$ in DMF (2 mL), as described for 53 (3 h), gave after work up and chromatography ${ }^{100}$ (hexan/EtOAc, 9:1), 54 (306 mg, $\left.0.742 \mathrm{mmol}, 92 \%\right)$ as a white solid. $\mathrm{mp}=187-188{ }^{\circ} \mathrm{C}$; ${ }^{1} \mathrm{H}$ NMR $\left(400 \mathrm{MHz}, \mathrm{CDCl}_{3}\right) \delta 8.60(\mathrm{~d}, J=1.2, \mathrm{~Hz}, 1 \mathrm{H}), 8.29(\mathrm{dd}, J=7.6,1.2 \mathrm{~Hz} \mathrm{1H}), 7.68(\mathrm{~d}, J=8.0 \mathrm{~Hz}$, $1 \mathrm{H}), 5.18(\mathrm{~s}, 1 \mathrm{H}), 5.20(\mathrm{~s}, 2 \mathrm{H}), 4.92(\mathrm{~s}, 1 \mathrm{H}), 3.98(\mathrm{~s}, 3 \mathrm{H}) ;{ }^{13} \mathrm{C} \mathrm{NMR}\left(100 \mathrm{MHz}, \mathrm{CDCl}_{3}\right) \delta$ 164.7, 148.9, 144.2, 139.0, 133.5, 132.6, 131.2, 125.3, 118.9, 52.8; IR (ATR) 1721, 1532, 1358, 1288, $1219 \mathrm{~cm}^{-1}$; HRMS (ESI, negative ion mode) calcd for $\mathrm{C}_{20} \mathrm{H}_{16} \mathrm{~N}_{2} \mathrm{O}_{8}\left(\mathrm{M}^{-}\right)$412.0907, found 412.0937.

2,3-Di(4-methoxy-2-nitrophenyl)-1,3-butadiene (55). Treatment of 51 (100 mg, $0.214 \mathrm{mmol}$ ) in the presence of $\mathrm{CuCl}(55 \mathrm{mg}, 0.56 \mathrm{mmol})$, in $\mathrm{DMF}(1.5 \mathrm{~mL})$, as described for $53(2 \mathrm{~h})$, gave after work up and chromatography ${ }^{100}$ (hexane/EtOAc, 9:1) 55 (37.1 mg, $\left.0.104 \mathrm{mmol}, 98 \%\right)$ as a white solid. mp=163.5$164{ }^{\circ} \mathrm{C} ;{ }^{1} \mathrm{H}$ NMR $\left(400 \mathrm{MHz}, \mathrm{CDCl}_{3}\right) \delta 7.48$ (d, J=9.2, Hz, 1H), 7.47 (d, J=2.4, Hz 1H), 7.17 (dd, $J=8.8$ $2.8 \mathrm{~Hz}, 1 \mathrm{H}), 5.09$ (s, 1H), $4.90(\mathrm{~s}, 1 \mathrm{H}), 3.90$ (s, 3H); ${ }^{13} \mathrm{C} \mathrm{NMR}\left(100 \mathrm{MHz}, \mathrm{CDCl}_{3}\right) \delta 159.3,149.5,145.3$, 133.2, 127.6, 119.3, 118.0, 108.8, 55.9; IR (ATR) 1521, 1340, 1308, 1278, $1028 \mathrm{~cm}^{-1}$; HRMS (ESI) calcd for $\mathrm{C}_{18} \mathrm{H}_{17} \mathrm{~N}_{2} \mathrm{O}_{6}\left(\mathrm{M}+\mathrm{H}^{+}\right)$357.1087, found 357.1081.

3,3'-Bi-1H-indole (56). ${ }^{101}$ Treatment of a solution of $\mathbf{5 2}(68 \mathrm{mg}, 0.229 \mathrm{mmol})$ in the presence of $\mathrm{Pd}(\mathrm{OAc})_{2}(3 \mathrm{mg}, 0.014 \mathrm{mmol})$, dppp (6 mg, $\left.0.014 \mathrm{mmol}\right)$, and phen $(6 \mathrm{mg}, 0.028 \mathrm{mmol})$, and in DMF (3 $\mathrm{mL})$ with $\mathrm{CO}(6 \mathrm{~atm})$, as described for $38\left(120{ }^{\circ} \mathrm{C}, 39 \mathrm{~h}\right)$ gave, after chromatography (hexane/EtOAc, 8:2), 56 (36 mg, $0.155 \mathrm{mmol}, 68 \%$ ) as a white solid. All data in accordance with previously reported values.

5,5'-Dibenzyloxy-3,3'-Bi-1H-indole (57). Treatment of a solution of 7 (100 mg, $0.197 \mathrm{mmol})$ in the presence of Pd(dba) $)_{2}(9 \mathrm{mg}, 0.016 \mathrm{mmol})$, dppp (6 mg, $\left.0.016 \mathrm{mmol}\right)$ phen $(6 \mathrm{mg}, 0.031 \mathrm{mmol})$ in DMF (2 $\mathrm{mL}$ ) with CO (6 atm), as described for $38(72 \mathrm{~h})$, gave after work up and chromatography (hexane/EtOAc; 7:3) 57 (76.3 mg, $0.172 \mathrm{mmol}, 87 \%)$ as a white solid. mp=125-126 ${ }^{\circ} \mathrm{C} ;{ }^{1} \mathrm{H}$ NMR (400 $\left.\mathrm{MHz} \mathrm{CDCl}_{3}\right) \delta 8.10(\mathrm{br}, \mathrm{s}, 1 \mathrm{H}), 7.46(\mathrm{~d}, J=6.8, \mathrm{~Hz}, 2 \mathrm{H}), 7.39-7.31(\mathrm{~m}, 6 \mathrm{H}), 7.02(\mathrm{dd}, J=8.8,2.4 \mathrm{~Hz}, 1 \mathrm{H})$, $5.07(\mathrm{~s}, 2 \mathrm{H}) ;{ }^{13} \mathrm{C}$ NMR $\left(100 \mathrm{MHz}, \mathrm{CDCl}_{3}\right) \delta 153.5,137.6,131.6,128.4,127.7,127.6,127.3,122.4,113.2$, 111.9, 110.7, 103.6, 71.0; IR (ATR) 3406, 1452, 1186, 1151, $794 \mathrm{~cm}^{-1}$; HRMS (ESI) calcd for $\mathrm{C}_{30} \mathrm{H}_{24} \mathrm{~N}_{2} \mathrm{O}_{2}\left(\mathrm{M}^{-}\right)$444.1838, found 444.1834.

6,6'-Dichloro-3,3'-Bi-1H-indole (58). Treatment of a solution of 53 (23 mg, $0.064 \mathrm{mmol})$ in the presence of $\mathrm{Pd}(\mathrm{dba})_{2}$ (4 mg, $\left.0.006 \mathrm{mmol}\right)$, dppp (4 mg, $\left.0.006 \mathrm{mmol}\right)$, and phen (2 mg, $\left.0.013 \mathrm{mmol}\right)$ in DMF (1.5 
$\mathrm{mL})$ with $\mathrm{CO}(6 \mathrm{~atm})$, as described for 38 (72 h), gave after work up and chromatography (hexane/EtOAc, 7:3) 58 (190 mg, $0.063 \mathrm{mmol}, 100 \%)$ as a white solid. $\mathrm{mp}=225-226{ }^{\circ} \mathrm{C} ;{ }^{1} \mathrm{H} \mathrm{NMR}\left(400 \mathrm{MHz}\right.$, Acetone- $\left.\mathrm{d}_{6}\right)$ $\delta 10.52$ (s, 1H), 7.77 (d, J=8.4 Hz, 1H), 7.69 (d, J=2.4 Hz, 1H), 7.53 (d, J=2.0 Hz, 1H), 7.09 (dd, J=8.4, $2.0 \mathrm{~Hz}, 1 \mathrm{H}) ;{ }^{13} \mathrm{C}$ NMR $\left(100 \mathrm{MHz}\right.$, Acetone-d $\left.\mathrm{d}_{6}\right) \delta 138.1,127.9,126.3,123.9,121.7,120.4,112.2,111.1$; IR (ATR) 3411, 1448, 1376, 1227, 914, $802 \mathrm{~cm}^{-1}$; HRMS (ESI) calcd for $\mathrm{C}_{16} \mathrm{H}_{11} \mathrm{Cl}_{2} \mathrm{~N}_{2}\left(\mathrm{M}+\mathrm{H}^{+}\right) 301.0299$, found 301.0292 .

6,6'-Carbomethoxy-3,3'-Bi-1H-indole (59). Treatment of a solution of 54 (100 mg, $0.243 \mathrm{mmol})$ in the presence of $\mathrm{Pd}(\mathrm{dba})_{2}(10 \mathrm{mg}, 0.017 \mathrm{mmol})$, dppp $(7 \mathrm{mg}, 0.017 \mathrm{mmol})$ and phen $(6 \mathrm{mg}, 0.034 \mathrm{mmol})$ in DMF (2 mL) with CO (6 atm), as described for 38 (44 h), gave after work up and chromatography (hexane/EtOAc, 1:1) 59 (84.3 mg, $0.242 \mathrm{mmol}, 100 \%)$ as a white solid. $\mathrm{mp}=300-301{ }^{\circ} \mathrm{C} ;{ }^{1} \mathrm{H}$ NMR (400 $\left.\mathrm{MHz}, \mathrm{CDCl}_{3} / \mathrm{DMSO}_{-} \mathrm{d}_{6}\right) \delta 11.67$ (br, s, 1H), 8.13 (s with further fine splittings, $1 \mathrm{H}$ ), 7.96 (d, J=2.4 Hz, 1H), $7.86(\mathrm{~d}, J=8.4 \mathrm{~Hz}, 1 \mathrm{H}), 7.69(\mathrm{dd}, J=8.4,1.6 \mathrm{~Hz}, 1 \mathrm{H}), 3.87$ (s, 3H); ${ }^{13} \mathrm{C} \mathrm{NMR}(100 \mathrm{MHz}$, $\left.\mathrm{CDCl}_{3} / \mathrm{DMSO}^{-} \mathrm{d}_{6}\right) \delta 167.1,135.5,129.2,125.7,122.3,119.5,118.9,113.6,109.5,51.4$; IR (ATR) 3305, 1689, 1436, 1319, $1227 \mathrm{~cm}^{-1}$; HRMS (ESI) calcd for $\mathrm{C}_{20} \mathrm{H}_{17} \mathrm{~N}_{2} \mathrm{O}_{4}\left(\mathrm{M}+\mathrm{H}^{+}\right)$349.1188, found 349.1182.

6,6'-Dimethoxy-3,3'-Bi-1H-indole (60). Treatment of a solution of 55 (421 mg, $1.18 \mathrm{mmol}$ ) in the presence of $\mathrm{Pd}(\mathrm{dba})_{2}(47 \mathrm{mg}, 0.083 \mathrm{mmol})$, dppp (34 mg, $\left.0.083 \mathrm{mmol}\right)$, and phen (30 mg, $\left.0.17 \mathrm{mmol}\right)$ in DMF (2.5 mL) with CO (6 atm), as described for $38(30 \mathrm{~h})$, gave after work up and chromatography (hexane/EtOAc, 1:1) 60 (221 mg, $0.757 \mathrm{mmol}, 64 \%)$ as a white solid. $\mathrm{mp}=282-284{ }^{\circ} \mathrm{C}$; ${ }^{1} \mathrm{H}$ NMR (400 MHz, DMSO-d $\left.{ }_{6}\right) \delta 10.90$ (s, 1H), 7.62 (d, J=8.8 Hz, 1H), 7.46 (d, J=2.0 Hz, 1H), 6.92 (d, J=2.4 Hz, 1H), $6.70(\mathrm{dd}, J=8.4,1.6 \mathrm{~Hz}, 1 \mathrm{H}), 3.79$ (s, 3H); ${ }^{13} \mathrm{C}$ NMR (100 MHz, DMSO-d 6 ) $\delta 155.5,137.1,120.3,120.2$, 120.1, 109.8, 108.9, 94.5, 55.1; IR (ATR) 3379, 1626, 1298, 1155, $1026 \mathrm{~cm}^{-1}$; HRMS (ESI) calcd for $\mathrm{C}_{18} \mathrm{H}_{17} \mathrm{~N}_{2} \mathrm{O}_{2}\left(\mathrm{M}+\mathrm{H}^{+}\right)$293.1290, found 293.1284.

Tributyl(1-(3-nitro-2-pyridyl)ethenyl)tin (62). Treatment of a solution of $\mathbf{6 1}^{102}$ (100 $\left.\mathrm{mg}, 0.675 \mathrm{mmol}\right)$ in THF (2 mL) with tributyltin hydride $(295 \mathrm{mg}, 1.01 \mathrm{mmol})$ in the presence of $\mathrm{PdCl}_{2}\left(\mathrm{PPh}_{3}\right)_{2}(47 \mathrm{mg}$, $0.068 \mathrm{mmol}$ ), as described for 48 (36 h), gave after solvent removal and chromatography (hexane/EtOAc, 9:1), 62 (181 mg, $0.412 \mathrm{mmol}, 61 \%)$ as a brown viscous oil. ${ }^{1} \mathrm{H}$ NMR (400 $\left.\mathrm{MHz}, \mathrm{CDCl}_{3}\right) \delta 8.70(\mathrm{dd}$, $J=4.8,1.6 \mathrm{~Hz}, 1 \mathrm{H}), 8.10(\mathrm{dd}, \mathrm{J}=8.4,1.6 \mathrm{~Hz}, 1 \mathrm{H}), 7.24$ (dd, J=8.0, 4.4 Hz, 1H), 5.95 (d, J=2.8 Hz, 1H), $5.64(\mathrm{~d}, J=2.4 \mathrm{~Hz}, 1 \mathrm{H}), 1.49-1.43(\mathrm{~m}, 6 \mathrm{H}), 1.30-125(\mathrm{~m}, 6 \mathrm{H}), 1.00-0.83(\mathrm{~m}, 15 \mathrm{H}) ;{ }^{13} \mathrm{C}$ NMR $(100 \mathrm{MHz}$, $\left.\mathrm{CDCl}_{3}\right) \delta 159.6,153.4,152.0,143.8,131.8,129.1,120.7,28.7,27.2,13.6,10.9$; (ATR) 2956, 2924, 1526, $1352,808 \mathrm{~cm}^{-1}$; HRMS (ESI) calcd for $\mathrm{C}_{19} \mathrm{H}_{33} \mathrm{~N}_{2} \mathrm{O}_{2}{ }^{120} \mathrm{Sn}\left(\mathrm{M}+\mathrm{H}^{+}\right) 441.1564$, found 441.1558 .

2,3-Di(3-nitro-2-pyridyl)-1,3-butadiene (63). Treatment of 62 (500 mg, $1.14 \mathrm{mmol})$ with CuCl (282 mg, $2.85 \mathrm{mmol})$ in DMF $(3 \mathrm{~mL})$, as described for $\mathbf{5 3}(5 \mathrm{~h})$, gave after work up and chromatography ${ }^{100}$ 
(hexane/EtOAc, 1:1) 63 (91.4 mg, $0.306 \mathrm{mmol}, 54 \%)$ as a white solid. mp=198-199 ${ }^{\circ} \mathrm{C} ;{ }^{1} \mathrm{H}$ NMR $(400$ $\left.\mathrm{MHz}, \mathrm{CDCl}_{3}\right) \delta 8.88(\mathrm{dd}, J=4.8,1.6 \mathrm{~Hz}, 1 \mathrm{H}), 8.22(\mathrm{dd}, J=8.4,1.6 \mathrm{~Hz} 1 \mathrm{H}), 7.48(\mathrm{dd}, J=8.4,4.8 \mathrm{~Hz}, 1 \mathrm{H})$, $5.40(\mathrm{~s}, 1 \mathrm{H}), 5.24(\mathrm{~s}, 1 \mathrm{H}) ;{ }^{13} \mathrm{C} \mathrm{NMR}\left(100 \mathrm{MHz}, \mathrm{CDCl}_{3}\right) \delta 152.6,152.4,146.4,143.6,132.0,123.2,120.0$; IR (ATR) 3019, 1522, 1361, 1217, $932 \mathrm{~cm}^{-1}$; HRMS (ESI) calcd for $\mathrm{C}_{14} \mathrm{H}_{11} \mathrm{~N}_{4} \mathrm{O}_{4}\left(\mathrm{M}+\mathrm{H}^{+}\right)$299.0780, found 299.0774 .

3,3'-Bi-1H-pyrrolo[3,2-b]pyridine (64). Treatment of a solution of 63 (90 mg, $0.302 \mathrm{mmol}$ ) in the presence of $\mathrm{Pd}(\mathrm{dba})_{2}(12 \mathrm{mg}, 0.021 \mathrm{mmol})$, dppp $(9 \mathrm{mg}, 0.021 \mathrm{mmol})$, and phen $(8 \mathrm{mg}, 0.042 \mathrm{mmol})$ in DMF (2 mL) with CO (6 atm), as described for 38 (36 h), gave after work up and chromatography (EtOAc) 64 (52.3 mg, $0.223 \mathrm{mmol}, 74 \%)$ as a white solid. mp=309-310 ${ }^{\circ} \mathrm{C} ;{ }^{1} \mathrm{H}$ NMR (400 MHz, DMSO$\left.\mathrm{d}_{6}\right) \delta 11.26(\mathrm{~s}, 1 \mathrm{H}), 8.63(\mathrm{~d}, J=2.4 \mathrm{~Hz}, 1 \mathrm{H}), 8.44(\mathrm{dd}, J=4.8,1.6 \mathrm{~Hz}, 1 \mathrm{H}), 7.81(\mathrm{dd}, J=8.0,1.2 \mathrm{~Hz}, 1 \mathrm{H})$, $7.16(\mathrm{dd}, J=8.4,4.8 \mathrm{~Hz}, 1 \mathrm{H}) ;{ }^{13} \mathrm{C}$ NMR (100 MHz, DMSO-d $\left.{ }_{6}\right) \delta 143.7,142.1,128.6,125.6,118.5,116.3$, 108 .9; IR (ATR) 3088, 3019, 1522, 1361, $1217 \mathrm{~cm}^{-1}$; HRMS (ESI) calcd for $\mathrm{C}_{14} \mathrm{H}_{11} \mathrm{~N}_{4}\left(\mathrm{M}+\mathrm{H}^{+}\right)$235.0984, found 235.0978.

2-(1-Bromo-1-ethenyl)-5-methoxy-1-nitrobenzene (66). A mixture of 2-(2-trimethylsilyl-1-ethynyl)-5methoxy-1-nitrobenzene $(\mathbf{6 5})^{103}(756 \mathrm{mg}, 3.03 \mathrm{mmol})$ and $\mathrm{HBr}$ (aq.-48\%, $\left.1.10 \mathrm{~mL}\right)$ in 3-pentanone (7 $\mathrm{mL}$ ) was stirred at $100{ }^{\circ} \mathrm{C}$ for $2 \mathrm{~h}$. After cooling to ambient temperature, the dark brown solution was diluted with EtOAc $(15 \mathrm{~mL})$ and the organic phase was washed with water $(3 \times 15 \mathrm{~mL})$, dried $\left(\mathrm{MgSO}_{4}\right)$, filtered, and the filtrate was concentrated under reduced pressure. The resulting brown oil was purified by chromatography (hexane/EtOAc, 95:5) to give 66 (446 mg, $1.73 \mathrm{mmol}, 57 \%$ ) as a brown oil. ${ }^{1} \mathrm{H}$ NMR $\left(400 \mathrm{MHz}, \mathrm{CDCl}_{3}\right) \delta 7.42(\mathrm{~d}, J=2.0 \mathrm{~Hz}, 1 \mathrm{H}), 7.37$ (d, $\left.J=8.4 \mathrm{~Hz}, 1 \mathrm{H}\right), 7.10$ (dd, $\left.J=8.4,2.8 \mathrm{~Hz}, 1 \mathrm{H}\right), 5.84$ $(\mathrm{d}, J=2.4 \mathrm{~Hz}, 1 \mathrm{H}), 5.82(\mathrm{~d}, J=2.4 \mathrm{~Hz}, 1 \mathrm{H}), 3.89(\mathrm{~s}, 3 \mathrm{H}) ;{ }^{13} \mathrm{C} \mathrm{NMR}\left(100 \mathrm{MHz}, \mathrm{CDCl}_{3}\right) \delta 160.2,148.2$, 132.2, 127.5, 124.3, 121.2, 118.8, 109.4, 56.0; IR (ATR) 1611, 1526, 1352, 1245, $1029 \mathrm{~cm}^{-1}$; HRMS (ESI) calcd for $\mathrm{C}_{9} \mathrm{H}_{9} \mathrm{BrNO}_{3}\left(\mathrm{M}+\mathrm{H}^{+}\right)$257.9765, found 257.9763.

2-(4-Methoxy-2-nitrophenyl)-3--(2-nitrophenyl)-1,3-butadiene (67). To a mixture of 66 (320 mg, 1.24 mmol), CuI (307 mg, $1.61 \mathrm{mmol}), \mathrm{CsF}(382 \mathrm{mg}, 2.52 \mathrm{mmol})$ and $\mathrm{Pd}\left(\mathrm{PPh}_{3}\right)_{4}(143 \mathrm{mg}, 0.124 \mathrm{mmol})$ in DMF (3 mL) was added, under a $\mathrm{N}_{2}$ atmosphere, a solution of 48 (706 mg, $\left.1.61 \mathrm{mmol}\right)$ in DMF (3 mL). The resulting mixture was stirred at $70{ }^{\circ} \mathrm{C}$ for $12 \mathrm{~h}$. The mixture was cooled to ambient temperature and EtOAc $(30 \mathrm{~mL})$ was added. The mixture was washed with water $(5 \times 20 \mathrm{~mL})$ and brine $(20 \mathrm{~mL})$, and the organic phase was dried $\left(\mathrm{MgSO}_{4}\right)$, filtered, and the filtrate was concentrated under reduced pressure. The resulting brown oil was purified by chromatography ${ }^{100}$ (hexane/EtOAc, 95:5) to give, in order of elution, 52 (66 mg, $0.22 \mathrm{mmol}, 28 \%)$ as a white solid and $\mathbf{6 7}(247 \mathrm{mg}, 0.756 \mathrm{mmol}, 61 \%)$ as a yellow solid. $\mathrm{mp}=91-95^{\circ} \mathrm{C} ;{ }^{1} \mathrm{H} \mathrm{NMR}\left(400 \mathrm{MHz}, \mathrm{CDCl}_{3}\right) \delta 7.96(\mathrm{dd}, J=8.4,1.2 \mathrm{~Hz}, 1 \mathrm{H}), 7.65(\mathrm{dt}, J=8.4,1.2 \mathrm{~Hz}, 1 \mathrm{H})$, 
$7.59(\mathrm{dd}, J=8.0,1.6 \mathrm{~Hz}, 1 \mathrm{H}), 7.52-7.47(\mathrm{~m}, 3 \mathrm{H}), 7.19$ (dd, J=8.4, $2.8 \mathrm{~Hz}, 1 \mathrm{H}), 5.12$ (d, $J=2.8 \mathrm{~Hz}, 2 \mathrm{H})$, 4.93 (s, 1H), 4.88 (s, 1H), 3.91 (s, 3H); ${ }^{13} \mathrm{C}$ NMR (100 MHz, $\left.\mathrm{CDCl}_{3}\right) \delta$ 159.3, 149.4,148.9, 145.4, 145.0, 135.4, 133.1, 132.9, 132.3, 128.6, 127.3, 123.9, 119.2, 118.1, 117.9, 108.8, 55.8; IR (ATR) 1520, 1351, 1233, 1027, $910 \mathrm{~cm}^{-1}$; HRMS (ESI) calcd for $\mathrm{C}_{17} \mathrm{H}_{15} \mathrm{~N}_{2} \mathrm{O}_{5}\left(\mathrm{M}+\mathrm{H}^{+}\right)$327.0981, found 327.0979.

6-Methoxy-3,3'-Bi-1H-indole (68). Treatment of a solution of 67 (200 $\mathrm{mg}, 0.613 \mathrm{mmol})$ in the presence of $\mathrm{Pd}_{2}(\mathrm{dba})_{3}$ (56 mg, $\left.0.061 \mathrm{mmol}\right)$, dppp (26 mg, $0.063 \mathrm{mmol}$ ), and phen (22 mg, $0.123 \mathrm{mmol}$ ) in DMF $(1.5 \mathrm{~mL})$ with $\mathrm{CO}(6 \mathrm{~atm})$, as described for $38(30 \mathrm{~h})$, gave after work up and chromatography (hexane/EtOAc, 7:3 then 1:1) $\mathbf{6 8}(161 \mathrm{mg}, 0.613 \mathrm{mmol}, 100 \%)$ as a white solid. $\mathrm{mp}=252{ }^{\circ} \mathrm{C}(\mathrm{dec}.) ;{ }^{1} \mathrm{H}$ NMR (400 MHz, DMSO-d ${ }_{6}$ ) $\delta 11.11$ (br s, 1H), 10.93 (br s, 1H), 7.77 (d, J=7.6 Hz, 1H), 7.64 (d, J=8.4 $\mathrm{Hz}, 1 \mathrm{H}), 7.61$ (d, J=2.4 Hz, 1H), 7.49 (d, J=2.4 Hz, 1H), 7.43 (d, J=8.0 Hz, 1H), 7.13 (t, J=7.2 Hz, 1H), 7.05 (t, $J=7.2 \mathrm{~Hz}, 1 \mathrm{H}), 6.94(\mathrm{~d}, J=2.4 \mathrm{~Hz}, 1 \mathrm{H}), 6.72(\mathrm{dd}, J=8.4,2.4 \mathrm{~Hz}, 1 \mathrm{H}), 3.80(\mathrm{~s}, 3 \mathrm{H}) ;{ }^{13} \mathrm{C}$ NMR $(100$ MHz, DMSO-d 6 ) $\delta 155.6,137.1,136.4,126.0,121.6,121.2,120.4,120.3,120.2,119.6,118.8,111.5$, 109.9, 109.7, 108.9, 94.5, 55.2; IR (ATR) 3395, 1629, 1454, 1239, $1105 \mathrm{~cm}^{-1}$; HRMS (ESI) calcd for $\mathrm{C}_{17} \mathrm{H}_{15} \mathrm{~N}_{2} \mathrm{O}\left(\mathrm{M}+\mathrm{H}^{+}\right)$263.1184, found 263.1180.

[(5-Bromo-2-nitrophenyl)methyl] triphenylphosphonium bromide (69). ${ }^{104}$ Treatment of 5-bromo-2nitrobenzyl bromide (486 mg, $1.65 \mathrm{mmol})$ with $\mathrm{PPh}_{3}(519 \mathrm{mg}, 1.977 \mathrm{mmol})$ in toluene $(6 \mathrm{~mL})$ as described for $28\left(100{ }^{\circ} \mathrm{C}, 10 \mathrm{~h}\right)$, gave after work up $\mathbf{6 9}(918 \mathrm{mg}, 1.647 \mathrm{mmol}, 100 \%)$ as a white solid. $\mathrm{mp}=249-250{ }^{\circ} \mathrm{C},{ }^{1} \mathrm{H}$ NMR $\left(600 \mathrm{MHz}, \mathrm{DMSO}_{\mathrm{d}}\right) \delta 7.97(\mathrm{~d}, J=9.0 \mathrm{~Hz}, 1 \mathrm{H}), 7.93(\mathrm{td}, J=8.4,1.8 \mathrm{~Hz}, 3 \mathrm{H})$, $7.87(\mathrm{dt}, J=7.2,2.4 \mathrm{~Hz}, 1 \mathrm{H}), 7.75$ (sext, $J=3.6 \mathrm{~Hz}, 6 \mathrm{H}), 7.67-7.64(\mathrm{~m}, 6 \mathrm{H}), 7.56$ (d, J=2.4 Hz, 1H), 5.47 $(\mathrm{d}, J=15.0 \mathrm{~Hz}, 2 \mathrm{H}) ;{ }^{13} \mathrm{C}$ NMR $\left(150 \mathrm{MHz}, \mathrm{DMSO}-\mathrm{d}_{6}\right) \delta 147.3\left(\mathrm{~d}, J^{\mathrm{CP}}=5.7 \mathrm{~Hz}\right), 136.3\left(\mathrm{~d}, J^{\mathrm{CP}}=10.3 \mathrm{~Hz}\right)$, $136.3,135.4,134.0\left(\mathrm{~d}, J^{\mathrm{CP}}=10.3 \mathrm{~Hz}\right), 133.3,127.9\left(\mathrm{~d}, J^{\mathrm{CP}}=4.5 \mathrm{~Hz}\right), 127.8,125.9\left(\mathrm{~d}, J^{\mathrm{CP}}=9.1 \mathrm{~Hz}\right), 116.9(\mathrm{~d}$, $\left.J^{\mathrm{CP}}=85.8 \mathrm{~Hz}\right), 26.6\left(\mathrm{~d}, J^{\mathrm{CP}}=49.2 \mathrm{~Hz}\right) ;$ IR 1519, 1330, 1109, 884, $754 \mathrm{~cm}^{-1}$; HRMS (ESI) calcd for $\mathrm{C}_{25} \mathrm{H}_{20} \mathrm{Br}_{2} \mathrm{NO}_{2} \mathrm{P}(\mathrm{M}-\mathrm{Br})$ 476.0415, found 476.0416.

EZ/EE-1,3-Di(2-nitrophenyl)-1,3-butadiene (71). Treatment of a solution of 2-(2-nitrophenyl)propenal $\mathbf{7 0}^{105}(250 \mathrm{mg}, 1.41 \mathrm{mmol})$ and $27(810 \mathrm{mg}, 1.69 \mathrm{mmol})$ in EtOH $(10 \mathrm{~mL})$ in the presence of $\mathrm{NaOEt}(1.8$ $\mathrm{M}$ in EtOH, $5.5 \mathrm{~mL}, 9.90 \mathrm{mmol})$, as described for 34 (24 h), gave after work up and chromatography (hexane/EtOAc, 17:3) 71 (171 mg, $0.579 \mathrm{mmol}, 41 \%$, EZ/EE=10:1) as a faint yellow solid. $\mathrm{mp}=132-138$ ${ }^{\circ} \mathrm{C}$; Data from the mixture of isomers of 71, major isomer: ${ }^{1} \mathrm{H} \mathrm{NMR}\left(600 \mathrm{MHz}, \mathrm{CDCl}_{3}\right) \delta 7.77(\mathrm{dd}, J=7.9$, $1.3 \mathrm{~Hz}, 1 \mathrm{H}), 7.53$ (dd, $J=8.0,1.3 \mathrm{~Hz}, 1 \mathrm{H}), 7.22-7.06$ (m, 6H), 6.83 (d, $J=12.1 \mathrm{~Hz}, 1 \mathrm{H}), 6.58$ (d, $J=12.3$ $\mathrm{Hz}, 1 \mathrm{H}), 5.51(\mathrm{~d}, J=0.8 \mathrm{~Hz}, 1 \mathrm{H}), 5.20(\mathrm{~d}, J=0.4 \mathrm{~Hz} 1 \mathrm{H})$; partial data of minor isomer: ${ }^{1} \mathrm{H} \mathrm{NMR}(600 \mathrm{MHz}$, $\left.\mathrm{CDCl}_{3}\right) \delta 8.01(\mathrm{dd}, J=8.2,1.1 \mathrm{~Hz}, 1 \mathrm{H}), 7.90(\mathrm{dd}, J=8.2,1.3 \mathrm{~Hz}, 1 \mathrm{H}), 7.68-7.65(\mathrm{~m}, 2 \mathrm{H}), 7.57$ (t with further fine splittings, $J=7.2 \mathrm{~Hz}, 1 \mathrm{H}), 7.43(\mathrm{dd}, J=7.6,1.3 \mathrm{~Hz}, 1 \mathrm{H}), 7.37$ (dt, $J=7.8,1.4 \mathrm{~Hz}, 1 \mathrm{H}), 6.99$ (d, 
$J=16.0 \mathrm{~Hz}, 1 \mathrm{H}), 5.59(\mathrm{~s}, 1 \mathrm{H}), 5.30(\mathrm{~s}, 1 \mathrm{H}) ;{ }^{13} \mathrm{C} \mathrm{NMR}\left(150 \mathrm{MHz}, \mathrm{CDCl}_{3}\right) \delta 148.6,147.8,147.6,146.9$, 144.6, 142.0, 135.4, 134.4, 134.2, 133.2, 133.1, 132.5, 132.4, 132.3, 132.1, 132.1, 132.0, 131.0, 131.0, $128.9,128.4,128.2$, 128.0, 127.9, 127.5, 126.8, 127.7, 124.3, 123.8, 123.7, 122.4, 120.2; IR (ATR) 1568, 1514, 1339, 912, $786 \mathrm{~cm}^{-1}$; HRMS (ESI) calcd for $\mathrm{C}_{16} \mathrm{H}_{13} \mathrm{~N}_{2} \mathrm{O}_{4}\left(\mathrm{M}+\mathrm{H}^{+}\right)$297.0875, found 297.0870.

E-1-(3-methoxy-2-nitrophenyl)-3-(2-nitrophenyl)-1,3-butadiene (72). Treatment of a solution of 70 $(69.7 \mathrm{mg}, 0.393 \mathrm{mmol})$ and $29(200 \mathrm{mg}, 0.393 \mathrm{mmol})$ in absolute EtOH $(2 \mathrm{~mL})$ in the presence of NaOEt (2 M, $1.1 \mathrm{~mL}, 2.2 \mathrm{mmol})$, as described for $\mathbf{3 4}(30 \mathrm{~h})$, gave after work up and chromatography (hexane/EtOAc, 85:15) 72 (49.1 mg, $0.150 \mathrm{mmol}, 38 \%$ ) as a yellow-orange oil. ${ }^{1} \mathrm{H}$ NMR (400 MHz, $\left.\mathrm{CDCl}_{3}\right) \delta 7.65(\mathrm{dd}, J=8.4,1.6 \mathrm{~Hz}, 1 \mathrm{H}), 7.27-7.23(\mathrm{~m}, 1 \mathrm{H}), 7.21-7.15(\mathrm{~m}, 2 \mathrm{H}), 6.92(\mathrm{t}, J=8.0 \mathrm{HZ}, 1 \mathrm{H}), 6.74$ (dt, J=7.6, 1.2 Hz, 1H), 6.67 (d, J=8.4 Hz, 1H), 6.55 (d, J=12.4 Hz, 1H), 6.44 (d, J=12.0 Hz 1H), 6.52 (d, $J=0.8 \mathrm{~Hz}, 1 \mathrm{H}), 5.23(\mathrm{~s}, 1 \mathrm{H}), 3.80(\mathrm{~s}, 3 \mathrm{H}) ;{ }^{13} \mathrm{C} \mathrm{NMR}\left(150 \mathrm{MHz}, \mathrm{CDCl}_{3}\right) \delta 150.2,150.2,148.2,141.6$, 139.8, 135.0, 133.8, 131.9, 131.4, 130.5, 129.9, 128.0, 123.7, 123.6, 123.0, 121.9, 56.4; IR (ATR) 1522, 1337, 1107, 8333, 756, 721, $690 \mathrm{~cm}^{-1}$; HRMS (ESI) calcd for $\mathrm{C}_{17} \mathrm{H}_{15} \mathrm{~N}_{2} \mathrm{O}_{5}\left(\mathrm{M}+\mathrm{H}^{+}\right) 327.0981$, found 327.0977 .

E-1-(4-bromo-2-nitrophenyl)-3-(2-nitrophenyl)-1,3-butadiene (73). Treatment of a solution of 70 (320 $\mathrm{mg}, 1.80 \mathrm{mmol})$ with $30(1.20 \mathrm{~g}, 2.23 \mathrm{mmol})$ in the presence of NaOEt $(2.0 \mathrm{M}, 4.2 \mathrm{~mL}, 8.41 \mathrm{mmol})$ in EtOH (15 mL), as described for $34(26 \mathrm{~h})$, gave after work up and chromatography (hexane/EtOAc, 9:1) 73 (139 mg, $0.370 \mathrm{mmol}, 21 \%)$ as a faint yellow solid. mp=119-121 ${ }^{\circ} \mathrm{C} ;{ }^{1} \mathrm{H} \mathrm{NMR}\left(400 \mathrm{MHz}, \mathrm{CDCl}_{3}\right) \delta$ $7.92(\mathrm{~d}, J=2.0 \mathrm{~Hz}, 1 \mathrm{H}), 7.58$ (dd, J=7.2, $1.2 \mathrm{~Hz}, 1 \mathrm{H}), 7.27-7.19$ (m, 3H), 7.10-7.05 (m, 2H), 6.74 (d, $J=12.0 \mathrm{~Hz}, 1 \mathrm{H}), 6.60(\mathrm{~d}, J=12.4 \mathrm{~Hz}, 1 \mathrm{H}), 5.54(\mathrm{~s}, 1 \mathrm{H}), 5.25(\mathrm{~s}, 1 \mathrm{H}) ;{ }^{13} \mathrm{C} \mathrm{NMR}\left(100 \mathrm{MHz}, \mathrm{CDCl}_{3}\right) \delta 147.9$, 147.2, 141.7, 135.2, 135.1, 133.3, 132.2, 131.8, 131.3, 131.1, 128.1, 126.8, 126.3, 123.9, 123.0, 121.0; IR (ATR) $1517,1338,1147,859,784 \mathrm{~cm}^{-1}$; HRMS (ESI) calcd for $\mathrm{C}_{16} \mathrm{H}_{11} \mathrm{BrN}_{2} \mathrm{O}_{4}$ (M) 373.9902, found 373.9898 .

E-1-(5-bromo-2-nitrophenyl)-3-(2-nitrophenyl)-1,3-butadiene (74). Treatment of a solution of 70 (246 $\mathrm{mg}, 1.39 \mathrm{mmol})$ and $69(850 \mathrm{mg}, 1.53 \mathrm{mmol})$ in EtOH $(8 \mathrm{~mL})$ in the presence of NaOEt $(2.0 \mathrm{M}, 3.1 \mathrm{~mL}$, $6.24 \mathrm{mmol}$ ), as described for 34 (26 h) gave after work up and chromatography (hexane/EtOAc, 9:1) 74 (113 mg, $0.301 \mathrm{mmol}, 22 \%)$ as a yellow solid. $\mathrm{mp}=129-134{ }^{\circ} \mathrm{C} ;{ }^{1} \mathrm{H} \mathrm{NMR}\left(600 \mathrm{MHz}, \mathrm{CDCl}_{3}\right) \delta 7.66(\mathrm{~d}$, $J=8.4 \mathrm{~Hz}, 1 \mathrm{H}), 7.60$ (dd, $J=8.4,1.2 \mathrm{~Hz}, 1 \mathrm{H}), 7.29$ (d, $J=2.4 \mathrm{~Hz}, 1 \mathrm{H}), 7.24$ (dd, J=7.2, $1.2 \mathrm{~Hz}, 1 \mathrm{H}), 7.20$ (dd, $J=9.0,2.4 \mathrm{~Hz}, 1 \mathrm{H}), 7.13(\mathrm{td}, J=8.4,1.8 \mathrm{~Hz}, 1 \mathrm{H}), 7.08$ (dd, $J=7.8,1.8 \mathrm{~Hz}, 1 \mathrm{H}), 6.78$ (d, J=12.0 Hz, $1 \mathrm{H}), 6.63(\mathrm{~d}, J=12.6 \mathrm{~Hz}, 1 \mathrm{H}), 5.58(\mathrm{~s}, 1 \mathrm{H}), 5.28(\mathrm{~s}, 1 \mathrm{H}) ;{ }^{13} \mathrm{C} \mathrm{NMR}\left(150 \mathrm{MHz}, \mathrm{CDCl}_{3}\right) \delta 147.7,145.6$, $141.8,135.1,135.1,133.8,132.3,132.0,130.9,130.8,128.2,127.3,125.8,125.3,124.0,123.4$; IR (ATR) 
1598, 1518, 1340, 855, $754 \mathrm{~cm}^{-1}$; HRMS (ESI, negative ion mode) calcd for $\mathrm{C}_{16} \mathrm{H}_{11} \mathrm{BrN}_{2} \mathrm{O}_{4}\left(\mathrm{M}^{-}\right)$ 373.9902, found 373.9898 .

2,3'-Bi-1H-indole (75). Treatment of a solution of $71(200 \mathrm{mg}, 0.675 \mathrm{mmol})$ in the presence of $\mathrm{Pd}(\mathrm{dba})_{2}$ (19 mg, $0.034 \mathrm{mmol})$, dppp (14 mg, $0.034 \mathrm{mmol})$, and phen (12 mg, 0.067mmol) in DMF (2 mL) with $\mathrm{CO}$ (6 atm), as described for $\mathbf{3 8}(41 \mathrm{~h})$, gave after work up and chromatography (hexane/EtOAc, 7:3) 75 (96 mg, $0.41 \mathrm{mmol}, 61 \%$ ) as a white solid. $\mathrm{mp}=206-207{ }^{\circ} \mathrm{C} ;{ }^{1} \mathrm{H} \mathrm{NMR}\left(600 \mathrm{MHz}, \mathrm{CDCl}_{3}\right) \delta 11.32(\mathrm{~s}, 1 \mathrm{H})$, 11.14 (s, 1H), 8.00 (d, J=7.8 Hz, 1H), 7.85 (d, J=1.8 Hz, 1H), 7.50 (d, J=7.8 Hz, 1H), 7.47 (d, J=7.8 Hz, 1H), 7.36 (d, J=8.4 Hz, 1H), 7.18 (t, J=6.0 Hz, 1H), 7.16 (t, J=6.0 Hz, 1H), 7.03 (t, J=7.2 Hz, 1H), 6.96 (t, $J=7.2 \mathrm{~Hz}, 1 \mathrm{H}), 6.74(\mathrm{~d}, J=1.2 \mathrm{~Hz}, 1 \mathrm{H}) ;{ }^{13} \mathrm{C} \mathrm{NMR}\left(150 \mathrm{MHz}, \mathrm{CDCl}_{3}\right) \delta 136.6,135.9,134.1,129.1,124.6$, 123.0, 121.5, 120.1, 119.6, 119.6, 118.9, 118.7, 111.8, 110.3, 108.4, 96.7; IR (ATR) 3405, 3054, 1596, 1456, 1308, $743 \mathrm{~cm}^{-1}$; HRMS (ESI) calcd for $\mathrm{C}_{16} \mathrm{H}_{13} \mathrm{~N}_{2}\left(\mathrm{M}+\mathrm{H}^{+}\right) 233.1078$, found 233.1073.

7-Methoxy-2,3'-Bi-1H-indole (76). Reaction of a solution of 72 (46 mg, $0.14 \mathrm{mmol})$ in the presence of $\operatorname{Pd}(\mathrm{dba})_{2}(8.1 \mathrm{mg}, 0.014 \mathrm{mmol})$, dppp $(6.0 \mathrm{mg}, 0.015 \mathrm{mmol})$ and phen $(5.1 \mathrm{mg}, 0.028 \mathrm{mmol})$ in DMF $(0.8$ $\mathrm{mL})$ with $\mathrm{CO}(6 \mathrm{~atm})$, as described for $38\left(120{ }^{\circ} \mathrm{C}, 48 \mathrm{~h}\right)$, gave after work up and chromatography (hexane/EtOAc, 7:3) 76 (26.7 mg, $0.102 \mathrm{mmol}, 72 \%)$ as a yellowish-brown oil. ${ }^{1} \mathrm{H}$ NMR (600 MHz, $\left.\mathrm{CDCl}_{3}\right) \delta 8.45$ (br s, $\left.1 \mathrm{H}\right), 8.29$ (br s, 1H), 7.48 (d, J=2.6 Hz, 1H), 7.45 (d, J=8.0 Hz, 1H), 7.29 (dt, J=7.0, $1.2 \mathrm{~Hz}, 1 \mathrm{H}),(7.25-7.23(\mathrm{~m}, 3 \mathrm{H}), 7.05(\mathrm{t}, J=7.9 \mathrm{~Hz}, 1 \mathrm{H}), 6.77$ (d, J=2.2 Hz, 1H), $6.66(\mathrm{~d}, J=7.6 \mathrm{~Hz}, 1 \mathrm{H})$, 3.99 (s, 3H); ${ }^{13} \mathrm{C}$ NMR (150 MHz, $\left.\mathrm{CDCl}_{3}\right) \delta 145.7,136.4,132.8,130.7,126.3,125.4,122.9,121.3,120.7$, 120.3, 120.0, 112.9, 111.5, 110.4, 101.6, 99.7, 55.4; IR (ATR) 3395, 1703, 1598, 1576, 1254, 1094, 789, $742 \mathrm{~cm}^{-1}$; HRMS (ESI) calcd for $\mathrm{C}_{17} \mathrm{H}_{15} \mathrm{~N}_{2} \mathrm{O}\left(\mathrm{M}+\mathrm{H}^{+}\right) \quad 263.1184$, found 263.1182 .

6-Bromo-2,3'-Bi-1H-indole (77). Reaction of a solution of $73(50 \mathrm{mg}, 0.133 \mathrm{mmol})$ in the presence of $\mathrm{Pd}(\mathrm{dba})_{2}$ (4.6 mg, $\left.0.008 \mathrm{mmol}\right)$, dppp (3.3 mg, $\left.0.008 \mathrm{mmol}\right)$ and phen $(3 \mathrm{mg}, 0.016 \mathrm{mmol})$ in DMF (1.5 $\mathrm{mL})$ with $\mathrm{CO}(6 \mathrm{~atm})$, as described for $38\left(120{ }^{\circ} \mathrm{C}, 43 \mathrm{~h}\right)$, gave after work up and chromatography (hexane/EtOAc, 7:3) 77 (27.4 mg, $0.088 \mathrm{mmol}, 66 \%)$ as a white solid. mp=205-206 ${ }^{\circ} \mathrm{C} ;{ }^{1} \mathrm{H}$ NMR (400 MHz, $\mathrm{CDCl}_{3} / \mathrm{DMSO}_{\mathrm{d}}$ ) $\delta 11.41$ (br, s, 1H), 11.34 (br, s, 1H), 7.99 (d, J=7.8 Hz, 1H), 7.90 (d, J=2.4 Hz, 1H), 7.67 (d, J=1.8 Hz, 1H), 7.49 (d, J=8.4 Hz, 1H), 7.32 (d, J=8.4 Hz, 1H), 7.22-7.13 (m , 3H), 6.77 (d, $J=1.8 \mathrm{~Hz}, 1 \mathrm{H}) ;{ }^{13} \mathrm{C} \mathrm{NMR}\left(100 \mathrm{MHz}, \mathrm{CDCl}_{3} / \mathrm{DMSO}_{-} \mathrm{d}_{6}\right) \delta 136.9,136.6,135.2,128.2,124.5,123.4,121.7$, 121.5, 120.5, 119.8, 119.4, 112.8, 112.6, 111.9, 107.9, 96.8; IR (ATR) 3410, 3381, 1588, 1317, $747 \mathrm{~cm}^{-1}$; HRMS (ESI) calcd for $\mathrm{C}_{16} \mathrm{H}_{12} \mathrm{BrN}_{2}\left(\mathrm{M}+\mathrm{H}^{+}\right)$311.0184, found 311.0183 .

5-Bromo-2,3'-Bi-1H-indole (78). Reaction of a solution of $74(121 \mathrm{mg}, 0.323 \mathrm{mmol})$ in the presence of $\mathrm{Pd}(\mathrm{dba})_{2}(13 \mathrm{mg}, 0.023 \mathrm{mmol}), \mathrm{dppp}(9.4 \mathrm{mg}, 0.023 \mathrm{mmol})$ and phen $(8.2 \mathrm{mg}, 0.045 \mathrm{mmol})$ in DMF (1.5 $\mathrm{mL})$ with $\mathrm{CO}(6 \mathrm{~atm})$, as described for $38\left(120{ }^{\circ} \mathrm{C}, 44 \mathrm{~h}\right)$, gave after work up and chromatography 
(hexane/EtOAc, 7:3) $78(67.3 \mathrm{mg}, \quad 0.216 \mathrm{mmol}, 67 \%)$ as a white solid. $\mathrm{mp}=205-206{ }^{\circ} \mathrm{C}{ }^{1} \mathrm{H}$ NMR $(600$ MHz, DMSO-d $\left.{ }_{6}\right) \delta 11.46$ (br, s, 1H), 11.42 (br, s, 1H), 7.99 (d, J=7.8 Hz, 1H), 7.90 (d, J=2.4 Hz, 1H), 7.67 (d, J=1.8 Hz, 1H), 7.49 (d, J=8.4 Hz, 1H), 7.32 (d, J=8.4 Hz, 1H), 7.22-7.15 (m, 3H), 7.14 (dd, $J=7.8,1.8 \mathrm{~Hz}, 1 \mathrm{H}), 7.27$ (d, J=1.8 Hz, 1H); ${ }^{13} \mathrm{C}$ NMR (150 MHz, DMSO-d $\left.{ }_{6}\right) \delta 136.6,135.8,134.7,131.2$, 124.5, 123.7, 122.6, 121.9, 121.1, 119.9, 119.5, 112.3, 112.0, 111.3, 107.9, 96.4; IR (ATR) 3412, 3356, 1589, 1234, $748 \mathrm{~cm}^{-1}$; HRMS (ESI) calcd for $\mathrm{C}_{16} \mathrm{H}_{12} \mathrm{BrN}_{2}\left(\mathrm{M}+\mathrm{H}^{+}\right)$311.0184, found 311.0182.

Indolo[1,2-c $]$ quinazolin-6(5H)-one $(81)^{87}$ and 5,11-Dihydro-6H-Indolo[3,2-c] quinolin-6-one (82). ${ }^{106}$ Treatment of a solution of trans-2,2'-di(2-nitrophenyl)ethene (79) ${ }^{107}(200 \mathrm{mg}, 0.740 \mathrm{mmol})$ in the presence of $\mathrm{Pd}(\mathrm{dba})_{2}(25.6 \mathrm{mg}, 0.044 \mathrm{mmol})$, dppp (18.3 mg, $\left.0.044 \mathrm{mmol}\right)$ and phen (16 mg, $\left.0.089 \mathrm{mmol}\right)$ in DMF (2 mL) with CO (6 atm), as described for $38\left(120{ }^{\circ} \mathrm{C}, 56 \mathrm{~h}\right)$, gave after work up and chromatography (hexane/EtOAc, 9:1, 8:2, and 1:1), in order of elution, 81 (132 mg, $0.565 \mathrm{mmol}, 76 \%$ ) and $82(21 \mathrm{mg}, 0.090 \mathrm{mmol}, 12 \%)$ both as white solids. Analytical data for 81: $\mathrm{mp}=320-321{ }^{\circ} \mathrm{C} ;{ }^{1} \mathrm{H}$ NMR (600 MHz, CDCl 3 /DMSO-d $\left.{ }_{6}\right) \delta 11.23$ (br, s, 1H), 8.56-8.55 (m, 1H), 7.96 (d, J=9.6 Hz, 1H), 7.68$7.66(\mathrm{~m}, 1 \mathrm{H}), 7.34(\mathrm{t}, J=8.4 \mathrm{~Hz}, 1 \mathrm{H}), 7.31-7.27$ (m, 2H), 7.24 (d, $J=8.4 \mathrm{~Hz}, 1 \mathrm{H}), 7.19-7.15(\mathrm{~m}, 2 \mathrm{H}) ;{ }^{13} \mathrm{C}$ NMR (150 MHz, $\left.\mathrm{CDCl}_{3} / \mathrm{DMSO}_{6}\right) \delta 147.0,134.1,133.9,133.3,129.4,128.9,123.1,123.0,122.5$, 122.5, 119.8, 115.4, 115.2, 113.5, 97.7; IR (ATR) 2920, 1697, 1596, 1339, $739 \mathrm{~cm}^{-1}$; HRMS (ESI) calcd for $\mathrm{C}_{15} \mathrm{H}_{11} \mathrm{~N}_{2} \mathrm{O}\left(\mathrm{M}+\mathrm{H}^{+}\right)$235.0871, found 235.0868.

Analytical data for 82: $\mathrm{mp}=396-397{ }^{\circ} \mathrm{C}$ (dec.); ${ }^{1} \mathrm{H}$ NMR (600 MHz, DMSO-d $\left.{ }_{6}\right) \delta 12.55$ (br, s, $\left.1 \mathrm{H}\right), 11.42$ (br, s, 1H), 8.20 (dd, J=7.8, 1.2 Hz, 2H), 7.62 (d, J=7.8 Hz, 1H), 7.51 (td, J=8.4, $1.2 \mathrm{~Hz}, 1 \mathrm{H}), 7.47(\mathrm{dd}$, $J=8.4,0.6 \mathrm{~Hz}, 1 \mathrm{H}), 7.38-7.35(\mathrm{~m}, 1 \mathrm{H}), 7.30-7.25(\mathrm{~m}, 2 \mathrm{H}) ;{ }^{13} \mathrm{C}$ NMR $(150 \mathrm{MHz}$, DMSO-d 6$) \delta 159.8$, $140.7,137.9,137.7,129.1,124.4,124.0,122.1,121.5,121.0,120.7,116.0,111.9,111.7,106.4$; IR (ATR) 3050, 2925, 1698, 1596, $1395 \mathrm{~cm}^{-1}$; HRMS (ESI) calcd for $\mathrm{C}_{15} \mathrm{H}_{11} \mathrm{~N}_{2} \mathrm{O}\left(\mathrm{M}+\mathrm{H}^{+}\right)$235.0871, found 235.0869 .

1-(5-Chloro-2-nitrophenyl)-2-(2-nitrophenyl)ethane (88). Treatment of a solution of 2nitrobenzaldehyde (531 mg, $3.51 \mathrm{mmol})$ with 28 (2.20 mg, $4.29 \mathrm{mmol})$ in EtOH (18 mL) in the presence of NaOEt (1.8 M, $10.5 \mathrm{~mL}, 18.9 \mathrm{mmol})$, as described for $34(24 \mathrm{~h})$ gave after work up and chromatography (hexane/EtOAc, 1:1) 88 (300 mg, $0.985 \mathrm{mmol}, 28 \%$ ) as a faint yellow solid. $\mathrm{mp}=139$ $140{ }^{\circ} \mathrm{C} ;{ }^{1} \mathrm{H}$ NMR $\left(600 \mathrm{MHz}, \mathrm{CDCl}_{3}\right) \delta 8.05(\mathrm{~d}, J=8.4 \mathrm{~Hz}, 1 \mathrm{H}), 7.80(\mathrm{~d}, J=8.4 \mathrm{~Hz}, 1 \mathrm{H}), 7.79(\mathrm{~d}, J=9.6 \mathrm{~Hz}$, $1 \mathrm{H}), 7.70(\mathrm{~d}, J=8.4 \mathrm{~Hz}, 1 \mathrm{H}), 7.68(\mathrm{t}, J=7.8 \mathrm{~Hz}, 1 \mathrm{H}), 7.50(\mathrm{t}, J=7.2 \mathrm{~Hz}, 1 \mathrm{H}), 7.41(\mathrm{t}, J=8.4 \mathrm{~Hz}, 1 \mathrm{H}), 7.30$ $(\mathrm{d}, J=16.8 \mathrm{~Hz}, 1 \mathrm{H}), 7.09$ (d, $J=16.8 \mathrm{~Hz}, 1 \mathrm{H}) ;{ }^{13} \mathrm{C} \mathrm{NMR}\left(150 \mathrm{MHz}, \mathrm{CDCl}_{3}\right) \delta 150.4,147.7,135.5,133.9$, 133.6, 132.3, 132.1, 130.8, 129.2, 129.1, 128.8, 125.5, 124.8, 122.6; IR (ATR) 1525, 1347, 1217, 738 $\mathrm{cm}^{-1}$; HRMS (ESI, negative ion mode) calcd for $\mathrm{C}_{14} \mathrm{H}_{9} \mathrm{ClNO}_{2}\left(\mathrm{M}^{-}\right) 304.0251$, found 304.0272. 
2-(2-Nitro-6-chlorophenyl)indole (89) and 4-Chloro-5,10-dihydroindolo[3,2-b]indole (90). Reaction of 88 (250 mg, $0.822 \mathrm{mmol})$ in the presence of Pd(dba) $)_{2}(23.6 \mathrm{mg}, 0.041 \mathrm{mmol})$, dppp (16.9 mg, 0.041 $\mathrm{mmol})$ and phen $(14.8 \mathrm{mg}, 0.082 \mathrm{mmol})$ in DMF (2 mL), as described for $38\left(120{ }^{\circ} \mathrm{C}, 76 \mathrm{~h}\right)$, gave, after work up and chromatography (hexane/EtOAc, 9:1, 8:2, 1:1), in order of elution, 90 (26 mg, $0.107 \mathrm{mmol}$, $13 \%)$ and 89 (153 mg, $0.570 \mathrm{mmol}, 69 \%)$ both as white solids. Analytic data for 89: $\mathrm{mp}=269-270{ }^{\circ} \mathrm{C} ;{ }^{1} \mathrm{H}$ NMR (400 MHz, DMSO-d $\left.{ }_{6}\right) \delta 11.52$ (br, s, 1H), 8.52 (dd, J=8.2, $\left.0.8 \mathrm{~Hz}, 1 \mathrm{H}\right), 8.24$ (dd, J=7.6, 1.2 Hz, $1 \mathrm{H}), 7.48-7.44(\mathrm{~m}, 3 \mathrm{H}), 7.35(\mathrm{t}, J=8.2 \mathrm{~Hz}, 1 \mathrm{H}), 7.28(\mathrm{~d}, J=8.2 \mathrm{~Hz}, 1 \mathrm{H}), 7.26(\mathrm{t}, J=7.8 \mathrm{~Hz}, 1 \mathrm{H}) ;{ }^{13} \mathrm{C}$ NMR $\left(100 \mathrm{MHz}, \mathrm{DMSO}-\mathrm{d}_{6}\right) \delta 146.8,135.2,134.4,134.0,130.0,128.2,124.1,124.0,123.8,123.1,123.0$, 115.4, 114.4, 113.2, 96.0; IR (ATR) 3053, 1712, 1419, 1398, $748 \mathrm{~cm}^{-1}$; HRMS (ESI) calcd for $\mathrm{C}_{15} \mathrm{H}_{10} \mathrm{ClN}_{2} \mathrm{O}\left(\mathrm{M}+\mathrm{H}^{+}\right) 269.0482$, found 269.0475 .

Analytic data for 90: $\mathrm{mp}=182-183{ }^{\circ} \mathrm{C} ;{ }^{1} \mathrm{H}$ NMR (400 MHz, DMSO-d $\left.\mathrm{d}_{6}\right) \delta 11.33$ (br, s, $1 \mathrm{H}$ ), 11.05 (br, s, $1 \mathrm{H}), 7.75(\mathrm{~d}, J=8.0 \mathrm{~Hz}, 1 \mathrm{H}), 7.54(\mathrm{~d}, J=8.0 \mathrm{~Hz}, 1 \mathrm{H}), 7.41$ (d, J=7.6 Hz, 1H), 7.18 (t, J=7.6 Hz, 1H), 7.117.04 (m, 3H); ${ }^{13} \mathrm{C}$ NMR (100 MHz, DMSO-d 6 ) $\delta 141.0,140.7,125.9,123.3,122.6,122.0,122.0,118.2$, 117.7, 117.4, 113.9, 113.5, 112.5, 110.9; IR (ATR) 3424, 1455, 1391, 1322, $730 \mathrm{~cm}^{-1}$; HRMS (ESI) calcd for $\mathrm{C}_{14} \mathrm{H}_{9} \mathrm{ClN}_{2}\left(\mathrm{M}^{+}\right)$240.0454, found 240.0447 .

Z/E-1-(3-Methoxy-2-nitrophenyl)-2-(2-nitrophenyl)ethene (91). Treatment of a solution of 2nitrobenzaldehyde (500 mg, $3.31 \mathrm{mmol})$ with 29 (1.68 mg, $3.31 \mathrm{mmol})$ in EtOH (16 mL) in the presence of $\mathrm{NaOEt}(1.8 \mathrm{M}, 12 \mathrm{~mL}, 21.6 \mathrm{mmol}$ ), as described for $\mathbf{3 4}$ (ambient temperature, $24 \mathrm{~h}$ ) gave after work up and chromatography (hexane/EtOAc, 7:3 then 1:1) 91 (891 mg, $2.97 \mathrm{mmol}, 90 \%, Z / E=1.8: 1)$ as an orange solid. NMR data from the $E / Z=1.8: 1$ mixture of 91, major isomer: ${ }^{1} \mathrm{H}$ NMR $\left(600 \mathrm{MHz}, \mathrm{CDCl}_{3}\right) \delta 8.10$ $8.08(\mathrm{~m}, 1 \mathrm{H}), 7.17(\mathrm{~d}, J=7.5 \mathrm{~Hz}, 1 \mathrm{H}), 7.48$ (t, $J=8.3 \mathrm{~Hz}, 1 \mathrm{H}), 7.40-7.36(\mathrm{~m}, 2 \mathrm{H}), 7.09$ (t, $J=8.5 \mathrm{~Hz}, 1 \mathrm{H})$, $6.87(\mathrm{~d}, J=8.3 \mathrm{~Hz}, 1 \mathrm{H}), 6.68(\mathrm{~d}, J=11.7 \mathrm{~Hz}, 1 \mathrm{H}), 6.48(\mathrm{~d}, J=7.7 \mathrm{~Hz}, 1 \mathrm{H}), 3.89(\mathrm{~s}, 3 \mathrm{H})$; minor isomer: ${ }^{1} \mathrm{H}$ $\operatorname{NMR}\left(600 \mathrm{MHz}, \mathrm{CDCl}_{3}\right) \delta 8.02(\mathrm{~d}, J=8.2 \mathrm{~Hz}, 1 \mathrm{H}), 7.69$ (d, J=15.7 Hz, 1H), $7.63(\mathrm{t}, J=8.2 \mathrm{~Hz}, 1 \mathrm{H}), 7.45$ $(\mathrm{d}, J=8.2 \mathrm{~Hz}, 1 \mathrm{H}), 7.19-7.16$ (m, 2H), $7.11(\mathrm{~d}, J=12.1 \mathrm{~Hz}, 1 \mathrm{H}), 7.01(\mathrm{~d}, J=8.2 \mathrm{~Hz}, 1 \mathrm{H}), 6.88(\mathrm{~d}, J=15.8$ $\mathrm{Hz}, 1 \mathrm{H}), 3.92(\mathrm{~s}, 3 \mathrm{H})$; Analytical data for both isomers from the mixture: $\mathrm{mp}=135-140{ }^{\circ} \mathrm{C} ;{ }^{13} \mathrm{C}$ NMR $(150$ $\left.\mathrm{MHz}, \mathrm{CDCl}_{3}\right) \delta 151.0,150.8,148.0,147.9,141.1,140.7,133.5,133.3,132.4,132.4,132.1,132.0,131.1$, 130.6, 130.3, 130.0, 129.4, 129.0, 128.8, 128.7, 125.4, 124.9, 124.7, 124.6, 122.1, 118.1, 112.1, 111.5, 56.5, 56.4; IR (ATR) 1518, 1342, 1285, 1063, $852 \mathrm{~cm}^{-1}$; HRMS (ESI) calcd for $\mathrm{C}_{15} \mathrm{H}_{13} \mathrm{~N}_{2} \mathrm{O}_{5}\left(\mathrm{M}+\mathrm{H}^{+}\right)$ 301.0824, found 301.0817.

7-Methoxy-2-(2-nitrophenyl)indole (92), 2-(3-Methoxy-2-nitrophenyl)indole (93), and 5,11-Dihydro4-methoxy-6H-indolo[3,2-c]quinolin-6-one (94). Reaction of 91 (439 mg, $1.46 \mathrm{mmol})$ in the presence of $\mathrm{Pd}(\mathrm{dba})_{2}(50.4 \mathrm{mg}, 0.088 \mathrm{mmol})$, dppp (36.2 mg, $\left.0.088 \mathrm{mmol}\right)$ and phen $(31.6 \mathrm{mg}, 0.175 \mathrm{mmol})$ in DMF 
(3 mL) with CO (6 atm) as described for $38\left(120{ }^{\circ} \mathrm{C}, 56 \mathrm{~h}\right)$, gave, after work up and chromatography (hexane/EtOAc, 9:1, 8:2, 1:1, 3:7), in order of elution 92 (38 mg, $0.14 \mathrm{mmol}, 10 \%$ ) as a viscous oil, 93 (290 mg, $1.08 \mathrm{mmol}, 74 \%)$ as a yellow solid and 94 (23.2 mg, $0.088 \mathrm{mmol}, 6 \%)$ as a white solid.

Analytic data for 92: ${ }^{1} \mathrm{H}$ NMR (400 MHz, $\left.\mathrm{CDCl}_{3}\right) \delta 8.69$ (d, J=7.2 Hz, 1H), 8.11 (br s, 1H), 7.71 (dd, $J=6.8,2.4 \mathrm{~Hz}, 1 \mathrm{H}), 7.52$ (d, J=8.0 Hz, 1H), 7.42-7.36 (m, 2H), 7.17 (t, J=8.0 Hz, 1H), 7.09 (s, 1H), 6.91 $(\mathrm{d}, J=8.0 \mathrm{~Hz}, 1 \mathrm{H}), 3.99$ (s, 3H); ${ }^{13} \mathrm{C}$ NMR (100 MHz, DMSO-d 6 ) $\delta 146.3,134.0,133.4,129.6,123.8$, 123.5, 123.2, 123.0, 120.2, 115.6, 115.3, 114.1, 110.7, 98.4, 56.0; IR (ATR) 3424, 2230, 1611, 1535, $1052 \mathrm{~cm}^{-1}$; HRMS (ESI) calcd for $\mathrm{C}_{15} \mathrm{H}_{13} \mathrm{~N}_{2} \mathrm{O}_{3}\left(\mathrm{M}+\mathrm{H}^{+}\right)$269.0926, found 269.0919.

Analytic data for 93: $\mathrm{mp}=158-160{ }^{\circ} \mathrm{C} ;{ }^{1} \mathrm{H} \mathrm{NMR}\left(600 \mathrm{MHz}, \mathrm{CDCl}_{3} / \mathrm{DMSO}_{-} \mathrm{d}_{6}\right) \delta 11.57$ (br s, $\left.1 \mathrm{H}\right), 7.59$ (t, $J=8.4 \mathrm{~Hz}, 1 \mathrm{H}), 7.56(\mathrm{~d}, J=7.6 \mathrm{~Hz}, 1 \mathrm{H}), 7.46(\mathrm{~d}, J=8.4 \mathrm{~Hz}, 1 \mathrm{H}), 7.41$ (d, $J=7.6 \mathrm{~Hz}, 1 \mathrm{H}), 7.23$ (d, $J=8.0 \mathrm{~Hz}$, $1 \mathrm{H}), 7.17(\mathrm{t}, J=7.6 \mathrm{~Hz}, 1 \mathrm{H}), 7.04(\mathrm{t}, J=8.0 \mathrm{~Hz}, 1 \mathrm{H}), 6.54(\mathrm{~d}, J=1.6 \mathrm{~Hz}, 1 \mathrm{H}), 3.92(\mathrm{~s}, 3 \mathrm{H}) ;{ }^{13} \mathrm{C}$ NMR $(150$ $\mathrm{MHz}, \mathrm{CDCl}_{3} / \mathrm{DMSO}_{-}$) $\delta 150.4,139.1,137.0,131.0,130.6,128.2,125.2,122.4,120.4,120.3,119.6$, 112.0, 111.5, 101.1, 56.5; IR (ATR) 3402, 1609, 1527, 1275, $1112 \mathrm{~cm}^{-1}$; HRMS (ESI) calcd for $\mathrm{C}_{15} \mathrm{H}_{13} \mathrm{~N}_{2} \mathrm{O}_{3}\left(\mathrm{M}+\mathrm{H}^{+}\right)$269.0926, found 269.0919.

Analytic data for 94: $\mathrm{mp}=355-360{ }^{\circ} \mathrm{C} ;{ }^{1} \mathrm{H}$ NMR (600 MHz, DMSO-d $\left.\mathrm{d}_{6}\right) \delta 12.57$ (br, s, 1H), 10.16 (br, s, $1 \mathrm{H}), 8.20$ (d, J=7.8 Hz, 1H), 7.79 (d, J=7.8 Hz, 1H), 7.62 (d, J=8.4 Hz, 1H), 7.37 (td, J=8.4, $1.2 \mathrm{~Hz}, 1 \mathrm{H})$, 7.28-7.25 (m, 2H), 7.17 (d, J=7.8 Hz, 1H), 3.96 (s, 3H); ${ }^{13} \mathrm{C}$ NMR (100 MHz, DMSO-d 6 ) $\delta 159.1,146.3$, 140.7, 137.7, 127.6, 124.4, 124.1, 121.7, 121.1, 120.8, 113.9, 112.4, 111.7, 110.0, 106.7, 56.1; IR (ATR) 3407, 1523, 1377, 1275, $1110 \mathrm{~cm}^{-1}$; HRMS (ESI) calcd for $\mathrm{C}_{16} \mathrm{H}_{13} \mathrm{~N}_{2} \mathrm{O}_{2}\left(\mathrm{M}+\mathrm{H}^{+}\right)$265.0977, found 265.0971.

1,1-Bis(2-nitrophenyl)ethene (95) and 2,3-di(2-nitrophenyl)-1,3-butadiene (52). To a solution of 2iodonitrobenzene (473 mg, $1.90 \mathrm{mmol})$ in DMF (10 mL) was added 48 (1.00 g, $2.28 \mathrm{mmol}), \mathrm{PdCl}_{2}\left(\mathrm{PPh}_{3}\right)_{2}$ (67 mg, $0.095 \mathrm{mmol}), \mathrm{PPh}_{3}(50 \mathrm{mg}, 0.190 \mathrm{mmol})$ and $\mathrm{CuI}(271 \mathrm{mg}, 1.43 \mathrm{mmol})$. The reaction was stirred at ambient temperature for $24 \mathrm{~h}$. $\mathrm{Et}_{2} \mathrm{O}(30 \mathrm{~mL})$ was added and the organic phase was washed with $\mathrm{NH}_{4} \mathrm{OH}$ (10\%-aqueous, $3 \times 30 \mathrm{~mL}), \mathrm{H}_{2} \mathrm{O}(30 \mathrm{~mL})$, and brine $(30 \mathrm{~mL})$. The organic phase was dried $\left(\mathrm{MgSO}_{4}\right)$, filtered, and the solvents were removed under reduced pressure. The residue was purified by chromatography (hexane/EtOAc, 9:1) to afford, in order of elution 52 (30 mg, $0.10 \mathrm{mmol}, 11 \%)$ and 95 (320 mg, $1.18 \mathrm{mmol}, 62 \%)$ both as a white solid. $\mathrm{mp}=132-134{ }^{\circ} \mathrm{C} ;{ }^{1} \mathrm{H}$ NMR $\left(600 \mathrm{MHz}, \mathrm{CDCl}_{3}\right) \delta 5.62(\mathrm{~s}$, $2 \mathrm{H}), 7.43-7.46(\mathrm{~m}, 2 \mathrm{H}), 7.58-7.59(\mathrm{~m}, 4 \mathrm{H}), 7.71(\mathrm{dt}, J=7.8,0.6 \mathrm{~Hz}, 2 \mathrm{H}) ;{ }^{13} \mathrm{C} \mathrm{NMR}\left(150 \mathrm{MHz}, \mathrm{CDCl}_{3}\right) \delta$ 121.3, 123.9, 129.1, 132.4, 133.1, 133.9, 142.4, 148.7; IR (ATR) 716, 774, 1351, $1516 \mathrm{~cm}^{-1}$; HRMS (ESI) calcd for $\mathrm{C}_{14} \mathrm{H}_{10} \mathrm{~N}_{2} \mathrm{NaO}_{4}\left(\mathrm{M}+\mathrm{Na}^{+}\right)$293.0538, found 293.0534. 
Indolo[2,3-b]indole (96) and 6H-Indolo[2,3-c]quinolin-6-one (97). ${ }^{108}$ Reaction of 95 (210 mg, 0.777 $\mathrm{mmol})$ in the presence of $\mathrm{Pd}(\mathrm{OAc})_{2}(17.5 \mathrm{mg}, 0.078 \mathrm{mmol})$, dppp (32 mg, $\left.0.078 \mathrm{mmol}\right)$, phen (28 $\mathrm{mg}$, $0.155 \mathrm{mmol})$, and CO (6 atm) in DMF (3 mL), as described for 38 (41 h), gave, after chromatography (hexane/EtOAc, 4:6), in order of elution, 96 (22.6 mg, $0.110 \mathrm{mmol}, 14 \%)$ and 97 (106 mg, $0.451 \mathrm{mmol}$, $58 \%$ ) both as white solids. Analytical data for 96: mp 340-343 ${ }^{\circ} \mathrm{C} ;{ }^{1} \mathrm{H}$ NMR (600 MHz, DMSO- $\left.d_{6}\right) \delta$ 11.35 (br, s, 1H), 7.79 (d, $J=7.2 \mathrm{~Hz}, 1 \mathrm{H}), 7.40$ (d, $J=7.8 \mathrm{~Hz}, 1 \mathrm{H}), 7.10$ (dt, $J=7.2,0.6 \mathrm{~Hz}, 1 \mathrm{H}) ; 7.05$ $(\mathrm{dt}, J=8.4,1.8 \mathrm{~Hz}, 1 \mathrm{H}) ;{ }^{13} \mathrm{C}$ NMR $\left.\left(150 \mathrm{MHz}, \mathrm{DMSO}-d_{6}\right)\right) \delta 144.5,138.6,121.9,119.2,119.0,117.4$, 111.5, 99.7; IR (ATR) 3413, 3363, 1450, 736, $696 \mathrm{~cm}^{-1}$; HRMS (ESI) calcd for $\mathrm{C}_{14} \mathrm{H}_{11} \mathrm{~N}_{2}\left(\mathrm{M}+\mathrm{H}^{+}\right)$ 207.0922, found 207.0917.

Analytical data for 97: $\mathrm{mp} 312-314{ }^{\circ} \mathrm{C} ;{ }^{1} \mathrm{H}$ NMR (600 MHz, DMSO- $d_{6}$ ) $\delta 12.34$ (br s, $1 \mathrm{H}$ ), 11.85 (br s, $1 \mathrm{H}), 8.47(\mathrm{~d}, J=7.8 \mathrm{~Hz}, 1 \mathrm{H}), 8.44(\mathrm{~d}, J=7.2 \mathrm{~Hz}, 1 \mathrm{H}), 7.65(\mathrm{~d}, J=7.8 \mathrm{~Hz}, 1 \mathrm{H}), 7.51(\mathrm{~d}, J=8.4 \mathrm{~Hz}, 1 \mathrm{H})$, $7.47(\mathrm{t}, J=7.8 \mathrm{~Hz}, 1 \mathrm{H}), 7.41(\mathrm{t}, J=7.2 \mathrm{~Hz}, 1 \mathrm{H}), 7.34(\mathrm{t}, J=7.2 \mathrm{~Hz}, 1 \mathrm{H}), 7.32(\mathrm{t}, J=7.2 \mathrm{~Hz}, 1 \mathrm{H}) ;{ }^{13} \mathrm{C}$ NMR (150 MHz, DMSO- $\left.d_{6}\right) \delta 155.7,138.8,134.9,127.6,125.9,125.7,123.0,122.3,122.3,122.2,120.7$, 118.2, 118.0, 116.1, 113.0, ; IR (ATR) 3316, 3158, 1648, 1620, 1328, $729 \mathrm{~cm}^{-1}$; HRMS (ESI) calcd for $\mathrm{C}_{15} \mathrm{H}_{11} \mathrm{~N}_{2} \mathrm{O}\left(\mathrm{M}+\mathrm{H}^{+}\right)$235.0871, found 235.0867.

1-(5-Benzyloxy-2-nitrophenyl)-2-(4-methoxy-2-nitrophenyl)ethene (99). To a solution of 98 (500 mg, $1.79 \mathrm{mmol})$ in DMF (4 mL) was added CuI (34 mg, $0.18 \mathrm{mmol}), \mathrm{CsF}$ (546 mg, $3.62 \mathrm{mmol}), \mathrm{Pd}_{\left(\mathrm{PPh}_{3}\right)_{4}}$ $(124 \mathrm{mg}, 0.108 \mathrm{mmol})$ and $4(1.10 \mathrm{~g}, 2.01 \mathrm{mmol})$. The mixture was stirred at $50{ }^{\circ} \mathrm{C}$ under $\mathrm{N}_{2}$ for $8 \mathrm{~h}$. After cooling to ambient temperature, the crude mixture was diluted with EtOAc $(35 \mathrm{~mL})$, washed with $\mathrm{H}_{2} \mathrm{O}(3 \times 35 \mathrm{~mL})$ and brine $(2 \times 35 \mathrm{~mL})$. The organic layer was dried $\left(\mathrm{MgSO}_{4}\right)$, filtered, and the solvents were removed under reduced pressure. The residue was purified by chromatography on $\mathrm{SiO}_{2} / \mathrm{K}_{2} \mathrm{CO}_{3}(9: 1)$ using (hexane/EtOAc, 9:1 then 85:15) to give, in order of elution, 7 (69.2 mg, $0.136 \mathrm{mmol}, 15 \%)$ and 99 (503 mg, $1.24 \mathrm{mmol}, 69 \%$ ) both as white solids. Analytical data for 99: $\mathrm{mp}=127-128{ }^{\circ} \mathrm{C} ;{ }^{1} \mathrm{H}$ NMR $(400$ $\left.\mathrm{MHz}, \mathrm{CDCl}_{3}\right) \delta 7.81(\mathrm{~d}, J=9.2 \mathrm{~Hz}, 1 \mathrm{H}), 7.50(\mathrm{~d}, J=8.8 \mathrm{~Hz}, 1 \mathrm{H}), 7.45-7.34(\mathrm{~m}, 5 \mathrm{H}), 7.17$ (d, J=2.8 Hz, $1 \mathrm{H}), 7.11(\mathrm{~d}, J=2.8 \mathrm{~Hz}, 1 \mathrm{H}), 7.09$ (dd, $J=8.8,2.8 \mathrm{~Hz}, 1 \mathrm{H}), 6.95$ (dd, $J=8.8,2.4 \mathrm{~Hz}, 1 \mathrm{H}), 5.55$ (s, 1H), 5.52 $(\mathrm{s}, 1 \mathrm{H}), 5.16(\mathrm{~s}, 2 \mathrm{H}), 3.85(\mathrm{~s}, 3 \mathrm{H}) ;{ }^{13} \mathrm{C} \mathrm{NMR}\left(100 \mathrm{MHz}, \mathrm{CDCl}_{3}\right) \delta 161.6,159.5,149.1,142.8,141.6$, 137.2, 135.5, 134.2, 128.7, 128.4, 127.7, 126.7, 126.0, 120.3, 119.1, 118.5, 114.7, 108.7, 70.7, 55.8; IR (ATR) 1515, 1348, 1228, 1004, $826 \mathrm{~cm}^{-1}$; HRMS (ESI) calcd for $\mathrm{C}_{22} \mathrm{H}_{19} \mathrm{~N}_{2} \mathrm{O}_{6}\left(\mathrm{M}+\mathrm{H}^{+}\right) 407.1243$, found 407.1236.

6-Methoxy-indolo[2,3-b]-5-benzyloxyindole indolo[2,3-c]quinolin-6-one (101), and c] quinolin-6-one (102). Reaction of 99 (300 mg, $0.738 \mathrm{mmol})$ in the presence of $\mathrm{Pd}(\mathrm{dba})_{2}$, dppp (29.7

(100), 2-benzyloxy-5,7-dihydro-9-methoxy-6H10-benzyloxy-5,7-dihydro-3-methoxy-6H-indolo[2,3- 
mg, $0.0527 \mathrm{mmol}$ ), phen (21.3 mg, $0.052 \mathrm{mmol})$ in DMF $(2.5 \mathrm{~mL})$, as described for 38 (pCO=6 atm, 41 h), gave, after chromatography (hexane/EtOAc, in order 7:3, 1:1, 3:7, 2:8), in order of elution, 100 (75.8 mg, $0.221 \mathrm{mmol}, 30 \%), 101$ (84.7 mg, $0.229 \mathrm{mmol}, 31 \%)$ and $\mathbf{1 0 2}$ (104 mg, $0.281 \mathrm{mmol}, 38 \%)$ all as white solids.

Analytical data for 100: $\mathrm{mp}=206-207{ }^{\circ} \mathrm{C} ;{ }^{1} \mathrm{H}$ NMR (400 MHz, DMSO- $\left.d_{6}\right) \delta 10.59(\mathrm{~s}, 1 \mathrm{H}), 10.50(\mathrm{~s}, 1 \mathrm{H})$, $8.16(\mathrm{~d}, J=8.4 \mathrm{~Hz}, 1 \mathrm{H}), 8.01-797$ (m, 2H), 7.95 (d, $J=2.8 \mathrm{~Hz}, 1 \mathrm{H}), 7.87-7.81(\mathrm{~m}, 2 \mathrm{H}), 7.79-7.75(\mathrm{~m}, 1 \mathrm{H})$, $7.74(\mathrm{~d}, J=8.8 \mathrm{~Hz}, 1 \mathrm{H}), 7.47$ (d, J=2.4 Hz, 1H), 7.25 (dd, J=8.8 Hz, 2.4 Hz, 1H), 7.22 (dd, J=9.2, $2.4 \mathrm{~Hz}$, $1 \mathrm{H}), 5.65$ (s, 2H), 4.26 (s, 3H); ${ }^{13} \mathrm{C}$ NMR (100 MHz, DMSO-d 6 ) $\delta$ 153.9, 152.6, 144.9, 139.6, 138.0, 133.3, 128.3, 127.6, 127.6, 122.2, 117.8, 116.2, 111.7, 107.4, 107.0, 102.8, 99.6, 96.9, 69.9, 55.3; IR (ATR) 3373, 1577, 1452, 1148, $1022 \mathrm{~cm}^{-1}$; HRMS (ESI) calcd for $\mathrm{C}_{22} \mathrm{H}_{18} \mathrm{~N}_{2} \mathrm{O}_{2}\left(\mathrm{M}^{+}\right) 342.1368$, found 342.1361 .

Analytical data for $101 \mathrm{mp}=285-286{ }^{\circ} \mathrm{C} ;{ }^{1} \mathrm{H}$ NMR $\left(400 \mathrm{MHz}, \mathrm{DMSO}-d_{6}\right) \delta 12.05(\mathrm{~s}, 1 \mathrm{H}), 11.66(\mathrm{~s}, 1 \mathrm{H})$, $8.34(\mathrm{~d}, J=8.4 \mathrm{~Hz}, 1 \mathrm{H}), 7.92$ (d, J=2.4 Hz,1H), 7.56 (d, J=6.8 Hz, 2H), 7.53 (d, J=9.2 Hz, 1H), 7.42 (t, $J=7.2 \mathrm{~Hz}, 2 \mathrm{H}), 7.34$ (tt, $J=6.8,1.2 \mathrm{~Hz}, 1 \mathrm{H}), 7.19$ (dd, J=9.2, $2.4 \mathrm{~Hz}, 1 \mathrm{H}), 7.05$ (d, J=2.8 Hz, 1H), 6.95 (dd, $J=8.4,2.4 \mathrm{~Hz}, 1 \mathrm{H}), 5.27$ (s, 2H), 3.83 (s, 3H); ${ }^{13} \mathrm{C}$ NMR (100 MHz, DMSO-d 6 ) $\delta 157.5,155.9,153.2$, 137.5, 136.3, 134.2, 128.4, 127.8, 127.7, 126.6, 124.2, 122.0, 118.3, 117.0, 113.8, 111.9, 110.2, 104.8, 99.8, 70.1, 55.2; IR (ATR) 3437, 3026, 1621, 1288, $811 \mathrm{~cm}^{-1}$; HRMS (ESI) calcd for $\mathrm{C}_{23} \mathrm{H}_{19} \mathrm{~N}_{2} \mathrm{O}_{3}\left(\mathrm{M}+\mathrm{H}^{+}\right.$) 371.1396 , found 371.1389 .

Analytical data for 102: $\mathrm{mp}=276-277{ }^{\circ} \mathrm{C} ;{ }^{1} \mathrm{H}$ NMR (400 MHz, DMSO- $\left.d_{6}\right) \delta 12.16(\mathrm{~s}, 1 \mathrm{H}), 11.65(\mathrm{~s}, 1 \mathrm{H})$, $8.27(\mathrm{~d}, J=8.8 \mathrm{~Hz}, 1 \mathrm{H}), 7.80$ (d, $J=2.8 \mathrm{~Hz}, 1 \mathrm{H}), 7.56$ (d, J=7.2 Hz, 2H), 7.44-7.40 (m, 3H), 7.34 (t, $J=7.6$ $\mathrm{Hz}, 1 \mathrm{H}), 7.12$ (dd, J=8.8, $2.4 \mathrm{~Hz}, 1 \mathrm{H}), 7.05$ (d, J=2.4 Hz, 1H), 6.94 (dd, J=8.8, 2.4 Hz, 1H), 5.29 (s, 2H), $3.86(\mathrm{~s}, 3 \mathrm{H}) ;{ }^{13} \mathrm{C}$ NMR (100 MHz, DMSO-d 6 ) $\delta 158.3,154.9,153.7,140.2,137.4,129.4,128.4,127.8$, 127.7, 127.2, 123.1, 118.4, 118.4, 117.2, 116.2, 115.0, 111.5, 106.7, 94.8, 69.8, 55.2; IR (ATR) 3448, 1652, 1458, 1264, $1228 \mathrm{~cm}^{-1}$; HRMS (ESI) calcd for $\mathrm{C}_{23} \mathrm{H}_{19} \mathrm{~N}_{2} \mathrm{O}_{3}\left(\mathrm{M}+\mathrm{H}^{+}\right) 371.1396$, found 371.1389. 
2-(5-Bromo-2-nitrophenyl)-1-trimethylsilylethyne (113). To a mixture of 5-bromo-2-nitro-1iodobenzene (112) (3.50 g, $10.7 \mathrm{mmol})$, CuI (203 mg, $1.07 \mathrm{mmol})$ and bis(triphenylphosphine)palladium dichloride $\left(\mathrm{PdCl}_{2}\left(\mathrm{PPh}_{3}\right)_{2}, 374 \mathrm{mg}, 0.533 \mathrm{mmol}\right)$ at ambient temperature under a $\mathrm{N}_{2}$ atmosphere was added tetrahydrofuran (THF, $10 \mathrm{~mL}$ ) followed by the addition of triethylamine $\left(\mathrm{Et}_{3} \mathrm{~N}, 16 \mathrm{~mL}\right)$. After stirring the mixture for 5 minutes, the solution of trimethylsilylethyne $(1.40 \mathrm{~g}, 13.4 \mathrm{mmol})$ in $\mathrm{Et}_{3} \mathrm{~N}(4 \mathrm{~mL})$ was added via syringe. The resulting dark brown mixture was stirred at this temperature for $36 \mathrm{~h}$. The reaction mixture was then filtered through Celite, and the Celite was washed with EtOAc $(30 \mathrm{~mL})$. The solvents were removed from the filtrate under reduced pressure. The resulting residue was purified by chromatography (hexane/EtOAc, 95:5) to give 113 (2.93 mg, $9.82 \mathrm{mmol}, 92 \%$ ) as a yellow oil. ${ }^{1} \mathrm{H}$ NMR $\left(600 \mathrm{MHz}, \mathrm{CDCl}_{3}\right) \delta 7.89$ (d, J=8.4 Hz, 1H), 7.79 (d, J=1.8 Hz, 1H), 7.56 (dd, J=8.4, $\left.1.8 \mathrm{~Hz}, 1 \mathrm{H}\right), 0.27$ (s, 9H); ${ }^{13} \mathrm{C}$ NMR (150 MHz, $\left.\mathrm{CDCl}_{3}\right) \delta 148.7,137.6,131.9,127.4,125.8,120.1,105.7,98.0,0.5$; IR (ATR) 2958, 1596, 1523, 1341, 1248, $833 \mathrm{~cm}^{-1}$; HRMS (ESI) calcd for $\mathrm{C}_{11} \mathrm{H}_{13} \mathrm{BrNO}_{2} \mathrm{Si}\left(\mathrm{M}+\mathrm{H}^{+}\right)$297.9899, found 297.9883.

1-(5-Bromo-2-nitrophenyl)ethyne (114). A heterogeneous mixture of 113 (2.56 g, $8.58 \mathrm{mmol})$ and $\mathrm{K}_{2} \mathrm{CO}_{3}(2.37 \mathrm{~g}, 17.2 \mathrm{mmol})$ in $\mathrm{MeOH} / \mathrm{Et}_{2} \mathrm{O}(1: 1,40 \mathrm{~mL})$ was stirred at ambient temperature under a $\mathrm{N}_{2}$ atmosphere for $2 \mathrm{~h}$. The mixture was diluted with EtOAc $(30 \mathrm{~mL})$ and the organic layer was washed with water $(3 \times 20 \mathrm{~mL})$. The resulting organic layer was dried $\left(\mathrm{MgSO}_{4}\right)$, filtered and the solvents were removed under reduced pressure. The crude product was purified by chromatography (hexane/EtOAc, 9:1) to give $114(1.76 \mathrm{~g}, 7.81 \mathrm{mmol}, 91 \%)$ as a white solid. mp=89-90 ${ }^{\circ} \mathrm{C} ;{ }^{1} \mathrm{H} \mathrm{NMR}\left(600 \mathrm{MHz}, \mathrm{CDCl}_{3}\right) \delta 7.93(\mathrm{~d}$, $J=8.4 \mathrm{~Hz}, 1 \mathrm{H}), 7.83(\mathrm{~d}, J=2.4 \mathrm{~Hz}, 1 \mathrm{H}), 7.63(\mathrm{dd}, J=9.0,2.4 \mathrm{~Hz}, 1 \mathrm{H}), 3.58(\mathrm{~s}, 1 \mathrm{H}),{ }^{13} \mathrm{C}$ NMR $(150 \mathrm{MHz}$, $\left.\mathrm{CDCl}_{3}\right) \delta 148.9,138.0,132.5,127.6,125.9,119.2,86.6,77.3$; IR (ATR) 3276, 2111, 1598, 1513, 1324, $836 \mathrm{~cm}^{-1}$; HRMS (ESI) calcd for $\mathrm{C}_{8} \mathrm{H}_{5} \mathrm{BrNO}_{2}\left(\mathrm{M}+\mathrm{H}^{+}\right)$225.9504, found 225.9502 .

Tributyl(1-(5-bromo-2-nitrophenyl)ethenyl)tin (115). Tributyltin hydride (1.98 g, $6.79 \mathrm{mmol})$ was added drop wise, at ambient temperature, to a solution of $\mathrm{PdCl}_{2}\left(\mathrm{PPh}_{3}\right)_{2}(318 \mathrm{mg}, 0.453 \mathrm{mmol})$ and 114 $(1.02 \mathrm{~g}, 4.53 \mathrm{mmol})$ in THF $(10 \mathrm{~mL})$. The dark brown reaction mixture was stirred for $36 \mathrm{~h}$ followed by removal of the solvent under reduced pressure. Purification by chromatography (hexane/EtOAc, 98:2) gave 115 (2.16 g, $4.185 \mathrm{mmol}, 92 \%)$ as a brown viscous oil. ${ }^{1} \mathrm{H}$ NMR (600 $\left.\mathrm{MHz}, \mathrm{CDCl}_{3}\right) \delta 7.89$ (d, J=9.0 $\mathrm{Hz}, 1 \mathrm{H}), 7.44(\mathrm{dd}, J=8.4,1.8 \mathrm{~Hz}, 1 \mathrm{H}), 7.27$ (d, $J=1.8 \mathrm{~Hz}, 1 \mathrm{H}), 5.75$ (d, $J=2.4 \mathrm{~Hz}, 1 \mathrm{H}), 5.46$ (d, J=2.4 Hz, $1 \mathrm{H}), 1.48-1.42$ (m, 6H), 1.26 (sext, $J=7.8 \mathrm{~Hz}, 6 \mathrm{H}), 0.94-0.91(\mathrm{~m}, 6 \mathrm{H}), 0.86(\mathrm{t}, J=7.2 \mathrm{~Hz}, 9 \mathrm{H}) ;{ }^{13} \mathrm{C}$ NMR $\left(150 \mathrm{MHz}, \mathrm{CDCl}_{3}\right) \delta 153.8,146.2,144.6,132.4,129.4,128.0,126.7,125.8,28.8,27.3$, 13.6, 11.0; IR (ATR) 2955, 2923, 1522, 1339, $878 \mathrm{~cm}^{-1}$; HRMS (ESI) calcd for $\mathrm{C}_{20} \mathrm{H}_{33} \mathrm{BrNO}_{2} \mathrm{Sn}\left(\mathrm{M}+\mathrm{H}^{+}\right)$518.0717, found 518.0730. 
2,3-Di(5-bromo-2-nitrophenyl)-1,3-butadiene (116). To a slurry of copper chloride (437 mg, 4.42 $\mathrm{mmol})$ in DMF (5 mL) in a round bottomed flask covered with aluminum foil to exclude light was added a solution of 115 (914 mg, $1.77 \mathrm{mmol})$ in DMF (3 mL). The resulting mixture was stirred at ambient temperature for $3 \mathrm{~h}$. A saturated solution of $\mathrm{NH}_{4} \mathrm{Cl}$ (aqueous, $6 \mathrm{~mL}$ ) was added and the mixture was allowed to stir for an additional hour. The mixture was diluted with EtOAc $(50 \mathrm{~mL})$, was washed with $\mathrm{H}_{2} \mathrm{O}(3 \times 40 \mathrm{~mL})$ and brine $(40 \mathrm{~mL})$. The organic phase was dried $\left(\mathrm{MgSO}_{4}\right)$, filtered, and the solvent was removed under reduced pressure. The residue was purified by chromatography (hexane/EtOAc, 9:1) affording 116 (223 mg, $0.491 \mathrm{mmol}, 56 \%$ ) as a white solid. $\mathrm{mp}=235{ }^{\circ} \mathrm{C}$ (decomposed); ${ }^{1} \mathrm{H}$ NMR (600 $\left.\mathrm{MHz}, \mathrm{CDCl}_{3}\right) \delta 7.90(\mathrm{~d}, J=9.0 \mathrm{~Hz}, 1 \mathrm{H}), 7.75(\mathrm{~d}, J=1.8 \mathrm{~Hz}, 1 \mathrm{H}), 7.67$ (dd, J=8.4, $\left.1.8 \mathrm{~Hz}, 1 \mathrm{H}\right), 5.18(\mathrm{~s}, 1 \mathrm{H})$, 4.94 (s, 1H); ${ }^{1} \mathrm{H}$ NMR (100 MHz, $\left.\mathrm{CDCl}_{3}\right) \delta$ 147.6, 143.8, 136.7, 135.0, 132.1, 128.0, 125.7, 119.0; IR (ATR) 1558, 1516, 1333, 1084, $868 \mathrm{~cm}^{-1}$; HRMS (ESI) calcd for $\mathrm{C}_{16} \mathrm{H}_{11} \mathrm{Br}_{2} \mathrm{~N}_{2} \mathrm{O}_{4}\left(\mathrm{M}+\mathrm{H}^{+}\right) 452.9085$, found 452.9068 .

5,5'-Dibromo-3,3'-bi-1H-indole (111). 2,3-Di(5-bromo-2-nitrophenyl)-1,3-butadiene (116) (207 mg, $0.456 \mathrm{mmol}), \mathrm{Pd}(\mathrm{dba})_{2}(26.2 \mathrm{mg}, 0.046 \mathrm{mmol}), \mathrm{dppp}(22.6 \mathrm{mg}, 0.055 \mathrm{mmol})$ and phen $(20.0 \mathrm{mg}, 0.111$ mmol) were dissolved in anhydrous DMF (3 mL) in a threaded ACE glass pressure tube. The tube was fitted with a pressure head, and the solution was saturated with carbon monoxide (four cycles of 6 atm of $\mathrm{CO})$. The reaction mixture was heated at $120{ }^{\circ} \mathrm{C}$ under $\mathrm{CO}(6 \mathrm{~atm})$ for $60 \mathrm{~h}$. After cooling to ambient temperature, the crude was diluted with EtOAc $(30 \mathrm{~mL})$ and was washed with water $(20 \mathrm{mLx} 4)$ and brine (20 mLx1) respectively. The organic layer was dried $\left(\mathrm{MgSO}_{4}\right)$, filtered and the solvent was removed from the filtrate under reduced pressure. The resulting residue was purified by chromatograpy (hexane/EtOAc, 7:3) to give 111 (80 mg, $0.205 \mathrm{mmol}, 45 \%$ ) as a white solid. ${ }^{1} \mathrm{H}$ NMR (600 MHz, DMSO-d 6 ) $\delta 11.42$ (br $\mathrm{s}, 1 \mathrm{H}), \mathrm{d}, J=1.8 \mathrm{~Hz}, 1 \mathrm{H}), 7.74(\mathrm{~d}, J=2.4 \mathrm{~Hz}, 1 \mathrm{H}), 7.42(\mathrm{~d}, J=8.4 \mathrm{~Hz}, 1 \mathrm{H}), 7.25(\mathrm{dd}, J=9.0,1.8 \mathrm{~Hz}, 1 \mathrm{H}) ;{ }^{1} \mathrm{H}$ NMR (100 MHz, DMSO-d 6 ) $\delta$ 135.0, 127.7, 123.9, 123.8, 121.4, 113.6, 111.6, 108.6; IR (ATR) 3423, $1658,1455,1098,794 \mathrm{~cm}^{-1}$.

2,2',5,5'-Tetrabromo-3,3'-bi-1H-indole (103). A mixture of $111(51.3 \mathrm{mg}, 0.132 \mathrm{mmol}$ ) and Nbromosuccinimide (NBS, $46.8 \mathrm{mg}, 0.263 \mathrm{mmol})$ in carbon tetrachloride $(2 \mathrm{~mL})$ was stirred at reflux $(78$ ${ }^{\circ} \mathrm{C}$ ) for $2 \mathrm{~h}$. After cooling to ambient temperature, the dark brown mixture was filtered through silica gel and the silica gel was washed with EtOAc $(3 \mathrm{~mL})$. The combined filtrate was concentrated under reduced pressure and purified by chromatography (hexane/EtOAc, 9:1) to give $\mathbf{1 0 3}$ (42.8 mg, $0.078 \mathrm{mmol}, 59 \%$ ) as a colorless oil. ${ }^{1} \mathrm{H} \mathrm{NMR}\left(400 \mathrm{MHz}, \mathrm{CDCl}_{3}\right) \delta 8.43(\mathrm{br} \mathrm{s}, 1 \mathrm{H}), 7.45(\mathrm{~d}, J=1.2 \mathrm{~Hz}, 1 \mathrm{H}), 7.31$ (dd, $J=8.8$, $2.0 \mathrm{~Hz}, 1 \mathrm{H}), 7.24$ (d, J=2.8 Hz, 1H); ${ }^{13} \mathrm{C} \mathrm{NMR}\left(100 \mathrm{MHz}, \mathrm{CDCl}_{3}\right) \delta$ 134.7, 129.6, 125.6, 122.0, 114.0, 112.0, 111.1, 108.2; IR (ATR) 3404, 1452, 1432, 1397, 1327, $794 \mathrm{~cm}^{-1}$; HRMS (ESI) calcd for $\mathrm{C}_{16} \mathrm{H}_{8} \mathrm{Br}_{4} \mathrm{~N}_{2}\left(\mathrm{M}^{+}\right)$547.7380, found 547.7359. 
2,2',5,5',6,6'-Hexabromo-3,3'-bi-1H-indole (105). Reaction of 111 (70.1 mg, $0.180 \mathrm{mmol}$ ) and NBS $(192 \mathrm{mg}, 1.08 \mathrm{mmol})$ in $\mathrm{THF} / \mathrm{CH}_{2} \mathrm{Cl}_{2}(1: 1,4 \mathrm{~mL})$, as described for $\mathbf{1 0 3}\left(70{ }^{\circ} \mathrm{C}, 13 \mathrm{~h}\right)$, gave after work up and chromatography (hexane/EtOAc, 9:1) $105(58.0 \mathrm{mg}, 0.082 \mathrm{mmol}, 46 \%)$ as a colorless oil. ${ }^{1} \mathrm{H}$ NMR $\left(400 \mathrm{MHz}, \mathrm{CDCl}_{3}\right) \delta 8.43$ (br s, $\left.1 \mathrm{H}\right), 7.67(\mathrm{~s}, 1 \mathrm{H}), 7.55(\mathrm{~s}, 1 \mathrm{H}) ;{ }^{13} \mathrm{C} \mathrm{NMR}\left(100 \mathrm{MHz}, \mathrm{CDCl}_{3}\right) \delta 135.5$, 128.4, 123.5, 118.1, 116.3, 115.3, 112.0, 107.8; IR (ATR) 3410, 1431, 1364, 1308, 936, $731 \mathrm{~cm}^{-1}$; HRMS (ESI) calcd for $\mathrm{C}_{16} \mathrm{H}_{6} \mathrm{Br}_{6} \mathrm{~N}_{2}\left(\mathrm{M}^{+}\right)$705.5570, found 705.5597.

Tributyl(1-(4-bromo-2-nitrophenyl)ethenyl)tin (118). Tributyltin hydride (2.56 g, $8.81 \mathrm{mmol})$ was added drop wise, at ambient temperature, to a solution of $\mathrm{PdCl}_{2}\left(\mathrm{PPh}_{3}\right)_{2}$ (412 mg, $\left.0.587 \mathrm{mmol}\right)$ and 1-(4bromo-2-nitrophenyl)ethyne (117) $(1.28 \mathrm{~g}, 5.88 \mathrm{mmol})$ in THF (13 mL). The dark brown reaction mixture was stirred for $24 \mathrm{~h}$ followed by removal of the solvent under reduced pressure. Purification by chromatography (hexane) gave 118 (2.95 g, $5.70 \mathrm{mmol}, 97 \%)$ as a brown oil. ${ }^{1} \mathrm{H}$ NMR (600 MHz, $\left.\mathrm{CDCl}_{3}\right) \delta 8.13(\mathrm{~d}, J=1.8 \mathrm{~Hz}, 1 \mathrm{H}), 7.63(\mathrm{dd}, J=8.4,1.8 \mathrm{~Hz}, 1 \mathrm{H}), 6.98(\mathrm{~d}, J=7.8 \mathrm{~Hz}, 1 \mathrm{H}), 5.73$ (d, J=2.4 Hz, $1 \mathrm{H}), 5.46$ (d, $J=2.4 \mathrm{~Hz}, 1 \mathrm{H}), 1.47-1.42(\mathrm{~m}, 6 \mathrm{H}), 1.26$ (sext, $J=7.2 \mathrm{~Hz}, 6 \mathrm{H}), 0.93-0.90(\mathrm{~m}, 6 \mathrm{H}), 0.86$ (t, $J=7.2 \mathrm{~Hz}, 9 \mathrm{H}) ;{ }^{13} \mathrm{C}$ NMR $\left(150 \mathrm{MHz}, \mathrm{CDCl}_{3}\right) \delta 153.7,146.3,143.3,136.1,131.1,127.1,126.7,119.0$, 28.8, 27.3, 13.6, 10.9; IR (ATR) 2921, 1522, 1464, 1339, $878 \mathrm{~cm}^{-1}$; HRMS (ESI, negative ion mode) calcd for $\mathrm{C}_{20} \mathrm{H}_{32} \mathrm{BrNO}_{2} \mathrm{Sn}\left(\mathrm{M}^{-}\right)$517.0638, found 517.0638.

2,3-Di(4-bromo-2-nitrophenyl)-1,3-butadiene (119). To a slurry of copper chloride ( $\mathrm{CuCl}, 579 \mathrm{mg}$, $5.85 \mathrm{mmol}$ ) in DMF ( $3 \mathrm{~mL}$ ) in a round bottomed flask covered with aluminum foil to exclude light was added a solution of $118(1.21 \mathrm{~g}, 2.34 \mathrm{mmol})$ in DMF (3 mL). The resulting mixture was stirred at ambient temperature for $2 \mathrm{~h}$. A saturated solution of $\mathrm{NH}_{4} \mathrm{Cl}$ (aqueous, $6 \mathrm{~mL}$ ) was added and the mixture was allowed to stir for an additional hour. The mixture was diluted with EtOAc $(50 \mathrm{~mL})$, was washed with $\mathrm{H}_{2} \mathrm{O}(3 \times 40 \mathrm{~mL})$ and brine $(40 \mathrm{~mL})$. The organic phase was dried $\left(\mathrm{MgSO}_{4}\right)$, filtered, and the solvent was removed under reduced pressure. The residue was purified by chromatography (hexane/EtOAc, 9:1) to give 119 (438 mg, $0.965 \mathrm{mmol}, 82 \%$ ) as a white solid. $\mathrm{mp}=185-187{ }^{\circ} \mathrm{C} ;{ }^{1} \mathrm{H} \mathrm{NMR}\left(400 \mathrm{MHz}, \mathrm{CDCl}_{3}\right) \delta$ $8.13(\mathrm{~d}, J=2.0 \mathrm{~Hz}, 1 \mathrm{H}), 7.79$ (dd, J=8.0, $2.0 \mathrm{~Hz}, 1 \mathrm{H}), 7.47$ (d, $J=8.4 \mathrm{~Hz}, 1 \mathrm{H}), 5.15$ (s, 1H), 4.92 (s, 1H); ${ }^{13} \mathrm{C}$ NMR $\left(100 \mathrm{MHz}, \mathrm{CDCl}_{3}\right) \delta 149.2,144.1,136.1,133.8,133.6,127.2,122.1,118.9$; IR (ATR) 1555, 1522, 1336, 913, $840 \mathrm{~cm}^{-1}$; HRMS (ESI, negative ion mode) calcd for $\mathrm{C}_{16} \mathrm{H}_{10} \mathrm{Br}_{2} \mathrm{~N}_{2} \mathrm{O}_{4}$ (M) 453.8987 , found 453.9017.

6,6'-Dibromo-3,3'-Bi-1H-indole (120). 2,3-Di(4-bromo-2-nitrophenyl)-1,3-butadiene (119) (320 mg, $0.705 \mathrm{mmol}), \mathrm{Pd}(\mathrm{dba})_{2}(28.4 \mathrm{mg}, 0.049 \mathrm{mmol})$, dppp (20.4 mg, $\left.0.049 \mathrm{mmol}\right)$ and phen $(17.8 \mathrm{mg}, 0.099$ mmol) were dissolved in anhydrous DMF (3 mL) in a threaded ACE glass pressure tube. The tube was fitted with a pressure head, and the solution was saturated with carbon monoxide (four cycles of 6 atm of 
$\mathrm{CO})$. The reaction mixture was heated at $120{ }^{\circ} \mathrm{C}$ under $\mathrm{CO}(6 \mathrm{~atm})$ for $42 \mathrm{~h}$. The combined organic phases were dried $\left(\mathrm{MgSO}_{4}\right)$, filtered, and the solvent was removed under reduced pressure. The resulting crude product was purified by chromatography (hexane/EtOAc, 19:1) to afford 120 (176 mg, $0.451 \mathrm{mmol}, 64 \%)$ as a white solid. mp=239-240 ${ }^{\circ} \mathrm{C} ;{ }^{1} \mathrm{H}$ NMR (400 MHz, DMSO-d $\left.{ }_{6}\right) \delta 11.34$ (br s, 1H), $7.70(\mathrm{~d}, \mathrm{~J}=8.8 \mathrm{~Hz}$, $1 \mathrm{H}), 7.68(\mathrm{~d}, J=2.4 \mathrm{~Hz}, 1 \mathrm{H}), 7.61(\mathrm{~d}, J=1.6 \mathrm{~Hz}, 1 \mathrm{H}), 7.18(\mathrm{dd}, J=8.4,2.0 \mathrm{~Hz}, 1 \mathrm{H}) ;{ }^{13} \mathrm{C} \mathrm{NMR}(150 \mathrm{MHz}$, DMSO-d $\left.{ }_{6}\right) \delta 137.2,124.9,123.0,121.7,121.2,114.1,114.0,109.3$; IR (ATR) 3382, 1589, 1519, 1335, $801 \mathrm{~cm}^{-1}$; HRMS (ESI) calcd for $\mathrm{C}_{16} \mathrm{H}_{11} \mathrm{Br}_{2} \mathrm{~N}_{2}\left(\mathrm{M}+\mathrm{H}^{+}\right)$388.9289, found 388.9287 .

2,2',6,6'-Tetrabromo-3,3'-bi-1H-indole (104). A mixture of 120 (41.2 mg, $0.106 \mathrm{mmol}$ ) and Nbromosuccinimide (NBS, $28.6 \mathrm{mg}, 0.161 \mathrm{mmol})$ in carbon tetrachloride $(1 \mathrm{~mL})$ was stirred at reflux $(78$ ${ }^{\circ} \mathrm{C}$ ) for $2 \mathrm{~h}$. After cooling to ambient temperature, the dark brown mixture was filtered through silica gel and the silica gel was washed with EtOAc $(3 \mathrm{~mL})$. The combined filtrate was concentrated under reduced pressure and purified by chromatography (hexane/EtOAc, 9:1) to give 104 (32.9 mg, $0.060 \mathrm{mmol}, 57 \%$ ) as a colorless oil. ${ }^{1} \mathrm{H}$ NMR $\left(400 \mathrm{MHz}, \mathrm{CDCl}_{3}\right) \delta 8.37$ (br s, $\left.1 \mathrm{H}\right), 7.55$ (d, $\left.J=1.2 \mathrm{~Hz}, 1 \mathrm{H}\right), 7.22$ (dd, $J=8.4$, $1.6 \mathrm{~Hz}, 1 \mathrm{H}), 7.20$ (d, J=8.4 Hz, 1H); ${ }^{13} \mathrm{C} \mathrm{NMR}\left(100 \mathrm{MHz}, \mathrm{CDCl}_{3}\right) \delta 136.7,126.9,123.9,120.9,116.3$, 113.5, 110.1, 108.9; IR (ATR) 3425, 1432, 1371, 1308, 910, $815 \mathrm{~cm}^{-1}$; HRMS (ESI) calcd for $\mathrm{C}_{16} \mathrm{H}_{8} \mathrm{Br}_{4} \mathrm{~N}_{2}$ $\left(\mathrm{M}^{+}\right)$547.7380, found 547.7364

2,2',5,5',6,6'-Hexabromo-3,3'-bi-1H-indole (105) and 2,2',5,6,6'-pentabromo-3,3'-bi-1H-indole (121). Treatment of 120 (76.1 mg, $0.195 \mathrm{mmol})$ with NBS (174 mg, $0.978 \mathrm{mmol})$ in $\mathrm{THF} / \mathrm{CH}_{2} \mathrm{Cl}_{2}(1: 1,4$ $\mathrm{mL})$, as described for $103\left(70{ }^{\circ} \mathrm{C}, 6 \mathrm{~h}\right)$, gave after work up and chromatography (hexane/EtOAc, 9:1), in order of elution, 121 (40.3 mg, $0.064 \mathrm{mmol}, 33 \%)$ and 105 (64.9 mg, $0.098 \mathrm{mmol}, 47 \%)$ both as a colorless oil. Analytical data for 121: ${ }^{1} \mathrm{H}$ NMR (400 MHz, $\left.\mathrm{CDCl}_{3}\right) \delta 8.41$ (br s, $\left.1 \mathrm{H}\right), 8.40(\mathrm{~s}, 1 \mathrm{H}), 7.68$ (s, $1 \mathrm{H}), 7.59(\mathrm{~s}, 1 \mathrm{H}), 7.55(\mathrm{~d}, J=1.2 \mathrm{~Hz}, 1 \mathrm{H}), 7.24(\mathrm{dd}, J=8.4,1.6 \mathrm{~Hz}, 1 \mathrm{H}), 7.18(\mathrm{~d}, J=8.4 \mathrm{~Hz}, 1 \mathrm{H}) ;{ }^{13} \mathrm{C} \mathrm{NMR}$ $\left(400 \mathrm{MHz}, \mathrm{CDCl}_{3}\right) \delta 136.6,135.5,128.6,126.7,124.1,123.8,120.7,118.0,116.4,116.2,115.3,113.5$, 111.7, 110.3, 108.4, 108.3; IR (ATR) 3411, 1433, 1372, 1303, $805 \mathrm{~cm}^{-1}$; HRMS (ESI) calcd for $\mathrm{C}_{16} \mathrm{H}_{8} \mathrm{Br}_{5} \mathrm{~N}_{2}\left(\mathrm{M}+\mathrm{H}^{+}\right)$622.6604, found 622.6589 .

2,2',6,6'-Tetrabromo-3,3'-bi-1H-indole (104) and 2,2',5,6,6'-Pentabromo-3,3'-bi-1H-indole (121). Compound 56 (40.1 mg, $0.173 \mathrm{mmol})$ was treated with NBS (123 mg, $0.690 \mathrm{mmol})$ in $\mathrm{THF} / \mathrm{CH}_{2} \mathrm{Cl}_{2}(1: 1$, $3 \mathrm{~mL}$ ), as described for $103\left(70{ }^{\circ} \mathrm{C}, 15 \mathrm{~h}\right)$. Work up and chromatography (hexane/EtOAc, 95:5) gave, in order of elution, 104 (7.8 mg, $0.014 \mathrm{mmol}, 8 \%)$ and 121 (8.5 mg, $0.014 \mathrm{mmol}, 8 \%)$ both as a colorless oil.

2,2',5,6,6'-Pentabromo-3,3'-bi-1H-indole (121) and 2,2',5,5',6,6'-Hexabromo-3,3'-bi-1H-indole (105). Compound $56(32.2 \mathrm{mg}, 0.139 \mathrm{mmol})$ was treated with NBS (126 mg, $0.708 \mathrm{mmol})$ in 
$\mathrm{THF} / \mathrm{CH}_{2} \mathrm{Cl}_{2}(1: 1,3 \mathrm{~mL})$, as described for $103\left(70{ }^{\circ} \mathrm{C}, 16 \mathrm{~h}\right)$. Work up and chromatography (hexane/EtOAc, 95:5) gave, in order of elution, 121 (10.8 mg, $0.017 \mathrm{mmol}, 12 \%)$ and 105 (10.1 mg, $0.014 \mathrm{mmol}, 10 \%$ ) both as a colorless oil.

2,2',5,5',6,6'-Hexabromo-3,3'-bi-1H-indole (105). Compound 56 (40.8 mg, $0.176 \mathrm{mmol})$ was treated with NBS (250 mg, $1.40 \mathrm{mmol})$ in $\mathrm{THF} / \mathrm{CH}_{2} \mathrm{Cl}_{2}(1: 1,3 \mathrm{~mL})$, as described for $\mathbf{1 0 3}\left(70{ }^{\circ} \mathrm{C}, 22 \mathrm{~h}\right)$. Work up and chromatography (hexane/EtOAc, 95:5) gave 105 (19.3 mg, $0.027 \mathrm{mmol}, 16 \%)$ as a colorless oil. 
5-Methoxy-2-nitro- $N$-(2-methyl-1-propen-1-yl)benzenamine (145). To a solution of 5-methoxy-2nitrobenzenamine $(1.00 \mathrm{~g}, 5.95 \mathrm{mmol})$ and 2-methylpropanal $(515 \mathrm{mg}, 7.14 \mathrm{mmol})$ in dichloromethane (DCM, $12 \mathrm{~mL}$ ) at ambient temperature under a nitrogen atmosphere was added $4 \AA$ molecular sieves ( $3 \mathrm{~g}$, activated by heating at $120^{\circ} \mathrm{C}$ under vacuum overnight, then stored under nitrogen). The reaction mixture was allowed to sit without agitation or stirring for $48 \mathrm{~h}$. The mixture was then filtered and the sieves were washed with DCM $(20 \mathrm{~mL})$. The filtrate was concentrated under reduced pressure and the resulting residue was purified by chromatography (hexane/EtOAc, 9:1) to give 145 (484 $\mathrm{mg}, 2.18 \mathrm{mmol}, 37 \%$ ) as a red solid. $\mathrm{mp}=83-84{ }^{\circ} \mathrm{C} ;{ }^{1} \mathrm{H} \mathrm{NMR}\left(600 \mathrm{MHz}, \mathrm{CDCl}_{3}\right) \delta 9.86(\mathrm{~d}, J=7.2 \mathrm{~Hz}, 1 \mathrm{H}), 8.14(\mathrm{~d}, J=9.6 \mathrm{~Hz}, 1 \mathrm{H})$, 6.32 (d, $J=3.0 \mathrm{~Hz}, 1 \mathrm{H}), 6.29$ (dd, $J=9.6,2.4 \mathrm{~Hz}, 1 \mathrm{H}), 6.20$ (dpent, $J=9.6,1.2 \mathrm{~Hz}, 1 \mathrm{H}$ ), 3.88 (s, 3H), 1.83 (s, 3H), $1.80(\mathrm{~s}, 3 \mathrm{H}) ;{ }^{13} \mathrm{C}$ NMR $\left(150 \mathrm{MHz}, \mathrm{CDCl}_{3}\right) \delta 165.7,143.5,129.0,126.0,119.0,118.0,106.1,95.0$, 55.7, 22.5, 16.8; IR (ATR) 2923, 1616, 1585, 1497, $1239 \mathrm{~cm}^{-1}$; HRMS (ESI) calcd for $\mathrm{C}_{11} \mathrm{H}_{14} \mathrm{~N}_{2} \mathrm{O}_{3} \mathrm{Na}$ $\left(\mathrm{M}+\mathrm{Na}^{+}\right)$245.0902, found 245.0896.

4-Bromo-2-nitro- $N$-(2-methyl-1-propen-1-yl)benzenamine (133). Treatment of a solution of 4-bromo2-nitrobenzenamine (1.04 g, $4.78 \mathrm{mmol})$ and 2-methylpropanal (414 mg, $5.74 \mathrm{mmol})$ in dichloromethane (DCM, $12 \mathrm{~mL}$ ) at ambient temperature under a nitrogen atmosphere in the presence of $4 \AA$ molecular sieves ( $3 \mathrm{~g}$ ), as described for 145 (24 h), gave after work up and chromatography, (hexane/EtOAc, 95:5), 133 (726 mg, $2.68 \mathrm{mmol}, 56 \%)$ as a violet solid. $\mathrm{mp}=119-120{ }^{\circ} \mathrm{C} ;{ }^{1} \mathrm{H} \mathrm{NMR}\left(400 \mathrm{MHz}, \mathrm{CDCl}_{3}\right) \delta 9.55(\mathrm{~d}$, $J=8.0 \mathrm{~Hz}, 1 \mathrm{H}), 8.29$ (d, J=2.4 Hz, 1H), 7.47 (dd, $J=9.2,2.4 \mathrm{~Hz}, 1 \mathrm{H}), 6.93$ (d, J=9.6 Hz, 1H), 6.20 (d, $J=9.6 \mathrm{~Hz}, 1 \mathrm{H}), 1.81(\mathrm{~s}, 3 \mathrm{H}), 1.78(\mathrm{~s}, 3 \mathrm{H}) ;{ }^{13} \mathrm{C} \mathrm{NMR}\left(100 \mathrm{MHz}, \mathrm{CDCl}_{3}\right) \delta 140.3,138.8,128.8,119.9$, 117.9, 116.0, 107.6, 22.5, 16.8; IR (ATR) 2912, 1564, 1340, $1165 \mathrm{~cm}^{-1}$; HRMS (ESI) calcd for $\mathrm{C}_{10} \mathrm{H}_{12} \mathrm{BrN}_{2} \mathrm{O}_{2}\left(\mathrm{M}+\mathrm{H}^{+}\right)$271.0082, found 271.0078.

5-Bromo-2-nitro- $N$-(2-methyl-1-propen-1-yl)benzenamine (150). Treatment of a solution of 5-bromo2-nitrobenzenamine (455 mg, $2.10 \mathrm{mmol})$ with 2-methylpropanal (182 mg, $2.52 \mathrm{mmol})$ in benzene (6 $\mathrm{mL})$, in the presence of $4 \AA$ molecular sieves $(4 \mathrm{~g})$, at ambient temperature under a nitrogen atmosphere, as described for 145 (24 h), gave after work up and chromatography (hexane/EtOAc, 95:5), 150 (416 mg, $1.534 \mathrm{mmol}, 73 \%)$ as a red solid. $\mathrm{mp}=94-95{ }^{\circ} \mathrm{C} ;{ }^{1} \mathrm{H} \mathrm{NMR}\left(600 \mathrm{MHz}, \mathrm{CDCl}_{3}\right) \delta 9.56(\mathrm{~d}, J=7.2 \mathrm{~Hz}, 1 \mathrm{H})$, $8.03(\mathrm{~d}, J=9.0 \mathrm{~Hz}, 1 \mathrm{H}), 7.20(\mathrm{~d}, J=1.2 \mathrm{~Hz}, 1 \mathrm{H}), 6.81(\mathrm{dd}, J=9.0,1.8 \mathrm{~Hz}, 1 \mathrm{H}), 6.18$ (d with further fine splitting, $J=9.6 \mathrm{~Hz}, 1 \mathrm{H}), 1.83(\mathrm{~s}, 3 \mathrm{H}), 1.79(\mathrm{~s}, 3 \mathrm{H}) ;{ }^{13} \mathrm{C} \mathrm{NMR}\left(150 \mathrm{MHz}, \mathrm{CDCl}_{3}\right) \delta 141.8,131.6,130.7$, 128.1, 120.7, 119.8, 117.8, 117.0, 22.5, 16.9; IR (ATR) 3495, 3381, 1616, 1560, 1489, $1218 \mathrm{~cm}^{-1}$; HRMS (ESI) calcd for $\mathrm{C}_{10} \mathrm{H}_{12} \mathrm{BrN}_{2} \mathrm{O}_{2}\left(\mathrm{M}+\mathrm{H}^{+}\right)$271.0082, found 271.0078. 
4-Fluoro-2-nitro- $N$-(2-methyl-1-propen-1-yl)benzenamine (136). Treatment of a solution of 4-fluoro2-nitrobenzenamine ( $845 \mathrm{mg}, 5.41 \mathrm{mmol}$ ) with 2-methylpropanal (390 mg, $5.41 \mathrm{mmol})$ in DCM (12 mL), in the presence of $5 \AA$ molecular (12 g), at ambient temperature under a nitrogen atmosphere, as described for 145, (24 h), gave after work up and chromatography (hexane/EtOAc, 97:3), 136 (788 mg, $3.75 \mathrm{mmol}$, $69 \%)$ as a reddish-brown solid. $\mathrm{mp}=79-80{ }^{\circ} \mathrm{C} ;{ }^{1} \mathrm{H}$ NMR $\left(400 \mathrm{MHz}, \mathrm{CDCl}_{3}\right) \delta 9.48(\mathrm{~d}, J=7.6 \mathrm{~Hz}, 1 \mathrm{H}), 7.87$ (dd, $J=8.8,2.8 \mathrm{~Hz}, 1 \mathrm{H}), 7.27-7.22$ (ddd, $J=9.6,6.4,2.4 \mathrm{~Hz}, 1 \mathrm{H}), 7.02$ (dd, J=9.6, 4.8 Hz, 1H), 6.21 (d, with further fine splitting $J=9.6 \mathrm{~Hz}, 1 \mathrm{H}), 1.81(\mathrm{~s}, 3 \mathrm{H}), 1.78(\mathrm{~s}, 3 \mathrm{H}) ;{ }^{13} \mathrm{C} \mathrm{NMR}\left(100 \mathrm{MHz}, \mathrm{CDCl}_{3}\right) \delta 153.0$ $\left(\mathrm{d}, J^{\mathrm{C}-\mathrm{F}}=239.0 \mathrm{~Hz}\right), 138.6\left(\mathrm{~d}, J^{\mathrm{C}-\mathrm{F}}=1.0 \mathrm{~Hz}\right), 130.5\left(\mathrm{~d}, J^{\mathrm{C}-\mathrm{F}}=8.0 \mathrm{~Hz}\right), 124.9\left(\mathrm{~d}, J^{\mathrm{C}-\mathrm{F}}=24 \mathrm{~Hz}\right), 119.2,118.2$, $115.7\left(\mathrm{~d}, J^{\mathrm{C}-\mathrm{F}}=8.0 \mathrm{~Hz}\right), 111.8\left(\mathrm{~d}, J^{\mathrm{C}-\mathrm{F}}=26.0 \mathrm{~Hz}\right), 22.3,16.7$; IR (ATR) 1580, 1519, 1404, 1185, 1158, 1112 $\mathrm{cm}^{-1}$; HRMS (ESI) $\mathrm{C}_{10} \mathrm{H}_{12} \mathrm{FN}_{2} \mathrm{O}_{2}\left(\mathrm{M}+\mathrm{H}^{+}\right) 211.0883$, found 211.0897.

5-Methyl-2-nitro- $N$-(2-methyl-1-propen-1-yl)benzenamine (153). Treatment of a solution of 5-methyl2-nitrobenzenamine (200 mg, $1.31 \mathrm{mmol})$ and 2-methylpropanal (114 mg, $1.58 \mathrm{mmol})$ in benzene (3 mL), in the presence of $4 \AA$ molecular sieves ( $1 \mathrm{~g}$ ) at ambient temperature under a nitrogen atmosphere, as described for 145 (24 h), gave after work up and chromatography (hexane/EtOAc, 95:5), 153 (106 mg, $0.514 \mathrm{mmol}, 39 \%)$ as a red solid. $\mathrm{mp}=75-76{ }^{\circ} \mathrm{C} ;{ }^{1} \mathrm{H} \mathrm{NMR}\left(600 \mathrm{MHz}, \mathrm{CDCl}_{3}\right) \delta 9.63(\mathrm{~d}, J=7.8 \mathrm{~Hz}, 1 \mathrm{H})$, $8.06(\mathrm{~d}, J=9.0 \mathrm{~Hz}, 1 \mathrm{H}), 6.81(\mathrm{~s}, 1 \mathrm{H}), 6.51(\mathrm{dd}, J=9.0,1.2 \mathrm{~Hz}, 1 \mathrm{H}), 6.27$ (d with further fine splitting, $J=9.0 \mathrm{~Hz}, 1 \mathrm{H}), 2.35(\mathrm{~s}, 3 \mathrm{H}), 1.82(\mathrm{~s}, 3 \mathrm{H}), 1.79$ (s, 3H); ${ }^{13} \mathrm{C} \mathrm{NMR}\left(150 \mathrm{MHz}, \mathrm{CDCl}_{3}\right) \delta 147.7,141.4$, 129.9, 126.8, 118.5, 118.4, 118.2, 113.8, 22.5, 22.1, 16.8; IR (ATR) 1618, 1578, 1332, 1210, $1183 \mathrm{~cm}^{-1}$; HRMS (ESI) calcd for $\mathrm{C}_{11} \mathrm{H}_{15} \mathrm{~N}_{2} \mathrm{O}_{2}\left(\mathrm{M}+\mathrm{H}^{+}\right)$207.1133, found 207.1128 .

4-Bromo-2-nitro- $N$-(2-phenyl-1-propen-1-yl)benzenamine (160). Treatment of a solution of 4-bromo2-nitrobenzenamine (430 mg, $1.98 \mathrm{mmol})$ with 2-phenylpropanal (239 mg, $1.78 \mathrm{mmol})$ in DCM (7 mL), in the presence of $5 \AA$ molecular sieves ( $3.5 \mathrm{~g}$ ), at ambient temperature under a nitrogen atmosphere, as described for 145 (24 h), gave after work up and chromatography (hexane/EtOAc, 95:5), 160 (446 mg, $1.34 \mathrm{mmol}, 67 \%)$ as a violet solid. $\mathrm{mp}=106-107{ }^{\circ} \mathrm{C} ;{ }^{1} \mathrm{H}$ NMR $\left(400 \mathrm{MHz}, \mathrm{CDCl}_{3}\right) \delta 9.93$ (d, J=10.4 Hz, $1 \mathrm{H}), 8.37$ (d, J=2.4 Hz, 1H), 7.56 (dd, J=9.2, 2.4 Hz, 1H), 7.42-7.34 (m, 4H), 7.29-7.25 (m, 1H), 7.09 (d, $J=9.2 \mathrm{~Hz}, 1 \mathrm{H}), 6.87(\mathrm{dq}, J=10.4,1.2 \mathrm{~Hz}, 1 \mathrm{H}), 2.21(\mathrm{~d}, J=1.2 \mathrm{~Hz}, 3 \mathrm{H}) ;{ }^{13} \mathrm{C} \mathrm{NMR}\left(100 \mathrm{MHz}, \mathrm{CDCl}_{3}\right) \delta$ $140.9,139.4,138.9,132.7,129.0,128.5,126.8,125.3,120.6,120.5,116.0,108.8,14.7$; IR (ATR) 1618, 1564, 1175, 1126, $749 \mathrm{~cm}^{-1}$; HRMS (ESI) calcd for $\mathrm{C}_{15} \mathrm{H}_{14} \mathrm{BrN}_{2} \mathrm{O}_{2}\left(\mathrm{M}+\mathrm{H}^{+}\right) 333.0238$, found 333.0234.

A detailed experimental procedure for the experiment shown in Entry 1 in Table 1 is shown below. All other entries in Tables 1-3 are slight variations of this procedure. Detailed experimental procedures for all entries discussed can be found below the analytical data section. 


\section{Table 4.2, Entry 11}

1-Methoxybenzimidazole (124), ${ }^{133}$ 1-Methoxy-2-(2-methoxy-2-propyl)benzimidazole (125), and 2-(2Hydroxy-2-propyl)-1-methoxybenzimidazole (126). A mixture of 2-nitro- $N$-(2-methyl-1-propen-1yl)benzenamine (122) $)^{134}(160 \mathrm{mg}, 0.833 \mathrm{mmol})$ and sodium hydride ${ }^{135}(\mathrm{NaH}, 74.4 \mathrm{mg}, 3.10 \mathrm{mmol})$ in dimethylsulfoxide (DMSO, $10 \mathrm{~mL}$ ) was stirred at ambient temperature under a nitrogen atmosphere. After stirring for $1 \mathrm{~h}$, the mixture was cooled to $0{ }^{\circ} \mathrm{C}$ and methyl iodide $(379 \mathrm{mg}, 2.67 \mathrm{mmol})$ was added via a syringe. The reaction vessel was removed from the cold bath and stirred for $1 \mathrm{~h}$. The resulting mixture was diluted with EtOAc $(20 \mathrm{~mL})$, washed with water $(5 \times 15 \mathrm{~mL})$, dried $\left(\mathrm{MgSO}_{4}\right)$, and filtered. The solvents were removed from the filtrate under reduced pressure and the resulting residue was purified by chromatography (hexane/EtOAc, 7:3 then 3:7) to give, in order of elution 125 (105 mg, $0.477 \mathrm{mmol}$, $57 \%)$ as a colorless oil, 126 (25.3 $\mathrm{mg}, 0.123 \mathrm{mmol}, 15 \%)$ as a white solid, and $\mathbf{1 2 4}$ (6.3 $\mathrm{mg}, 0.043 \mathrm{mmol}$, $5 \%)$ as a colorless oil.

\section{Analytical data for all compounds:}

1-Methoxybenzimidazole (124). Colorless oil; ${ }^{1} \mathrm{H}$ NMR $\left(400 \mathrm{MHz}, \mathrm{CDCl}_{3}\right) \delta 8.08(\mathrm{~s}, 1 \mathrm{H}), 7.78(\mathrm{~d}$, $J=8.0 \mathrm{~Hz}, 1 \mathrm{H}), 7.49$ (d, $J=8.0 \mathrm{~Hz}, 1 \mathrm{H}), 7.34$ (td, $J=7.2,1.2 \mathrm{~Hz}, 1 \mathrm{H}), 7.29$ (td, $J=8.0,1.2 \mathrm{~Hz}, 1 \mathrm{H}), 4.17$ (s, $3 \mathrm{H}) ;{ }^{13} \mathrm{C}$ NMR (100 MHz, $\left.\mathrm{CDCl}_{3}\right) \delta 139.4,137.4,129.2,123.6,122.5,120.9,108.3,67.2$; IR (ATR) 1476, 1449, 1318, 1074, $962 \mathrm{~cm}^{-1}$; HRMS (ESI) calcd for $\mathrm{C}_{8} \mathrm{H}_{9} \mathrm{~N}_{2} \mathrm{O}\left(\mathrm{M}+\mathrm{H}^{+}\right)$149.0715, found 149.0710.

1-Methoxy-2-(2-methoxy-2-propyl)benzimidazole (125). Colorless oil; ${ }^{1} \mathrm{H} \mathrm{NMR}\left(400 \mathrm{MHz}, \mathrm{CDCl}_{3}\right) \delta$ $7.75(\mathrm{~d}, J=8.0 \mathrm{~Hz}, 1 \mathrm{H}), 7.45(\mathrm{~d}, J=8.0 \mathrm{~Hz}, 1 \mathrm{H}), 7.32(\mathrm{td}, J=7.2,1.2 \mathrm{~Hz}, 1 \mathrm{H}), 7.26(\mathrm{td}, J=7.2,1.2 \mathrm{~Hz}, 1 \mathrm{H})$, 4.20 (s, 3H), 3.19 (s, 3H), 1.80 (s, 6H); $\left.{ }^{13} \mathrm{C} \mathrm{NMR} \mathrm{(100} \mathrm{MHz,} \mathrm{CDCl}_{3}\right) \delta 151.3,136.9,131.4,123.4,122.2$, 120.4, 108.3, 74.5, 65.2, 51.3, 24.8; IR (ATR) 1244, 1176, 1151, 1066, $969 \mathrm{~cm}^{-1}$; HRMS (ESI) calcd for $\mathrm{C}_{12} \mathrm{H}_{17} \mathrm{~N}_{2} \mathrm{O}_{2}\left(\mathrm{M}+\mathrm{H}^{+}\right)$221.1290, found 221.1285.

1-Methoxy-2-(2-hydroxy-2-propyl)benzimidazole (126). White solid; mp=121-122 ${ }^{\circ} \mathrm{C} ;{ }^{1} \mathrm{H}$ NMR (400 $\left.\mathrm{MHz}, \mathrm{CDCl}_{3}\right) \delta 7.71(\mathrm{~d}, J=7.6 \mathrm{~Hz}, 1 \mathrm{H}), 7.43(\mathrm{~d}, J=8.4 \mathrm{~Hz}, 1 \mathrm{H}), 7.31(\mathrm{td}, J=7.2,1.2 \mathrm{~Hz}, 1 \mathrm{H}), 7.26(\mathrm{td}$, $J=8.0,1.6 \mathrm{~Hz}, 1 \mathrm{H}), 4.22$ (s, 3H), 3.51 (br s, $1 \mathrm{H}), 1.77(\mathrm{~s}, 6 \mathrm{H}) ;{ }^{13} \mathrm{C} \mathrm{NMR}\left(100 \mathrm{MHz}, \mathrm{CDCl}_{3}\right) \delta 154.2$, 136.9, 131.2, 123.3, 122.5, 120.4, 108.5, 69.8, 65.7, 28.8; IR (ATR) 3240, 1438, 1359, 1234, 1176, 1147 $\mathrm{cm}^{-1}$; HRMS (ESI) calcd for $\mathrm{C}_{11} \mathrm{H}_{15} \mathrm{~N}_{2} \mathrm{O}_{2}\left(\mathrm{M}+\mathrm{H}^{+}\right)$207.1133, found 207.1118.

1,6-Dimethoxybenzimidazole (128). Colorless oil; ${ }^{1} \mathrm{H}$ NMR $\left(400 \mathrm{MHz}, \mathrm{CDCl}_{3}\right) \delta 7.97(\mathrm{~s}, 1 \mathrm{H}), 7.64(\mathrm{~d}$, $J=9.6 \mathrm{~Hz}, 1 \mathrm{H}), 6.93-6.90(\mathrm{~m}, 2 \mathrm{H}), 4.17$ (s, 3H), 3.89 (s, 3H); ${ }^{13} \mathrm{C} \mathrm{NMR}\left(100 \mathrm{MHz}, \mathrm{CDCl}_{3}\right) \delta 157.3,136.6$, 
133.9, 129.9, 121.6, 112.4, 91.3, 67.0, 55.8; IR (ATR) 1493, 1236, 1020, $815 \mathrm{~cm}^{-1}$; HRMS (ESI) calcd for $\mathrm{C}_{9} \mathrm{H}_{11} \mathrm{~N}_{2} \mathrm{O}_{2}\left(\mathrm{M}+\mathrm{H}^{+}\right)$179.0820, found 179.0814.

1,6-Dimethoxy-2-(2-methoxy-2-propyl)benzimidazole (129). Pale yellow oil; ${ }^{1} \mathrm{H}$ NMR (400 MHz, $\left.\mathrm{CDCl}_{3}\right) \delta 7.61(\mathrm{~d}, J=9.6 \mathrm{~Hz}, 1 \mathrm{H}), 6.88(\mathrm{dd}, J=7.2,2.4 \mathrm{~Hz}, 1 \mathrm{H}), 6.87$ (s, 1H), 4.17 (s, 3H), 3.88 (s, 3H), 3.16 (s, 3H), 1.77 (s, 6H); ${ }^{13} \mathrm{C}$ NMR (100 MHz, $\left.\mathrm{CDCl}_{3}\right) \delta 157.2,150.5,132.1,131.3,121.1,111.8,91.4$, 74.5, 65.0, 55.8, 51.3, 24.9; IR (ATR) 2929, 1738, 1215, 1063, $817 \mathrm{~cm}^{-1}$; HRMS (ESI) calcd for $\mathrm{C}_{13} \mathrm{H}_{19} \mathrm{~N}_{2} \mathrm{O}_{3}\left(\mathrm{M}+\mathrm{H}^{+}\right)$251.1395, found 251.1389.

2-(2-Hydroxy-2-propyl)-1,6-dimethoxybenzimidazole (130). Data from a 37:1 mixture of $\mathbf{1 3 0}$ and $\mathbf{1 2 8}$ : White solid; mp=119-120 ${ }^{\circ} \mathrm{C},{ }^{1} \mathrm{H}$ NMR $\delta 7.59(\mathrm{~d}, J=8.6 \mathrm{~Hz}, 1 \mathrm{H}), 6.90(\mathrm{dd}, J=8.8,2.5 \mathrm{~Hz}, 1 \mathrm{H}), 6.87(\mathrm{~d}$, $J=2.2 \mathrm{~Hz}, 1 \mathrm{H}), 4.21$ (s, 3H), 3.89 (s, 3H), 3.26 (br s, 1H), 1.76 (s, 6H); ${ }^{13} \mathrm{C}$ NMR $\delta 157.1,153.4,131.8$, 131.4, 121.0, 111.8, 92.0, 69.8, 65.5, 55.9, 28.8; IR (neat) 3234, 1625, 1497, 1242, 1217, 1178, 1147, $1018,956,823 \mathrm{~cm}^{-1}$; HRMS (ESI) calcd for $\mathrm{C}_{12} \mathrm{H}_{17} \mathrm{~N}_{2} \mathrm{O}_{3}\left(\mathrm{M}+\mathrm{H}^{+}\right)$237.1239, found 237.1229.

6-Chloro-1-methoxy-2-(2-methoxy-2-propyl)benzimidazole (132). Orange solid; $\mathrm{mp}=66-67{ }^{\circ} \mathrm{C} ;{ }^{1} \mathrm{H}$ NMR (400 MHz, $\left.\mathrm{CDCl}_{3}\right) \delta 7.62(\mathrm{~d}, J=8.4 \mathrm{~Hz}, 1 \mathrm{H}), 7.41(\mathrm{~d}, J=1.6 \mathrm{~Hz}, 1 \mathrm{H}), 7.19$ (dd, $\left.J=8.4,1.6 \mathrm{~Hz}, 1 \mathrm{H}\right)$, 4.16 (s, 3H), 3.16 (s, 3H), 1.76 (s, 6H); $\left.{ }^{13} \mathrm{C} \mathrm{NMR} \mathrm{(100} \mathrm{MHz,} \mathrm{CDCl}_{3}\right) \delta 152.4,135.5,132.1,129.4,123.1$, 121.4, 108.4, 74.5, 65.5, 51.3, 24.8; IR (ATR) 2944, 1677, 1496, 1340, 1268, $1228 \mathrm{~cm}^{-1}$; HRMS (ESI) calcd for $\mathrm{C}_{12} \mathrm{H}_{16} \mathrm{ClN}_{2} \mathrm{O}_{2}\left(\mathrm{M}+\mathrm{H}^{+}\right) 255.0900$, found 255.0893 .

6-Bromo-1-methoxybenzimidazole (134). White solid; mp $=46-47{ }^{\circ} \mathrm{C} ;{ }^{1} \mathrm{H}$ NMR $\left(400 \mathrm{MHz}, \mathrm{CDCl}_{3}\right) \delta$ 8.05 (s, 1H), 7.66 (d, J=2.0 Hz, 1H), 7.63 (d, J=8.8 Hz, 1H), 7.38 (dd, $J=8.4,1.6 \mathrm{~Hz}, 1 \mathrm{H}), 4.17$ (s, 3H); ${ }^{13} \mathrm{C}$ NMR $\left(100 \mathrm{MHz}, \mathrm{CDCl}_{3}\right) \delta 138.3,138.1,130.3,126.0,122.3,117.1,111.5,67.4$; IR (ATR) 1462, 1347, 1220, 1086, $952 \mathrm{~cm}^{-1}$; HRMS (ESI) calcd for $\mathrm{C}_{8} \mathrm{H}_{8} \mathrm{BrN}_{2} \mathrm{O}\left(\mathrm{M}+\mathrm{H}^{+}\right) 226.9820$, found 226.9814 .

6-Bromo-1-methoxy-2-(2-methoxy-2-propyl)benzimidazole (135). Orange oil; ${ }^{1} \mathrm{H}$ NMR (600 MHz, $\left.\mathrm{CDCl}_{3}\right) \delta 7.57$ (s, 1H), $7.56(\mathrm{~d}, J=8.4 \mathrm{~Hz}, 1 \mathrm{H}), 7.32$ (dd, J=8.4, $\left.1.8 \mathrm{~Hz}, 1 \mathrm{H}\right), 4.15$ (s, 3H), 3.15 (s, 3H), $1.74(\mathrm{~s}, 6 \mathrm{H}) ;{ }^{13} \mathrm{C}$ NMR $\left(150 \mathrm{MHz}, \mathrm{CDCl}_{3}\right) \delta 152.3,136.0,132.6,125.8,121.8,116.9,111.5,74.6,65.6$, 51.4, 24.8; IR (ATR) 2941, 1461, 1355, 1240, $1177 \mathrm{~cm}^{-1}$; HRMS (ESI) calcd for $\mathrm{C}_{12} \mathrm{H}_{16} \mathrm{BrN}_{2} \mathrm{O}_{2}\left(\mathrm{M}+\mathrm{H}^{+}\right)$ 299.0395, found 299.0390 .

6-Fluoro-1-methoxy-2-(2-methoxy-2-propyl)benzimidazole (137). Pale yellow oil; ${ }^{1} \mathrm{H}$ NMR (400 $\left.\mathrm{MHz}, \mathrm{CDCl}_{3}\right) \delta 7.62(\mathrm{dd}, J=8.8,4.8 \mathrm{~Hz}, 1 \mathrm{H}), 7.07(\mathrm{dd}, J=8.0,2.4 \mathrm{~Hz}, 1 \mathrm{H}), 6.95(\mathrm{td}, J=9.6,2.4 \mathrm{~Hz}, 1 \mathrm{H})$, 
$4.13(\mathrm{~s}, 3 \mathrm{H}), 3.14(\mathrm{~s}, 3 \mathrm{H}), 1.73(\mathrm{~s}, 6 \mathrm{H}) ;{ }^{13} \mathrm{C} \mathrm{NMR}\left(100 \mathrm{MHz}, \mathrm{CDCl}_{3}\right) \delta 159.9\left(\mathrm{~d}, J^{\mathrm{C}-\mathrm{F}}=241.0 \mathrm{~Hz}\right), 152.2(\mathrm{~d}$, $\left.J^{\mathrm{C}-\mathrm{F}}=3.0 \mathrm{~Hz}\right), 133.2,131.5\left(\mathrm{~d}, J^{\mathrm{C}-\mathrm{F}}=13.0 \mathrm{~Hz}\right), 121.4\left(\mathrm{~d}, J^{\mathrm{C}-\mathrm{F}}=10.0 \mathrm{~Hz}\right), 110.8\left(\mathrm{~d},{ }^{J \mathrm{C}-\mathrm{F}}=25.0 \mathrm{~Hz}\right), 95.0\left(\mathrm{~d}, J^{\mathrm{C}-}\right.$ $\mathrm{F}=28.0 \mathrm{~Hz}$ ), 74.4, 65.2, 51.3, 24.7; IR (ATR) 1486, 1448, 1172, 1067, 965, $811 \mathrm{~cm}^{-1}$; HRMS (ESI) calcd for $\mathrm{C}_{12} \mathrm{H}_{16} \mathrm{FN}_{2} \mathrm{O}_{2}\left(\mathrm{M}+\mathrm{H}^{+}\right)$239.1196, found 239.1191.

1-Methoxy-6-methylbenzimidazole (139). Colorless oil; ${ }^{1} \mathrm{H}$ NMR (400 MHz, $\left.\mathrm{CDCl}_{3}\right) \delta 8.02$ (br s, $\left.1 \mathrm{H}\right)$, 7.65 (d, $J=8.4 \mathrm{~Hz}, 1 \mathrm{H}), 7.29$ (s, 1H), 7.11 (dd, $J=8.0,1.2 \mathrm{~Hz}, 1 \mathrm{H}), 4.17$ (s, 3H), 2.51 (s, 3H); ${ }^{13} \mathrm{C}$ NMR $\left(100 \mathrm{MHz}, \mathrm{CDCl}_{3}\right) \delta 137.5,137.0,133.8,129.4,124.2,120.4,108.1,67.1,21.7$; IR (ATR) 2922, 1457, $1060,965,806 \mathrm{~cm}^{-1}$; HRMS (ESI) calcd for $\mathrm{C}_{9} \mathrm{H}_{11} \mathrm{~N}_{2} \mathrm{O}\left(\mathrm{M}+\mathrm{H}^{+}\right)$163.0871, found 163.0867.

1-Methoxy-2-(2-methoxy-2-propyl)-6-methylbenzimidazole (140). Colorless oil; ${ }^{1} \mathrm{H}$ NMR (600 MHz, $\left.\mathrm{CDCl}_{3}\right) \delta 7.60(\mathrm{~d}, J=8.4 \mathrm{~Hz}, 1 \mathrm{H}), 7.21(\mathrm{~d}, J=1.2 \mathrm{~Hz}, 1 \mathrm{H}), 7.06(\mathrm{dd}, J=8.4,1.2 \mathrm{~Hz}, 1 \mathrm{H}), 4.15(\mathrm{~s}, 3 \mathrm{H}), 3.15$ (s, 3H), 2.48 (s, 3H), $1.76(\mathrm{~s}, 6 \mathrm{H}) ;{ }^{13} \mathrm{C}$ NMR (150 MHz, $\left.\mathrm{CDCl}_{3}\right) \delta 150.8,135.1,133.6,131.6,123.9$, 119.9, 108.1, 74.5, 65.1, 51.3, 24.9, 21.7; IR (ATR) 2947, 1242, 1176, $810 \mathrm{~cm}^{-1}$; HRMS (ESI) calcd for $\mathrm{C}_{13} \mathrm{H}_{19} \mathrm{~N}_{2} \mathrm{O}_{2}\left(\mathrm{M}+\mathrm{H}^{+}\right)$235.1446, found 235.1441.

2-(2-Hydroxy-2-propyl)-1-methoxy-6-methylbenzimidazole (141). White solid; $\mathrm{mp}=99-100{ }^{\circ} \mathrm{C} ;{ }^{1} \mathrm{H}$ NMR (600 MHz, $\left.\mathrm{CDCl}_{3}\right) \delta 7.58(\mathrm{~d}, J=8.4 \mathrm{~Hz}, 1 \mathrm{H}), 7.22(\mathrm{~s}, 1 \mathrm{H}), 7.08(\mathrm{dd}, J=8.4,1.2 \mathrm{~Hz}, 1 \mathrm{H}), 4.21(\mathrm{~s}$, 3H), 3.44 (br s, 1H), 2.50 (s, 3H), $1.76(\mathrm{~s}, 6 \mathrm{H}) ;{ }^{13} \mathrm{C} \mathrm{NMR}\left(100 \mathrm{MHz}, \mathrm{CDCl}_{3}\right) \delta 153.8,135.0,133.5,131.4$, 124.1, 119.9, 108.3, 69.8, 65.6, 28.8, 21.8; IR (ATR) 3241, 1145, 951, $815 \mathrm{~cm}^{-1}$; HRMS (ESI) calcd for $\mathrm{C}_{12} \mathrm{H}_{17} \mathrm{~N}_{2} \mathrm{O}_{2}\left(\mathrm{M}+\mathrm{H}^{+}\right)$221.1290, found 221.1285.

Methyl 1-methoxybenzimidazole-6-carboxylate (143). White solid; mp $=126-127{ }^{\circ} \mathrm{C} ;{ }^{1} \mathrm{H}$ NMR $(600$ $\left.\mathrm{MHz}, \mathrm{CDCl}_{3}\right) \delta 8.23$ (br s, $\left.1 \mathrm{H}\right), 8.19(\mathrm{~s}, 1 \mathrm{H}), 7.99$ (dd, J=8.4, $\left.1.2 \mathrm{~Hz}, 1 \mathrm{H}\right), 7.78$ (d, J=8.4 Hz, 1H), 4.22 (s, $3 \mathrm{H}), 3.95(\mathrm{~s}, 3 \mathrm{H}) ;{ }^{13} \mathrm{C}$ NMR $\left(150 \mathrm{MHz}, \mathrm{CDCl}_{3}\right) \delta 167.0,142.6,140.0,129.0,125.6,123.9,120.7,110.9$, 67.7, 52.2; IR (ATR) 1698, 1441, 1320, 1280, $1226 \mathrm{~cm}^{-1}$; HRMS (ESI) calcd for $\mathrm{C}_{10} \mathrm{H}_{11} \mathrm{~N}_{2} \mathrm{O}_{3}\left(\mathrm{M}+\mathrm{H}^{+}\right)$ 207.0769, found 207.0763.

1,5-Dimethoxybenzimidazole (146). Colorless oil; ${ }^{1} \mathrm{H}$ NMR $\left(600 \mathrm{MHz}, \mathrm{CDCl}_{3}\right) \delta 8.01(\mathrm{~s}, 1 \mathrm{H}), 7.35(\mathrm{~d}$, $J=8.4 \mathrm{~Hz}, 1 \mathrm{H}), 7.23(\mathrm{~d}, J=2.4 \mathrm{~Hz}, 1 \mathrm{H}), 6.98(\mathrm{dd}, J=8.4,2.4 \mathrm{~Hz}, 1 \mathrm{H}), 4.15$ (s, 3H), $3.85(\mathrm{~s}, 3 \mathrm{H}) ;{ }^{13} \mathrm{C}$ NMR $\left(150 \mathrm{MHz}, \mathrm{CDCl}_{3}\right) \delta 156.4,140.3,137.4,123.9,114.1,108.8,102.7,67.2,55.7$; IR (ATR) 1213, 1125, $969,803 \mathrm{~cm}^{-1}$; HRMS (ESI) calcd for $\mathrm{C}_{9} \mathrm{H}_{11} \mathrm{~N}_{2} \mathrm{O}_{2}\left(\mathrm{M}+\mathrm{H}^{+}\right)$179.0820, found 179.0820. 
5-Chloro-1-methoxybenzimidazole (148). Yellow oil; ${ }^{1} \mathrm{H}$ NMR $\left(600 \mathrm{MHz}, \mathrm{CDCl}_{3}\right) \delta 8.09$ (s, 1H), 7.75 $(\mathrm{d}, J=1.8 \mathrm{~Hz}, 1 \mathrm{H}), 7.39$ (d, J=8.4 Hz, 1H), 7.29 (dd, J=9.0, $1.8 \mathrm{~Hz}, 1 \mathrm{H}), 4.16(\mathrm{~s}, 3 \mathrm{H}) ;{ }^{13} \mathrm{C}$ NMR $(150$ $\left.\mathrm{MHz}, \mathrm{CDCl}_{3}\right) \delta 140.0,138.5,128.1,127.8,124.1,120.5,109.1,67.3$; IR (ATR) 1457, 1309, 1054, 965, $898 \mathrm{~cm}^{-1}$; HRMS (ESI) calcd for $\mathrm{C}_{8} \mathrm{H}_{7} \mathrm{ClN}_{2} \mathrm{O}\left(\mathrm{M}+\mathrm{Na}^{+}\right)$205.0144, found 205.0139.

5-Chloro-1-methoxy-2-(2-methoxy-2-propyl)benzimidazole (149). Yellow oil; ${ }^{1} \mathrm{H}$ NMR (600 MHz, $\left.\mathrm{CDCl}_{3}\right) \delta 7.71(\mathrm{~d}, J=1.2 \mathrm{~Hz}, 1 \mathrm{H}), 7.35(\mathrm{~d}, J=8.4 \mathrm{~Hz}, 1 \mathrm{H}), 7.28(\mathrm{dd}, J=8.4,1.8 \mathrm{~Hz}, 1 \mathrm{H}), 4.18$ (s, 3H), 3.18 $(\mathrm{s}, 3 \mathrm{H}), 1.77(\mathrm{~s}, 6 \mathrm{H}) ;{ }^{13} \mathrm{C} \mathrm{NMR}\left(100 \mathrm{MHz}, \mathrm{CDCl}_{3}\right) \delta 152.7,137.8,130.2,128.0,124.0,120.3,109.2,74.6$, 65.5, 51.4, 24.8; IR (ATR) 2938, 1700, 1608, 1575, 1493, $1245 \mathrm{~cm}^{-1}$; HRMS (ESI) calcd for $\mathrm{C}_{12} \mathrm{H}_{16} \mathrm{ClN}_{2} \mathrm{O}_{2}\left(\mathrm{M}+\mathrm{H}^{+}\right)$255.0900, found 255.0901.

5-Bromo-1-methoxybenzimidazole (151). Faint yellow oil; ${ }^{1} \mathrm{H} \mathrm{NMR}\left(400 \mathrm{MHz}, \mathrm{CDCl}_{3}\right) \delta 8.05(\mathrm{~s}, 1 \mathrm{H})$, $7.91(\mathrm{~d}, J=1.6 \mathrm{~Hz}, 1 \mathrm{H}), 7.43(\mathrm{dd}, J=8.8,2.0 \mathrm{~Hz}, 1 \mathrm{H}), 7.35$ (d, $J=8.8 \mathrm{~Hz}, 1 \mathrm{H}), 4.16(\mathrm{~s}, 3 \mathrm{H}) ;{ }^{13} \mathrm{C}$ NMR $(100$ $\left.\mathrm{MHz}, \mathrm{CDCl}_{3}\right) \delta 140.6,138.4,128.2,126.8,123.7,115.6,109.7,67.4$; IR (ATR) 1454, 1308, 1175, 1066, $729 \mathrm{~cm}^{-1}$; HRMS (ESI) calcd for $\mathrm{C}_{8} \mathrm{H}_{8} \mathrm{BrN}_{2} \mathrm{O}\left(\mathrm{M}+\mathrm{H}^{+}\right)$226.9820, found 226.9815 .

5-Bromo-1-methoxy-2-(2-methoxy-2-propyl)benzimidazole (152). Red oil; ${ }^{1} \mathrm{H}$ NMR (400 MHz, $\left.\mathrm{CDCl}_{3}\right) \delta 7.87(\mathrm{~d}, J=1.6 \mathrm{~Hz}, 1 \mathrm{H}), 7.41(\mathrm{dd}, J=8.4,1.6 \mathrm{~Hz}, 1 \mathrm{H}), 7.31$ (d, $\left.J=8.8 \mathrm{~Hz}, 1 \mathrm{H}\right), 4.17$ (s, 3H), 3.17 (s, 3H), 1.77 (s, 6H); ${ }^{13} \mathrm{C}$ NMR (100 MHz, $\left.\mathrm{CDCl}_{3}\right) \delta 152.6,138.3,130.5,126.6,123.4,115.2$, 109.6, 74.6, 65.5, 51.4, 24.8; IR (ATR) 1454, 1308, 1246, 1176, $1066 \mathrm{~cm}^{-1}$; HRMS (ESI) calcd for $\mathrm{C}_{12} \mathrm{H}_{16} \mathrm{BrN}_{2} \mathrm{O}_{2}$ $\left(\mathrm{M}+\mathrm{H}^{+}\right)$299.0395, found 299.0376.

1-Methoxy-5-methylbenzimidazole (154). Orange oil; ${ }^{1} \mathrm{H}$ NMR (600 MHz, $\left.\mathrm{CDCl}_{3}\right) \delta 8.04$ (s, 1H), 7.56 (s, 1H), 7.37 (d, J=8.4 Hz, 1H), 7.17 (d, J=7.8 Hz, 1H), 4.17 (s, 3H), 2.48 (s, 3H); ${ }^{13} \mathrm{C}$ NMR (150 MHz, $\left.\mathrm{CDCl}_{3}\right) \delta 139.9,137.4,132.3,127.4,125.2,120.6,107.9,21.5$; IR (ATR) 2931, 1686, 1607, 1523, 1341, $1077 \mathrm{~cm}^{-1}$; HRMS (ESI) calcd for $\mathrm{C}_{9} \mathrm{H}_{11} \mathrm{~N}_{2} \mathrm{O}\left(\mathrm{M}+\mathrm{H}^{+}\right)$163.0871, found 163.0866.

1-Methoxy-5-methyl-(2-methoxy-2-propyl)benzimidazole (155). Orange oil; ${ }^{1} \mathrm{H}$ NMR (400 MHz, $\left.\mathrm{CDCl}_{3}\right) \delta 7.53($ br s, $1 \mathrm{H}), 7.32(\mathrm{~d}, J=8.0 \mathrm{~Hz}, 1 \mathrm{H}), 7.14(\mathrm{dd}, J=8.0,0.8 \mathrm{~Hz}, 1 \mathrm{H}), 4.17$ (s, 3H), 3.17 (s, 3H), $2.47(\mathrm{~s}, 3 \mathrm{H}), 1.78(\mathrm{~s}, 6 \mathrm{H}) ;{ }^{13} \mathrm{C} \mathrm{NMR}\left(100 \mathrm{MHz}, \mathrm{CDCl}_{3}\right) \delta 151.2,137.4,132.0,129.6,124.9,120.2,107.9$, 74.6, 65.2, 51.4, 25.0, 21.5; IR (ATR) 2939, 1455, 1314, 1248, $1067 \mathrm{~cm}^{-1}$; HRMS (ESI) calcd for $\mathrm{C}_{13} \mathrm{H}_{19} \mathrm{~N}_{2} \mathrm{O}_{2}\left(\mathrm{M}+\mathrm{H}^{+}\right)$235.1446, found 235.1439. 
1-Methoxy-(2-hydroxy-2-propyl)-5-methylbenzimidazole (156). White solid; mp=99-100 ${ }^{\circ} \mathrm{C}$; ${ }^{1} \mathrm{H}$ NMR $\left(400 \mathrm{MHz}, \mathrm{CDCl}_{3}\right) \delta 7.50$ (br s, 1H), 7.31 (d, J=8.0 Hz, 1H), 7.14 (dd, J=8.4, $\left.1.2 \mathrm{~Hz}, 1 \mathrm{H}\right), 4.20$ (s, 3H), 3.45 (br s, 1H), 2.47 (s, 3H), 1.76 (s, 3H); $\left.{ }^{13} \mathrm{C} \mathrm{NMR} \mathrm{(100} \mathrm{MHz,} \mathrm{CDCl}_{3}\right) \delta$ 154.2, 137.3, 132.3, 129.3, 124.7, 120.1, 108.0, 69.7, 65.6, 28.7, 21.5; IR (ATR) 3242, 1372, 1309, 1236, $1145 \mathrm{~cm}^{-1}$; HRMS (ESI) calcd for $\mathrm{C}_{12} \mathrm{H}_{17} \mathrm{~N}_{2} \mathrm{O}_{2}\left(\mathrm{M}+\mathrm{H}^{+}\right)$221.1290, found 221.1285.

6-Bromo-1-methoxy-(1-methoxy-1-phenylethyl)benzimidazole (161). Orange oil; ${ }^{1} \mathrm{H}$ NMR (400 MHz, $\left.\mathrm{CDCl}_{3}\right) \delta 7.67(\mathrm{dd}, J=8.8,0.4 \mathrm{~Hz}, 1 \mathrm{H}), 7.51(\mathrm{~d}, J=1.6 \mathrm{~Hz}, 1 \mathrm{H}), 7.38(\mathrm{dd}, J=8.8,1.6 \mathrm{~Hz}, 1 \mathrm{H}), 7.42-7.40$ $(\mathrm{m}, 2 \mathrm{H}), 7.35-7.24(\mathrm{~m}, 3 \mathrm{H}), 3.53(\mathrm{~s}, 3 \mathrm{H}), 3.29(\mathrm{~s}, 3 \mathrm{H}), 2.04(\mathrm{~s}, 3 \mathrm{H}) ;{ }^{13} \mathrm{C} \mathrm{NMR}\left(100 \mathrm{MHz}, \mathrm{CDCl}_{3}\right) \delta 152.1$, 144.0, 136.1, 128.2, 127.4, 125.9, 125.2, 122.0, 117.0, 111.6, 110.0, 78.3, 64.8, 51.4, 25.0; IR (ATR) 1437, 1373, 1245, 1185, 981, $792 \mathrm{~cm}^{-1}$; HRMS (ESI) calcd for $\mathrm{C}_{17} \mathrm{H}_{18} \mathrm{~N}_{2} \mathrm{O}_{2}\left(\mathrm{M}+\mathrm{H}^{+}\right) 361.0551$, found 361.0549 .

6-Bromo-(1-hydroxy-1-phenylethyl)-1-methoxybenzimidazole (162). Colorless oil; ${ }^{1} \mathrm{H}$ NMR (400 $\left.\mathrm{MHz}, \mathrm{CDCl}_{3}\right) \delta 7.65(\mathrm{~d}, J=8.4 \mathrm{~Hz}, 1 \mathrm{H}), 7.50(\mathrm{~d}, J=1.6 \mathrm{~Hz}, 1 \mathrm{H}), 7.48-7.46(\mathrm{~m}, 2 \mathrm{H}), 7.39$ (dd, $J=8.4,1.6$ $\mathrm{Hz}, 1 \mathrm{H}), 7.38-7.27$ (m, 3H), 3.90 (br s, $1 \mathrm{H}), 3.39$ (s, 3H), 2.08 (s, 3H); ${ }^{13} \mathrm{C} \mathrm{NMR}\left(100 \mathrm{MHz}, \mathrm{CDCl}_{3}\right) \delta$ 154.2, 144.4, 136.0, 132.0, 128.6, 127.9, 126.1, 125.2, 122.0, 116.9, 111.5, 73.4, 65.0, 28.6; IR (ATR) 3233, 1449, 1364, 1251, $1089 \mathrm{~cm}^{-1}$; HRMS (ESI) calcd for $\mathrm{C}_{16} \mathrm{H}_{16} \mathrm{~N}_{2} \mathrm{O}_{2}\left(\mathrm{M}+\mathrm{H}^{+}\right)$347.0395, found 347.0395 .

1,6-Dimethoxy-2-(1-methoxycyclohexyl)benzimidazole (158). Colorless oil; ${ }^{1} \mathrm{H}$ NMR (400 MHz, $\left.\mathrm{CDCl}_{3}\right) \delta 7.62(\mathrm{~d}, J=9.6 \mathrm{~Hz}, 1 \mathrm{H}), 6.88(\mathrm{dd}, J=9.6,2.4 \mathrm{~Hz}, 1 \mathrm{H}), 6.87(\mathrm{~s}, 1 \mathrm{H}), 4.16(\mathrm{~s}, 3 \mathrm{H}), 3.89$ (s, 3H), 3.10 (s, 3H), 2.34-2.31 (m, 2H), 2.16-2.10 (m, 2H), 1.76-1.64 (m, 2H), 1.62-1.56 (m, 3H), 1.42-1.35 (m, $1 \mathrm{H}) ;{ }^{13} \mathrm{C} \mathrm{NMR}\left(150 \mathrm{MHz}, \mathrm{CDCl}_{3}\right) \delta 157.0,149.9,131.8,131.4,120.9,111.7,91.3$, 75.8, 64.9, 55.7, 50.4, 32.5, 25.5, 21.7; IR (ATR) 2944, 1739, 1450, 1365, $1207 \mathrm{~cm}^{-1}$; HRMS (ESI) calcd for $\mathrm{C}_{16} \mathrm{H}_{23} \mathrm{~N}_{2} \mathrm{O}_{3}$ $\left(\mathrm{M}+\mathrm{H}^{+}\right)$291.1708, found 291.1701.

1,6-Dimethoxy-2-(1-hydroxycyclohexyl)benzimidazole (159). White solid; mp=132-133 ${ }^{\circ} \mathrm{C} ;{ }^{1} \mathrm{H}$ NMR $\left(400 \mathrm{MHz}, \mathrm{CDCl}_{3}\right) \delta 7.58(\mathrm{dd}, J=8.4,1.2 \mathrm{~Hz}, 1 \mathrm{H}), 6.87$ (dd, J=8.4, $2.4 \mathrm{~Hz}, 1 \mathrm{H}, 6.86(\mathrm{~s}, 1 \mathrm{H}), 4.19$ (s, 3H), 3.88 (s, 3H), 2.87 (br s, 1H), 2.17 (td, J=12.8, 4.0 Hz, 2H), 2.02-1.99 (m, 2H), 1.85-1.77 (m, 2H), 1.72$1.62(\mathrm{~m}, 3 \mathrm{H}), 1.42-1.33(\mathrm{~m}, 1 \mathrm{H}) ;{ }^{13} \mathrm{C} \mathrm{NMR}\left(100 \mathrm{MHz}, \mathrm{CDCl}_{3}\right) \delta 157.0,153.2,131.7,131.5,121.0,111.7$, 91.8, 71.3, 65.5, 55.9, 36.1, 25.3, 21.6; IR (ATR) 3281, 2940, 1504, 1451, 1435, $1237 \mathrm{~cm}^{-1}$; HRMS (ESI) calcd for $\mathrm{C}_{15} \mathrm{H}_{21} \mathrm{~N}_{2} \mathrm{O}_{3}\left(\mathrm{M}+\mathrm{H}^{+}\right)$277.1552; found: 277.1547 . 
6-Chloro-2-(2-hydroxy-2-propyl)-1-methoxybenzimidazole (163). White solid; mp=94-95 ${ }^{\circ} \mathrm{C}$; ${ }^{1} \mathrm{H}$ NMR (400 MHz, $\left.\mathrm{CDCl}_{3}\right) \delta 7.61(\mathrm{~d}, J=8.8 \mathrm{~Hz}, 1 \mathrm{H}), 7.43(\mathrm{~d}, J=2.0 \mathrm{~Hz}, 1 \mathrm{H}), 7.23(\mathrm{dd}, J=8.8,2.0 \mathrm{~Hz}, 1 \mathrm{H})$, 4.22 (s, 3H), 3.19 (br s, 1H), 1.77 (s, 6H); $\left.{ }^{13} \mathrm{C} \mathrm{NMR} \mathrm{(100} \mathrm{MHz,} \mathrm{CDCl}_{3}\right) \delta$ 155.1, 135.6, 131.8, 129.2, 123.3, 121.3, 108.6, 69.9, 66.0, 28.7; IR (ATR) 3286, 1457, 1309, 1054, $965 \mathrm{~cm}^{-1}$; HRMS (ESI) calcd for $\mathrm{C}_{11} \mathrm{H}_{14} \mathrm{ClN}_{2} \mathrm{O}_{2}\left(\mathrm{M}+\mathrm{H}^{+}\right)$241.0743, found 241.0739.

6-Bromo-2-(2-hydroxy-2-propyl)-1-methoxybenzimidazole (164). White solid; $\mathrm{mp}=118-119{ }^{\circ} \mathrm{C} ;{ }^{1} \mathrm{H}$ NMR (400 MHz, $\left.\mathrm{CDCl}_{3}\right) \delta 7.58(\mathrm{~s}, 1 \mathrm{H}), 7.56(\mathrm{~d}, J=8.4 \mathrm{~Hz}, 1 \mathrm{H}), 7.36(\mathrm{dd}, J=8.4,0.8 \mathrm{~Hz}, 1 \mathrm{H}), 4.21$ (s, $3 \mathrm{H}), 3.36$ (br s, 1H), 1.76 (s, 6H); ${ }^{13} \mathrm{C} \mathrm{NMR}\left(100 \mathrm{MHz}, \mathrm{CDCl}_{3}\right) \delta 155.0,135.9,132.2,126.0,121.7$, 116.6, 111.5, 69.8, 66.0, 28.7; IR (ATR) 3332, 1458, 1269, 1104, $729 \mathrm{~cm}^{-1}$; HRMS (ESI) calcd for $\mathrm{C}_{11} \mathrm{H}_{14} \mathrm{BrN}_{2} \mathrm{O}_{2}\left(\mathrm{M}+\mathrm{H}^{+}\right)$285.0238, found 285.0233.

6-Fluoro-2-(2-hydroxy-2-propyl)-1-methoxybenzimidazole (165). White solid; mp=123-124 ${ }^{\circ} \mathrm{C} ;{ }^{1} \mathrm{H}$ NMR (400 MHz, $\left.\mathrm{CDCl}_{3}\right) \delta 7.61(\mathrm{dd}, J=9.2,4.8 \mathrm{~Hz}, 1 \mathrm{H}), 7.10(\mathrm{dd}, J=8.0,2.4 \mathrm{~Hz}, 1 \mathrm{H}), 6.99$ (ddd, $J=9.6$, 8.8, $2.4 \mathrm{~Hz}, 1 \mathrm{H}), 4.19$ (s, 3H), 3.46 (br s, $1 \mathrm{H}), 1.75(\mathrm{~s}, 6 \mathrm{H}) ;{ }^{13} \mathrm{C} \mathrm{NMR}\left(100 \mathrm{MHz}, \mathrm{CDCl}_{3}\right) \delta 159.8\left(\mathrm{~d}, J^{\mathrm{C}-}\right.$ F $=241.0 \mathrm{~Hz}), 155.0\left(\mathrm{~d}, J^{\mathrm{C}-\mathrm{F}}=3.0 \mathrm{~Hz}\right), 133.2,131.2\left(\mathrm{~d}, J^{\mathrm{C}-\mathrm{F}}=14.0 \mathrm{~Hz}\right), 121.3\left(\mathrm{~d}, J^{\mathrm{C}-\mathrm{F}}=10.0 \mathrm{~Hz}\right), 111.0\left(\mathrm{~d}, J^{\mathrm{C}-}\right.$ F $=25.0 \mathrm{~Hz}), 95.3\left(\mathrm{~d}, J^{\mathrm{C}-\mathrm{F}}=28.0 \mathrm{~Hz}\right), 69.8,65.7,28.7$; IR (ATR) 3232, 1488, 1438, 1363, 1172, 960, 833 $\mathrm{cm}^{-1}$; HRMS (ESI) calcd for $\mathrm{C}_{11} \mathrm{H}_{14} \mathrm{FN}_{2} \mathrm{O}_{2}\left(\mathrm{M}+\mathrm{H}^{+}\right)$225.1039, found 225.1035.

5-Chloro-2-(2-hydroxy-2-propyl)-1-methoxybenzimidazole (166). Yellow oil; ${ }^{1} \mathrm{H}$ NMR (400 MHz, $\left.\mathrm{CDCl}_{3}\right) \delta 7.67(\mathrm{~d}, J=2.0 \mathrm{~Hz}, 1 \mathrm{H}), 7.34(\mathrm{~d}, J=8.8 \mathrm{~Hz}, 1 \mathrm{H}), 7.26(\mathrm{dd}, J=8.8,2.0 \mathrm{~Hz}, 1 \mathrm{H}), 4.21$ (s, 3H), 3.71 (br s, 1H), $1.76(\mathrm{~s}, 6 \mathrm{H}) ;{ }^{13} \mathrm{C}$ NMR $\left(100 \mathrm{MHz}, \mathrm{CDCl}_{3}\right) \delta 155.4,1376,129.8,128.1,123.7,120.1,109.2$, 69.8, 65.9, 28.6; IR (ATR) $\mathrm{cm}^{-1}$; HRMS (ESI) calcd for $\mathrm{C}_{11} \mathrm{H}_{14} \mathrm{ClN}_{2} \mathrm{O}_{2}\left(\mathrm{M}+\mathrm{H}^{+}\right)$241.0744, found 241.0727.

5-Bromo-2-(2-hydroxy-2-propyl)-1-methoxybenzimidazole (167). White solid; $\mathrm{mp}=109-110{ }^{\circ} \mathrm{C} ;{ }^{1} \mathrm{H}$ NMR (400 MHz, $\left.\mathrm{CDCl}_{3}\right) \delta 7.83(\mathrm{~s}, 1 \mathrm{H}), 7.40(\mathrm{dd}, J=8.8,1.6 \mathrm{~Hz}, 1 \mathrm{H}), 7.29(\mathrm{~d}, J=8.8 \mathrm{~Hz}, 1 \mathrm{H}), 4.20(\mathrm{~s}$, 3H), 3.49 (br s, 1H), 1.75 (s, 6H); ${ }^{13} \mathrm{C}$ NMR (100 MHz, $\left.\mathrm{CDCl}_{3}\right) \delta 155.3,138.1,130.1,126.4,123.2$, 115.4, 109.7, 69.8, 66.0, 28.7; IR (ATR) 3257, 1455, 1309, 1175, 918, 731 $\mathrm{cm}^{-1}$; HRMS (ESI) calcd for $\mathrm{C}_{11} \mathrm{H}_{14} \mathrm{BrN}_{2} \mathrm{O}_{2}\left(\mathrm{M}+\mathrm{H}^{+}\right)$285.0239, found 285.0233.

1,6-Dimethoxy-2-(1-hydroxy-1,1-diphenylmethyl)benzimidazole (169). Red viscous oil; ${ }^{1} \mathrm{H}$ NMR (600 $\left.\mathrm{MHz}, \mathrm{CDCl}_{3}\right) \delta$ 7.24-7.21 (m, 2H), 7.19-7.16 (m, 1H), 7.15 (d, J=1.8 Hz, 1H), 7.14-7.13 (m, 1H), 7.09- 
$7.02(\mathrm{~m}, 6 \mathrm{H}), 7.01(\mathrm{~d}, J=9.0 \mathrm{~Hz}, 1 \mathrm{H}), 6.81(\mathrm{dd}, J=9.0,3.0 \mathrm{~Hz}, 1 \mathrm{H}), 6.33$ (br s, 1H), $3.72(\mathrm{~s}, 3 \mathrm{H}), 3.12$ (s, $3 \mathrm{H}) ;{ }^{13} \mathrm{C}$ NMR $\left(100 \mathrm{MHz}, \mathrm{CDCl}_{3}\right) \delta 154.8,142.9,142.6,138.7,135.9,134.1,130.4,128.0,127.6,127.3$, 127.0, 126.3, 126.0, 122.6, 120.5, 108.9, 55.9, 42.7; IR (ATR) 2835, 1529, 1493, 1228, $1040 \mathrm{~cm}^{-1}$; HRMS (ESI) calcd for $\mathrm{C}_{22} \mathrm{H}_{21} \mathrm{~N}_{2} \mathrm{O}_{3}\left(\mathrm{M}+\mathrm{H}^{+}\right)$361.1552, found 361.1548 .

1-Benzyloxy-2-(2-benzyloxy-2-propyl)-6-methoxybenzimidazole (171). Orange oil; ${ }^{1} \mathrm{H}$ NMR (400 $\left.\mathrm{MHz}, \mathrm{CDCl}_{3}\right) \delta 7.64(\mathrm{~d}, J=8.8 \mathrm{~Hz}, 1 \mathrm{H}), 7.38-7.20(\mathrm{~m}, 10 \mathrm{H}), 6.87(\mathrm{dd}, J=8.8,2.4 \mathrm{~Hz}, 1 \mathrm{H}), 6.66(\mathrm{~d}, J=2.8$ $\mathrm{Hz}, 1 \mathrm{H}), 5.18$ (s, 2H), 4.48 (s, 2H), 3.78 (s, 3H), 1.93 (s, 6H); ${ }^{13} \mathrm{C}$ NMR (100 MHz, $\left.\mathrm{CDCl}_{3}\right) \delta 157.1$, 150.9, 138.4, 134.2, 132.9, 131.2, 129.3, 129.1, 128.6, 128.2, 127.3, 126.9, 121.0, 1119, 92.0, 79.8, 74.7, 65.6, 55.7, 25.5; IR (ATR) 2938, 1625, 1490, 1454, 1381, $1306 \mathrm{~cm}^{-1}$; HRMS (ESI) calcd for $\mathrm{C}_{25} \mathrm{H}_{27} \mathrm{~N}_{2} \mathrm{O}_{3}$ $\left(\mathrm{M}+\mathrm{H}^{+}\right)$403.2022, found 403.2014.

1-Benzyloxy-2-(2-hydroxyl-2-propyl)-6-methoxybenzimidazole (172). White solid; $\mathrm{mp}=118-119{ }^{\circ} \mathrm{C}$; ${ }^{1} \mathrm{H}$ NMR (400 MHz, $\left.\mathrm{CDCl}_{3}\right) \delta 7.57(\mathrm{~d}, J=8.8 \mathrm{~Hz}, 1 \mathrm{H}), 7.53-7.50(\mathrm{~m}, 2 \mathrm{H}), 7.45-7.42(\mathrm{~m}, 3 \mathrm{H}), 6.85(\mathrm{dd}$, $J=8.8,2.4 \mathrm{~Hz}, 1 \mathrm{H}), 6.69$ (d, J=2.4 Hz, 1H), 5.36 (s, 2H), 3.79 (s, 3H), 3.46 (br s, 1H), 1.80 (s, 6H); ${ }^{13} \mathrm{C}$ NMR $\left(100 \mathrm{MHz}, \mathrm{CDCl}_{3}\right) \delta 156.9,153.5,133.9,132.4,131.2,129.5,129.4,128.9,120.8,111.7,92.2$, 80.3, 69.9, 55.7, 28.9; IR (ATR) 3240, 1627, 1491, 1452, 1250, $1213 \mathrm{~cm}^{-1}$; HRMS (ESI) calcd for $\mathrm{C}_{18} \mathrm{H}_{21} \mathrm{~N}_{2} \mathrm{O}_{3}\left(\mathrm{M}+\mathrm{H}^{+}\right)$313.1552, found 313.1547.

1-(2-Propen-1-yloxy)-6-methoxybenzimidazole (173). Pale yellow oil; ${ }^{1} \mathrm{H}$ NMR (400 $\left.\mathrm{MHz}, \mathrm{CDCl}_{3}\right) \delta$ 7.88 (s, 1H), 7.61 (dd, J=9.6, $1.2 \mathrm{~Hz}, 1 \mathrm{H}), 6.90-6.87$ (m, 2H), 6.07 (ddt, J=17.2, 10.4, $6.8 \mathrm{~Hz}, 1 \mathrm{H}), 5.33$ (dq, $J=17.2,1.2 \mathrm{~Hz}, 1 \mathrm{H}), 4.71(\mathrm{dt}, J=6.4,1.2 \mathrm{~Hz}, 1 \mathrm{H}), 3.85(\mathrm{~s}, 3 \mathrm{H}) ;{ }^{13} \mathrm{C} \mathrm{NMR}\left(100 \mathrm{MHz}, \mathrm{CDCl}_{3}\right) \delta 157.2$, 137.6, 133.7, 130.5, 130.3, 123.1, 121.3, 112.1, 91.6, 79.7, 55.7; IR (ATR) 1624, 1495, 1224, 1061, 932, $815 \mathrm{~cm}^{-1}$; HRMS (ESI) calcd for $\mathrm{C}_{11} \mathrm{H}_{13} \mathrm{~N}_{2} \mathrm{O}_{2}\left(\mathrm{M}+\mathrm{H}^{+}\right)$205.0977, found 205.0961.

1-(2-Propen-1-yloxy)-2-(2-(2-propen-1-yloxy)-2-propyl)-6-methoxybenzimidazole (174). Yellow oil; ${ }^{1} \mathrm{H}$ NMR $\left(400 \mathrm{MHz}, \mathrm{CDCl}_{3}\right) \delta 7.60(\mathrm{dd}, J=8.8,0.8 \mathrm{~Hz}, 1 \mathrm{H}), 6.87(\mathrm{dd}, J=8.4,2.4 \mathrm{~Hz}, 1 \mathrm{H}), 6.85(\mathrm{~d}, J=2.0$ $\mathrm{Hz}, 1 \mathrm{H}), 6.15$ (ddt, $J=16.8,10.4,6.4,1 \mathrm{H}), 5.87$ (ddt, $J=17.2,10.4,5.2 \mathrm{~Hz}, 1 \mathrm{H}), 5.50$ (dq, J=17.2, $1.2 \mathrm{~Hz}$, $1 \mathrm{H}), 5.41(\mathrm{dd}, J=17.2,1.2 \mathrm{~Hz}, 1 \mathrm{H}), 5.23$ (dq, $J=17.2,1.6 \mathrm{~Hz}, 1 \mathrm{H}), 5.10$ (dq, $J=10.4,1.6 \mathrm{~Hz}, 1 \mathrm{H}), 4.84$ (dt, $J=6.4,1.2 \mathrm{~Hz}, 2 \mathrm{H}), 3.87(\mathrm{~s}, 3 \mathrm{H}), 3.85(\mathrm{dt}, J=5.2,1.6 \mathrm{~Hz}, 2 \mathrm{H}), 1.82(\mathrm{~s}, 6 \mathrm{H}) ;{ }^{13} \mathrm{C}$ NMR $\left(100 \mathrm{MHz}, \mathrm{CDCl}_{3}\right)$ $\delta 157.1,150.7,134.8,132.7,131.1,130.9,121.0,120.9,116.1,111.7,91.9,78.7,74.2,64.5,55.7,25.3$; IR (ATR) 2939, 1626, 1490, 1212, 1158, $1026 \mathrm{~cm}^{-1}$; HRMS (ESI) calcd for $\mathrm{C}_{17} \mathrm{H}_{23} \mathrm{~N}_{2} \mathrm{O}_{3}\left(\mathrm{M}+\mathrm{H}^{+}\right)$ 303.1708, found 303.1702. 
2-(2-Hydroxy-2-propyl)-6-methoxy-1-(2-propen-1-yloxy)benzimidazole (175). White solid; mp=110$111{ }^{\circ} \mathrm{C} ;{ }^{1} \mathrm{H}$ NMR $\left(400 \mathrm{MHz}, \mathrm{CDCl}_{3}\right) \delta 7.56(\mathrm{dd}, J=8.4,0.8 \mathrm{~Hz}, 1 \mathrm{H}), 6.87(\mathrm{dd}, J=8.8,2.4 \mathrm{~Hz}, 1 \mathrm{H}), 6.84(\mathrm{~d}$, $J=2.0 \mathrm{~Hz}, 1 \mathrm{H}), 6.13$ (ddt, $J=16.8,10.4,6.4 \mathrm{~Hz}, 1 \mathrm{H}), 5.52$ (dq, $J=17.2,1.2 \mathrm{~Hz}, 1 \mathrm{H}), 5.44$ (dq, $J=10.8,1.2$ $\mathrm{Hz}, 1 \mathrm{H}), 4.86(\mathrm{~d}, J=6.4 \mathrm{~Hz}, 2 \mathrm{H}), 3.86(\mathrm{~s}, 3 \mathrm{H}), 3.48$ (br s, $1 \mathrm{H}), 1.75(\mathrm{~s}, 6 \mathrm{H}) ;{ }^{13} \mathrm{C} \mathrm{NMR}\left(100 \mathrm{MHz}, \mathrm{CDCl}_{3}\right)$ $\delta 156.9,153.6,132.3,131.2,130.4,121.5,120.9,111.7,92.3,79.0,69.7,55.8,28.8$; IR (ATR) 3208, 2991, 1490, 1252, 1252, $1176 \mathrm{~cm}^{-1}$; HRMS (ESI) calcd for $\mathrm{C}_{14} \mathrm{H}_{19} \mathrm{~N}_{2} \mathrm{O}_{3}\left(\mathrm{M}+\mathrm{H}^{+}\right)$263.1396, found 263.1390 .

8-Methoxy-4,4-dimethyl-4H-benzo[4,5]imidazo[1,2-b]dioxazine (176). White solid; $\mathrm{mp}=121-122{ }^{\circ} \mathrm{C}$; ${ }^{1} \mathrm{H}$ NMR $\left(400 \mathrm{MHz}, \mathrm{CDCl}_{3}\right) \delta 7.55(\mathrm{dd}, J=8.8,1.2 \mathrm{~Hz}, 1 \mathrm{H}), 6.88(\mathrm{dd}, J=8.4,2.4 \mathrm{~Hz}, 1 \mathrm{H}), 6.86(\mathrm{~d}, J=2.4$ $\mathrm{Hz}, 1 \mathrm{H}), 5.44(\mathrm{~s}, 2 \mathrm{H}), 3.83(\mathrm{~s}, 3 \mathrm{H}), 1.76(\mathrm{~s}, 6 \mathrm{H}) ;{ }^{13} \mathrm{C} \mathrm{NMR}\left(100 \mathrm{MHz}, \mathrm{CDCl}_{3}\right) \delta 156.6,146.3,131.6$, 129.0, 120.4, 112.6, 91.3, 91.2, 75.8, 55.8, 27.4; IR (ATR) 1523, 1451, 1234, 1209, $1148 \mathrm{~cm}^{-1}$; HRMS (ESI) calcd for $\mathrm{C}_{12} \mathrm{H}_{15} \mathrm{~N}_{2} \mathrm{O}_{3}\left(\mathrm{M}+\mathrm{H}^{+}\right)$235.1082, found 235.1077 .

6-Methoxy-1-(2-propyn-1-yloxy)benzimidazole (177). Pale yellow oil; ${ }^{1} \mathrm{H}$ NMR $\left(400 \mathrm{MHz}, \mathrm{CDCl}_{3}\right) \delta$ $8.03(\mathrm{~s}, 1 \mathrm{H}), 7.62(\mathrm{~d}, J=8.8 \mathrm{~Hz}, 1 \mathrm{H}), 6.92(\mathrm{~d}, J=2.0 \mathrm{~Hz}, 1 \mathrm{H}), 6.90(\mathrm{dd}, J=8.8,2.4 \mathrm{~Hz}, 1 \mathrm{H}), 4.86(\mathrm{~d}, J=2.4$ $\mathrm{Hz}, 2 \mathrm{H}), 3.85(\mathrm{~s}, 3 \mathrm{H}), 2.68(\mathrm{t}, J=2.4 \mathrm{~Hz}, 1 \mathrm{H}) ;{ }^{13} \mathrm{C} \mathrm{NMR}\left(100 \mathrm{MHz}, \mathrm{CDCl}_{3}\right) \delta 157.3,137.8,133.6,130.1$, 121.5, 112.4, 91.6, 79.1, 76.4, 66.0, 55.8; IR (ATR) 3286, 1626, 1496, 1238, 1021; HRMS (ESI) calcd for $\mathrm{C}_{11} \mathrm{H}_{11} \mathrm{~N}_{2} \mathrm{O}_{2}\left(\mathrm{M}+\mathrm{H}^{+}\right)$203.0820, found 203.0811.

2-(2-Hydroxy-2-propyl)-6-methoxy-1-(2-propyn-1-yloxy)benzimidazole (178). White solid; mp=150$151{ }^{\circ} \mathrm{C}$ (dec.); ${ }^{1} \mathrm{H}$ NMR (600 MHz, $\left.\mathrm{CDCl}_{3}\right) \delta 7.57(\mathrm{~d}, J=8.4 \mathrm{~Hz}, 1 \mathrm{H}), 6.89(\mathrm{~d}, J=1.8 \mathrm{~Hz}, 1 \mathrm{H}), 6.87$ (dd, $J=8.4,2.4 \mathrm{~Hz}, 1 \mathrm{H}), 5.26$ (d, J=2.4 Hz, 2H), 3.87 (s, 3H), 2.89 (br s, 1H), 2.33 (t, J=3.0 Hz, 1H), 1.77 (s, $6 \mathrm{H}) ;{ }^{13} \mathrm{C} \mathrm{NMR}\left(150 \mathrm{MHz}, \mathrm{CDCl}_{3}\right) \delta 156.8,156.2,136.4,135.7,120.2,111.4,93.6,77.8,73.2,71.2,55.9$, 34.5, 29.3; IR (ATR) 3282, 1627, 1490, 1214, $1146 \mathrm{~cm}-1$; HRMS (ESI) calcd for $\mathrm{C}_{14} \mathrm{H}_{16} \mathrm{~N}_{2} \mathrm{O}_{3}\left(\mathrm{M}+\mathrm{Na}^{+}\right)$ 283.1058, found 283.1042.

1-(3-Butyn-2-yloxy)-6-methoxybenzimidazole (179). Colorless oil; ${ }^{1} \mathrm{H}$ NMR (400 MHz, $\left.\mathrm{CDCl}_{3}\right) \delta 8.04$ (s, 1H), 7.62 (d, J=9.6 Hz, 1H), 6.89 (d, J=2.8 Hz, 1H), 6.88 (dd, J=9.6, 2.8 Hz, 1H), 5.02 (dq, J=6.4, 2.0 $\mathrm{Hz}, 1 \mathrm{H}), 3.85(\mathrm{~s}, 3 \mathrm{H}), 2.63(\mathrm{~d}, J=2.0 \mathrm{~Hz}, 1 \mathrm{H}), 1.70(\mathrm{~d}, J=6.8 \mathrm{~Hz}, 3 \mathrm{H}) ;{ }^{13} \mathrm{C} \mathrm{NMR}\left(100 \mathrm{MHz}, \mathrm{CDCl}_{3}\right) \delta$ 157.2, 138.3, 133.6, 130.7, 121.3, 112.1, 92.0, 80.6, 76.7, 74.1, 55.8, 20.3; IR (ATR) 1624, 1495, 1234, 1061, 816; HRMS (ESI) calcd for $\mathrm{C}_{12} \mathrm{H}_{13} \mathrm{~N}_{2} \mathrm{O}_{3}\left(\mathrm{M}+\mathrm{H}^{+}\right)$217.0977, found 217.0968. 
1-(3-Butyn-2-yloxy)-2-(2-hydroxy-2-propyl)-6-methoxybenzimidazole (180). White solid; mp=131$132{ }^{\circ} \mathrm{C} ;{ }^{1} \mathrm{H}$ NMR $\left(400 \mathrm{MHz}, \mathrm{CDCl}_{3}\right) \delta 7.54(\mathrm{~d}, J=8.8 \mathrm{~Hz}, 1 \mathrm{H}), 7.13(\mathrm{~d}, J=2.4 \mathrm{~Hz}, 1 \mathrm{H}), 6.86(\mathrm{dd}, J=8.8$, $2.4 \mathrm{~Hz}, 1 \mathrm{H}), 5.52(\mathrm{dq}, J=6.8,2.0 \mathrm{~Hz}, 1 \mathrm{H}), 3.86$ (s, 3H), 2.78 (br s, 1H), 2.56 (d, J=2.0 Hz, 1H), 1.83 (s, $3 \mathrm{H}), 2.74(\mathrm{~d}, J=1.2 \mathrm{~Hz}, 6 \mathrm{H}) ;{ }^{13} \mathrm{C}$ NMR $\left(100 \mathrm{MHz}, \mathrm{CDCl}_{3}\right) \delta 156.8,153.7,133.9,131.0,120.5,112.0$, 93.9, 81.9, 74.3, 70.2, 55.8, 29.7, 28.4, 20.7; IR (ATR) 3168, 1489, 1251, 1217, $1179 \mathrm{~cm}^{-1}$; HRMS (ESI) calcd for $\mathrm{C}_{15} \mathrm{H}_{19} \mathrm{~N}_{2} \mathrm{O}_{3}\left(\mathrm{M}+\mathrm{H}^{+}\right)$275.1396, found 275.1391.

1-Acetoxy-2-(2-acetoxy-2-propyl)-6-methoxybenzimidazole (181). Yellow oil; ${ }^{1} \mathrm{H}$ NMR (600 MHz, $\left.\mathrm{CDCl}_{3}\right) \delta 7.60(\mathrm{~d}, J=8.4 \mathrm{~Hz}, 1 \mathrm{H}), 6.87(\mathrm{dd}, J=8.4,2.4 \mathrm{~Hz}, 1 \mathrm{H}), 6.57(\mathrm{~d}, J=2.4 \mathrm{~Hz}, 1 \mathrm{H}), 3.84(\mathrm{~s}, 3 \mathrm{H}), 2.46$ (s, 3H), $1.82(\mathrm{~s}, 6 \mathrm{H}), 1.76(\mathrm{~s}, 3 \mathrm{H}) ;{ }^{13} \mathrm{C} \mathrm{NMR}\left(150 \mathrm{MHz}, \mathrm{CDCl}_{3}\right) \delta 167.1,157.5,150.4,133.4,130.8$, 120.9, 112.0, 91.6, 55.8, 43.0, 31.5, 27.1, 18.9, 12.4; IR (ATR) 1809, 1630, 1491, 1213, $1158 \mathrm{~cm}^{-1}$; HRMS (ESI) calcd for $\mathrm{C}_{15} \mathrm{H}_{19} \mathrm{~N}_{2} \mathrm{O}_{5}\left(\mathrm{M}+\mathrm{H}^{+}\right) 307.1294$, found 307.1275 .

1-Acetoxy-2-(1-propen-2-yl)-6-methoxybenzimidazole (182). Pale yellow oil; ${ }^{1} \mathrm{H}$ NMR (600 MHz, $\left.\mathrm{CDCl}_{3}\right) \delta 7.63(\mathrm{~d}, J=9.0 \mathrm{~Hz}, 1 \mathrm{H}), 6.89(\mathrm{dd}, J=9.0,2.4 \mathrm{~Hz}, 1 \mathrm{H}), 6.63(\mathrm{~d}, J=2.4 \mathrm{~Hz}, 1 \mathrm{H}), 5.69$ (pent, $J=1.2$ $\mathrm{Hz}, 1 \mathrm{H}), 5.50$ (pent, $J=1.2 \mathrm{~Hz}, 1 \mathrm{H}), 3.84$ (s, 3H), 2.40 (s, 3H), 2.27 (dd, $J=1.8,1.2 \mathrm{~Hz}, 3 \mathrm{H}) ;{ }^{13} \mathrm{C}$ NMR $\left(150 \mathrm{MHz}, \mathrm{CDCl}_{3}\right) \delta 167.2,157.7,148.3,133.3,133.0,132.3,121.2,118.3,112.4,91.6,55.8,21.2,18.3$; IR (ATR) 1805, 1623, 1491, 1221, 1157, 813; HRMS (ESI) calcd for $\mathrm{C}_{13} \mathrm{H}_{15} \mathrm{~N}_{2} \mathrm{O}_{3}\left(\mathrm{M}+\mathrm{H}^{+}\right)$247.1083, found 247.1066 .

2,4-Dinitro- $\boldsymbol{N}$-methyl- $\boldsymbol{N}$-(2-methyl-1-propen-1-yl)benzenamine. Red oil; ${ }^{1} \mathrm{H}$ NMR (400 $\mathrm{MHz}, \mathrm{CDCl}_{3}$ ) $\delta 8.54(\mathrm{~d}, J=2.8 \mathrm{~Hz}, 1 \mathrm{H}), 8.20$ (dd, $J=9.6,2.8 \mathrm{~Hz}, 1 \mathrm{H}), 7.04$ (d, J=9.6 Hz, 1H), 5.71 (sept, $J=1.2 \mathrm{~Hz}, 1 \mathrm{H})$, 3.09 (s, 3H), 1.71 (d, $J=1.2 \mathrm{~Hz}, 3 \mathrm{H}), 1.42(\mathrm{~d}, J=1.2 \mathrm{~Hz}, 3 \mathrm{H}) ;{ }^{13} \mathrm{C} \mathrm{NMR}\left(100 \mathrm{MHz}, \mathrm{CDCl}_{3}\right) \delta 147.3,141.3$, 136.8, 132.3, 127.7, 127.0, 123.2, 117.3, 40.7, 21.4, 17.5; ; IR (ATR) 1601, 1580, 1501, 1311, $1137 \mathrm{~cm}^{-1}$; HRMS (ESI) calcd for $\mathrm{C}_{11} \mathrm{H}_{14} \mathrm{~N}_{3} \mathrm{O}_{4}\left(\mathrm{M}+\mathrm{H}^{+}\right) 252.0984$, found 252.0980.

Methyl 4- $N$-methyl- $N$-(2-methyl-1-propen-1-yl)amino-3-nitrobenzoate. Red oil; ${ }^{1} \mathrm{H}$ NMR (600 MHz, $\left.\mathrm{CDCl}_{3}\right) \delta 8.27(\mathrm{~d}, J=1.8 \mathrm{~Hz}, 1 \mathrm{H}), 7.96$ (dd, $\left.J=9.0,2.4 \mathrm{~Hz}, 1 \mathrm{H}\right), 6.99$ (d, J=9.0 Hz, 1H), 5.64 (sept, $J=1.2$ $\mathrm{Hz}, 1 \mathrm{H}), 3.87(\mathrm{~s}, 3 \mathrm{H}), 3.02(\mathrm{~s}, 3 \mathrm{H}), 1.67(\mathrm{~s}, 3 \mathrm{H}), 1.38(\mathrm{~s}, 3 \mathrm{H}) ;{ }^{13} \mathrm{C} \mathrm{NMR}\left(150 \mathrm{MHz}, \mathrm{CDCl}_{3}\right) \delta 165.5$, 146.3, 138.1, 133.5, 130.3, 128.2, 127.6, 118.7, 117.5, 52.0, 40.3, 21.4, 17.4; IR (ATR) 1704, 1607, 1525, 1290, 1259, $1126 \mathrm{~cm}^{-1}$; HRMS (ESI) calcd for $\mathrm{C}_{13} \mathrm{H}_{17} \mathrm{~N}_{2} \mathrm{O}_{4}\left(\mathrm{M}+\mathrm{H}^{+}\right)$265.1188, found 265.1171. 
5-Chloro-2-nitro- $N$-methyl- $N$-(2-methyl-1-propen-1-yl)benzenamine. Yellow oil; ${ }^{1} \mathrm{H}$ NMR (600 MHz, $\left.\mathrm{CDCl}_{3}\right) \delta 7.58(\mathrm{~d}, J=8.4 \mathrm{~Hz}, 1 \mathrm{H}), 7.01(\mathrm{~d}, J=1.8 \mathrm{~Hz}, 1 \mathrm{H}), 6.74(\mathrm{dd}, J=8.4,1.8 \mathrm{~Hz}, 1 \mathrm{H}), 5.60$ (sept, $J=1.8$ $\mathrm{Hz}, 1 \mathrm{H}), 2.97(\mathrm{~s}, 3 \mathrm{H}), 1.68(\mathrm{~d}, J=1.2 \mathrm{~Hz}, 3 \mathrm{H}), 1.43(\mathrm{~d}, J=1.2 \mathrm{~Hz}, 3 \mathrm{H}) ;{ }^{13} \mathrm{C} \mathrm{NMR}\left(150 \mathrm{MHz}, \mathrm{CDCl}_{3}\right) \delta$ 144.4, 138.8, 137.7, 129.1, 127.7, 127.2, 118.5, 117.7, 40.5, 21.5, 17.5; IR (ATR) 1596, 1514, 1487, 1340, $1288 \mathrm{~cm}^{-1}$; HRMS (ESI) calcd for $\mathrm{C}_{11} \mathrm{H}_{14} \mathrm{ClN}_{2} \mathrm{O}_{2}\left(\mathrm{M}+\mathrm{H}^{+}\right)$241.0743, found 241.0733.

\section{Table 4.1, Entry 1}

4-Methoxy- $N$-(2-methyl-1-propen-1-yl)-2-nitrobenzenamine (127)

A mixture of $127(120 \mathrm{mg}, 0.540 \mathrm{mmol})$ and $\mathrm{NaH}(80 \%, 17.0 \mathrm{mg}, 0.567 \mathrm{mmol})$ in DMSO (10 mL) was stirred at ambient temperature under a nitrogen atmosphere. After $1 \mathrm{~h}$, the mixture was cooled to $0{ }^{\circ} \mathrm{C}$ and MeI (35 $\mu \mathrm{L}, 0.57 \mathrm{mmol})$ was added via a syringe. The reaction vessel was removed from the cold bath and stirred for an additional $1 \mathrm{~h}$. EtOAc $(15 \mathrm{~mL})$ was added and the mixture was washed with water $(6 \times 10$ $\mathrm{mL})$. The organic phase was dried $\left(\mathrm{MgSO}_{4}\right)$, filtered and the solvent was removed from the filtrate under reduced pressure. The residue was purified by chromatography (hexane/EtOAc, 95:5) to give 127 (57.9 $\mathrm{mg}, 0.261 \mathrm{mmol}, 48 \%)$ as a deep red solid.

\section{Table 4.1, Entry 2}

4-Methoxy- $N$-(2-methyl-1-propen-1-yl)-2-nitrobenzenamine (127), 1,6-dimethoxybenzimidazole (128), and 2-(2-hydroxy-2-propyl)-1,6-dimethoxybenzimidazole (130)

A mixture of $127(70 \mathrm{mg}, 0.32 \mathrm{mmol})$ and $\mathrm{NaH}(80 \%, 9.9 \mathrm{mg}, 0.33 \mathrm{mmol})$ in DMSO (9 mL) was stirred at ambient temperature under a nitrogen atmosphere. After $1 \mathrm{~h}$, the mixture was cooled to $0{ }^{\circ} \mathrm{C}$ and MeI (41 $\mu \mathrm{L}, 0.66 \mathrm{mmol}$ ) was added via a syringe. The reaction vessel was removed from the cold bath and stirred for an additional $1 \mathrm{~h}$. EtOAc $(12 \mathrm{~mL})$ was added and the mixture was washed with water $(8 \mathrm{x} 7$ $\mathrm{mL})$. The organic phase was dried $\left(\mathrm{MgSO}_{4}\right)$, filtered and the solvent was removed from the filtrate under reduced pressure. The residue was purified by chromatography (hexane/EtOAc, 7:3) to give, in order of elution, 127 (28 mg, $0.126 \mathrm{mmol}, 40 \%$ ) as a deep red solid and an inseparable mixture of $\mathbf{1 2 8}$ and $\mathbf{1 3 0}$ (26.1 mg, $128(1 \%)$ and $130(34 \%)$ as calculated from ${ }^{1} \mathrm{H}$ NMR spectrum) as a white solid.

\section{Table 4.1, Entry 3}

\section{4-Methoxy- $N$-(2-methyl-1-propen-1-yl)-2-nitrobenzenamine $\quad(127), \quad$ 1,6-dimethoxybenzimidazole} (128), and 2-(2-hydroxy-2-propyl)-1,6-dimethoxybenzimidazole (130)

A mixture of 127 (80 mg, $0.36 \mathrm{mmol})$ and $\mathrm{NaH}(80 \%, 11.4 \mathrm{mg}, 0.379 \mathrm{mmol})$ in DMSO (6 mL) was stirred at ambient temperature under a nitrogen atmosphere. After $1 \mathrm{~h}$, the mixture was cooled to $0{ }^{\circ} \mathrm{C}$ and 
MeI $(71 \mu \mathrm{L}, 1.1 \mathrm{mmol})$ was added via a syringe. The reaction vessel was removed from the cold bath and stirred for an additional $1 \mathrm{~h}$. EtOAc $(13 \mathrm{~mL})$ was added and the mixture was washed with water $(7 \mathrm{x} 8$ $\mathrm{mL})$. The organic phase was dried $\left(\mathrm{MgSO}_{4}\right)$, filtered and the solvent was removed from the filtrate under reduced pressure. The residue was purified by chromatography (hexane/EtOAc, 7:3) to give, in order of elution, 127 (28.4 mg, $0.128 \mathrm{mmol}, 35 \%)$ as a deep red solid and an inseparable mixture of $\mathbf{1 2 8}$ and $\mathbf{1 3 0}$ (27.8 mg, 128 (3\%) and $\mathbf{1 3 0}(30 \%)$ as calculated from ${ }^{1} \mathrm{H}$ NMR spectrum) as a white solid.

\section{Table 4.1, Entry 4 See Table 4.3, Entry 1}

Table 4.1, Entry 5

1,6-dimethoxybenzimidazole (128), 1,6-dimethoxy-2-(2-methoxy-2-propyl)benzimidazole (129), and 2-(2-hydroxy-2-propyl)-1,6-dimethoxybenzimidazole (130)

A mixture of $127(120 \mathrm{mg}, 0.540 \mathrm{mmol})$ and $\mathrm{NaH}(80 \%, 34.0 \mathrm{mg}, 1.13 \mathrm{mmol})$ in DMSO (10 mL) was stirred at ambient temperature under a nitrogen atmosphere. After $1 \mathrm{~h}$, the mixture was cooled to $0{ }^{\circ} \mathrm{C}$ and MeI $(71 \mu \mathrm{L}, 1.1 \mathrm{mmol})$ was added via a syringe. The reaction vessel was removed from the cold bath and stirred for an additional $1 \mathrm{~h}$. EtOAc $(15 \mathrm{~mL})$ was added and the mixture was washed with water $(6 \times 10$ $\mathrm{mL})$. The organic phase was dried $\left(\mathrm{MgSO}_{4}\right)$, filtered and the solvent was removed from the filtrate under reduced pressure. The residue was purified by chromatography (hexane/EtOAc, 7:3, then 1:1) to give, in order of elution, 129 (23.4 mg, $0.093 \mathrm{mmol}, 17 \%)$ as a pale yellow oil and an inseparable mixture of 128 and 130 (75.5 mg, 128 (5\%) and 130 (56\%) as calculated from ${ }^{1} \mathrm{H}$ NMR spectrum) as a white solid.

\section{Table 4.1, Entry 6}

1,6-dimethoxybenzimidazole (128), 1,6-dimethoxy-2-(2-methoxy-2-propyl)benzimidazole (129), and 2-(2-hydroxy-2-propyl)-1,6-dimethoxybenzimidazole (130)

A mixture of $127(70 \mathrm{mg}, 0.32 \mathrm{mmol})$ and $\mathrm{NaH}(80 \%, 19.9 \mathrm{mg}, 0.663 \mathrm{mmol})$ in DMSO (9 mL) was stirred at ambient temperature under a nitrogen atmosphere. After $1 \mathrm{~h}$, the mixture was cooled to $0{ }^{\circ} \mathrm{C}$ and MeI $(62 \mu \mathrm{L}, 0.10 \mathrm{mmol})$ was added via a syringe. The reaction vessel was removed from the cold bath and stirred for an additional $1 \mathrm{~h}$. EtOAc $(14 \mathrm{~mL})$ was added and the mixture was washed with water $(8 \mathrm{x} 8$ $\mathrm{mL})$. The organic phase was dried $\left(\mathrm{MgSO}_{4}\right)$, filtered and the solvent was removed from the filtrate under reduced pressure. The residue was purified by chromatography (hexane/EtOAc, 7:3) to give, in order of elution, 129 (14.2 mg, $0.056 \mathrm{mmol}, 18 \%$ ) as a pale yellow oil and an inseparable mixture of 128 and 130 (38.6 mg, 128 (7\%) and 130 (46\%) as calculated from ${ }^{1} \mathrm{H}$ NMR spectrum) as a white solid. 
Table 4.1, Entry 7

1,6-Dimethoxy-2-(2-methoxy-2-propyl)benzimidazole (129). A mixture of 127 (115 mg, $0.518 \mathrm{mmol}$ ) and $\mathrm{NaH}(80 \%, 58.1 \mathrm{mg}, 1.94 \mathrm{mmol})$ in DMSO $(10 \mathrm{~mL})$ was stirred at ambient temperature under a nitrogen atmosphere for $9 \mathrm{~h}$. MeI $(171 \mu \mathrm{L}, 2.75 \mathrm{mmol})$ was added and the mixture was stirred for $1 \mathrm{~h}$. EtOAc $(15 \mathrm{~mL})$ was added and the mixture was washed with water $(5 \times 10 \mathrm{~mL})$. The organic phase was dried $\left(\mathrm{MgSO}_{4}\right)$, filtered, and the solvent was removed from the filtrate under reduced pressure. The crude product was purified by chromatography (hexane/EtOAc, 7:3) to give $\mathbf{1 2 9}(89.1 \mathrm{mg}, 0.356 \mathrm{mmol}, 69 \%)$ as a pale yellow oil.

\section{Table 4.1, Entry 8}

1,6-dimethoxybenzimidazole (128), 1,6-dimethoxy-2-(2-methoxy-2-propyl)benzimidazole (129), and 2-(2-hydroxy-2-propyl)-1,6-dimethoxybenzimidazole (130)

A mixture of $127(62.3 \mathrm{mg}, 0.280 \mathrm{mmol})$ and $\mathrm{NaH}(80 \%, 31.5 \mathrm{mg}, 1.05 \mathrm{mmol})$ in DMSO (2 mL) was stirred at ambient temperature under a nitrogen atmosphere. After stirring for $1 \mathrm{~h}$, MeI $(58 \mu \mathrm{L}, 0.93$ mmol) was added via a syringe. The resulting reaction mixture was stirred for an additional $1 \mathrm{~h}$. EtOAc $(10 \mathrm{~mL})$ was added and the mixture was washed with water $(7 \times 6 \mathrm{~mL})$. The organic phase was dried $\left(\mathrm{MgSO}_{4}\right)$, filtered and the solvent was removed from the filtrate under reduced pressure. The residue was purified by chromatography (hexane/EtOAc, 7:3) to give, in order of elution, 129 (45.0 mg, 0.180 mmol, $64 \%)$ as a pale yellow oil and an inseparable mixture of 128 and 130 (4.0 mg, 128 (5\%) and 130 (2\%) as calculated from ${ }^{1} \mathrm{H}$ NMR spectrum) as a white solid.

\section{Table 4.1, Entry 9}

\section{1,6-dimethoxy-2-(2-methoxy-2-propyl)benzimidazole (129)}

To a mixture of $127(165 \mathrm{mg}, 0.741 \mathrm{mmol})$ and $\mathrm{NaH}(80 \%, 111 \mathrm{mg}, 3.70 \mathrm{mmol})$ placed in ice bath at 0 ${ }^{\circ} \mathrm{C}$ under a nitrogen atmosphere was added DMSO $(10 \mathrm{~mL})$ followed by the immediate addition of MeI $(231 \mu \mathrm{L}, 3.71 \mathrm{mmol})$ via syringes. The reaction vessel was removed from the cold bath and the yellow solution was stirred at ambient temperature for $2 \mathrm{~h}$. EtOAc $(20 \mathrm{~mL})$ was added and the mixture was washed with water $(15 \times 5 \mathrm{~mL})$ and brine $(15 \mathrm{~mL})$. The organic phase was dried $\left(\mathrm{MgSO}_{4}\right)$, filtered and the solvent was removed from the filtrate under reduced pressure. The residue was purified by chromatography (hexane/EtOAc, 7:3) to give $\mathbf{1 2 9}$ (120 mg, $0.479 \mathrm{mmol}, 65 \%)$ as a pale yellow oil. 
Table 4.1, Entry 10

1,6-Dimethoxybenzimidazole (128) and 1,6-Dimethoxy-2-(2-methoxy-2-propyl)benzimidazole (129). A mixture of 127 (85.0 mg, $0.382 \mathrm{mmol})$ and $\mathrm{NaH}(80 \%, 42.9 \mathrm{mg}, 1.43 \mathrm{mmol})$ in DMF (8 mL) was stirred at ambient temperature under a nitrogen atmosphere for 1-2 min. MeI (72 $\mu \mathrm{L}, 1.16 \mathrm{mmol})$ was added and the mixture was stirred for $2 \mathrm{~h}$. EtOAc $(10 \mathrm{~mL})$ was added and the mixture was washed with water $(4 \mathrm{x} 8 \mathrm{~mL})$. The organic phase was dried $\left(\mathrm{MgSO}_{4}\right)$, filtered, and the solvent was removed from the filtrate under reduced pressure. The crude product was purified by chromatography (hexane/EtOAc, 7:3 then 1:1) to give, in order of elution, $\mathbf{1 2 9}(8.0 \mathrm{mg}, 0.032 \mathrm{mmol}, 8 \%)$ as a pale yellow oil followed by $\mathbf{1 2 8}$ (14.1 $\mathrm{mg}, 0.079 \mathrm{mmol}, 21 \%)$ as a colorless oil.

\section{Table 4.1, Entry 11}

1,6-Dimethoxy-2-(2-methoxy-2-propyl)benzimidazole (129). A mixture of 127 (75.4 mg, $0.339 \mathrm{mmol}$ ) and $\mathrm{NaH}(80 \%, 38.1 \mathrm{mg}, 1.27 \mathrm{mmol})$ in NMP $(7 \mathrm{~mL})$ was stirred at ambient temperature under a nitrogen atmosphere for $2 \mathrm{~min}$. MeI (70 $\mu \mathrm{L}, 1.12 \mathrm{mmol})$ was added and the mixture was stirred for $2 \mathrm{~h}$. EtOAc (10 $\mathrm{mL})$ was added and the mixture was washed with water $(5 \times 8 \mathrm{~mL})$ and brine $(8 \mathrm{~mL})$. The organic phase was dried $\left(\mathrm{MgSO}_{4}\right)$, filtered, and the solvent was removed from the filtrate under reduced pressure. The crude product was purified by chromatography (hexane/EtOAc, 7:3 then 1:1) to give $\mathbf{1 2 9}$ (25.0 mg, 0.100 mmol, 29\%) as a pale yellow oil.

\section{Table 4.1, Entry 12}

1,6-Dimethoxybenzimidazole (128) and 1,6-Dimethoxy-2-(2-methoxy-2-propyl)benzimidazole (129). A mixture of $127(150 \mathrm{mg}, 0.675 \mathrm{mmol})$ and $\mathrm{NaH}(80 \%, 75.7 \mathrm{mg}, 2.52 \mathrm{mmol})$ in acetonitrile (MeCN, 10 $\mathrm{mL}$ ) was stirred at ambient temperature under a nitrogen atmosphere for approximately $2 \mathrm{~min}$. To the brown mixture was added MeI (134 $\mu \mathrm{L}, 2.15 \mathrm{mmol})$ and it was stirred for $2 \mathrm{~h}$. An orange solution was obtained after 18-20 min. EtOAc (15 mL) was added and the mixture was washed with water (3x15 mL). The organic phase was dried $\left(\mathrm{MgSO}_{4}\right)$, filtered, and the solvent was removed from the filtrate under reduced pressure. The crude product was purified by chromatography (hexane/EtOAc, 7:3 then 1:1) to give, in order of elution 129 (21.5 mg, $0.086 \mathrm{mmol}, 13 \%)$ as a pale yellow oil and $\mathbf{1 2 8}$ (78.1 $\mathrm{mg}, 0.438$ mmol, $65 \%$ ) as a colorless oil.

\section{Table 4.2, Entry 1}

1,6-Dimethoxy-2-(2-methoxy-2-propyl)benzimidazole (129). A mixture of 4-methoxy-2-nitro- $N$-(2methyl-1-propen-1-yl)benzenamine 127 (191 mg, $0.859 \mathrm{mmol})$ and $\mathrm{NaH}(80 \%, 96.4 \mathrm{mg}, 3.21 \mathrm{mmol})$ in 
DMSO $(10 \mathrm{~mL})$ was stirred at ambient temperature under a nitrogen atmosphere for $1 \mathrm{~h}$. The orange solution was cooled to $0{ }^{\circ} \mathrm{C}$ and $\mathrm{MeI}(171 \mu \mathrm{L}, 2.75 \mathrm{mmol})$ was added via a syringe. The reaction vessel was removed from the cold bath and the orange solution was stirred for $1 \mathrm{~h}$. EtOAc $(20 \mathrm{~mL})$ was added and the mixture was washed with water $(5 \times 15 \mathrm{~mL})$. The organic phase was dried $\left(\mathrm{MgSO}_{4}\right)$, filtered, and the solvent was removed from the filtrate under reduced pressure. The crude product was purified by chromatography (hexane/EtOAc, 7:3) to give $\mathbf{1 2 9}$ (153 $\mathrm{mg}, 0.609 \mathrm{mmol}, 71 \%)$ as a pale yellow oil.

\section{Table 4.2, Entry 2}

1,6-Dimethoxy-2-(2-methoxy-2-propyl)benzimidazole (129). A mixture of 127 (146 mg, $0.659 \mathrm{mmol}$ ) and $\mathrm{NaH}(80 \%, 98.7 \mathrm{mg}, 3.29 \mathrm{mmol})$ in DMSO $(10 \mathrm{~mL})$ was stirred at ambient temperature under a nitrogen atmosphere for $2 \mathrm{~min}$. MeI $(205 \mu \mathrm{L}, 3.29 \mathrm{mmol})$ was added and the mixture was stirred for $2 \mathrm{~h}$. EtOAc $(15 \mathrm{~mL})$ was added and the mixture was washed with water $(4 \times 10 \mathrm{~mL})$ and brine $10 \mathrm{~mL})$. The organic phase was dried $\left(\mathrm{MgSO}_{4}\right)$, filtered, and the solvent was removed from the filtrate under reduced pressure. The crude product was purified by chromatography (hexane/EtOAc, 7:3) to give 129 (118 mg, $0.471 \mathrm{mmol}, 71 \%$ ) as a pale yellow oil.

\section{Table 4.2, Entry 3}

1,6-Dimethoxy-2-(2-methoxy-2-propyl)benzimidazole (129). A mixture of 127 (115 mg, $0.518 \mathrm{mmol}$ ) and $\mathrm{NaH}(80 \%, 58.1 \mathrm{mg}, 1.94 \mathrm{mmol})$ in DMSO $(10 \mathrm{~mL})$ was stirred at ambient temperature under a nitrogen atmosphere for $9 \mathrm{~h}$. MeI $(171 \mu \mathrm{L}, 2.75 \mathrm{mmol})$ was added and the mixture was stirred for $1 \mathrm{~h}$. EtOAc $(15 \mathrm{~mL})$ was added and the mixture was washed with water $(5 \times 10 \mathrm{~mL})$. The organic phase was dried $\left(\mathrm{MgSO}_{4}\right)$, filtered, and the solvent was removed from the filtrate under reduced pressure. The crude product was purified by chromatography (hexane/EtOAc, 7:3) to give $\mathbf{1 2 9}(89.1 \mathrm{mg}, 0.356 \mathrm{mmol}, 69 \%)$ as a pale yellow oil.

\section{Table 4.2, Entry 4}

6-Chloro-1-methoxy-2-(2-methoxy-2-propyl)benzimidazole (132). A mixture of 4-chloro-2-nitro- $N$-(2methyl-1-propen-1-yl)benzenamine (131) (164 mg, $0.724 \mathrm{mmol}$ ) and $\mathrm{NaH}(80 \%, 110 \mathrm{mg}, 3.66 \mathrm{mmol})$ in DMSO (13 mL) was stirred at ambient temperature under a nitrogen atmosphere $1 \mathrm{~h}$. The mixture was cooled to $0{ }^{\circ} \mathrm{C}$ and $\mathrm{MeI}(144 \mu \mathrm{L}, 2.31 \mathrm{mmol})$ was added. The reaction vessel was removed from the cold bath and the resulting orange solution was stirred for $1 \mathrm{~h}$. EtOAc $(20 \mathrm{~mL})$ was added and the mixture was washed with water $(5 \times 15 \mathrm{~mL})$. The organic phase was dried $\left(\mathrm{MgSO}_{4}\right)$, filtered, and the solvent was 
removed from the filtrate under reduced pressure. The crude product was purified by chromatography (hexane/EtOAc, 1:1) to give 132 (159 $\mathrm{mg}, 0.624 \mathrm{mmol}, 86 \%$ ) as an orange solid.

\section{Table 4.2, Entry 5}

6-Chloro-1-methoxy-2-(2-methoxy-2-propyl)benzimidazole (132). A mixture of 131 (133 mg, 0.587 $\mathrm{mmol})$ and $\mathrm{NaH}(80 \%, 65.9 \mathrm{mg}, 2.20 \mathrm{mmol})$ in DMSO $(10 \mathrm{~mL})$ was stirred at ambient temperature for 1 h. MeI (121 $\mu \mathrm{L}, 1.94 \mathrm{mmol})$ was added and the resulting orange solution was stirred for an additional $1 \mathrm{~h}$. EtOAc $(15 \mathrm{~mL})$ was added and the mixture was washed with water $(5 \times 10 \mathrm{~mL})$ and brine $(10 \mathrm{~mL})$. The organic phase was dried $\left(\mathrm{MgSO}_{4}\right)$, filtered, and the solvent was removed from the filtrate under reduced pressure. The crude product was purified by chromatography (hexane/EtOAc, 7:3) to give 132 (141 mg, $0.555 \mathrm{mmol}, 94 \%$ ) as an orange solid.

\section{Table 4.2, Entry 6}

6-Bromo-1-methoxy-2-(2-methoxy-2-propyl)benzimidazole (135). A mixture of 4-bromo-2-nitro- $N$-(2methyl-1-propen-1-yl)benzenamine (133) $(56 \mathrm{mg}, 0.207 \mathrm{mmol}$ ) and $\mathrm{NaH}$ (80\%, $23.0 \mathrm{mg}, 0.767 \mathrm{mmol})$ in DMSO (4 mL) was stirred at ambient temperature under a nitrogen atmosphere for $1 \mathrm{~h}$. The brown reaction mixture was cooled to $0{ }^{\circ} \mathrm{C}$ and $\mathrm{MeI}(41 \mu \mathrm{L}, 0.66 \mathrm{mmol})$ was added. The reaction vessel was removed from the cold bath and the resulting orange solution was stirred for $1 \mathrm{~h}$. EtOAc $(10 \mathrm{~mL})$ was added and the mixture was washed with water $(5 \times 10 \mathrm{~mL})$ and brine $(10 \mathrm{~mL})$. The organic phase was concentrated under reduced pressure and the residue was purified by chromatography (hexane/EtOAc, 7:3) to give 135 (48.2 $\mathrm{mg}, 0.161 \mathrm{mmol}, 78 \%$ ) as an orange oil.

\section{Table 4.2, Entry 7}

6-Bromo-1-methoxy-2-(2-methoxy-2-propyl)benzimidazole (135). A mixture of 133 (96.2 mg, 0.355 $\mathrm{mmol})$ and $\mathrm{NaH}(80 \%, 39.8 \mathrm{mg}, 1.33 \mathrm{mmol})$ in DMSO $(10 \mathrm{~mL})$ was stirred at ambient temperature under a nitrogen atmosphere for approximately $6 \mathrm{~min}$. An immediate color change from purple to brown was observed. MeI $(73 \mu \mathrm{L}, 1.17 \mathrm{mmol})$ was added and the resulting orange solution was stirred for $4 \mathrm{~h}$. EtOAc $(15 \mathrm{~mL})$ was added and the mixture was washed with water $(5 \times 10 \mathrm{~mL})$ and brine $(10 \mathrm{~mL})$. The organic phase was dried $\left(\mathrm{MgSO}_{4}\right)$, filtered, and the solvent was removed from the filtrate under reduced pressure. The crude product was purified by chromatography (hexane/EtOAc, 7:3) to give 135 (104 mg, $0.329 \mathrm{mmol}, 93 \%$ ) as an orange oil. 
Table 4.2, Entry 8

6-Fluoro-1-methoxy-2-(2-methoxy-2-propyl)benzimidazole (137). A mixture of 136 (134 mg, 0.636 $\mathrm{mmol})$ and $\mathrm{NaH}(80 \%, 71.8 \mathrm{mg}, 2.39 \mathrm{mmol})$ in DMSO $(10 \mathrm{~mL})$ was stirred at ambient temperature under a nitrogen atmosphere. After stirring for $1 \mathrm{~h}$, MeI $(132 \mu \mathrm{L}, 2.12 \mathrm{mmol})$ was added via a syringe and the resulting yellow-orange solution was stirred for an additional $1 \mathrm{~h}$. EtOAc $(15 \mathrm{~mL})$ was added and the mixture was washed with water $(7 \times 10 \mathrm{~mL})$. The organic phase was dried $\left(\mathrm{MgSO}_{4}\right)$, filtered and the solvent was removed from the filtrate under reduced pressure. The residue was purified by chromatography (hexane/EtOAc, 7:3) to give $\mathbf{1 3 7}$ (113 $\mathrm{mg}, 0.474 \mathrm{mmol}, 75 \%)$ as a pale yellow oil.

\section{Table 4.2, Entry 9}

\section{1-Methoxy-6-methylbenzimidazole (139), 1-Methoxy-2-(2-methoxy-2-propyl)-6-} methylbenzimidazole (140), and 2-(2-Hydroxy-2-propyl)-1-methoxy-6-methylbenzimidazole (141). A mixture of 4-methyl-2-nitro- $N$-(2-methyl-1-propen-1-yl)benzenamine (138) (145 mg, $0.705 \mathrm{mmol}$ ) and $\mathrm{NaH}(80 \%, 78.9 \mathrm{mg}, 2.63 \mathrm{mmol})$ in DMSO $(10 \mathrm{~mL})$ was stirred at ambient temperature under a nitrogen atmosphere for $1 \mathrm{~h}$. The resulting orange mixture was cooled to $0{ }^{\circ} \mathrm{C}$ and $\mathrm{MeI}(140 \mu \mathrm{L}, 2.25 \mathrm{mmol})$ was added. The reaction was removed from the cold bath and the resulting solution was stirred for an additional $1 \mathrm{~h}$. EtOAc $(20 \mathrm{~mL})$ was added and the mixture was washed with water $(5 \mathrm{x} 15 \mathrm{~mL})$ and brine $(10 \mathrm{~mL})$. The organic phase was dried $\left(\mathrm{MgSO}_{4}\right)$, filtered, and the solvent was removed from the filtrate under reduced pressure. The crude product was purified by chromatography (hexane/EtOAc, in order 7:3, 1:1, 3:7) to give, in order of elution, $\mathbf{1 4 0}(86.3 \mathrm{mg}, 0.368 \mathrm{mmol}, 52 \%)$ as a colorless oil, $141(0.008 \mathrm{mg}$, $0.036 \mathrm{mmol}, 5 \%)$ as a white solid and $\mathbf{1 3 9}(32.5 \mathrm{mg}, 0.200 \mathrm{mmol}, 28 \%)$ as a colorless oil.

\section{Table 4.2, Entry 10}

1-Methoxy-6-methylbenzimidazole (139) and 1-Methoxy-2-(2-methoxy-2-propyl)-6-

methylbenzimidazole (140) A mixture of 138 (114 mg, $0.555 \mathrm{mmol})$ and $\mathrm{NaH}(80 \%, 62.3 \mathrm{mg}, 2.08$ $\mathrm{mmol})$ in DMSO $(10 \mathrm{~mL})$ was stirred at ambient temperature under a nitrogen atmosphere for $1 \mathrm{~h}$. MeI $(111 \mu \mathrm{L}, 1.78 \mathrm{mmol})$ was added and the resulting orange solution was stirred for $1 \mathrm{~h}$. EtOAc $(15 \mathrm{~mL})$ was added and the mixture was washed with water $(6 \mathrm{X} 10 \mathrm{~mL})$. The organic phase was dried $\left(\mathrm{MgSO}_{4}\right)$ filtered, and the solvent was removed from the filtrate under reduced pressure. The crude was purified by chromatography (hexane/EtOAc, 1:1) to give, in order of elution, 140 (88.6 mg, $0.378 \mathrm{mmol}, 68 \%)$ and 139 (24.6 $\mathrm{mg}, 0.152 \mathrm{mmol}, 27 \%)$ both as a colorless oil. 


\section{Table 4.2, Entry 11}

1-Methoxybenzimidazole (124) and 1-Methoxy-2-(2-methoxy-2-propyl)benzimidazole (125) and 2(2-Hydroxy-2-propyl)-1-methoxybenzimidazole (126). A mixture of 122 (160.2 mg, $0.833 \mathrm{mmol}$ ) and $\mathrm{NaH}(80 \%, 93 \mathrm{mg}, 3.10 \mathrm{mmol})$ in DMSO $(10 \mathrm{~mL})$ was stirred at ambient temperature under a nitrogen atmosphere. After stirring for $1 \mathrm{~h}$, the mixture was cooled to $0{ }^{\circ} \mathrm{C}$ and $\mathrm{MeI}(166 \mu \mathrm{L}, 2.67 \mathrm{mmol})$ was added via a syringe. The reaction vessel was removed from the cold bath and stirred for an additional $1 \mathrm{~h}$. EtOAc $(30 \mathrm{~mL})$ was added and the mixture was washed with water $(20 \times 5 \mathrm{~mL})$ and brine $(20 \mathrm{~mL})$. The organic phase was dried $\left(\mathrm{MgSO}_{4}\right)$, filtered and the solvent was removed from the filtrate under reduced pressure. The residue was purified by chromatography (hexane/EtOAc, 7:3, then 3:7) to give, in order of elution, 124 (6.3 mg, $0.043 \mathrm{mmol}, 5 \%)$ and, 125 (105.1 mg, $0.477 \mathrm{mmol}, 57 \%)$ both as a colorless oil and 126 (25.3 mg, $0.123 \mathrm{mmol}, 15 \%)$ as a white solid.

\section{Table 4.2, Entry 12}

\section{1-Methoxybenzimidazole (124) and 1-Methoxy-2-(2-methoxy-2-propyl)benzimidazole (125).}

A mixture of $122(160 \mathrm{mg}, 0.832 \mathrm{mmol})$ and $\mathrm{NaH}(80 \%, 93.4 \mathrm{mg}, 3.11 \mathrm{mmol})$ in DMSO (10 mL) was stirred at ambient temperature under a nitrogen atmosphere for $1 \mathrm{~h}$. Methyl iodide (MeI, $166 \mu \mathrm{L}, 2.67$ mmol) was added via a syringe and the resulting orange solution was stirred for $1 \mathrm{~h}$. EtOAc $(20 \mathrm{~mL})$ was added and the mixture was washed with water $(6 \times 15 \mathrm{~mL})$. The organic phase was dried $\left(\mathrm{MgSO}_{4}\right)$, filtered and the solvent was removed from the filtrate under reduced pressure. The residue was purified by chromatography (hexane/EtOAc, 7:3 then 1:1) to give, in order of elution, 125 (62.6 mg, $0.284 \mathrm{mmol}$, $34 \%$ ) and 124 (37.8 mg, $0.255 \mathrm{mmol}, 31 \%$ ) both as a colorless oil.

\section{Table 4.2, Entry 13}

Methyl 4- $N$-methyl- $N$-(2-methyl-1-propen-1-yl)amino-3-nitrobenzoate. A mixture of methyl 4-( $N$-2methyl-1-propen-1-yl)amino-3-nitrobenzoate (142) (195 mg, $0.779 \mathrm{mmol}$ ) and $\mathrm{NaH}$ (80\%, $87.4 \mathrm{mg}, 2.91$ $\mathrm{mmol})$ in DMSO $(10 \mathrm{~mL})$ was stirred at ambient temperature under a nitrogen atmosphere for $10 \mathrm{~min}$. MeI $(104 \mu \mathrm{L}, 1.67 \mathrm{mmol})$ was added and the mixture was stirred for an additional $1 \mathrm{~h} 50 \mathrm{~min}$. EtOAc (20 $\mathrm{mL})$ was added to the yellow-orange solution and the mixture was washed with water $(5 \mathrm{x} 15 \mathrm{~mL})$ and brine $(15 \mathrm{~mL})$. The organic phase was dried $\left(\mathrm{MgSO}_{4}\right)$, filtered and the solvent was removed from the filtrate under reduced pressure. The crude product was purified by chromatography (hexane/EtOAc, 19:1) to give methyl 4- $N$-methyl- $N$-(2-methyl-1-propen-1-yl)amino-3-nitrobenzoate (128 mg, $0.484 \mathrm{mmol}$, $62 \%$ ) as a red viscous oil. 


\section{Table 4.2, Entry 14}

Methyl 1-methoxybenzimidazole-6-carboxylate (143). A mixture of 142 (83.5 mg, $0.334 \mathrm{mmol}$ ) and $\mathrm{NaH}(80 \%, 50.1 \mathrm{mg}, 1.67 \mathrm{mmol})$ in DMSO $(10 \mathrm{~mL})$ was stirred at ambient temperature under a nitrogen atmosphere for $24 \mathrm{~h}$. To the resulting colorless solution was added MeI (104 $\mu \mathrm{L}, 1.67 \mathrm{mmol})$ and it was stirred for $1 \mathrm{~h}$. EtOAc $(15 \mathrm{~mL})$ was added and the mixture was washed with water $(5 \times 10 \mathrm{~mL})$ and brine $(10 \mathrm{~mL})$. The organic phase was dried $\left(\mathrm{MgSO}_{4}\right)$, filtered and the solvent was removed from the filtrate under reduced pressure. The crude product was purified by chromatography (hexane/EtOAc, 1:1) to give 143 (51.0 mg, $0.247 \mathrm{mmol}, 74 \%)$ as a white solid.

\section{Table 4.2, Entry 15}

$N$-Methyl- $N$-(2-methyl-1-propen-1-yl)-2,4-dinitrobenzenamine. A mixture of $N$-(2-methyl-1-propen1-yl)-2,4-dinitrobenzenamine (144) $(45.1 \mathrm{mg}, 0.190 \mathrm{mmol})$ and $\mathrm{NaH}(80 \%, 21.3 \mathrm{mg}, 0.708 \mathrm{mmol})$ in DMSO (4 mL) was stirred at ambient temperature under a nitrogen atmosphere for $1 \mathrm{~h}$. The red solution was cooled to $0{ }^{\circ} \mathrm{C}$ and $\mathrm{MeI}(38 \mu \mathrm{L}, 0.61 \mathrm{mmol})$ was added via a syringe. The reaction vessel was removed from the cold bath and the resulting solution was stirred for $1 \mathrm{~h}$. EtOAc $(15 \mathrm{~mL})$ was added and the mixture was washed with water $(5 \times 10 \mathrm{~mL})$ and brine $(2 \times 10 \mathrm{~mL})$. The organic phase was dried $\left(\mathrm{MgSO}_{4}\right)$, filtered, and the solvent was removed from the filtrate under reduced pressure. The crude product was purified by chromatography (hexane/EtOAc, 8:2) to give $N$-methyl- $N$-(2-methyl-1-propen-1yl)-2,4-dinitrobenzenamine (40.6 $\mathrm{mg}, 0.162 \mathrm{mmol}, 85 \%)$ as a red oil.

\section{Table 4.2, Entry 16}

A mixture of $144(36 \mathrm{mg}, 0.15 \mathrm{mmol})$ and $\mathrm{NaH}(80 \%, 22.7 \mathrm{mg}, 0.758 \mathrm{mmol})$ in DMSO (5 mL) was stirred at ambient temperature under a nitrogen atmosphere for $24 \mathrm{~h}$. MeI (47 $\mu \mathrm{L}, 0.76 \mathrm{mmol})$ was added via a syringe and the resulting solution was stirred for an additional $2 \mathrm{~h}$. EtOAc $(10 \mathrm{~mL})$ was added and the mixture was washed with water $(5 \times 7 \mathrm{~mL})$ and brine $(2 \times 7 \mathrm{~mL})$. The organic phase was dried $\left(\mathrm{MgSO}_{4}\right)$, filtered, and the solvent was removed from the filtrate under reduced pressure. No product or unreacted starting material was observed in the ${ }^{1} \mathrm{H}$ NMR of the crude reaction mixture.

\section{Table 4.2, Entry 17}

5-Methoxy- $N$-methyl-2-nitrobenzenamine. A mixture of 5-methoxy-2-nitro- $N$-(2-methyl-1-propen-1yl)benzenamine (145) (156 mg, $0.703 \mathrm{mmol})$ and $\mathrm{NaH}(80 \%, 84.4 \mathrm{mg}, 2.81 \mathrm{mmol})$ in DMSO (10 mL) was stirred at ambient temperature under a nitrogen atmosphere for $1 \mathrm{~h}$. To the resulting orange mixture was added MeI $(145 \mu \mathrm{L}, 2.33 \mathrm{mmol})$ and the solution was stirred for $1 \mathrm{~h}$. EtOAc $(20 \mathrm{~mL})$ was added and 
the mixture was washed with water $(5 \times 15 \mathrm{~mL})$. The organic phase was dried $\left(\mathrm{MgSO}_{4}\right)$, filtered, and the solvent was removed from the filtrate under reduced pressure. The crude was purified by chromatography (hexane/EtOAc, 9:1) to give the 5-methoxy- $N$-methyl-2-nitrobenzenamine (71.0 mg, $0.390 \mathrm{mmol}, 55 \%$ ) as an orange solid.

\section{Table 4.2, Entry 18}

1,5-Dimethoxybenzimidazole (146). A mixture of $145(53 \mathrm{mg}, 0.24 \mathrm{mmol})$ and $\mathrm{NaH}(80 \%, 35.7 \mathrm{mg}$, $1.19 \mathrm{mmol})$ in DMSO (5 mL) was stirred at ambient temperature under a nitrogen atmosphere for $24 \mathrm{~h}$. To the resulting almost clear solution was added $\mathrm{MeI}(58 \mu \mathrm{L}, 0.93 \mathrm{mmol})$ and the solution was stirred for $1 \mathrm{~h}$. EtOAc $(10 \mathrm{~mL})$ was added and the mixture was washed with water $(7 \mathrm{x} 8 \mathrm{~mL})$. The organic phase was dried $\left(\mathrm{MgSO}_{4}\right)$, filtered, and the solvent was removed from the filtrate under reduced pressure. The crude was purified by chromatography (hexane/EtOAc, 1:1) to give $146(21.6 \mathrm{mg}, 0.121 \mathrm{mmol}, 51 \%)$ as a colorless oil.

\section{Table 4.2, Entry 19}

5-Chloro-2-nitro- $N$-methyl- $N$-(2-methyl-1-propen-1-yl)benzenamine and 5-chloro-1-methoxy-2-(2methoxy-2-propyl)benzimidazole (149). A mixture of 147 (175 mg, $0.775 \mathrm{mmol})$ and $\mathrm{NaH}(80 \%, 87.0$ $\mathrm{mg}, 2.90 \mathrm{mmol})$ in DMSO $(10 \mathrm{~mL})$ was stirred at ambient temperature under a nitrogen atmosphere for 6 min. MeI (160 $\mu \mathrm{L}, 2.57 \mathrm{mmol})$ was added and the resulting solution was stirred for $2 \mathrm{~h}$. EtOAc $(20 \mathrm{~mL})$ was added and the mixture was washed with water $(5 \times 15 \mathrm{~mL})$ and brine $(15 \mathrm{~mL})$. The organic phase was dried $\left(\mathrm{MgSO}_{4}\right)$, filtered, and the solvent was removed from the filtrate under reduced pressure. The crude product was purified by chromatography (hexane/EtOAc, 8:2 then 1:1) to give, in order of elution, 5chloro-2-nitro- $N$-methyl- $N$-(2-methyl-1-propen-1-yl)benzenamine (109 mg, $0.452 \mathrm{mmol}, 52 \%)$ and 149 (17.8 $\mathrm{mg}, 0.070 \mathrm{mmol}, 9 \%)$ both as a yellow oil.

Table 4.2, Entry 20

\section{5-Chloro-1-methoxybenzimidazole (148) and 5-chloro-1-methoxy-2-(2-methoxy-2-} propyl)benzimidazole (149). A mixture of 5-chloro-2-nitro- $N$-(2-methyl-1-propen-1-yl)benzenamine (147) $(225 \mathrm{mg}, 0.991 \mathrm{mmol})$ and $\mathrm{NaH}(80 \%, 111 \mathrm{mg}, 3.70 \mathrm{mmol})$ in DMSO (15 mL) was stirred at ambient temperature under a nitrogen atmosphere for $1 \mathrm{~h}$. The orange solution was cooled to $0{ }^{\circ} \mathrm{C}$ and MeI (205 $\mu \mathrm{L}, 3.29 \mathrm{mmol})$ was added. The reaction vessel was removed from the cold bath and stirred for $1 \mathrm{~h}$. EtOAc $(20 \mathrm{~mL})$ was added and the mixture was washed with water $(5 \times 15 \mathrm{~mL})$ and brine $(15 \mathrm{~mL})$. The organic phase was dried $\left(\mathrm{MgSO}_{4}\right)$, filtered, and the solvent was removed from the filtrate under 
reduced pressure. The crude product was purified by chromatography (hexane/EtOAc, 7:3 then 1:1) to give, in order of elution, $149(68.2 \mathrm{mg}, 0.268 \mathrm{mmol}, 27 \%)$ and $148(101 \mathrm{mg}, 0.555 \mathrm{mmol}, 56 \%)$ both as a yellow oil.

\section{Table 4.2, Entry 21}

\section{5-Chloro-1-methoxybenzimidazole (148) and 5-chloro-1-methoxy-2-(2-methoxy-2-} propyl)benzimidazole (149). A mixture of $147(53.3 \mathrm{mg}, 0.235 \mathrm{mmol})$ and $\mathrm{NaH}(80 \%, 26.4 \mathrm{mg}, 0.879$ mmol) in DMSO (2 mL) was stirred at ambient temperature under a nitrogen atmosphere for $1 \mathrm{~h}$. MeI (49 $\mu \mathrm{L}, 0.783 \mathrm{mmol}$ ) was added. The reaction mixture was stirred for an additional $1 \mathrm{~h}$. EtOAc (10 mL) was added and the mixture was washed with water $(5 \times 7 \mathrm{~mL})$. The organic phase was dried $\left(\mathrm{MgSO}_{4}\right)$, filtered, and the solvent was removed from the filtrate under reduced pressure. The crude product was purified by chromatography (hexane/EtOAc, 7:3) to give, in order of elution, 149 (18.8 mg, $0.074 \mathrm{mmol}, 31 \%$ ) and 148 (22.5 mg, $0.123 \mathrm{mmol}, 52 \%)$ both as a yellow oil.

\section{Table 4.2, Entry 22}

5-Bromo-1-methoxybenzimidazole

propyl)benzimidazole (152). A mixture of 5-bromo-2-nitro- $N$-(2-methyl-1-propen-1-yl)benzenamine (150) (141 mg, $0.519 \mathrm{mmol})$ and $\mathrm{NaH}(80 \%, 58.4 \mathrm{mg}, 1.95 \mathrm{mmol})$ in DMSO (10 mL) was stirred at ambient temperature under a nitrogen atmosphere for approximately $10 \mathrm{~min}$. An immediate color change from purple to brown was observed. MeI $(107 \mu \mathrm{L}, 1.72 \mathrm{mmol})$ was added and the resulting orange solution was stirred for $2 \mathrm{~h}$. EtOAc $(15 \mathrm{~mL})$ was added and the mixture was washed with water $(5 \times 10$ $\mathrm{mL})$ and brine $(10 \mathrm{~mL})$. The organic phase was dried $\left(\mathrm{MgSO}_{4}\right)$, filtered, and the solvent was removed from the filtrate under reduced pressure. The crude product was purified by chromatography (hexane/EtOAc, 7:3 then 1:1) to give, in order of elution, 152 (43.4 mg, $0.145 \mathrm{mmol}, 28 \%$ ) as a red oil and 151 (79.3 mg, $0.349 \mathrm{mmol}, 67 \%)$ as a pale yellow oil.

Table 4.2, Entry 23

\section{5-Bromo-1-methoxybenzimidazole (151) and 5-bromo-1-methoxy-2-(2-methoxy-2-} propyl)benzimidazole (152). A mixture of 150 (76.8 $\mathrm{mg}, 0.283 \mathrm{mmol})$ and $\mathrm{NaH}(80 \%, 32 \mathrm{mg}, 1.07$ mmol) in DMSO (9 mL) was stirred at ambient temperature under a nitrogen atmosphere for approximately $1 \mathrm{~h}$. To the resulting orange solution was added MeI $(59 \mu \mathrm{L}, 0.943 \mathrm{mmol})$ was added and stirred for an additional $1 \mathrm{~h}$. EtOAc $(12 \mathrm{~mL})$ was added and the mixture was washed with water $(7 \mathrm{x} 8$ $\mathrm{mL})$. The organic phase was dried $\left(\mathrm{MgSO}_{4}\right)$, filtered, and the solvent was removed from the filtrate under 
reduced pressure. The crude product was purified by chromatography (hexane/EtOAc, 7:3 then 1:1) to give, in order of elution, 152 (24.8 $\mathrm{mg}, 0.083 \mathrm{mmol}, 29 \%)$ as a red oil and 151 (35.4 mg, $0.156 \mathrm{mmol}$, $55 \%$ ) as a pale yellow oil.

Table 4.2, Entry 24

1-Methoxy-5-methylbenzimidazole

(154),

1-Methoxy-2-(2-methoxy-2-propyl)-5methylbenzimidazole (155), and 2-(2-Hydroxy-2-propyl)-1-methoxy-5-methylbenzimidazole (156). A mixture of 5-methyl-2-nitro- $N$-(2-methyl-1-propen-1-yl)benzenamine (153) (136 mg, $0.657 \mathrm{mmol}$ ) and $\mathrm{NaH}(80 \%, 73.4 \mathrm{mg}, 2.45 \mathrm{mmol})$ in DMSO $(10 \mathrm{~mL})$ was stirred at ambient temperature under a nitrogen atmosphere for $1 \mathrm{~h}$. The yellowish-brown solution was cooled to $0{ }^{\circ} \mathrm{C}$ and $\mathrm{MeI}(136 \mu \mathrm{L}, 2.18 \mathrm{mmol})$ was added. The reaction vessel was removed from the cold bath and the resulting orange solution was stirred for $1 \mathrm{~h}$. EtOAc $(20 \mathrm{~mL})$ was added and the mixture was washed with water $\left(5_{\mathrm{X}} 15 \mathrm{~mL}\right)$ and brine $(15 \mathrm{~mL})$. The organic phase was dried $\left(\mathrm{MgSO}_{4}\right)$, filtered, and the solvent was removed from the filtrate under reduced pressure. The crude product was purified by chromatography (hexane/EtOAc, 7:3 then 3:7) to give, in order of elution, 155 (33.6 mg, $0.143 \mathrm{mmol}, 22 \%)$ as an orange oil, 156 (29.2 $\mathrm{mg}, 0.133 \mathrm{mmol}$, $20 \%)$ as a white solid and $154(22.7 \mathrm{mg}, 0.140 \mathrm{mmol}, 21 \%)$ as an orange oil.

\section{Table 4.2, Entry 25}

\section{1-Methoxy-5-methylbenzimidazole \\ and 1-Methoxy-2-(2-methoxy-2-propyl)-5-}

methylbenzimidazole (155). A mixture of 5-methyl-2-nitro- $N$-(2-methyl-1-propen-1-yl)benzenamine (153) $(47.1 \mathrm{mg}, 0.228 \mathrm{mmol})$ and $\mathrm{NaH}(80 \%, 25.6 \mathrm{mg}, 0.854 \mathrm{mmol})$ in DMSO (5 mL) was stirred at ambient temperature under a nitrogen atmosphere for approximately $10 \mathrm{~min}$. To the yellowish brown solution was added MeI (47 $\mu \mathrm{L}, 0.76 \mathrm{mmol})$ and the resulting orange solution was stirred for $2 \mathrm{~h}$. EtOAc $(8 \mathrm{~mL})$ was added and the mixture was washed with water $(5 \times 5 \mathrm{~mL})$ and brine $(5 \mathrm{~mL})$. The organic phase was dried $\left(\mathrm{MgSO}_{4}\right)$, filtered, and the solvent was removed from the filtrate under reduced pressure. The crude product was purified by chromatography (hexane/EtOAc, 7:3 then 1:1) to give, in order of elution, 155 (13.8 mg, $0.059 \mathrm{mmol}, 26 \%)$ and 154 (11.5 mg, $0.071 \mathrm{mmol}, 31 \%)$ both as an orange oil.

\section{Table 4.2, Entry 26}

1,6-Dimethoxy-2-(1-methoxycyclohexyl)benzimidazole (158). A mixture of 4-methoxy-2-nitro- $N$ (cyclohexylmethylene)benzenamine (157) (149 mg, $0.569 \mathrm{mmol})$ and $\mathrm{NaH}(80 \%, 63.5 \mathrm{mg}, 2.12 \mathrm{mmol})$ in DMSO $(10 \mathrm{~mL})$ was stirred at ambient temperature under a nitrogen atmosphere for $1 \mathrm{~h}$. The orangeyellow solution was cooled to $0{ }^{\circ} \mathrm{C}$ and $\mathrm{MeI}(113 \mu \mathrm{L}, 1.82 \mathrm{mmol})$ was added. The reaction vessel was 
removed from the cold bath and the resulting orange solution was stirred for $1 \mathrm{~h}$. EtOAc $(20 \mathrm{~mL})$ was added and the mixture was washed with water $(5 \times 20 \mathrm{~mL})$ and brine $(20 \mathrm{~mL})$. The organic phase was concentrated under reduced pressure and the residue was purified by chromatography (hexane/EtOAc, 7:3) to give 158 (129 mg, $0.446 \mathrm{mmol}, 78 \%$ ) as a colorless oil.

Table 4.2, Entry 27

\section{1,6-Dimethoxy-2-(1-methoxycyclohexyl)benzimidazole}

(158),

1,6-Dimethoxy-2-(1hydroxycyclohexyl)benzimidazole (159). A mixture of 157 (104 mg, $0.396 \mathrm{mmol})$ and $\mathrm{NaH}(80 \%, 44.5$ $\mathrm{mg}, 1.48 \mathrm{mmol})$ in DMSO $(10 \mathrm{~mL})$ was stirred at ambient temperature under a nitrogen atmosphere for 2 min. An immediate color change from purple to brown was observed. MeI ( $82 \mu \mathrm{L}, 1.32 \mathrm{mmol})$ was added and the resulting orange solution was stirred for $2 \mathrm{~h}$. EtOAc $(20 \mathrm{~mL})$ was added and the mixture was washed with water $(5 \times 20 \mathrm{~mL})$ and brine $(15 \mathrm{~mL})$. The organic phase was dried $\left(\mathrm{MgSO}_{4}\right)$, filtered, and the solvent was removed from the filtrate under reduced pressure. The crude product was purified by chromatography (hexane/EtOAc, 7:3 then 4:6) to give, in order of elution, 158 (97 mg, $0.33 \mathrm{mmol}, 84 \%$ ) as a colorless oil and $\mathbf{1 5 9}(12.2 \mathrm{mg}, 0.044 \mathrm{mmol}, 11 \%)$ as a white solid.

\section{Table 4.2, Entry 28}

6-Bromo-1-methoxybenzimidazole

(134),

6-Bromo-1-methoxy-(1-methoxy-1phenylethyl)benzimidazole (161), and 6-bromo-(1-hydroxy-1-phenylethyl)-1-methoxybenzimidazole (162). A mixture of 4-bromo-2-nitro- $N$-(2-phenyl-1-propen-1-yl)benzenamine (160) (157 mg, 0.471 $\mathrm{mmol})$ and $\mathrm{NaH}(80 \%, 52.6 \mathrm{mg}, 1.75 \mathrm{mmol})$ in DMSO $(10 \mathrm{~mL})$ was stirred at ambient temperature under a nitrogen atmosphere for $1 \mathrm{~h}$. The brown reaction mixture was cooled to $0{ }^{\circ} \mathrm{C}$ and $\mathrm{MeI}(94 \mu \mathrm{L}, 1.51$ mmol) was added. The reaction vessel was removed from the cold bath and the resulting orange solution was stirred for $1 \mathrm{~h}$. EtOAc $(20 \mathrm{~mL})$ was added and the mixture was washed with water $(5 \times 15 \mathrm{~mL})$ and brine $(15 \mathrm{~mL})$. The organic phase was dried $\left(\mathrm{MgSO}_{4}\right)$, filtered, and the solvent was removed from the filtrate under reduced pressure. The crude product was purified by chromatography (hexane/EtOAc, 7:3 then 3:7) to give, in order of elution, 161 (37.2 $\mathrm{mg}, 0.103 \mathrm{mmol}, 22 \%)$ as an orange oil, 162 (20.5 $\mathrm{mg}$, $0.059 \mathrm{mmol}, 13 \%)$ as a colorless oil and $134(25.7 \mathrm{mg}, 0.113 \mathrm{mmol}, 22 \%)$ as a white solid.

\section{Table 4.2, Entry 29}

6-Bromo-1-methoxybenzimidazole (134). A mixture of $160(50.0 \mathrm{mg}, 0.150 \mathrm{mmol})$ and $\mathrm{NaH}(80 \%$, $16.9 \mathrm{mg}, 0.563 \mathrm{mmol})$ in DMSO $(5 \mathrm{~mL})$ was stirred at ambient temperature under a nitrogen atmosphere for approximately $38 \mathrm{~min}$. Color change was not observed in the specified period of time. MeI $(31 \mu \mathrm{L}$, 
$0.50 \mathrm{mmol}$ ) was added and the resulting faint yellow solution was stirred for an $1 \mathrm{~h} 22 \mathrm{~m}$. EtOAc (10 mL) was added and the mixture was washed with water $(5 \times 5 \mathrm{~mL})$ and brine $(5 \mathrm{~mL})$. The organic phase was dried $\left(\mathrm{MgSO}_{4}\right)$, filtered, and the solvent was removed from the filtrate under reduced pressure. The crude product was purified by chromatography (hexane/EtOAc, 7:3 then 3:7) to give 134 (34.1 $\mathrm{mg}, 0.150$ mmol, $92 \%$ ) as a white solid.

\section{Scheme 4.3}

2-(2-Hydroxy-2,2-diphenylmethyl)-1,6-dimethoxybenzimidazole (169) and benzophenone (170). A mixture of 4-methoxy-2-nitro- $N$-(2,2-diphenylethenyl)benzenamine (168) (124 mg, $0.358 \mathrm{mmol})$ and $\mathrm{NaH}(80 \%, 40.1 \mathrm{mg}, 1.34 \mathrm{mmol})$ in DMSO $(10 \mathrm{~mL})$ was stirred at ambient temperature under a nitrogen atmosphere for $35 \mathrm{~min}$. MeI ( $74 \mu \mathrm{L}, 1.19 \mathrm{mmol})$ was added and the resulting red solution was stirred for $1 \mathrm{~h} 25 \mathrm{~min}$. EtOAc $(20 \mathrm{~mL})$ was added and the mixture was washed with water $(5 \times 15 \mathrm{~mL})$ and brine $(15$ $\mathrm{mL})$. The organic phase was dried $\left(\mathrm{MgSO}_{4}\right)$, filtered, and the solvent was removed from the filtrate under reduced pressure. The crude product was purified by chromatography (hexane/EtOAc, 7:3 then 3:7) to give, in order of elution, 170 (5.7 mg, $0.03 \mathrm{mmol}, 9 \%)$ as a colorless oil and $\mathbf{1 6 9}$ (115 mg, $0.320 \mathrm{mmol}$, $89 \%$ ) as a red viscous oil.

\section{Scheme 4.3}

1,6-Dimethoxylbenzimidazole (128) and benzophenone (170). A mixture of 168 (124 mg, $0.385 \mathrm{mmol})$ and t-BuOK (201 mg, $1.79 \mathrm{mmol})$ in $\mathrm{t}-\mathrm{BuOH}(5 \mathrm{~mL})$ was stirred at ambient temperature under a nitrogen atmosphere for $24 \mathrm{~h}$. MeI $(89 \mu \mathrm{L}, 1.43 \mathrm{mmol})$ was added and the mixture was stirred for $1 \mathrm{~h}$. The resulting mixture was filtered through silica gel and silica gel was washed with EtOAc. The solvent was removed from the filtrate under reduced pressure and the resulting residue was purified by chromatography (hexane/EtOAc, 9:1 then 1:1) to give, in order of elution, 170 (63.6 mg, $0.349 \mathrm{mmol}$, $97 \%$ ) and 128 (49.8 $\mathrm{mg}, 0.279 \mathrm{mmol}, 78 \%$ ) both as a colorless oil.

\section{Table 4.3, Entry 1}

2-(2-Hydroxy-2-propyl)-1-methoxybenzimidazole (126). A mixture of 122 (83.1 $\mathrm{mg}, 0.432 \mathrm{mmol})$ and $\mathrm{NaH}(80 \%, 27.3 \mathrm{mg}, 0.908 \mathrm{mmol})$ in DMSO $(8 \mathrm{~mL})$ was stirred at ambient temperature under a nitrogen atmosphere. After stirring for $1 \mathrm{~h}$, the mixture was cooled to $0{ }^{\circ} \mathrm{C}$ and $\mathrm{MeI}(28 \mu \mathrm{L}, 0.454 \mathrm{mmol})$ was added via a syringe. The reaction vessel was removed from the cold bath and stirred for an additional $1 \mathrm{~h}$. EtOAc $(13 \mathrm{~mL})$ was added and the mixture was washed with water $(7 \times 8 \mathrm{~mL})$. The organic phase was dried $\left(\mathrm{MgSO}_{4}\right)$, filtered and the solvent was removed from the filtrate under reduced pressure. The residue 
was purified by chromatography (hexane/EtOAc, 7:3 then 1:1) to give $\mathbf{1 2 6}$ (49.4 mg, $0.240 \mathrm{mmol}, 55 \%)$ as a white solid.

\section{Table 4.3, Entry 2}

1,6-Dimethoxybenzimidazole (128) and 2-(2-Hydroxy-2-propyl)-1,6-dimethoxy-benzimidazole (130). A mixture of 127 (120 mg, $0.540 \mathrm{mmol})$ and $\mathrm{NaH}(80 \%, 34.0 \mathrm{mg}, 1.13 \mathrm{mmol})$ in DMSO (10 mL) was stirred at ambient temperature under a nitrogen atmosphere. After $1 \mathrm{~h}$, the mixture was cooled to $0{ }^{\circ} \mathrm{C}$ and MeI (35 $\mu \mathrm{L}, 0.57 \mathrm{mmol})$ was added via a syringe. The reaction vessel was removed from the cold bath and stirred for an additional $1 \mathrm{~h}$. EtOAc $(15 \mathrm{~mL})$ was added and the mixture was washed with water $(6 \times 10$ $\mathrm{mL})$. The organic phase was dried $\left(\mathrm{MgSO}_{4}\right)$, filtered and the solvent was removed from the filtrate under reduced pressure. The residue was purified by chromatography (hexane/EtOAc, 1:1) to give an inseparable mixture of $\mathbf{1 2 8}$ and $\mathbf{1 3 0}\left(96.1 \mathrm{mg}, \mathbf{1 2 8}(2 \%)\right.$ and $\mathbf{1 3 0}(74 \%)$ as calculated from ${ }^{1} \mathrm{H}$ NMR spectrum) as a white solid.

\section{Table 4.3, Entry 3}

6-Chloro-1-methoxy-2-(2-methoxy-2-propyl)benzimidazole (132) and 6-Chloro-2-(2-hydroxy-2propyl)-1-methoxybenzimidazole (163). A mixture of 131 (121 mg, $0.534 \mathrm{mmol})$ and $\mathrm{NaH}$ (80\%, 33.6 $\mathrm{mg}, 1.12 \mathrm{mmol})$ in DMSO (10 mL) was stirred at ambient temperature under a nitrogen atmosphere. After stirring for $1 \mathrm{~h}$, the mixture was cooled to $0{ }^{\circ} \mathrm{C}$ and $\mathrm{MeI}(35 \mu \mathrm{L}, 0.56 \mathrm{mmol})$ was added via a syringe. The reaction vessel was removed from the cold bath and stirred for an additional $1 \mathrm{~h}$. EtOAc $(20 \mathrm{~mL})$ was added and the mixture was washed with water $(8 \times 15 \mathrm{~mL})$. The organic phase was dried $\left(\mathrm{MgSO}_{4}\right)$, filtered and the solvent was removed from the filtrate under reduced pressure. The residue was purified by chromatography (hexane/EtOAc, 7:3, then 6:4)) to give, in order of elution, 132 (27.5 mg, $0.108 \mathrm{mmol}$, $20 \%)$ as a orange solid and $\mathbf{1 6 3}(76.0 \mathrm{mg}, 0.316 \mathrm{mmol}, 59 \%)$ as a white solid.

\section{Table 4.3, Entry 4}

\section{6-Bromo-1-methoxy-2-(2-methoxy-2-propyl)benzimidazole (135) and 6-Bromo-2-(2-hydroxy-2-} propyl)-1-methoxybenzimidazole (164). A mixture of 133 (71.3 mg, $0.263 \mathrm{mmol})$ and $\mathrm{NaH}(80 \%, 16.6$ $\mathrm{mg}, 0.554 \mathrm{mmol})$ in DMSO (8 mL) was stirred at ambient temperature under a nitrogen atmosphere. After stirring for $1 \mathrm{~h}$, the mixture was cooled to $0{ }^{\circ} \mathrm{C}$ and $\mathrm{MeI}(17 \mu \mathrm{L}, 0.275 \mathrm{mmol})$ was added via a syringe. The reaction vessel was removed from the cold bath and stirred for an additional $1 \mathrm{~h}$. EtOAc (12 mL) was added and the mixture was washed with water $(7 \mathrm{x} 8 \mathrm{~mL})$. The organic phase was dried $\left(\mathrm{MgSO}_{4}\right)$, filtered and the solvent was removed from the filtrate under reduced pressure. The residue was purified by 
chromatography (hexane/EtOAc, 6:4) to give, in order of elution, 135 (17.1 mg, $0.057 \mathrm{mmol}, 22 \%)$ as an orange oil and 164 (51.0 $\mathrm{mg}, 0.178 \mathrm{mmol}, 68 \%)$ as a white solid.

\section{Table 4.3, Entry 5}

\section{6-Fluoro-1-methoxy-2-(2-methoxy-2-propyl)benzimidazole (137) and 6-Fluoro-2-(2-hydroxy-2-} propyl)-1-methoxybenzimidazole (165). A mixture of 136 (80 mg, $0.381 \mathrm{mmol})$ and $\mathrm{NaH}(80 \%, 24 \mathrm{mg}$, $0.80 \mathrm{mmol})$ in DMSO $(8 \mathrm{~mL})$ was stirred at ambient temperature under a nitrogen atmosphere. After stirring for $1 \mathrm{~h}$, the mixture was cooled to $0{ }^{\circ} \mathrm{C}$ and $\mathrm{MeI}(25 \mu \mathrm{L}, 0.40 \mathrm{mmol})$ was added via a syringe. The reaction vessel was removed from the cold bath and stirred for an additional $1 \mathrm{~h}$. EtOAc $(10 \mathrm{~mL})$ was added and the mixture was washed with water $(7 \times 8 \mathrm{~mL})$. The organic phase was dried $\left(\mathrm{MgSO}_{4}\right)$, filtered and the solvent was removed from the filtrate under reduced pressure. The residue was purified by chromatography (hexane/EtOAc, 6:4)) to give, in order of elution, 137 (14 mg, $0.06 \mathrm{mmol}, 15 \%)$ as a pale yellow oil and 165 (48.1 $\mathrm{mg}, 0.215 \mathrm{mmol}, 56 \%)$ as a white solid.

\section{Table 4.3, Entry 6}

\section{1-Methoxy-6-methylbenzimidazole \\ and \\ 2-(2-Hydroxy-2-propyl)-1-methoxy-6-} methylbenzimidazole (141). A mixture of $138(83.0 \mathrm{mg}, 0.402 \mathrm{mmol})$ and $\mathrm{NaH}(80 \%, 25.4 \mathrm{mg}, 0.908$ $\mathrm{mmol})$ in DMSO $(8 \mathrm{~mL})$ was stirred at ambient temperature under a nitrogen atmosphere. After stirring for $1 \mathrm{~h}$, the mixture was cooled to $0{ }^{\circ} \mathrm{C}$ and $\mathrm{MeI}(26 \mu \mathrm{L}, 0.422 \mathrm{mmol})$ was added via a syringe. The reaction vessel was removed from the cold bath and stirred for an additional $1 \mathrm{~h}$. EtOAc $(13 \mathrm{~mL})$ was added and the mixture was washed with water $(7 \times 8 \mathrm{~mL})$. The organic phase was dried $\left(\mathrm{MgSO}_{4}\right)$, filtered and the solvent was removed from the filtrate under reduced pressure. The residue was purified by chromatography (hexane/EtOAc, 6:4) to give an inseparable mixture of 139 and 141 (57.5 mg, 139 (11\%) and 141 (45\%) as calculated from ${ }^{1} \mathrm{H}$ NMR spectrum) as a white solid.

\section{Table 4.3, Entry 7}

Methyl 4- $N$-methyl- $N$-(2-methyl-1-propen-1-yl)amino-3-nitrobenzoate. A mixture of 142 (83 mg, $0.33 \mathrm{mmol})$ and $\mathrm{NaH}(80 \%, 21 \mathrm{mg}, 0.70 \mathrm{mmol})$ in DMSO $(8 \mathrm{~mL})$ was stirred at ambient temperature under a nitrogen atmosphere for $1 \mathrm{~h}$. The dirty brown mixture was cooled to $0{ }^{\circ} \mathrm{C}$ and $\mathrm{MeI}(22 \mu \mathrm{L}, 0.35$ mmol) was added. The reaction vessel was removed from the cold bath and the resulting solution was stirred for an additional $1 \mathrm{~h}$. EtOAc $(12 \mathrm{~mL})$ was added and the mixture was washed with water $(8 \mathrm{x} 8$ $\mathrm{mL})$. The organic phase was dried $\left(\mathrm{MgSO}_{4}\right)$, filtered and the solvent was removed from the filtrate under 
reduced pressure. An 3:1 mixture of methyl 4- $N$-methyl- $N$-(2-methyl-1-propen-1-yl)amino-3nitrobenzoate and 159 was observed in the ${ }^{1} \mathrm{H}$ NMR of the crude reaction mixture.

\section{Table 4.3, Entry 8}

Methyl 1-methoxybenzimidazole-6-carboxylate (143). A mixture of $142(110.2 \mathrm{mg}, 0.440 \mathrm{mmol})$ and $\mathrm{NaH}(80 \%, 27.7 \mathrm{mg}, 0.925 \mathrm{mmol})$ in DMSO (8 mL) was stirred at ambient temperature under a nitrogen atmosphere. After $24 \mathrm{~h}$, the mixture was cooled to $0{ }^{\circ} \mathrm{C}$ and $\mathrm{MeI}(29 \mu \mathrm{L}, 0.465 \mathrm{mmol})$ was added via a syringe. The reaction vessel was removed from the cold bath and stirred for an additional $1 \mathrm{~h}$. EtOAc (15 $\mathrm{mL}$ ) was added and the mixture was washed with water $(7 \times 8 \mathrm{~mL})$. The organic phase was dried $\left(\mathrm{MgSO}_{4}\right)$, filtered and the solvent was removed from the filtrate under reduced pressure. The residue was purified by chromatography (hexane/EtOAc, 1:1 then 3:7) to give 143 (66.2 $\mathrm{mg}, 0.321 \mathrm{mmol}, 73 \%$ ) as a white solid.

\section{Table 4.3, Entry 10}

1,5-Dimethoxybenzimidazole (146). A mixture of 145 (98.5 mg, $0.443 \mathrm{mmol})$ and $\mathrm{NaH}(80 \%, 27.9 \mathrm{mg}$, $0.930 \mathrm{mmol})$ in DMSO $(8 \mathrm{~mL})$ was stirred at ambient temperature under a nitrogen atmosphere. After 24 $\mathrm{h}$, the mixture was cooled to $0{ }^{\circ} \mathrm{C}$ and $\mathrm{MeI}(29 \mu \mathrm{L}, 0.465 \mathrm{mmol})$ was added via a syringe. The reaction vessel was removed from the cold bath and stirred for an additional $1 \mathrm{~h}$. EtOAc $(15 \mathrm{~mL})$ was added and the mixture was washed with water $(7 \times 8 \mathrm{~mL})$. The organic phase was dried $\left(\mathrm{MgSO}_{4}\right)$, filtered and the solvent was removed from the filtrate under reduced pressure. The residue was purified by chromatography (hexane/EtOAc, 1:1 then 3:7) to give 146 (50.8 mg, $0.285 \mathrm{mmol}, 64 \%$ ) as a colorless oil.

\section{Table 4.3, Entry 11}

5-Chloro-1-methoxy-2-(2-methoxy-2-propyl)benzimidazole (149) and 5-Chloro-2-(2-hydroxy-2propyl)-1-methoxybenzimidazole (166). A mixture of $147(118 \mathrm{mg}, 0.521 \mathrm{mmol})$ and $\mathrm{NaH}(80 \%, 32.7$ $\mathrm{mg}, 1.09 \mathrm{mmol})$ in DMSO $(10 \mathrm{~mL})$ was stirred at ambient temperature under a nitrogen atmosphere. After stirring for $1 \mathrm{~h}$, the mixture was cooled to $0{ }^{\circ} \mathrm{C}$ and $\mathrm{MeI}(34 \mu \mathrm{L}, 0.55 \mathrm{mmol})$ was added via a syringe. The reaction vessel was removed from the cold bath and stirred for an additional $1 \mathrm{~h}$. EtOAc $(15 \mathrm{~mL})$ was added and the mixture was washed with water $(7 \times 10 \mathrm{~mL})$. The organic phase was dried $\left(\mathrm{MgSO}_{4}\right)$, filtered and the solvent was removed from the filtrate under reduced pressure. The residue was purified by chromatography (hexane/EtOAc, 7:3, then 1:1)) to give, in order of elution, 149 (20.1 mg, $0.079 \mathrm{mmol}$, $15 \%)$ and 166 (69.1 $\mathrm{mg}, 0.287 \mathrm{mmol}, 55 \%)$ both as yellow oils. 
Table 4.3, Entry 12

5-Bromo-1-methoxy-2(2-methoxy-2-propyl)benzimidazole (152) and 5-Bromo-2(2-hydroxy-2propyl)-1-methoxybenzimidazole (167) A mixture of 150 (73 mg, $0.27 \mathrm{mmol})$ and $\mathrm{NaH}(80 \%, 17 \mathrm{mg}$, $0.57 \mathrm{mmol}$ ) in DMSO (8 $\mathrm{mL}$ ) was stirred at ambient temperature under a nitrogen atmosphere for $1 \mathrm{~h}$. The reddish-brown solution was cooled to $0{ }^{\circ} \mathrm{C}$ and MeI $(18 \mu \mathrm{L}, 0.28 \mathrm{mmol})$ was added. The reaction vessel was removed from the cold bath and the mixture was stirred for $1 \mathrm{~h}$. EtOAc $(13 \mathrm{~mL})$ was added and the mixture was washed with water $(7 \times 8 \mathrm{~mL})$. The organic phase was dried $\left(\mathrm{MgSO}_{4}\right)$, filtered, and the solvent was removed from the filtrate under reduced pressure. The crude product was purified by chromatography (hexane/EtOAc, 6:4) to give, in order of elution, $152(12.9 \mathrm{mg}, 0.043 \mathrm{mmol}, 16 \%)$ as a red oil and 167 (48 mg, $0.17 \mathrm{mmol}, 63 \%$ ) as a white solid.

\section{Table 4.3, Entry 13}

2-(2-Hydroy-2-propyl)-1-methoxy-5-methylbenzimidazole (156). A mixture of 153 (100 mg, 0.485 $\mathrm{mmol})$ and $\mathrm{NaH}(80 \%, 30.5 \mathrm{mg}, 1.017 \mathrm{mmol})$ in DMSO $(10 \mathrm{~mL})$ was stirred at ambient temperature under a nitrogen atmosphere. After stirring for $1 \mathrm{~h}$, the mixture was cooled to $0{ }^{\circ} \mathrm{C}$ and $\mathrm{MeI}(32 \mu \mathrm{L}, 0.51$ mmol) was added via a syringe. The reaction vessel was removed from the cold bath and stirred for an additional $1 \mathrm{~h}$. EtOAc $(15 \mathrm{~mL})$ was added and the mixture was washed with water $(7 \times 10 \mathrm{~mL})$. The organic phase was dried $\left(\mathrm{MgSO}_{4}\right)$, filtered and the solvent was removed from the filtrate under reduced pressure. The residue was purified by chromatography (hexane/EtOAc, 6:4)) to give $\mathbf{1 5 6}$ (65.2 mg, 0.296 mmol, $61 \%$ ) as a white solid.

\section{Table 4.3, Entry 14}

\section{1,6-Dimethoxy-2-(1-methoxycyclohexyl)benzimidazole}

(158),

1,6-Dimethoxy-2-(1-

hydroxycyclohexyl)benzimidazole (159). A mixture of 157 (73 mg, $0.28 \mathrm{mmol})$ and $\mathrm{NaH}(80 \%, 17.5$ $\mathrm{mg}, 0.583 \mathrm{mmol})$ in DMSO $(8 \mathrm{~mL})$ was stirred at ambient temperature under a nitrogen atmosphere. After stirring for $1 \mathrm{~h}$, the mixture was cooled to $0{ }^{\circ} \mathrm{C}$ and $\mathrm{MeI}(18 \mu \mathrm{L}, 0.29 \mathrm{mmol})$ was added via a syringe. The reaction vessel was removed from the cold bath and stirred for an additional $1 \mathrm{~h}$. EtOAc (12 mL) was added and the mixture was washed with water $(7 \times 8 \mathrm{~mL})$. The organic phase was dried $\left(\mathrm{MgSO}_{4}\right)$, filtered and the solvent was removed from the filtrate under reduced pressure. The residue was purified by chromatography (hexane/EtOAc, 6:4) to give, in order of elution, 158 (15.4 mg, $0.053 \mathrm{mmol}, 19 \%)$ as a colorless oil and 159 (39.4 mg, $0.143 \mathrm{mmol}, 51 \%)$ as a white solid. 
Table 4.3, Entry 15

\section{6-Bromo-1-methoxybenzimidazole}

(134)

and

6-Bromo-2-(2-hydroxy-2-phenylethyl)-1methoxybenzimidazole (162). A mixture of $160(73 \mathrm{mg}, 0.22 \mathrm{mmol})$ and $\mathrm{NaH}(80 \%, 13.7 \mathrm{mg}, 0.458$ $\mathrm{mmol})$ in DMSO $(8 \mathrm{~mL})$ was stirred at ambient temperature under a nitrogen atmosphere. After stirring for $1 \mathrm{~h}$, the mixture was cooled to $0{ }^{\circ} \mathrm{C}$ and $\mathrm{MeI}(14 \mu \mathrm{L}, 0.23 \mathrm{mmol})$ was added via a syringe. The reaction vessel was removed from the cold bath and stirred for an additional $1 \mathrm{~h}$. EtOAc $(13 \mathrm{~mL})$ was added and the mixture was washed with water $(7 \times 9 \mathrm{~mL})$. The organic phase was dried $\left(\mathrm{MgSO}_{4}\right)$, filtered and the solvent was removed from the filtrate under reduced pressure. The residue was purified by chromatography (hexane/EtOAc, 6:4) to give, in order of elution, 162 (39.4 mg, $0.114 \mathrm{mmol}, 52 \%)$ and 134 (10.6 mg, $0.047 \mathrm{mmol}, 22 \%)$ both as a white solid.

\section{Table 4.4, Entry 1}

1-Benzyloxy-2-(2-benzyloxy-2-propyl)-6-methoxybenzimidazole (171) and 1-benzyloxy-2-(2hydroxy-2-propyl)-6-methoxybenzimidazole (172) A mixture of 127 (108 mg, $0.484 \mathrm{mmol}$ ) and $\mathrm{NaH}$ $(80 \%, 54.0 \mathrm{mg}, 1.80 \mathrm{mmol})$ in DMSO $(10 \mathrm{~mL})$ was stirred at ambient temperature under a nitrogen atmosphere for $1 \mathrm{~h}$. The orange mixture was cooled to $0{ }^{\circ} \mathrm{C}$ and benzyl bromide $(\mathrm{BnBr}, 191 \mu \mathrm{L}, 1.61$ mmol) was added via a syringe. The resulting almost colorless solution was stirred for an additional $1 \mathrm{~h}$. EtOAc $(20 \mathrm{~mL})$ was added and the resulting mixture was washed with water $(5 \times 10 \mathrm{~mL})$ and brine $(10$ $\mathrm{mL})$. The organic phase was dried $\left(\mathrm{MgSO}_{4}\right)$, filtered, and the solvent was removed from the filtrate under reduced pressure. The crude product was purified by chromatography (hexane/EtOAc, 7:3 then 1:1) to give, in order of elution, 171 (56.3 $\mathrm{mg}, 0.140 \mathrm{mmol}, 29 \%)$ as an orange oil and 172 (56.8 $\mathrm{mg}, 0.182$ mmol, $38 \%$ ) as a white solid.

\section{Table 4.4, Entry 2}

1-Benzyloxy-2-(2-benzyloxy-2-propyl)-6-methoxybenzimidazole (171) and 1-Benzyloxy-2-(2hydroxyl-2-propyl)-6-methoxybenzimidazole (172). A mixture of 127 (104 mg, 0.469 mmol) and NaH $(80 \%, 52.7 \mathrm{mg}, 1.76 \mathrm{mmol})$ in DMSO $(10 \mathrm{~mL})$ was stirred at ambient temperature under a nitrogen atmosphere for approximately $2 \mathrm{~min}$. An immediate color change from purple to brown was observed. Benzyl bromide $(185 \mu \mathrm{L}, 1.56 \mathrm{mmol})$ was added via a syringe and the mixture was stirred for an additional $2 \mathrm{~h}$. EtOAc $(15 \mathrm{~mL})$ was added and the resulting mixture was washed with water $(5 \times 10 \mathrm{~mL})$ and brine $(10 \mathrm{~mL})$. The organic phase was dried $\left(\mathrm{MgSO}_{4}\right)$, filtered, and the solvent was removed from the filtrate under reduced pressure. The crude product was purified by chromatography (hexane/EtOAc, 7:3 
then 1:1) to give, in order of elution, $171(94.5 \mathrm{mg}, 0.235 \mathrm{mmol}, 50 \%)$ as an orange oil and $172(27.9 \mathrm{mg}$, $0.089 \mathrm{mmol}, 19 \%$ ) as a white solid.

Table 4.4, Entry 3

1-(2-Propen-1-yloxy)-2-(2-(2-propen-1-yloxy)-2-propyl)-6-methoxybenzimidazole (174) and 2-(2Hydroxyl-2-propyl)-1-(2-propen-1-yloxy)-6-methoxybenzimidazole (175) and 1-(2-propen-1-yloxy)7-methoxybenzimidazole (173). A mixture of $127(80.3 \mathrm{mg}, 0.389 \mathrm{mmol})$ and $\mathrm{NaH}(80 \%, 43.5 \mathrm{mg}, 1.45$ mmol) in DMSO ( $8 \mathrm{~mL}$ ) was stirred at ambient temperature under a nitrogen atmosphere for $1 \mathrm{~h}$. The orange mixture was cooled to $0{ }^{\circ} \mathrm{C}$ and allyl bromide $(108 \mu \mathrm{L}, 1.25 \mathrm{mmol})$ was added via syringe. The reaction vessel was removed from the cold bath and the resulting orange solution was stirred for an additional $1 \mathrm{~h}$. EtOAc $(15 \mathrm{~mL})$ was added and the mixture was washed with water $(5 \times 10 \mathrm{~mL})$ and brine $(10 \mathrm{~mL})$. The organic phase was dried $\left(\mathrm{MgSO}_{4}\right)$, filtered, and the solvent was removed from the filtrate under reduced pressure. The crude product was purified by chromatography (hexane/EtOAc, 7:3 then 1:1) to give, in order of elution, $174(16.7 \mathrm{mg}, 0.055 \mathrm{mmol}, 14 \%)$ as a pale yellow oil and a mixture of 173 and 175 (36.3 mg, 173 (5\%) and 175 (31\%) as calculated from ${ }^{1} \mathrm{H}$ NMR spectrum) as a white solid.

\section{Table 4.4, Entry 4}

1-(2-Propen-1-yloxy)-2-(2-(2-propen-1-yloxy)-2-propyl)-6-methoxybenzimidazole (174). A mixture of 127 (178 mg, $0.803 \mathrm{mmol})$ and $\mathrm{NaH}(80 \%, 96.4 \mathrm{mg}, 3.21 \mathrm{mmol})$ in DMSO (10 mL) was stirred at ambient temperature under a nitrogen atmosphere for approximately $1 \mathrm{~min}$. An immediate color change from purple to brown was observed. Allyl bromide $(236 \mu \mathrm{L}, 2.73 \mathrm{mmol})$ was added via syringe and the resulting orange mixture was stirred for an addition $2 \mathrm{~h}$. EtOAc $(20 \mathrm{~mL})$ was added and the mixture was washed with water $(5 \times 15 \mathrm{~mL})$ and brine $(15 \mathrm{~mL})$. The organic phase was dried $\left(\mathrm{MgSO}_{4}\right)$, filtered, and the solvent was removed from the filtrate under reduced pressure. The crude product was purified by chromatography (hexane/EtOAc) to give 174 (142.6 mg, $0.472 \mathrm{mmol}, 59 \%)$ as a yellow oil.

\section{Table 4.4, Entry 5}

8-Methoxy-4,4-dimethyl-4H-benzo[4,5]imidazo[1,2-b]dioxazine (176). A mixture of 127 (143 mg, $0.642 \mathrm{mmol})$ and $\mathrm{NaH}(80 \%, 72.0 \mathrm{mg}, 2.40 \mathrm{mmol})$ in DMSO $(10 \mathrm{~mL})$ was stirred at ambient temperature under a nitrogen atmosphere for approximately $2 \mathrm{~min}$. An immediate color change from purple to brown was observed. Diiodomethane $(172 \mu \mathrm{L}, 2.13 \mathrm{mmol})$ was added via a syringe and the resulting reddishbrown solution was stirred for an additional $2 \mathrm{~h}$. EtOAc $(20 \mathrm{~mL})$ was added and the mixture was washed with water $(5 \times 15 \mathrm{~mL})$ and brine $(15 \mathrm{~mL})$. The organic phase was dried $\left(\mathrm{MgSO}_{4}\right)$, filtered, and the solvent 
was removed from the filtrate under reduced pressure. The crude product was purified by chromatography (hexane/EtOAc, 1:1) to give $\mathbf{1 7 6}(59.6 \mathrm{mg}, 0.254 \mathrm{mmol}, 40 \%$ ) as a white solid.

Table 4.4, Entry 6

6-Methoxy-1-(2-propyn-1-yloxy)benzimidazole (177) and 2-(2-Hydroxy-2-propyl)-6-methoxy-1-(2propyn-1-yloxy)benzimidazole (178). A mixture of $127(311 \mathrm{mg}, 1.400 \mathrm{mmol})$ and $\mathrm{NaH}(80 \%, 157 \mathrm{mg}$, $5.23 \mathrm{mmol})$ in DMSO (15 mL) was stirred at ambient temperature under a nitrogen atmosphere for approximately $1 \mathrm{~min}$. An immediate color change from purple to brown was observed. Propargyl bromide (414 $\mu \mathrm{L}, 4.65 \mathrm{mmol}$ ) was added via a syringe and the resulting reddish-brown solution was stirred for an additional $2 \mathrm{~h}$. EtOAc $(20 \mathrm{~mL})$ was added and the mixture was washed with water $(7 \times 10 \mathrm{~mL})$. The organic phase was dried $\left(\mathrm{MgSO}_{4}\right)$, filtered, and the solvent was removed from the filtrate under reduced pressure. The crude product was purified by chromatography (hexane/EtOAc, 9:1, then 85:15) to give, in order of elution, 178 (171 mg, $0.656 \mathrm{mmol}, 47 \%)$ as a white solid and 177 (17 mg, $0.084 \mathrm{mmol}, 6 \%)$ as a pale yellow oil.

\section{Table 4.4, Entry 7}

1-(3-Butyn-2-yloxy)-6-methoxybenzimidazole (179) and 1-(3-Butyn-2-yloxy)-2-(2-hydroxy-2propyl)-6-methoxybenzimidazole (180). A mixture of 127 (100 mg, $0.450 \mathrm{mmol})$ and $\mathrm{NaH}(80 \%, 50.7$ $\mathrm{mg}, 1.69 \mathrm{mmol})$ in DMSO $(10 \mathrm{~mL})$ was stirred at ambient temperature under a nitrogen atmosphere. After $1 \mathrm{~h}$, the reaction mixture was cooled to $0{ }^{\circ} \mathrm{C}$ and 3-bromo-1-butyne $(135 \mu \mathrm{L}, 1.49 \mathrm{mmol})$ was added via syringe and the reaction vessel was removed from the cold bath and light brown solution was stirred for an additional $1 \mathrm{~h}$. EtOAc $(20 \mathrm{~mL})$ was added and the mixture was washed with water $(5 \times 15 \mathrm{~mL})$ and brine $(15 \mathrm{~mL})$. The organic phase was dried $\left(\mathrm{MgSO}_{4}\right)$, filtered, and the solvent was removed from the filtrate under reduced pressure. The crude product was purified by chromatography (hexane/EtOAc, 1:1) to give, in order of elution, 180 (50.1 $\mathrm{mg}, 0.183 \mathrm{mmol}, 41 \%)$ as a white solid and $\mathbf{1 7 9}$ (9 $\mathrm{mg}, 0.042 \mathrm{mmol}$, $9 \%)$ as a colorless liquid.

\section{Table 4.4, Entry 8}

1-(3-Butyn-2-yloxy)-2-(2-hydroxy-2-propyl)-6-methoxybenzimidazole (180). A mixture of 127 (180 $\mathrm{mg}, 0.871 \mathrm{mmol})$ and $\mathrm{NaH}(80 \%, 97.3 \mathrm{mg}, 3.24 \mathrm{mmol})$ in DMSO $(10 \mathrm{~mL})$ was stirred at ambient temperature under a nitrogen atmosphere. After $1 \mathrm{~h}$, the reaction mixture was cooled to $0{ }^{\circ} \mathrm{C}$ and 3 bromo-1-butyne $(261 \mu \mathrm{L}, 2.79 \mathrm{mmol})$ was added via syringe and the reaction vessel was removed from the cold bath and light brown solution was stirred for an additional $1 \mathrm{~h}$. EtOAc $(20 \mathrm{~mL})$ was added and 
the mixture was washed with water $(5 \times 15 \mathrm{~mL})$ and brine $(15 \mathrm{~mL})$. The organic phase was dried $\left(\mathrm{MgSO}_{4}\right)$, filtered, and the solvent was removed from the filtrate under reduced pressure. The crude product was purified by chromatography (hexane/EtOAc, 7:3) to give $\mathbf{1 8 0}(157 \mathrm{mg}, 0.572 \mathrm{mmol}, 66 \%$ ) as a white solid.

\section{Scheme 4.4}

1-Acetoxy-2-(2-acetoxy-2-propyl)-6-methoxybenzimidazole (181) and 1-Acetoxy-2-(2-propen-2-yl)-

6-methoxybenzimidazole (182). A mixture of $127(334 \mathrm{mg}, 1.50 \mathrm{mmol})$ and $\mathrm{NaH}(80 \%, 169 \mathrm{mg}, 5.62$ $\mathrm{mmol})$ in DMSO $(15 \mathrm{~mL})$ was stirred at ambient temperature under a nitrogen atmosphere for $1 \mathrm{~h}$. The reaction mixture was cooled to $0{ }^{\circ} \mathrm{C}$ and acetyl chloride $(355 \mu \mathrm{L}, 4.97 \mathrm{mmol})$ was added via a syringe. The reaction vessel was removed from the cold bath and the resulting mixture was stirred for an additional $1 \mathrm{~h}$. EtOAc $(20 \mathrm{~mL})$ was added and the mixture was washed with water $(7 \times 15 \mathrm{~mL})$. The organic phase was dried $\left(\mathrm{MgSO}_{4}\right)$, filtered, and the solvent was removed from the filtrate under reduced pressure. The crude product was purified by chromatography (hexane/EtOAc, 7:3, then 1:1) to give, in order of elution, 182 (20.6 mg, $0.084 \mathrm{mmol}, 6 \%)$ as a pale yellow oil and $\mathbf{1 8 1}$ (80.9 mg, $0.264 \mathrm{mmol}$, $18 \%$ ) as a yellow oil. 
4-Methoxy-2-nitro- $N$-(2-phenyl-1-propen-1-yl)benzenamine (190). To a solution of 4-methoxy-2nitrobenzenamine (500 $\mathrm{mg}, 2.974 \mathrm{mmol}$ ) and 2-phenylpropanal (399.1 $\mathrm{mg}, 2.974 \mathrm{mmol})$ in dichloromethane (DCM, $12 \mathrm{~mL}$ ) at ambient temperature under a nitrogen atmosphere was added $4 \AA$ molecular sieves $\left(3 \mathrm{~g}\right.$, activated by heating at $120{ }^{\circ} \mathrm{C}$ under vacuum overnight, then stored under nitrogen). The reaction mixture was allowed to sit without agitation or stirring for $48 \mathrm{~h}$. The mixture was then filtered and the sieves were washed with DCM $(20 \mathrm{~mL})$. The filtrate was concentrated under reduced pressure and the resulting residue was purified by chromatography (hexane/EtOAc, 95:5) to give 190 (501 mg, $1.762 \mathrm{mmol}, 59 \%)$ as a red solid. $\mathrm{mp}=85-86{ }^{\circ} \mathrm{C} ;{ }^{1} \mathrm{H} \mathrm{NMR}\left(400 \mathrm{MHz}, \mathrm{CDCl}_{3}\right) \delta 9.92$ (d, $J=10.8$ $\mathrm{Hz}, 1 \mathrm{H}), 9.83$ (d, J=10.8 Hz, 1H), 7.57 (d, J=2.8 Hz, 1H), 7.49 (d, J=2.0 Hz, 1H), 7.38-7.32 (m, 5H), 7.29-7.21 (m, 3H), 7.18-7.12 (m, 2H), 7.10-7.04 (m, 4H), 6.85 (dq, J=10.8, 0.8 Hz, 1H), 6.50 (dq, J=10.8, $0.8 \mathrm{~Hz}, 1 \mathrm{H}), 3.74(\mathrm{~s}, 3 \mathrm{H}), 3.71(\mathrm{~s}, 3 \mathrm{H}), 2.12(\mathrm{~d}, J=1.2 \mathrm{~Hz}, 3 \mathrm{H}), 2.06(\mathrm{~d}, J=1.2 \mathrm{~Hz}, 1 \mathrm{H}) ;{ }^{13} \mathrm{C}$ NMR $(100$ $\left.\mathrm{MHz}, \mathrm{CDCl}_{3}\right) \delta 151.1,150.7,141.4,139.1,136.1,136.0,131.8,131.3,129.0,128.5,127.3,127.3,127.0$, 126.9, 126.4, 125.2, 121.4, 119.9, 118.8, 118.4, 115.8, 115.6, 107.1, 107.0, 55.8, 55.8, 22.0, 14.6; IR (ATR) 3311, 1640, 1571, 1513, 1163, $1060 \mathrm{~cm}^{-1}$; HRMS (ESI) calcd for $\mathrm{C}_{16} \mathrm{H}_{17} \mathrm{~N}_{2} \mathrm{O}_{3}\left(\mathrm{M}+\mathrm{H}^{+}\right) 285.1239$, found 285.1225 .

1-Methoxybenzimidazole (124). ${ }^{136}$ A mixture of $N$-(2-methyl-1-propen-1-yl)-2-nitrobenzenamine (122) $)^{134}(63.8 \mathrm{mg}, 0.332 \mathrm{mmol})$ and $t$-BuOK $(149 \mathrm{mg}, 1.33 \mathrm{mmol})$ in $t$-BuOH $(5 \mathrm{~mL})$ was stirred at ambient temperature under a nitrogen atmosphere for $24 \mathrm{~h}$. MeI $(62 \mu \mathrm{L}, 0.996 \mathrm{mmol})$ was added via a syringe and the mixture was stirred for an additional $1 \mathrm{~h}$. The resulting orange mixture containing floating white solids was filtered through silica gel and the filtrate was washed with EtOAc $(10 \mathrm{~mL})$. The solvent was removed from the filtrate under reduced pressure. The crude product was purified by chromatography (hexane/EtOAc, 1:1) to give $\mathbf{1 2 4}(0.049 \mathrm{mg}, 0.332 \mathrm{mmol}, 100 \%)$ as a colorless oil.

1,6-Dimethoxybenzimidazole (128). ${ }^{136}$ Reaction of a solution of 4-methoxy-2-nitro- $N$-(2-methyl-1propen-1-yl)benzenamine $(\mathbf{1 2 7})^{134}(216 \mathrm{mg}, 0.973 \mathrm{mmol})$ with $\mathrm{MeI}(194 \mu \mathrm{L}, 3.15 \mathrm{mmol})$ in $t$-BuOH $(10$ $\mathrm{mL})$ in the presence of $t$-BuOK $(437 \mathrm{mg}, 3.89 \mathrm{mmol})$, at ambient temperature under a nitrogen atmosphere, as described for 124 (25 h), gave after work up and chromatography (hexane/EtOAc, 1:1), 128 (131 mg, $0.735 \mathrm{mmol}, 76 \%)$ as a colorless oil.

1-Methoxy-6-methylbenzimidazole (139). ${ }^{136}$ Reaction of a solution of 4-methyl-2-nitro- $N$-(2-methyl-1propen-1-yl)benzenamine $(\mathbf{1 3 8})^{136}(76 \mathrm{mg}, 0.368 \mathrm{mmol})$ with $\mathrm{MeI}(69 \mu \mathrm{L}, 1.105 \mathrm{mmol})$ in $t$ - $\mathrm{BuOH}(5$ 
$\mathrm{mL})$ in the presence of $t$ - $\mathrm{BuOK}(165.4 \mathrm{mg}, 1.474 \mathrm{mmol})$, at ambient temperature under a nitrogen atmosphere, as described for 124 (25 h), gave after work up and chromatography (hexane/EtOAc, 1:1), 139 (45.5 mg, $0.281 \mathrm{mmol}, 76 \%$ ) as a colorless oil.

6-Chloro-1-methoxybenzimidazole (191). Reaction of a solution of 4-chloro-2-nitro- $N$-(2-methyl-1propen-1-yl)benzenamine (131) ${ }^{134}(75 \mathrm{mg}, 0.331 \mathrm{mmol})$ with $\mathrm{MeI}(62 \mu \mathrm{L}, 0.993 \mathrm{mmol})$ in $t$-BuOH $(5$ $\mathrm{mL})$ in the presence of $t$-BuOK $(148.5 \mathrm{mg}, 1.323 \mathrm{mmol})$, at ambient temperature under a nitrogen atmosphere, as described for 1234 (25 h), gave after work up and chromatography (hexane/EtOAc, 1:1), 191 (55.6 mg, $0.304 \mathrm{mmol}, 92 \%)$ as a colorless oil. ${ }^{1} \mathrm{H}$ NMR (400 MHz, $\left.\mathrm{CDCl}_{3}\right) \delta 8.05(\mathrm{~s}, 1 \mathrm{H}), 7.66(\mathrm{~d}$, $J=8.8 \mathrm{~Hz}, 1 \mathrm{H}), 7.47(\mathrm{~d}, J=2.0 \mathrm{~Hz}, 1 \mathrm{H}), 7.23(\mathrm{dd}, J=8.8,2.0 \mathrm{~Hz}, 1 \mathrm{H}), 4.15(\mathrm{~s}, 3 \mathrm{H}) ;{ }^{13} \mathrm{C} \mathrm{NMR}(100 \mathrm{MHz}$, $\left.\mathrm{CDCl}_{3}\right) \delta 138.2,137.9,129.8,129.6,123.4,121.8,108.5,67.4$; IR (neat) 3293, 1623, 1473, 1346, 1214 $\mathrm{cm}^{-1} \mathrm{HRMS}$ (ESI) calcd for $\mathrm{C}_{8} \mathrm{H}_{7} \mathrm{ClN}_{2} \mathrm{O}\left(\mathrm{M}+\mathrm{Na}^{+}\right)$205.0144, found 205.0139.

6-Bromo-1-methoxybenzimidazole (134). ${ }^{136}$ Reaction of a solution of 4-bromo-2-nitro- $N$-(2-methyl-1propen-1-yl)benzenamine (133) ${ }^{136}(136.2 \mathrm{mg}, 0.502 \mathrm{mmol})$ with $\mathrm{MeI}(94 \mu \mathrm{L}, 1.506 \mathrm{mmol})$ in $t$-BuOH $(8$ $\mathrm{mL})$ in the presence of $t$-BuOK $(225.5 \mathrm{mg}, 2.010 \mathrm{mmol})$, at ambient temperature under a nitrogen atmosphere, as described for 124 (25 h), gave after work up and chromatography (hexane/EtOAc, 1:1), 134 (96.9 $\mathrm{mg}, 0.427 \mathrm{mmol}, 85 \%)$ as a colorless oil.

6-Fluoro-1-methoxybenzimidazole (192). Reaction of a solution of 4-fluoro-2-nitro- $N$-(2-methyl-1propen-1-yl)benzenamine (136) ${ }^{136}(118.1 \mathrm{mg}, 0.562 \mathrm{mmol})$ with $\mathrm{MeI}(75 \mu \mathrm{L}, 1.197 \mathrm{mmol})$ in $t$-BuOH $(10$ $\mathrm{mL})$ in the presence of $t$-BuOK $(252.2 \mathrm{mg}, 2.248 \mathrm{mmol})$, at ambient temperature under a nitrogen atmosphere, as described for 124 (25 h), gave after work up and chromatography (hexane/EtOAc, 1:1), $192(62.6 \mathrm{mg}, 0.377 \mathrm{mmol}, 67 \%)$ as a colorless oil. ${ }^{1} \mathrm{H} \mathrm{NMR}\left(400 \mathrm{MHz}, \mathrm{CDCl}_{3}\right) \delta 8.06(\mathrm{~s}, 1 \mathrm{H}), 7.70(\mathrm{dd}$, $J=5.6,2.8 \mathrm{~Hz}, 1 \mathrm{H}), 7.16(\mathrm{dd}, J=5.6,1.6 \mathrm{~Hz}, 1 \mathrm{H}), 7.03(\mathrm{td}, J=6.4,1.6 \mathrm{~Hz}, 1 \mathrm{H}), 4.17(\mathrm{~s}, 3 \mathrm{H}) ;{ }^{13} \mathrm{C} \mathrm{NMR}$ $\left(100 \mathrm{MHz}, \mathrm{CDCl}_{3}\right) \delta 161.4,158.9,138.1,135.8,122.0\left(\mathrm{~d}, J^{\mathrm{C}-\mathrm{F}}=10 \mathrm{~Hz}, 1 \mathrm{C}\right), 111.3\left(\mathrm{~d}, J^{\mathrm{C}-\mathrm{F}}=25 \mathrm{~Hz}, 1 \mathrm{C}\right)$, $95.1\left(\mathrm{~d}, J^{\mathrm{C}-\mathrm{F}}=28 \mathrm{~Hz}, 1 \mathrm{C}\right), 67.2$; IR (neat) 3395, 1626, 1493, 1357, $1225 \mathrm{~cm}^{-1}$; HRMS (ESI) calcd for $\mathrm{C}_{8} \mathrm{H}_{8} \mathrm{~N}_{2} \mathrm{O}_{2}\left(\mathrm{M}+\mathrm{H}^{+}\right)$167.0621, found 167.0614.

1,5-Dimethoxybenzimidazole (146). ${ }^{136}$ Reaction of a solution of 5-methoxy-2-nitro- $N$-(2-methyl-1propen-1-yl)benzenamine (12) ${ }^{136}(77.2 \mathrm{mg}, 0.347 \mathrm{mmol})$ with $\mathrm{MeI}(65 \mu \mathrm{L}, 1.041 \mathrm{mmol})$ in $t$ - $\mathrm{BuOH}(5$ 
$\mathrm{mL})$ in the presence of $t$ - $\mathrm{BuOK}(155.9 \mathrm{mg}, 1.389 \mathrm{mmol})$, at ambient temperature under a nitrogen atmosphere, as described for 124 (25 h), gave after work up and chromatography (hexane/EtOAc, 1:1), 146 (45.4 mg, $0.255 \mathrm{mmol}, 73 \%$ ) as a colorless oil.

1-Methoxy-5-methylbenzimidazole (154). ${ }^{136}$ Reaction of a solution of 5-methyl-2-nitro- $N$-(2-methyl-1propen-1-yl)benzenamine (153) ${ }^{136}(183 \mathrm{mg}, 0.887 \mathrm{mmol})$ with $\mathrm{MeI}(166 \mu \mathrm{L}, 2.662 \mathrm{mmol})$ in $t$-BuOH $(10$ $\mathrm{mL})$ in the presence of $t$-BuOK $(398.3 \mathrm{mg}, 3.550 \mathrm{mmol})$, at ambient temperature under a nitrogen atmosphere, as described for 124 (25 h), gave after work up and chromatography (hexane/EtOAc, 1:1), 154 (102.3 $\mathrm{mg}, 0.631 \mathrm{mmol}, 71 \%)$ as an orange oil.

5-Chloro-1-methoxybenzimidazole (148). ${ }^{136}$ Reaction of a solution of 5-chloro-2-nitro- $N$-(2-methyl-1propen-1-yl)benzenamine (147) ${ }^{134}(197.9 \mathrm{mg}, 0.873 \mathrm{mmol})$ with $\mathrm{MeI}(163 \mu \mathrm{L}, 2.619 \mathrm{mmol})$ in $t$-BuOH $(10 \mathrm{~mL})$ in the presence of $t$-BuOK $(391.9 \mathrm{mg}, 3.493 \mathrm{mmol})$, at ambient temperature under a nitrogen atmosphere, as described for $\mathbf{1 2 4}(25 \mathrm{~h})$, gave after work up and chromatography (hexane/EtOAc, 1:1), 148 (113.5 mg, $0.622 \mathrm{mmol}, 71 \%)$ as a yellow oil.

5-Bromo-1-methoxybenzimidazole (151). ${ }^{136}$ Reaction of a solution of 5-bromo-2-nitro- $N$-(2-methyl-1propen-1-yl)benzenamine (150) ${ }^{136}(86.9 \mathrm{mg}, 0.321 \mathrm{mmol})$ with $\mathrm{MeI}(60 \mu \mathrm{L}, 0.962 \mathrm{mmol})$ in $t$-BuOH $(5$ $\mathrm{mL})$ in the presence of $t$-BuOK $(143.9 \mathrm{mg}, 1.282 \mathrm{mmol})$, at ambient temperature under a nitrogen atmosphere, as described for 124 (25 h), gave after work up and chromatography (hexane/EtOAc, 1:1), 151 (53.5 mg, $0.236 \mathrm{mmol}, 73 \%)$ as a faint yellow oil.

1,6-Dimethoxybenzimidazole (128). ${ }^{136}$ Reaction of a solution of 4-methoxy-2-nitro- $N$ (cyclohexylmethylene)benzenamine $(\mathbf{1 5 7})^{134}(339.5 \mathrm{mg}, 1.295 \mathrm{mmol})$ with $\mathrm{MeI}(258 \mu \mathrm{L}, 4.144 \mathrm{mmol})$ in $t$ - $\mathrm{BuOH}(10 \mathrm{~mL})$ in the presence of $t$-BuOK $(580.9 \mathrm{mg}, 5.177 \mathrm{mmol})$, at ambient temperature under a nitrogen atmosphere, as described for $124(25 \mathrm{~h})$, gave after work up and chromatography (hexane/EtOAc, 1:1), 128 (176.1 mg, $0.988 \mathrm{mmol}, 76 \%$ ) as a colorless oil. 
1,6-Dimethoxybenzimidazole (128). Reaction of a solution of 4-methoxy-2-nitro- $N$-(2,2diphenylethylene)benzenamine $(\mathbf{1 6 8})^{134}(123.9 \mathrm{mg}, 0.358 \mathrm{mmol})$ with $\mathrm{MeI}(89 \mu \mathrm{L}, 1.432 \mathrm{mmol})$ in $t$ $\mathrm{BuOH}(5 \mathrm{~mL})$ in the presence of $t$-BuOK $(200.7 \mathrm{mg}, 1.789 \mathrm{mmol})$, at ambient temperature under a nitrogen atmosphere, as described for $124(25 \mathrm{~h})$, gave after work up and chromatography (hexane/EtOAc, 9:1, then,1:1), in order of elution, benzophenone (170, $63.6 \mathrm{mg}, 0.349 \mathrm{mmol}, 98 \%$ ) and 128 (49.8 $\mathrm{mg}, 0.279 \mathrm{mmol}, 78 \%)$ both as a colorless oil.

6-Bromo-1-methoxybenzimidazole (134). ${ }^{136}$ Reaction of a solution of 4-bromo-2-nitro- $N$-(2-phenyl-1propen-1-yl)benzenamine $(\mathbf{1 6 0})^{136}(50.8 \mathrm{mg}, 0.153 \mathrm{mmol})$ with $\mathrm{MeI}(29 \mu \mathrm{L}, 0.459 \mathrm{mmol})$ in $t$-BuOH $(3$ $\mathrm{mL})$ in the presence of $t$-BuOK $(68.6 \mathrm{mg}, 0.611 \mathrm{mmol})$, at ambient temperature under a nitrogen atmosphere, as described for $\mathbf{1 2 4}$ (25 h), gave after work up and chromatography (hexane/EtOAc, 1:1), 134 (32.5 mg, $0.143 \mathrm{mmol}, 94 \%$ ) as a colorless oil that solidified upon standing.

1-Benzyloxy-6-methoxybenzimidazole (193). Reaction of a solution of 127 (109.7 mg, $0.494 \mathrm{mmol})$ with benzyl bromide $(\mathrm{BnBr}, 152 \mu \mathrm{L}, 1.283 \mathrm{mmol})$ in $t$-BuOH $(8 \mathrm{~mL})$ in the presence of $t$-BuOK $(208.3$ $\mathrm{mg}, 1.856 \mathrm{mmol})$, at ambient temperature under a nitrogen atmosphere, as described for $\mathbf{1 2 4}(25 \mathrm{~h})$, gave after work up and chromatography (hexane/EtOAc, 95:5, 6:4) 193 (103.4 mg, $0.407 \mathrm{mmol}, 82 \%)$ as a colorless oil. ${ }^{1} \mathrm{H}$ NMR $\left(400 \mathrm{MHz}, \mathrm{CDCl}_{3}\right) \delta 7.59$ (d, $\left.J=8.8 \mathrm{~Hz}, 1 \mathrm{H}\right), 7.57$ (s, 1H), 7.41-7.34 (m, 3H), 7.31$7.28(\mathrm{~m}, 2 \mathrm{H}), 6.87(\mathrm{dd}, J=8.8,2.4 \mathrm{~Hz}, 1 \mathrm{H}), 6.72(\mathrm{~d}, J=2.4 \mathrm{~Hz}, 1 \mathrm{H}), 5.19(\mathrm{~s}, 2 \mathrm{H}), 3.80(\mathrm{~s}, 3 \mathrm{H})$; ${ }^{13} \mathrm{C}$ NMR $\left(100 \mathrm{MHz}, \mathrm{CDCl}_{3}\right) \delta 157.1,137.6,133.8,133.6,130.3,129.8,129.6,128.8,121.3,112.2,91.5,80.9$, 55.6; IR (neat) 1624, 1494, 1234, 1061, $816 \mathrm{~cm}^{-1}$; HRMS (ESI) calcd for $\mathrm{C}_{15} \mathrm{H}_{15} \mathrm{~N}_{2} \mathrm{O}_{2}\left(\mathrm{M}+\mathrm{H}^{+}\right) 255.1133$, found 255.1123 .

6-methoxy-1-(2-propen-1-yloxy)benzimidazole (194). Reaction of a solution of 127 (52.2 mg, 0.235 mmol) with allyl bromide $(41 \mu \mathrm{L}, 0.470 \mathrm{mmol})$ in $t$-BuOH $(5 \mathrm{~mL})$ in the presence of $t$-BuOK $(105.4 \mathrm{mg}$, $0.939 \mathrm{mmol})$, at ambient temperature under a nitrogen atmosphere, as described for $\mathbf{1 2 4}(25 \mathrm{~h})$, gave after work up and chromatography (hexane/EtOAc, 1:1) 194 (47.9 mg, $0.235 \mathrm{mmol}, 100 \%)$ as a colorless oil.

1-(2-Methyl-2-propen-1-yloxy)-6-methoxybenzimidazole (195). Reaction of a solution of 127 (106.4 $\mathrm{mg}, 0.479 \mathrm{mmol})$ with 3-bromo-2-methyl-1-propene $(102 \mu \mathrm{L}, 1.016 \mathrm{mmol})$ in $t$-BuOH $(10 \mathrm{~mL})$ in the presence of $t$-BuOK $(214.9 \mathrm{mg}, 1.915 \mathrm{mmol})$, at ambient temperature under a nitrogen atmosphere, as 
described for $124(25 \mathrm{~h}$ ), gave after work up and chromatography (hexane/EtOAc, 7:3) 195 (94.3 mg, $0.432 \mathrm{mmol}, 90 \%)$ as a colorless oil. ${ }^{1} \mathrm{H} \mathrm{NMR}\left(400 \mathrm{MHz}, \mathrm{CDCl}_{3}\right) \delta 7.85(\mathrm{~s}, 1 \mathrm{H}), 7.59$ (d, J=9.2 Hz, 1H), $6.88(\mathrm{~d}, J=2.4 \mathrm{~Hz}, 1 \mathrm{H}), 6.86$ (dd, $J=9.6,2.4 \mathrm{~Hz}, 1 \mathrm{H}), 5.05$ (pentet, $J=1.6 \mathrm{~Hz}, 1 \mathrm{H}$ ), 4.93 (pentet, $J=0.8 \mathrm{~Hz}$, $1 \mathrm{H}), 4.59$ (s, with further fine splitting, $2 \mathrm{H}), 3.83(\mathrm{~s}, 3 \mathrm{H}), 1.94(\mathrm{t}, J=1.2 \mathrm{~Hz}, 3 \mathrm{H}) ;{ }^{13} \mathrm{C} \mathrm{NMR}(100 \mathrm{MHz}$, $\left.\mathrm{CDCl}_{3}\right) \delta 157.1,138.2,137.4,133.6,130.0,121.3,118.4,112.0,91.5,82.9,55.6,19.6$; IR (neat) 1629, 1499, 1449, 1230, 917, $822 \mathrm{~cm}^{-1}$; HRMS (ESI) calcd for $\mathrm{C}_{12} \mathrm{H}_{15} \mathrm{~N}_{2} \mathrm{O}_{2}\left(\mathrm{M}+\mathrm{H}^{+}\right)$219.1133, found 219.1123.

6-Methoxy-1-(1,2-propadien-1-yloxy)benzimidazole (196). Reaction of a solution of 127 (131.3 mg, $0.591 \mathrm{mmol})$ with propargyl bromide $(112 \mu \mathrm{L}, 1.259 \mathrm{mmol})$ in $t$ - $\mathrm{BuOH}(8 \mathrm{~mL})$ in the presence of $t$-BuOK (259.2 $\mathrm{mg}, 2.310 \mathrm{mmol}$ ), at ambient temperature under a nitrogen atmosphere, as described for 124 (25 h), gave after work up and chromatography (hexane/EtOAc, 1:1) 196 (17.4 mg, $0.086 \mathrm{mmol}, 15 \%)$ as a yellow oil. ${ }^{1} \mathrm{H}$ NMR (400 MHz, $\left.\mathrm{CDCl}_{3}\right) \delta 7.91(\mathrm{~s}, 1 \mathrm{H}), 7.54(\mathrm{~d}, J=8.8 \mathrm{~Hz}, 1 \mathrm{H}), 7.28$ (d, J=2.4 Hz, 1H), $7.13(\mathrm{t}, J=6.4 \mathrm{~Hz}, 1 \mathrm{H}), 6.95(\mathrm{dd}, J=9.2,2.4 \mathrm{~Hz}, 1 \mathrm{H}), 5.69$ (d, $J=6.4 \mathrm{~Hz}, 2 \mathrm{H}), 3.87(\mathrm{~s}, 3 \mathrm{H})$; ${ }^{13} \mathrm{C}$ NMR $(100$ $\left.\mathrm{MHz}, \mathrm{CDCl}_{3}\right) \delta 203.0,156.5,145.1,141.8,127.3,113.5,111.2,102.6,95.3,88.2,55.8$; IR (neat) 3367, 1488, 1239, 1144, $850 \mathrm{~cm}^{-1}$; HRMS (ESI) calcd for $\mathrm{C}_{11} \mathrm{H}_{11} \mathrm{~N}_{2} \mathrm{O}_{2}\left(\mathrm{M}+\mathrm{H}^{+}\right)$208.0820, found 208.0811.

1-(1,2-Butadien-1-yloxy)-6-methoxybenzimidazole (197). Reaction of a solution of 127 (222.2 mg, $1.000 \mathrm{mmol})$ with 3-bromo-1-butyne $(181 \mu \mathrm{L}, 2.000 \mathrm{mmol})$ in $t$-BuOH $(12 \mathrm{~mL})$ in the presence of $t$ BuOK (449.6 mg, $4.007 \mathrm{mmol}$ ), at ambient temperature under a nitrogen atmosphere, as described for 124 (25 h), gave after work up and chromatography (hexane/EtOAc, 1:1) 197 (26.1 mg, $0.121 \mathrm{mmol}$, $12 \%)$ as a yellow oil. ${ }^{1} \mathrm{H}$ NMR $\left(400 \mathrm{MHz}, \mathrm{CDCl}_{3}\right) \delta 8.01(\mathrm{~s}, 1 \mathrm{H}), 7.63(\mathrm{~d}, J=8.8 \mathrm{~Hz}, 1 \mathrm{H}), 6.93(\mathrm{~d}, J=2.4$ $\mathrm{Hz}, 1 \mathrm{H}), 6.90(\mathrm{dd}, J=8.8,2.4 \mathrm{~Hz}, 1 \mathrm{H}), 4.82$ (q, J=2.4 Hz, 2H), $3.86(\mathrm{~s}, 3 \mathrm{H}), 1.85(\mathrm{t}, J=2.4 \mathrm{~Hz}, 3 \mathrm{H}) ;{ }^{13} \mathrm{C}$ NMR (100 MHz, $\left.\mathrm{CDCl}_{3}\right) \delta 157.2,138.4,137.9,133.7,130.3,121.4,112.3,91.7,87.7,72.3,66.9,55.8$; IR (neat) 1624, 1495, 1234, 1061, $816 \mathrm{~cm}^{-1}$; HRMS (ESI) calcd for $\mathrm{C}_{12} \mathrm{H}_{13} \mathrm{~N}_{2} \mathrm{O}_{2}\left(\mathrm{M}+\mathrm{H}^{+}\right)$217.0977, found 217.0968.

1-Acetoxy-6-methoxybenzimidazole (198). Reaction of a solution of 127 (130.3 mg, $0.586 \mathrm{mmol})$ with acetyl chloride $(88 \mu \mathrm{L}, 1.236 \mathrm{mmol})$ in $t$ - $\mathrm{BuOH}(10 \mathrm{~mL})$ in the presence of $t$-BuOK $(263.2 \mathrm{mg}, 2.346$ $\mathrm{mmol})$, at ambient temperature under a nitrogen atmosphere, as described for $\mathbf{1 2 4}(25 \mathrm{~h})$, gave after work up and chromatography (hexane/EtOAc, 6:4) 198 (65.4 mg, $0.317 \mathrm{mmol}, 54 \%)$ as a white solid. $\mathrm{mp}=231$ $231.5{ }^{\circ} \mathrm{C},{ }^{1} \mathrm{H}$ NMR (400 MHz, DMSO-d 6 ) $\delta 11.28$ (br, s, 1H), 7.84 (d, J=8.8 Hz, 1H), 6.61 (dd, J=8.8, 2.4 $\mathrm{Hz}, 1 \mathrm{H}), 6.56(\mathrm{~d}, \mathrm{~J}=2.4 \mathrm{~Hz}, 1 \mathrm{H}), 3.73(\mathrm{~s}, 3 \mathrm{H}), 2.57$ (s, 3H); ${ }^{13} \mathrm{C}$ NMR $\left(100 \mathrm{MHz}, \mathrm{DSMO}-\mathrm{d}_{6}\right) \delta 169.9$, 156.6, 152.7, 129.5, 120.8, 115.6, 106.8, 95.4, 55.4, 25.0; IR (neat) 1709, 1634, 1377, 1332, 1158, 1014 $\mathrm{cm}^{-1}$; HRMS (ESI) calcd for $\mathrm{C}_{10} \mathrm{H}_{10} \mathrm{~N}_{2} \mathrm{O}_{3}\left(\mathrm{M}+\mathrm{Na}^{+}\right)$229.0589, found 229.0596. 
1-(Heptyloxy)-6-methoxybenzimidazole (199). Reaction of a solution of 127 (113.6 mg, $0.511 \mathrm{mmol})$ with 1-bromoheptane $(169 \mu \mathrm{L}, 1.073 \mathrm{mmol})$ in $t$-BuOH $(10 \mathrm{~mL})$ in the presence of $t$-BuOK $(229.4 \mathrm{mg}$, $2.044 \mathrm{mmol}$ ), at ambient temperature under a nitrogen atmosphere, as described for $\mathbf{1 2 4}(25 \mathrm{~h})$, gave after work up and chromatography (hexane/EtOAc, 7:3) 199 (21.8 $\mathrm{mg}, 0.083 \mathrm{mmol}, 16 \%)$ as a colorless oil. ${ }^{1} \mathrm{H}$ NMR $\left(400 \mathrm{MHz}, \mathrm{CDCl}_{3}\right) \delta 7.93(\mathrm{~s}, 1 \mathrm{H}), 7.63(\mathrm{~d}, J=9.2 \mathrm{~Hz}, 1 \mathrm{H}), 6.91(\mathrm{dd}, J=8.4,2.4 \mathrm{~Hz}, 1 \mathrm{H}), 6.88(\mathrm{~d}$, $J=2.0 \mathrm{~Hz}, 1 \mathrm{H}), 4.28(\mathrm{t}, J=6.8 \mathrm{~Hz}, 2 \mathrm{H}), 3.88(\mathrm{~s}, 3 \mathrm{H}), 1.84-1.76(\mathrm{~m}, 3 \mathrm{H}), 1.52-1.48(\mathrm{~m}, 1 \mathrm{H}), 1.39-1.29(\mathrm{~m}$, $6 \mathrm{H}), 0.90$ (t, with further splitting, $J=7.2 \mathrm{~Hz}, 3 \mathrm{H}) ;{ }^{13} \mathrm{C} \mathrm{NMR}\left(100 \mathrm{MHz}, \mathrm{CDCl}_{3}\right) \delta 157.2,137.3,133.9$, 130.4, 121.4, 112.2, 91.5, 79.7, 55.8, 31.6, 28.9, 28.2, 25.6, 22.5, 14.0; IR (neat) 2928, 1625, 1496, 1236, $1064 \mathrm{~cm}^{-1}$; HRMS (ESI) calcd for $\mathrm{C}_{15} \mathrm{H}_{23} \mathrm{~N}_{2} \mathrm{O}_{2}\left(\mathrm{M}+\mathrm{H}^{+}\right)$263.1759, found 263.1749.

1-(1-butyloxy)-6-methoxybenzimidazole (200). Reaction of a solution of $\mathbf{1 2 7}$ (111.5 $\mathrm{mg}, 0.502 \mathrm{mmol})$ with 1-iodobutane $(120 \mu \mathrm{L}, 1.054 \mathrm{mmol})$ in $t$ - $\mathrm{BuOH}(8 \mathrm{~mL})$ in the presence of $t$-BuOK $(224.1 \mathrm{mg}, 1.997$ mmol), at ambient temperature under a nitrogen atmosphere, as described for $\mathbf{1 2 4}(25 \mathrm{~h})$, gave after work up and chromatography (hexane/EtOAc, 6:4) 200 (16.8 mg, $0.076 \mathrm{mmol}, 15 \%)$ as a colorless oil. ${ }^{1} \mathrm{H}$ NMR $\left(400 \mathrm{MHz}, \mathrm{CDCl}_{3}\right) \delta 7.94(\mathrm{~s}, 1 \mathrm{H}), 7.63(\mathrm{~d}, J=8.8 \mathrm{~Hz}, 1 \mathrm{H}), 6.91(\mathrm{dd}, J=8.4,2.4 \mathrm{~Hz}, 1 \mathrm{H}), 6.88(\mathrm{~d}$, $J=2.0 \mathrm{~Hz}, 1 \mathrm{H}), 4.29$ (t, $J=6.4 \mathrm{~Hz}, 2 \mathrm{H}), 3.88(\mathrm{~s}, 3 \mathrm{H}), 1.84-1.75(\mathrm{~m}, 2 \mathrm{H}), 1.58-1.52(\mathrm{~m}, 1 \mathrm{H}), 1.01(\mathrm{t}, J=7.2$ $\mathrm{Hz}, 3 \mathrm{H}) ;{ }^{13} \mathrm{C} \mathrm{NMR}\left(100 \mathrm{MHz}, \mathrm{CDCl}_{3}\right) \delta 157.2,137.3,133.9,130.4,121.4,112.2,91.5,79.5,55.8,30.1$, 18.9, 13.7; IR (neat) 2959, 1625, 1496, 1236, 1063, 1022, $816 \mathrm{~cm}^{-1}$; HRMS (ESI) calcd for $\mathrm{C}_{12} \mathrm{H}_{17} \mathrm{~N}_{2} \mathrm{O}_{2}$ $\left(\mathrm{M}+\mathrm{H}^{+}\right)$221.1290, found 221.1280.

6-methoxy-1-(2-methylethyloxy)benzimidazole (201). Reaction of a solution of 127 (143.6 mg, 0.646 mmol) with 2-iodopropane $(136 \mu \mathrm{L}, 1.357 \mathrm{mmol})$ in $t$-BuOH $(8 \mathrm{~mL})$ in the presence of $t$-BuOK $(290 \mathrm{mg}$, $2.584 \mathrm{mmol}$ ), at ambient temperature under a nitrogen atmosphere, as described for $\mathbf{1 2 4}(25 \mathrm{~h})$, gave after work up and chromatography (hexane/EtOAc, 6:4) $201(10.9 \mathrm{mg}, 0.053 \mathrm{mmol}, 8 \%)$ as a colorless oil. ${ }^{1} \mathrm{H}$ NMR (400 MHz, $\left.\mathrm{CDCl}_{3}\right) \delta 7.90(\mathrm{~s}, 1 \mathrm{H}), 7.63$ (d, J=8.8 Hz, 1H), 6.90 (dd, J=8.8, 2.8 Hz, 1H), 6.87 (d, $J=2.4 \mathrm{~Hz}, 1 \mathrm{H}$ ), 4.57 (heptet, $J=6.0 \mathrm{~Hz}, 1 \mathrm{H}), 3.88$ (s, 3H), 1.39 (d, $J=6.4 \mathrm{~Hz}, 6 \mathrm{H}) ;{ }^{13} \mathrm{C}$ NMR $(100 \mathrm{MHz}$, $\left.\mathrm{CDCl}_{3}\right) \delta 157.2,138.2,133.9,131.2,121.4,112.0,92.0,81.3,55.8,21.0 ;$ IR (neat) 1625, 1497, 1105, 817 $\mathrm{cm}^{-1}$; HRMS (ESI) calcd for $\mathrm{C}_{11} \mathrm{H}_{15} \mathrm{~N}_{2} \mathrm{O}_{2}\left(\mathrm{M}+\mathrm{H}^{+}\right)$207.1133, found 207.1124. 
2,4-Dinitro-5-bromophenyl trifluoromethanesulfonate (204) To a solution of fuming $\mathrm{HNO}_{3}(2 \mathrm{~mL})$ in $\mathrm{H}_{2} \mathrm{SO}_{4}(2 \mathrm{~mL})$ was added 3-bromophenyl trifluoromethanesulfonate (203) ${ }^{172}(297 \mathrm{mg}, 0.974 \mathrm{mmol})$ and the mixture was heated at $60{ }^{\circ} \mathrm{C}$ for $7 \mathrm{~h}$. The resulting mixture was poured onto ice, the ice was allowed to melt, and the resulting mixture was extracted with EtOAc $(3 \times 20 \mathrm{~mL})$. The combined organic phases were dried $\left(\mathrm{MgSO}_{4}\right)$, filtered, and the solvents were removed under reduced pressure. The crude product was purified by chromatography (hexanes/EtOAc, 9:1) affording 204 (307 $\mathrm{mg}, 0.777 \mathrm{mmol}, 80 \%)$ as a pale yellow oil that solidified upon standing. $\mathrm{mp}=33-35^{\circ} \mathrm{C} ;{ }^{1} \mathrm{H} \mathrm{NMR}\left(400 \mathrm{MHz}, \mathrm{CDCl}_{3}\right) \delta 8.73(\mathrm{~s}, 1 \mathrm{H})$, $7.90(\mathrm{~s}, 1 \mathrm{H}) ;{ }^{13} \mathrm{C} \mathrm{NMR}\left(100 \mathrm{MHz}, \mathrm{CDCl}_{3}\right) \delta 148.2,142.6,140.1,131.2,124.0,122.2,118.4\left(\mathrm{q}, J^{C-F}=319\right.$ $\mathrm{Hz}$ ); IR (ATR) 1591, 1541, 1436, 1337, 1213, $1130 \mathrm{~cm}^{-1}$.

5-Ethenyl-2,4-dinitro trifluoromethanesulfonate (205). A mixture of 204 (238 $\mathrm{mg}, 0.60 \mathrm{mmol}$ ), ethenyltributyltin (187 mg, $0.59 \mathrm{mmol}), 2,6$-di-t-butyl-4-methylphenol (22 mg, $0.10 \mathrm{mmol}), \mathrm{LiCl}(87 \mathrm{mg}$, $2.05 \mathrm{mmol}), \mathrm{PPh}_{3}(25.2 \mathrm{mg}, 0.098 \mathrm{mmol})$, and $\mathrm{PdCl}_{2}\left(\mathrm{PPh}_{3}\right)_{2}(33.7 \mathrm{mg}, 0.048 \mathrm{mmol})$ in toluene $(4 \mathrm{~mL})$ was heated at $80{ }^{\circ} \mathrm{C}$ for $17 \mathrm{~h}$. The solvent was removed at reduced pressure and the crude product was purified by chromatography (hexanes/EtOAc, 9:1) to give 205 (160 mg, $0.468 \mathrm{mmol}, 78 \%$ ) as a faint brown oil. ${ }^{1} \mathrm{H}$ NMR (600 MHz, $\left.\mathrm{CDCl}_{3}\right) \delta 8.82(\mathrm{~s}, 1 \mathrm{H}), 7.69$ (s, 1H), $7.26(\mathrm{dd}, J=17.4,10.8 \mathrm{~Hz}, 1 \mathrm{H}), 5.96$ $(\mathrm{d}, J=17.4 \mathrm{~Hz}, 1 \mathrm{H}), 5.84(\mathrm{~d}, J=11.4 \mathrm{~Hz}, 1 \mathrm{H}) ;{ }^{13} \mathrm{C} \mathrm{NMR}\left(150 \mathrm{MHz}, \mathrm{CDCl}_{3}\right) \delta 145.3,143.6,140.5,129.8$, 127.4, 125.0, 124.4, 123.9, $118.5\left(\mathrm{q}, J^{C-F}=319 \mathrm{~Hz}\right) ;{ }^{19} \mathrm{~F}$ NMR (376 $\left.\mathrm{MHz}, \mathrm{CDCl}_{3}\right) \delta-72.9$; IR (ATR) $1614,1590,1539,1437,1345,1223,1134 \mathrm{~cm}^{-1} .173$

4-Bromo-3-nitrophenyl trifluoromethanesulfonate (215). To a solution of 4-bromo-3-nitrophenol ${ }^{174}$ (269 mg, $1.23 \mathrm{mmol})$ in $\mathrm{CH}_{2} \mathrm{Cl}_{2}(5 \mathrm{~mL})$ at $0{ }^{\circ} \mathrm{C}$ was added pyridine $(200 \mu \mathrm{L}, 2.48 \mathrm{mmol})$ and trifluoromethanesulfonic anhydride $\left(\mathrm{Tf}_{2} \mathrm{O}, 250 \mu \mathrm{L}, 1.48 \mathrm{mmol}\right)$. The mixture was removed from the cold bath and allowed to stir at ambient temperature for $30 \mathrm{~min}$. The resulting mixture was filtered through a small plug of silica gel and the solvent was removed under reduced pressure from the filtrate. Purification by chromatography (hexanes/EtOAc, 9:1) afforded 215 (391 mg, $1.12 \mathrm{mmol}, 90 \%)$ as a yellow oil. ${ }^{1} \mathrm{H}$ $\operatorname{NMR}\left(600 \mathrm{MHz}, \mathrm{CDCl}_{3}\right) \delta 7.88(\mathrm{~d}, J=9.0 \mathrm{~Hz}, 1 \mathrm{H}), 7.82(\mathrm{~d}, J=2.4 \mathrm{~Hz}, 1 \mathrm{H}), 7.41(\mathrm{dd}, J=9.0,3.0 \mathrm{~Hz}$, $1 \mathrm{H}) ;{ }^{13} \mathrm{C}$ NMR $\left(150 \mathrm{MHz}, \mathrm{CDCl}_{3}\right) \delta 150.1,147.9,136.8,126.2,119.3,118.6\left(\mathrm{q}, J^{C-F}=319 \mathrm{~Hz}\right), 114.6$; IR (ATR) 3103, 1541, 1428, 1208, $1132 \mathrm{~cm}^{-1}$; HRMS (ESI) calcd for $\mathrm{C}_{7} \mathrm{H}_{3} \mathrm{BrNNaO}_{5} \mathrm{~F}_{3} \mathrm{~S}\left(\mathrm{M}+\mathrm{Na}^{+}\right) 371.8765$; found 371.8760 .

3-Bromo-2-nitrophenyl trifluoromethanesulfonate (216). Treatment of 3-bromo-2-nitrophenol ${ }^{175}$ (298 $\mathrm{mg}, 1.37 \mathrm{mmol})$ in $\mathrm{CH}_{2} \mathrm{Cl}_{2}(5 \mathrm{~mL})$ with pyridine $(250 \mu \mathrm{L}, 3.10 \mathrm{mmol})$ and $\mathrm{Tf}_{2} \mathrm{O}(300 \mu \mathrm{L}, 1.77 \mathrm{mmol})$, as described for 213, gave after chromatography (hexanes/EtOAc, 8:2) 216 (394 mg, $1.13 \mathrm{mmol}, \mathbf{8 0 \%}$ ) as a 
red solid. mp 52-53 ${ }^{\circ} \mathrm{C} ;{ }^{1} \mathrm{H}$ NMR (400 MHz, $\left.\mathrm{CDCl}_{3}\right) \delta$ 7.74-7.72 (m, 1H), 7.50-7.49 (m, 2H); ${ }^{13} \mathrm{C}$ NMR $\left(150 \mathrm{MHz}, \mathrm{CDCl}_{3}\right) \delta 140.6,133.4,132.1,121.6,119.4,117.3\left(\mathrm{q}, J^{C-F}=319 \mathrm{~Hz}\right), 115.2 ;{ }^{19} \mathrm{~F}$ NMR $(376$ $\mathrm{MHz} \mathrm{CDCl}_{3}$ ) $\delta$-73.2; IR (ATR) 3099, 1538, 1434, 1360, 1219, $1132 \mathrm{~cm}^{-1}$; HRMS (ESI) calcd for $\mathrm{C}_{7} \mathrm{H}_{3} \mathrm{BrNNaO}_{5} \mathrm{~F}_{3} \mathrm{~S}\left(\mathrm{M}+\mathrm{Na}^{+}\right)$371.8765; found 371.8767 .

2-Bromo-6-nitrophenyl trifluoromethanesulfonate (217). Treatment of 2-bromo-6-nitrophenol ${ }^{176}$ (189 $\mathrm{mg}, 0.87 \mathrm{mmol})$ in $\mathrm{CH}_{2} \mathrm{Cl}_{2}(5 \mathrm{~mL})$ with pyridine $(150 \mu \mathrm{L}, 1.85 \mathrm{mmol})$ and $\mathrm{Tf}_{2} \mathrm{O}(200 \mu \mathrm{L}, 1.18 \mathrm{mmol})$, as described for 215, gave after chromatography (hexanes/EtOAc, 7:3) 217 (299 $\mathrm{mg}, 0.85 \mathrm{mmol} 98 \%$ ) as a colorless oil. ${ }^{1} \mathrm{H}$ NMR $\left(600 \mathrm{MHz}, \mathrm{CDCl}_{3}\right) \delta 8.03(\mathrm{dd}, J=7.8,1.2 \mathrm{~Hz}, 1 \mathrm{H}), 7.98(\mathrm{dd}, J=8.4,1.8 \mathrm{~Hz}, 1 \mathrm{H})$, $7.45(\mathrm{dt}, J=8.4,1.2 \mathrm{~Hz}, 1 \mathrm{H}) ;{ }^{13} \mathrm{C} \mathrm{NMR}\left(150 \mathrm{MHz}, \mathrm{CDCl}_{3}\right) \delta 143.6,139.4,139.1,129.3,125.6,119.0$, $118.4\left(\mathrm{q}, J^{C-F}=319 \mathrm{~Hz}\right) ;{ }^{19} \mathrm{~F}$ NMR $\left(376 \mathrm{MHz}, \mathrm{CDCl}_{3}\right) \delta-73.2$; IR (ATR) 3093, 1588, 1540, 1431, 1347 , $1207 \mathrm{~cm}^{-1}$; HRMS (ESI) calcd for $\mathrm{C}_{7} \mathrm{H}_{3} \mathrm{BrNNaO}_{5} \mathrm{~F}_{3} \mathrm{~S}\left(\mathrm{M}+\mathrm{Na}^{+}\right)$371.8765; found 371.8761 .

3-Bromo-5-nitrophenyl trifluoromethanesulfonate (219) Treatment of 3-bromo-5-nitrophenol ${ }^{177}$ (329 $\mathrm{mg}, 1.51 \mathrm{mmol})$ in $\mathrm{CH}_{2} \mathrm{Cl}_{2}(10 \mathrm{~mL})$ with pyridine $(250 \mu \mathrm{L}, 3.10 \mathrm{mmol})$ and $\mathrm{Tf}_{2} \mathrm{O}(300 \mu \mathrm{L}, 1.78 \mathrm{mmol})$, as described for 215, gave after chromatography (hexanes/EtOAc, 7:3) 219 (316 mg, $0.90 \mathrm{mmol}, 60 \%$ ) as a red oil. ${ }^{1} \mathrm{H}$ NMR $\left(600 \mathrm{MHz}, \mathrm{CDCl}_{3}\right) \delta 8.44(\mathrm{t}, J=1.8 \mathrm{~Hz}, 1 \mathrm{H}), 8.11(\mathrm{t}, J=2.4 \mathrm{~Hz}, 1 \mathrm{H}), 7.80(\mathrm{t}, J=1.8$ $\mathrm{Hz}, 1 \mathrm{H}) ;{ }^{13} \mathrm{C}$ NMR $\left(150 \mathrm{MHz}, \mathrm{CDCl}_{3}\right) \delta 149.2,130.8,126.8,123.8,118.6$ (q, $\left.J^{C-F}=319 \mathrm{~Hz}\right), 116.1$; IR (ATR) 3097, 1732, 1542, 1427, 1344, 1210, $1134 \mathrm{~cm}^{-1}$; HRMS (ESI) calcd for $\mathrm{C}_{7} \mathrm{H}_{3} \mathrm{BrNNaO}_{5} \mathrm{~F}_{3} \mathrm{~S}$ $\left(\mathrm{M}+\mathrm{Na}^{+}\right)$371.8765; found 371.8764 .

4-Bromo-2-nitrophenyl trifluoromethanesulfonate (220). ${ }^{164}$ Treatment of 4-bromo-3-nitrophenol ${ }^{178}$ (353 mg, $1.60 \mathrm{mmol})$ in $\mathrm{CH}_{2} \mathrm{Cl}_{2}(5 \mathrm{~mL})$ with pyridine $(260 \mu \mathrm{L}, 3.22 \mathrm{mmol})$ and $\mathrm{Tf}_{2} \mathrm{O}(330 \mu \mathrm{L}, 1.95$ mmol), as described for 215, gave after chromatography (hexanes/EtOAc, 7:3) 220 (540 mg, $1.54 \mathrm{mmol}$, 97\%) as a yellow oil. ${ }^{1} \mathrm{H} \mathrm{NMR}\left(600 \mathrm{MHz}, \mathrm{CDCl}_{3}\right) \delta 8.30(\mathrm{~d}, J=2.4 \mathrm{~Hz}, 1 \mathrm{H}), 7.88(\mathrm{dd}, J=8.4,2.4 \mathrm{~Hz}$, $1 \mathrm{H}), 7.36(\mathrm{~d}, J=9.0 \mathrm{~Hz}, 1 \mathrm{H}) ;{ }^{13} \mathrm{C} \mathrm{NMR}\left(150 \mathrm{MHz}, \mathrm{CDCl}_{3}\right) \delta 141.9,140.5,138.2,129.7,125.6,122.3$, $118.5\left(\mathrm{q}, J^{C-F}=319 \mathrm{~Hz}\right.$ ); IR (ATR) 3105, 1540, 1431, 1207, $1131 \mathrm{~cm}^{-1}$; HRMS (ESI) calcd for $\mathrm{C}_{7} \mathrm{H}_{3} \mathrm{BrNNaO}_{5} \mathrm{~F}_{3} \mathrm{~S}\left(\mathrm{M}+\mathrm{Na}^{+}\right)$371.8765; found 371.8764 .

2-Bromo-5-nitrophenyl trifluoromethanesulfonate (222). Treatment of 2-bromo-5-nitrophenol ${ }^{179}$ (119 $\mathrm{mg}, 0.55 \mathrm{mmol})$ in $\mathrm{CH}_{2} \mathrm{Cl}_{2}(5 \mathrm{~mL})$ with pyridine $(90 \mu \mathrm{L}, 1.12 \mathrm{mmol})$ and $\mathrm{Tf}_{2} \mathrm{O}(120 \mu \mathrm{L}, 0.71 \mathrm{mmol})$, as described for 215, gave without further purification $222(188 \mathrm{mg}, 0.54 \mathrm{mmol}, 98 \%)$ as a brown oil. ${ }^{1} \mathrm{H}$ NMR $\left(400 \mathrm{MHz}, \mathrm{CDCl}_{3}\right) \delta 8.22(\mathrm{~d}, J=2.4 \mathrm{~Hz}, 1 \mathrm{H}), 8.16(\mathrm{dd}, J=8.8,2.4 \mathrm{~Hz}, 1 \mathrm{H}), 7.93(\mathrm{~d}, J=8.8 \mathrm{~Hz}$, $1 \mathrm{H}) ;{ }^{13} \mathrm{C} \mathrm{NMR}\left(100 \mathrm{MHz}, \mathrm{CDCl}_{3}\right) \delta 147.6,146.9,135.2,124.0,123.9,118.5\left(\mathrm{q}, J^{C-F}=320 \mathrm{~Hz}\right), 118.3$; IR 
(ATR) $3104,1534,1431,1348,1211,1134 \mathrm{~cm}^{-1}$; HRMS (ESI) calcd for $\mathrm{C}_{7} \mathrm{H}_{3} \mathrm{BrNNaO}_{5} \mathrm{~F}_{3} \mathrm{~S}\left(\mathrm{M}+\mathrm{Na}^{+}\right)$ 371.8765; found 371.8764 .

\section{Conditions A}

4-Ethenylphenyl trifluoromethanesulfonate (206). ${ }^{138}$ To a solution of $\mathrm{PPh}_{3}(7.5 \mathrm{mg}, 0.029 \mathrm{mmol})$ and $\operatorname{Pd}(\mathrm{dba})_{2}(4.3 \mathrm{mg}, 0.007 \mathrm{mmol})$ in dioxane $(1.5 \mathrm{~mL})$, stirred for $5 \mathrm{~min}$ under an atmosphere of $\mathrm{N}_{2}$, was added 4-bromophenyl trifluoromethanesulphonate $(\mathbf{2 0 2})^{138}(102 \mathrm{mg}, 0.34 \mathrm{mmol})$ followed by ethenyltributyltin $(128 \mathrm{mg}, 0.40 \mathrm{mmol})$. The solution was heated at reflux for $24 \mathrm{~h}$. The solvent was removed under reduced pressure and the residue was dissolved in EtOAc $(10 \mathrm{~mL})$ and washed with $\mathrm{NH}_{4} \mathrm{OH}(10 \%$ aqueous, $3 \times 20 \mathrm{~mL}), \mathrm{H}_{2} \mathrm{O}(20 \mathrm{~mL})$, and brine $(20 \mathrm{~mL})$. The organic phase was dried $\left(\mathrm{MgSO}_{4}\right)$, filtered, and solvents were removed under reduced pressure. The crude product was purified by chromatography (hexanes/EtOAc, 97:3) to give 206 (25.2 $\mathrm{mg}, 0.10 \mathrm{mmol}, 30 \%)$ as a colorless oil. Only 206 was observed by ${ }^{1} \mathrm{H}$ NMR $\left(600 \mathrm{MHz}, \mathrm{CDCl}_{3}\right)$ of the crude reaction mixture.

\section{Conditions B}

4-Ethenylphenyl trifluoromethanesulfonate (206) and 4-ethenyl-1-bromobenzene (207). ${ }^{138}$ To a solution of $\mathrm{LiCl}(45.5 \mathrm{mg}, 1.07 \mathrm{mmol})$ and $\mathrm{Pd}\left(\mathrm{PPh}_{3}\right)_{2} \mathrm{Cl}_{2}(4.7 \mathrm{mg}, 0.007 \mathrm{mmol})$ in DMF $(1.5 \mathrm{~mL})$, under an atmosphere of $\mathrm{N}_{2}$, was added $202(108 \mathrm{mg}, 0.35 \mathrm{mmol})$ followed by ethenyltributyltin (141 $\mathrm{mg}, 0.44$ mmol). After stirring at ambient temperature for $24 \mathrm{~h}$, the solvent was removed by bulb-to-bulb distillation. The resulting residue was dissolved in EtOAc $(15 \mathrm{~mL})$ and washed with $\mathrm{NH}_{4} \mathrm{OH}(10 \%$ aqueous, $3 \times 20 \mathrm{~mL})$ and brine $(20 \mathrm{~mL})$. The organic phase was dried $\left(\mathrm{MgSO}_{4}\right)$, filtered, and solvents were removed under reduced pressure. The terminal cis-alkene protons were clearly resolved in the ${ }^{1} \mathrm{H}$ NMR spectrum at $600 \mathrm{MHz}$ and these signals were used to determine the ratio of $\mathbf{2 0 6}(\delta 5.35, \mathrm{~d}, J=10.8$ $\mathrm{Hz}, 1 \mathrm{H})$ to $207(\delta 5.27, \mathrm{~d}, J=10.8 \mathrm{~Hz}, 1 \mathrm{H})$. A 6.7:1 ratio of 207/206 was observed by NMR. The products decomposed upon attempted purification on silica gel.

\section{Conditions C}

4-Ethenylphenyl trifluoromethanesulfonate (206). To a solution of $\mathrm{PdCl}_{2}\left(\mathrm{PPh}_{3}\right)_{2}$ (4.3 mg, $\left.0.007 \mathrm{mmol}\right)$ in dioxane $(4 \mathrm{~mL})$, stirred for $5 \mathrm{~min}$ under an atmosphere of $\mathrm{N}_{2}$, was added 202 (310 $\left.\mathrm{mg}, 1.02 \mathrm{mmol}\right)$ followed by ethenyltributyltin $(340 \mathrm{mg}, 1.07 \mathrm{mmol})$. The solution was heated at reflux for $24 \mathrm{~h}$. The solvent was removed under reduced pressure. The product was purified by chromatography on $\mathrm{SiO}_{2} / \mathrm{K}_{2} \mathrm{CO}_{3}\left(10 \% \mathrm{~K}_{2} \mathrm{CO}_{3}\right.$, hexanes/EtOAc, 98:2) to give 206 (231 mg, $\left.0.917 \mathrm{mmol}, 90 \%\right)$ as a colorless oil. Only 206 was observed by ${ }^{1} \mathrm{H}$ NMR $\left(600 \mathrm{MHz}, \mathrm{CDCl}_{3}\right)$ of the crude reaction mixture. 
2-Ethenylphenyl trifluoromethanesulfonate (209). ${ }^{180}$ Cross coupling of 2-bromophenyl trifluoromethanesulphonate $(\mathbf{2 0 8})^{181}(95.6 \mathrm{mg}, 0.31 \mathrm{mmol})$ with ethenyltributyltin $(121 \mathrm{mg}, 0.38 \mathrm{mmol})$ in the presence of $\mathrm{PPh}_{3}(6.9 \mathrm{mg}, 0.026 \mathrm{mmol})$ and $\mathrm{Pd}(\mathrm{dba})_{2}(3.8 \mathrm{mg}, 0.007 \mathrm{mmol})$ in dioxane $(1.5 \mathrm{~mL})$ was performed as described for $\mathbf{2 0 6}$ under Conditions A. Work up and chromatography chromatography (hexanes/EtOAc, 9:1) gave 209 (4.1 $\mathrm{mg}, 0.016 \mathrm{mmol}, 5 \%$ ) as a colorless oil. Only 209 was observed by ${ }^{1} \mathrm{H}$ NMR $\left(600 \mathrm{MHz}, \mathrm{CDCl}_{3}\right)$ of the crude reaction mixture after work up.

2-Ethenylphenyl trifluoromethanesulfonate (209) and 2-ethenyl-1-bromobenzene (210). ${ }^{182}$ Cross coupling of 208 ( $88.3 \mathrm{mg}, 0.25 \mathrm{mmol})$ with ethenyltributyltin $(98.0 \mathrm{mg}, 0.31 \mathrm{mmol})$ in the presence of $\mathrm{LiCl}(32.2 \mathrm{mg}, 0.76 \mathrm{mmol})$ and $\mathrm{Pd}\left(\mathrm{PPh}_{3}\right)_{2} \mathrm{Cl}_{2}(3.8 \mathrm{mg}, 0.005 \mathrm{mmol})$ in DMF $(1.0 \mathrm{~mL})$ was performed as described for 208 under Conditions B. A 5.9:1 ratio of 210/209 was observed by ${ }^{1} \mathrm{H}$ NMR (600 MHz, $\mathrm{CDCl}_{3}$ ) of the crude reaction mixture after standard work up. The products decomposed upon attempted purification on silica gel. The terminal cis-alkene protons were clearly resolved in the ${ }^{1} \mathrm{H}$ NMR spectrum at $600 \mathrm{MHz}$ and these signals were used to determine the ratios of $209(\delta 5.49, \mathrm{~d}, J=11.1 \mathrm{~Hz}, 1 \mathrm{H})$ to 210 $(\delta 5.37, \mathrm{dd}, J=10.9,1.0 \mathrm{~Hz}, 1 \mathrm{H})$.

3-Ethenylphenyl trifluoromethanesulfonate (211) and 3-Ethenylphenyl-1-bromobenzene (212). ${ }^{182}$ Cross coupling of 3-bromophenyl trifluoromethane sulphonate (203) (105 mg, $0.34 \mathrm{mmol}$ ) with ethenyltributyltin $(127 \mathrm{mg}, 0.40 \mathrm{mmol})$ in the presence of $\mathrm{PPh}_{3}(7.6 \mathrm{mg}, 0.03 \mathrm{mmol})$ and $\mathrm{Pd}(\mathrm{dba})_{2}(4.0$ $\mathrm{mg}, 0.007 \mathrm{mmol})$ in dioxane $(1.5 \mathrm{~mL})$ was performed as described for $\mathbf{2 0 6}$ under Conditions A. Work up and chromatography chromatography (hexanes/EtOAc, 9:1) to give an inseparable mixture of 203 and 211 (49.2 mg, calculated from ${ }^{1} \mathrm{H}$ NMR spectrum: $20.2 \mathrm{mg} 203$ and $28.8 \mathrm{mg} \mathbf{2 1 1}, 34 \%$ ) as a colorless oil. A $~ 30: 1$ ratio of 211/212 was observed by ${ }^{1} \mathrm{H}$ NMR $\left(600 \mathrm{MHz}, \mathrm{CDCl}_{3}\right)$ of the crude reaction mixture after standard work up. The terminal cis-alkene protons were clearly resolved in the ${ }^{1} \mathrm{H}$ NMR spectrum at 600 $\mathrm{MHz}$ and these signals were used to determine the ratios of $211(\delta 5.38, \mathrm{~d}, J=11.4 \mathrm{~Hz}, 1 \mathrm{H})$ to $212(\delta$ $5.30, \mathrm{~d}, J=10.8 \mathrm{~Hz}, 1 \mathrm{H})$.

3-Ethenylphenyl trifluoromethanesulfonate (211) and 3-Ethenylphenyl-1-bromobenzene (212). Cross coupling of 203 (108 mg, $0.36 \mathrm{mmol}$ ) with ethenyltributyltin (140 mg, $0.44 \mathrm{mmol})$ in the presence of $\mathrm{LiCl}(45.6 \mathrm{mg}, 1.08 \mathrm{mmol})$ and $\mathrm{Pd}\left(\mathrm{PPh}_{3}\right)_{2} \mathrm{Cl}_{2}(5.4 \mathrm{mg}, 0.008 \mathrm{mmol})$ in $\mathrm{DMF}(1.5 \mathrm{~mL})$ was performed as described for $\mathbf{2 0 7}$ under Conditions B. A 5.3:6.3:1 ratio of $\mathbf{2 0 3 / 2 1 2 / 2 1 1}$ was observed by ${ }^{1} \mathrm{H}$ NMR $\left(600 \mathrm{MHz}, \mathrm{CDCl}_{3}\right)$ of the crude reaction mixture after standard work up. The products decomposed upon attempted purification on silica gel or basic alumina. 
3-Ethenylphenyl trifluoromethanesulfonate (211) Cross coupling of 203 (310 $\mathrm{mg}, 1.02 \mathrm{mmol}$ ) with ethenyltributyltin $(490 \mathrm{mg}, 1.54 \mathrm{mmol})$ in the presence of $\mathrm{Pd}\left(\mathrm{PPh}_{3}\right)_{2} \mathrm{Cl}_{2}(13.3 \mathrm{mg}, 0.019 \mathrm{mmol})$ in DMF $(4 \mathrm{~mL})$ was performed as described for 206 under Conditions $\mathrm{C}\left(100{ }^{\circ} \mathrm{C}, 50 \mathrm{~h}\right)$. Work up and chromatography on $\mathrm{SiO}_{2} / \mathrm{K}_{2} \mathrm{CO}_{3}\left(10 \% \mathrm{~K}_{2} \mathrm{CO}_{3}\right.$, hexanes/EtOAc, 98:2) gave 211 (121 mg, $0.480 \mathrm{mmol}$, $47 \%)$ as a colorless oil. Only 211 was observed by ${ }^{1} \mathrm{H}$ NMR $\left(600 \mathrm{MHz}, \mathrm{CDCl}_{3}\right)$ of the crude reaction mixture Needs to be reintegrated some 212 can be seen. ${ }^{1} \mathrm{H}$ NMR $\left(600 \mathrm{MHz}, \mathrm{CDCl}_{3}\right) \delta 7.42-7.39(\mathrm{~m}, 2 \mathrm{H})$, $7.29(\mathrm{~d}, \mathrm{~J}=1.2 \mathrm{~Hz}, 1 \mathrm{H}), 7.17-7.15(\mathrm{~m}, 1 \mathrm{H}), 6.70(\mathrm{dd}, J=17.4,10.8 \mathrm{~Hz}, 1 \mathrm{H}), 5.81(\mathrm{~d}, J=17.4 \mathrm{~Hz}, 1 \mathrm{H})$, $5.39(\mathrm{~d}, J=10.8 \mathrm{~Hz}, 1 \mathrm{H}) ;{ }^{13} \mathrm{C} \mathrm{NMR}\left(150 \mathrm{MHz}, \mathrm{CDCl}_{3}\right) \delta 149.9,140.3,135.0,130.2,126.1,120.2,118.8$, $118.7\left(\mathrm{q}, J^{C-F}=319 \mathrm{~Hz}\right), 116.5$; IR (ATR) 1574, 1420, 1205, 1136, 1117, 924, $825 \mathrm{~cm}^{-1}$; HRMS (ESI) calcd for $\mathrm{C}_{9} \mathrm{H}_{8} \mathrm{~F}_{3} \mathrm{O}_{3} \mathrm{~S}\left(\mathrm{M}+\mathrm{H}^{+}\right)$253.0146; found 253.0147.

2-Ethenyl-3-nitrophenyl trifluoromethanesulfonate (223). ${ }^{183}$ Cross coupling of 2-bromo-3-nitrophenyl trifluoromethanesulfonate $(\mathbf{2 1 3})^{183}(105 \mathrm{mg}, 0.30 \mathrm{mmol})$ with ethenyltributyltin $(119 \mathrm{mg}, 0.38 \mathrm{mmol})$ in the presence of in the presence of $\mathrm{PPh}_{3}(6.5 \mathrm{mg}, 0.03 \mathrm{mmol})$ and $\mathrm{Pd}(\mathrm{dba})_{2}(3.7 \mathrm{mg}, 0.006 \mathrm{mmol})$ in dioxane $(1.5 \mathrm{~mL})$ was performed as described for $\mathbf{2 0 6}$ under Conditions A. Work up and chromatography (hexanes/EtOAc, 97:3) gave , in order of elution, 223 (32.3 mg, $0.11 \mathrm{mmol}, 36 \%$ ) as a white solid and impure 2-bromo-3-nitrophenol ${ }^{182}(41 \mathrm{mg})$.

2-Ethenyl-3-nitrophenyl trifluoromethanesulfonate (223), 2-bromo-3-nitrophenol, and 2-bromo-3ethenyl-nitrobenzene (224). Cross coupling of 213 (110 mg, $0.32 \mathrm{mmol})$ with ethenyltributyltin (123 $\mathrm{mg}, 0.39 \mathrm{mmol})$ in the presence of $\mathrm{LiCl}(40.9 \mathrm{mg}, 0.96 \mathrm{mmol})$ and $\mathrm{Pd}\left(\mathrm{PPh}_{3}\right)_{2} \mathrm{Cl}_{2}(4.8 \mathrm{mg}, 0.007 \mathrm{mmol})$ in DMF (1.5 mL) was performed as described for 207 under Conditions B. Work up and chromatography (hexanes/EtOAc, 97:3) gave , in order of elution, 223 (18.5 mg, $0.06 \mathrm{mmol}, 20 \%), 224$ (27.6 mg, 0.12 $\mathrm{mmol}, 38 \%$ ) as a colorless oil and an impure mixture of $\mathbf{2 1 3}$ and 2-bromo-3-nitrophenol (38.2 $\mathrm{mg}$ ). Spectral data for 224: ${ }^{1} \mathrm{H} \mathrm{NMR}\left(600 \mathrm{MHz}, \mathrm{CDCl}_{3}\right) \delta 7.70(\mathrm{~d}, J=7.8 \mathrm{~Hz}, 1 \mathrm{H}), 7.57(\mathrm{~d}, J=7.8 \mathrm{~Hz}, 1 \mathrm{H})$, $7.41(\mathrm{t}, J=7.8 \mathrm{~Hz}, 1 \mathrm{H}), 7.10(\mathrm{dd}, J=17.4,10.8 \mathrm{~Hz}, 1 \mathrm{H}), 5.76(\mathrm{~d}, J=17.4 \mathrm{~Hz}, 1 \mathrm{H}), 5.52$ (d, $J=10.8 \mathrm{~Hz}$, $1 \mathrm{H}) ;{ }^{13} \mathrm{C} \mathrm{NMR}\left(150 \mathrm{MHz}, \mathrm{CDCl}_{3}\right) \delta 147.2,128.9,128.3,126.2,125.7,124.8,123.8,119.5$; IR (ATR) 3110, 1533, 1423, 1358, 1210, $1135 \mathrm{~cm}^{-1}$; HRMS (ESI) calcd for $\mathrm{C}_{8} \mathrm{H}_{6} \mathrm{NNaO}_{2} \mathrm{Br}\left(\mathrm{M}+\mathrm{Na}^{+}\right)$249.9474; found 249.9473 .

2-Ethenyl-3-nitrophenyl trifluoromethanesulfonate (223). Cross coupling of 213 (127 mg, 0.362 mmol) with ethenyltributyltin $(149 \mathrm{mg}, 0.471 \mathrm{mmol})$ in the presence of $\mathrm{Pd}\left(\mathrm{PPh}_{3}\right)_{2} \mathrm{Cl}_{2}(5.1 \mathrm{mg}, 0.007$ $\mathrm{mmol})$ in dioxane $(1 \mathrm{~mL})$ was performed as described for 206 under Conditions $\mathrm{C}(23 \mathrm{~h})$. Work up and chromatography on $\mathrm{SiO}_{2} / \mathrm{K}_{2} \mathrm{CO}_{3}\left(10 \% \mathrm{~K}_{2} \mathrm{CO}_{3}\right.$, hexanes/EtOAc, 98:2) gave 223 (83 mg, $0.28 \mathrm{mmol}, 77 \%$ ). 
2-Ethenyl-3-nitrophenyl trifluoromethanesulfonate (223) and 1-bromo-2-ethenyl-nitrobenzene (224) 2,3-Diethenyl-nitrobenzene (225), and 2-ethenyl-3-nitrophenol. Cross coupling of 213 (143 mg, $0.41 \mathrm{mmol})$ with ethenyltributyltin $(123 \mathrm{mg}, 0.39 \mathrm{mmol})$ in the presence of $\mathrm{LiCl}(53.8 \mathrm{mg}, 1.27 \mathrm{mmol})$ and 1,3-bis(diphenylphosphino)propanepalladium dichloride $(5.3 \mathrm{mg}, 0.009 \mathrm{mmol}$ ) in DMF (2 mL) was performed as described for $\mathbf{2 0 7}$ under Conditions B. Work up and chromatography (hexanes/EtOAc, 97:3) gave in order of elution, 225 (7.0 mg, $0.04 \mathrm{mmol}, 10 \%), 223$ (22.9 mg, $0.077 \mathrm{mmol}, 19 \%), 224$ (45.7 mg, $0.20 \mathrm{mmol}, 49 \%$ ) as a colorless oil, and 2-ethenyl-3-nitrophenol (7.7 mg, $0.046 \mathrm{mmol}, 11 \%)$. Spectral data for 225: ${ }^{1} \mathrm{H}$ NMR (400 MHZ, $\left.\mathrm{CDCl}_{3}\right) \delta 7.75(\mathrm{dd}, J=8.0,1.2 \mathrm{~Hz}, 1 \mathrm{H}), 7.71(\mathrm{dd}, J=8.0,1.2$ $\mathrm{HZ}, 1 \mathrm{H}), 7.37$ (t, $J=8.0 \mathrm{~Hz}, 1 \mathrm{H}), 6.96$ (dd, $J=17.6,10.8 \mathrm{~Hz}, 1 \mathrm{H}), 6.89$ (dd, $J=17.6,11.6 \mathrm{~Hz}, 1 \mathrm{H}), 5.71$ (dd, $J=17.6,0.8 \mathrm{~Hz}, 1 \mathrm{H}), 5.63(\mathrm{dd}, J=11.6,1.2 \mathrm{~Hz}, 1 \mathrm{H}), 5.37$ (dd, $J=11.2,1.2 \mathrm{~Hz}, 1 \mathrm{H}), 5.32$ (d, J=18.0, 1.2 $\mathrm{Hz}, 1 \mathrm{H}) ;{ }^{13} \mathrm{C}$ NMR $\left(100 \mathrm{MHZ}, \mathrm{CDCl}_{3}\right) \delta 149.8,138.7,134.5,131.4,130.5,130.0,127.6,122.8,122.6$, 117.3; IR (ATR) 1521, 1348, 985, 921, 810, 774, 753, $735 \mathrm{~cm}^{-1}$; HRMS (ESI) calculated from $\mathrm{C}_{10} \mathrm{H}_{10} \mathrm{NO}_{2}$ $\left(\mathrm{M}+\mathrm{H}^{+}\right)$176.0711, found 176.0709.

2,3-Diethenyl-nitrobenzene (225). Cross coupling of 213 (70.2 mg, $0.201 \mathrm{mmol})$ with ethenyltributyltin (203 mg, $0.641 \mathrm{mmol})$ in the presence of $\mathrm{Pd}\left(\mathrm{PPh}_{3}\right)_{2} \mathrm{Cl}_{2}(6.0 \mathrm{mg}, 0.008 \mathrm{mmol})$ and $\mathrm{LiCl}(6.0 \mathrm{mg}, 0.008$ $\mathrm{mmol})$ in dioxane $(1 \mathrm{~mL})$ was performed as described for 207 under Conditions B $\left(100{ }^{\circ} \mathrm{C}, 24 \mathrm{~h}\right)$. Work up and chromatography on $\mathrm{SiO}_{2} / \mathrm{K}_{2} \mathrm{CO}_{3}\left(10 \% \mathrm{~K}_{2} \mathrm{CO}_{3}\right.$, hexanes/EtOAc, 98:2) gave 223 (33.7 mg, 0.192 mmol, 96\%).

3-Ethenyl-4-nitrophenyl trifluoromethanesulfonate (226) and 2,4-diethenyl-nitrobenzene (227). Cross coupling of 3-bromo-4-nitrophenyl trifluoromethanesulfonate (214) ${ }^{184}(67.6 \mathrm{mg}, 0.19 \mathrm{mmol})$ with ethenyltributyltin $(72.8 \mathrm{mg}, 0.23 \mathrm{mmol})$ in the presence of in the presence of $\mathrm{PPh}_{3}(4.5 \mathrm{mg}, 0.02 \mathrm{mmol})$ and $\mathrm{Pd}(\mathrm{dba})_{2}(2.2 \mathrm{mg}, 0.004 \mathrm{mmol})$ in dioxane $(1 \mathrm{~mL})$ was performed as described for 206 under Conditions A. Work up and chromatography (hexanes/EtOAc, 97:3), gave in order of elution, 227 (3.0 $\mathrm{mg}, 0.02 \mathrm{mmol}, 9 \%)$ and $226(47.3 \mathrm{mg}, 0.16 \mathrm{mmol}, 82 \%)$ as a colorless oil. Analytical data for $\mathbf{2 2 6}$ : ${ }^{1} \mathrm{H}$ NMR (600 MHZ, $\left.\mathrm{CDCl}_{3}\right) \delta 8.06(\mathrm{~d}, J=9.0 \mathrm{~Hz}, 1 \mathrm{H}), 7.51(\mathrm{~d}, J=2.4 \mathrm{~Hz}, 1 \mathrm{H}), 7.33(\mathrm{dd}, J=9.0,3.0 \mathrm{~Hz}$, $1 \mathrm{H}), 7.18(\mathrm{dd}, J=17.4,11.4 \mathrm{~Hz}, 1 \mathrm{H}), 5.81(\mathrm{~d}, J=17.4 \mathrm{~Hz}, 1 \mathrm{H}), 5.66(\mathrm{~d}, J=11.4 \mathrm{~Hz}, 1 \mathrm{H}) ;{ }^{13} \mathrm{C} \mathrm{NMR}(150$ $\left.\mathrm{MHZ}, \mathrm{CDCl}_{3}\right) \delta 151.7,146.6,136.4,131.0,127.0,121.4,121.3,121.0,118.6\left(\mathrm{q}, J^{C-F}=319 \mathrm{~Hz}\right) ; \mathrm{IR}(\mathrm{ATR})$ $3118,1530,1424,1350,1207,1131 \mathrm{~cm}^{-1}$; HRMS (ESI) calcd for $\mathrm{C}_{9} \mathrm{H}_{6} \mathrm{NNaO}_{5} \mathrm{~F}_{3} \mathrm{~S}\left(\mathrm{M}+\mathrm{Na}^{+}\right) 319.9811$; found 319.9809 .

Analytical data for 227: ${ }^{1} \mathrm{H}$ NMR $\left(400 \mathrm{MHz}, \mathrm{CDCl}_{3}\right) \delta 7.95(\mathrm{~d}, J=8.4 \mathrm{~Hz}, 1 \mathrm{H}), 7.57(\mathrm{~d}, J=1.6 \mathrm{~Hz}), 7.44$ $(\mathrm{dd}, J=8.4,2.0 \mathrm{~Hz}, 1 \mathrm{H}), 7.23(\mathrm{dd}, J=17.2,10.8 \mathrm{~Hz}, 1 \mathrm{H}), 6.76(\mathrm{dd}, J=17.6,10.8 \mathrm{~Hz}, 1 \mathrm{H}), 5.92(\mathrm{~d}, J=$ $17.6 \mathrm{~Hz}, 1 \mathrm{H}), 5.75(\mathrm{dd}, J=17.2,0.8 \mathrm{~Hz}, 1 \mathrm{H}), 5.51(\mathrm{dd}, J=11.2,0.8 \mathrm{~Hz}, 1 \mathrm{H}), 5.49$ (dd, $J=11.2,0.8 \mathrm{~Hz}$, 
$1 \mathrm{H}) ;{ }^{13} \mathrm{C} \mathrm{NMR}\left(150 \mathrm{MHz}, \mathrm{CDCl}_{3}\right) \delta 146.6,142.4,134.9,134.1,132.9,126.5,125.5,125.1,118.9,118.2$;

IR (ATR) 1600, 1575, 1509, 1337, 914, $835 \mathrm{~cm}^{-1}$; HRMS (ESI) calculated for $\mathrm{C}_{10} \mathrm{H}_{10} \mathrm{NO}_{2}\left(\mathrm{M}+\mathrm{H}^{+}\right)$ 176.0711, found 176.0709 .

3-Ethenyl-4-nitrophenyl trifluoromethanesulfonate (226), 2,4-diethenyl-nitrobenzene (227), and 2bromo-4-ethenyl-nitrobenzene (228). Cross coupling of $214(75.2 \mathrm{mg}, 0.22 \mathrm{mmol})$ with ethenyltributyltin $(85.5 \mathrm{mg}, 0.27 \mathrm{mmol})$ in the presence of $\mathrm{LiCl}(28.1 \mathrm{mg}, 0.66 \mathrm{mmol})$ and $\mathrm{Pd}\left(\mathrm{PPh}_{3}\right)_{2} \mathrm{Cl}_{2}$ (3.6 mg, $0.005 \mathrm{mmol})$ in DMF (1 mL) was performed as described for 207 under Conditions B. Work up and chromatography (hexanes/EtOAc, 97:3) gave, in order of elution, 227 (5.5 mg, $0.03 \mathrm{mmol}, 15 \%$ ) followed by a mixture of $\mathbf{2 2 8}$ and $\mathbf{2 2 6}$ (23.8 $\mathrm{mg}$, calculated from ${ }^{1} \mathrm{H}$ NMR spectrum: $18.0 \mathrm{mg}$ of $\mathbf{2 2 8}, 37 \%$ , $5.8 \mathrm{mg}$ of 226, 9\%) as a yellow oil. Spectral data for 228 from the mixture: ${ }^{1} \mathrm{H}$ NMR $\left(600 \mathrm{MHz}, \mathrm{CDCl}_{3}\right)$ $\delta 7.86(\mathrm{~d}, J=8.4 \mathrm{~Hz}, 1 \mathrm{H}), 7.74(\mathrm{~d}, J=1.8 \mathrm{~Hz}, 1 \mathrm{H}), 7.45(\mathrm{dd}, J=8.4,1.2 \mathrm{~Hz}, 1 \mathrm{H}), 6.68(\mathrm{dd}, J=17.4,10.8$ $\mathrm{Hz}, 1 \mathrm{H}), 5.90,(\mathrm{~d}, J=17.4 \mathrm{~Hz}, 1 \mathrm{H}), 5.52(\mathrm{~d}, J=10.8 \mathrm{~Hz}, 1 \mathrm{H}) ;{ }^{13} \mathrm{C} \mathrm{NMR}\left(150 \mathrm{MHz}, \mathrm{CDCl}_{3}\right) \delta 142.9$, 136.4, 133.7, 132.6, 126.1, 125.5, 119.3, 115.1; IR (ATR) 3095, 1573, 1526, 1346, 1217, $1139 \mathrm{~cm}^{-1}$; HRMS (ESI) calcd for $\mathrm{C}_{8} \mathrm{H}_{6} \mathrm{NNaO}_{2} \mathrm{Br}\left(\mathrm{M}+\mathrm{Na}^{+}\right)$249.9474; found

4-Ethenyl-3-nitrophenyl trifluoromethanesulfonate (229) and 2,5-diethenyl-nitrobenzene (230). ${ }^{185}$ Cross coupling of 215 (110 mg, $0.32 \mathrm{mmol})$ with ethenyltributyltin (134 mg, $0.42 \mathrm{mmol})$ in the presence of in the presence of $\mathrm{PPh}_{3}(6.9 \mathrm{mg}, 0.03 \mathrm{mmol})$ and $\mathrm{Pd}(\mathrm{dba})_{2}(3.6 \mathrm{mg}, 0.006 \mathrm{mmol})$ in dioxane $(1.5 \mathrm{~mL})$ was performed as described for $\mathbf{2 0 6}$ under Conditions A. Work up and chromatography (hexanes/EtOAc, 97:3) gave, in order of elution, 230 (1.2 $\mathrm{mg}, 0.0068 \mathrm{mmol}, 2 \%)$ and 229 (63.3 $\mathrm{mg}, 0.21 \mathrm{mmol}, 68 \%)$ as colorless oils. Spectral data for $\mathbf{2 3 0}$ were in accordance with literature values. Analytical data for 229: ${ }^{1} \mathrm{H}$ NMR (600 MHz, $\left.\mathrm{CDCl}_{3}\right) \delta 7.90(\mathrm{~d}, J=2.4 \mathrm{~Hz}, 1 \mathrm{H}), 7.74(\mathrm{~d}, J=8.4 \mathrm{~Hz}, 1 \mathrm{H}), 7.53$ (dd, $J=8.4,2.4 \mathrm{~Hz}$, $1 \mathrm{H}), 7.18(\mathrm{dd}, J=17.4,11.4 \mathrm{~Hz}, 1 \mathrm{H}), 5.79(\mathrm{~d}, J=17.4 \mathrm{~Hz}, 1 \mathrm{H}), 5.60(\mathrm{~d}, J=11.4 \mathrm{~Hz}, 1 \mathrm{H}) ;{ }^{13} \mathrm{C}$ NMR $(150$ $\left.\mathrm{MHz}, \mathrm{CDCl}_{3}\right) \delta 148.0,147.7,133.8,131.1,130.5,126.2,121.0,118.6\left(\mathrm{q}, J^{C-F}=319 \mathrm{~Hz}\right), 118.0$; IR (ATR) 3110, 1533, 1426, 1351, 1208, $1133 \mathrm{~cm}^{-1}$; HRMS (ESI) calcd for $\mathrm{C}_{9} \mathrm{H}_{6} \mathrm{NNaO}_{5} \mathrm{~F}_{3} \mathrm{~S}\left(\mathrm{M}+\mathrm{Na}^{+}\right) 319.9811$; found 319.9810 .

4-Ethenyl-3-nitrophenyl trifluoromethanesulfonate (229), 2,5-diethenyl-nitrobenzene (230), and 2bromo-5-ethenyl-nitrobenzene (231). ${ }^{186}$ Cross coupling of 215 (120 mg, $\left.0.34 \mathrm{mmol}\right)$ with ethenyltributyltin $(135 \mathrm{mg}, 0.43 \mathrm{mmol})$ in the presence of $\mathrm{LiCl}(48.2 \mathrm{mg}, 1.13 \mathrm{mmol})$ and $\mathrm{Pd}\left(\mathrm{PPh}_{3}\right)_{2} \mathrm{Cl}_{2}$ (4.8 mg, $0.007 \mathrm{mmol})$ in DMF (1.5 mL) was performed as described for 207 under Conditions B. Work up and chromatography (hexanes/EtOAc, 97:3) gave, in order of elution, 230 (1.7 mg, $0.0097 \mathrm{mmol}, 3 \%$ ), 231 (27.8 $\mathrm{mg}, 0.12 \mathrm{mmol}, 36 \%$ ) as a colorless oil and a mixture of 229 and 215 (42 $\mathrm{mg}$, calculated from 
${ }^{1}$ H NMR spectrum: $22922 \mathrm{mg}, 23 \%$ and $21520 \mathrm{mg}$, 17\%). Spectral data for 231 were in accordance with literature values.

3-Ethenyl-2-nitrophenyl trifluoromethanesulfonate (232), 2-bromo-6-ethenyl-nitrobenzene (233), and 2,6-diethenyl-nitrobenzene (234). Cross coupling of 216 (104 mg, 0.30 mmol) with ethenyltributyltin $(121 \mathrm{mg}, 0.38 \mathrm{mmol})$ in the presence of in the presence of $\mathrm{PPh}_{3}(6.7 \mathrm{mg}, 0.03 \mathrm{mmol})$ and $\mathrm{Pd}(\mathrm{dba})_{2}(3.7 \mathrm{mg}, 0.006 \mathrm{mmol})$ in dioxane $(1.5 \mathrm{~mL})$ was performed as described for 206 under Conditions A. Work up and chromatography (hexanes/EtOAc, 97:3) gave, in order of elution, 234 (16.0 $\mathrm{mg}, 0.09 \mathrm{mmol}, 30 \%)$ a colorless oil, 233 (2.3 mg, $0.01 \mathrm{mmol}, 3 \%)$ and 232 (52.9 $\mathrm{mg}, 0.18 \mathrm{mmol}, 60 \%)$ as faint yellow solids.

Analytical data for 232: $\mathrm{mp}=38-39{ }^{\circ} \mathrm{C} ;{ }^{1} \mathrm{H}$ NMR $\left(400 \mathrm{MHZ}, \mathrm{CDCl}_{3}\right) \delta 7.67(\mathrm{~d}, J=8.0,0.8 \mathrm{~Hz}, 1 \mathrm{H}), 7.57$ (t, $J=8.4 \mathrm{~Hz}, 1 \mathrm{H}), 7.40(\mathrm{dd}, J=8.4,1.2 \mathrm{~Hz}, 1 \mathrm{H}), 6.68(\mathrm{dd}, J=17.6,11.2 \mathrm{~Hz}, 1 \mathrm{H}), 5.91(\mathrm{~d}, J=17.2 \mathrm{~Hz}$, 1H), $5.61(\mathrm{~d}, J=11.2 \mathrm{~Hz}, 1 \mathrm{H}) ;{ }^{13} \mathrm{C}$ NMR (150 MHZ, $\left.\mathrm{CDCl}_{3}\right) \delta 142.0,140.2,133.1,131.6,128.5,126.4$, 121.9, 121.4, $118.4\left(\mathrm{q}, J^{C-F}=319 \mathrm{~Hz}\right.$ );IR (ATR) 3090, 1533, 1427, 1361, 1211, $1138 \mathrm{~cm}^{-1}$; HRMS (ESI) calcd for $\mathrm{C}_{9} \mathrm{H}_{6} \mathrm{NNaO}_{5} \mathrm{~F}_{3} \mathrm{~S}\left(\mathrm{M}+\mathrm{Na}^{+}\right) 319.9811$; found 319.9809 .

Analytical data for 233: $\mathrm{mp}=42-44{ }^{\circ} \mathrm{C} ;{ }^{1} \mathrm{H}$ NMR $\left(600 \mathrm{MHz}, \mathrm{CDCl}_{3}\right) \delta 7.57(\mathrm{t}, J=7.2 \mathrm{~Hz}, 2 \mathrm{H}), 7.32(\mathrm{t}, J$ $=8.4 \mathrm{~Hz}, 1 \mathrm{H}), 6.57(\mathrm{dd}, J=17.4,11.4 \mathrm{~Hz}, 1 \mathrm{H}), 5.85(\mathrm{~d}, J=17.4 \mathrm{~Hz}, 1 \mathrm{H}), 5.51(\mathrm{~d}, J=10.8 \mathrm{~Hz}, 1 \mathrm{H}) ;{ }^{13} \mathrm{C}$ NMR $\left(150 \mathrm{MHz}, \mathrm{CDCl}_{3}\right) \delta 150.1,132.6,131.6,130.9,129.0,125.5,120.8,112.9$; IR (ATR) 3077, 1557, 1521, 1460, 1365, $1187 \mathrm{~cm}^{1}$. HRMS (ESI) calcd for $\mathrm{C}_{8} \mathrm{H}_{6} \mathrm{NNaO}_{2} \mathrm{Br}\left(\mathrm{M}+\mathrm{Na}^{+}\right)$251.9454; found 251.9454. Analytical data for 234: ${ }^{1} \mathrm{H}$ NMR $\left(400 \mathrm{MHz}, \mathrm{CDCl}_{3}\right) \delta 7.54(\mathrm{~d}, J=8.0 \mathrm{~Hz}, 2 \mathrm{H}), 7.43(\mathrm{t}, J=7.2 \mathrm{~Hz}, 1 \mathrm{H})$, $6.59(\mathrm{dd}, J=17.2,10.8 \mathrm{~Hz}, 2 \mathrm{H}), 5.82(\mathrm{~d}, J=17.6 \mathrm{~Hz}, 2 \mathrm{H}), 5.47(\mathrm{~d}, J=11.2 \mathrm{~Hz}, 2 \mathrm{H}) ;{ }^{13} \mathrm{C}$ NMR $(150$ $\mathrm{MHz}_{\mathrm{CDCl}}$ ) $\delta 148.6,130.2,129.8,129.6,125.8,119.6$; IR (ATR) 1513, 1365, 926, 850, 809, $731 \mathrm{~cm}^{-1}$; HRMS (ESI) calcd for $\mathrm{C}_{10} \mathrm{H}_{10} \mathrm{NO}_{2}\left(\mathrm{M}+\mathrm{H}^{+}\right)$176.0711; found 176.0709 .

2-Bromo-6-ethenyl-nitrobenzene (233). Cross coupling of 216 (119 mg, 0.34 mmol) with ethenyltributyltin (138 mg, $0.44 \mathrm{mmol})$ in the presence of $\mathrm{LiCl}(45.6 \mathrm{mg}, 1.08 \mathrm{mmol})$ and $\mathrm{Pd}\left(\mathrm{PPh}_{3}\right)_{2} \mathrm{Cl}_{2}$ (5.0 mg, $0.007 \mathrm{mmol})$ in DMF (1.5 mL) was performed as described for 207 under Conditions B. Work up and chromatography (hexanes/EtOAc, 97:3) gave 233 (47.1 $\mathrm{mg}, 0.21 \mathrm{mmol}, 61 \%$ ) as an off-white solid.

3-Ethenyl-2-nitrophenyl trifluoromethanesulfonate (232), 2-bromo-6-ethenyl-nitrobenzene (233), and 2,6-diethenyl-nitrobenzene (234). Cross coupling of 216 (105 mg, 0.300 mmol) with ethenyltributyltin $(140 \mathrm{mg}, 0.442 \mathrm{mmol})$ in the presence of $\mathrm{Pd}\left(\mathrm{PPh}_{3}\right)_{2} \mathrm{Cl}_{2}(4.2 \mathrm{mg}, 0.006 \mathrm{mmol})$ in 1,4dioxane $(1 \mathrm{~mL})$ was performed as described in Conditions $\mathrm{C}\left(100{ }^{\circ} \mathrm{C}, 27 \mathrm{~h}\right)$. Work up and purification 
gave after chromatography on $\mathrm{SiO}_{2} / \mathrm{K}_{2} \mathrm{CO}_{3}\left(10 \% \mathrm{~K}_{2} \mathrm{CO}_{3}\right.$, hexanes/EtOAc, 19:1), in order of elution, 234 (11.9 mg, $0.068 \mathrm{mmol}, 23 \%)$ a colorless oil, 233 (0.9 mg, $0.004 \mathrm{mmol}, 1 \%)$ and 232 (60.6 mg, 0.204 mmol, 68\%).

6-Ethenyl-2-nitrophenyl trifluoromethanesulfonate (235). Cross coupling of 217 (135 mg, $0.39 \mathrm{mmol}$ ) with ethenyltributyltin $(129 \mathrm{mg}, 0.41 \mathrm{mmol})$ in the presence of in the presence of $\mathrm{PPh}_{3}(8.2 \mathrm{mg}, 0.03$ $\mathrm{mmol})$ and $\mathrm{Pd}(\mathrm{dba})_{2}(4.5 \mathrm{mg}, 0.008 \mathrm{mmol})$ in dioxane $(2 \mathrm{~mL})$ was performed as described for 206 under Conditions A. Work up and chromatography (hexanes/EtOAc, 97:3) to afford in order of elution, 236 and 225 (8.5 mg, 1:1 mixture), 2-bromo-6-nitrophenol ${ }^{183}$ (22.7 mg mixed with dba), and a mixture of 217 and $235(95.1 \mathrm{mg})$. The latter fraction was repurified by chromatograhy (hexanes/EtOAc, 97:3) to give in order of elution, 235 as a colorless oil (27.8 mg, 0.09 mmol, 24\%) and 217 (12.3 mg, $0.04 \mathrm{mmol}, 9 \%$ ). Analytical data for 235: ${ }^{1} \mathrm{H}$ NMR $\left(600 \mathrm{MHz}, \mathrm{CDCl}_{3}\right) \delta 7.98(\mathrm{dd}, J=8.4,1.8 \mathrm{~Hz}, 1 \mathrm{H}), 7.90(\mathrm{dd}, J=7.8$, $1.8 \mathrm{~Hz}, 1 \mathrm{H}), 7.52(\mathrm{t}, J=7.8 \mathrm{~Hz}, 1 \mathrm{H}), 6.99(\mathrm{dd}, J=18.0,11.4 \mathrm{~Hz}, 1 \mathrm{H}), 5.95(\mathrm{~d}, J=18.0 \mathrm{~Hz}, 1 \mathrm{H}), 5.67(\mathrm{~d}$, $J=11.4 \mathrm{~Hz}, 1 \mathrm{H}) ;{ }^{13} \mathrm{C}$ NMR $\left(100 \mathrm{MHz}, \mathrm{CDCl}_{3}\right) \delta 143.1,137.9,134.4,132.0,128.5,128.0,125.5,121.4$, $118.3\left(\mathrm{q}, J^{C-F}=319 \mathrm{~Hz}\right.$ ); IR (ATR) 3103, 1539, 1429, 1351, 1210, $1131 \mathrm{~cm}^{-1}$; HRMS (ESI) calcd for $\mathrm{C}_{9} \mathrm{H}_{6} \mathrm{NNaO}_{5} \mathrm{~F}_{3} \mathrm{~S}\left(\mathrm{M}+\mathrm{Na}^{+}\right)$319.9811; found 319.9808.

3-Bromo-2-ethenyl-nitrobenzene (236). ${ }^{187}$ Cross coupling of 217 (99.8 mg, 0.29 mmol) with ethenyltributyltin $(97.5 \mathrm{mg}, 0.31 \mathrm{mmol})$ in the presence of $\mathrm{LiCl}(36.6 \mathrm{mg}, 0.86 \mathrm{mmol})$ and $\mathrm{Pd}\left(\mathrm{PPh}_{3}\right)_{2} \mathrm{Cl}_{2}$ (4.1 mg, $0.006 \mathrm{mmol}$ ) in DMF (1.5 mL) was performed as described for 207 under Conditions B. Work up and chromatography (hexanes/EtOAc, 9:1) gave, in order of elution, 236 as a yellow oil (28.3 mg, 0.12 mmol, 43\%), 2-bromo-6-nitrophenol (7.4 mg, $0.03 \mathrm{mmol}, 12 \%$ ), and 217 (5.0 mg, $0.01 \mathrm{mmol}, 5 \%$ ). Spectral data for $\mathbf{2 3 6}$ were in accordance with literature values.

\section{3-Ethenyl-6-nitrophenyl trifluoromethanesulfonate (235), 3-bromo-6-ethenylnitrobenzene (236),} and 2,3-diethenyl-nitrobenzene (225). Cross coupling of 217 (117 mg, $0.334 \mathrm{mmol})$ with ethenyltributyltin $(159 \mathrm{mg}, 0.501 \mathrm{mmol})$ in the presence of $\mathrm{Pd}\left(\mathrm{PPh}_{3}\right)_{2} \mathrm{Cl}_{2}(5.1 \mathrm{mg}, 0.007 \mathrm{mmol})$ in 1,4dioxane $(1 \mathrm{~mL})$ was performed as described in Conditions $\mathrm{C}\left(100{ }^{\circ} \mathrm{C}, 24 \mathrm{~h}\right)$. Work up and purification gave after chromatography on $\mathrm{SiO}_{2} / \mathrm{K}_{2} \mathrm{CO}_{3}\left(10 \% \mathrm{~K}_{2} \mathrm{CO}_{3}\right.$, hexanes/EtOAc, 97:3), in order of elution, a mixture of 236 and 225 (16.1 mg, 1:2 mixture, 8\% and 4\%, respectively) and 235 (65.3 mg, $0.220 \mathrm{mmol}$, $66 \%)$.

2-Ethenyl-4-nitrophenyl trifluoromethanesulfonate (237). Cross coupling of 2-bromo-4-nitrophenyl trifluoromethanesulfonate $(\mathbf{2 1 8})^{164}(102 \mathrm{mg}, 0.29 \mathrm{mmol})$ with ethenyltributyltin $(116 \mathrm{mg}, 0.37 \mathrm{mmol})$ in 
the presence of in the presence of $\mathrm{PPh}_{3}(6.5 \mathrm{mg}, 0.03 \mathrm{mmol})$ and $\mathrm{Pd}(\mathrm{dba})_{2}(4.1 \mathrm{mg}, 0.007 \mathrm{mmol})$ in dioxane $(1.5 \mathrm{~mL})$ was performed as described for 206 under Conditions A. Work up and chromatography (hexanes/EtOAc, 8:2) gave, in order of elution, a mixture of 218 and 237 (45.1 mg) followed by 2-bromo4-nitrophenol ${ }^{176}(5.2 \mathrm{mg}, 0.02 \mathrm{mmol}, 7 \%)$. The mixture was repurified by chromatographed (hexanes/EtOAc, 97:3) to afford, in order of elution, 237 (19.3 mg, $0.06 \mathrm{mmol}, 22 \%)$ as a light pink oil and 218 (7.0 mg, $0.02 \mathrm{mmol}, 7 \%)$. Analytical data for 237: ${ }^{1} \mathrm{H}$ NMR (400 MHz, $\left.\mathrm{CDCl}_{3}\right) \delta 8.52(\mathrm{~d}, J=2.8$ $\mathrm{Hz}, 1 \mathrm{H}), 8.20$ (dd, $J=9.2,2.8 \mathrm{~Hz}, 1 \mathrm{H}), 7.48$ (d, $J=9.2 \mathrm{~Hz}, 1 \mathrm{H}), 6.94(\mathrm{dd}, J=17.6,11.2 \mathrm{~Hz}, 1 \mathrm{H}), 6.04$ (d, $J=17.6 \mathrm{~Hz}, 1 \mathrm{H}), 5.70(\mathrm{~d}, J=11.2 \mathrm{~Hz}, 1 \mathrm{H}) ;{ }^{13} \mathrm{C}\left(100 \mathrm{MHz}, \mathrm{CDCl}_{3}\right) \delta 149.9,147.2,132.7,127.2,124.0$, 122.9, 122.6, 121.7, 118.5 (q, $J^{C-F}=319 \mathrm{~Hz}$ ); IR (ATR) 3107, 1536, 1424, 1347, 1209, $1134 \mathrm{~cm}^{-1}$; HRMS (ESI) calcd for $\mathrm{C}_{9} \mathrm{H}_{6} \mathrm{NNaO}_{5} \mathrm{~F}_{3} \mathrm{~S}\left(\mathrm{M}+\mathrm{Na}^{+}\right) 319.9811$; found 319.9809 .

3-Bromo-4-ethenyl-nitrobenzene (238). Cross coupling of 218 (110 mg, $0.32 \mathrm{mmol})$ with ethenyltributyltin $(138 \mathrm{mg}, 0.43 \mathrm{mmol})$ in the presence of $\mathrm{LiCl}(42.6 \mathrm{mg}, 1.0 \mathrm{mmol})$ and $\mathrm{Pd}\left(\mathrm{PPh}_{3}\right)_{2} \mathrm{Cl}_{2}$ (4.5 mg, $0.006 \mathrm{mmol})$ in DMF (1.5 mL) was performed as described for 207 under Conditions B. Work up and chromatography (hexanes/EtOAc, 85:15) to give a mixture of $\mathbf{2 1 8}$ and $\mathbf{2 3 8}$. The mixture was repurified by chromatography (hexanes/EtOAc, 97:3) to afford in order of elution, 238 (40.7 mg, 0.18 mmol, 57\%) as a yellow oil, $218(8.7 \mathrm{mg}, 0.02 \mathrm{mmol}, 8 \%)$, and 3-bromo-4-nitrophenol (11.1 $\mathrm{mg}, 0.05$ mmol, 16\%). Analytical data for 238: ${ }^{1} \mathrm{H} \mathrm{NMR}\left(600 \mathrm{MHz}, \mathrm{CDCl}_{3}\right) \delta 8.43(\mathrm{~d}, J=2.4 \mathrm{~Hz}, 1 \mathrm{H}), 8.14(\mathrm{ddd}, J$ = 8.4, 2.4, 0.6 Hz, 1H), $7.69(\mathrm{~d}, J=9.0 \mathrm{~Hz}, 1 \mathrm{H}), 7.08(\mathrm{dd}, J=17.4,10.8 \mathrm{~Hz}, 1 \mathrm{H}), 5.88(\mathrm{~d}, J=17.4 \mathrm{~Hz}$, $1 \mathrm{H}), 5.60(\mathrm{dd}, J=10.8,0.6 \mathrm{~Hz}, 1 \mathrm{H}) ;{ }^{13} \mathrm{C} \mathrm{NMR}\left(150 \mathrm{MHz}, \mathrm{CDCl}_{3}\right) \delta 147.2,143.7,134.3,128.2,127.0$, 123.3, 122.4, 120.8; IR (ATR) 3099, 1520, 1342, 1116, $1035 \mathrm{~cm}^{-1}$; HRMS (ESI) calcd for $\mathrm{C}_{8} \mathrm{H}_{6} \mathrm{NNaO}_{2} \mathrm{Br}$ $\left(\mathrm{M}+\mathrm{Na}^{+}\right)$249.9479; found 249.9478 .

2-Ethenyl-4-nitrophenyl trifluoromethanesulfonate (237). Cross coupling of 218 (89.1 mg, 0.255 mmol) with ethenyltributyltin $(105 \mathrm{mg}, 0.331 \mathrm{mmol})$ in the presence of $\mathrm{Pd}\left(\mathrm{PPh}_{3}\right)_{2} \mathrm{Cl}_{2}(3.6 \mathrm{mg}, 0.005$ $\mathrm{mmol}$ ) in 1,4-dioxane $(0.8 \mathrm{~mL})$ was performed as described for $\mathbf{2 0 6}$ as described under Conditions C (100 $\left.{ }^{\circ} \mathrm{C}, 24 \mathrm{~h}\right)$. Work up and purification gave after chromatography on $\mathrm{SiO}_{2} / \mathrm{K}_{2} \mathrm{CO}_{3}\left(10 \% \mathrm{~K}_{2} \mathrm{CO}_{3}\right.$, hexanes/EtOAc, 98:2) gave 237 (71.4 mg, $0.240 \mathrm{mmol}, 94 \%$ ).

3-Ethenyl-4-nitrophenyl trifluoromethanesulfonate (239). Cross coupling of 219 (106 mg, $0.30 \mathrm{mmol}$ ) with ethenyltributyltin $(130 \mathrm{mg}, 0.41 \mathrm{mmol})$ in the presence of in the presence of $(6.4 \mathrm{mg}, 0.02 \mathrm{mmol})$ and $\mathrm{Pd}(\mathrm{dba})_{2}(3.4 \mathrm{mg}, 0.006 \mathrm{mmol})$ in dioxane $(1.5 \mathrm{~mL})$ was performed as described for 206 under Conditions A. Work up and chromatography (hexanes/EtOAc, 97:3) gave, in order of elution, 219 (4.1 $\mathrm{mg}, 0.01 \mathrm{mmol}, 4 \%)$ and $239(70.0 \mathrm{mg}, 0.24 \mathrm{mmol}, 78 \%)$ as a colorless oil. Analytical data for $239:{ }^{1} \mathrm{H}$ 
$\operatorname{NMR}\left(400 \mathrm{MHz}, \mathrm{CDCl}_{3}\right) \delta 8.30(\mathrm{t}, J=1.6 \mathrm{~Hz}, 1 \mathrm{H}), 8.02(\mathrm{t}, J=2.4 \mathrm{~Hz}, 1 \mathrm{H}), 7.60(\mathrm{t}, J=2.0 \mathrm{~Hz}, 1 \mathrm{H}), 6.77$ $(\mathrm{dd}, J=17.2,10.8 \mathrm{~Hz}, 1 \mathrm{H}), 5.98(\mathrm{~d}, J=17.6 \mathrm{~Hz}, 1 \mathrm{H}), 5.60(\mathrm{~d}, J=10.8 \mathrm{~Hz}, 1 \mathrm{H}) ;{ }^{13} \mathrm{C} \mathrm{NMR}(150 \mathrm{MHz}$, $\left.\mathrm{CDCl}_{3}\right) \delta 149.4,149.2,141.7,133.2,124.6,120.6,119.8,118.6\left(\mathrm{q}, J^{C-F}=319 \mathrm{~Hz}\right), 115.6$; IR (ATR) 3103, 1540, 1425, 1348, 1213, $1132 \mathrm{~cm}^{-1}$; HRMS (ESI) calcd for $\mathrm{C}_{9} \mathrm{H}_{7} \mathrm{NO}_{5} \mathrm{~F}_{3} \mathrm{~S}\left(\mathrm{M}+\mathrm{H}^{+}\right)$297.9997; found 297.9997.

3-Bromo-5-ethenyl-nitrobenzene (240). Cross coupling of 219 (109 mg, $0.31 \mathrm{mmol})$ with ethenyltributyltin $(139 \mathrm{mg}, 0.44 \mathrm{mmol})$ in the presence of $\mathrm{LiCl}(45.0 \mathrm{mg}, 1.1 \mathrm{mmol})$ and $\mathrm{Pd}\left(\mathrm{PPh}_{3}\right)_{2} \mathrm{Cl}_{2}$ (4.7 mg, $0.007 \mathrm{mmol})$ in DMF (1.5 mL) was performed as described for 207 under Conditions B. Work up and chromatography (hexanes/EtOAc, 97:3) gave 240 (47.1 $\mathrm{mg}, 0.21 \mathrm{mmol}, 66 \%$ ) as an off-white solid. Analytical data for 240: $\mathrm{mp}=37-39{ }^{\circ} \mathrm{C} ;{ }^{1} \mathrm{H} \mathrm{NMR}\left(600 \mathrm{MHz}, \mathrm{CDCl}_{3}\right) \delta 8.23(\mathrm{t}, J=1.8 \mathrm{~Hz}, 1 \mathrm{H})$, $8.16(\mathrm{t}, J=1.8 \mathrm{~Hz}, 1 \mathrm{H}), 7.82(\mathrm{t}, J=1.8 \mathrm{~Hz}, 1 \mathrm{H}), 6.70(\mathrm{dd}, J=17.4,10.8 \mathrm{~Hz}, 1 \mathrm{H}), 5.90(\mathrm{~d}, J=18.0 \mathrm{~Hz}$, 1H), $5.50(\mathrm{~d}, J=10.8 \mathrm{~Hz}, 1 \mathrm{H}) ;{ }^{13} \mathrm{C} \mathrm{NMR}\left(150 \mathrm{MHz}, \mathrm{CDCl}_{3}\right) \delta 149.0,140.8,134.8,133.6,125.3,122.9$, 119.6, 118.5; IR (ATR) 3079, 1531, 1339, 1301, $1214 \mathrm{~cm}^{1}$; HRMS (ESI) calcd for $\mathrm{C}_{8} \mathrm{H}_{6} \mathrm{NNaO}_{2} \mathrm{Br}$ $\left(\mathrm{M}+\mathrm{Na}^{+}\right)$251.9454; found 251.9452.

3-Ethenyl-5-nitrophenyl trifluoromethanesulfonate (239). Cross coupling of 219 (104 mg, 0.296 mmol) with ethenyltributyltin $(122 \mathrm{mg}, 0.385 \mathrm{mmol})$ in the presence of $\mathrm{Pd}\left(\mathrm{PPh}_{3}\right)_{2} \mathrm{Cl}_{2}(4.3 \mathrm{mg}, 0.006$ $\mathrm{mmol})$ in 1,4-dioxane (1 mL) was performed as described for $\mathbf{2 0 6}$ as described under Conditions C (100 $\left.{ }^{\circ} \mathrm{C}, 24 \mathrm{~h}\right)$. Work up and purification gave after chromatography on $\mathrm{SiO}_{2} / \mathrm{K}_{2} \mathrm{CO}_{3}\left(10 \% \mathrm{~K}_{2} \mathrm{CO}_{3}\right.$, hexanes/EtOAc, 98:2) gave 239 (78.5 mg, $0.264 \mathrm{mmol}, 89 \%)$.

4-Ethenyl-2-nitrophenyl trifluoromethanesulfonate (241). Cross coupling of 220 (103 mg, $0.29 \mathrm{mmol}$ ) with ethenyltributyltin $(112 \mathrm{mg}, 0.35 \mathrm{mmol})$ in the presence of in the presence of $(6.8 \mathrm{mg}, 0.03 \mathrm{mmol})$ and $\mathrm{Pd}(\mathrm{dba})_{2}(3.4 \mathrm{mg}, 0.006 \mathrm{mmol})$ in dioxane $(1.5 \mathrm{~mL})$ was performed as described for 206 under Conditions A. Work up and chromatography (hexanes/EtOAc, 9:1) gave, in order of elution, a mixture of 220 and 5-bromo-2-nitrophenol (11.3 mg) followed by 241 as a yellow oil mixed with dibenzylideneacetone (34.6 mg, $0.12 \mathrm{mmol}, 41 \%$ of $\mathbf{2 4 1}$, calculated from ${ }^{1} \mathrm{H}$ NMR spectrum).

5-Bromo-2-ethenyl-nitrobenzene (242). Cross coupling of 220 (111 mg, $0.32 \mathrm{mmol})$ with ethenyltributyltin $(131 \mathrm{mg}, 0.41 \mathrm{mmol})$ in the presence of $\mathrm{LiCl}(44.5 \mathrm{mg}, 0.86 \mathrm{mmol})$ and $\mathrm{Pd}\left(\mathrm{PPh}_{3}\right)_{2} \mathrm{Cl}_{2}$ (4.2 mg, $0.006 \mathrm{mmol})$ in DMF (1.5 mL) was performed as described for 207 under Conditions B. Work up and chromatography (hexanes/EtOAc, 9:1) gave 242 as a yellow solid (56.3 $\mathrm{mg}, 0.25 \mathrm{mmol}, 78 \%$ ). Analytical data for 242: $\mathrm{mp}=40-41{ }^{\circ} \mathrm{C} ;{ }^{1} \mathrm{H} \mathrm{NMR}\left(600 \mathrm{MHz}, \mathrm{CDCl}_{3}\right) \delta 8.43(\mathrm{~d}, J=2.4 \mathrm{~Hz}, 1 \mathrm{H}), 8.14(\mathrm{ddd}$, 
$J=8.4,2.4,0.6 \mathrm{~Hz}, 1 \mathrm{H}), 7.69(\mathrm{~d}, J=9.0 \mathrm{~Hz}, 1 \mathrm{H}), 7.08(\mathrm{dd}, J=17.4,10.8 \mathrm{~Hz}, 1 \mathrm{H}), 5.88(\mathrm{~d}, J=17.4 \mathrm{~Hz}$, $1 \mathrm{H}), 5.60(\mathrm{~d}, J=10.8 \mathrm{~Hz}, 1 \mathrm{H}) ;{ }^{13} \mathrm{C} \mathrm{NMR}\left(150 \mathrm{MHz}, \mathrm{CDCl}_{3}\right) \delta 148.0,136 . .1,132.2,131.5,129.7,127.3$, 121.4, 119.7; IR (ATR) 3097, 1552, 1514, 1341, $1149 \mathrm{~cm}^{-1}$; HRMS (ESI) calcd for $\mathrm{C}_{8} \mathrm{H}_{6} \mathrm{NNaO}_{2} \mathrm{Br}$ $\left(\mathrm{M}+\mathrm{Na}^{+}\right)$251.9454; found 251.9455 .

4-Ethenyl-2-nitrophenyl trifluoromethanesulfonate (241). Cross coupling of 220 (122 mg, 0.348 mmol) with ethenyltributyltin $(162 \mathrm{mg}, 0.511 \mathrm{mmol})$ in the presence of $\mathrm{Pd}\left(\mathrm{PPh}_{3}\right)_{2} \mathrm{Cl}_{2}(4.9 \mathrm{mg}, 0.007$ mmol) in 1,4-dioxane (2.5 mL) was performed as described for 206 as described under Conditions C (100 $\left.{ }^{\circ} \mathrm{C}, 26 \mathrm{~h}\right)$. Work up and purification gave after chromatography on $\mathrm{SiO}_{2} / \mathrm{K}_{2} \mathrm{CO}_{3}\left(10 \% \mathrm{~K}_{2} \mathrm{CO}_{3}\right.$, hexanes/EtOAc, 98:2) gave 241 (54.5 mg, $0.311 \mathrm{mmol}$, 89\%). Analytical data for 241: ${ }^{1} \mathrm{H}$ NMR (600 $\left.\left.\mathrm{MHz}, \mathrm{CDCl}_{3}\right) \delta \mathrm{d}, J=2.4 \mathrm{~Hz}, 1 \mathrm{H}\right), 7.72(\mathrm{dd}, J=8.4,1.8 \mathrm{~Hz}, 1 \mathrm{H}), 7.41(\mathrm{~d}, J=9.0 \mathrm{~Hz}, 1 \mathrm{H}), 6.74(\mathrm{dd}, J=17.4$, $10.8 \mathrm{~Hz}, 1 \mathrm{H}), 5.92$ (d, $J=17.4 \mathrm{~Hz}, 1 \mathrm{H}), 5.55$ (d, $J=10.8 \mathrm{~Hz}, 1 \mathrm{H}) ;{ }^{13} \mathrm{C}$ NMR $\left(150 \mathrm{MHz}, \mathrm{CDCl}_{3}\right) \delta 141.6$, 140.2, 139.2, 133.1, 132.2, 124.3, 123.8, 119.1, 118.5 (q, $J^{C-F}=319 \mathrm{~Hz}$ ), IR (ATR) 3117, 1581, 1519, 1431, 1343, $1207 \mathrm{~cm}^{-1}$; HRMS (ESI) calculated for $\mathrm{C}_{9} \mathrm{H}_{7} \mathrm{~F}_{3} \mathrm{NO}_{5} \mathrm{~S}\left(\mathrm{M}+\mathrm{H}^{+}\right)$297.9997, found 297.9994 .

3-Ethenyl-6-nitrophenyl trifluoromethanesulfonate (243). Cross coupling of 3-bromo-6-nitrophenyl trifluoromethanesulfonate $(\mathbf{2 2 1})(100 \mathrm{mg}, 0.29 \mathrm{mmol})$ with ethenyltributyltin $(95.1 \mathrm{mg}, 0.30 \mathrm{mmol})$ in the presence of in the presence of $(6.0 \mathrm{mg}, 0.02 \mathrm{mmol})$ and $\mathrm{Pd}(\mathrm{dba})_{2}(3.3 \mathrm{mg}, 0.006 \mathrm{mmol})$ in dioxane $(1.5$ $\mathrm{mL}$ ) was performed as described for $\mathbf{2 0 6}$ under Conditions A. Work up and chromatography (hexanes/EtOAc, 97:3) gave, in order of elution, a mixture of $\mathbf{2 2 1}$ and dba (17.2 $\mathrm{mg}$ ) followed by $\mathbf{2 4 3}$ (34.6 mg, $0.12 \mathrm{mmol}, 41 \%$ ) as a yellow oil. Analytical data for 243: ${ }^{1} \mathrm{H}$ NMR $\left(600 \mathrm{MHz}, \mathrm{CDCl}_{3}\right) \delta 8.15$ $(\mathrm{d}, J=8.4 \mathrm{~Hz}, 1 \mathrm{H}), 7.55(\mathrm{dd}, J=9.0,1.8 \mathrm{~Hz}, 1 \mathrm{H}), 7.41(\mathrm{~d}, J=1.2 \mathrm{~Hz}, 1 \mathrm{H}), 6.75(\mathrm{dd}, J=17.4,10.8 \mathrm{~Hz}$, $1 \mathrm{H}), 5.97(\mathrm{~d}, J=17.4 \mathrm{~Hz}, 1 \mathrm{H}), 5.63(\mathrm{~d}, J=10.8 \mathrm{~Hz}, 1 \mathrm{H}) ;{ }^{13} \mathrm{C} \mathrm{NMR}\left(150 \mathrm{MHz}, \mathrm{CDCl}_{3}\right) \delta 145.3,141.9$, 139.9, 133.3, 127.1, 126.3, 121.7, 120.9, 118.5 (q, $J^{C-F}=318$ Hz); IR (ATR) 3114, 1587, 1529, 1429, 1341, $1209 \mathrm{~cm}^{-1}$; HRMS (ESI) calcd for $\mathrm{C}_{9} \mathrm{H}_{7} \mathrm{NO}_{5} \mathrm{~F}_{3} \mathrm{~S}\left(\mathrm{M}+\mathrm{H}^{+}\right)$297.9997; found 297.9995.

\section{3-Ethenyl-6-nitrophenyl trifluoromethanesulfonate (243) and 4-Bromo-2-ethenyl-nitrobenzene} (244). ${ }^{188}$ Cross coupling of 221 (99.5 mg, $\left.0.29 \mathrm{mmol}\right)$ with ethenyltributyltin $(98.1 \mathrm{mg}, 0.31 \mathrm{mmol})$ in the presence of $\mathrm{LiCl}(37.0 \mathrm{mg}, 0.87 \mathrm{mmol})$ and $\mathrm{Pd}\left(\mathrm{PPh}_{3}\right)_{2} \mathrm{Cl}_{2}(4.0 \mathrm{mg}, 0.006 \mathrm{mmol})$ in DMF $(1.5 \mathrm{~mL})$ was performed as described for 207 under Conditions B. Work up and chromatography (hexanes/EtOAc, 9:1) gave, in order of elution, 244 (11.0 mg, $0.05 \mathrm{mmol}, 17 \%)$ as an off-white solid, 221 (22.9 mg, $0.07 \mathrm{mmol}$, $22 \%$ ) and 243 (19.4 $\mathrm{mg}, 0.07 \mathrm{mmol}, 23 \%)$. Spectral data for $\mathbf{2 4 4}$ were in accordance with literature values. 
3-Ethenyl-6-nitrophenyl trifluoromethanesulfonate (243) Cross coupling of 221 (96.8 mg, 0.277 mmol) with ethenyltributyltin $(114 \mathrm{mg}, 0.360 \mathrm{mmol})$ in the presence of $\mathrm{Pd}\left(\mathrm{PPh}_{3}\right)_{2} \mathrm{Cl}_{2}(4.0 \mathrm{mg}, 0.006$ $\mathrm{mmol})$ in 1,4-dioxane $(0.7 \mathrm{~mL})$ was performed as described for 206 as described under Conditions C (100 $\left.{ }^{\circ} \mathrm{C}, 24 \mathrm{~h}\right)$. Work up and purification by chromatography on $\mathrm{SiO}_{2} / \mathrm{K}_{2} \mathrm{CO}_{3}\left(10 \% \mathrm{~K}_{2} \mathrm{CO}_{3}\right.$, hexanes/EtOAc, 19:1) gave, in order of elution, a 6:1 mixture of 227 and $244(5.1 \mathrm{mg}, 0.024 \mathrm{mmol}, 9 \%$ of 227 and $0.9 \mathrm{mg}$, $0.004 \mathrm{mmol}, 14 \%$ of $\mathbf{2 4 4}$, calculated from ${ }^{1} \mathrm{H}$ NMR spectrum) and $\mathbf{2 4 3}$ (60.9 mg, $0.205 \mathrm{mmol}, \mathbf{7 4 \%}$ ).

2-Ethenyl-5-nitrophenyl trifluoromethanesulfonate (245). Cross coupling of 222 (74.7 $\mathrm{mg}, 0.21$ mmol) with ethenyltributyltin $(83.2 \mathrm{mg}, 0.26 \mathrm{mmol})$ in the presence of in the presence of $\mathrm{PPh}_{3}(5.0 \mathrm{mg}$, $0.02 \mathrm{mmol})$ and $\mathrm{Pd}(\mathrm{dba})_{2}(2.7 \mathrm{mg}, 0.005 \mathrm{mmol})$ in dioxane $(1.5 \mathrm{~mL})$ was performed as described for 206 under Conditions A. Work up and chromatography (hexanes/EtOAc, 97:3) gave, in order of elution, 222 (14.8 $\mathrm{mg}, 0.04 \mathrm{mmol}, 20 \%)$ and 245 (30.2 $\mathrm{mg}, 0.10 \mathrm{mmol}, 48 \%)$ as a yellow oil. Analytical data for $\mathbf{2 4 5}$ : ${ }^{1} \mathrm{H}$ NMR $\left(600 \mathrm{MHz}, \mathrm{CDCl}_{3}\right) \delta 8.24(\mathrm{dd}, J=8.4,2.4 \mathrm{~Hz}, 1 \mathrm{H}), 8.17(\mathrm{~d}, J=1.8 \mathrm{~Hz}, 1 \mathrm{H}), 7.83(\mathrm{~d}, J=8.4 \mathrm{~Hz}$, $1 \mathrm{H}), 6.97(\mathrm{dd}, J=17.4,11.4 \mathrm{~Hz}, 1 \mathrm{H}), 6.05(\mathrm{~d}, J=17.4 \mathrm{~Hz}, 1 \mathrm{H}), 5.75(\mathrm{~d}, J=10.8 \mathrm{~Hz}, 1 \mathrm{H}) ;{ }^{13} \mathrm{C}$ NMR $(150$ $\left.\mathrm{MHz} \mathrm{CDCl}_{3}\right) \delta 147.4,145.9,137.5,127.8,127.5,123.2,122.9,118.5\left(\mathrm{q}, J^{\mathrm{C}-\mathrm{F}}=319 \mathrm{~Hz}\right), 117.7$; IR (ATR) $3118,1528,1425,1346,1210,1132 \mathrm{~cm}^{-1}$; HRMS (ESI) calcd for $\mathrm{C}_{9} \mathrm{H}_{7} \mathrm{NO}_{5} \mathrm{~F}_{3} \mathrm{~S}\left(\mathrm{M}+\mathrm{H}^{+}\right)$297.9997; found 297.9994.

2-Ethenyl-5-nitrophenyl trifluoromethanesulfonate (245) and 4-bromo-3-ethenyl-nitrobenzene (246). Cross coupling of $222(85.9 \mathrm{mg}, 0.25 \mathrm{mmol})$ with ethenyltributyltin $(98.2 \mathrm{mg}, 0.31 \mathrm{mmol})$ in the presence of $\mathrm{LiCl}(32.2 \mathrm{mg}, 0.76 \mathrm{mmol})$ and $\mathrm{Pd}\left(\mathrm{PPh}_{3}\right)_{2} \mathrm{Cl}_{2}(3.6 \mathrm{mg}, 0.005 \mathrm{mmol})$ in DMF (1.5 mL) was performed as described for $\mathbf{2 0 7}$ under Conditions B. Work up and chromatography (hexanes/EtOAc, 97:3) gave, in order of elution, $246(3.8 \mathrm{mg}, 0.02 \mathrm{mmol}, 7 \%)$ as an off-white solid, $222(27.0 \mathrm{mg}, 0.08$ $\mathrm{mmol}, 31 \%)$ and 245 (22.4 mg, $0.08 \mathrm{mmol}, 31 \%)$. Analytical data for 246: $\mathrm{mp}=38-40{ }^{\circ} \mathrm{C} ;{ }^{1} \mathrm{H}$ NMR $(400$ $\left.\mathrm{MHz}, \mathrm{CDCl}_{3}\right) \delta 8.38(\mathrm{~d}, J=2.8 \mathrm{~Hz}, 1 \mathrm{H}), 7.96(\mathrm{dd}, J=8.8,2.8 \mathrm{~Hz}, 1 \mathrm{H}), 7.73(\mathrm{~d}, J=8.4 \mathrm{~Hz}, 1 \mathrm{H}), 7.05(\mathrm{dd}$, $J=17.2,10.8 \mathrm{~Hz}, 1 \mathrm{H}), 5.88(\mathrm{~d}, J=17.2 \mathrm{~Hz}, 1 \mathrm{H}), 5.56(\mathrm{~d}, J=10.8 \mathrm{~Hz}, 1 \mathrm{H}) ;{ }^{13} \mathrm{C} \mathrm{NMR}\left(100 \mathrm{MHz}, \mathrm{CDCl}_{3}\right)$ $\delta$ 147.4, 139.0, 134.1, 133.9, 130.3, 123.1, 121.5, 119.7; IR (ATR) 3099, 2926, 1525, 1341, $1030 \mathrm{~cm}^{1}$; HRMS (ESI) calcd for $\mathrm{C}_{8} \mathrm{H}_{6} \mathrm{NNaO}_{2} \mathrm{Br}\left(\mathrm{M}+\mathrm{Na}^{+}\right)$251.9454; found 251.9452.

2-Ethenyl-5-nitrophenyl trifluoromethanesulfonate (245) A solution of 222 (162 $\mathrm{mg}, 0.463 \mathrm{mmol})$ in dioxane $(2 \mathrm{~mL})$ was treated with ethenyltributyltin $(191 \mathrm{mg}, 0.602 \mathrm{mmol})$ in the presence of $\mathrm{PdCl}_{2}\left(\mathrm{PPh}_{3}\right)_{2}$ (13.0 mg, $0.019 \mathrm{mmol}$ ), was performed as described for $\mathbf{2 0 6}$ as described under Conditions C (100 ${ }^{\circ} \mathrm{C}, 24$ h). Work up and purification, gave after chromatography $\mathrm{SiO}_{2} / \mathrm{K}_{2} \mathrm{CO}_{3}\left(10 \% \mathrm{~K}_{2} \mathrm{CO}_{3}\right.$, hexanes/EtOAc, 19:1) 245 (122 mg, $0.412 \mathrm{mmol}, 89 \%)$ as a pale yellow oil. ${ }^{1} \mathrm{H} \mathrm{NMR}\left(600 \mathrm{MHz}, \mathrm{CDCl}_{3}\right) \delta 8.24(\mathrm{dd}, J=$ 
8.4, $2.4 \mathrm{~Hz}, 1 \mathrm{H}), 8.17$ (d, $J=1.8 \mathrm{~Hz}, 1 \mathrm{H}), 7.83(\mathrm{~d}, J=8.4 \mathrm{~Hz}, 1 \mathrm{H}), 6.97(\mathrm{dd}, J=17.4,11.4 \mathrm{~Hz}, 1 \mathrm{H}), 6.05$ $(\mathrm{d}, J=17.4 \mathrm{~Hz}, 1 \mathrm{H}), 5.75(\mathrm{~d}, J=10.8 \mathrm{~Hz}, 1 \mathrm{H}) ;{ }^{13} \mathrm{C} \mathrm{NMR}\left(150 \mathrm{MHz}, \mathrm{CDCl}_{3}\right) \delta 147.4,145.9,137.5$, 127.8, 127.5, 123.2, 122.9,118.5 (q, $\left.J^{C-F}=319 \mathrm{~Hz}\right), 117.7$; IR (ATR) 1526, 1425, 1345, 1209, 1134, 944, $836 \mathrm{~cm}^{-1}$; HRMS (ESI) calcd for $\mathrm{C}_{9} \mathrm{H}_{6} \mathrm{NNaO}_{5} \mathrm{~F}_{3} \mathrm{~S}\left(\mathrm{M}+\mathrm{Na}^{+}\right) 319.9811$; found 319.9812 .

\section{3-Ethenyl-6-trifluoromethanesulfonate-nitrobenzene (241), 3-Ethenyl-6-hydroxy-1-nitrobenzene} (248), and 2,5-Diethenyl-1-nitrobenzene (230). Cross coupling of 4-iodo-6-nitrophenyl trifluoromethanesulfonate $(\mathbf{2 4 7})^{189}(102 \mathrm{mg}, 0.26 \mathrm{mmol})$ with ethenyltributyltin (106 $\left.\mathrm{mg}, 0.33 \mathrm{mmol}\right)$ in the presence of in the presence of $\mathrm{PPh}_{3}(5.8 \mathrm{mg}, 0.02 \mathrm{mmol})$ and $\mathrm{Pd}(\mathrm{dba})_{2}(3.5 \mathrm{mg}, 0.006 \mathrm{mmol})$ in dioxane $(1.5 \mathrm{~mL})$ was performed as described for $\mathbf{2 0 6}$ under Conditions A. Work up and chromatography (hexanes/EtOAc, 97:3) gave, in order of elution, a mixture of $\mathbf{2 4 8}$ and $\mathbf{2 3 0}$ (8.2 mg; $5.8 \mathrm{mg}, 0.035 \mathrm{mmol}$, $14 \%$ of $\mathbf{2 4 8}$ and $2.4 \mathrm{mg}, 0.014 \mathrm{mmol}, 5 \%$ of $\mathbf{2 3 0}$, calculated from ${ }^{1} \mathrm{H}$ NMR spectrum) followed by 241 $(29.5 \mathrm{mg}, 0.099 \mathrm{mmol}, 38 \%)$ as a yellow oil.

3-Ethenyl-6-trifluoromethanesulfonate-nitrobenzene (241), 3-Iodo-6-ethenyl-nitrobenzene (249) and 5-Iodo-2-hydroxynitrobenzene. Cross coupling of 247 (116 mg, $0.29 \mathrm{mmol})$ with ethenyltributyltin $(120 \mathrm{mg}, 0.38 \mathrm{mmol})$ in the presence of $\mathrm{LiCl}(39.7 \mathrm{mg}, 0.94 \mathrm{mmol})$ and $\mathrm{Pd}\left(\mathrm{PPh}_{3}\right)_{2} \mathrm{Cl}_{2}(4.1 \mathrm{mg}, 0.006$ mmol) in DMF (1.5 mL) was performed as described for 207 under Conditions B. Work up and chromatography (hexanes/EtOAc, 97:3) gave in order of elution, 249 (3.2 $\mathrm{mg}, 0.01 \mathrm{mmol}, 4 \%$ ) as a brown oil, 5-iodo-2-hydroxynitrobenzene ( $8.7 \mathrm{mg}, 0.03 \mathrm{mmol}, 14 \%$ ), and a mixture of 241 and 247 (67.1 $\mathrm{mg} ; 43.7 \mathrm{mg}, 0.147 \mathrm{mmol}, 50 \%$ of $\mathbf{2 4 1}$ and $23.3 \mathrm{mg}, 0.059 \mathrm{mmol}, 20 \%$ of $\mathbf{2 4 7}$, calculated from ${ }^{1} \mathrm{H}$ NMR spectrum). Analytical data for 249: ${ }^{1} \mathrm{H}$ NMR $\left(600 \mathrm{MHz}, \mathrm{CDCl}_{3}\right) \delta 8.25(\mathrm{~d}, J=1.8 \mathrm{~Hz}, 1 \mathrm{H}), 7.89(\mathrm{dd}, J=$ $7.8,1.8 \mathrm{~Hz}, 1 \mathrm{H}), 7.35(\mathrm{~d}, J=7.8 \mathrm{~Hz}, 1 \mathrm{H}), 7.09(\mathrm{dd}, J=17.4,11.4 \mathrm{~Hz}, 1 \mathrm{H}), 5.76(\mathrm{~d}, J=167.4 \mathrm{~Hz}, 1 \mathrm{H})$, $5.52(\mathrm{~d}, J=11.4 \mathrm{~Hz}, 1 \mathrm{H}) ;{ }^{13} \mathrm{C} \mathrm{NMR}\left(150 \mathrm{MHz}, \mathrm{CDCl}_{3}\right) \delta 148.0,141.9,133.0,132.8,131.6,129.8,119.7$, 91.8; IR (ATR) 3094, 2925, 1519, 1341, 1261, $1086 \mathrm{~cm}^{-1}$; HRMS (ESI) calcd for $\mathrm{C}_{8} \mathrm{H}_{6} \mathrm{NNaO}_{2} \mathrm{I}\left(\mathrm{M}+\mathrm{Na}^{+}\right)$ 297.9335; found 297.9333 .

\section{3-Ethenyl-6-trifluoromethanesulfonate-nitrobenzene (241) and 2,5-Diethenyl-1-nitrobenzene (230).}

Cross coupling of 247 (205 mg, $0.517 \mathrm{mmol})$ with ethenyltributyltin $(213 \mathrm{mg}, 0.671 \mathrm{mmol})$ in the presence of $\mathrm{PdCl}_{2}\left(\mathrm{PPh}_{3}\right)_{2}(7.3 \mathrm{mg}, 0.010 \mathrm{mmol})$ in dioxane $(2 \mathrm{~mL})$ was performed as described for 206 under Conditions $\mathrm{C}\left(100{ }^{\circ} \mathrm{C}, 24 \mathrm{~h}\right)$. Work up and purification gave after chromatography $\mathrm{SiO}_{2} / \mathrm{K}_{2} \mathrm{CO}_{3}$ $\left(10 \% \mathrm{~K}_{2} \mathrm{CO}_{3}\right.$, hexanes/EtOAc, 19:1), in order of elution, 230 (10.0 mg, $\left.0.057 \mathrm{mmol}, 8 \%\right)$ and 241 (133 $\mathrm{mg}, 0.449 \mathrm{mmol}, 87 \%$ ) as faint yellow oil. 
3-Bromo-6-trifluoromethanesulfonate acetophenone (253). ${ }^{190}$ To a solution of 1-hydroxy-5bromoacetophenone ${ }^{182}(381 \mathrm{mg}, 1.77 \mathrm{mmol})$ in $\mathrm{CH}_{2} \mathrm{Cl}_{2}(10 \mathrm{~mL})$ at $0{ }^{\circ} \mathrm{C}$ was added pyridine $(275 \mu \mathrm{L}, 3.41$ mmol) and $\mathrm{Tf}_{2} \mathrm{O}(325 \mu \mathrm{L}, 1.92 \mathrm{mmol})$. The mixture was removed from the cold bath and allowed to stir at ambient temperature for $1 \mathrm{~h}$. The resulting mixture was filtered through a small plug of silica gel and the solvent was removed under reduced pressure from the filtrate to give 253 (590 $\mathrm{mg}, 1.70 \mathrm{mmol}, 96 \%)$ as a pale orange oil. The compound was used as such without further purification. ${ }^{1} \mathrm{H}$ NMR (400 MHz, $\left.\mathrm{CDCl}_{3}\right) \delta 7.90(\mathrm{~d}, J=2.8 \mathrm{~Hz}, 1 \mathrm{H}), 7.70(\mathrm{dd}, J=8.8 \mathrm{~Hz}, 2.8 \mathrm{~Hz}, 1 \mathrm{H}), 7.22(\mathrm{~d}, J=8.8 \mathrm{~Hz}, 1 \mathrm{H}), 2.62(\mathrm{~s}$, $3 \mathrm{H}) ;{ }^{13} \mathrm{C}$ NMR NMR $\left(100 \mathrm{MHz}, \mathrm{CDCl}_{3}\right) \delta 195.1,145.5,136.4,133.5,133.4,124.3,122.0,118.4\left(\mathrm{~d}, J^{C-F}\right.$ $=213 \mathrm{~Hz}$ ) 29.2; IR (ATR) 1701, 1424, 1202, 1133, 879, $796 \mathrm{~cm}^{-1}$; HRMS (ESI) calcd for $\mathrm{C}_{9} \mathrm{H}_{7} \mathrm{BrO}_{4} \mathrm{~F}_{3} \mathrm{~S}$ $\left(\mathrm{M}+\mathrm{H}^{+}\right)$348.9175; found 348.9177 .

3-Ethenyl-6-trifluoromethanesulfonate acetophenone (254). Cross coupling of 253 (101 mg, 0.29 mmol) with ethenyltributyltin $(116 \mathrm{mg}, 0.37 \mathrm{mmol})$ in the presence of in the presence of $\mathrm{PPh}_{3}(6.4 \mathrm{mg}$, $0.02 \mathrm{mmol})$ and $\mathrm{Pd}(\mathrm{dba})_{2}(3.8 \mathrm{mg}, 0.007 \mathrm{mmol})$ in dioxane $(1.5 \mathrm{~mL})$ was performed as described for 206 under Conditions A. Work up and chromatography (hexanes/EtOAc, 9:1) gave 254 (11.6 mg, 0.04 mmol, $14 \%)$ as a colorless oil. ${ }^{1} \mathrm{H} \mathrm{NMR}\left(600 \mathrm{MHz}, \mathrm{CDCl}_{3}\right) \delta 7.78(\mathrm{~d}, J=2.4 \mathrm{~Hz}, 1 \mathrm{H}), 7.60(\mathrm{dd}, J=8.4,2.4 \mathrm{~Hz}$, $1 \mathrm{H}), 7.30(\mathrm{~d}, J=9.0 \mathrm{~Hz}, 1 \mathrm{H}), 6.73(\mathrm{dd}, J=17.4,10.8 \mathrm{~Hz}, 1 \mathrm{H}), 5.83(\mathrm{~d}, J=17.4 \mathrm{~Hz}, 1 \mathrm{H}), 5.44(\mathrm{~d}, J=$ $10.8 \mathrm{~Hz}, 1 \mathrm{H}), 2.65(\mathrm{~s}, 3 \mathrm{H}) ;{ }^{13} \mathrm{C} \mathrm{NMR}\left(100 \mathrm{MHz}, \mathrm{CDCl}_{3}\right) \delta 196.5,145.8,138.1,134.2,132.2,130.7$, 128.3, 122.8, $118.5\left(\mathrm{q}, J^{C-F}=319 \mathrm{~Hz}\right), 117.2,29.4$; IR (ATR) 3096, 1698, 1421, 1202, $1134 \mathrm{~cm}^{-1}$; HRMS (ESI) calcd for $\mathrm{C}_{11} \mathrm{H}_{10} \mathrm{~F}_{3} \mathrm{O}_{4} \mathrm{~S}\left(\mathrm{M}+\mathrm{H}^{+}\right)$295.0252; found 295.0249.

6-Ethenyl-3-bromoacetophenone (255). Cross coupling of 253 (112 mg, $0.32 \mathrm{mmol})$ with ethenyltributyltin $(129 \mathrm{mg}, 0.41 \mathrm{mmol})$ in the presence of $\mathrm{LiCl}(43.5 \mathrm{mg}, 1.03 \mathrm{mmol})$ and $\mathrm{Pd}\left(\mathrm{PPh}_{3}\right)_{2} \mathrm{Cl}_{2}$ (4.8 mg, $0.007 \mathrm{mmol})$ in DMF (1.5 mL) was performed as described for 207 under Conditions B. Work up and chromatography (hexanes/EtOAc, 8:2) gave 255 (42.6 mg, $0.19 \mathrm{mmol}, 58 \%$ ) as a colorless oil. ${ }^{1} \mathrm{H}$ $\operatorname{NMR}\left(600 \mathrm{MHz}, \mathrm{CDCl}_{3}\right) \delta 7.72(\mathrm{~d}, J=1.8 \mathrm{~Hz}, 1 \mathrm{H}), 7.56(\mathrm{dd}, J=8.4,2.4 \mathrm{~Hz}, 1 \mathrm{H}), 7.42(\mathrm{~d}, J=8.4 \mathrm{~Hz}$, 1H), 7.09 (dd, $J=17.4,10.8 \mathrm{~Hz}, 1 \mathrm{H}), 5.63(\mathrm{dd}, J=17.4,1.2 \mathrm{~Hz}, 1 \mathrm{H}), 5.37$ (dd, $J=11.4,1.2 \mathrm{~Hz}, 1 \mathrm{H})$, 2.56 (s, 3H); ${ }^{13} \mathrm{C}$ NMR $\left(100 \mathrm{MHz}, \mathrm{CDCl}_{3}\right) \delta 200.5,138.9,136.3,134.7,134.4,131.3,129.1,121.1,117.4$, 29.8; IR (ATR) 3088, 1686, 1472, 1355, 1235, $830 \mathrm{~cm}^{-1}$; HRMS (ESI) calcd for $\mathrm{C}_{10} \mathrm{H}_{10} \mathrm{BrO}\left(\mathrm{M}+\mathrm{H}^{+}\right)$ 224.9915; found 224.9914.

3-Ethenyl-6-trifluoromethanesulfonate acetophenone (254). Cross coupling of 253 (70.2 $\mathrm{mg}, 0.202$ mmol) with ethenyltributyltin $(83.4 \mathrm{mg}, 0.263 \mathrm{mmol})$ in the presence of $\mathrm{PdCl}_{2}\left(\mathrm{PPh}_{3}\right)_{2}(3.0 \mathrm{mg}, 0.004$ $\mathrm{mmol})$ in dioxane $(0.6 \mathrm{~mL})$ was performed as described for 206 under Conditions $\mathrm{C}\left(100{ }^{\circ} \mathrm{C}, 24 \mathrm{~h}\right)$. Work 
up and purification gave after chromatography $\mathrm{SiO}_{2} / \mathrm{K}_{2} \mathrm{CO}_{3}\left(10 \% \mathrm{~K}_{2} \mathrm{CO}_{3}\right.$, hexanes/EtOAc, 19:1) 254 (33.8 mg, $0.115 \mathrm{mmol}, 57 \%)$.

3-Ethenyl-4-trifluoromethanesulfonate-1-methoxybenzene (251). Cross coupling of 3-bromo-4trifluoromethanesulfonate-1-methoxybenzene $(\mathbf{2 5 0})^{191}(106 \mathrm{mg}, 0.32 \mathrm{mmol})$ with ethenyltributyltin (126 $\mathrm{mg}, 0.40 \mathrm{mmol})$ in the presence of in the presence of $\mathrm{PPh}_{3}(7.0 \mathrm{mg}, 0.03 \mathrm{mmol})$ and $\mathrm{Pd}(\mathrm{dba})_{2}(3.7 \mathrm{mg}$, $0.006 \mathrm{mmol})$ in dioxane $(1.5 \mathrm{~mL})$ was performed as described for 206 under Conditions A. Work up and chromatography (hexanes/EtOAc, 9:1) gave $251(29.6 \mathrm{mg}, 0.10 \mathrm{mmol}, 33 \%)$ as a colorless oil. ${ }^{1} \mathrm{H} \mathrm{NMR} \delta$ $7.18(\mathrm{~d}, J=9.0 \mathrm{~Hz}, 1 \mathrm{H}), 7.09$ (d, $J=3.0 \mathrm{~Hz}, 1 \mathrm{H}), 6.89$ (dd, $J=17.4,11.4 \mathrm{~Hz}, 1 \mathrm{H}), 6.84$ (dd, $J=9.0,3.0$ $\mathrm{Hz}, 1 \mathrm{H}), 5.83(\mathrm{~d}, J=18.0 \mathrm{~Hz}, 1 \mathrm{H}), 5.49(\mathrm{~d}, J=11.4 \mathrm{~Hz}, 1 \mathrm{H}), 3.84(\mathrm{~s}, 1 \mathrm{H}) ;{ }^{13} \mathrm{C} \mathrm{NMR} \delta 158.9,140.5$, 132.1, 129.1, 122.6, 118.6, $118.3\left(\mathrm{q}, J^{C-F}=319 \mathrm{~Hz}\right), 114.5,111.6,55.7$; IR (ATR) 2968, 1485, 1419, 1206, 1137, $868 \mathrm{~cm}^{-1}$; HRMS (ESI) calcd for $\mathrm{C}_{10} \mathrm{H}_{8} \mathrm{~F}_{3} \mathrm{O}_{4} \mathrm{~S}$ (M-H) 281.0095; found 281.0100.

\section{3-Ethenyl-4-trifluoromethanesulfonate-1-methoxybenzene (251) and 3-Bromo-4-ethenylanisole} (252). ${ }^{192}$ Cross coupling of $\mathbf{2 5 0}$ (103 mg, $\left.0.31 \mathrm{mmol}\right)$ with ethenyltributyltin (119 $\left.\mathrm{mg}, 0.38 \mathrm{mmol}\right)$ in the presence of $\mathrm{LiCl}(38.9 \mathrm{mg}, 0.92 \mathrm{mmol})$ and $\mathrm{Pd}\left(\mathrm{PPh}_{3}\right)_{2} \mathrm{Cl}_{2}(4.5 \mathrm{mg}, 0.006 \mathrm{mmol})$ in $\mathrm{DMF}(2 \mathrm{~mL})$ was performed as described for $\mathbf{2 0 7}$ under Conditions B. Work up and chromatography (hexanes/EtOAc, 9:1) gave, in order of elution, 251 (26.3 mg, $0.12 \mathrm{mmol}, 39 \%)$ as a faint orange oil and 252 (3.3 $\mathrm{mg}, 0.01$ $\mathrm{mmol}, \mathbf{4 \%}$ ). Spectral data for $\mathbf{2 5 2}$ were in accordance with literature values. 
4-Bromo-2,3-dinitrophenol (257). To a solution of 256 (730 mg, $2.24 \mathrm{mmol}$ ) in THF (8 mL) and $\mathrm{H}_{2} \mathrm{O}$ $(0.5 \mathrm{~mL})$ in an ACE Glass pressure tube was added $\mathrm{NaOH}(1.04 \mathrm{~g}, 26.1 \mathrm{mmol})$. The pressure tube was sealed with a Teflon screw cap and the solution was heated at $80^{\circ} \mathrm{C}(18 \mathrm{~h})$. The resulting brown solution was diluted with $\mathrm{H}_{2} \mathrm{O}(25 \mathrm{~mL})$, acidified with $\mathrm{HCl}$, and extracted with EtOAc $(3 \times 30 \mathrm{~mL})$. The combined organic layers were dried $\left(\mathrm{MgSO}_{4}\right)$, filtered, and solvents removed from the filtrate under reduced pressure. The crude brown oil was purified by chromatography (hexanes/EtOAc, 7:3 with 5\% AcOH) to afford 257 (554 mg, $2.11 \mathrm{mmol}, 94 \%)$ as a yellow solid. mp=59-61 ${ }^{\circ} \mathrm{C} ;{ }^{1} \mathrm{H}$ NMR $\left(400 \mathrm{MHz}, \mathrm{CDCl}_{3}\right) \delta$ $7.55(\mathrm{~d}, J=8.4 \mathrm{~Hz}, 1 \mathrm{H}), 7.20(\mathrm{~d}, J=8.8 \mathrm{~Hz}, 1 \mathrm{H}) ;{ }^{13} \mathrm{C}$ NMR $\left(150 \mathrm{MHz}, \mathrm{DMSO}-\mathrm{d}_{6}\right) \delta 151.3,143.8,137.6$, 132.4, 123.1, 101.9; IR (ATR) 3391, 1566, 1535, 1456, $1374 \mathrm{~cm}^{-1}$; HRMS (ESI) calcd for $\mathrm{C}_{6} \mathrm{H}_{3} \mathrm{~N}_{2} \mathrm{O} 5 \mathrm{Br}\left(\mathrm{M}-\mathrm{H}^{+}\right)$260.9147, found 260.9148 .

4-Bromo-2,3-dinitrophenyltrifluoromethanesulfonate (258) To an ice-cooled solution of 257 (1003 $\mathrm{mg}, 3.812 \mathrm{mmol})$ in $\mathrm{CH}_{2} \mathrm{Cl}_{2}(10 \mathrm{~mL})$ under a nitrogen atmosphere was added pyridine $(933 \mu \mathrm{L}, 11.441$ mmol) followed by addition of trifluoromethanesulfonic anhydride $\left(\mathrm{Tf}_{2} \mathrm{O}, 834 \mu \mathrm{L}, 4.957 \mathrm{mmol}\right)$ and the solution was stirred while warming to ambient temperature over 30 minutes. Solvents were removed under reduced pressure and the crude product was purified by chromatography (hexane/EtOAc, 7:3) to afford 258 (1229 mg, $3.111 \mathrm{mmol}, 82 \%)$ as a colorless viscous oil. $\mathrm{mp}=59-60{ }^{\circ} \mathrm{C} ;{ }^{1} \mathrm{H} \mathrm{NMR}(400 \mathrm{MHz}$, $\left.\mathrm{CDCl}_{3}\right) \delta 8.06(\mathrm{~d}, J=9.2 \mathrm{~Hz}, 1 \mathrm{H}), 7.59(\mathrm{~d}, J=9.2 \mathrm{~Hz}, 1 \mathrm{H}) ;{ }^{13} \mathrm{C} \mathrm{NMR}\left(100 \mathrm{MHz}, \mathrm{CDCl}_{3}\right) \delta 144.9,139.8$, 138.3, 137.4, 126.3,118.3 (q, $\left.J^{\mathrm{C}-\mathrm{F}}=319 \mathrm{~Hz}\right), 115.1,{ }^{19} \mathrm{~F} \mathrm{NMR}\left(376.10 \mathrm{MHz}, \mathrm{CDCl}_{3}\right) \delta-72.6$; IR (ATR) 1547, 1436, 1350, 1222, $1130 \mathrm{~cm}^{-1}$; HRMS (ESI) calcd for $\mathrm{C}_{7} \mathrm{H}_{3} \mathrm{BrF}_{3} \mathrm{~N}_{2} \mathrm{O}_{7} \mathrm{~S}\left(\mathrm{M}+\mathrm{H}^{+}\right) 394.8796$ $(\mathrm{M}+\mathrm{Na}=416.8616)$, found

4-Bromo-2,5-dinitrophenol (262) and 4-bromo-2,3-dinitrophenol (257). 4-Bromo-3-nitrophenol (259) $(5.0 \mathrm{~g}, 22.94 \mathrm{mmol})$ was added to concentrated nitric acid $(130 \mathrm{~mL})$ and the solution was stirred at ambient temperature for $1 \mathrm{~h}$ whereby yellow solid started separating out. The mixture was quenched with ice-cold water $(100 \mathrm{~mL})$ and allowed to cool to ambient temperature. The mixture was diluted with EtOAc $(180 \mathrm{~mL})$ and the organic layer was washed with ice-cold water $(100 \mathrm{mLx} 5)$. The organic layer was dried (MgSO4), filtered and was concentrated under reduced pressure. The crude was purified by chromatography (Hexane/EtOAc, 95:5, then, 8:2 acidified with few drops of AcOH) to give, in order of elution, 262 (2.25 g, $8.56 \mathrm{mmol}, 37 \%)$ and 257 (3.06 g, $11.62 \mathrm{mmol}, 51 \%)$ both as a yellow solid. Analytica data for 262: $\mathrm{mp}=113-114{ }^{\circ} \mathrm{C} ;{ }^{1} \mathrm{H}$ NMR $\left(400 \mathrm{MHz}, \mathrm{CDCl}_{3}\right) \delta 10.50(\mathrm{~s}, 1 \mathrm{H}), 8.49(\mathrm{~s}, 1 \mathrm{H}), 7.58$ (s, 1H); ${ }^{13} \mathrm{C}$ NMR $\left(100 \mathrm{MHz}, \mathrm{CDCl}_{3}\right) \delta 154.4,154.1,134.8,131.1,117.0,103.0$; IR (ATR) 3286, 1538, $1254 \mathrm{~cm}^{-1}$; HRMS (ESI) calculated for $\mathrm{C}_{6} \mathrm{H}_{4} \mathrm{BrN}_{2} \mathrm{O}_{5}\left(\mathrm{M}+\mathrm{H}^{+}\right) 262.9304$, found 
4-Iodo-2,5-dinitrophenol (263) $)^{218}$ and 4-iodo-2,3-dinitrophenol (261). 4-Iodo-3-nitrophenol (260) (5.0 $\mathrm{g}, 18.87 \mathrm{mmol})$ was added to concentrated nitric acid $\left(\mathrm{HNO}_{3}, 100 \mathrm{~mL}\right)$ and the solution was stirred at ambient temperature for $1 \mathrm{~h}$ whereby yellow solid started separating out. The mixture was quenched with ice-cold water $(150 \mathrm{~mL})$ and allowed to cool to ambient temperature. The mixture was diluted with EtOAc $(150 \mathrm{~mL})$ and the organic layer was washed with ice-cold water $(100 \mathrm{mLx})$. The organic layer was dried (MgSO4), filtered and was concentrated under reduced pressure. The crude was purified by chromatography (Hexane/DCM, 7:3, acidified with few drops of AcOH) to give, in order of elution, 263 (1.79 g, $5.77 \mathrm{mmol}, 31 \%)$ and $261(2.90 \mathrm{~g}, 9.36 \mathrm{mmol}, 50 \%)$ both as a yellow solid. Analytical data for 261: $\mathrm{mp}=139-140{ }^{\circ} \mathrm{C} ;{ }^{1} \mathrm{H}$ NMR (400 MHz, $\left.\mathrm{CDCl}_{3}\right) \delta 11.25$ (br, s, 1H), 7.72 (d, J=9.2 Hz, 1H), 6.98 (d, $J=8.8 \mathrm{~Hz}, 1 \mathrm{H}) ;{ }^{13} \mathrm{C} \mathrm{NMR}\left(100 \mathrm{MHz}, \mathrm{CDCl}_{3} / \mathrm{DMSO}^{\left.-\mathrm{d}_{6}\right)} \delta 151.8,147.7,142.5,132.5,122.6,72.8\right.$; IR (ATR) $3237,1544,1433,1215,1137 \mathrm{~cm}^{-1}$; HRMS (ESI) calcd for $\mathrm{C}_{6} \mathrm{H}_{4} \mathrm{IN}_{2} \mathrm{O}_{5}\left(\mathrm{M}+\mathrm{H}^{+}\right) 310.9165$, found

4-Iodo-2,3-dinitrophenyltrifluoromethanesulfonate (264). Treatment of 261 (1.923 g, $6.203 \mathrm{mmol})$ with $\mathrm{Tf}_{2} \mathrm{O}(1.40 \mathrm{~mL}, 8.32 \mathrm{mmol})$ in $\mathrm{CH}_{2} \mathrm{Cl}_{2}(20 \mathrm{~mL})$ in the presence of pyridine $(1.50 \mathrm{~mL}, 18.39 \mathrm{mmol})$ under a nitrogen atmosphere, as described for 258 ( $0{ }^{\circ} \mathrm{C}-\mathrm{rt}, 30 \mathrm{mins}$.), gave after solvent removal and chromatography (Hexane/EtOAc, 7:3), 264 (2.523 g, $5.707 \mathrm{mmol}, 92 \%)$ as a yellow solid. $\mathrm{mp}=61-62{ }^{\circ} \mathrm{C}$; ${ }^{1} \mathrm{H}$ NMR (400 MHz, $\left.\mathrm{CDCl}_{3}\right) \delta 8.26(\mathrm{~d}, J=8.8 \mathrm{~Hz}, 1 \mathrm{H}), 7.42(\mathrm{~d}, J=8.8 \mathrm{~Hz}, 1 \mathrm{H}) ;{ }^{13} \mathrm{C} \mathrm{NMR}(100 \mathrm{MHz}$, $\left.\mathrm{CDCl}_{3}\right) \delta 148.5,144.5,140.7,137.2,126.3,118.3\left(\mathrm{q}, J^{\mathrm{C}-\mathrm{F}}=319 \mathrm{~Hz}\right), 86.8 ;{ }^{19} \mathrm{~F}$ NMR $\left(376.10 \mathrm{MHz}, \mathrm{CDCl}_{3}\right)$ $\delta$-72.5; IR (ATR) 1546, 1435, 1349, 1213, $1131 \mathrm{~cm}^{-1}$; HRMS (ESI) calculated for $\mathrm{C}_{7} \mathrm{H}_{3} \mathrm{~F}_{3} \mathrm{IN}_{2} \mathrm{O}_{7} \mathrm{~S}$ $\left(\mathrm{M}+\mathrm{H}^{+}\right) 442.8658(\mathrm{M}+\mathrm{Na}=464.8477)$, found

4-Bromo-2,5-dinitrophenyltrifluoromethanesulfonate (265). Treatment of a solution of 262 (1.500 g, $5.705 \mathrm{mmol})$ with Tf2O $(1.25 \mathrm{~mL}, 7.416 \mathrm{mmol})$ in $\mathrm{CH}_{2} \mathrm{Cl}_{2}$ in the presence of pyridine $(1.40 \mathrm{~mL}, 17.114$ mmol) under a nitrogen atmosphere, as described for 258 ( $0{ }^{\circ} \mathrm{C}-\mathrm{rt}, 30 \mathrm{mins}$.) gave after solvent removal and chromatography (Hexane/EtOAc, 9:1), 265 (1.803 g, $4.564 \mathrm{mmol}, 80 \%)$ as a white solid. mp=62-63 ${ }^{\circ} \mathrm{C} ;{ }^{1} \mathrm{H}$ NMR (400 MHz, $\left.\mathrm{CDCl}_{3}\right) \delta 8.54(\mathrm{~s}, 1 \mathrm{H}), 7.94(\mathrm{~s}, 1 \mathrm{H}) ;{ }^{13} \mathrm{C} \mathrm{NMR}\left(100 \mathrm{MHz}, \mathrm{CDCl}_{3}\right) \delta 152.0,142.8$, $140.3,133.1,121.7,118.4\left(\mathrm{q}, J^{\mathrm{C}-\mathrm{F}}=320 \mathrm{~Hz}\right), 115.1 ;{ }^{19} \mathrm{~F} \mathrm{NMR}\left(376 \mathrm{MHz}, \mathrm{CDCl}_{3}\right) \delta-72.5$; IR (ATR) 1539 , 1426, 1353, 1231, $1141 \mathrm{~cm}^{-1}$; HRMS (ESI) calcd for $\mathrm{C}_{7} \mathrm{H}_{3} \mathrm{BrF}_{3} \mathrm{~N}_{2} \mathrm{O}_{7} \mathrm{~S} \quad\left(\mathrm{M}+\mathrm{H}^{+}\right) \quad 394.8796$ $(\mathrm{M}+\mathrm{Na}=416.8616)$, found.

4-Iodo-2,5-dinitrophenyltrifluoromethanesulfonate (266). Treatment of 263 (1.389 g, $4.480 \mathrm{mmol})$ with $\mathrm{Tf}_{2} \mathrm{O}(0.98 \mathrm{~mL}, 5.82 \mathrm{mmol})$ in $\mathrm{CH}_{2} \mathrm{Cl}_{2}(16 \mathrm{~mL})$ in the presence of pyridine $(1.10 \mathrm{~mL}, 13.44 \mathrm{mmol})$ under a nitrogen atmosphere, as described for 258 ( $0{ }^{\circ} \mathrm{C}-\mathrm{rt}, 25 \mathrm{mins}$.), gave after solvent removal and chromatography (Hexane/EtOAc, 95:5), 266 (1.596 g, $3.610 \mathrm{mmol}, 81 \%$ ) as a yellow oil. $\mathrm{mp}=60-61{ }^{\circ} \mathrm{C}$; 
${ }^{1} \mathrm{H}$ NMR $\left(400 \mathrm{MHz}, \mathrm{CDCl}_{3}\right) \delta 8.77$ (s, 1H), $7.92(\mathrm{~s}, 1 \mathrm{H}) ;{ }^{13} \mathrm{C} \mathrm{NMR}\left(100 \mathrm{MHz}, \mathrm{CDCl}_{3}\right) \delta 155.4,142.5$, 141.2, 139.6, 121.2, $118.4\left(\mathrm{q}, J^{\mathrm{C}-\mathrm{F}}=319 \mathrm{~Hz}\right), 85.8 ;{ }^{19} \mathrm{~F}$ NMR $\left(376.10 \mathrm{MHz}, \mathrm{CDCl}_{3}\right) \delta-72.4$; IR (ATR) 1532, 1434, 1336, 1195, $1132 \mathrm{~cm}^{-1}$; HRMS (ESI) calcd for $\mathrm{C}_{7} \mathrm{H}_{3} \mathrm{~F}_{3} \mathrm{IN}_{2} \mathrm{O}_{7} \mathrm{~S}\left(\mathrm{M}+\mathrm{H}^{+}\right) 442.8658$ $(\mathrm{M}+\mathrm{Na}=464.8477)$, found

5-Bromo-2,4-dinitrophenol (270) and 3-bromo-2,6-dinitrophenol (271). Treatment of a solution of 5bromo-2-nitrophenol (268) (579 mg, $2.658 \mathrm{mmol})$ in DCM (2 mL) with $\mathrm{NaNO}_{3}(271 \mathrm{mg}, 3.190 \mathrm{mmol})$ in concentrated $\mathrm{H}_{2} \mathrm{SO}_{4}(5 \mathrm{~mL})$ at $0{ }^{\circ} \mathrm{C}$, as described for 274 and $275(10 \mathrm{~h})$, gave, after work up and chromatography (Hexane/EtOAc, 9:1), a mixture of $\mathbf{2 7 0}$ and 271 (693 mg, $2.634 \mathrm{mmol}, 99 \%$ ) as a yellow oil. ${ }^{1} \mathrm{H}$ NMR (400 MHz, $\left.\mathrm{CDCl}_{3}\right) \delta 10.93$ (s, 1H), 10.82 (s, 1H), 8.82 (s, 1H), 8.14 (d, J=9.2 Hz, 1H), 7.63 (s, 1H), 7.35 (d, J=9.2 Hz, 1H); IR (ATR) 3233, 1542, 1236, $854 \mathrm{~cm}^{-1}$; HRMS (ESI) calcd for, found

\section{5-Bromo-2,5-dinitrophenyltrifluoromethanesulfonate (204) and 3-bromo-2,6-} dinitrophenyltrifluromethanesulfonate (273). Treatment of the mixture of 270 and 271 (1.700 g, 6.465 mmol) with $\mathrm{Tf}_{2} \mathrm{O}(1.41 \mathrm{~mL}, 8.40 \mathrm{mmol})$ in $\mathrm{CH}_{2} \mathrm{Cl}_{2}(18 \mathrm{~mL})$ in the presence of triethylamine (Et $3 \mathrm{~N}, 2.34$ $\mathrm{mL}, 16.81 \mathrm{mmol})$ under a nitrogen atmosphere, as described for $258\left(0{ }^{\circ} \mathrm{C}-\mathrm{rt}, 1 \mathrm{~h}\right)$, gave, in order of elution, after solvent removal and chromatography (Hexane/EtOAc, 9:1), 204 (1.458 g, $3.691 \mathrm{mmol}$, 57\%) as a yellow oil and 273 (409 $\mathrm{mg}, 1.034 \mathrm{mmol}, 16 \%)$ as a white solid. Analytical data for 273: $\mathrm{mp}=93-94{ }^{\circ} \mathrm{C} ;{ }^{1} \mathrm{H}$ NMR $\left(400 \mathrm{MHz}, \mathrm{CDCl}_{3}\right) \delta 8.18(\mathrm{~d}, \mathrm{~J}=8.8 \mathrm{~Hz}, 1 \mathrm{H}), 7.95(\mathrm{~d}, \mathrm{~J}=9.2 \mathrm{~Hz}, 1 \mathrm{H}) ;{ }^{13} \mathrm{C} \mathrm{NMR}$ $\left(100 \mathrm{MHz}, \mathrm{CDl}_{3}\right) \delta 145.8,141.4,134.0,133.9,127.7,121.4,118.1$ (q, JC-F=320 Hz); ${ }^{19} \mathrm{~F}$ NMR $(376.10$ $\left.\mathrm{MHz}, \mathrm{CDCl}_{3}\right) \delta-71.8$ IR (ATR) 1534, 1437, 1350, 1218, $1131 \mathrm{~cm}^{-1}$; HRMS (ESI) calcd for, found

5-Iodo-2,4-dinitrophenol (274) $)^{215}$ and 3-Iodo-2,6-dinitrophenol (275). To an ice-cooled solution of $\mathrm{NaNO}_{3}(648 \mathrm{mg}, 7.629 \mathrm{mmol})$ in concentrated $\mathrm{H}_{2} \mathrm{SO}_{4}(13 \mathrm{~mL})$ was added drop wise the solution of 5iodo-2-nitrophenol (269) (1.264 g, $4.768 \mathrm{mmol})$ in DCM (5 mL). The resulting solution was maintained at $0{ }^{\circ} \mathrm{C}$ overnight $(10 \mathrm{~h})$. The solution was quenched with ice-cold water and allowed to cool to ambient temperature. The solution was extracted in EtOAc $(3 \mathrm{X} 30 \mathrm{~mL})$, dried $\left(\mathrm{MgSO}_{4}\right)$, filtered and was concentrated under reduced pressure. The crude oil was purified by chromatography (Hexane/DCM, 7:3, acidified with $\mathrm{AcOH})$ to give a mixture of 274 and 275 (1.168 g, $3.768 \mathrm{mmol}, 79 \%)$ as a yellow oil. Analytical data for 274 and 275 from the mixture: ${ }^{1} \mathrm{H}$ NMR $\left(600 \mathrm{MHz}, \mathrm{CDCl}_{3}\right) \delta 10.94(\mathrm{~s}, 1 \mathrm{H}), 10.74(\mathrm{~s}$, $1 \mathrm{H}), 8.79(\mathrm{~s}, 1 \mathrm{H}), 7.79(\mathrm{~s}, 1 \mathrm{H}), 7.95(\mathrm{~d}, J=9.0 \mathrm{~Hz}, 1 \mathrm{H}), 7.57$ (d, $J=9.0 \mathrm{~Hz}, 1 \mathrm{H}) ;{ }^{13} \mathrm{C} \mathrm{NMR}(150 \mathrm{MHz}$, $\left.\mathrm{CDCl}_{3}\right) \delta 155.9,147.0,146.9,144.3,134.1,133.9,132.2,130.2,126.2,122.6,97.2,96.9$; IR (ATR) 3276, $1538,1239,835 \mathrm{~cm}^{-1}$; HRMS (ESI) calcd. for, found 

$3.764 \mathrm{mmol})$ with $\mathrm{Tf}_{2} \mathrm{O}(0.86 \mathrm{~mL}, 5.08 \mathrm{mmol})$ in $\mathrm{CH}_{2} \mathrm{Cl}_{2}(12 \mathrm{~mL})$ in the presence of triethylamine $\left(\mathrm{Et}_{3} \mathrm{~N}\right.$, $1.40 \mathrm{~mL}, 9.79 \mathrm{mmol})$ under a nitrogen atmosphere, as described for 258 (0 ${ }^{\circ} \mathrm{C}-\mathrm{rt}, 30 \mathrm{mins}$.), gave, in order of elution, after solvent removal and chromatography (Hexane/EtOAc, 9:1), 276 (1.035 g, 2.341 mmol, 62\%) as a yellow oil and 277 (466 mg, $1.054 \mathrm{mmol}, 28 \%)$ as a white solid. Analytical data for 276: $\left.1 \mathrm{H} \mathrm{NMR} \mathrm{(400} \mathrm{MHz,} \mathrm{CDCl}_{3}\right) \delta 8.68$ (s, 1H), 8.16 (s, 1H); 151.5, 142.1, 140.9, 138.0, 123.2, 118.4 $\left(\mathrm{q}, J^{\mathrm{C}-\mathrm{F}}=319.5 \mathrm{~Hz}\right), 94.3 ;{ }^{19} \mathrm{~F} \mathrm{NMR}\left(\mathrm{MHz}, \mathrm{CDCl}_{3}\right) \delta-72.4$; IR (ATR) 1527, 1433, 1343, 1197, $837 \mathrm{~cm}^{-1}$; HRMS (ESI) calcd for, found

Analytical data for 277: $\mathrm{mp}=121-122{ }^{\circ} \mathrm{C} ;{ }^{1} \mathrm{H} \mathrm{NMR}\left(400 \mathrm{MHz}, \mathrm{CDCl}_{3}\right) \delta 8.18(\mathrm{~d}, J=8.4 \mathrm{~Hz}, 1 \mathrm{H}), 7.98(\mathrm{~d}$, $J=9.0 \mathrm{~Hz}, 1 \mathrm{H}) ;{ }^{13} \mathrm{C} \mathrm{NMR}\left(100 \mathrm{MHz}, \mathrm{CDl}_{3}\right) \delta 149.6,142.2,140.3,133.0,127.8,118.1\left(\mathrm{q}, J^{\mathrm{C}-\mathrm{F}}=319.5 \mathrm{~Hz}\right)$, 94.3; ${ }^{19} \mathrm{~F}$ NMR (376.10 MHz, $\mathrm{CDCl}_{3}$ ) $\delta$-71.9; IR (ATR) 1532, 1436, 1352, 1216, $897 \mathrm{~cm}^{-1}$; HRMS (ESI) calcd for, found

2-Bromo-3,6-dinitrophenol (279) and 2-Bromo-3,4-dinitrophenol (280). To an ice-cooled solution of $\mathrm{NaNO}_{3}(869 \mathrm{mg}, 10.23 \mathrm{mmol})$ in concentrated $\mathrm{H}_{2} \mathrm{SO}_{4}(20 \mathrm{~mL})$ was added drop wise the solution of 2bromo-3-nitrophenol (278) (1.716 g, $7.872 \mathrm{mmol})$ in $\mathrm{DCM} / \mathrm{MeOH}(5 / 5 \mathrm{~mL})$. The resulting solution was maintained at $0{ }^{\circ} \mathrm{C} 3 \mathrm{~h}$ and then was allowed to warm to ambient temperature over a period of $1 \mathrm{~h}$. The solution was quenched with ice-cold water and allowed to cool to ambient temperature. The solution was extracted in EtOAc $(3 \mathrm{X} 20 \mathrm{~mL})$, dried $\left(\mathrm{MgSO}_{4}\right)$, filtered and was concentrated under reduced pressure. The crude oil was purified by chromatography (Hexane/EtOAc, 9:1, then, 7:3, acidified with AcOH) to give, in order of elution, 279 (1.263 g, $4.802 \mathrm{mmol}, 61 \%)$ and $\mathbf{2 8 0}$ (213 g, $0.811 \mathrm{mmol}, 10 \%)$ both as a yellow solid.

Analytical data for 279: $\mathrm{mp}=92-93{ }^{\circ} \mathrm{C} ;{ }^{1} \mathrm{H} \mathrm{NMR}\left(600 \mathrm{MHz}, \mathrm{CDCl}_{3}\right) \delta 11.43(\mathrm{~s}, 1 \mathrm{H}), 8.29(\mathrm{~d}, J=9.0 \mathrm{~Hz}$, $1 \mathrm{H}), 7.31(\mathrm{~d}, J=9.0 \mathrm{~Hz}, 1 \mathrm{H}) ;{ }^{13} \mathrm{C} \mathrm{NMR}\left(100 \mathrm{MHz}, \mathrm{CDCl}_{3}\right) \delta 155.4,153.6,134.7,125.2,114.7,107.0$; IR (ATR) 3239, 1536, 1445, 1141, $816 \mathrm{~cm}^{-1}$; HRMS (ESI) calcd for $\mathrm{C}_{6} \mathrm{H}_{4} \mathrm{BrN}_{2} \mathrm{O}_{5}\left(\mathrm{M}+\mathrm{H}^{+}\right) 262.9304$ $(\mathrm{M}+\mathrm{Na}=284.9123)$, found

Analytical data for 280: $\mathrm{mp}=149{ }^{\circ} \mathrm{C}$ (decomposition); ${ }^{1} \mathrm{H}$ NMR $\left(400 \mathrm{MHz}, \mathrm{CDCl}_{3}\right) \delta 8.25(\mathrm{~d}, \mathrm{~J}=9.2 \mathrm{~Hz}$, $1 \mathrm{H}), 7.28$ (d, J=9.2 Hz, 1H), 6.55 (br, s, 1H); $\left.{ }^{13} \mathrm{C} \mathrm{NMR} \mathrm{(100} \mathrm{MHz,} \mathrm{CDCl}_{3} / \mathrm{DMSO}_{6} \mathrm{~d}_{6}\right) \delta 161.8,146.2$, 130.9, 125.9, 115.8, 103.6; IR (ATR) 3234, 1553, 1313, $1091 \mathrm{~cm}^{-1}$; HRMS (ESI) calcd for $\mathrm{C}_{6} \mathrm{H}_{4} \mathrm{BrN}_{2} \mathrm{O}_{5}$ $\left(\mathrm{M}+\mathrm{H}^{+}\right) 262.9304(\mathrm{M}+\mathrm{Na}=284.9123)$, found

2-Bromo-3,6-dininitrophenyltrifluoromethanesulfonate (281). Treatment of a solution of 279 (1.2360 $\mathrm{g}, 4.700 \mathrm{mmol})$ with $\mathrm{Tf}_{2} \mathrm{O}(1.12 \mathrm{~mL}, 6.657 \mathrm{mmol})$ in $\mathrm{CH}_{2} \mathrm{Cl}_{2}(15 \mathrm{~mL})$ in the presence of $\mathrm{Et}_{3} \mathrm{~N}(1.70 \mathrm{~mL}$, 
$12.220 \mathrm{mmol})$ under a nitrogen atmosphere, as described for 258 ( $0{ }^{\circ} \mathrm{C}$-rt, $\left.30 \mathrm{~min}\right)$, gave, after solvent removal and chromatography (Hexane/EtOAc, 7:3), 281 (1.6934 g, $4.286 \mathrm{mmol}, 91 \%)$ as a white solid. $\mathrm{mp}=108-109{ }^{\circ} \mathrm{C} ;{ }^{1} \mathrm{H}$ NMR $\left(600 \mathrm{MHz}, \mathrm{CDCl}_{3}\right) \delta 8.20(\mathrm{~d}, J=9.0 \mathrm{~Hz}, 1 \mathrm{H}), 7.93(\mathrm{~d}, J=9.0 \mathrm{~Hz}, 1 \mathrm{H}) ;{ }^{13} \mathrm{C} \mathrm{NMR}$ $\left(150 \mathrm{MHz}, \mathrm{CDCl}_{3}\right) \delta 153.7,144.7,140.7,126.0,124.2,118.3\left(\mathrm{q}, J^{\mathrm{C}-\mathrm{F}}=319.5 \mathrm{~Hz}\right), 113.3 ;{ }^{19} \mathrm{~F} \mathrm{NMR}(\mathrm{MHz}$, $\left.\mathrm{CDCl}_{3}\right) \delta$-72.3; IR (ATR) 1544, 1434, 1351, 1214, $1131 \mathrm{~cm}^{-1}$; HRMS (ESI) calcd for $\mathrm{C}_{7} \mathrm{H}_{3} \mathrm{BrF}_{3} \mathrm{~N}_{2} \mathrm{O}_{7} \mathrm{~S}$ $\left(\mathrm{M}+\mathrm{H}^{+}\right) 394.8796(\mathrm{M}+\mathrm{Na}=416.8616)$, found

1,4-Diethenyl-2,3-dinitrobenzene (285). To a yellow mixture of 2,3-dinitro-1.4-dibromobenzene 256 (538.3 mg, $1.652 \mathrm{mmol})$ and $\mathrm{PdCl}_{2}\left(\mathrm{PPh}_{3}\right)_{2}$ in 1,4-dioxane $(4 \mathrm{~mL})$ at ambient temperature under a nitrogen atmosphere was added a solution of ethenyl tributyltin (282) $(1153 \mathrm{mg}, 3.634 \mathrm{mmol})$ in 1,4-dioxane (1 $\mathrm{mL})$ via syringe. The resulting solution was heated at reflux $\left(100{ }^{\circ} \mathrm{C}, 21 \mathrm{~h}\right)$. The solvent was removed at reduced pressure and the crude product was purified by chromatography $\left(10 \% \mathrm{~K}_{2} \mathrm{CO}_{3}-\mathrm{SiO}_{2}\right.$, hexane/EtOAc, 95:5) to give 285 (245.1 mg, $1.113 \mathrm{mmol}, 67 \%)$ as a yellow solid. $\mathrm{mp}=113-114{ }^{\circ} \mathrm{C} ;{ }^{1} \mathrm{H}$ NMR (400 MHz, $\left.\mathrm{CDCl}_{3}\right) \delta 7.77(\mathrm{~s}, 2 \mathrm{H}), 6.74(\mathrm{dd}, J=17.2,11.2 \mathrm{~Hz}, 2 \mathrm{H}), 5.93(\mathrm{~d}, J=17.2 \mathrm{~Hz}, 2 \mathrm{H}), 5.64$ (d, $J=11.2 \mathrm{~Hz}, 2 \mathrm{H}) ;{ }^{13} \mathrm{C}$ NMR (100 MHz, $\left.\mathrm{CDCl}_{3}\right) \delta 142.0,131.4,129.2,128.4,122.1$; IR (ATR) 1525,1478 , 1352, 975, 934, $848 \mathrm{~cm}^{-1}$; HRMS (ESI) calcd for $\mathrm{C}_{10} \mathrm{H}_{8} \mathrm{~N}_{2} \mathrm{O}_{4} \mathrm{Na}\left(\mathrm{M}+\mathrm{Na}^{+}\right)$243.0382, found 243.0375 .

2,3-Dinitro-1,4-di(1-propen-1-yl)benzene (286). To a solution of 256 (106 mg, $0.327 \mathrm{mmol}$ ), $\mathrm{PdCl}_{2}\left(\mathrm{PPh}_{3}\right)_{2}(4.7 \mathrm{mg}, 0.01 \mathrm{mmol})$ and $\mathrm{PPh}_{3}(3.4 \mathrm{mg}, 0.01 \mathrm{mmol})$ in dioxane $(3 \mathrm{~mL})$, under a nitrogen atmosphere, was added the solution of 1-propen-1-yl tributyltin (283) (282 $\mathrm{mg}, 0.852 \mathrm{mmol}$ ) in dioxane $(1 \mathrm{~mL})$ via a syringe. The solution was heated at reflux $\left(100{ }^{\circ} \mathrm{C}, 24 \mathrm{~h}\right)$. The solvent was removed under reduced pressure and the resulting residue was purified by chromatography $\left(10 \% \mathrm{~K}_{2} \mathrm{CO}_{3}-\mathrm{SiO}_{2}\right.$, hexane/EtOAc, 9:1) to afford $286(69.0 \mathrm{mg}, 0.278 \mathrm{mmol}, 85 \%, \sim 2: 1$ trans-cis/trans-trans mixture $)$ as a yellow solid. $\mathrm{mp}=125-127{ }^{\circ} \mathrm{C} ;{ }^{1} \mathrm{H}$ NMR $\left(400 \mathrm{MHz}, \mathrm{CDCl}_{3}\right) \delta 7.69(\mathrm{~d}, J=8.3 \mathrm{~Hz}, 1 \mathrm{H}), 7.64(\mathrm{~s}, 1 \mathrm{H}), 7.50$ (s, 1H), $7.45(\mathrm{~d}, J=8.3 \mathrm{~Hz}, 1 \mathrm{H}), 6.47-6.33(\mathrm{~m}, 6 \mathrm{H}), 6.06(\mathrm{ddq}, J=11.5,9.7,7.2 \mathrm{~Hz}, 2 \mathrm{H}), 1.94(\mathrm{~m}, 6 \mathrm{H})$, $1.78(\mathrm{dd}, J=7.0,1.6 \mathrm{~Hz}, 3 \mathrm{H}), 1.76(\mathrm{dd}, J=7.0,1.6 \mathrm{~Hz}, 3 \mathrm{H}) ;{ }^{13} \mathrm{C}$ NMR $\left(150 \mathrm{MHz}, \mathrm{DMSO}-\mathrm{d}_{6}\right) \delta 141.8$, $141.7,140.5,140.4,135.8,135.6,133.8,133.7,133.5,133.4,130.4$, 130.2, 129.9, 129.9, 129.8, 129.8, 121.7, 121.7, 121.5, 18.7, 14.3; IR (ATR) 3041, 2954, 1649, 1528, 1358, 859, $820 \mathrm{~cm}^{-1}$; HRMS (ESI) calcd for $\mathrm{C}_{12} \mathrm{H}_{12} \mathrm{~N}_{2} \mathrm{O}_{4} \mathrm{Na}\left(\mathrm{M}+\mathrm{Na}^{+}\right) 271.0689$, found 271.0688.

2,3-Dinitro-1,4-di(1-propen-2-yl)benzene (287). To a solution of 256 (352 $\mathrm{mg}, 1.082 \mathrm{mmol}$ ) and $\mathrm{PdCl}_{2}\left(\mathrm{PPh}_{3}\right)_{2}(30.4 \mathrm{mg}, 0.043 \mathrm{mmol})$ in 1,4-dioxane $(3 \mathrm{~mL})$, stirred for $5 \mathrm{~min}$ under a nitrogen atmosphere, was added the solution of isopropenytributyltin (284) (824 $\mathrm{mg}, 2.488 \mathrm{mmol}$ ) in dioxane $(1 \mathrm{~mL})$ via a syringe. The solution was heated at reflux $\left(100^{\circ} \mathrm{C}\right)$ for $24 \mathrm{~h}$. The solvent was removed under 
reduced pressure and the resulting crude was purified by chromatography $\left(10 \% \mathrm{~K}_{2} \mathrm{CO}_{3}-\mathrm{SiO}_{2}\right.$, hexane/EtOAc, 95:5) to afford $287(189 \mathrm{mg}, 0.761 \mathrm{mmol}, 70 \%)$ as a white solid. $\mathrm{mp}=138-139{ }^{\circ} \mathrm{C} ;{ }^{1} \mathrm{H}$ NMR (400 MHz, $\left.\mathrm{CDCl}_{3}\right) \delta 7.45(\mathrm{~s}, 2 \mathrm{H}), 5.27(\mathrm{~s}, 2 \mathrm{H}), 5.02(\mathrm{~s}, 2 \mathrm{H}), 2.10(\mathrm{~s}, 6 \mathrm{H}) ;{ }^{13} \mathrm{C} \mathrm{NMR}(100 \mathrm{MHz}$, $\left.\mathrm{CDCl}_{3}\right) \delta 141.9,139.2,137.4,131.5,118.1,23.1$; IR (ATR) 3094, 2973, 1643, 1543, 1526, 1355, 911, $847 \mathrm{~cm}^{-1}$; HRMS (ESI) calcd for $\mathrm{C}_{12} \mathrm{H}_{13} \mathrm{~N}_{2} \mathrm{O}_{4}\left(\mathrm{M}+\mathrm{H}^{+}\right) 249.0875(\mathrm{M}+\mathrm{Na}=271.0695)$, found xxxxxxxxx.

1,5-Diethenyl-2,4-dinitrobenzene (289). Treatment of a solution of 288 (500 $\mathrm{mg}, 1.535 \mathrm{mmol}$ ) with 282 $(1.071 \mathrm{~g}, 3.38 \mathrm{mmol})$ in the presence of $\mathrm{PdCl}_{2}\left(\mathrm{PPh}_{3}\right)_{2}(43.1 \mathrm{mg}, 0.061 \mathrm{mmol})$ in dioxane $(6.5 \mathrm{~mL})$, as described for 285 ( $90{ }^{\circ} \mathrm{C}, 24 \mathrm{~h}$ ), gave after solvent removal and chromatography (Hexane/EtOAc, 95:5) $289(247 \mathrm{mg}, 1.121 \mathrm{mmol}, 73 \%)$ as a white solid. $\mathrm{mp}=60-61{ }^{\circ} \mathrm{C} ;{ }^{1} \mathrm{H} \mathrm{NMR}\left(400 \mathrm{MHz}, \mathrm{CDCl}_{3}\right) \delta 8.65(\mathrm{~s}$, $1 \mathrm{H}), 7.83(\mathrm{~s}, 1 \mathrm{H}), 7.27(\mathrm{dd}, J=17.2,10.8 \mathrm{~Hz}, 2 \mathrm{H}), 5.90(\mathrm{~d}, J=17.2 \mathrm{~Hz}, 2 \mathrm{H}), 5.70(\mathrm{~d}, J=11.2 \mathrm{~Hz}, 2 \mathrm{H}) ;{ }^{13} \mathrm{C}$ NMR $\left(100 \mathrm{MHz}, \mathrm{CDCl}_{3}\right) \delta 145.6,137.9,131.2,129.1,122.4,121.6$; IR (ATR) 1578, 1517, 1340, $940,907 \mathrm{~cm}^{-1}$; HRMS (ESI) calcd for $\mathrm{C}_{10} \mathrm{H}_{9} \mathrm{~N}_{2} \mathrm{O}_{4}\left(\mathrm{M}+\mathrm{H}^{+}\right) 221.0562(\mathrm{M}+\mathrm{Na}=243.0382)$, found

2,4-Dinitro-1,5-di(1-propen-1-yl)benzene (290). Treatment of a solution of $\mathbf{2 8 8}$ (136.1 mg, $0.418 \mathrm{mmol})$ with 283 (360 mg, $1.086 \mathrm{mmol})$ in the presence of $\mathrm{PdCl}_{2}\left(\mathrm{PPh}_{3}\right)_{2}(5.9 \mathrm{mg}, 0.008 \mathrm{mmol})$ and $\mathrm{PPh}_{3}(4.4 \mathrm{mg}$, $0.017 \mathrm{mmol})$ in dioxane $(4 \mathrm{~mL})$, as described for $285\left(90{ }^{\circ} \mathrm{C}, 24 \mathrm{~h}\right)$, gave after solvent removal and chromatography (Hexane/EtOAc, 95:5) 290 (96.7 mg, $0.390 \mathrm{mmol}, 93 \%)$ as a yellow solid. mp=98-99 ${ }^{\circ} \mathrm{C} ;{ }^{1} \mathrm{H}$ NMR $\left(400 \mathrm{MHz}, \mathrm{CDCl}_{3}\right) \delta 8.75(\mathrm{~s}, 1 \mathrm{H}), 8.65(\mathrm{~s}, 1 \mathrm{H}), 8.56(\mathrm{~s}, 1 \mathrm{H}), 7.73(\mathrm{~s}, 1 \mathrm{H}), 7.57(\mathrm{~s}, 1 \mathrm{H}), 7.39$ (s, 1H), 6.99-6.93 (m, 2H), 6.78-6.74 (m, 2H), 6.46-6.35 (m, 2H), 6.13-6.04 (m, 2H), 2.02-1.99 (m, 6H), $1.79(\mathrm{dd}, J=7.2,2.0 \mathrm{~Hz}, 6 \mathrm{H}) ;{ }^{13} \mathrm{C}$ NMR $\left(100 \mathrm{MHz}, \mathrm{CDCl}_{3}\right) \delta 145.9,145.5,144.7,144.5,137.6,137.2$, 137.0, 136.9, 135.9, 135.6, 135.4, 131.8, 131.3, 131.2, 128.4, 125.2, 125.0, 124.9, 124.7, 121.8, 121.7, 121.6, 19.1, 19.0, 14.5, 14.5; IR (ATR) 1537, 1352, 1277, $938 \mathrm{~cm}^{-1}$; HRMS (ESI) calcd for $\mathrm{C}_{12} \mathrm{H}_{13} \mathrm{~N}_{2} \mathrm{O}_{4}$ $\left(\mathrm{M}+\mathrm{H}^{+}\right) 249.0875(\mathrm{M}+\mathrm{Na}=271.0695)$, found

2,4-Dinitro-1,5-di(1-propen-1-yl)benzene (290). Treatment of a solution of 204 (400.3 mg, $1.013 \mathrm{mmol})$ with 283 (772 mg, $2.331 \mathrm{mmol})$ in the presence of $\mathrm{PdCl}_{2}\left(\mathrm{PPh}_{3}\right)_{2}(28.3 \mathrm{mg}, 0.040 \mathrm{mmol})$, BHT (20.1 mg, $0.091 \mathrm{mmol})$ and $\mathrm{LiCl}(133.1 \mathrm{mg}, 3.140 \mathrm{mmol})$ in dioxane $(3 \mathrm{~mL})$, as described for $285\left(90{ }^{\circ} \mathrm{C}, 23 \mathrm{~h}\right)$, gave after solvent removal and chromatography (Hexane/EtOAc, 95:5) 290 (104.2 mg, 0.420 mmol, 41\%) as a yellow solid.

2,4-Dinitro-1,5-di(1-propen-2-yl)benzene (291). Treatment of a solution of 288 (175.6 $\mathrm{mg}, 0.539 \mathrm{mmol})$ with 284 (464 mg, $1.401 \mathrm{mmol})$ in the presence of $\mathrm{PdCl}_{2}\left(\mathrm{PPh}_{3}\right)_{2}(15.1 \mathrm{mg}, 0.022 \mathrm{mmol})$ and $\mathrm{PPh}_{3}(11.3$ $\mathrm{mg}, 0.043 \mathrm{mmol})$ in dioxane $(5 \mathrm{~mL})$, as described for $285\left(90^{\circ} \mathrm{C}, 24 \mathrm{~h}\right)$, gave after solvent removal and 
chromatography (Hexane/EtOAc, 9:1) 291 (116.5 mg, $0.469 \mathrm{mmol}, 87 \%)$ as a white solid. $\mathrm{mp}=69-70{ }^{\circ} \mathrm{C}$; ${ }^{1} \mathrm{H}$ NMR (400 MHz, $\left.\mathrm{CDCl}_{3}\right) \delta 8.45(\mathrm{~s}, 1 \mathrm{H}), 7.31(\mathrm{~s}, 1 \mathrm{H}), 5.28(\mathrm{~s}, 2 \mathrm{H}), 5.02(\mathrm{~s}, 2 \mathrm{H}), 2.11(\mathrm{~s}, 6 \mathrm{H}) ;{ }^{13} \mathrm{C}$ NMR (100 MHz, $\left.\mathrm{CDCl}_{3}\right) \delta 146.3,143.3,141.1,133.0,120.6,117.1,22.9$; IR (ATR) 1584, 1525, 1344, 908, $734 \mathrm{~cm}^{-1}$; HRMS (ESI) calcd for $\mathrm{C}_{12} \mathrm{H}_{13} \mathrm{~N}_{2} \mathrm{O}_{4}\left(\mathrm{M}+\mathrm{H}^{+}\right) 249.0875(\mathrm{M}+\mathrm{Na}=271.0695)$, found

1,4-Diethenyl-2,5-dinitrobenzene (292). To a solution of 266 (310.3 mg, $0.740 \mathrm{mmol})$ in DMF (5 mL) under nitrogen atmosphere were added BHT (18.4 mg, $0.084 \mathrm{mmol}), \mathrm{PdCl}_{2}\left(\mathrm{PPh}_{3}\right)_{2}(9.9 \mathrm{mg}, 0.014 \mathrm{mmol})$, $\mathrm{LiCl}(95.3 \mathrm{mg}, 2.248 \mathrm{mmol})$ and $282(534.2 \mathrm{mg}, 1.685 \mathrm{mmol})$ respectively. The reaction mixture was stirred at ambient temperature for $27 \mathrm{~h}$. EtOAc $(20 \mathrm{~mL})$ was added and the mixture was washed with water $(6 \mathrm{X} 15 \mathrm{~mL})$. The organic layer was dried $(\mathrm{MgSO} 4)$, filtered and the solvent was evaporated under reduced pressure. The resulting residue was purified by chromatography (Hexane/EtOAc, 98:2) to give 292 (82 mg, $0.372 \mathrm{mmol}, 53 \%)$ as a yellow solid. $\mathrm{mp}=148-149{ }^{\circ} \mathrm{C} ;{ }^{1} \mathrm{H} \mathrm{NMR}\left(400 \mathrm{MHz}, \mathrm{CDCl}_{3}\right) \delta 8.14(\mathrm{~s}$, 2H), 7.09 (dd, J=17.2, $11.2 \mathrm{~Hz}, 2 \mathrm{H}), 5.90$ (d, $J=17.6 \mathrm{~Hz}, 2 \mathrm{H}), 5.65$ (d, $J=11.2 \mathrm{~Hz}, 2 \mathrm{H}) ;{ }^{13} \mathrm{C}$ NMR (100 $\left.\mathrm{MHz}, \mathrm{CDCl}_{3}\right) \delta 149.3,133.0,129.9,124.3,121.6$; IR (ATR) 1518, 1348, 1283, $937 \mathrm{~cm}^{-1}$; HRMS (ESI) calcd for $\mathrm{C}_{10} \mathrm{H}_{9} \mathrm{~N}_{2} \mathrm{O}_{4}\left(\mathrm{M}+\mathrm{H}^{+}\right) 221.0562(\mathrm{M}+\mathrm{Na}=243.0382)$, found

2,5-Dinitro-1,4-di(1-propen-1-yl)benzene (293). A mixture of 266 (397.6 mg, $0.899 \mathrm{mmol}$ ) and 283 (774.3 mg, $2.338 \mathrm{mmol})$ in DMF (6 mL) in the presence of $\mathrm{PdCl}_{2}\left(\mathrm{PPh}_{3}\right)_{2}(25.3 \mathrm{mg}, 0.036 \mathrm{mmol})$, BHT (21.8 mg, $0.099 \mathrm{mmol})$ and $\mathrm{LiCl}(137.3 \mathrm{mg}, 3.239 \mathrm{mmol})$ was stirred at $65^{\circ} \mathrm{C}$ for $14 \mathrm{~h}$. After cooling to ambient temperature, the solution was diluted with EtOAc $(15 \mathrm{~mL})$ and was washed with water $(7 \times 10$ $\mathrm{mL})$. The resulting crude was purified by chromatography $\left(10 \% \mathrm{~K}_{2} \mathrm{CO}_{3}\right.$ silica gel, Hexane/EtOAc, 97:3) to give 293 (89.1 mg, $0.359 \mathrm{mmol}, 40 \%)$ as a yellow solid. mp=114-115 ${ }^{\circ} \mathrm{C} ;{ }^{1} \mathrm{H} \mathrm{NMR}\left(600 \mathrm{MHz}, \mathrm{CDCl}_{3}\right)$ $\delta 8.14(\mathrm{~s}, 1 \mathrm{H}), 8.01(\mathrm{~s}, 1 \mathrm{H}), 7.95(\mathrm{~s}, 1 \mathrm{H}), 7.82(\mathrm{~s}, 1 \mathrm{H}), 6.78(\mathrm{dq}, J=16.8,1.2 \mathrm{~Hz}, 1 \mathrm{H}), 6.74$ (dq, J=15.6, 1.8 $\mathrm{Hz}, 1 \mathrm{H}), 6.64$ (dq, $J=11.4,1.8 \mathrm{~Hz}, 1 \mathrm{H}), 6.61$ (dq, $J=11.4,1.8 \mathrm{~Hz}, 1 \mathrm{H}), 6.46-6.36$ (m, 2H), 6.11-6.02 (m, 2H), 1.99 (dd, J=6.6, 1.8 Hz, 3H), 1.97 (dd, J=6.6, 1.8 Hz, 3H), 1.81 (dd, J=7.2, $1.8 \mathrm{~Hz}, 3 \mathrm{H}$ ), 1.79 (dd, $J=7.2,1.8 \mathrm{~Hz}, 3 \mathrm{H}) ;{ }^{13} \mathrm{C} \mathrm{NMR}\left(100 \mathrm{MHz}, \mathrm{CDCl}_{3}\right) \delta 150.0,149.6,148.9,148.4,134.6,134.2,132.5,131.9$, $131.8,131.8,131.6,131.2$, 127.6, 127.5, 124.0, 123.9, 123.8, 123.5, 123.5, 18.9, 18.9, 14.5, 14.5; IR (ATR) 1519, 1350, 1270, $963 \mathrm{~cm}^{-1}$; HRMS (ESI) calcd for $\mathrm{C}_{12} \mathrm{H}_{13} \mathrm{~N}_{2} \mathrm{O}_{4}\left(\mathrm{M}+\mathrm{H}^{+}\right) 249.0875$ $(\mathrm{M}+\mathrm{Na}=271.0695)$, found

2,5-Dinitro-1,4-di(1-propen-1-yl)benzene (293). Treatment of a solution of 266 (213 $\mathrm{mg}, 0.482 \mathrm{mmol})$ with 283 (416.5 mg, $1.258 \mathrm{mmol})$ in the presence of $\mathrm{PdCl}_{2}\left(\mathrm{PPh}_{3}\right)_{2}(6.8 \mathrm{mg}, 0.010 \mathrm{mmol}), \mathrm{PPh}_{3}(5.2 \mathrm{mg}$, $0.020 \mathrm{mmol})$, BHT $(12.6 \mathrm{mg}, 0.057 \mathrm{mmol})$ and $\mathrm{LiCl}(73.5 \mathrm{mg}, 1.734 \mathrm{mmol})$ in dioxane $(2.5 \mathrm{~mL})$, as 
described for $285\left(100{ }^{\circ} \mathrm{C}, 24 \mathrm{~h}\right)$, gave after solvent removal and chromatography (Hexane/EtOAc, 9:1) 293 (62.2 $\mathrm{mg}, 0.251 \mathrm{mmol}, 52 \%)$ as a yellow solid.

2,5-Dinitro-1,4-di(1-propen-1-yl)benzene (293). Treatment of a solution of 265 (313.1 $\mathrm{mg}, 0.793 \mathrm{mmol})$ with 283 (685 mg, $2.069 \mathrm{mmol})$ in the presence of $\mathrm{PdCl}_{2}\left(\mathrm{PPh}_{3}\right)_{2}(11.1 \mathrm{mg}, 0.016 \mathrm{mmol}), \mathrm{PPh}_{3}(8.3 \mathrm{mg}$, $0.032 \mathrm{mmol})$, BHT (20.9 mg, $0.0595 \mathrm{mmol})$ and $\mathrm{LiCl}(121.6 \mathrm{mg}, 2.855 \mathrm{mmol})$ in dioxane (3 $\mathrm{mL})$, as described for $285\left(100{ }^{\circ} \mathrm{C}, 24 \mathrm{~h}\right)$, gave after solvent removal and chromatography (Hexane/EtOAc, 95:5) 293 (124 mg, $0.500 \mathrm{mmol}, 63 \%)$ as a yellow solid.

2,5-Dinitro-1,4-di(1-propen-2-yl)benzene (294). Treatment of a solution of $\mathbf{2 6 6}$ (239.2 $\mathrm{mg}, 0.541 \mathrm{mmol})$ with 284 (466 mg, $1.407 \mathrm{mmol})$ in the presence of $\mathrm{PdCl}_{2}\left(\mathrm{PPh}_{3}\right)_{2}(7.6 \mathrm{mg}, 0.011 \mathrm{mmol}), \mathrm{PPh}_{3}(5.7 \mathrm{mg}$, $0.022 \mathrm{mmol})$, BHT (11.9 $\mathrm{mg}, 0.054 \mathrm{mmol})$, and $\mathrm{LiCl}(78 \mathrm{mg}, 1.840 \mathrm{mmol})$ in dioxane $(5 \mathrm{~mL})$, as described for $285\left(100{ }^{\circ} \mathrm{C}, 30 \mathrm{~h}\right)$, gave after solvent removal and chromatography (Hexane/EtOAc, 95:5) $294(71.9 \mathrm{mg}, 0.290 \mathrm{mmol}, 54 \%)$ as a white solid. $\mathrm{mp}=118-119{ }^{\circ} \mathrm{C} ;{ }^{1} \mathrm{H}$ NMR $\left(600 \mathrm{MHz}, \mathrm{CDCl}_{3}\right) \delta 7.77$ $(\mathrm{s}, 2 \mathrm{H}), 5.29$ (s, with further fine splitting, $2 \mathrm{H}$ ), 5.05 (s, with further fine splitting, $2 \mathrm{H}$ ), 2.11 (dd, $J=2.4$, $1.2 \mathrm{~Hz}, 6 \mathrm{H}) ;{ }^{13} \mathrm{C}$ NMR $\left(150 \mathrm{MHz}, \mathrm{CDCl}_{3}\right) \delta 149.6,140.1,138.5,125.9,117.6,22.85$; IR (ATR) 1533, 1350, 905, $847 \mathrm{~cm}^{-1}$; HRMS (ESI) calcd for $\mathrm{C}_{12} \mathrm{H}_{13} \mathrm{~N}_{2} \mathrm{O}_{4}\left(\mathrm{M}+\mathrm{H}^{+}\right) 249.0875(\mathrm{M}+\mathrm{Na}=271.0695)$, found

2,5-Dinitro-1,4-di(1-propen-2-yl)benzene (294). Treatment of a solution of 265 (203.4 $\mathrm{mg}, 0.515 \mathrm{mmol})$ with 284 (445 mg, $1.344 \mathrm{mmol})$ in the presence of $\mathrm{PdCl}_{2}\left(\mathrm{PPh}_{3}\right)_{2}(7.3 \mathrm{mg}, 0.010 \mathrm{mmol}), \mathrm{PPh}_{3}(5.4 \mathrm{mg}$, $0.021 \mathrm{mmol})$, BHT $(13.2 \mathrm{mg}, 0.060 \mathrm{mmol})$ and $\mathrm{LiCl}(78.6 \mathrm{mg}, 1.854 \mathrm{mmol})$ in dioxane $(2 \mathrm{~mL})$, as described for $285\left(100{ }^{\circ} \mathrm{C}, 26 \mathrm{~h}\right)$, gave after solvent removal and chromatography (Hexane/EtOAc, 97:3) 294 (81.9 $\mathrm{mg}, 0.330 \mathrm{mmol}, 64 \%)$ as a white solid.

1,2-Diethenyl-3,6-dinitrobenzene (295) Treatment of a solution of 288 (103 $\mathrm{mg}, 0.261 \mathrm{mmol}$ ) with 282 (206.6 mg, $0.652 \mathrm{mmol}$ ) in the presence of $\mathrm{PdCl}_{2}\left(\mathrm{PPh}_{3}\right)_{2}(3.7 \mathrm{mg}, 0.005 \mathrm{mmol}), \mathrm{PPh}_{3}(2.7 \mathrm{mg}, 0.010$ $\mathrm{mmol})$, BHT $(6.9 \mathrm{mg}, 0.031 \mathrm{mmol})$ and $\mathrm{LiCl}(39.8 \mathrm{mg}, 0.940 \mathrm{mmol})$ in dioxane $(1.5 \mathrm{~mL})$, as described for $285\left(100{ }^{\circ} \mathrm{C}, 24 \mathrm{~h}\right.$ ), gave after solvent removal and chromatography (Hexane/EtOAc, 9:1) 295 (21.2 $\mathrm{mg}, 0.096 \mathrm{mmol}, 37 \%)$ as a yellow oil. ${ }^{1} \mathrm{H}$ NMR (400 MHz, $\left.\mathrm{CDCl}_{3}\right) \delta 7.71(\mathrm{~s}, 2 \mathrm{H}), 6.73$ (dd, $J=18.0,11.6$ $\mathrm{Hz}, 2 \mathrm{H}), 5.60(\mathrm{dd}, J=11.2,0.4 \mathrm{~Hz}, 2 \mathrm{H}), 5.41(\mathrm{dd}, J=18.0,0.8 \mathrm{~Hz}, 2 \mathrm{H}) ;{ }^{13} \mathrm{C} \mathrm{NMR}\left(100 \mathrm{MHz}, \mathrm{CDCl}_{3}\right) \delta$ 151.0, 133.6, 129.6, 123.3, 122.8; IR (ATR) 1523, 1350, 804, $726 \mathrm{~cm}^{-1}$; HRMS (ESI) calcd for $\mathrm{C}_{10} \mathrm{H}_{9} \mathrm{~N}_{2} \mathrm{O}_{4}\left(\mathrm{M}+\mathrm{H}^{+}\right) 221.0562(\mathrm{M}+\mathrm{Na}=243.0382)$, found 
3,6-Dinitro-1,3-di(1-propen-1-yl)benzene (296) Treatment of a solution of $\mathbf{2 8 8}$ (482.2 $\mathrm{mg}, 1.221 \mathrm{mmol}$ ) with 283 (1.0104 g, $3.051 \mathrm{mmol})$ in the presence of $\mathrm{PdCl}_{2}\left(\mathrm{PPh}_{3}\right)_{2}(17.1 \mathrm{mg}, 0.024 \mathrm{mmol}), \mathrm{PPh}_{3}(12.7 \mathrm{mg}$, $0.048 \mathrm{mmol})$, BHT (32.3 mg, $0.147 \mathrm{mmol})$ and $\mathrm{LiCl}(186.2 \mathrm{mg}, 4.396 \mathrm{mmol})$ in dioxane $(4 \mathrm{~mL})$, as described for $285\left(100{ }^{\circ} \mathrm{C}, 20 \mathrm{~h}\right)$, gave after solvent removal and chromatography (Hexane/EtOAc, 9:1) 296 (143.6 mg, $0.578 \mathrm{mmol}, 47 \%)$ as a yellow oil. ${ }^{1} \mathrm{H}$ NMR (400 MHz, $\left.\mathrm{CDCl}_{3}\right) \delta 7.80(\mathrm{~s}, 1 \mathrm{H}), 7.71(8.8$ $\mathrm{Hz}, 1 \mathrm{H}), 7.63$ (d, J=8.8 Hz, 1H), 7.56 (s, 1H), 6.36-6.28 (m, 4H), 5.96-5.77 (m, 4H), 1.85 (dd, J=6.8, 1.6 $\mathrm{Hz}, 3 \mathrm{H}), 1.82(\mathrm{dd}, J=6.8,2.0 \mathrm{~Hz}, 3 \mathrm{H}), 1.43(\mathrm{dd}, J=6.8,1.6 \mathrm{~Hz}, 3 \mathrm{H}), 1.41(\mathrm{dd}, J=7.2,1.6 \mathrm{~Hz}, 3 \mathrm{H}) ;{ }^{13} \mathrm{C}$ NMR (100 MHz, $\left.\mathrm{CDCl}_{3}\right) \delta 151.6,151.3,151.3,151.2,135.0,134.5,133.8,133.8,133.3,133.0,131.3$, $131.3,122.9,122.8,122.6,122.5,122.5,122.3,122.1,121.9,18.9,18.9,14.5,14.4$; IR (ATR) 1528, 1350, 959, $826 \mathrm{~cm}^{-1}$; HRMS (ESI) calcd for $\mathrm{C}_{12} \mathrm{H}_{13} \mathrm{~N}_{2} \mathrm{O}_{4}\left(\mathrm{M}+\mathrm{H}^{+}\right) 249.0875(\mathrm{M}+\mathrm{Na}=271.0695)$, found

3,6-Dinitro-1,2-di-(1-propen-2-yl)benzene (297) Treatment of a solution of 288 (400.1 mg, 1.013 mmol) with 32 (831.6 g, $2.512 \mathrm{mmol})$ in the presence of $\mathrm{PdCl}_{2}\left(\mathrm{PPh}_{3}\right)_{2}(14.2 \mathrm{mg}, 0.020 \mathrm{mmol}), \mathrm{PPh}_{3}(11$ $\mathrm{mg}, 0.042 \mathrm{mmol})$, BHT (26.8 mg, $0.122 \mathrm{mmol})$ and $\mathrm{LiCl}(154.4 \mathrm{mg}, 3.642 \mathrm{mmol})$ in dioxane (4 mL), as described for $285\left(100{ }^{\circ} \mathrm{C}, 20 \mathrm{~h}\right.$ ), gave after solvent removal and chromatography (Hexane/EtOAc, 9:1) 297 (121.2 mg, $0.488 \mathrm{mmol}, 48 \%$ ) as a yellow oil. ${ }^{1} \mathrm{H} \mathrm{NMR}\left(400 \mathrm{MHz}, \mathrm{CDCl}_{3}\right) \delta 7.75$ (s, 2H), 5.30 (s, 2H), 4.92 (s, 2H), $2.11(\mathrm{~s}, 6 \mathrm{H}) ;{ }^{13} \mathrm{C}$ NMR (100 MHz, $\left.\mathrm{CDCl}_{3}\right) \delta 151.1,139.1$, 138.7, 122.8, 118.7, 24.1; IR (ATR) 1531, 1348, 912, $735 \mathrm{~cm}^{-1}$; HRMS (ESI) calcd for $\mathrm{C}_{12} \mathrm{H}_{13} \mathrm{~N}_{2} \mathrm{O}_{4}\left(\mathrm{M}+\mathrm{H}^{+}\right) 249.0875$ $(\mathrm{M}+\mathrm{Na}=271.0695)$, found

1,3-Diethenyl-2,6-dinitrobenzene (298). Treatment of a solution of 273 (463 mg, $1.172 \mathrm{mmol}$ ) with 282 (929 mg, $2.930 \mathrm{mmol})$ in the presence of $\mathrm{PdCl}_{2}\left(\mathrm{PPh}_{3}\right)_{2}(32.9 \mathrm{mg}, 0.047 \mathrm{mmol}), \mathrm{PPh}_{3}(24.6 \mathrm{mg}, 0.094$ $\mathrm{mmol})$, BHT (28.4 mg, $0.129 \mathrm{mmol})$, and $\mathrm{LiCl}(159 \mathrm{mg}, 3.75 \mathrm{mmol})$ in dioxane $(8 \mathrm{~mL})$, as described for $285\left(90^{\circ} \mathrm{C}, 24 \mathrm{~h}\right.$ ), gave after solvent removal and chromatography (Hexane/EtOAc, 95:5) 298 (82.6 mg, $0.375 \mathrm{mmol}, 32 \%)$ as a yellow oil. ${ }^{1} \mathrm{H} \mathrm{NMR}\left(600 \mathrm{MHz}, \mathrm{CDCl}_{3}\right) \delta 8.08(\mathrm{~d}, J=8.4 \mathrm{~Hz}, 1 \mathrm{H}), 7.71(\mathrm{~d}, J=9.0$ $\mathrm{Hz}, 1 \mathrm{H}), 6.89$ (dd, $J=17.4,10.8 \mathrm{~Hz}, 1 \mathrm{H}), 6.61$ (dd, $J=17.4,11.4 \mathrm{~Hz}, 1 \mathrm{H}), 6.50$ (d, J=17.4 Hz, 1H), 5.69 (d, $J=10.8 \mathrm{~Hz}, 1 \mathrm{H}), 5.56(\mathrm{~d}, J=12.0 \mathrm{~Hz}, 1 \mathrm{H}), 5.46(\mathrm{~d}, J=18.0 \mathrm{~Hz}, 1 \mathrm{H}) ;{ }^{13} \mathrm{C} \mathrm{NMR}\left(100 \mathrm{MHz}, \mathrm{CDCl}_{3}\right) \delta 149.7$, 146.9, 134.2, 128.1, 127.3, 127.2, 126.0, 125.5, 123.4, 122.3; IR (ATR) 1516, 1346, 937, $914 \mathrm{~cm}^{-1}$; HRMS (ESI) calcd for $\mathrm{C}_{10} \mathrm{H}_{9} \mathrm{~N}_{2} \mathrm{O}_{4}\left(\mathrm{M}+\mathrm{H}^{+}\right) 221.0562(\mathrm{M}+\mathrm{Na}=243.0382)$, found.

Scheme 7.12 Selective coupling of 258 with 282. Treatment of a solution of 258 (203.2 $\mathrm{mg}, 0.514$ mmol) with $282(196.2 \mathrm{mg}, 0.619 \mathrm{mmol})$ in the presence of $\mathrm{PdCl}_{2}\left(\mathrm{PPh}_{3}\right)_{2}(7.2 \mathrm{mg}, 0.010 \mathrm{mmol}), \mathrm{PPh}_{3}$ (5.4 mg, $0.021 \mathrm{mmol})$, and BHT (10 mg, $0.045 \mathrm{mmol})$ in dioxane $(4 \mathrm{~mL})$, as described for $285\left(100{ }^{\circ} \mathrm{C}\right.$, $24 \mathrm{~h}$ ), gave, in order of elution, after solvent removal and chromatography (Hexane/DCM, 95:5, then 9:1), 
inseparable mixtures of $\mathbf{2 8 5}$ and 4-bromo-1-ethenyl-2,3-dinitrobenzene $(\mathbf{2 8 5}+\mathbf{2 9 9}, 22.7 \mathrm{mg})$ as a white solid and $\mathbf{2 5 8}$ and $\mathbf{3 0 0}(\mathbf{2 5 8}+\mathbf{3 0 0}, 69.8 \mathrm{mg})$ as a colorless oil.

4-Ethenyl-2,3-Dinitrophenyltrifluoromethanesulfonate (300). Treatment of a solution of 264 (884.1 mg, $2.000 \mathrm{mmol})$ with 282 (761 mg, $2.400 \mathrm{mmol})$ in the presence of $\mathrm{PdCl}_{2}\left(\mathrm{PPh}_{3}\right)_{2}$ (28.1 $\mathrm{mg}, 0.040$ $\mathrm{mmol}), \mathrm{PPh}_{3}(21 \mathrm{mg}, 0.080 \mathrm{mmol})$, and BHT (44 mg, $\left.0.200 \mathrm{mmol}\right)$ in dioxane $(8 \mathrm{~mL})$, as described for $285\left(98^{\circ} \mathrm{C}, 24 \mathrm{~h}\right)$, gave in order of elution, after solvent removal and chromatography (Hexane/EtOAc, 9:1) 285 ( $8.8 \mathrm{mg}, 0.040 \mathrm{mmol}, 2 \%)$ and $\mathbf{3 0 0}$ (444 $\mathrm{mg}, 1.297 \mathrm{mmol}, 65 \%)$ both as a white solid. $\mathrm{mp}=58-59$ ${ }^{\circ} \mathrm{C} ;{ }^{1} \mathrm{H}$ NMR $\left(400 \mathrm{MHz}, \mathrm{CDCl}_{3}\right) \delta 7.93(\mathrm{~d}, J=8.8 \mathrm{~Hz}, 1 \mathrm{H}), 7.65(\mathrm{~d}, J=8.8 \mathrm{~Hz}, 1 \mathrm{H}), 6.75(\mathrm{dd}, J=17.2,10.8$ $\mathrm{Hz}, 1 \mathrm{H}), 5.97(\mathrm{~d}, J=17.6 \mathrm{~Hz}, 1 \mathrm{H}), 5.76(\mathrm{~d}, J=10.8 \mathrm{~Hz}, 1 \mathrm{H}) ;{ }^{13} \mathrm{C} \mathrm{NMR}\left(100 \mathrm{MHz}, \mathrm{CDCl}_{3}\right) \delta 142.3,139.5$, 136.9, 132.6, 131.2, 127.4, $118.3\left(\mathrm{q}, J^{\mathrm{C}-\mathrm{F}}=319 \mathrm{~Hz}\right) ;{ }^{19} \mathrm{~F}$ NMR $\left(376.10 \mathrm{MHz}, \mathrm{CDCl}_{3}\right) \delta-72.7$; IR (ATR) 1521, 1421, 1326, 1200, $1132 \mathrm{~cm}^{-1}$; HRMS (ESI) calcd for $\mathrm{C}_{9} \mathrm{H}_{6} \mathrm{~F}_{3} \mathrm{~N}_{2} \mathrm{O}_{7} \mathrm{~S}\left(\mathrm{M}+\mathrm{H}^{+}\right) 342.9848$ $(\mathrm{M}+\mathrm{Na}=364.9667)$, found

2,3-Dinitro-4-(1-propen-1-yl)phenyltrifluoromethanesulfonate (301). Treatment of a solution of 264 (720 mg, $1.629 \mathrm{mmol})$ with 283 (663 mg, $2.003 \mathrm{mmol})$ in the presence of $\mathrm{PdCl}_{2}\left(\mathrm{PPh}_{3}\right)_{2}(22.8 \mathrm{mg}, 0.032$ $\mathrm{mmol}), \mathrm{PPh}_{3}(17.1 \mathrm{mg}, 0.065 \mathrm{mmol})$, and BHT (35 mg, $\left.0.159 \mathrm{mmol}\right)$ in dioxane $(6 \mathrm{~mL})$, as described for $285\left(100^{\circ} \mathrm{C}, 24 \mathrm{~h}\right)$, gave, after solvent removal and chromatography (Hexane/EtOAc, 9:1) 301 (370.2 mg, $1.039 \mathrm{mmol}, 64 \%)$ as a yellow solid. $\mathrm{mp}=64-65{ }^{\circ} \mathrm{C} ;{ }^{1} \mathrm{H} \mathrm{NMR}\left(400 \mathrm{MHz}, \mathrm{CDCl}_{3}\right) \delta 7.84(\mathrm{~d}, J=9.0 \mathrm{~Hz}$, $1 \mathrm{H}), 7.67(\mathrm{~d}, J=8.8 \mathrm{~Hz}, 1 \mathrm{H}), 7.64(\mathrm{~d}, J=8.8 \mathrm{~Hz}, 1 \mathrm{H}), 7.58(\mathrm{~d}, J=9.0 \mathrm{~Hz}, 1 \mathrm{H}), 6.54-6.37$ (m, 3H), 6.18 $(\mathrm{dq}, J=11.6,7.2 \mathrm{~Hz}, 1 \mathrm{H}), 1.99(\mathrm{~d}, J=5.1 \mathrm{~Hz}, 3 \mathrm{H}), 1.79(\mathrm{dd}, J=7.2,1.8 \mathrm{~Hz}, 2 \mathrm{H}) ;{ }^{13} \mathrm{C} \mathrm{NMR}(100 \mathrm{MHz}$, $\left.\mathrm{CDCl}_{3}\right) \delta 143.1,141.6,138.8,138.6,137.9,136.9,136.8,135.4,135.2,133.0,132.6,131.3,125.4,125.2$, $121.1,120.6,118.3\left(\mathrm{q}, J^{\mathrm{C}-\mathrm{F}}=319 \mathrm{~Hz}, 2 \mathrm{C}\right), 18.9,14.5$; IR (ATR) 1547, 1436, 1350, 1233, $1137 \mathrm{~cm}^{-1}$; HRMS (ESI) calcd for $\mathrm{C}_{10} \mathrm{H}_{8} \mathrm{~F}_{3} \mathrm{~N}_{2} \mathrm{O}_{7} \mathrm{~S}\left(\mathrm{M}+\mathrm{H}^{+}\right) 357.0004(\mathrm{M}+\mathrm{Na}=378.9824)$, found

2,3-Dinitro-4-(1-propen-2-yl)phenyltrifluoromethanesulfonate (302). Treatment of a solution of 264 (923.1 mg, $2.088 \mathrm{mmol}$ ) with 284 (830 mg, $2.506 \mathrm{mmol})$ in the presence of $\mathrm{PdCl}_{2}\left(\mathrm{PPh}_{3}\right)_{2}(29.3 \mathrm{mg}, 0.042$ $\mathrm{mmol}), \mathrm{PPh}_{3}(21.9 \mathrm{mg}, 0.083 \mathrm{mmol})$, and BHT (46 mg, $\left.0.209 \mathrm{mmol}\right)$ in dioxane (10 mL), as described for $285\left(98^{\circ} \mathrm{C}, 24 \mathrm{~h}\right)$, gave, in order of elution, after solvent removal and chromatography (Hexane/EtOAc, 9:1) 287 (15.4 mg, $0.062 \mathrm{mmol}, 2 \%)$ and 302 (479 mg, $1.344 \mathrm{mmol}, 64 \%)$ both as a white solid. mp=69$70{ }^{\circ} \mathrm{C} ; \quad{ }^{1} \mathrm{H}$ NMR $\left(600 \mathrm{MHz}, \mathrm{CDCl}_{3}\right) \delta 7.67(\mathrm{~d}, J=8.4 \mathrm{~Hz}, 1 \mathrm{H}), 7.63(\mathrm{~d}, J=8.4 \mathrm{~Hz}, 1 \mathrm{H}), 5.34(\mathrm{~s}, 1 \mathrm{H}), 5.08$ (s, 1H), $2.12(\mathrm{~s}, 3 \mathrm{H}) ;{ }^{13} \mathrm{C} \mathrm{NMR}\left(150 \mathrm{MHz}, \mathrm{CDCl}_{3}\right) \delta 142.8,139.2,138.9,138.1,136.6,133.7,125.3$, 119.5, 118.3 (q, $J^{\mathrm{C}-\mathrm{F}}=319.5 \mathrm{~Hz}$ ); IR (ATR) 1544, 1429, 1219, 1135, $834 \mathrm{~cm}^{-1}$; HRMS (ESI) calcd for $\mathrm{C}_{10} \mathrm{H}_{8} \mathrm{~F}_{3} \mathrm{~N}_{2} \mathrm{O}_{7} \mathrm{~S}\left(\mathrm{M}+\mathrm{H}^{+}\right) 357.0004(\mathrm{M}+\mathrm{Na}=378.9824)$, found 
Ethyl 4(-trifluoromethylsulfonyloxy)-2,3-dinitrophenylprop-2-enoate (303). Treatment of a solution of 264 (367.8 $\mathrm{mg}, 0.832 \mathrm{mmol}$ ) with ethyl 2-tributylstannyl-2-propenoate (369 $\mathrm{mg}, 0.948 \mathrm{mmol}$ ) in the presence of $\mathrm{PdCl}_{2}\left(\mathrm{PPh}_{3}\right)_{2}(11.7 \mathrm{mg}, 0.017 \mathrm{mmol}), \mathrm{PPh}_{3}(8.7 \mathrm{mg}, 0.033 \mathrm{mmol})$, and BHT (20.2 mg, 0.092 mmol) in dioxane $(4 \mathrm{~mL})$, as described for $285\left(98{ }^{\circ} \mathrm{C}, 23 \mathrm{~h}\right)$, gave, after solvent removal and chromatography (Hexane/DCM, 6:4) 303 (170.7 mg, $0.412 \mathrm{mmol}, 50 \%)$ as a yellow oil. ${ }^{1} \mathrm{H}$ NMR (400 $\left.\mathrm{MHz}, \mathrm{CDCl}_{3}\right) \delta 7.73(\mathrm{~d}, J=8.8 \mathrm{~Hz}, 1 \mathrm{H}), 7.67$ (d, J=8.8 Hz, 1H), 6.73 (s, 1H), 6.05 (s, 1H), 4.23 (quartet, $7.2 \mathrm{~Hz}, 2 \mathrm{H}), 1.27$ (t, J=7.2 Hz, 3H); ${ }^{13} \mathrm{C} \mathrm{NMR}\left(100 \mathrm{MHz}, \mathrm{CDCl}_{3}\right) \delta 163.1,142.5,140.1,137.6,135.8$, 134.8, 133.2, 131.8, 125.9, 119.9, 116.7, 62.3, 13.8; $\left.{ }^{19} \mathrm{~F} \mathrm{NMR} \mathrm{(376.10} \mathrm{MHz,} \mathrm{CDCl}_{3}\right) \delta-72.3$; IR (ATR) 1716, 1552, 1212, 1131, $832 \mathrm{~cm}^{-1}$; HRMS (ESI) calcd for $\mathrm{C}_{12} \mathrm{H}_{10} \mathrm{~F}_{3} \mathrm{~N}_{2} \mathrm{O}_{9} \mathrm{~S}\left(\mathrm{M}+\mathrm{H}^{+}\right) 415.0059$ $(\mathrm{M}+\mathrm{Na}=436.9879)$, found

5-Ethenyl-2,4-Dinitrophenyltrifluoromethanesulfonate (205). Treatment of a solution of 276 (112.2 $\mathrm{mg}, 0.254 \mathrm{mmol})$ with $282(98 \mathrm{mg}, 0.309 \mathrm{mmol})$ in the presence of $\mathrm{PdCl}_{2}\left(\mathrm{PPh}_{3}\right)_{2}(3.6 \mathrm{mg}, 0.005 \mathrm{mmol})$, $\mathrm{PPh}_{3}(2.7 \mathrm{mg}, 0.010 \mathrm{mmol})$, and BHT $(6.7 \mathrm{mg}, 0.030 \mathrm{mmol})$ in dioxane $(2 \mathrm{~mL})$, as described for 285 (98 ${ }^{\circ} \mathrm{C}, 32 \mathrm{~h}$ ), gave, after solvent removal and chromatography (Hexane/EtOAc, 95:5) 205 (30 mg, 0.088 mmol, 35\%) as a faint yellow oil. ${ }^{1} \mathrm{H}$ NMR $\left(600 \mathrm{MHz}, \mathrm{CDCl}_{3}\right) \delta 8.80(\mathrm{~s}, 1 \mathrm{H}), 7.74(\mathrm{~s}, 1 \mathrm{H}), 7.26(\mathrm{dd}$, $J=17.4,10.8 \mathrm{~Hz}, 1 \mathrm{H}), 6.02(\mathrm{~d}, J=16.8 \mathrm{~Hz}, 1 \mathrm{H}), 5.87$ (d, $J=10.8 \mathrm{~Hz}, 1 \mathrm{H}) ;{ }^{13} \mathrm{C} \mathrm{NMR}\left(150 \mathrm{MHz}, \mathrm{CDCl}_{3}\right) \delta$ 145.3, 143.5, 140.6, 139.6, 129.7, 124.9, 124.3, 123.9, 118.5 (q, $\left.J^{\mathrm{C}-\mathrm{F}}=213 \mathrm{~Hz}\right) ;{ }^{19} \mathrm{~F} \mathrm{NMR}\left(\mathrm{MHz}, \mathrm{CDCl}_{3}\right) \delta$ -72.8; IR (ATR) 1536, 1434, 1338, 1210, $1131 \mathrm{~cm}^{-1}$; HRMS (ESI) calcd for $\mathrm{C}_{9} \mathrm{H}_{6} \mathrm{~F}_{3} \mathrm{~N}_{2} \mathrm{O}_{7} \mathrm{~S}\left(\mathrm{M}+\mathrm{H}^{+}\right)$ $342.9848(\mathrm{M}+\mathrm{Na}=364.9667)$, found

2,4-Dinitro-5-(1-propen-1-yl)phenyl trifluoromethanesulfonate (305). Treatment of a solution of 204 (703.2 $\mathrm{mg}, 1.780 \mathrm{mmol}$ ) with 283 (766 mg, $2.314 \mathrm{mmol})$ in the presence of $\mathrm{PdCl}_{2}\left(\mathrm{PPh}_{3}\right)_{2}$ (25 $\mathrm{mg}, 0.036$ $\mathrm{mmol}), \mathrm{PPh}_{3}(18.7 \mathrm{mg}, 0.071 \mathrm{mmol})$, and BHT $(43.1 \mathrm{mg}, 0.196 \mathrm{mmol})$ in dioxane $(8 \mathrm{~mL})$, as described for 285 (90 $\left.{ }^{\circ} \mathrm{C}, 24 \mathrm{~h}\right)$, gave, in order of elution, after solvent removal and chromatography (Hexane/EtOAc, 95:5) 290 (52.6 mg, $0.212 \mathrm{mmol}, 12 \%$ ) as a yellow solid and $\mathbf{3 0 5}$ (476.3 mg, 1.337 mmol, $75 \%)$ as a yellow oil. ${ }^{1} \mathrm{H}$ NMR $\left(400 \mathrm{MHz}, \mathrm{CDCl}_{3}\right) \delta 8.85$ (s, 1H), 8.75 (s, 1H), 7.64 (s, 1H), 7.51 (s, 1H), $6.95(\mathrm{dq}, J=15.6,2.0 \mathrm{~Hz}, 1 \mathrm{H}), 6.76(\mathrm{dq}, \mathrm{J}=12.0,2.0 \mathrm{~Hz}, 1 \mathrm{H}), 6.54(\mathrm{dq}, J=15.6,6.8 \mathrm{~Hz}, 1 \mathrm{H}), 6.24$ (dq, $J=12.0,7.2 \mathrm{~Hz}, 1 \mathrm{H}), 2.06(\mathrm{dd}, J=6.8,1.6 \mathrm{~Hz}, 3 \mathrm{H}), 1.84(\mathrm{dd}, J=7.2,2.0 \mathrm{~Hz}, 3 \mathrm{H}) ;{ }^{13} \mathrm{C} \mathrm{NMR}(100 \mathrm{MHz}$, $\left.\mathrm{CDCl}_{3}\right) \delta 146.1,144.8,143.3,142.9,140.6,140.2,139.4,139.1,138.6,134.4,127.6,123.9,123.7,123.7$, $123.5,123.1,118.5\left(\mathrm{q}, J^{\mathrm{C}-\mathrm{F}}=320 \mathrm{~Hz}\right), 118.4\left(\mathrm{q}, J^{\mathrm{C}-\mathrm{F}}=319 \mathrm{~Hz}\right) ;{ }^{19} \mathrm{~F}$ NMR $\left(376.10 \mathrm{MHz}, \mathrm{CDCl}_{3}\right) \delta-73.0,-$ 72.9; IR (ATR) 1534, 1434, 1339, 1215, $1131 \mathrm{~cm}^{-1}$; HRMS (ESI) calcd for $\mathrm{C}_{10} \mathrm{H}_{8} \mathrm{~F}_{3} \mathrm{~N}_{2} \mathrm{O}_{7} \mathrm{~S}\left(\mathrm{M}+\mathrm{H}^{+}\right)$ $357.0004(\mathrm{M}+\mathrm{Na}=378.9824)$, found 
2,4-Dinitro-5-(1-propen-2-yl)trifluoromethanesulfonate (306). Treatment of a solution of 276 (312.7 $\mathrm{mg}, 0.707 \mathrm{mmol})$ with 284 (284 mg, $0.858 \mathrm{mmol})$ in the presence of $\mathrm{PdCl}_{2}\left(\mathrm{PPh}_{3}\right)_{2}(9.9 \mathrm{mg}, 0.014 \mathrm{mmol})$, $\mathrm{PPh}_{3}(7.4 \mathrm{mg}, 0.028 \mathrm{mmol})$, and BHT $(17.1 \mathrm{mg}, 0.078 \mathrm{mmol})$ in dioxane $(3 \mathrm{~mL})$, as described for 285 (90 ${ }^{\circ} \mathrm{C}, 23 \mathrm{~h}$ ), gave, after solvent removal and chromatography (Hexane/EtOAc, 97:3), in order of elution, 291 (9.9 mg, $0.040 \mathrm{mmol}, 6 \%)$ as a white solid and $306(129 \mathrm{mg}, 0.362 \mathrm{mmol}, 51 \%)$ as a colorless oil. ${ }^{1} \mathrm{H}$ NMR $\left(400 \mathrm{MHz}, \mathrm{CDCl}_{3}\right) \delta 8.70(\mathrm{~s}, 1 \mathrm{H}), 7.44(\mathrm{~s}, 1 \mathrm{H}), 5.38(\mathrm{~s}$, with further fine splitting, 1H), $5.11(\mathrm{~s}$, $1 \mathrm{H}), 2.12(\mathrm{~s}, 3 \mathrm{H}) ;{ }^{13} \mathrm{C} \mathrm{NMR}\left(100 \mathrm{MHz}, \mathrm{CDCl}_{3}\right) \delta 146.3,146.3,143.1,139.8,139.6,126.7,123.2,118.9$, $118.5\left(\mathrm{q}, J^{\mathrm{C}-\mathrm{F}}=320 \mathrm{~Hz}\right) ;{ }^{19} \mathrm{~F}$ NMR $\left(376.10 \mathrm{MHz}, \mathrm{CDCl}_{3}\right) \delta-72.6$; IR (ATR) 1539, 1435, 1340, 1213, 1131 $\mathrm{cm}^{-1}$; HRMS (ESI) calcd for $\mathrm{C}_{10} \mathrm{H}_{8} \mathrm{~F}_{3} \mathrm{~N}_{2} \mathrm{O}_{7} \mathrm{~S}\left(\mathrm{M}+\mathrm{H}^{+}\right) 357.0004(\mathrm{M}+\mathrm{Na}=378.9824)$, found

4-Ethenyl-2,5-dinitrophenyltrifluoromethanesulfonate (307). Treatment of a solution of 266 (782.4 mg, $1.770 \mathrm{mmol})$ with $282(670.5 \mathrm{mg}, 2.114 \mathrm{mmol})$ in the presence of $\mathrm{PdCl}_{2}\left(\mathrm{PPh}_{3}\right)_{2}(24.8 \mathrm{mg}, 0.035$ $\mathrm{mmol}), \mathrm{PPh}_{3}(18.6 \mathrm{mg}, 0.071 \mathrm{mmol})$, and BHT (39 mg, $\left.0.177 \mathrm{mmol}\right)$ in dioxane $(10 \mathrm{~mL})$, as described for $285\left(80^{\circ} \mathrm{C}, 24 \mathrm{~h}\right)$, gave, in order of elution, after solvent removal and chromatography (Hexane/EtOAc, 98:2, then 95:5) 292 (12.2 mg, $0.055 \mathrm{mmol}, 3 \%$ ) as a yellow solid and $\mathbf{3 0 7}$ (463.3 mg, $1.354 \mathrm{mmol}, 76 \%$ ) as yellow oil. ${ }^{1} \mathrm{H}$ NMR $\left(600 \mathrm{MHz}, \mathrm{CDCl}_{3}\right) \delta 8.41(\mathrm{~s}, 1 \mathrm{H}), 8.00(\mathrm{~s}, 1 \mathrm{H}), 7.13(\mathrm{dd}, J=17.4,10.8 \mathrm{~Hz}, 1 \mathrm{H})$, $6.01(\mathrm{~d}, J=17.4 \mathrm{~Hz}, 1 \mathrm{H}), 5.78(\mathrm{~d}, J=10.8 \mathrm{~Hz}, 1 \mathrm{H}) ;{ }^{13} \mathrm{C} \mathrm{NMR}\left(150 \mathrm{MHz}, \mathrm{CDCl}_{3}\right) \delta 149.0,143.5,139.7$, 134.5, 129.0, 126.7, 123.7, 120.8, $118.4\left(\mathrm{q}, J^{\mathrm{C}-\mathrm{F}}=213 \mathrm{~Hz}\right) ;{ }^{19} \mathrm{~F}$ NMR $\left(\mathrm{MHz}, \mathrm{CDCl}_{3}\right) \delta-72.9$; IR (ATR) 1553, 1437, 1342, 1213, $1130 \mathrm{~cm}^{-1}$; HRMS (ESI) calcd for $\mathrm{C}_{9} \mathrm{H}_{6} \mathrm{~F}_{3} \mathrm{~N}_{2} \mathrm{O}_{7} \mathrm{~S}\left(\mathrm{M}+\mathrm{H}^{+}\right) 342.9848$ $(\mathrm{M}+\mathrm{Na}=364.9667)$, found

2,5-Dinitro-4-(1-propen-1-yl)phenyltrifluoromethanesulfonate (308). Treatment of a solution of 266 (723 mg, $1.636 \mathrm{mmol})$ with 283 (763.2 $\mathrm{mg}, 2.305 \mathrm{mmol})$ in the presence of $\mathrm{PdCl}_{2}\left(\mathrm{PPh}_{3}\right)_{2}(23 \mathrm{mg}, 0.033$ $\mathrm{mmol})$, and $\mathrm{PPh}_{3}(17.2 \mathrm{mg}, 0.065 \mathrm{mmol})$ in dioxane $(7 \mathrm{~mL})$, as described for $285\left(90{ }^{\circ} \mathrm{C}, 24 \mathrm{~h}\right)$, gave, in order of elution, after solvent removal and chromatography (Hexane/EtOAc, 98:2) 293 (29.6 mg, 0.119 mmol, 7\%) as a yellow solid and $\mathbf{3 0 8}$ (406.9 mg, $1.142 \mathrm{mmol}, 70 \%)$ as yellow oil. ${ }^{1} \mathrm{H}$ NMR (400 MHz, $\left.\mathrm{CDCl}_{3}\right) \delta 8.34(\mathrm{~s}, 1 \mathrm{H}), 8.17(\mathrm{~s}, 1 \mathrm{H}), 8.06(\mathrm{~s}, 1 \mathrm{H}), 7.94(\mathrm{~s}, 1 \mathrm{H}), 6.84(\mathrm{dq}, J=15.6,1.6 \mathrm{~Hz}, 1 \mathrm{H}), 6.72-6.62$ $(\mathrm{m}, 1 \mathrm{H}), 6.52(\mathrm{dq}, J=15.6,6.8 \mathrm{~Hz}, 1 \mathrm{H}), 6.20(\mathrm{dq}, J=11.6,7.2 \mathrm{~Hz}, 1 \mathrm{H}), 2.03(\mathrm{dd}, J=6.4,1.6 \mathrm{~Hz}, 3 \mathrm{H})$, $1.83(\mathrm{dd}, J=7.2,1.8 \mathrm{~Hz}, 3 \mathrm{H}) ;{ }^{13} \mathrm{C} \mathrm{NMR}\left(100 \mathrm{MHz}, \mathrm{CDCl}_{3}\right) \delta 150.0,148.5,143.3,142.9,139.3,138.8$, 137.6, 134.6, 133.9, 133.6, 129.8, 126.4, 122.8, 122.4, 120.8, 120.7, $118.4\left(\mathrm{q}, J^{\mathrm{C}-\mathrm{F}}=319 \mathrm{~Hz}\right) ;{ }^{19} \mathrm{~F}$ NMR (376.10 MHz, $\left.\mathrm{CDl}_{3}\right) \delta$-72.6, -72.6; IR (ATR) 1532, 1336, 1202, $1123 \mathrm{~cm}^{-1}$; HRMS (ESI) calcd for $\mathrm{C}_{10} \mathrm{H}_{8} \mathrm{~F}_{3} \mathrm{~N}_{2} \mathrm{O}_{7} \mathrm{~S}\left(\mathrm{M}+\mathrm{H}^{+}\right) 357.0004(\mathrm{M}+\mathrm{Na}=378.9824)$, found 
2-Ethenyl-3,6-dinitrophenyltrifluoromethanesulfonate (309). Treatment of a solution of 281 (987.8 mg, $2.500 \mathrm{mmol})$ with $282(961.1 \mathrm{mg}, 3.031 \mathrm{mmol})$ in the presence of $\mathrm{PdCl}_{2}\left(\mathrm{PPh}_{3}\right)_{2}(35.1 \mathrm{mg}, 0.050$ $\mathrm{mmol}), \mathrm{PPh}_{3}(26.2 \mathrm{mg}, 0.100 \mathrm{mmol})$ and BHT $(66.1 \mathrm{mg}, 0.300 \mathrm{mmol})$ in dioxane $(7 \mathrm{~mL})$, as described for $285\left(98^{\circ} \mathrm{C}, 24 \mathrm{~h}\right)$, gave, in order of elution, after solvent removal and chromatography (Hexane/EtOAc, 95:5) 295 (55.6 mg, $0.253 \mathrm{mmol}, 10 \%)$ as a yellow oil and 309 (301.2 mg, $0.880 \mathrm{mmol}, 35 \%)$ as a faint yellow solid. mp=61-62 ${ }^{\circ} \mathrm{C} ;{ }^{1} \mathrm{H}$ NMR $\left(400 \mathrm{MHz}, \mathrm{CDCl}_{3}\right) \delta 8.11(\mathrm{~d}, J=8.8 \mathrm{~Hz}, 1 \mathrm{H}), 7.98(\mathrm{~d}, J=8.8 \mathrm{~Hz}$, $1 \mathrm{H}), 6.77(\mathrm{dd}, J=17.6,11.6 \mathrm{~Hz}, 1 \mathrm{H}), 5.90(\mathrm{~d}, J=11.6 \mathrm{~Hz}, 1 \mathrm{H}), 7.75(\mathrm{~d}, J=18.0 \mathrm{~Hz}, 1 \mathrm{H}) ;{ }^{13} \mathrm{C}$ NMR $(100$ $\left.\mathrm{MHz}, \mathrm{CDCl}_{3}\right) \delta 151.5,144.8,139.4,131.1,127.2,125.4,124.6,123.8,118.2\left(\mathrm{q}, J^{\mathrm{C}-\mathrm{F}}=319 \mathrm{~Hz}\right) ;{ }^{19} \mathrm{~F}$ NMR $\left(\mathrm{MHz}, \mathrm{CDCl}_{3}\right) \delta$-73.0; IR (ATR) 1548, 1341, 1207, 1129, $971 \mathrm{~cm}^{-1}$; HRMS (ESI) calcd for $\mathrm{C}_{9} \mathrm{H}_{6} \mathrm{~F}_{3} \mathrm{~N}_{2} \mathrm{O}_{7} \mathrm{~S}$ $\left(\mathrm{M}+\mathrm{H}^{+}\right) 342.9848(\mathrm{M}+\mathrm{Na}=364.9667)$, found

3,6-dinitro-2-(1-propen-1-yl)phenyltrifluoromethanesulfonate (310). Treatment of a solution of 281 (1.2523 g, $3.170 \mathrm{mmol}$ ) with 283 (1.2500 g, $3.775 \mathrm{mmol})$ in the presence of $\mathrm{PdCl}_{2}\left(\mathrm{PPh}_{3}\right)_{2}$ (44.5 mg, 0.063 $\mathrm{mmol}), \mathrm{PPh}_{3}(33.3 \mathrm{mg}, 0.127 \mathrm{mmol})$ and BHT $(83.8 \mathrm{mg}, 0.300 \mathrm{mmol})$ in dioxane $(10 \mathrm{~mL})$, as described for $285\left(100{ }^{\circ} \mathrm{C}, 25 \mathrm{~h}\right)$, gave, in order of elution, after solvent removal and chromatography (Hexane/EtOAc, 96:4) 296 (48.8 mg, $0.197 \mathrm{mmol}, 6 \%)$ and 310 (509.3 mg, $1.430 \mathrm{mmol}, 45 \%)$ both as a yellow oil. ${ }^{1} \mathrm{H}$ NMR $\left(400 \mathrm{MHz}, \mathrm{CDCl}_{3}\right) \delta 8.12(\mathrm{~d}, J=8.8 \mathrm{~Hz}, 1 \mathrm{H}), 8.04(\mathrm{~d}, J=8.8 \mathrm{~Hz}, 1 \mathrm{H}), 8.03(\mathrm{~d}, J=8.8$ $\mathrm{Hz}, 1 \mathrm{H}), 7.91$ (d, J=8.8 Hz, 1H), 6.44-6.39 (m, 2H), 6.30-6.19 (m, 2H), 1.97 (dd, J=6.8, 2.0 Hz, 3H), 1.63 $(\mathrm{dd}, J=6.8,1.6 \mathrm{~Hz}, 3 \mathrm{H}) ;{ }^{13} \mathrm{C} \mathrm{NMR}\left(100 \mathrm{MHz}, \mathrm{CDCl}_{3}\right) \delta 152.0,151.6,144.6,140.3,139.6,139.4,136.4$, 130.9, 129.9, 125.4, 124.7, 124.0, 123.6, 118.1 (q, JC-F=320 Hz), 118.1 (q, JC-F=319 Hz), 117.7, 117.2, 19.0, 15.1; ${ }^{19} \mathrm{~F}$ NMR $\left(\mathrm{MHz}, \mathrm{CDCl}_{3}\right) \delta$-73.4, -73.5; IR (ATR) 1557, 1423, 1347, 1203, $839 \mathrm{~cm}^{-1}$; HRMS (ESI) calcd for $\mathrm{C}_{10} \mathrm{H}_{8} \mathrm{~F}_{3} \mathrm{~N}_{2} \mathrm{O}_{7} \mathrm{~S}\left(\mathrm{M}+\mathrm{H}^{+}\right) 357.0004(\mathrm{M}+\mathrm{Na}=378.9824)$, found

3,6-Dinitro-2-(1-propen-2-yl)phenyltrifluoromethanesulfonate (311). Treatment of a solution of 281 (1.5178 g, $3.842 \mathrm{mmol}$ ) with 284 (1.5100 g, $4.560 \mathrm{mmol})$ in the presence of $\mathrm{PdCl}_{2}\left(\mathrm{PPh}_{3}\right)_{2}$ (53.9 $\mathrm{mg}, 0.077$ $\mathrm{mmol}), \mathrm{PPh}_{3}(40.3 \mathrm{mg}, 0.154 \mathrm{mmol})$ and $\mathrm{BHT}(101.6 \mathrm{mg}, 0.461 \mathrm{mmol})$ in dioxane (10 mL), as described for $285\left(100{ }^{\circ} \mathrm{C}, 25 \mathrm{~h}\right)$, gave, in order of elution, after solvent removal and chromatography (Hexane/EtOAc, 96:4) 297 (32.9 mg, $0.133 \mathrm{mmol}, 3 \%$ ) as a yellow oil and 311 (698 mg, $1.959 \mathrm{mmol}$, $51 \%$ ) as a white solid. $\mathrm{mp}=94-95{ }^{\circ} \mathrm{C} ;{ }^{1} \mathrm{H} \mathrm{NMR}\left(400 \mathrm{MHz}, \mathrm{CDCl}_{3}\right) \delta 8.08(\mathrm{~d}, J=8.8 \mathrm{~Hz}, 1 \mathrm{H}), 7.89$ (d, J=8.8 $\mathrm{Hz}, 1 \mathrm{H}), 5.34(\mathrm{~s}, 1 \mathrm{H}), 5.18(\mathrm{~s}, 1 \mathrm{H}), 2.19(\mathrm{~s}, 3 \mathrm{H}) ;{ }^{13} \mathrm{C} \mathrm{NMR}\left(100 \mathrm{MHz}, \mathrm{CDCl}_{3}\right) \delta 152.1,144.5,139.0$, 135.6, 134.5, 125.4, 123.5, 122.7, $118.1\left(\mathrm{q}, J^{\mathrm{C}-\mathrm{F}}=319 \mathrm{~Hz}\right), 22.4 ;{ }^{19} \mathrm{~F}$ NMR $\left(\mathrm{MHz}, \mathrm{CDCl}_{3}\right) \delta-72.8 ; \mathrm{IR}$ (ATR) 1548, 1419, 1336, 1197, $837 \mathrm{~cm}^{-1}$; HRMS (ESI) calcd for $\mathrm{C}_{10} \mathrm{H}_{8} \mathrm{~F}_{3} \mathrm{~N}_{2} \mathrm{O}_{7} \mathrm{~S}\left(\mathrm{M}+\mathrm{H}^{+}\right) 357.0004$ $(\mathrm{M}+\mathrm{Na}=378.9824)$, found 
3-Ethenyl-2,6-dinitrophenyltrifluoromethanesulfonate (312). Treatment of a solution of 273 (803.2 $\mathrm{mg}, 2.033 \mathrm{mmol})$ with $282(762 \mathrm{mg}, 2.403 \mathrm{mmol})$ in the presence of $\mathrm{PdCl}_{2}\left(\mathrm{PPh}_{3}\right)_{2}(28.5 \mathrm{mg}, 0.041$ $\mathrm{mmol}), \mathrm{PPh}_{3}(21.3 \mathrm{mg}, 0.081 \mathrm{mmol})$ and BHT $(44.7 \mathrm{mg}, 0.203 \mathrm{mmol})$ in dioxane $(6 \mathrm{~mL})$, as described for $282\left(98^{\circ} \mathrm{C}, 24 \mathrm{~h}\right)$, gave, in order of elution, after solvent removal and chromatography (Hexane/EtOAc, 9:1) an inseparable mixture of $\mathbf{2 9 8}$ and 3-bromo-2,6-dinitrobenzene (80.2 $\mathrm{mg}$ ) as a colorless oil and $\mathbf{3 1 2}$ (305.2 mg, $0.892 \mathrm{mmol}, 44 \%$ ) as white solid. $\mathrm{mp}=72-73{ }^{\circ} \mathrm{C} ;{ }^{1} \mathrm{H}$ NMR (400 MHz, $\left.\mathrm{CDCl}_{3}\right) \delta 8.26$ (d, $J=8.8$ $\mathrm{Hz}, 1 \mathrm{H}), 7.87$ (d, J=8.8 Hz, 1H), 6.66 (dd, J=17.2, $11.2 \mathrm{~Hz}, 1 \mathrm{H}), 6.10$ (d, J=17.2 Hz, 1H), 5.82 (d, $J=10.8$ $\mathrm{Hz}, 1 \mathrm{H}) ;{ }^{13} \mathrm{C} \mathrm{NMR}\left(100 \mathrm{MHz}, \mathrm{CDCl}_{3}\right) \delta 143.0,140.7,137.8,133.5,127.6,127.4,126.7,125.6,118.1$ (q, $\left.J^{\mathrm{C}-\mathrm{F}}=320 \mathrm{~Hz}\right) ;{ }^{19} \mathrm{~F}$ NMR $\left(376.10 \mathrm{MHz}, \mathrm{CDCl}_{3}\right) \delta-72.3$; IR (ATR) 1546, 1435, 1349, 1209, $1132 \mathrm{~cm}^{-1}$; HRMS (ESI) calcd for $\mathrm{C}_{9} \mathrm{H}_{6} \mathrm{~F}_{3} \mathrm{~N}_{2} \mathrm{O}_{7} \mathrm{~S}\left(\mathrm{M}+\mathrm{H}^{+}\right) 342.9848(\mathrm{M}+\mathrm{Na}=364.9667)$, found

2,6-Dinitro-3-(1-propen-2-yl)phenyltrifluoromethanesulfonate (313). Treatment of a solution of 273 $(1.126 \mathrm{~g}, 2.850 \mathrm{mmol})$ with $284(1.213 \mathrm{~g}, 3.663 \mathrm{mmol})$ in the presence of $\mathrm{PdCl}_{2}\left(\mathrm{PPh}_{3}\right)_{2}(40 \mathrm{mg}, 0.057$ $\mathrm{mmol}), \mathrm{PPh}_{3}(29.9 \mathrm{mg}, 0.114 \mathrm{mmol})$ and BHT $(62.8 \mathrm{mg}, 0.285 \mathrm{mmol})$ in dioxane $(7 \mathrm{~mL})$, as described for $285\left(100^{\circ} \mathrm{C}, 22 \mathrm{~h}\right)$, gave, in order of elution, after solvent removal and chromatography (Hexane/EtOAc, 96:4), an inseparable mixture of decoupling and C-OTf coupling products along with unknown impurities (125.3 mg) and $\mathbf{3 1 3}$ (459 mg, $1.288 \mathrm{mmol}, 45 \%)$ both as a yellow oil. Analytical data for 313: ${ }^{1} \mathrm{H}$ NMR $\left(400 \mathrm{MHz}, \mathrm{CDCl}_{3}\right) \delta 8.07(\mathrm{~d}, J=8.6 \mathrm{~Hz}, 1 \mathrm{H}), 7.44(\mathrm{~d}, J=8.6 \mathrm{~Hz}, 1 \mathrm{H}), 5.19(\mathrm{~s}, 1 \mathrm{H}), 4.95(\mathrm{~s}, 1 \mathrm{H}), 1.94(\mathrm{~s}$, $3 \mathrm{H}) ;{ }^{13} \mathrm{C}$ NMR $\left(100 \mathrm{MHz}, \mathrm{CDCl}_{3}\right) \delta 144.4,143.4,140.7,138.2,133.1,129.6,127.5,119.7,118.1\left(\mathrm{q}, J^{\mathrm{C}-}\right.$ F=319 Hz), 22.5; $\left.{ }^{19} \mathrm{~F} \mathrm{NMR} \mathrm{(376} \mathrm{MHz,} \mathrm{CDCl}_{3}\right) \delta-72.6$; IR (ATR) 1546, 1454, 1353, 1216, $1139 \mathrm{~cm}^{-1}$; HRMS (ESI) calcd for $\mathrm{C}_{10} \mathrm{H}_{8} \mathrm{~F}_{3} \mathrm{~N}_{2} \mathrm{O}_{7} \mathrm{~S}\left(\mathrm{M}+\mathrm{H}^{+}\right) 357.0004(\mathrm{M}+\mathrm{Na}=378.9824)$, found

4-Chloro-2,3-Dinitro-1-ethenylbenzene (314). Reaction of $\mathbf{3 0 0}$ (260 mg, $0.760 \mathrm{mmol})$ with 284 (317 $\mathrm{mg}, 0.957 \mathrm{mmol})$ in DMF (3 mL) in the presence of $\mathrm{PdCl}_{2}\left(\mathrm{PPh}_{3}\right)_{2}(10.6 \mathrm{mg}, 0.015 \mathrm{mmol})$, BHT (18.4 mg, $0.084 \mathrm{mmol}$ ), and $\mathrm{LiCl}(103.4 \mathrm{mg}, 2.440 \mathrm{mmol}$ ), as described for 292 (rt, $16 \mathrm{~h}$ ), gave, after work up and chromatography (Hexane/EtOAc, 95:5, then 9:1) 314 (109.3 mg, $0.478 \mathrm{mmol}, 63 \%$ ) as a white solid. $\mathrm{mp}=100-101{ }^{\circ} \mathrm{C} ; \quad{ }^{1} \mathrm{H}$ NMR $\left(400 \mathrm{MHz}, \mathrm{CDCl}_{3}\right) \delta 7.77(\mathrm{~d}, J=6.0 \mathrm{~Hz}, 1 \mathrm{H}), 7.68(\mathrm{~d}, J=6.0 \mathrm{~Hz}, 1 \mathrm{H}), 6.72$ (dd, $J=17.2,10.8 \mathrm{~Hz}, 1 \mathrm{H}), 5.93(\mathrm{~d}, J=17.2 \mathrm{~Hz}, 1 \mathrm{H}), 5.67(\mathrm{~d}, J=11.2 \mathrm{~Hz}, 1 \mathrm{H}) ;{ }^{13} \mathrm{C}$ NMR $(100 \mathrm{MHz}$, $\left.\mathrm{CDCl}_{3}\right) \delta 142.5,142.2,133.3,131.4,129.8,128.0,126.3,122.9$; IR (ATR) 1548, 1354, 947, $841 \mathrm{~cm}^{-1}$; HRMS (ESI) calcd for $\mathrm{C}_{8} \mathrm{H}_{6} \mathrm{ClN}_{2} \mathrm{O}_{4}\left(\mathrm{M}+\mathrm{H}^{+}\right) 229.0016(\mathrm{M}+\mathrm{Na}=250.9836)$, found

4-Chloro-2,5-Dinitro-1-ethenylbenzene (315). Reaction of 307 (158.3 mg, $0.463 \mathrm{mmol}$ ) with 284 (188.4 $\mathrm{mg}, 0.569 \mathrm{mmol})$ in DMF (2 mL) in the presence of $\mathrm{PdCl}_{2}\left(\mathrm{PPh}_{3}\right)_{2}(6.4 \mathrm{mg}, 0.009 \mathrm{mmol})$, BHT (12.2 mg, $0.056 \mathrm{mmol}$ ), and $\mathrm{LiCl}$ (57 mg, $1.345 \mathrm{mmol})$, as described for 292 ( $\mathrm{rt}, 21 \mathrm{~h}$ ), gave, after work up and 
chromatography (Hexane/EtOAc, 98:2) $315(56 \mathrm{mg}, 0.245 \mathrm{mmol}, 53 \%)$ as a white solid. $\mathrm{mp}=102-103{ }^{\circ} \mathrm{C}$; ${ }^{1} \mathrm{H}$ NMR (400 MHz, $\left.\mathrm{CDCl}_{3}\right) \delta 8.09$ (s, 2H), 7.08 (dd, $\left.J=17.2,10.8 \mathrm{~Hz}, 1 \mathrm{H}\right), 5.90$ (d, J=17.2 Hz, 1H), 5.69 $(\mathrm{d}, J=11.2 \mathrm{~Hz}, 1 \mathrm{H}) ;{ }^{13} \mathrm{C}$ NMR $\left(100 \mathrm{MHz}, \mathrm{CDCl}_{3}\right) \delta 149.9,148.4,133.1,129.5,127.8,126.4,125.1,122.4$; IR (ATR) 1547, 1524, 1347, 1274, $851 \mathrm{~cm}^{-1}$; HRMS (ESI) calcd for $\mathrm{C}_{8} \mathrm{H}_{6} \mathrm{ClN}_{2} \mathrm{O}_{4}\left(\mathrm{M}+\mathrm{H}^{+}\right) 229.0016$ $(\mathrm{M}+\mathrm{Na}=250.9836)$, found

1-Ethenyl-2,5-dinitro-4-(1-propen-2-yl)benzene (316) Treatment of a solution of 315 (95 mg, 0.416 mmol) with 284 (206 mg, $0.623 \mathrm{mmol})$ in the presence of $\mathrm{PdCl}_{2}\left(\mathrm{PPh}_{3}\right)_{2}(5.8 \mathrm{mg}, 0.008 \mathrm{mmol}), \mathrm{PPh}_{3}(8.7$ $\mathrm{mg}, 0.033 \mathrm{mmol})$, and BHT (11 mg, $0.050 \mathrm{mmol})$ in dioxane $(4 \mathrm{~mL})$, as described for $285\left(90{ }^{\circ} \mathrm{C}, 24 \mathrm{~h}\right)$, gave, after solvent removal and chromatography (Hexane/EtOAc, 98:2) 316 (76.9 mg, 0.328 mmol, 79\%) as a faint yellow solid. $\mathrm{mp}=89-90{ }^{\circ} \mathrm{C} ;{ }^{1} \mathrm{H} \mathrm{NMR}\left(400 \mathrm{MHz}, \mathrm{CDCl}_{3}\right) \delta 8.04(\mathrm{~s}, 1 \mathrm{H}), 7.86(\mathrm{~s}, 1 \mathrm{H}), 7.09$ (dd, $J=17.2,10.8 \mathrm{~Hz}, 1 \mathrm{H}), 5.88(\mathrm{~d}, J=17.2 \mathrm{~Hz}, 1 \mathrm{H}), 5.64(\mathrm{~d}, J=11.2 \mathrm{~Hz}, 1 \mathrm{H}), 5.29$ (s, with further fine splitting, 1H), 5.05 (s, with further fine splitting, $1 \mathrm{H}) ; 2.10(\mathrm{dd}, J=1.2,0.8 \mathrm{~Hz}, 3 \mathrm{H}) ;{ }^{13} \mathrm{C} \mathrm{NMR}(100 \mathrm{MHz}$, $\left.\mathrm{CDCl}_{3}\right) \delta 150.2,148.7,140.1,138.5,133.0,130.0,126.4,123.8,121.5,117.7,22.8$; IR (ATR) 1528, $1350,912,853 \mathrm{~cm}^{-1}$; HRMS (ESI) calcd for $\mathrm{C}_{11} \mathrm{H}_{11} \mathrm{~N}_{2} \mathrm{O}_{4}\left(\mathrm{M}+\mathrm{H}^{+}\right) 235.0719(\mathrm{M}+\mathrm{Na}=257.0537)$, found

1-Ethenyl-2,3-dinitro-4-(1-propen-1-yl)benzene (317). Treatment of a solution of $\mathbf{3 0 0}$ (203.5 mg, 0.595 mmol) with 283 (256 mg, $0.773 \mathrm{mmol})$ in the presence of $\mathrm{PdCl}_{2}\left(\mathrm{PPh}_{3}\right)_{2}(8.3 \mathrm{mg}, 0.012 \mathrm{mmol}), \mathrm{PPh}_{3}(6.2$ $\mathrm{mg}, 0.024 \mathrm{mmol})$, BHT (15.7 mg, $0.071 \mathrm{mmol})$ and $\mathrm{LiCl}(95.8 \mathrm{mg}, 2.260 \mathrm{mmol})$ in dioxane $(6 \mathrm{~mL})$, as described for $285\left(98^{\circ} \mathrm{C}, 30 \mathrm{~h}\right)$, gave after solvent removal and chromatography (Hexane/EtOAc, 85:15) 317 (91.2 $\mathrm{mg}, 0.389 \mathrm{mmol}, 65 \%)$ as a faint yellow solid. mp=95-97 ${ }^{\circ} \mathrm{C} ;{ }^{1} \mathrm{H} \mathrm{NMR}\left(400 \mathrm{MHz}, \mathrm{CDCl}_{3}\right) \delta$ $7.77(\mathrm{~d}, J=8.4 \mathrm{~Hz}, 1 \mathrm{H}), 7.71(\mathrm{~s}, 2 \mathrm{H}), 7.52$ (d, $J=8.0 \mathrm{~Hz}, 1 \mathrm{H}), 6.70$ (dd, $J=17.2,11.2 \mathrm{~Hz}, 1 \mathrm{H}), 6.68$ (dd, $J=17.2,11.2 \mathrm{~Hz}, 1 \mathrm{H}), 6.49-6.35$ (m, 3H), 6.07 (dq, $J=14.0,6.8 \mathrm{~Hz}, 1 \mathrm{H}), 5.93$ (d, J=10.4 Hz, 1H), 5.88 (d, $J=10.4 \mathrm{~Hz}, 1 \mathrm{H}), 5.61$ (d, J=10.4 Hz, 1H), 5.58 (d, $J=11.2 \mathrm{~Hz}, 1 \mathrm{H}), 1.93$ (dd, J=6.0, $0.8 \mathrm{~Hz}, 3 \mathrm{H}), 1.76$ (dd, $J=7.2,2.0 \mathrm{~Hz}, 3 \mathrm{H}) ;{ }^{13} \mathrm{C} \mathrm{NMR}\left(100 \mathrm{MHz}, \mathrm{CDCl}_{3}\right) \delta 142.7,142.0,141.9,141.4,135.2,133.6,133.2,131.6$, $131.4,130.7,130.2,129.1,128.8,128.7,128.4,128.4,122.3,121.9,121.8,121.5,18.9,14.6$; IR (ATR) 1533, 1436, 1347, 1216, 1128, $902 \mathrm{~cm}^{-1}$; HRMS (ESI) calcd for $\mathrm{C}_{11} \mathrm{H}_{11} \mathrm{~N}_{2} \mathrm{O}_{4}\left(\mathrm{M}+\mathrm{H}^{+}\right) 235.0719$ $(\mathrm{M}+\mathrm{Na}=257.0537)$, found

1-Ethenyl-2,3-dinitro-4-(1-propen-2-yl)benzene (318). Treatment of a solution of $\mathbf{3 0 0}$ (189.3 mg, 0.553 mmol) with $284(234.4 \mathrm{mg}, 0.708 \mathrm{mmol})$ in the presence of $\mathrm{PdCl}_{2}\left(\mathrm{PPh}_{3}\right)_{2}(7.8 \mathrm{mg}, 0.011 \mathrm{mmol}), \mathrm{PPh}_{3}$ (5.8 $\mathrm{mg}, 0.022 \mathrm{mmol})$, BHT (14.6 $\mathrm{mg}, 0.066 \mathrm{mmol})$ and $\mathrm{LiCl}(89.1 \mathrm{mg}, 2.102 \mathrm{mmol})$ in dioxane $(4.5$ $\mathrm{mL})$, as described for $285\left(100{ }^{\circ} \mathrm{C}, 28 \mathrm{~h}\right)$, gave after solvent removal and chromatography (Hexane/EtOAc, 9:1) 318 (83.2 mg, $0.355 \mathrm{mmol}, 64 \%)$ as a white solid. $\mathrm{mp}=99-100{ }^{\circ} \mathrm{C} ;{ }^{1} \mathrm{H}$ NMR (400 
$\left.\mathrm{MHz}, \mathrm{CDCl}_{3}\right) \delta 7.75(\mathrm{~d}, J=8.4 \mathrm{~Hz}, 1 \mathrm{H}), 7.48$ (d, J=8.4 Hz, 1H), 6.72 (dd, J=17.2, $\left.10.8 \mathrm{~Hz}, 1 \mathrm{H}\right), 5.91$ (d, $J=17.2 \mathrm{~Hz}, 1 \mathrm{H}), 5.62(\mathrm{~d}, J=11.2 \mathrm{~Hz}, 1 \mathrm{H}), 5.25$ (s, with further fine splitting, 1H), 5.02 (s, with further fine splitting, 1H), 2.09 (dd, $\left.J=1.6,1.2 \mathrm{~Hz}, 3 \mathrm{H}) ;{ }^{13} \mathrm{C} \mathrm{NMR} \mathrm{(100} \mathrm{MHz,} \mathrm{CDCl}_{3}\right) \delta 142.2,141.6,139.2,137.8$, 131.8, 131.0, 129.0, 128.4, 122.0, 118.1, 22.9IR (ATR) 1536, 1302, 1169, $803 \mathrm{~cm}^{-1}$; HRMS (ESI) calcd for $\mathrm{C}_{11} \mathrm{H}_{11} \mathrm{~N}_{2} \mathrm{O}_{4}\left(\mathrm{M}+\mathrm{H}^{+}\right) 235.0719(\mathrm{M}+\mathrm{Na}=257.0537)$, found

2,3-Dinitro-4-(1-propen-1-yl)-1-(1-propen-2-yl)benzene (319). Treatment of a solution of 302 (199.8 $\mathrm{mg}, 0.561 \mathrm{mmol})$ with $283(232.2 \mathrm{mg}, 0.701 \mathrm{mmol})$ in the presence of $\mathrm{PdCl}_{2}\left(\mathrm{PPh}_{3}\right)_{2}(7.8 \mathrm{mg}, 0.011$ $\mathrm{mmol})$, BHT (13.6 mg, $0.062 \mathrm{mmol})$, and $\mathrm{LiCl}(76.1 \mathrm{mg}, 1.795 \mathrm{mmol})$ in DMF (2.5 mL), as described for 292 (rt, 18 h), gave after work up and chromatography (Hexane/EtOAc, 95:5) 319 (63 mg, 0.254 mmol, $45 \%)$ as a white solid. $\mathrm{mp}=62-63{ }^{\circ} \mathrm{C} ;{ }^{1} \mathrm{H}$ NMR $\left(600 \mathrm{MHz}, \mathrm{CDCl}_{3}\right) \delta 7.67(\mathrm{~d}, J=7.8 \mathrm{~Hz}, 1 \mathrm{H}), 7.48(\mathrm{~d}$, $J=8.4 \mathrm{~Hz}, 1 \mathrm{H}), 7.45$ (d, $J=7.8 \mathrm{~Hz}, 1 \mathrm{H}), 7.40$ (d, $J=8.4 \mathrm{~Hz}, 1 \mathrm{H}), 6.42-6.38$ (m, 3H), 6.07 (dq, $J=14.4,7.2$ Hz, 1H), $5.26(\mathrm{~s}, 1 \mathrm{H}), 5.24$ (s, 1H), 5.03 (s, 1H), 5.01 (s, 1H), 2.11 (s, 3H), 2.09 (s, 3H), 1.94 (dd, J=4.8, $2.4 \mathrm{~Hz}, 3 \mathrm{H}), 1.77(\mathrm{dd}, J=7.2,1.8 \mathrm{~Hz}, 3 \mathrm{H}) ;{ }^{13} \mathrm{C} \mathrm{NMR}\left(150 \mathrm{MHz}, \mathrm{CDCl}_{3}\right) \delta 142.5,142.3,142.2,141.3$, 139.3, 139.3, 137.1, 136.8, 134.9, 133.4, 132.9, 131.5, 131.3, 131.3, 131.0, 128.9, 122.4, 121.9, 118.1, 117.9, 23.1, 23.0, 18.9, 14.6; IR (ATR) 1547, 1409, 1326, 1174, $837 \mathrm{~cm}^{-1}$; HRMS (ESI) calcd for $\mathrm{C}_{11} \mathrm{H}_{11} \mathrm{~N}_{2} \mathrm{O}_{4}\left(\mathrm{M}+\mathrm{H}^{+}\right) 235.0719(\mathrm{M}+\mathrm{Na}=257.0538)$, found

Ethyl 4-(ethenyl-2,3-dinitrophenyl)prop-2-enoate (320). Treatment of a solution of 300 (403.2 mg, $1.178 \mathrm{mmol})$ with ethyl 2-tributylstannyl-2-propenoate $(531.2 \mathrm{mg}, 1.365 \mathrm{mmol})$ in the presence of $\mathrm{PdCl}_{2}\left(\mathrm{PPh}_{3}\right)_{2}(16.5 \mathrm{mg}, 0.023 \mathrm{mmol}), \mathrm{PPh}_{3}(12.4 \mathrm{mg}, 0.047 \mathrm{mmol})$, BHT (31 mg, $\left.0.141 \mathrm{mmol}\right)$ and $\mathrm{LiCl}$ (179.8 $\mathrm{mg}, 4.242 \mathrm{mmol})$ in dioxane $(4 \mathrm{~mL})$, as described for $285\left(100{ }^{\circ} \mathrm{C}, 24 \mathrm{~h}\right)$, gave after solvent removal and chromatography (Hexane/EtOAc, 9:1) 320 (174.5 mg, $0.597 \mathrm{mmol}, 51 \%)$ as a colorless oil. ${ }^{1} \mathrm{H}$ NMR $\left(400 \mathrm{MHz}, \mathrm{CDCl}_{3}\right) \delta 7.84(\mathrm{~d}, J=8.40 \mathrm{~Hz}, 1 \mathrm{H}), 7.50$ (d, J=8.4 Hz, 1H), 6.67 (dd, J=17.2, $11.2 \mathrm{~Hz}$, 1H), $6.64(\mathrm{~s}, 1 \mathrm{H}), 5.98$ (s, 1H), 5.95 (d, J=17.2 Hz, 1H), 5.64 (d, J=11.2 Hz, 1H), 4.19 (quartet, J=7.2 Hz, 2H), $1.24(\mathrm{t}, J=7.2 \mathrm{~Hz}, 3 \mathrm{H}) ;{ }^{13} \mathrm{C} \mathrm{NMR}\left(100 \mathrm{MHz}, \mathrm{CDCl}_{3}\right) \delta 163.6,142.5,141.8,136.8,133.3,132.3$, 132.0, 130.6, 129.9, 127.9, 122.6, 61.9, 13.8; IR (ATR) 1703, 1523, 1363, 1156, $802 \mathrm{~cm}^{-1}$; HRMS (ESI) calcd for $\mathrm{C}_{13} \mathrm{H}_{13} \mathrm{~N}_{2} \mathrm{O}_{6}\left(\mathrm{M}+\mathrm{H}^{+}\right) 293.0774(\mathrm{M}+\mathrm{Na}=315.0593)$, found

Ethyl 2,3-dinitro-4-(1-propen-1-yl)phenylprop-2-enoate (321). Treatment of a solution of 301 (360.1 $\mathrm{mg}, 1.011 \mathrm{mmol})$ with ethyl 2-tributylstannyl-2-propenoate $(444.6 \mathrm{mg}, 1.142 \mathrm{mmol})$ in the presence of $\mathrm{PdCl}_{2}\left(\mathrm{PPh}_{3}\right)_{2}(14.2 \mathrm{mg}, 0.020 \mathrm{mmol}), \mathrm{PPh}_{3}(10.6 \mathrm{mg}, 0.040 \mathrm{mmol}), \mathrm{BHT}(22.1 \mathrm{mg}, 0.100 \mathrm{mmol})$ and $\mathrm{LiCl}$ $(150 \mathrm{mg}, 3.539 \mathrm{mmol})$ in dioxane $(4 \mathrm{~mL})$, as described for $285\left(100{ }^{\circ} \mathrm{C}, 24 \mathrm{~h}\right)$, gave after solvent removal and chromatography (Hexane/EtOAc, 9:1) 321 (157.9 mg, $0.516 \mathrm{mmol}, 51 \%$ ) as a faint yellow oil. ${ }^{1} \mathrm{H}$ 
NMR (600 MHz, $\left.\mathrm{CDCl}_{3}\right) \delta 7.75(\mathrm{~d}, J=8.4 \mathrm{~Hz}, 1 \mathrm{H}), 7.57(\mathrm{~d}, J=8.4 \mathrm{~Hz}, 1 \mathrm{H}), 7.48(\mathrm{~d}, J=8.4 \mathrm{~Hz}, 1 \mathrm{H})$, $7.43(\mathrm{~d}, J=8.4 \mathrm{~Hz}, 1 \mathrm{H}), 6.66(\mathrm{~s}, 1 \mathrm{H}), 6.63(\mathrm{~s}, 1 \mathrm{H}), 6.46(\mathrm{dq}, J=15.6,6.6 \mathrm{~Hz}, 1 \mathrm{H}), 6.40-6.35(\mathrm{~m}, 2 \mathrm{H})$, $6.11(\mathrm{dq}, J=11.6,7.2 \mathrm{~Hz}, 1 \mathrm{H}), 5.99(\mathrm{~s}, 1 \mathrm{H}), 5.95(\mathrm{~s}, 1 \mathrm{H}), 4.21(\mathrm{dq}, J=8.0,7.2 \mathrm{~Hz}, 4 \mathrm{H}), 1.95$ (dd, $J=6.6$, $1.2 \mathrm{~Hz}, 4 \mathrm{H}), 1.79(\mathrm{dd}, J=7.2,1.8 \mathrm{~Hz}, 3 \mathrm{H}), 1.26(\mathrm{t}, J=7.2 \mathrm{~Hz}, 3 \mathrm{H}), 1.25(\mathrm{t}, J=7.2 \mathrm{~Hz}, 3 \mathrm{H}) ;{ }^{13} \mathrm{C}$ NMR $(150$ $\left.\mathrm{MHz} \mathrm{CDCl}_{3}\right) \delta 163.7,143.5,142.1,141.9,141.9,137.0,137.0,135.6,134.1,133.8,133.0,132.7,132.4$, 132.1, 131.6, 131.2, 130.5, 130.3, 129.8, 122.1, 121.5, 62.0, 62.0, 19.0, 14.6, 13.9, 13.9; IR (ATR) 1708, 1547, 1355, 1207, $1097 \mathrm{~cm}^{-1}$; HRMS (ESI) calcd for $\mathrm{C}_{14} \mathrm{H}_{15} \mathrm{~N}_{2} \mathrm{O}_{6}\left(\mathrm{M}+\mathrm{H}^{+}\right) 307.0930(\mathrm{M}+\mathrm{Na}=329.0750)$, found

Ethyl 2,3-dinitro-4-(1-propen-1-yl)phenylprop-2-enoate (321). Treatment of a solution of 303 (100.3 $\mathrm{mg}, 0.242 \mathrm{mmol}$ ) with 283 (104 $\mathrm{mg}, 0.314 \mathrm{mmol})$ in the presence of $\mathrm{PdCl}_{2}\left(\mathrm{PPh}_{3}\right)_{2}(3.4 \mathrm{mg}, 0.005 \mathrm{mmol})$, $\mathrm{PPh}_{3}(2.5 \mathrm{mg}, 0.010 \mathrm{mmol})$, BHT $(5.3 \mathrm{mg}, 0.024 \mathrm{mmol})$, and $\mathrm{LiCl}(36.9 \mathrm{mg}, 0.871 \mathrm{mmol})$ in dioxane (2 $\mathrm{mL})$, as described for $285\left(100{ }^{\circ} \mathrm{C}, 26 \mathrm{~h}\right)$, gave after solvent removal and chromatography (Hexane/EtOAc, 7:3) 321 (32.1 mg, $0.105 \mathrm{mmol}, 43 \%$ ) as a faint yellow oil.

Ethyl 4-(1-propen-2-yl-2,3-dinitrophenyl)prop-2-enoate (322). Treatment of a solution of 302 (465.5 $\mathrm{mg}, 1.307 \mathrm{mmol})$ with ethyl 2-tributylstannyl-2-propenoate $(661 \mathrm{mg}, 1.698 \mathrm{mmol})$ in the presence of $\mathrm{PdCl}_{2}\left(\mathrm{PPh}_{3}\right)_{2}(18.3 \mathrm{mg}, 0.026 \mathrm{mmol}), \mathrm{PPh}_{3}(13.7 \mathrm{mg}, 0.052 \mathrm{mmol})$, BHT (34.5 mg, $\left.0.157 \mathrm{mmol}\right)$, and $\mathrm{LiCl}$ (199.4 mg, $4.704 \mathrm{mmol})$ in dioxane $(4 \mathrm{~mL})$, as described for $285\left(100{ }^{\circ} \mathrm{C}, 24 \mathrm{~h}\right)$, gave after solvent removal and chromatography (Hexane/DCM, 6:4) $322(218.2 \mathrm{mg}, 1.058 \mathrm{mmol}, 55 \%)$ as a colorless oil. ${ }^{1} \mathrm{H}$ NMR (400 MHz, $\left.\mathrm{CDCl}_{3}\right) \delta 7.53(\mathrm{~d}, J=8.0 \mathrm{~Hz}, 1 \mathrm{H}), 7.48(\mathrm{~d}, J=8.0 \mathrm{~Hz}, 1 \mathrm{H}), 6.63(\mathrm{~s}, 1 \mathrm{H}), 5.97(\mathrm{~s}, 1 \mathrm{H}), 5.27$ (s, with further fine splitting, $1 \mathrm{H}$ ), 5.02 (s, with further fine splitting, $1 \mathrm{H}$ ), 4.20 (quartet, $J=7.2 \mathrm{~Hz}, 2 \mathrm{H}$ ), 2.09 (s, 3H), 1.25 (t, J=7.2 Hz, 3H); ${ }^{13} \mathrm{C}$ NMR (100 MHz, $\left.\mathrm{CDCl}_{3}\right) \delta 163.6,142.7,141.5,138.8,138.4$, 136.8, 133.1, 132.5, 131.8, 130.6, 118.3, 61.9, 23.1, 13.8; IR (ATR) 1650, 1534, 1372, 1169, $802 \mathrm{~cm}^{-1}$; HRMS (ESI) calcd for $\mathrm{C}_{14} \mathrm{H}_{15} \mathrm{~N}_{2} \mathrm{O}_{6}\left(\mathrm{M}+\mathrm{H}^{+}\right) 307.0930(\mathrm{M}+\mathrm{Na}=329.0750)$, found

1-Ethenyl-2,4-dinitro-5-(1-propen-2-yl)benzene (323). Treatment of a solution of 205 (310.3 mg, 0.907 mmol) with $284(381.3 \mathrm{mg}, 1.152 \mathrm{mmol})$ in the presence of $\mathrm{PdCl}_{2}\left(\mathrm{PPh}_{3}\right)_{2}(12.7 \mathrm{mg}, 0.018 \mathrm{mmol}), \mathrm{PPh}_{3}$ $(9.5 \mathrm{mg}, 0.036 \mathrm{mmol}), \mathrm{BHT}(23.9 \mathrm{mg}, 0.108 \mathrm{mmol})$ and $\mathrm{LiCl}(130.7 \mathrm{mg}, 3.084 \mathrm{mmol})$ in dioxane $(6 \mathrm{~mL})$, as described for $\mathbf{2 8 5}\left(90^{\circ} \mathrm{C}, 24 \mathrm{~h}\right)$, gave after solvent removal and chromatography (Hexane/EtOAc, 98:2) $323(135.2 \mathrm{mg}, 0.577 \mathrm{mmol}, 64 \%)$ as a faint yellow oil. ${ }^{1} \mathrm{H}$ NMR (600 MHz, $\left.\mathrm{CDCl}_{3}\right) \delta 8.53(\mathrm{~s}, 1 \mathrm{H}), 7.58$ (s, 1H), 7.23 (dd, $J=17.4,11.4 \mathrm{~Hz}, 1 \mathrm{H}), 5.89$ (d, $J=17.4 \mathrm{~Hz}, 1 \mathrm{H}), 5.67$ (d, $J=11.4 \mathrm{~Hz}, 1 \mathrm{H}$ ), 5.29 (s, with further fine splitting, 1H), 5.03 (s, with further fine splitting, 1H), 2.11 (s, with further fine splitting, 3H); ${ }^{13} \mathrm{C} \mathrm{NMR}\left(150 \mathrm{MHz}, \mathrm{CDCl}_{3}\right) \delta 146.4,145.6,143.7,141.3,137.5,131.0,131.0,122.4,121.2,117.0,22.9$; 
IR (ATR) 1582, 1522, 1344, 1265, 911, $733 \mathrm{~cm}^{-1}$; HRMS (ESI) calcd for $\mathrm{C}_{11} \mathrm{H}_{11} \mathrm{~N}_{2} \mathrm{O}_{4}\left(\mathrm{M}+\mathrm{H}^{+}\right) 235.0719$ $(\mathrm{M}+\mathrm{Na}=257.0538)$, found

2,4-Dinitro-5-(1-propen-1-yl)-1-(1-propen-2-yl)benzene (324). Treatment of a solution of 305 (404.4 $\mathrm{mg}, 1.135 \mathrm{mmol})$ with $284(473.6 \mathrm{mg}, 1.430 \mathrm{mmol})$ in the presence of $\mathrm{PdCl}_{2}\left(\mathrm{PPh}_{3}\right)_{2}(15.9 \mathrm{mg}, 0.023$ $\mathrm{mmol}), \mathrm{PPh}_{3}(11.9 \mathrm{mg}, 0.045 \mathrm{mmol}), \mathrm{BHT}(25 \mathrm{mg}, 0.114 \mathrm{mmol})$ and $\mathrm{LiCl}(154 \mathrm{mg}, 3.633 \mathrm{mmol})$ in dioxane $(8 \mathrm{~mL})$, as described for $285\left(80{ }^{\circ} \mathrm{C}, 24 \mathrm{~h}\right)$, gave after solvent removal and chromatography (Hexane/EtOAc, 97:3) 324 (203.2 mg, $0.819 \mathrm{mmol}, 72 \%)$ as a faint yellow oil. ${ }^{1} \mathrm{H}$ NMR (600 MHz, $\left.\mathrm{CDCl}_{3}\right) \delta 8.57(\mathrm{~s}, 1 \mathrm{H}), 8.48(\mathrm{~s}, 1 \mathrm{H}), 7.54(\mathrm{~s}, 1 \mathrm{H}), 7.36(\mathrm{~s}, 1 \mathrm{H}), 6.91(\mathrm{dq}, J=15.6,1.8 \mathrm{~Hz}, 1 \mathrm{H}), 6.73(\mathrm{dq}$, $J=11.4,1.8 \mathrm{~Hz}, 1 \mathrm{H}), 6.45$ (dq, $J=15.6,6.6 \mathrm{~Hz}, 1 \mathrm{H}), 6.08$ (dq, $J=11.6,7.2 \mathrm{~Hz}, 1 \mathrm{H}), 5.27$ (s, with further fine splitting, 1H), 5.25 (s, with further fine splitting, $1 \mathrm{H}), 5.01$ (s, with further fine splitting, $1 \mathrm{H}), 4.99(\mathrm{~s}$, with further fine splitting, 1H), $2.10(\mathrm{~s}, 3 \mathrm{H}), 2.09$ (s, 3H), 1.99 (dd, J=3.0, $1.2 \mathrm{~Hz}, 3 \mathrm{H}), 1.78$ (dd, J=7.2, $1.8 \mathrm{~Hz}, 3 \mathrm{H}) ;{ }^{13} \mathrm{C} \mathrm{NMR}\left(100 \mathrm{MHz}, \mathrm{CDCl}_{3}\right) \delta 146.2,145.8,145.4,145.2,143.4,143.0,141.6,141.2,137.6$, 137.0, 136.0, 134.3, 131.7, 130.7, 124.7, 124.4, 121.2, 121.1, 116.9, 116.6, 22.9, 22.8, 19.0, 14.5; IR (ATR) 1579, 1517, 1339, 909, $832 \mathrm{~cm}^{-1}$; HRMS (ESI) calcd for $\mathrm{C}_{12} \mathrm{H}_{13} \mathrm{~N}_{2} \mathrm{O}_{4}\left(\mathrm{M}+\mathrm{H}^{+}\right) 249.0875$ $(\mathrm{M}+\mathrm{Na}=271.0695)$, found

1-Ethenyl-2,5-dinitro-4-(1-propen-1-yl)benzene (325). Treatment of a solution of $\mathbf{3 0 7}$ (302.7 mg, 0.885 mmol) 283 (369.5 mg, $1.116 \mathrm{mmol}$ ) in the presence of $\mathrm{PdCl}_{2}\left(\mathrm{PPh}_{3}\right)_{2}(12.4 \mathrm{mg}, 0.018 \mathrm{mmol}), \mathrm{PPh}_{3}(9.3$ $\mathrm{mg}, 0.035 \mathrm{mmol})$, BHT (23.4 mg, $0.106 \mathrm{mmol})$ and $\mathrm{LiCl}(142.6 \mathrm{mg}, 3.364 \mathrm{mmol})$ in dioxane (4 mL), as described for $285\left(100^{\circ} \mathrm{C}, 30 \mathrm{~h}\right)$, gave after solvent removal and chromatography (Hexane/DCM, 9:1) 325 (132.6 mg, $0.566 \mathrm{mmol}, 64 \%$ ) as a yellow solid. $\mathrm{mp}=111-113{ }^{\circ} \mathrm{C} ;{ }^{1} \mathrm{H} \mathrm{NMR}\left(400 \mathrm{MHz}, \mathrm{CDCl}_{3}\right) \delta 8.19$ (s, 1H), 8.08 (s, 1H), 8.06 (s, 1H), 7.90 (s, 1H), 7.10 (dd, J=17.2, $10.8 \mathrm{~Hz}, 1 \mathrm{H}), 7.07$ (dd, J=17.2, $10.8 \mathrm{~Hz}$, 1H), 6.77 (dq, J=15.6, $1.2 \mathrm{~Hz}, 1 \mathrm{H}), 6.64$ (dq, J=11.6, $1.6 \mathrm{~Hz}, 1 \mathrm{H}), 6.43$ (dq, J=15.2, $6.8 \mathrm{~Hz}, 1 \mathrm{H}), 6.08$ (dq, J=14.4, 7.2 Hz, 1H), 5.90 (d, J=16.8 Hz, 1H), 5.86 (d, J=17.2 Hz, 1H), 5.65 (d, J=10.8, 5.61 (d, $J=11.2 \mathrm{~Hz}, 1 \mathrm{H}), 1.98(\mathrm{dd}, J=6.8,1.6 \mathrm{~Hz}, 3 \mathrm{H}), 1.80(\mathrm{dd}, J=7.2,2.0 \mathrm{~Hz}, 3 \mathrm{H}) ;{ }^{13} \mathrm{C} \mathrm{NMR}\left(100 \mathrm{MHz}, \mathrm{CDCl}_{3}\right)$ $\delta 150.1,149.2$, 149.0, 148.7, 134.8, 133.0, 132.4, 132.3, 132.0, 131.8, 130.0, 127.6, 124.2, 124.1, 124.0, 123.7, 123.4, 121.4, 121.0, 18.9, 14.5; IR (ATR) 1538, 1520, 1351, 1275, $938 \mathrm{~cm}^{-1}$; HRMS (ESI) calcd for $\mathrm{C}_{11} \mathrm{H}_{11} \mathrm{~N}_{2} \mathrm{O}_{4}\left(\mathrm{M}+\mathrm{H}^{+}\right) 235.0719(\mathrm{M}+\mathrm{Na}=257.0538)$, found

(325). Treatment of a solution of $\mathbf{3 0 7}(325.9 \mathrm{mg}, 0.952 \mathrm{mmol})$ with $\mathbf{2 8 3}$ (387.8 $\mathrm{mg}, 1.171 \mathrm{mmol})$ in the presence of $\mathrm{PdCl}_{2}\left(\mathrm{PPh}_{3}\right)_{2}(13.4 \mathrm{mg}, 0.019 \mathrm{mmol})$, BHT (23 mg, $\left.0.105 \mathrm{mmol}\right)$ and $\mathrm{LiCl}(129.1 \mathrm{mg}, 3.046$ mmol) in DMF (4 mL), as described for 292 (rt, $21 \mathrm{~h}$ ), gave after work up and chromatography 
(Hexane/EtOAc, 98:2) 325 (37 mg, $0.158 \mathrm{mmol}, 17 \%)$ as a yellow solid and $\mathbf{3 1 5}$ (13.4 mg, $0.059 \mathrm{mmol}$, $6 \%)$ as a white solid.

2,5-Dinitro-4-(1-propen-1-yl)-1-(1-propen-2-yl)benzene (326). Treatment of a solution of 308 (362.2 $\mathrm{mg}, 1.017 \mathrm{mmol})$ with $284(424.2 \mathrm{mg}, 1.281 \mathrm{mmol})$ in the presence of $\mathrm{PdCl}_{2}\left(\mathrm{PPh}_{3}\right)_{2}(14.3 \mathrm{mg}, 0.020$ $\mathrm{mmol})$, BHT (26.9 mg, $0.122 \mathrm{mmol})$ and $\mathrm{LiCl}(176.8 \mathrm{mg}, 4.171 \mathrm{mmol})$ in DMF (7 mL), as described for 292 (rt, $21 \mathrm{~h}$ ), gave after work up and chromatography (Hexane/EtOAc, 98:2) 326 (66.3 mg, $0.267 \mathrm{mmol}$, $26 \%$ ) as a yellow oil. ${ }^{1} \mathrm{H}$ NMR $\left(600 \mathrm{MHz}, \mathrm{CDCl}_{3}\right) \delta 7.99(\mathrm{~s}, 1 \mathrm{H}), 7.90(\mathrm{~s}, 1 \mathrm{H}), 7.80(\mathrm{~s}, 1 \mathrm{H}), 7.78(\mathrm{~s}, 1 \mathrm{H})$, $6.76(\mathrm{dq}, \mathrm{J}=15.6,1.2 \mathrm{~Hz}, 1 \mathrm{H}), 6.62$ (dq, J=11.4, $1.2 \mathrm{~Hz}, 1 \mathrm{H}), 6.41$ (dq, J=15.6, $2.4 \mathrm{~Hz}, 1 \mathrm{H}), 6.07$ (dq, $\mathrm{J}=14.4,7.2 \mathrm{~Hz}, 1 \mathrm{H}), 5.28$ (s, with further splitting, 1H), 5.26 (s, with further splitting, 1H), 5.06 (s, with further splitting, 1H), 5.03 (s, with further splitting, 1H), 2.11 (s, with further splitting, 3H), 2.08 (s, with further splitting, 3H), $1.97(\mathrm{dd}, \mathrm{J}=6.6,1.8 \mathrm{~Hz}, 3 \mathrm{H}), 1.80(\mathrm{dd}, \mathrm{J}=7.2,1.8 \mathrm{~Hz}, 3 \mathrm{H}) ;{ }^{13} \mathrm{C} \mathrm{NMR}(100 \mathrm{MHz}$, $\left.\mathrm{CDCl}_{3}\right) \delta 150.0,149.5,148.4,140.2,137.9,137.4,134.7,133.0,132.3,131.8,127.1,126.4,126.3,123.7$, 123.6, 123.4, 117.6, 117.4, 22.8, 22.8, 18.9, 14.4; IR (ATR) 1540, 1345, 1265, $733 \mathrm{~cm}^{-1}$; HRMS (ESI) calcd for $\mathrm{C}_{12} \mathrm{H}_{13} \mathrm{~N}_{2} \mathrm{O}_{4}\left(\mathrm{M}+\mathrm{H}^{+}\right) 249.0875(\mathrm{M}+\mathrm{Na}=271.0695)$, found

1-Ethenyl-3,6-dinitro-2-(1-propen-1-yl)benzene (327). Treatment of a solution of $\mathbf{3 0 9}$ (301 $\mathrm{mg}, 0.880$ mmol) with $283(363.7 \mathrm{mg}, 1.098 \mathrm{mmol})$ in the presence of $\mathrm{PdCl}_{2}\left(\mathrm{PPh}_{3}\right)_{2}(12.9 \mathrm{mg}, 0.018 \mathrm{mmol}), \mathrm{PPh}_{3}$ (9.2 mg, $0.035 \mathrm{mmol})$, BHT (22.3 mg, $0.101 \mathrm{mmol})$ and $\mathrm{LiCl}(135.6 \mathrm{mg}, 3.199 \mathrm{mmol})$ in dioxane (3 mL), as described for $285\left(100{ }^{\circ} \mathrm{C}, 30 \mathrm{~h}\right)$, gave after solvent removal and chromatography (Hexane/EtOAc, 97:3) 327 (130 mg, $0.555 \mathrm{mmol}, 63 \%)$ as a yellow oil. ${ }^{1} \mathrm{H}$ NMR (400 MHz, $\left.\mathrm{CDCl}_{3}\right) \delta 7.78(\mathrm{~d}, \mathrm{~J}=8.8 \mathrm{~Hz}$, 1H), 7.70 (d, J=8.8 Hz, 1H), 7.63 (s, 2H), 6.69 (dd, J=17.6, 11.6 Hz, 1H), 6.67 (dd, J=17.6, 11.6 Hz, 1H), 6.38-6.34 (m, 2H), 5.98-5.80 (m, 2H), 5.57 (d, J=11.6 Hz, 1H), 5.50 (d, J=11.6 Hz, 1H), 5.38 (d, J=17.6 $\mathrm{Hz}, 2 \mathrm{H}), 1.85(\mathrm{dd}, \mathrm{J}=6.8,1.6 \mathrm{~Hz}, 3 \mathrm{H}), 1.42(\mathrm{dd}, \mathrm{J}=6.8,1.6 \mathrm{~Hz}, 3 \mathrm{H}) ;{ }^{13} \mathrm{C} \mathrm{NMR}\left(100 \mathrm{MHz}, \mathrm{CDCl}_{3}\right) \delta$ 151.3, 151.2, 151.0, 135.6, 134.1, 133.5, 133.3, 133.1, 131.7, 129.8, 129.4, 122.8, 122.8, 122.7, 122.7, 122.6, 122.3, 122.0, 18.9, 14.6; IR (ATR) 1531, 1351, 906, $726 \mathrm{~cm}^{-1}$; HRMS (ESI) calcd for $\mathrm{C}_{11} \mathrm{H}_{11} \mathrm{~N}_{2} \mathrm{O}_{4}$ $\left(\mathrm{M}+\mathrm{H}^{+}\right) 235.0719(\mathrm{M}+\mathrm{Na}=257.0538)$, found

1-Ethenyl-3,6-dinitro-2-(1-propen-2-yl)benzene (328). Treatment of a solution of $\mathbf{3 0 9}(211.8 \mathrm{mg}, 0.619$ mmol) with $284(276.7 \mathrm{mg}, 0.836 \mathrm{mmol})$ in the presence of $\mathrm{PdCl}_{2}\left(\mathrm{PPh}_{3}\right)_{2}(8.7 \mathrm{mg}, 0.012 \mathrm{mmol}), \mathrm{PPh}_{3}$ (6.5 mg, $0.025 \mathrm{mmol})$, BHT (16.4 mg, $0.074 \mathrm{mmol})$ and $\mathrm{LiCl}(95 \mathrm{mg}, 2.241 \mathrm{mmol})$ in dioxane (2.5 mL), as described for $285\left(100{ }^{\circ} \mathrm{C}, 30 \mathrm{~h}\right)$, gave after solvent removal and chromatography (Hexane/EtOAc, 95:5) 328 (93 mg, $0.397 \mathrm{mmol}, 64 \%)$ as a yellow oil. ${ }^{1} \mathrm{H}$ NMR (400 MHz, $\left.\mathrm{CDCl}_{3}\right) \delta 7.74(\mathrm{~d}, \mathrm{~J}=8.8 \mathrm{~Hz}$, 1H), 7.70 (d, J=8.8 Hz, 1H), 6.75 (dd, J=18.0, 11.6 Hz, 1H), 5.53 (d, J=11.6 Hz, 1H), 5.44 (d, J=18.0 Hz, 
1H), 5.26 (s, with further fine splitting, $1 \mathrm{H}), 4.90$ (s, with further fine splitting, 1H), 2.05 (s, with further fine splitting, 3H) $\left.{ }^{13} \mathrm{C} \mathrm{NMR} \mathrm{(100} \mathrm{MHz,} \mathrm{CDCl}_{3}\right) \delta 151.0,150.8,139.2,139.0,132.9,128.8,122.8,122.7$, 118.2, 23.2; IR (ATR) 1531, 1349, 804, $726 \mathrm{~cm}^{-1}$; HRMS (ESI) calcd for $\mathrm{C}_{11} \mathrm{H}_{11} \mathrm{~N}_{2} \mathrm{O}_{4}\left(\mathrm{M}+\mathrm{H}^{+}\right) 235.0719$ $(\mathrm{M}+\mathrm{Na}=257.0538)$, found

3,6-Dinitro-1-(1-propen-1-yl)-2-(1-propen-2-yl)benzene (329). Treatment of a solution of 311 (303.1 $\mathrm{mg}, 0.851 \mathrm{mmol})$ with $283(366.3 \mathrm{mg}, 1.106 \mathrm{mmol})$ in the presence of $\mathrm{PdCl}_{2}\left(\mathrm{PPh}_{3}\right)_{2}(11.9 \mathrm{mg}, 0.017$ $\mathrm{mmol}), \mathrm{PPh}_{3}(8.9 \mathrm{mg}, 0.034 \mathrm{mmol}), \mathrm{BHT}(22.5 \mathrm{mg}, 0.102 \mathrm{mmol})$ and $\mathrm{LiCl}(1299 \mathrm{mg}, 3.064 \mathrm{mmol})$ in dioxane $(3 \mathrm{~mL})$, as described for $285\left(100{ }^{\circ} \mathrm{C}, 30 \mathrm{~h}\right)$, gave after solvent removal and chromatography (Hexane/EtOAc, 97:3) 329 (106 mg, $0.427 \mathrm{mmol}, 50 \%)$ as a yellow oil. ${ }^{1} \mathrm{H}$ NMR (400 MHz, $\left.\mathrm{CDCl}_{3}\right) \delta$ 7.77 (s, 2H), 7.67 (d, J=8.8 Hz, 1H), 7.63 (d, J=8.8 Hz, 1H), 6.41-6.34 (m, 2H), 5.95-5.85 (m, 2H), 5.25$5.24(\mathrm{~m}, 1 \mathrm{H}), 5.18-5.16(\mathrm{~m}, 1 \mathrm{H}), 4.88-4.87(\mathrm{~m}, 1 \mathrm{H}), 4.82(\mathrm{~m}, 1 \mathrm{H}), 2.05-2.04(\mathrm{~m}, 6 \mathrm{H}), 1.83(\mathrm{dd}, \mathrm{J}=6.4,1.6$ $\mathrm{Hz}, 3 \mathrm{H}), 1.42(\mathrm{dd}, \mathrm{J}=6.8,1.6 \mathrm{~Hz}, 3 \mathrm{H}) ;{ }^{13} \mathrm{C} \mathrm{NMR}\left(100 \mathrm{MHz}, \mathrm{CDCl}_{3}\right) \delta 151.7,151.3,150.9,150.8$, $139.9,139.7,139.5,138.9,134.8,132.6,132.6,131.6,122.8,122.6,122.5,122.1,122.0,121.9,117.9$, 116.8, 23.0, 22.8, 18.9, 14.6; IR (ATR) 1537, 1435, 1341, 1216, 1332, $793 \mathrm{~cm}^{-1}$; HRMS (ESI) calcd for $\mathrm{C}_{12} \mathrm{H}_{13} \mathrm{~N}_{2} \mathrm{O}_{4}\left(\mathrm{M}+\mathrm{H}^{+}\right) 249.0875(\mathrm{M}+\mathrm{Na}=271.0695)$, found

3-Ethenyl-2,6-dinitro-1-(1-propen-2-yl)benzene (330). Treatment of a solution of 313 (415.8 mg, 0.1.167 mmol) with 282 (666.2 $\mathrm{mg}, 2.101 \mathrm{mmol})$ in the presence of $\mathrm{PdCl}_{2}\left(\mathrm{PPh}_{3}\right)_{2}$ (16.4 $\left.\mathrm{mg}, 0.023 \mathrm{mmol}\right)$, $\mathrm{PPh}_{3}(12.2 \mathrm{mg}, 0.047 \mathrm{mmol})$, BHT $(25.7 \mathrm{mg}, 0.117 \mathrm{mmol})$ and $\mathrm{LiCl}(178 \mathrm{mg}, 4.199 \mathrm{mmol})$ in dioxane (3.5 mL), as described for $285\left(100{ }^{\circ} \mathrm{C}, 24 \mathrm{~h}\right)$, gave after solvent removal and chromatography (Hexane/DCM, 95:5) 330 (122.7 mg, $0.524 \mathrm{mmol}, 45 \%)$ as a yellow oil. ${ }^{1} \mathrm{H}$ NMR (400 $\left.\mathrm{MHz}, \mathrm{CDCl}_{3}\right) \delta$ $8.05(\mathrm{~d}, J=8.4 \mathrm{~Hz}, 1 \mathrm{H}), 7.41(\mathrm{~d}, J=8.4 \mathrm{~Hz}, 1 \mathrm{H}), 6.90(\mathrm{dd}, J=17.8,11.6 \mathrm{~Hz}, 1 \mathrm{H}), 5.55$ (dd, $J=11.6,0.5$ $\mathrm{Hz}, 1 \mathrm{H}), 5.44(\mathrm{dd}, J=17.8,0.5 \mathrm{~Hz}, 1 \mathrm{H}), 5.31(\mathrm{~s}, 1 \mathrm{H}), 5.07(\mathrm{~s}, 1 \mathrm{H}), 2.10(\mathrm{~d}, J=0.4 \mathrm{~Hz}, 3 \mathrm{H}) ;{ }^{13} \mathrm{C}$ NMR $\left(100 \mathrm{MHz}, \mathrm{CDCl}_{3}\right) \delta 149.7,146.8,140.9,138.8,128.7,127.3,127.1,125.2,122.2,118.7,23.2$; IR (ATR) 1546, 1387, 1178, $837 \mathrm{~cm}^{-1}$; HRMS (ESI) calcd for $\mathrm{C}_{11} \mathrm{H}_{11} \mathrm{~N}_{2} \mathrm{O}_{4}\left(\mathrm{M}+\mathrm{H}^{+}\right) 235.0719(\mathrm{M}+\mathrm{Na}=257.0538)$, found

1-Ethenyl-2,6-dinitro-3-(1-propen-2-yl)benzene (331). Treatment of a solution of 312 (293.1 mg, 0.856 mmol) with 284 (357.2 $\mathrm{mg}, 1.079 \mathrm{mmol})$ in the presence of $\mathrm{PdCl}_{2}\left(\mathrm{PPh}_{3}\right)_{2}(12 \mathrm{mg}, 0.017 \mathrm{mmol}), \mathrm{PPh}_{3}(9$ $\mathrm{mg}, 0.034 \mathrm{mmol})$, BHT (18.8 mg, $0.086 \mathrm{mmol})$ and $\mathrm{LiCl}(130.7 \mathrm{mg}, 3.083 \mathrm{mmol})$ in dioxane $(4 \mathrm{~mL})$, as described for $285\left(100{ }^{\circ} \mathrm{C}, 24 \mathrm{~h}\right)$, gave after solvent removal and chromatography (Hexane/DCM, 85:15) 331 (54.8 mg, $0.234 \mathrm{mmol}, 27 \%)$ as a colorless oil. ${ }^{1} \mathrm{H}$ NMR $\left(400 \mathrm{MHz}, \mathrm{CDCl}_{3}\right) \delta 8.10(\mathrm{~d}, J=8.4 \mathrm{~Hz}, 1 \mathrm{H})$, $7.46(\mathrm{~d}, J=8.4 \mathrm{~Hz}, 1 \mathrm{H}), 6.60$ (dd, $J=17.2,11.2 \mathrm{~Hz}, 1 \mathrm{H}), 6.00$ (d, $J=17.2 \mathrm{~Hz}, 1 \mathrm{H}), 5.68$ (d, $J=11.2 \mathrm{~Hz}, 1 \mathrm{H})$, 
$2.11(\mathrm{dd}, J=1.6,1.2 \mathrm{~Hz}, 3 \mathrm{H}) ;{ }^{13} \mathrm{C}$ NMR $\left(100 \mathrm{MHz}, \mathrm{CDCl}_{3}\right) \delta 149.8,146.7,136.8,134.2,132.0,128.4$, 125.9, 125.8, 123.3, 118.7, 23.3; IR (ATR) 1546, 1397, 1179, $836 \mathrm{~cm}^{-1}$; HRMS (ESI) calcd for $\mathrm{C}_{11} \mathrm{H}_{11} \mathrm{~N}_{2} \mathrm{O}_{4}\left(\mathrm{M}+\mathrm{H}^{+}\right) 235.0719(\mathrm{M}+\mathrm{Na}=257.0538)$, found

1H,6 H-Pyrrolo[2,3-g]indole (336). A solution of 285 (35.2 $\mathrm{mg}, 0.160 \mathrm{mmol})$, palladium (II) acetate $\left(\mathrm{Pd}(\mathrm{OAc})_{2}, 2.9 \mathrm{mg}, 0.013 \mathrm{mmol}\right)$, and 1,10-phenanthroline $(2.4 \mathrm{mg}, 0.013 \mathrm{mmol})$ was prepared in anhydrous $N, N$-dimethylformamide (DMF, $2 \mathrm{~mL}$ ) in a threaded ACE glass pressure tube. The tube was fitted with a pressure head, the solution was saturated with carbon monoxide (four cycles of 6 atm of CO). The reaction mixture was stirred at $120^{\circ} \mathrm{C}$ for $93 \mathrm{~h}$. The solution was concentrated under reduced pressure at $80{ }^{\circ} \mathrm{C}$ and the resulting crude was purified by chromatography (Hexane/EtOAc, 7:3) to give 336 (17.5 $\mathrm{mg}, 0.112 \mathrm{mmol}, 70 \%$ ) as a white solid. $\mathrm{mp}=199-200{ }^{\circ} \mathrm{C}$ (decomposition); ${ }^{1} \mathrm{H} \mathrm{NMR}\left(400 \mathrm{MHz}, \mathrm{CDCl}_{3}\right) \delta$ 7.43 (s, 2H), 6.88 (br, s, 2H), 6.79 (t, J=2.8 Hz, 2H), 6.64 (dd, J=3.2, 2.0 Hz, 2H); ${ }^{13} \mathrm{C}$ NMR (100 MHz, $\left.\mathrm{CDCl}_{3}\right) \delta 123.1,121.9,121.7,113.8,103.2$; IR (ATR) 3353,1516, 1135, 1053, $754 \mathrm{~cm}^{-1}$; HRMS (ESI) calcd for $\mathrm{C}_{10} \mathrm{H}_{9} \mathrm{~N}_{2}\left(\mathrm{M}+\mathrm{H}^{+}\right)$157.0766 $(\mathrm{M}+\mathrm{Na}=179.0585)$, found

1H,6H-Pyrrolo[3,2-g]indole (336). Reaction of a solution of 285 (14.1 mg, $0.064 \mathrm{mmol})$, bis(dibenzylideneacetone)palladium $(0)\left(\mathrm{Pd}(\mathrm{dba})_{2}, 3.8 \mathrm{mg}, 0.007 \mathrm{mmol}\right)$, and triphenylphosphine $(6.8 \mathrm{mg}$, $0.026 \mathrm{mmol})$ in DMF $(1.2 \mathrm{~mL})$, as described for 336 above $\left(\mathrm{pCO}=6 \mathrm{~atm}, 120{ }^{\circ} \mathrm{C}, 126 \mathrm{~h}\right)$, gave after solvent removal and chromatography (Hexane/EtOAc, 7:3) 336 (6.4 mg, $0.041 \mathrm{mmol}, 64 \%)$ as a white solid.

6-Ethenyl-7-nitroindole (337). Reaction of a solution of 285 (27.8 mg, $0.126 \mathrm{mmol}), \mathrm{Pd}(\mathrm{OAc})_{2}(2.8 \mathrm{mg}$, $0.012 \mathrm{mmol}$ ), and 1,3-bis(diphenylphosphino)propane (dppp, $5.2 \mathrm{mg}, 0.013 \mathrm{mmol}$ ) in DMF (1.5 mL), as described for $336\left(\mathrm{pCO}=6 \mathrm{~atm}, 120{ }^{\circ} \mathrm{C}, 94 \mathrm{~h}\right)$, gave after solvent removal and chromatography (Hexane/EtOAc, 7:3) 337 (11.4 mg, $0.061 \mathrm{mmol}, 48 \%)$ as a yellow solid. mp=90-91 ${ }^{\circ} \mathrm{C} ; \quad{ }^{1} \mathrm{H}$ NMR (400 $\mathrm{MHz}_{\mathrm{CDCl}}$ ) $\delta 9.93$ (br, s, 1H), 7.87 (d, J=8.0 Hz, 1H), 7.56 (dd, J=17.2, $\left.11.2 \mathrm{~Hz}, 1 \mathrm{H}\right), 7.37$ (dd, $J=2.8$, $0.4 \mathrm{~Hz}, 1 \mathrm{H}), 7.33$ (d, J=8.4 Hz, 1H), 6.67 (dd, $J=3.2,2.0 \mathrm{~Hz}, 1 \mathrm{H}), 5.72$ (dd, J=17.2, $1.2 \mathrm{~Hz}, 1 \mathrm{H}$ ), 5.49 (dd, $J=10.8,0.8 \mathrm{~Hz}, 1 \mathrm{H}) ;{ }^{13} \mathrm{C}$ NMR $\left(100 \mathrm{MHz}, \mathrm{CDCl}_{3}\right) \delta 135.2,135.2,131.5,130.9,130.0,127.6,126.6$, 120.7, 117.9, 103.9; IR (ATR) 3407, 1330, 1265, $1092 \mathrm{~cm}^{-1}$; HRMS (ESI) calcd for $\mathrm{C}_{10} \mathrm{H}_{9} \mathrm{~N}_{2} \mathrm{O}_{2}\left(\mathrm{M}+\mathrm{H}^{+}\right)$ 189.0664 $(\mathrm{M}+\mathrm{Na}=211.0483)$, found 
2,5-Dimethyl-1H,6H-pyrrolo[2,3-g]indole (338). Reaction of a solution of 286 (45.5 mg, $0.183 \mathrm{mmol}$ ), $\mathrm{Pd}(\mathrm{OAc})_{2}(4.1 \mathrm{mg}, 0.018 \mathrm{mmol})$, and 1,10-phen $(3.3 \mathrm{mg}, 0.018 \mathrm{mmol})$ in DMF (1.5 mL), as described for $336\left(\mathrm{pCO}=6 \mathrm{~atm}, 120{ }^{\circ} \mathrm{C}, 144 \mathrm{~h}\right)$, gave after solvent removal and chromatography (Hexane/EtOAc, 7:3) 338 (22.6 mg, $0.119 \mathrm{mmol}, 67 \%)$ as a white solid. $\mathrm{mp}=209{ }^{\circ} \mathrm{C}$ (decomposition); ${ }^{1} \mathrm{H} \mathrm{NMR} \mathrm{(400} \mathrm{MHz,}$ $\left.\mathrm{CDCl}_{3}\right) \delta 7.24$ (s, 2H), $7.11(\mathrm{br}, \mathrm{s}, 2 \mathrm{H}), 6.25(\mathrm{dd}, J=2.0,1.2 \mathrm{~Hz}, 2 \mathrm{H}), 2.27(\mathrm{~s}, 3 \mathrm{H}) ;{ }^{13} \mathrm{C} \mathrm{NMR}(100 \mathrm{MHz}$, $\left.\mathrm{CDCl}_{3}\right) \delta 131.9,123.9,121.7,112.6,101.5,13.5$; IR (ATR) 3354, 1547, 1432, 1333, $1234 \mathrm{~cm}^{-1}$; HRMS (ESI) calcd for $\mathrm{C}_{12} \mathrm{H}_{13} \mathrm{~N}_{2}\left(\mathrm{M}+\mathrm{H}^{+}\right) 185.1079$, found

2-Methyl-7-nitro-6-(1-propen-1-yl)indole (339). Reaction of a solution of 286 (46.7 mg, $0.188 \mathrm{mmol}$ ), $\mathrm{Pd}(\mathrm{OAc})_{2}(4.2 \mathrm{mg}, 0.019 \mathrm{mmol})$, and dppp $(7.8 \mathrm{mg}, 0.019 \mathrm{mmol})$ in DMF $(2 \mathrm{~mL})$, as described for 336 $\left(\mathrm{pCO}=6 \mathrm{~atm}, 120{ }^{\circ} \mathrm{C}, 57 \mathrm{~h}\right)$, gave, in orde of elution, after solvent removal and chromatography (Hexane/EtOAc, 7:3) 339 (28.1 mg, $0.130 \mathrm{mmol}, 69 \%)$ as a yellow solid and 338 (3.1 $\mathrm{mg}, 0.017 \mathrm{mmol}$, $9 \%$ ) as a white solid. $\mathrm{mp}=116-117^{\circ} \mathrm{C} ; \quad{ }^{1} \mathrm{H}$ NMR (600 MHz, $\left.\mathrm{CDCl}_{3}\right) \delta 9.72$ (br, s, 1H), $9.63(\mathrm{br}, \mathrm{s}, 1 \mathrm{H})$, 7.70 (d, J=8.4 Hz, 1H), 7.66 (d, J=7.8 Hz, 1H ), 7.23 (d, J=18.6 Hz, 1H), 7.20 (d, J=8.4 Hz, 1H), 7.02 (d, $J=7.8 \mathrm{~Hz}, 1 \mathrm{H}), 6.96$ (d, $J=11.4 \mathrm{~Hz}, 1 \mathrm{H}), 6.33$ (d, J=1.2 Hz, 1H), 6.30 (d, J=1.2 Hz, 1H), 6.18 (dq, J=15.6, $6.6 \mathrm{~Hz}, 1 \mathrm{H}), 5.91$ (dq, J=14.4, 7.2 Hz, 1H), 2.52 (s, 3H), 2.50 (s, 3H), 1.98 (dd, J=6.6, $1.2 \mathrm{~Hz}, 3 \mathrm{H}), 1.75$ $(\mathrm{dd}, J=7.2,1.8 \mathrm{~Hz}, 3 \mathrm{H}) ;{ }^{13} \mathrm{C} \mathrm{NMR}\left(150 \mathrm{MHz}, \mathrm{CDCl}_{3}\right) \delta 137.7,137.4,131.4,131.3,131.3,130.5,130.4$, $130.3,129.8,129.1,128.9,128.8,126.5,126.2,125.7,123.2,120.5,101.5,101.4,18.9,14.5,13.7$; IR (ATR) 3399, 1507, 1333, 1217, $764 \mathrm{~cm}^{-1}$; HRMS (ESI) calcd for $\mathrm{C}_{12} \mathrm{H}_{13} \mathrm{~N}_{2} \mathrm{O}_{2}\left(\mathrm{M}+\mathrm{H}^{+}\right.$) 217.0977, found

2,5-Dimethyl-1H,6H-pyrrolo[3,2-g]indole (338). Reaction of a solution of 286 (42.7 $\mathrm{mg}, 0.172 \mathrm{mmol}$ ), $\mathrm{Pd}(\mathrm{OAc})_{2}(9.9 \mathrm{mg}, 0.017 \mathrm{mmol})$, and dppp (7.1 mg, $\left.0.017 \mathrm{mmol}\right)$ in DMF (1.5 mL), as described for 336 $\left(\mathrm{pCO}=6 \mathrm{~atm}, 120^{\circ} \mathrm{C}, 48 \mathrm{~h}\right.$ ), gave, after solvent removal and chromatography (Hexane/EtOAc, 85:15) 338 (11.4 mg, $0.062 \mathrm{mmol}, 36 \%)$ as a white solid.

3,6-Dimethyl-1H,6H-pyrrolo[2,3-g]indole (340). Reaction of a solution of 287 (29.2 $\mathrm{mg}, 0.118 \mathrm{mmol}$ ), $\mathrm{Pd}(\mathrm{OAc})_{2}(2.6 \mathrm{mg}, 0.012 \mathrm{mmol})$, and 1,10-phen $(2.6 \mathrm{mg}, 0.014 \mathrm{mmol})$ in DMF (1.5 mL), as described for $336\left(\mathrm{pCO}=6 \mathrm{~atm}, 120^{\circ} \mathrm{C}, 98 \mathrm{~h}\right.$ ), gave, after solvent removal and chromatography (Hexane/EtOAc, 7:3) 340 (11.7 $\mathrm{mg}, 0.061 \mathrm{mmol}, 54 \%)$ as a white solid. $\mathrm{mp}=245{ }^{\circ} \mathrm{C}$ (decomposition); ${ }^{1} \mathrm{H} \mathrm{NMR}$ (400 MHz, Acetone-d $\left.{ }_{6}\right) \delta 9.73(\mathrm{br}, \mathrm{s}, 2 \mathrm{H}), 7.19(\mathrm{~s}, 2 \mathrm{H}), 6.92(\mathrm{~m}, 2 \mathrm{H}), 2.32(\mathrm{~d}, J=1.2 \mathrm{~Hz}, 6 \mathrm{H}) ;{ }^{13} \mathrm{C} \mathrm{NMR}(100 \mathrm{MHz}$, Acetone-d ${ }_{6}$ ) $\delta 124.9,124.3,119.5,112.4,111.4,10.3$; IR (ATR) 3367, 1548, 1433, 1323, $1232 \mathrm{~cm}^{-1}$; HRMS (ESI) calcd for $\mathrm{C}_{12} \mathrm{H}_{13} \mathrm{~N}_{2}\left(\mathrm{M}+\mathrm{H}^{+}\right)$185.1079, found 
3,6-Dimethyl-1H,6H-pyrrolo[3,2-g]indole (340). Reaction of a solution of 287 (39.6 mg, $0.160 \mathrm{mmol})$, $\operatorname{Pd}(\mathrm{dba})_{2}(9.2 \mathrm{mg}, 0.016 \mathrm{mmol})$, dppp (6.6 mg, $\left.0.016 \mathrm{mmol}\right)$, and 1,10-phen (5.7 $\left.\mathrm{mg}, 0.032 \mathrm{mmol}\right)$ in DMF (1.5 mL), as described for $336\left(\mathrm{pCO}=6 \mathrm{~atm}, 120{ }^{\circ} \mathrm{C}, 110 \mathrm{~h}\right)$, gave, after solvent removal and chromatography (Hexane/EtOAc, 7:3) 340 (19.6 mg, $0.106 \mathrm{mmol}, 67 \%)$ as a white solid.

3,6-Dimethyl-1H,6H-pyrrolo[3,2-g]indole (340). Reaction of a solution of 287 (34.6 mg, $0.139 \mathrm{mmol}$ ), $\mathrm{Pd}(\mathrm{dba})_{2}(8 \mathrm{mg}, 0.014 \mathrm{mmol})$, and $\mathrm{PPh}_{3}(14.7 \mathrm{mg}, 0.056 \mathrm{mmol})$ in DMF (1.5 mL), as described for 336 $\left(\mathrm{pCO}=6 \mathrm{~atm}, 120^{\circ} \mathrm{C}, 60 \mathrm{~h}\right.$ ), gave, after solvent removal and chromatography (Hexane/EtOAc, 7:3) 340 (15.8mg, $0.086 \mathrm{mmol}, 62 \%)$ as a white solid.

3-Methyl-7-nitro-6-(1-propen-2-yl)indole (341). Reaction of a solution of 287 (21.3 $\mathrm{mg}, 0.086 \mathrm{mmol})$, $\mathrm{Pd}(\mathrm{OAc})_{2}(1.3 \mathrm{mg}, 0.006 \mathrm{mmol})$, and dppp (2.5 mg, $\left.0.006 \mathrm{mmol}\right)$ in DMF (1 mL), as described for 336 $\left(\mathrm{pCO}=6 \mathrm{~atm}, 120^{\circ} \mathrm{C}, 72 \mathrm{~h}\right)$, gave, in order of elution, after solvent removal and chromatography (Hexane/EtOAc, 9:1) 341 (14.5 mg, $0.067 \mathrm{mmol}, 78 \%$ ) as a yellow oil) and 340 (2.6 mg, $0.014 \mathrm{mmol}$, $16 \%$ ) as a white solid. Analytical data for $341: \mathrm{mp}=179{ }^{\circ} \mathrm{C}$ (decomposition); ${ }^{1} \mathrm{H} \mathrm{NMR}\left(400 \mathrm{MHz}, \mathrm{CDCl}_{3}\right.$ ) $\delta 9.62$ (br, s, 1H), $7.78(\mathrm{~d}, \mathrm{~J}=7.6 \mathrm{~Hz}, 1 \mathrm{H}), 7.12$ (s, with further fine splitting, 1H), $7.01(\mathrm{~d}, \mathrm{~J}=7.6 \mathrm{~Hz}, 1 \mathrm{H})$, 5.19 (s, with further fine splitting, 1H), 4.93 (s, with further fine splitting, 1H), 2.35 (s, with further fine splitting, 3H), 2.13 (s, with further fine splitting, 3H); $\left.{ }^{13} \mathrm{C} \mathrm{NMR} \mathrm{(100} \mathrm{MHz,} \mathrm{CDCl}_{3}\right) \delta 145.4,136.7,131.1$, 130.3, 125.5, 123.8, 121.7, 113.7, 112.9, 23.7, 9.4; IR (ATR) 3330, 1493, 1301, 1268, 1096, $894 \mathrm{~cm}^{-1}$; HRMS (ESI) calcd for $\mathrm{C}_{12} \mathrm{H}_{13} \mathrm{~N}_{2} \mathrm{O}_{2}\left(\mathrm{M}+\mathrm{H}^{+}\right)$217.0977, found

3,6-Dimethyl-1H,6H-pyrrolo[3,2-g]indole (342). Reaction of a solution of 317 (56 mg, $0.239 \mathrm{mmol}$ ), $\mathrm{Pd}(\mathrm{OAc})_{2}(4.3 \mathrm{mg}, 0.019 \mathrm{mmol})$, and 1,10-phen $(3.5 \mathrm{mg}, 0.019 \mathrm{mmol})$ in DMF (3 mL), as described for $336\left(\mathrm{pCO}=6 \mathrm{~atm}, 120^{\circ} \mathrm{C}, 100 \mathrm{~h}\right)$, gave, after solvent removal and chromatography (Hexane/EtOAc, 7:3) 342 (25.7 mg, $0.151 \mathrm{mmol}, 63 \%)$ as a white solid. $\mathrm{mp}=179{ }^{\circ} \mathrm{C}$ (decomposition); ${ }^{1} \mathrm{H} \mathrm{NMR}(400 \mathrm{MHz}$, $\left.\mathrm{CDCl}_{3}\right) \delta 7.38(\mathrm{~d}, J=8.4 \mathrm{~Hz}, 1 \mathrm{H}), 7.32(\mathrm{~d}, J=8.4 \mathrm{~Hz}, 1 \mathrm{H}), 6.67$ (br, s, 1H), 6.65-6.63 (m, 1H), 6.59-6.58 $(\mathrm{m}, 1 \mathrm{H}), 6.55$ (br, s, 1H), 6.32-6.31 (m, 1H), $2.33(\mathrm{~s}, 3 \mathrm{H}) ;{ }^{13} \mathrm{C} \mathrm{NMR}\left(100 \mathrm{MHz}, \mathrm{CDCl}_{3}\right) \delta 132.5,124.0$, 122.6, 121.7, 121.6, 121.5, 113.3, 113.1, 102.9, 101.3, 13.5; IR (ATR) 3356, 1532, 1473, 1302, $1272 \mathrm{~cm}^{-}$ ${ }^{1}$; HRMS (ESI) calcd for

3,6-Dimethyl-1H,6H-pyrrolo[3,2-g]indole (342). Reaction of a solution of 317 (31 $\mathrm{mg}, 0.132 \mathrm{mmol}$ ), $\mathrm{Pd}(\mathrm{OAc})_{2}(1.5 \mathrm{mg}, 0.007 \mathrm{mmol})$, and $\mathrm{PPh}_{3}(6.9 \mathrm{mg}, 0.026 \mathrm{mmol})$ in DMF (1.5 mL), as described for 336 $\left(\mathrm{pCO}=6 \mathrm{~atm}, 120^{\circ} \mathrm{C}, 31 \mathrm{~h}\right)$, gave, after solvent removal and chromatography (Hexane/EtOAc, 7:3), 342 (5.7 $\mathrm{mg}, 0.033 \mathrm{mmol}, 25 \%)$ as a white solid. 
3,6-Dimethyl-1H,6H-pyrrolo[3,2-g]indole (343). Reaction of a solution of $\mathbf{3 1 8}$ (52.3 $\mathrm{mg}, 0.223 \mathrm{mmol})$, $\mathrm{Pd}(\mathrm{OAc})_{2}(3 \mathrm{mg}, 0.013 \mathrm{mmol})$, and 1,10-phen (2.4 mg, $\left.0.013 \mathrm{mmol}\right)$ in DMF (3 mL), as described for 336 $\left(\mathrm{pCO}=6 \mathrm{~atm}, 120^{\circ} \mathrm{C}, 98 \mathrm{~h}\right.$ ), gave, after solvent removal and chromatography (Hexane/DCM, 3:7) 343 (24.5 mg, $0.144 \mathrm{mmol}, 65 \%$ ) as a white solid. $\mathrm{mp}=181{ }^{\circ} \mathrm{C}$ (decomposition); ${ }^{1} \mathrm{H} \mathrm{NMR}\left(400 \mathrm{MHz}, \mathrm{CDCl}_{3}\right)$ $\delta 7.43(\mathrm{~d}, J=8.4 \mathrm{~Hz}, 1 \mathrm{H}), 7.35$ (d, J=8.4 Hz, 1H), 6.74-6.73 (m, 1H), $6.63(\mathrm{~m}, 2 \mathrm{H}), 6.55(\mathrm{~s}, 1 \mathrm{H}), 6.45$ (br, $\mathrm{s}, 1 \mathrm{H}), 2.42(\mathrm{~s}, 3 \mathrm{H}) ;{ }^{13} \mathrm{C} \mathrm{NMR}\left(100 \mathrm{MHz}, \mathrm{CDCl}_{3}\right) \delta 123.4,123.1,121.9,121.9,121.7,119.4,113.1$, 112.3, 112.0, 103.0, 10.0; , IR (ATR) 3380, 1548, 1403, 1375, $1205 \mathrm{~cm}^{-1}$; HRMS (ESI) calcd for

3,6-Dimethyl-1H,6H-pyrrolo[3,2-g]indole (344). Reaction of a solution of 319 (41.3 mg, $0.166 \mathrm{mmol})$, $\mathrm{Pd}(\mathrm{OAc})_{2}(2.7 \mathrm{mg}, 0.012 \mathrm{mmol})$, and 1,10-phen $(2.1 \mathrm{mg}, 0.012 \mathrm{mmol})$ in DMF $(2 \mathrm{~mL})$, as described for $336\left(\mathrm{pCO}=6 \mathrm{~atm}, 120^{\circ} \mathrm{C}, 100 \mathrm{~h}\right.$ ), gave, after solvent removal and chromatography (Hexane/EtOAc, 8:2) 344 (20.6 mg, $0.112 \mathrm{mmol}, 67 \%$ ) as a white solid. $\mathrm{mp}=210{ }^{\circ} \mathrm{C}$ (decomposition); ${ }^{1} \mathrm{H}$ NMR (400 MHz, $\mathrm{CDCl}_{3} / \mathrm{DMSO}_{\mathrm{d}}$ ) $\delta 10.36(\mathrm{br}, \mathrm{s}, 1 \mathrm{H}), 9.92(\mathrm{br}, \mathrm{s}, 1 \mathrm{H}), 7.01(\mathrm{~s}, 2 \mathrm{H}), 6.87-6.86(\mathrm{~m}, 1 \mathrm{H}), 6.10-6.09(\mathrm{~m}, 1 \mathrm{H})$, $2.41(\mathrm{~d}, J=0.8 \mathrm{~Hz}, 3 \mathrm{H}), 2.25(\mathrm{~d}, J=0.8 \mathrm{~Hz}, 3 \mathrm{H}) ;{ }^{13} \mathrm{C} \mathrm{NMR}\left(100 \mathrm{MHz}, \mathrm{CDCl}_{3} / \mathrm{DMSO}_{-} \mathrm{d}_{6}\right) \delta 130.5,123.4$, 122.5, 122.5, 122.2, 118.3, 111.2, 110.5, 110.2, 100.3, 13.3, 9.9; IR (ATR) 3396, 1601, 1406, 1343, 1263 $\mathrm{cm}^{-1}$; HRMS (ESI) calcd for $\mathrm{C}_{12} \mathrm{H}_{13} \mathrm{~N}_{2}\left(\mathrm{M}+\mathrm{H}^{+}\right)$185.1979, found

3,6-Dimethyl-1H,6H-pyrrolo[3,2-g]indole (345). Reaction of a solution of 320 (79.8 mg, $0.273 \mathrm{mmol}$ ), $\mathrm{Pd}(\mathrm{OAc})_{2}(3.7 \mathrm{mg}, 0.016 \mathrm{mmol})$, and 1,10-phen (3 mg, $\left.0.017 \mathrm{mmol}\right)$ in DMF (3 mL), as described for 336 $\left(\mathrm{pCO}=6 \mathrm{~atm}, 120{ }^{\circ} \mathrm{C}, 109 \mathrm{~h}\right.$ ), gave, after solvent removal and chromatography (Hexane/EtOAc, 6:4) 345 (42.1 mg, $0.184 \mathrm{mmol}, 68 \%$ ) as a white solid. $\mathrm{mp}=199{ }^{\circ} \mathrm{C}$ (decomposition); ${ }^{1} \mathrm{H}$ NMR (400 MHz, DMSO-d $\left._{6}\right) \delta 11.45$ (br, s, 1H), 10.68 (br, s, 1H), 7.94 (d, J=2.8 Hz, 1H), 7.68 (d, J=8.4 Hz, 1H), 7.35 (d, $\mathrm{J}=8.8 \mathrm{~Hz}, 1 \mathrm{H}), 7.27(\mathrm{t}, \mathrm{J}=2.8 \mathrm{~Hz}, 1 \mathrm{H}), 6.51(\mathrm{dd}, \mathrm{J}=2.8,2.0 \mathrm{~Hz}, 1 \mathrm{H}), 4.29$ (quartet, J=7.2 Hz, 2H), $1.34(\mathrm{t}$, $\mathrm{J}=7.2 \mathrm{~Hz}, 3 \mathrm{H}) ;{ }^{13} \mathrm{C}$ NMR (100 MHz, DMSO-d 6 ) $\delta 164.8,128.7,123.6,123.0,122.3,122.3,120.6,114.8$, 112.5, 108.0, 102.5, 58.8, 14.5; IR (ATR) 3325, 1642, 1433, $1175 \mathrm{~cm}^{-1}$; HRMS (ESI) calcd for $\mathrm{C}_{13} \mathrm{H}_{13} \mathrm{~N}_{2} \mathrm{O}_{2}\left(\mathrm{M}+\mathrm{H}^{+}\right)$229.0977, found

3,6-Dimethyl-1H,6H-pyrrolo[3,2-g]indole (346). Reaction of a solution of 321 (59.2 mg, $0.193 \mathrm{mmol}$ ), $\mathrm{Pd}(\mathrm{OAc})_{2}(3.1 \mathrm{mg}, 0.014 \mathrm{mmol})$, and 1,10-phen $(2.5 \mathrm{mg}, 0.014 \mathrm{mmol})$ in DMF (3 mL), as described for $336\left(\mathrm{pCO}=6 \mathrm{~atm}, 120^{\circ} \mathrm{C}, 108 \mathrm{~h}\right.$ ), gave, after solvent removal and chromatography (Hexane/EtOAc, 1:1) 346 (34.2 $\mathrm{mg}, 0.141 \mathrm{mmol}, 73 \%$ ) as a white solid. $\mathrm{mp}=235{ }^{\circ} \mathrm{C}$ (decomposition); ${ }^{1} \mathrm{H} \mathrm{NMR}(400 \mathrm{MHz}$, DMSO-d $\left._{6}\right) \delta 11.16$ (br, s, 1H), 10.56 (br, s, 1H), 7.89 (d, J=3.2 Hz, 1H), 7.61 (d, J=8.4 Hz, 1H), 7.22 (d, $\mathrm{J}=8.4 \mathrm{~Hz}, 1 \mathrm{H}$ ), 6.19 (s, with further fine splitting, 1H), 4.28 (quartet, $\mathrm{J}=7.2 \mathrm{~Hz}, 2 \mathrm{H}), 2.44(\mathrm{~s}, 3 \mathrm{H}), 1.34(\mathrm{t}$, 
$\mathrm{J}=7.2 \mathrm{~Hz}, 3 \mathrm{H}) ;{ }^{13} \mathrm{C}$ NMR (100 MHz, DMSO-d 6 ) $\delta 164.8,131.9$, 128.7, 124.3, 122.6, 122.0, 120.2, 114.1, 112.1, 107.9, 100.6, 58.8, 14.5, 13.4; IR (ATR) 3343, 1651, 1403, 1313, 1170, $803 \mathrm{~cm}^{-1}$; HRMS (ESI) calcd for $\mathrm{C}_{14} \mathrm{H}_{15} \mathrm{~N}_{2} \mathrm{O}_{2}\left(\mathrm{M}+\mathrm{H}^{+}\right) 243.1134$, found

3,6-Dimethyl-1H,6H-pyrrolo[3,2-g]indole (347). A solution of 322 (85.3 mg, $0.279 \mathrm{mmol}), \mathrm{Pd}(\mathrm{OAc})_{2}$ (3.2 mg, $0.014 \mathrm{mmol})$, and 1,10-phen (2.5 mg, $0.014 \mathrm{mmol}$ ) in DMF (3 mL) was stirred under CO (pCO $=6 \mathrm{~atm})$ at $120^{\circ} \mathrm{C}$ for $110 \mathrm{~h}$. After coling to ambient temperature, pressure was released and the reaction mixture was diluted with EtOAc $(30 \mathrm{~mL})$. The organic layer was washed with brine $(6 \times 10 \mathrm{~mL})$, dried $\left(\mathrm{MgSO}_{4}\right)$ and was filtered. The filtrate was concentrated and the resulting crude was purified by chromatography (Hexane/EtOAc, 1:1) to give 347 (50.2 $\mathrm{mg}, 0.207 \mathrm{mmol}, 74 \%$ ) as a white solid. $\mathrm{mp}=233$ ${ }^{\circ} \mathrm{C}$ (decomposition); ${ }^{1} \mathrm{H}$ NMR (400 MHz, DMSO-d 6 ) $\delta 11.43$ (br, s, 1H), 10.33 (br, s, 1H), 7.93 (d, J=2.8 $\mathrm{Hz}, 1 \mathrm{H}), 7.68(\mathrm{~d}, J=8.4 \mathrm{~Hz}, 1 \mathrm{H}), 7.29$ (d, J=8.4 Hz, 1H), 7.04 (s, with further fine splitting, 1H), 4.28 (quartet, $J=7.2 \mathrm{~Hz}, 2 \mathrm{H}), 2.29(\mathrm{~s}, 3 \mathrm{H}), 1.34(\mathrm{t}, J=7.2 \mathrm{~Hz}, 3 \mathrm{H}) ;{ }^{13} \mathrm{C}$ NMR (100 MHz, DMSO-d 6 ) $\delta 164.8$, 128.7, 123.8, 123.0, 122.5, 120.8, 119.8, 113.0, 111.8, 110.7, 107.9, 58.8, 14.5, 9.9; IR (ATR) 3350, $1644,1428,1184,1133 \mathrm{~cm}^{-1}$; HRMS (ESI) calcd for $\mathrm{C}_{14} \mathrm{H}_{15} \mathrm{~N}_{2} \mathrm{O}_{2}\left(\mathrm{M}+\mathrm{H}^{+}\right) 243.1134$, found

2-Methyl-6-nitro-5-(1-propen-1-yl)indole (348). Reaction of a solution of 290 (35.9 mg, $0.145 \mathrm{mmol}$ ), $\mathrm{Pd}(\mathrm{OAc})_{2}(2.3 \mathrm{mg}, 0.010 \mathrm{mmol})$, and 1,10-phen $(1.8 \mathrm{mg}, 0.010 \mathrm{mmol})$ in DMF $(1.5 \mathrm{~mL})$, as described for $336\left(\mathrm{pCO}=6 \mathrm{~atm}, 120{ }^{\circ} \mathrm{C}, 46 \mathrm{~h}\right)$, gave, after solvent removal and chromatography (Hexane/EtOAc, 85:15) 348 (11.6 mg, $0.054 \mathrm{mmol}, 37 \%)$ as a yellow solid. $\mathrm{mp}=118-119{ }^{\circ} \mathrm{C} ;{ }^{1} \mathrm{H} \mathrm{NMR}\left(400 \mathrm{MHz}, \mathrm{CDCl}_{3}\right)$ $\delta 8.29(\mathrm{~s}, 1 \mathrm{H}), 8.22(\mathrm{~s}, 1 \mathrm{H}), 8.15(\mathrm{~s}, 1 \mathrm{H}), 8.03(\mathrm{~s}, 1 \mathrm{H}), 7.56(\mathrm{~s}, 1 \mathrm{H}), 7.37(\mathrm{~s}, 1 \mathrm{H}), 7.03-6.94(\mathrm{~m}, 1 \mathrm{H}), 6.87$ $-6.81(\mathrm{~m}, 1 \mathrm{H}), 6.30-6.27(\mathrm{~m}, 2 \mathrm{H}), 6.09(\mathrm{dq}, J=15.6,6.8 \mathrm{~Hz}, 1 \mathrm{H}), 5.86(\mathrm{dq}, J=11.6,7.2 \mathrm{~Hz}, 1 \mathrm{H}), 2.51$ $(\mathrm{d}, J=0.8 \mathrm{~Hz}, 3 \mathrm{H}), 2.49$ (d, $J=0.8 \mathrm{~Hz}, 3 \mathrm{H}), 1.93(\mathrm{dd}, J=6.8,2.0 \mathrm{~Hz}, 3 \mathrm{H}), 1.74(\mathrm{dd}, J=7.2,2.0 \mathrm{~Hz}, 3 \mathrm{H})$; ${ }^{13} \mathrm{C}$ NMR (100 MHz, DMSO-d 6 ) $\delta$ 143.4, 143.3, 141.3, 141.0, 133.6, 133.5, 132.9, 132.4, 128.3, 126.6, 125.1, 124.8, 122.9, 120.8, 117.9, 108.1, 107.7, 100.5, 100.4, 18.5, 14.1, 13.7, 13.6; IR (ATR) 3353, 1490, 1437, $1297 \mathrm{~cm}^{-1}$; HRMS (ESI) calcd for $\mathrm{C}_{12} \mathrm{H}_{13} \mathrm{~N}_{2} \mathrm{O}_{2}\left(\mathrm{M}+\mathrm{H}^{+}\right) 217.0977$, found

3,6-Dimethyl-1H,6H-pyrrolo[3,2-g]indole (348). Reaction of a solution of 290 (35.6 mg, $0.143 \mathrm{mmol}$ ), $\mathrm{Pd}(\mathrm{dba})_{2}(6.6 \mathrm{mg}, 0.010 \mathrm{mmol})$, and $\mathrm{PPh}_{3}(12 \mathrm{mg}, 0.046 \mathrm{mmol})$ in DMF $(1.5 \mathrm{~mL})$, as described for 336 $\left(\mathrm{pCO}=6 \mathrm{~atm}, 120^{\circ} \mathrm{C}, 92 \mathrm{~h}\right.$ ), gave, after solvent removal and chromatography (Hexane/EtOAc, 7:3) 348 (19.9 mg, $0.092 \mathrm{mmol}, 64 \%$ ) as a yellow solid. 
3,6-Dimethyl-1H,6H-pyrrolo[3,2-g]indole (349). Reaction of a solution of 291 (45.6 mg, $0.184 \mathrm{mmol}$ ), $\mathrm{Pd}(\mathrm{dba})_{2}(5.3 \mathrm{mg}, 0.009 \mathrm{mmol})$, dppp $(3.8 \mathrm{mg}, 0.009 \mathrm{mmol})$, and 1,10-phen (3.3 $\left.\mathrm{mg}, 0.018 \mathrm{mmol}\right)$ in DMF (2 mL), as described for $347\left(\mathrm{pCO}=6 \mathrm{~atm}, 120{ }^{\circ} \mathrm{C}, 82 \mathrm{~h}\right.$ ), gave, after solvent removal and chromatography (Hexane/EtOAc, 7:3) $349(18.1 \mathrm{mg}, 0.098 \mathrm{mmol}, 53 \%)$ as a white solid. $\mathrm{mp}=200{ }^{\circ} \mathrm{C}$ (decomposition); ${ }^{1} \mathrm{H}$ NMR (400 MHz, $\left.\mathrm{CDCl}_{3}\right) \delta 7.65$ (s, 1H), 7.57 (br, s, 2H), 7.19 (s, 1H), 6.93 (s, 2H), $2.41(\mathrm{~s}, 6 \mathrm{H}) ;{ }^{13} \mathrm{C} \mathrm{NMR}\left(100 \mathrm{MHz}, \mathrm{CDCl}_{3}\right) \delta 135.2,124.7,121.1,111,0,106.7,90.9,10.0$; IR (ATR) $3329,1474,1301,1092 \mathrm{~cm}^{-1}$; HRMS (ESI) calcd for $\mathrm{C}_{12} \mathrm{H}_{13} \mathrm{~N}_{2}\left(\mathrm{M}+\mathrm{H}^{+}\right)$185.1079, found

3-Methyl-6-nitro-5-(1-propen-2-yl)indole (350). Reaction of a solution of 291 (56.5 mg, $0.228 \mathrm{mmol}$ ), $\mathrm{Pd}(\mathrm{OAc})_{2}(3.1 \mathrm{mg}, 0.014 \mathrm{mmol})$, and 1,10-phen $(2.5 \mathrm{mg}, 0.014 \mathrm{mmol})$ in DMF (2 mL), as described for $336\left(\mathrm{pCO}=6 \mathrm{~atm}, 120{ }^{\circ} \mathrm{C}, 50 \mathrm{~h}\right)$, gave, after solvent removal and chromatography (Hexane/EtOAc, 7:3) 350 (9.7 mg, $0.045 \mathrm{mmol}, 20 \%)$ as a yellow solid. $\mathrm{mp}=117-118{ }^{\circ} \mathrm{C} ;{ }^{1} \mathrm{H}$ NMR $\left(600 \mathrm{MHz}, \mathrm{CDCl}_{3}\right) \delta 8.29$ (br, s, 1H), 8.08 (s, 1H), ${ }^{13} \mathrm{C}$ NMR (150 MHz, $\left.\mathrm{CDCl}_{3}\right) \delta 145.2,143.2,133.7,131.6,131.2,127.4,120.0$, 114.0, 112.7, 108.5, 24.1, 9.5; IR (ATR) 3335, 1206, 1100, $896 \mathrm{~cm}^{-1}$; HRMS (ESI) calcd for $\mathrm{C}_{12} \mathrm{H}_{13} \mathrm{~N}_{2} \mathrm{O}_{2}$ $\left(\mathrm{M}+\mathrm{H}^{+}\right)$217.0977, found

3,6-Dimethyl-1H,6H-pyrrolo[3,2-g]indole (350). Reaction of a solution of 291 (51.7 mg, $0.208 \mathrm{mmol}$ ), $\mathrm{Pd}(\mathrm{dba})_{2}(7.1 \mathrm{mg}, 0.012 \mathrm{mmol})$, and $\mathrm{PPh}_{3}(13.1 \mathrm{mg}, 0.050 \mathrm{mmol})$ in DMF (2 mL), as described for 336 $\left(\mathrm{pCO}=6 \mathrm{~atm}, 120{ }^{\circ} \mathrm{C}, 121 \mathrm{~h}\right.$ ), gave, after solvent removal and chromatography (Hexane/EtOAc, 7:3) 350 (38.1 $\mathrm{mg}, 0.176 \mathrm{mmol}, 85 \%)$ as a yellow solid.

3,6-Dimethyl-1H,6H-pyrrolo[3,2-g]indole (351). Reaction of a solution of 292 (29.3 mg, $0.133 \mathrm{mmol})$, $\mathrm{Pd}(\mathrm{OAc})_{2}(2.1 \mathrm{mg}, 0.009 \mathrm{mmol})$, and 1,10-phen $(1.7 \mathrm{mg}, 0.009 \mathrm{mmol})$ in DMF $(1 \mathrm{~mL})$, as described for $336\left(\mathrm{pCO}=6 \mathrm{~atm}, 120^{\circ} \mathrm{C}, 48 \mathrm{~h}\right.$ ), gave, after solvent removal and chromatography (Hexane/EtOAc, 7:3) 351 (12.5 mg, $0.080 \mathrm{mmol}, 60 \%)$ as a white solid. $\mathrm{mp}=239{ }^{\circ} \mathrm{C}$ (decomposition); ${ }^{1} \mathrm{H}$ NMR (600 MHz, $\left.\mathrm{CDCl}_{3}\right) \delta 7.91(\mathrm{br}, \mathrm{s}, 2 \mathrm{H}), 7.58(\mathrm{~s}, 2 \mathrm{H}),{ }^{13} \mathrm{C} \mathrm{NMR}\left(100 \mathrm{MHz}, \mathrm{CDCl}_{3}\right) \delta 133.2,126.0,125.0,101.4,100.0$; IR (ATR) 3336, 1156, 1053, $756 \mathrm{~cm}^{-1}$; HRMS (ESI) calcd for $\mathrm{C}_{10} \mathrm{H}_{9} \mathrm{~N}_{2}\left(\mathrm{M}+\mathrm{H}^{+}\right)$157.0766, found

3,6-Dimethyl-1H,6H-pyrrolo[3,2-g]indole (XX). Reaction of a solution of 292 (29.3 mg, $0.133 \mathrm{mmol})$, $\mathrm{Pd}(\mathrm{dba})_{2}(4.6 \mathrm{mg}, 0.008 \mathrm{mmol})$, and $\mathrm{PPh}_{3}(8.5 \mathrm{mg}, 0.032 \mathrm{mmol})$ in DMF (1 mL), as described for 336 $\left(\mathrm{pCO}=6 \mathrm{~atm}, 120{ }^{\circ} \mathrm{C}, 64 \mathrm{~h}\right.$ ), gave, after solvent removal and chromatography (Hexane/EtOAc, 7:3) XX $(14 \mathrm{mg}, 0.074 \mathrm{mmol}, 56 \%)$ as a yellow solid. ${ }^{1} \mathrm{H} \mathrm{NMR}\left(400 \mathrm{MHz}, \mathrm{CDCl}_{3}\right) \delta 77^{13} \mathrm{C} \mathrm{NMR}(100 \mathrm{MHz}$, $\left.\mathrm{CDCl}_{3}\right) \delta 1 \mathrm{IR}(\mathrm{ATR}) \mathrm{cm}^{-1}$; HRMS (ESI) calcd for 
2-Methyl-5-nitro-6-(1-propen-1-yl)indole (352). Reaction of a solution of 293 (39 mg, $0.157 \mathrm{mmol}$ ), $\mathrm{Pd}(\mathrm{OAc})_{2}(2.5 \mathrm{mg}, 0.011 \mathrm{mmol})$, and 1,10-phen (2 mg, $\left.0.011 \mathrm{mmol}\right)$ in DMF (2 mL), as described for 336 $\left(\mathrm{pCO}=6 \mathrm{~atm}, 120^{\circ} \mathrm{C}, 49 \mathrm{~h}\right)$, gave, after solvent removal and chromatography (Hexane/EtOAc, 7:3) 352 (10.5 mg, $0.049 \mathrm{mmol}, 31 \%$ ) as a yellow solid. $\mathrm{mp}=111-112{ }^{\circ} \mathrm{C} ;{ }^{1} \mathrm{H}$ NMR $\left(600 \mathrm{MHz}, \mathrm{CDCl}_{3}\right) \delta 8.31$ (s, 1H), 8.18 (s, 1H), 8.18 (br, s, 1H), 8.15 (br, s, 1H), 7.32 (s, 1H), 7.15 (s, 1H), 6.97 (d, J=15.6 Hz, 1H), $6.84(\mathrm{~d}, J=11.4 \mathrm{~Hz}, 1 \mathrm{H}), 6.34$ (s, 1H), 6.29 (s, 1H), 6.07 (dq, J=15.6, $6.0 \mathrm{~Hz}, 1 \mathrm{H}), 5.85$ (dq, J=11.4, 6.6 $\mathrm{Hz}, 1 \mathrm{H}), 2.48$ (s, 3H), 2.47 (s, 3H), 1.93 (d, J=6.6 Hz, 3H), 1.72 (d, J=6.6 Hz, 3H); ${ }^{13} \mathrm{C}$ NMR (150 MHz, $\left.\mathrm{CDCl}_{3}\right) \delta 142.3,142.0,138.8,138.7,138.5,138.0,128.5,128.2,127.8,127.8,127.4,127.4,126.1,126.1$, 117.6, 117.2, 112.4, 109.2, 102.1, 102.0, 18.6, 14.3, 13.8; IR (ATR) 3321, 1462, 1285, $823 \mathrm{~cm}^{-1}$; HRMS (ESI) calcd for $\mathrm{C}_{12} \mathrm{H}_{13} \mathrm{~N}_{2} \mathrm{O}_{2}\left(\mathrm{M}+\mathrm{H}^{+}\right) 217.0977$, found

3,6-Dimethyl-1H,6H-pyrrolo[3,2-g]indole (352). Reaction of a solution of 293 (45.6 mg, $0.184 \mathrm{mmol}$ ), $\mathrm{Pd}(\mathrm{dba})_{2}(6.4 \mathrm{mg}, 0.011 \mathrm{mmol})$, and $\mathrm{PPh}_{3}(11.6 \mathrm{mg}, 0.044 \mathrm{mmol})$ in DMF (1.5 mL), as described for 336 $\left(\mathrm{pCO}=6 \mathrm{~atm}, 120^{\circ} \mathrm{C}, 72 \mathrm{~h}\right.$ ), gave, after solvent removal and chromatography (Hexane/EtOAc, 7:3) 352 (39.7 mg, $0.184 \mathrm{mmol}, 100 \%)$ as a yellow solid.

3-Methyl-5-nitro-6-(1-propen-2-yl)indole (353). Reaction of a solution of 294 (45.2 mg, $0.182 \mathrm{mmol}$ ), $\mathrm{Pd}(\mathrm{dba})_{2}(6.3 \mathrm{mg}, 0.011 \mathrm{mmol})$, and $\mathrm{PPh}_{3}(11.5 \mathrm{mg}, 0.044 \mathrm{mmol})$ in DMF (2 mL), as described for 347 $\left(\mathrm{pCO}=6 \mathrm{~atm}, 120^{\circ} \mathrm{C}, 72 \mathrm{~h}\right.$ ), gave, after solvent removal and chromatography (Hexane/EtOAc, 9:1) 353 (28.7 mg, $0.133 \mathrm{mmol}, 73 \%)$ as a yellow solid. $\mathrm{mp}=114-115{ }^{\circ} \mathrm{C} ;{ }^{1} \mathrm{H} \mathrm{NMR}\left(400 \mathrm{MHz}, \mathrm{CDCl}_{3}\right) \delta 8.34$ (br, $\mathrm{s}, 1 \mathrm{H}), 8.30(\mathrm{~s}, 1 \mathrm{H}), 7.20(\mathrm{~s}, 1 \mathrm{H}), 7.10(\mathrm{~s}, 1 \mathrm{H}), 5.14(\mathrm{~s}, 1 \mathrm{H}), 4.95(\mathrm{~s}, 1 \mathrm{H}), 2.34(\mathrm{~s}, 3 \mathrm{H}), 2.11(\mathrm{~s}, 3 \mathrm{H}) ;{ }^{13} \mathrm{C}$ NMR (100 MHz, $\left.\mathrm{CDCl}_{3}\right) \delta 145.1,141.5,138.1,133.9,126.8,124.7,117.0,114.0,113.8,112.2,23.8,9.5$; IR (ATR) 3337, 1208, 1113, $836 \mathrm{~cm}^{-1}$; HRMS (ESI) calcd for $\mathrm{C}_{12} \mathrm{H}_{13} \mathrm{~N}_{2} \mathrm{O}_{2}\left(\mathrm{M}+\mathrm{H}^{+}\right)$217.0977, found

3,6-Dimethyl-1H,6H-pyrrolo[3,2-g]indole (YY). Reaction of a solution of 294 (24.9 mg, $0.100 \mathrm{mmol}$ ), $\mathrm{Pd}(\mathrm{dba})_{2}(3.5 \mathrm{mg}, 0.006 \mathrm{mmol})$, and $\mathrm{PPh}_{3}(6.3 \mathrm{mg}, 0.024 \mathrm{mmol})$ in DMF $(1.5 \mathrm{~mL})$, as described for 336 $\left(\mathrm{pCO}=6 \mathrm{~atm}, 120^{\circ} \mathrm{C}, 68 \mathrm{~h}\right.$ ), gave, after solvent removal and chromatography (Hexane/EtOAc, 7:3) YY (4.8 $\mathrm{mg}, 0.026 \mathrm{mmol}, 26 \%)$ as a white solid. $\mathrm{mp}=214{ }^{\circ} \mathrm{C}$ (decomposition); ${ }^{1} \mathrm{H}$ NMR (400 MHz, $\mathrm{CDCl}_{3} / \mathrm{DMSO}_{\mathrm{d}}$ ) $\delta 9.88(\mathrm{br}, \mathrm{s}, 2 \mathrm{H}), 7.25$ (s, 2H), $6.91(\mathrm{~s}, 2 \mathrm{H}), 2.25$ (s, 3H); ${ }^{13} \mathrm{C}$ NMR (100 MHz, $\mathrm{CDCl}_{3} / \mathrm{DMSO}_{-}$) $\delta$ 132.9, 125.9, 122.2, 107.6, 97.4, 9.8; IR (ATR) 3315, 1476, 1300, $1175 \mathrm{~cm}^{-1}$; HRMS (ESI) calcd for $\mathrm{C}_{12} \mathrm{H}_{13} \mathrm{~N}_{2}\left(\mathrm{M}+\mathrm{H}^{+}\right) 185.1079$, found

4-Ethenyl-5-nitroindole(354). A solution of $295(81.4 \mathrm{mg}, 0.370 \mathrm{mmol}), \mathrm{Pd}(\mathrm{OAc})_{2}(4.2 \mathrm{mg}, 0.018$ mmol), and 1,10-phen (3.4 mg, $0.019 \mathrm{mmol})$ in DMF (3 mL) was stirred under CO (pCO = $6 \mathrm{~atm})$ at 120 
${ }^{\circ} \mathrm{C}$ for $120 \mathrm{~h}$. After coling to ambient temperature, pressure was released and the reaction mixture was concentrated under reduced pressure via bulb-to-bulb distillation. The resulting crude was purified by chromatography (Hexane/EtOAc, 7:3) to give 354 (30.1 mg, $0.160 \mathrm{mmol}, 43 \%$ ) as a yellow solid solid. $\mathrm{mp}=143-144{ }^{\circ} \mathrm{C} ;{ }^{1} \mathrm{H}$ NMR (400 MHz, $\mathrm{CDCl}_{3} / \mathrm{DMSO}_{\mathrm{d}}$ ) $\delta 11.35$ (br, s, 1H), 7.77 (d, J=9.2 Hz, 1H), 7.33 (d, J=9.2 Hz, 1H), 7.32 (dd, J=5.6, 2.8 Hz, 1H), 7.20 (dd, J=17.6, $11.2 \mathrm{~Hz}, 1 \mathrm{H}), 6.72-6.71(\mathrm{~m}, 1 \mathrm{H}), 5.66$ $(\mathrm{dd}, \mathrm{J}=17.6,1.6 \mathrm{~Hz}, 1 \mathrm{H}), 5.60(\mathrm{dd}, \mathrm{J}=11.6,1.6 \mathrm{~Hz}, 1 \mathrm{H}) ;{ }^{13} \mathrm{C} \mathrm{NMR}\left(100 \mathrm{MHz}, \mathrm{CDCl}_{3} / \mathrm{DMSO}^{-\mathrm{d}_{6}}\right) \delta 139.4$, 137.9, 132.4, 127.8, 127.5, 126.0, 119.3, 117.5, 110.4, 103.4; IR (ATR) 3323, 1311, 929, $739 \mathrm{~cm}^{-1}$; HRMS (ESI) calcd for $\mathrm{C}_{10} \mathrm{H}_{9} \mathrm{~N}_{2} \mathrm{O}_{4}\left(\mathrm{M}+\mathrm{H}^{+}\right)$189.0664, found

(354). Reaction of a solution of 295 (71.1 mg, $0.323 \mathrm{mmol}), \mathrm{Pd}(\mathrm{dba})_{2}(9.5 \mathrm{mg}, 0.016 \mathrm{mmol})$, and $\mathrm{PPh}_{3}$ (16.9 mg, $0.065 \mathrm{mmol})$ in DMF (2.5 mL), as described for 354 (pCO = $6 \mathrm{~atm}, 120^{\circ} \mathrm{C}, 110 \mathrm{~h}$ ), gave, after solvent removal and chromatography (Hexane/EtOAc, 7:3) 354 (41.1 mg, $0.218 \mathrm{mmol}, 68 \%$ ) as a yellow solid.

2-Methyl-5-nitro-4-(1-propen-1-yl)indole(355). Reaction of a solution of 296 (70.4 mg, $0.284 \mathrm{mmol}$ ), $\mathrm{Pd}(\mathrm{OAc})_{2}(3.3 \mathrm{mg}, 0.015 \mathrm{mmol})$, and 1,10-phen $(2.7 \mathrm{mg}, 0.015 \mathrm{mmol})$ in DMF (3 mL), as described for $336\left(\mathrm{pCO}=6 \mathrm{~atm}, 120^{\circ} \mathrm{C}, 70 \mathrm{~h}\right.$ ), gave, after solvent removal and chromatography (Hexane/EtOAc, 7:3) 355 (45.3 mg, $0.209 \mathrm{mmol}, 74 \%)$ as a yellow solid. $\mathrm{mp}=101-103{ }^{\circ} \mathrm{C} ;{ }^{1} \mathrm{H} \mathrm{NMR}\left(600 \mathrm{MHz}, \mathrm{CDCl}_{3}\right) \delta 8.34$ (br, s, 2H), $7.93(\mathrm{~d}, \mathrm{~J}=9.0 \mathrm{~Hz}, 1 \mathrm{H}), 7.82(\mathrm{~d}, \mathrm{~J}=9.0 \mathrm{~Hz}, 1 \mathrm{H}), 7.22(\mathrm{~d}, \mathrm{~J}=9.0 \mathrm{~Hz}, 1 \mathrm{H}), 7.16(\mathrm{~d}, \mathrm{~J}=9.0 \mathrm{~Hz}, 1 \mathrm{H})$, 6.98 (dq, J=16.2, $1.8 \mathrm{~Hz}, 1 \mathrm{H}), 6.85$ (dq, J=11.4, $1.8 \mathrm{~Hz}, 1 \mathrm{H}), 6.50-6.49$ (m, 1H), 6.24-6.18 (m, 2H), 5.97 (dq, J=13.8, 7.2 Hz, 1H), $2.47(\mathrm{~m}, 6 \mathrm{H}), 2.01(\mathrm{dd}, \mathrm{J}=7.2,1.8 \mathrm{~Hz}, 3 \mathrm{H}), 1.50(\mathrm{dd}, \mathrm{J}=6.6,1.8 \mathrm{~Hz}, 3 \mathrm{H}) ;{ }^{13} \mathrm{C}$ $\mathrm{NMR}\left(150 \mathrm{MHz}, \mathrm{CDCl}_{3}\right) \delta 141.1,140.6,138.1,137.9,137.7,137.7,132.0,128.5,128.0,127.9,127.8$, $126.8,126.4,125.4,118.3,118.3,108.9,108.7,103.1,102.9,19.0,15.1,13.8,13.7$; IR (ATR) 3351, $1508,1316,1172 \mathrm{~cm}^{-1}$; HRMS (ESI) calcd for $\mathrm{C}_{12} \mathrm{H}_{13} \mathrm{~N}_{2} \mathrm{O}_{2}\left(\mathrm{M}+\mathrm{H}^{+}\right) 217.0977$, found

(355). Reaction of a solution of 296 (34.5 mg, $0.139 \mathrm{mmol}), \mathrm{Pd}(\mathrm{dba})_{2}(4 \mathrm{mg}, 0.007 \mathrm{mmol})$, and $\mathrm{PPh}_{3}(7.3$ $\mathrm{mg}, 0.028 \mathrm{mmol}$ ) in DMF ( $2 \mathrm{~mL}$ ), as described for 336 (pCO = $6 \mathrm{~atm}, 120{ }^{\circ} \mathrm{C}, 72 \mathrm{~h}$ ), gave, after solvent removal and chromatography (Hexane/EtOAc, 8:2) 355 (20.9 mg, $0.097 \mathrm{mmol}, 70 \%$ ) as a yellow solid.

3-Methyl-5-nitro-4-(1-propen-2-yl)indole(356). Reaction of a solution of 297 (80.3 mg, $0.323 \mathrm{mmol})$, $\mathrm{Pd}(\mathrm{OAc})_{2}(3.7 \mathrm{mg}, 0.016 \mathrm{mmol})$, and 1,10-phen $(3.0 \mathrm{mg}, 0.017 \mathrm{mmol})$ in DMF (3 mL), as described for $354\left(\mathrm{pCO}=6 \mathrm{~atm}, 120^{\circ} \mathrm{C}, 100 \mathrm{~h}\right)$, gave, after solvent removal and chromatography (Hexane/EtOAc, 7:3) 356 (60.3 mg, $0.279 \mathrm{mmol}, 86 \%)$ as a yellow solid. mp=98-99 ${ }^{\circ} \mathrm{C} ;{ }^{1} \mathrm{H} \mathrm{NMR}\left(400 \mathrm{MHz}, \mathrm{CDCl}_{3}\right) \delta 8.43$ (br, s, 1H), 7.84 (d, J=8.8 Hz, 1H), 7.28 (d, J=8.8 Hz, 1H), 7.09-7.08 (m, 1H), 5.32-5.30 (m, 1H), 4.91- 
$4.90(\mathrm{~m}, 1 \mathrm{H}), 2.38$ (d, J=0.8 Hz, 3H), 2.30-2.29 (m, 3H); ${ }^{13} \mathrm{C}$ NMR (100 MHz, $\left.\mathrm{CDCl}_{3}\right) \delta 141.3,140.9$, 138.6, 134.3, 125.2, 124.8, 118.7, 115.1, 114.8, 110.0, 25.3, 11.6; IR (ATR) 3369, 1406, $799 \mathrm{~cm}^{-1}$; HRMS (ESI) calcd for $\mathrm{C}_{12} \mathrm{H}_{13} \mathrm{~N}_{2} \mathrm{O}_{2}\left(\mathrm{M}+\mathrm{H}^{+}\right)$217.0977, found

(356). Reaction of a solution of $297(57.7 \mathrm{mg}, 0.232 \mathrm{mmol}), \mathrm{Pd}(\mathrm{dba})_{2}(6.7 \mathrm{mg}, 0.012 \mathrm{mmol})$, dppp (4.8 $\mathrm{mg}, 0.012 \mathrm{mmol})$, and 1,10-phen $(4.2 \mathrm{mg}, 0.023 \mathrm{mmol})$ in DMF $(2.5 \mathrm{~mL})$, as described for $354(\mathrm{pCO}=6$ atm, $120{ }^{\circ} \mathrm{C}, 91 \mathrm{~h}$ ), gave, after solvent removal and chromatography (Hexane/EtOAc, 7:3) 356 (25.7 mg, $0.119 \mathrm{mmol}, 51 \%$ ) as a yellow solid.

5-Nitro-4-(1-propen-1-yl)indole (357) and 4-ethenyl-2-methyl-5-nitroindole (358). Reaction of a solution of 327 (75.2 $\mathrm{mg}, 0.321 \mathrm{mmol}), \mathrm{Pd}(\mathrm{dba})_{2}(11.1 \mathrm{mg}, 0.019 \mathrm{mmol})$, dppp (7.9 mg, $\left.0.019 \mathrm{mmol}\right)$, and 1,10-phen (6.9 mg, $0.038 \mathrm{mmol}$ ) in DMF (3 mL), as described for 336 (pCO = 6 atm, $120{ }^{\circ} \mathrm{C}, 74 \mathrm{~h}$ ), gave, after solvent removal and chromatography (Hexane/EtOAc, 85:15) an inseparable mixture of 357 and 358 (28.9 $\mathrm{mg}, 0.143 \mathrm{mmol}, 45 \%, \mathbf{3 5 7 / 3 5 8}, 1: 1)$ as a yellow oil.

5-Nitro-4-(1-propen-2-yl)indole (359) and 4-ethenyl-3-methyl-5-nitroindole (360). Reaction of a solution of $328(83.9 \mathrm{mg}, 0.358 \mathrm{mmol}), \mathrm{Pd}(\mathrm{OAc})_{2}(4.8 \mathrm{mg}, 0.021 \mathrm{mmol})$, and 1,10-phen (3.9 mg, 0.022 $\mathrm{mmol})$ in DMF (3 mL), as described for $\mathbf{3 3 6}\left(\mathrm{pCO}=6 \mathrm{~atm}, 120{ }^{\circ} \mathrm{C}, 96 \mathrm{~h}\right)$, gave, after solvent removal and chromatography (Hexane/EtOAc, 8:2) an inseparable mixture of $\mathbf{3 5 9}$ and $\mathbf{3 6 0}$ (51 mg, 0.252 mmol, 70\%, 359/360, 1:1) as a yellow oil.

5-Nitro-3-methyl-4-(1-propen-1-yl)indole (361) and 5-nitro-2-methyl-4-(1-propen-2-yl)indole (362). Reaction of a solution of $\mathbf{3 2 9}(81.5 \mathrm{mg}, 0.328 \mathrm{mmol}), \mathrm{Pd}(\mathrm{OAc})_{2}(5.2 \mathrm{mg}, 0.023 \mathrm{mmol})$, and 1,10-phen (4.2 mg, $0.023 \mathrm{mmol}$ ) in DMF (3 mL), as described for 336 (pCO = $6 \mathrm{~atm}, 120{ }^{\circ} \mathrm{C}, 122 \mathrm{~h}$ ), gave, after solvent removal and chromatography (Hexane/EtOAc, 8:2) an inseparable mixture of $\mathbf{3 6 1}$ and 362 (54.6 $\mathrm{mg}, 0.253 \mathrm{mmol}, 77 \%, \mathbf{3 6 1 / 3 6 2}, 1: 1)$ as a yellow oil.

3,6-Dimethyl-1H,6H-pyrrolo[3,2-g]indole (363) and (364). Reaction of a solution of 298 (78.5 mg, $0.357 \mathrm{mmol}), \mathrm{Pd}(\mathrm{OAc})_{2}(4.8 \mathrm{mg}, 0.021 \mathrm{mmol})$, and 1,10-phen $(3.9 \mathrm{mg}, 0.022 \mathrm{mmol})$ in DMF (3 mL), as described for $336\left(\mathrm{pCO}=6 \mathrm{~atm}, 120{ }^{\circ} \mathrm{C}, 101 \mathrm{~h}\right)$, gave, in order of elution, after solvent removal and chromatography (Hexane/DCM, 1:1) 364 (13.7 mg, $0.073 \mathrm{mmol}, 20 \%$ ) as a yellow solid and 363 (28 $\mathrm{mg}$, $0.179 \mathrm{mmol}, 50 \%)$ as a white solid. Analytical data for 363: $\mathrm{mp}=138-139{ }^{\circ} \mathrm{C} ;{ }^{1} \mathrm{H}$ NMR $(400 \mathrm{MHz}$, $\left.\mathrm{CDCl}_{3} / \mathrm{DMSO}_{-} \mathrm{d}_{6}\right) \delta 11.06$ (br, s, 1H), 10.89 (br, s, 1H), 7.22 (d, $\left.J=8.8 \mathrm{~Hz}, 1 \mathrm{H}\right), 7.13(\mathrm{t}, J=2.8 \mathrm{~Hz}, 1 \mathrm{H})$, $7.07(\mathrm{~d}, J=8.8 \mathrm{~Hz}, 1 \mathrm{H}), 7.05(\mathrm{t}, J=2.8 \mathrm{~Hz}, 1 \mathrm{H}), 6.62(\mathrm{t}, J=2.4 \mathrm{~Hz}, 1 \mathrm{H}), 6.40(\mathrm{dd}, J=2.8,2.0 \mathrm{~Hz}, 1 \mathrm{H})$. 
${ }^{13} \mathrm{C}$ NMR (100 MHz, $\left.\mathrm{CDCl}_{3} / \mathrm{DMSO}_{-} \mathrm{d}_{6}\right) \delta 132.4,128.6,121.6,120.0,119.6,114.2,113.4,104.9,101.9$, 97.9; IR (ATR) 3426, 3363, 1389, $718 \mathrm{~cm}^{-1} ; \mathrm{C}_{10} \mathrm{H}_{9} \mathrm{~N}_{2}\left(\mathrm{M}+\mathrm{H}^{+}\right)$157.0766, found $\mathrm{cm}^{-1}$; HRMS (ESI) calcd for Analytical data for 364: $\mathrm{mp}=97-98{ }^{\circ} \mathrm{C}$; ${ }^{1} \mathrm{H} \mathrm{NMR}\left(400 \mathrm{MHz}, \mathrm{CDCl}_{3}\right) \delta 8.58$ (br, s, 1H), 7.59 (d, J=8.4 $\mathrm{Hz}, 1 \mathrm{H}), 7.43$ (d, J=8.4 Hz, 1H), 7.38 (t, $J=2.4 \mathrm{~Hz}, 1 \mathrm{H}), 7.25$ (dd, $J=17.2,10.8 \mathrm{~Hz}, 1 \mathrm{H}), 6.92$ (s, with further fine splitting, $1 \mathrm{H}), 5.73(\mathrm{~d}, J=17.2 \mathrm{~Hz}, 1 \mathrm{H}), 5.43(\mathrm{~d}, J=10.8 \mathrm{~Hz}, 1 \mathrm{H}) ;{ }^{13} \mathrm{C} \mathrm{NMR}\left(100 \mathrm{MHz}, \mathrm{CDCl}_{3}\right)$ $\delta 140.0,136.8,133.3,127.7,126.5,122.2,121.4,117.0,116.0,102.6$; IR (ATR) 3461, 1520, 1264, 731 $\mathrm{cm}^{-1}$; HRMS (ESI) calcd for $\mathrm{C}_{10} \mathrm{H}_{9} \mathrm{~N}_{2} \mathrm{O}_{2}\left(\mathrm{M}+\mathrm{H}^{+}\right) 189.0664$, found

7-Ethenyl-6-nitroindole (364). Reaction of a solution of 298 (45.6 mg, $0.207 \mathrm{mmol}), \operatorname{Pd}(\mathrm{dba})_{2}(7.1 \mathrm{mg}$, $0.012 \mathrm{mmol})$, and $\mathrm{PPh}_{3}(13 \mathrm{mg}, 0.050 \mathrm{mmol})$ in DMF $(2 \mathrm{~mL})$, as described for $354\left(\mathrm{pCO}=6 \mathrm{~atm}, 120{ }^{\circ} \mathrm{C}\right.$, 126 h), gave, after solvent removal and chromatography (Hexane/EtOAc, 7:3) 364 (19.2 mg, 0.102 mmol, $49 \%$ ) as a yellow solid.

5-Ethenyl-3-methyl-4-nitroindole (365). Reaction of a solution of 331 (49.1 mg, $0.210 \mathrm{mmol}$ ), $\mathrm{Pd}(\mathrm{OAc})_{2}(2.4 \mathrm{mg}, 0.011 \mathrm{mmol})$, and 1,10-phen $(1.9 \mathrm{mg}, 0.011 \mathrm{mmol})$ in DMF (2.5 mL), as described for $354\left(\mathrm{pCO}=6 \mathrm{~atm}, 120{ }^{\circ} \mathrm{C}, 71 \mathrm{~h}\right)$, gave, after solvent removal and chromatography (Hexane/EtOAc, 7:3) 365 (31 mg, $0.153 \mathrm{mmol}, 73 \%)$ as a yellow sticky oil. $\mathrm{mp}=64-65{ }^{\circ} \mathrm{C} ;{ }^{1} \mathrm{H} \mathrm{NMR}\left(400 \mathrm{MHz}, \mathrm{CDCl}_{3}\right) \delta 8.23$ (br, s, 1H), 7.41 (s, 2H), $7.04(\mathrm{~m}, 1 \mathrm{H}), 6.81$ (dd, J=17.2, $10.8 \mathrm{~Hz}, 1 \mathrm{H}), 5.77$ (dd, J=17.2, $0.8 \mathrm{~Hz}, 1 \mathrm{H}), 5.38$ (dd, J=10.8, $0.8 \mathrm{~Hz}, 1 \mathrm{H}), 2.18$ (d, J=0.8 Hz, 3H); ${ }^{13} \mathrm{C}$ NMR (100 MHz, $\left.\mathrm{CDCl}_{3}\right) \delta 142.1,137.6,130.4$, 125.1, 121.5, 119.4, 118.6, 116.8, 113.7, 110.4, 9.8; IR (ATR) 3392, 1504, 1331, $807 \mathrm{~cm}^{-1}$; HRMS (ESI) calcd for $\mathrm{C}_{11} \mathrm{H}_{11} \mathrm{~N}_{2} \mathrm{O}_{2}\left(\mathrm{M}+\mathrm{H}^{+}\right)$203.0821, found

4-Nitro-5-(1-propen-2-yl)indole (366). Reaction of a solution of 330 (57.8 mg, $0.247 \mathrm{mmol}), \mathrm{Pd}(\mathrm{OAc})_{2}$ (4.4 mg, $0.020 \mathrm{mmol})$, and 1,10-phen $(3.6 \mathrm{mg}, 0.020 \mathrm{mmol})$ in DMF $(2 \mathrm{~mL})$, as described for $354(\mathrm{pCO}=$ $6 \mathrm{~atm}, 120{ }^{\circ} \mathrm{C}, 108 \mathrm{~h}$ ), gave, after solvent removal and chromatography (Hexane/EtOAc, 7:3) 366 (30.3 $\mathrm{mg}, 0.150 \mathrm{mmol}, 61 \%)$ as a yellow solid. $\mathrm{mp}=100-101{ }^{\circ} \mathrm{C} ;{ }^{1} \mathrm{H} \mathrm{NMR}\left(400 \mathrm{MHz}, \mathrm{CDCl}_{3}\right) \delta 8.69(\mathrm{~s}, 1 \mathrm{H})$, $7.56(\mathrm{~d}, J=8.4 \mathrm{~Hz}, 1 \mathrm{H}), 7.39$ (s, 1H), 7.10 (d, $J=8.4 \mathrm{~Hz}, 1 \mathrm{H}), 6.89$ (s, 1H), 5.17 (s, 1H), 4.95 (s, 1H), $2.15(\mathrm{~s}, 3 \mathrm{H}) ;{ }^{13} \mathrm{C}$ NMR $\left(100 \mathrm{MHz}, \mathrm{CDCl}_{3}\right) \delta 143.8,139.7,136.4,132.2,127.9,123.3,122.0,115.5,114.7$, 101.9, 23.8; IR (ATR) 3387, 1488, 1333, $814 \mathrm{~cm}^{-1}$; HRMS (ESI) calcd for $\mathrm{C}_{11} \mathrm{H}_{11} \mathrm{~N}_{2} \mathrm{O}_{2}\left(\mathrm{M}+\mathrm{H}^{+}\right) 203.0821$, found

4-Nitro-5-(1-propen-2-yl)indole (366) and pyrroloindole (367). Reaction of a solution of 330 (62.8 mg, $0.268 \mathrm{mmol}), \mathrm{Pd}(\mathrm{dba})_{2}(9.3 \mathrm{mg}, 0.016 \mathrm{mmol})$, dppp (6.6 mg, $\left.0.016 \mathrm{mmol}\right)$ and 1,10-phen (5.8 mg, 0.032 $\mathrm{mmol})$ in DMF (2 mL), as described for $354\left(\mathrm{pCO}=6 \mathrm{~atm}, 120^{\circ} \mathrm{C}, 72 \mathrm{~h}\right)$, gave, after solvent removal and 
chromatography (Hexane/EtOAc, 8:2) an inseparable mixture of 366 and 367 (27.5 mg, 367 nearly $8 \%$ of the mixture) as a yellow oil.

1-Acetoxy-2-methyl-5-nitro-6-(1-propen-1-yl)indole(368). A solution of 352 (35.6 mg, $0.165 \mathrm{mmol}$ ), acetic anhydride $\left(\left(\mathrm{CH}_{3} \mathrm{CO}\right)_{2} \mathrm{O},(0.6 \mathrm{~mL}, 6.347 \mathrm{mmol})\right.$, 4-dimethylaminopyridine $(77 \mathrm{mg}, 0.630 \mathrm{mmol})$, and $\mathrm{Et}_{3} \mathrm{~N}(0.7 \mathrm{~mL}, 5.019 \mathrm{mmol})$ in 1,2-dichloroethane (DCE, $\left.2 \mathrm{~mL}\right)$ was stirred at $75{ }^{\circ} \mathrm{C}$ under a nitrogen atmosphere for $18 \mathrm{~h}$. After cooling to ambient temperature, the mixture was diluted with water and was extracted in EtOAc $(2 \times 10 \mathrm{~mL})$. The organic layer was dried $\left(\mathrm{MgSO}_{4}\right)$, filtered and was concentrated under reduced pressure. The resulting crude was purified by chromatography (Hexane/EtOAc, 8:2) to give 368 (42.4 mg, $0.164 \mathrm{mmol}, 100 \%)$ as a yellow oil. ${ }^{1} \mathrm{H} \mathrm{NMR}\left(600 \mathrm{MHz}, \mathrm{CDCl}_{3}\right) \delta 8.29(\mathrm{~s}, 1 \mathrm{H}), 8.16$ (s, 1H), $8.08(\mathrm{~s}, 1 \mathrm{H}), 8.03(\mathrm{~s}, 1 \mathrm{H}), 6.94(\mathrm{dd}, J=15.5,1.7 \mathrm{~Hz}, 1 \mathrm{H}), 6.82(\mathrm{dd}, J=11.5,1.5 \mathrm{~Hz}, 1 \mathrm{H}), 6.47(\mathrm{~d}$, $J=0.8 \mathrm{~Hz}, 1 \mathrm{H}), 6.43(\mathrm{~d}, J=0.8 \mathrm{~Hz}, 1 \mathrm{H}), 6.21(\mathrm{dq}, J=15.3,6.6 \mathrm{~Hz}, 1 \mathrm{H}), 5.93(\mathrm{dq}, J=11.4,7.1 \mathrm{~Hz}, 1 \mathrm{H})$, $2.74(\mathrm{~d}, J=3.5 \mathrm{~Hz}, 6 \mathrm{H}), 2.67(\mathrm{dd}, J=8.3,1.0 \mathrm{~Hz}, 6 \mathrm{H}), 1.95(\mathrm{dd}, J=6.7,1.7 \mathrm{~Hz}, 3 \mathrm{H}), 1.78(\mathrm{dd}, J=7.1$, $1.8 \mathrm{~Hz}, 3 \mathrm{H}) ;{ }^{13} \mathrm{C} \mathrm{NMR}\left(150 \mathrm{MHz}, \mathrm{CDCl}_{3}\right) \delta 170.04,170.0,144.4,143.9,139.9,139.7,139.0,138.4$, 129.8, 129.6, 128.2, 128.1, 128.0, 127.6, 127.5, 127.3, 118.0, 116.4, 116.1, 114.9, 109.7, 109.6, 27.2, 27.2, 18.7, 17.6, 17.6, 14.3; IR (ATR) 1710, 1510, 1365, $1295 \mathrm{~cm}^{-1}$; HRMS (ESI) calcd for $\mathrm{C}_{14} \mathrm{H}_{15} \mathrm{~N}_{2} \mathrm{O}_{3}$ $\left(\mathrm{M}+\mathrm{H}^{+}\right) 259.1083$, found

2-Methyl-5-nitro-6-(1-propen-1-yl)indole(352). Reaction of a solution of $\mathbf{3 6 8}$ (33.4 mg, $0.129 \mathrm{mmol}$ ), $\mathrm{Pd}(\mathrm{dba})_{2}(3.7 \mathrm{mg}, 0.006 \mathrm{mmol})$, dppp $(2.7 \mathrm{mg}, 0.007 \mathrm{mmol})$, and 1,10-phen $(2.3 \mathrm{mg}, 0.013 \mathrm{mmol})$ in DMF $(2 \mathrm{~mL})$, as described for $354\left(\mathrm{pCO}=6 \mathrm{~atm}, 120{ }^{\circ} \mathrm{C}, 36 \mathrm{~h}\right)$, gave, after solvent removal and chromatography (Hexane/EtOAc, 7:3) $352(11 \mathrm{mg}, 0.051 \mathrm{mmol}, 39 \%)$ as a yellow solid.

1-Acetoxy-2-methyl-5-nitro-4-(1-propen-2-yl)indole (369). Treatment of a solution of 356 (50 mg, $0.231 \mathrm{mmol})$ with $\left(\mathrm{CH}_{3} \mathrm{CO}\right)_{2} \mathrm{O}(0.84 \mathrm{~mL}, 8.996 \mathrm{mmol})$ in the presence of $\mathrm{Et}_{3} \mathrm{~N}(0.98 \mathrm{~mL}, 7.030 \mathrm{mmol})$, and DMAP (108 mg, $0.883 \mathrm{mmol})$ in DCE (3 mL) under nitrogen atmosphere, as described for 368 (75 ${ }^{\circ} \mathrm{C}, 18 \mathrm{~h}$ ), gave after work up and chromatography (Hexane/EtOAc, 8:2) 369 (56.3 mg, $0.218 \mathrm{mmol}$, 94\%) as a white solid. mp $=189-190{ }^{\circ} \mathrm{C} ;{ }^{1} \mathrm{H}$ NMR $\left(600 \mathrm{MHz}, \mathrm{CDCl}_{3}\right) \delta 8.49(\mathrm{~d}, J=9.1 \mathrm{~Hz}, 1 \mathrm{H}), 7.87(\mathrm{~d}, J$ $=9.1 \mathrm{~Hz}, 1 \mathrm{H}), 7.29(\mathrm{~s}, 1 \mathrm{H}), 5.33(\mathrm{~s}, 1 \mathrm{H}), 4.91(\mathrm{~s}, 1 \mathrm{H}), 2.62(\mathrm{~s}, 3 \mathrm{H}), 2.35(\mathrm{~s}, 3 \mathrm{H}), 2.26(\mathrm{~s}, 3 \mathrm{H}) ;{ }^{13} \mathrm{C} \mathrm{NMR}$ $\left(150 \mathrm{MHz}, \mathrm{CDCl}_{3}\right) \delta 168.3,144.3,140.1,138.0,132.8,128.3,125.5,121.1,119.4,116.6,115.5,25.5$, 23.9, 11.9; IR (ATR) 1718, 1533, 1371, $1303 \mathrm{~cm}^{-1}$;

3-Methyl-5-nitro-4-(1-propen-2-yl)indole (356). Reaction of a solution of $\mathbf{3 6 9}$ (27.5 mg, $0.106 \mathrm{mmol}$ ), $\operatorname{Pd}(\mathrm{dba})_{2}(3.1 \mathrm{mg}, 0.005 \mathrm{mmol})$, dppp $(2.2 \mathrm{mg}, 0.005 \mathrm{mmol})$, and 1,10-phen (1.9 $\left.\mathrm{mg}, 0.011 \mathrm{mmol}\right)$ in 
DMF (2 mL), as described for $354\left(\mathrm{pCO}=6 \mathrm{~atm}, 120{ }^{\circ} \mathrm{C}, 48 \mathrm{~h}\right.$ ), gave, after solvent removal and chromatography (Hexane/EtOAc, 7:3) a mixture of $\mathbf{3 5 6}$ and the pyrroloindole (7.2 $\mathrm{mg}$ ).

3-Methyl-5-nitro-4-(1-propen-2-yl)-1-tosylindole (370). To a slurry of $\mathrm{NaH}$ (60\%, $84.5 \mathrm{mg}, 2.112$ $\mathrm{mmol})$ in DMF $(2 \mathrm{~mL})$ at $0{ }^{\circ} \mathrm{C}$ under a nitrogen atmosphere was added the solution of 356 (228.3 $\mathrm{mg}$, $1.056 \mathrm{mmol})$ in DMF (2 mL) via a syringe and the red solution was allowed to stir for $20 \mathrm{~min}$. A solution of $p$-toluenesulfonyl chloride ( $\mathrm{TsCl}, 241.6 \mathrm{mg}, 1.267 \mathrm{mmol}$ ) in DMF (2 mL) was added drop wise via a syringe at the same temperature. The orange solution was allowed to warm to ambient temperature. After $8 \mathrm{~h}$, the reaction mixture was quenched with saturated solution of $\mathrm{NaHCO}_{3}$ and diluted with EtOAc (20 $\mathrm{mL})$. The organic layer was washed with water $(6 \times 10 \mathrm{~mL})$, dried $\left(\mathrm{MgSO}_{4}\right)$ and filtered. The filtrate was concentrated under reduced pressure and purified by chromatography (Hexane/EtOAc, 8:2) to afford 370 (306 mg, $0.826 \mathrm{mml}, 78 \%)$ as a white solid. $\mathrm{mp}=154-155{ }^{\circ} \mathrm{C} ;{ }^{1} \mathrm{H} \mathrm{NMR}\left(600 \mathrm{MHz}, \mathrm{CDCl}_{3}\right) \delta 8.00(\mathrm{~d}, J=$ $9.1 \mathrm{~Hz}, 1 \mathrm{H}), 7.85(\mathrm{~d}, J=9.1 \mathrm{~Hz}, 1 \mathrm{H}), 7.80(\mathrm{~d}, J=8.3 \mathrm{~Hz}, 2 \mathrm{H}), 7.47(\mathrm{~s}, 1 \mathrm{H}), 7.27(\mathrm{~d}, J=8.2 \mathrm{~Hz}, 2 \mathrm{H}), 5.29$ (s, 1H), 4.85 (s, 1H), 2.35 (s, 3H), 2.32 (s, 3H), 2.21 (s, 3H); $\left.{ }^{13} \mathrm{C} \mathrm{NMR} \mathrm{(150} \mathrm{MHz,} \mathrm{CDCl}_{3}\right) \delta$ 145.5, 144.0, 139.7, 136.9, 134.7, 133.4, 130.0, 128.4, 126.8, 126.2, 120.3, 119.3, 116.5, 112.3, 25.24, 21.44, 11.63; IR (ATR) $1518,1341,1176,906,728 \mathrm{~cm}^{-1}$;

(371). Reaction of a solution of $\mathbf{3 7 0}(53.6 \mathrm{mg}, 0.145 \mathrm{mmol}), \mathrm{Pd}(\mathrm{dba})_{2}(4.3 \mathrm{mg}, 0.008 \mathrm{mmol})$, dppp (3.2 $\mathrm{mg}, 0.008 \mathrm{mmol}$ ), and 1,10-phen $(2.7 \mathrm{mg}, 0.015 \mathrm{mmol})$ in DMF (2 mL), as described for 336 (pCO=6 atm, $120{ }^{\circ} \mathrm{C}, 48 \mathrm{~h}$ ), gave, after solvent removal and chromatography (Hexane/EtOAc, 7:3) 371 (43.2 mg, $0.128 \mathrm{mmol}, 88 \%)$ as an off white solid. $\mathrm{mp}=147-148{ }^{\circ} \mathrm{C} ;{ }^{1} \mathrm{H} \mathrm{NMR}\left(600 \mathrm{MHz}, \mathrm{CDCl}_{3}\right) \delta 8.07(\mathrm{~s}, 1 \mathrm{H})$, $7.88(\mathrm{~d}, J=8.9 \mathrm{~Hz}, 1 \mathrm{H}), 7.68(\mathrm{~d}, J=8.1 \mathrm{~Hz}, 2 \mathrm{H}), 7.35(\mathrm{~s}, 1 \mathrm{H}), 7.22(\mathrm{~d}, J=8.9 \mathrm{~Hz}, 1 \mathrm{H}), 7.07(\mathrm{~d}, J=8.0$ $\mathrm{Hz}, 2 \mathrm{H}), 6.92(\mathrm{~s}, 1 \mathrm{H}), 2.51(\mathrm{~s}, 3 \mathrm{H}), 2.49(\mathrm{~s}, 3 \mathrm{H}), 2.21(\mathrm{~s}, 3 \mathrm{H}) ;{ }^{13} \mathrm{C} \mathrm{NMR}\left(150 \mathrm{MHz}, \mathrm{CDCl}_{3}\right) \delta 144.3$, 135.3, 133.9, 130.6, 129.5, 126.5, 124.3, 123.1, 122.9, 120.3, 118.8, 111.1, 108.9, 108.5, 21.34, 14.97, 14.89; IR (ATR) 3417, 1353, 1165, $1087 \mathrm{~cm}^{-1}$;

(372). Treatment of $\mathbf{3 5 0}$ (119.6 mg, $0.553 \mathrm{mmol})$ with $\mathrm{TsCl}(137 \mathrm{mg}, 0.719 \mathrm{mmol})$ in $\mathrm{DMF}(4 \mathrm{~mL})$ in the presence of $\mathrm{NaH}(60 \%, 44.3 \mathrm{mg}, 1.108 \mathrm{mmol})$, as described for $370\left(0{ }^{\circ} \mathrm{C}\right.$-ambient temperature, $\left.8 \mathrm{~h}\right)$, gave after work up and chromatography $372(158.2 \mathrm{mg}, 0.427 \mathrm{mmol}, 77 \%)$ as a white solid. $\mathrm{mp}=177-178$ ${ }^{\circ} \mathrm{C} ;{ }^{1} \mathrm{H}$ NMR (600 MHz, $\left.\mathrm{CDCl}_{3}\right) \delta 8.57(\mathrm{~s}, 1 \mathrm{H}), 7.79(\mathrm{~d}, J=8.3 \mathrm{~Hz}, 2 \mathrm{H}), 7.53(\mathrm{~s}, 1 \mathrm{H}), 7.36(\mathrm{~s}, 1 \mathrm{H}), 7.26$ $(\mathrm{d}, J=8.3 \mathrm{~Hz}, 2 \mathrm{H}), 5.16(\mathrm{~s}, 1 \mathrm{H}), 4.92(\mathrm{~s}, 1 \mathrm{H}), 2.36(\mathrm{~s}, 3 \mathrm{H}), 2.28(\mathrm{~d}, J=0.7 \mathrm{~Hz}, 3 \mathrm{H}), 2.09(\mathrm{~s}, 3 \mathrm{H}) ;{ }^{13} \mathrm{C}$ $\mathrm{NMR}\left(150 \mathrm{MHz}, \mathrm{CDCl}_{3}\right) \delta 145.5,145.1,143.6,134.7,134.6,134.3,132.7,130.1,127.6,126.7,120.7$, 118.2, 114.9, 110.2, 23.6, 21.5, 9.4; IR (ATR) 1516, 1370, 1175, $906 \mathrm{~cm}^{-1}$; 
(373). Treatment of 348 (58.3 mg, $0.270 \mathrm{mmol})$ with $\mathrm{TsCl}(66.9 \mathrm{mg}, 0.351 \mathrm{mmol})$ in $\mathrm{DMF}(3 \mathrm{~mL})$ in the presence of $\mathrm{NaH}(60 \%, 21.6 \mathrm{mg}, 0.540 \mathrm{mmol})$, as described for $370\left(0{ }^{\circ} \mathrm{C}\right.$-ambient temperature, $\left.8 \mathrm{~h}\right)$, gave after work up and chromatography $373(69.4 \mathrm{mg}, 0.187 \mathrm{mmol}, 69 \%)$ as a faint yellow solid. $\mathrm{mp}=181-182{ }^{\circ} \mathrm{C} ;{ }^{1} \mathrm{H}$ NMR $\left(400 \mathrm{MHz}, \mathrm{CDCl}_{3}\right) \delta 8.89$ (s, 1H), $8.76(\mathrm{~s}, 1 \mathrm{H}), 7.73$ (d, J=8.4 Hz, 1H), 7.70 $(\mathrm{d}, J=8.4 \mathrm{~Hz}, 2 \mathrm{H}), 7.49$ (s, 1H), $7.30(\mathrm{~d}, J=11.2 \mathrm{~Hz}, 1 \mathrm{H}), 7.27-7.23(\mathrm{~m}, 3 \mathrm{H}), 6.88$ (dd, $J=15.5,1.4$ $\mathrm{Hz}, 1 \mathrm{H}), 6.75(\mathrm{dd}, J=11.4,1.1 \mathrm{~Hz}, 1 \mathrm{H}), 6.39(\mathrm{~s}, 1 \mathrm{H}), 6.36(\mathrm{~s}, 1 \mathrm{H}), 6.18-6.08(\mathrm{~m}, 1 \mathrm{H}), 5.89(\mathrm{dq}, J=$ 11.5, $7.0 \mathrm{~Hz}, 1 \mathrm{H}), 2.64(\mathrm{~s}, 3 \mathrm{H}), 2.62(\mathrm{~s}, 3 \mathrm{H}), 2.38(\mathrm{~s}, 3 \mathrm{H}), 2.37$ (s, 3H), $1.92(\mathrm{dd}, J=6.6,1.6 \mathrm{~Hz}, 3 \mathrm{H})$, $1.71(\mathrm{dd}, J=7.0,1.7 \mathrm{~Hz}, 3 \mathrm{H}) ;{ }^{13} \mathrm{C} \mathrm{NMR}\left(100 \mathrm{MHz}, \mathrm{CDCl}_{3}\right) \delta 145.6,145.5,144.7,144.2,142.8,142.8$, $135.5,134.5,134.4,133.3,132.8,130.2$, 130.2, 129.3, 129.6, 128.1, 127.5, 127.2, 126.9, 126.6, 126.5, 122.2, 119.1, 111.5, 111.2, 108.9, 108.8, 21.6, 18.7, 15.8, 14.3; IR (ATR) 1518, 1341, 1175, 1096, 811 $\mathrm{cm}^{-1}$;

(374). Treatment of 353 (159.2 mg, $0.736 \mathrm{mmol})$ with $\mathrm{TsCl}(168.4 \mathrm{mg}, 0.883 \mathrm{mmol})$ in $\mathrm{DMF}(4 \mathrm{~mL})$ in the presence of $\mathrm{NaH}(60 \%, 58.8 \mathrm{mg}, 1.472 \mathrm{mmol})$, as described for $370\left(0{ }^{\circ} \mathrm{C}\right.$-ambient temperature, $\left.8 \mathrm{~h}\right)$, gave after work up and chromatography $374(197.5 \mathrm{mg}, 0.533 \mathrm{mmol}, 72 \%)$ as a white solid. $\mathrm{mp}=169-170$ ${ }^{\circ} \mathrm{C} ;{ }^{1} \mathrm{H}$ NMR (600 MHz, $\left.\mathrm{CDCl}_{3}\right) \delta 8.05$ (s, 1H), 7.89 (s, 1H), 7.77 (d, J = 8.3 Hz, 2H), 7.44 (s, 1H), 7.26 $(\mathrm{d}, J=8.2 \mathrm{~Hz}, 2 \mathrm{H}), 5.21(\mathrm{~s}, 1 \mathrm{H}), 4.95(\mathrm{~s}, 1 \mathrm{H}), 2.36(\mathrm{~s}, 3 \mathrm{H}), 2.27(\mathrm{~s}, 3 \mathrm{H}), 2.12(\mathrm{~s}, 3 \mathrm{H}) ;{ }^{13} \mathrm{C}$ NMR $(150$ $\left.\mathrm{MHz} \mathrm{CDCl}_{3}\right) \delta 145.5,144.3,143.5,136.6,135.8,134.7,130.4,130.0,126.7,125.7,118.7,116.3,115.3$, 114.7, 23.6, 21.5, 9.4; IR (ATR) 1513, 1338, 1175, 905, $726 \mathrm{~cm}^{-1}$;

(375). Treatment of 352 (127 mg, $0.587 \mathrm{mmol})$ with $\mathrm{TsCl}$ (146.5 mg, $0.768 \mathrm{mmol})$ in DMF (4 mL) in the presence of $\mathrm{NaH}(60 \%, 47.2 \mathrm{mg}, 1.180 \mathrm{mmol})$, as described for $\mathbf{3 7 0}\left(0{ }^{\circ} \mathrm{C}\right.$-ambient temperature, $\left.8 \mathrm{~h}\right)$, gave after work up and chromatography (Hexane/EtOAc, 9:1), 375 (154.7 $\mathrm{mg}, 0.418 \mathrm{mmol}, 71 \%)$ as a faint yellow oil. ${ }^{1} \mathrm{H}$ NMR (600 MHz, $\left.\mathrm{CDCl}_{3}\right) \delta 8.32(\mathrm{~s}, 1 \mathrm{H}), 8.11(\mathrm{~d}, J=11.8 \mathrm{~Hz}, 2 \mathrm{H}), 7.95(\mathrm{~s}, 1 \mathrm{H}), 7.67$ $(\mathrm{dd}, J=8.2,3.2 \mathrm{~Hz}, 4 \mathrm{H}), 7.26(\mathrm{~d}, J=7.8 \mathrm{~Hz}, 4 \mathrm{H}), 6.91(\mathrm{~d}, J=15.4 \mathrm{~Hz}, 1 \mathrm{H}), 6.80(\mathrm{~d}, J=11.5 \mathrm{~Hz}, 1 \mathrm{H})$, $6.43(\mathrm{~s}, 1 \mathrm{H}), 6.38(\mathrm{~s}, 1 \mathrm{H}), 6.26(\mathrm{dq}, J=13.3,6.7 \mathrm{~Hz}, 1 \mathrm{H}), 5.94(\mathrm{dq}, J=14.1,7.0 \mathrm{~Hz}, 1 \mathrm{H}), 2.63(\mathrm{~s}, 3 \mathrm{H})$, $2.60(\mathrm{~s}, 3 \mathrm{H}), 2.37(\mathrm{~s}, 6 \mathrm{H}), 1.97(\mathrm{~d}, J=5.8 \mathrm{~Hz}, 3 \mathrm{H}), 1.78(\mathrm{dd}, J=6.8,0.9 \mathrm{~Hz}, 3 \mathrm{H}) ;{ }^{13} \mathrm{C} \mathrm{NMR}(150 \mathrm{MHz}$, $\left.\mathrm{CDCl}_{3}\right) \delta 145.5,145.5,144.7,144.3,140.7,140.6,139.0,138.4,135.7,130.1,130.1,129.4,128.1,127.9$, $127.8,127.2$, 127.0, 126.4, 126.3, 116.8, 116.6, 116.4, 113.3, 109.1, 109.1, 21.5, 18.7, 15.7, 14.3; IR (ATR) 1521, 1342, 1165, 1096, $843 \mathrm{~cm}^{-1}$;

(376). Treatment of 354 (41.6 mg, $0.221 \mathrm{mmol}$ ) with $\mathrm{TsCl}(50.6 \mathrm{mg}, 0.265 \mathrm{mmol})$ in $\mathrm{DMF}(4 \mathrm{~mL})$ in the presence of $\mathrm{NaH}(60 \%, 10.6 \mathrm{mg}, 0.442 \mathrm{mmol})$, as described for $370\left(0{ }^{\circ} \mathrm{C}\right.$-ambient temperature, $\left.12 \mathrm{~h}\right)$, gave after work up and chromatography (Hexane/EtOAc, 9:1) 376 (58.5 mg, $0.171 \mathrm{mmol}, 77 \%$ ) as a 
white solid. $\mathrm{mp}=148-149{ }^{\circ} \mathrm{C} ;{ }^{1} \mathrm{H} \mathrm{NMR}\left(600 \mathrm{MHz}, \mathrm{CDCl}_{3}\right) \delta 8.03-7.96(\mathrm{~m}, 2 \mathrm{H}), 7.79(\mathrm{~d}, J=8.3 \mathrm{~Hz}$, 2H), $7.71(\mathrm{~d}, J=3.7 \mathrm{~Hz}, 1 \mathrm{H}), 7.28(\mathrm{~d}, J=8.1 \mathrm{~Hz}, 2 \mathrm{H}), 7.17(\mathrm{dd}, J=17.6,11.4 \mathrm{~Hz}, 1 \mathrm{H}), 6.94(\mathrm{~d}, J=3.7$ $\mathrm{Hz}, 1 \mathrm{H}), 5.69(\mathrm{~d}, J=11.4 \mathrm{~Hz}, 1 \mathrm{H}), 5.58(\mathrm{~d}, J=17.7 \mathrm{~Hz}, 1 \mathrm{H}), 2.37(\mathrm{~s}, 3 \mathrm{H}) ;{ }^{13} \mathrm{C} \mathrm{NMR}\left(150 \mathrm{MHz}, \mathrm{CDCl}_{3}\right)$ $\delta 145.8,143.1,136.7,134.6,131.3,130.2,130.0,128.9,128.6,126.9,121.4,120.9$, 112.5, 109.3, 21.6; IR (ATR) $1513,1374,1168,823 \mathrm{~cm}^{-1}$;

(377). Treatment of 355 (128.7 mg, $0.595 \mathrm{mmol})$ with $\mathrm{TsCl}$ (147.5 mg, $0.774 \mathrm{mmol})$ in DMF (5 mL) in the presence of $\mathrm{NaH}(60 \%, 47.6 \mathrm{mg}, 1.190 \mathrm{mmol})$, as described for $370\left(0{ }^{\circ} \mathrm{C}\right.$-ambient temperature, $\left.10 \mathrm{~h}\right)$, gave after work up and chromatography (Hexane/EoOAc, 8:2), 377 (174.4 mg, $0.471 \mathrm{mmol}, 79 \%)$ as a yellow oil. ${ }^{1} \mathrm{H}$ NMR $\left(400 \mathrm{MHz}, \mathrm{CDCl}_{3}\right) \delta 8.17(\mathrm{~d}, J=9.2 \mathrm{~Hz}, 1 \mathrm{H}), 8.11(\mathrm{~d}, J=9.2 \mathrm{~Hz}, 1 \mathrm{H}), 7.98(\mathrm{~d}, J=$ $9.2 \mathrm{~Hz}, 1 \mathrm{H}), 7.86(\mathrm{~d}, J=9.2 \mathrm{~Hz}, 1 \mathrm{H}), 7.70(\mathrm{dd}, J=8.2,5.5 \mathrm{~Hz}, 3 \mathrm{H}), 7.26(\mathrm{dd}, J=8.1,4.1 \mathrm{~Hz}, 3 \mathrm{H}), 6.82$ $(\mathrm{dd}, J=15.9,1.5 \mathrm{~Hz}, 1 \mathrm{H}), 6.71(\mathrm{dd}, J=11.4,1.1 \mathrm{~Hz}, 1 \mathrm{H}), 6.60(\mathrm{~s}, 1 \mathrm{H}), 6.34(\mathrm{~s}, 1 \mathrm{H}), 6.00$ (ddq, $J=20.8$, 11.5, $6.8 \mathrm{~Hz}, 2 \mathrm{H}), 2.62(\mathrm{~s}, 6 \mathrm{H}), 2.36(\mathrm{~d}, J=2.7 \mathrm{~Hz}, 6 \mathrm{H}), 1.95(\mathrm{dd}, J=6.6,1.6 \mathrm{~Hz}, 3 \mathrm{H}), 1.42(\mathrm{dd}, J=7.0$, $1.6 \mathrm{~Hz}, 3 \mathrm{H}) ;{ }^{13} \mathrm{C} \mathrm{NMR}\left(100 \mathrm{MHz}, \mathrm{CDCl}_{3}\right) \delta 145.5,145.5,143.7,143.1,139.9,139.6,138.6,138.2,135.6$, $135.5,133.3,130.1,130.0,129.3,129.1,128.9,127.0,126.3,126.3,125.9,124.7,123.8,120.0,119.9$, 113.0, 112.6, 109.7, 109.6, 21.4, 18.8, 15.6, 14.9; IR (ATR) 1518, 1365, 1176, 1095, $810 \mathrm{~cm}^{-1}$;

(378). Treatment of $\mathbf{3 6 6}(50.8 \mathrm{mg}, 0.251 \mathrm{mmol})$ with $\mathrm{TsCl}(57.4 \mathrm{mg}, 0.301 \mathrm{mmol})$ in $\mathrm{DMF}(4 \mathrm{~mL})$ in the presence of $\mathrm{NaH}(60 \%, 20 \mathrm{mg}, 0.502 \mathrm{mmol})$, as described for $370\left(0{ }^{\circ} \mathrm{C}\right.$-ambient temperature, $\left.8 \mathrm{~h}\right)$, gave after work up and chromatography (Hexane/EtOAc, 9:1), 378 (69 mg, $0.194 \mathrm{mmol}, 77 \%$ ) as a white solid. $\mathrm{mp}=128-129{ }^{\circ} \mathrm{C} ;{ }^{1} \mathrm{H}$ NMR $\left(600 \mathrm{MHz}, \mathrm{CDCl}_{3}\right) \delta 8.14(\mathrm{~d}, J=8.5 \mathrm{~Hz}, 1 \mathrm{H}), 7.78(\mathrm{~d}, J=8.3 \mathrm{~Hz}, 2 \mathrm{H}), 7.72$ $(\mathrm{d}, J=3.7 \mathrm{~Hz}, 1 \mathrm{H}), 7.27(\mathrm{~d}, J=8.4 \mathrm{~Hz}, 2 \mathrm{H}), 7.24(\mathrm{~d}, J=8.5 \mathrm{~Hz}, 1 \mathrm{H}), 6.94(\mathrm{~d}, J=3.6 \mathrm{~Hz}, 1 \mathrm{H}), 5.17(\mathrm{~s}$, $1 \mathrm{H}), 4.92(\mathrm{~s}, 1 \mathrm{H}), 2.36(\mathrm{~s}, 3 \mathrm{H}), 2.10(\mathrm{~s}, 3 \mathrm{H}) ;{ }^{13} \mathrm{C} \mathrm{NMR}\left(150 \mathrm{MHz}, \mathrm{CDCl}_{3}\right) \delta 145.7,142.4,140.4,134.7$, 134.4, 130.2, 129.3, 126.9, 126.0, 124.9, 117.1, 115.8, 106.9, 23.5, 21.6; IR (ATR) 1512, 1373, 1161, $1128,662 \mathrm{~cm}^{-1}$;

(379). Treatment of $337(39.8 \mathrm{mg}, 0.211 \mathrm{mmol})$ with $\mathrm{TsCl}(52.4 \mathrm{mg}, 0.275 \mathrm{mmol})$ in $\mathrm{DMF}(3 \mathrm{~mL})$ in the presence of $\mathrm{NaH}(60 \%, 16.9 \mathrm{mg}, 0.422 \mathrm{mmol})$, as described for $370\left(0{ }^{\circ} \mathrm{C}\right.$-ambient temperature, $\left.10 \mathrm{~h}\right)$, gave after work up and chromatography (Hexane/EtOAc, 8:2) 379 (58.2 $\mathrm{mg}, 0.170 \mathrm{mmol}, 81 \%)$ as a colorless oil. ${ }^{1} \mathrm{H}$ NMR $\left(600 \mathrm{MHz}, \mathrm{CDCl}_{3}\right) \delta$ 7.72-7.70 (m, 3H), $7.60(\mathrm{~d}, J=8.3 \mathrm{~Hz}, 1 \mathrm{H}), 7.48(\mathrm{~d}, J=8.3$ $\mathrm{Hz}, 1 \mathrm{H}), 7.25(\mathrm{~d}, J=8.5 \mathrm{~Hz}, 2 \mathrm{H}), 6.74(\mathrm{~d}, J=3.7 \mathrm{~Hz}, 1 \mathrm{H}), 6.70(\mathrm{dd}, J=16.8,10.8 \mathrm{~Hz}, 1 \mathrm{H}), 5.80(\mathrm{~d}, J=$ $17.2 \mathrm{~Hz}, 1 \mathrm{H}), 5.45(\mathrm{~d}, J=11.0 \mathrm{~Hz}, 1 \mathrm{H}), 2.36(\mathrm{~s}, 3 \mathrm{H}) ;{ }^{13} \mathrm{C} \mathrm{NMR}\left(150 \mathrm{MHz}, \mathrm{CDCl}_{3}\right) \delta 145.3,137.6,134.8$, 133.8, 130.8, 130.2, 129.7, 127.2, 127.0, 123.9, 123.6, 121.8, 119.6, 109.4, 21.6; IR (ATR) 1593, 1514, $1344,1161,662 \mathrm{~cm}^{-1}$; 
(380). Reaction of a solution of 372 (76.4 mg, $0.206 \mathrm{mmol}), \operatorname{Pd}(\mathrm{dba})_{2}(7.1 \mathrm{mg}, 0.012 \mathrm{mmol})$, dppp (5.1 $\mathrm{mg}, 0.012 \mathrm{mmol}$ ), and 1,10-phen $(4.5 \mathrm{mg}, 0.025 \mathrm{mmol})$ in DMF $(2 \mathrm{~mL})$, as described for $336(\mathrm{pCO}=6$ atm, $120{ }^{\circ} \mathrm{C}, 24 \mathrm{~h}$ ), gave, after solvent removal and chromatography (Hexane/EtOAc, 7:3), 380 (55.4 mg, $0.164 \mathrm{mmol}, 79 \%)$ as an off white solid. $\mathrm{mp}=143-144{ }^{\circ} \mathrm{C} ;{ }^{1} \mathrm{H} \mathrm{NMR}\left(400 \mathrm{MHz}, \mathrm{CDCl}_{3}\right) \delta 7.98(\mathrm{~s}, 1 \mathrm{H})$, $7.94(\mathrm{~s}, 1 \mathrm{H}), 7.68(\mathrm{~d}, J=8.3 \mathrm{~Hz}, 2 \mathrm{H}), 7.49(\mathrm{~s}, 1 \mathrm{H}), 7.22$ (d, $J=0.8 \mathrm{~Hz}, 1 \mathrm{H}), 7.08$ (d, $J=8.1 \mathrm{~Hz}, 2 \mathrm{H}), 6.98$ $(\mathrm{s}, 1 \mathrm{H}), 2.33(\mathrm{~s}, 3 \mathrm{H}), 2.28(\mathrm{~d}, J=0.6 \mathrm{~Hz}, 3 \mathrm{H}), 2.23(\mathrm{~s}, 3 \mathrm{H}) ;{ }^{13} \mathrm{C} \mathrm{NMR}\left(100 \mathrm{MHz}, \mathrm{CDCl}_{3}\right) \delta 144.2,135.4$, 135.0, 133.0, 129.5, 126.8, 126.6, 126.3, 122.6, 122.1, 119.6, 111.0, 107.9, 95.9, 21.4, 10.0, 9.8; IR (ATR) $3417,1444,1353,1165,1087 \mathrm{~cm}^{-1}$;

(381). Reaction of a solution of $\mathbf{3 7 3}$ (41.1 mg, $0.111 \mathrm{mmol}), \mathrm{Pd}(\mathrm{dba})_{2}(3.8 \mathrm{mg}, 0.007 \mathrm{mmol})$, dppp (2.7 $\mathrm{mg}, 0.007 \mathrm{mmol})$, and 1,10-phen $(2.4 \mathrm{mg}, 0.013 \mathrm{mmol})$ in DMF $(2 \mathrm{~mL})$, as described for 336 (pCO=6 atm, $120{ }^{\circ} \mathrm{C}, 24 \mathrm{~h}$ ), gave, after solvent removal and chromatography (Hexane/EtOAc, 8:2) 381 (26.4 mg, $0.078 \mathrm{mmol}, 70 \%)$ as an off white solid. $\mathrm{mp}=153^{\circ} \mathrm{C}$ (dec.) ; ${ }^{1} \mathrm{H} \mathrm{NMR}\left(400 \mathrm{MHz}, \mathrm{CDCl}_{3}\right) \delta 8.11(\mathrm{~s}, 1 \mathrm{H})$, $7.88(\mathrm{~s}, 1 \mathrm{H}), 7.59(\mathrm{~d}, J=8.5 \mathrm{~Hz}, 2 \mathrm{H}), 7.41(\mathrm{~s}, 1 \mathrm{H}), 7.11(\mathrm{~d}, J=8.0 \mathrm{~Hz}, 2 \mathrm{H}), 6.35(\mathrm{~s}, 1 \mathrm{H}), 6.19(\mathrm{dt}, J=$ 1.9, $0.9 \mathrm{~Hz}, 1 \mathrm{H}), 2.57(\mathrm{~d}, J=1.2 \mathrm{~Hz}, 3 \mathrm{H}), 2.44(\mathrm{~d}, J=0.9 \mathrm{~Hz}, 3 \mathrm{H}), 2.28(\mathrm{~s}, 3 \mathrm{H}) ;{ }^{13} \mathrm{C} \mathrm{NMR}(100 \mathrm{MHz}$, $\left.\mathrm{CDCl}_{3}\right) \delta 144.2,136.2,136.1,136.0,134.7,134.0,129.6,127.0,126.1,124.7,110.7,109.0,99.5,96.5$, 21.5, 16.1, 13.9; IR (ATR) 3418, 1353, 1165, $1086 \mathrm{~cm}^{-1}$;

(382). Reaction of a solution of $374(61.2 \mathrm{mg}, 0.165 \mathrm{mmol}), \mathrm{Pd}(\mathrm{dba})_{2}(6.7 \mathrm{mg}, 0.012 \mathrm{mmol})$, dppp (4.8 $\mathrm{mg}, 0.012 \mathrm{mmol}$ ), and 1,10-phen $(4.2 \mathrm{mg}, 0.023 \mathrm{mmol})$ in DMF (2 mL), as described for 336 (pCO=6 atm, $120{ }^{\circ} \mathrm{C}, 24 \mathrm{~h}$ ), gave, after solvent removal and chromatography (Hexane/EtOAc, 7:3) 382 (49.4 mg, $0.146 \mathrm{mmol}, 88 \%)$ as an off white solid. $\mathrm{mp}=179-180{ }^{\circ} \mathrm{C} ;{ }^{1} \mathrm{H}$ NMR $\left(400 \mathrm{MHz}, \mathrm{CDCl}_{3}\right) \delta 8.12(\mathrm{~s}, 1 \mathrm{H})$, $7.78(\mathrm{~s}, 1 \mathrm{H}), 7.70(\mathrm{~d}, J=8.3 \mathrm{~Hz}, 2 \mathrm{H}), 7.23(\mathrm{~d}, J=1.0 \mathrm{~Hz}, 1 \mathrm{H}), 7.19(\mathrm{~s}, 1 \mathrm{H}), 7.07$ (d, $J=8.2 \mathrm{~Hz}, 2 \mathrm{H}), 6.97$ (s, 1H), $2.38(\mathrm{~s}, 3 \mathrm{H}), 2.22(\mathrm{~s}, 3 \mathrm{H}), 2.20(\mathrm{~d}, J=0.8 \mathrm{~Hz}, 3 \mathrm{H}) ;{ }^{13} \mathrm{C} \mathrm{NMR}\left(100 \mathrm{MHz}, \mathrm{CDCl}_{3}\right) \delta 144.1,135.1$, 134.3, 131.0, 129.5, 129.1, 127.4, 126.6, 123.1, 119.1, 111.5, 103.0, 99.8, 21.4, 9.9, 9.9; IR (ATR) 3436, $1349,1166,734 \mathrm{~cm}^{-1}$;

(383). Reaction of a solution of 375 (58.2 mg, $0.157 \mathrm{mmol}), \mathrm{Pd}(\mathrm{dba})_{2}(6.3 \mathrm{mg}, 0.011 \mathrm{mmol})$, dppp (4.5 $\mathrm{mg}, 0.011 \mathrm{mmol}$ ), and 1,10-phen (4 mg, $0.022 \mathrm{mmol}$ ) in DMF (2 mL), as described for 336 (pCO=6 atm, $120{ }^{\circ} \mathrm{C}, 25 \mathrm{~h}$ ), gave, after solvent removal and chromatography (Hexane/EtOAc, 8:2) 383 (40.5 mg, 0.120 mmol, $76 \%)$ as a faint yellow oil. ${ }^{1} \mathrm{H}$ NMR $\left(600 \mathrm{MHz}, \mathrm{CDCl}_{3}\right) \delta 8.28(\mathrm{~s}, 1 \mathrm{H}), 7.71(\mathrm{~s}, 1 \mathrm{H}), 7.62(\mathrm{~d}, J=$ $8.0 \mathrm{~Hz}, 2 \mathrm{H}), 7.11(\mathrm{~s}, 1 \mathrm{H}), 7.08(\mathrm{~d}, J=8.0 \mathrm{~Hz}, 2 \mathrm{H}), 6.31(\mathrm{~s}, 1 \mathrm{H}), 6.28(\mathrm{~s}, 1 \mathrm{H}), 2.61(\mathrm{~s}, 3 \mathrm{H}), 2.41(\mathrm{~s}, 3 \mathrm{H})$, $2.25(\mathrm{~s}, 3 \mathrm{H}) ;{ }^{13} \mathrm{C} \mathrm{NMR}\left(150 \mathrm{MHz}, \mathrm{CDCl}_{3}\right) \delta 144.1,136.7,136.2,136.1,134.2,133.0,129.5,127.3,126.1$, 125.8, 110.4, 104.7, 100.5, 99.6, 21.4, 16.2, 13.8; IR (ATR) 3436, 1359, $1166 \mathrm{~cm}^{-1}$; 
(384). Reaction of a solution of $\mathbf{3 7 6}(52.5 \mathrm{mg}, 0.153 \mathrm{mmol}), \mathrm{Pd}(\mathrm{dba})_{2}(5.3 \mathrm{mg}, 0.010 \mathrm{mmol})$, dppp (3.8 $\mathrm{mg}, 0.010 \mathrm{mmol}$ ), and 1,10-phen $(3.3 \mathrm{mg}, 0.018 \mathrm{mmol})$ in DMF $(2 \mathrm{~mL})$, as described for $336(\mathrm{pCO}=6$ atm, $120{ }^{\circ} \mathrm{C}, 25 \mathrm{~h}$ ), gave, after solvent removal and chromatography (Hexane/EtOAc, 7:3), 384 (42.4 mg, $0.137 \mathrm{mmol}, 89 \%)$ as a faint yellow oil. ${ }^{1} \mathrm{H} \mathrm{NMR}\left(400 \mathrm{MHz}, \mathrm{CDCl}_{3}\right) \delta 8.32(\mathrm{~s}, 1 \mathrm{H}), 7.87(\mathrm{~d}, J=9.0 \mathrm{~Hz}$, $1 \mathrm{H}), 7.72(\mathrm{~d}, J=8.4 \mathrm{~Hz}, 2 \mathrm{H}), 7.59(\mathrm{~d}, J=3.6 \mathrm{~Hz}, 1 \mathrm{H}), 7.30(\mathrm{~d}, J=9.0 \mathrm{~Hz}, 1 \mathrm{H}), 7.18(\mathrm{t}, J=2.8 \mathrm{~Hz}, 1 \mathrm{H})$, $7.11(\mathrm{~d}, J=8.4 \mathrm{~Hz}, 2 \mathrm{H}), 6.90(\mathrm{~d}, J=3.4 \mathrm{~Hz}, 1 \mathrm{H}), 6.70-6.64(\mathrm{~m}, 1 \mathrm{H}), 2.24(\mathrm{~s}, 3 \mathrm{H}) ;{ }^{13} \mathrm{C} \mathrm{NMR}(100 \mathrm{MHz}$, $\left.\mathrm{CDCl}_{3}\right) \delta 144.6,135.3,132.1,129.6,129.5,126.6,125.3,123.9,123.2,120.2,108.7,108.4,108.0,100.8$, 21.4; IR (ATR) 3401, 1348, 1160, 1102, $777 \mathrm{~cm}^{-1}$;

(385). Reaction of a solution of $\mathbf{3 7 7}(85.4 \mathrm{mg}, 0.230 \mathrm{mmol}), \mathrm{Pd}(\mathrm{dba})_{2}(8.0 \mathrm{mg}, 0.014 \mathrm{mmol})$, dppp (5.7 $\mathrm{mg}, 0.014 \mathrm{mmol})$, and 1,10-phen $(5.0 \mathrm{mg}, 0.028 \mathrm{mmol})$ in DMF $(2.5 \mathrm{~mL})$, as described for 336 (pCO=6 atm, $120{ }^{\circ} \mathrm{C}, 26 \mathrm{~h}$ ), gave, after solvent removal and chromatography (Hexane/EtOAc, 8:2), 385 (60.4 mg, $0.178 \mathrm{mmol}, 77 \%)$ as an off white solid. $\mathrm{mp}=134-135{ }^{\circ} \mathrm{C} ;{ }^{1} \mathrm{H}$ NMR $\left(400 \mathrm{MHz}, \mathrm{CDCl}_{3}\right) \delta 7.98(\mathrm{~d}, J=9.1$ $\mathrm{Hz}, 1 \mathrm{H}), 7.98(\mathrm{~s}, 1 \mathrm{H}), 7.62(\mathrm{~d}, J=8.2 \mathrm{~Hz}, 2 \mathrm{H}), 7.15(\mathrm{~d}, J=9.0 \mathrm{~Hz}, 1 \mathrm{H}), 7.10$ (d, $J=8.2 \mathrm{~Hz}, 2 \mathrm{H}), 6.56$ (s, $1 \mathrm{H}), 6.31(\mathrm{~s}, 1 \mathrm{H}), 2.66(\mathrm{~s}, 3 \mathrm{H}), 2.42(\mathrm{~s}, 3 \mathrm{H}), 2.27(\mathrm{~s}, 3 \mathrm{H}) ;{ }^{13} \mathrm{C} \mathrm{NMR}\left(100 \mathrm{MHz}, \mathrm{CDCl}_{3}\right) \delta 144.2,136.3$, 136.0, 134.8, 132.5, 131.5, 129.6, 126.0, 121.4, 120.4, 108.9, 108.5, 106.9, 98.7, 21.4, 16.1, 13.7; IR (ATR) 3401, 1348, 1160, $1102 \mathrm{~cm}^{-1}$;

(386). Reaction of a solution of $\mathbf{3 7 8}(59.9 \mathrm{mg}, 0.168 \mathrm{mmol}), \mathrm{Pd}(\mathrm{dba})_{2}(4.9 \mathrm{mg}, 0.010 \mathrm{mmol})$, dppp (3.5 $\mathrm{mg}, 0.010 \mathrm{mmol}$ ), and 1,10-phen (3.1 mg, $0.20 \mathrm{mmol})$ in DMF $(2 \mathrm{~mL})$, as described for 336 (pCO=6 atm, $120{ }^{\circ} \mathrm{C}, 26 \mathrm{~h}$ ), gave, after solvent removal and chromatography (Hexane/EtOAc, 8:2), 386 (47.6 mg, $0.147 \mathrm{mmol}, 87 \%$ ) as an off white solid. $\mathrm{mp}=137{ }^{\circ} \mathrm{C}$ (dec.); ${ }^{1} \mathrm{H} \mathrm{NMR}\left(400 \mathrm{MHz}, \mathrm{CDCl}_{3}\right) \delta 8.17(\mathrm{~s}, 1 \mathrm{H})$, $7.83(\mathrm{~d}, J=8.8 \mathrm{~Hz}, 1 \mathrm{H}), 7.76(\mathrm{~d}, J=8.2 \mathrm{~Hz}, 2 \mathrm{H}), 7.57(\mathrm{~d}, J=3.6 \mathrm{~Hz}, 1 \mathrm{H}), 7.50(\mathrm{~d}, J=8.8 \mathrm{~Hz}, 1 \mathrm{H}), 7.14$ $(\mathrm{d}, J=8.1 \mathrm{~Hz}, 2 \mathrm{H}), 6.90(\mathrm{~s}, 1 \mathrm{H}), 6.77(\mathrm{~d}, J=3.5 \mathrm{~Hz}, 1 \mathrm{H}), 2.35(\mathrm{~s}, 3 \mathrm{H}), 2.27$ (s, 3H); ${ }^{13} \mathrm{C} \mathrm{NMR}(100 \mathrm{MHz}$, $\left.\mathrm{CDCl}_{3}\right) \delta 144.7,135.1,131.7,129.7,128.5,126.7,124.8,123.7,119.8,116.0,115.9,112.5,106.1,105.3$, 21.4, 9.8; IR (ATR) 3415, 1353, 1165, $1087 \mathrm{~cm}^{-1}$;

(379). Reaction of a solution of $\mathbf{3 7 9}(52.7 \mathrm{mg}, 0.154 \mathrm{mmol}), \mathrm{Pd}(\mathrm{dba})_{2}(5.3 \mathrm{mg}, 0.010 \mathrm{mmol})$, dppp (3.8 $\mathrm{mg}, 0.010 \mathrm{mmol})$, and 1,10-phen $(3.3 \mathrm{mg}, 0.018 \mathrm{mmol})$ in DMF $(3 \mathrm{~mL})$, as described for 336 (pCO=6 atm, $120{ }^{\circ} \mathrm{C}, 36 \mathrm{~h}$ ), gave, after solvent removal and chromatography (Hexane/EtOAc, 7:3) 379 (37.1 mg, $0.108 \mathrm{mmol}, 70 \%$ ) as a colorless oil. 
(379). Reaction of a solution of $\mathbf{3 7 9}$ (31 mg, $0.091 \mathrm{mmol}), \mathrm{Pd}(\mathrm{OAc})_{2}(2.1 \mathrm{mg}, 0.009 \mathrm{mmol})$ and 1,10-phen $(1.7 \mathrm{mg}, 0.009 \mathrm{mmol})$ in DMF (2 mL), as described for 354 (pCO=6 atm, $\left.120{ }^{\circ} \mathrm{C}, 50 \mathrm{~h}\right)$, gave, after solvent removal and chromatography (Hexane/EtOAc, 7:3) 379 (30.2 $\mathrm{mg}, 0.088 \mathrm{mmol}, 82 \%)$ as a colorless oil. 


\section{References and Footnotes}

1. a) Bao, B.; Sun, Q.; Yao, X.; Hong, J.; Lee, C.-O.; Cho, H. Y.; Jung, J. H. J. Nat. Prod. 2007, 70, 28. b) Jung, J. H.; Shinde, P. B.; Hong, J.; Liu, Y.; Sim, C. J. Biochem. System. Ecol. 2007, 35, 4851. c) Bao, B.; Sun, Q.; Yao, X.; Hong, J.; Lee, C. O.; Sim, C. J.; Im, K. S.; Jung, J. H. J. Nat. Prod. 2005, 68, 711-715. d) Oh, K.-B.; Mar, W.; Kim, S.; Kim, J.-Y.; Oh, M.-N.; Kim, J.-G.; Shin, D.; Sim, C. J.; Shin, J. Bioorg. Med. Chem. Lett. 2005, 15, 4927-2931. e) Casapullo, A.; Bifulco, G.; Bruno, I.; Riccio, R. J. Nat. Prod. 2000, 63, 447-451. f) Gunasekera, S. P.; McCarthy, P. J.; Kelly-Borges, M. J. J. Nat. Prod. 1994, 57, 1437-1441.

2. a) Capon, R. J.; Rooney, F.; Murray, L. M.; Collins, E.; Sim, A. T. R.; Rostas, J. A. P.; Butler, M. S.; Carroll, A. R. J. Nat. Prod. 1998, 61, 660-662. b) Wright, A. E.; Pomponi, S. A.; Cross, S. S.; McCarthy, P. J. Org. Chem. 1992, 57, 4772-4775. c) Morris, S. A.; Andersen, R. J. Tetrahedron 1990, 46, 715-720. d) Kohmoto, S.; Kashman, Y.; McConnell, O. J.; Rinehart Jr., K. L.; Wright, A.; Koehn, F. J. Org. Chem. 1988, 53, 3116-3118.

3. a) Shin, J.; Seo, Y.; Cho, K. W.; Rho, J.-R.; Sim, C. J. J. Nat. Prod. 1999, 62, 647-649. b) Morris, S. A.; Andersen, R. J. Tetrahedron 1990, 46, 715-720. f) Morris, S. A.; Andersen, R. J. Can. J. Chem. 1989, 67, 677-681. c) Tsujii, S.; Rinehart, K. L.; Gunasekera, S. P.; Kashman, Y.; Cross, S. S.; Lui, M. S.; Pomponi, S. A.; Diaz, M. C. J. Org. Chem. 1988, 53, 5446-5453. d) Bartic, K.; Braekman, J. C.; Daloze, D.; Stoller, C.; Huysecom, J.; Vandevyver, G.; Ottinger, R. Can. J. Chem. 1987, 65, 2118-2121.

4. Sakemi, S.; Sun, H. H. J. Org. Chem. 1991, 56, 4304-4307.

5. Sato, H.; Tsuda, M.; Watanabe, K.; Kobayashi, J. Tetrahedron 1998, 54, 8687-8690.

6. For a recent extensive review of indoles isolated from marine organisms, see: Netz, N.; Opatz, T. Mar. Drugs 2015, 13, 4814-4914.

7. Oh, K.-B.; Mar, W.; Kim, S.; Kim, J.-Y.; Lee, T.-H.; Kim, J.-G.; Shin, D.; Sim, C. J.; Shin, J. Biol. Pharm. Bull. 2006, 29, 570-573.

8. Alvarado, S.; Roberts, B. F.; Wright, A. E.; Chakrabarti, D. Antimicrob. Agents Ch. 2013, 57, 23622364.

9. Zhu, L.-h.; Chen, C.; Wang, H.; Ye, W.-e.; Zhou, G.-x. Chem. Pharm. Bull. 2012, 60, 670-673.

10. Endo, T.; Tsuda, M.; Fromont, J.; Kobayashi, J. J. Nat. Prod. 2007, 70, 423-424.

11. Lee, Y.-L.; Lee, D.-G.; Rho, H. S.; Krasokhin, V. B.; Shin, H. J.; Lee, J. S.; Lee, H.-S. J. Heterocycl. Chem. 2013, 50, 1400-1404.

12. Kim, S. H.; Sperry, J. J. Nat. Prod. 2015, 78, 3080-3082. 
13. Kim, S. H.; Sperry, J. Tetrahedron Lett. 2015, 56, 5914-5915.

14. Tasch, B. O. A.; Merkul, E.; Müller, T. J. J. Eur. J. Org. Chem. 2011, 4532-4535.

15. Mosquera, A.; Riveiros, R.; Sestelo, J. P.; Sarandeses, L. A. Org. Lett. 2008, 10, 3745-3748.

16. For leading references see: a) Kuethe, J. T.; Davies, I. W. Tetrahedron 2006, 62, 11381-11390. b) Clawson Jr., R. W.; Deavers III, R. E.; Akhmedov, N. G.; Söderberg, B. C. G. Tetrahedron 2006, 62, 10829-10834. c) Akazome, M.; Kondo, T.; Watanabe, Y. J. Org. Chem. 1994, 59, 3375-3380.

d) Tollari, S.; Cenini, S, Crotti, C.; Gianella, E. J. Mol. Catal. 1994, 87, $203-214$.

17. Kuethe, J. T.; Wong, A.; Davies, I. W. Org. Lett. 2003, 5, 3721-3723.

18. Scott, T. L.; Söderberg, B. C. G. Tetrahedron 2003, 59, 6323-6332.

19. Dantale, S. W.; Söderberg, B. C. G. Tetrahedron 2003, 59, 5507-5514.

20. Scott, T. L.; Yu, X.; Gorunatula, S. P.; Carrero-Martínez, G.; Söderberg, B. C. G. Tetrahedron 2006, 62, 10835-10842.

21. Söderberg, B. C.; Chisnell, A. C.; O’Neil, S. N.; Shriver, J. A. J. Org. Chem. 1999, 64, 9731-9734.

22. Zhang, Y.; Hubbard, J. W.; Akhmedov, N. G.; Petersen, J. L.; Söderberg, B. C. G. J. Org. Chem. 2015, 80, 4783-4790.

23. a) Hamze, A.; Le Menez, P.; Provot, O.; Morvan, E.; Brion, J.-D.; Alami, M. Tetrahedron 2012, 66, 8698-8706. b) Hamze, A.; Veau, D.; Provot, O.; Brion, J.-D.; Alami, M. J. Org. Chem. 2009, 74, 1337. c) Alami, M.; Liron, F.; Gervais, M.; Peyrat, J.-F.; Brion, J.-D. Angew. Chem., Int. Ed. 2002, 41, 1578-1580. d) Liron, F.; Le Garrec, P.; Alami, M. Synlett 1999, 246-248.

24. Barlow, A. J.; Compton, B. J.; Weavers, R. T. J. Org. Chem. 2005, 70, 2470-2475.

25. For a review, see: Nikishkin, N. I.; Huskens, J.; Verboom, W. Org. Biomolec. Chem. 2013, 11, 3583-3602.

26. Pieterse, K.; Lauritsen, A.; Schenning, A. P. H. J.; Vekemans, J. A. J. M.; Meijer, E. W. Chem. Eur. J. 2003, 9, 5597-5604.

27. a) Lanoe, P.-H.; Tong, C. M.; Harrington, R. W.; Probert, M. R.; Clegg, W.; Williams, J. A. G.; Kozhevnikov, V. N. Chem. Commun. 2014, 50, 6831-6834. b) Hao, Q.; Yu, S.; Li, S.; Chen, J.; Zeng, Y; Yu, T.; Yang, G.; Li, Y. J. Org. Chem. 2014, 79, 459-464. c) Williams, J. D.; Bguyen, S. T.; Gu, S.; Ding, X.; Butler, M. M.; Tashjian, T. F.; Opperman, T. J.; Panchal, R. G.; Bavari, S.; Peet, N. P.; Moir, D. T.; Bowlin, T. L. Bioorg. Med. Chem. 2013, 21, 7790-7806.

28. Hasan, K.; Donato, L.; Shen, Y.; Slinker, J. D.; Zysman-Colman, E. Dalton Trans. 2014, 43, 13672-13682.

29. Pieterse, K.; Vekemans, J. A. J. M.; Kooijman, H.; Spek, A. L.; Meijer, E. W. Chem. Eur. J. 2000, $6,4597-4603$. 
30. Garg, N. K.; Stolz, B. M. Tetrahedron Lett. 2005, 46, 2423-2426.

31. Yang, C.-G.; Liu, G.; Jiang, B. J. Org. Chem. 2002, 67, 9392-9396.

32. For examples, see: a) Yoshida, H.; Shinke, A.; Kawano, Y.; Takai, K. Chem. Commun. 2015, 51, 10616-10619. b) Jeong, S.; Chen, X.; Harran, P. G. J. Org. Chem. 1998, 63, 8640-8641.

33. a) Jacqemard, U.; Dias, N.; Lansiaux, A.; Bailly, C.; Loge, C.; Robert, J.-M.; Lozach, O.; Meijer, L. Bioorg. Med. Chem. 2008, 16, 4932-4953. b) Krompiec, S.; Krompiec, M.; Ignasiak, H.; Lapkowski, M.; Baj, S.; Grabarczyk, D. Catal. Commun. 2007, 8, 1457-1462. c) Liu, C.-M.; Chen, B.-H.; Liu, W.-Y.; Wu, X.-L.; Ma, Y.-X. J. Organomet. Chem. 2000, 598, 348-352.

34. For a review of cine substitution, see: Peng, Y.; Li, W.-D. Z. Eur. J. Org. Chem. 2010, 6703-6718.

35. Chen, S.-H. Tetrahedron Lett. 1997, 38, 4741-4744.

36. Tanaka, K.; Kobayashi, T.; Mori, H.; Katsumura, S. J. Org. Chem. 2004, 69, 5906-5925.

37. Faye, D.; Vybornyi, M.; Boeda, F.; Legoupy, S. Tetrahedron 2013, 69, 5421-5425.

38. Solberg, J.; Undheim, K. Acta Chem. Scand. 1989, 43, 62-68.

39. For NMR data for 1-phenyl-1-(2-pyrimidyl)ethene, see: Itoh, M.; Hirano, K.; Satoh, T.; Miura, M. Org. Lett. 2014, 16, 2050-2053.

40. For related double Sonogashira reactions of 2,5-dibromopyridine, see: a) Miguel, D.; Alvarez de Cienfuegos, L.; Martin-Lasanta, A.; Morcillo, S. P.; Zotti, L. A.; Leary, E.; Burkle, M.; Asai, Y.; Jurado, R.; Cardenas, D. J.; Rubio-Bollinger, G.; Agrait, N.; Cuerva, J. M.; Gonzalez, M. T. J. Am. Chem. Soc. 2015, 137, 13818-13826. b) Wang, C.; Jung, G.-Y.; Batsanov, A. S.; Bryce, M. R.; Petty, M. C. J. Mat. Chem. 2002, 12, 173-180.

41. Compound $\mathbf{1 4}$ has been described in a patent but only a very cursory ${ }^{1} \mathrm{H}$ NMR was reported. Piotrowski, D. W.; Rogers, B. N.; McWhorter, W. W., Jr.; Walker, D. P.; Corbett, J. W.; Groppi, V. E., Jr.; Rudmann, D. G. PCT Int. Appl. WO 2003093250 [CAN139:395938].

42. The compound is commercially available however it was prepared in two steps from 4bromopyrimidine according to: Maji, A.; Hazra, A.; Maiti, D. Org. Lett. 2014, 16, 4524-4527.

43. Cadogan, J. I. G.; Cameron-Wood, M. Proc. Chem. Soc. 1962, 361.

44. Sundberg, R. J. J. Org. Chem. 1968, 33, 487-490.

45. For recent applications of this type of cyclization in total synthesis of indole alkaloids, see: a) Zhang, Y.; McArdle, I. W.; Hubbard, J. W.; Akhmedov, N. G.; Söderberg, B. C. G. Tetrahedron Lett. 2016, 57, 2865-2867. b) Cummings, M. M.; Clawson, R. W.; Sharma, S. B.; Byerly, R. A.; Akhmedov, N. G.; Söderberg, B. C. G. Tetrahedron 2011, 67, 4753-4757. c) Clawson, R. W.; Dacko, C. A.; Deavers, R. E.; Akhmedov, N. G.; Söderberg, B. C. G. Tetrahedron 2009, 65, 87868793. 
46. Lowinger, T. B.; Chu, J.; Spence, P. L. Tetrahedron Lett. 1995, 36, 8383-8386.

47. For reported spectral data see: Hudkins, D. L.; Diebold, J. L.; Marsh, F. D. J. Org. Chem. 1995, 60, 6218-6220.

48. Kumaran, E.; Leong, W. K. Tetrahedron Lett. 2014, 55, 5495-5498.

49. Arcadi, A.; Bianchi, G.; Marinelli, F. Synthesis 2004, 610-618.

50. a) Lopez, M. S.; Choy, J. W.; Peters, U.; Sos, M. L.; Morgan, D. O.; Shokat, K. M. J. Am. Chem. Soc. 2013, 135, 18135-18159. b) Lin, C-I.; Selvi, S.; Fang, J.-M.; Chou, P.-T.; Lai, C.-H.; Cheng, Y.-M. J. Org. Chem. 2007, 72, 3537-3542.

51. Parvatkar, P. T.; Kadam, H. K.; Parameswaran, P. S.; Tilve, S. G. Asian J. Chem. 2012, 24, 22132215.

52. Koza, D. J.; Euler, W. B. Heterocycl. Commun. 1999, 5, 399-402.

53. Bergman, J.; Koch, E.; Pelcman, B. Tetrahedron 1995, 51, 5631-5642.

54. $\mathrm{TMB}=2,4,6$-trimethylbenzoate; $\mathrm{TMphenanthroline}=3,4,7,8$-tetramethylphenanthroline.

55. Tolari, S.; Cenini, S.; Crotti, C.; Gianelli, E. J. Mol. Catal. 1994, 87, 203-214.

56. Davies, I. W.; Smitrovich, J. H.; Qu, C. PCT Int. Appl. 2005, WO 2005000804.

57. Araki, T.; Manabe, Y.; Fujioka, K.; Yokoe, H.; Kanematsu, M.; Yoshida, M.; Shishido, K. Tetrahedon Lett. 2013, 54, 1012-1014.

58. Ansari, N. H.; Söderberg, B. C. G. Tetrahedron 2016, 72, 4214-4221.

59. Spectral data for this compound has been reported, see: Berens, U.; Brown, J. M.; Long, J.; Selke, R. Tetrahedron Asymm. 1996, 7, 285-292.

60. a) Tanaka, J.; Higa, T.; Bernardinelli, G.; Jefford, C. W. Tetrahedron 1989, 45, 7301-7310. b) ElGamal, A. A.; Wang, W.-L.; Duh, C.-Y. J. Nat. Prod. 2005, 68, 815-817. c) Kubota, N. K.; Iwamoto, H.; Fukuzawa, Y.; Uchio, Y. Hetercycles 2005, 65, 2675-2682. d) Fang, H.-Y.; Chiou, S.-F.; Uvarani, C.; Wen, Z.-H.; Hsu, C.-H.; Wu, Y.-C.; Wang, W.-L.; Liaw, C.-C.; Sheu, J.-H. Bull. Chem. Soc. Jpn. 2014, 87, 1278-1280.

61. Su, H.; Yuan, Z. H.; Li, J.; Guo, S. J.; Deng, L. P.; Han, L. J.; Zhu, X. B.; Shi, D. Y. Chin. Chem. Lett. 2009, 20, 456-458.

62. Norton, R. S.; Wells, R. J. J. Am. Chem. Soc. 1982, 104, 3628-3635.

63. Shi, D. Y.; Han, L. J.; Sun, J.; Li, S.; Wang, S. Y.; Yang, Y. C.; Fan, X.; Shi, J. G. Chinese Chem. Lett. 2005, 16, 777-780.

64. Bertinetti, B. V.; Rodriguez, M. A.; Godeas, A. M.; Cabrera, G. M. J. Antibiot. 2010, 63, 681-683.

65. Tasch, B. O. A.; Antovic, D.; Merkul, E.; Müller, T. J. J. Eur. J. Org. Chem. 2013, 4564-4569.

66. Nakhai, A.; Bergman, J. Heterocycles 2014, 88, 309-318. 
67. Li, Y.; Wang, W.-H.; Yang, S.-D.; Li, B.-J.; Feng, C.; Shi, Z.-J. Chem. Commun. 2010, 46, 45534555 .

68. Piers, E.; Gladstone, P. L.; Yee, J. G. K.; McEachern, E. J. Tetrahedron 1998, 54, 10609-10626.

69. Ghosal, S.; Luke, G. P.; Kyler, K. S. J. Org. Chem. 1987, 52, 4296-4298.

70. Kang, S.-K.; Baik, T.-G.; Jiao, X. H.; Lee, Y.-T. Tetrahedron Lett. 1999, 40, 2383-2384.

71. Following the procedure: Lo, C.; Kumar, M. P.; Chang, H.; Lush, S.; Liu, R. J. Org. Chem. 2005, $70,10482-10487$.

72. Harrowven, D. C.; Curran, D. P.; Kostiuk, S. L.; Wallis-Guy, I. L.; Whiting, S.; Stenning, K. J.; Tang, B.; Packard, E.; Nanson, L. Chem. Commun. 2010, 46, 6335-6337.

73. For a synthesis of unsymmetrical 3,3'-biindoles, see: Ramesh, C.; Kavala, V.; Kuo, C.-W.; Raju, B. R.; Yao, C.-F. Eur. J. Org. Chem. 2010, 3796-3801.

74. Alford, P. E.; Fu, L.; Kubert, J. K.; Wang, L.; Gribble, G. W. Tetrahedron Lett. 2011, 52, 26422644.

75. Bocchi, V.; Palla, G. Tetrahedron 1986, 42, 5019-5024.

76. For a very recent example and references to previously reported reactions, see: Guo, T.; Han, S.-L.; Liu, Y.-C.; Liu, Y.; Liu, H.-M. Tetrahedron Lett. 2016, 57, 1097-1099.

77. a) Racemosine B: Liu, D.-Q.; Mao, S.-C.; Zhang, H.-Y.; Yu, X.-Q.; Feng, M.-T.; Wang, B.; Feng, L.-H.; Guo, Y.-W. Fitotherapia. 2013, 91, 15-20. b) Asteropusazole A and B: Russell, F.; Harmody, D.; McCarthy, P. J.; Pomponi, S. A.; Wright, A. E. J. Nat. Prod. 2013, 76, 1989-1992. c) Ancorinazole: Meragelman, K. M.; West, L. M.; Northcote, P. T.; Pannell, L. K.; McKee, T. C.; Boyd, M. R. J. Org. Chem. 2002, 67, 6671-6677.

78. Tanaka, H.; Murakami, Y.; Torii, S. Bull. Chem. Soc. Jpn. 1989, 62, 4061-4062.

79. Blanc, A.; Bochet, C. G. J. Org. Chem. 2003, 68, 1138-1141.

80. Murray, M. M.; Kaszynski, P.; Kaisaki, D. A.; Chang, W.; Dougherty, D. A. J. Am. Chem. Soc. 1994, 116, 8152-8161.

81. Umeda, R.; Morishita, S.; Nishiyama, Y. Heteroatom Chem. 2011, 22, 571-575.

82. Hayashi, K.; Choshi, T.; Chikaraishi, K.; Oda, A.; Yoshinaga, R.; Hatae, N.; Ishikura, M.; Hibino, S. Tetrahedron 2012, 68, 4274-4279.

83. a) Bassoli, A.; Cenini, S.; Farina, F.; Orlandi, M.; Rindone, B. J. Mol. Catal. 1994, 89, 121-142. b) Tollari, S.; Cenini, S.; Crotti, C.; Gianella, E. J. Mol. Catal. 1994, 87, 203-214. c) Crotti, C.; Cenini, S. Bassoli, A.; Rindone, B.; Demartin, F. J. Mol. Catal. 1991, 70, 175-187.

84. Crotti, C.; Cenini, S.; Rindone, B.; Tollari, S.; Demartin, F. J. Chem. Soc., Chem. Commun. 1986, 784-786. 
85. For a related alkyne hydrostannation cross coupling, see: Hamze, A.; Veau, D.; Provot, O.; Brion, J.-D.; Alami, M. J. Org. Chem. 2009, 74, 1337-1340.

86. Kim, H. S.; Kim, Y. B.; Lee, C. J.; Shin, J. Y.; La, J. G.; Kim, T. H.; Baek, Y. M. Repub. Korean Kongkae Taeho Kongbo, KR 2015119653, 2015. [CAN163:649442].

87. Kumar, S.; Rathore, V.; Verma, A.; Prasad, C. D.; Kumar, A.; Yadav, A.; Jana, S.; Sattar, M.; Meenakshi, Kumar, S. Org. Lett. 2015, 17, 82-85.

88. Franck, P.; Hostyn, S.; Dajka-Halasz, B.; Polonka-Balint, A.; Monsieurs, K.; Matyus, P.; Maes, B. U. W. Tetrahedron 2008, 64, 6030-6037.

89. Kanaoka, Y.; Itoh, K. Synthesis 1972, 36.

90. Li, Z.; Wang, W.; Zhang, X.; Hu, C.; Zhang, Wei. Synlett 2013, 24, 73-78.

91. Li, Y.; Zhang-Negrerie, D.; Du, Y.; Zhao, K. Tetrahedron 2015, 71, 2927-2935.

92. Clemo, G. R.; Felton, D. G. I. J. Chem. Soc. 1951, 671-677.

93. One carbon resonance was missing in the spectrum.

94. Compound $\mathbf{6}$ is commercially available however, it was prepared for this study according to: Hughes, I.; Raphael, R. A. Tetrahedron Lett. 1994, 24, 1441-1444.

95. Hudkins, D. L.; Diebold, J. L.; Marsh, F. D. J. Org. Chem. 1995, 60, 6218-6220.

96. Compound $\mathbf{1 7}$ is commercially available however, it was prepared for this study according to: Coffman, K. C.; Palazzo, T. A.; Hartley, T. P.; Fettinger, J. C.; Tantillo, D. J.; Kurth, M. J. Org. Lett. 2013, 15, 2062-2065.

97. Prepared as described by :Jadhav, A. M.; Bhunia, S.; Liao, H.-Y.; Liu, R. S.; J. Am. Chem. Soc. 2011, 133, 1769-1771.

98. Bai, H.; Zhao, X.; Gong, Y.; Zhong, J.; Zhu, Q.; Liu, Z.; Liu, L.; Zhou, Q. PCT Int. Appl. 2012, WO 2012058866.

99. Yamakawa, T.; Ideue, E.; Iwaki, Y.; Sato, A.; Tokuyama, H.; Shimokawa, J.; Fukuyama, T. Tetrahedron, 2011, 67, 6547-6560.

100. The chromatography was performed using silica gel/ $\mathrm{K}_{2} \mathrm{CO}_{3}\left(10 \% \mathrm{~K}_{2} \mathrm{CO}_{3}\right)$.

101. Li, Y.; Wang, W.-H.; Yang, S.-D.; Li, B.-J.; Feng, S.; Shi, Z.-J. Chem. Commun. 2010, 46, 45534555.

102. Prepared as described by: Cikotiene, I. Eur. J. Org. Chem. 2012, 2012, 2766-2773.

103. Yamakawa, T.; Ideue, E.; Iwaki, Y.; Sato, A.; Tokuyama, H.; Shimokawa, J.; Fukuyama, T. Tetrahedron 2011, 67, 6547-6560.

104. Prasitpan, N.; Patel, J. N.; De Croos, P. Z.; Stockwell, B. L.; Manavalan, P.; Kar, L.; Johnson, M. E.; Currie, B. L. J. Heterocycl. Chem. 1992, 29, 335-341.

105. Tanaka, H.; Murakami, Y.; Torii, S. Bull. Chem. Soc. Jpn. 1989, 62, 4061-4062. 
106. Chen, Y.-L.; Chung, C.-H.; Chen, I.-L.; Chen, P.-S.; Jeng, H.-Y. Bioorg. Med. Chem. 2002, 10, 2705-2712.

107. Blanc, A.; Bochet, C. G. J. Org. Chem. 2003, 68, 1138-1141.

108. For recent syntheses and complete analytical data, see: a) Li, Z.; Wang, W.; Zhang, X.; Hu, C.; Zhang, W. Synlett 2013, 24, 73-78. b) Putey, A.; Popowycz, F.; Do, O.-T.; Bernard, P.; Talapatra, S. K.; Kozielski, F.; Galamaini, C. M.; Joseph, B. J. Med. Chem. 2009, 52, 5916-5925.

109. Norton, R. S.; Wells, R. J. J. Am. Chem. Soc. 1982, 104, 3628-3635.

110. Hodder, A. R.; Capon, R. J. J. Nat. Prod. 1991, 54, 1616-1663.

111. Su, H.; Yuan, Z. H.; Li, J. Chin. Chem. Lett. 2009, 20, 456-458.

112. Guo, S.; Su, H.; Li, X.; Zhu, X. Faming Zhuanli Shenqing, CN 103833624, A 20140604, 2014.

113. Ansari, N. H.; Dacko, C. A.; Akhmedov, N. G.; Björn, C. G. S. J. Org. Chem. 2016, 81, 9337-9349.

114. Carter, M. D.; Hadden, M.; Weaver, D. F.; Jacobo, S. M. H.; Lu, E. PCT Int. Appl. WO 2006125324 [CAN146:45396].

115. Zhang, P.; Liu, R.; Cook, J. M. Tetrahedron Lett. 1995, 36, 3103-3106.

116. Suarez-Castillo, O. R.; Sanchez-Zavala, M.; Melendez-Rodrigues, M.; Aquino-Torres, M. S.; Morales-Rios, M. S.; Joseph-Nathan, P. Heterocycles 2007, 71, 1539-1551.

117. Mistry, A. G.; Smith, K.; Bye, M. R. Tetrahedron Lett. 1986, 27, 1051-1054.

118. Hino, T.; Tonuzka, M.; Nakagawa, M. Tetrahedron 1974, 30, 2123-2133.

119. Miyake, F. Y.; Yakushijin, K.; Horne, D. A. Org. Lett. 2004, 6, 4249-4251.

120. Da Settimo, A.; Santerini, V.; Primofore, G.; Biagi, G.; Veneziano, C. Gazz. Chim. Ital. 1977, 107, 367-372.

121. Loudon, V. J. D.; Tennant, G. J. Chem. Soc. 1963, 4268-4269.

122. a) Stacey, G. W.; Ettling, B. V.; Papa, A. J. J. Org. Chem. 1964, 29, 1537-1540. b) McNab, H.; Smith, D. M. J. Chem. Soc., Perkin Trans. 1 1973, 1310-1314. c) Machin, J.; Mackie, R. K.; McNab, H.; Reed, G. A.; Sagar, A. J. G.; Smith, D. M. J. Chem. Soc., Perkin Trans. 1 1976, 394399.

123. a) Gardiner, J. M.; Loyns, C. R. Synth. Commun. 1995, 25, 819-827. b) Gardiner, J. M.; Loyns, C. R.; Schwalbe, C. H.; Barrett, G. C.; Lowe, P. L. Tetrahedron 1995, 51, 4101-4110. c) Gardiner, J. M.; Lyons, C. R.; Burke, A.; Khan, A.; Mahmood, N. Bioorg. Med Chem. Lett. 1995, 5, 1251-1254. d) Evans, T. M.; Gardiner, J. M.; Mahmood, N.; Smis, M. Bioorg. Med Chem. Lett. 1997, 7, 409412. e) Gardiner, J. M.; Procter, Tetrahedron Lett. 2001, 42, 5109, 5111. f) Gardiner, J. M.; Goss, A. D.; Majid, T.; Morley, A. D.; Pritchard, R. G.; Warren, J. E. Tetrahedron Lett. 2002, 43, 77077710. g) Gardiner, J. M.; Goss, A. D.; Majid, T.; Morley, A. D. Tetrahedron Lett. 2003, 44, 511 513. 
124. For a cyclization in the absence of an alkylating reagents, see: Popov, I. I.; Kryshtalyuk, O. V. Khim. Geterotsilikl. 1991, 997-998.

125. See for example, Bowser, T. E.; Bartlett, V. J.; Grier, M. C.; Verma, A. K.; Warchol, T.; Levy,S. B.; Alekshun, M. N. Bioorg. Med. Chem. Lett. 2007, 17, 5652-5655.

126. a) Grochowski, E.; Kwastowa, E. J. Chem. Res., Synop. 1978, 300-301. b) Grochowski, E.; Stepowska, H. Synthesis 1988, 795-797.

127. Aguirre, G.; Boiani, M.; Cerecetto, H.; Gerpe, A.; Gonzales, M.; Sainz, Y. F.; Denicola, A. de Ortiz, C. O.; Nogal, J. J.; Montero, S.; Escario, J. A. Arch. Pharm. 2004, 337, 259-270.

128. Commercially available. For spectral data, see: Deiters, E.; Song, B.; Chauvin, A.-S.; Vandevyver, C. D. B.; Gumy, F.; Bünzli, J.-C. Chem. Eur. J. 2009, 15, 885-900.

129. Nazer, M. Z.; Haddadin, M. J.; Petridou, J. P.; Issidorides, C. H. Heterocycles 1977, 6, 541-545.

130. Nyerges, M.; Viranyi, A.; Zhang, W.; Groundwater, P. W.; Blasko, G.; Toke, L. Tetrahedron 2004, 60, 9937-9944.

131. Nyerges, M.; Somfai, B.; Toth, J.; Toke, L.; Dancso, A.; Blasko, G. Synthesis 2005, 2039-2045.

132. Banini, S. R.; Turner, M. R.; Cummings, M. M.; Söderberg, B. C. G. Tetrahedron 2011, 67, 36033611 .

133. Compound $\mathbf{1 2}$ is known but only the $1 \mathrm{H}$ NMR was reported. Chen Y. I.; Helberg, K.; Guarino, K.; et al. J. Antibiot. 1991, 44, 870-884.

134. Söderberg, B. C. G.; Wallace, J. M.; Tamariz, J. Org. Lett. 2002, 4, 1339-1342.

135. $\mathrm{NaH}(80 \%)$ in mineral oil without removal of oil was used in all experiments. The amount of $\mathrm{NaH}$ shown in each experimental procedure represents the mass of $\mathrm{NaH}$ and not the total mass of $\mathrm{NaH}$ plus mineral oil.

136. Ansari, N. H.; Jordan, A. L.; Sderberg, B. C. G. Tetrahedron, 2017, 73, 4811-4821.

137. Takahashi, S.; Kanio, H. Chem. Pharm. Bull. 1964, 12, 282-291.

138. Echavarren, A, M.; Stille, J. K. J. Am. Chem. Soc. 1987, 109, 5478-5486.

139. For the same substrate and selectivity, see: Saa, J. M.; Martorell, G. J. Org. Chem. 1993, 58, 1963-1966.

140. For iodo-selective Kosugi-Migita-Stille reactions, see also: a) DeBoos, G. A.; Fullbrook, J. J.; Percy, J. M. Org. Lett. 2001, 3, 2859-2861. b) Takayuki, D.; Inoue, H.; Tokita, M.; Watanabe, J.; Takahashi, T. J. Comb. Chem. 2008, 10, 135-141.

141. This catalyst system was used as a convenient substitute for $1.5 \% \mathrm{Pd}_{2}(\mathrm{dba})_{3}-3.0 \% \mathrm{P}(t-\mathrm{Bu})_{3}$, since $\mathrm{Pd}_{2}(\mathrm{dba})_{3}$ and $\mathrm{Pd}\left(\mathrm{P}(t-\mathrm{Bu})_{3}\right)_{2}$ are more easily handled and stored.

142. Littke, A. F.; Schwartz, L.; Fu, G. C. J. Am. Chem. Soc. 2002, 124, 6343-6348. 
143. For additional examples of the C-Cl vs C-OTf selectivity in Suzuki-Miyaura and Kosugi-MigitaStille reactions, see: a) Littke, A. F.; Dai, C.; Fu, G. C. J. Am. Chem. Soc. 2000, 122, 4020-4028. b) Proutiere, F.; Schoenebeck, F. Angew. Chem. In. Ed. 2011, 50, 8192-8195.

144. Jutand, A.; Mosleh, A. Organometallics 1995, 14, 1810-18-17.

145. For the same conclusion, see: Casado, A. L.; Espinet, P.; Gallego, A. M. J. Am. Chem. Soc. 2000, 122, 11771-11782.

146. Verbeeck, S.; Meyers, C.; Franck, P.; Jutand, A.; Maes, M. U. W. Chem. Eur. J. 2010, 16, 1283112837.

147. Casado, A. L.; Espinet, P. J. Am. Chem. Soc. 1998, 120, 8978-8985.

148. Echavarren, A, M.; Stille, J. K. J. Am. Chem. Soc. 1988, 110, 1557-1565.

149. Related C-OTf specific carbonylations of $\mathbf{1}$ and similar compounds containing both a C-OTf and a C-Br bond have been reported. a) Dolle, R. E.; Schmidt, S. J.; Kruse, L. I. J. Chem. Soc., Chem. Commun. 1987, 904-905. b) Cacchi, S.; Ciattini, P. G.; Morera, E.; Ortar, G. Tetrahedron Lett. 1986, 27, 3931-3934. c) Holt, D. A.; Levy, M. A.; Ladd, D. L.; Oh, H.-J.; Erb, J. M.; Heaslip, J. I.; Brandt, M.; Metcalf, B. W. J. Med. Chem. 1990, 33, 937-942. d) Brennfuehrer, A.; Neumann, H.; Beller, M. Synlett 2007, 2537-2540.

150. a) Chen, S.; Williams, R. M. Tetrahedron 2006, 62, 11572-11579. b) Huang, C.-W.; Shanmugasundaram, M.; Chang, H.-M.; Cheng, C.-H. Tetrahedron 2003, 59, 3635-3641. c) Takemura, S.; Hirayama, A.; Tokunaga, J.; Kawamura, F.; Inigaki, K.; Hashimoto, K.; Nakata, M. Tetrahedron Lett. 1999, 40, 7501-7505.

151. It has been shown that an increase in temperature from ambient to $70{ }^{\circ} \mathrm{C}$ resulted in erosion of $\mathrm{C}$ OTf versus C-Br selectivity in Suzuki couplings. Wang, B., Sun, H.-X.; Sun, Z.-H. Eur. J. Org. Chem. 2009, 3688-3692.

152. Espino, G.; Kurbangalieva, A.; Brown, J. M. Chem. Commun. 2007, 1742-1744.

153. Molander, G. A.; Petrillo, D. E.; Landzberg, N. R.; Rohanna, J. C.; Biolatto, B. Synlett 2005, 17631766.

154. Hassan, Z.; Hussein, M.; Villinger, A.; Langer, P. Tetrahedron 2012, 68, 6305-6313.

155. Fu, J. M.; Snieckus, V. Tetrahedron Lett. 1990, 31, 1665-1668.

156. Eleya, N.; Mahal, A.; Hein, M.; Villinger, A.; Langer, P. Adv. Synth. Catal. 2011, 353, 2761-2774.

157. Abid, O.; Ibad, M. F.; Nawaz, M.; Ali, A.; Sher, M.; Rama, N. H.; Villinger, A.; Langer, P. Tetrahedron Lett. 2010, 51, 1541.

158. Nawaz, M.; Farooq Ibad, M.; Obaid-Ur-Rahman, A.; Khera, R. A.; Villinger, A.; Langer, P. Synlett. 2010, 150 .

159. Oh-e, T.; Miyaura, N.; Suzuki, A. J. Org. Chem. 1993, 58, 2201-2208. 
160. Finke, A. D.; Elleby, E. C.; Boyd, M. J.; Weissman, H.; Moore, J. S. J. Org. Chem. 2009, 74, 88978900 .

161. Mio, M. J.; Kopel, L. C.; Braun, J. B.; Gadzikwa, T. L.; Hull, K. L.; Brisbois, R. G.; Markworth, C. J.; Grieco, P. A. Org. Lett. 2002, 4, 3199-3202.

162. Kamikawa, T.; Hayashi, T. J. Org. Chem. 1998, 63, 8922-8925.

163. Fujita, T.; Ichitsuka, T.; Fuchibe, K.; Ichiwara, J. Chem. Lett. 2011, 40, 986-988.

164. Herbert, J. M.; Kohler, A. D.; Le Strat, F.; Whitehead, D. J. Label. Compd. Radiopharm. 2007, 50, 440-441.

165. Kamikawa, T.; Hayashi, T. Tetrahedron Lett. 1997, 38, 7087-7090.

166. Jutand, A.; Mosleh, A. Organometallics 1995, 14, 1810-18-17.

167. Dong, Z.-B.; Manolikakes, G.; Shi, L.; Knochel, P.; Maye, H. Chem. Eur. J. 2010, 16, 248-253.

168. Kim, Y. M.; Yu, S. J. Am. Chem. Soc. 2003, 125, 1696-1697.

169. Bahmanyar, S.; Borer, B. C.; Kim, Y. M.; Kurtz, D. M.; Yu, S. Org. Lett. 2005, 7, 1011-1014.

170. Fürstner, A.; Ackerstaff, J. Chem. Commun. 2008, 2870-2872.

171. Tada, A.; Tokoro, Y.; Fukuzawa, S.-i. J. Org. Chem. 2014, 79, 7905-7909.

172. Xiaoquing, S.; Hyde, A. M.; Buckwald, S. L. J. Am. Chem. Soc. 2010, 132, 14076-14078.

173. We were unable to obtain a HRMS of this compound.

174. Li, D.; Zhao, B.; Sim, S.; Li, T.; Liu, A.; Liu, L. F.; LaVoie, E. J. Bioorg. Med. Chem. 2003, 11, $521-528$

175. Hodgson, H. H.; Moore, F. H. J. Chem. Soc. 1926, 155-161.

176. Habibi, D.; Zolfigol, M. A.; Shiri, M.; Sedaghat, A. S. Afr. J. Chem., 2006, 59, 93-96.

177. Lee, Y.; Kelly, M. J. Tet. Lett. 2006, 47, 4897-4901.

178. Ghorbani-Choghamarani, A.; Nikoorazm, M.; Goudarziafshar, H.; Naserifar, Z.; Zamani, P. Chinese. J. Chem. 2011, 29, 731-734.

179. Suresh, P.; Annalakshmi, S.; Pitchumani, K. Tetrahedron 2007, 63, 4959-4967.

180. Hu, J.; Hirao, H.; Li, Y.; Zhou, J. Angew. Chem. Int. Ed. 2013, 52, 8676-8680.

181. Matsumura, K.; Shimizu, H.; Saito, T.; Kumobayashi, H. Adv. Synth. Catal. 2003, 345, 180-184.

182. Commercially available.

183. Krolski, M. E.; Renaldo, A. F.; Rudisill, D. E.; Stille, J. K. J. Org. Chem. 1988, 53, 1170-1176.

184. Rudisill, D.; Stille, J. K. J. Org. Chem. 1989, 54, 5856-5866.

185. Eloy, N.; Pasquinet, E.; Grech, E.; David-quillot, F.; Besnard, O.; Poullain, D. Synthesis 2008, 1805-1807.

186. Jarkas, N.; McConathy, J.; Voll, R. J.; Goodman, M. M. J. Med. Chem. 2005, 48, 4254-4265. 
187. Harrington, P. J.; Hegedus, L. S. J. Org. Chem., 1984, 49, 2657-2662.

188. Oda, N.; Yoshida, Y.: Nagai, S.; Ueda, T.; Sakakibara, J. Chem. Pharm. Bull. 1987, 35, 1796-1802.

189. Rottaelander, M.: Palmer, N.; Knochel, P. Synlett 1996, 573-575.

190. The compound has been prepared previously but no analytical data were reported. Murugesan, N.; Tellew, J. E.; Macor, J. E.; Gu, Z. U.S. Pat Appl. (2002) US 20020143024.

191. Dai, M.; Wang, Z.; Danishefsky, S. J. Tetrahedron Lett. 2008, 49, 6613-6616.

192. Grigg, R. D.; Van Hoveln, R.; Schomaker, J. M. J. Am. Chem. Soc. 2012, 134, 16131-16134.

193. a) Samsoniya, S. A.; Trapaidze, M. V.; Targamadze, N. L; Chikvaidze, I. S.; Suvorov, R. R.; Ershova, N. N.; Chernov Soobshch. Akad. Nauk Gruz. SSR, 1980, 337, 100. b) Samsoniya, S. A.; Medvedev, B. A.; Kadzhrishvili, D. O.; Tabidze, D. M.; Mashkovskii, M. D.; Suvorov, N. N. Khim.-Farm. Zh. 1982, 1335. c) Samsoniya, S. A.; Lomtatidze, Z. S.; Dolidze, S. V.; Suvorov, N. N. Khim.-Farm. Zh. 1984, 1452.

194. Lee, S. M.; Li, X. F.; Jiang, H.; Cheng, J. G.; Seong, S.; Choi, H. D.; Son, B. W. Tetrahedron Lett. 2003, 44, 7707-7710.

195. Wang, C.; Sperry, J. Org. Lett. 2011, 13, 6444-6447.

196. Yudina, L. N.; Bergman, J. Tetrahedron, 2003, 59, 1265-1275.

197. Boudreault, P-L. T.; Wakim S.; Bloun, N.; Simard, M.; Tassier, C.; Tao, Y.; Leclerc, M. J. Am. Chem. Soc. 2007, 129, 9125-9136.

198. Wu, Y.; Li, Y.; Gardner, G.; Ong, B. S. J. Am. Chem. Soc. 2005, 127, 614-618.

199. a) Giuliano, R.; Leonardi, G. Ric. Sci. 1957, 27, 1843. b) Samsoniya, S. A.; Targamadze, N. L.; Tret'yakova, L. G.; Efimova, T. K.; Turchin, K. G.; Gverdtsiteli, I. M.; Suvorov, N. N. Khim. Geterotskl. Soedin. 1977, 938. c) Ruggli, P.; Petitjean, C. Helv. Chim. Acta 1936, 19, 928. d) Katti, H. A.; Siddappa, S. Ind. J. Chem. 1983, 228, 1205.

200. Baird, D. B.; Baxter, V.; Cameron, D. W.; Phillips, W. R. J. Chem. Soc. Perkin Trans. 1 1973, 832.

201. Ruggli, P.; Straub, O. Helv. Chim Acta 1936, 19, 326.

202. Ruggli, P.; Straub, O. Helv. Chim. Acta 1938, 21, 1084.

203. Berlin, A.; Bradamante, S.; Ferraccioli, R.; Pagani, G. A.; Sannicolo, F. J. Chem. Soc., Chem. Commun. 1987, 1176.

204. Chunchatprasert, L.; Shannon, P. V. R. J. Chem. Soc., Perkin Trans. 1 1996, 1787-1795.

205. Samsoniya, S. A.; Kadzhrishvili, D. O.; Chikvaidze, I. S. Pharm. Chem. J. 2011, 45, 22-25.

206. Samsoniya, S. A.; Kadzhrishvili, D. O.; Chikvaidze, I. S.; Targamadze, N. L. Heterocycl. Compd. 2010, 99-118.

207. Yoshikai, N.; Wei, Y. U.S. Pat. Appl. (2015), US 20150005494 A1 20150101.

208. Suzuki, Y.; Ohta, Y.; Oishi, S.; Fujii, N.; Ohno, H. J. Org. Chem. 2009, 74, 4246-4251. 
209. Clentsmith, G. K. B.; Field, L. D.; Messerle, B. A.; Shasha, A.; Turner, P. Tetrahedron Lett. 2009, $50,1469-1471$.

210. Prepared from 1,4-dibromobenzene according to: Sunde, C. J.; Johnson, G.; Kade, C. F. J. Org. Chem. 1939, 4, 548-554.

211. Prepared from 4-amino-3-nitrophenol according to: Gim, H. J.; Li, H.; Eun, L.; Ryu, J.-H.; Jeon, R. Bioorg. Med. Chem. Lett. 2013, 23, 513-517.

212. Prepared from 3-nitrophenol according to: Smith, C. J.; Ali, A.; Chen, L.; Hammond, M. L.; Anderson, M. S.; Chen, Y.; Eveland, S. S.; Guo, Q.; Hyland, S. A.; Milot, D. P.; Sparrow, C. P.; Wright, S. D.; Sinclair, P. J. Bioorg. Med. Chem. Lett. 2010, 20, 346-349.

213. Olah, G. A.; Wang, Q.; Sandford, G.; Prakash, G. K. S. J. Org. Chem. 1993, 58, 3194-3195.

214. Prepared from 3-iodophenol according to: Banwell, M. G.; Jones, M. T.; Loong, D. T. J.; Lupton, D. W.; Pinkerton, D. M.; Ray, J. K.; Willis, A. C. Tetrahedron 2010, 66, 9252-9262.

215. Hodgson, H. H.; Moore, F. H. J. Chem. Soc. 1927, 630-635.

216. Prepared according to: Kawakami, T.; Suzuki, H. Tetrahedron Lett. 2000, 41, 7093-7096.

217. Mundla, S. R. Tetrahedron Lett. 2000, 41, 6319-6321.

218. Previously prepared by iodination of 2,5-dinitrophenol: Hajipour, A. R.; Adibi, H. J. Chem. Res. 2004, 4, 294-295 

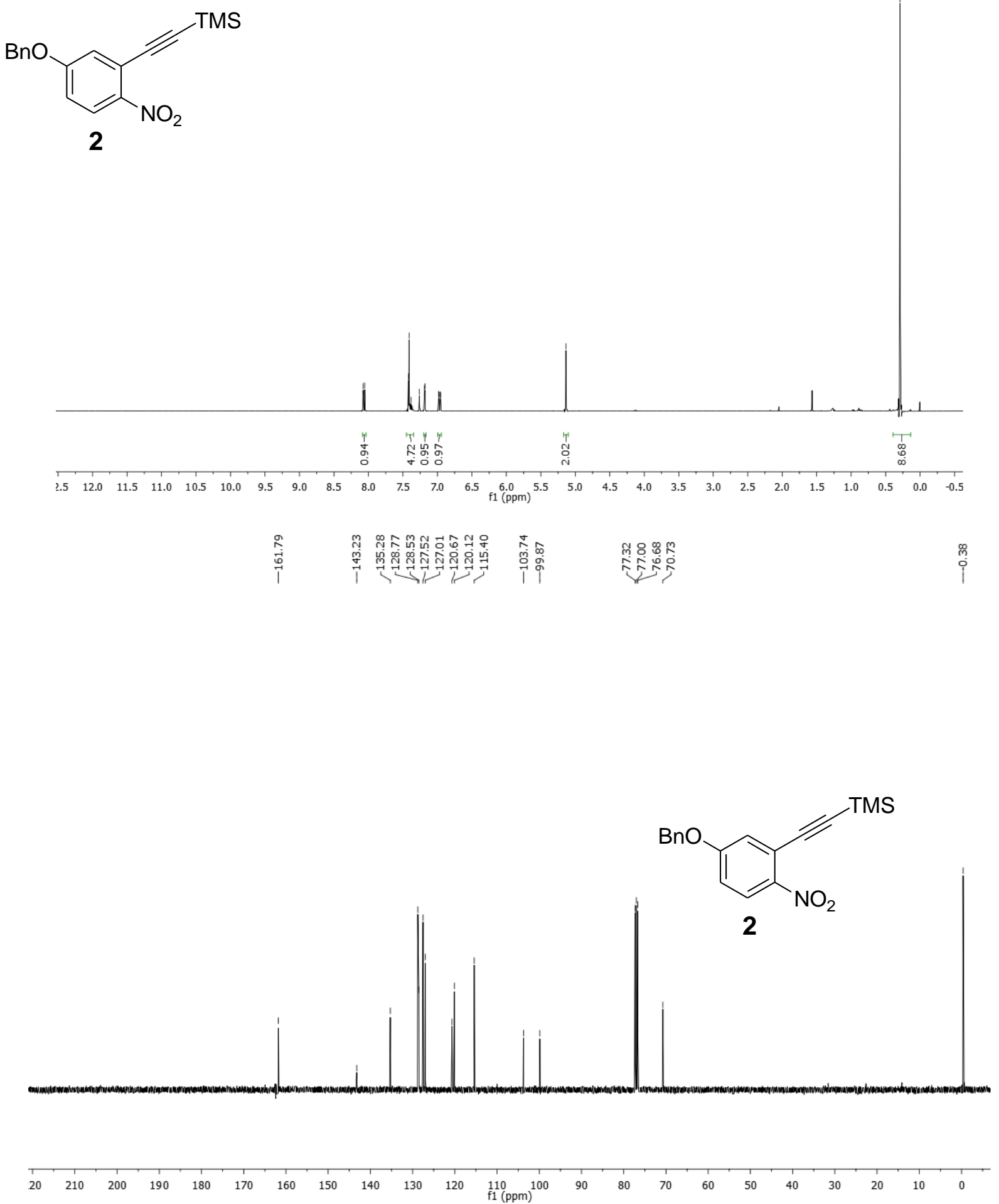

Figure $1.2{ }^{1} \mathrm{H}$ and ${ }^{13} \mathrm{C}$ NMR of compound 2 

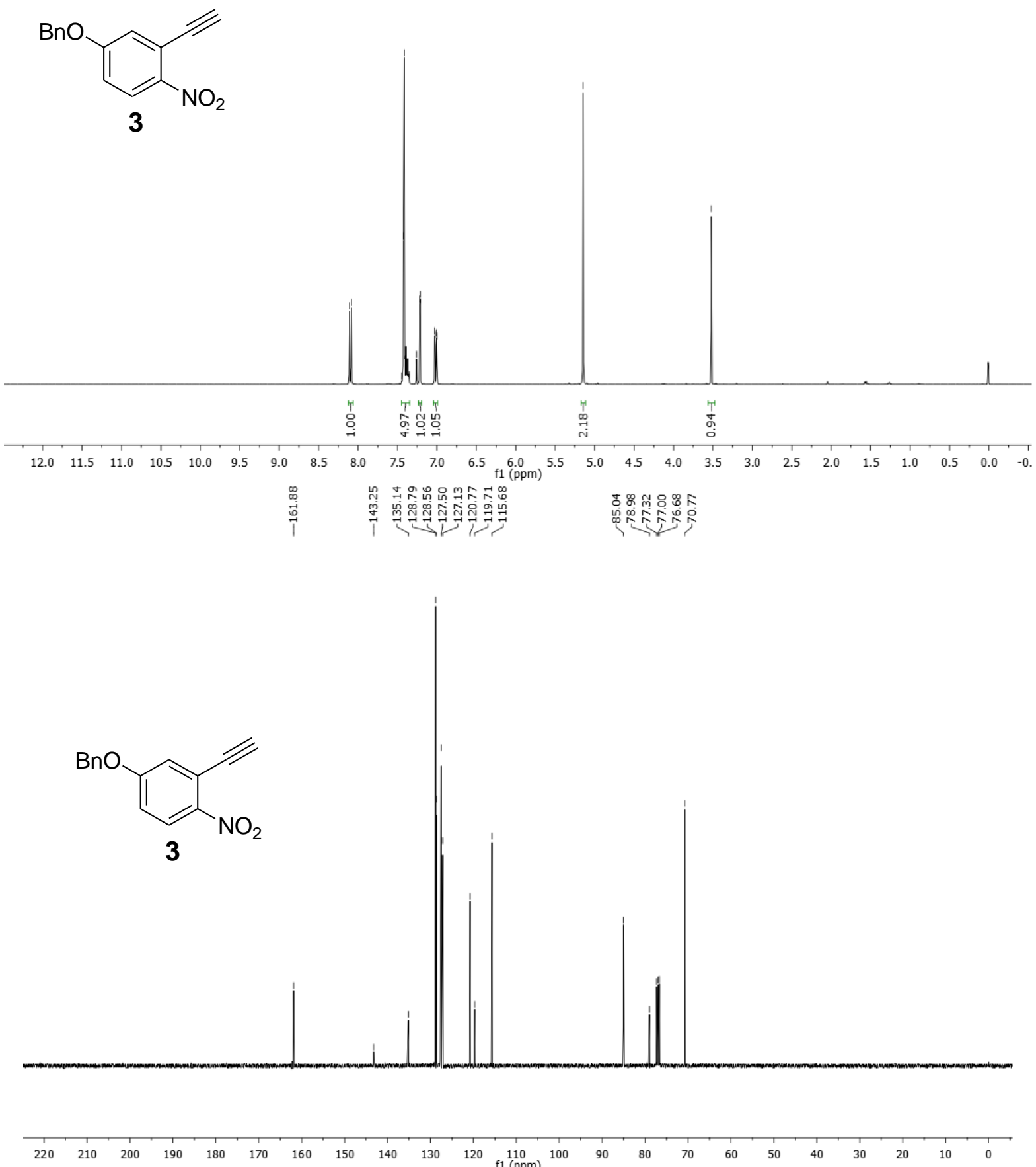

Figure $1.3{ }^{1} \mathrm{H}$ and ${ }^{13} \mathrm{C}$ NMR of compound 3 
<smiles>C=C(CCCC)c1cc(OCc2ccccc2)ccc1[N+](=O)[O-]</smiles>

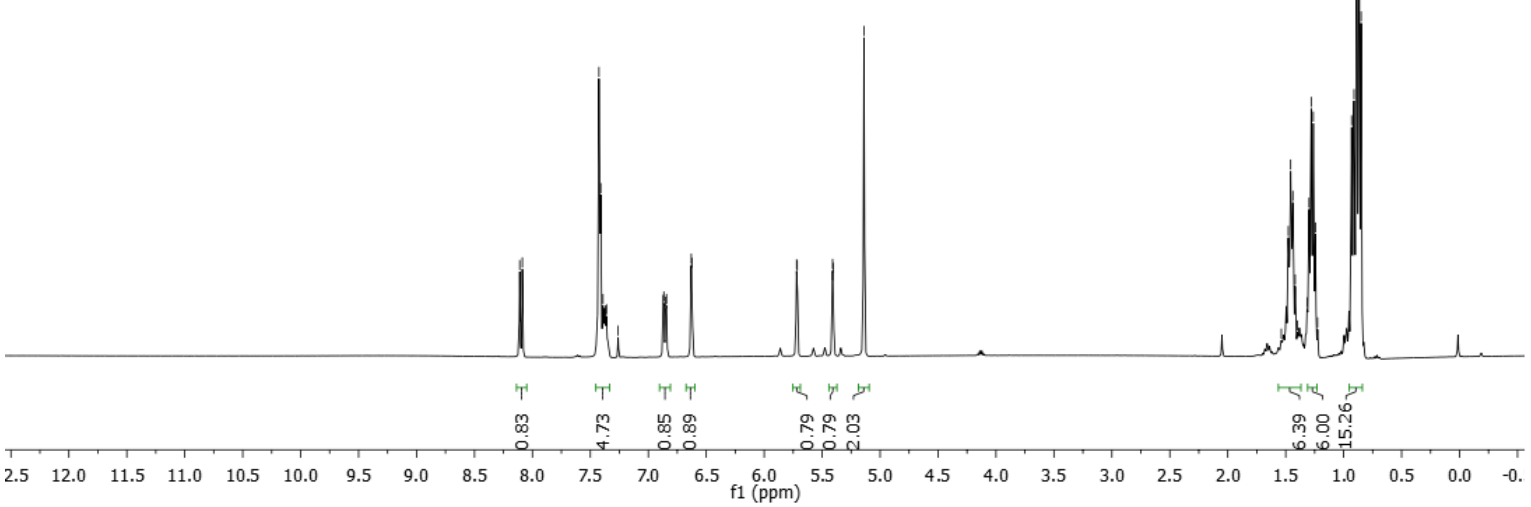

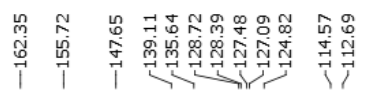

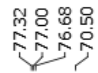

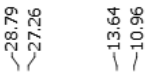<smiles>C=C(CCCC)c1cc(OCc2ccccc2)ccc1[N+](=O)[O-]</smiles>
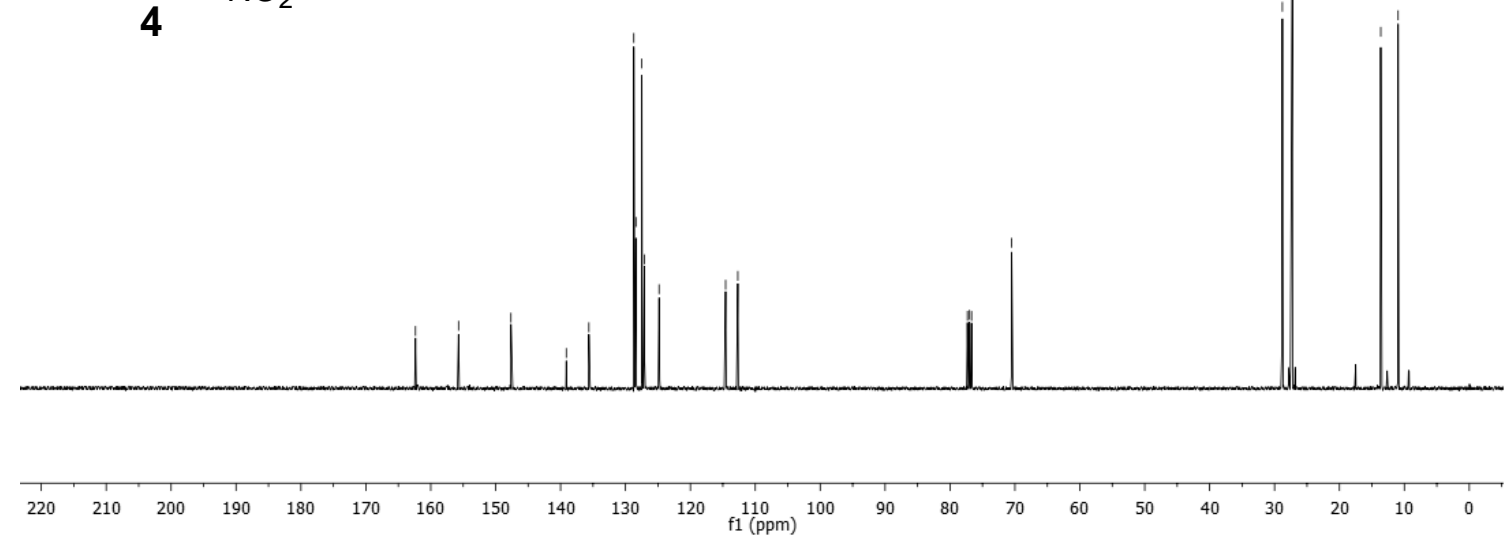

Figure $1.4{ }^{1} \mathrm{H}$ and ${ }^{13} \mathrm{C}$ NMR of compound 4 

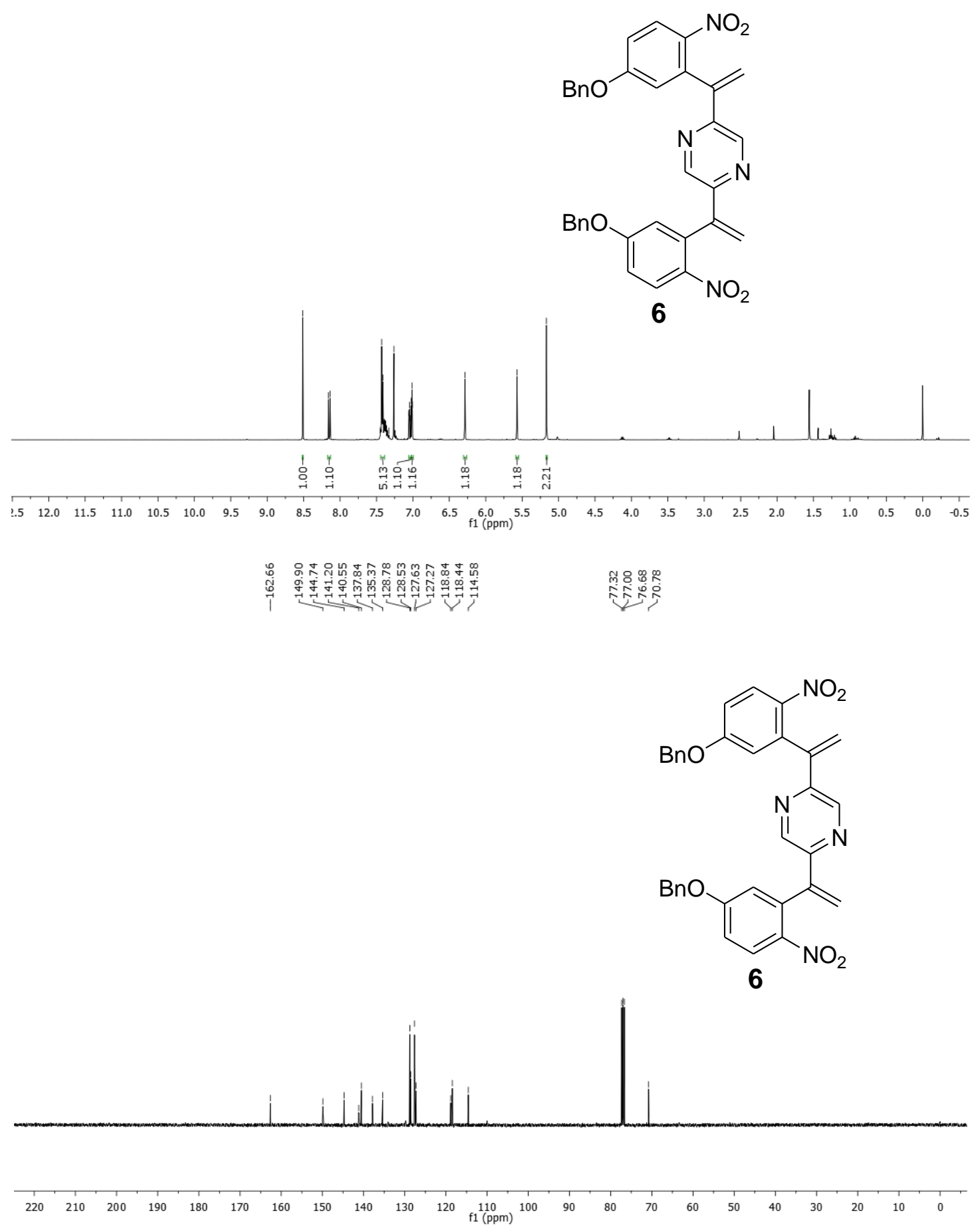

Figure $1.5{ }^{1} \mathrm{H}$ and ${ }^{13} \mathrm{C}$ NMR of compound 6 


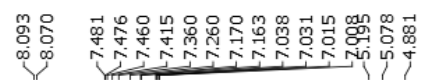
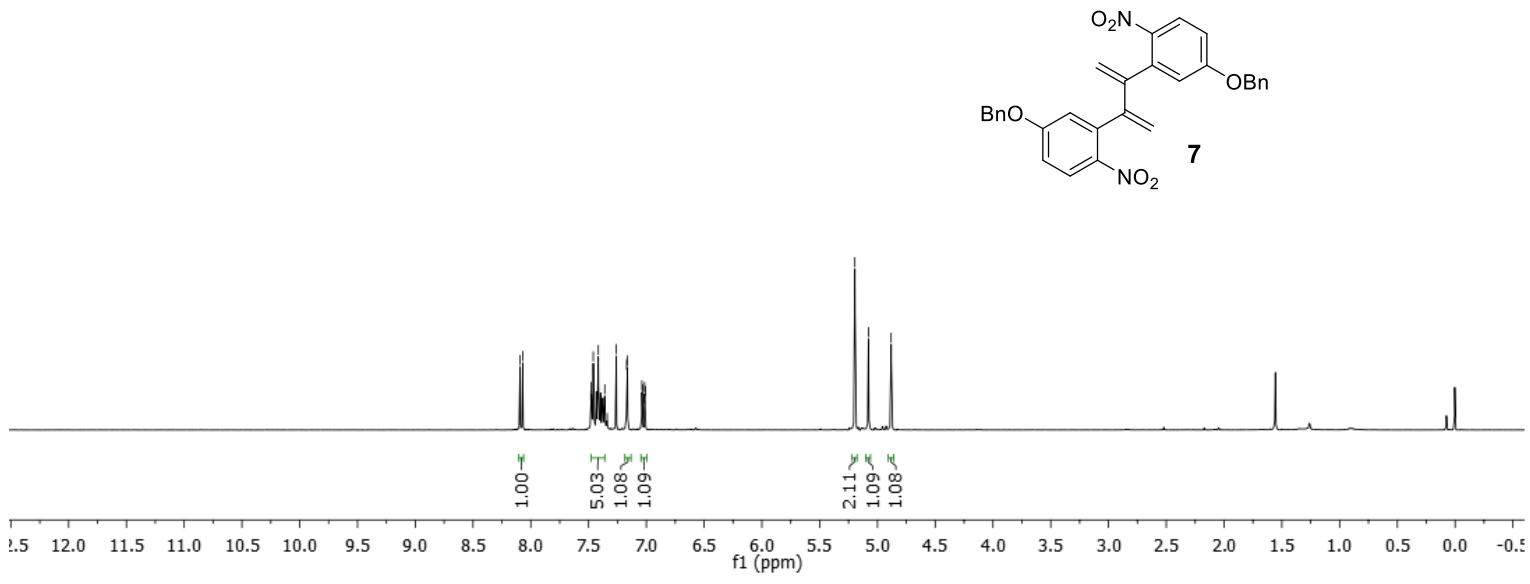

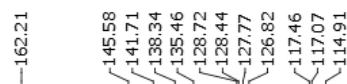

蜍学
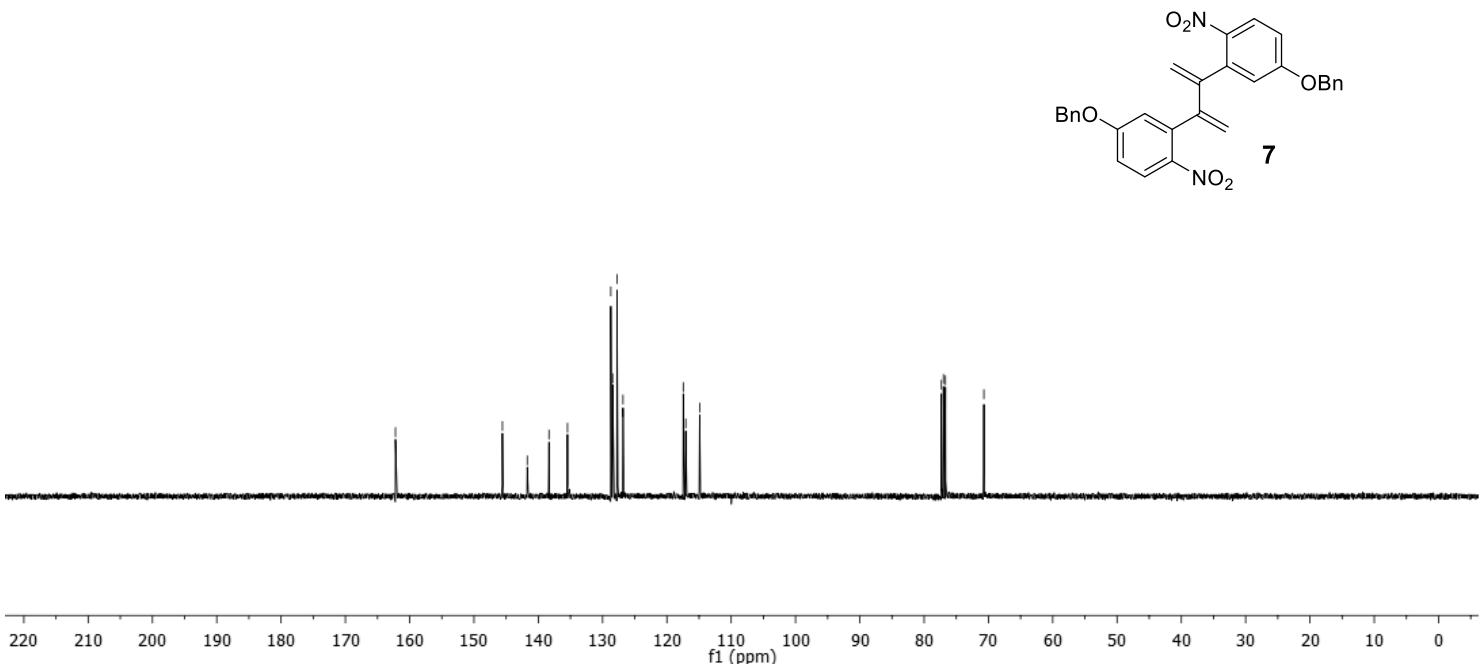

Figure 1.6 ${ }^{1} \mathrm{H}$ and ${ }^{13} \mathrm{C}$ NMR of compound 7 


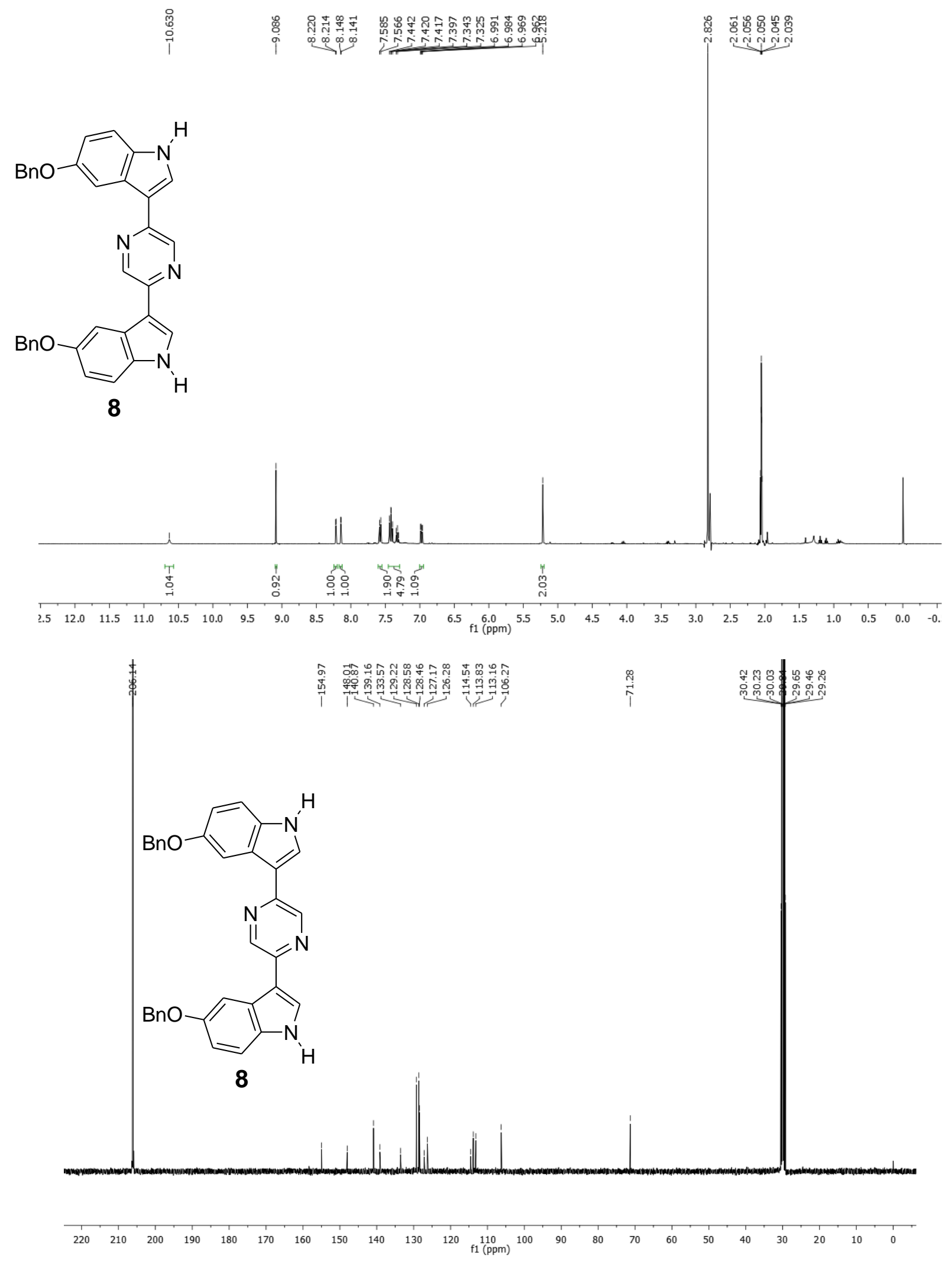

Figure $1.7{ }^{1} \mathrm{H}$ and ${ }^{13} \mathrm{C}$ NMR of compound 8 

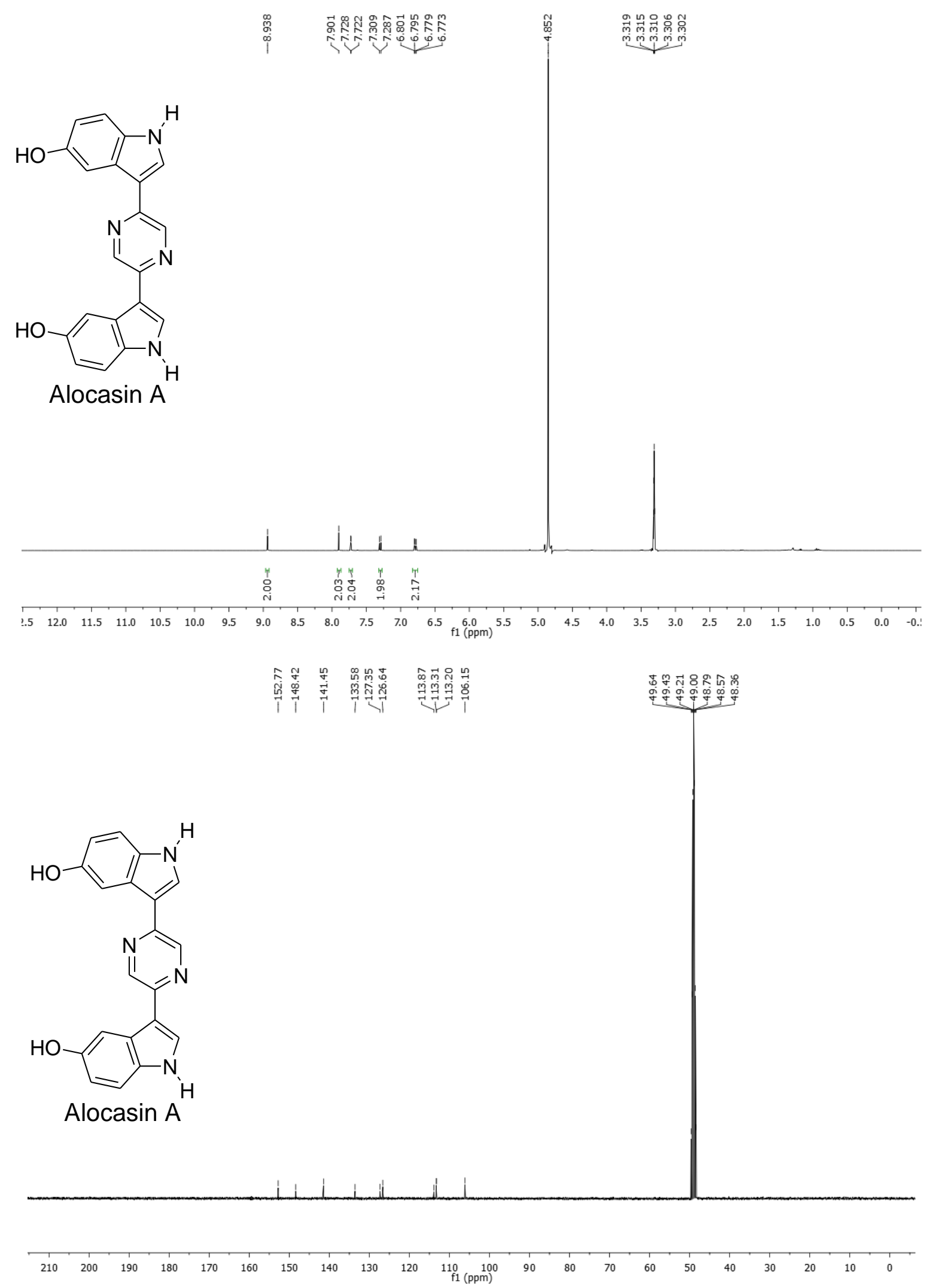

Figure $1.8{ }^{1} \mathrm{H}$ and ${ }^{13} \mathrm{C}$ NMR of Alocasin A 

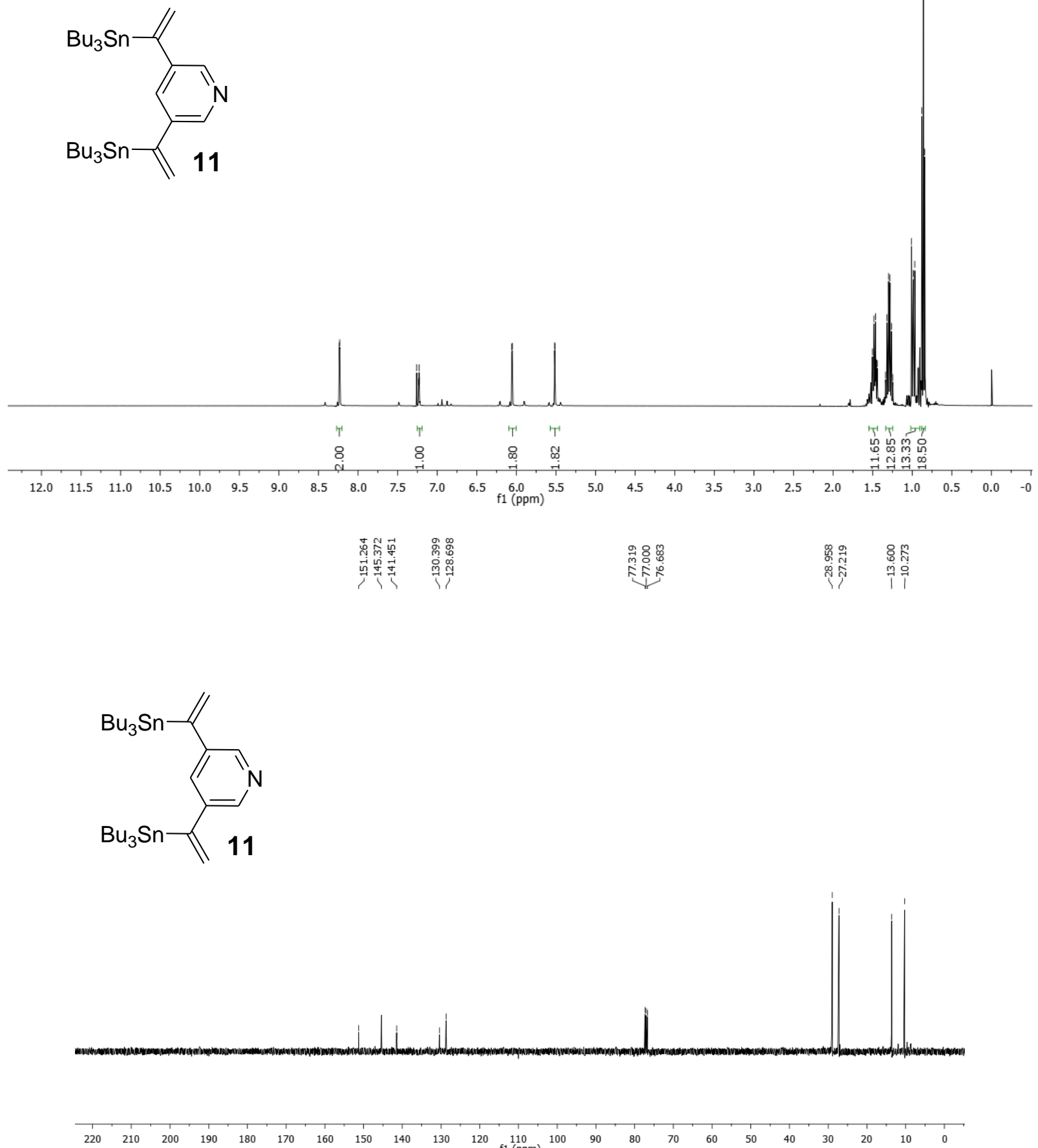

Figure $1.9{ }^{1} \mathrm{H}$ and ${ }^{13} \mathrm{C}$ NMR of compound $\mathbf{1 1}$ 

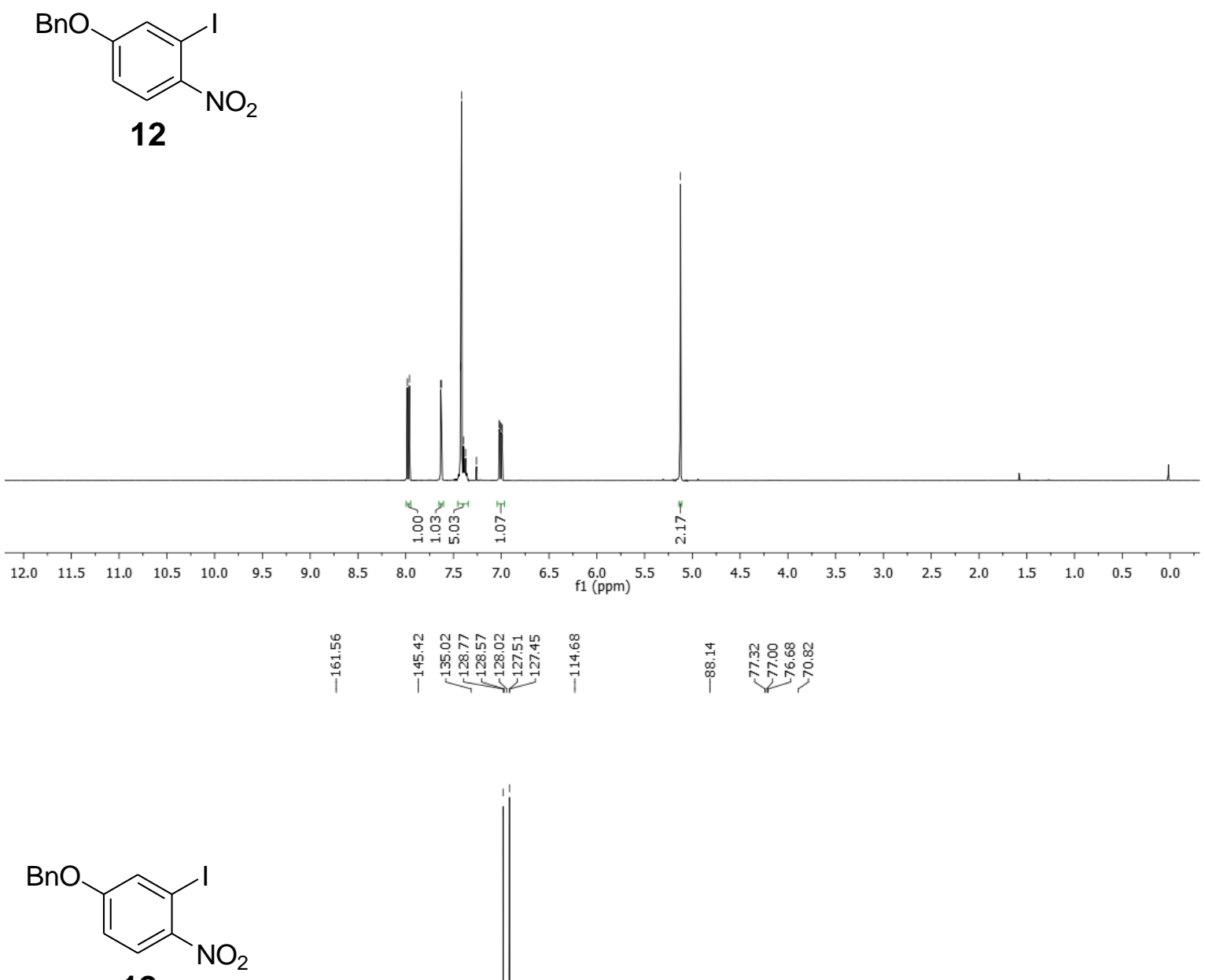

12

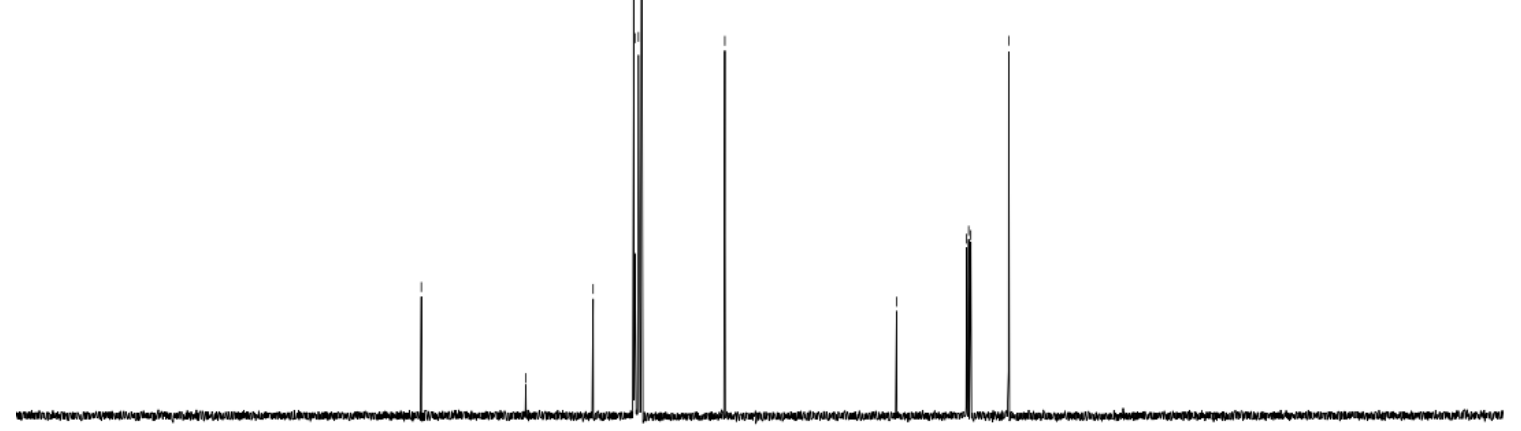

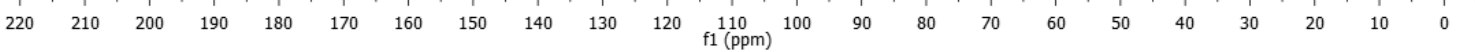

Figure 1.10 ${ }^{1} \mathrm{H}$ and ${ }^{13} \mathrm{C}$ NMR of compound 12 

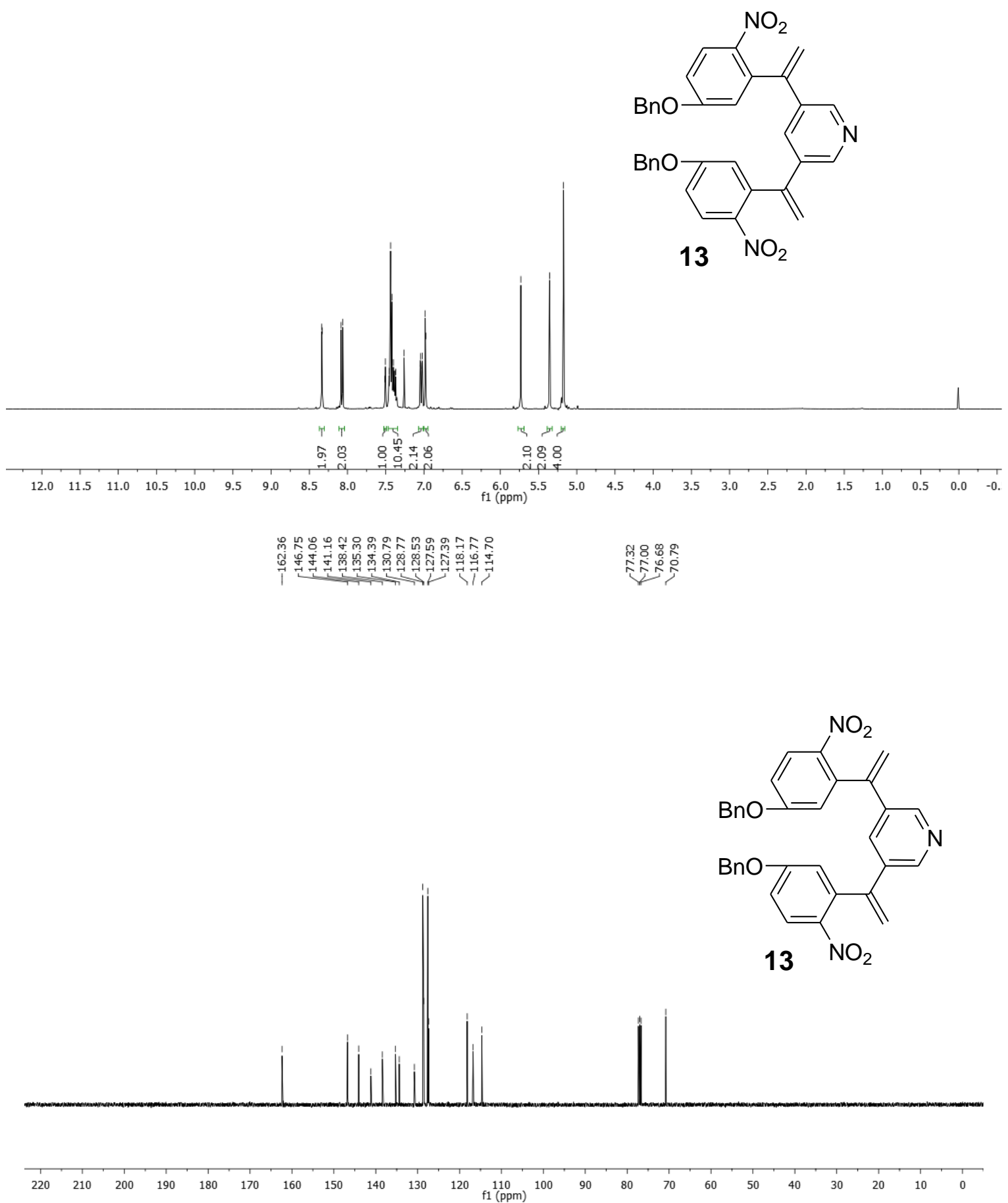

Figure $1.11{ }^{1} \mathrm{H}$ and ${ }^{13} \mathrm{C}$ NMR of compound $\mathbf{1 3}$ 

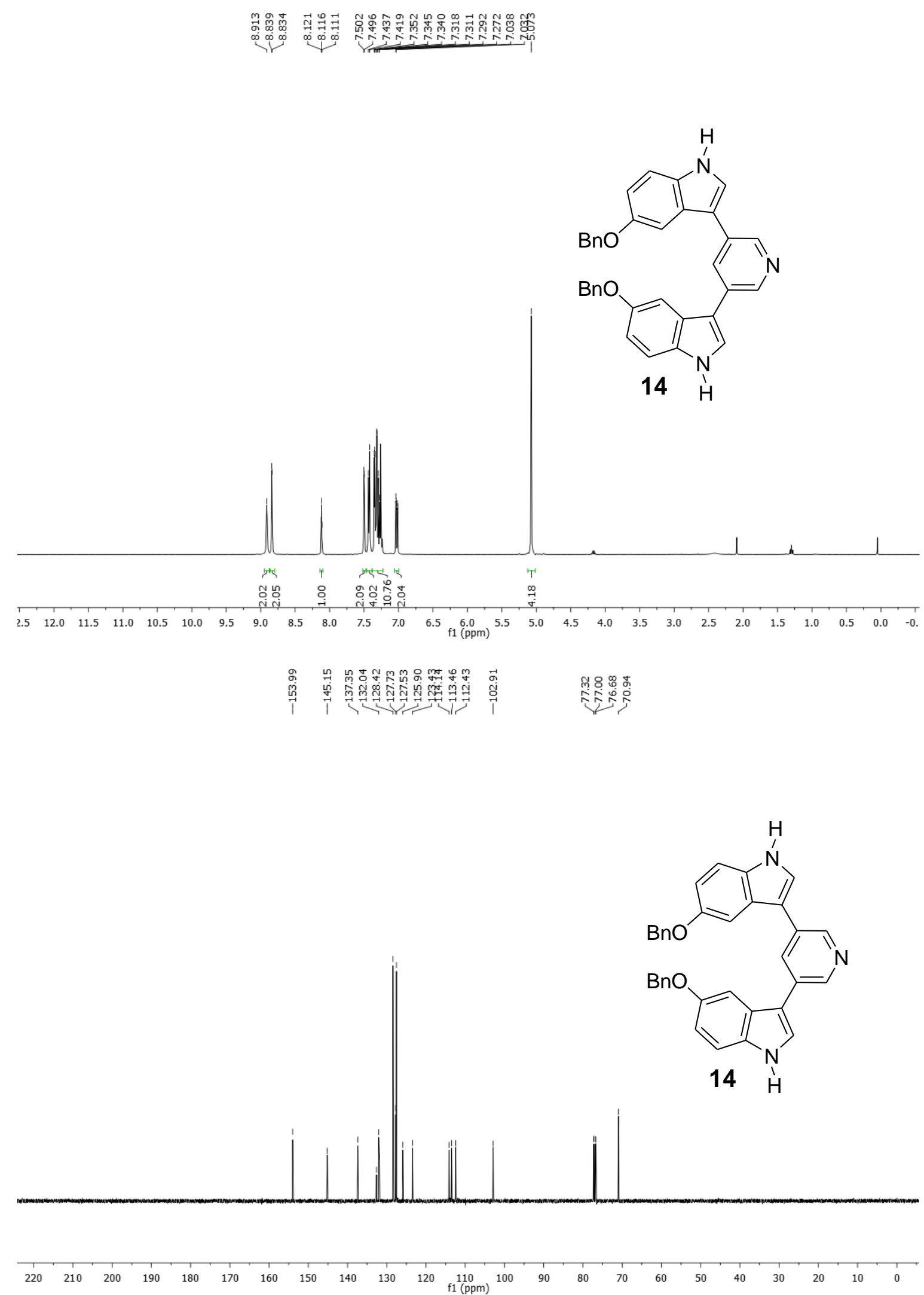

Figure $1.12{ }^{1} \mathrm{H}$ and ${ }^{13} \mathrm{C}$ NMR of compound 14 

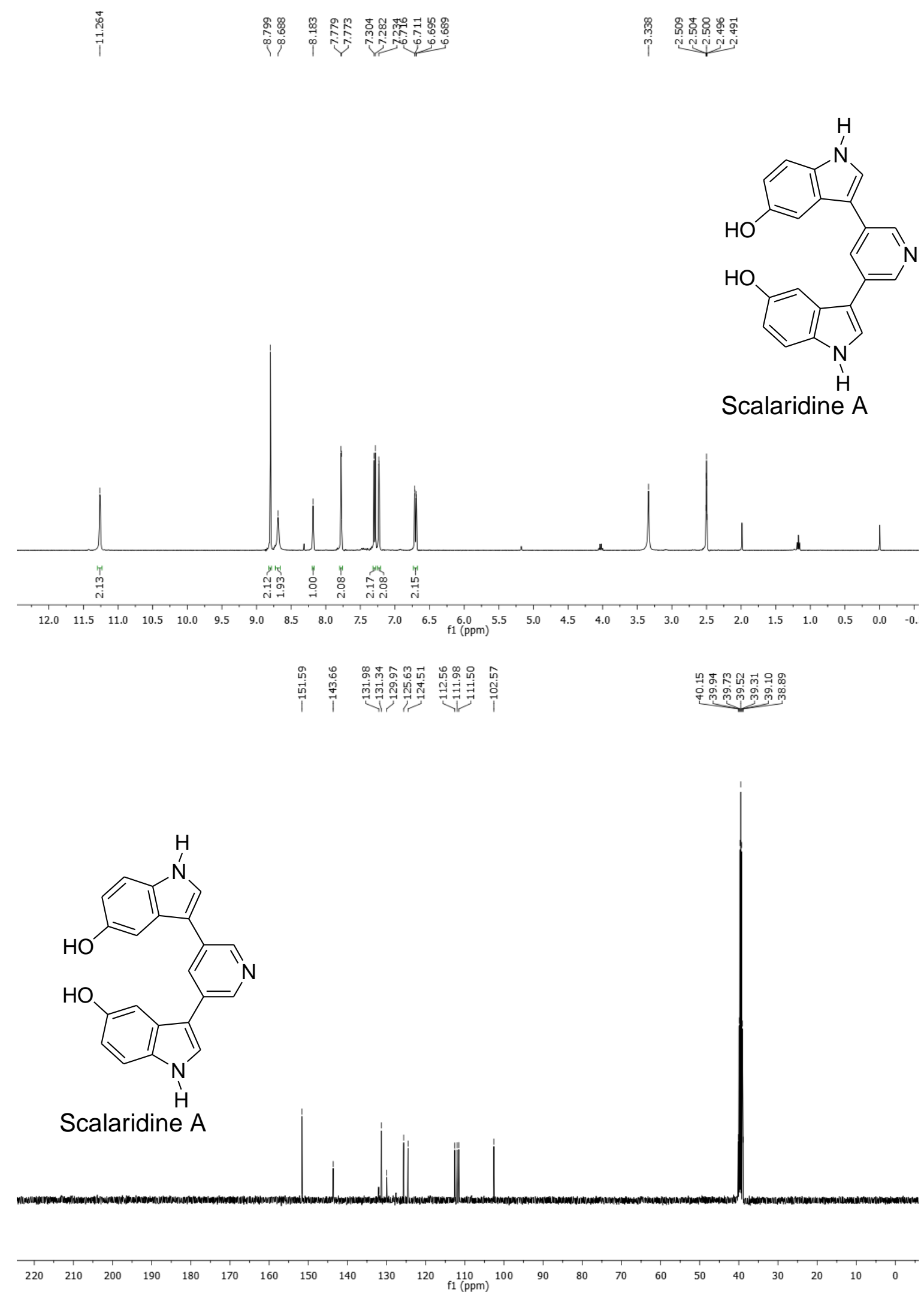

Figure $1.13{ }^{1} \mathrm{H}$ and ${ }^{13} \mathrm{C}$ NMR of scalaridine $\mathrm{A}$ 

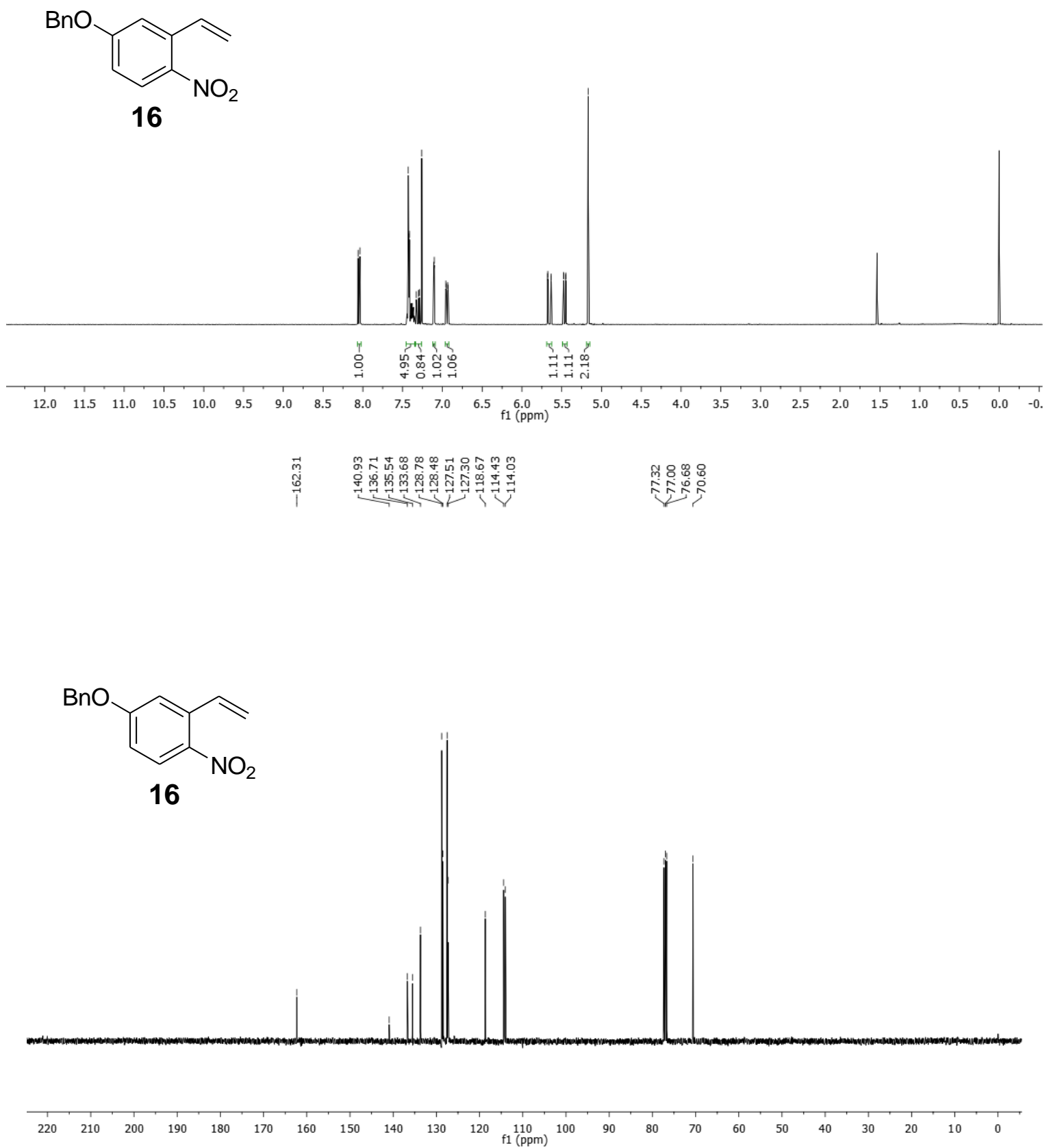

Figure $1.14{ }^{1} \mathrm{H}$ and ${ }^{13} \mathrm{C}$ NMR of compound 16 


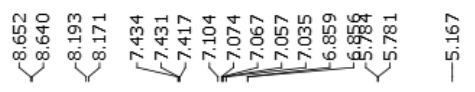

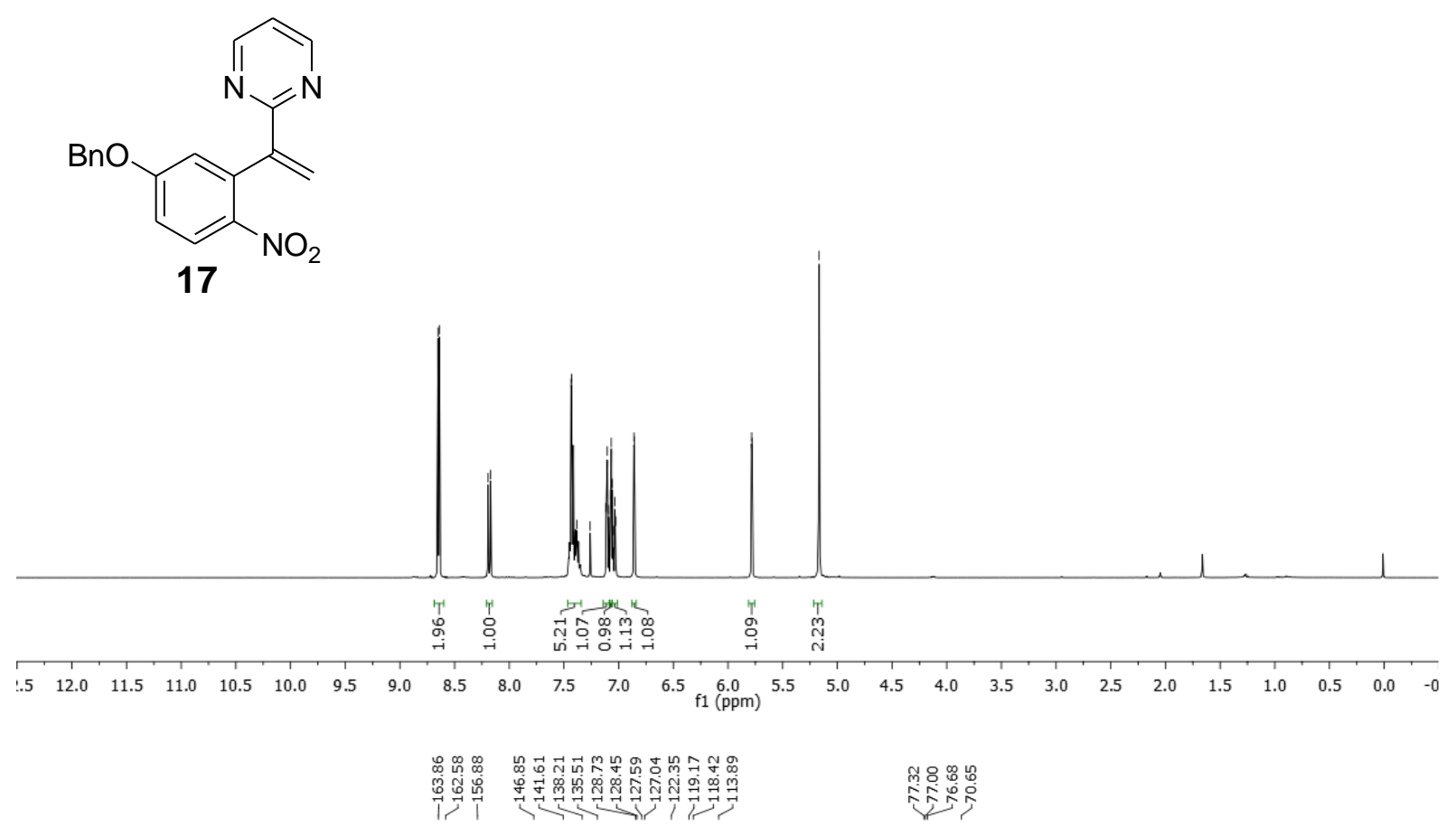<smiles>C=C(c1ncccn1)c1cc(OCc2ccccc2)ccc1[N+](=O)[O-]</smiles>
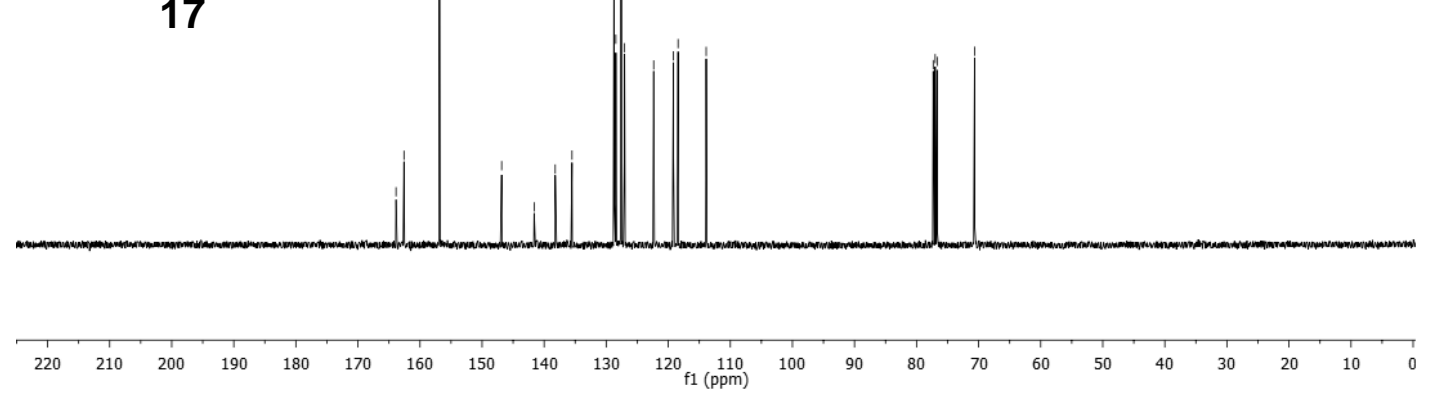

Figure $1.15{ }^{1} \mathrm{H}$ and ${ }^{13} \mathrm{C}$ NMR of compound 17 


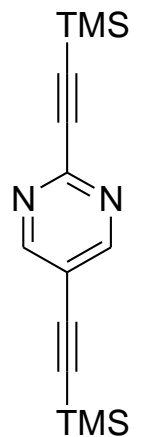

18

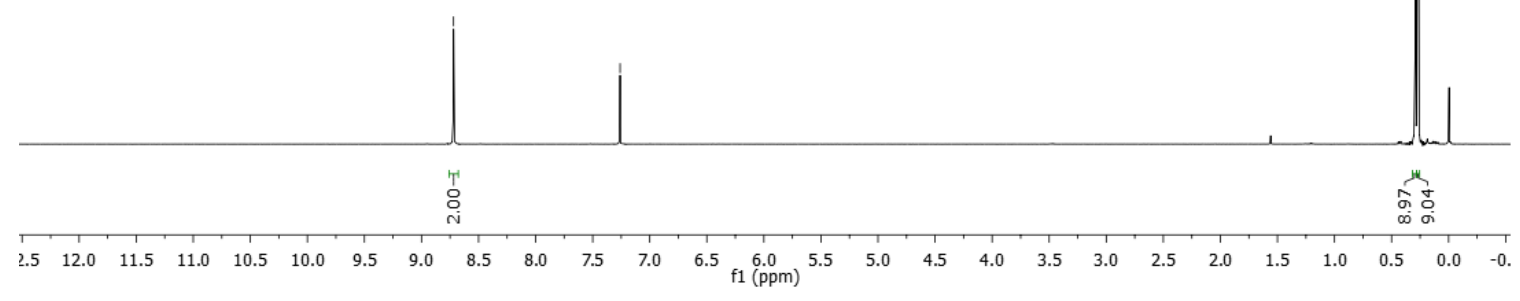

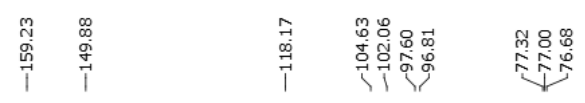

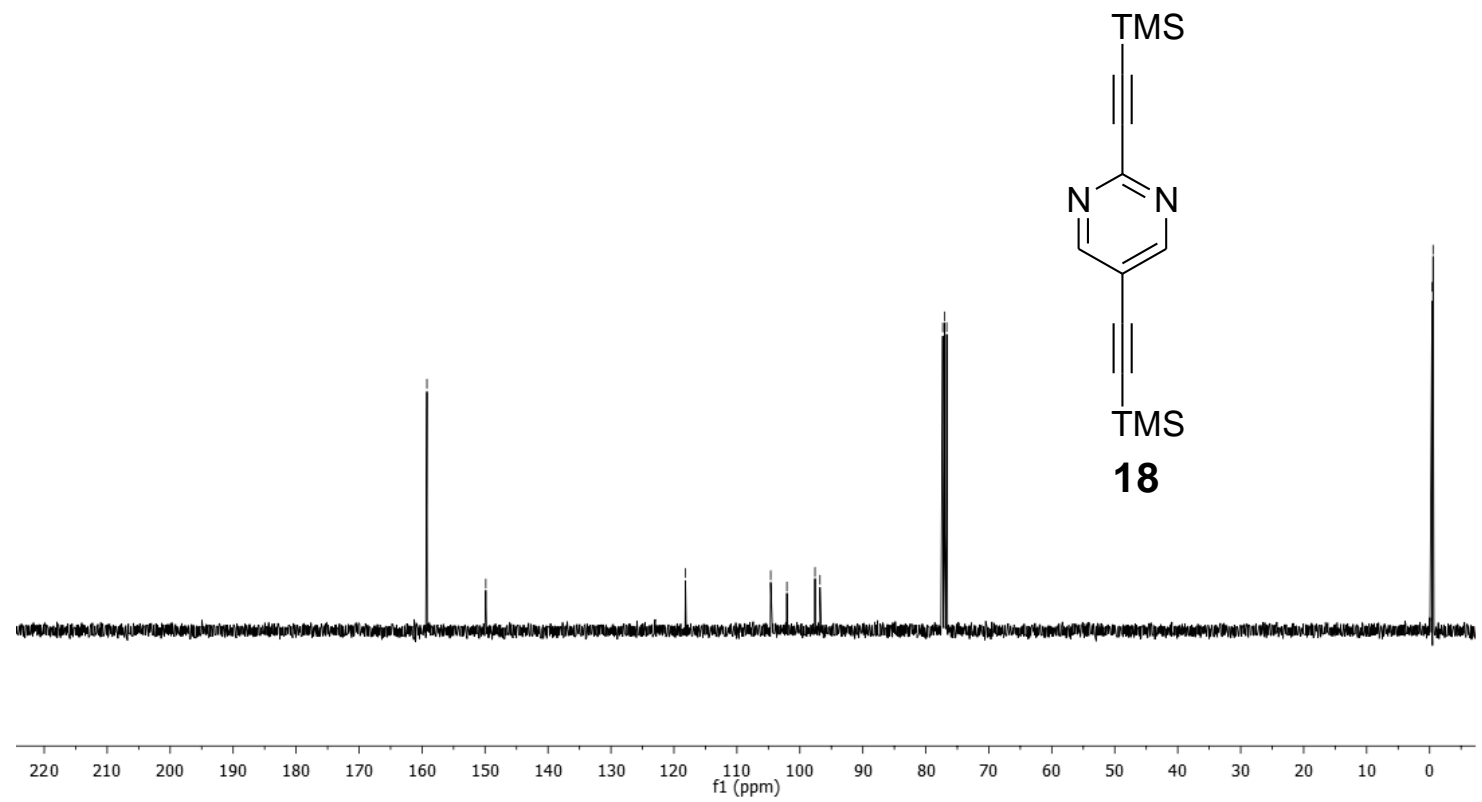

Figure $1.16{ }^{1} \mathrm{H}$ and ${ }^{13} \mathrm{C}$ NMR of compound 18 


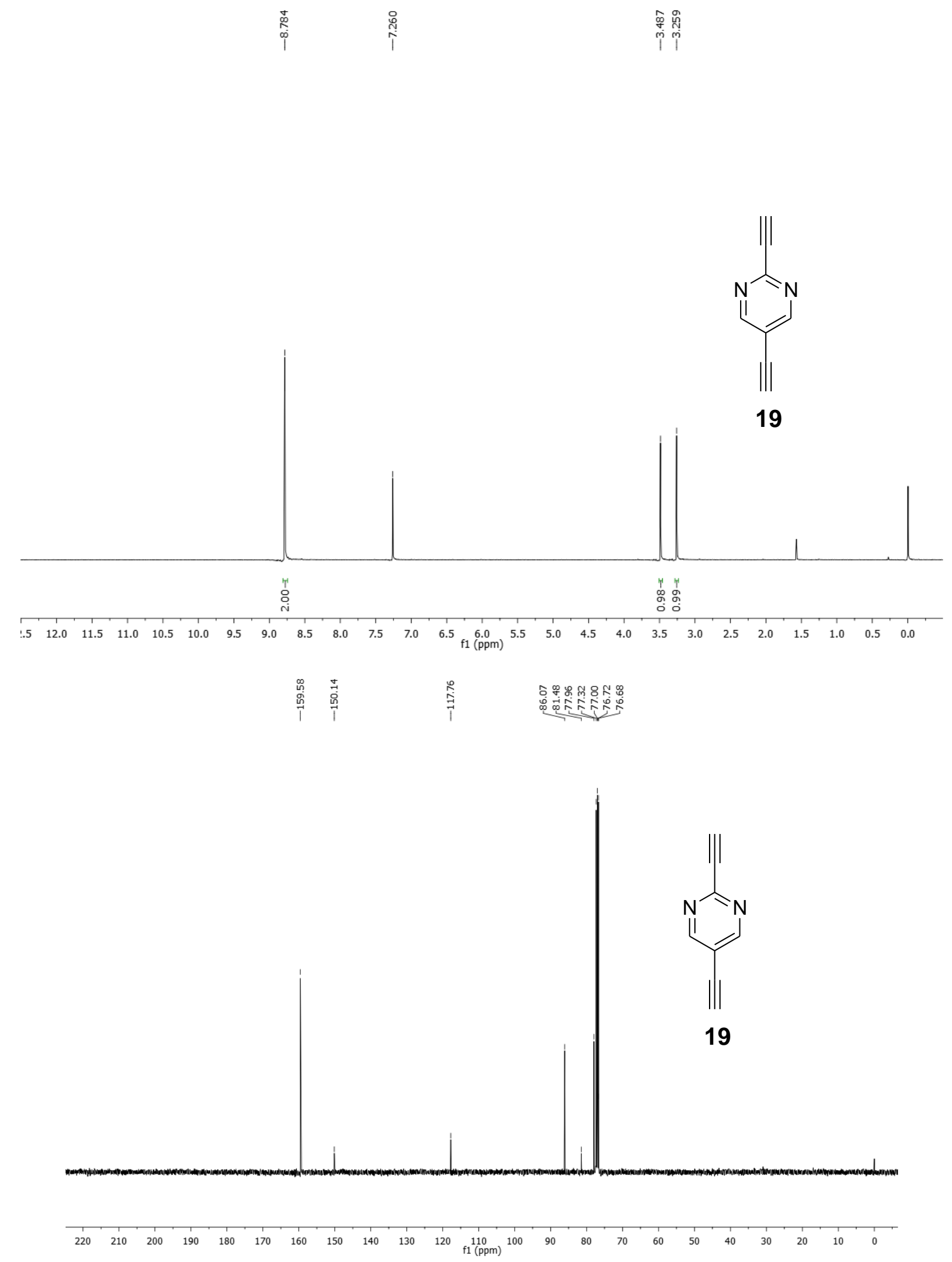

Figure $1.17{ }^{1} \mathrm{H}$ and ${ }^{13} \mathrm{C}$ NMR of compound 19 

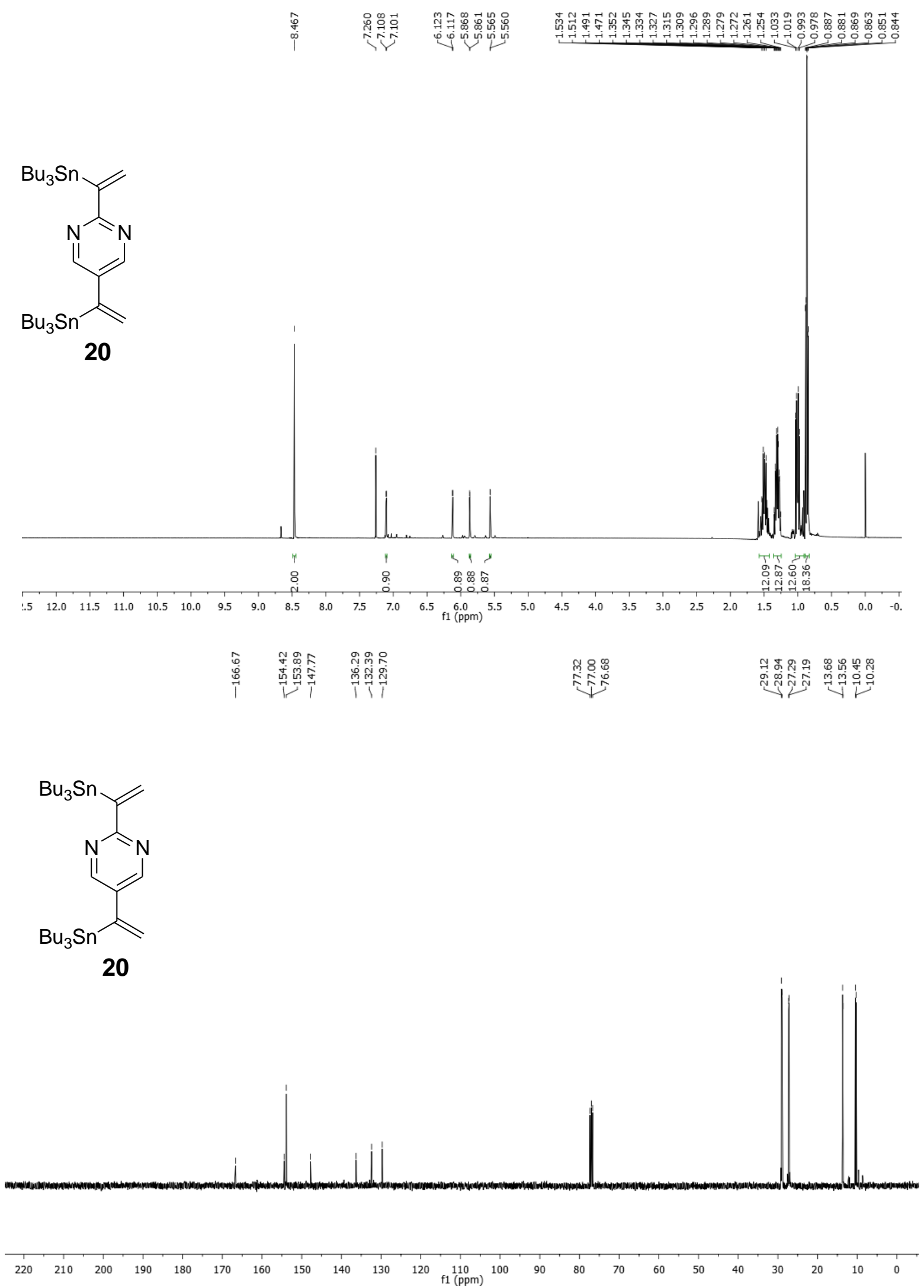

Figure 1.18 ${ }^{1} \mathrm{H}$ and ${ }^{13} \mathrm{C}$ NMR of compound 20 


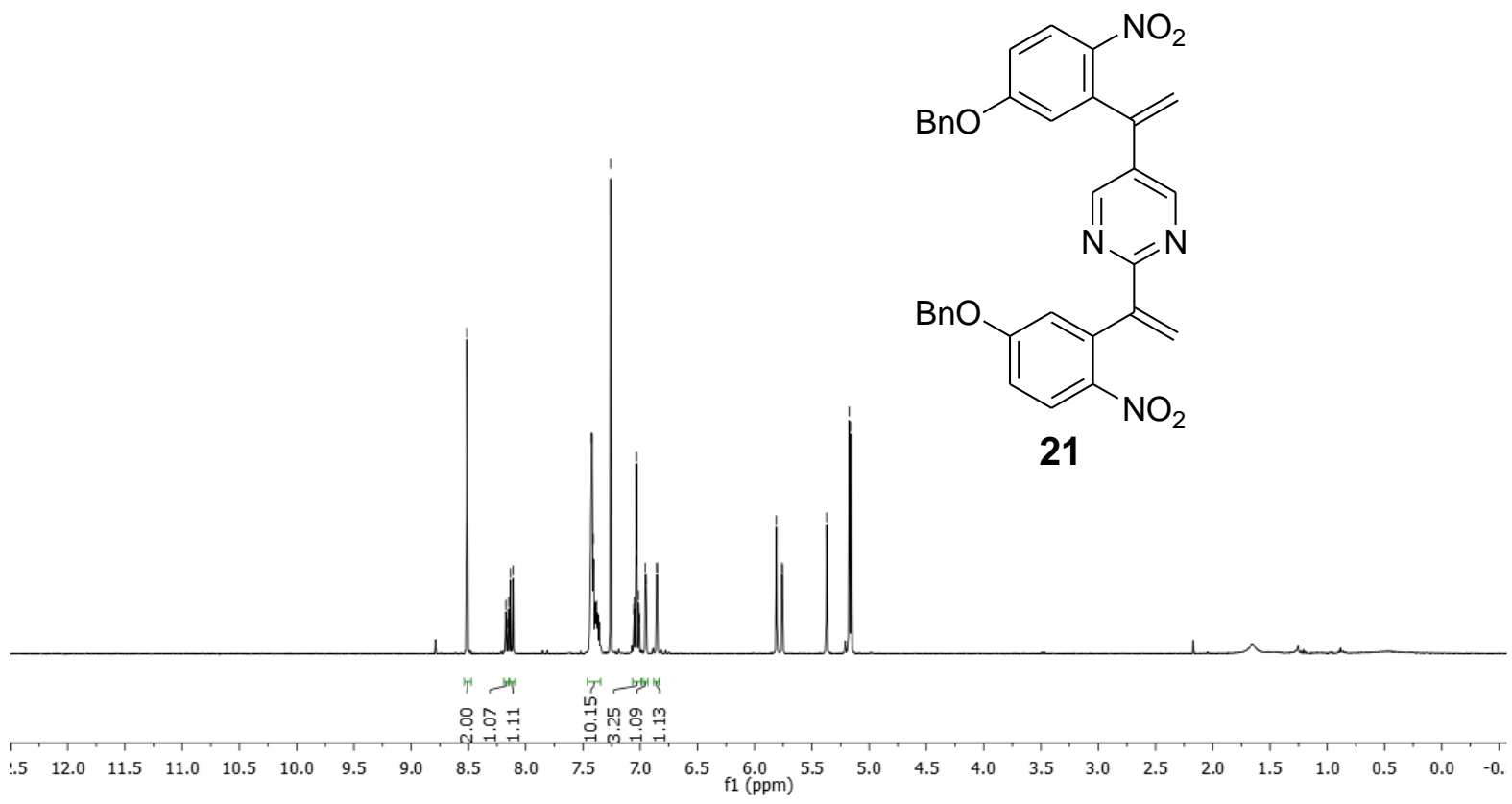

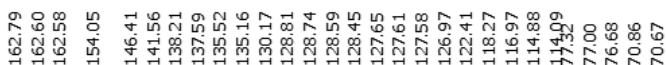

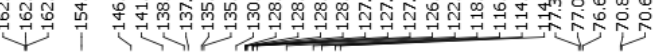
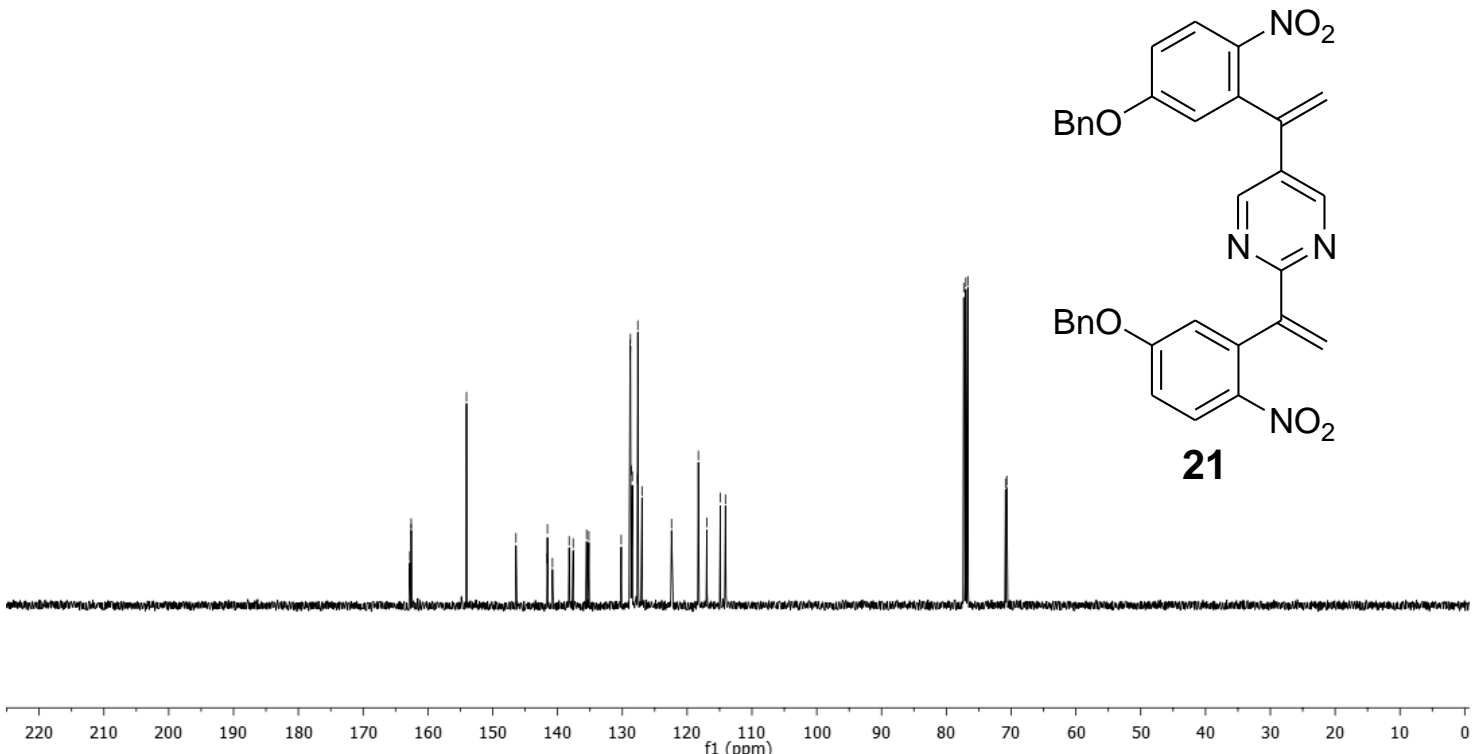

Figure $1.19{ }^{1} \mathrm{H}$ and ${ }^{13} \mathrm{C}$ NMR of compound 21 
荺量
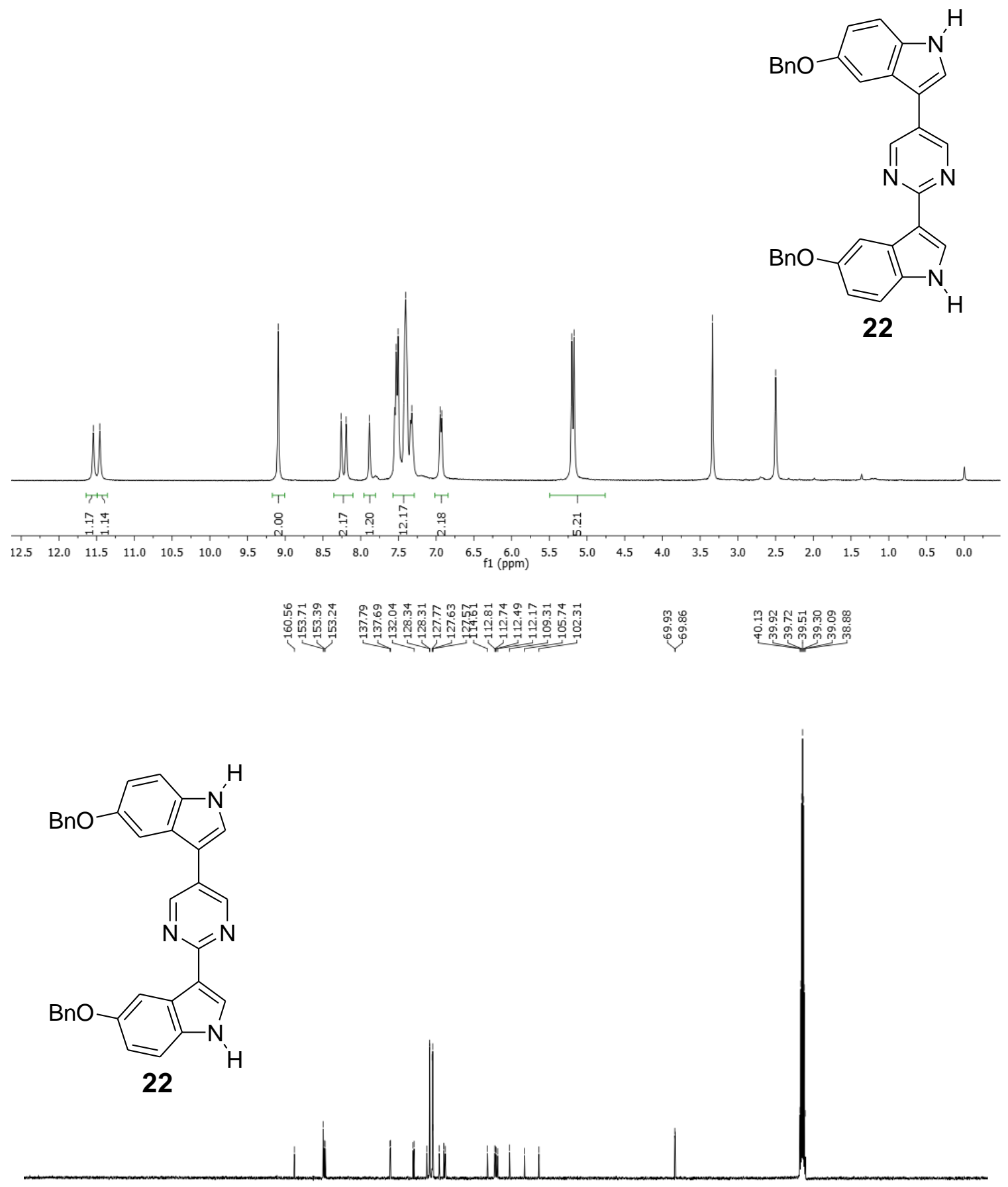

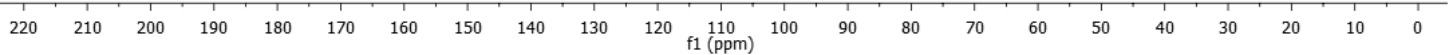

Figure 1.20 ${ }^{1} \mathrm{H}$ and ${ }^{13} \mathrm{C}$ NMR of compound 22 


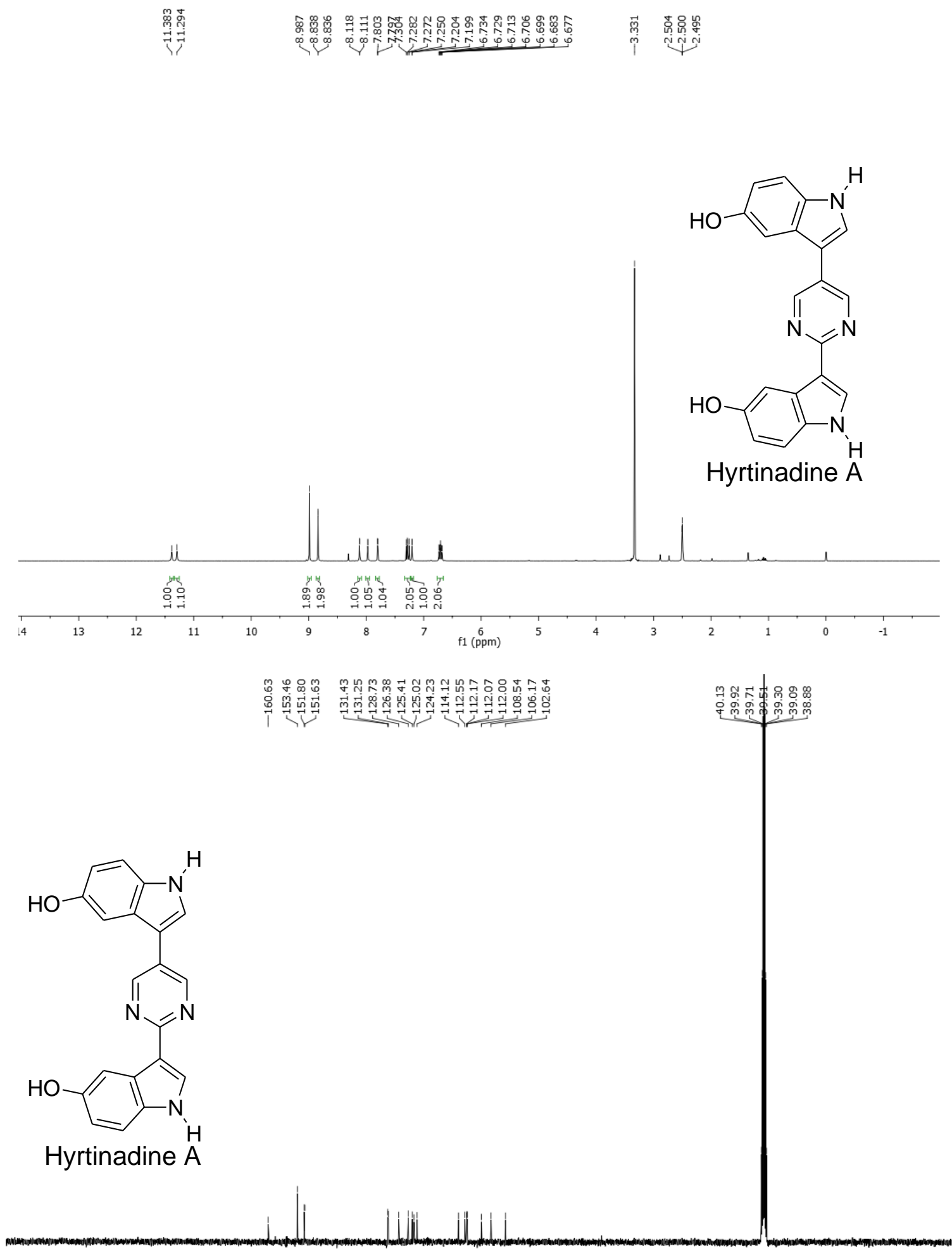

$\begin{array}{lllllllllllllllllllllllll}220 & 210 & 200 & 190 & 180 & 170 & 160 & 150 & 140 & 130 & 120 & \begin{array}{c}110 \\ \mathrm{f} 1(\mathrm{ppm})\end{array} & 100 & 90 & 80 & 70 & 60 & 50 & 40 & 30 & 20 & 10 & 0\end{array}$

Figure $1.21{ }^{1} \mathrm{H}$ and ${ }^{13} \mathrm{C}$ NMR of hyrtinadine A 


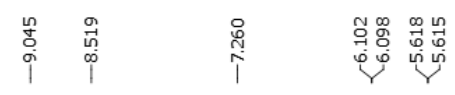
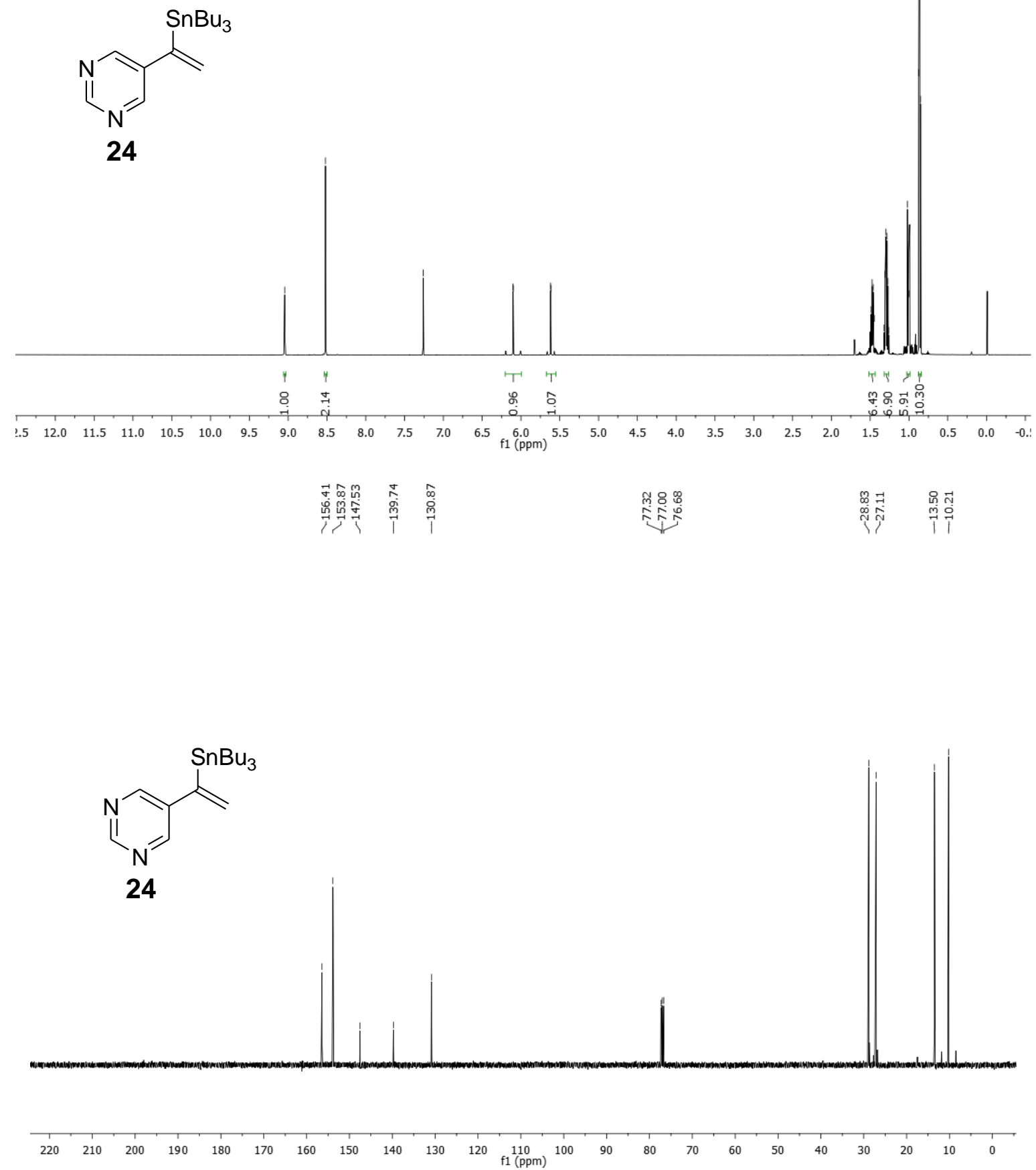

Figure $1.22{ }^{1} \mathrm{H}$ and ${ }^{13} \mathrm{C}$ NMR of compound 24 

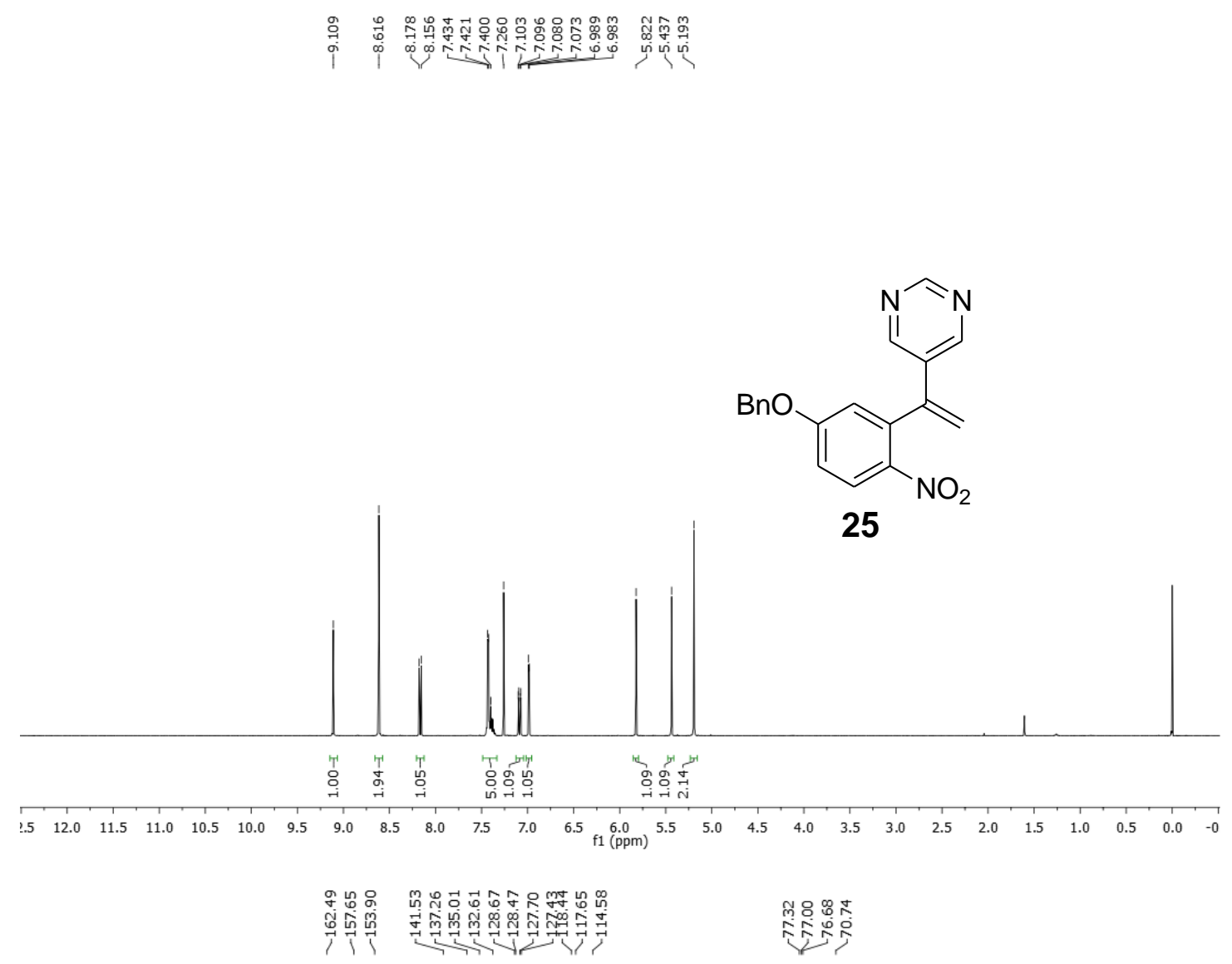<smiles>C=C(c1cncnc1)c1cc(OCc2ccccc2)ccc1[N+](=O)[O-]</smiles>

25
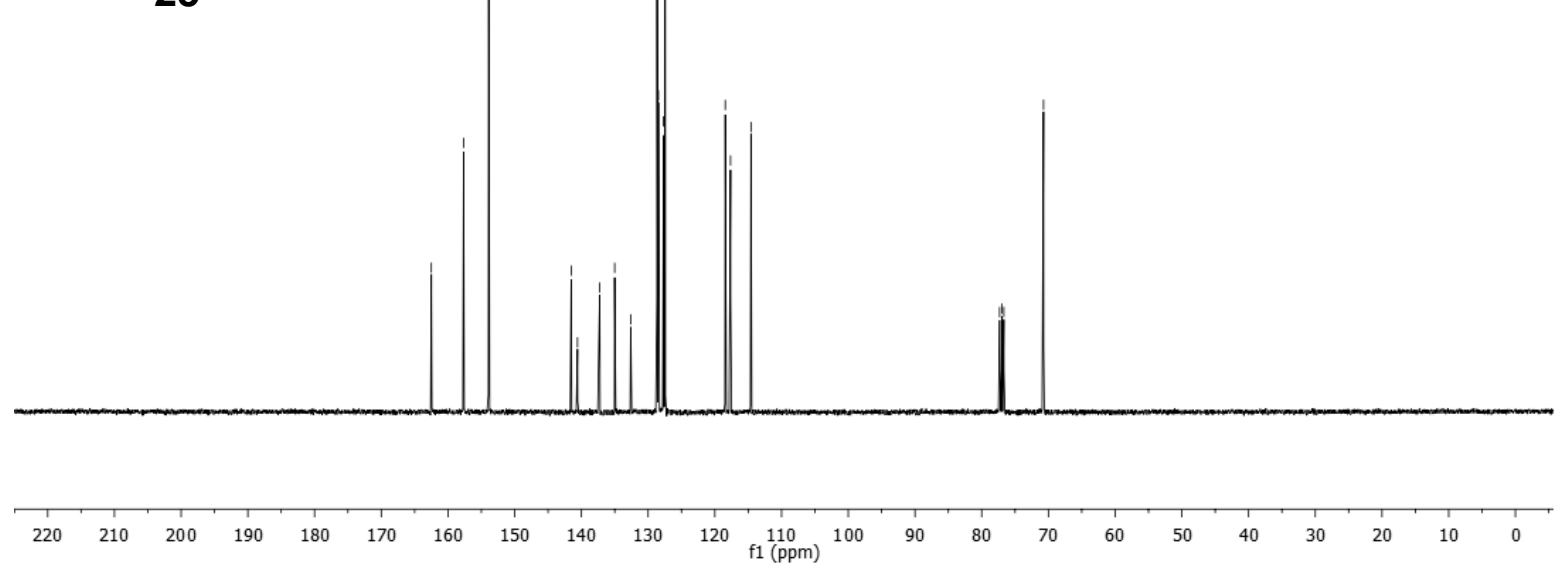

Figure 1.23 ${ }^{1} \mathrm{H}$ and ${ }^{13} \mathrm{C}$ NMR of compound 25 


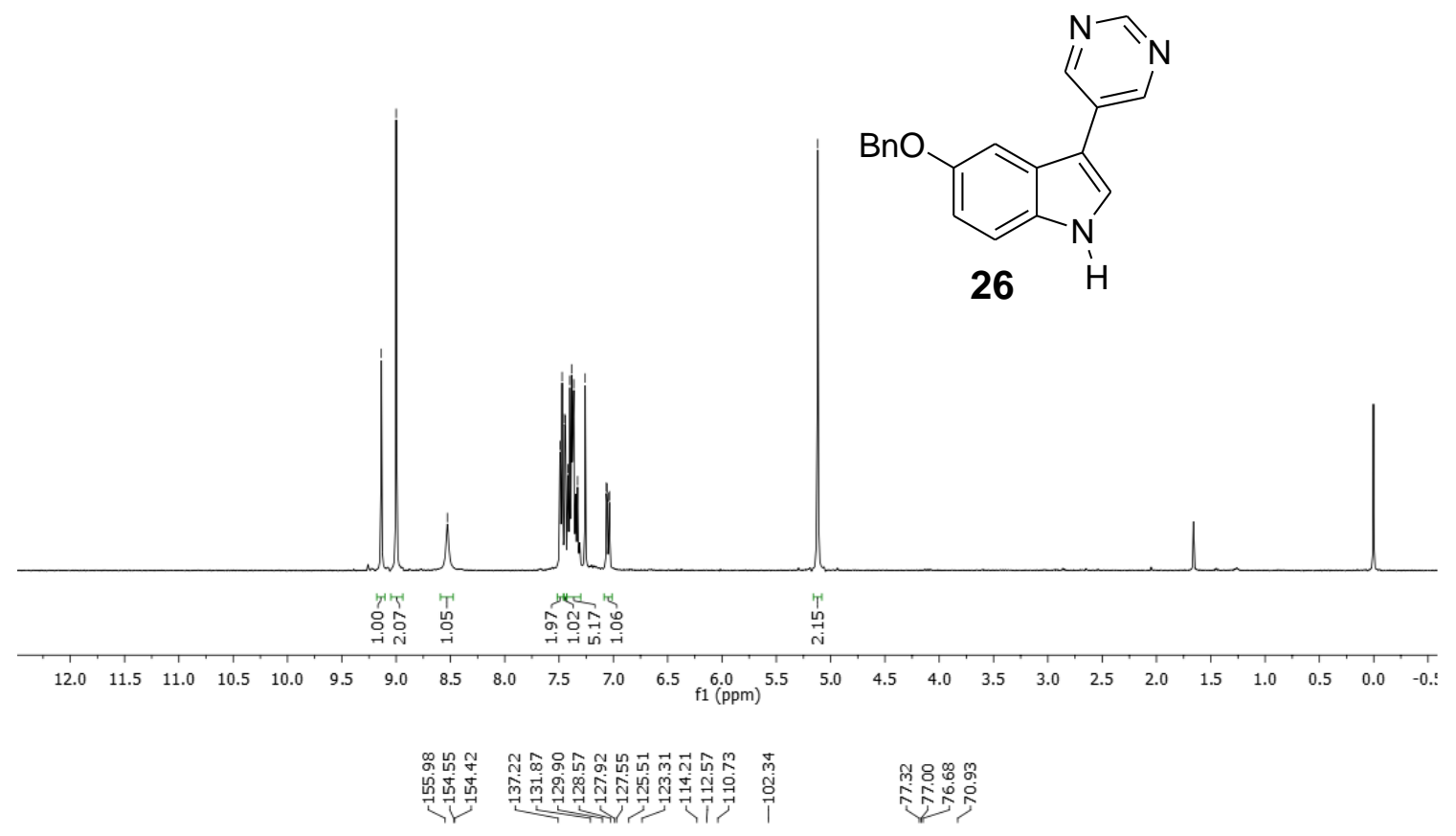<smiles></smiles>
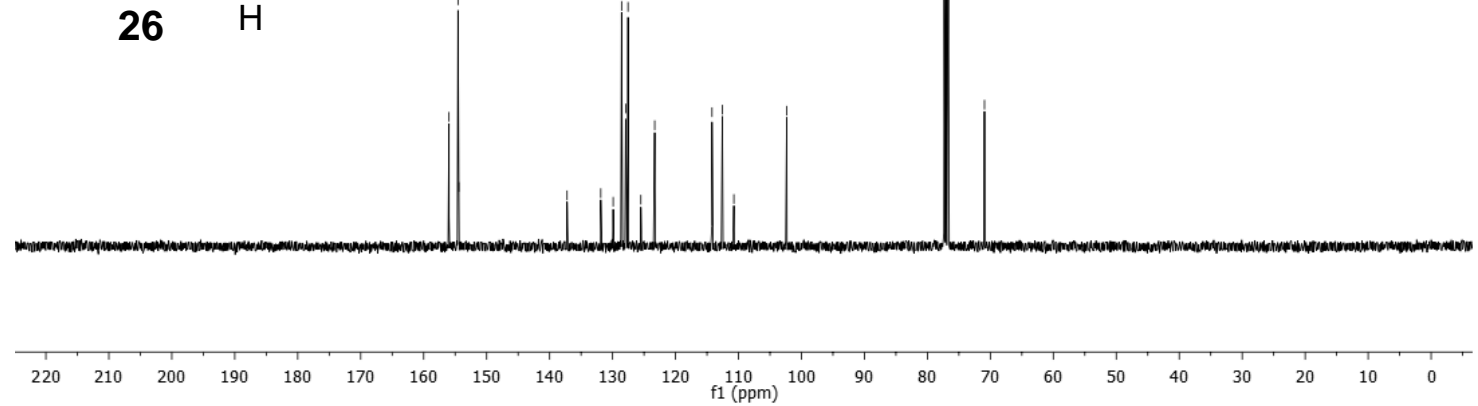

Figure 1.24 ${ }^{1} \mathrm{H}$ and ${ }^{13} \mathrm{C}$ NMR of compound 26 

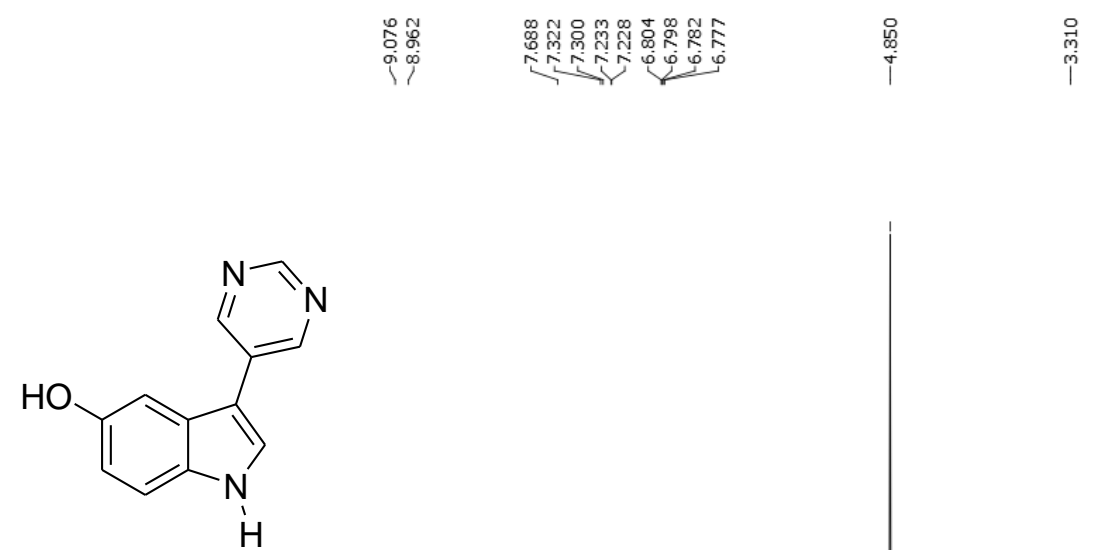

Hyrtinadine B

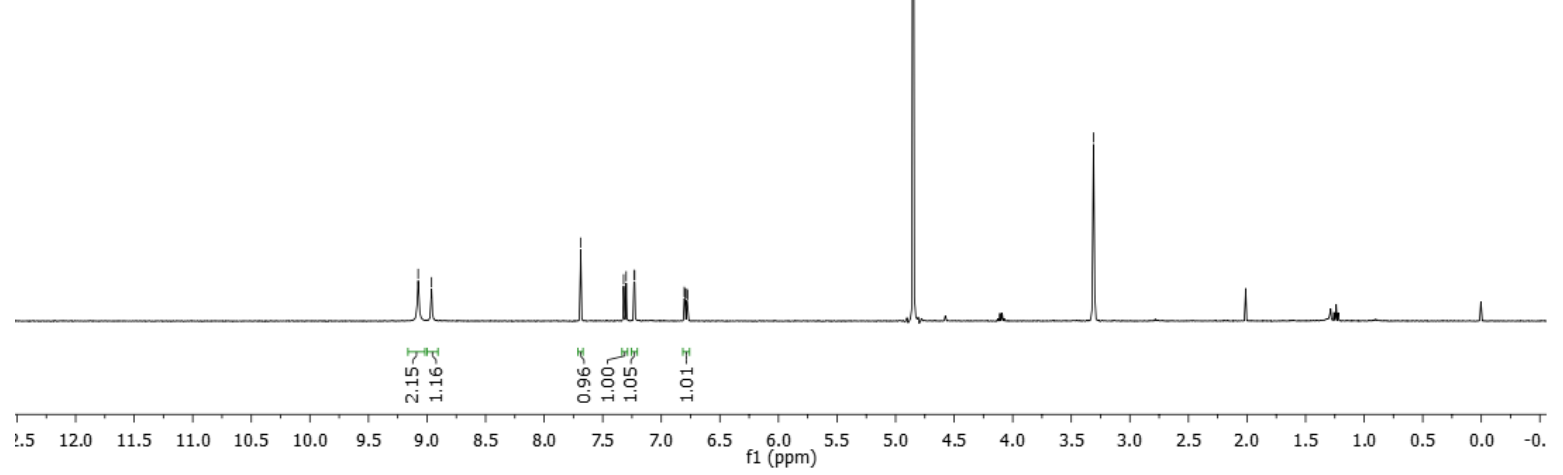<smiles>Oc1ccc2[nH]cc(-c3cncnc3)c2c1</smiles>

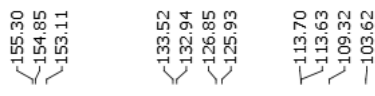

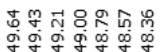

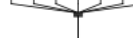

Hyrtinadine $B$

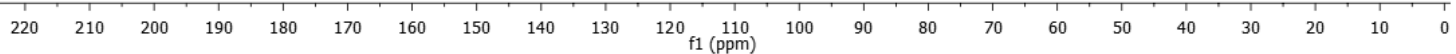

Figure $1.25{ }^{1} \mathrm{H}$ and ${ }^{13} \mathrm{C}$ NMR of hyrtinadine $\mathrm{B}$ 


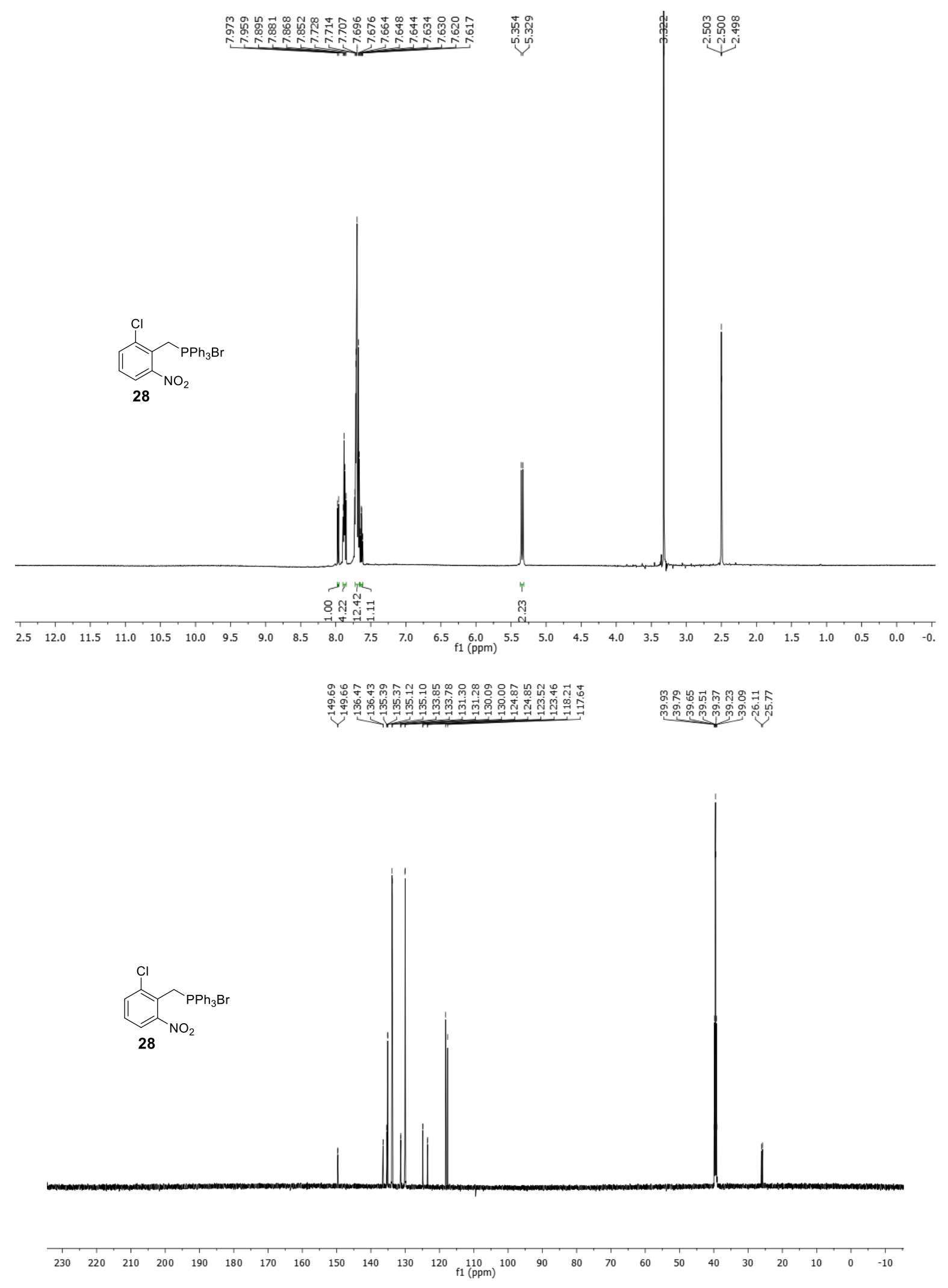

Figure $2.2{ }^{1} \mathrm{H}$ and ${ }^{13} \mathrm{C}$ NMR of compound 28 

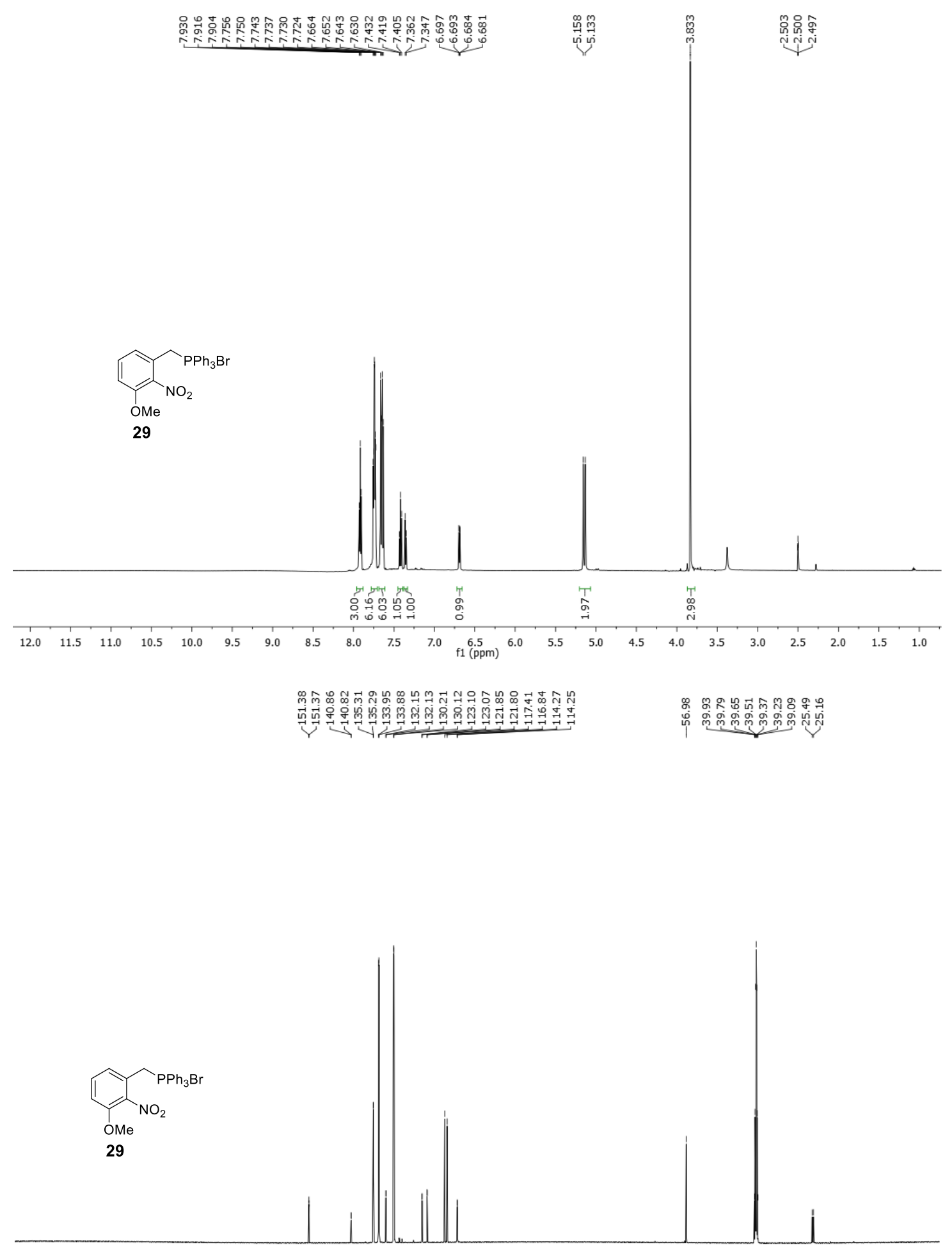

$\begin{array}{lllllllllllllllllllllllllllll}220 & 210 & 200 & 190 & 180 & 170 & 160 & 150 & 140 & 130 & 120 & 110 & 100 & 90 & 80 & 70 & 60 & 50 & 40 & 30 & 20 & 10 & 0\end{array}$

Figure $2.3{ }^{1} \mathrm{H}$ and ${ }^{13} \mathrm{C}$ NMR of compound 29 

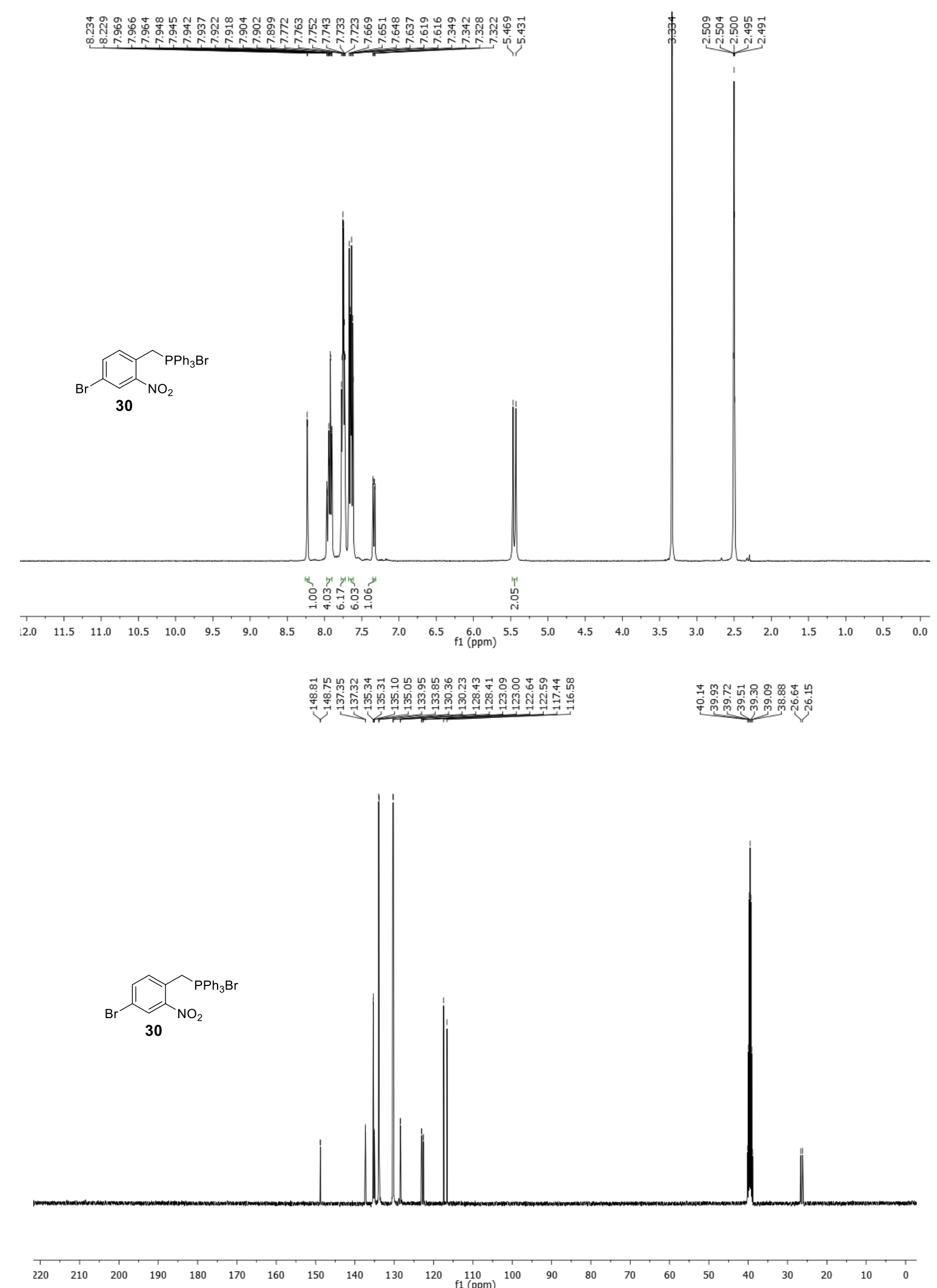

Figure $2.4{ }^{1} \mathrm{H}$ and ${ }^{13} \mathrm{C}$ NMR of compound 30 

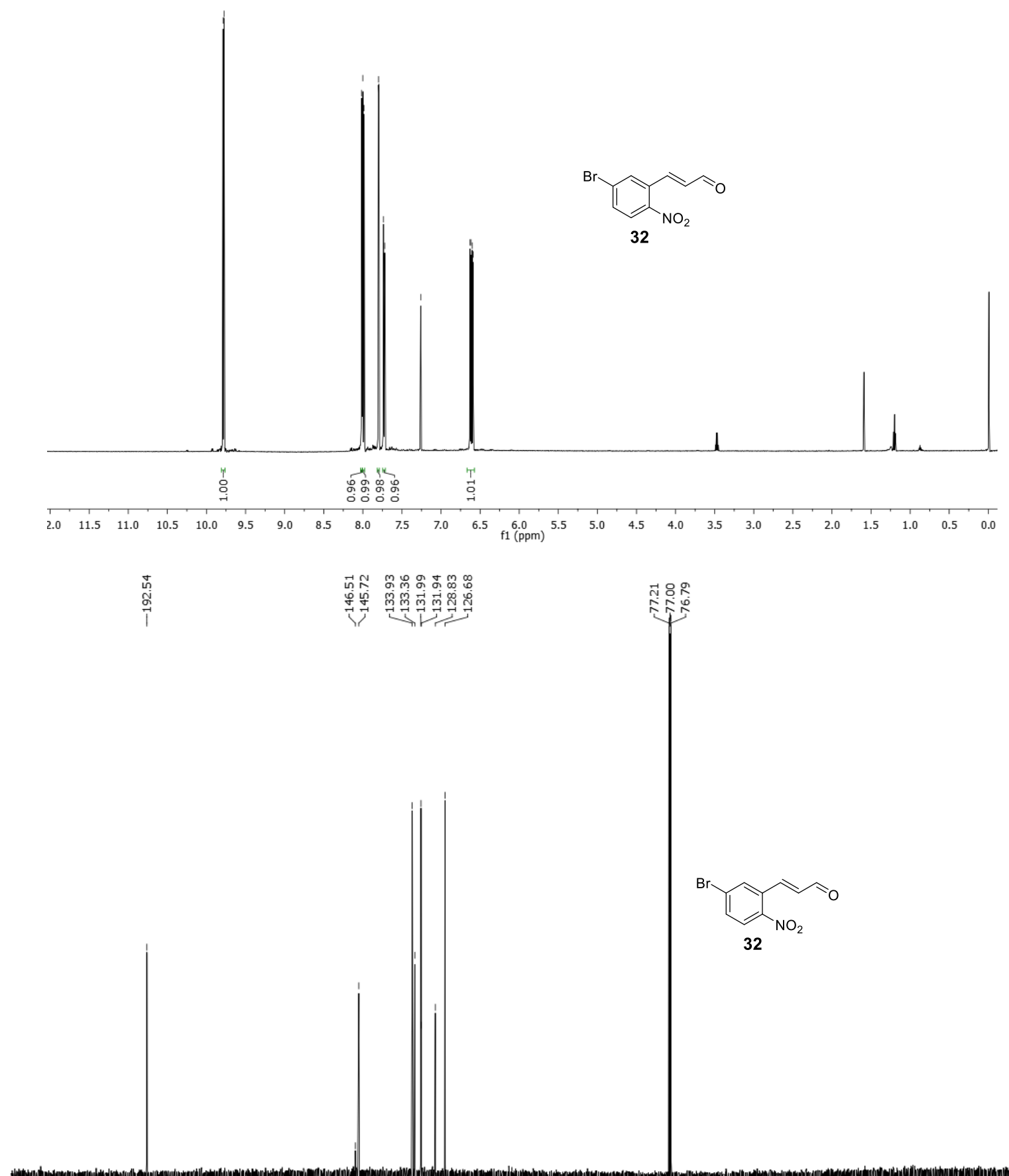

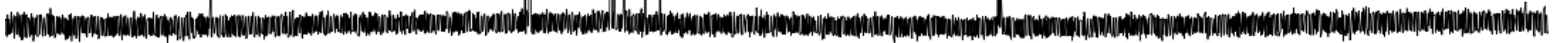

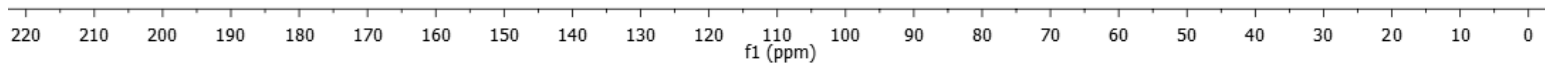

Figure $2.5{ }^{1} \mathrm{H}$ and ${ }^{13} \mathrm{C}$ NMR of compound 32 

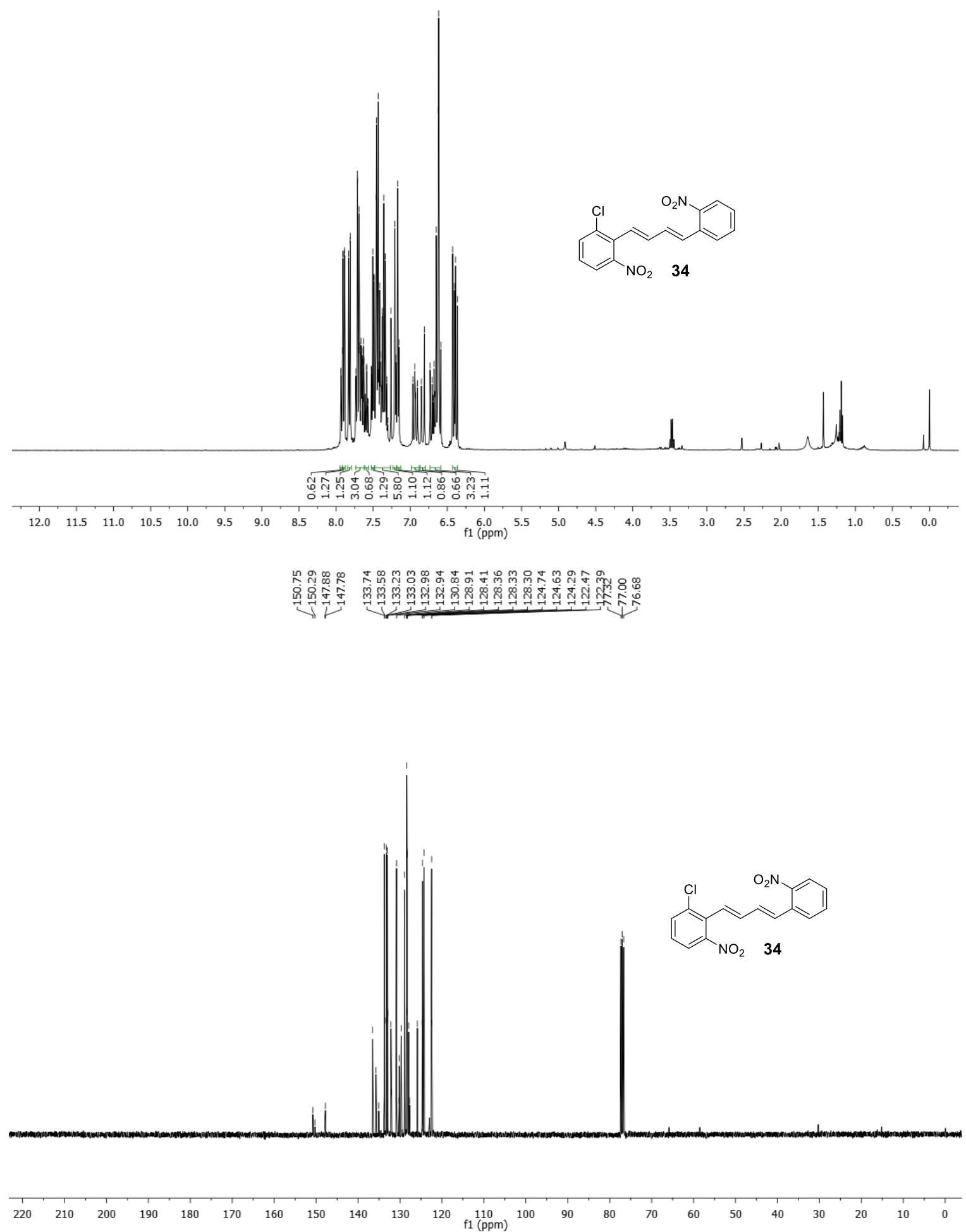

Figure 2.6 ${ }^{1} \mathrm{H}$ and ${ }^{13} \mathrm{C}$ NMR of compound 34 


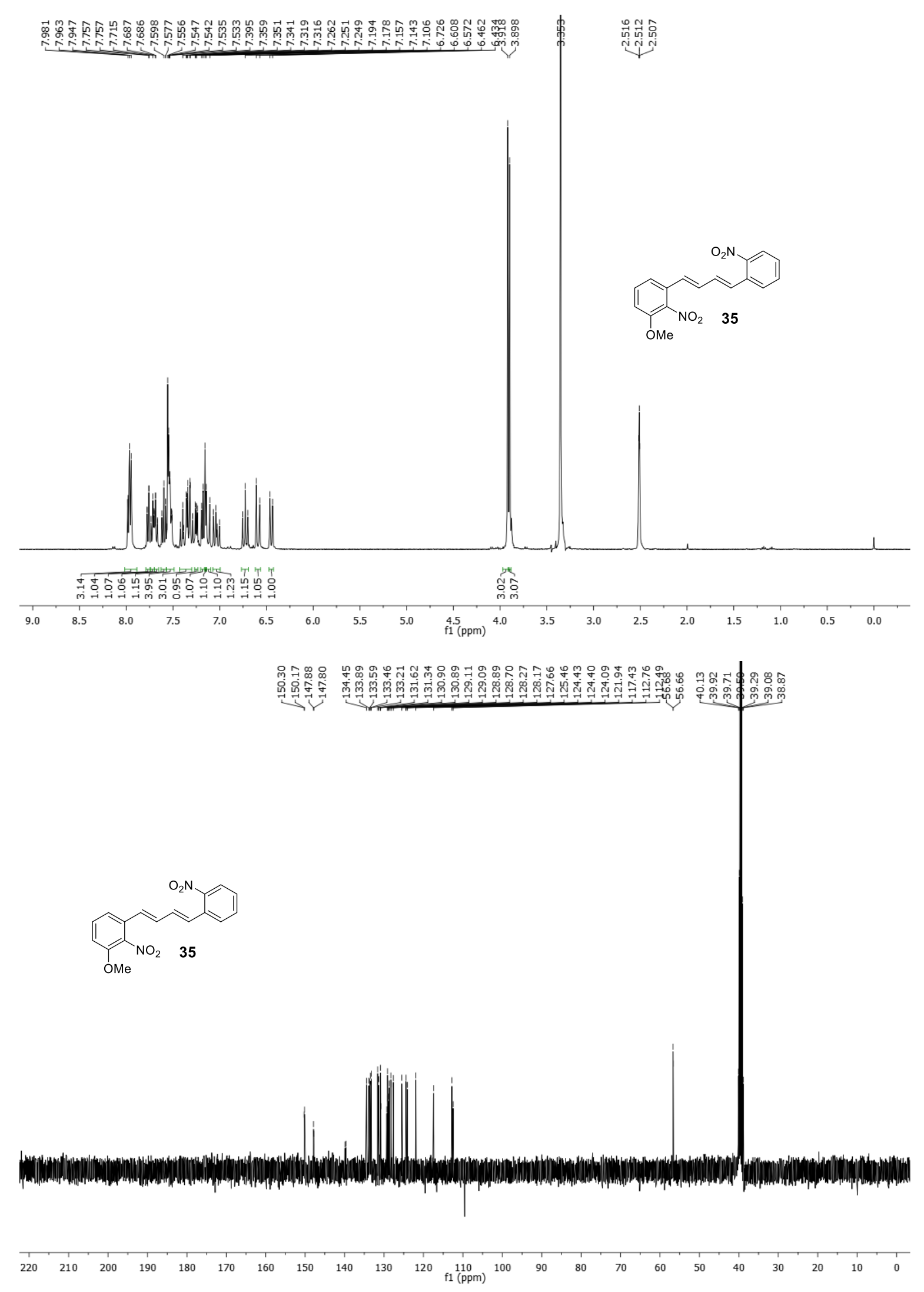

Figure $2.7{ }^{1} \mathrm{H}$ and ${ }^{13} \mathrm{C}$ NMR of compound 35 

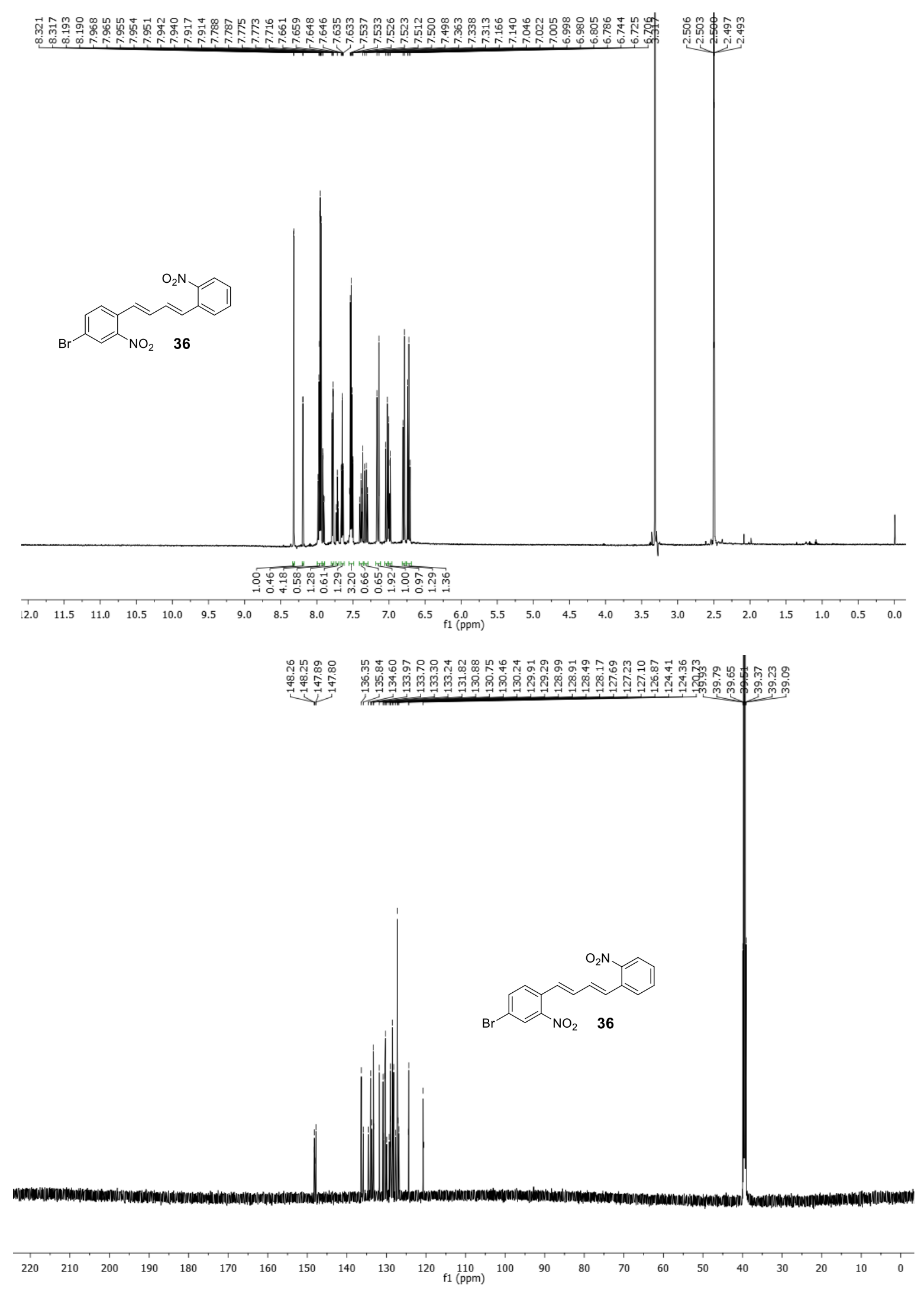

Figure $2.8{ }^{1} \mathrm{H}$ and ${ }^{13} \mathrm{C}$ NMR of compound $\mathbf{3 6}$ 


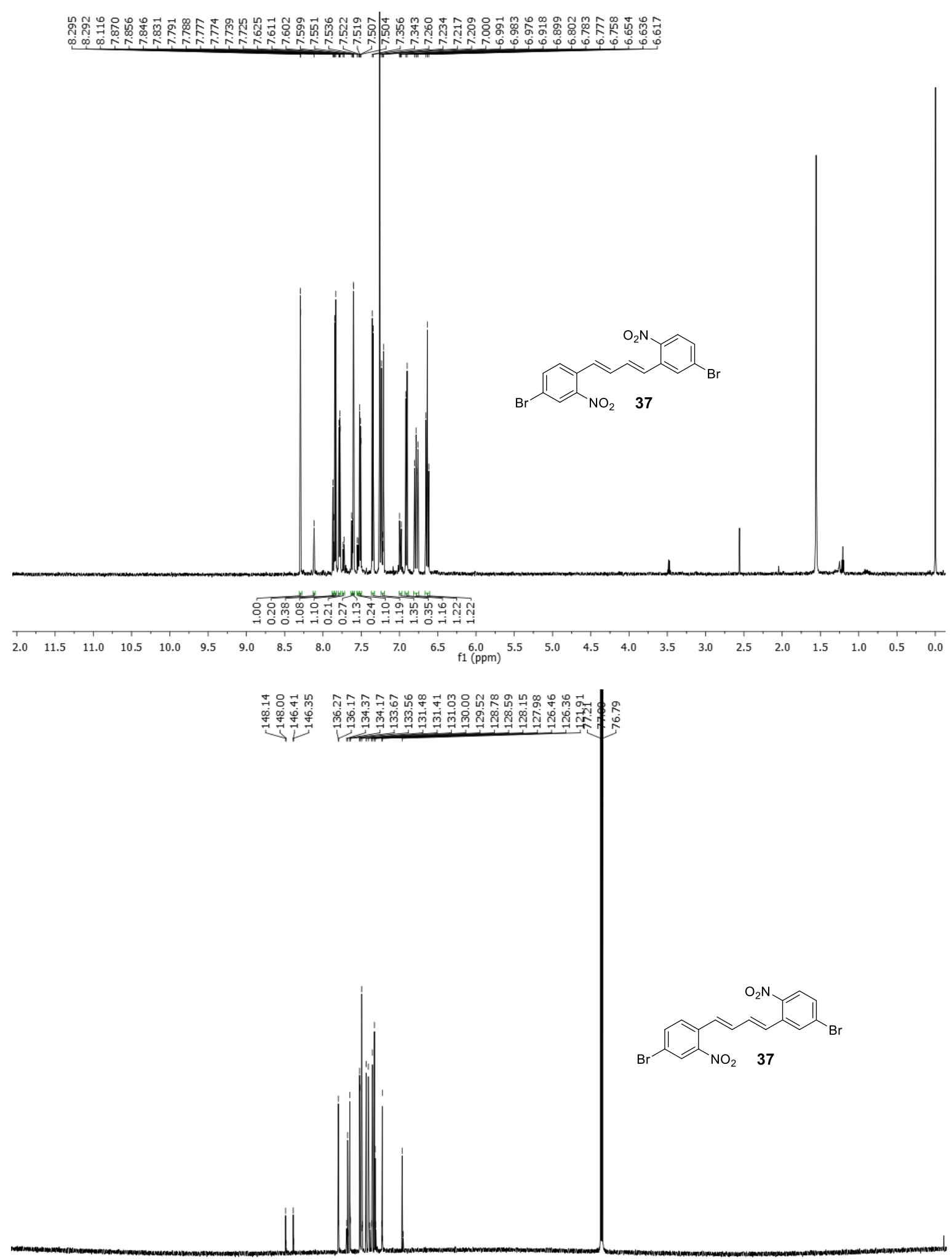

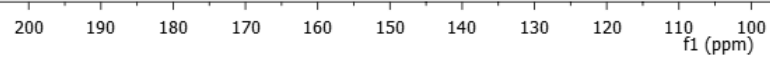

Figure $2.9{ }^{1} \mathrm{H}$ and ${ }^{13} \mathrm{C}$ NMR of compound 37 

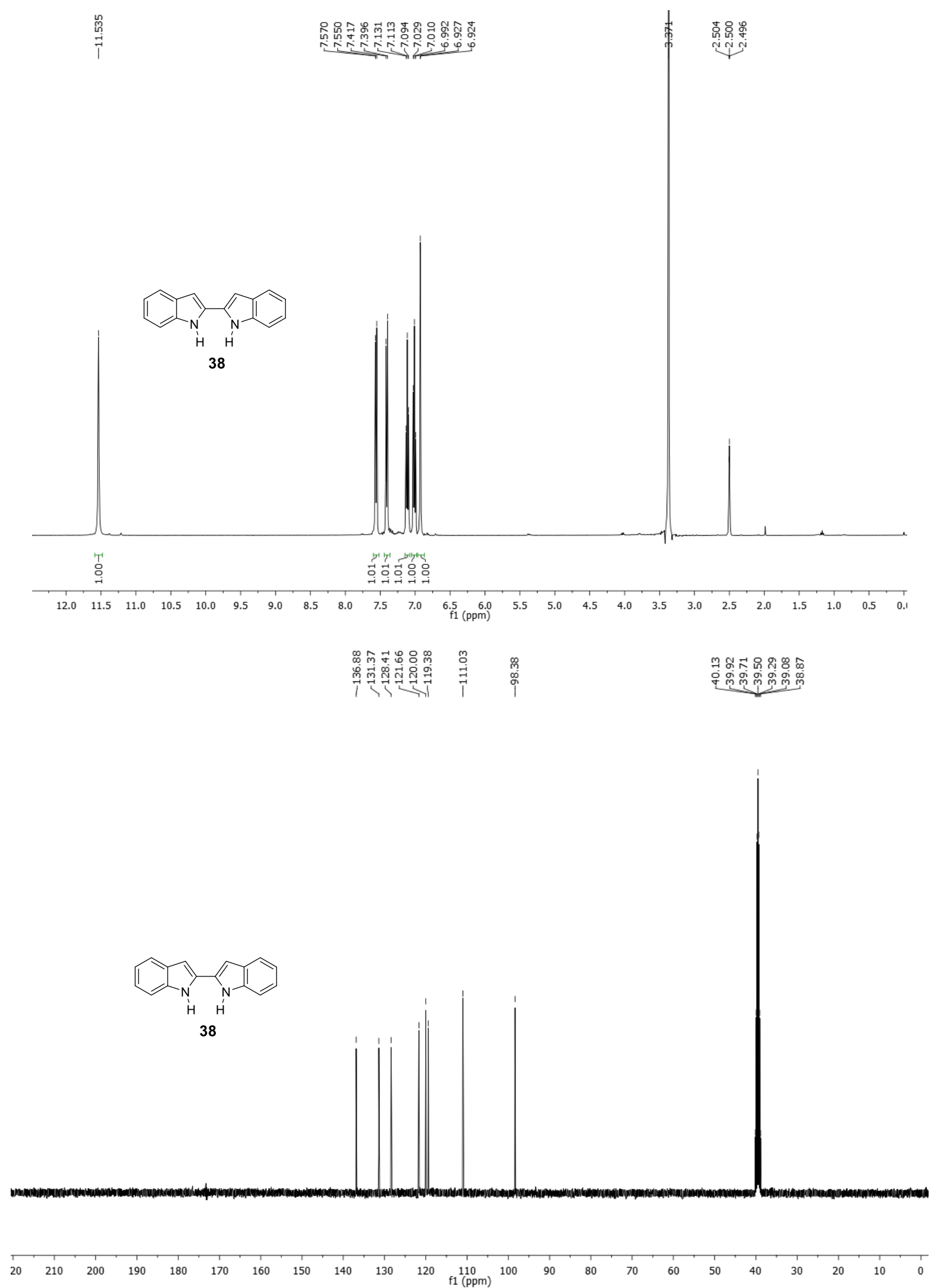

Figure 2.10 ${ }^{1} \mathrm{H}$ and ${ }^{13} \mathrm{C}$ NMR of compound 38 

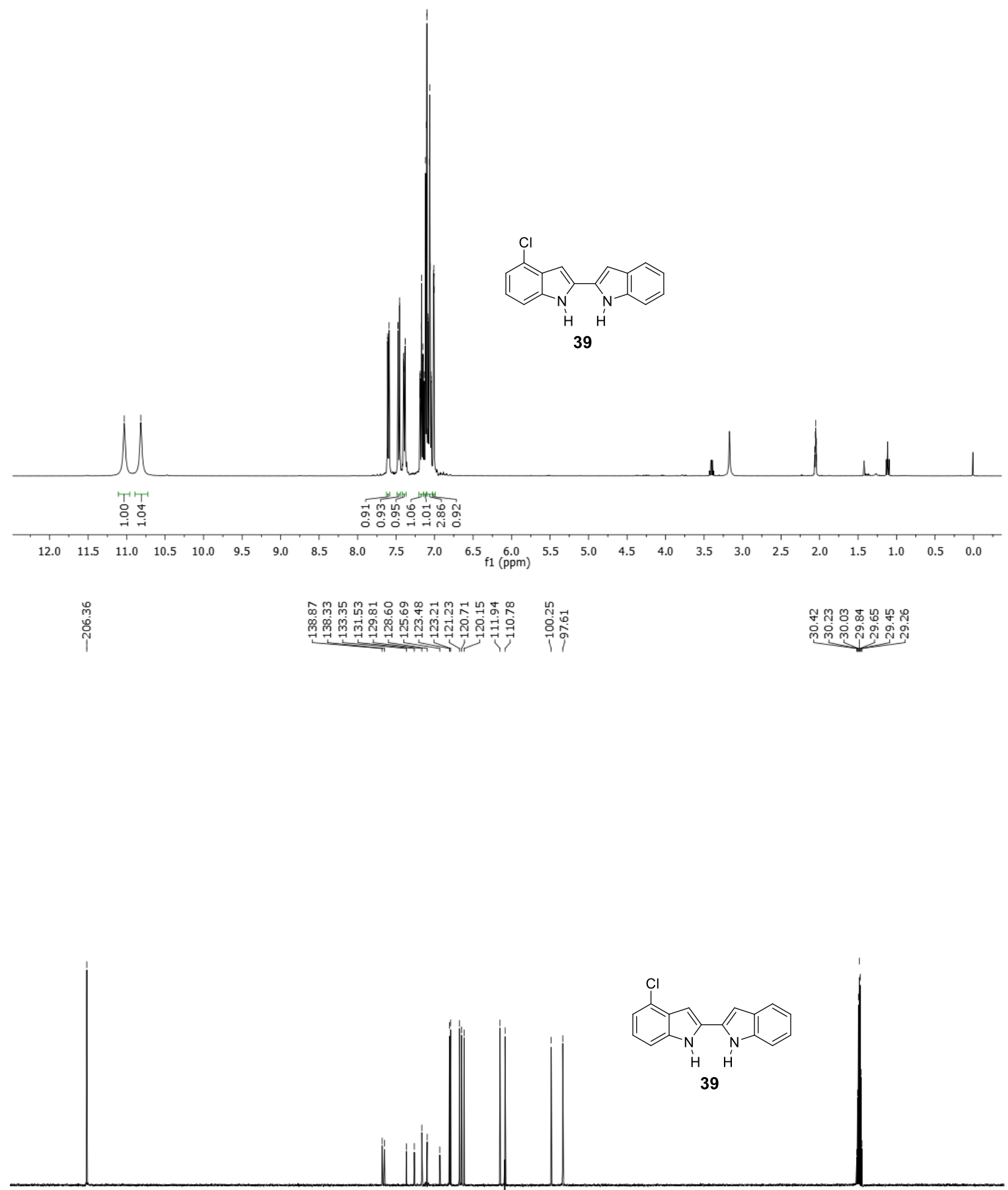

Figure 2.11 ${ }^{1} \mathrm{H}$ and ${ }^{13} \mathrm{C}$ NMR of compound 39 


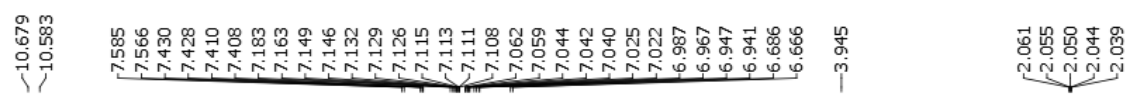
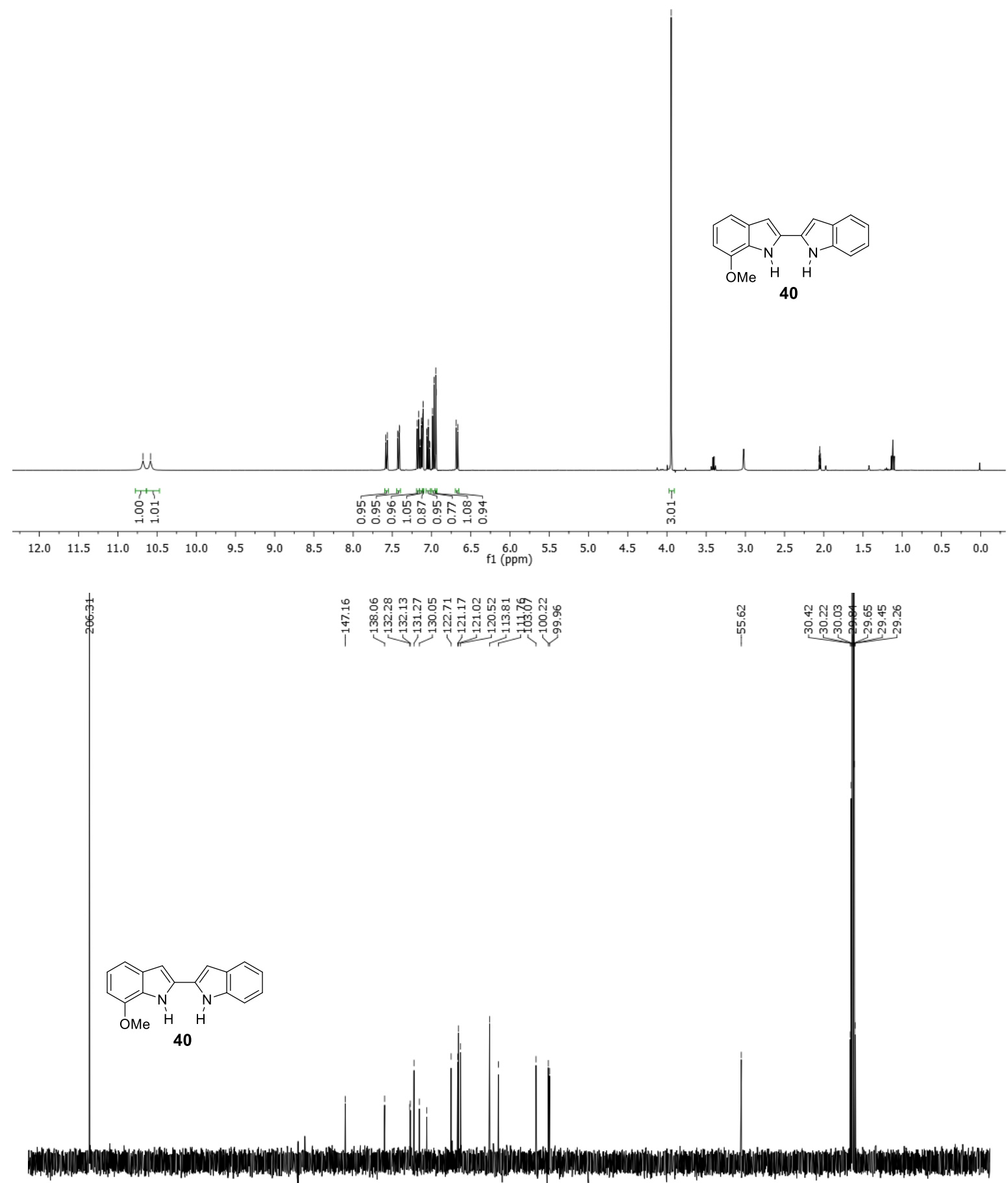

\begin{tabular}{llllllllllllllllllllllll}
\hline & 20 & 210 & 200 & 190 & 180 & 170 & 160 & 150 & 140 & 130 & 120 & 110 & 100 & 90 & 80 & 70 & 60 & 50 & 40 & 30 & 20 & 10 & 0
\end{tabular}

Figure 2.12 ${ }^{1} \mathrm{H}$ and ${ }^{13} \mathrm{C}$ NMR of compound 40 

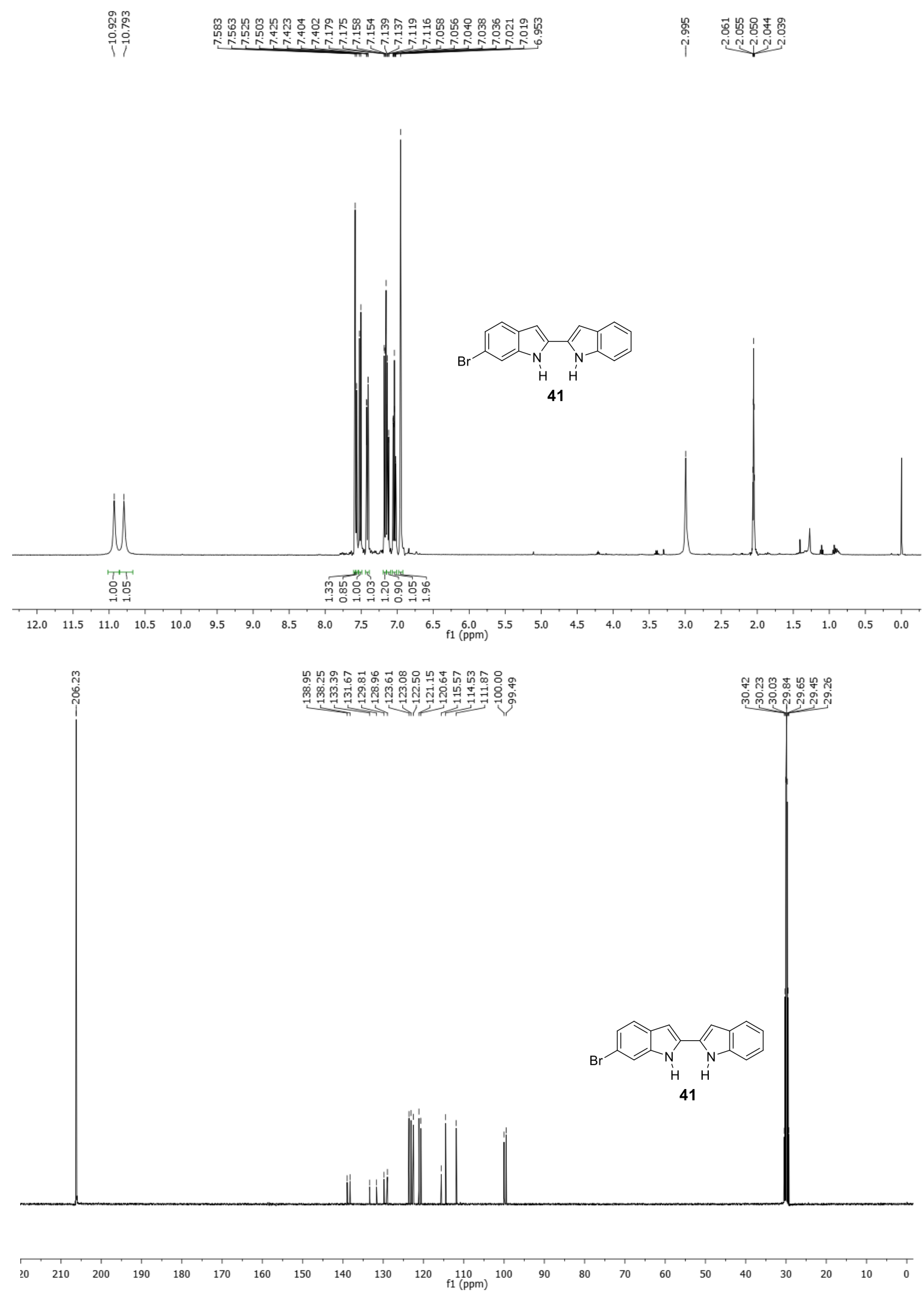

Figure 2.13 ${ }^{1} \mathrm{H}$ and ${ }^{13} \mathrm{C}$ NMR of compound 41 


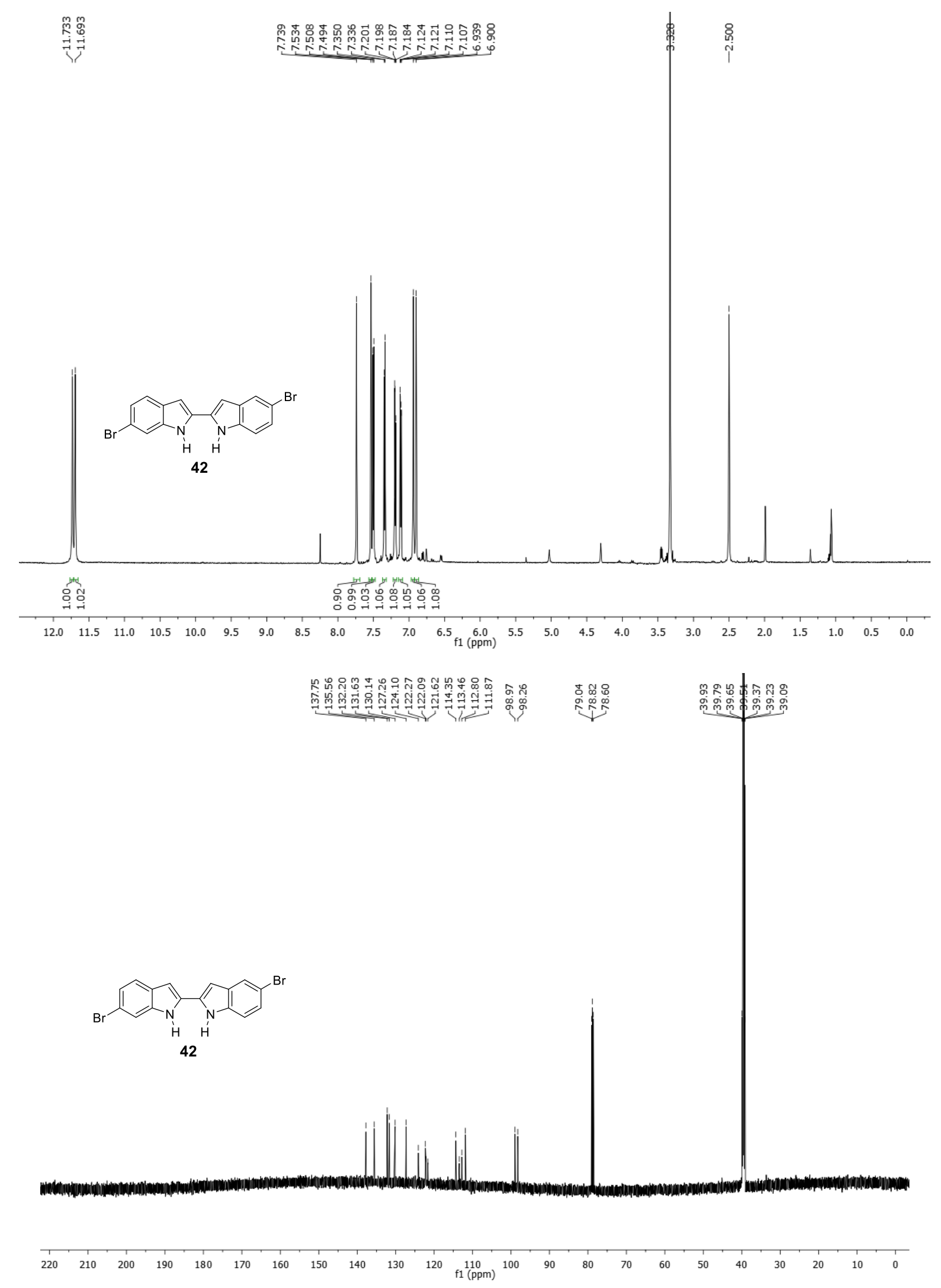

Figure 2.14 ${ }^{1} \mathrm{H}$ and ${ }^{13} \mathrm{C}$ NMR of compound 42 

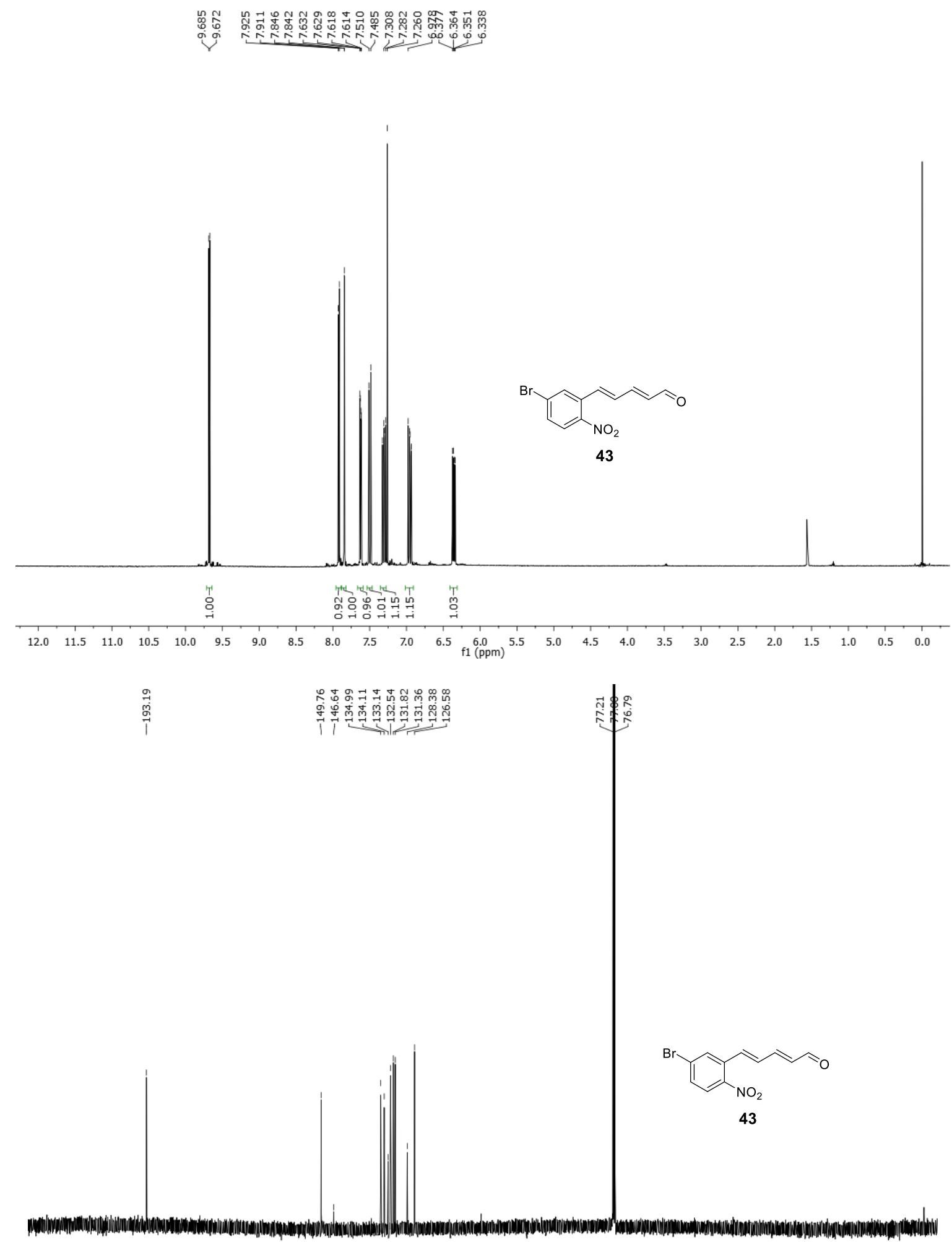

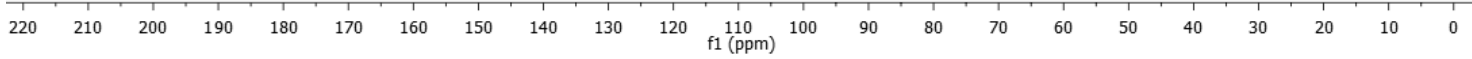

Figure 2.15 ${ }^{1} \mathrm{H}$ and ${ }^{13} \mathrm{C}$ NMR of compound 43 

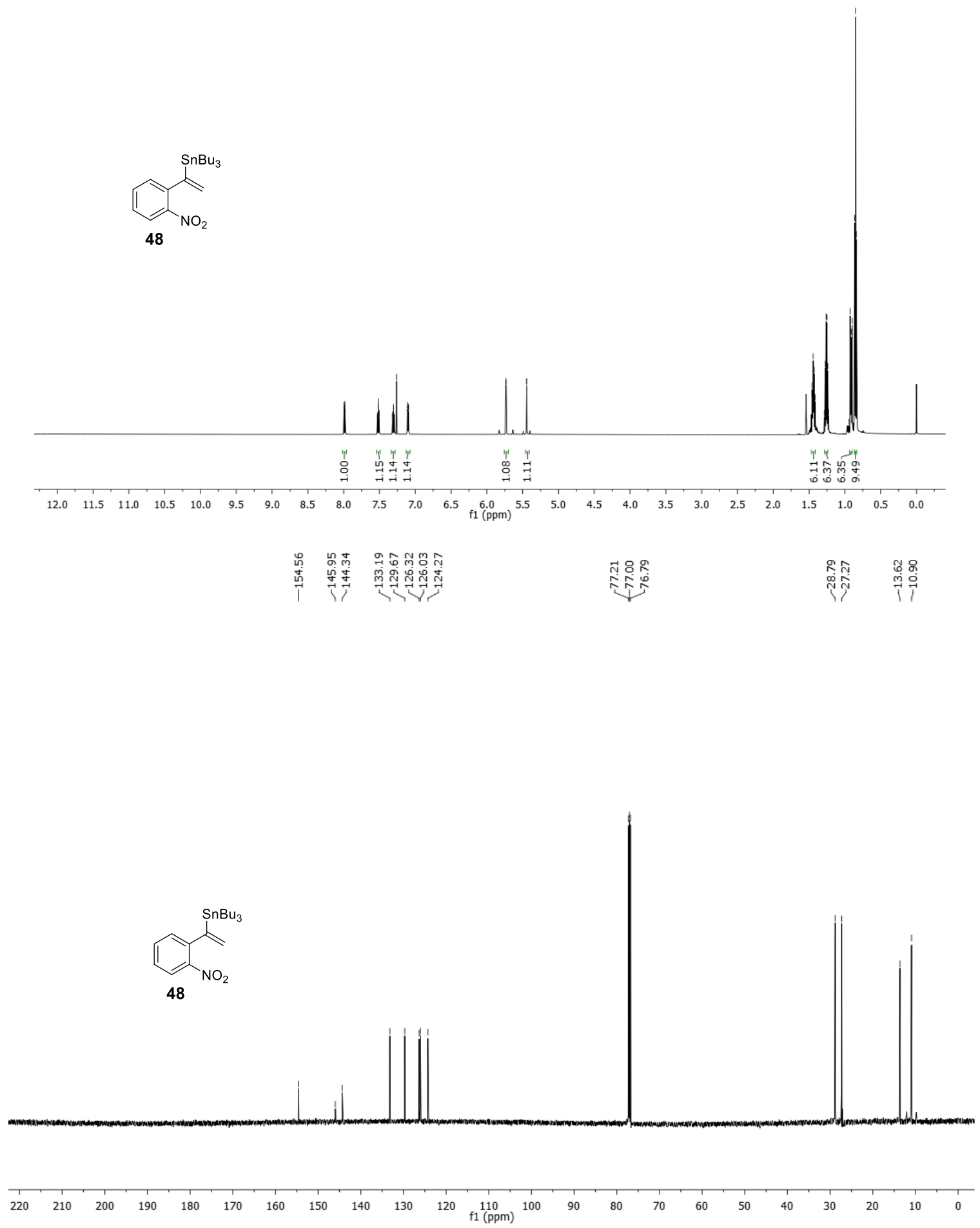

Figure 2.16 ${ }^{1} \mathrm{H}$ and ${ }^{13} \mathrm{C}$ NMR of compound 48 


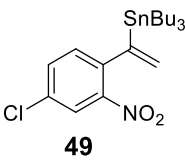

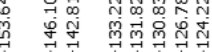

।

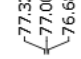

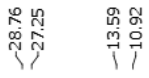

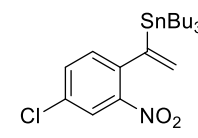

49

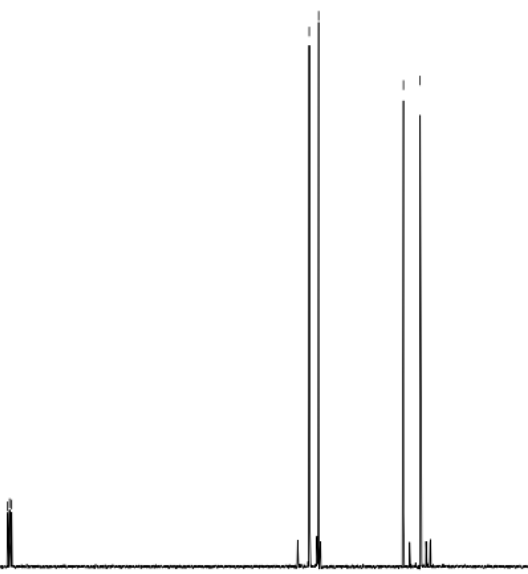

$220 \quad 210$

$\begin{array}{llll}200 & 190 & 180 & 170\end{array}$

Figure $2.17{ }^{1} \mathrm{H}$ and ${ }^{13} \mathrm{C}$ NMR of compound 49 

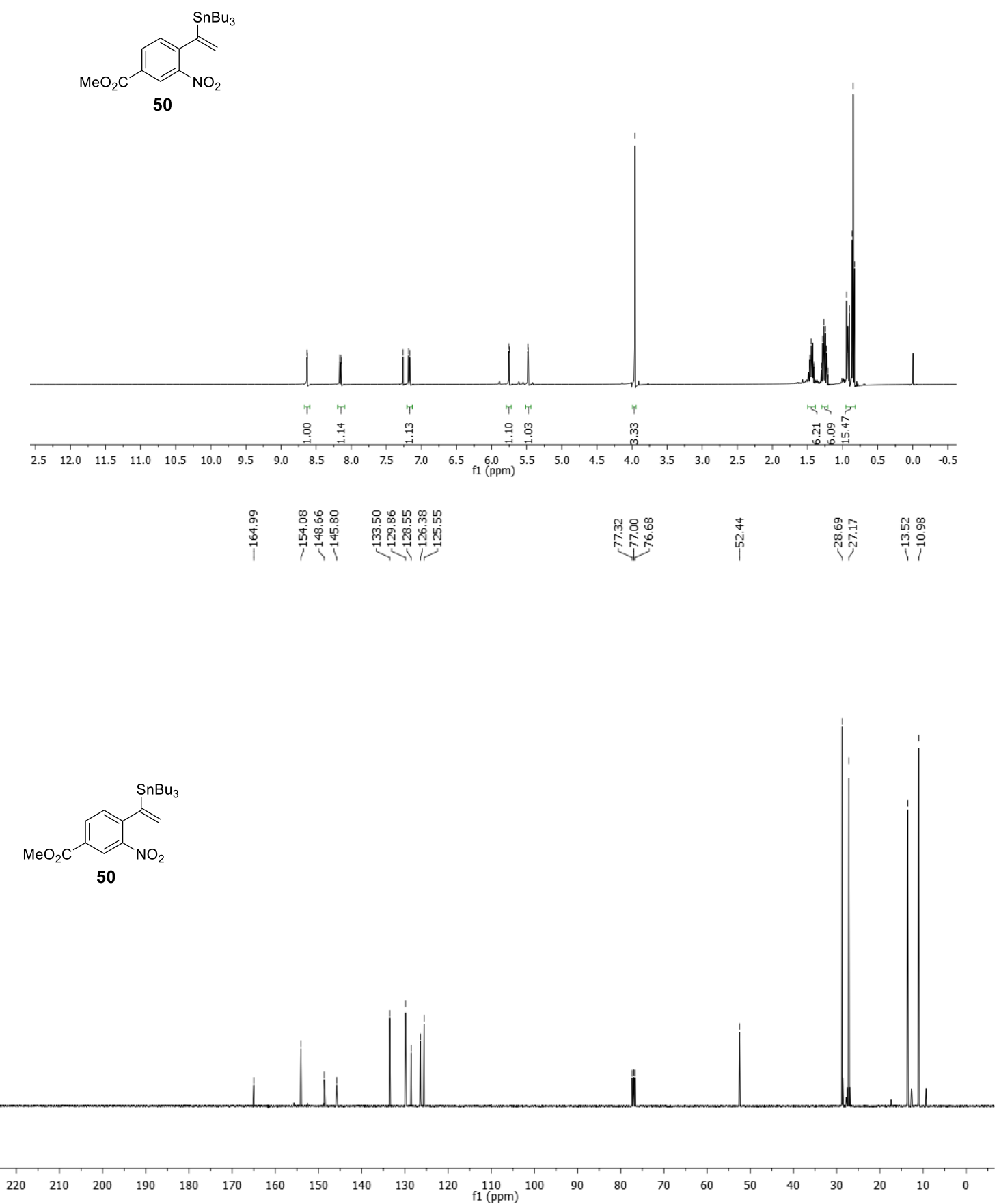

Figure $2.18{ }^{1} \mathrm{H}$ and ${ }^{13} \mathrm{C}$ NMR of compound $\mathbf{5 0}$ 

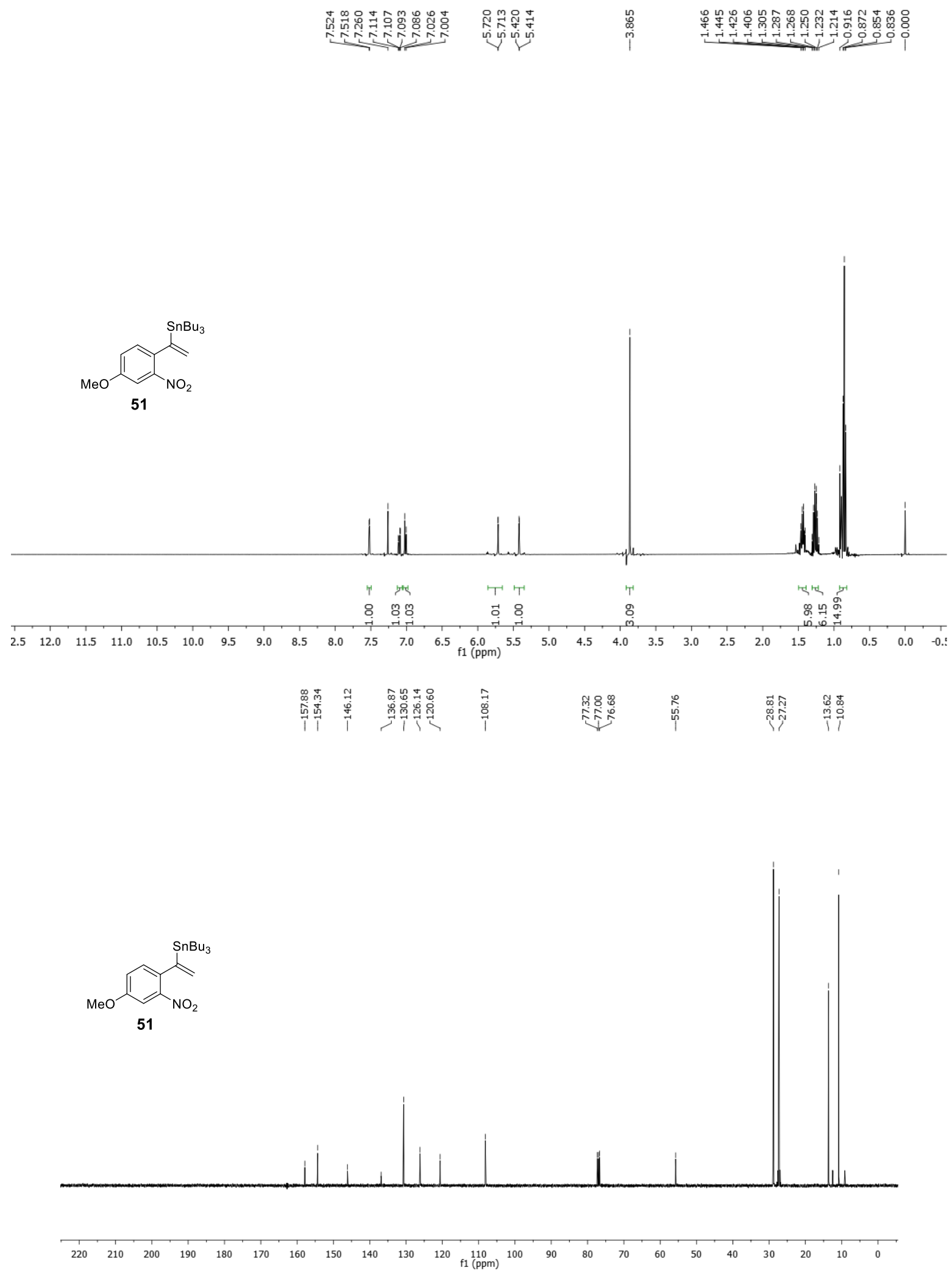

Figure 2.19 ${ }^{1} \mathrm{H}$ and ${ }^{13} \mathrm{C}$ NMR of compound 51 

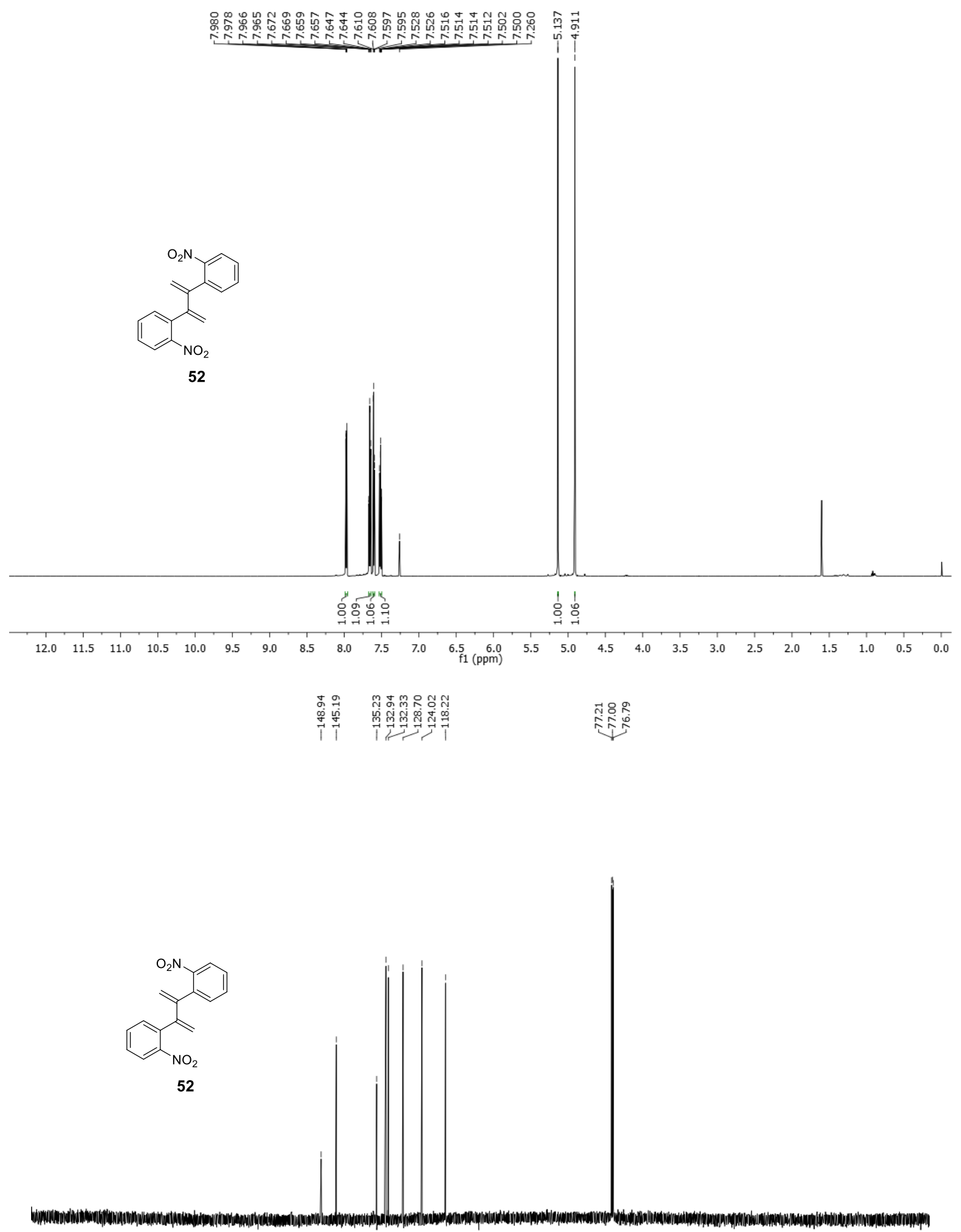

\begin{tabular}{lllllllllllllllllllllll}
\hline 20 & 210 & 200 & 190 & 180 & 170 & 160 & 150 & 140 & 130 & 120 & 110 & 100 & 90 & 80 & 70 & 60 & 50 & 40 & 30 & 20 & 10 & 0
\end{tabular}

Figure $2.20{ }^{1} \mathrm{H}$ and ${ }^{13} \mathrm{C}$ NMR of compound 52 


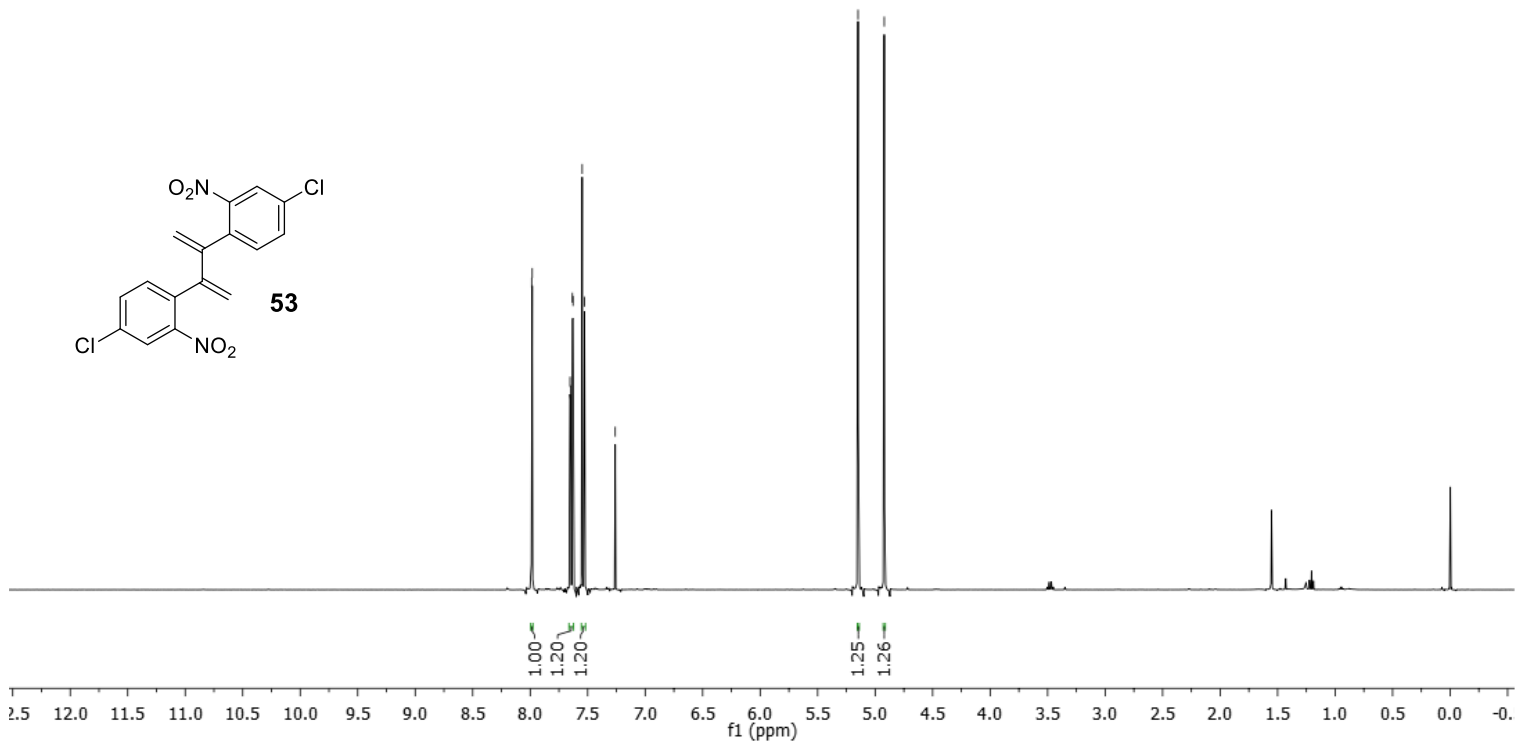

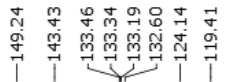
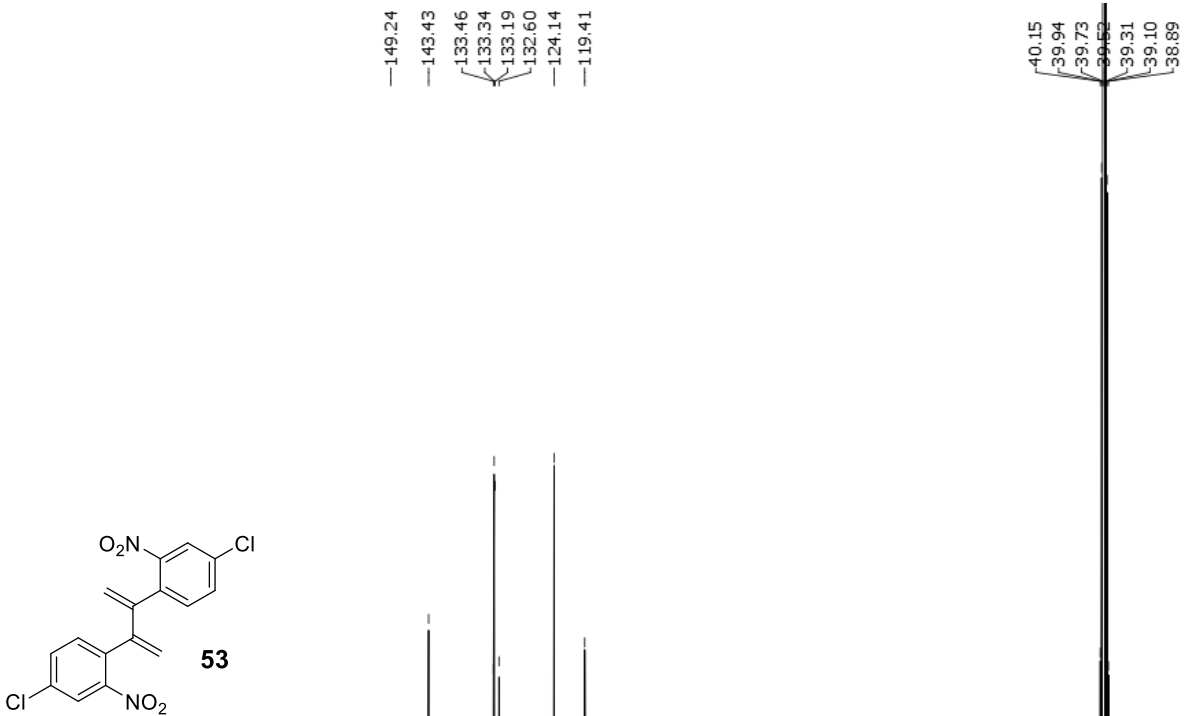

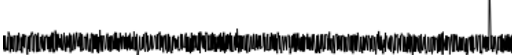

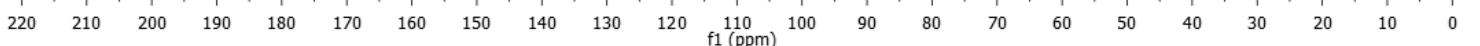

Figure 2.21 ${ }^{1} \mathrm{H}$ and ${ }^{13} \mathrm{C}$ NMR of compound 53 


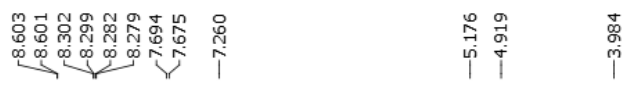
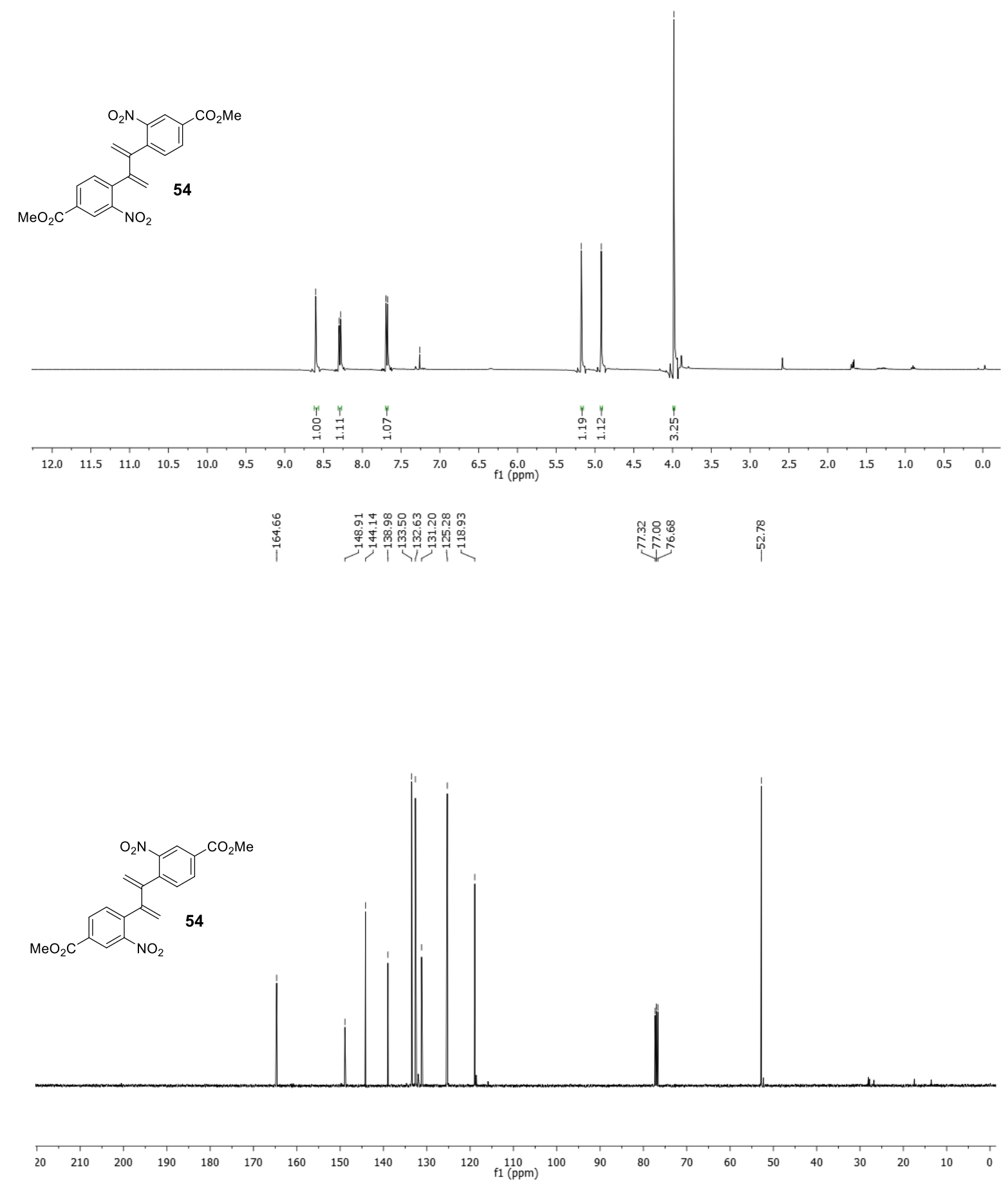

Figure $2.22{ }^{1} \mathrm{H}$ and ${ }^{13} \mathrm{C}$ NMR of compound 54 

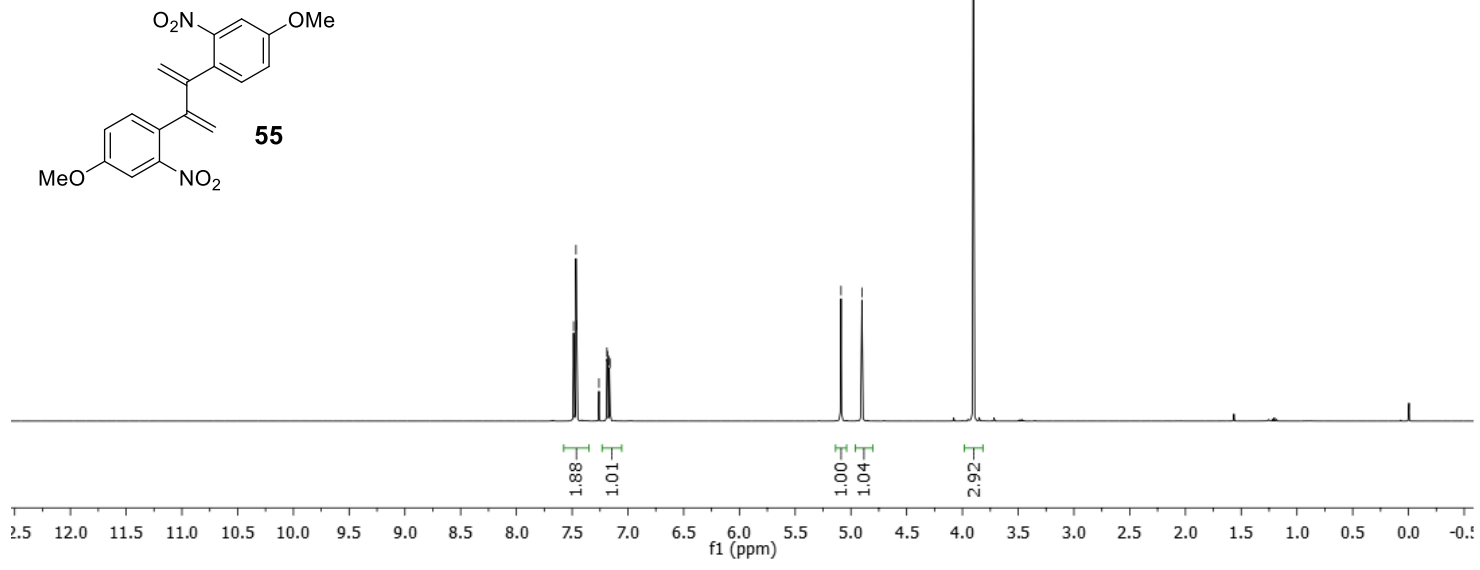

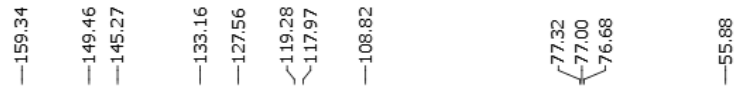

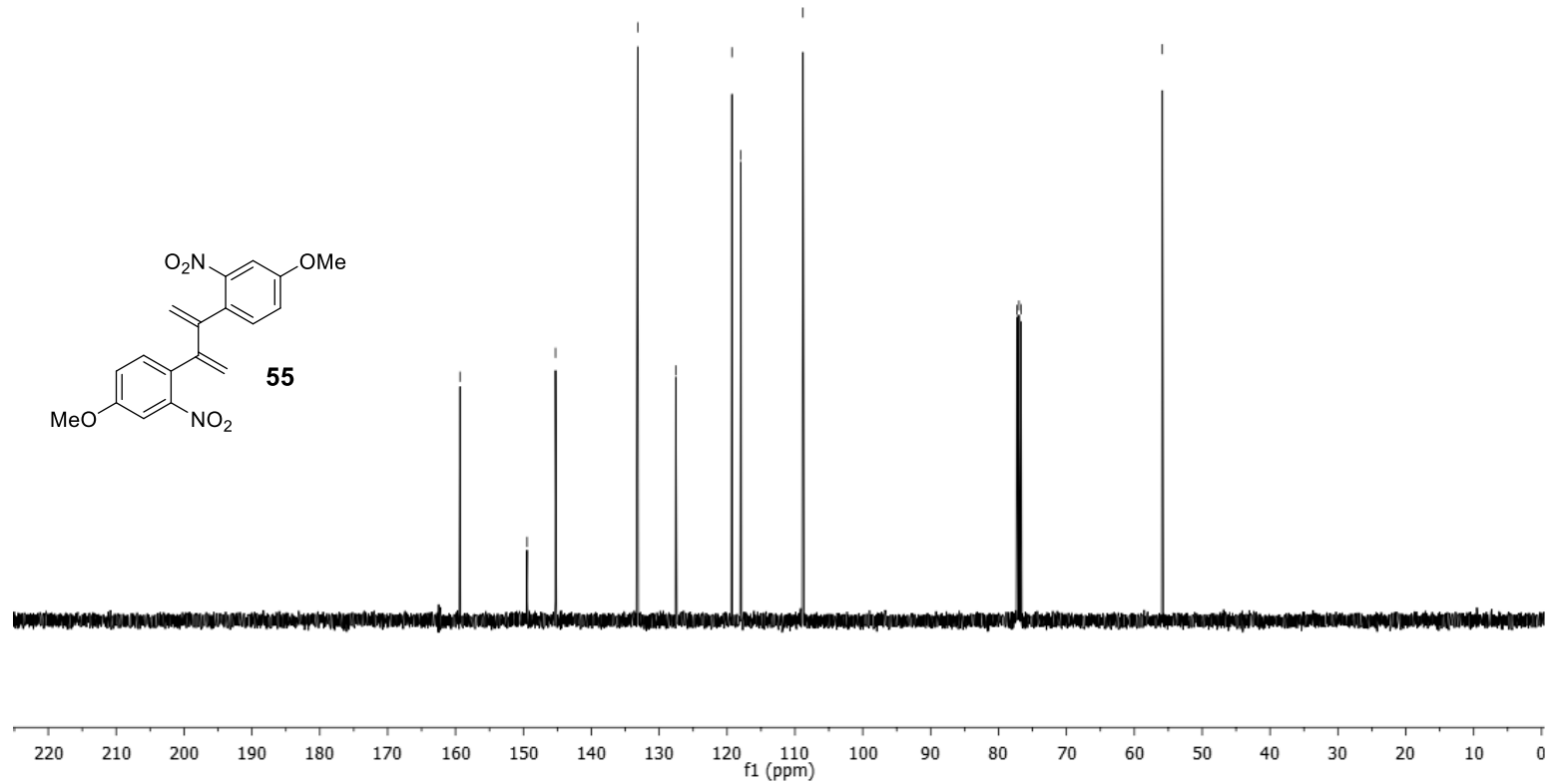

Figure $2.23{ }^{1} \mathrm{H}$ and ${ }^{13} \mathrm{C}$ NMR of compound 55 

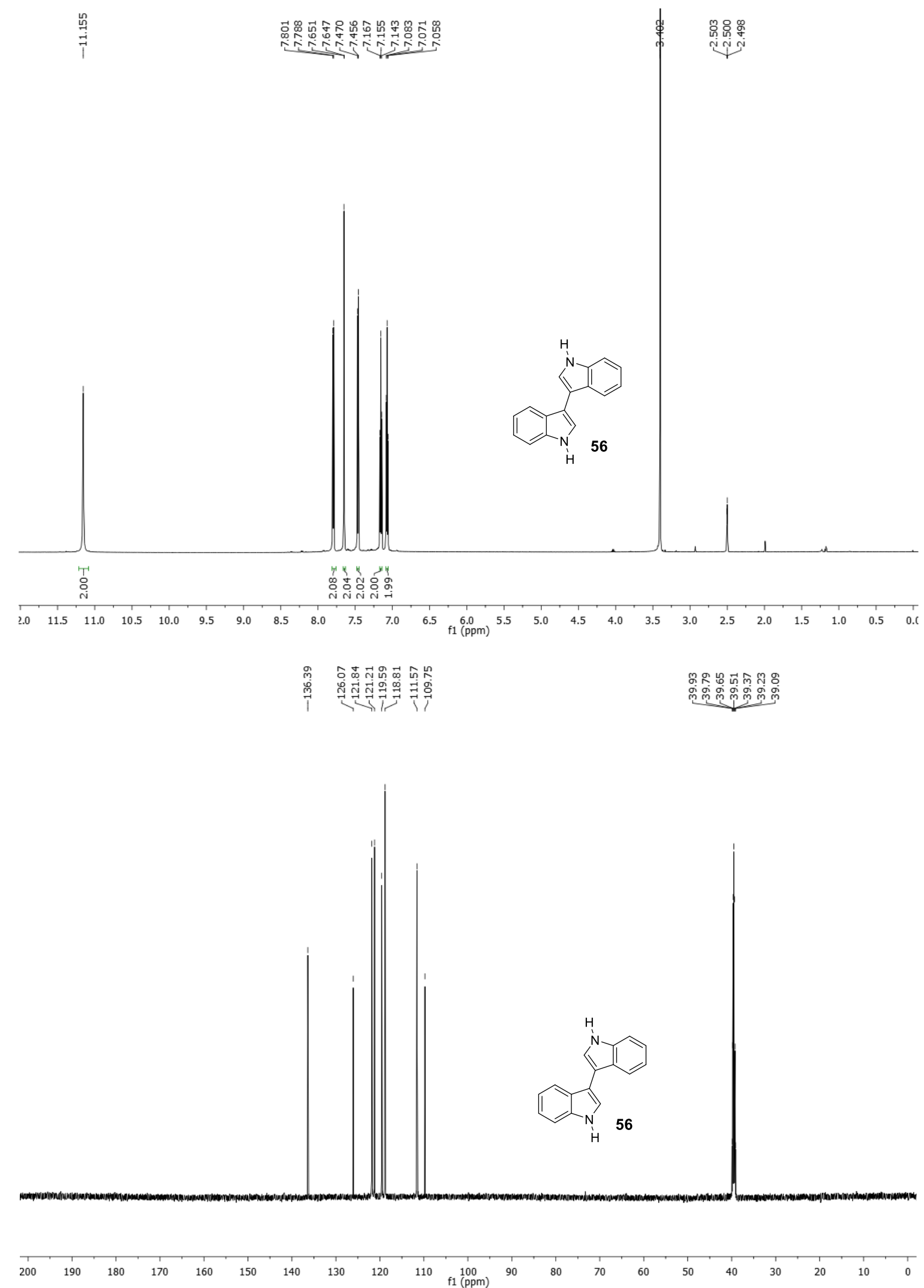

Figure $2.24{ }^{1} \mathrm{H}$ and ${ }^{13} \mathrm{C}$ NMR of compound $\mathbf{5 6}$ 


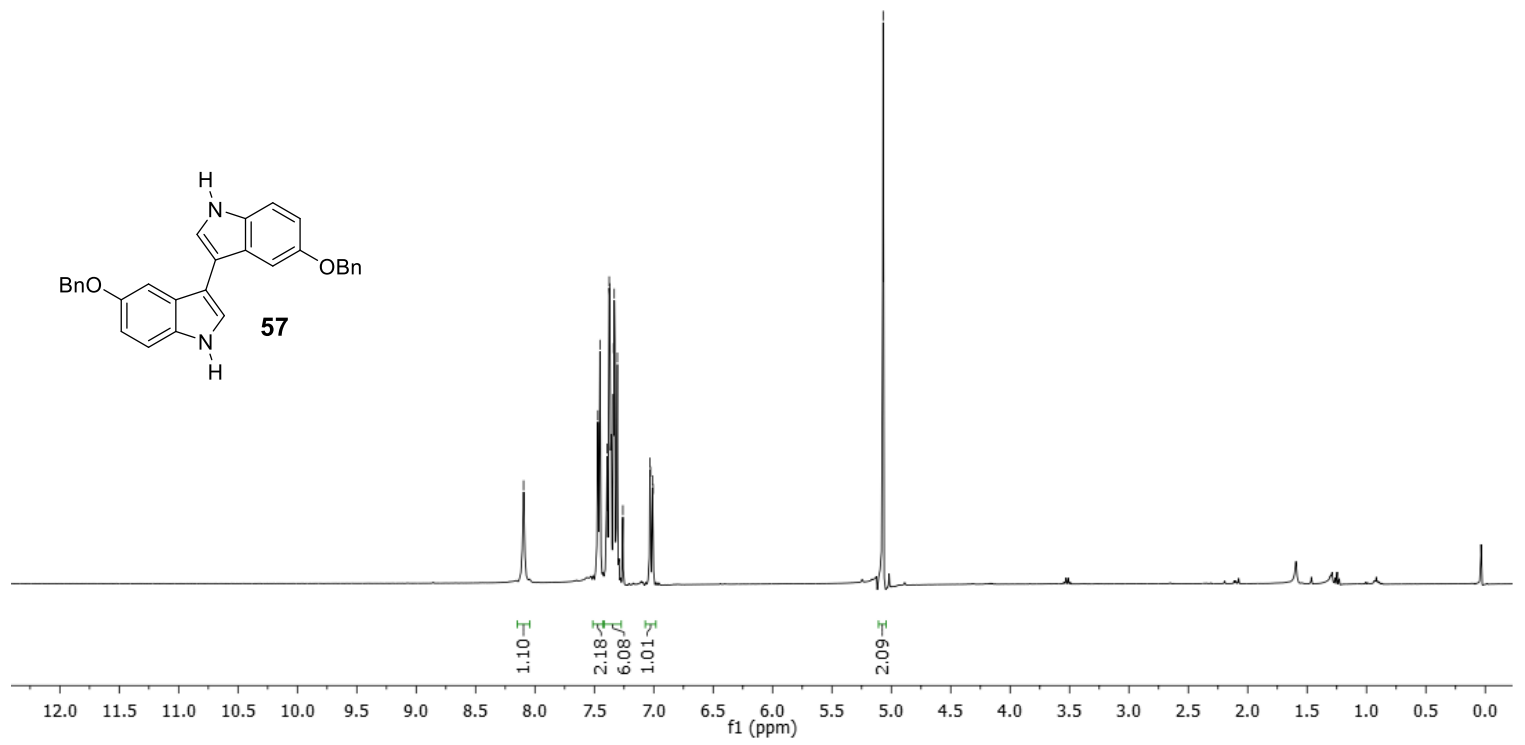

|

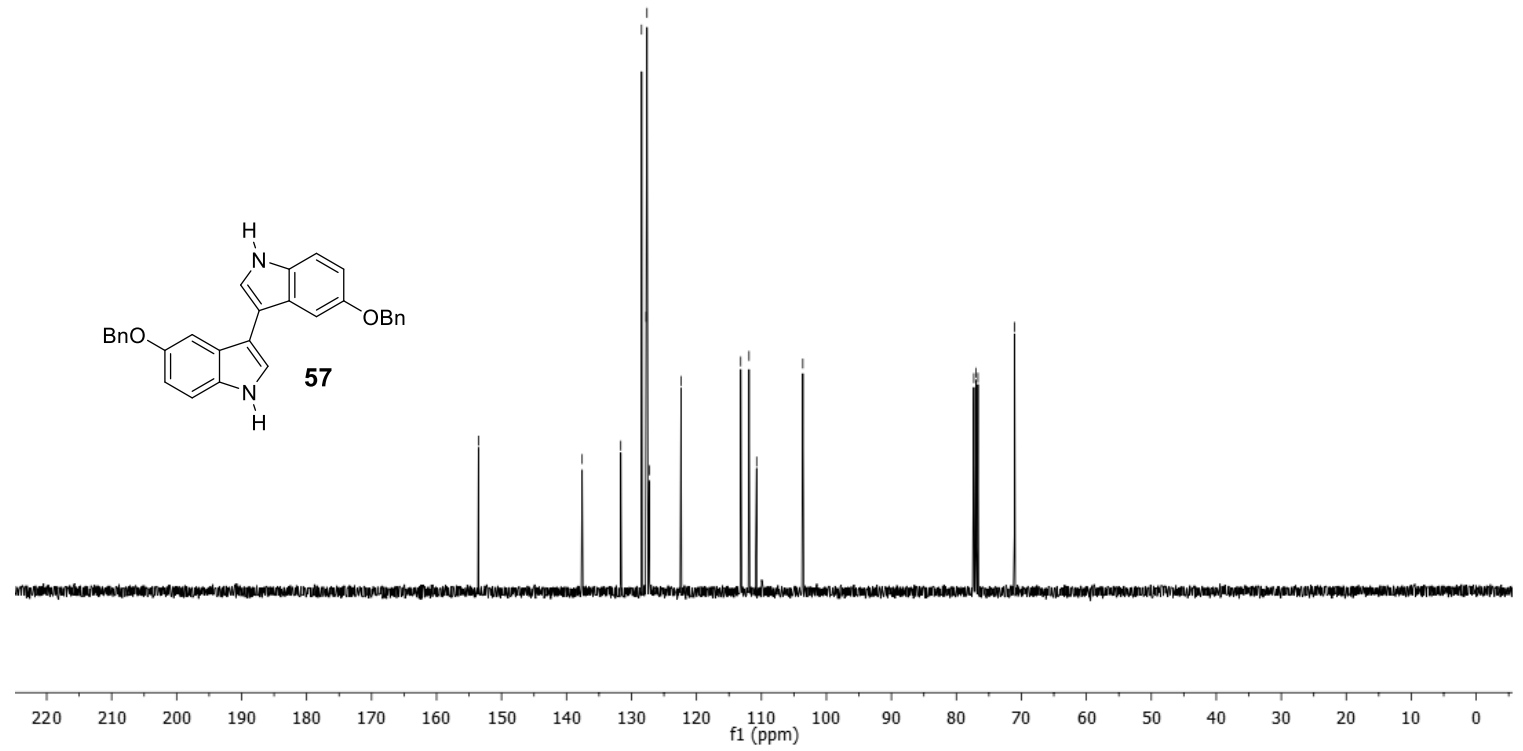

Figure $2.25{ }^{1} \mathrm{H}$ and ${ }^{13} \mathrm{C}$ NMR of compound 57 

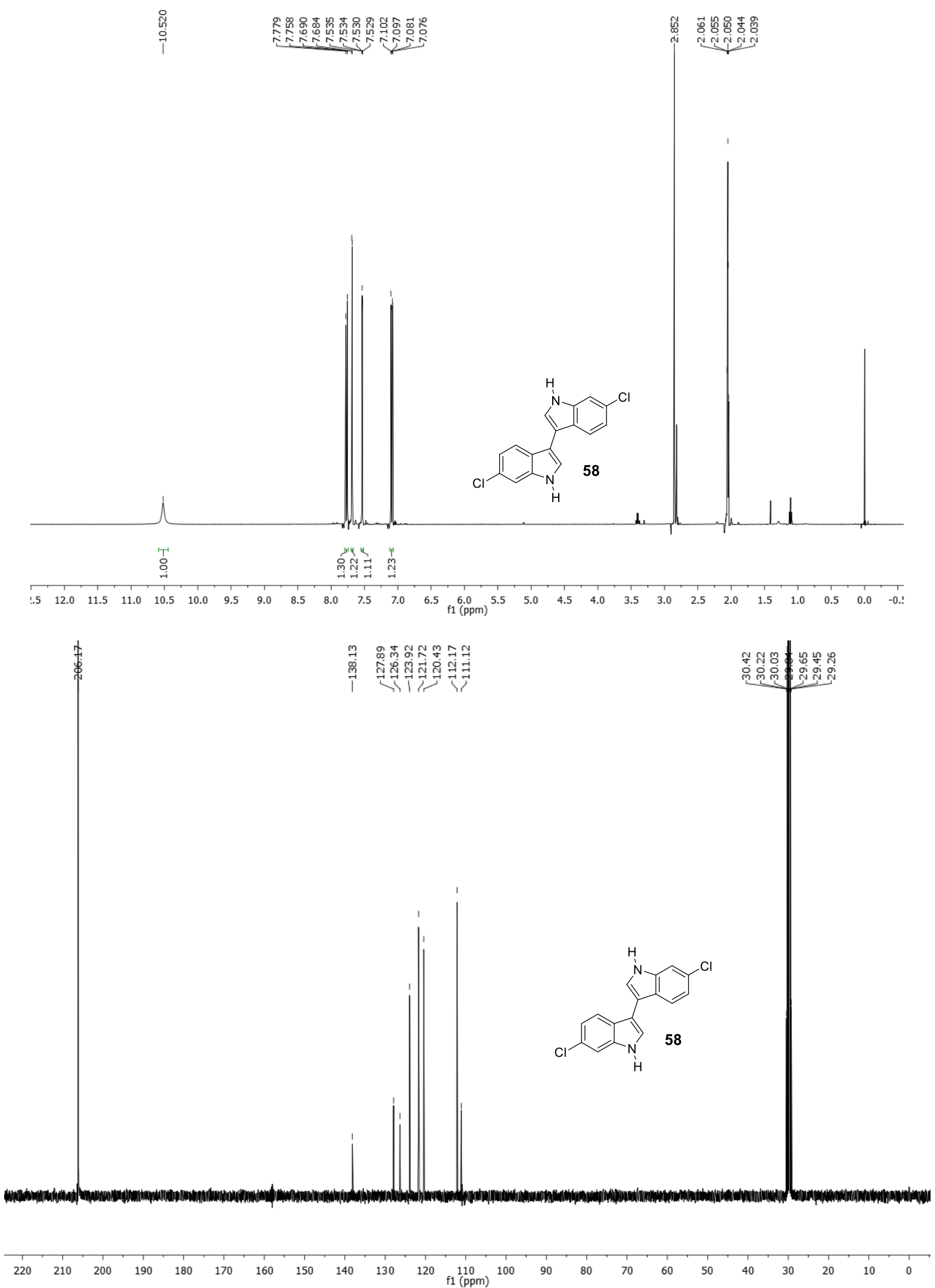

Figure $2.26{ }^{1} \mathrm{H}$ and ${ }^{13} \mathrm{C}$ NMR of compound $\mathbf{5 8}$ 


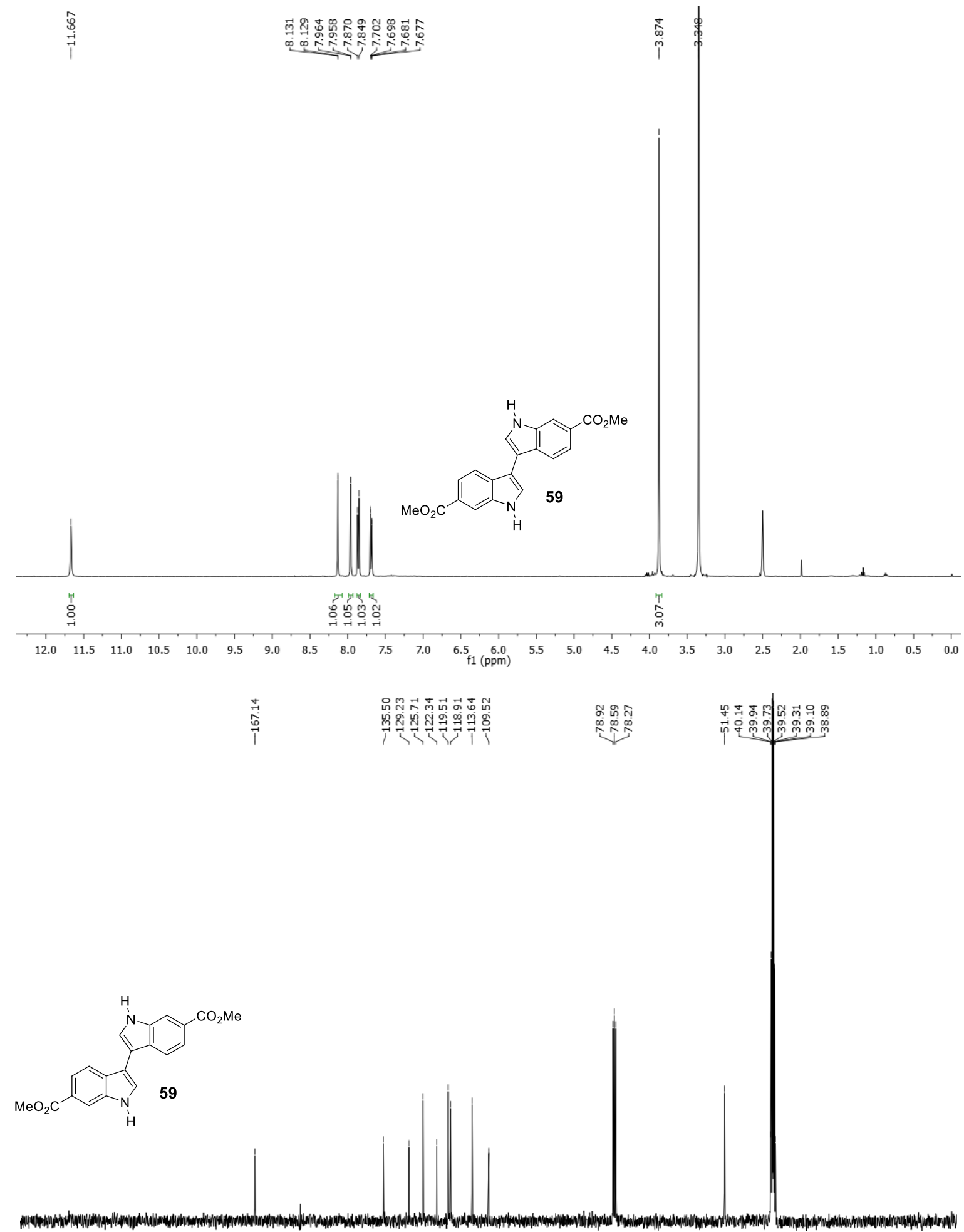

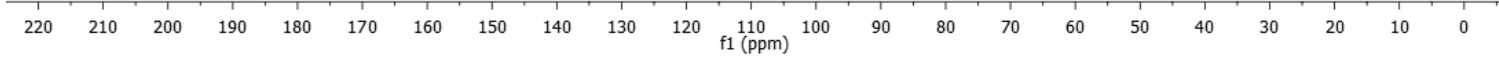

Figure $2.27{ }^{1} \mathrm{H}$ and ${ }^{13} \mathrm{C}$ NMR of compound 59 


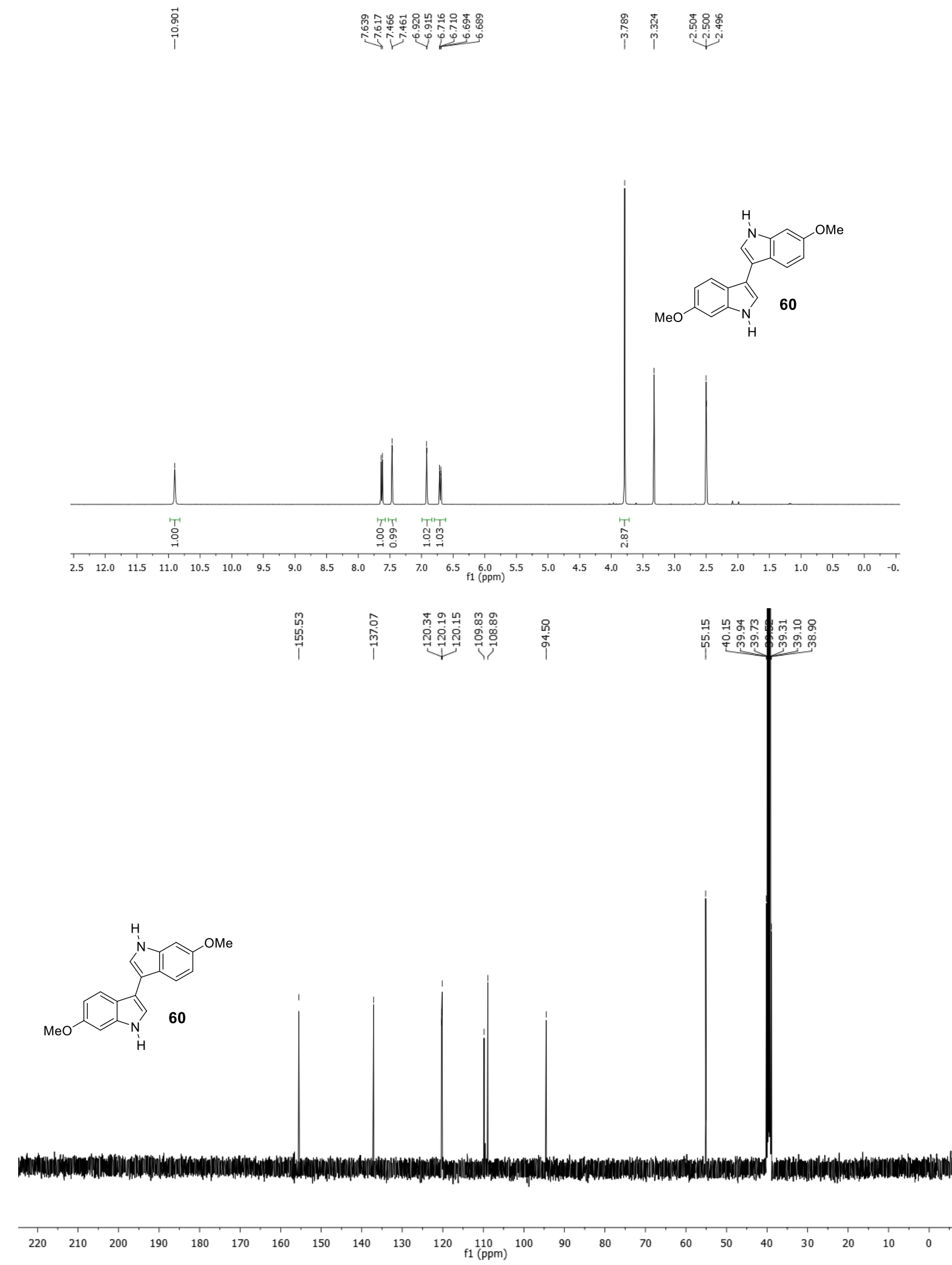

Figure $2.28{ }^{1} \mathrm{H}$ and ${ }^{13} \mathrm{C}$ NMR of compound 60 

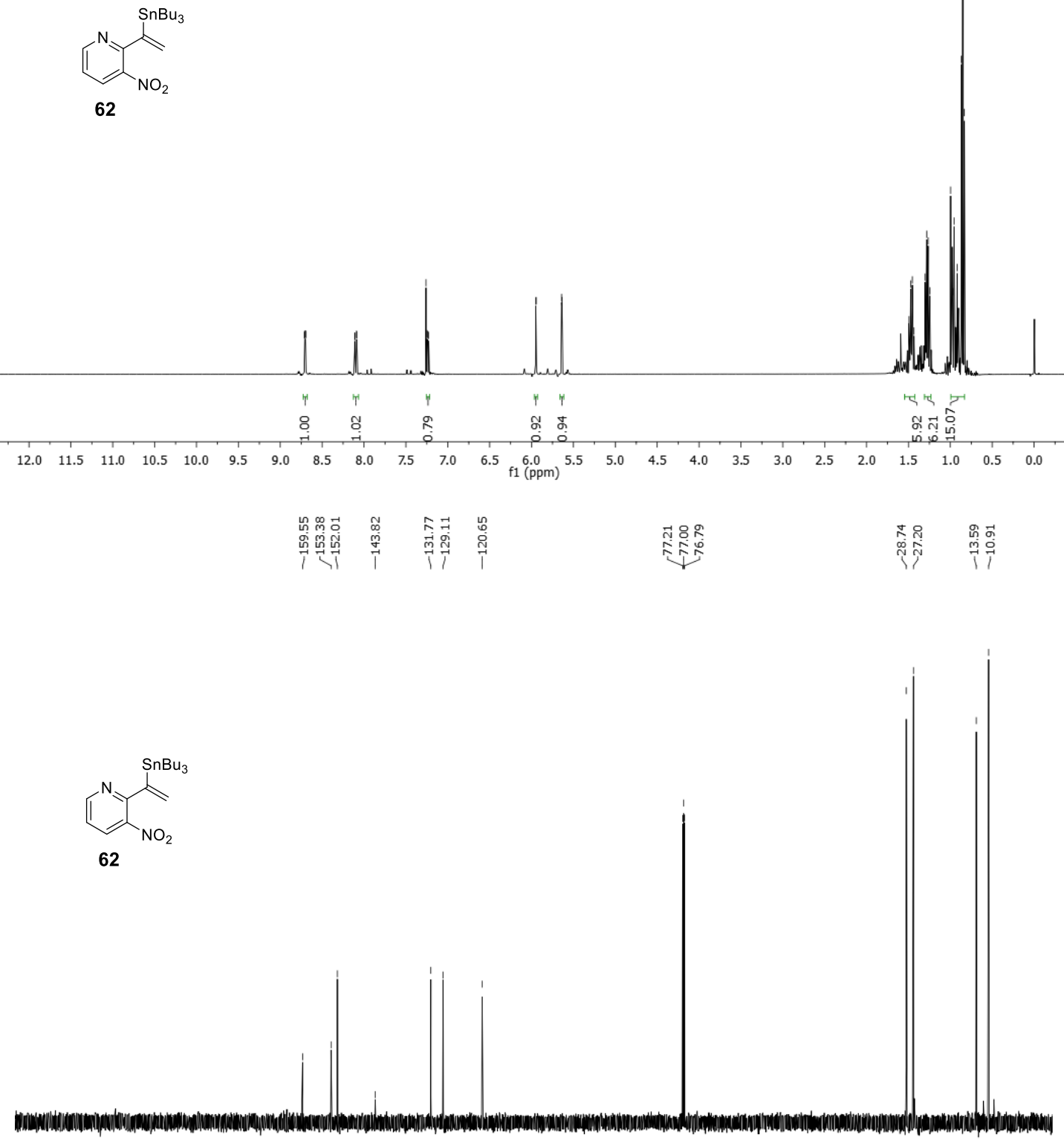

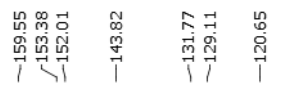

$\begin{array}{lllllll}220 & 210 & 200 & 190 & 180 & 170 & 160\end{array}$

Figure 2.29 ${ }^{1} \mathrm{H}$ and ${ }^{13} \mathrm{C}$ NMR of compound 62 

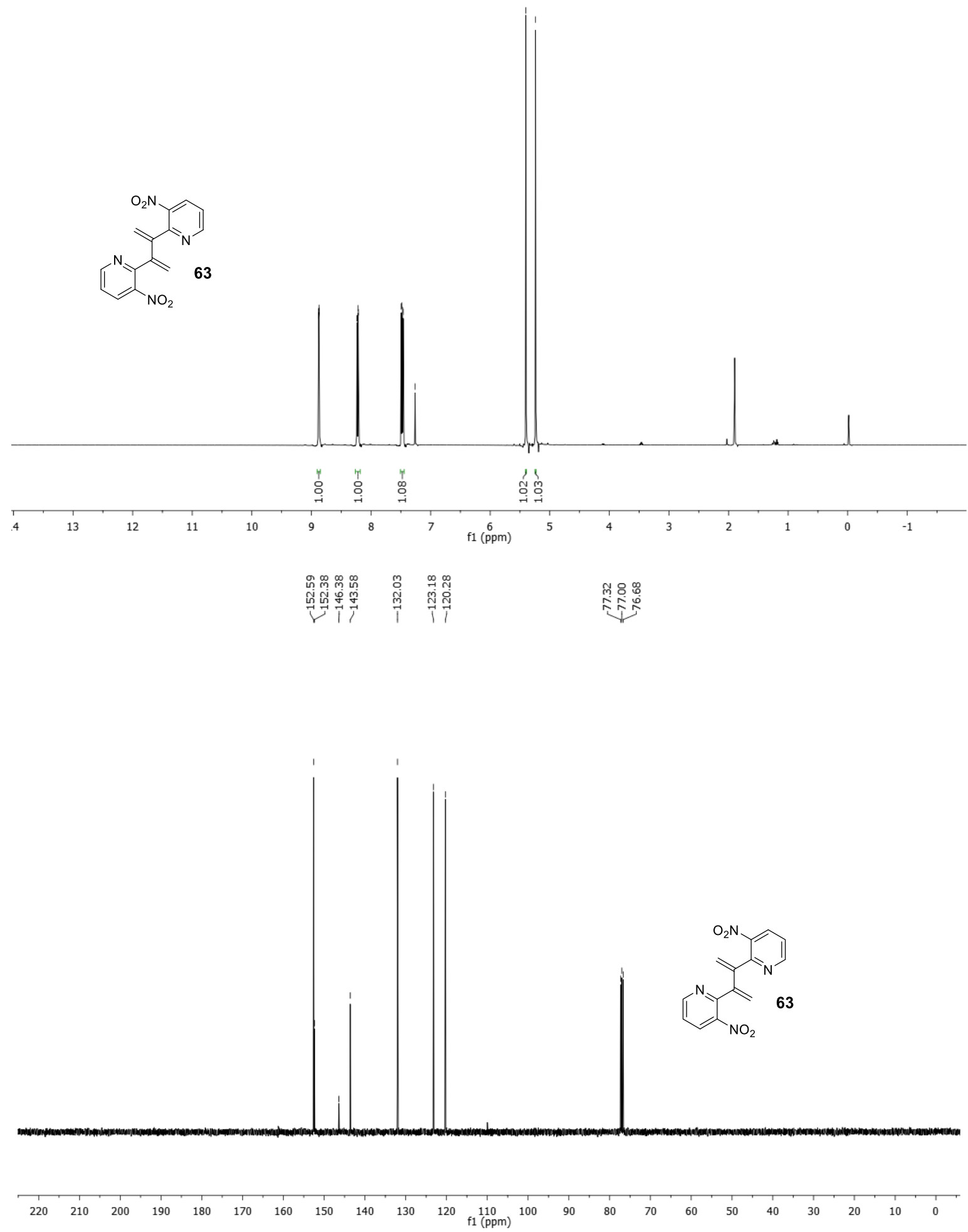

Figure $2.30{ }^{1} \mathrm{H}$ and ${ }^{13} \mathrm{C}$ NMR of compound 63 

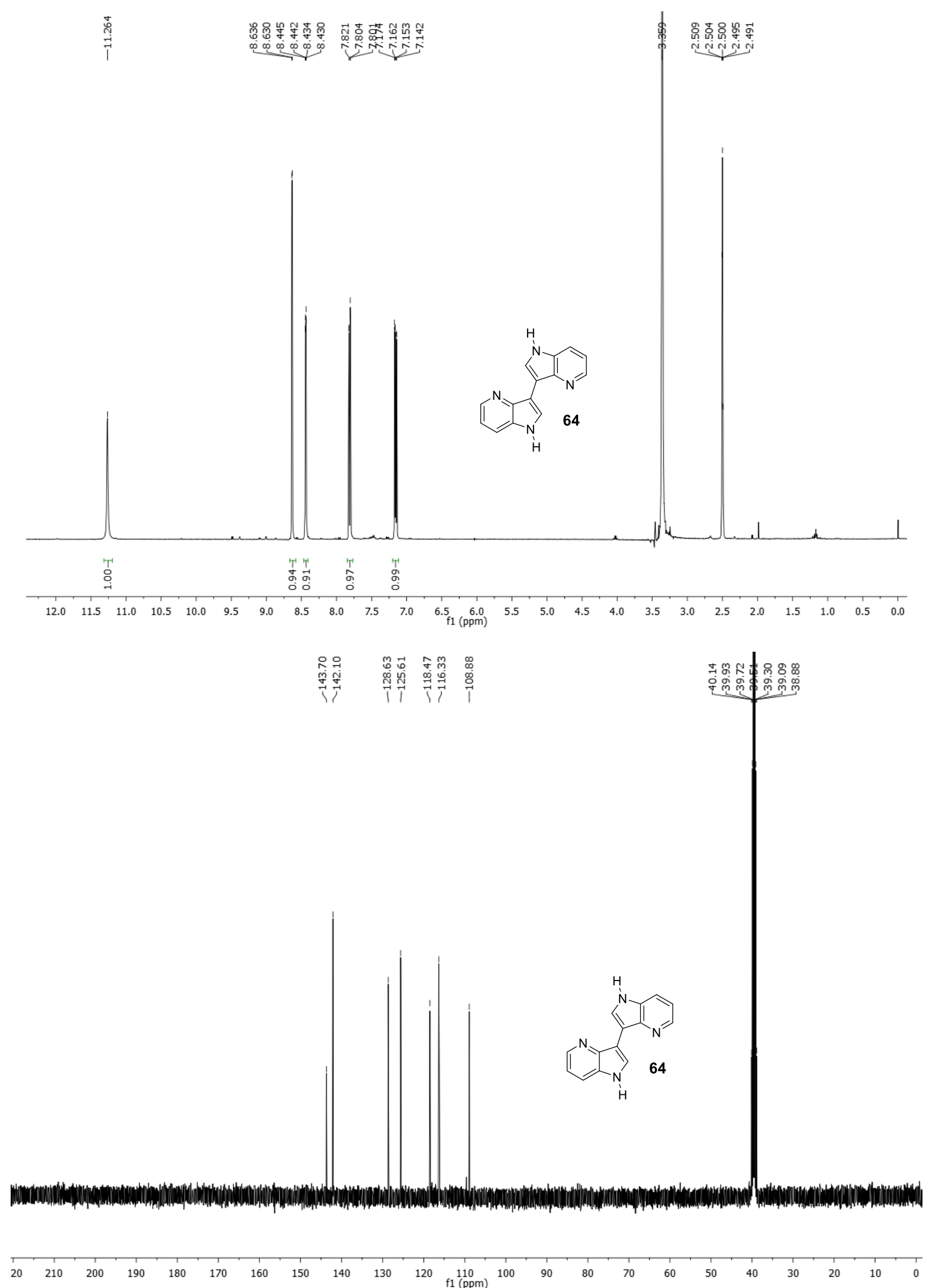

Figure 2.31 ${ }^{1} \mathrm{H}$ and ${ }^{13} \mathrm{C}$ NMR of compound 64 

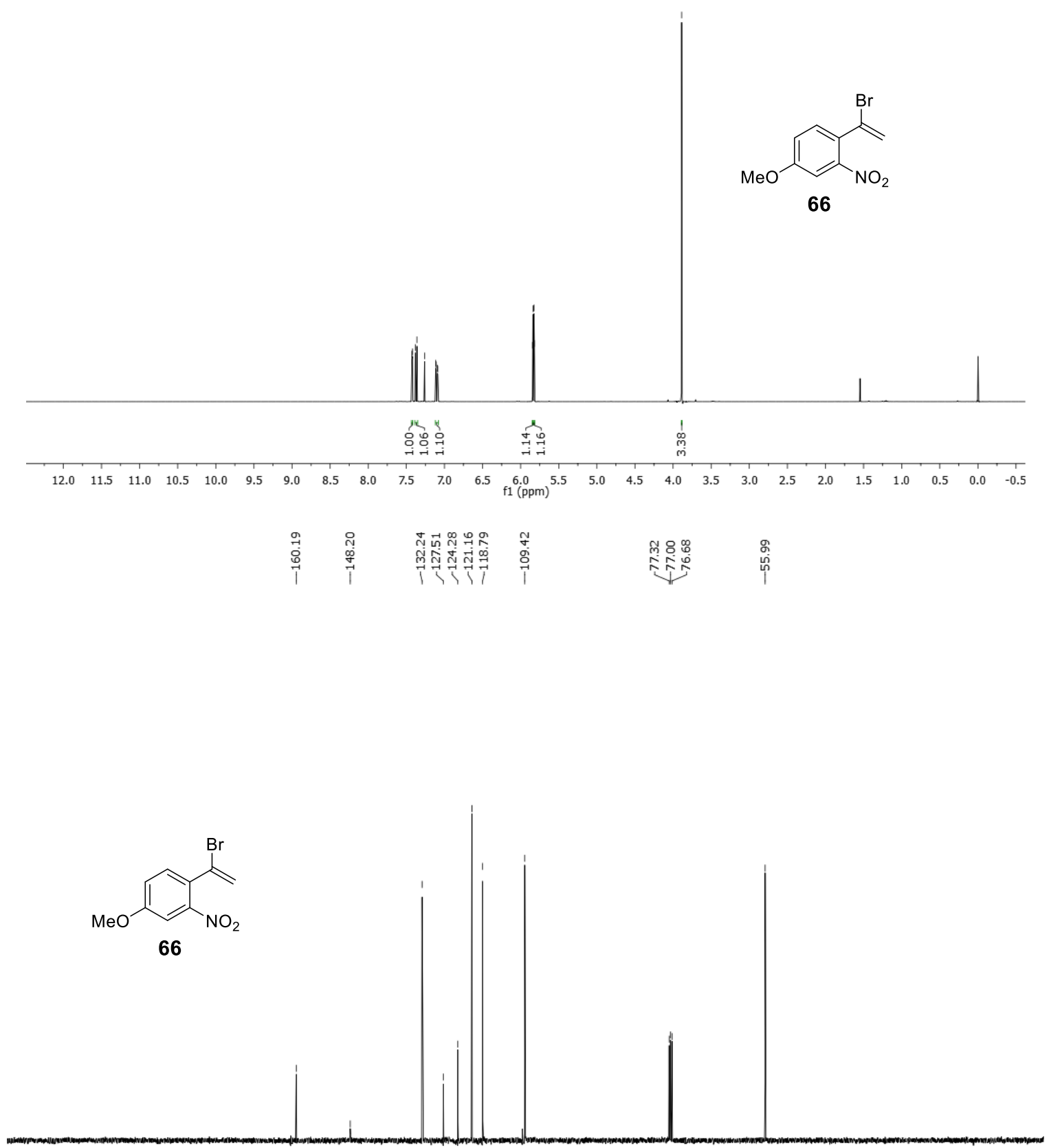

$\begin{array}{llllllllllllllllllllllllll}220 & 210 & 200 & 190 & 180 & 170 & 160 & 150 & 140 & 130 & 120 & 110 & 100 & 90 & 80 & 70 & 60 & 50 & 40 & 30 & 20 & 10 & 0\end{array}$

Figure $2.32{ }^{1} \mathrm{H}$ and ${ }^{13} \mathrm{C}$ NMR of compound 66 

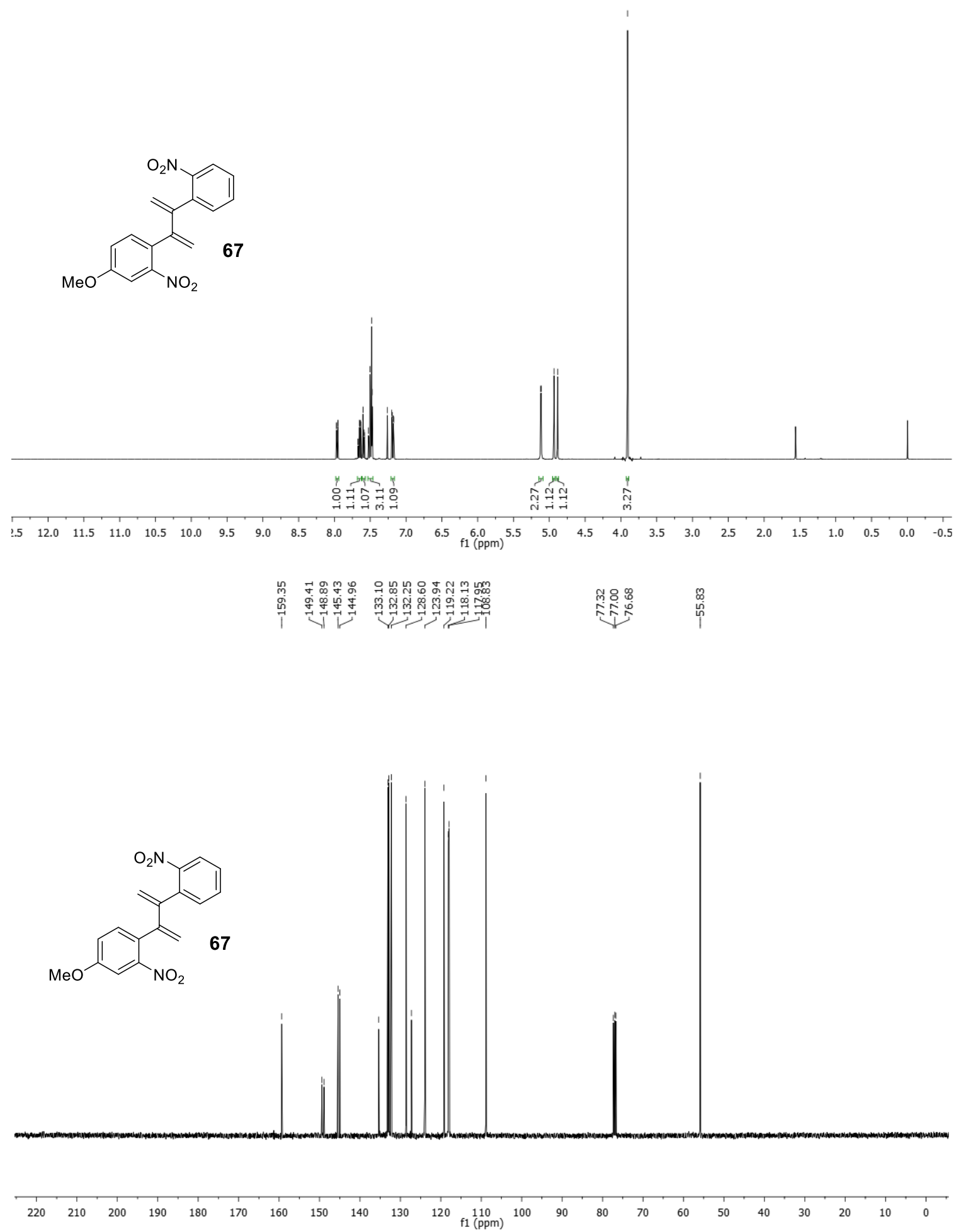

Figure $2.33{ }^{1} \mathrm{H}$ and ${ }^{13} \mathrm{C}$ NMR of compound 67 


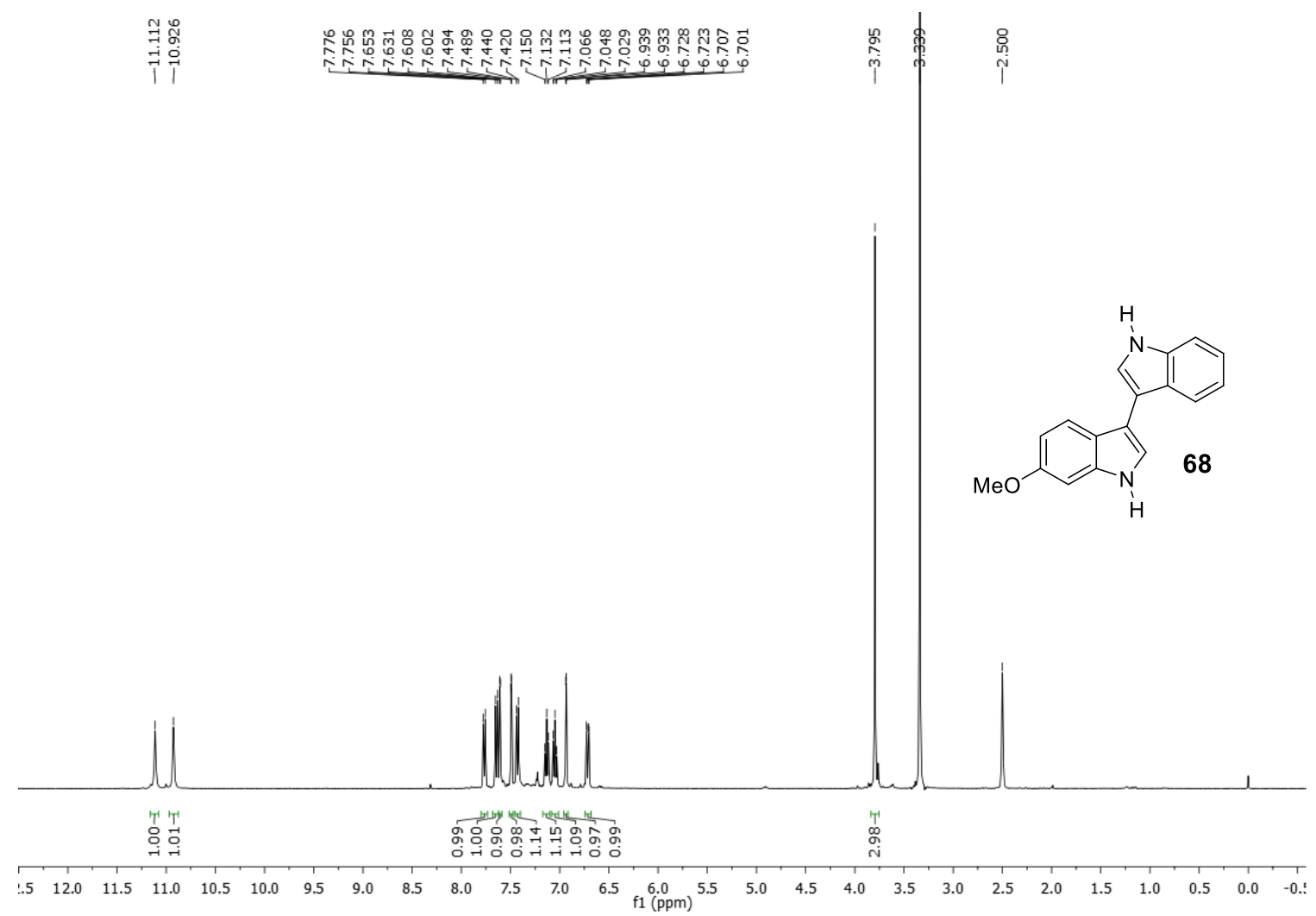

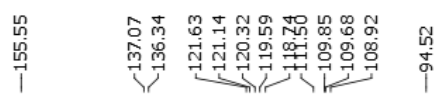

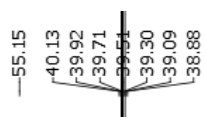
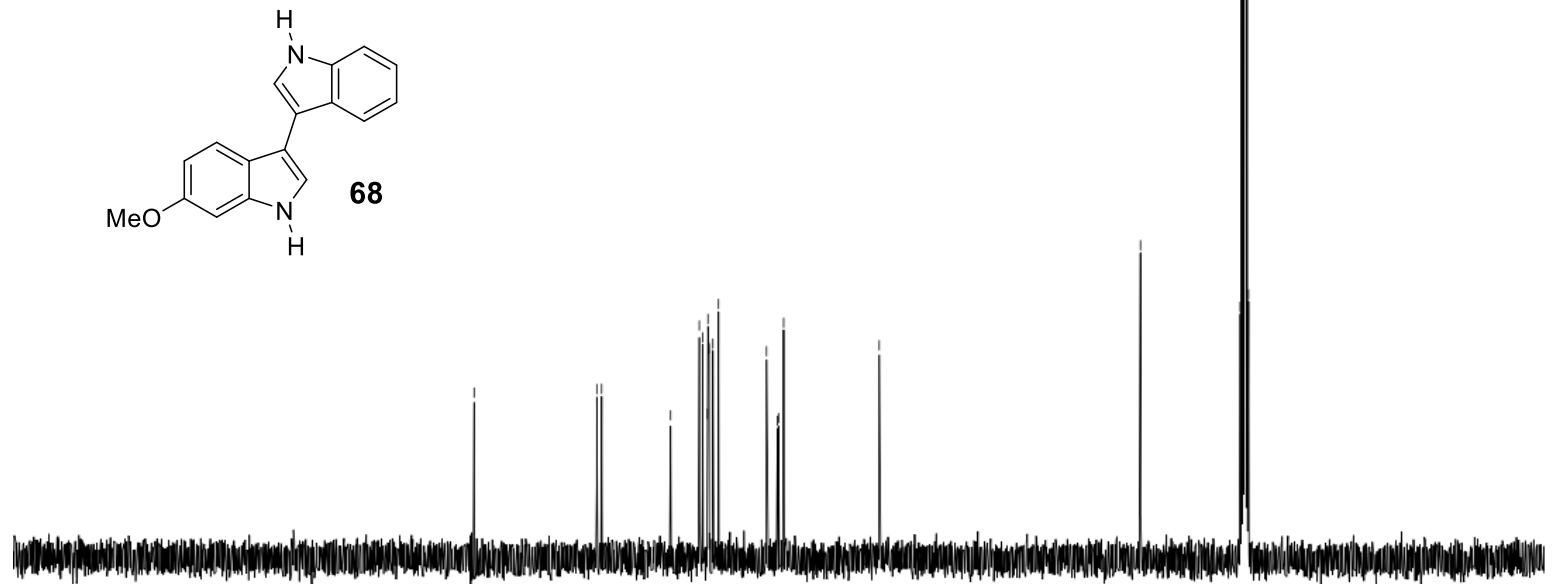

$\begin{array}{lllllllllllllllllllllllll}220 & 210 & 200 & 190 & 180 & 170 & 160 & 150 & 140 & 130 & 120 & 110 & 100 & 90 & 80 & 70 & 60 & 50 & 40 & 30 & 20 & 10 & 0\end{array}$

Figure $2.34{ }^{1} \mathrm{H}$ and ${ }^{13} \mathrm{C}$ NMR of compound 68 

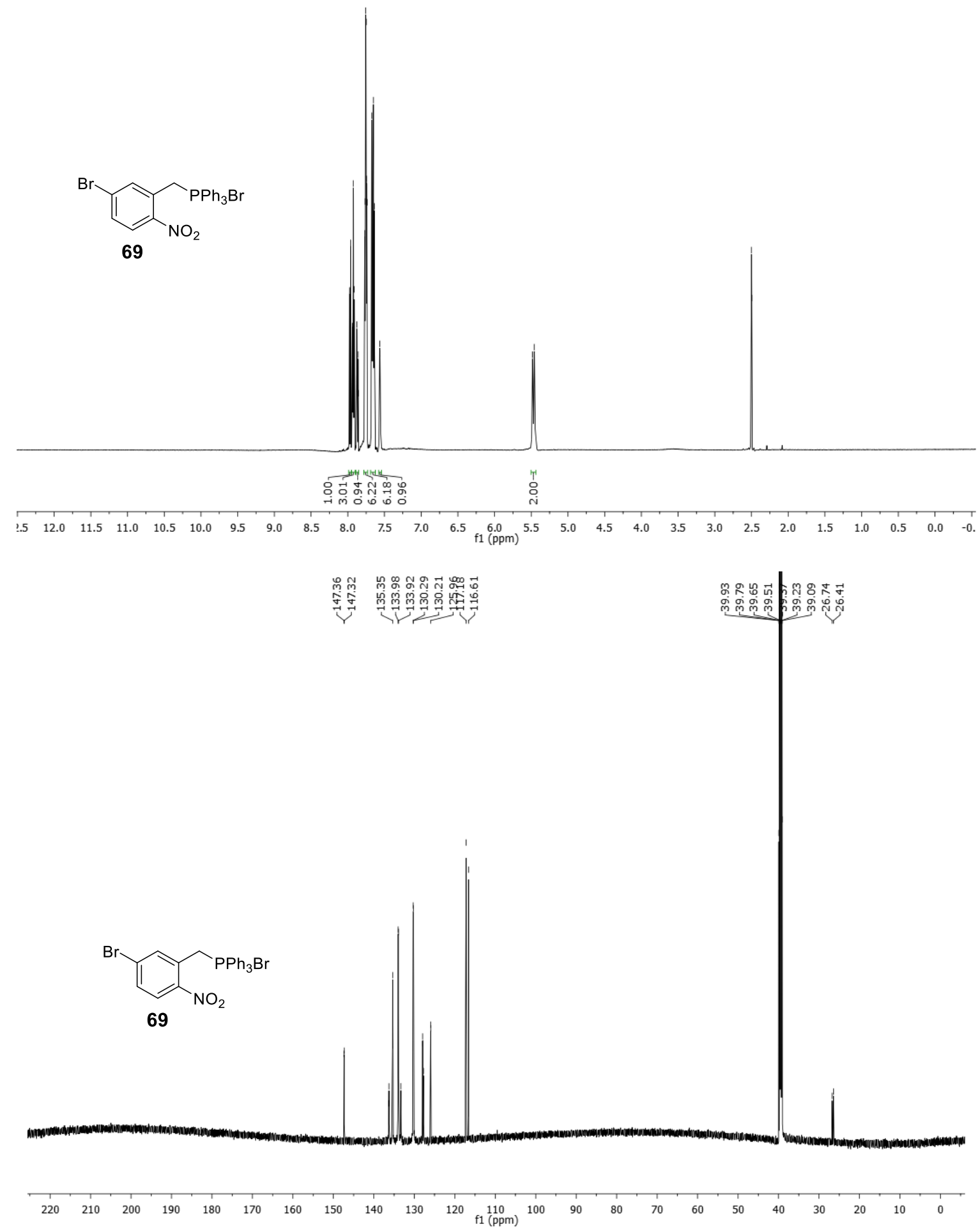

Figure $2.35{ }^{1} \mathrm{H}$ and ${ }^{13} \mathrm{C}$ NMR of compound 69 

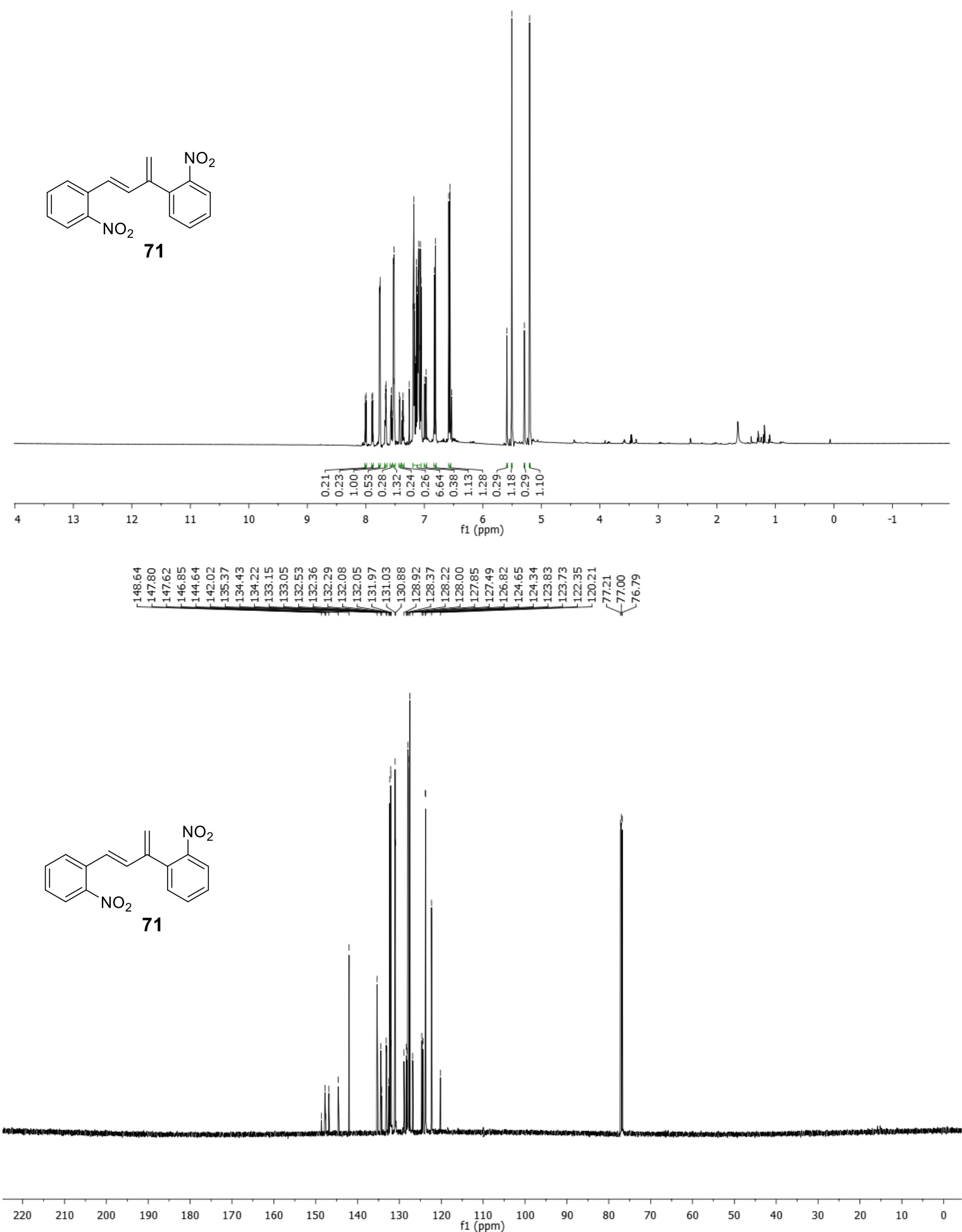

Figure $2.35{ }^{1} \mathrm{H}$ and ${ }^{13} \mathrm{C}$ NMR of compound 71 

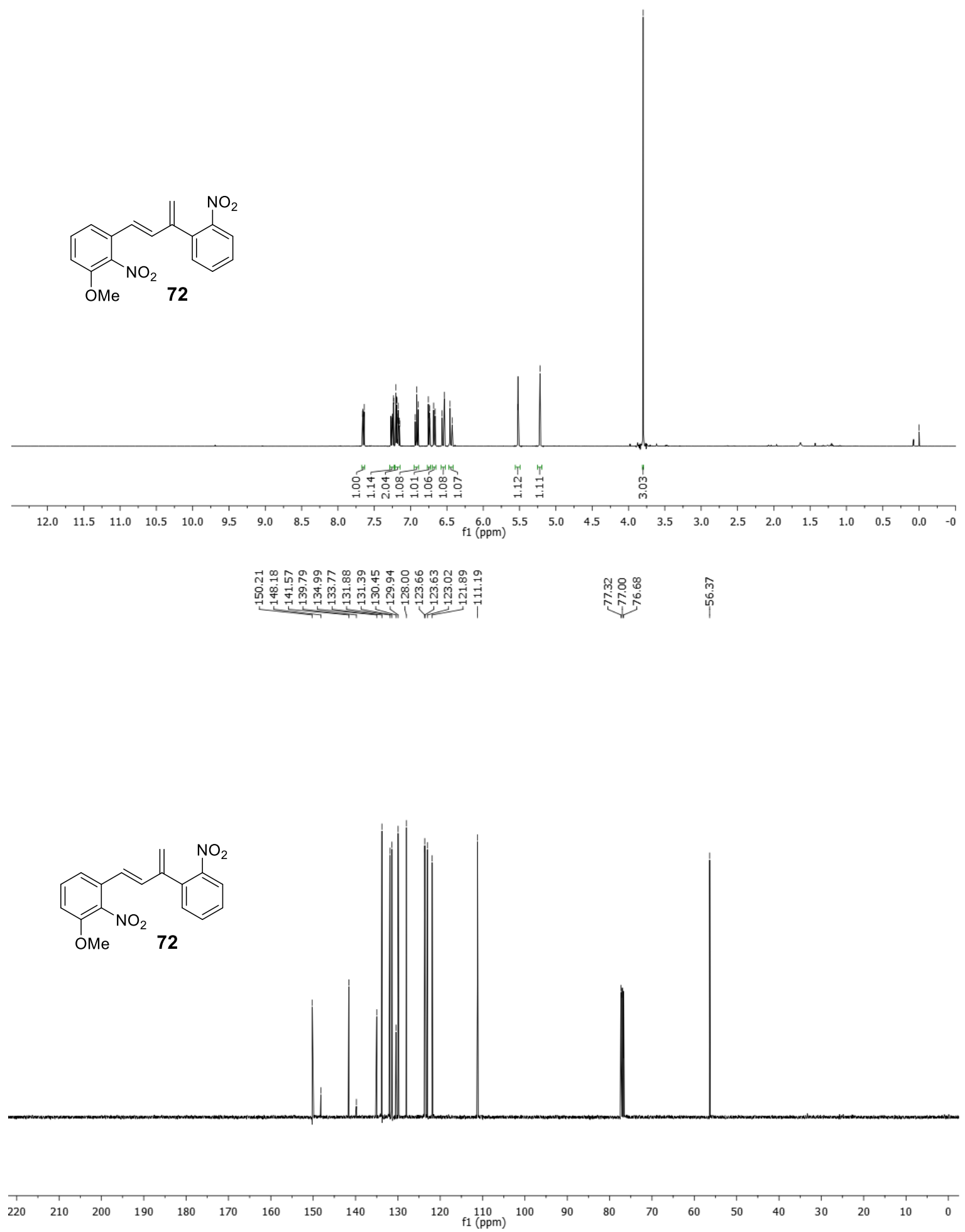

Figure $2.37{ }^{1} \mathrm{H}$ and ${ }^{13} \mathrm{C}$ NMR of compound 72 

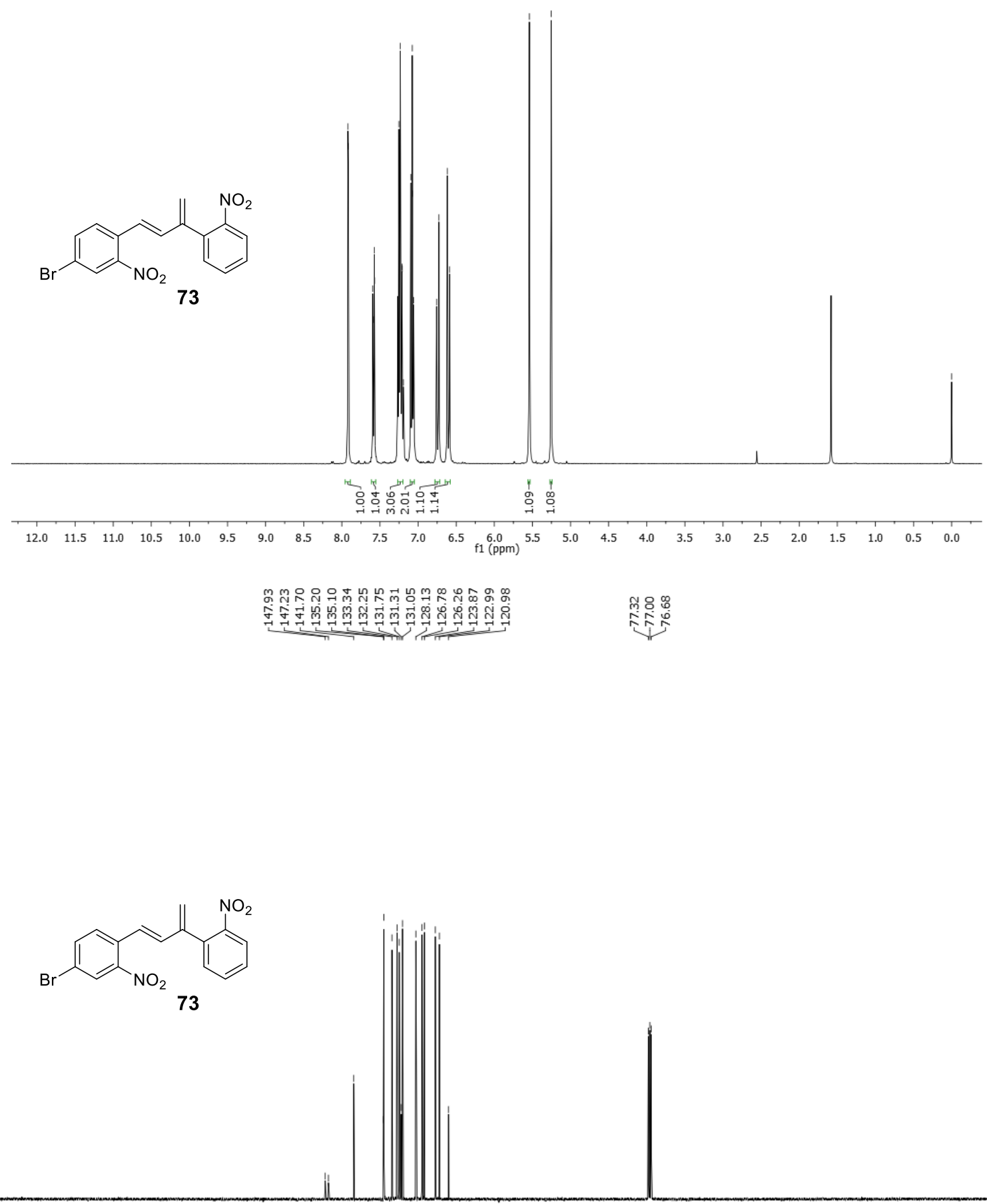

2202 200

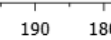
$170 \quad 160$

Figure 2.38 ${ }^{1} \mathrm{H}$ and ${ }^{13} \mathrm{C}$ NMR of compound 73 

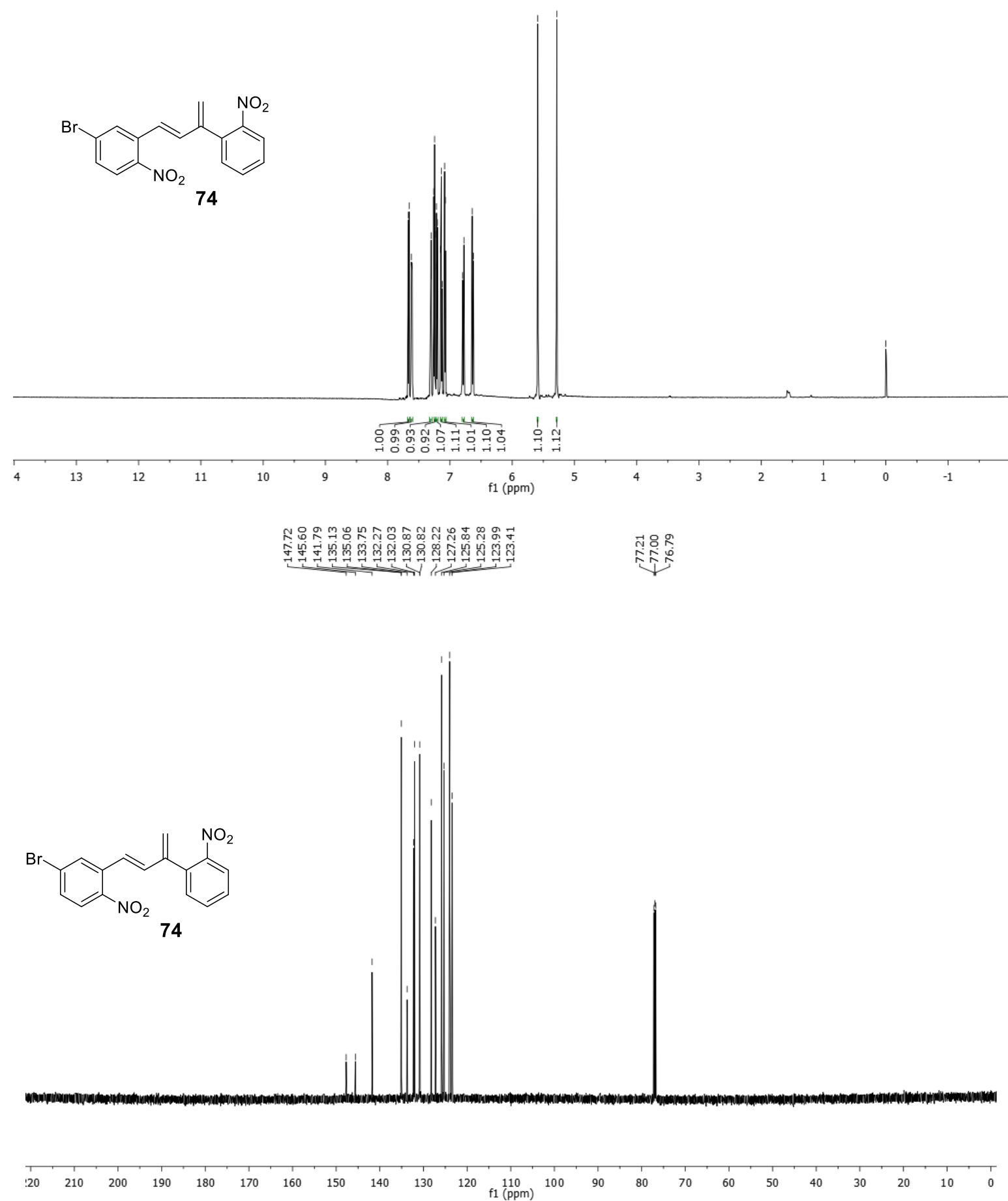

Figure $2.39{ }^{1} \mathrm{H}$ and ${ }^{13} \mathrm{C}$ NMR of compound 74 

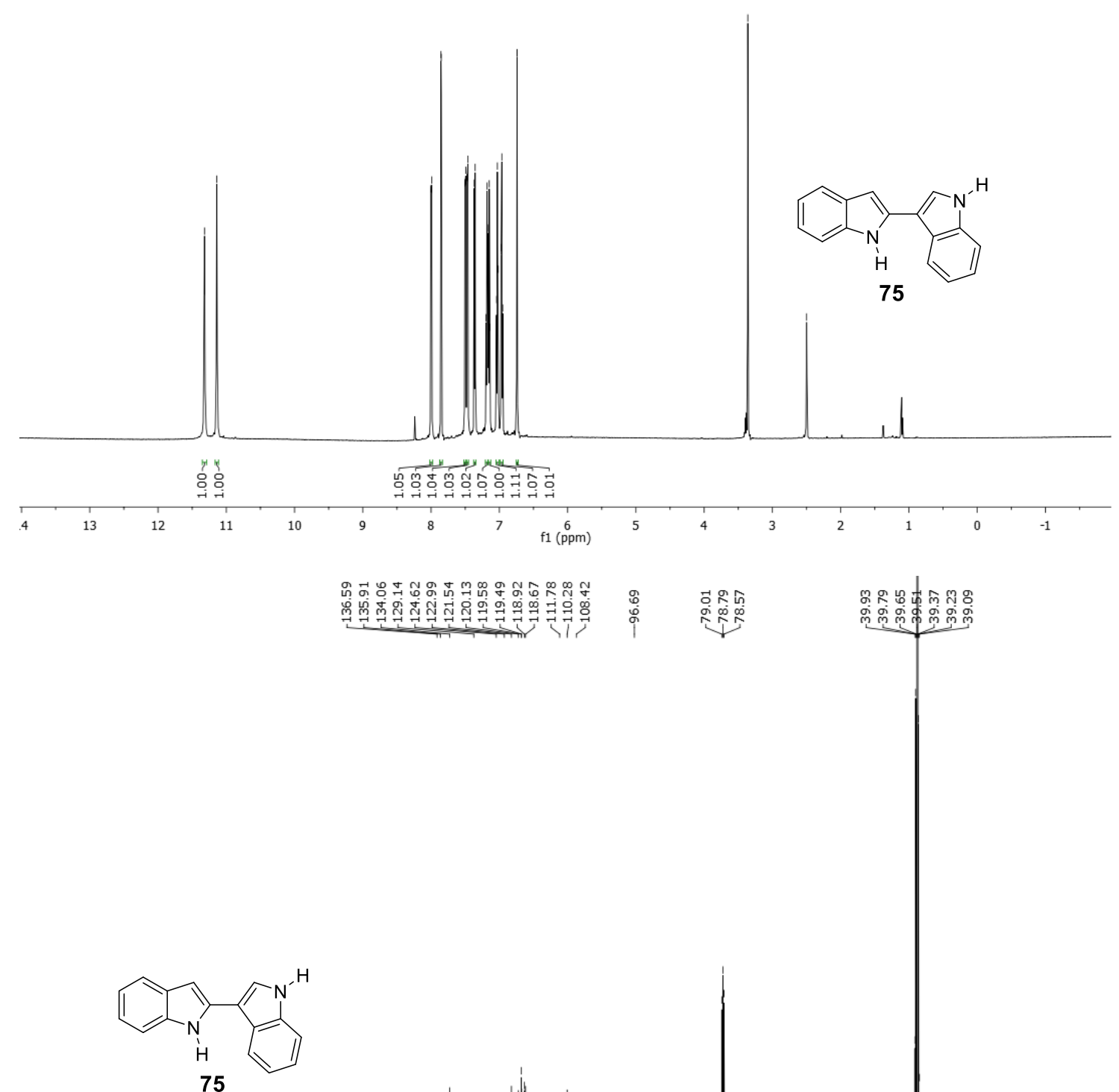

75

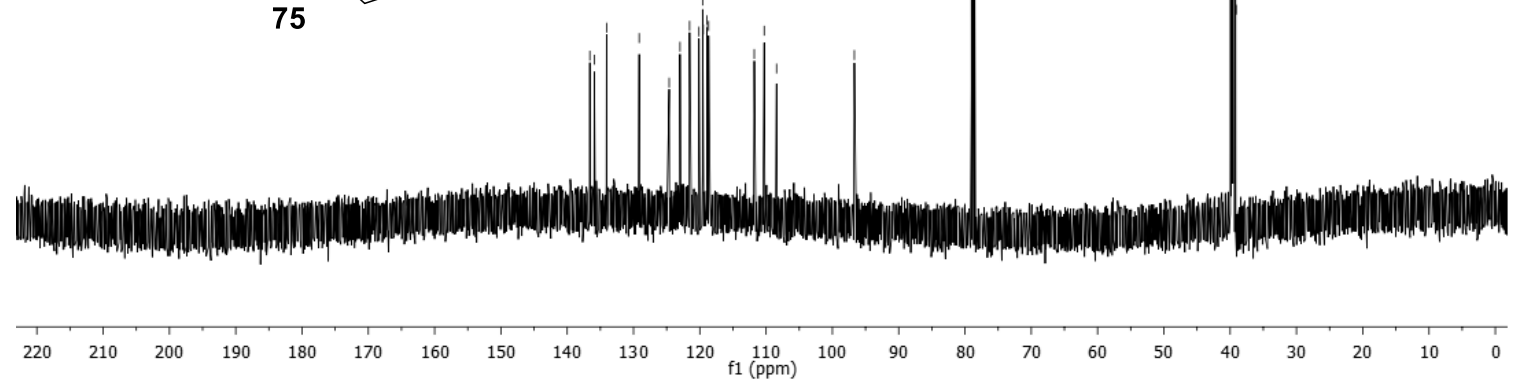

Figure 2.40 ${ }^{1} \mathrm{H}$ and ${ }^{13} \mathrm{C}$ NMR of compound 75 

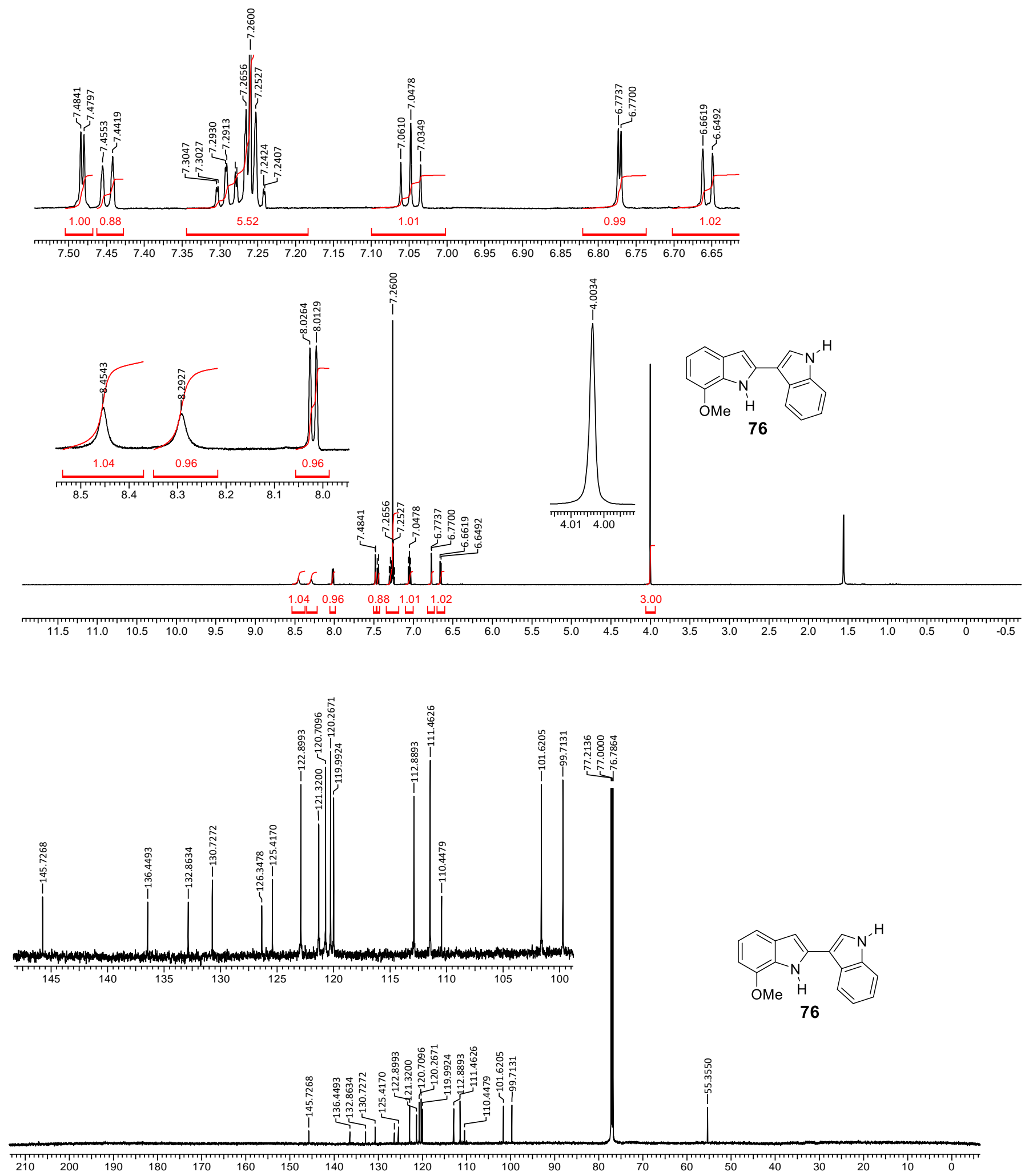

Figure 2.41 ${ }^{1} \mathrm{H}$ and ${ }^{13} \mathrm{C}$ NMR of compound 76 

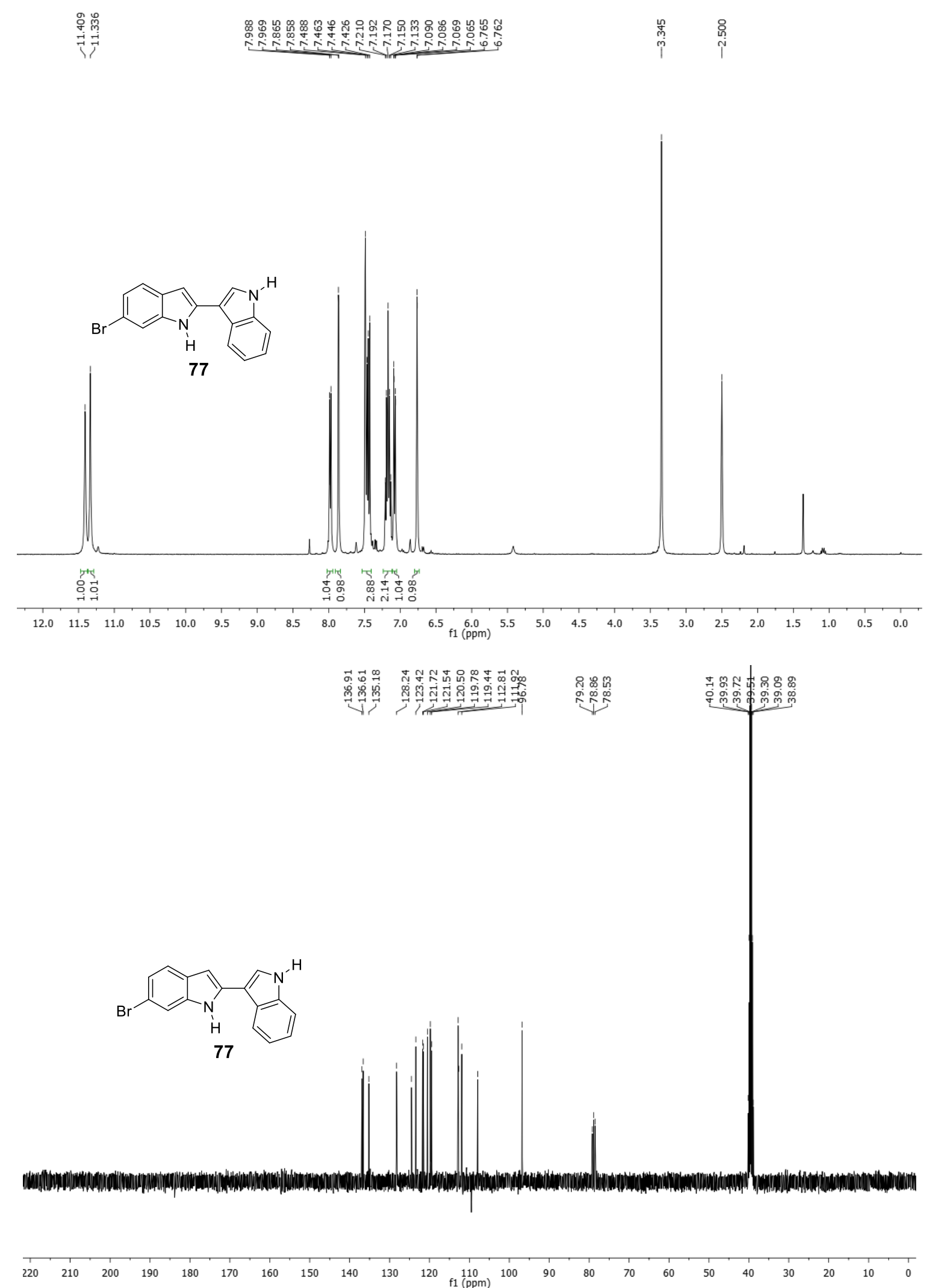

Figure $2.42{ }^{1} \mathrm{H}$ and ${ }^{13} \mathrm{C}$ NMR of compound 77 


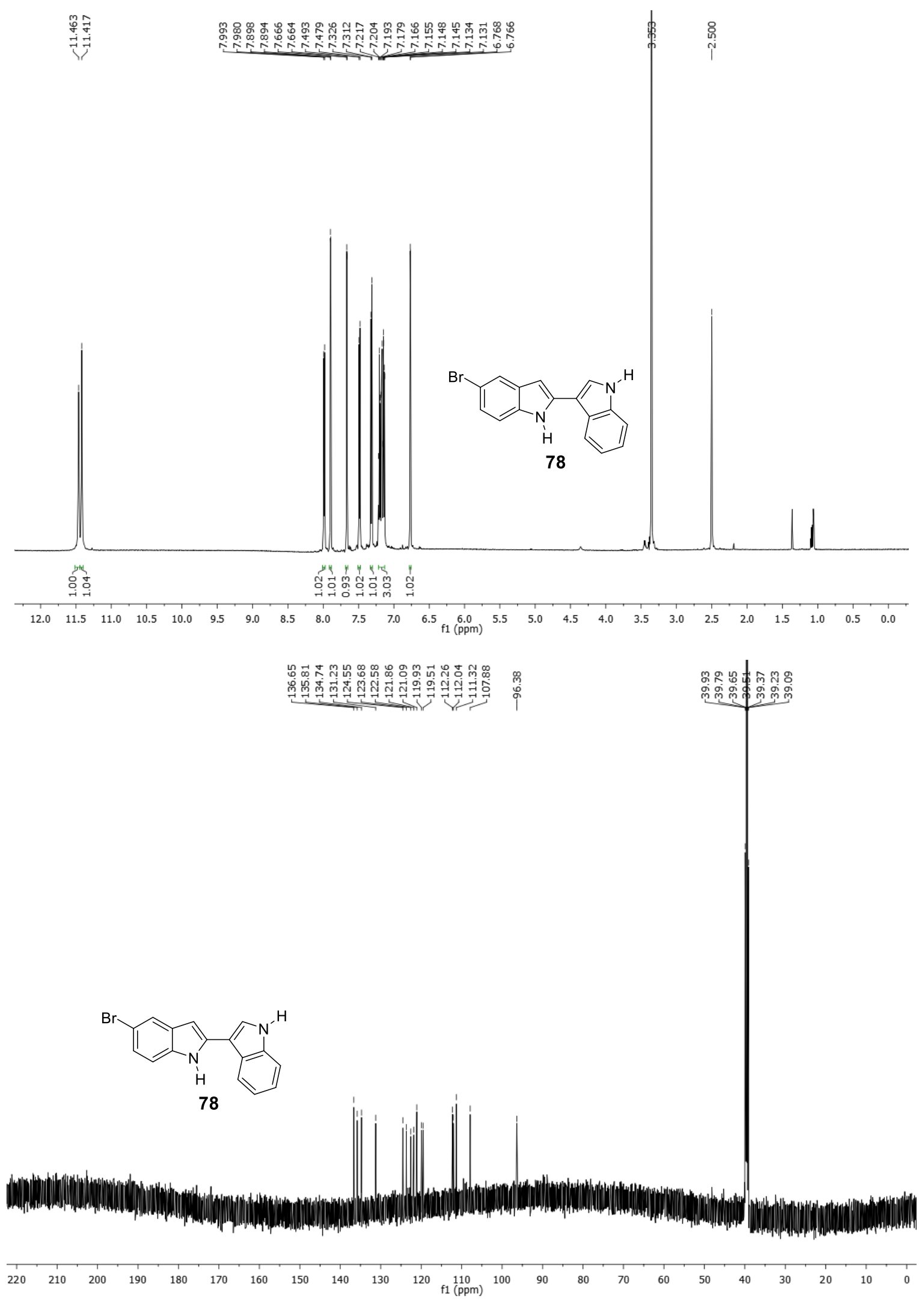

Figure $2.43{ }^{1} \mathrm{H}$ and ${ }^{13} \mathrm{C}$ NMR of compound 78 

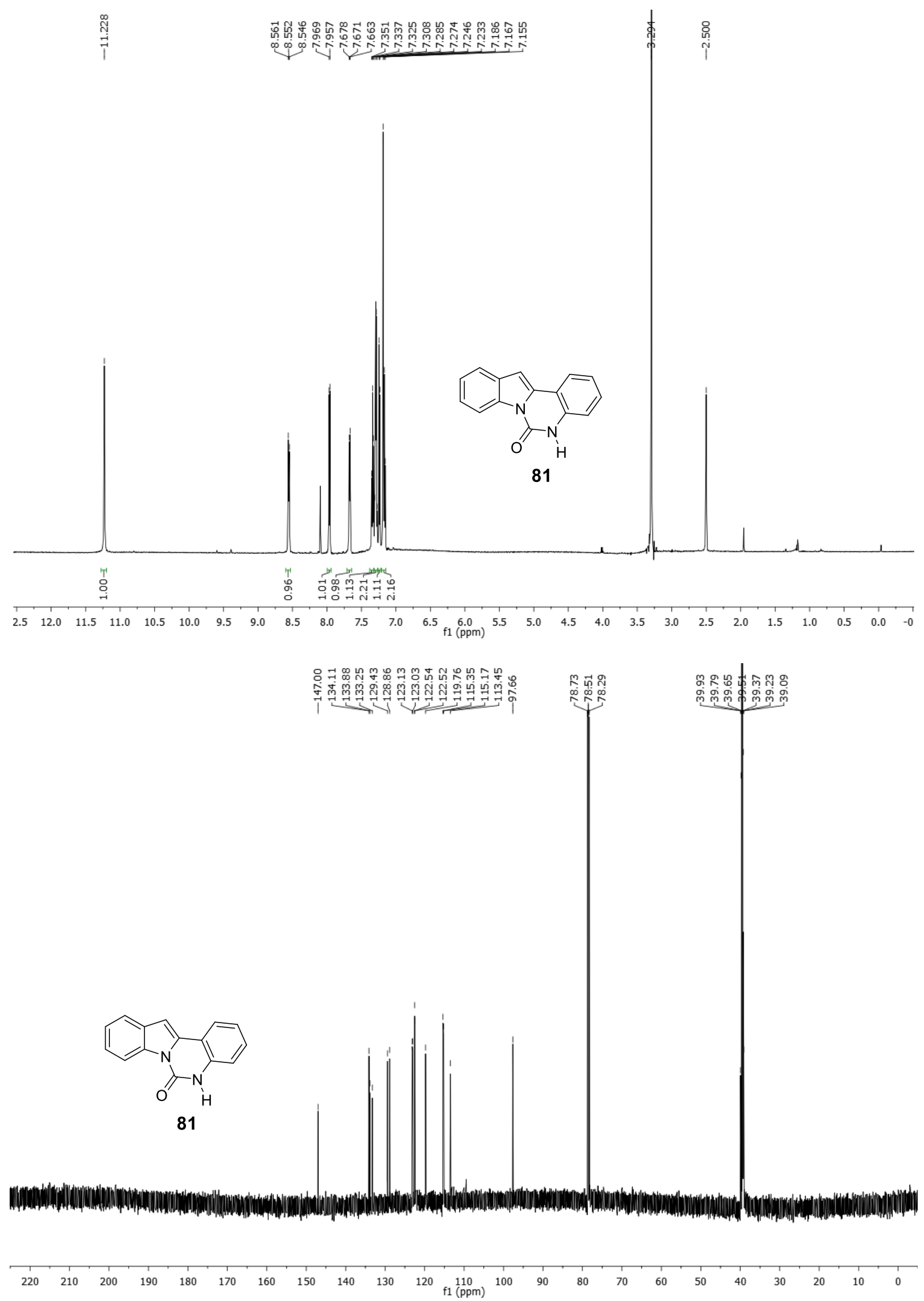

Figure $2.44{ }^{1} \mathrm{H}$ and ${ }^{13} \mathrm{C}$ NMR of compound 81 


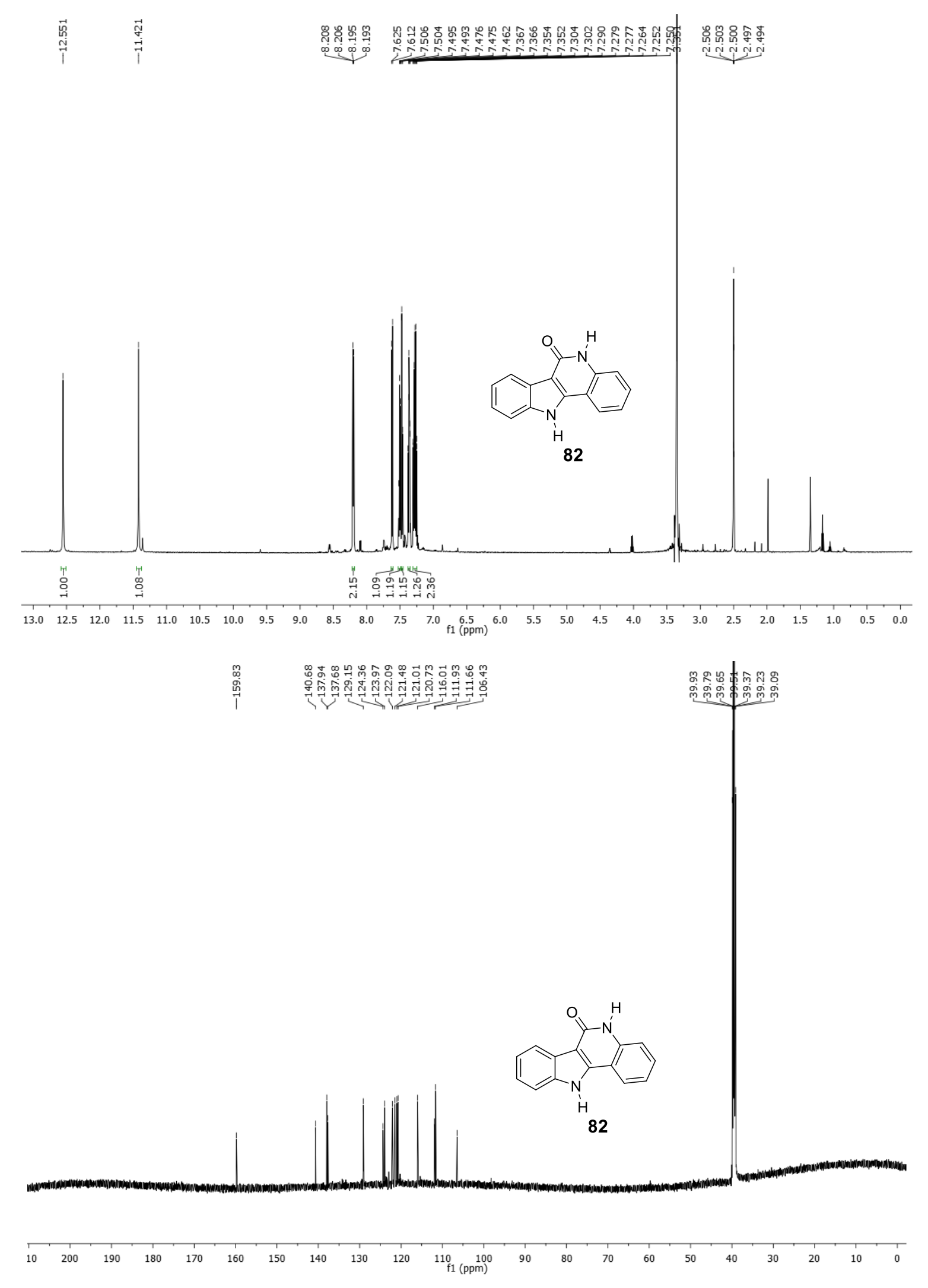

Figure $2.45{ }^{1} \mathrm{H}$ and ${ }^{13} \mathrm{C}$ NMR of compound 82 

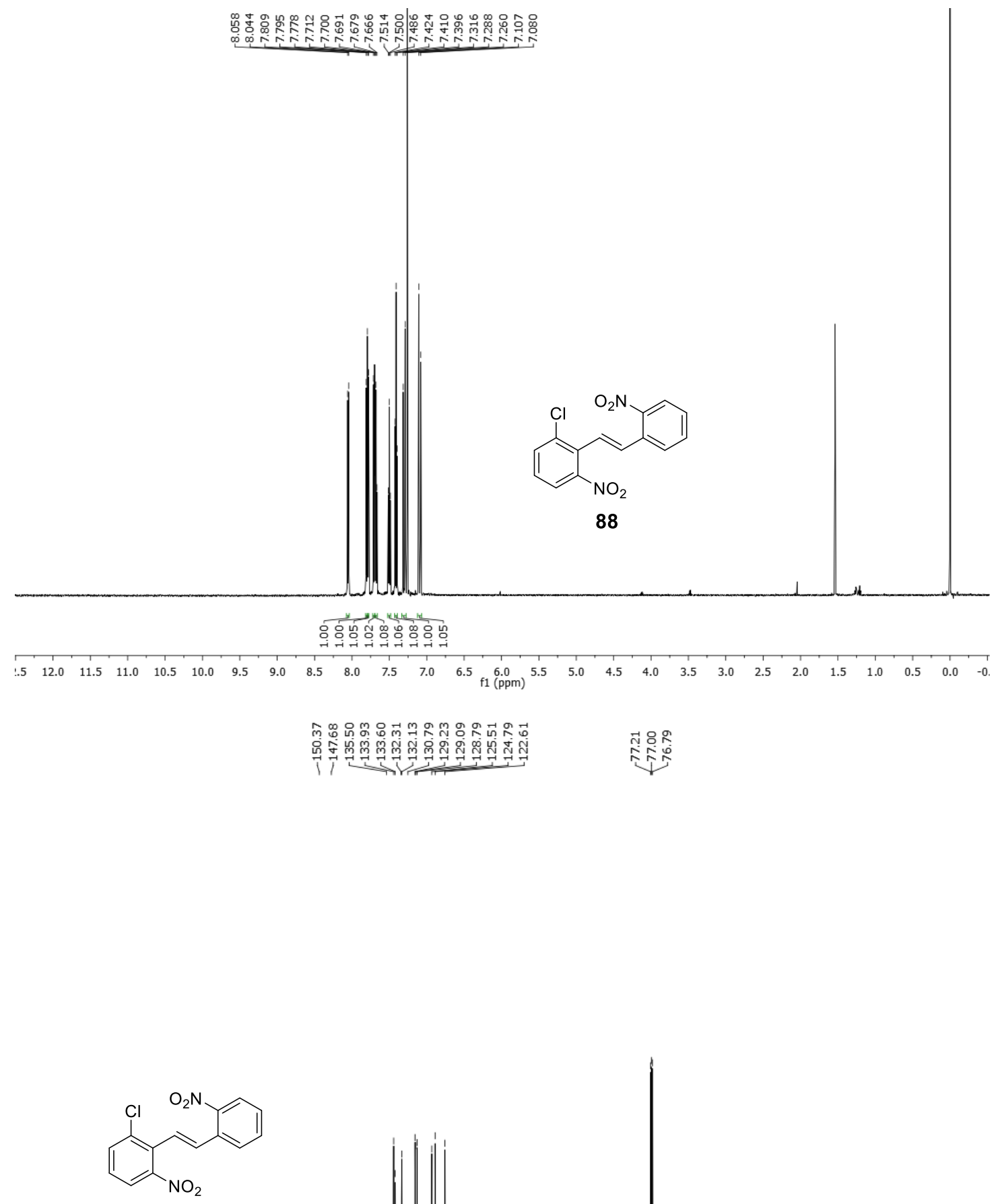

88

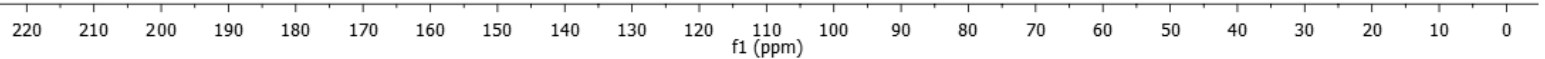

Figure 2.46 ${ }^{1} \mathrm{H}$ and ${ }^{13} \mathrm{C}$ NMR of compound $\mathbf{8 8}$ 

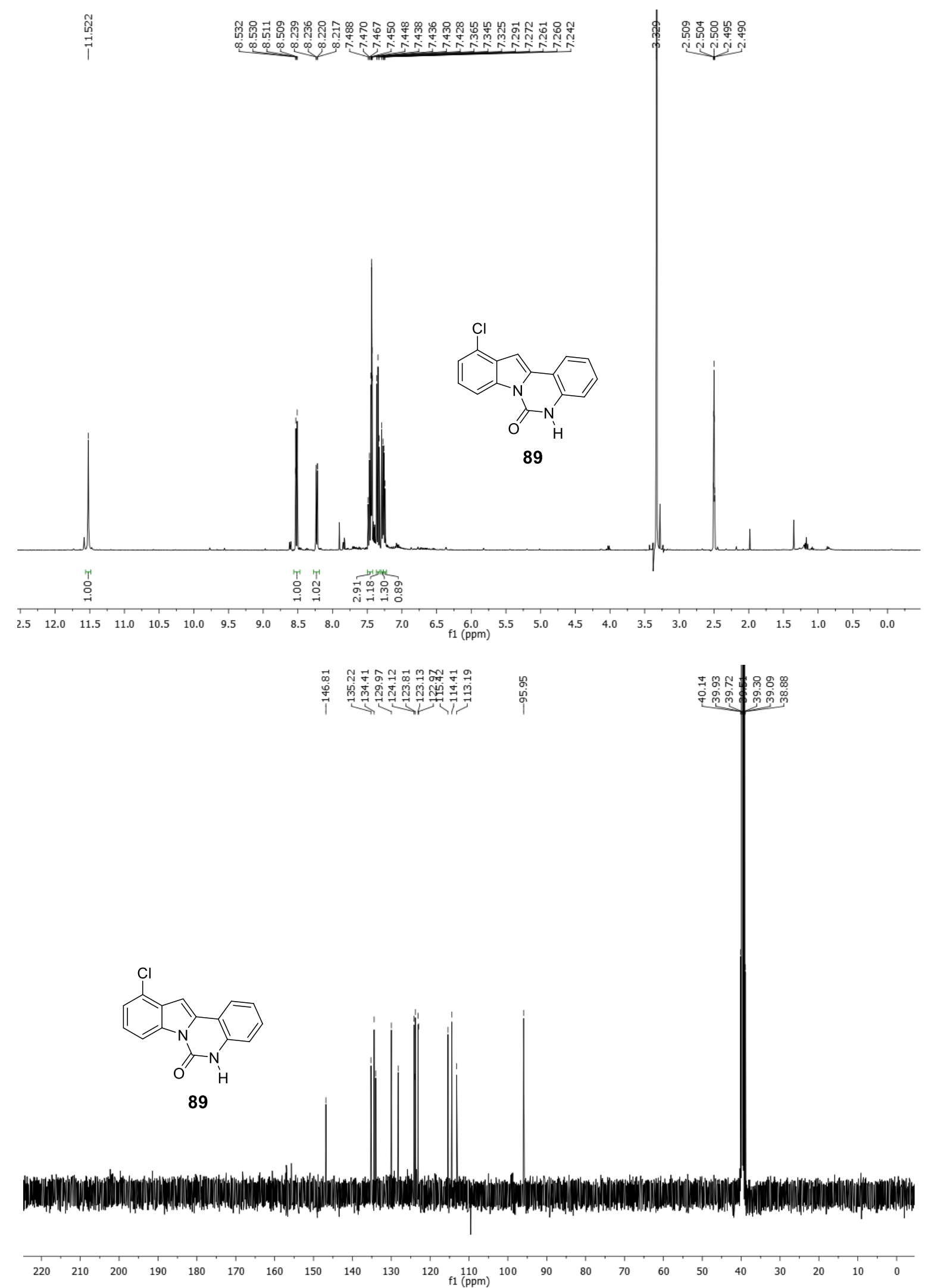

Figure $2.47{ }^{1} \mathrm{H}$ and ${ }^{13} \mathrm{C}$ NMR of compound 89 


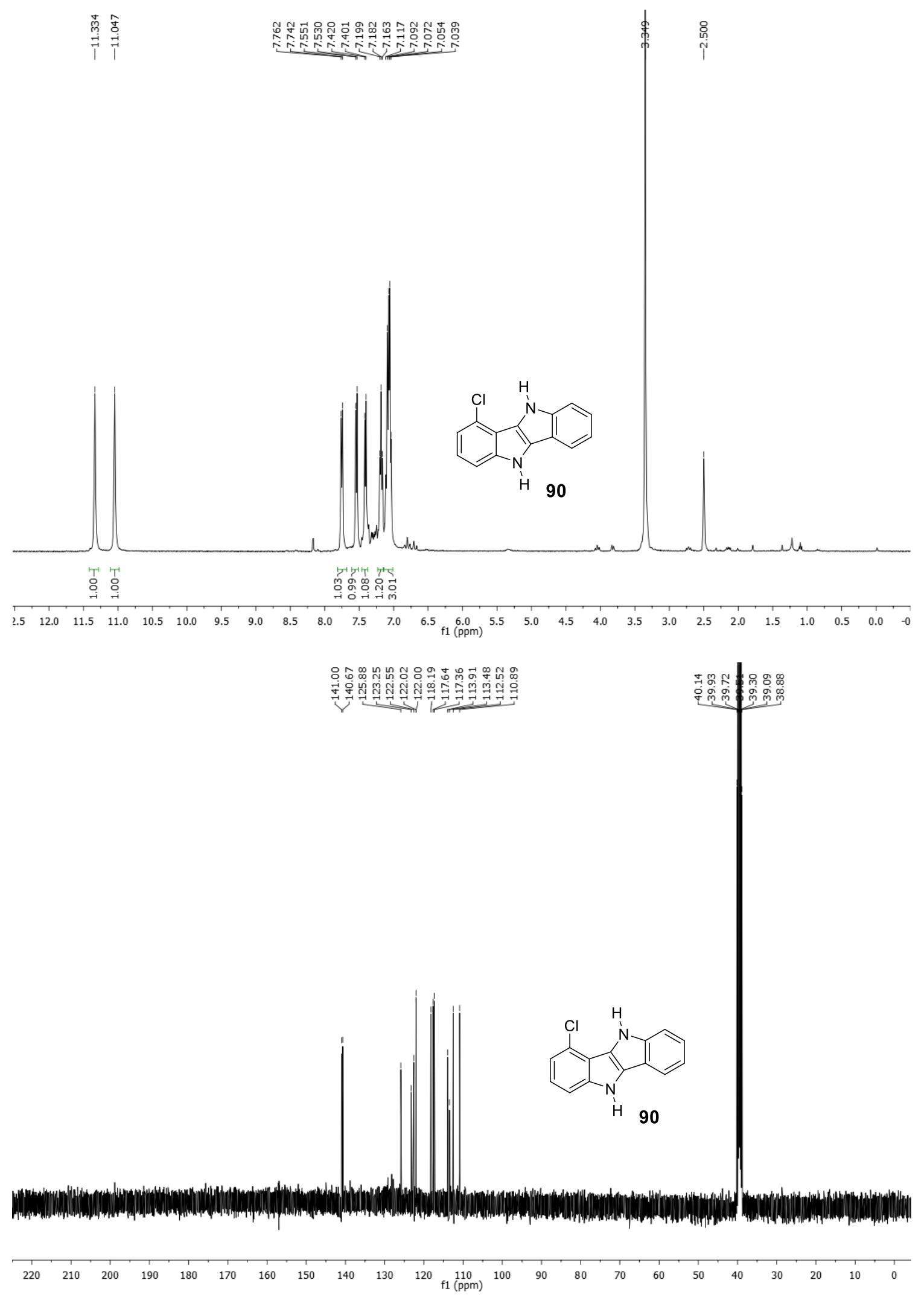

Figure $2.48{ }^{1} \mathrm{H}$ and ${ }^{13} \mathrm{C}$ NMR of compound 90 

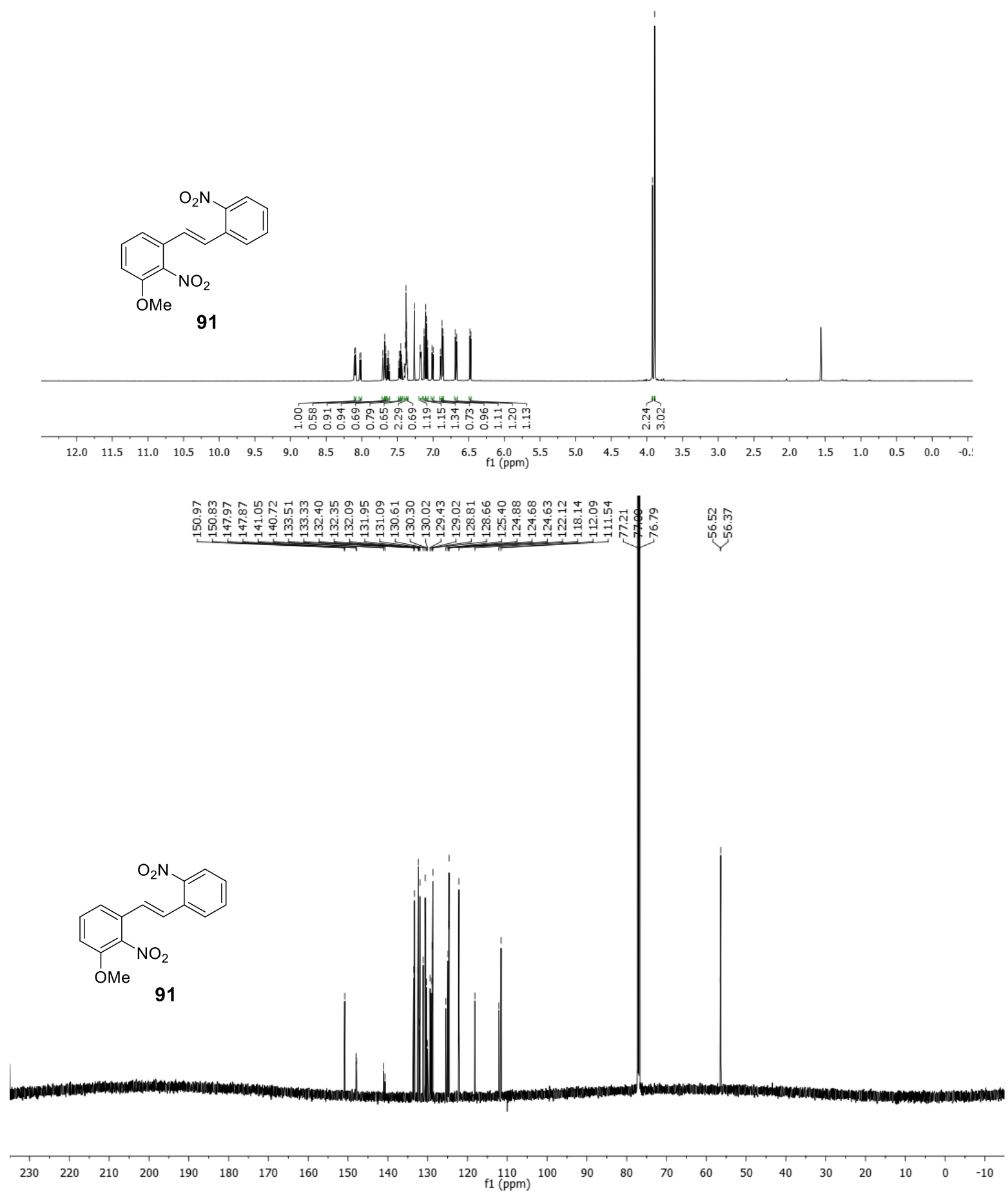

Figure 2.49 ${ }^{1} \mathrm{H}$ and ${ }^{13} \mathrm{C}$ NMR of compound 91 

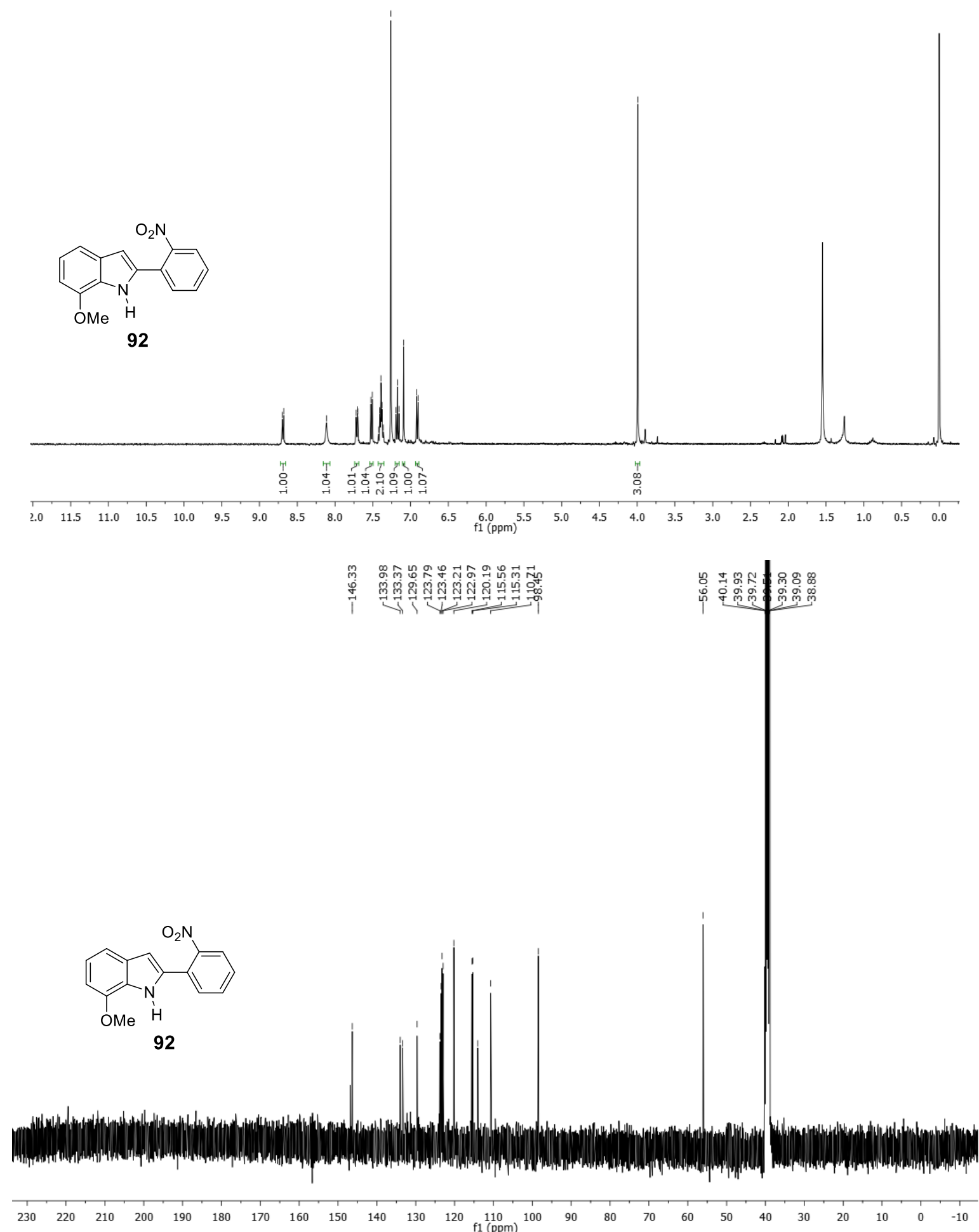

Figure $2.50{ }^{1} \mathrm{H}$ and ${ }^{13} \mathrm{C}$ NMR of compound 92 

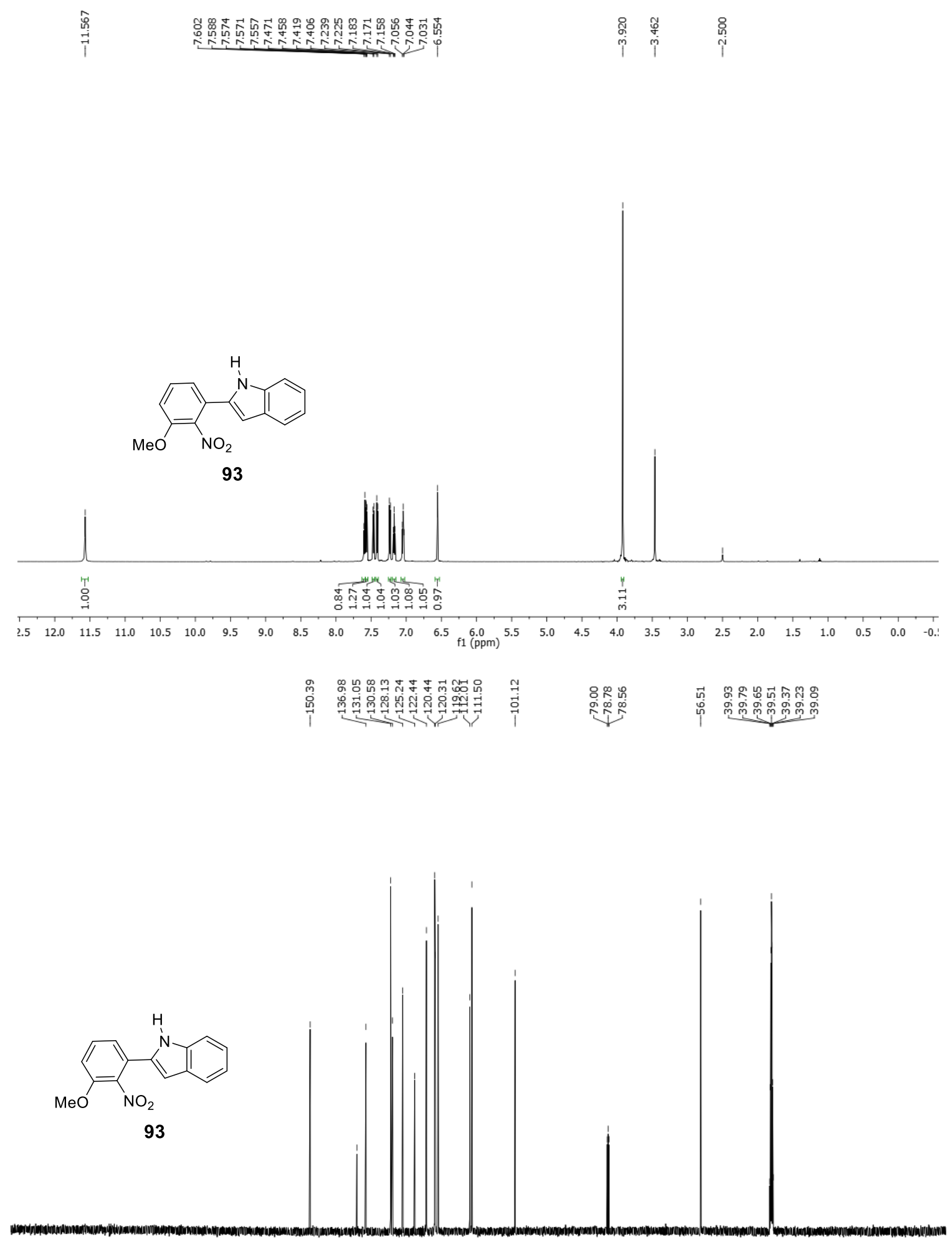

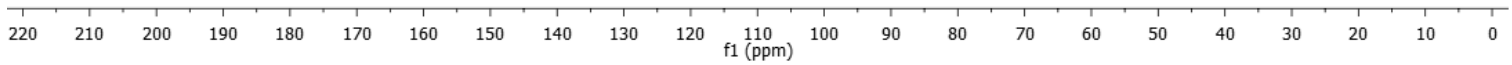

Figure $2.51{ }^{1} \mathrm{H}$ and ${ }^{13} \mathrm{C}$ NMR of compound 93 

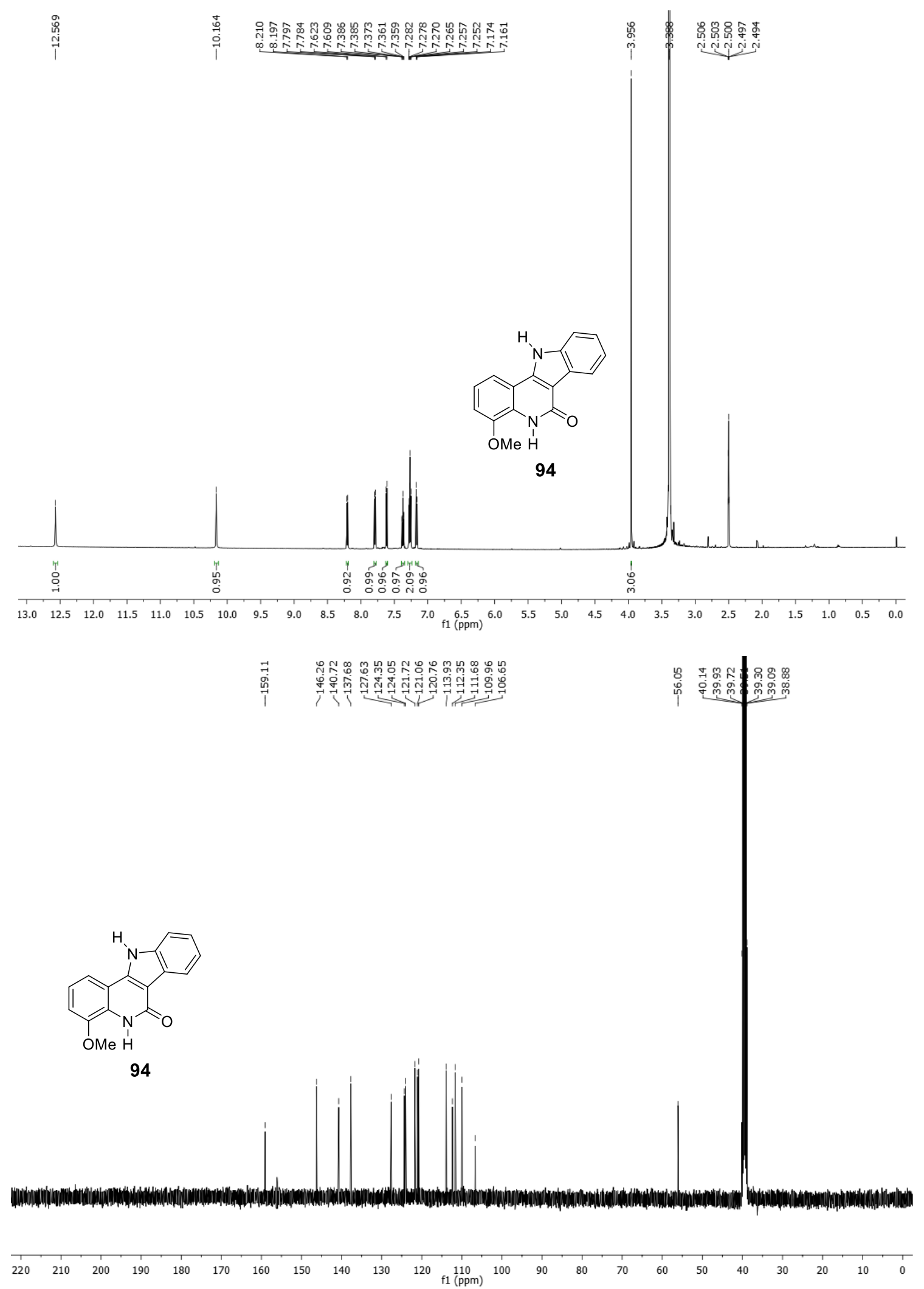

Figure 2.52 ${ }^{1} \mathrm{H}$ and ${ }^{13} \mathrm{C}$ NMR of compound 94 

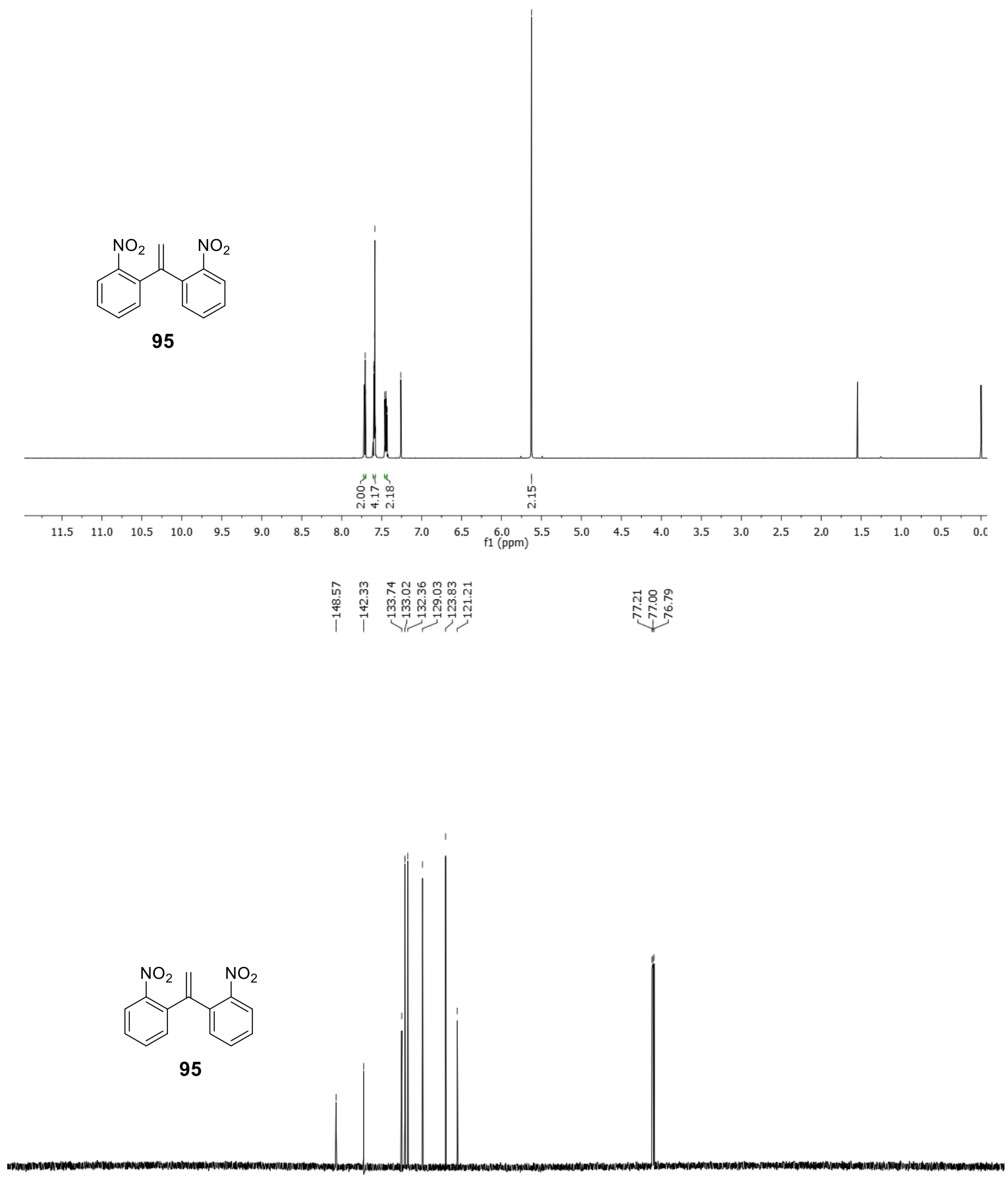

\begin{tabular}{llllllllllllllllllllllllllll}
\hline 220 & 210 & 200 & 190 & 180 & 170 & 160 & 150 & 140 & 130 & 120 & 110 & 100 & 90 & 80 & 70 & 60 & 50 & 40 & 30 & 20 & 10 & 0
\end{tabular}

Figure $2.53{ }^{1} \mathrm{H}$ and ${ }^{13} \mathrm{C}$ NMR of compound 95 

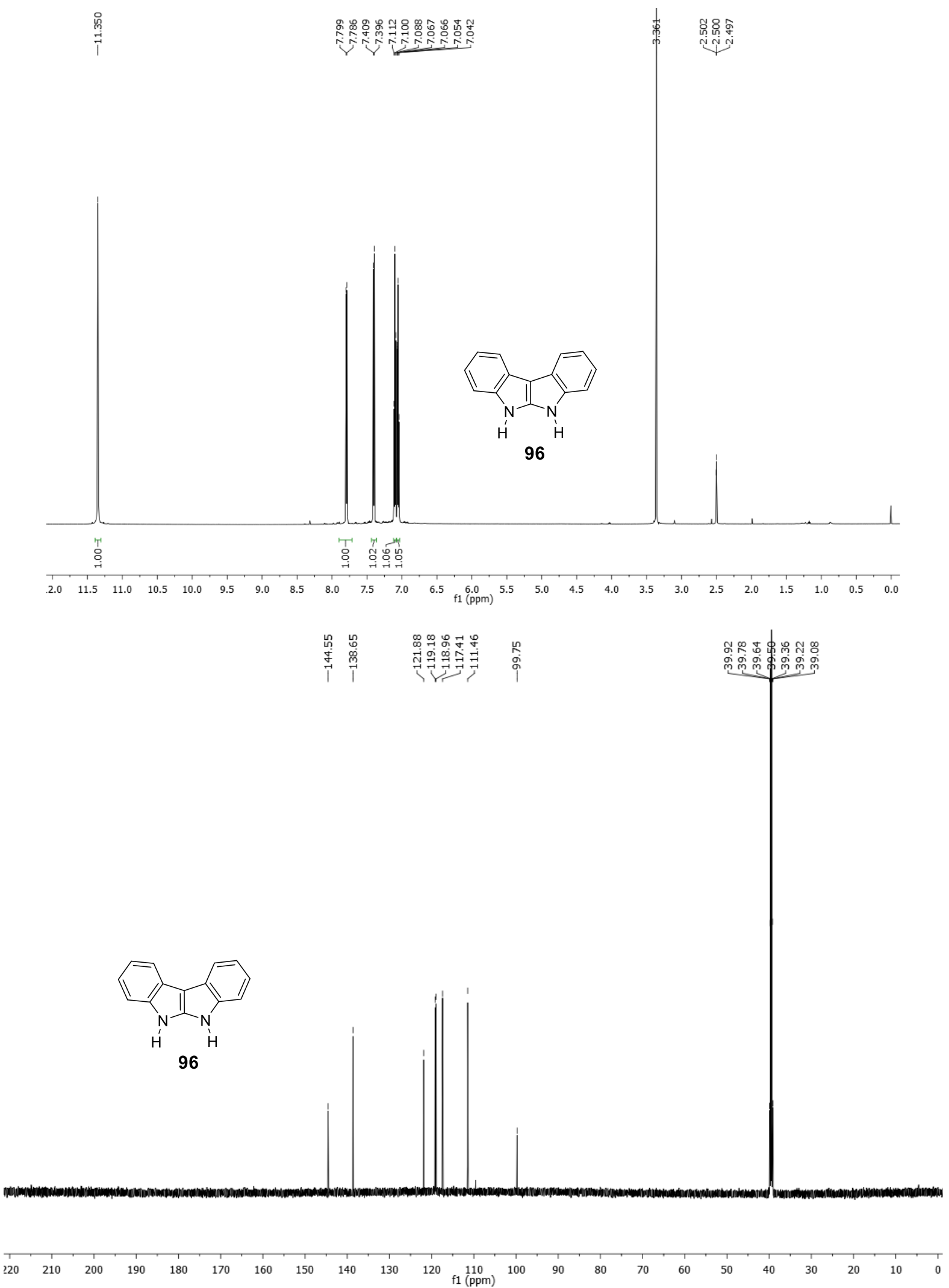

Figure $2.54{ }^{1} \mathrm{H}$ and ${ }^{13} \mathrm{C}$ NMR of compound 96 

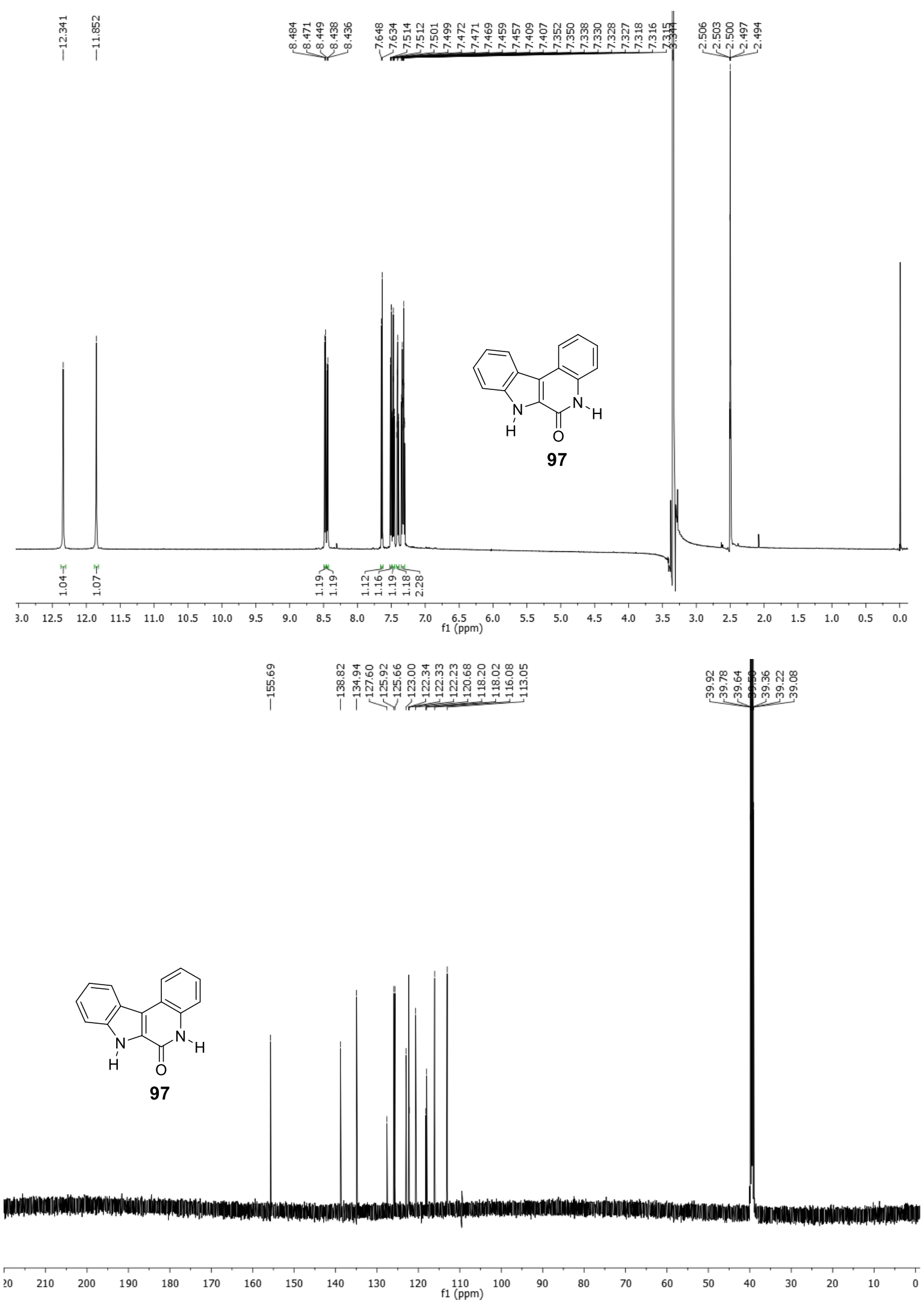

Figure $2.55{ }^{1} \mathrm{H}$ and ${ }^{13} \mathrm{C}$ NMR of compound 97 

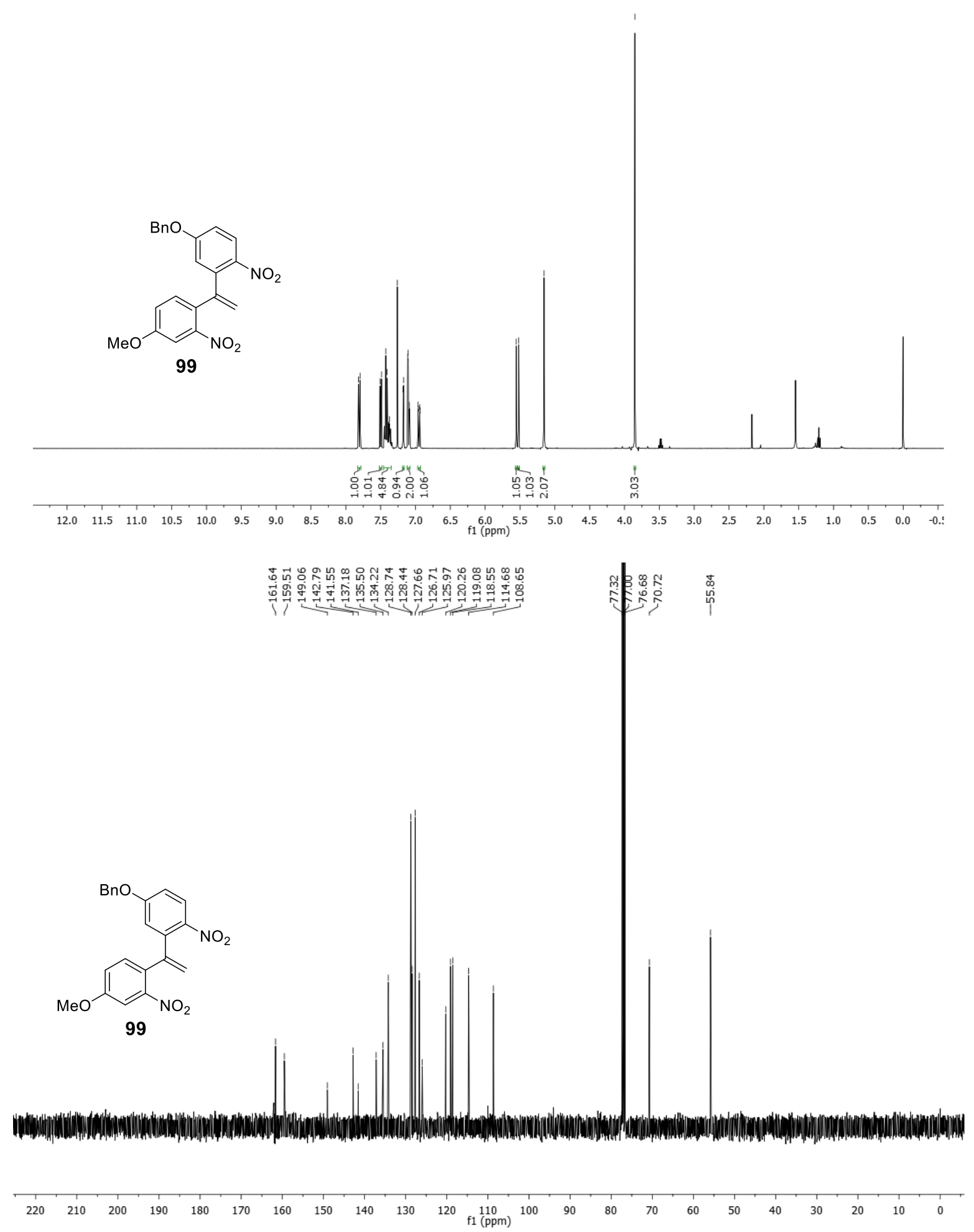

Figure $2.56{ }^{1} \mathrm{H}$ and ${ }^{13} \mathrm{C}$ NMR of compound 99 

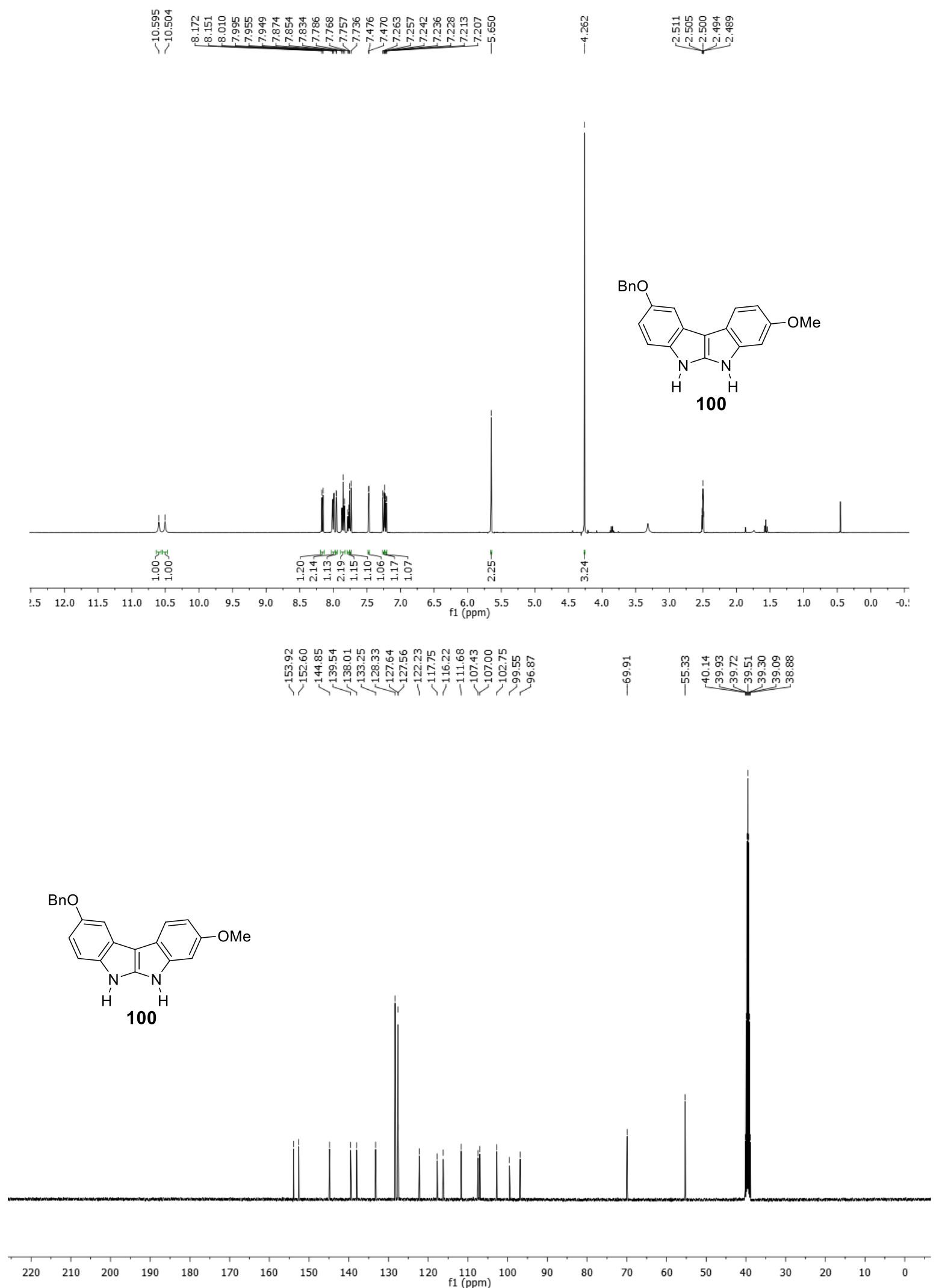

Figure $2.57{ }^{1} \mathrm{H}$ and ${ }^{13} \mathrm{C}$ NMR of compound $\mathbf{1 0 0}$ 

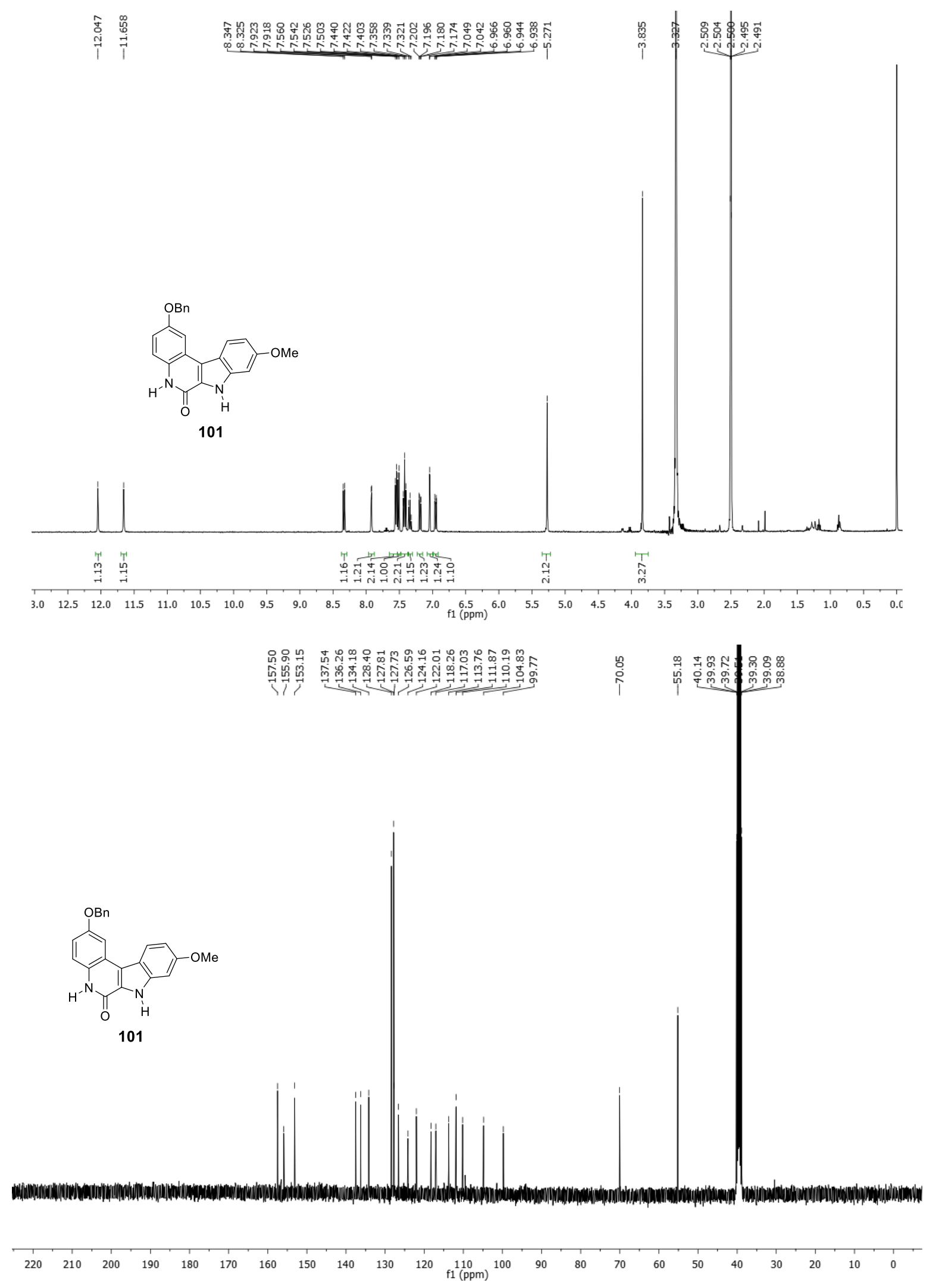

Figure $2.58{ }^{1} \mathrm{H}$ and ${ }^{13} \mathrm{C}$ NMR of compound $\mathbf{1 0 1}$ 

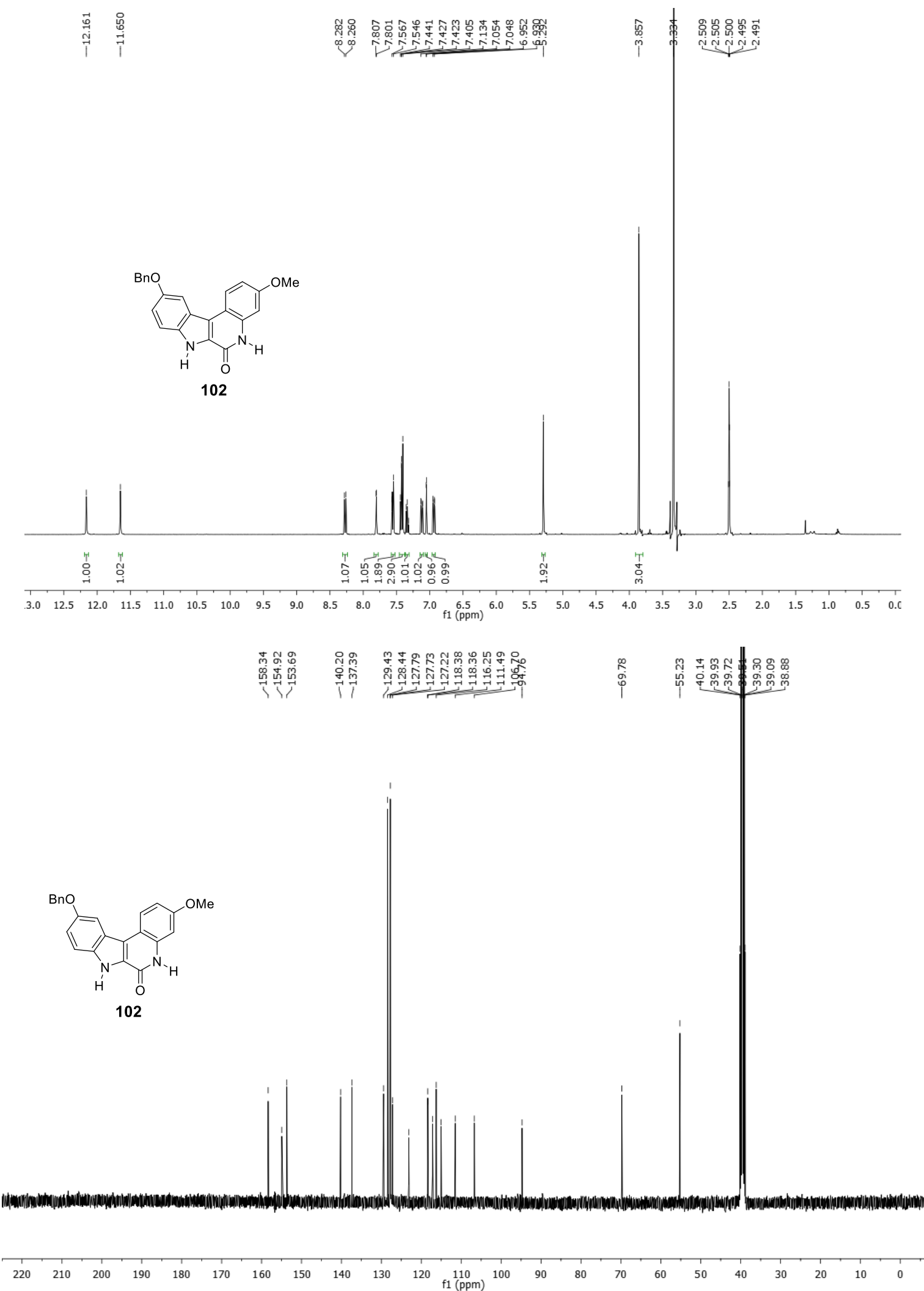

Figure $2.59{ }^{1} \mathrm{H}$ and ${ }^{13} \mathrm{C}$ NMR of compound $\mathbf{1 0 2}$ 


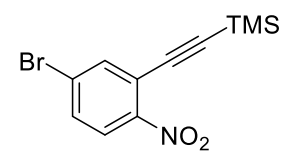

113
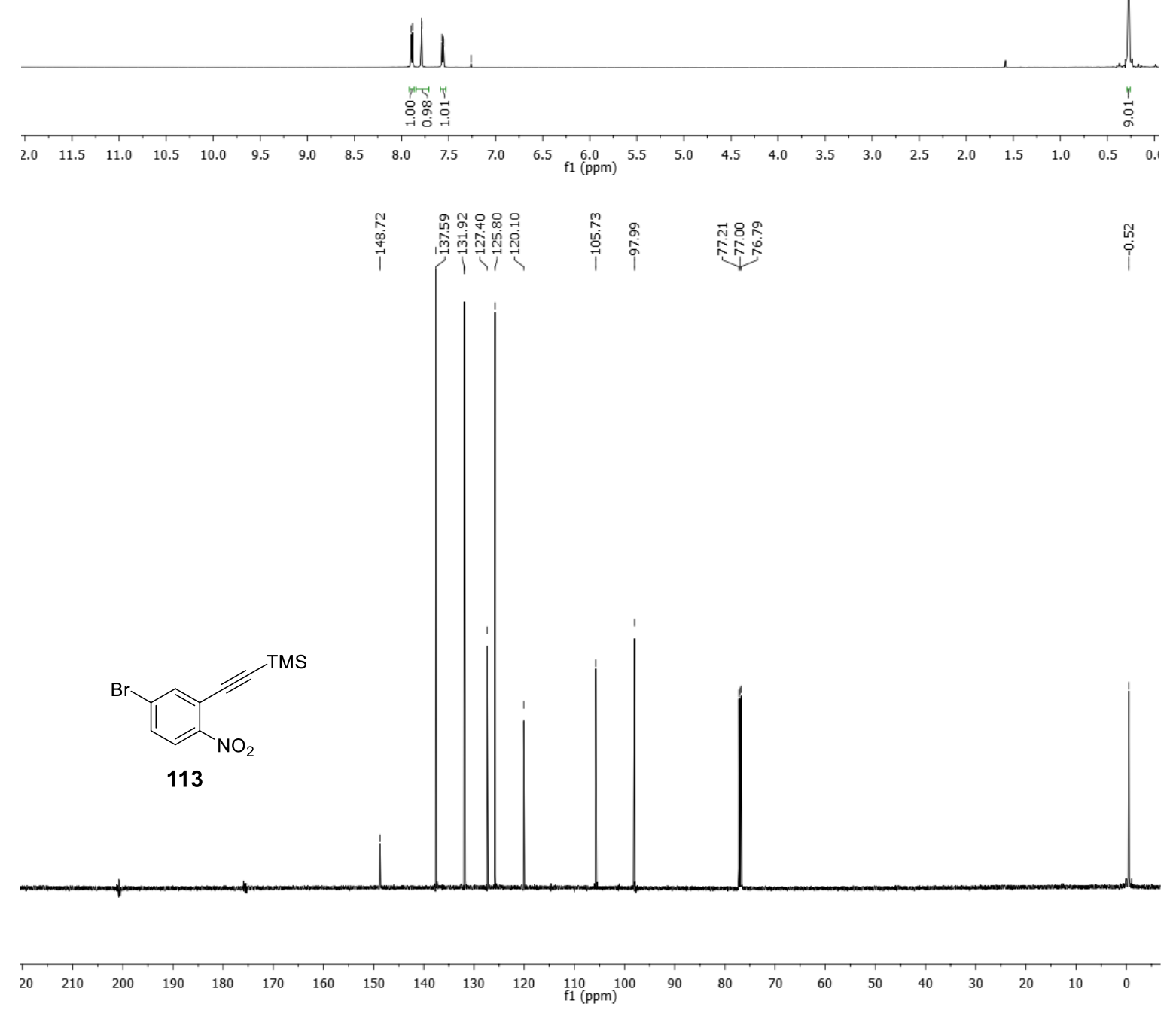

Figure 3.2 ${ }^{1} \mathrm{H}$ and ${ }^{13} \mathrm{C}$ NMR of compound $\mathbf{1 1 3}$ 

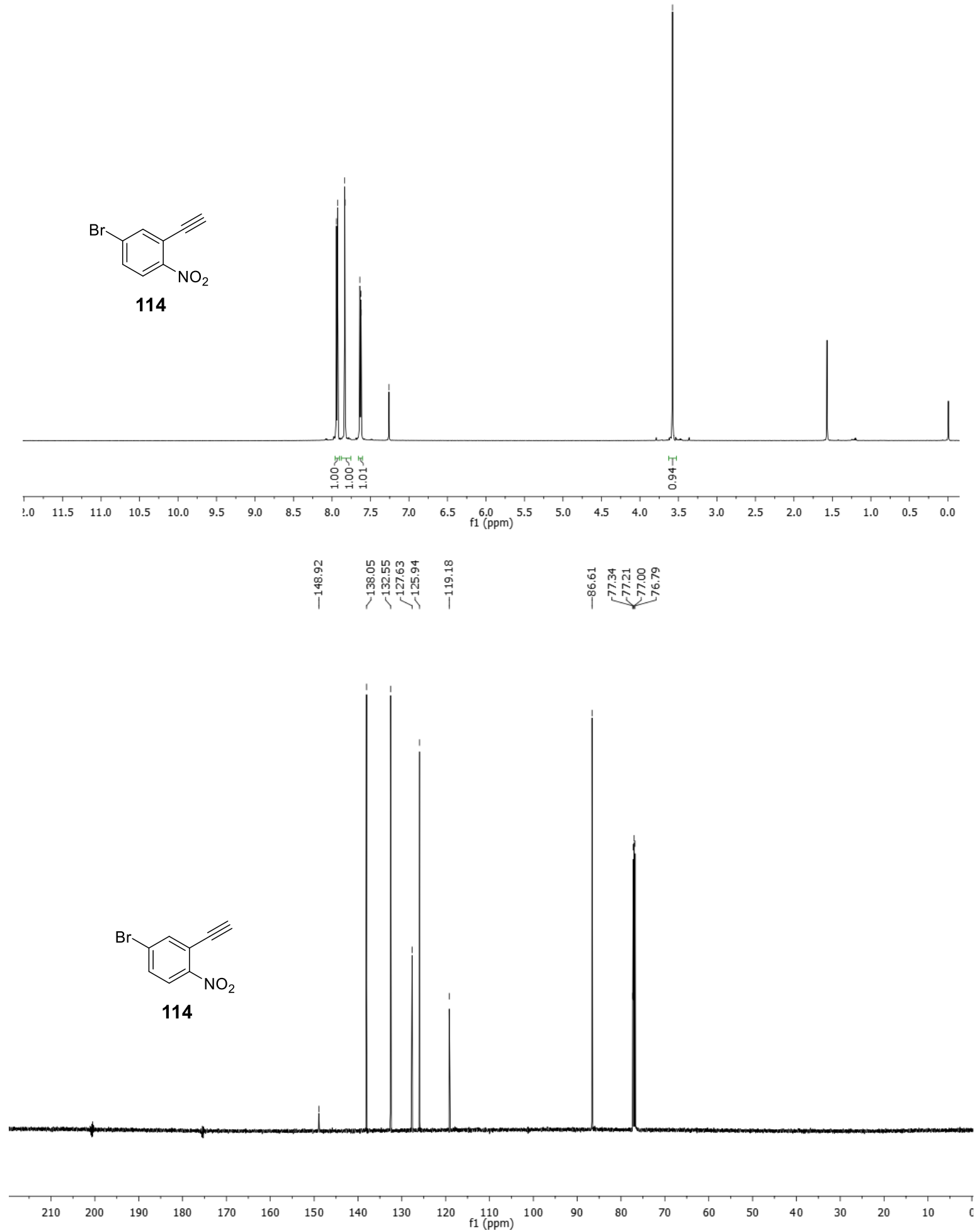

Figure $3.3{ }^{1} \mathrm{H}$ and ${ }^{13} \mathrm{C}$ NMR of compound 114 


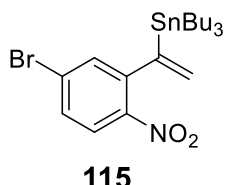

115
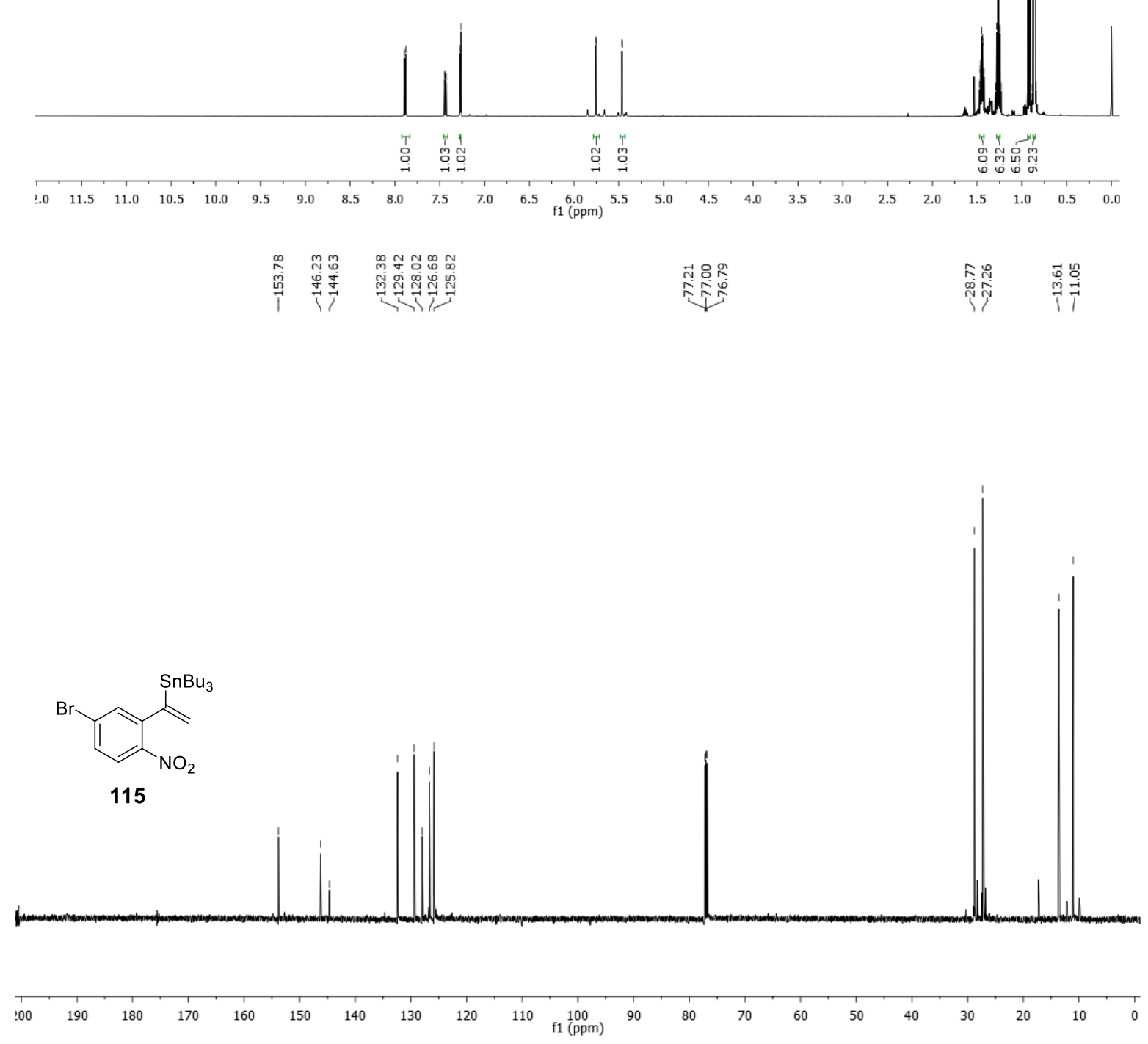

Figure 3.4 ${ }^{1} \mathrm{H}$ and ${ }^{13} \mathrm{C}$ NMR of compound 115 

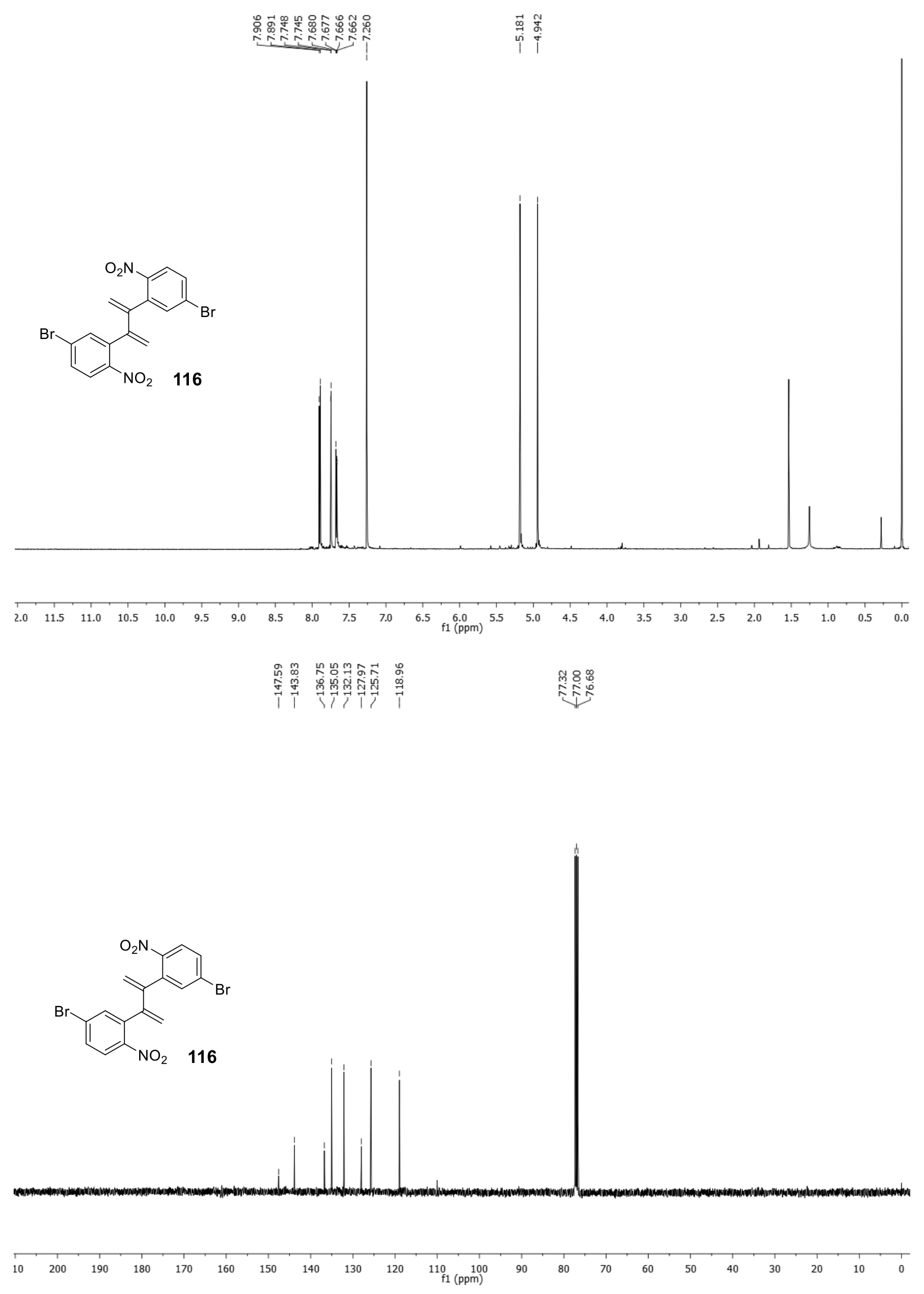

Figure $3.5{ }^{1} \mathrm{H}$ and ${ }^{13} \mathrm{C}$ NMR of compound 116 

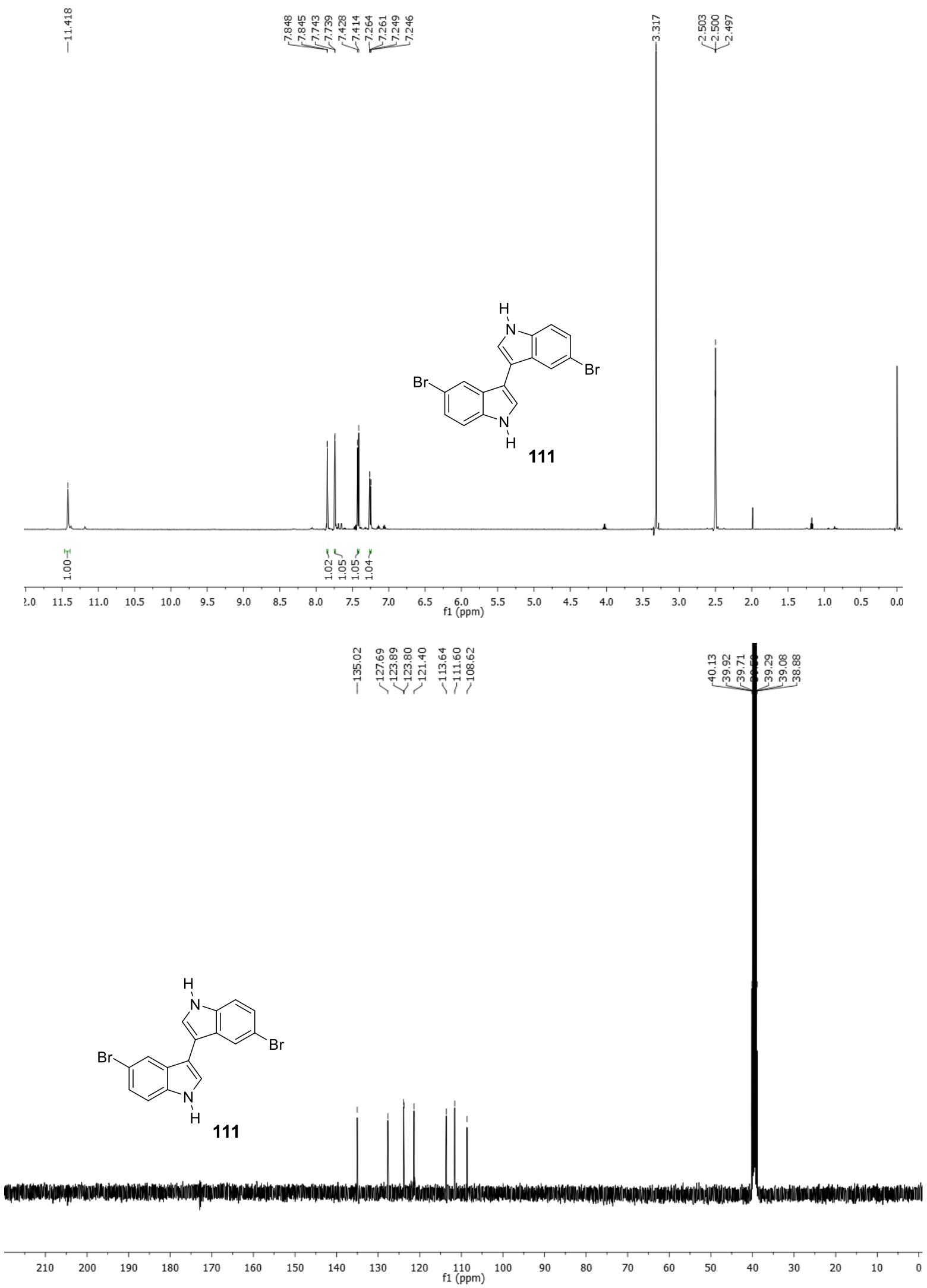

Figure 3.6 ${ }^{1} \mathrm{H}$ and ${ }^{13} \mathrm{C}$ NMR of compound 111 


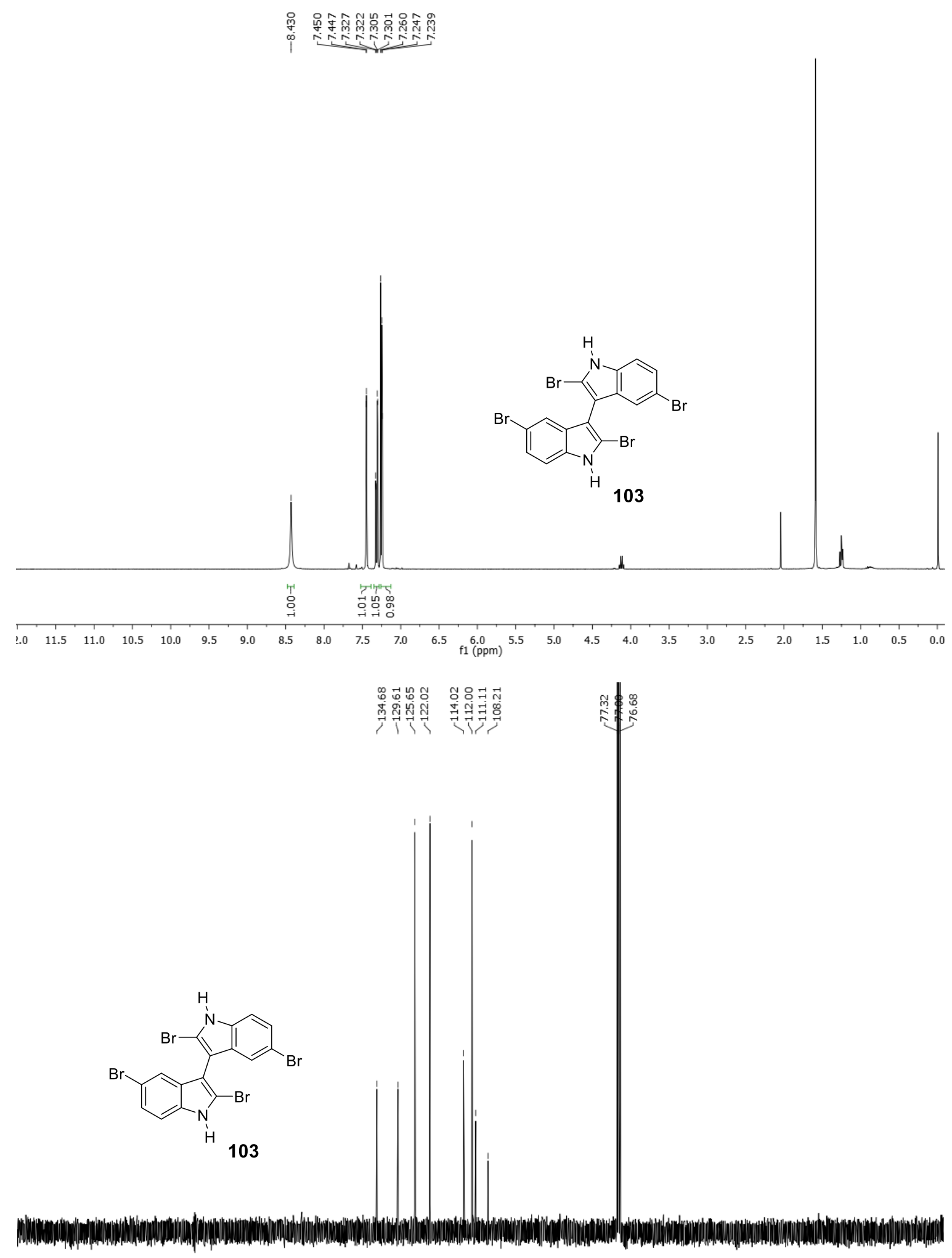

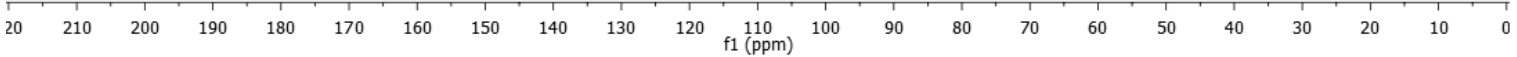

Figure $3.7{ }^{1} \mathrm{H}$ and ${ }^{13} \mathrm{C}$ NMR of compound 103 

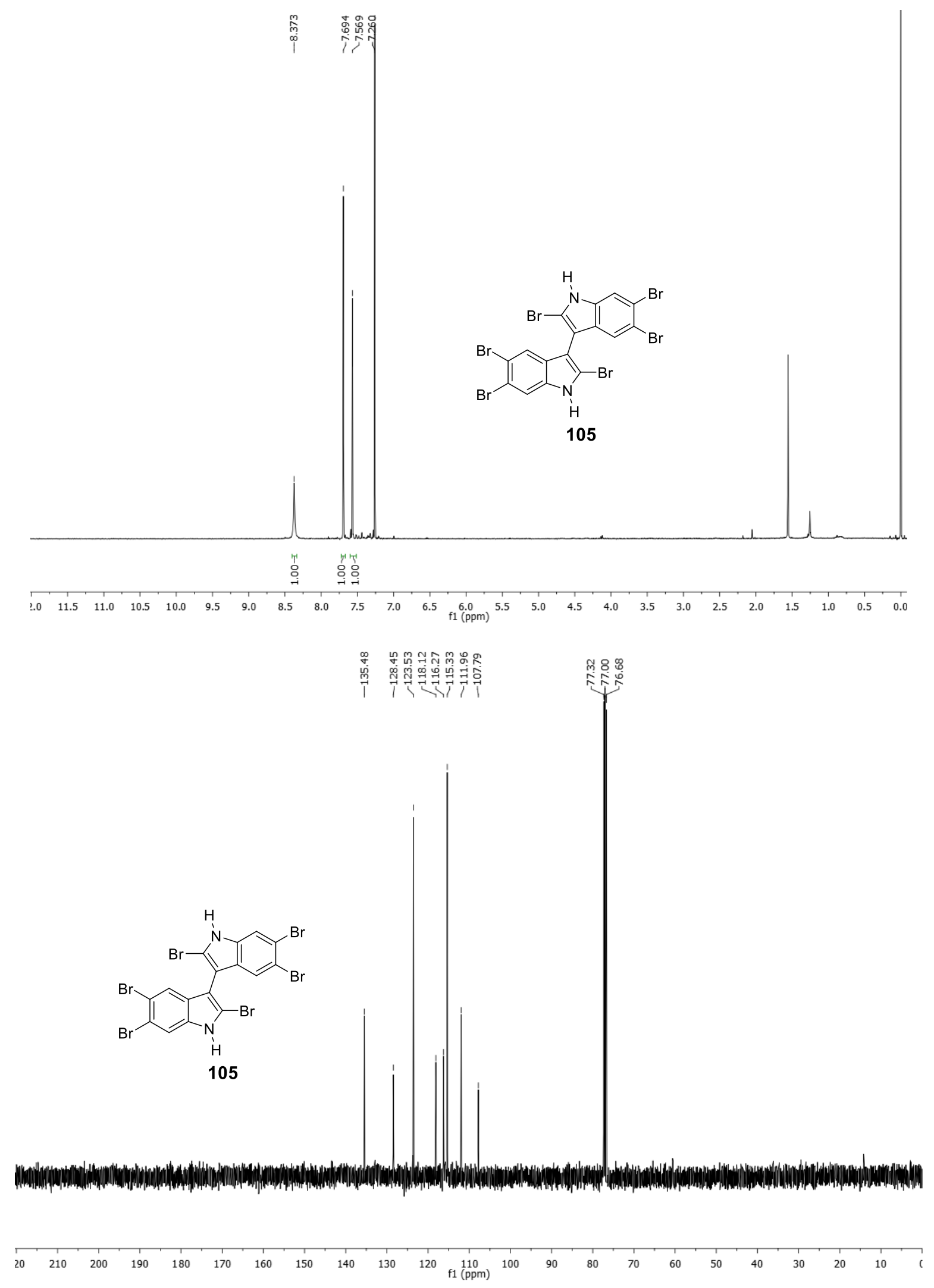

Figure 3.8 ${ }^{1} \mathrm{H}$ and ${ }^{13} \mathrm{C}$ NMR of compound $\mathbf{1 0 5}$ 


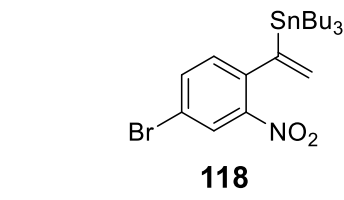

118
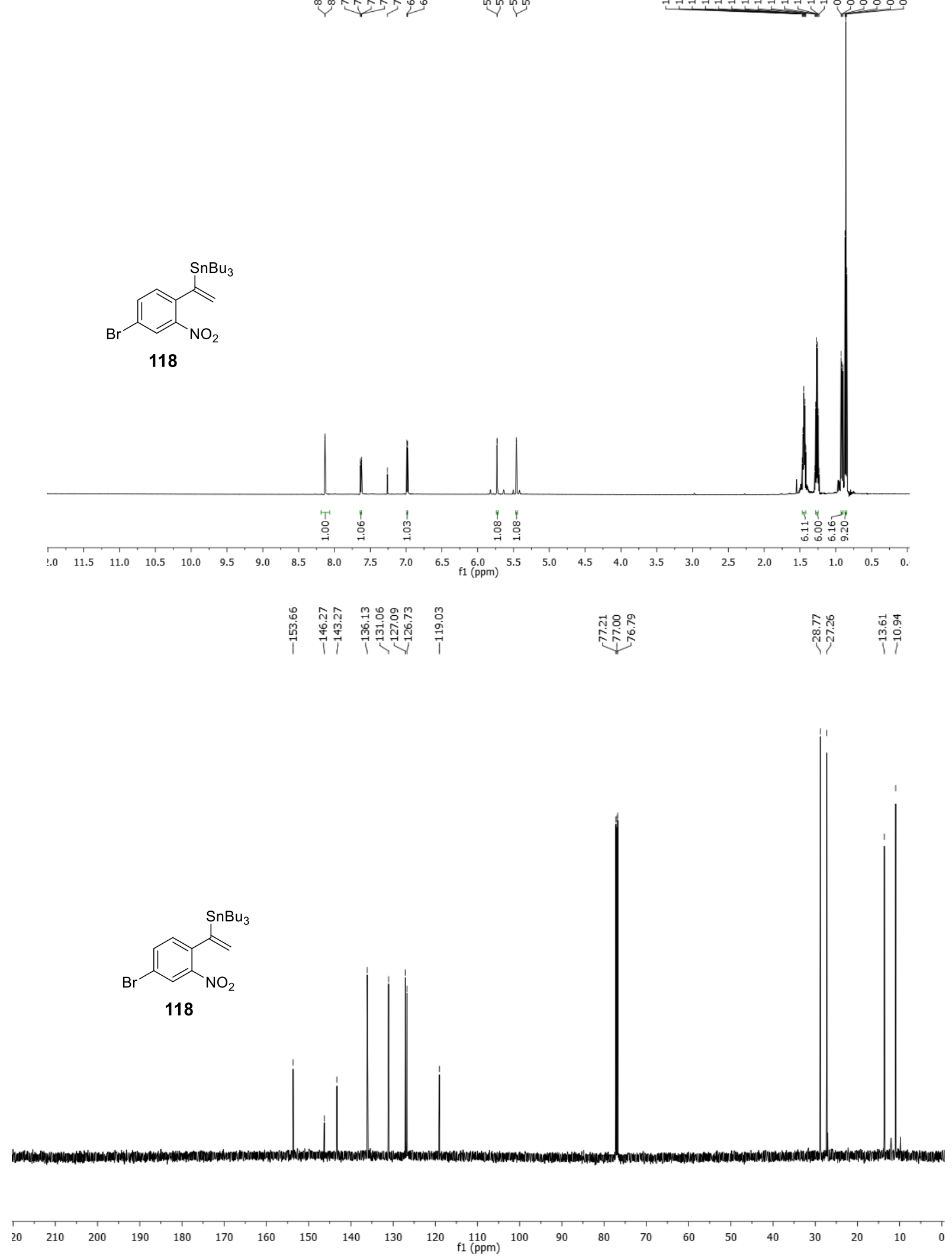

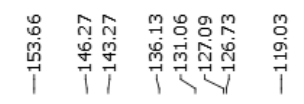

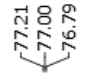$$
\text { în }
$$

Figure 3.9 ${ }^{1} \mathrm{H}$ and ${ }^{13} \mathrm{C}$ NMR of compound 118 

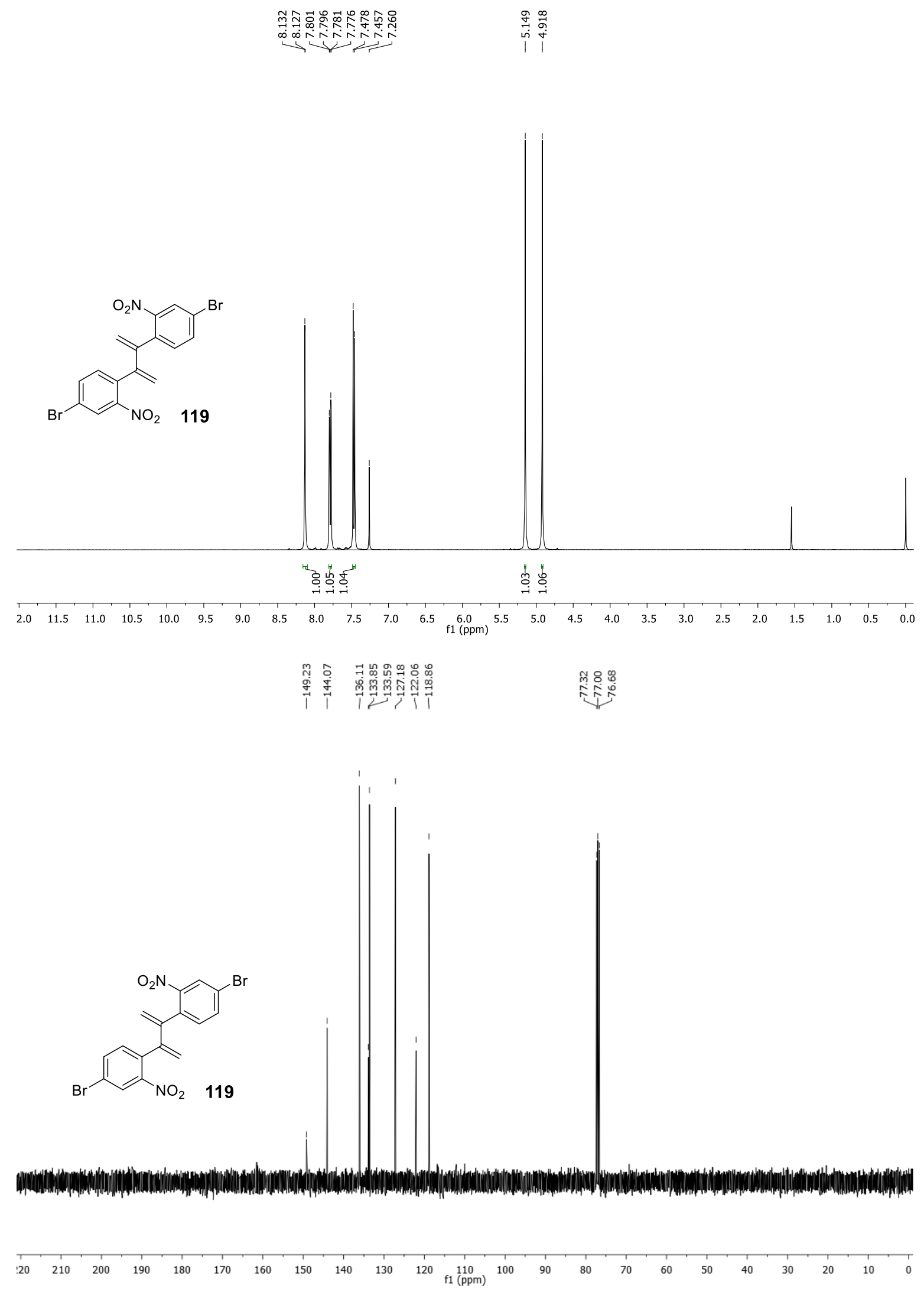

Figure 3.10 ${ }^{1} \mathrm{H}$ and ${ }^{13} \mathrm{C}$ NMR of compound 119 

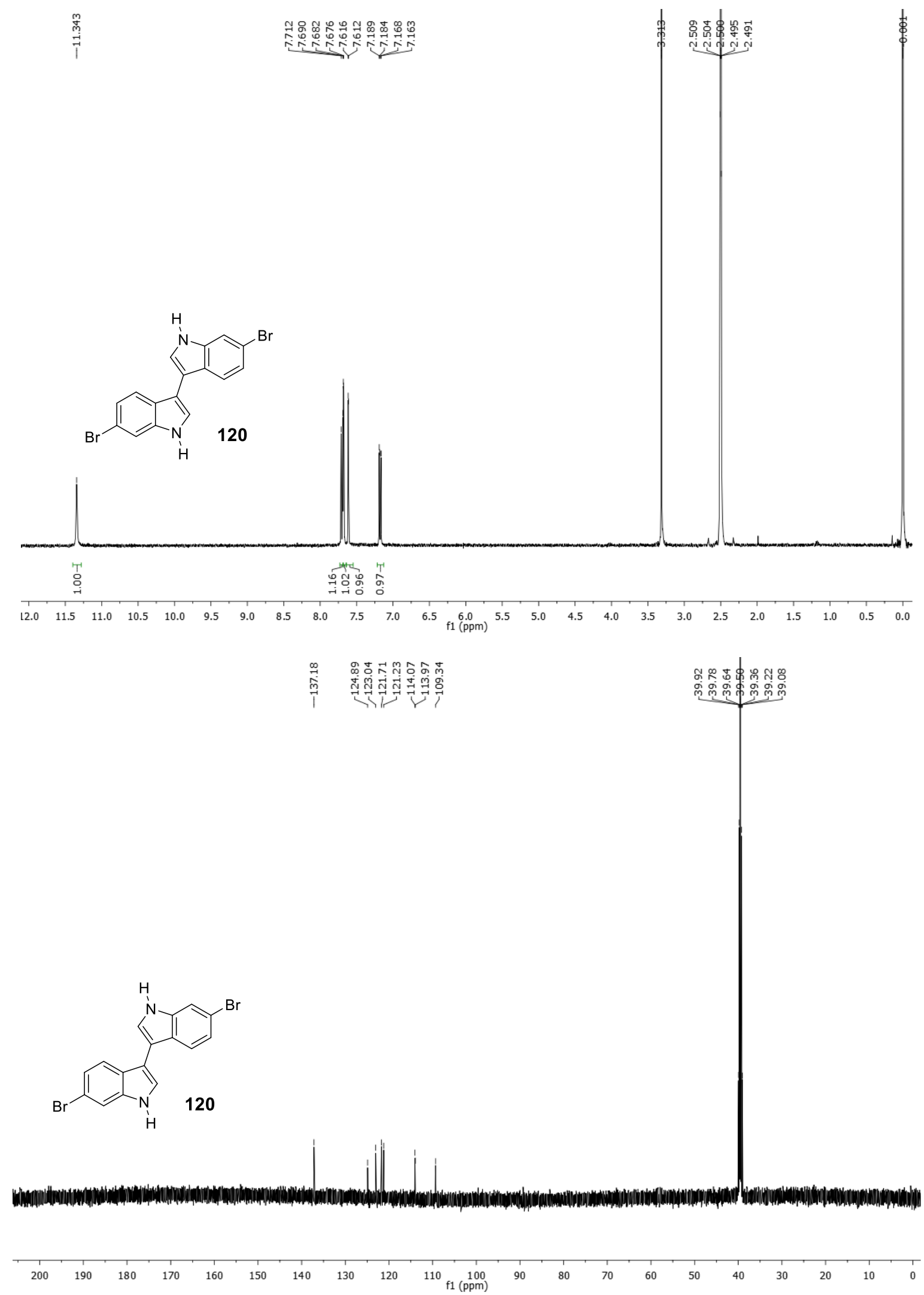

Figure $3.11{ }^{1} \mathrm{H}$ and ${ }^{13} \mathrm{C}$ NMR of compound $\mathbf{1 2 0}$ 


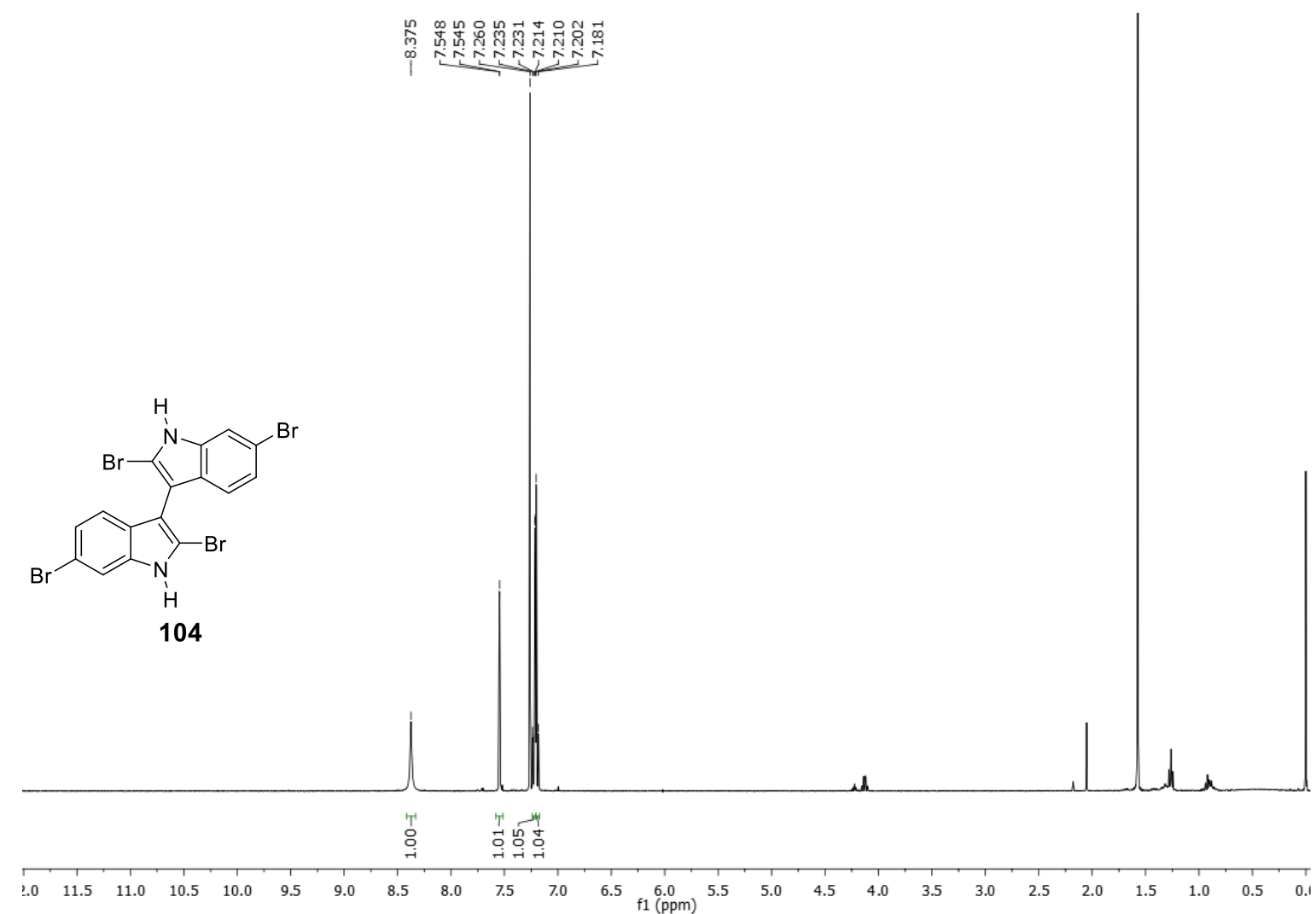

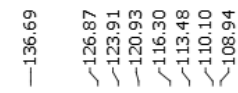
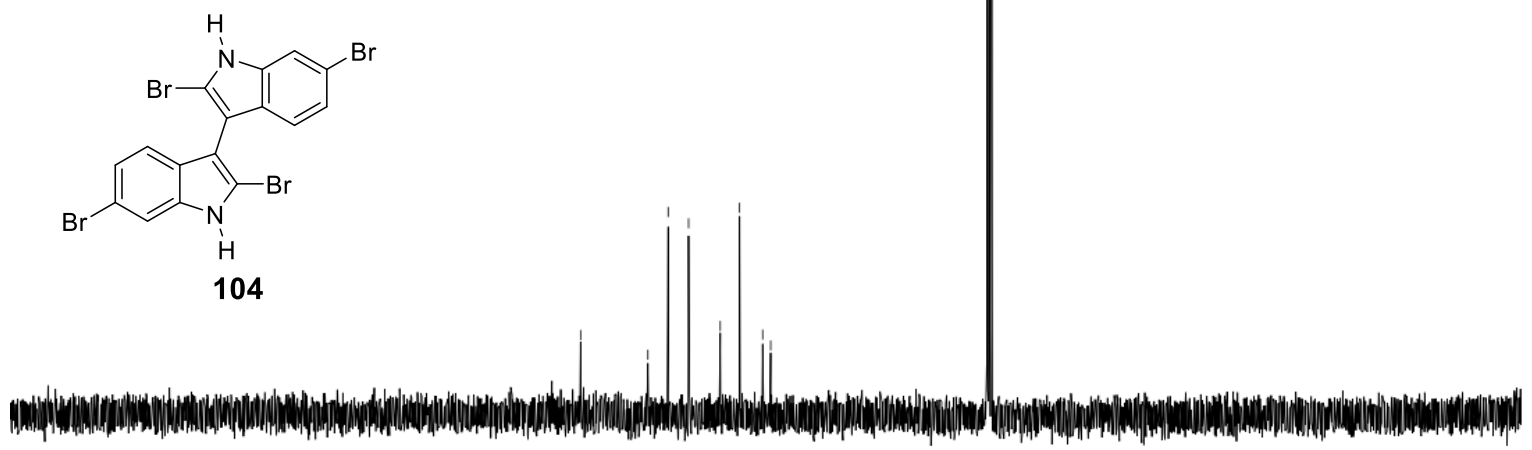

$\begin{array}{lllllllllllllllllllllllll}20 & 210 & 200 & 190 & 180 & 170 & 160 & 150 & 140 & 130 & 120 & \begin{array}{l}110 \\ \mathrm{f} 1(\mathrm{ppm})\end{array} & 100 & 90 & 80 & 70 & 60 & 50 & 40 & 30 & 20 & 10 & 0\end{array}$

Figure $3.12{ }^{1} \mathrm{H}$ and ${ }^{13} \mathrm{C}$ NMR of compound 104 

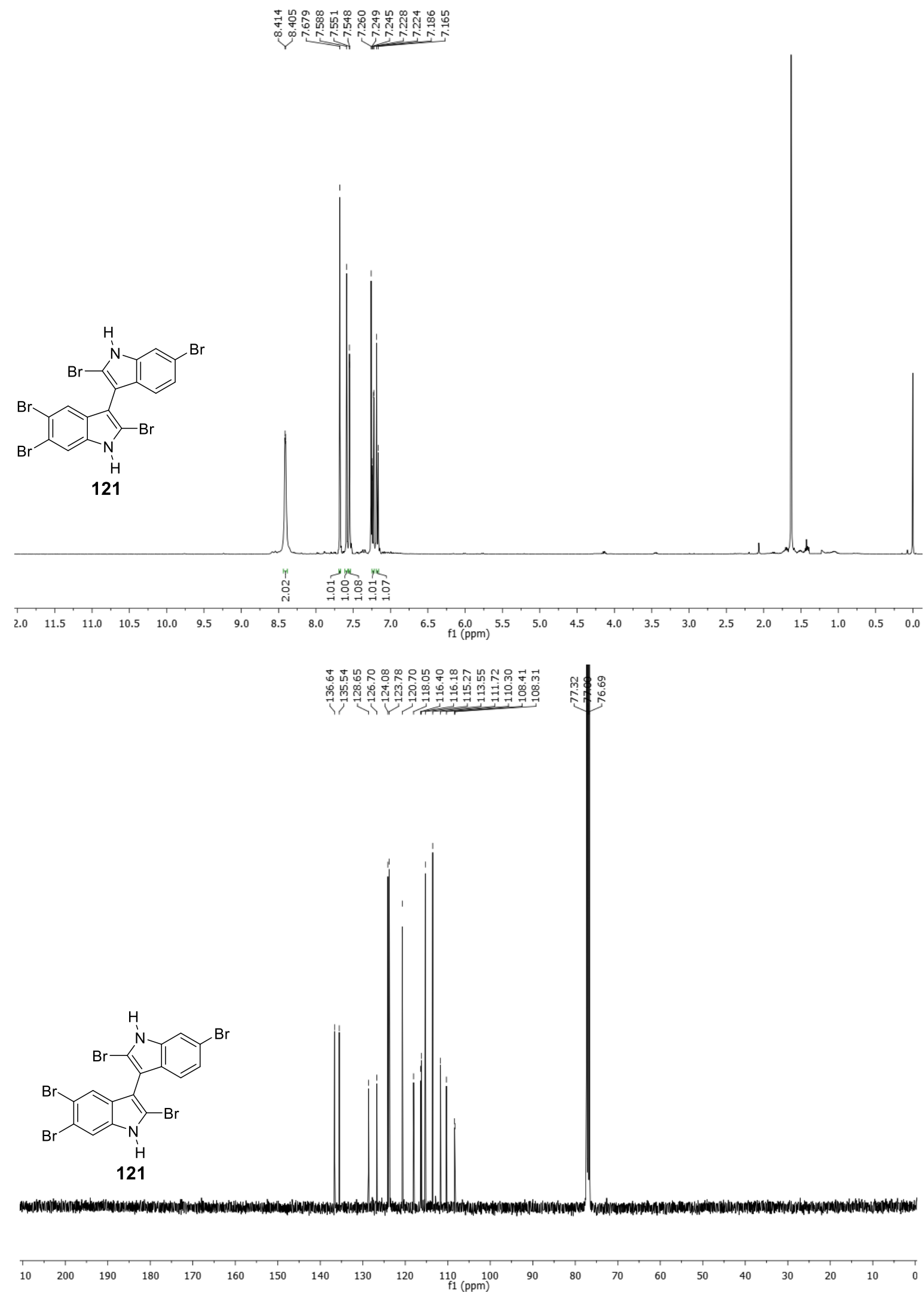

Figure 3.13 ${ }^{1} \mathrm{H}$ and ${ }^{13} \mathrm{C}$ NMR of compound 121 


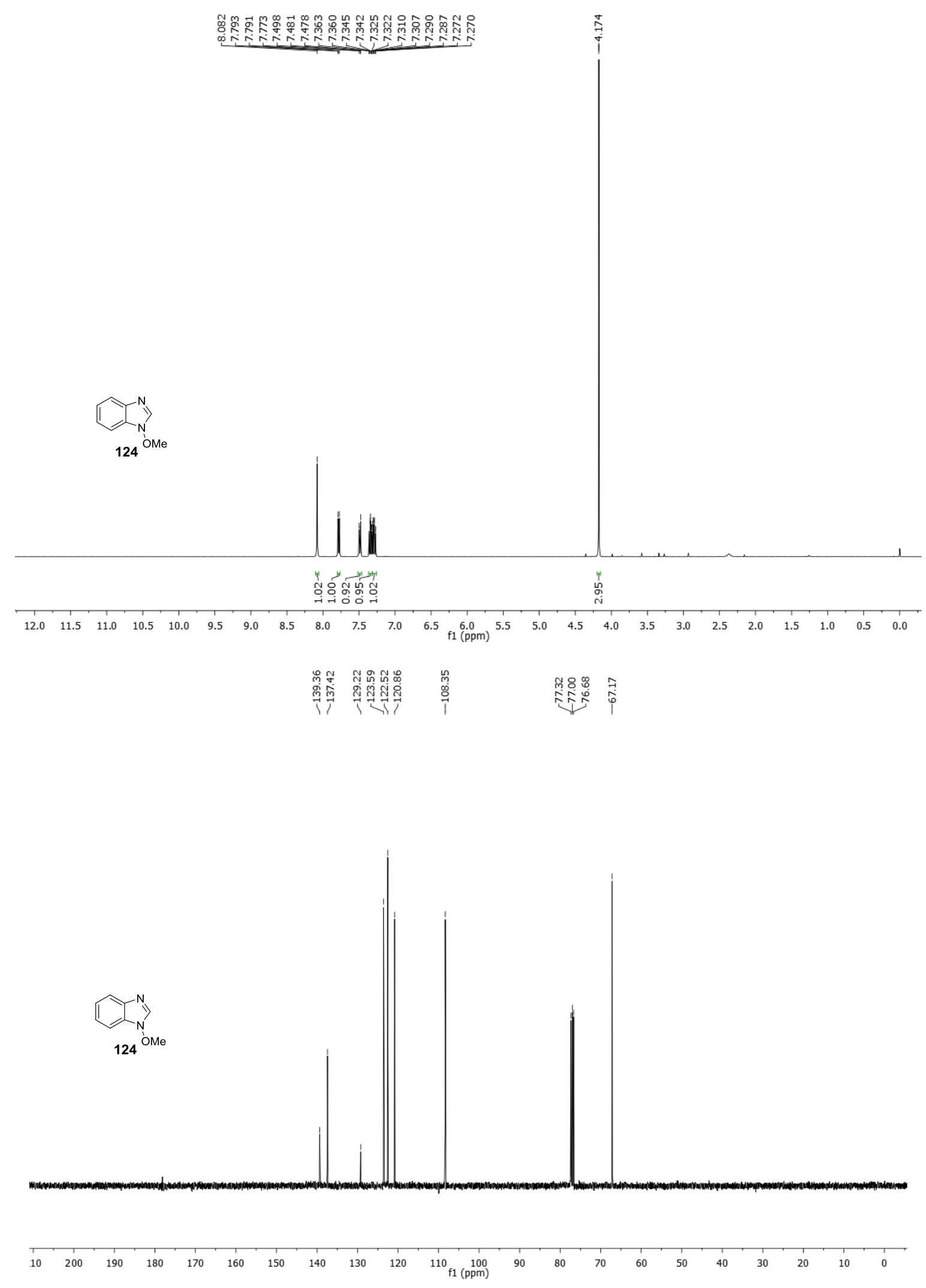

Figure 4.1 ${ }^{1} \mathrm{H}$ and ${ }^{13} \mathrm{C}$ NMR of compound 124 

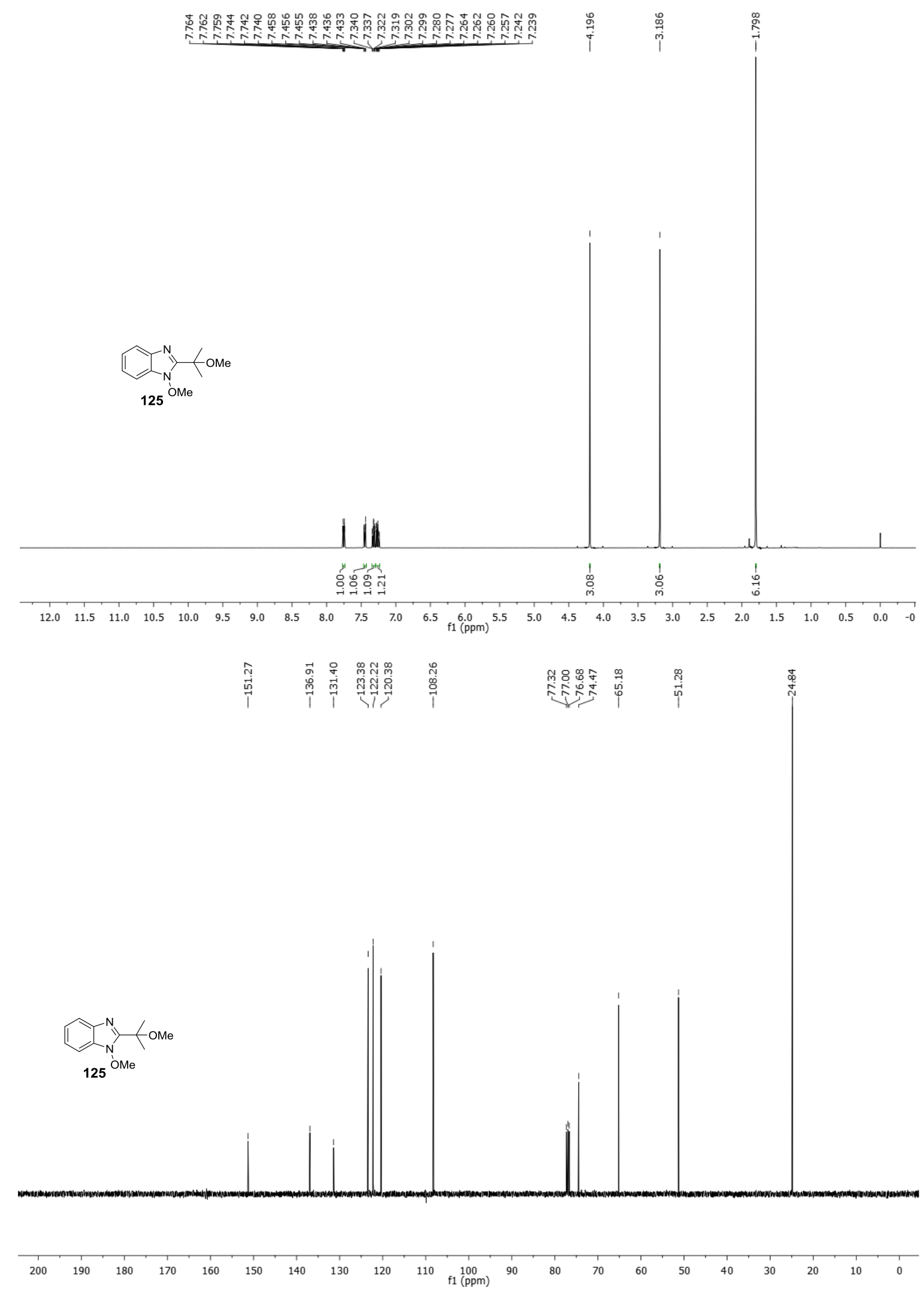

Figure 4.2 ${ }^{1} \mathrm{H}$ and ${ }^{13} \mathrm{C}$ NMR of compound 125 

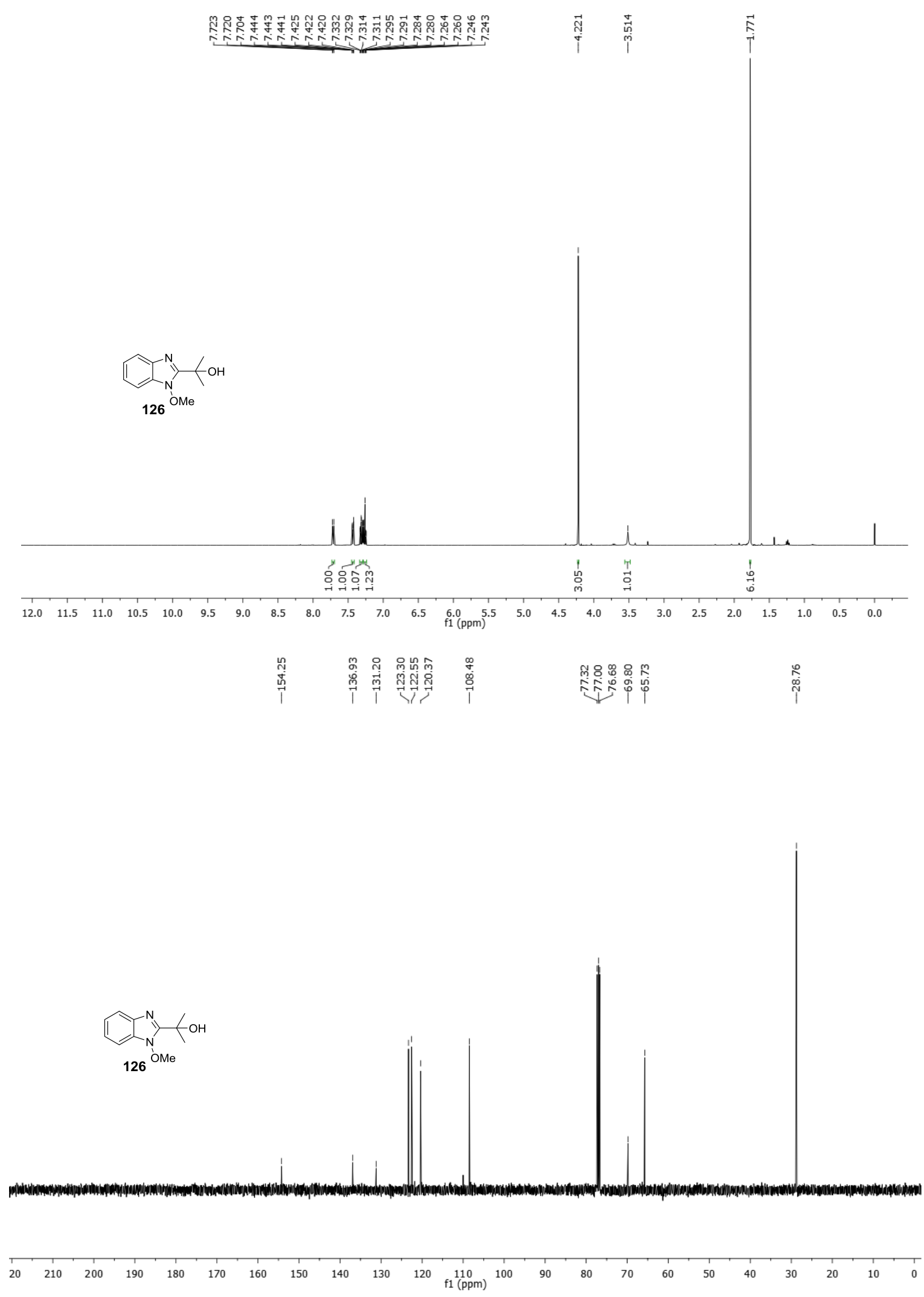

Figure $4.3{ }^{1} \mathrm{H}$ and ${ }^{13} \mathrm{C}$ NMR of compound 125 

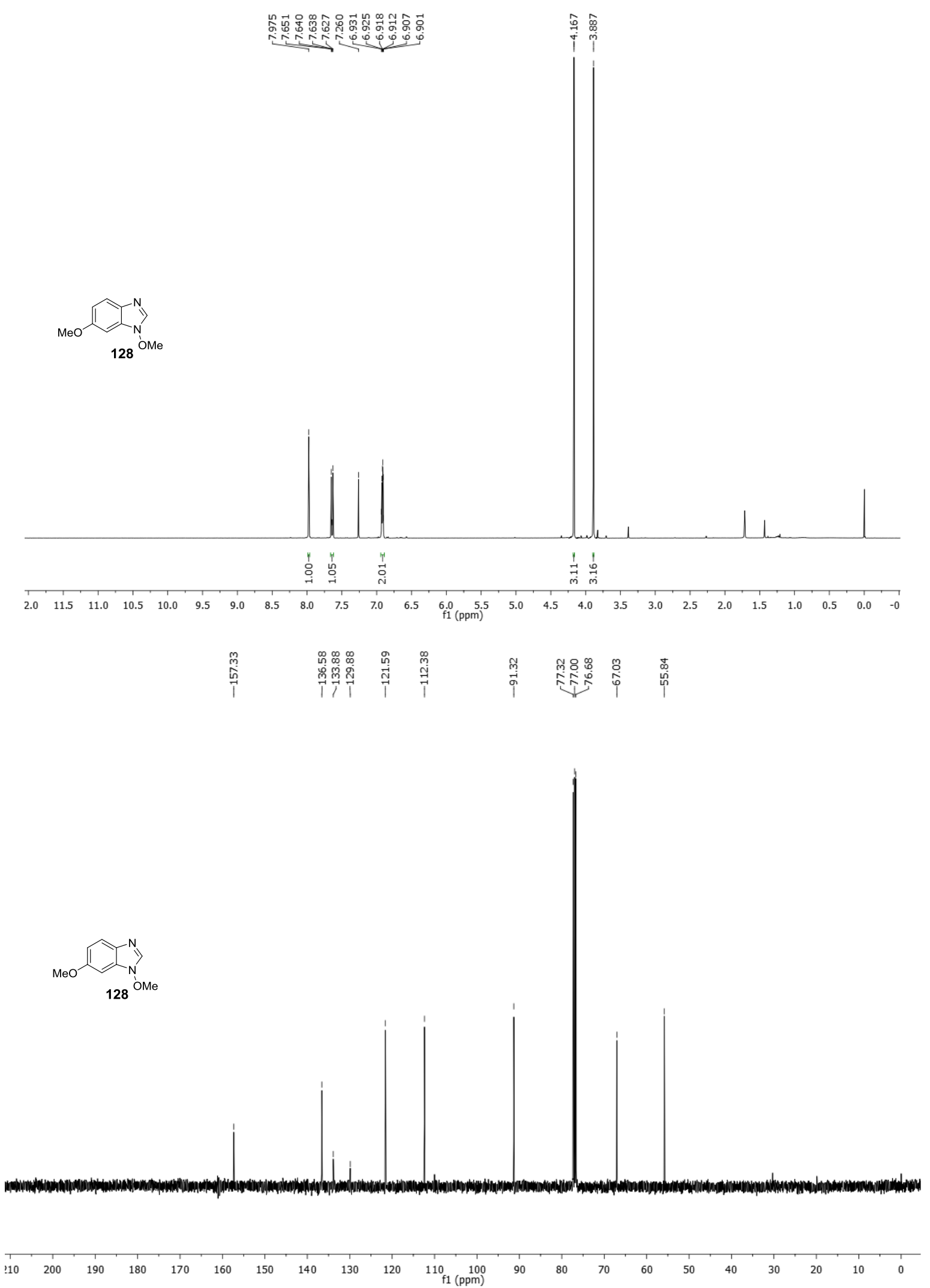

Figure 4.4 ${ }^{1} \mathrm{H}$ and ${ }^{13} \mathrm{C}$ NMR of compound 128 

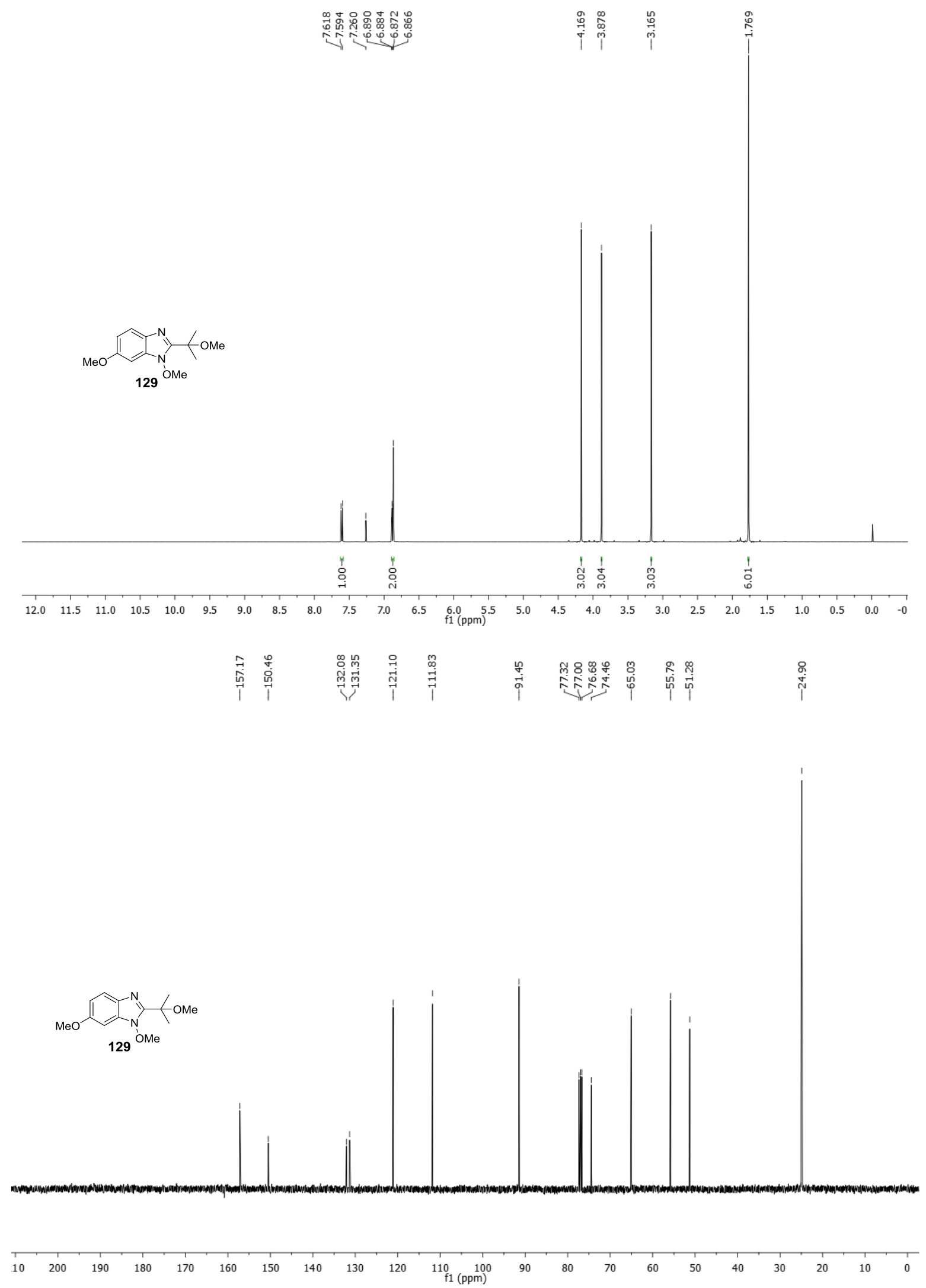

Figure $4.5{ }^{1} \mathrm{H}$ and ${ }^{13} \mathrm{C}$ NMR of compound 129 


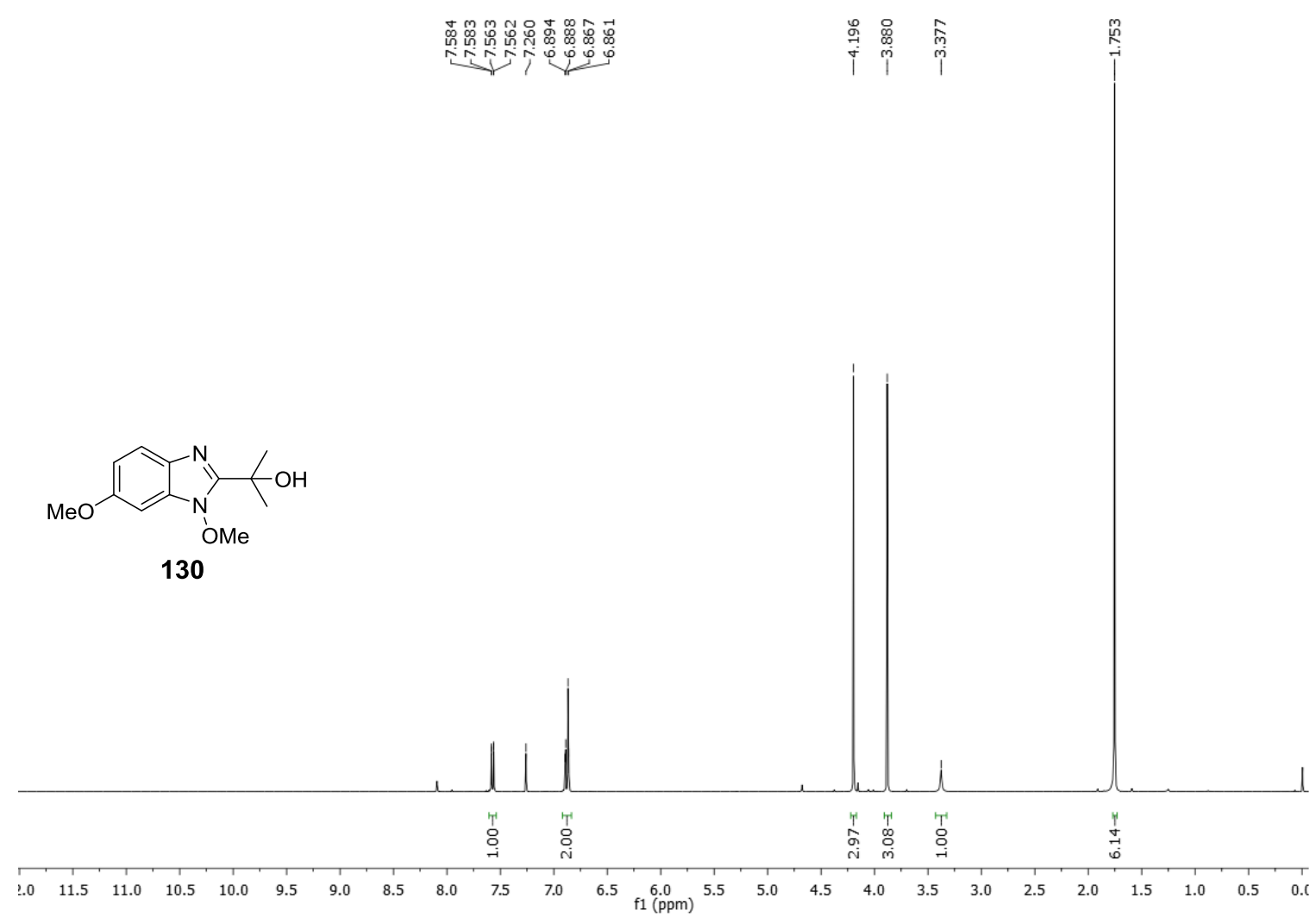

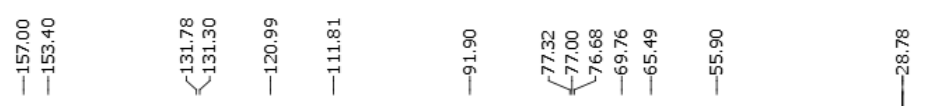

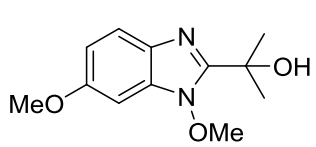

130

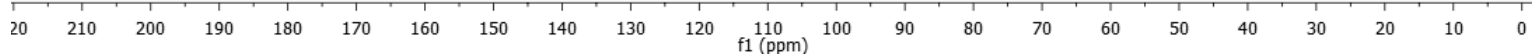

Figure 4.6 ${ }^{1} \mathrm{H}$ and ${ }^{13} \mathrm{C}$ NMR of compound 130 


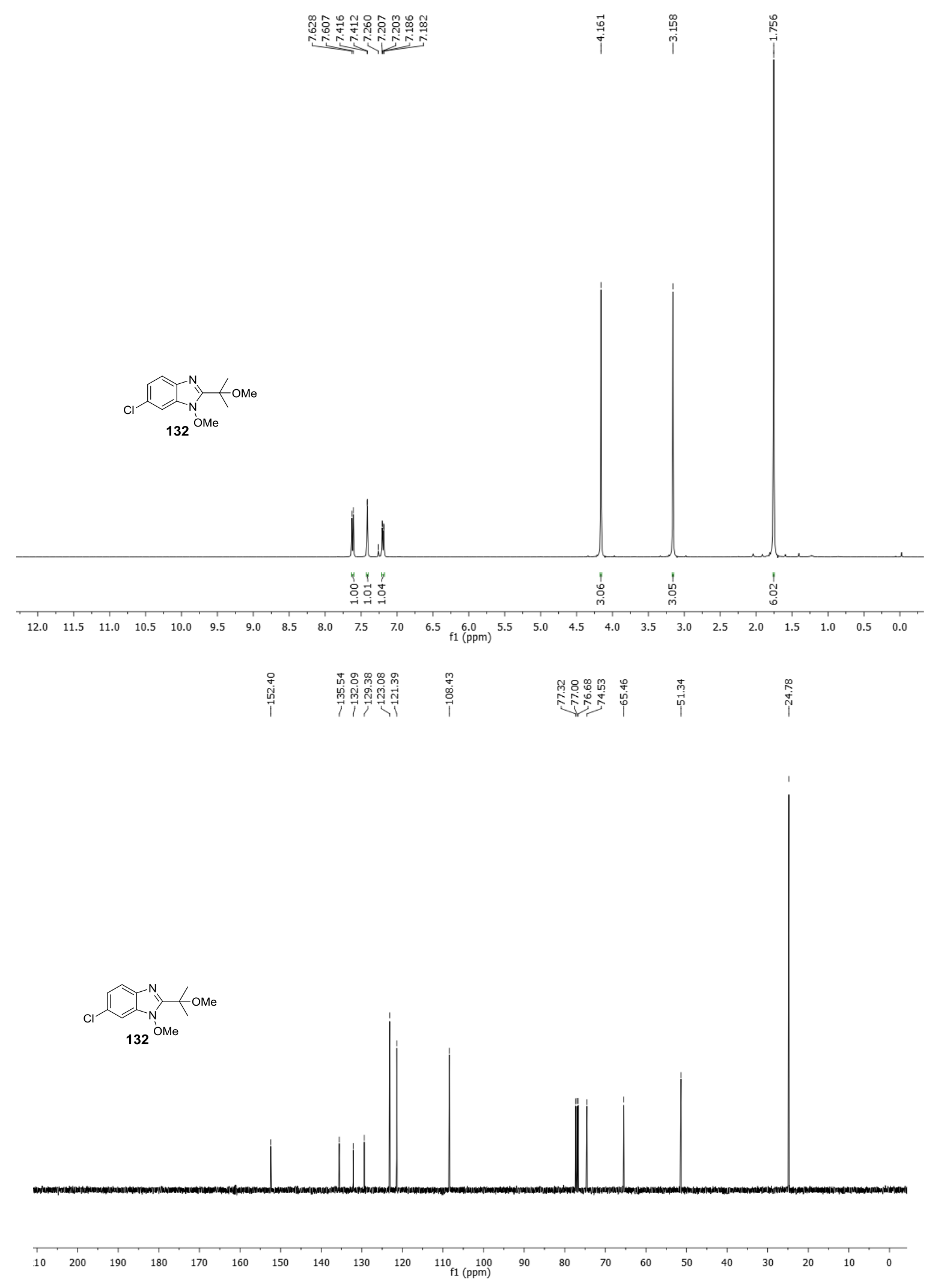

Figure $4.7{ }^{1} \mathrm{H}$ and ${ }^{13} \mathrm{C}$ NMR of compound 132 


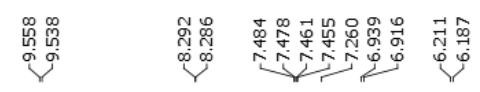
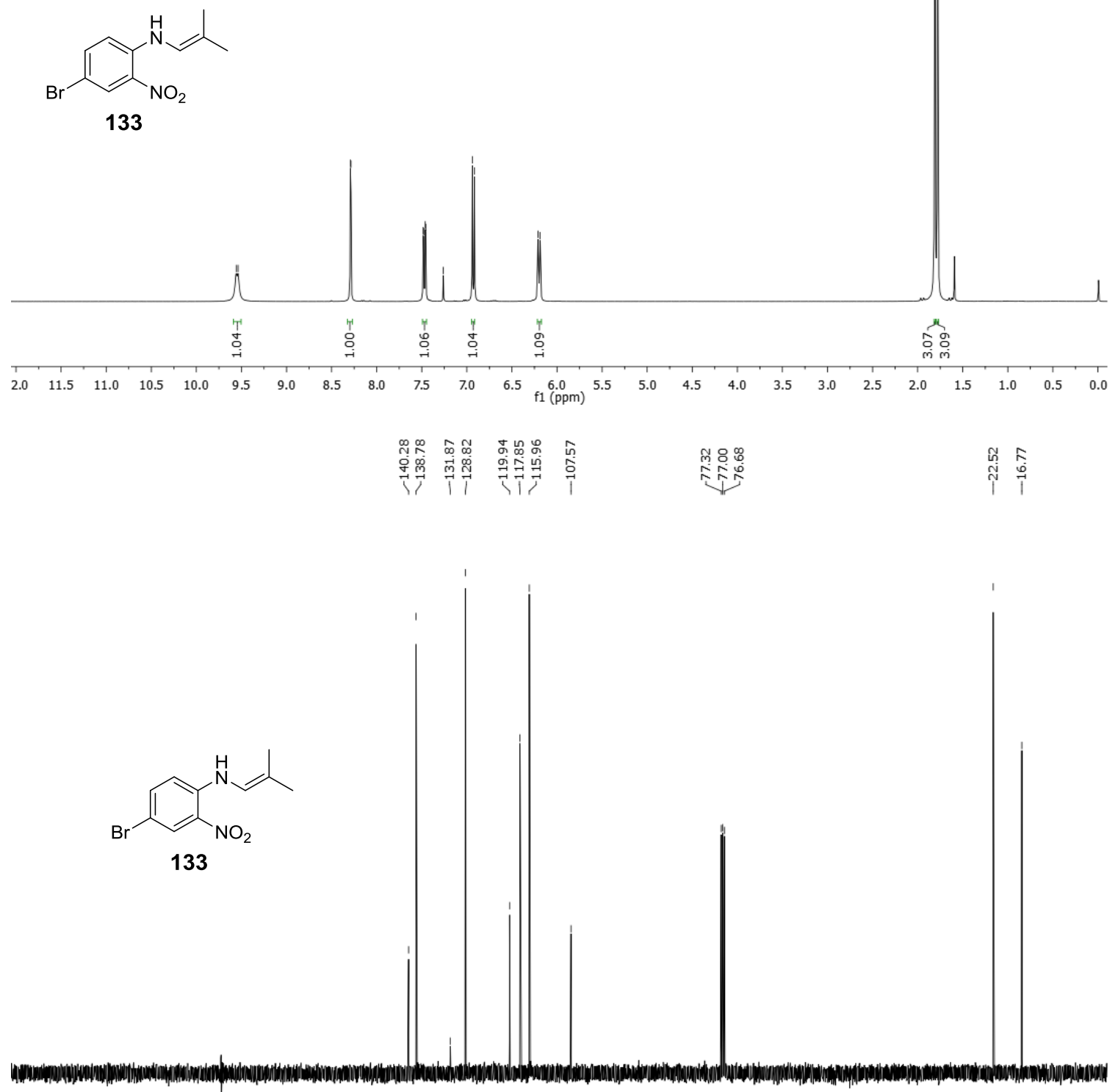

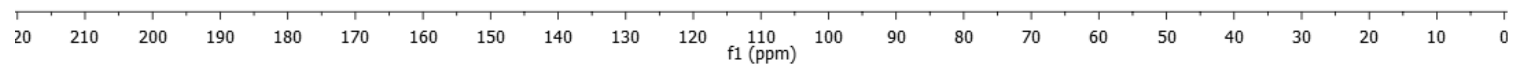

Figure $4.8{ }^{1} \mathrm{H}$ and ${ }^{13} \mathrm{C}$ NMR of compound 133 

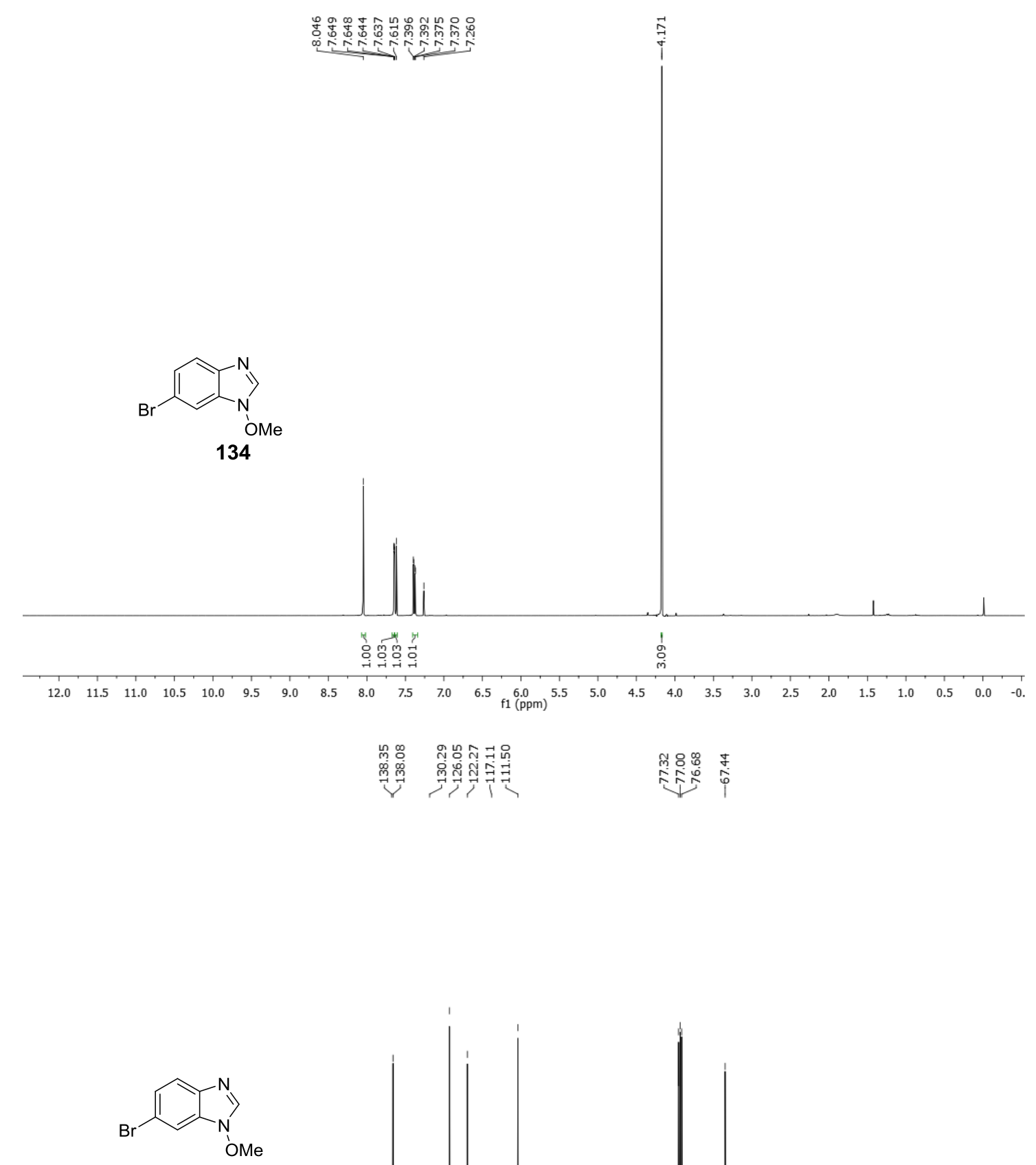
134

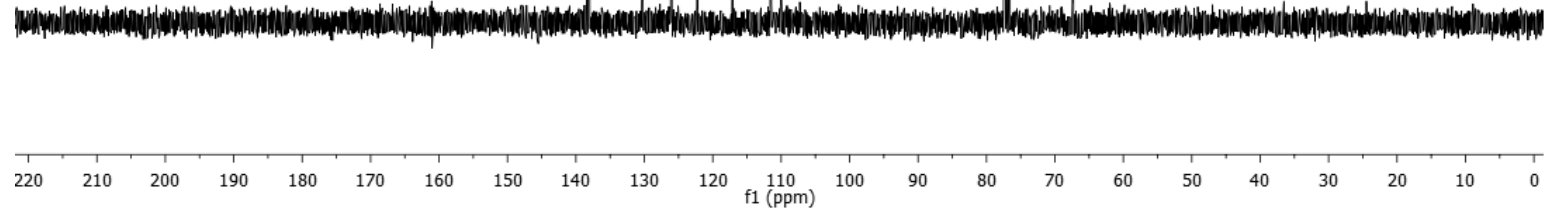

Figure 4.9 ${ }^{1} \mathrm{H}$ and ${ }^{13} \mathrm{C}$ NMR of compound 134 

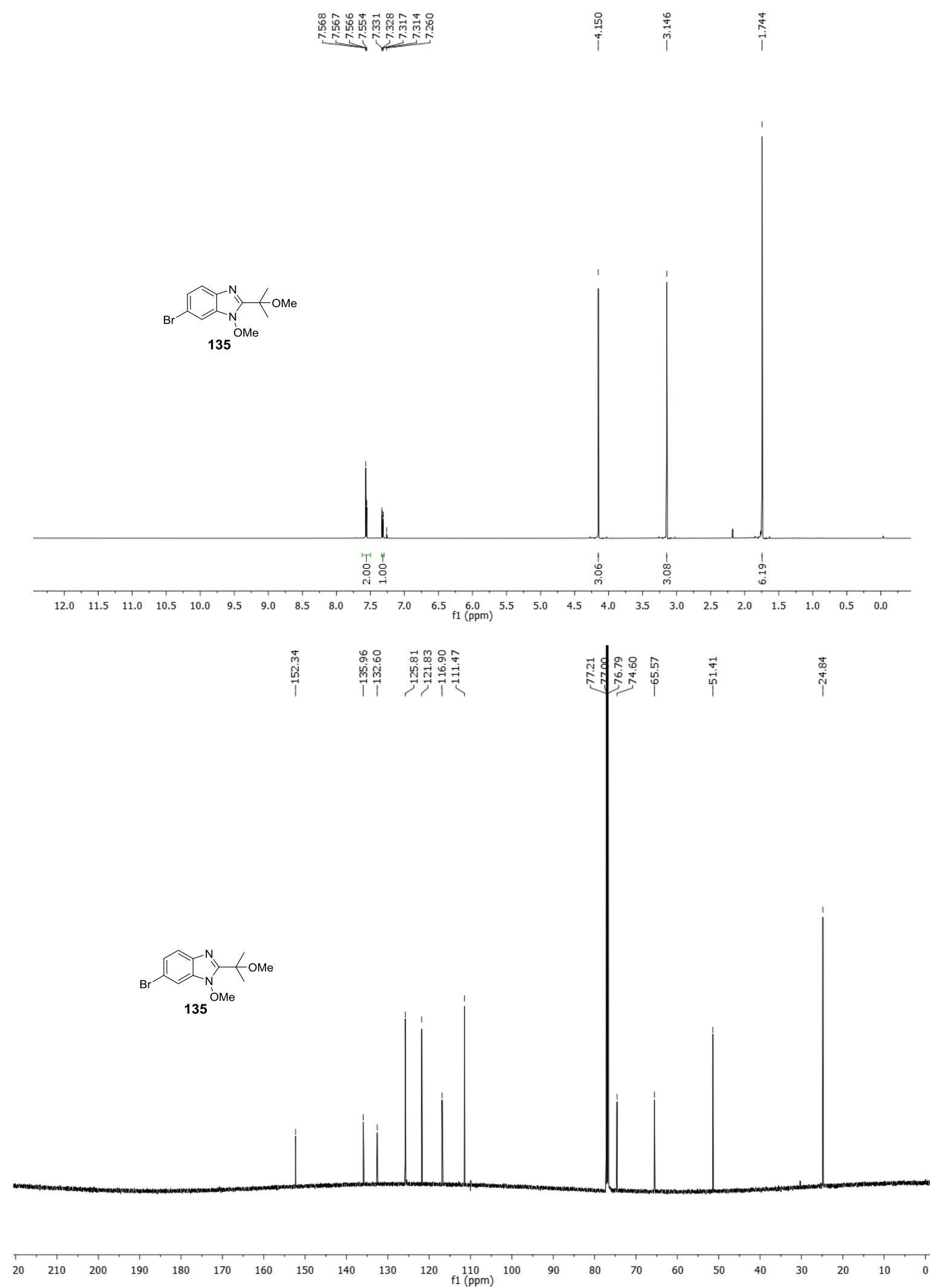

Figure 4.10 ${ }^{1} \mathrm{H}$ and ${ }^{13} \mathrm{C}$ NMR of compound 135 


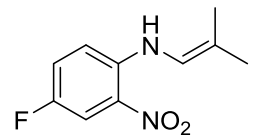

136

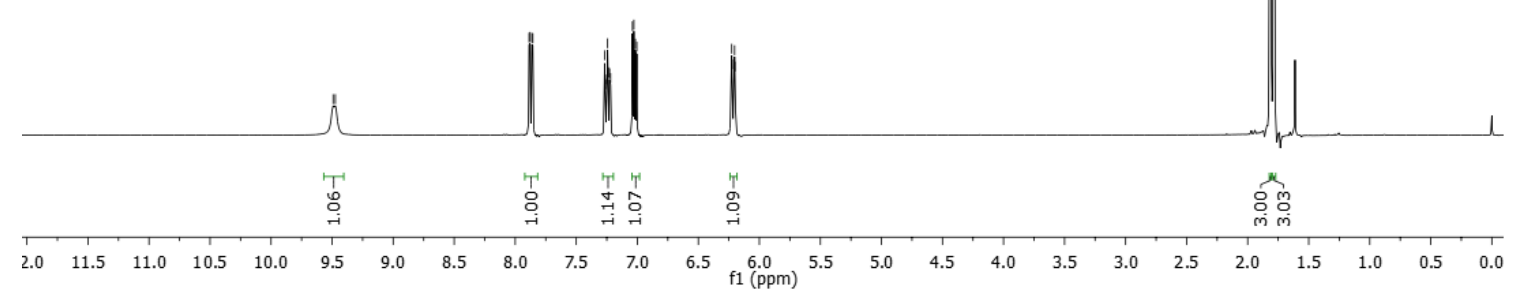

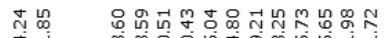

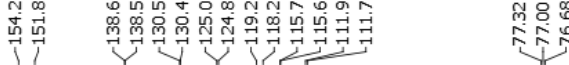

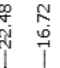<smiles>CC(C)=CNc1ccc(F)cc1[N+](=O)[O-]</smiles>

136

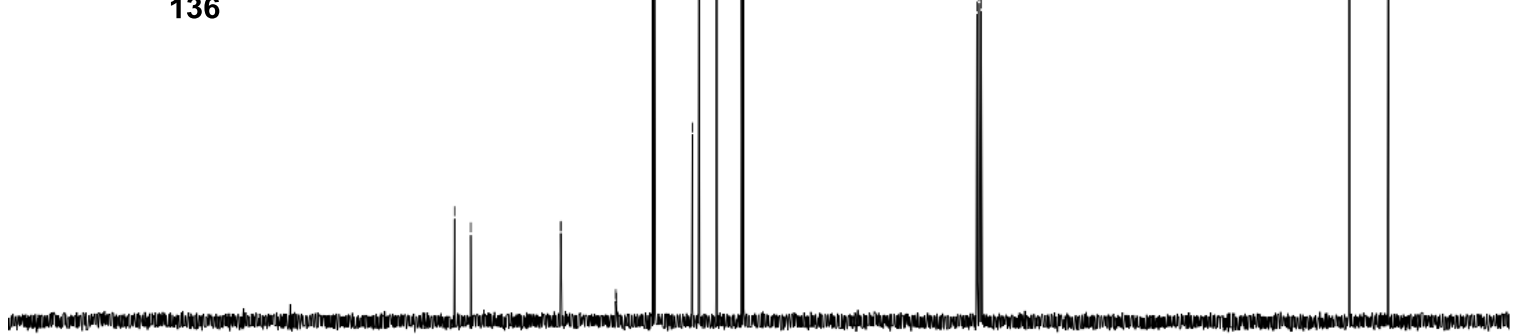

$\begin{array}{lllllllllllllllllllllllll}20 & 210 & 200 & 190 & 180 & 170 & 160 & 150 & 140 & 130 & 120 & 110 & 100 & 90 & 80 & 70 & 60 & 50 & 40 & 30 & 20 & 10 & 0\end{array}$

Figure 4.11 ${ }^{1} \mathrm{H}$ and ${ }^{13} \mathrm{C}$ NMR of compound 136 

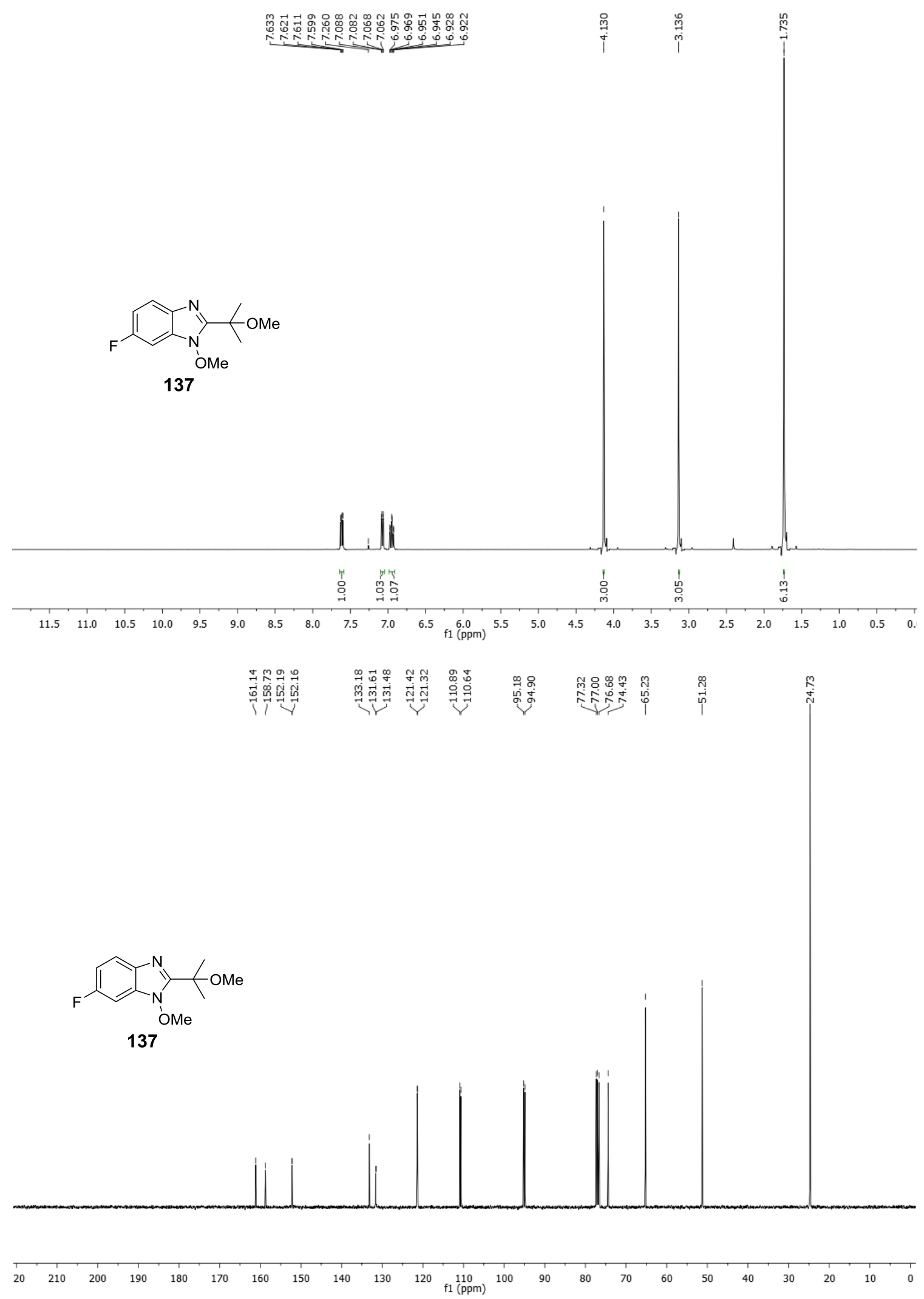

Figure 4.12 ${ }^{1} \mathrm{H}$ and ${ }^{13} \mathrm{C}$ NMR of compound 137 

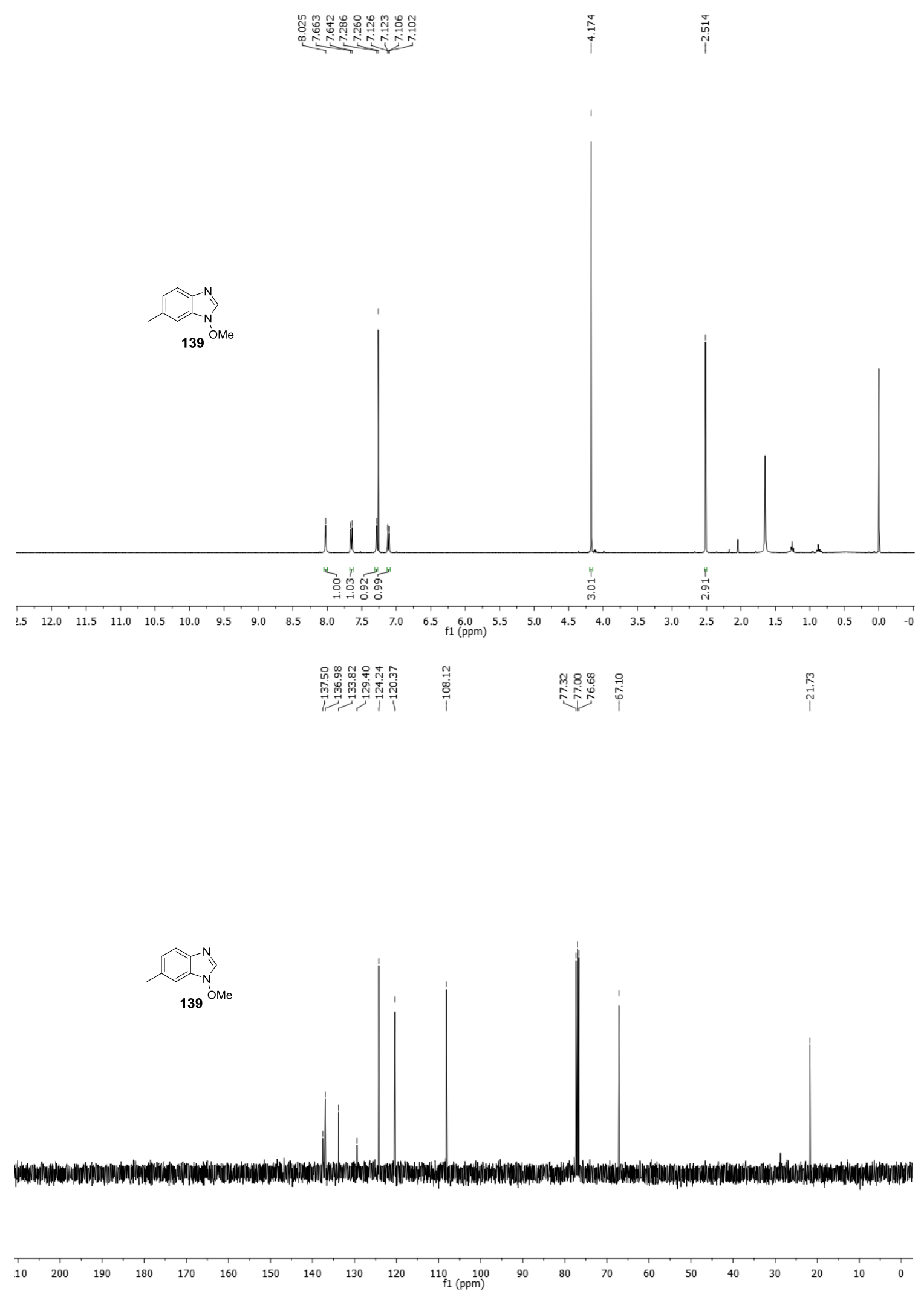

Figure 4.13 ${ }^{1} \mathrm{H}$ and ${ }^{13} \mathrm{C}$ NMR of compound 139 

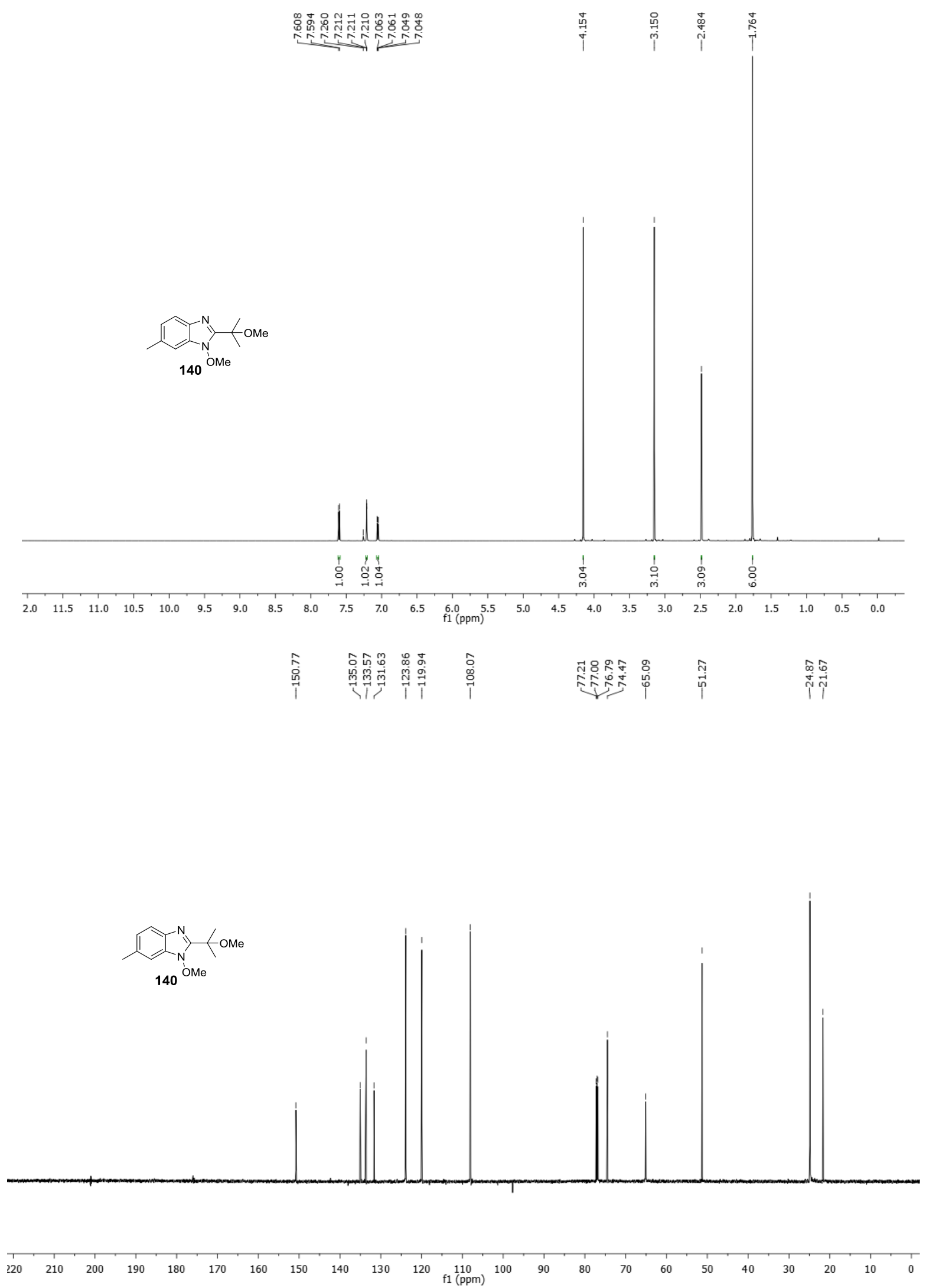

Figure 4.14 ${ }^{1} \mathrm{H}$ and ${ }^{13} \mathrm{C}$ NMR of compound 140 


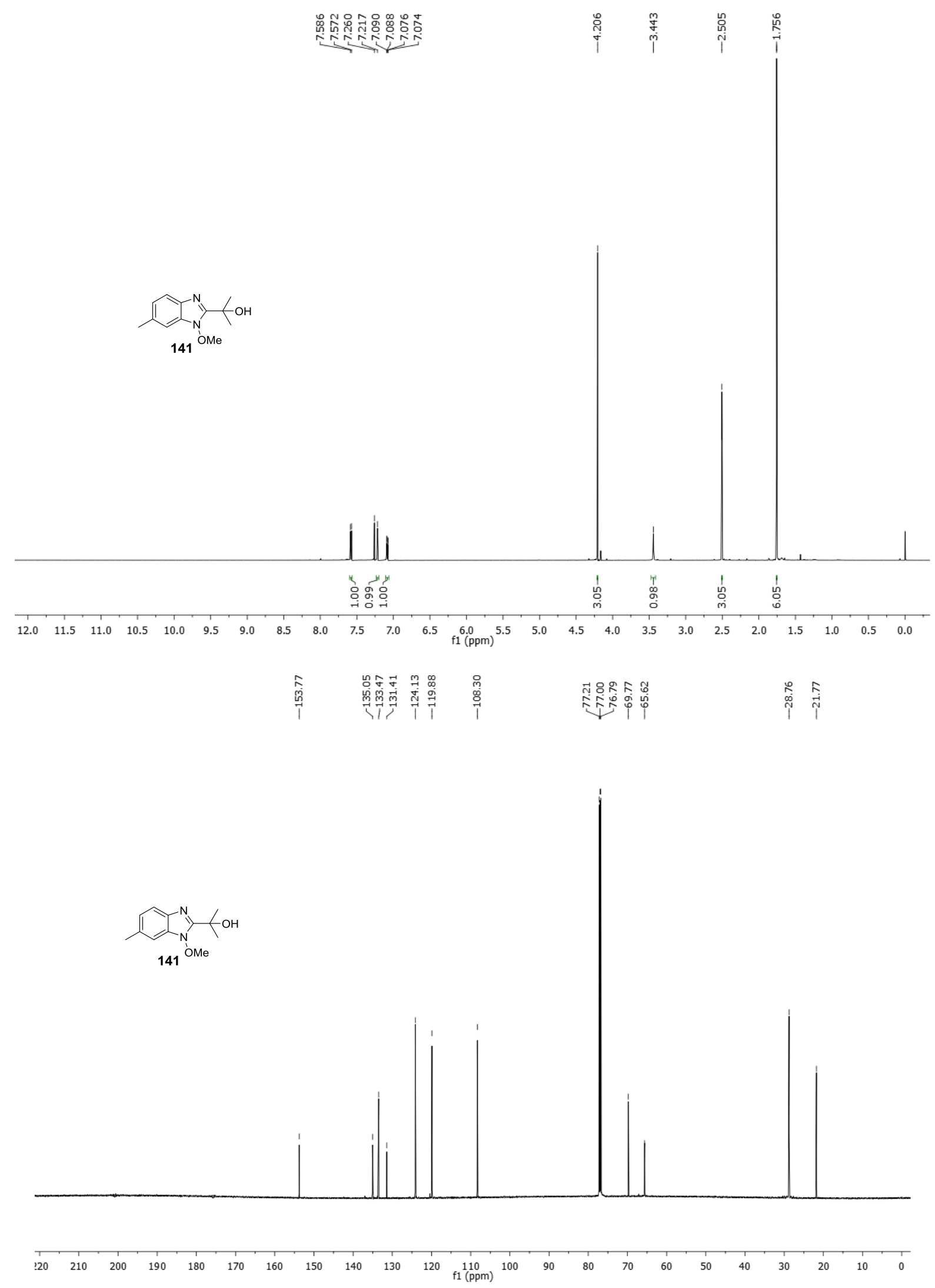

Figure 4.15 ${ }^{1} \mathrm{H}$ and ${ }^{13} \mathrm{C}$ NMR of compound 141 


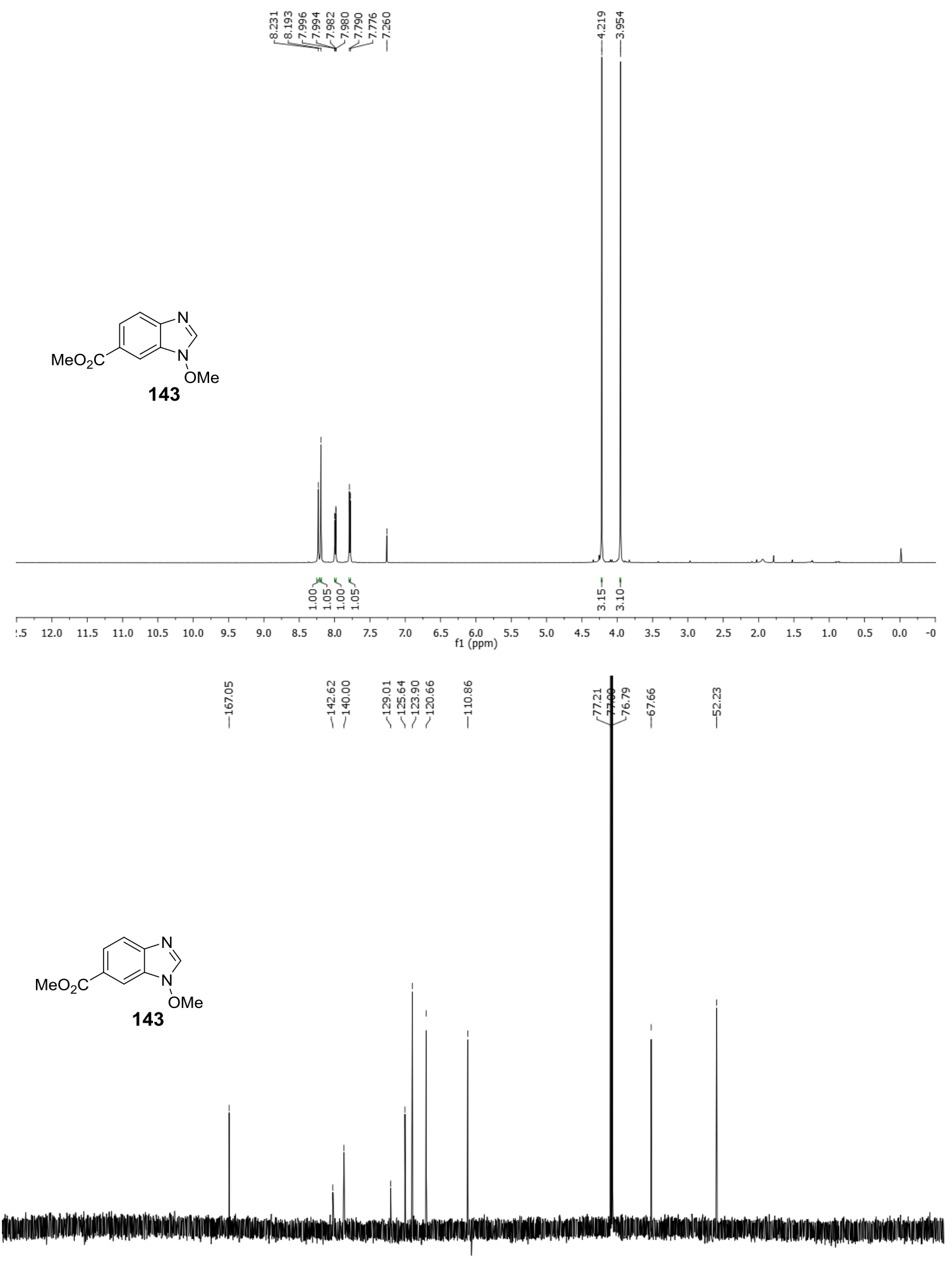

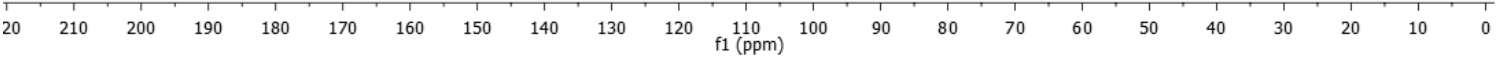

Figure 4.16 ${ }^{1} \mathrm{H}$ and ${ }^{13} \mathrm{C}$ NMR of compound 143 


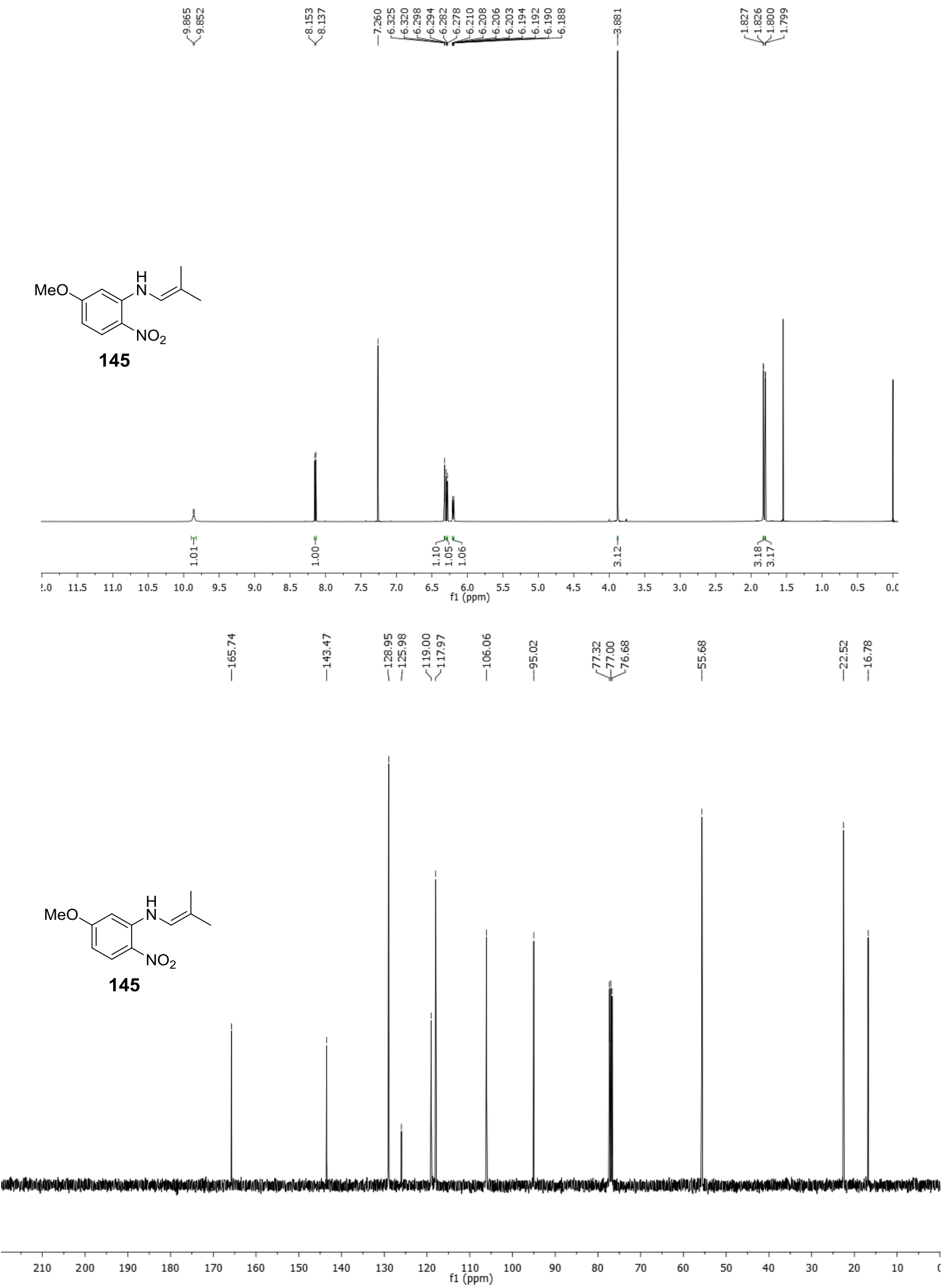

Figure 4.17 ${ }^{1} \mathrm{H}$ and ${ }^{13} \mathrm{C}$ NMR of compound $\mathbf{1 4 5}$ 


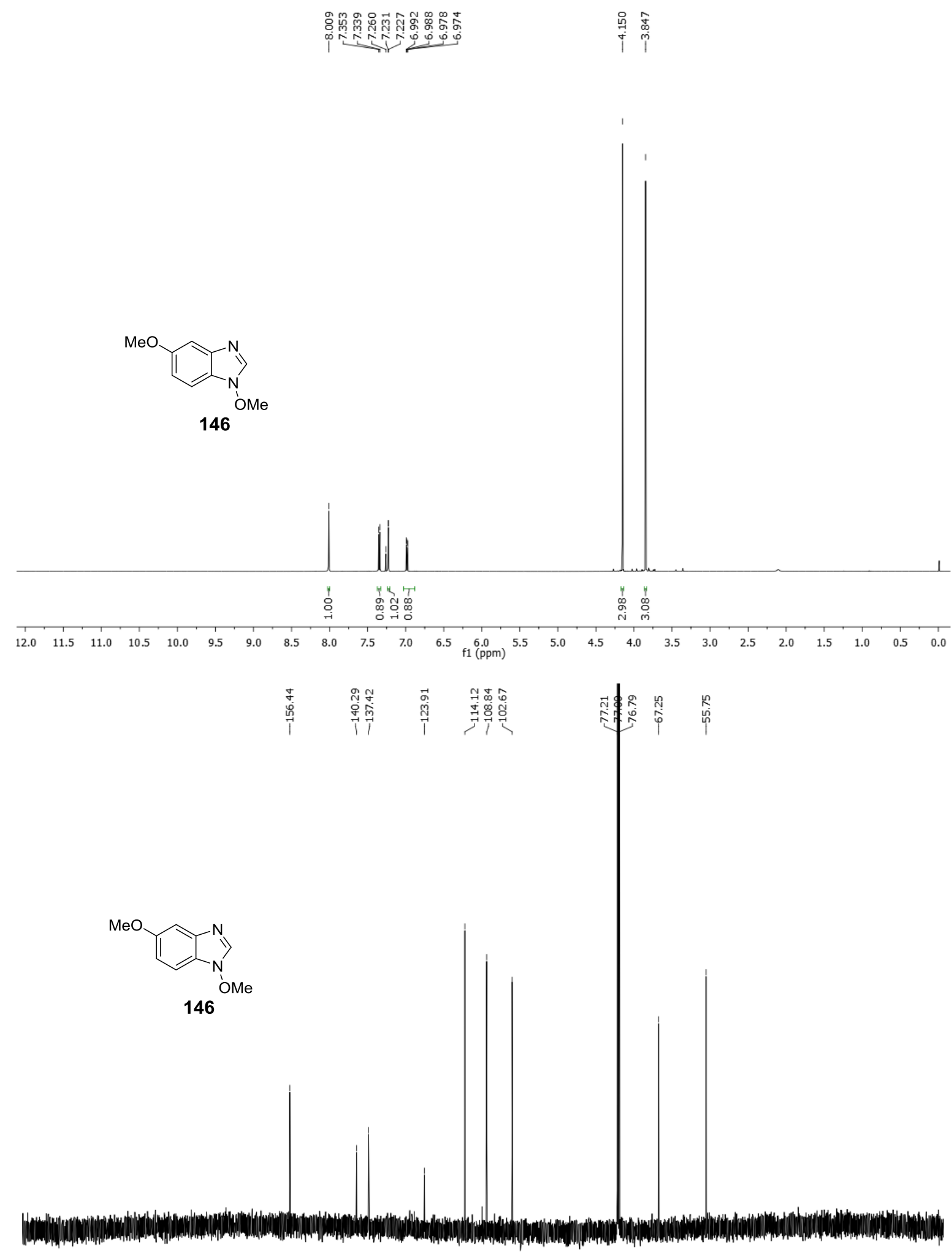

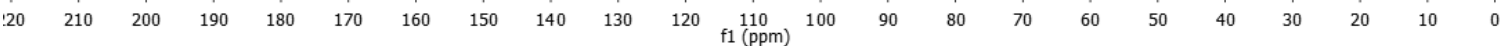

Figure 4.18 ${ }^{1} \mathrm{H}$ and ${ }^{13} \mathrm{C}$ NMR of compound 146 


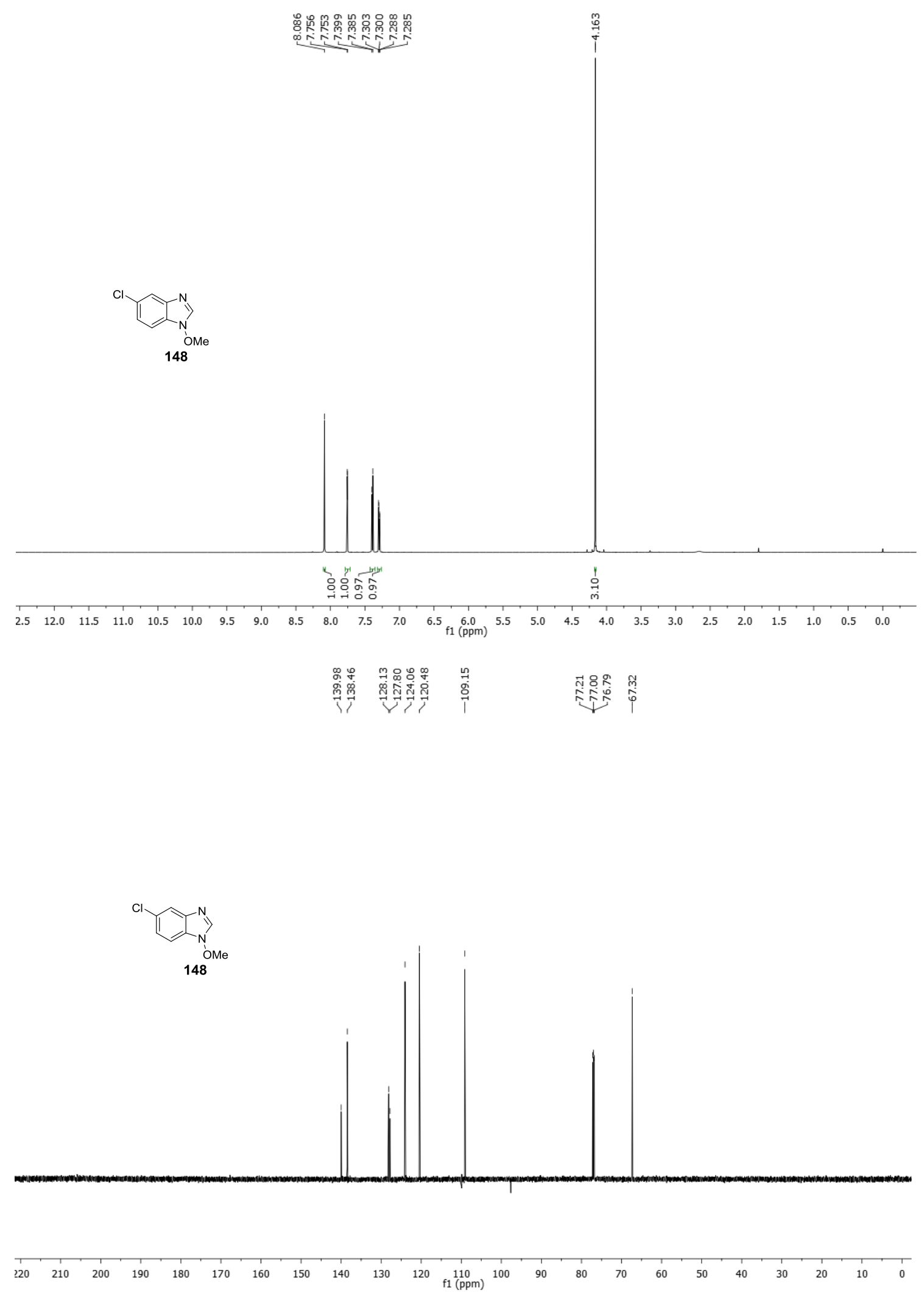

Figure 4.19 ${ }^{1} \mathrm{H}$ and ${ }^{13} \mathrm{C}$ NMR of compound 148 

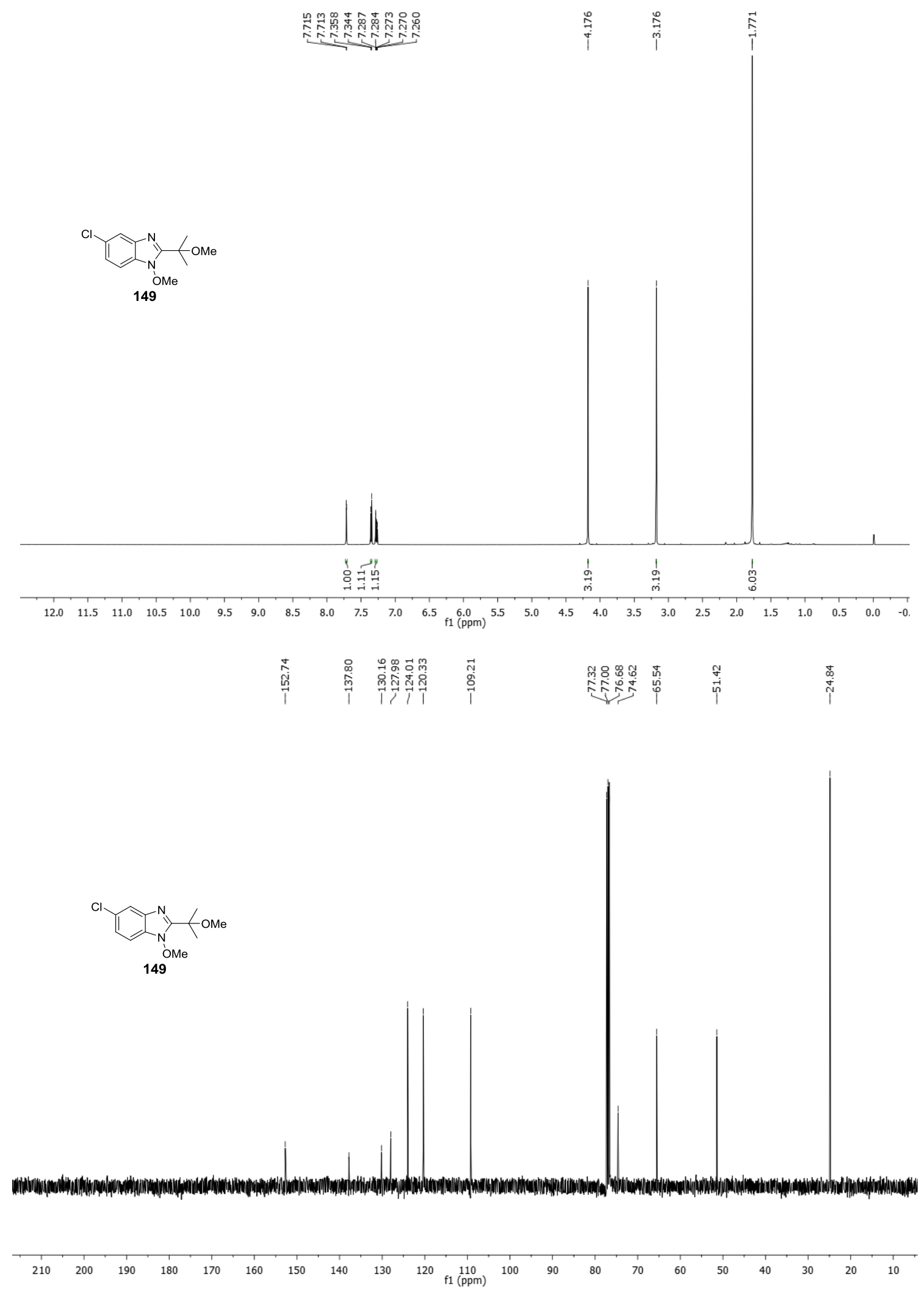

Figure 4.20 ${ }^{1} \mathrm{H}$ and ${ }^{13} \mathrm{C}$ NMR of compound 149 


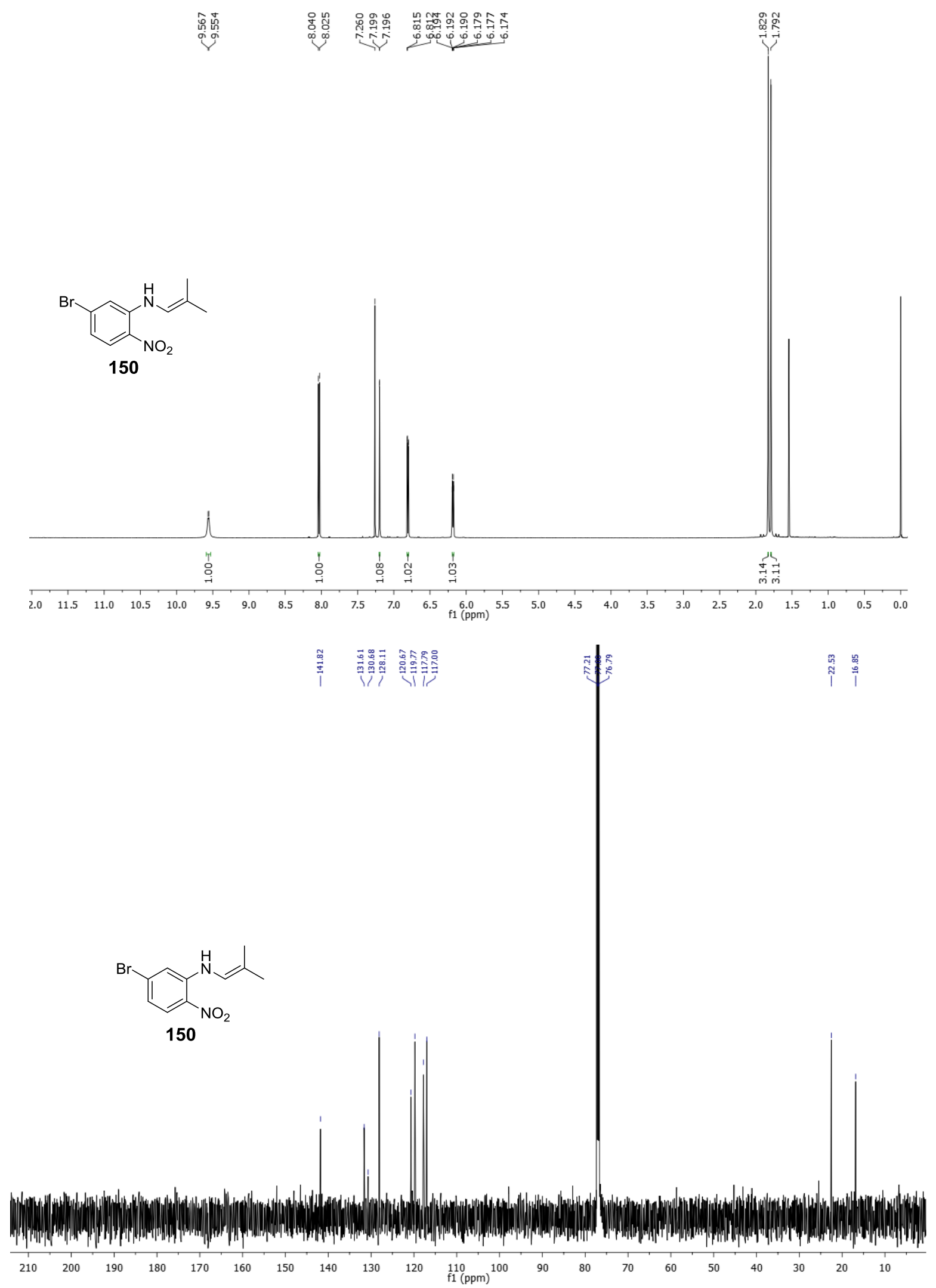

Figure 4.21 ${ }^{1} \mathrm{H}$ and ${ }^{13} \mathrm{C}$ NMR of compound 150 


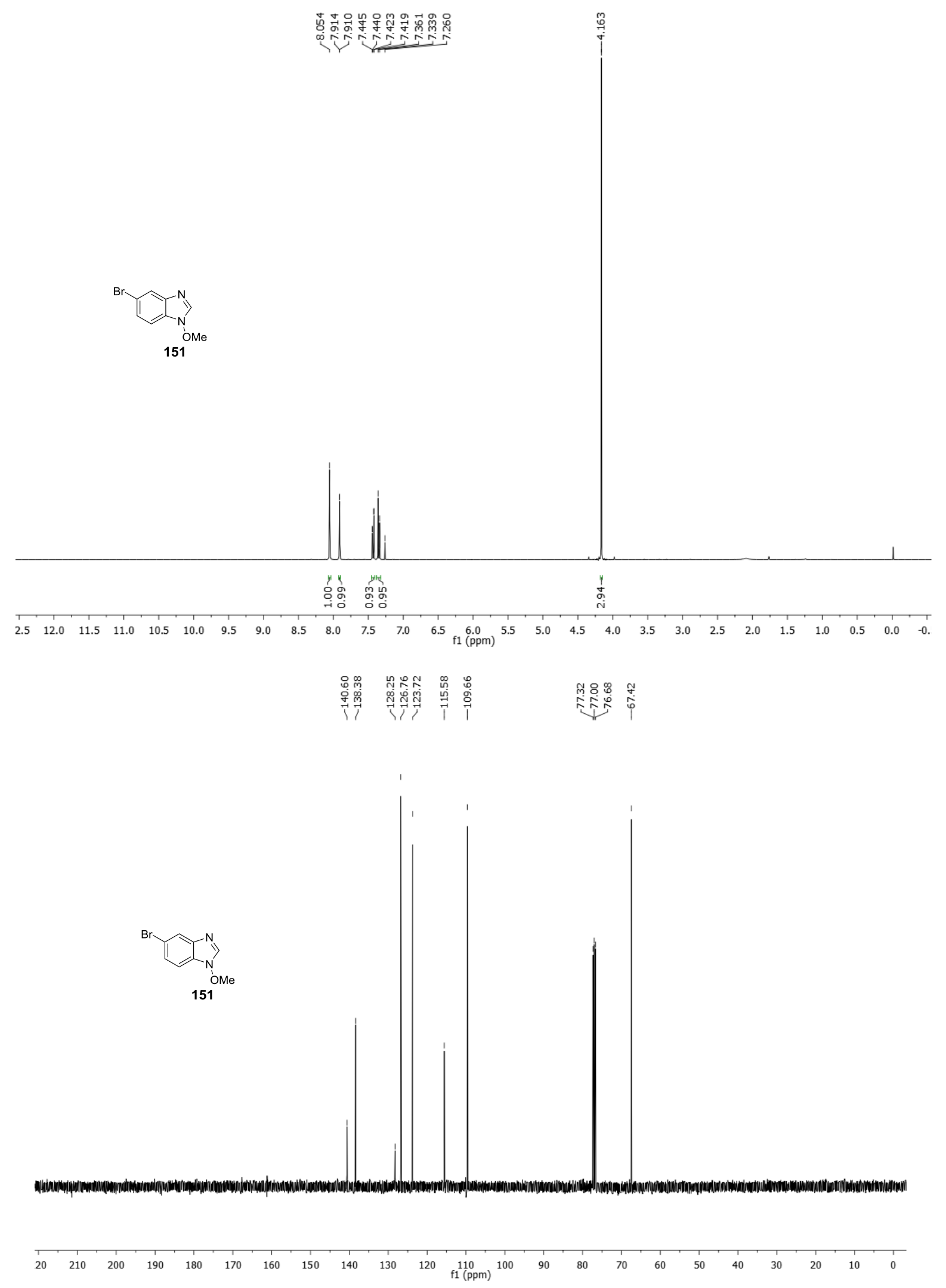

Figure 4.22 ${ }^{1} \mathrm{H}$ and ${ }^{13} \mathrm{C}$ NMR of compound 151 

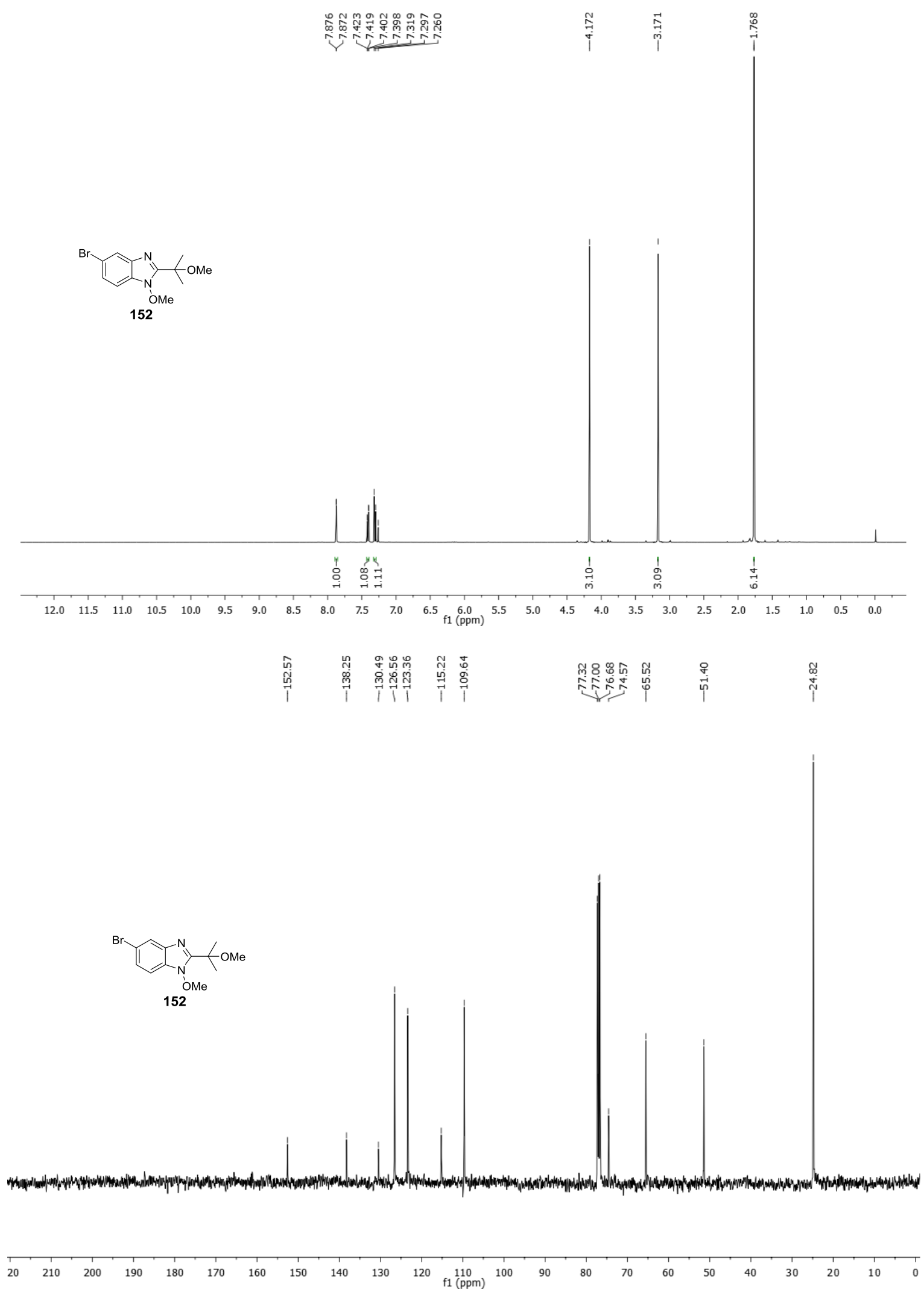

Figure 4.23 ${ }^{1} \mathrm{H}$ and ${ }^{13} \mathrm{C}$ NMR of compound 152 

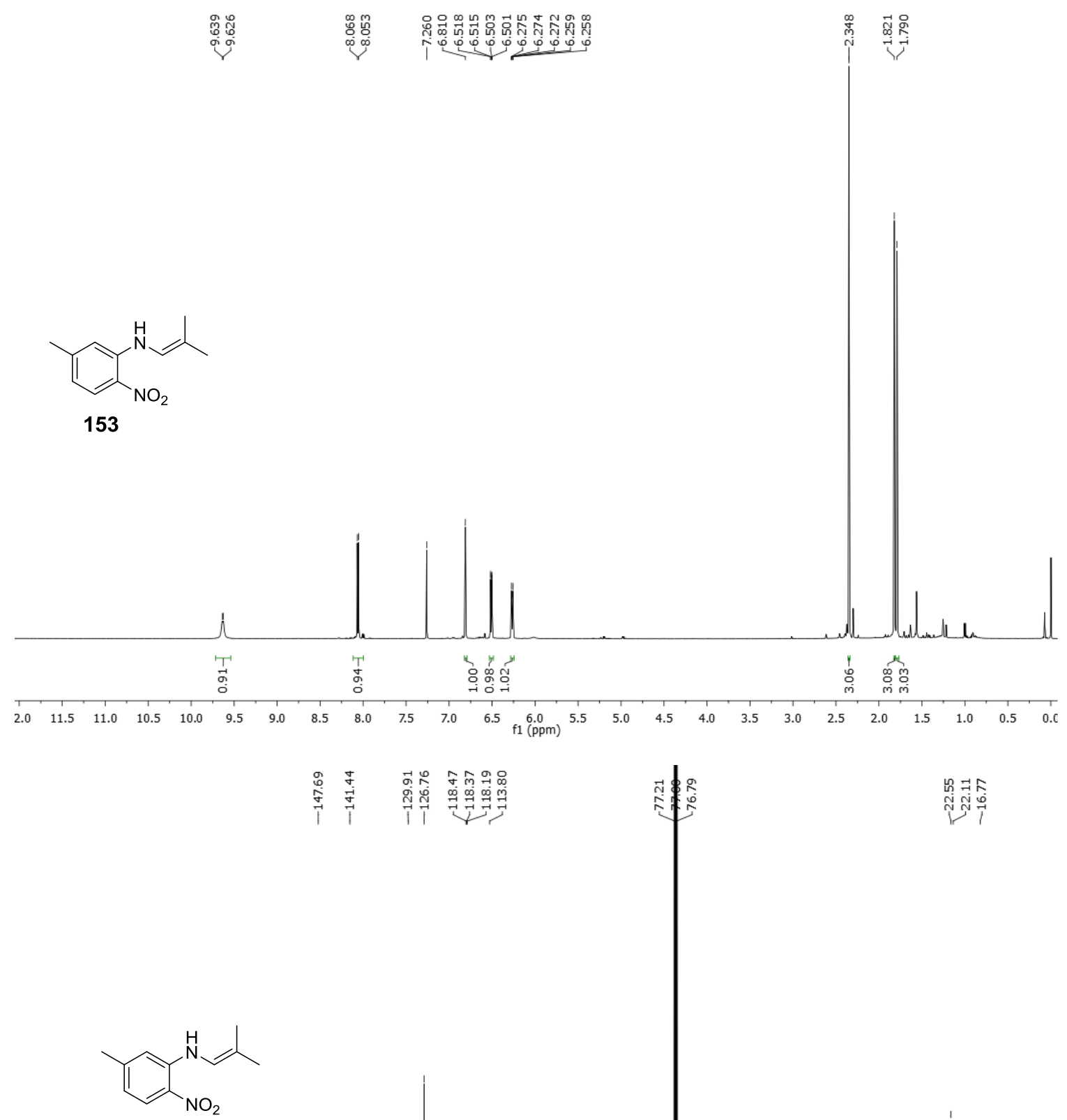

153

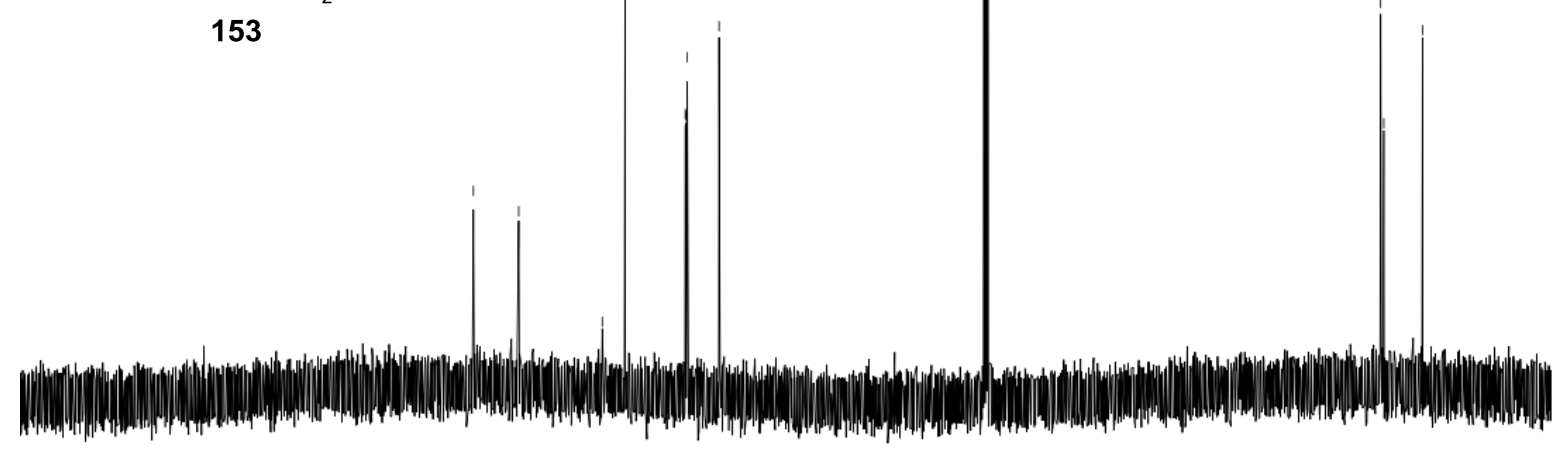

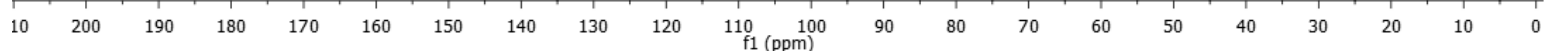

Figure 4.24 ${ }^{1} \mathrm{H}$ and ${ }^{13} \mathrm{C}$ NMR of compound 153 

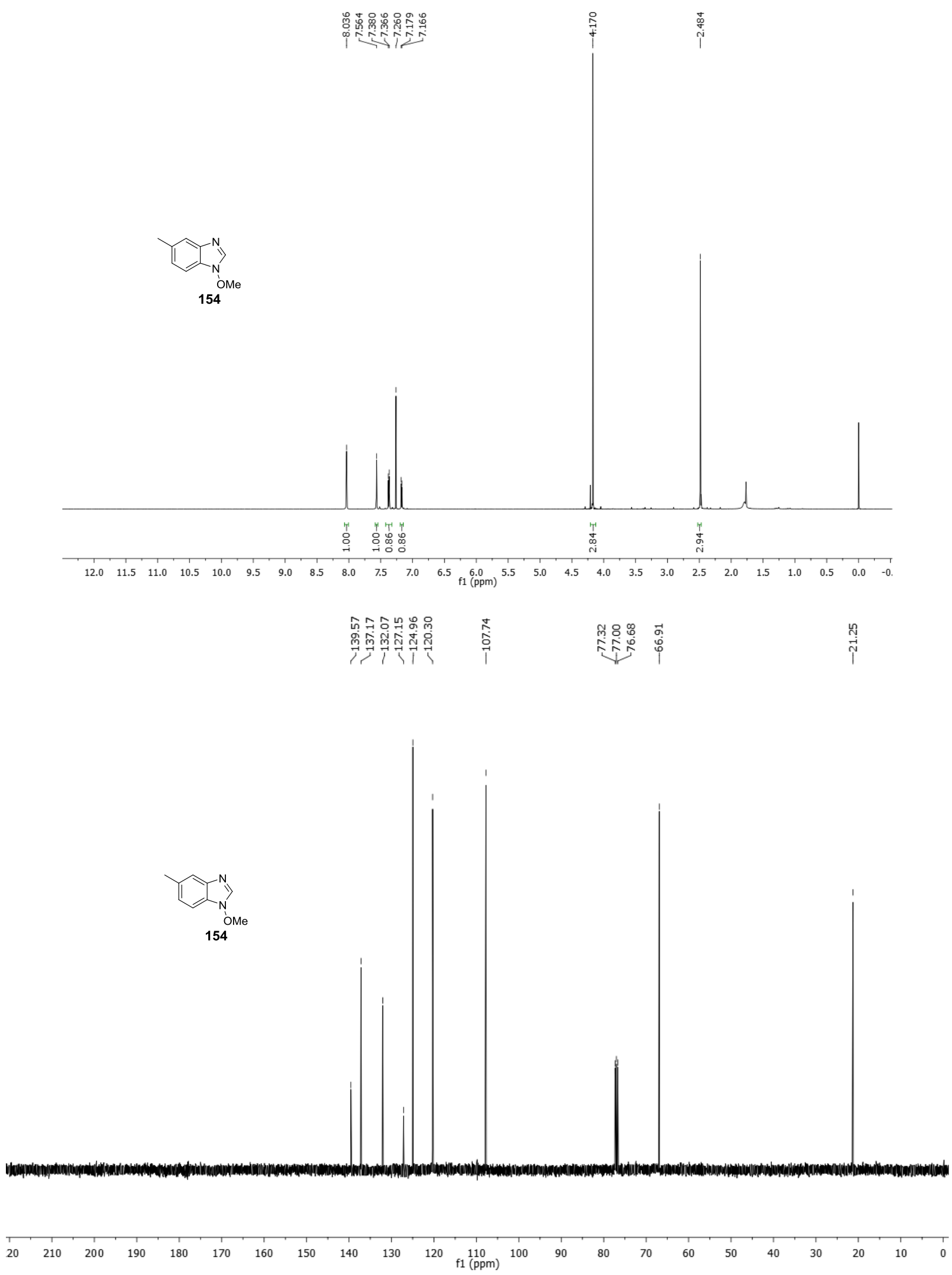

Figure 4.25 ${ }^{1} \mathrm{H}$ and ${ }^{13} \mathrm{C}$ NMR of compound 154 


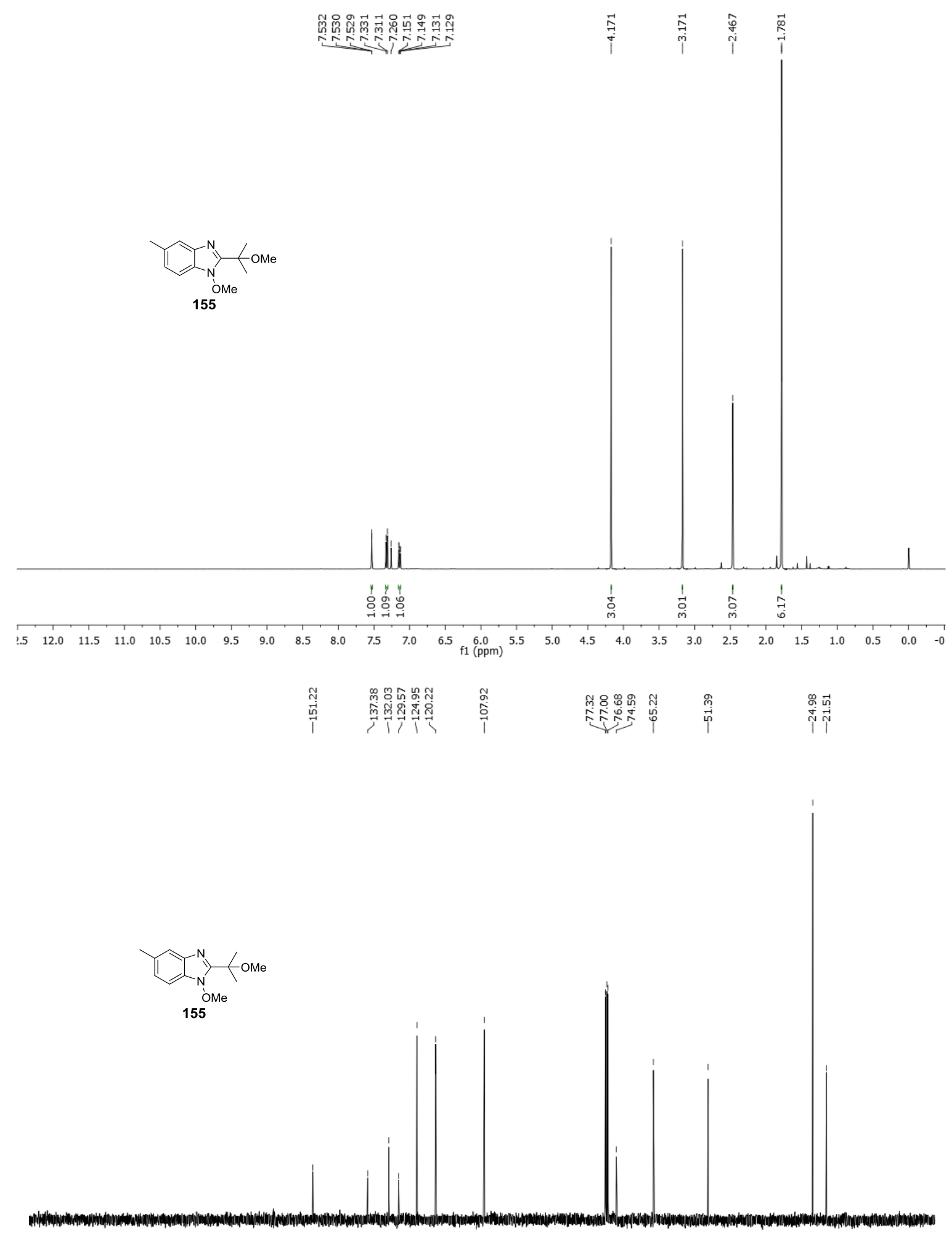

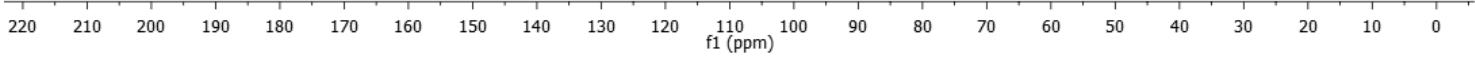

Figure 4.26 ${ }^{1} \mathrm{H}$ and ${ }^{13} \mathrm{C}$ NMR of compound 155 


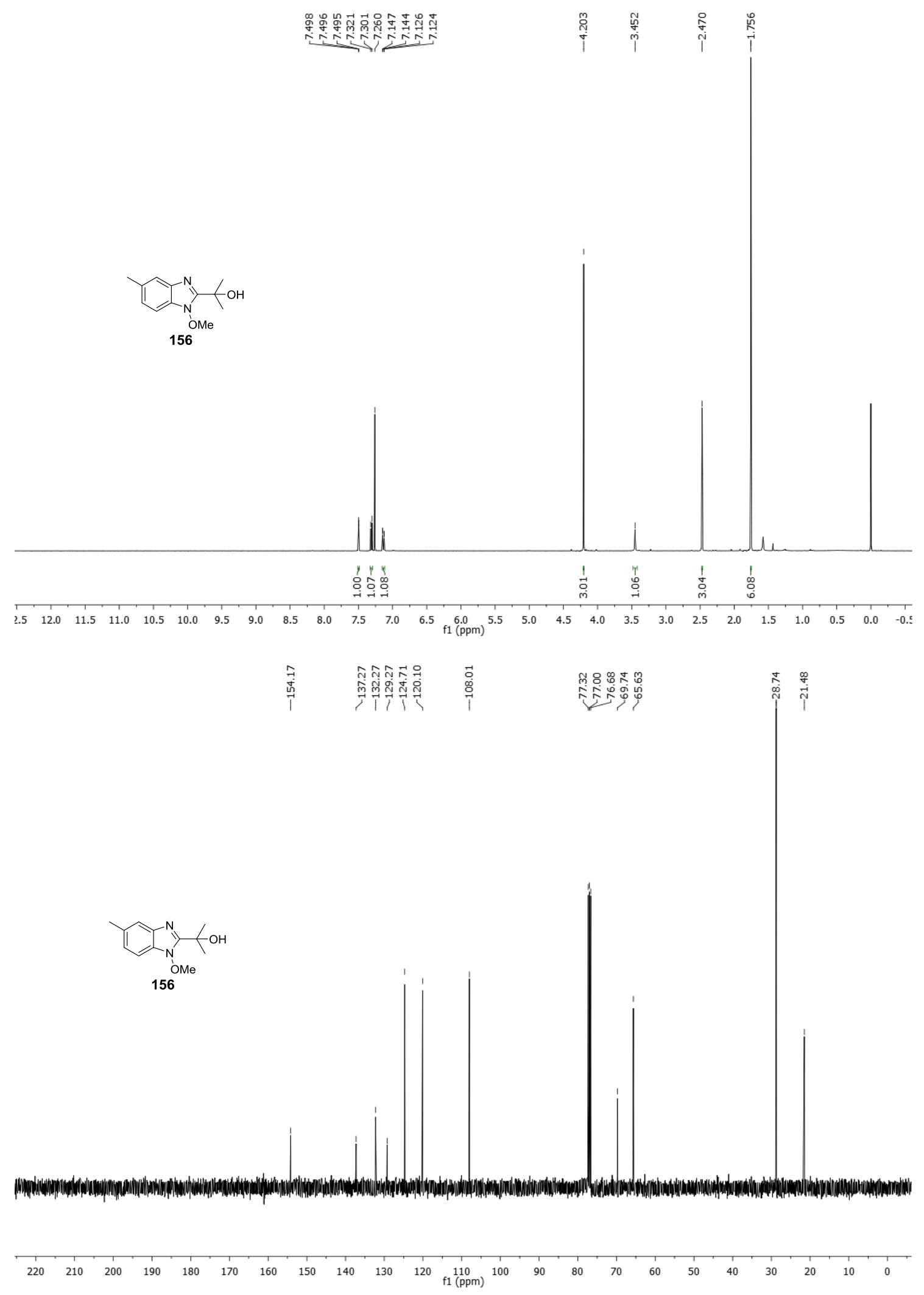

Figure 4.27 ${ }^{1} \mathrm{H}$ and ${ }^{13} \mathrm{C}$ NMR of compound 156 


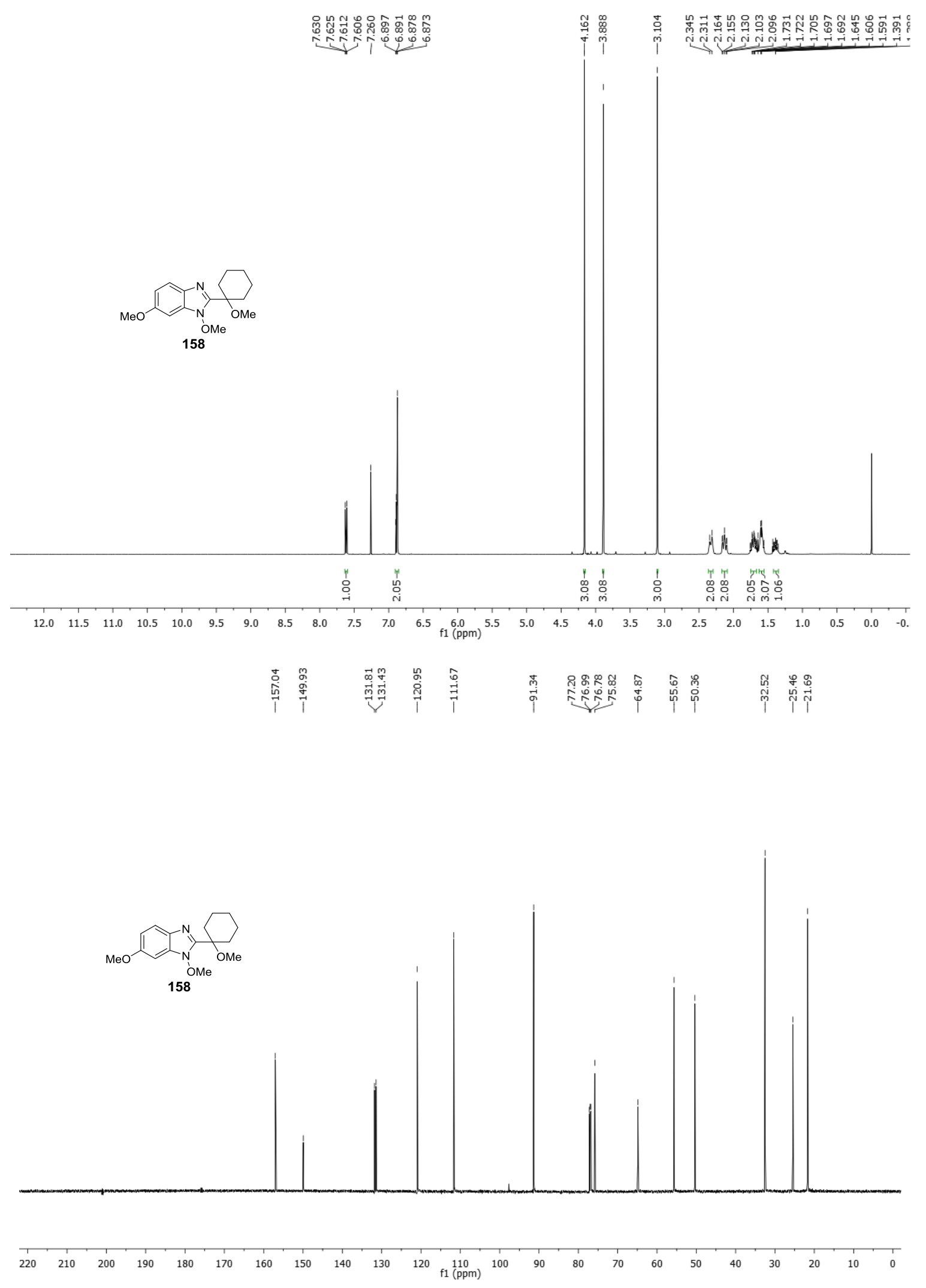

Figure 4.28 ${ }^{1} \mathrm{H}$ and ${ }^{13} \mathrm{C}$ NMR of compound 158 


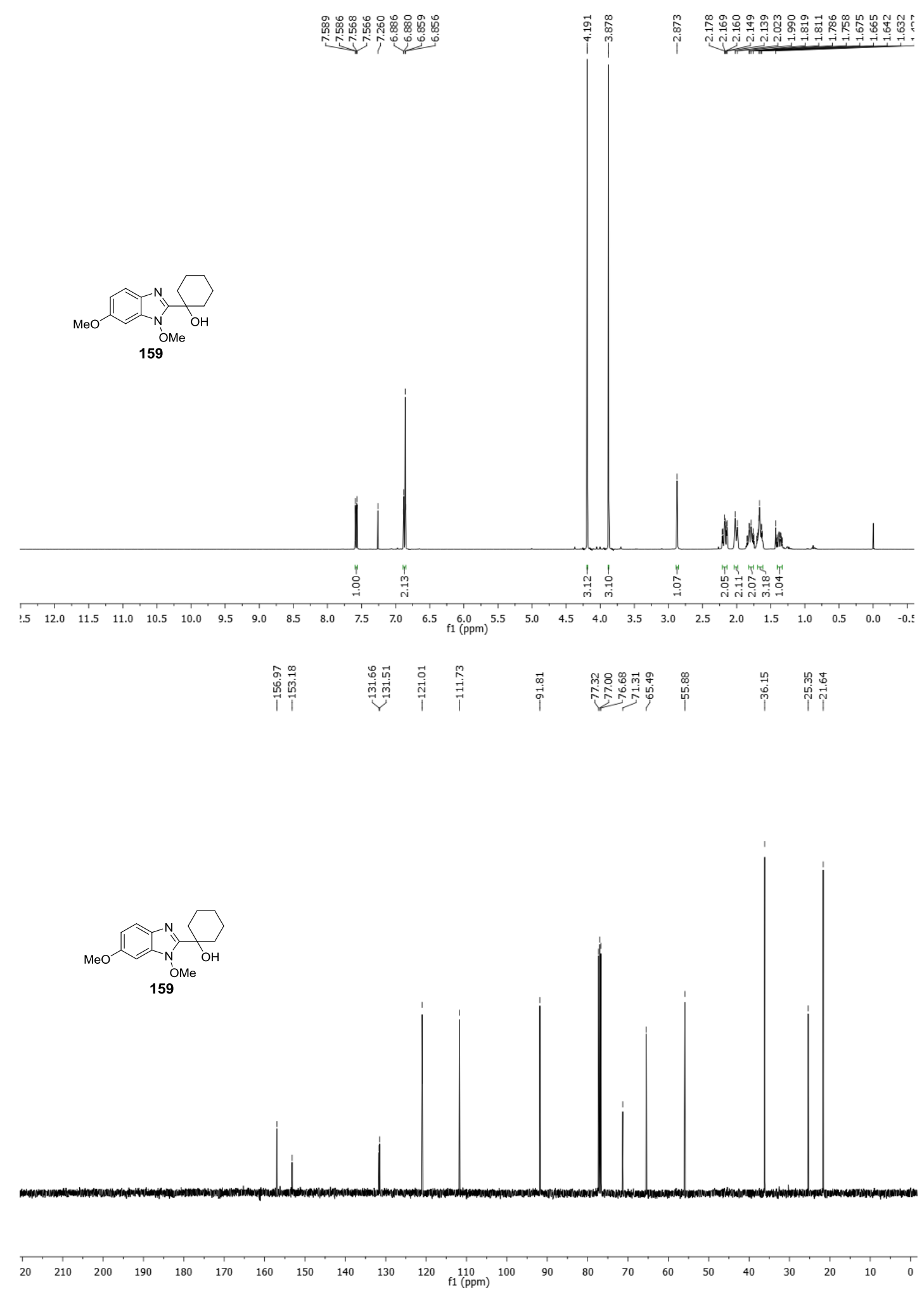

Figure 4.29 ${ }^{1} \mathrm{H}$ and ${ }^{13} \mathrm{C}$ NMR of compound 159 

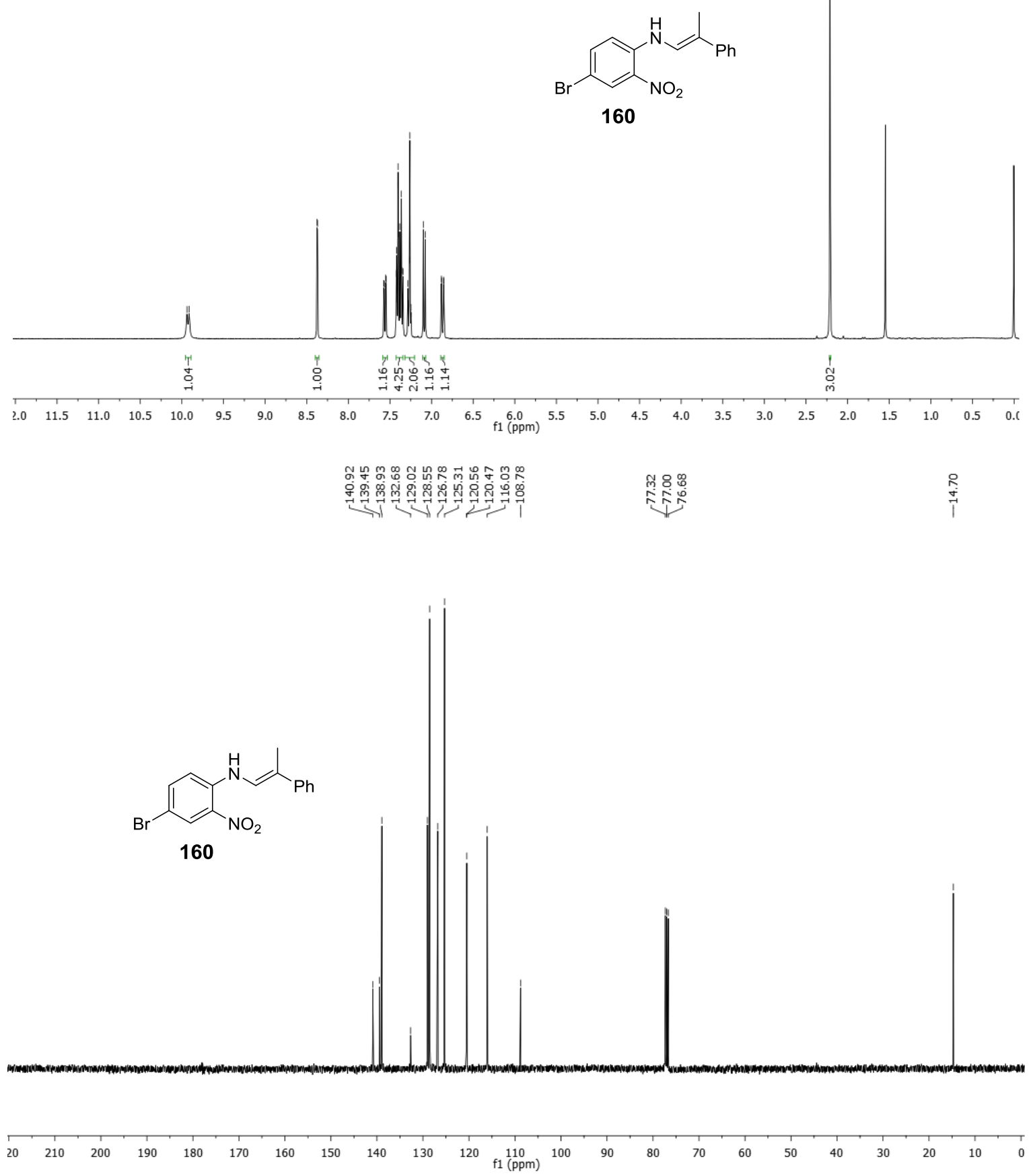

Figure 4.30 ${ }^{1} \mathrm{H}$ and ${ }^{13} \mathrm{C}$ NMR of compound $\mathbf{1 6 0}$ 

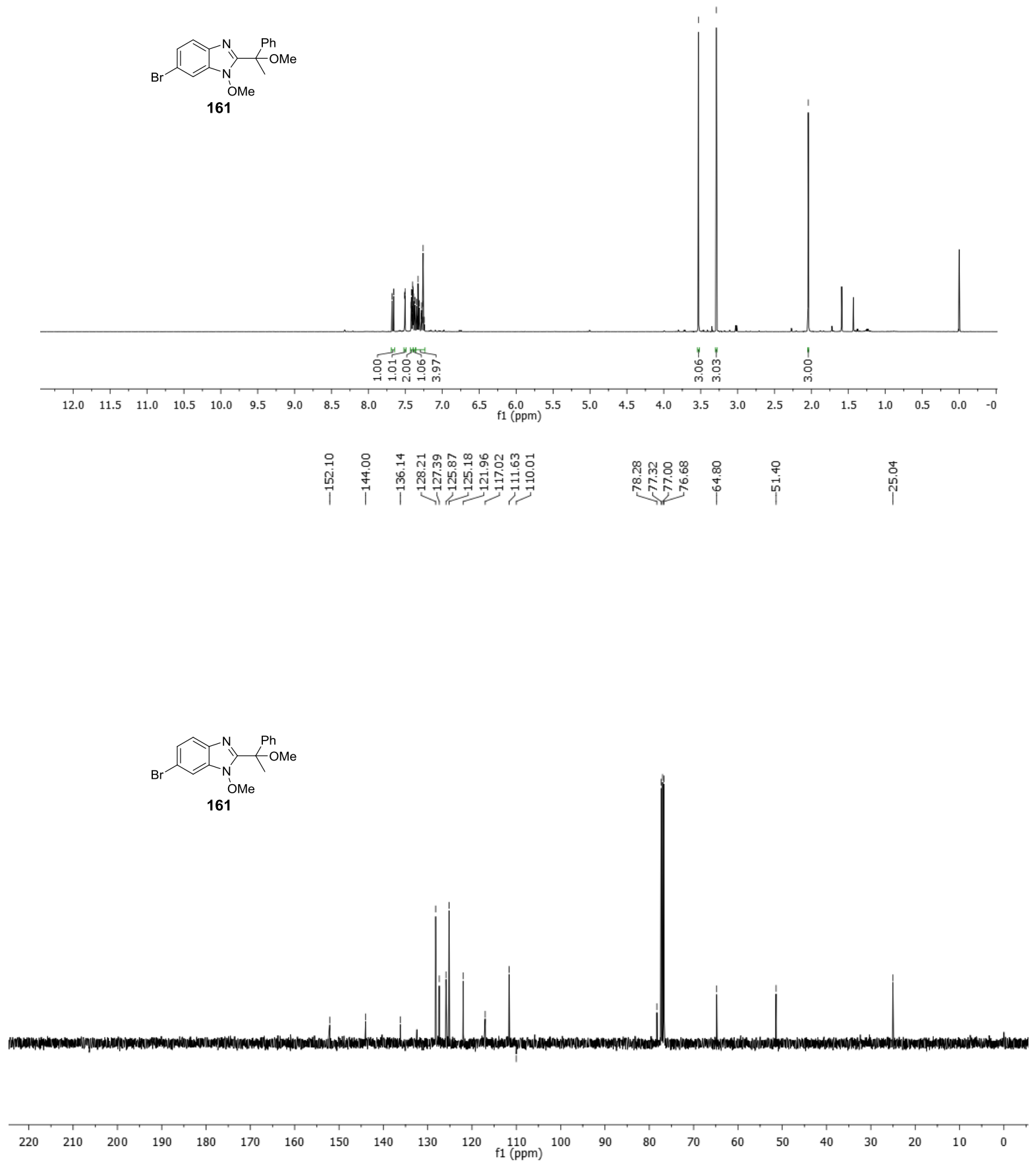

Figure 4.31 ${ }^{1} \mathrm{H}$ and ${ }^{13} \mathrm{C}$ NMR of compound 161 

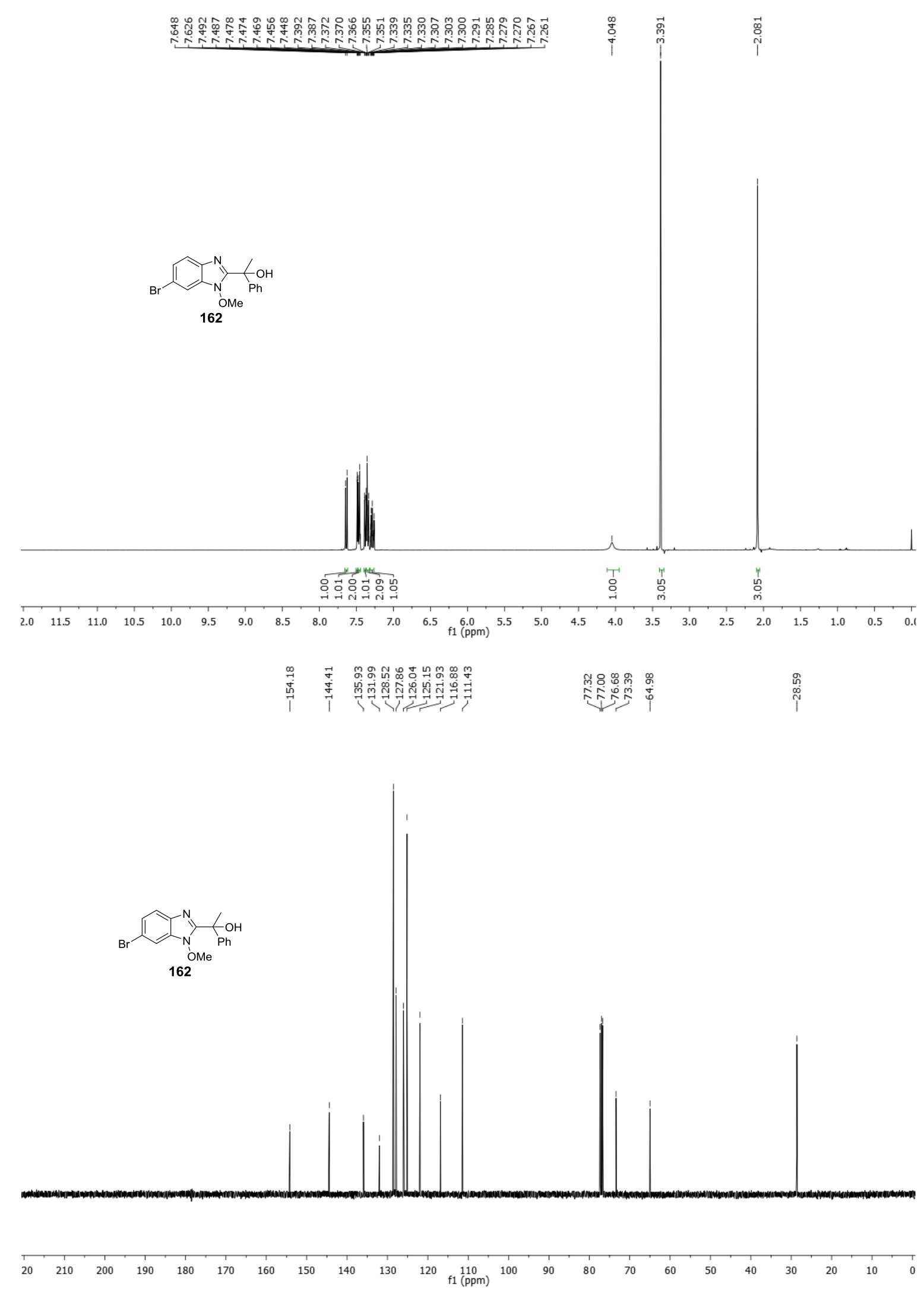

Figure 4.32 ${ }^{1} \mathrm{H}$ and ${ }^{13} \mathrm{C}$ NMR of compound 162 

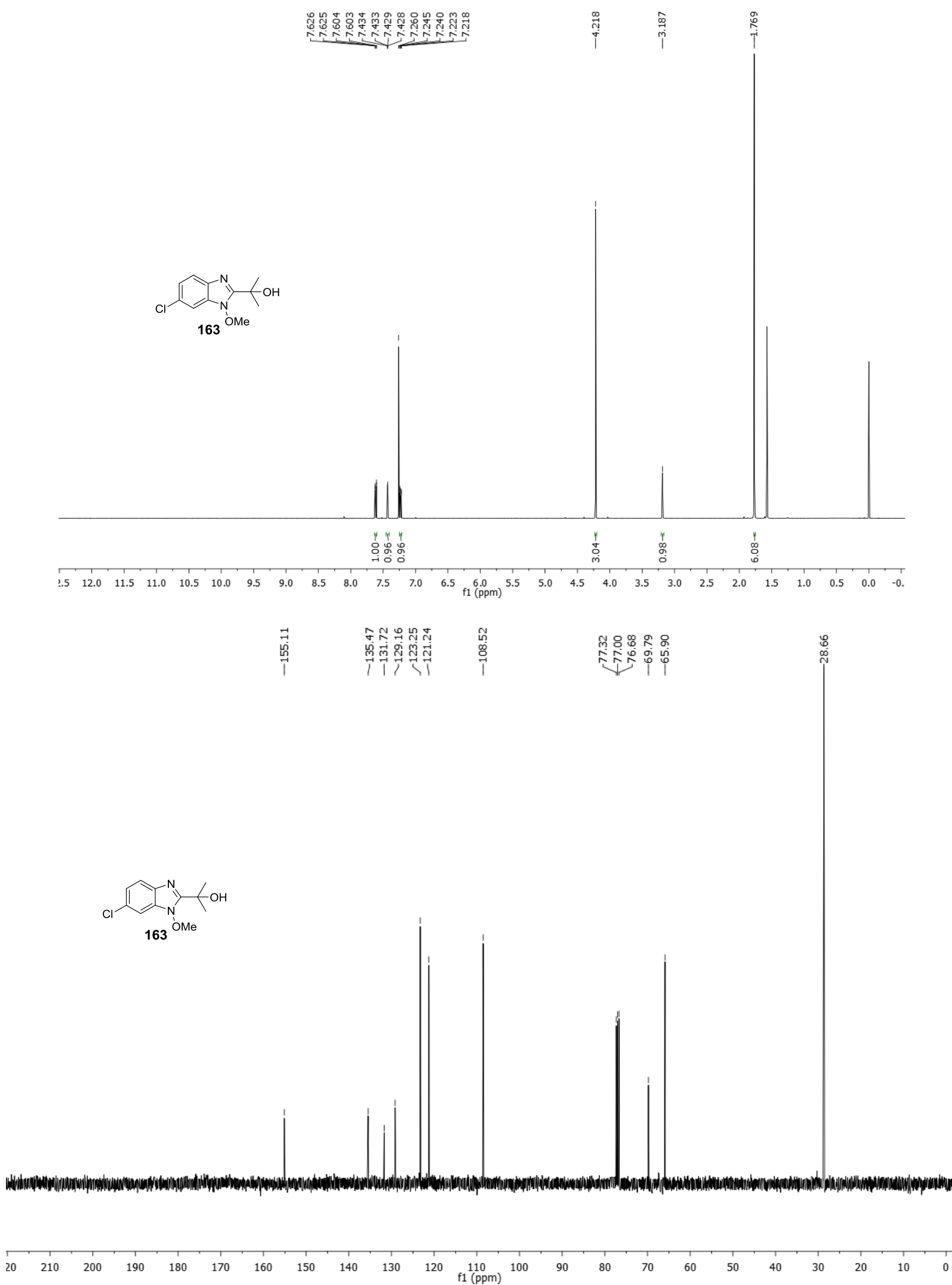

Figure $4.33{ }^{1} \mathrm{H}$ and ${ }^{13} \mathrm{C}$ NMR of compound 163 

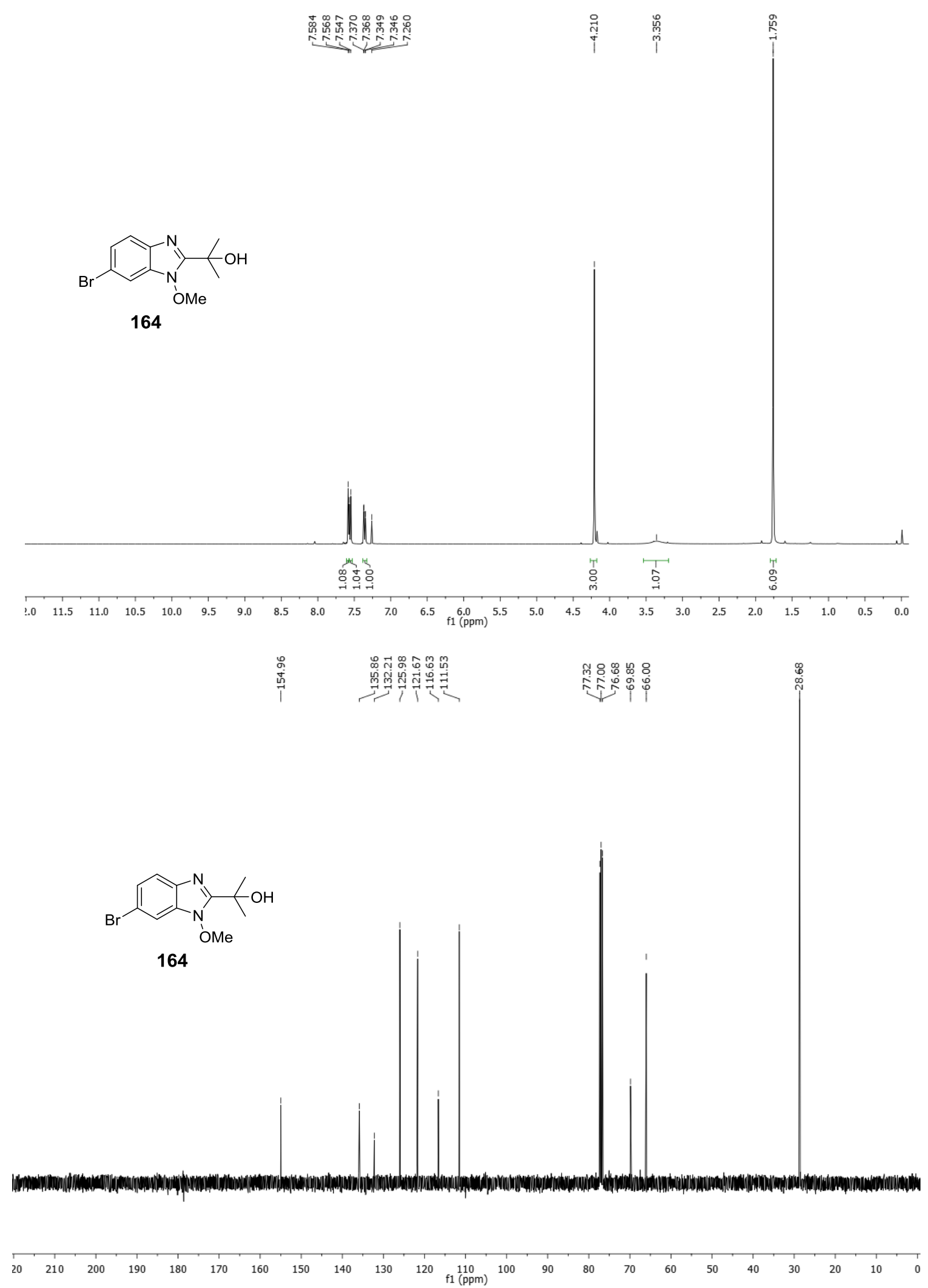

Figure 4.34 ${ }^{1} \mathrm{H}$ and ${ }^{13} \mathrm{C}$ NMR of compound 164 

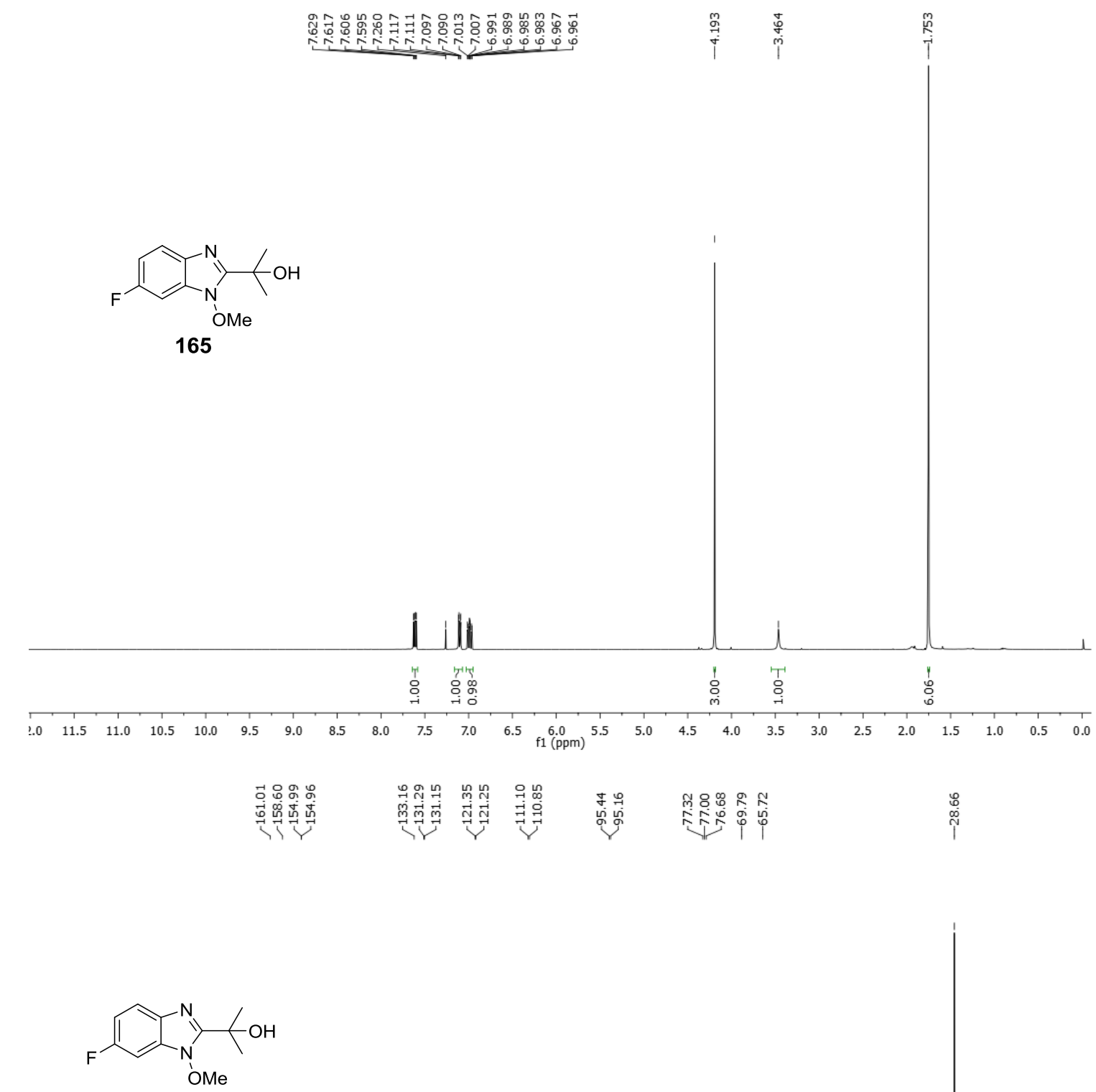

165
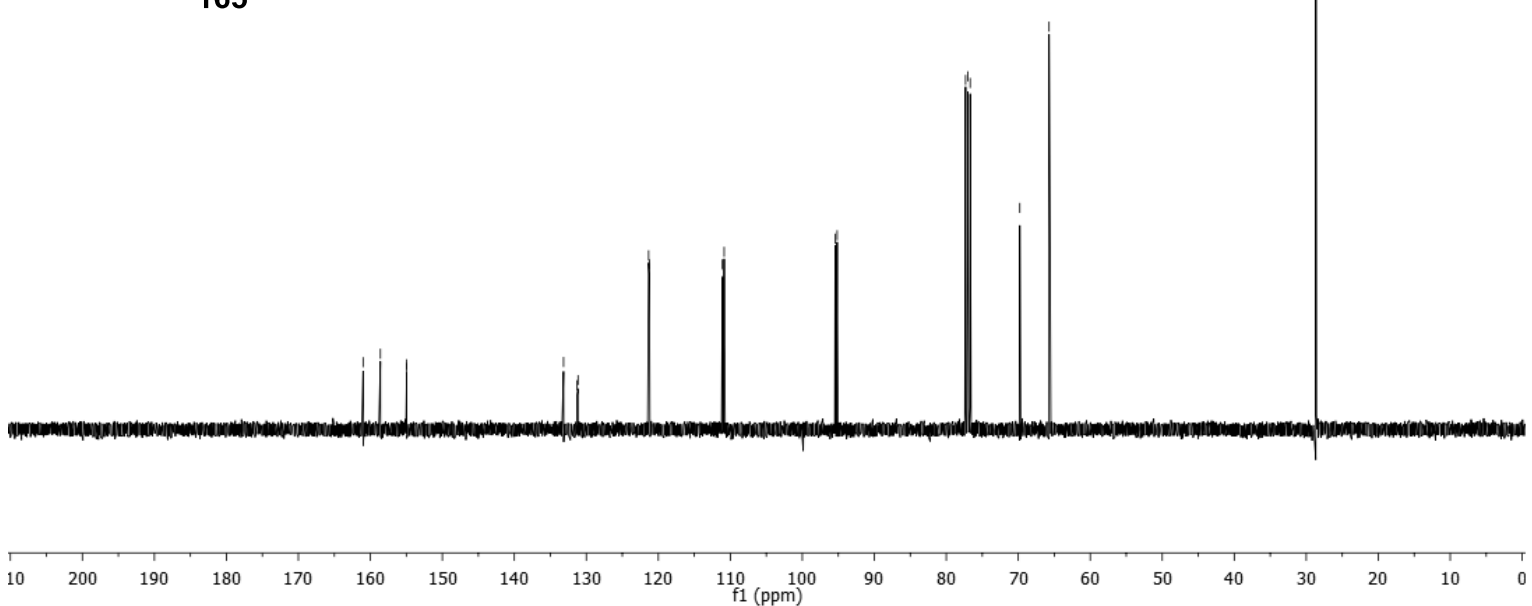

Figure $4.35{ }^{1} \mathrm{H}$ and ${ }^{13} \mathrm{C}$ NMR of compound 165 


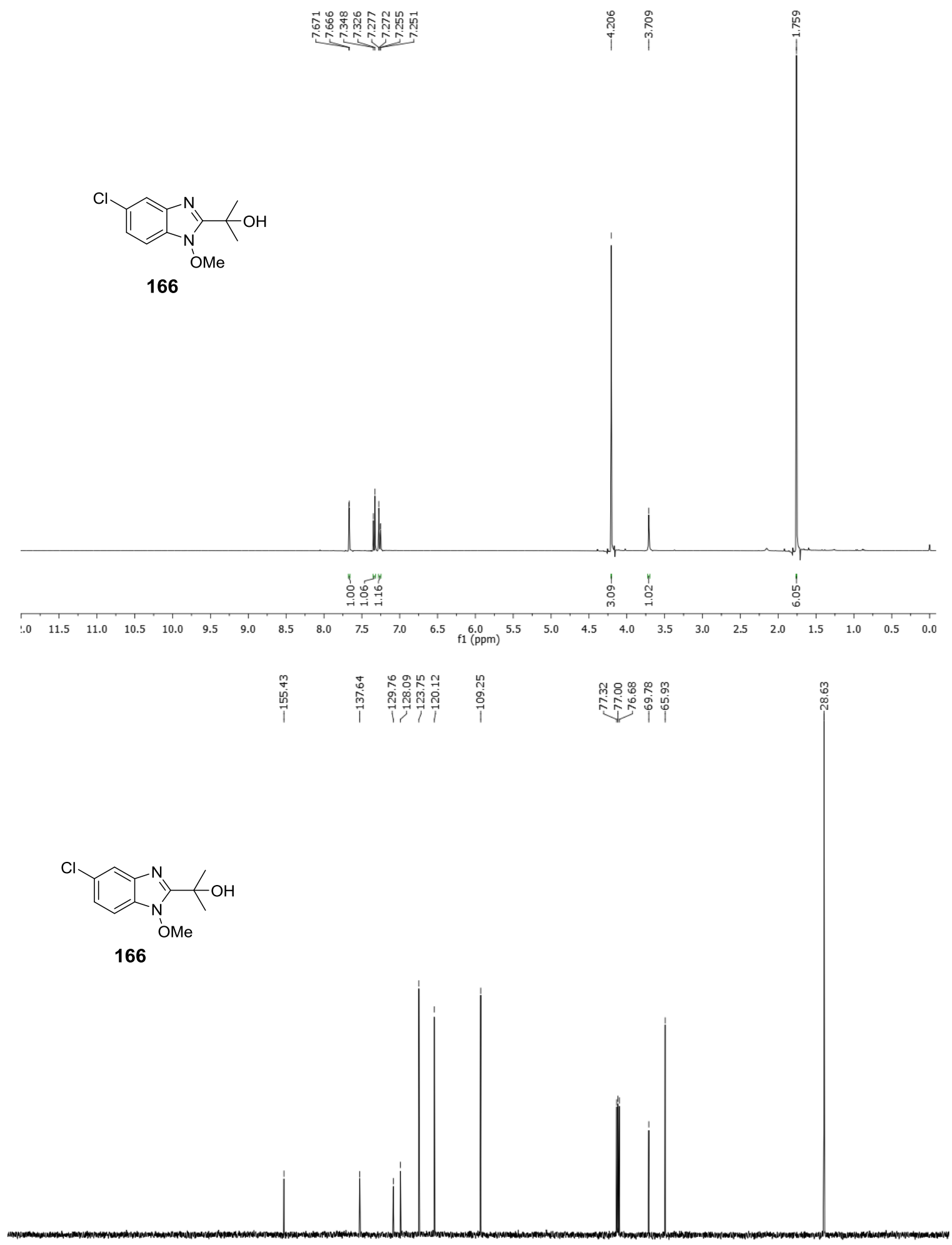

$\begin{array}{llllllllllllllllllllllll}20 & 210 & 200 & 190 & 180 & 170 & 160 & 150 & 140 & 130 & 120 & 110 & 100 & 90 & 80 & 70 & 60 & 50 & 40 & 30 & 20 & 10 & 0\end{array}$

Figure 4.36 ${ }^{1} \mathrm{H}$ and ${ }^{13} \mathrm{C}$ NMR of compound 166 


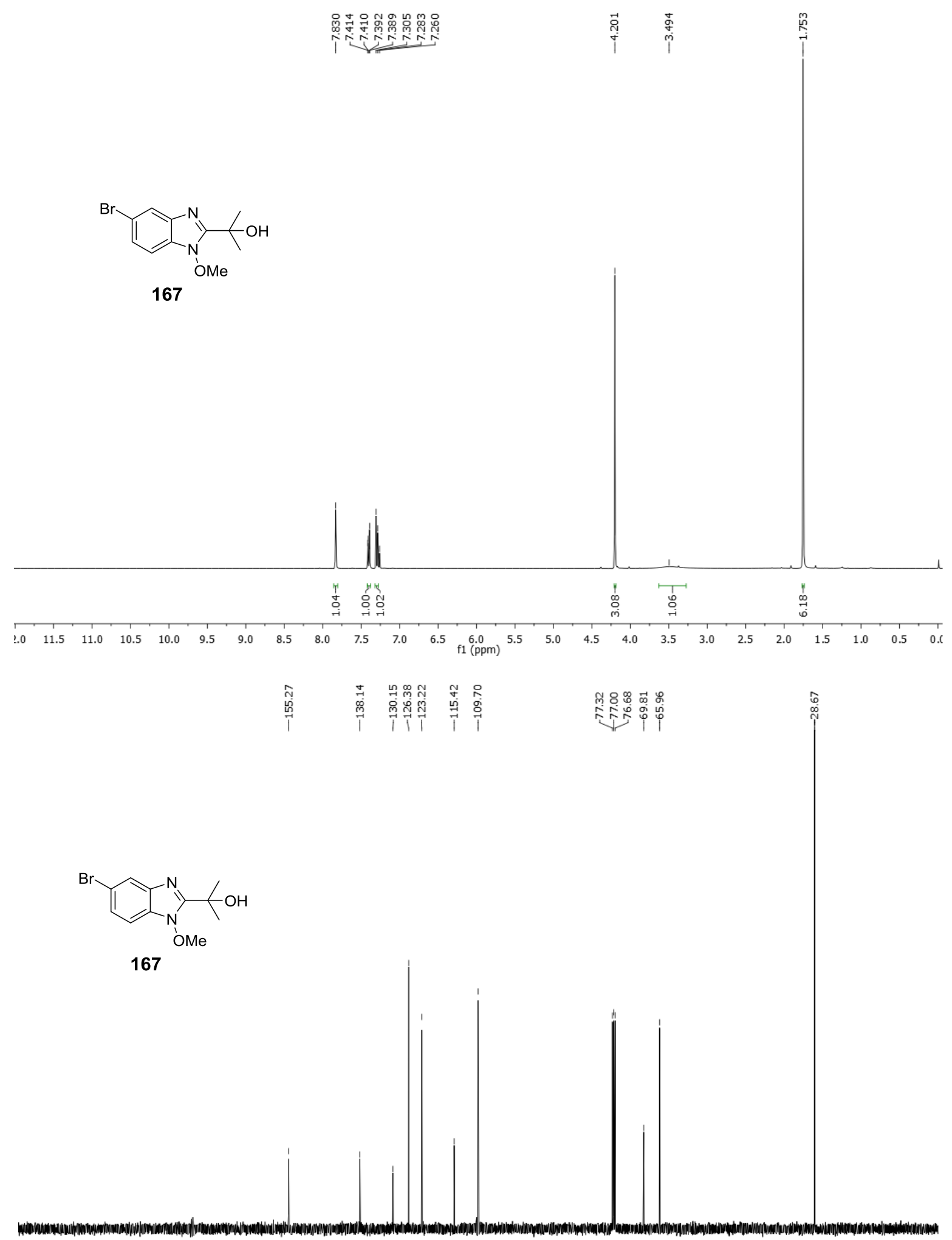

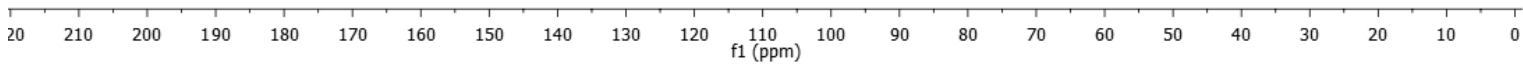

Figure 4.37 ${ }^{1} \mathrm{H}$ and ${ }^{13} \mathrm{C}$ NMR of compound 167 

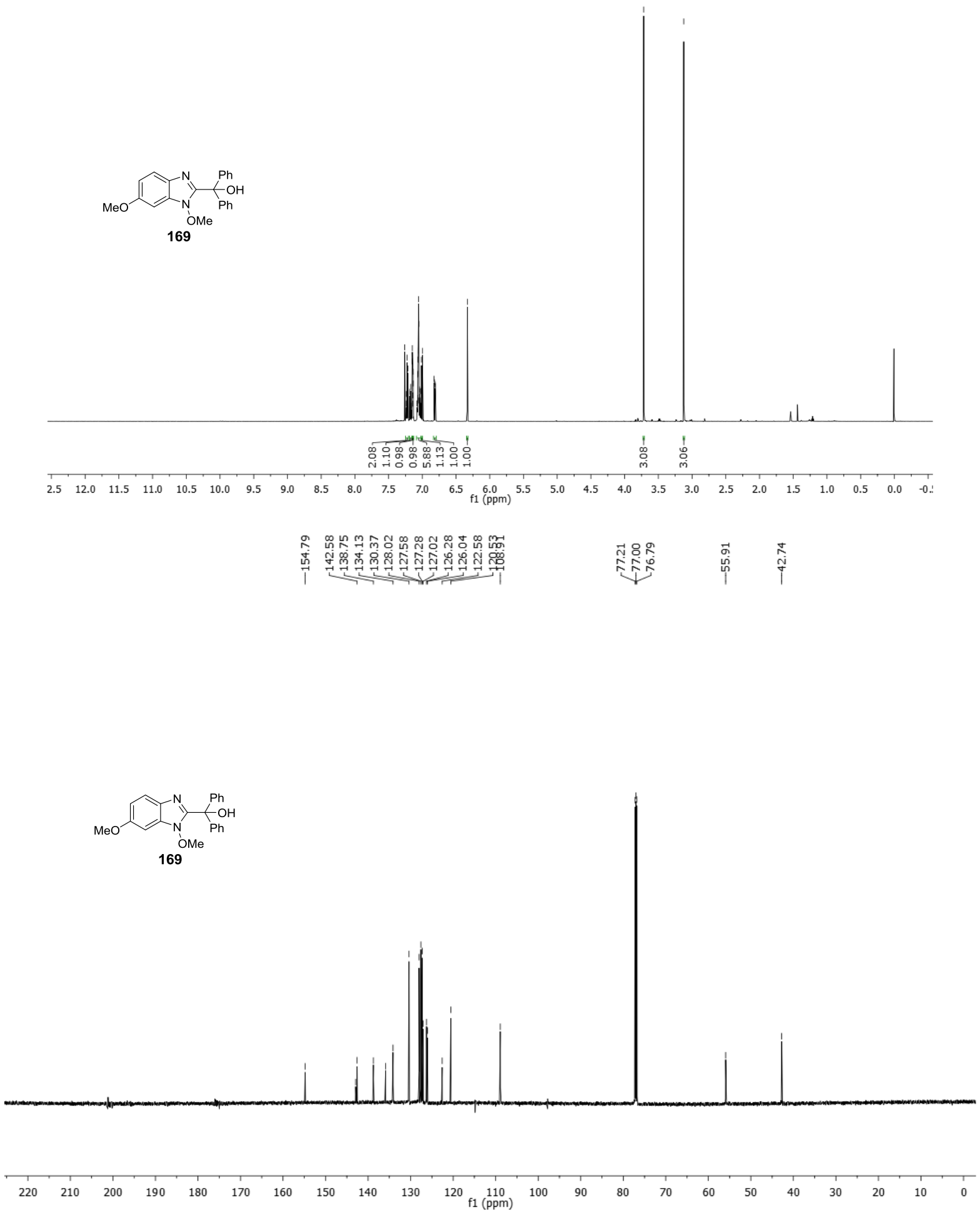

Figure 4.38 ${ }^{1} \mathrm{H}$ and ${ }^{13} \mathrm{C}$ NMR of compound 169 


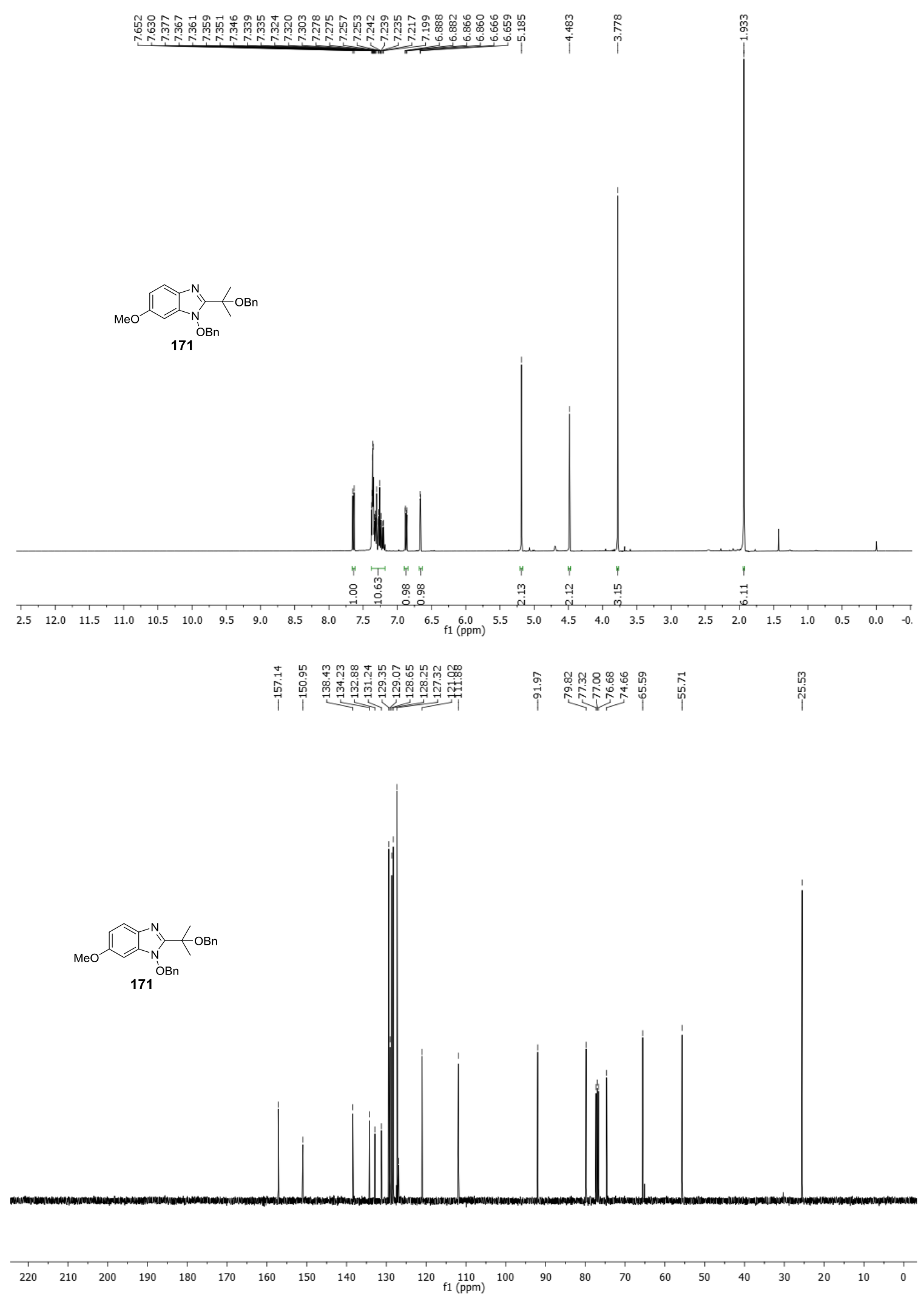

Figure 4.39 ${ }^{1} \mathrm{H}$ and ${ }^{13} \mathrm{C}$ NMR of compound 171 

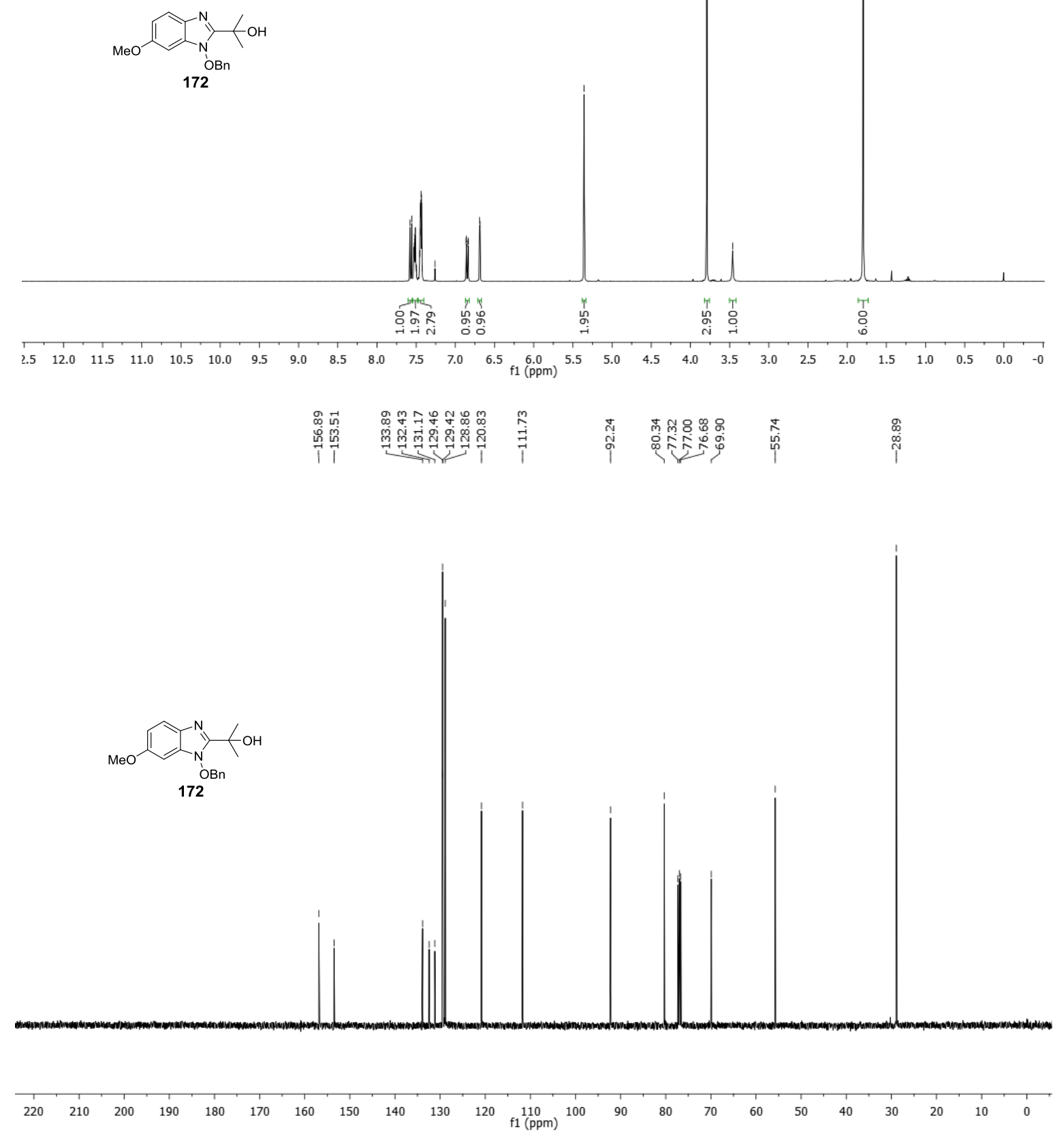

Figure 4.40 ${ }^{1} \mathrm{H}$ and ${ }^{13} \mathrm{C}$ NMR of compound 172 

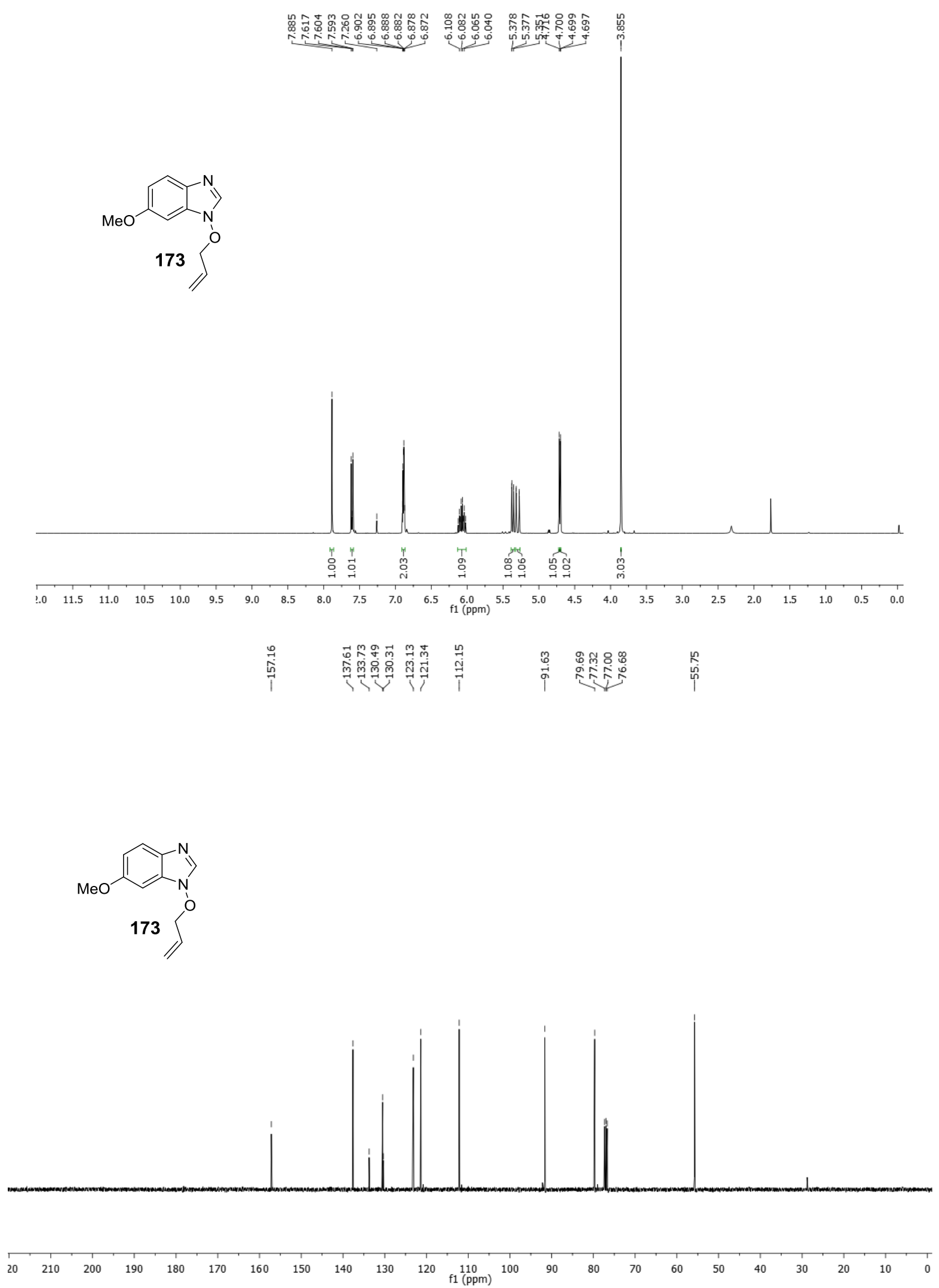

Figure 4.41 ${ }^{1} \mathrm{H}$ and ${ }^{13} \mathrm{C}$ NMR of compound 173 

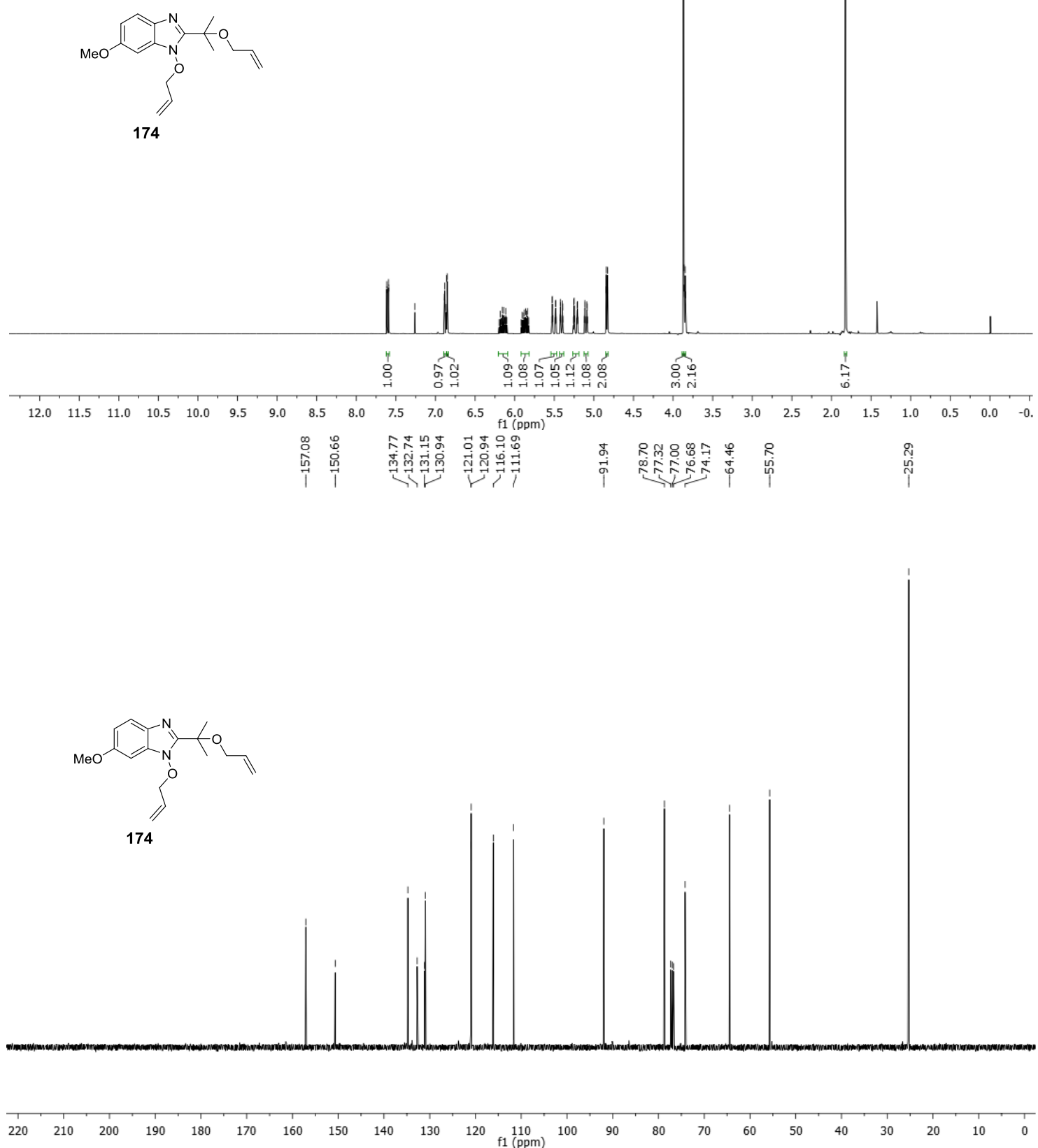

Figure 4.42 ${ }^{1} \mathrm{H}$ and ${ }^{13} \mathrm{C}$ NMR of compound 174 

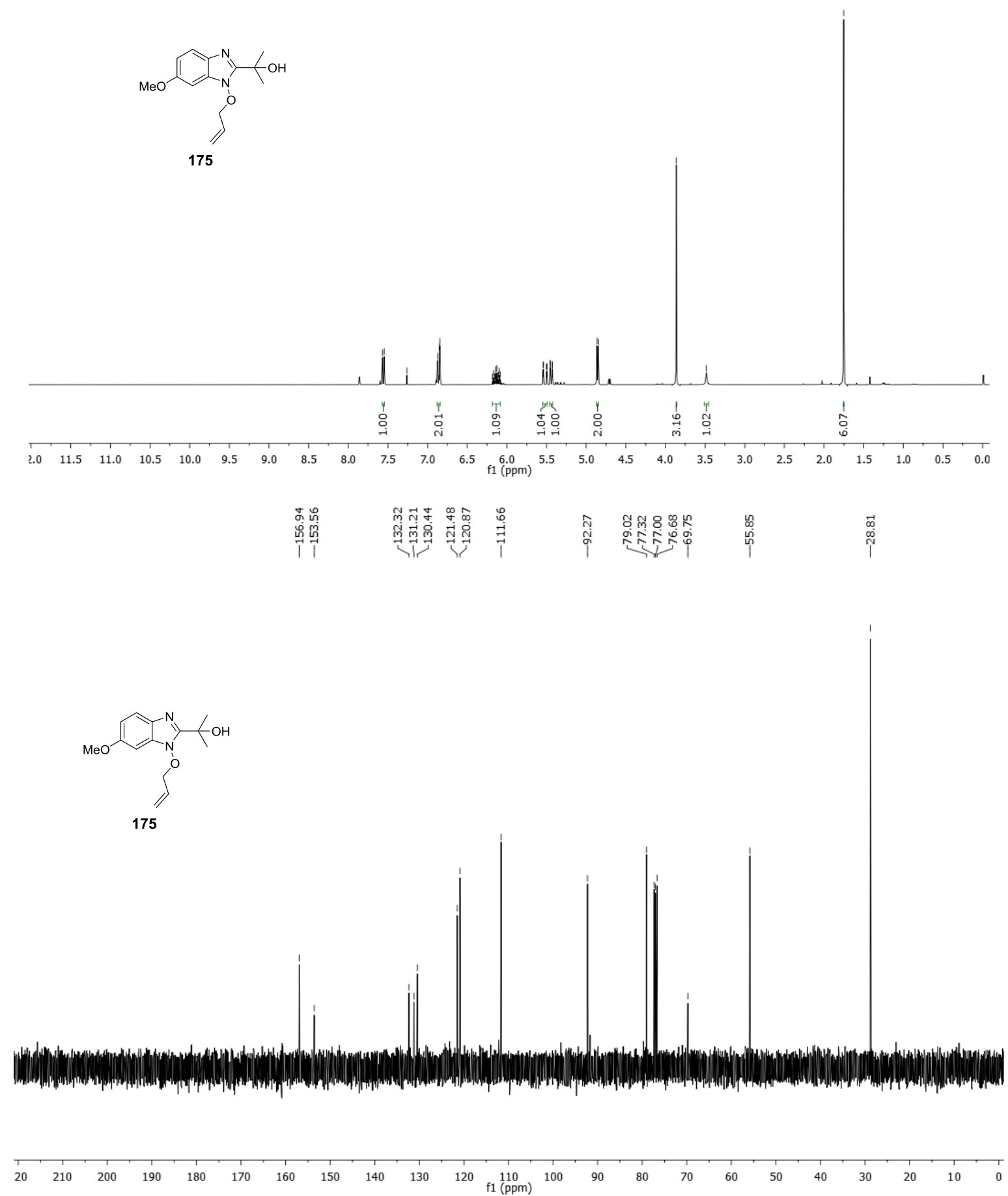

Figure $4.43{ }^{1} \mathrm{H}$ and ${ }^{13} \mathrm{C}$ NMR of compound 175 

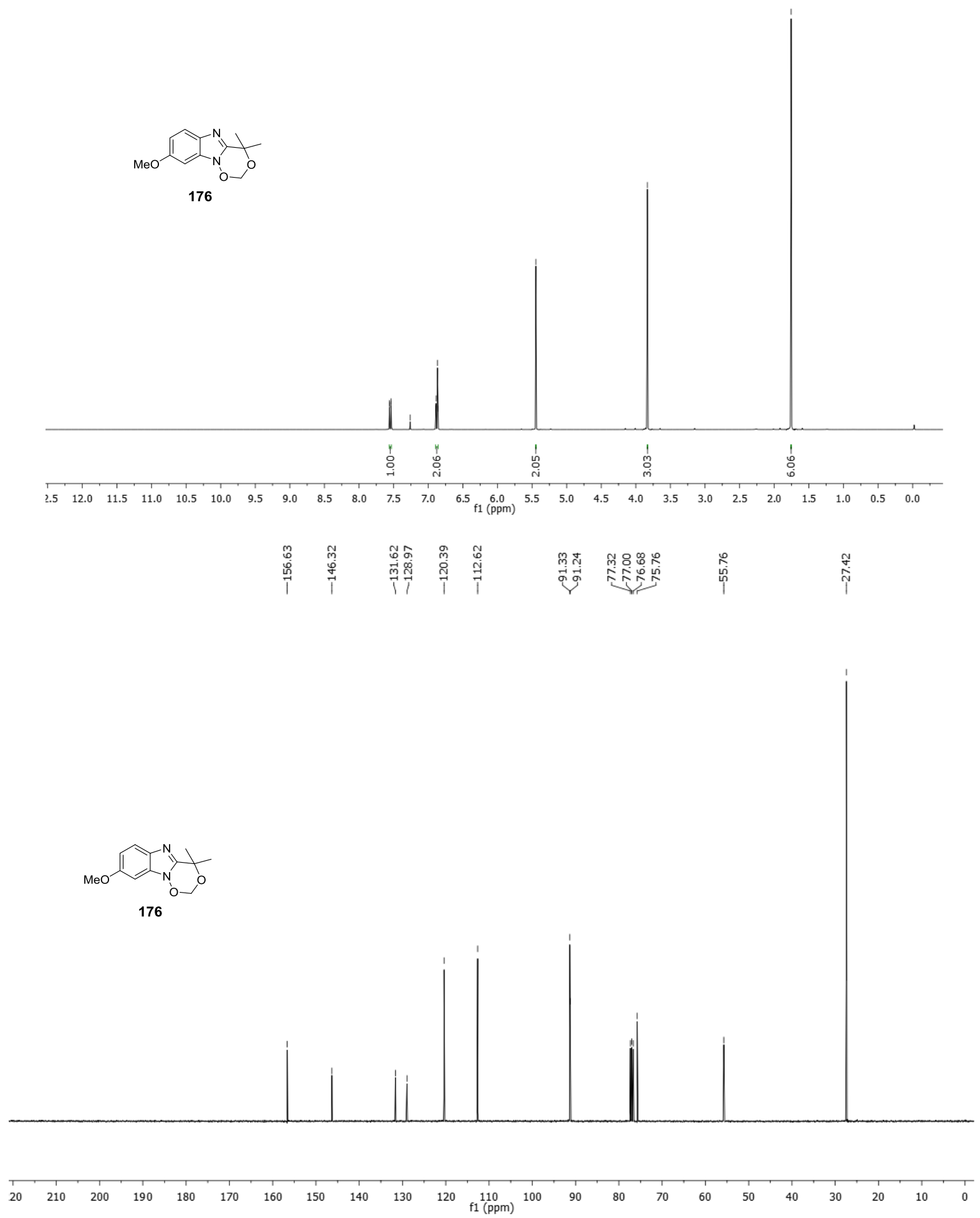

Figure $4.44{ }^{1} \mathrm{H}$ and ${ }^{13} \mathrm{C}$ NMR of compound 176 


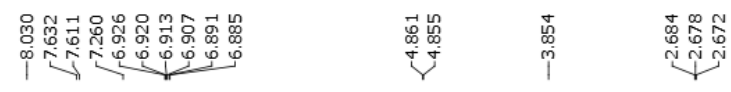
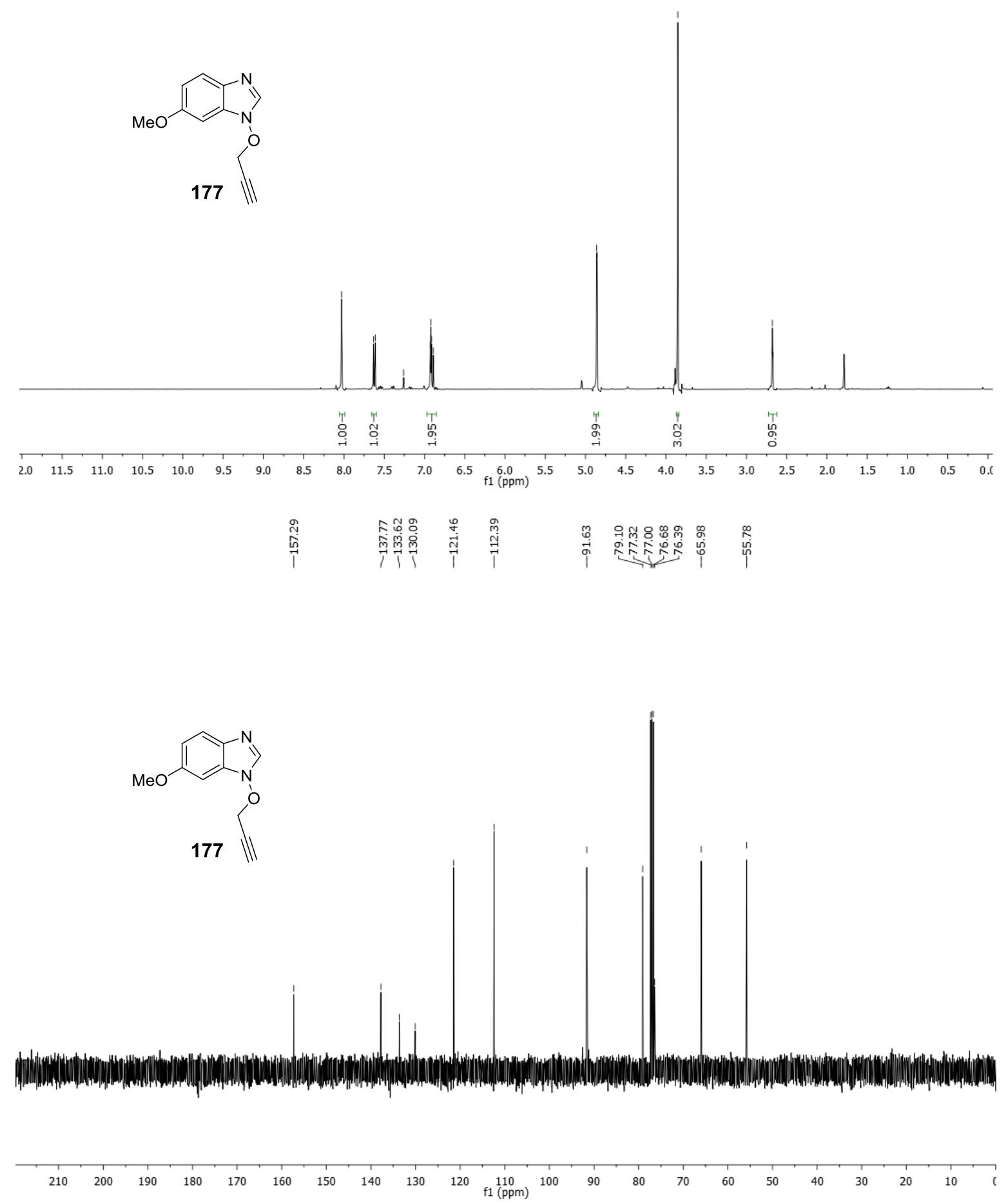

Figure 4.45 ${ }^{1} \mathrm{H}$ and ${ }^{13} \mathrm{C}$ NMR of compound 177 

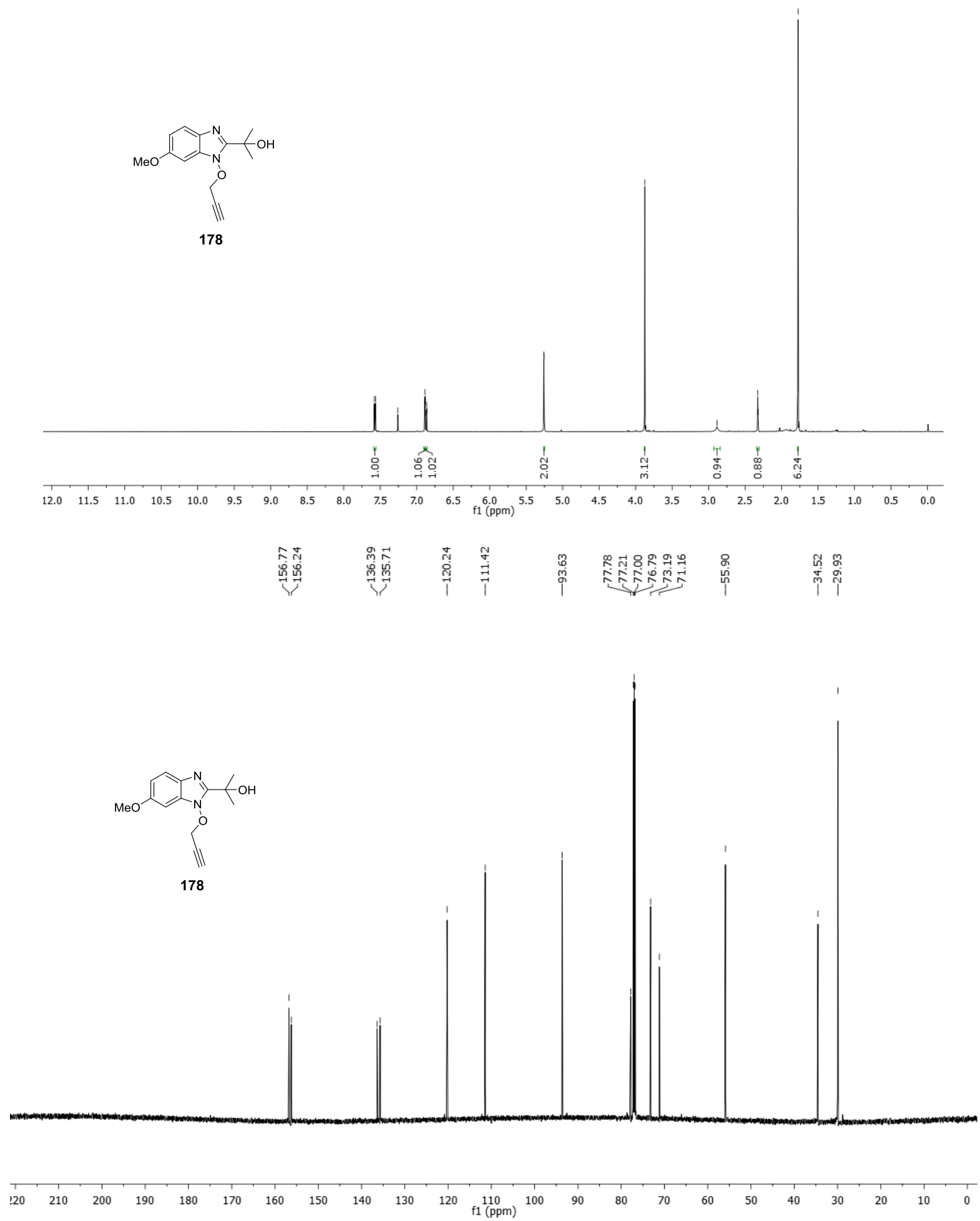

Figure 4.46 ${ }^{1} \mathrm{H}$ and ${ }^{13} \mathrm{C}$ NMR of compound 178 

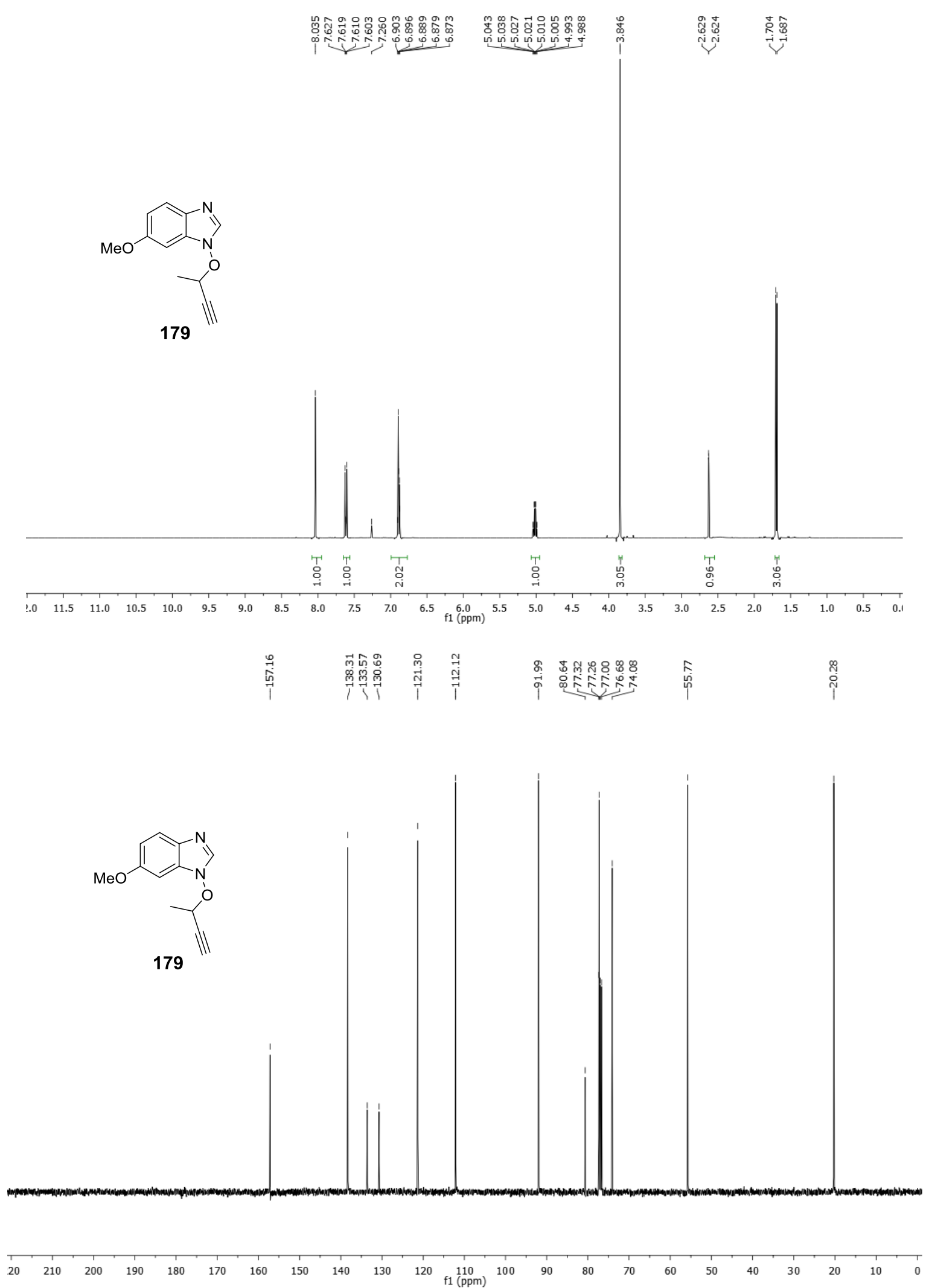

Figure 4.47 ${ }^{1} \mathrm{H}$ and ${ }^{13} \mathrm{C}$ NMR of compound 179 

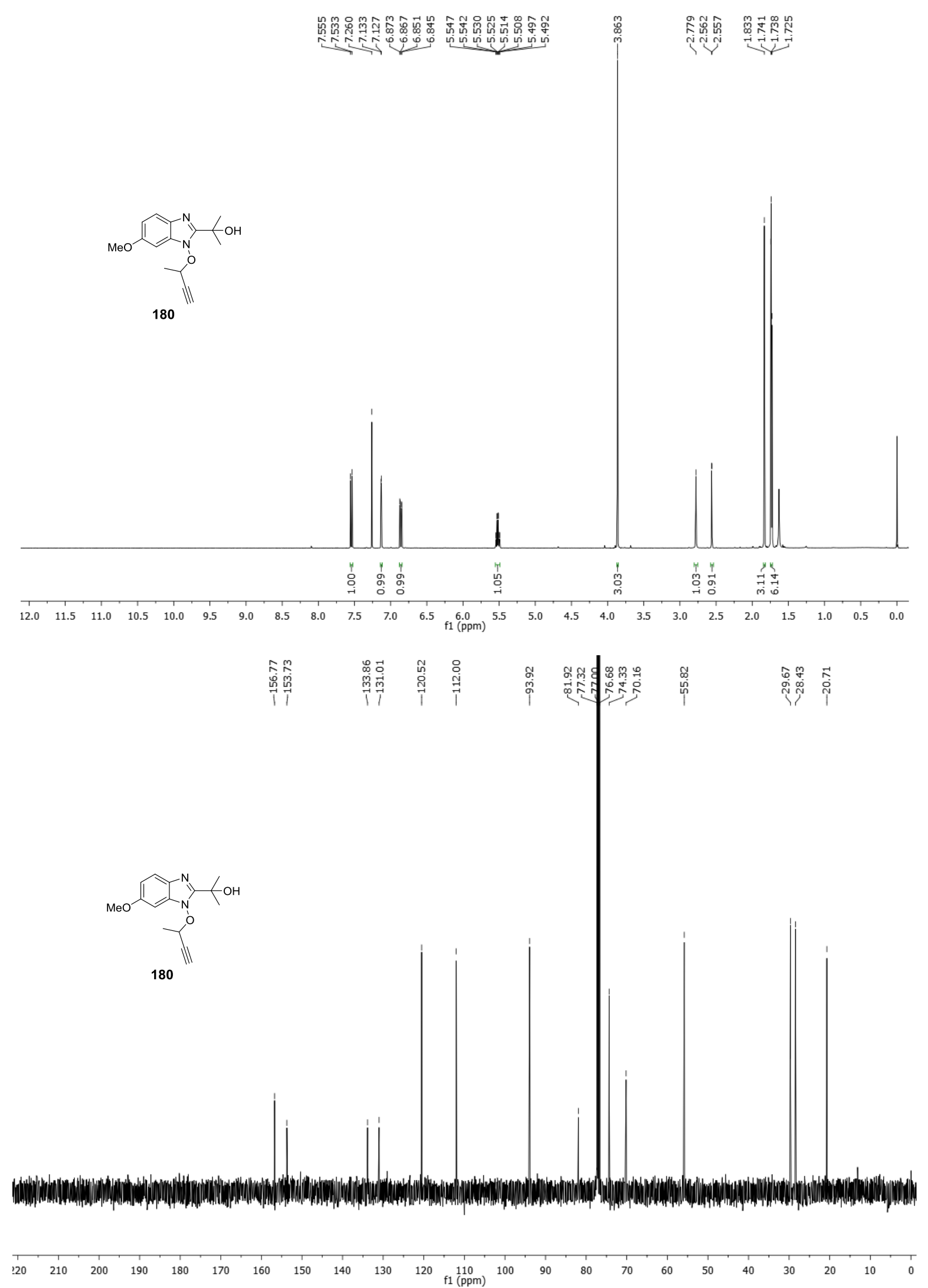

Figure 4.48 ${ }^{1} \mathrm{H}$ and ${ }^{13} \mathrm{C}$ NMR of compound 180 


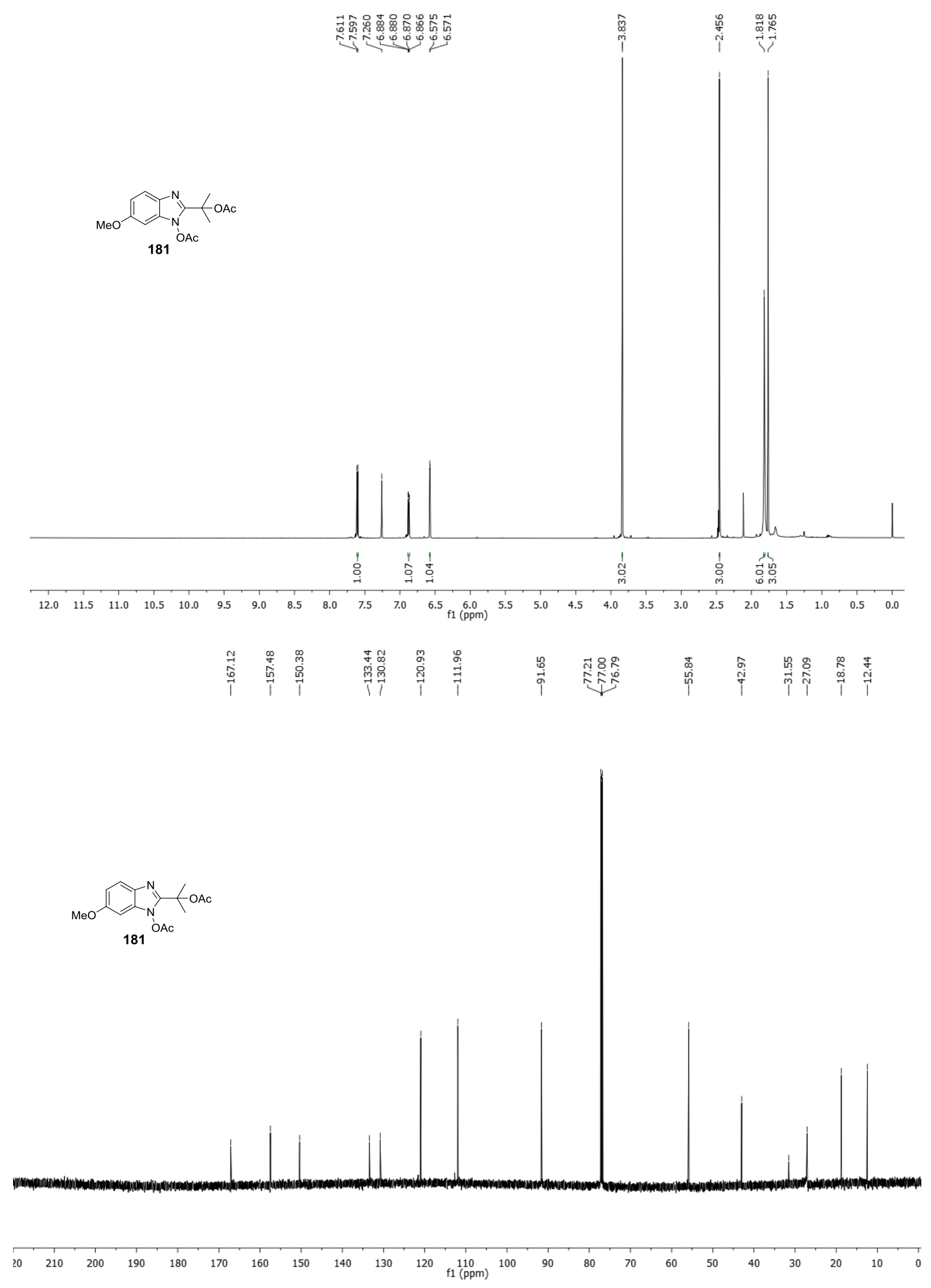

Figure 4.49 ${ }^{1} \mathrm{H}$ and ${ }^{13} \mathrm{C}$ NMR of compound 181 

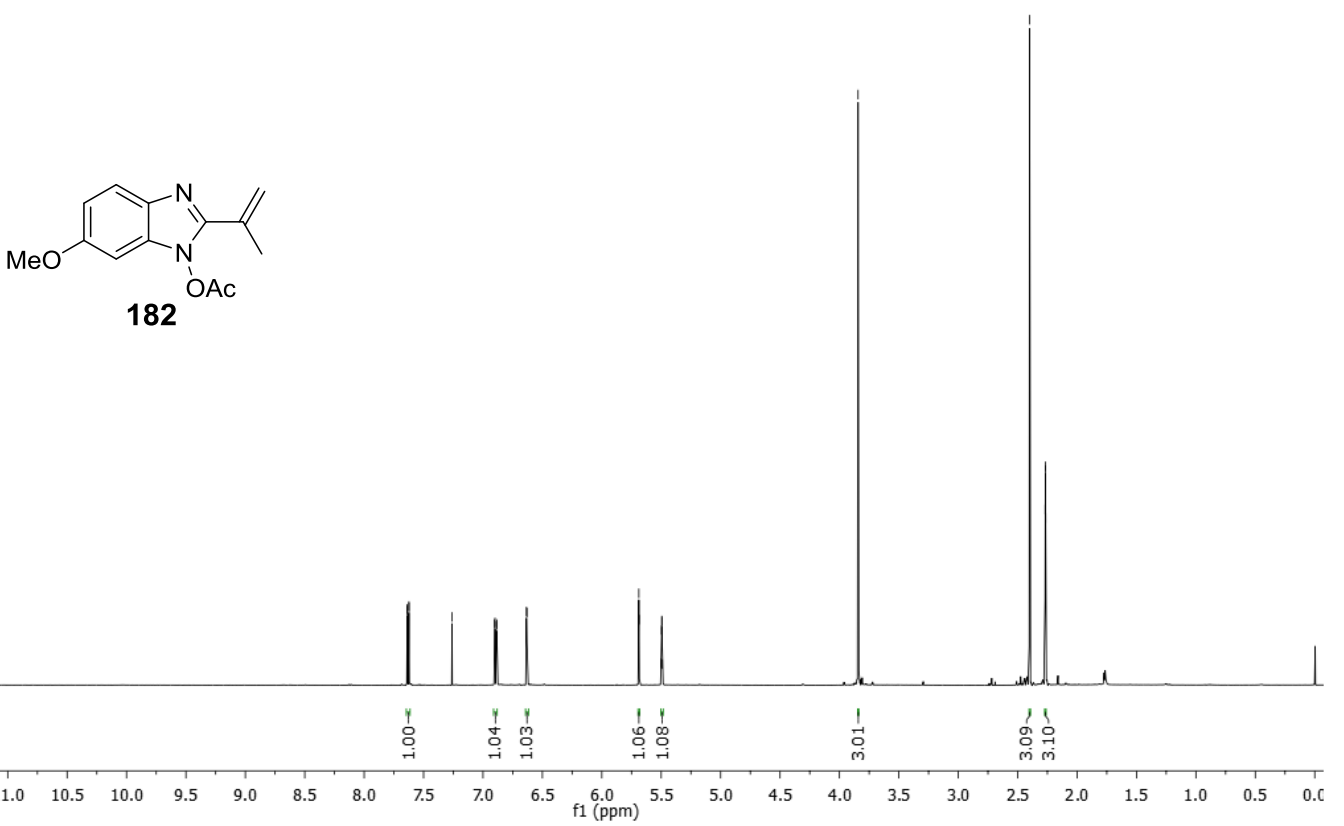

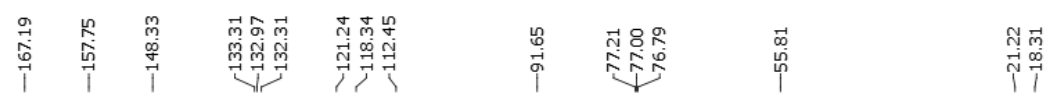
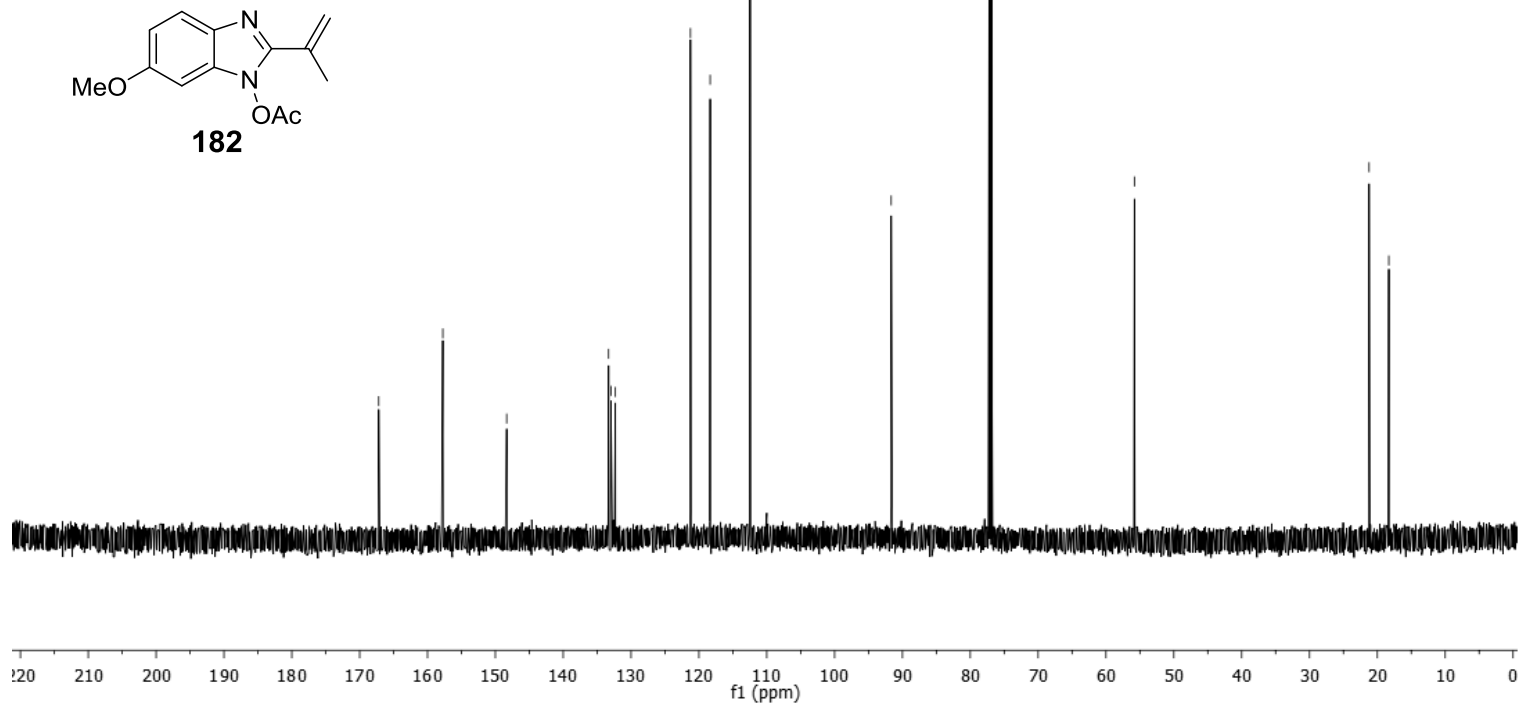

Figure 4.50 ${ }^{1} \mathrm{H}$ and ${ }^{13} \mathrm{C}$ NMR of compound 182 

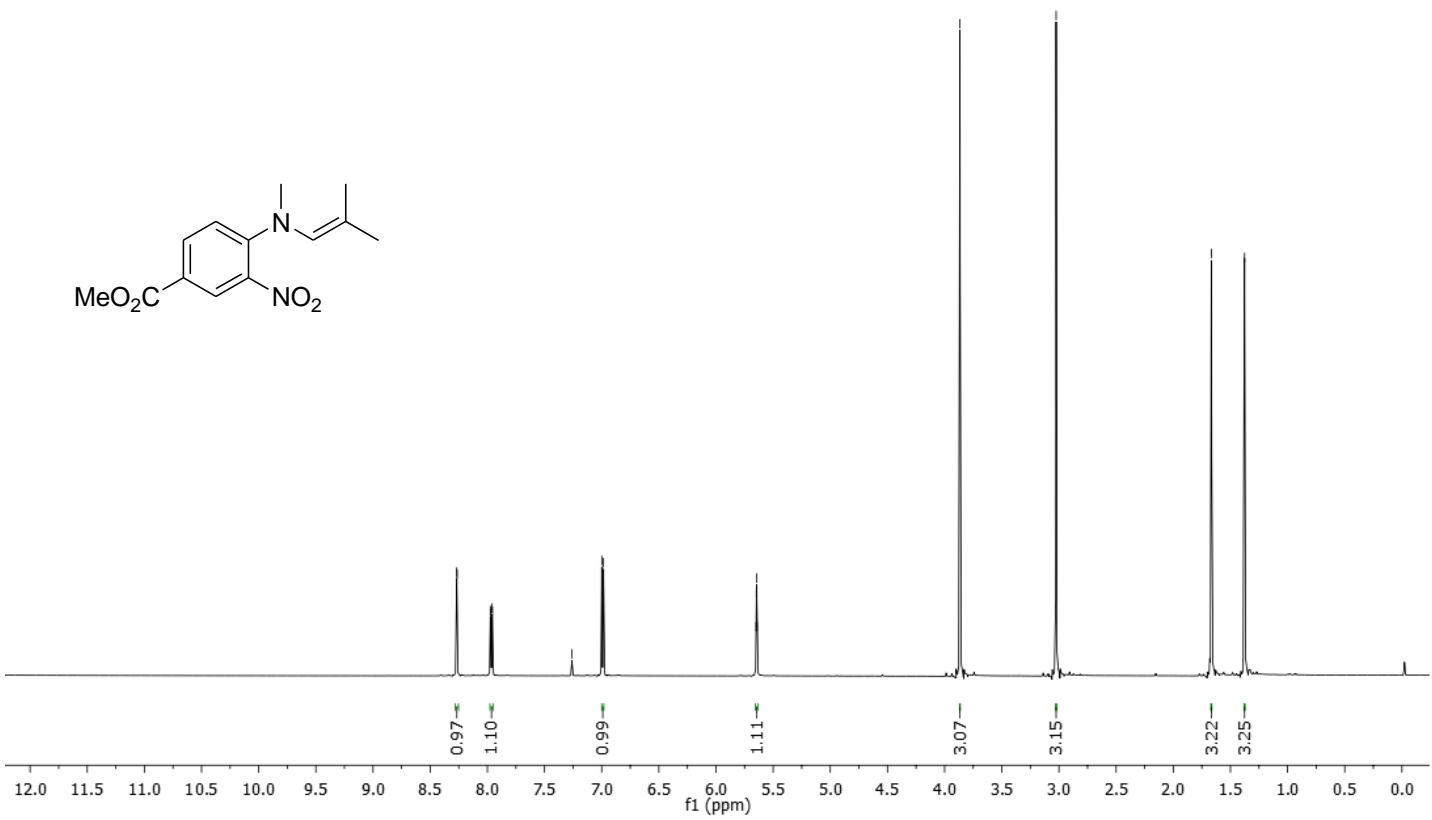

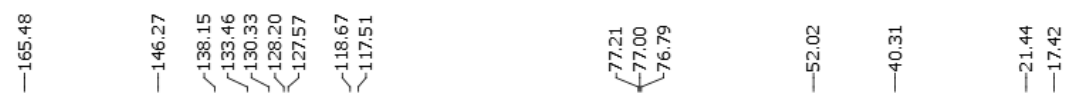

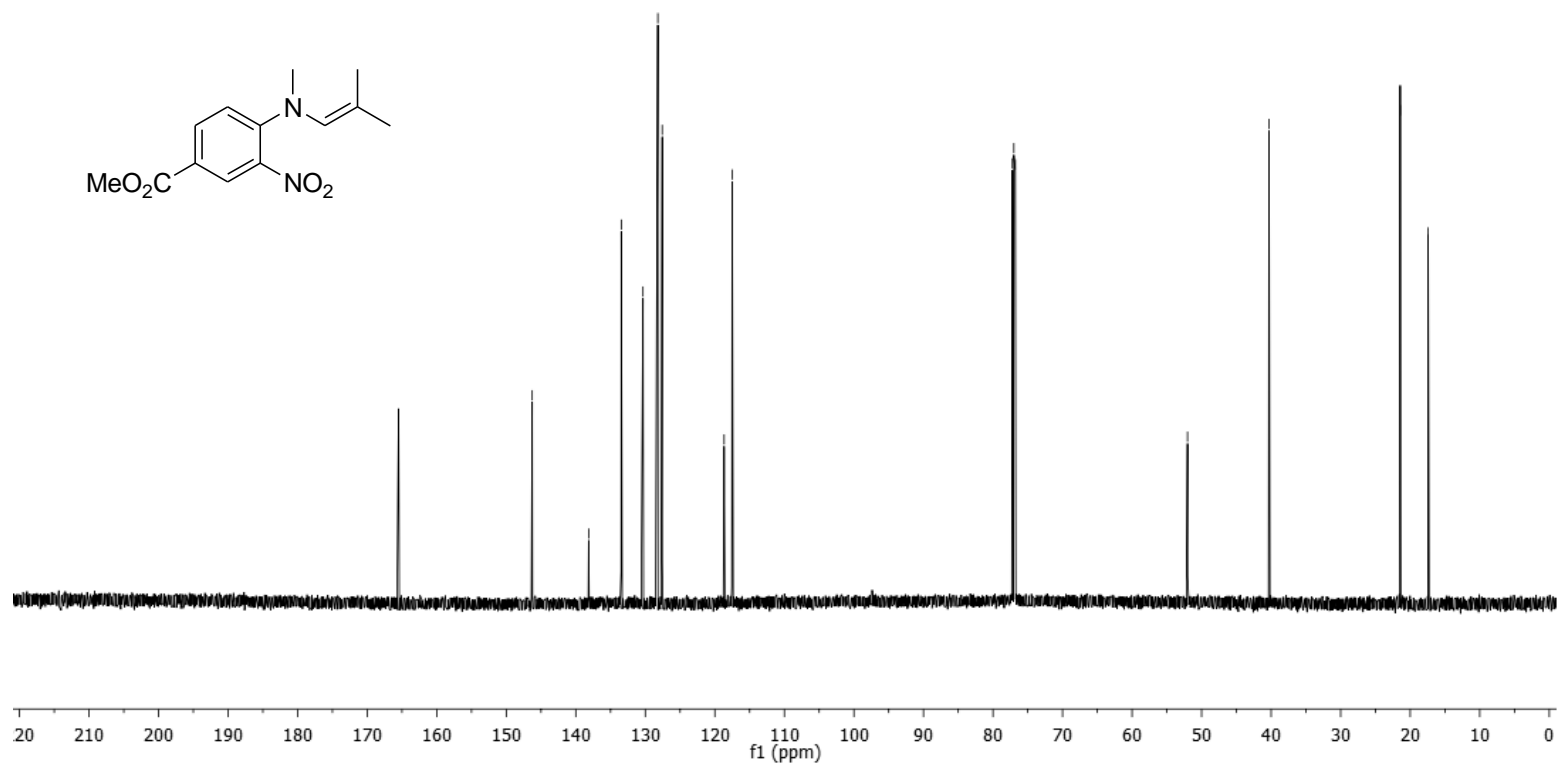

Figure 4.51 ${ }^{1} \mathrm{H}$ and ${ }^{13} \mathrm{C}$ NMR of 4-carbomethoxy- $N$-methyl- $N$-(2-methyl-1-propen-1-yl)-2-nitroaniline 


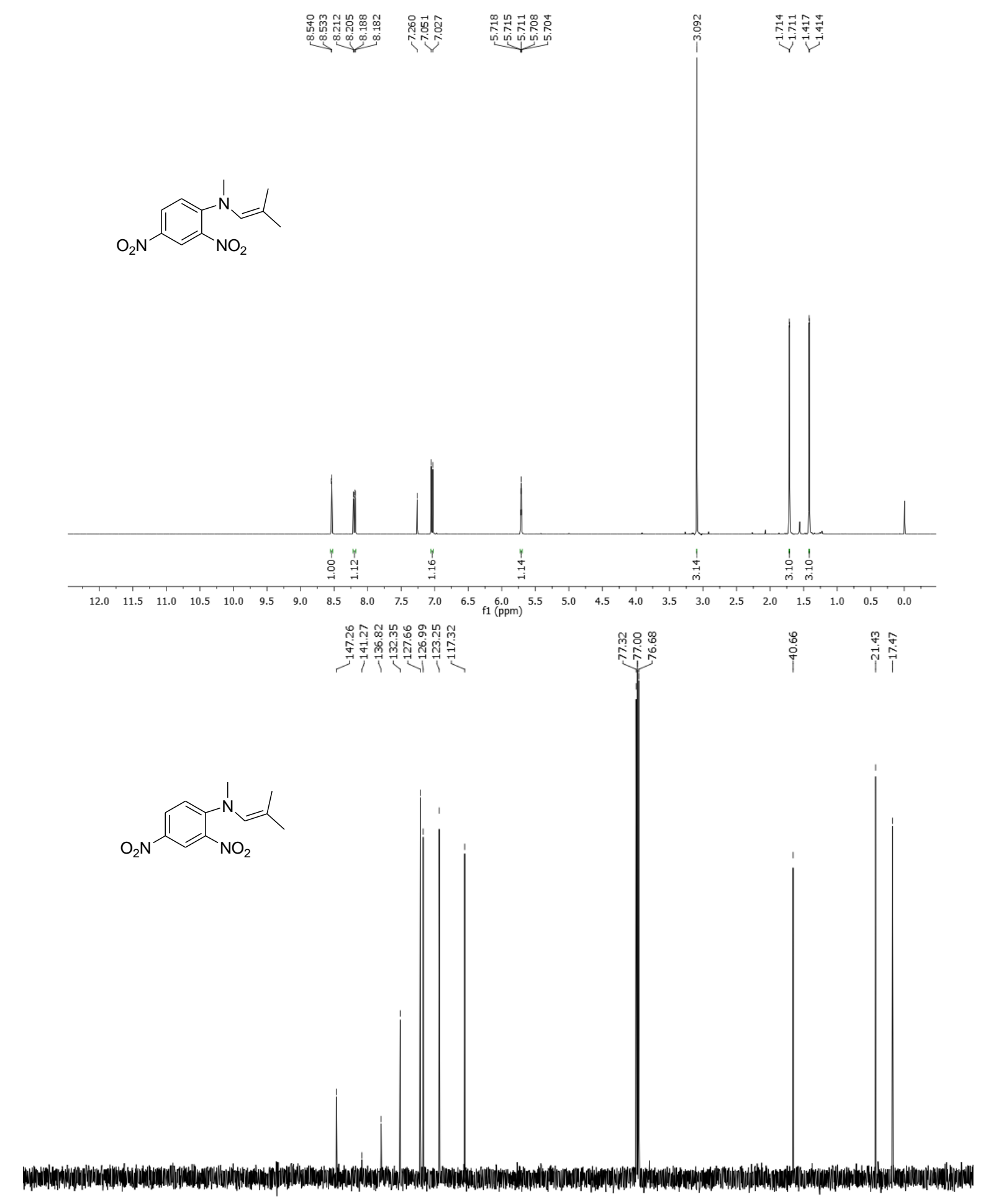

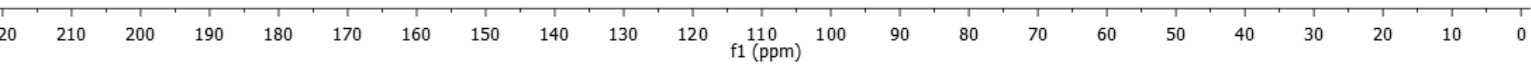

Figure 4.52 ${ }^{1} \mathrm{H}$ and ${ }^{13} \mathrm{C}$ NMR of 2,4-dinitro- $N$-methyl- $N$-(2-methyl-1-propen-1-yl)aniline 


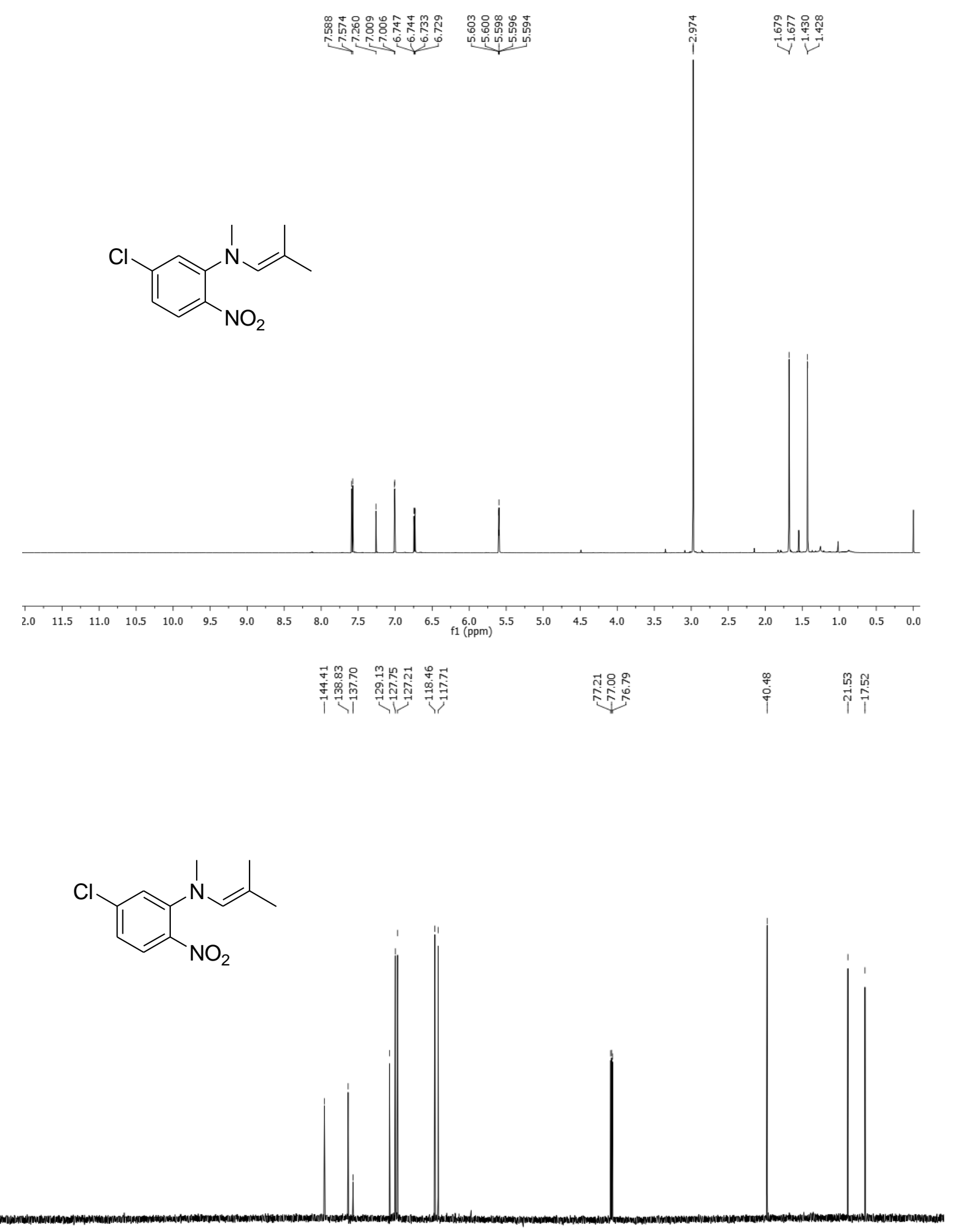

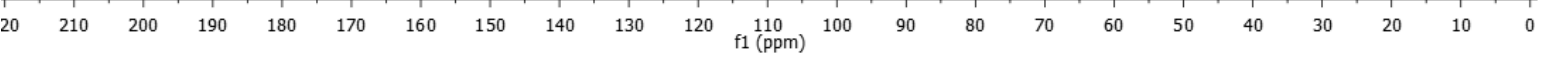

Figure 4.53 ${ }^{1} \mathrm{H}$ and ${ }^{13} \mathrm{C}$ NMR of 5-chloro- $N$-(2-methyl-1-propen-1-yl)-2-nitroaniline 

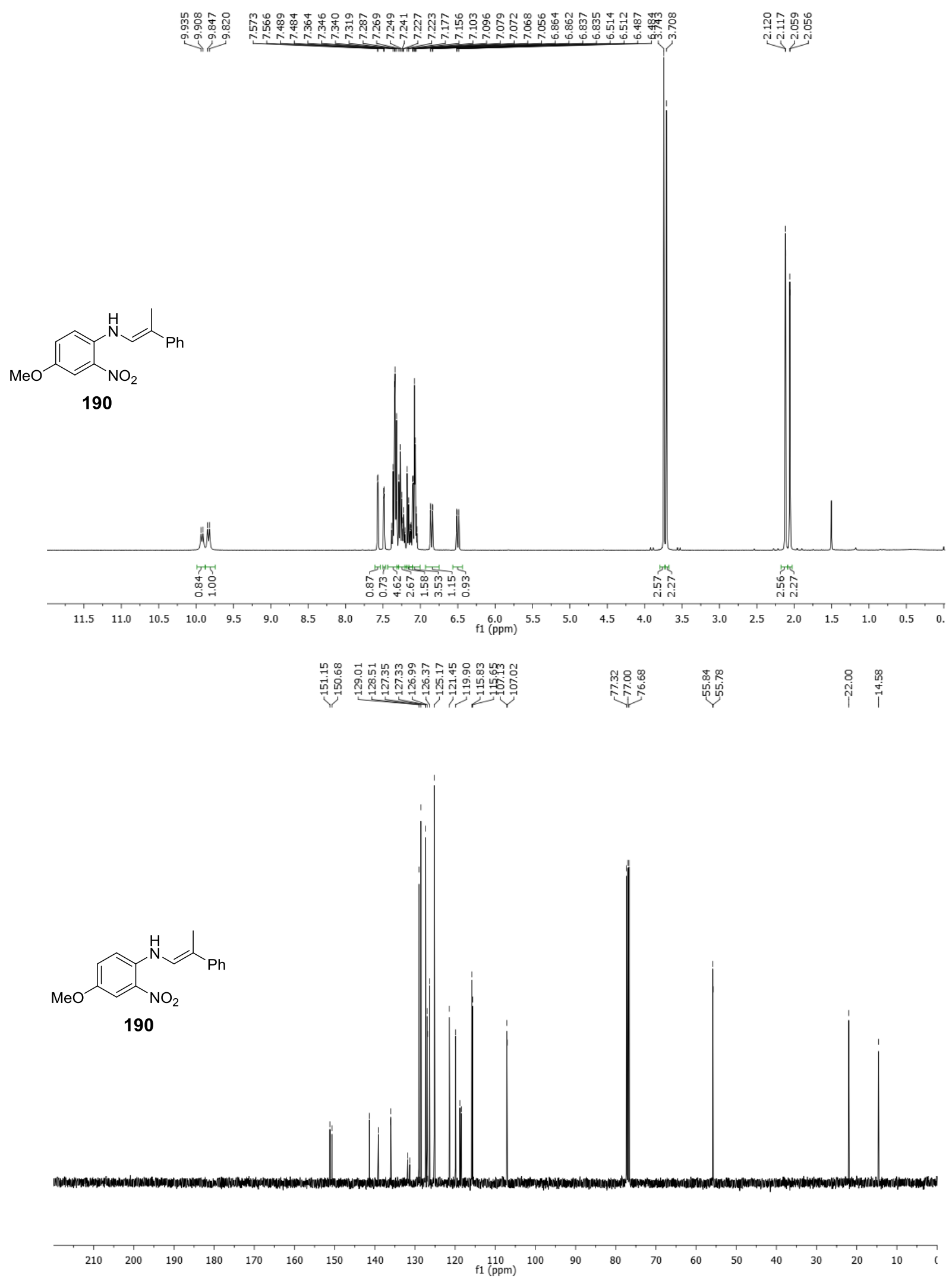

Figure 5.1 ${ }^{1} \mathrm{H}$ and ${ }^{13} \mathrm{C}$ NMR of compound 190 

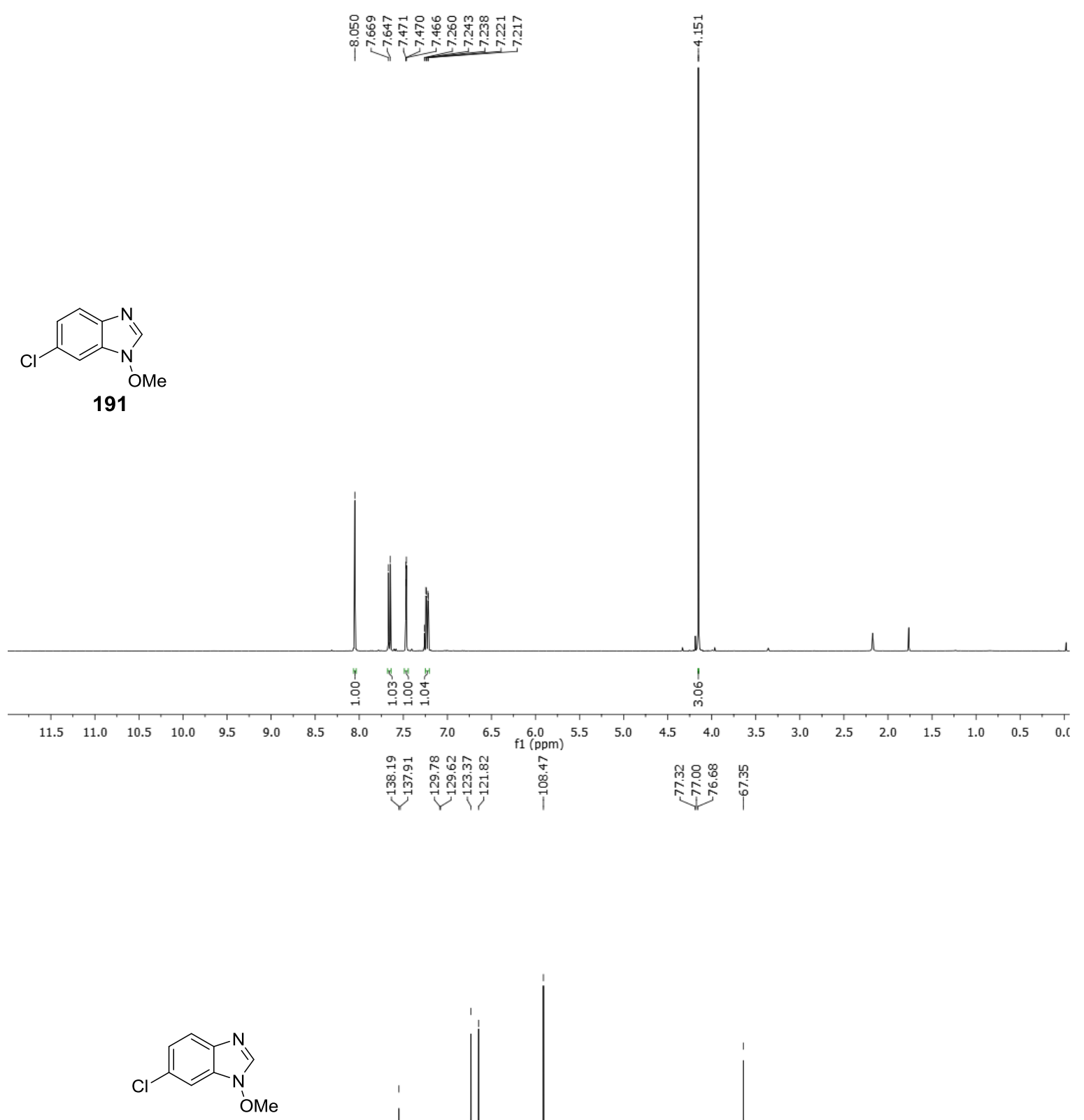

191
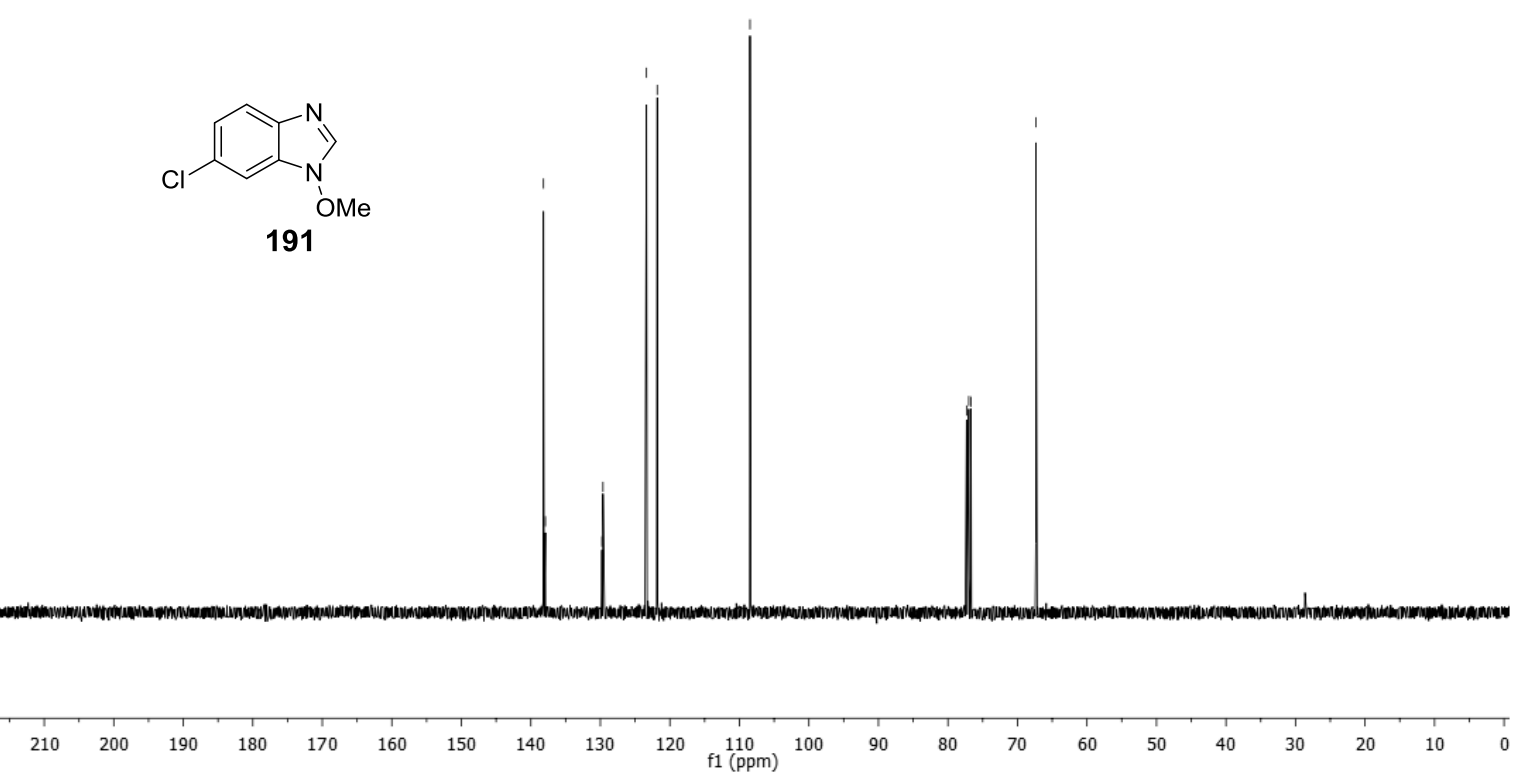

Figure 5.2 ${ }^{1} \mathrm{H}$ and ${ }^{13} \mathrm{C}$ NMR of compound 191 

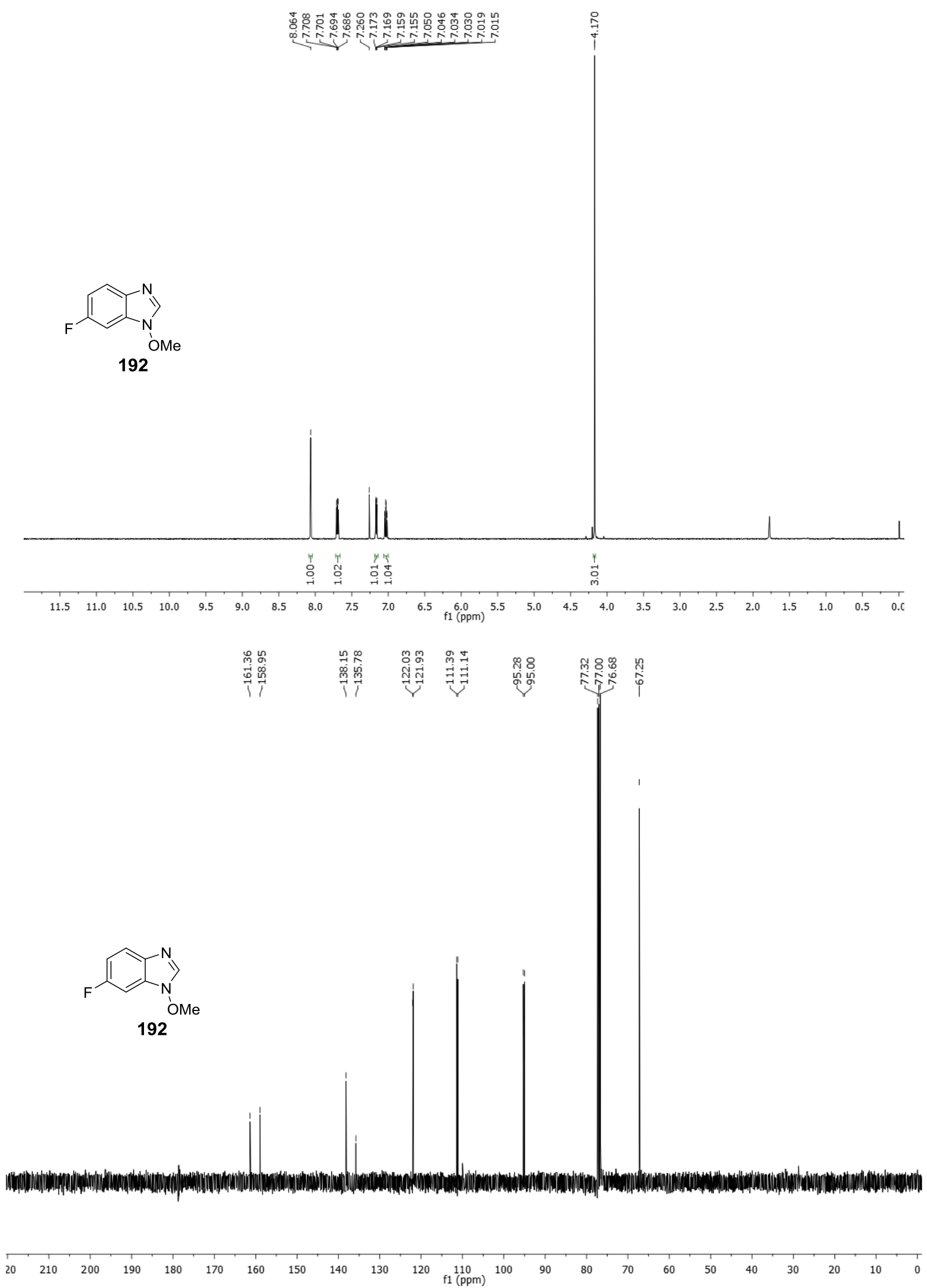

Figure 5.3 ${ }^{1} \mathrm{H}$ and ${ }^{13} \mathrm{C}$ NMR of compound 192 

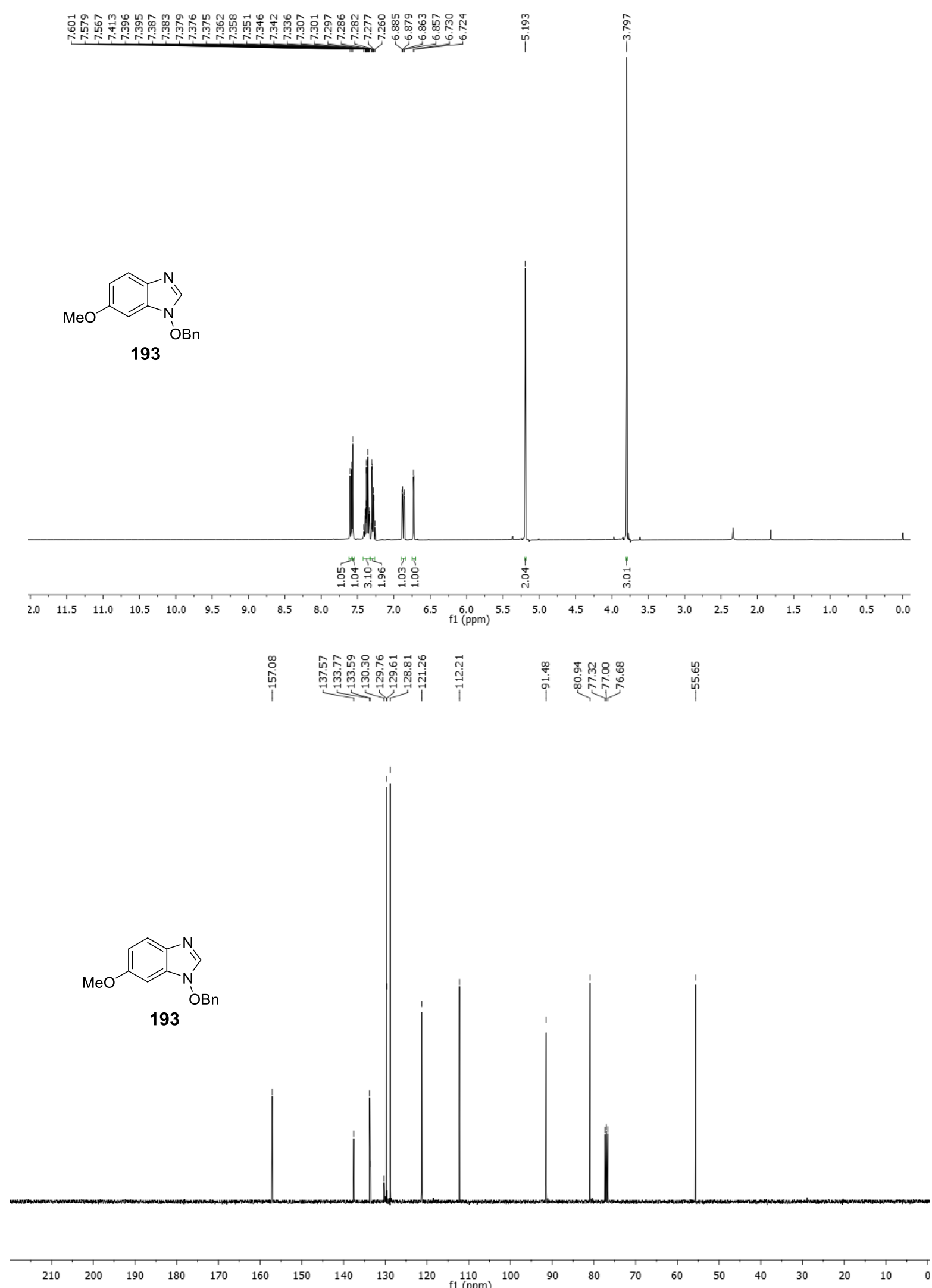

Figure 5.4 ${ }^{1} \mathrm{H}$ and ${ }^{13} \mathrm{C}$ NMR of compound 193 

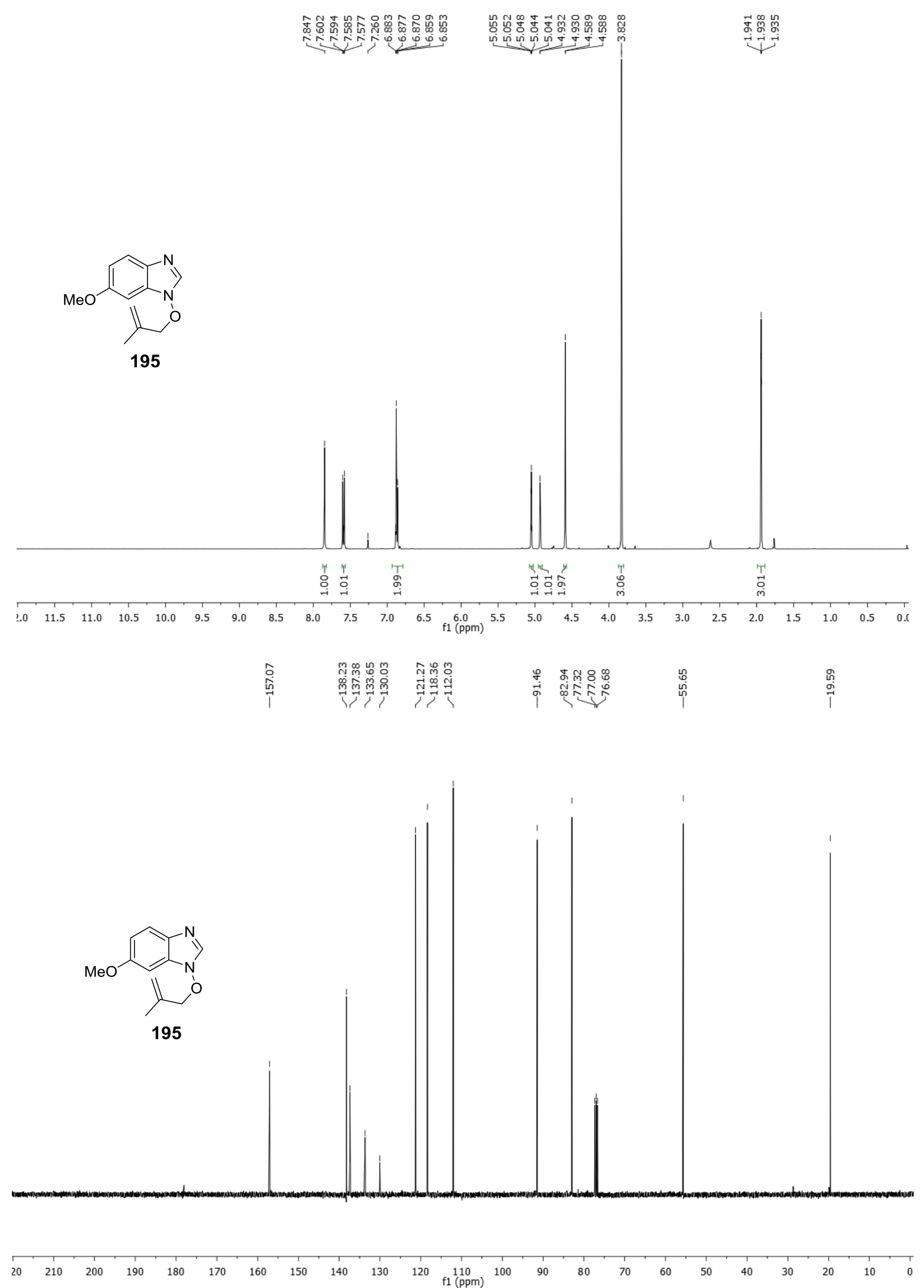

Figure 5.5 ${ }^{1} \mathrm{H}$ and ${ }^{13} \mathrm{C}$ NMR of compound 195 


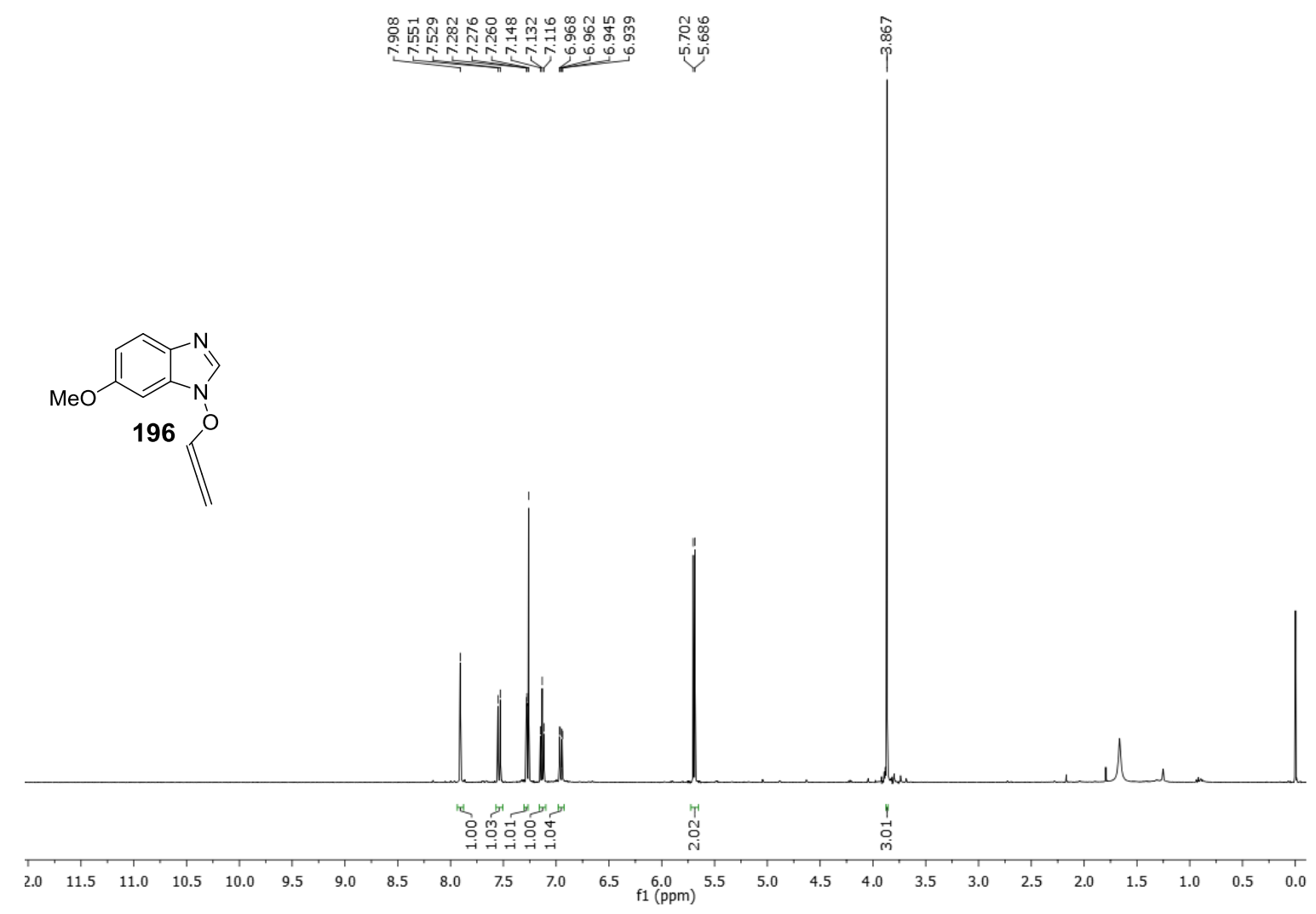

๙

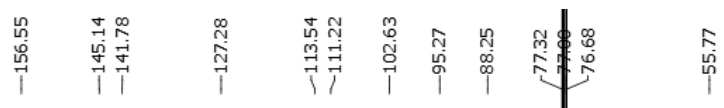

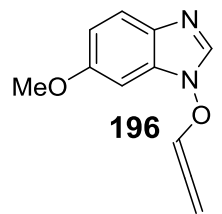

$20 \quad 210$

$190 \quad 1$

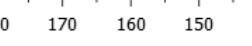

Figure 5.6 ${ }^{1} \mathrm{H}$ and ${ }^{13} \mathrm{C}$ NMR of compound 196 

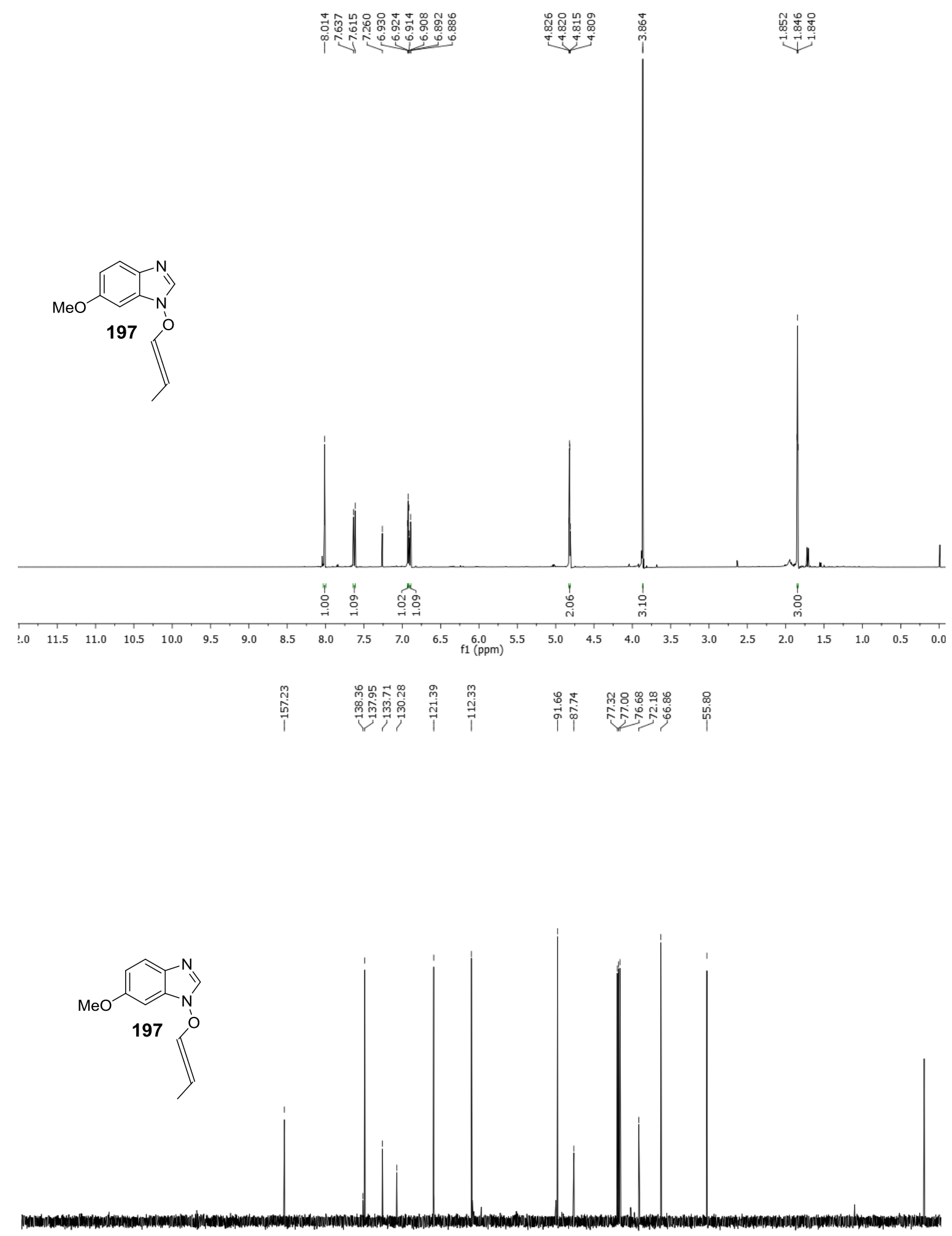

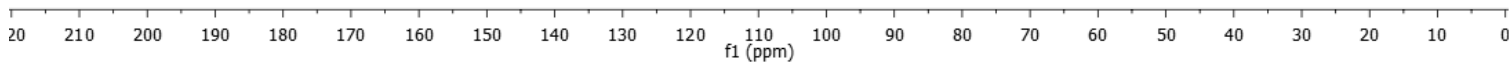

Figure $5.7{ }^{1} \mathrm{H}$ and ${ }^{13} \mathrm{C}$ NMR of compound 197 

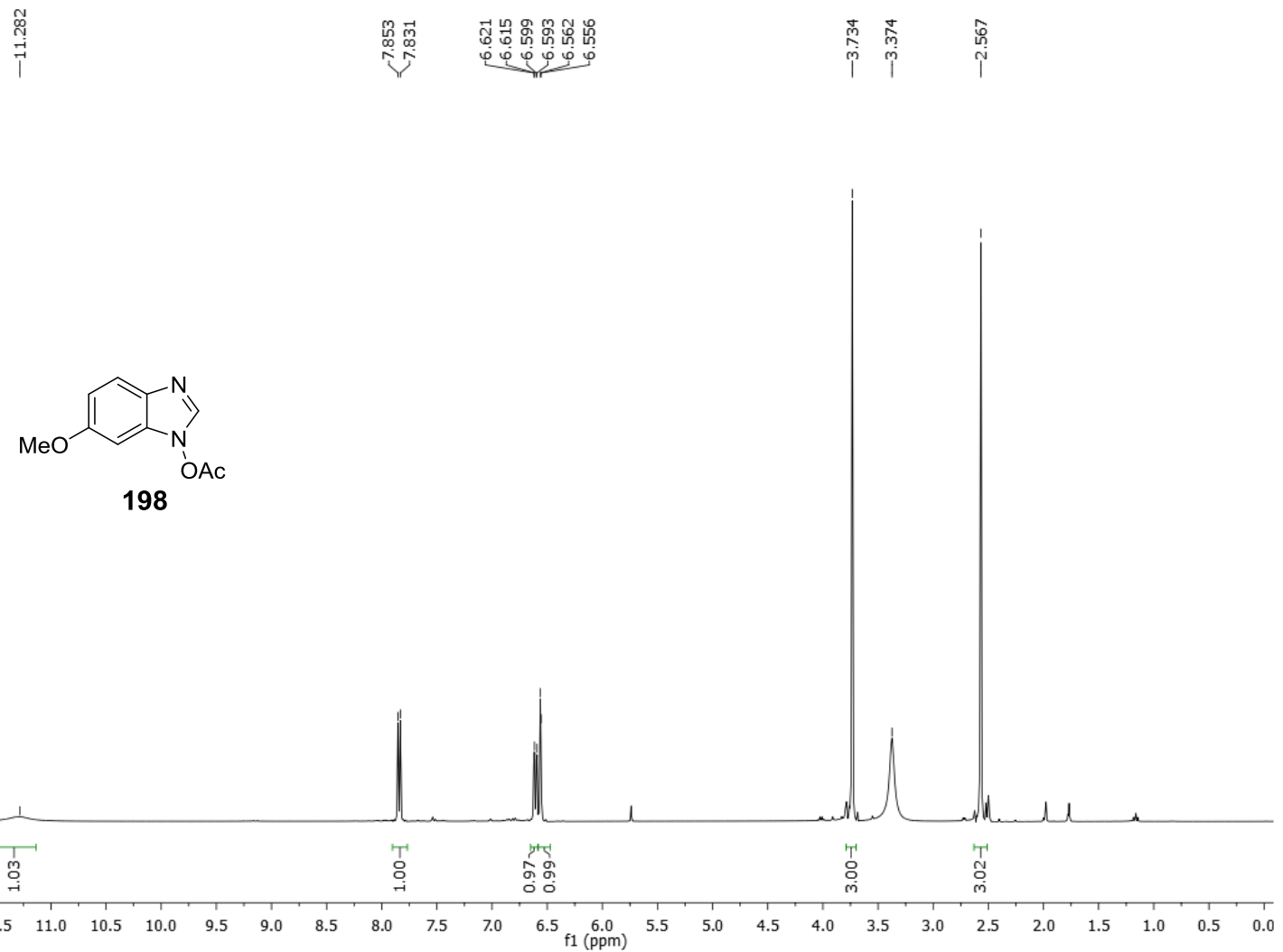

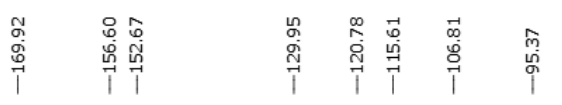

우

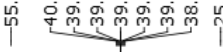

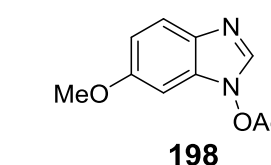

98

$20 \quad 210 \quad 200 \quad 190 \quad 180$
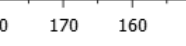

140

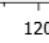

Figure $5.8{ }^{1} \mathrm{H}$ and ${ }^{13} \mathrm{C}$ NMR of compound 198 

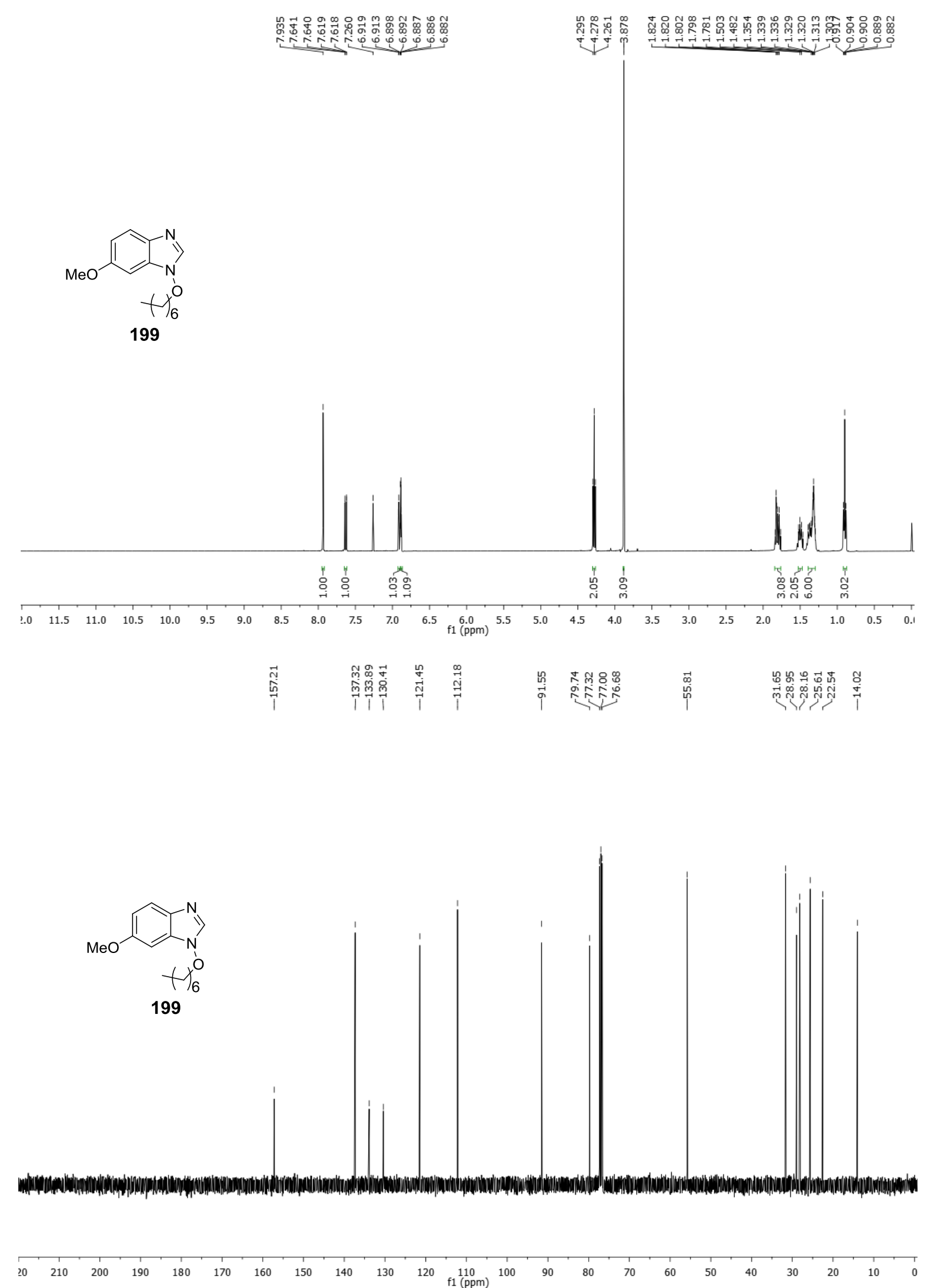

Figure 5.9 ${ }^{1} \mathrm{H}$ and ${ }^{13} \mathrm{C}$ NMR of compound 199 

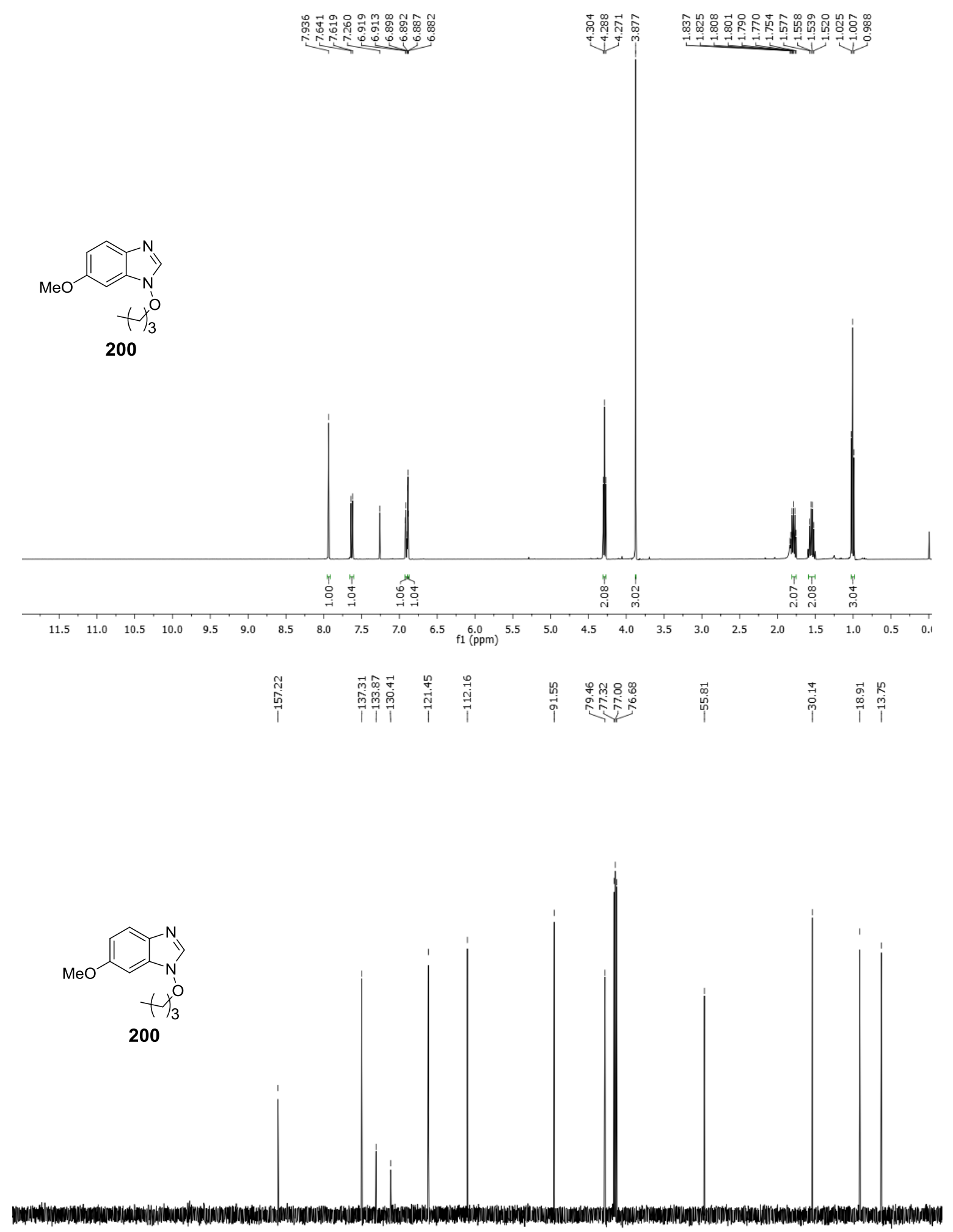

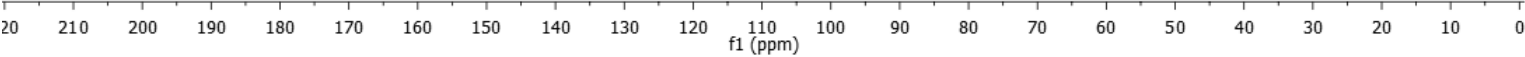

Figure 5.10 ${ }^{1} \mathrm{H}$ and ${ }^{13} \mathrm{C}$ NMR of compound 200 


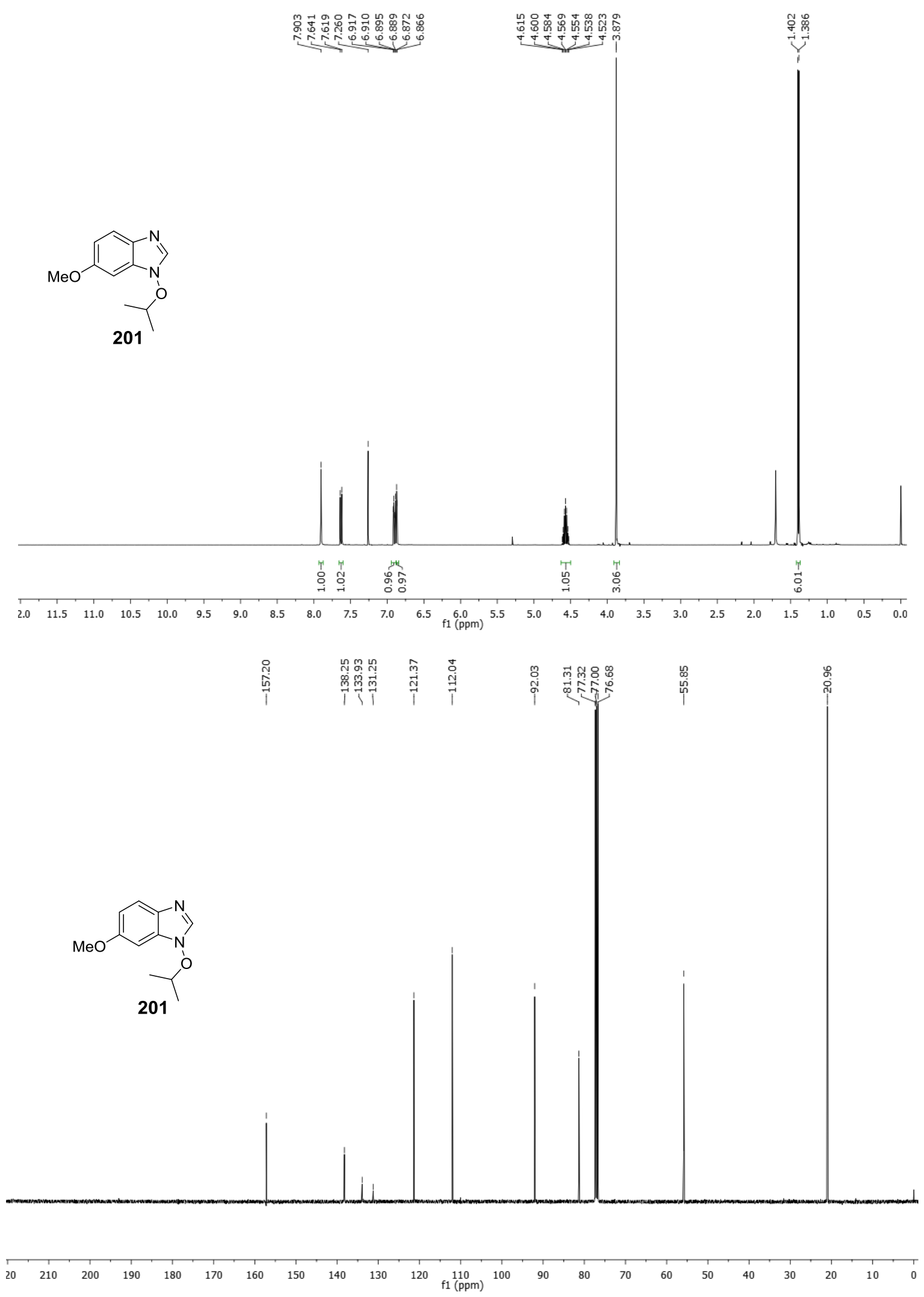

Figure 5.11 ${ }^{1} \mathrm{H}$ and ${ }^{13} \mathrm{C}$ NMR of compound 201 


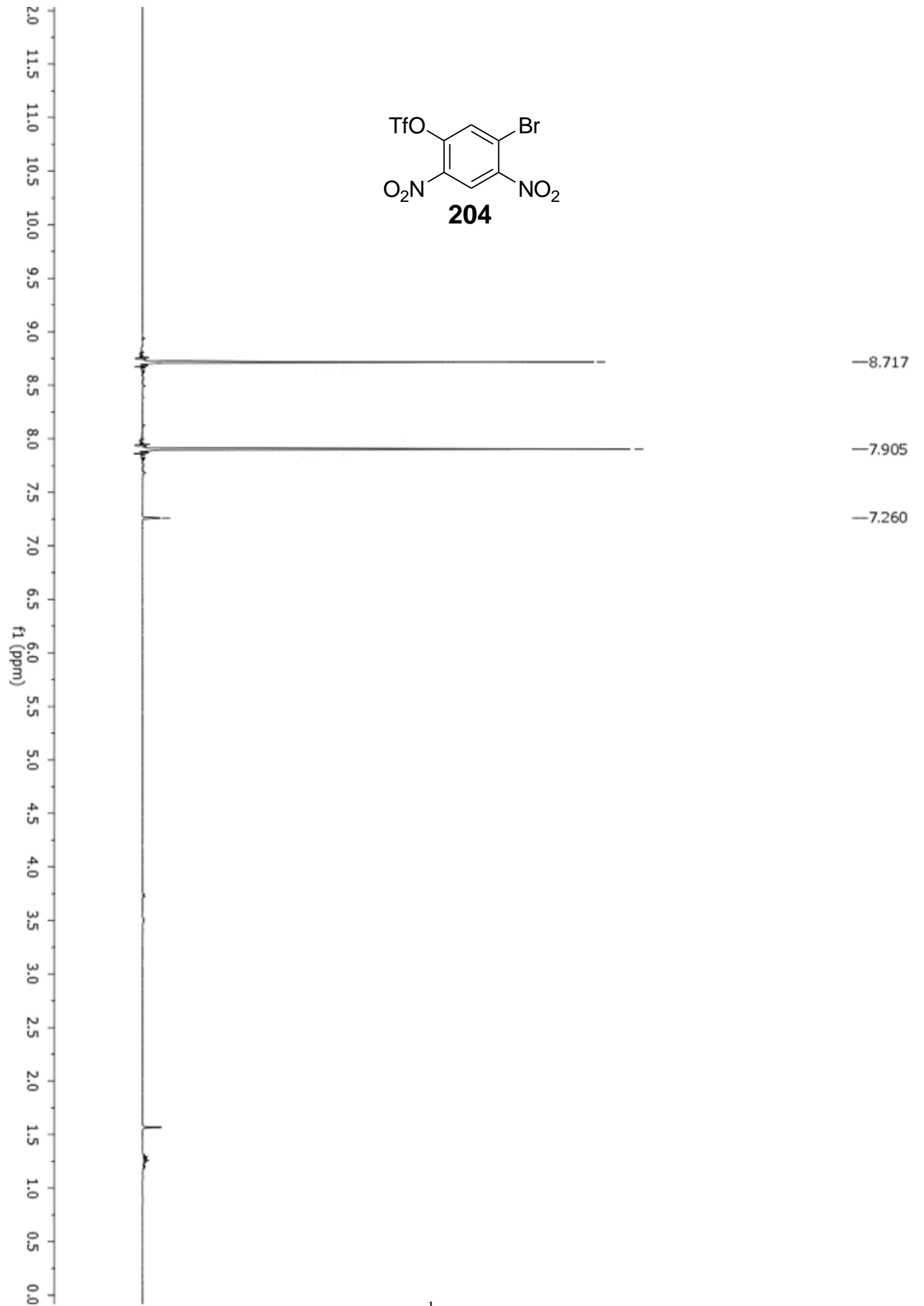

Figure 6.2 ${ }^{1} \mathrm{H}$ of compound 204 


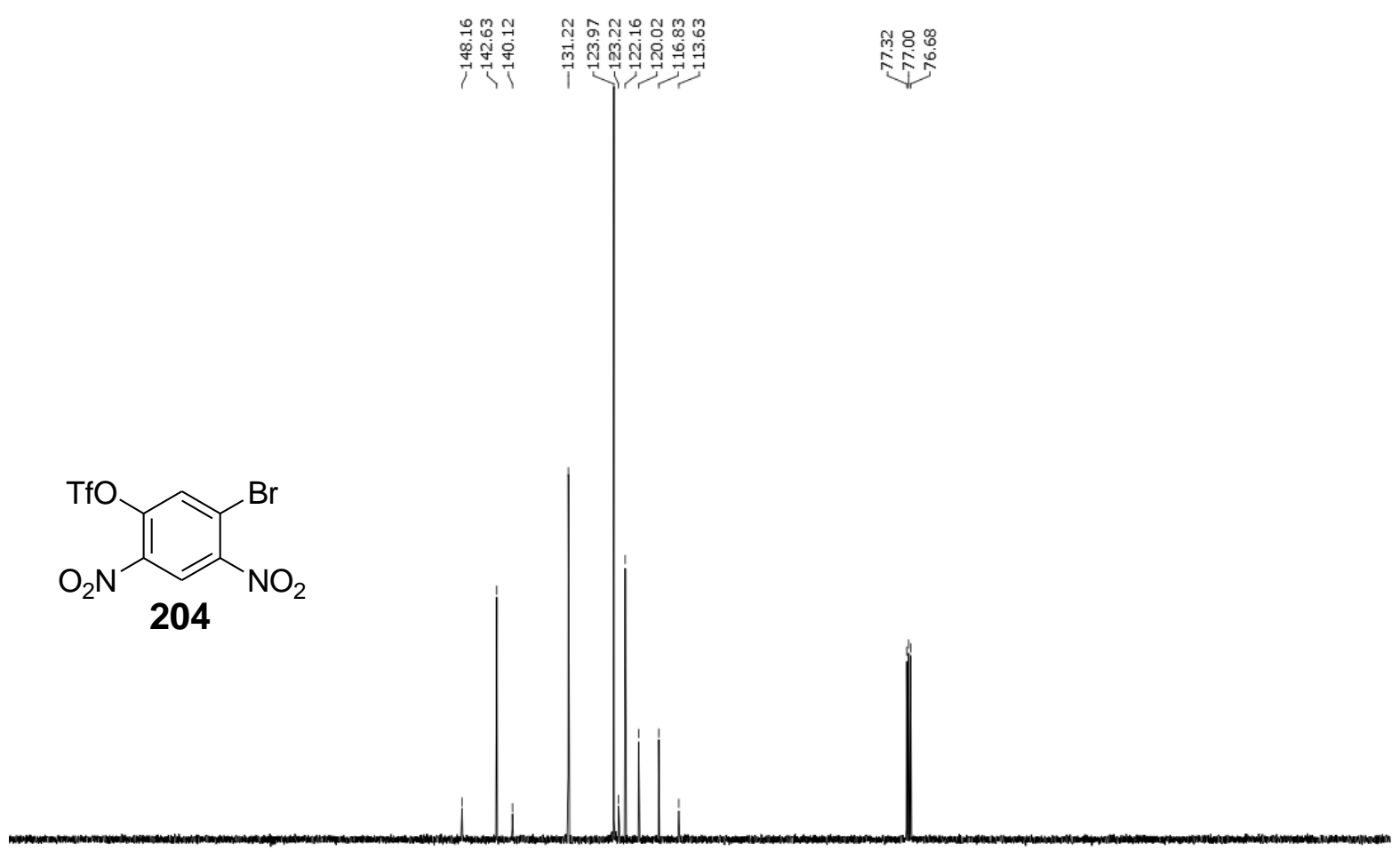

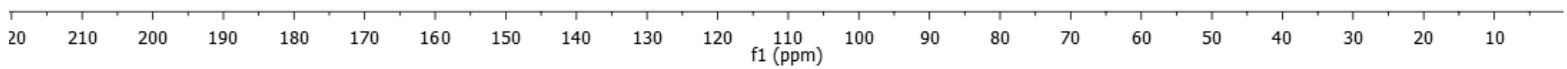

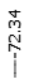
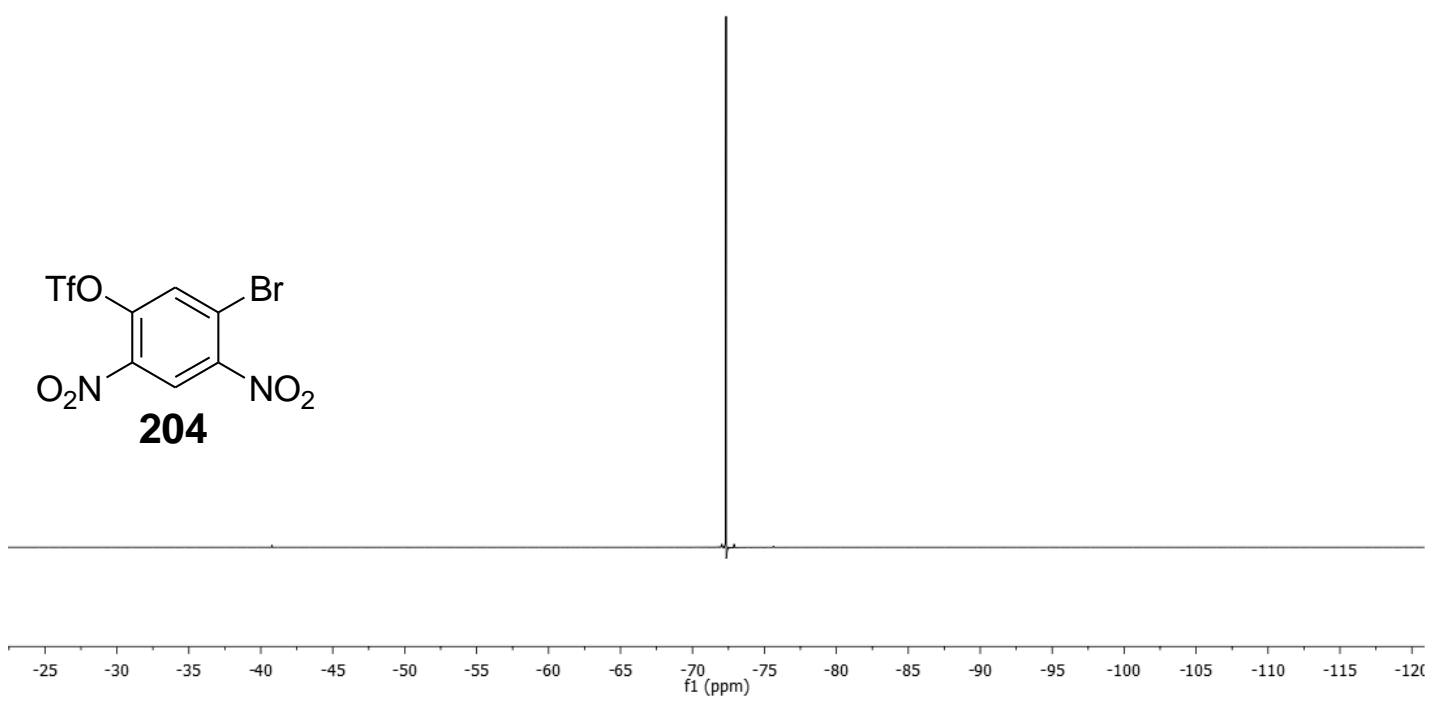

Figure 6.3 ${ }^{13} \mathrm{C}$ and ${ }^{19} \mathrm{~F}$ NMR of compound 204 


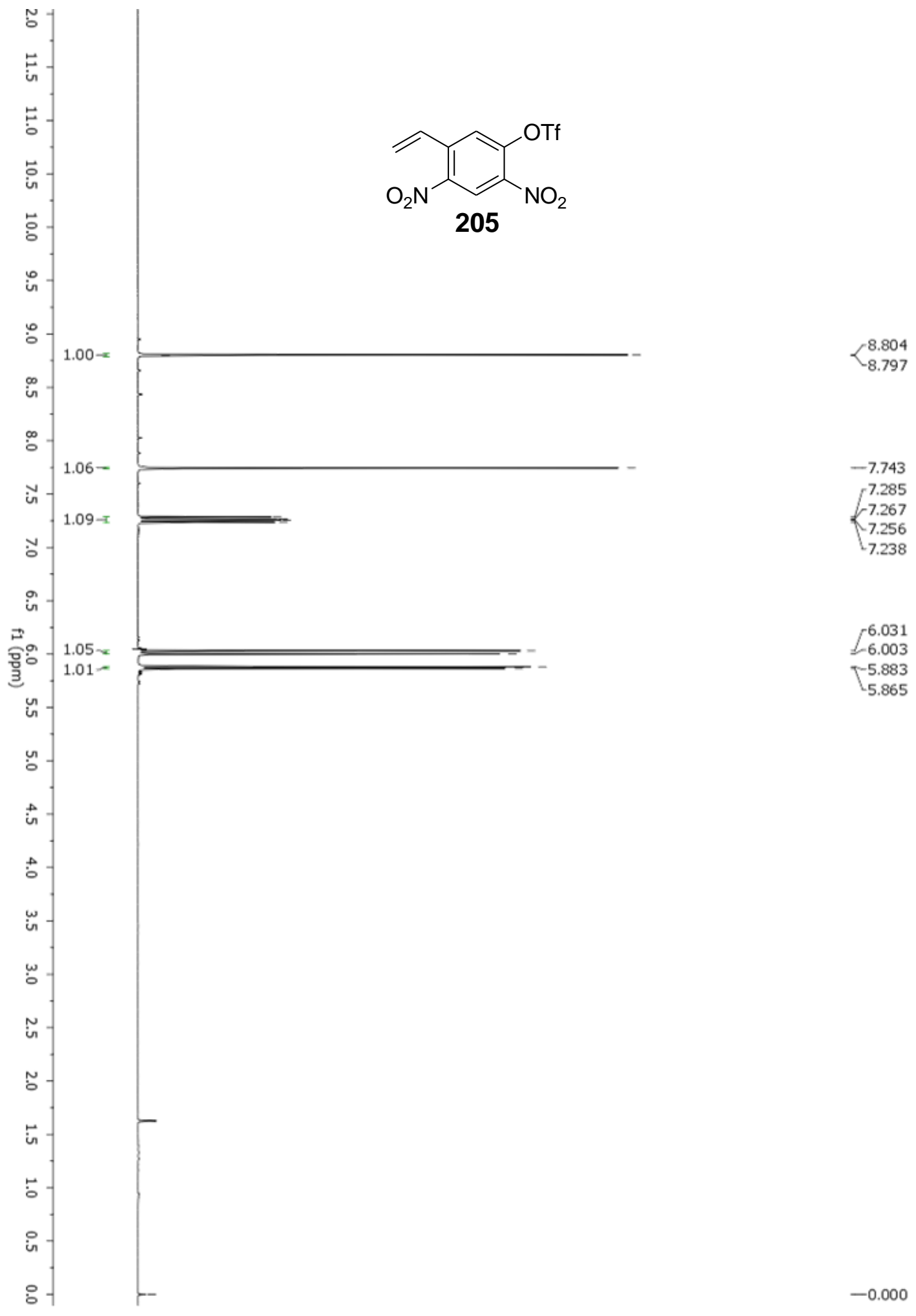

Figure 6.4 ${ }^{1} \mathrm{H}$ NMR of compound 205 

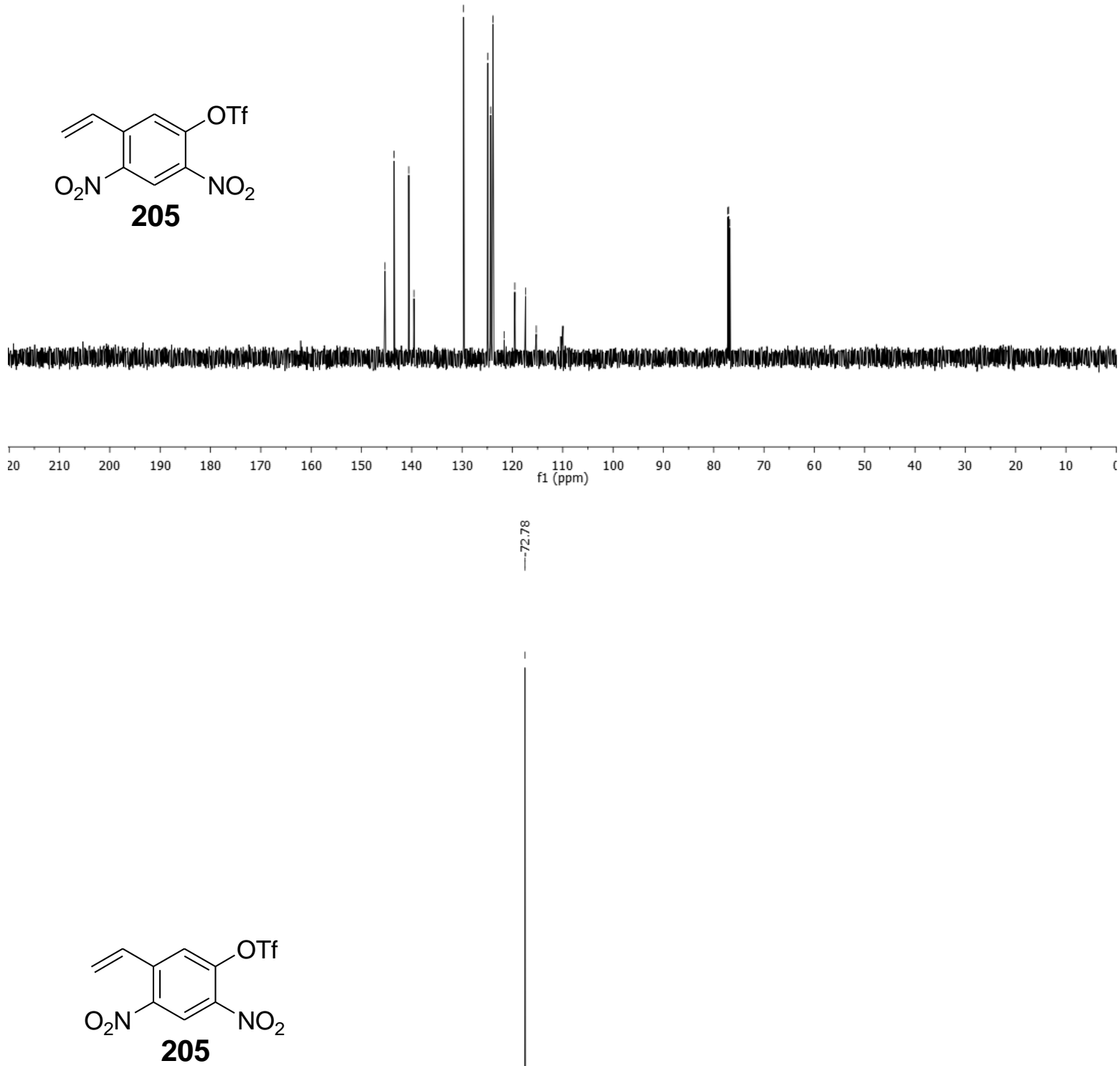

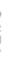

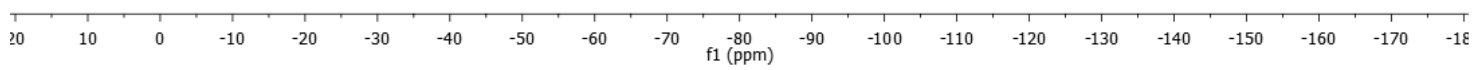

Figure $6.5{ }^{13} \mathrm{C}$ and ${ }^{19} \mathrm{~F}$ NMR of compound 205 

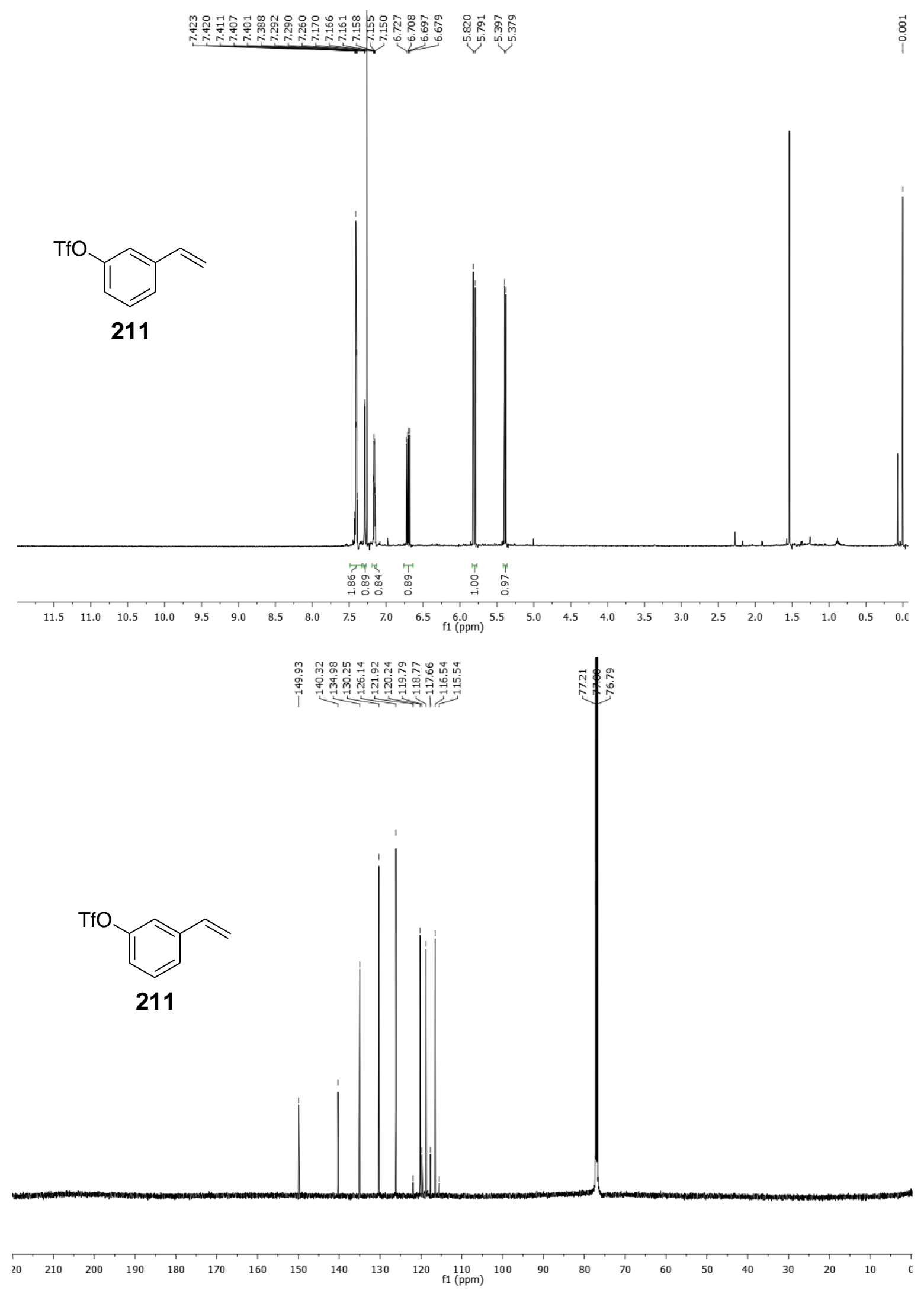

Figure 6.6 ${ }^{1} \mathrm{H}$ and ${ }^{13} \mathrm{C}$ NMR of compound 211 

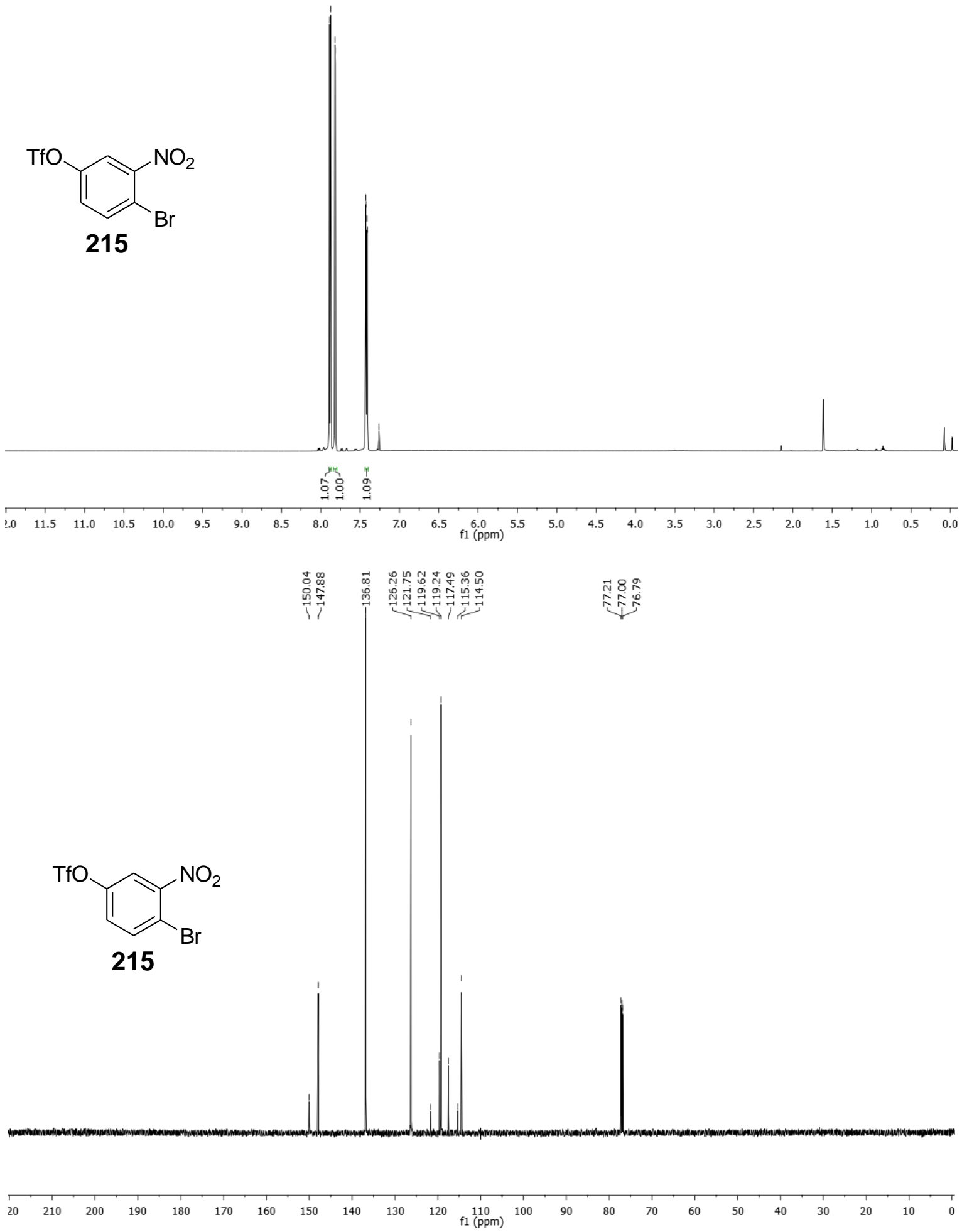

Figure 6.7 ${ }^{1} \mathrm{H}$ and ${ }^{13} \mathrm{C}$ NMR of compound 215 


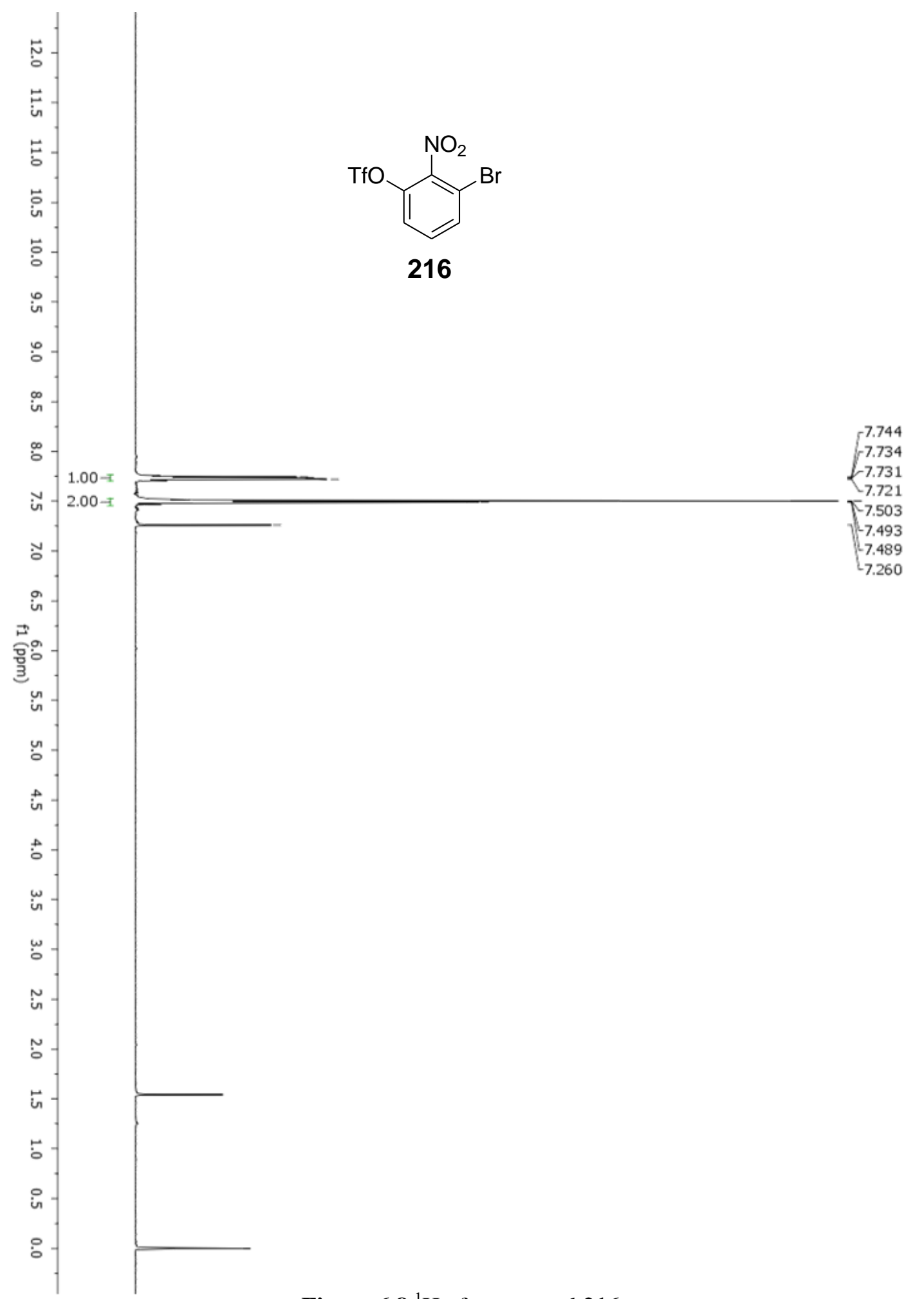

Figure $6.8{ }^{1} \mathrm{H}$ of compound 216 

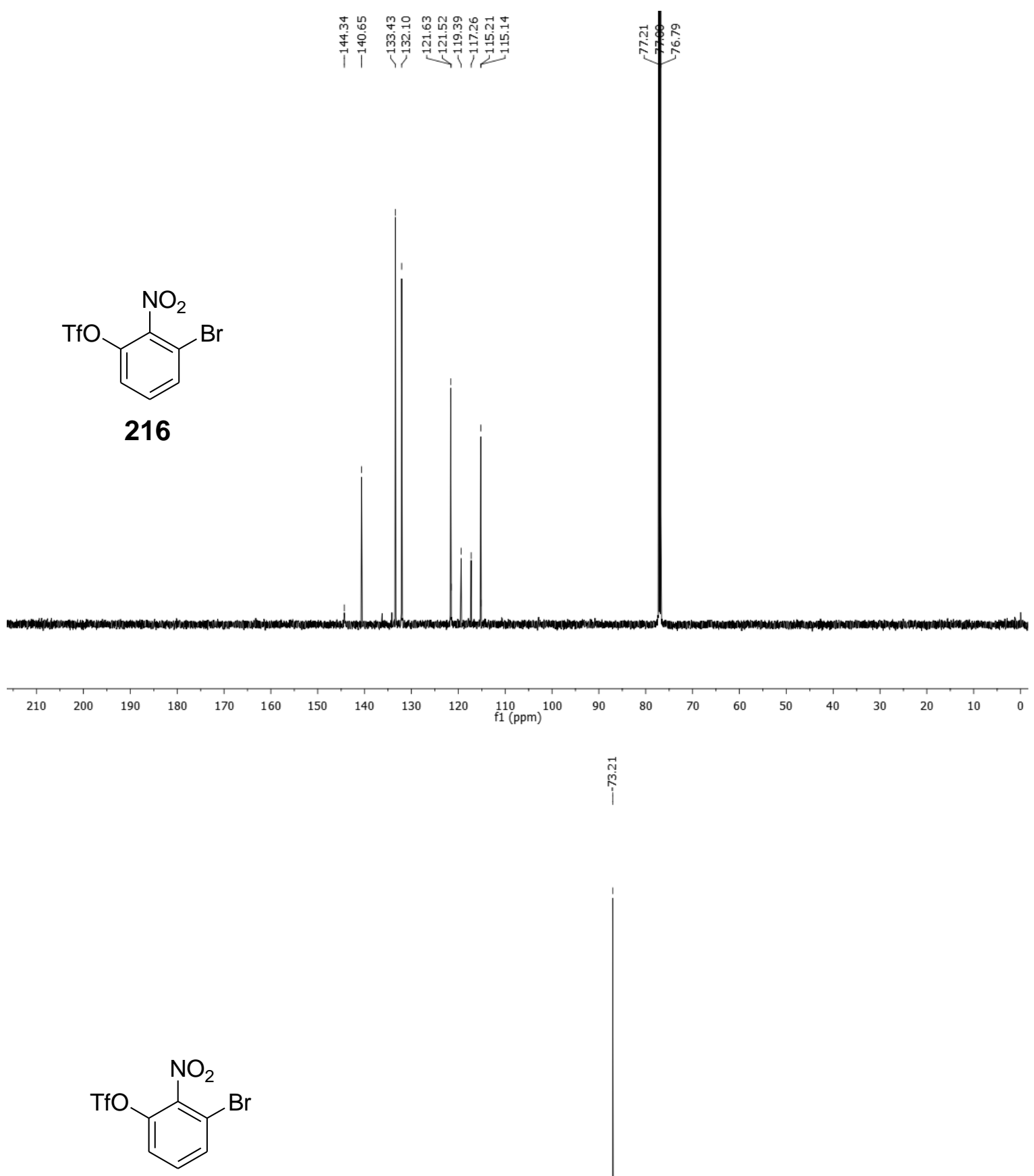

216

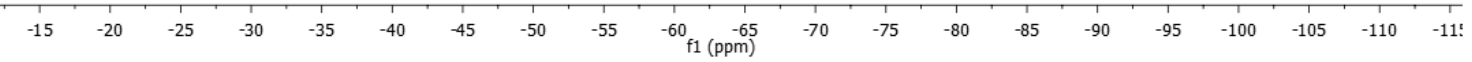

Figure 6.9 ${ }^{13} \mathrm{C}$ and ${ }^{19} \mathrm{~F}$ NMR of compound 216 


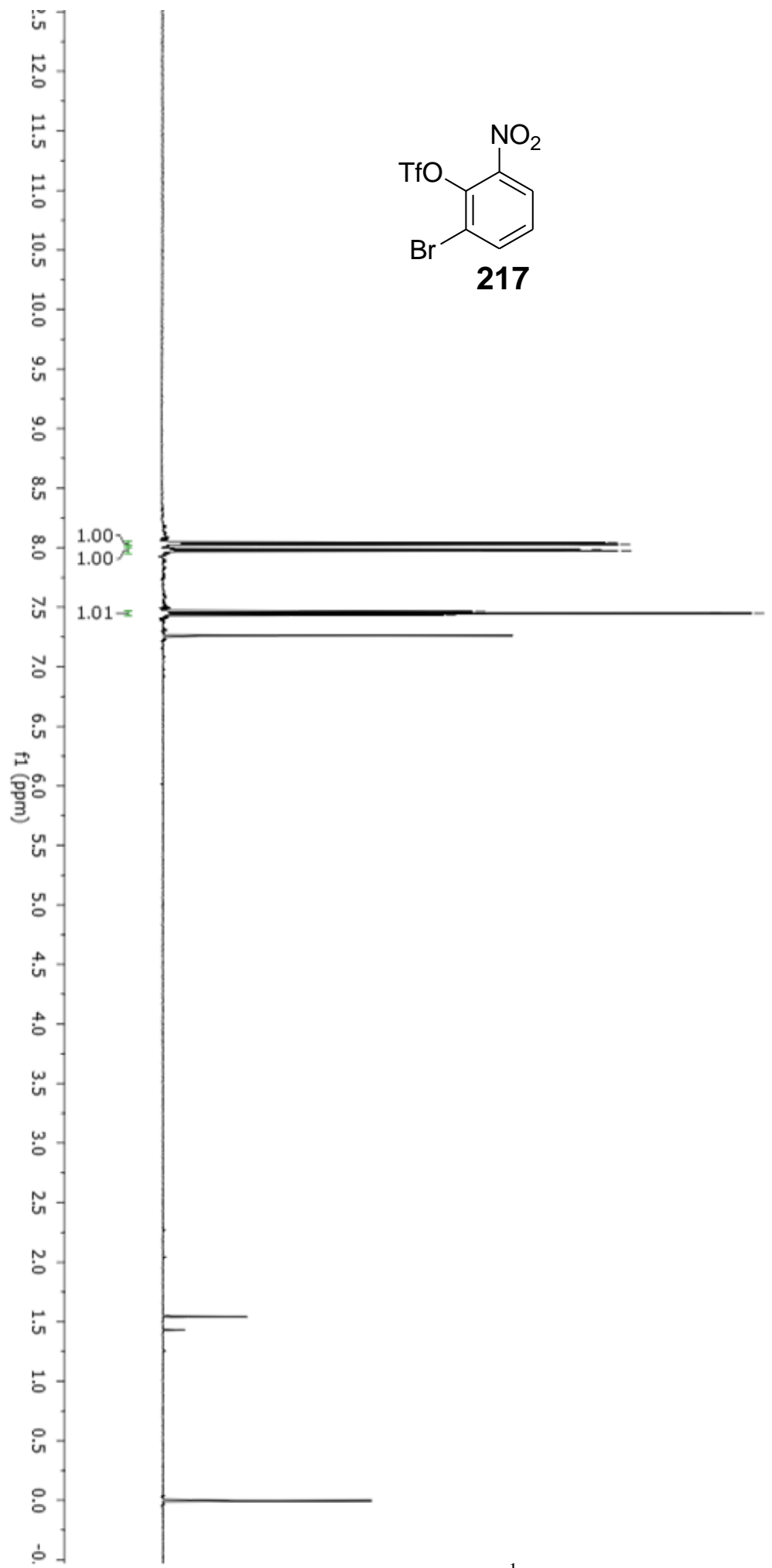

8.043
-8.041
-8.030
-8.027
-7.987
7.984
-7.973
7.971
-7.463
-7.461
-7.449
-7.448
-7.436
7.434

Figure 6.10 ${ }^{1} \mathrm{H}$ of compound 217 


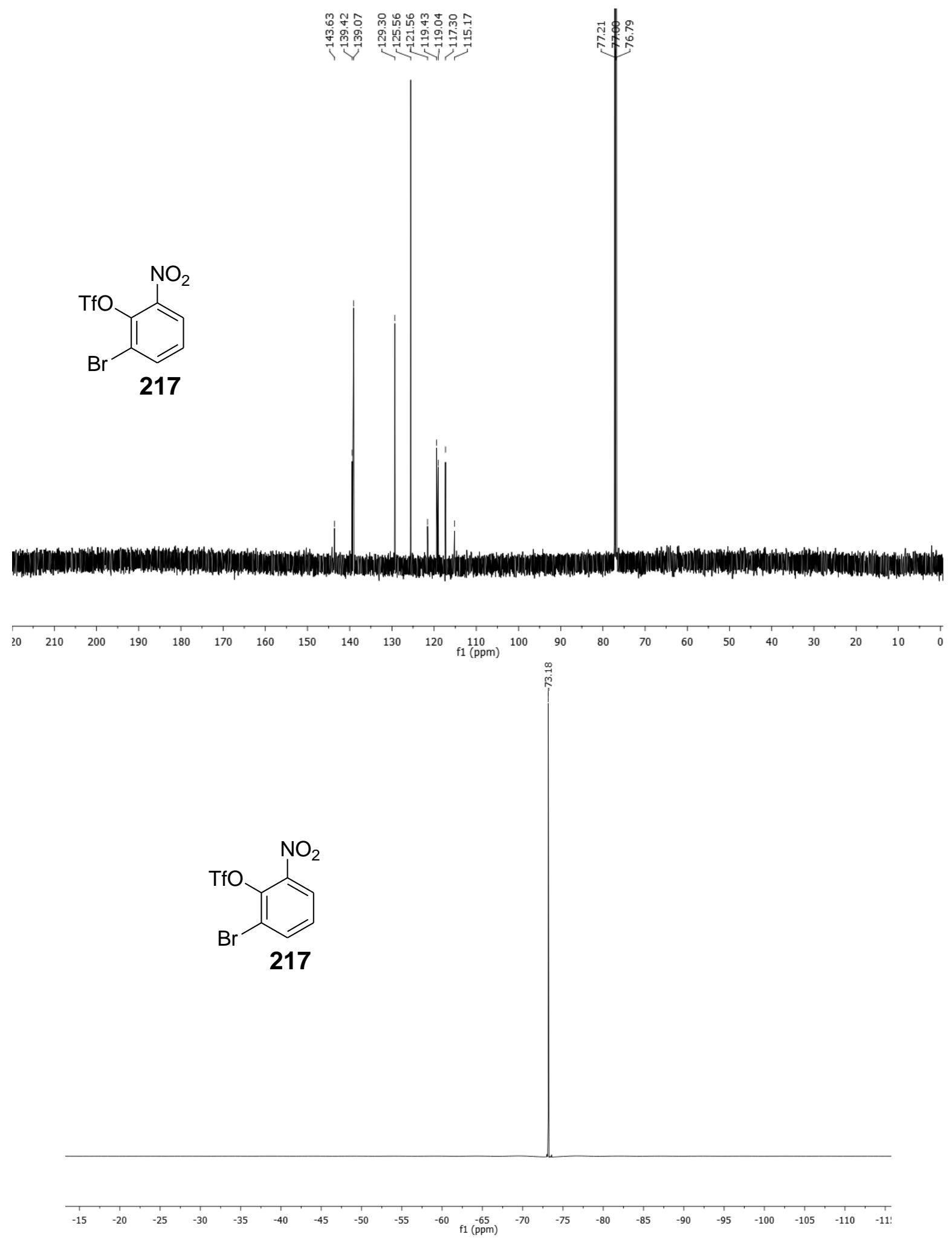

Figure 6.11 ${ }^{13} \mathrm{C}$ and ${ }^{19} \mathrm{~F}$ NMR of compound 217 


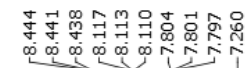
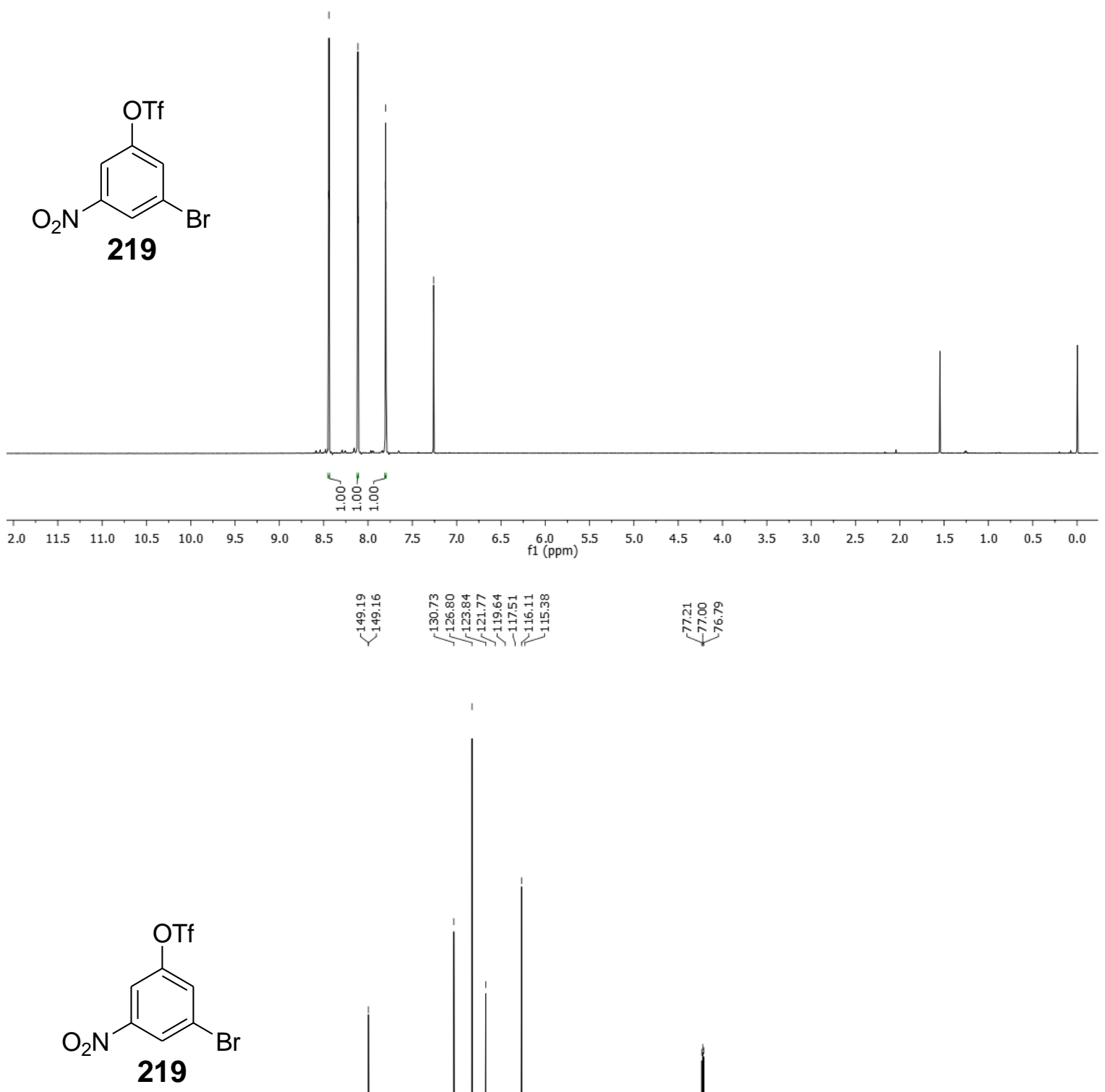

$20 \quad 210 \quad 200$
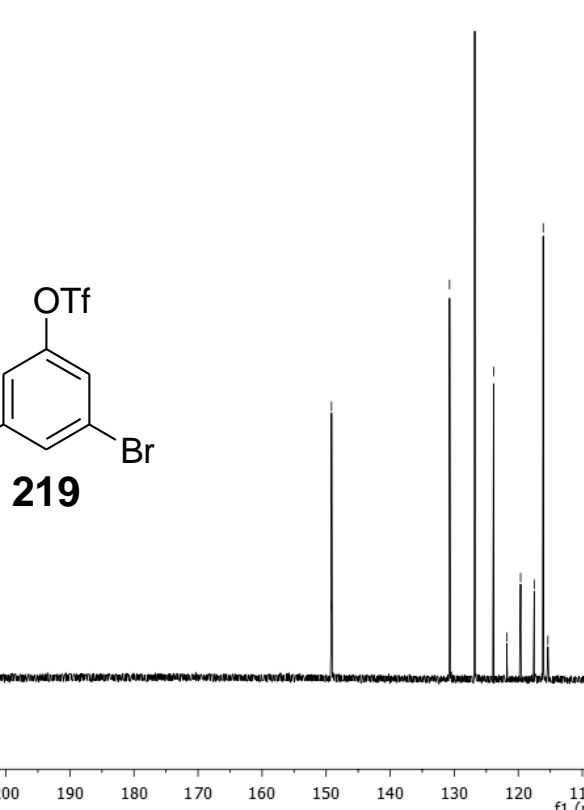

Figure 6.12 ${ }^{1} \mathrm{H}$ and ${ }^{13} \mathrm{C}$ NMR of compound 219 
<smiles>O=[N+]([O-])c1ccc(Br)c([O+])c1</smiles>
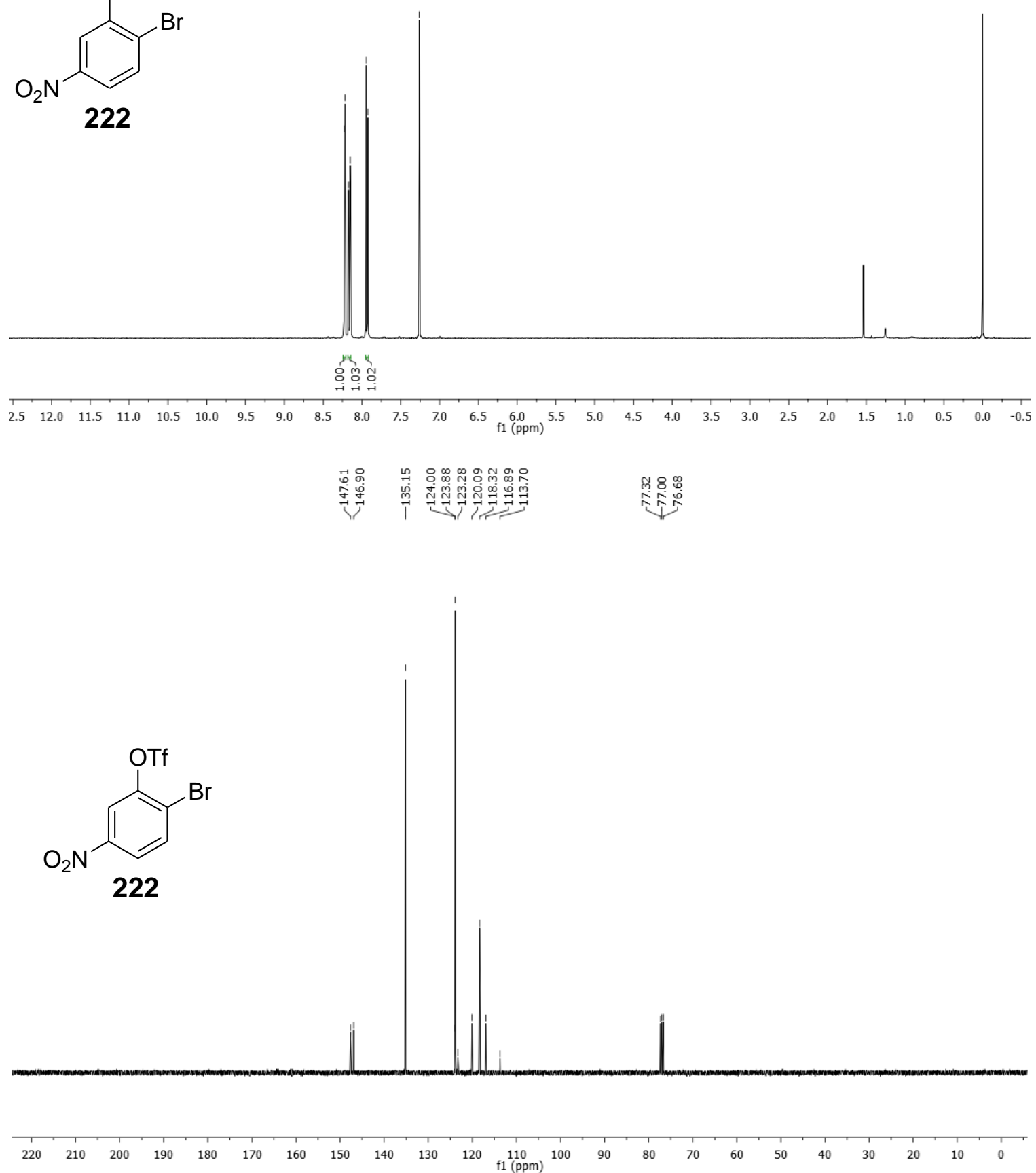

Figure 6.13 ${ }^{1} \mathrm{H}$ and ${ }^{13} \mathrm{C}$ NMR of compound 222 


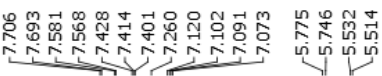
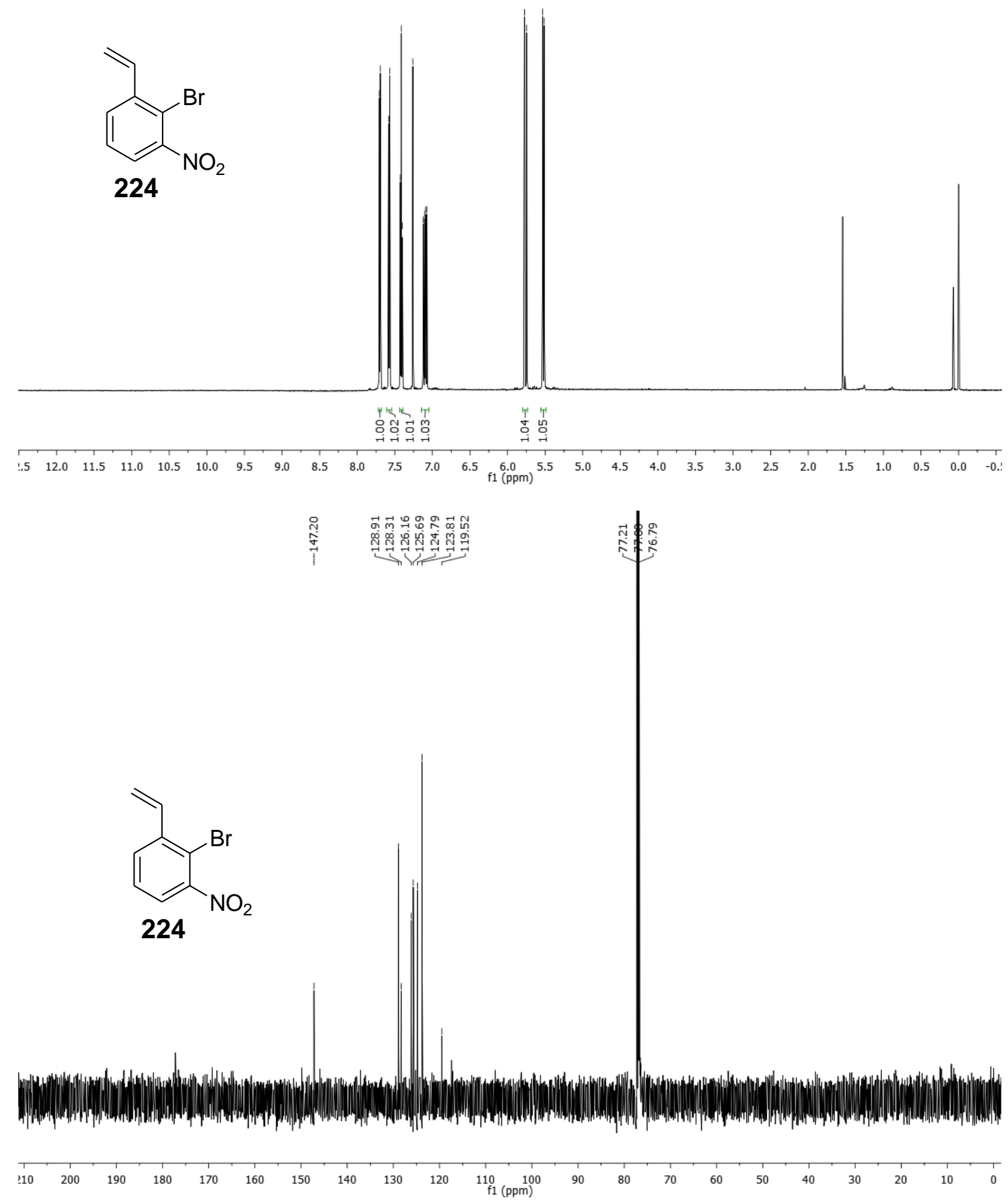

Figure 6.14 ${ }^{1} \mathrm{H}$ and ${ }^{13} \mathrm{C}$ NMR of compound 224 


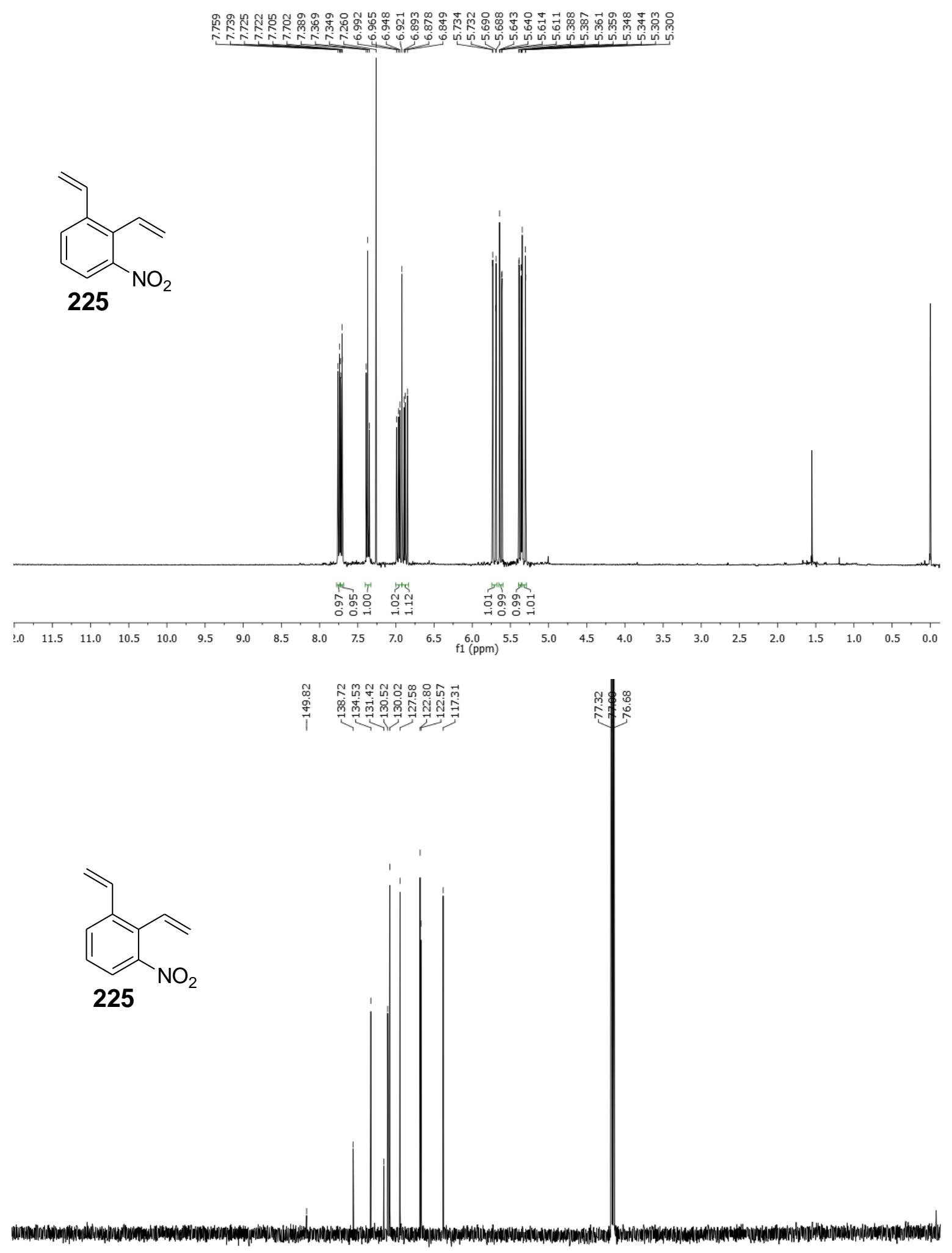

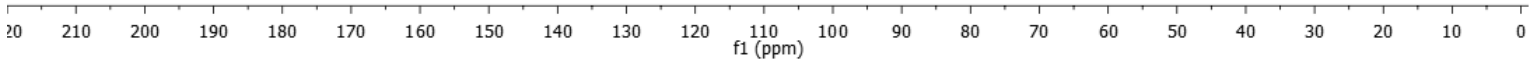

Figure 6.15 ${ }^{1} \mathrm{H}$ and ${ }^{13} \mathrm{C}$ NMR of compound 225 

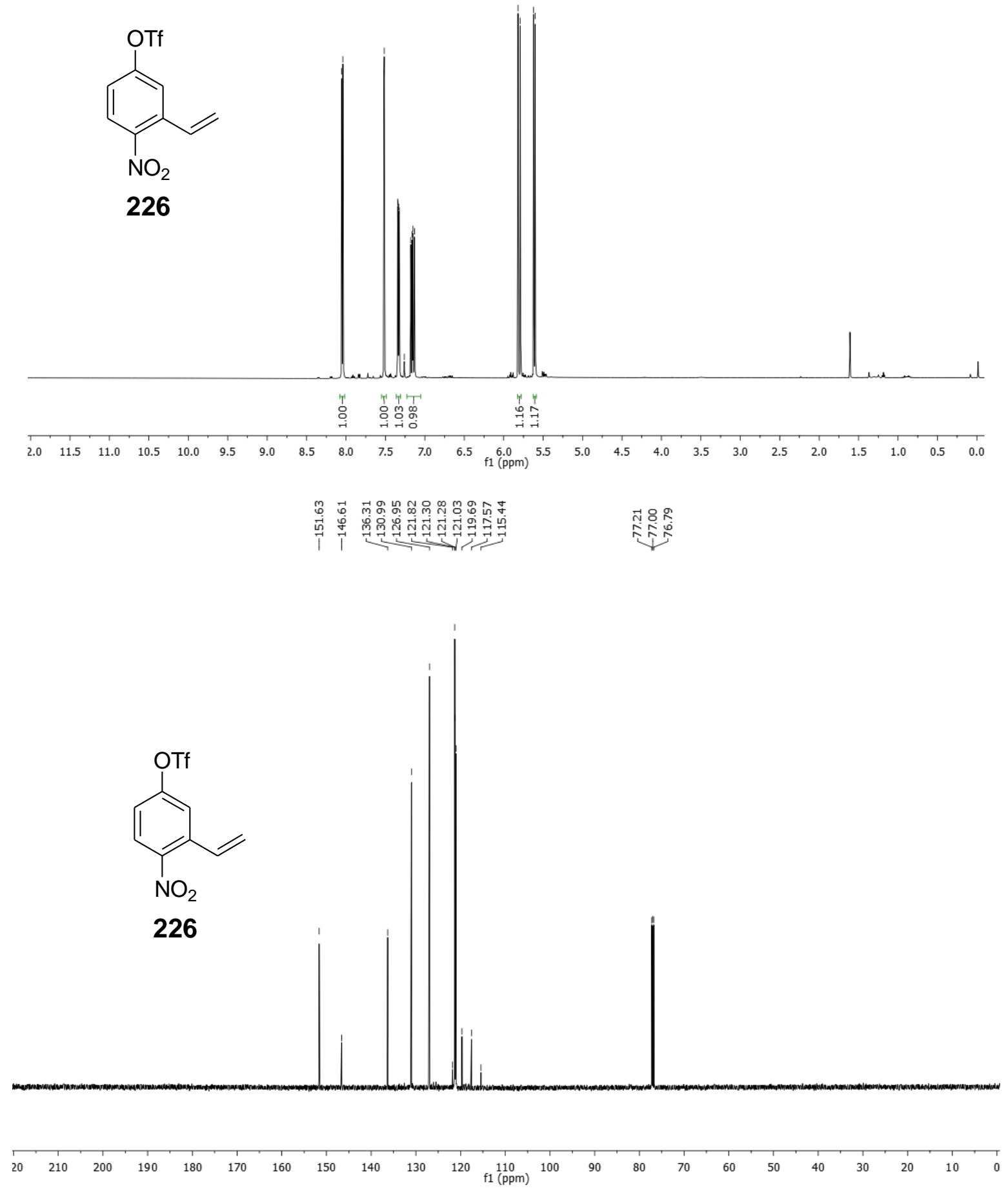

Figure 6.16 ${ }^{1} \mathrm{H}$ and ${ }^{13} \mathrm{C}$ NMR of compound 226 

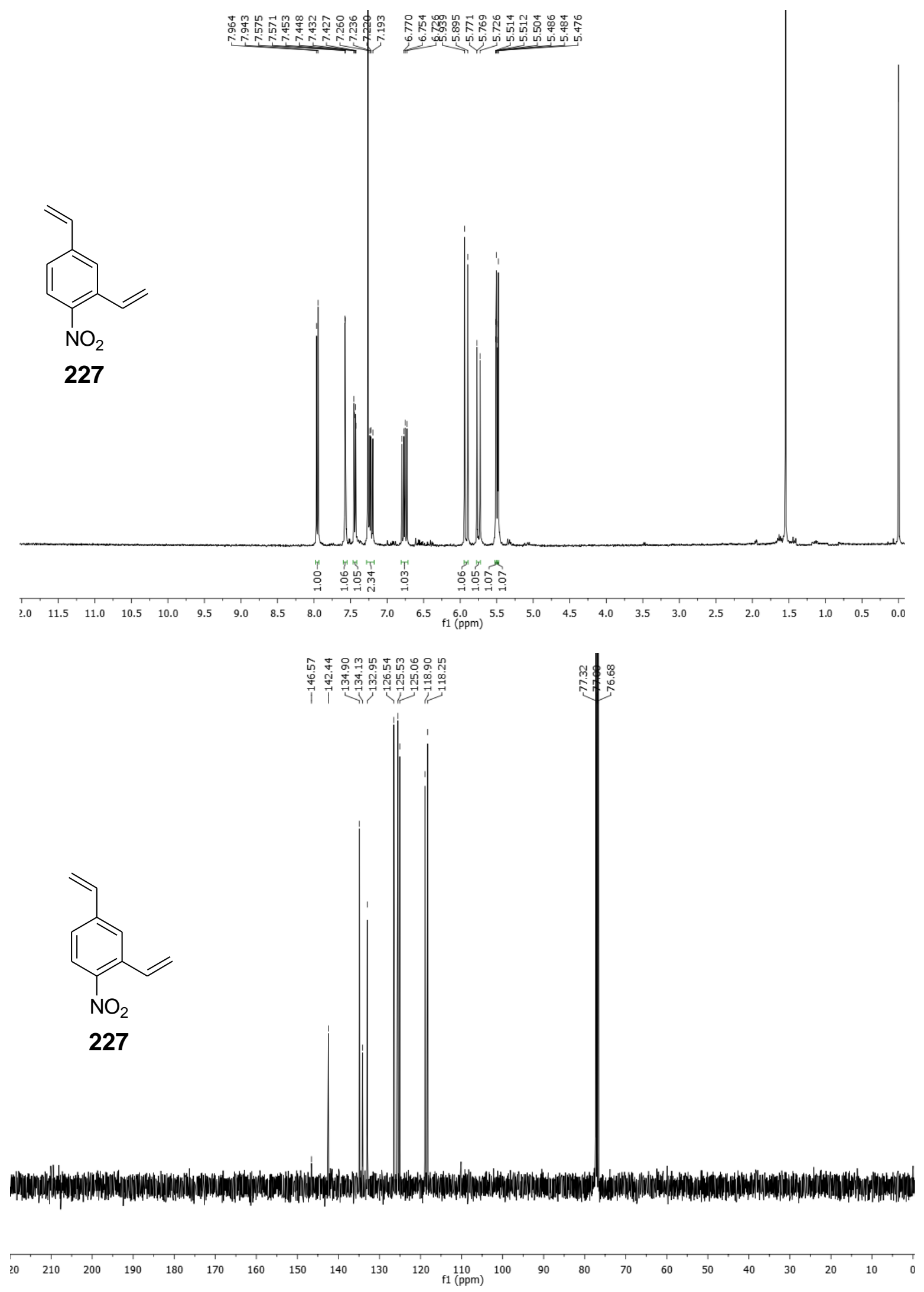

Figure 6.17 ${ }^{1} \mathrm{H}$ and ${ }^{13} \mathrm{C}$ NMR of compound 227 


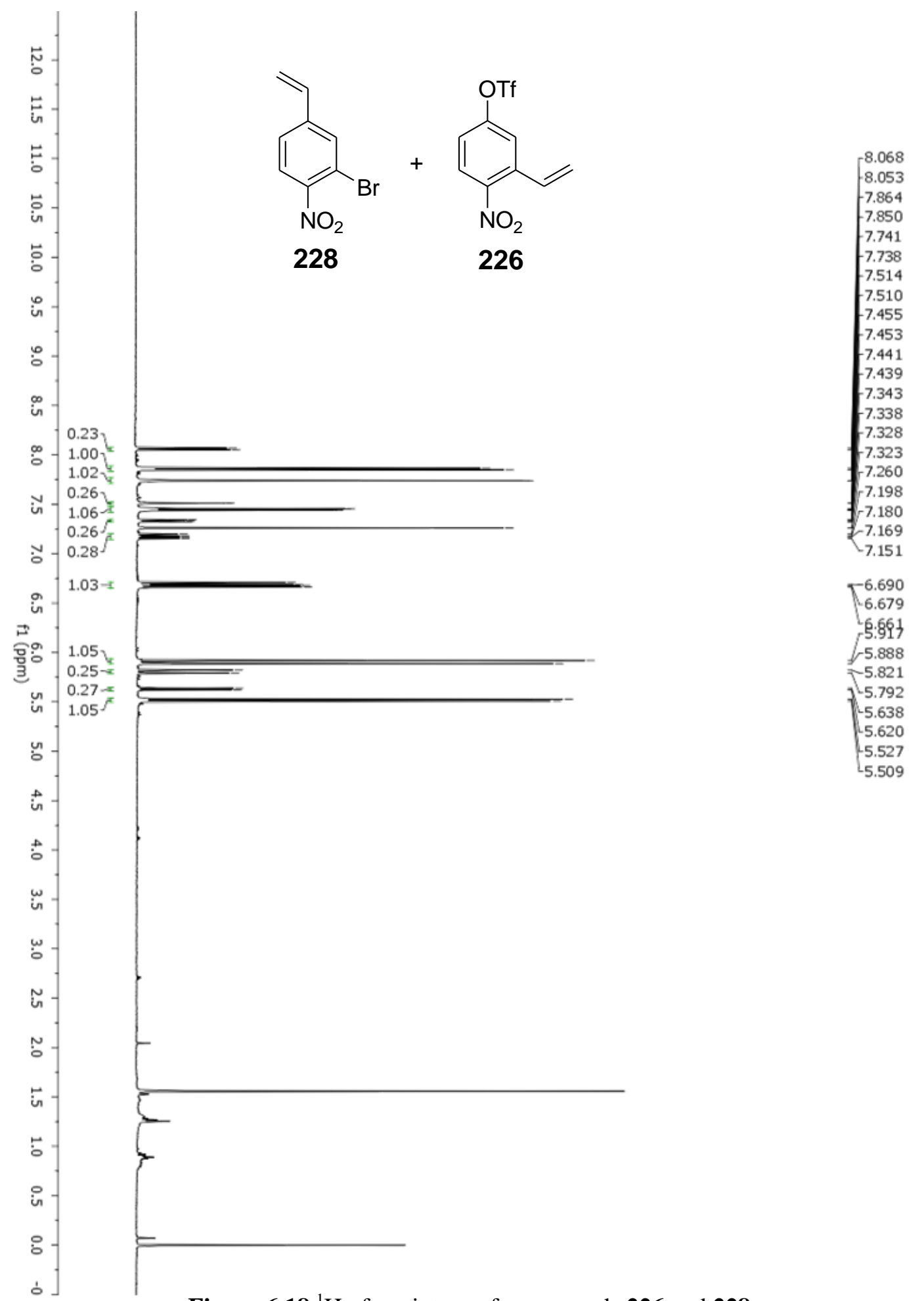

Figure 6.18 ${ }^{1} \mathrm{H}$ of a mixture of compounds 226 and 228 

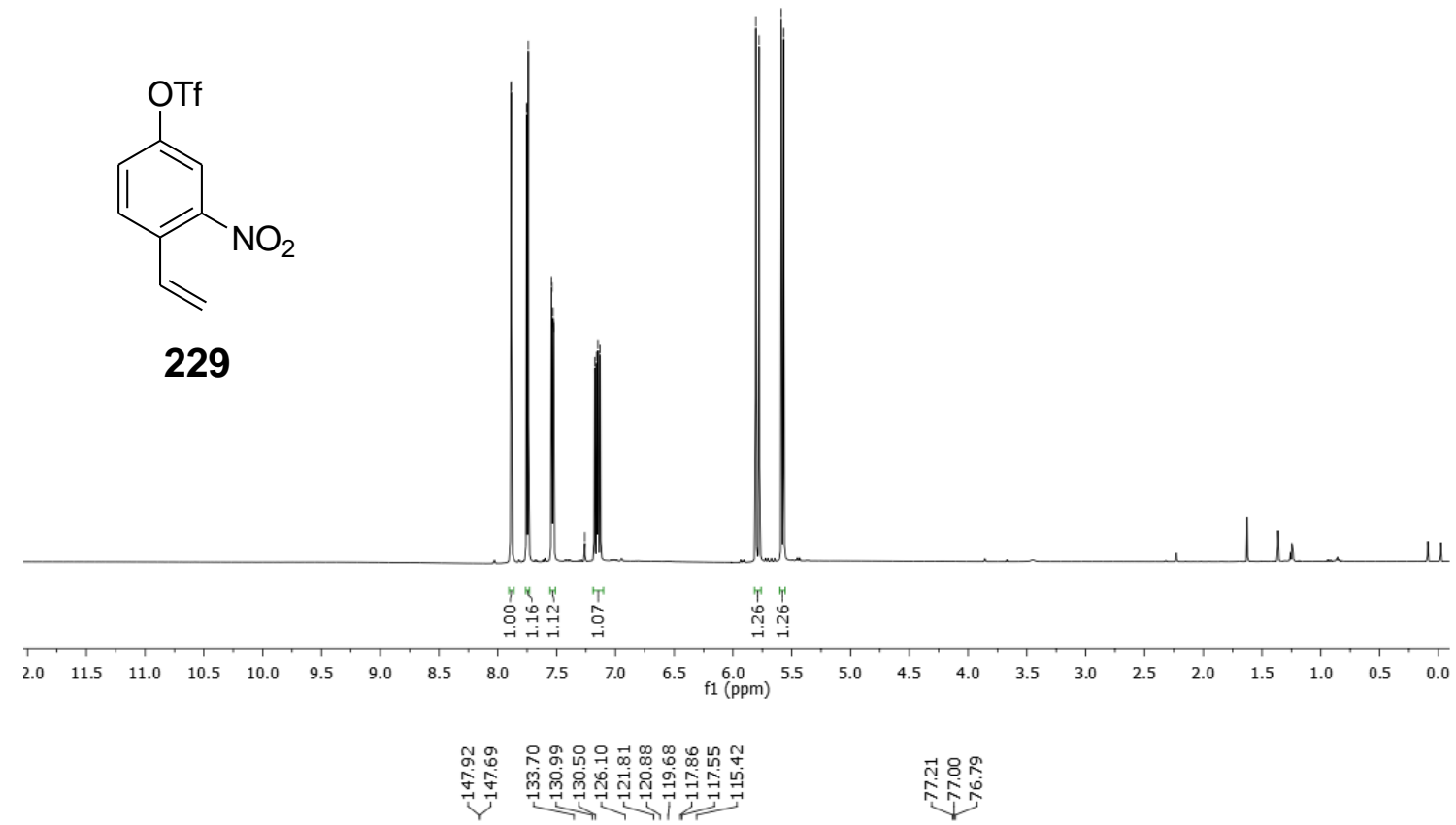

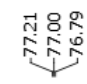

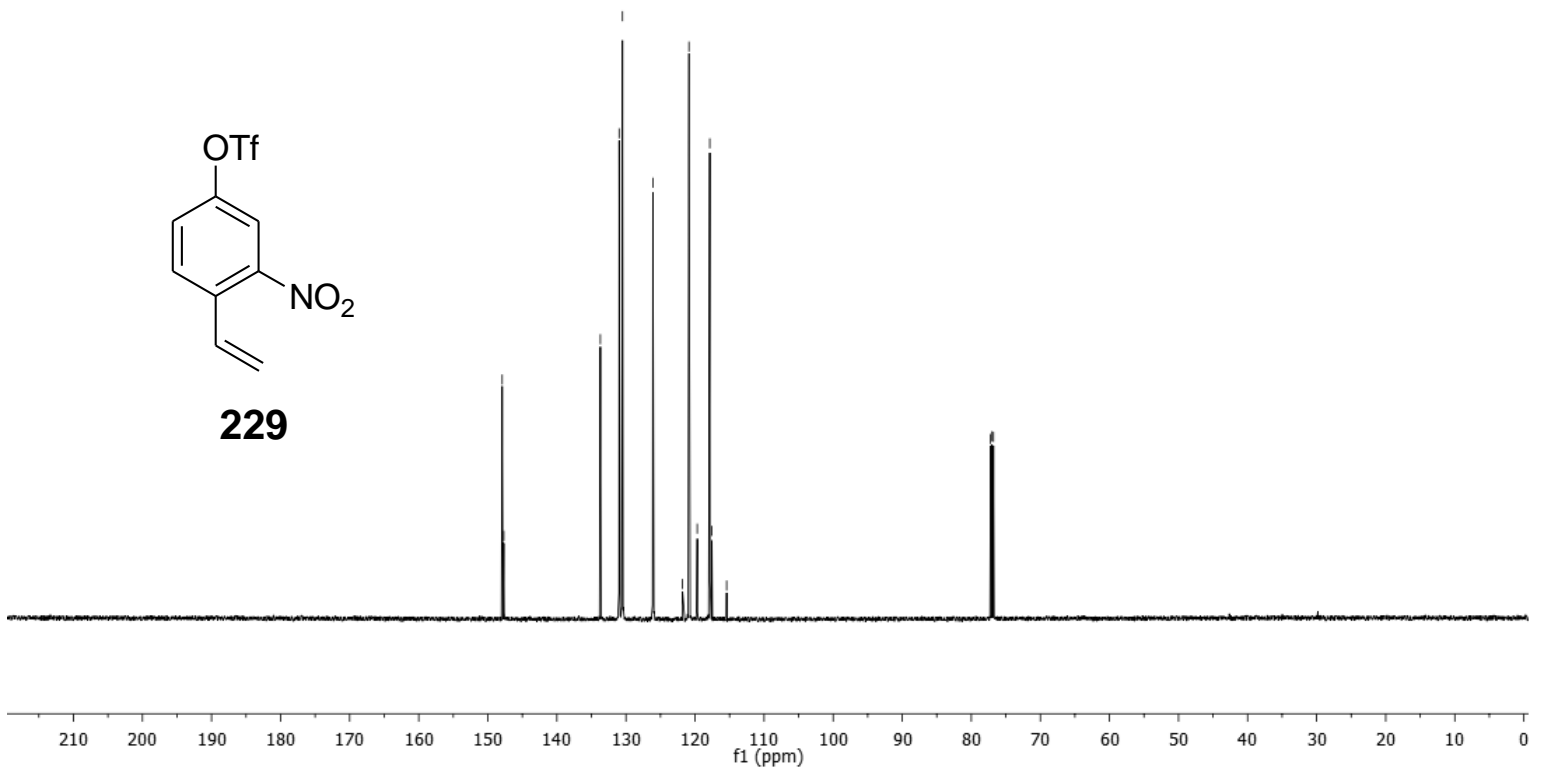

Figure 6.19 ${ }^{1} \mathrm{H}$ and ${ }^{13} \mathrm{C}$ NMR of compound 229 

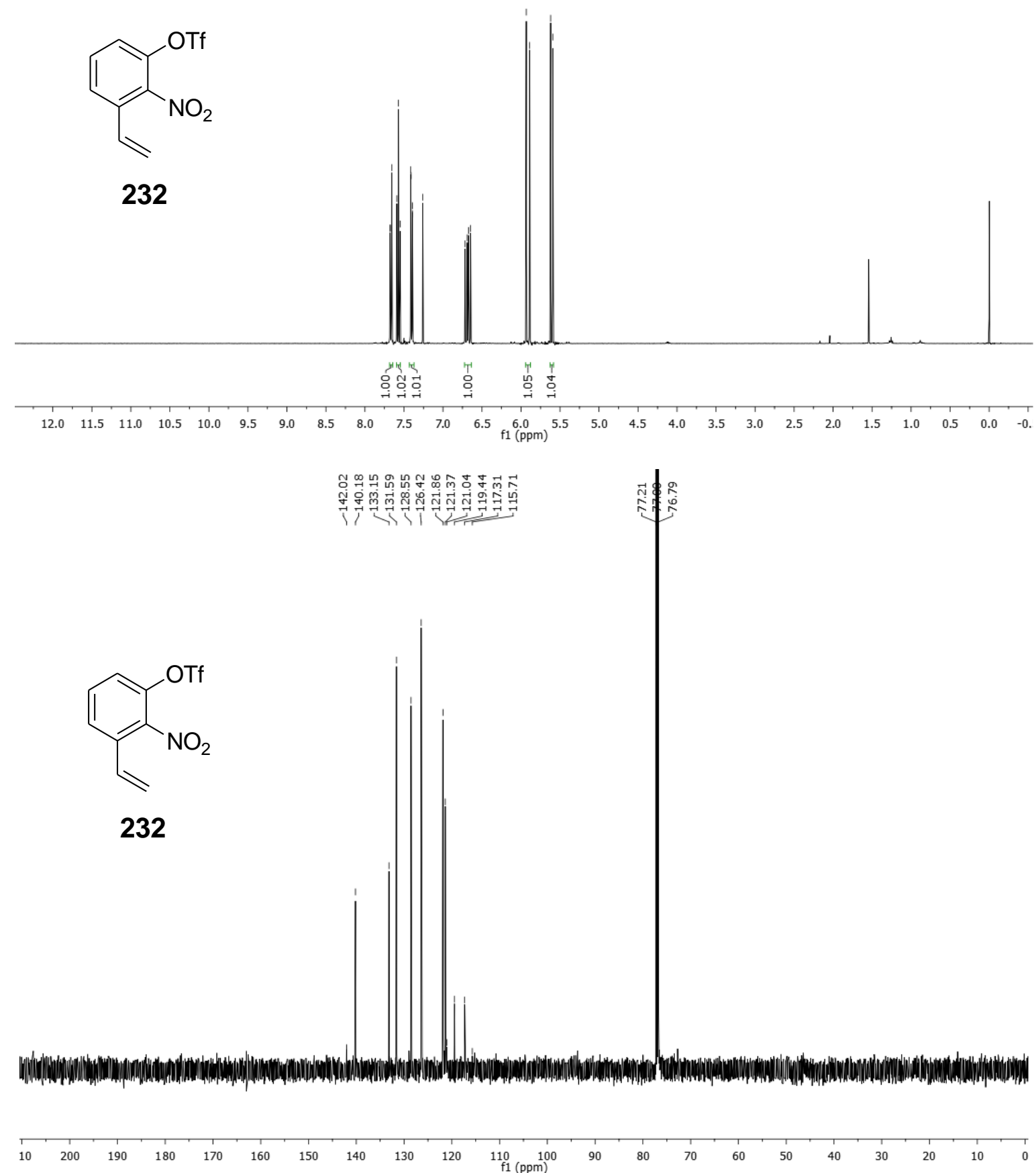

Figure 6.20 ${ }^{1} \mathrm{H}$ and ${ }^{13} \mathrm{C}$ NMR of compound 232 


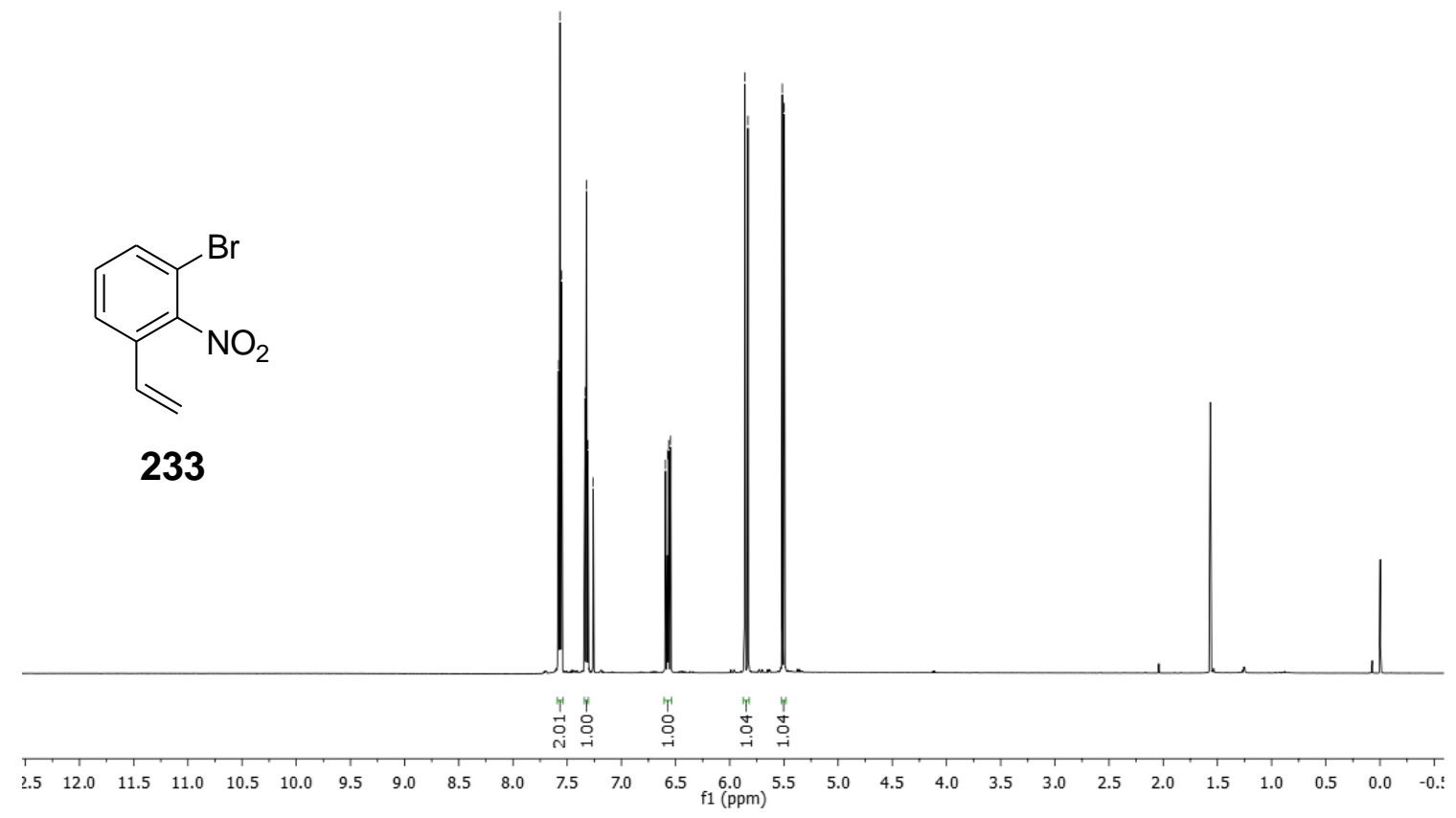

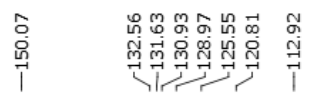

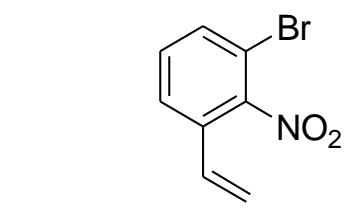

233
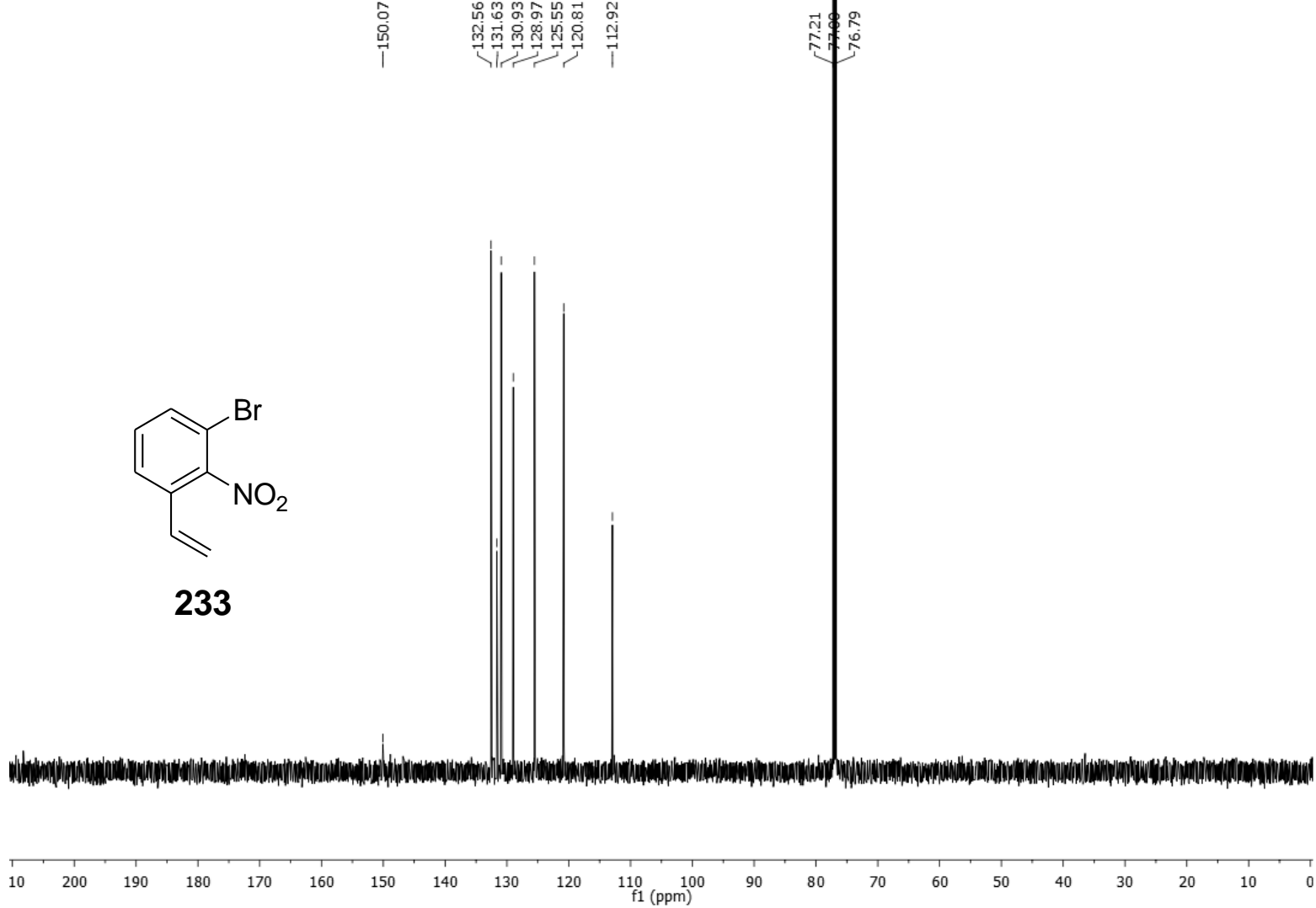

Figure 6.21 ${ }^{1} \mathrm{H}$ and ${ }^{13} \mathrm{C}$ NMR of compound 233 

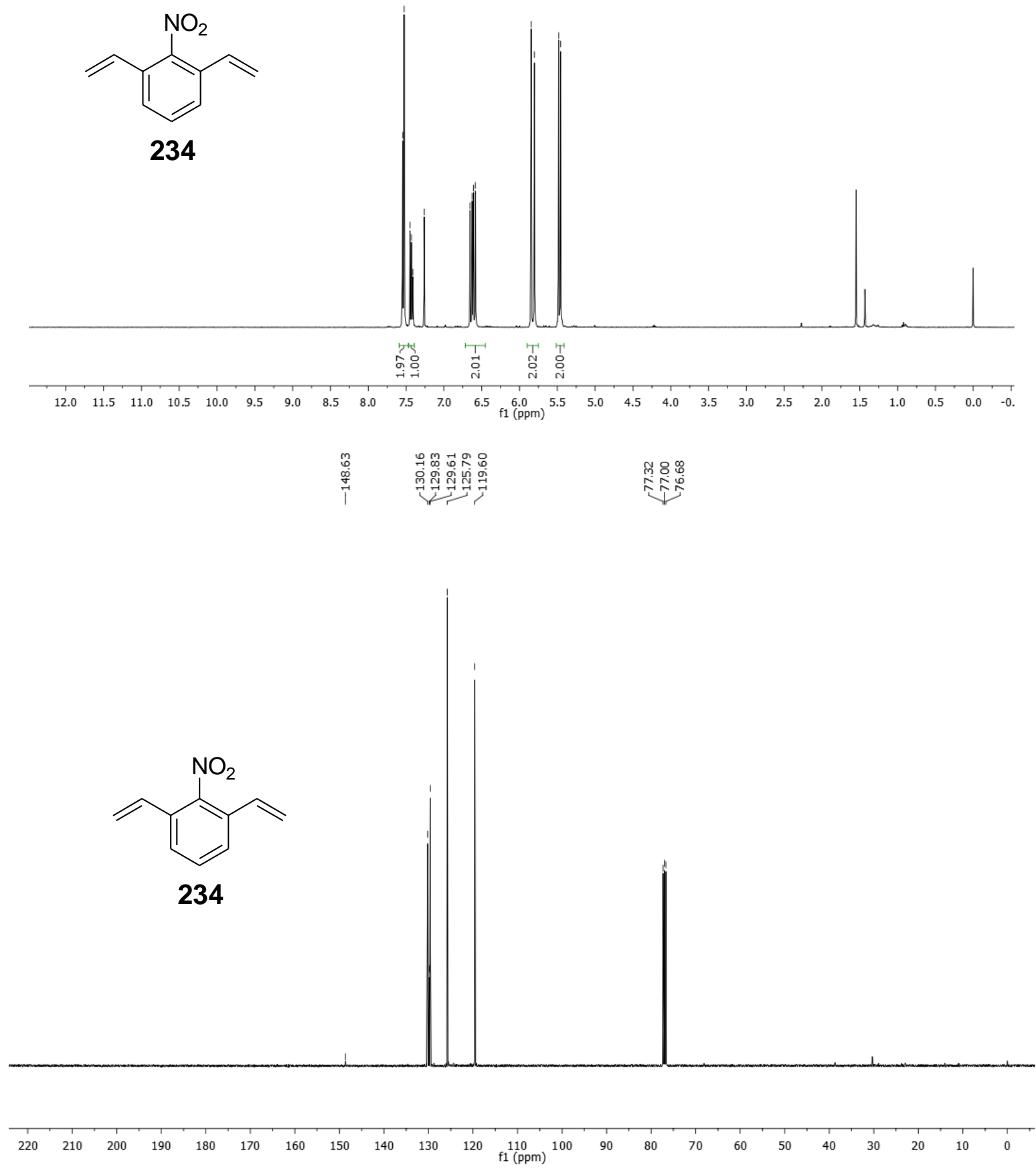

Figure 6.22 ${ }^{1} \mathrm{H}$ and ${ }^{13} \mathrm{C}$ NMR of compound 234 

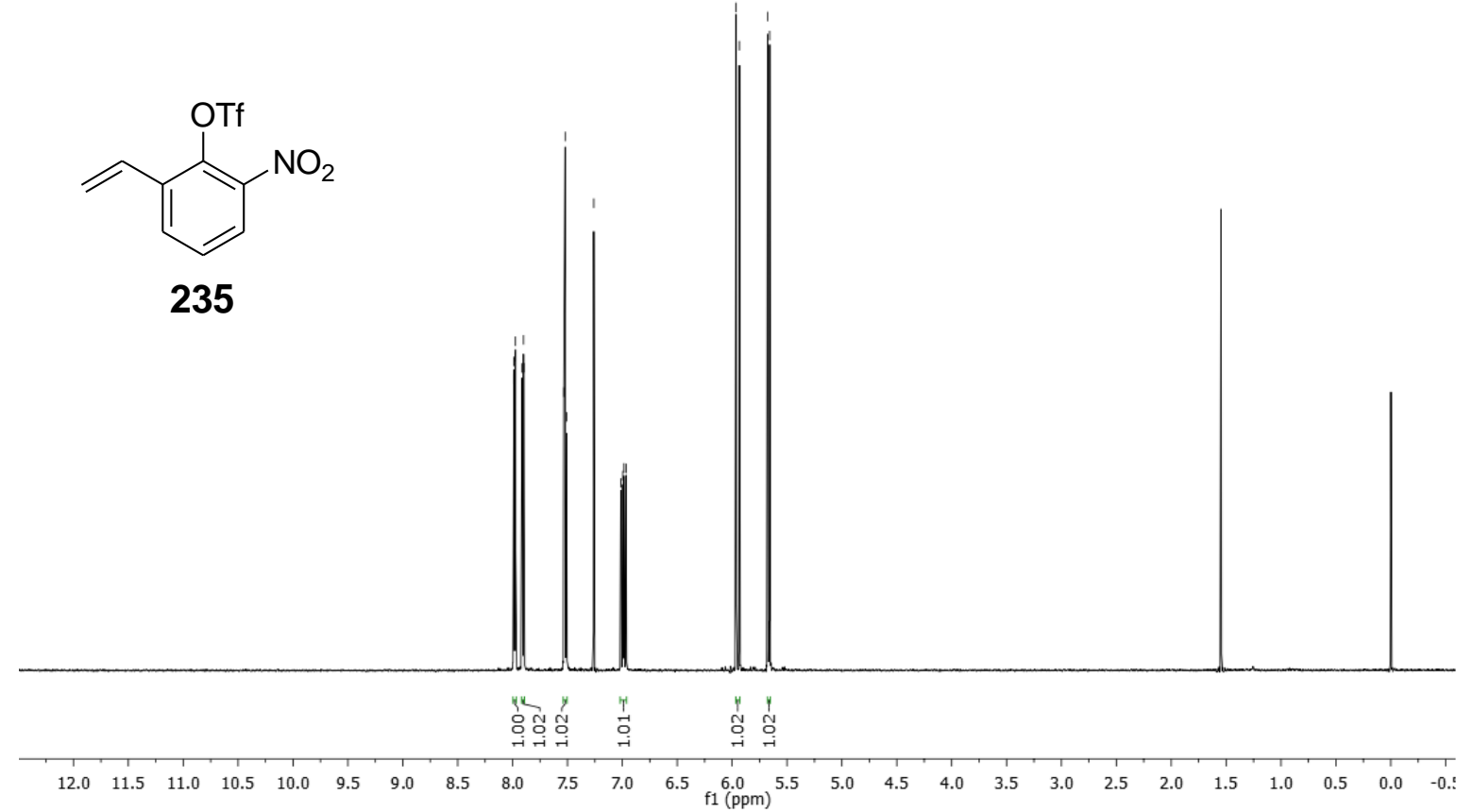

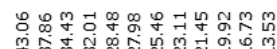

贶然

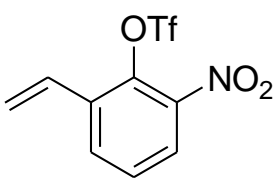

235
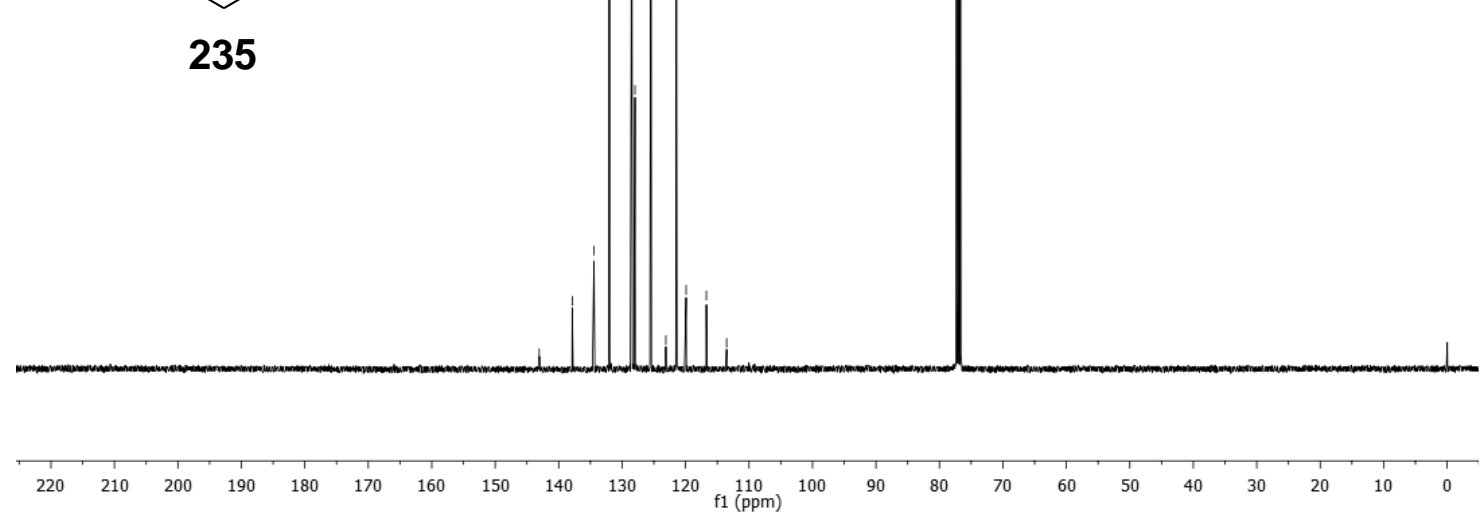

Figure 6.23 ${ }^{1} \mathrm{H}$ and ${ }^{13} \mathrm{C}$ NMR of compound 235 

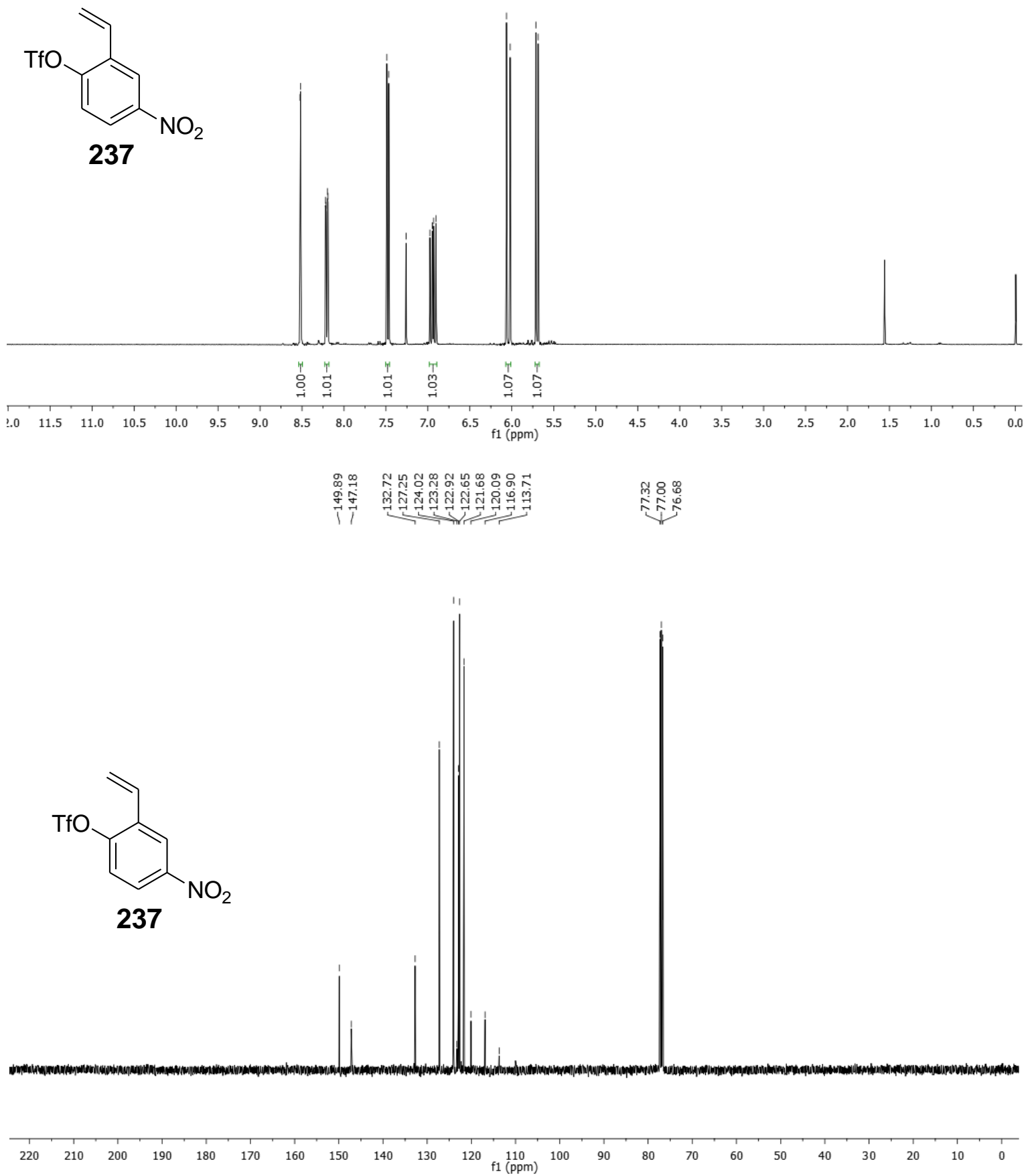

Figure 6.24 ${ }^{1} \mathrm{H}$ and ${ }^{13} \mathrm{C}$ NMR of compound 237 

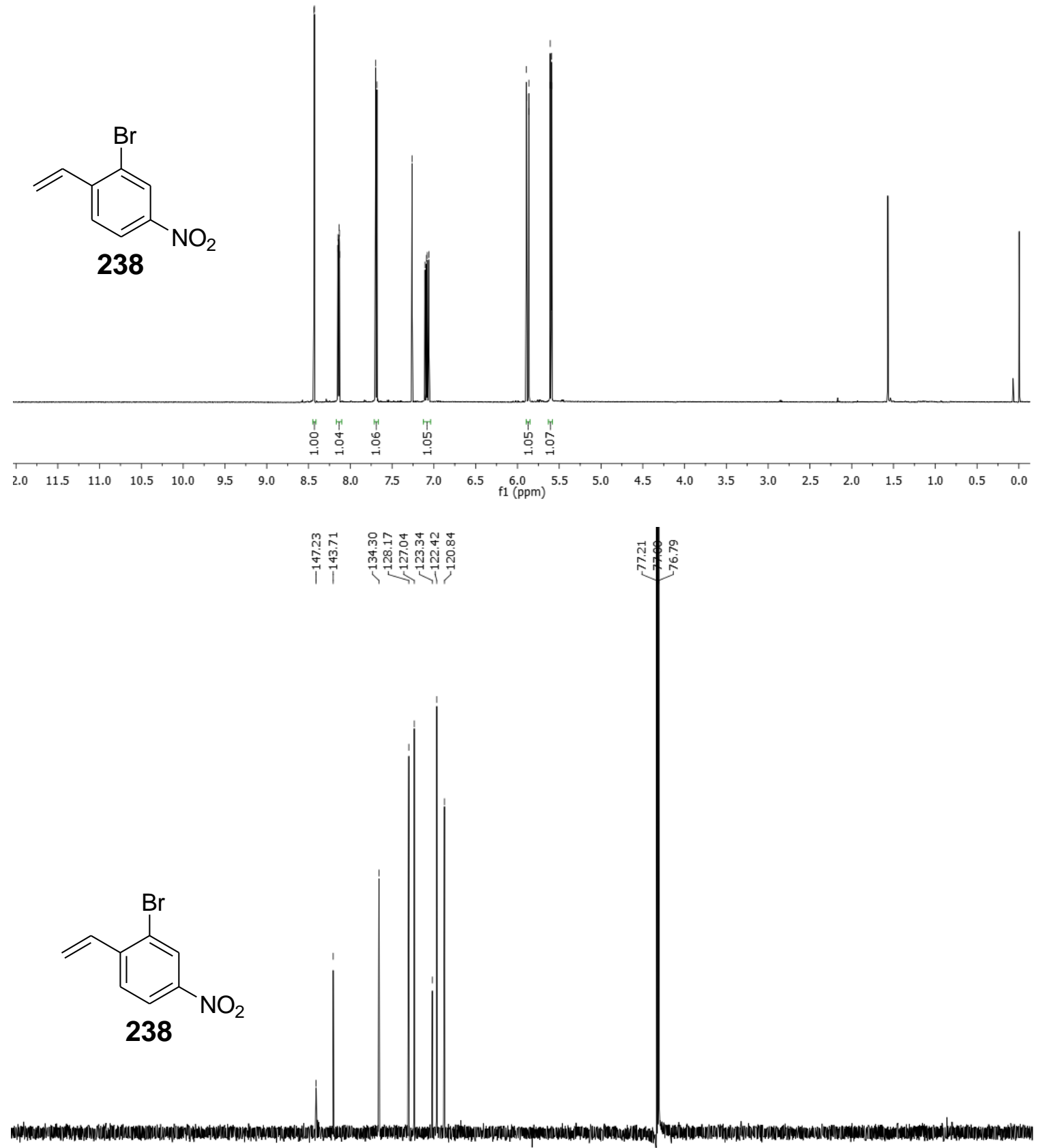

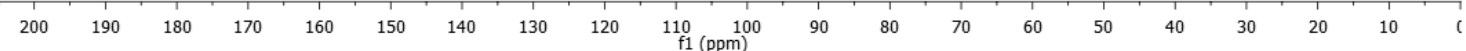

Figure 6.25 ${ }^{1} \mathrm{H}$ and ${ }^{13} \mathrm{C}$ NMR of compound 238 

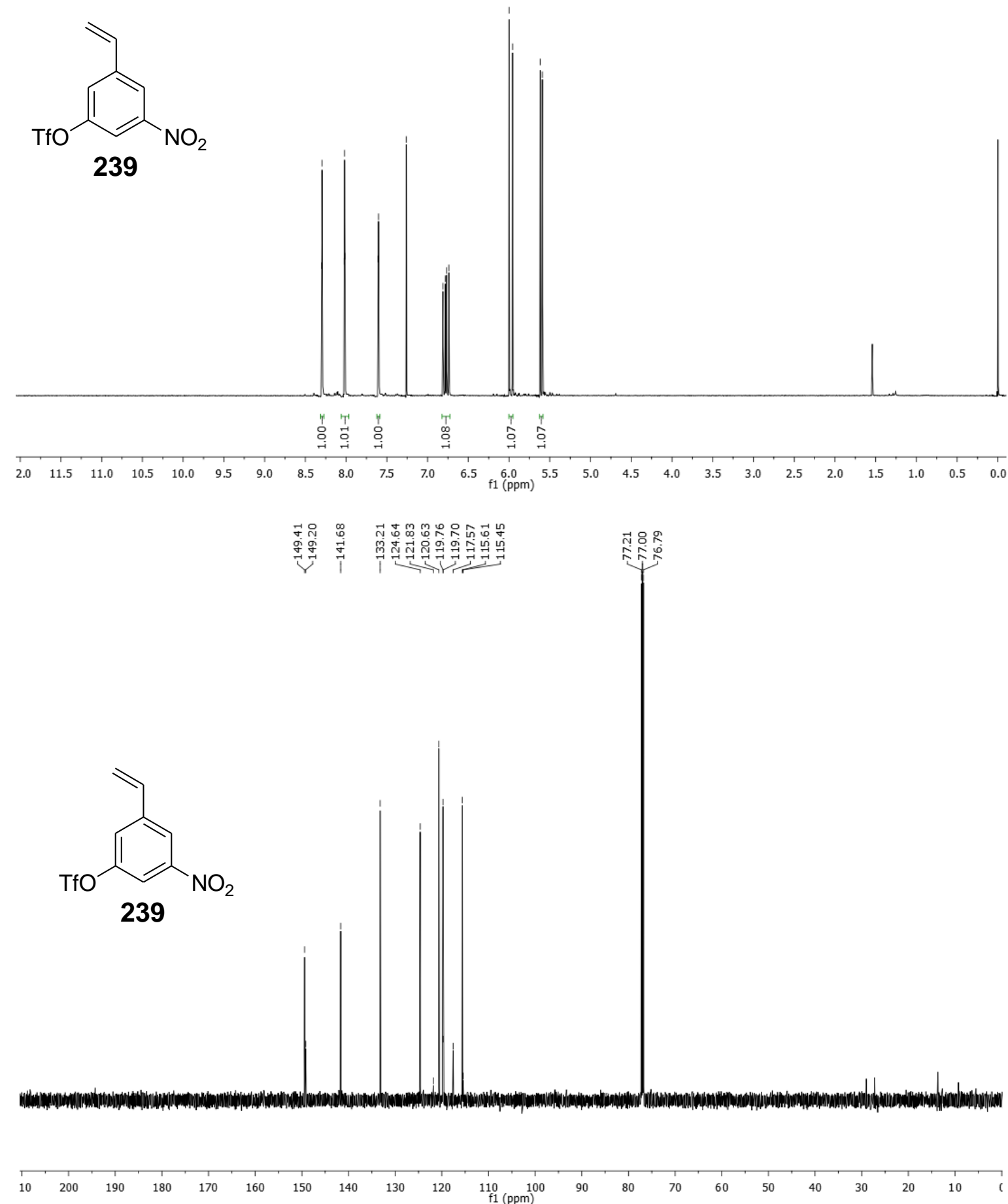

Figure 6.26 ${ }^{1} \mathrm{H}$ and ${ }^{13} \mathrm{C}$ NMR of compound 239 


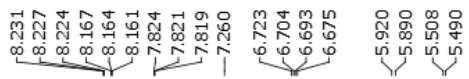

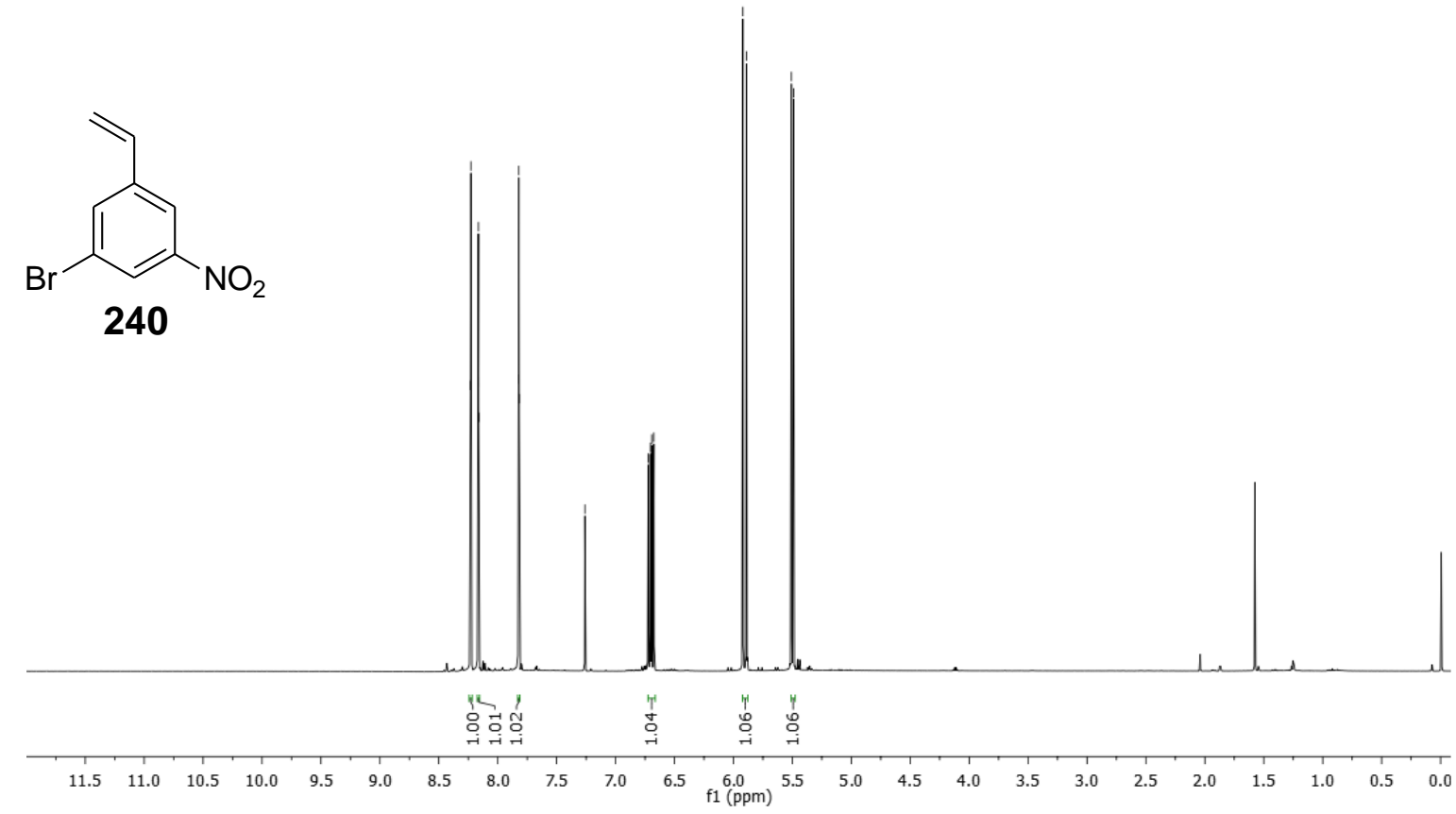

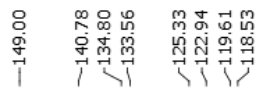
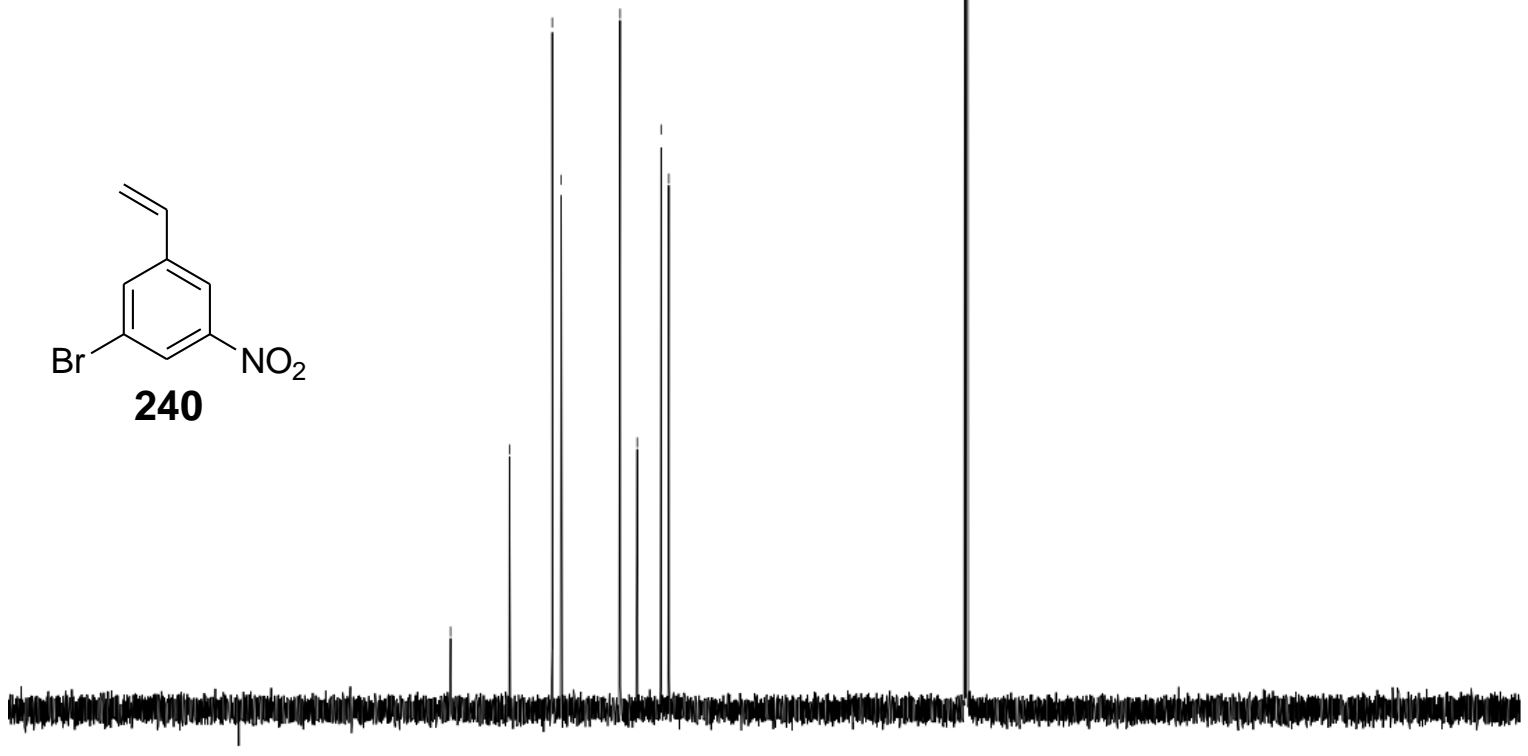

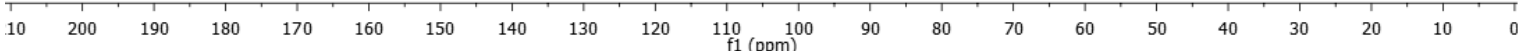

Figure 6.27 ${ }^{1} \mathrm{H}$ and ${ }^{13} \mathrm{C}$ NMR of compound 240 


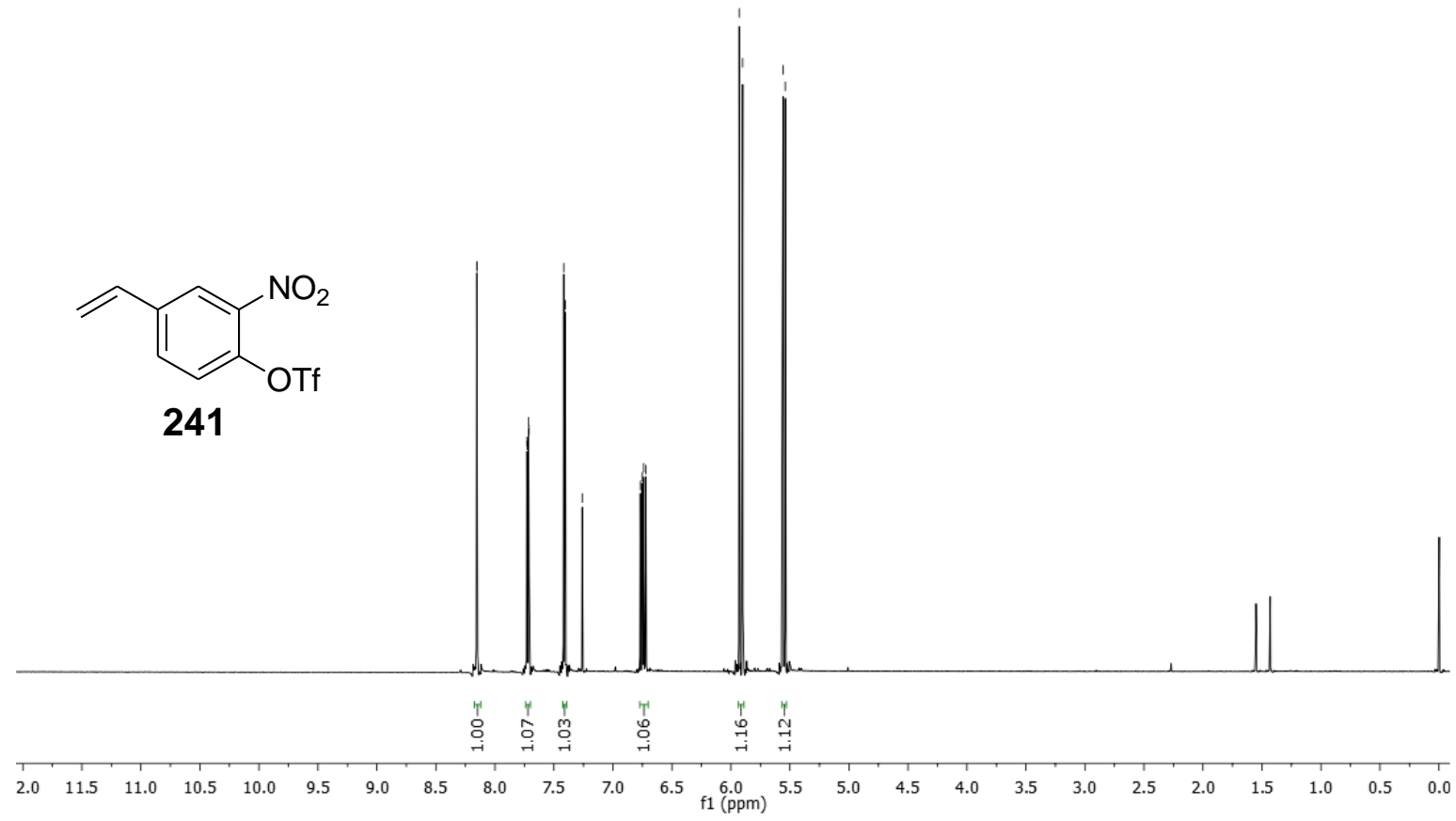

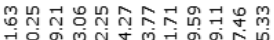

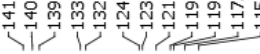

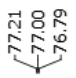<smiles>C=Cc1ccc(Br)c([N+](=O)[O-])c1</smiles>

241

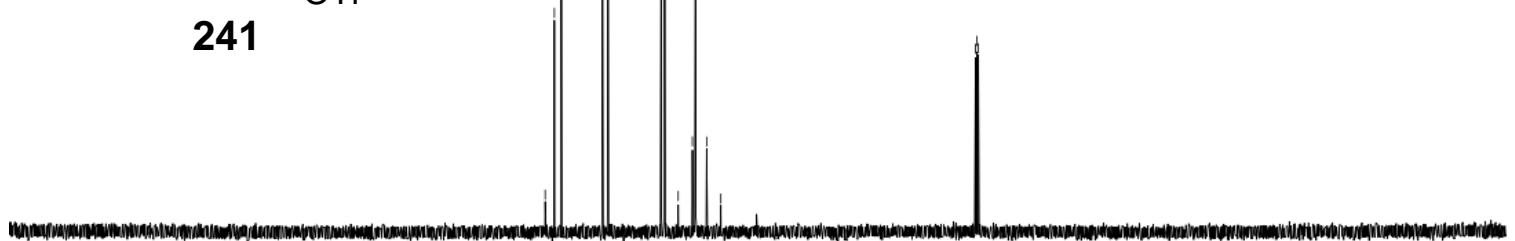

$\begin{array}{llllllllllllllllllllllll}220 & 210 & 200 & 190 & 180 & 170 & 160 & 150 & 140 & 130 & 120 & \underset{\mathrm{f} 1}{11(\mathrm{ppm})} & 100 & 90 & 80 & 70 & 60 & 50 & 40 & 30 & 20 & 10 & 0\end{array}$

Figure 6.28 ${ }^{1} \mathrm{H}$ and ${ }^{13} \mathrm{C}$ NMR of compound 241 

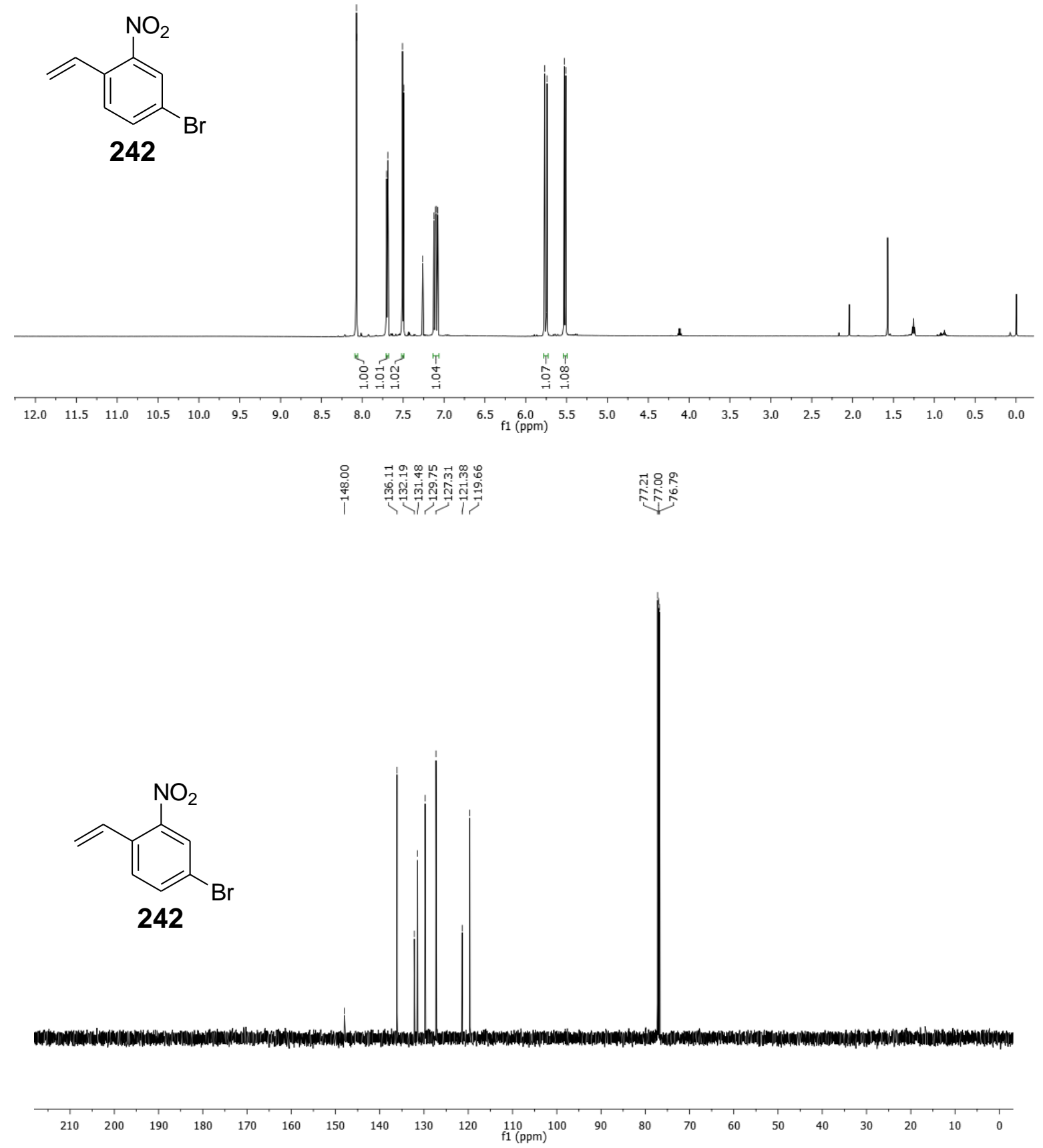

Figure 6.29 ${ }^{1} \mathrm{H}$ and ${ }^{13} \mathrm{C}$ NMR of compound 242 

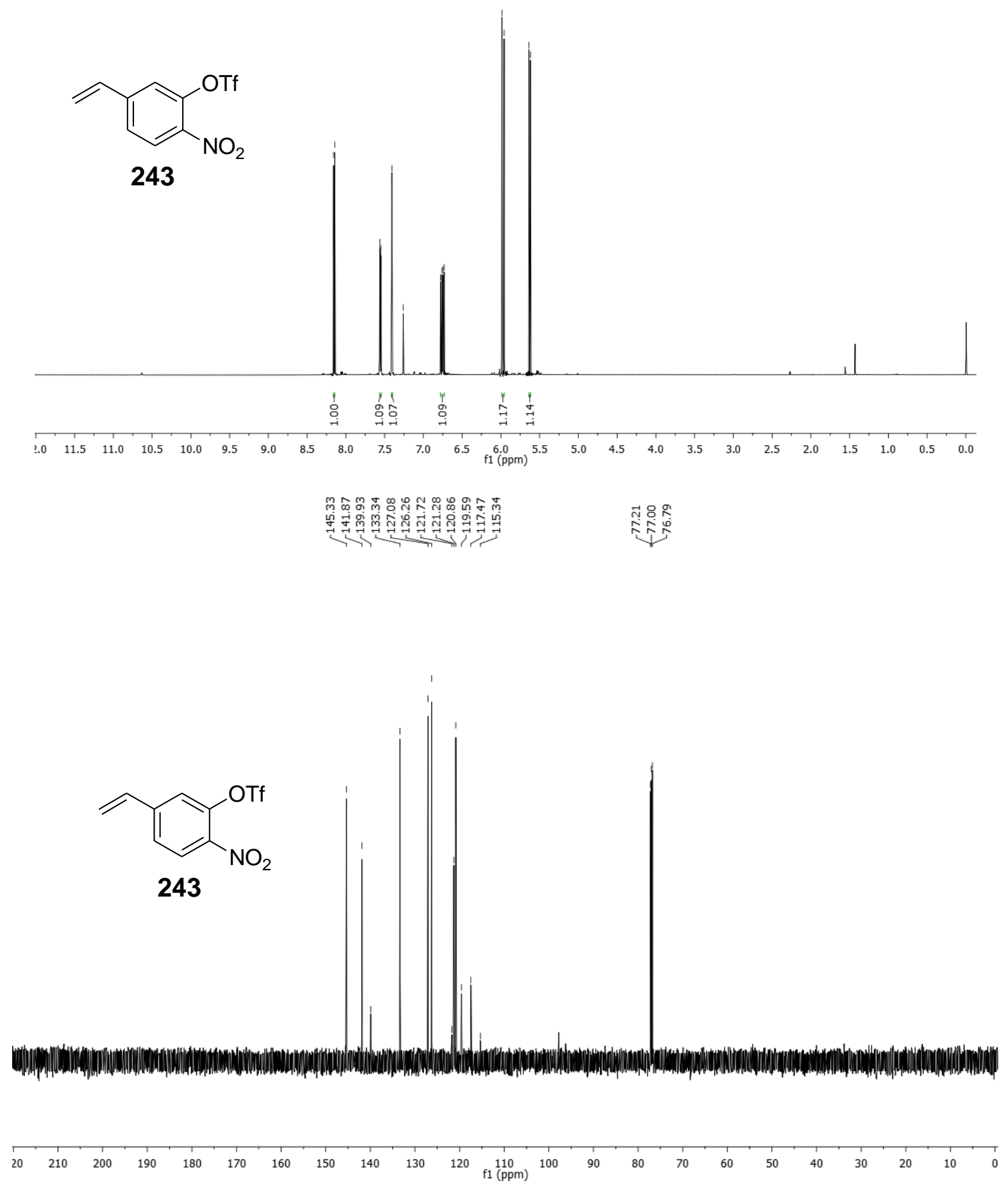

Figure 6.30 ${ }^{1} \mathrm{H}$ and ${ }^{13} \mathrm{C}$ NMR of compound 243 

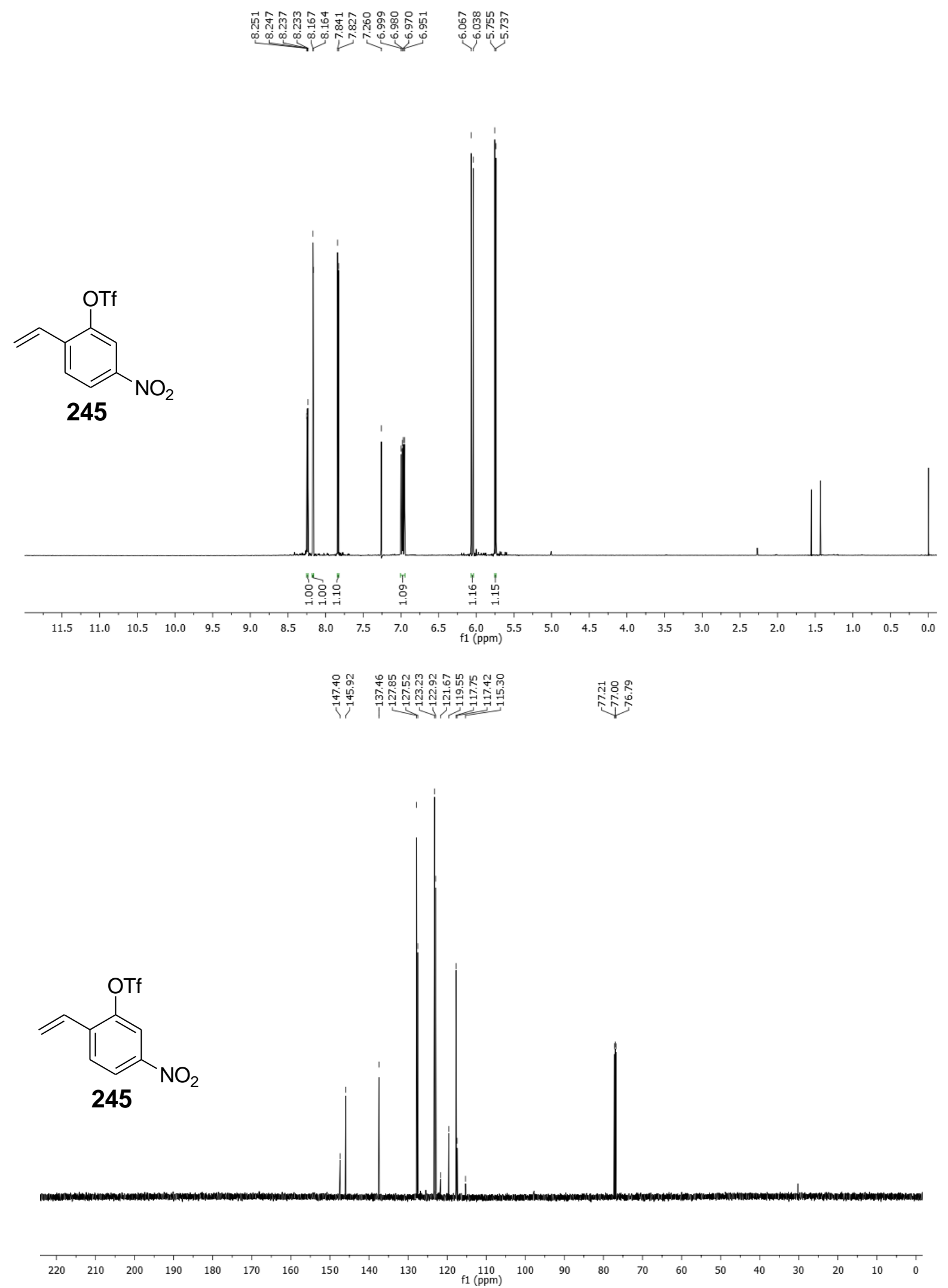

Figure 6.31 ${ }^{1} \mathrm{H}$ and ${ }^{13} \mathrm{C}$ NMR of compound 245 

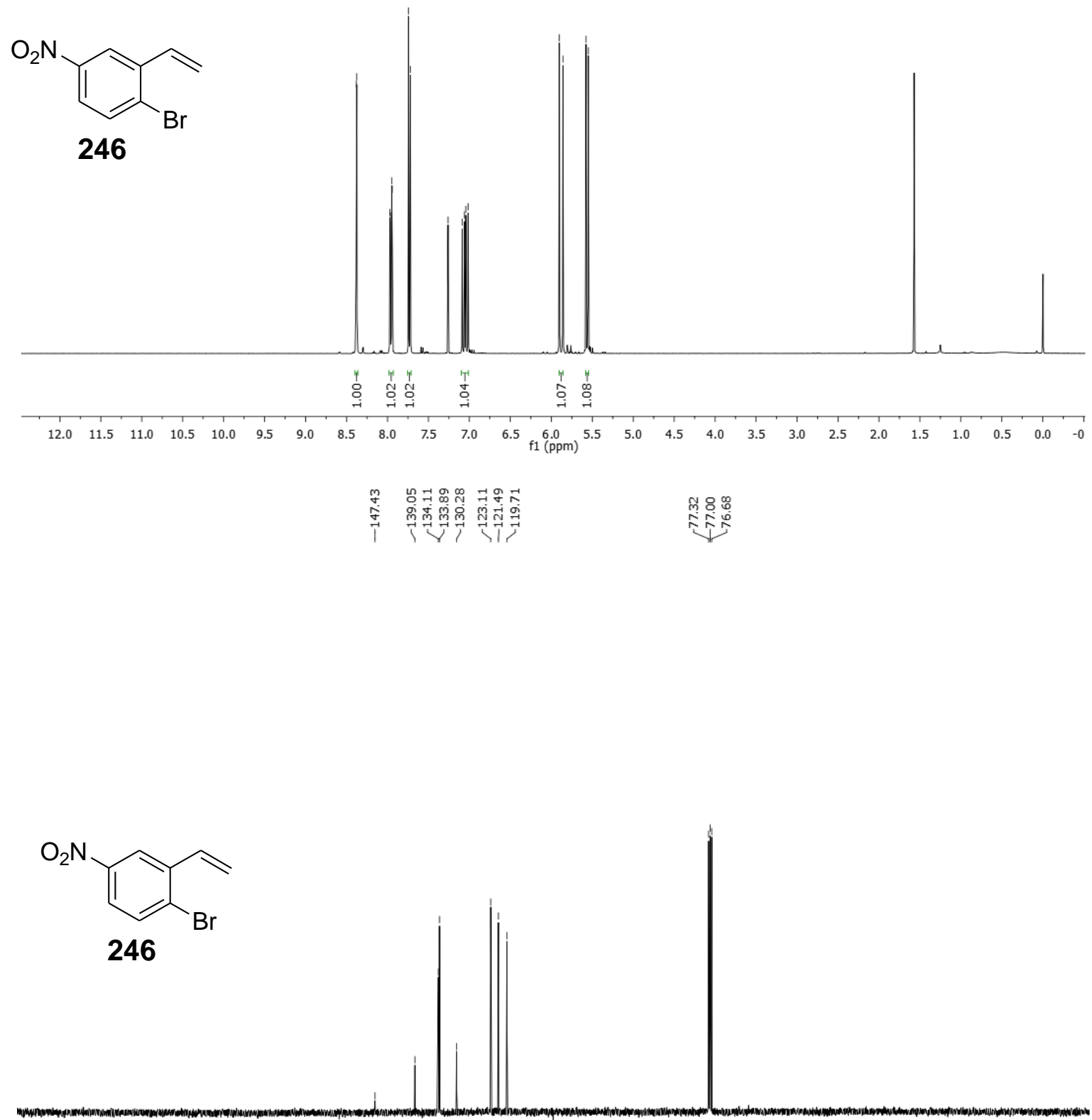

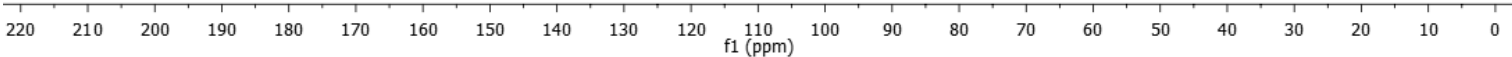

Figure 6.32 ${ }^{1} \mathrm{H}$ and ${ }^{13} \mathrm{C}$ NMR of compound 246 

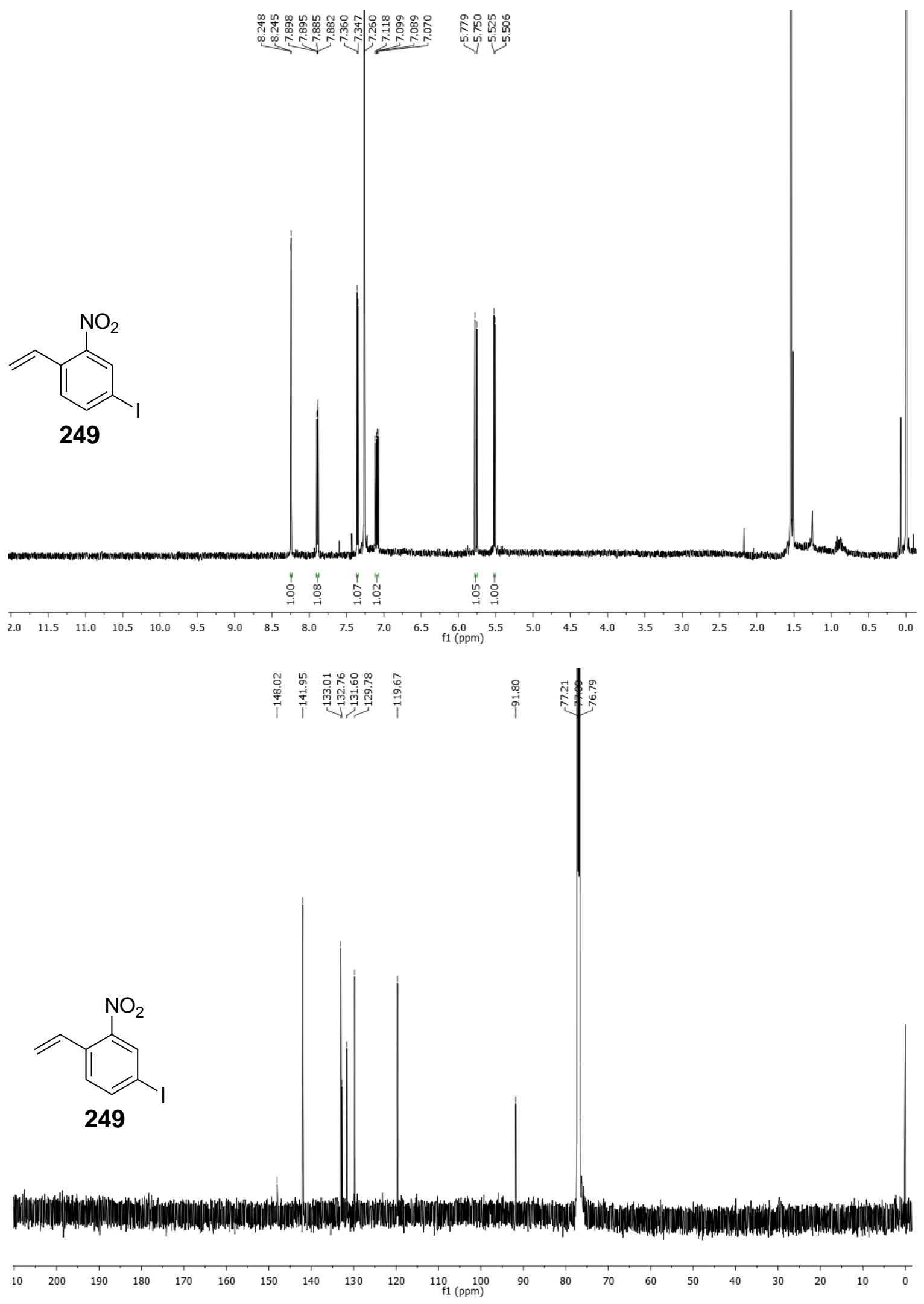

Figure 6.33 ${ }^{1} \mathrm{H}$ and ${ }^{13} \mathrm{C}$ NMR of compound 249 


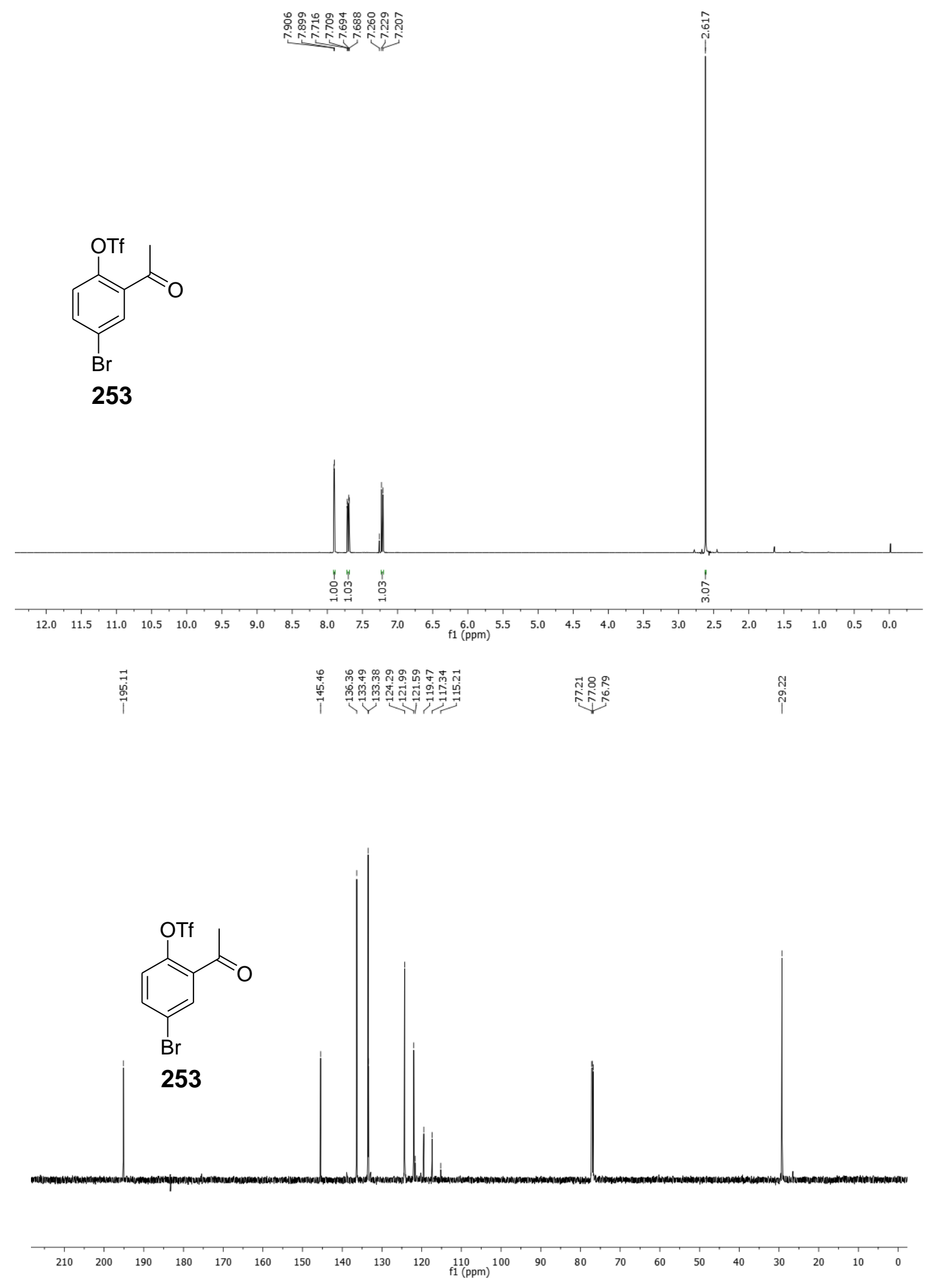

Figure 6.34 ${ }^{1} \mathrm{H}$ and ${ }^{13} \mathrm{C}$ NMR of compound 253 

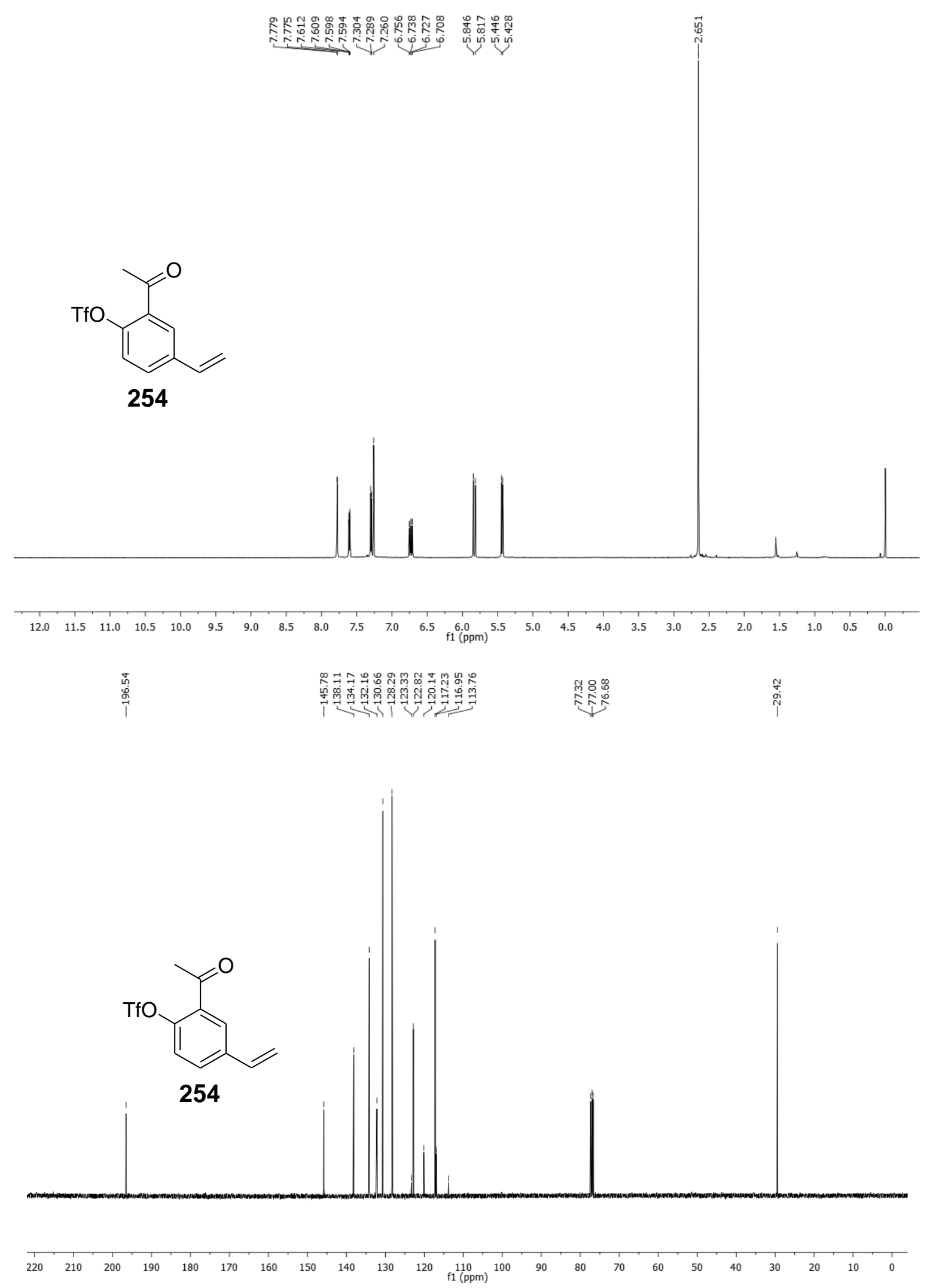

Figure 6.35 ${ }^{1} \mathrm{H}$ and ${ }^{13} \mathrm{C}$ NMR of compound 254 

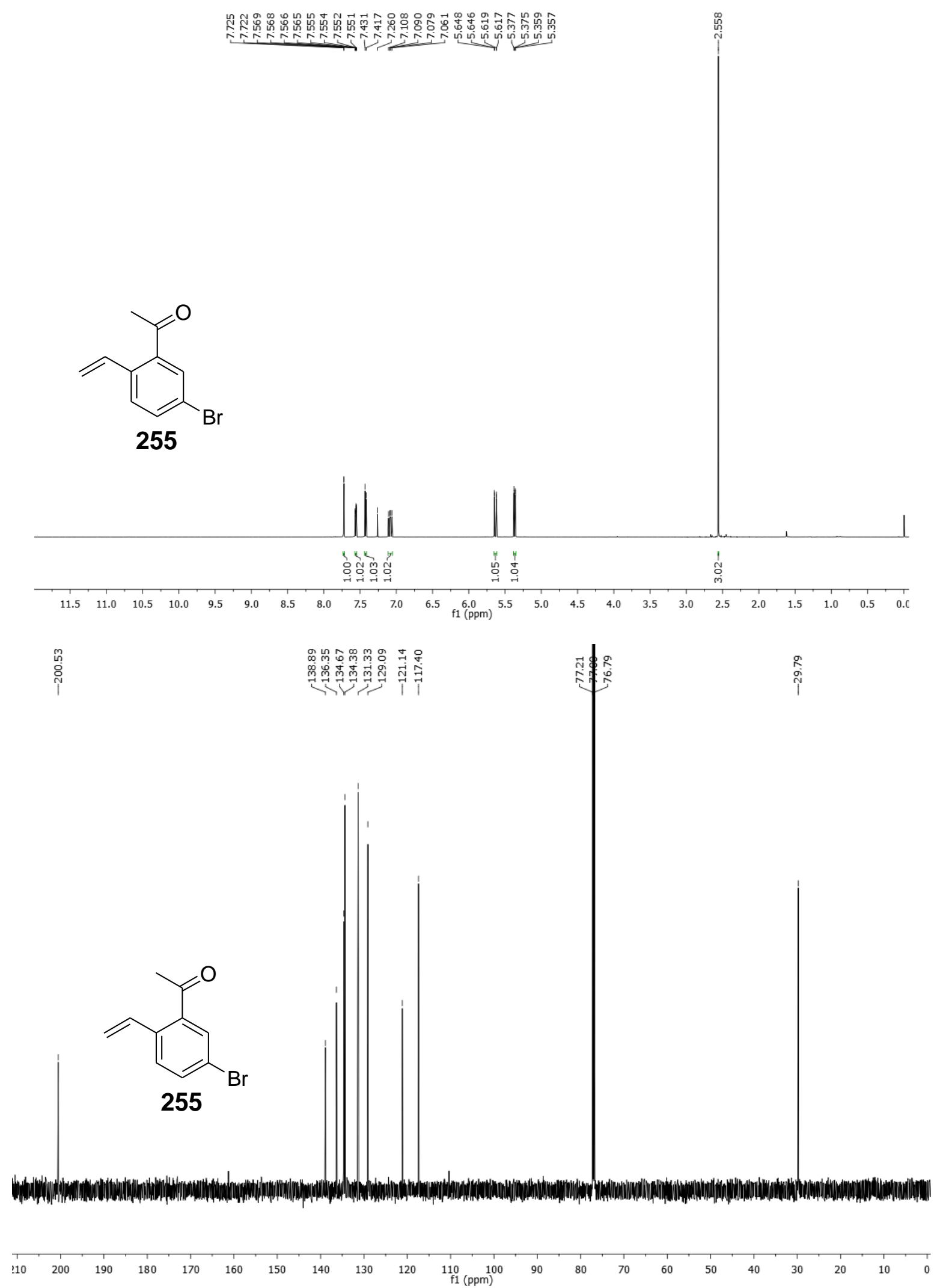

Figure 6.36 ${ }^{1} \mathrm{H}$ and ${ }^{13} \mathrm{C}$ NMR of compound 255 


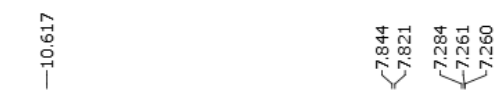

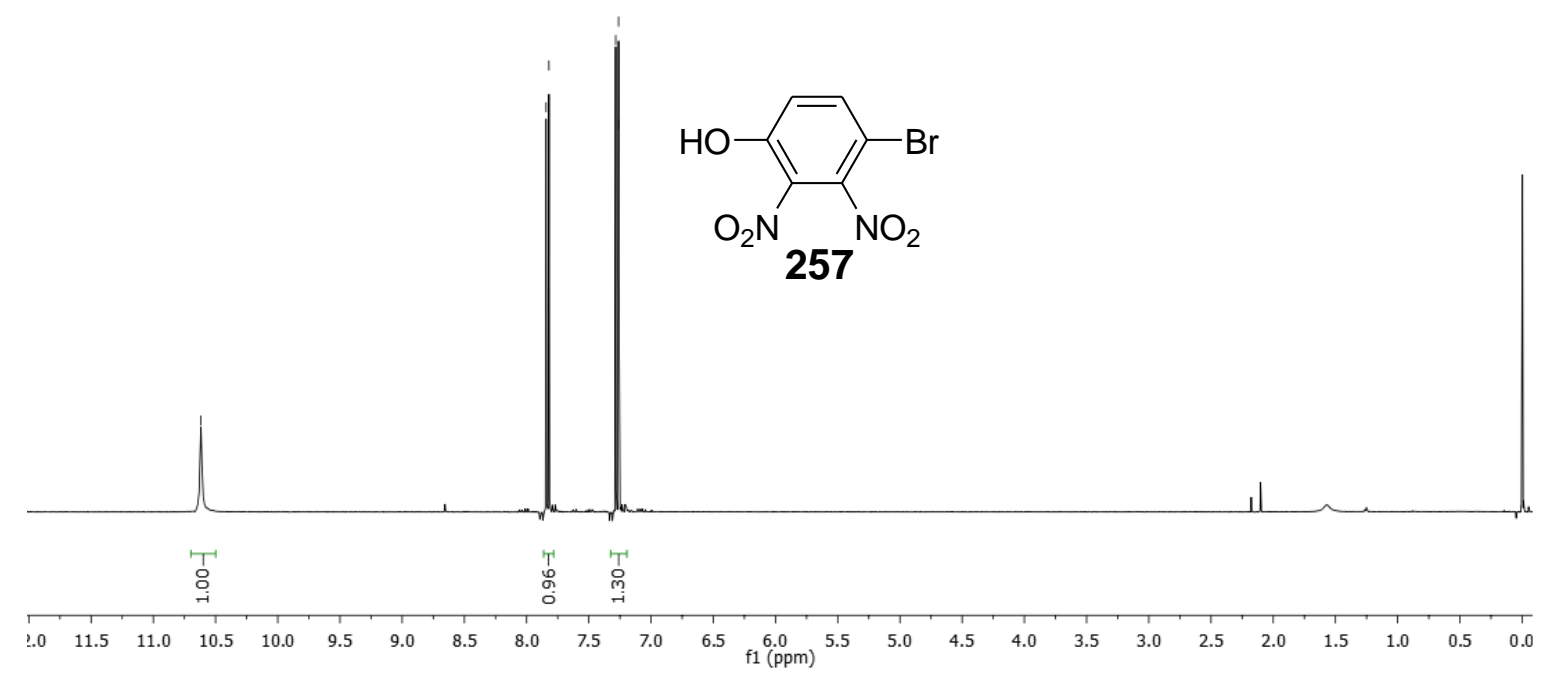

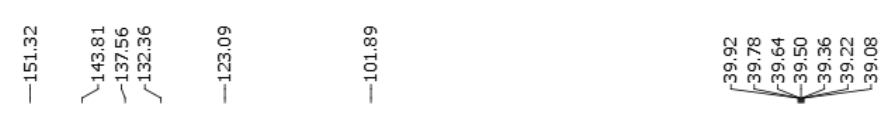

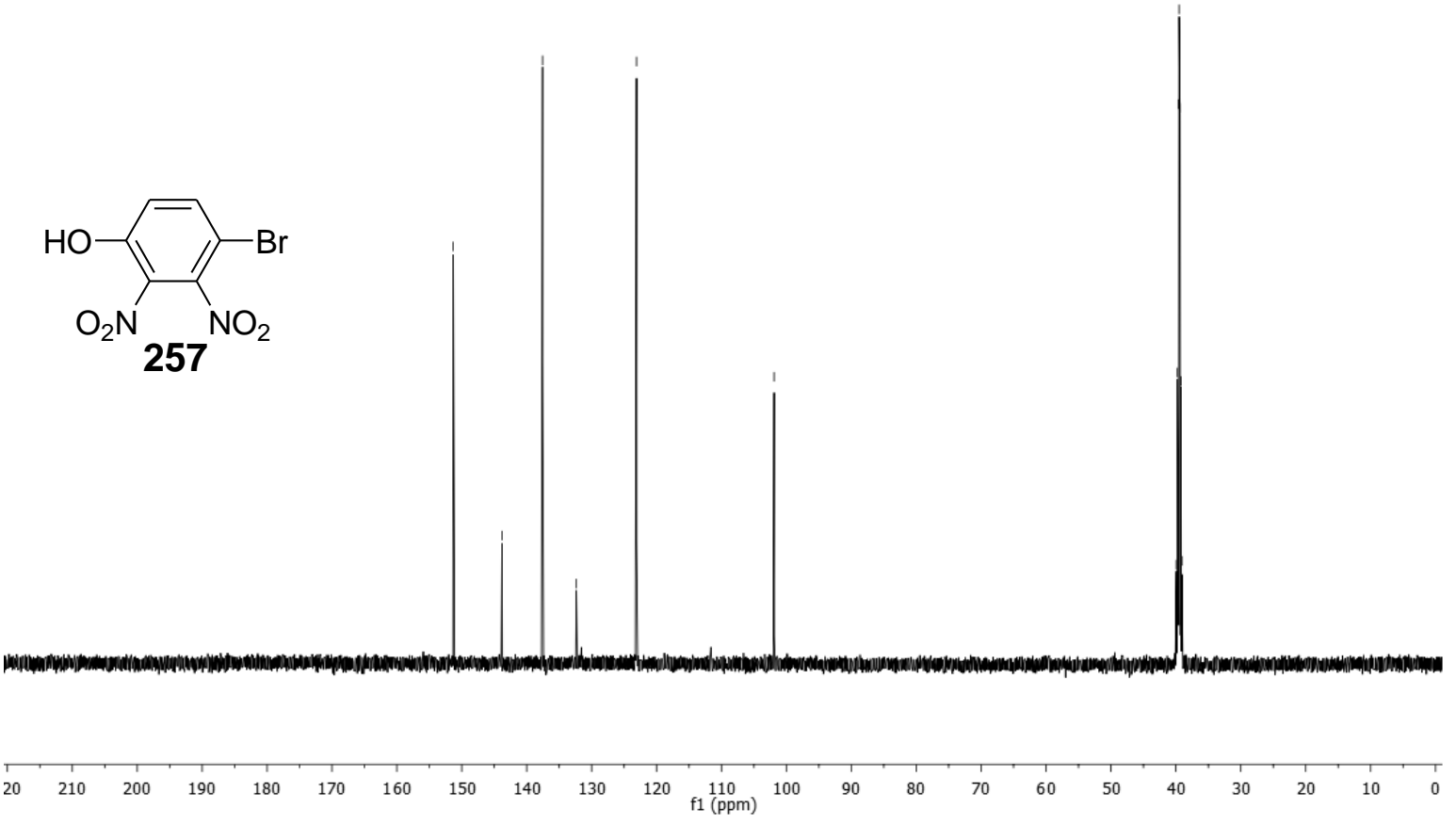

Figure 7.2 ${ }^{1} \mathrm{H}$ and ${ }^{13} \mathrm{C}$ NMR of compound 257 


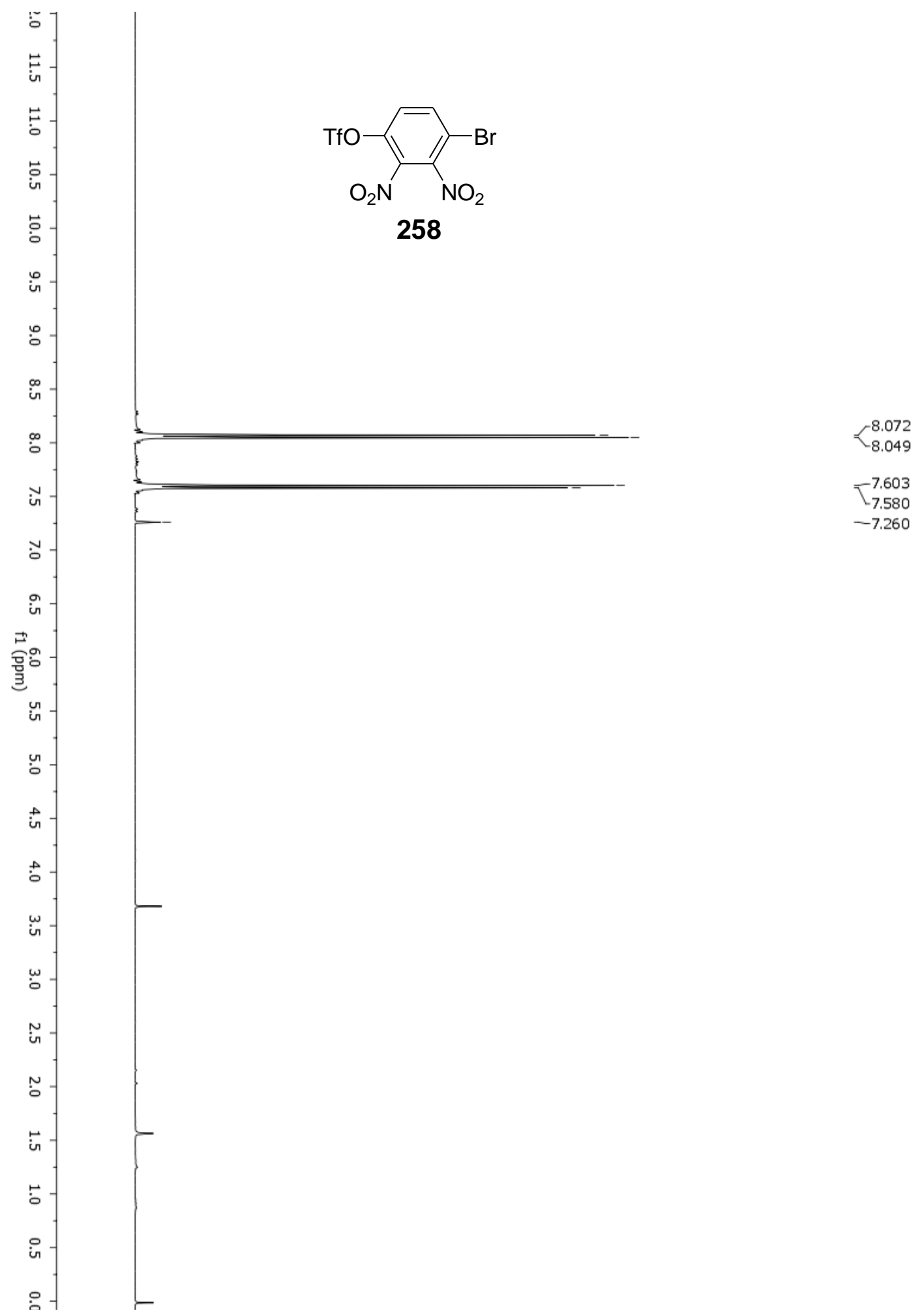

Figure $7.3{ }^{1} \mathrm{H}$ of compound 258 

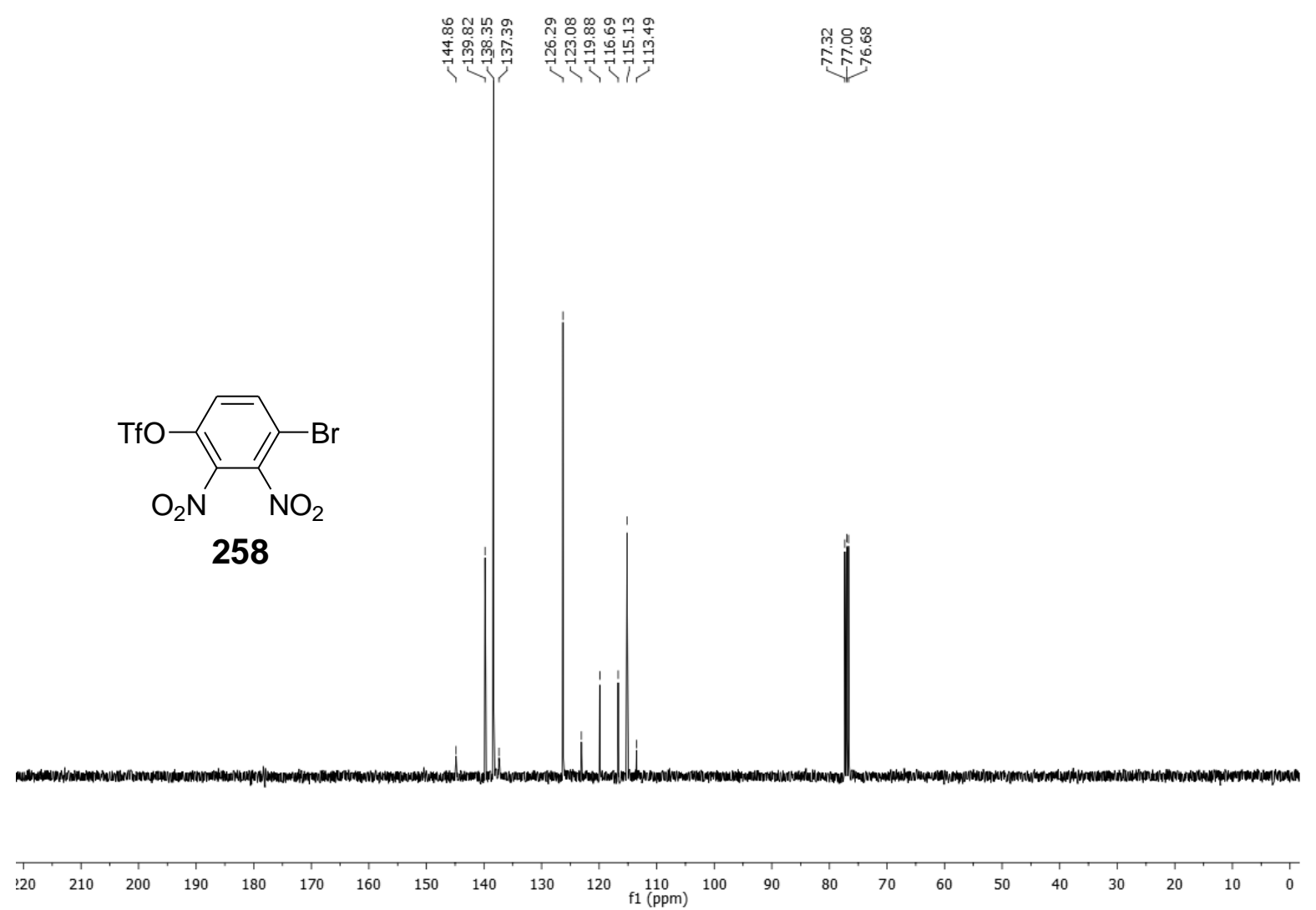

$\stackrel{\text { กิ }}{\text { i }}$

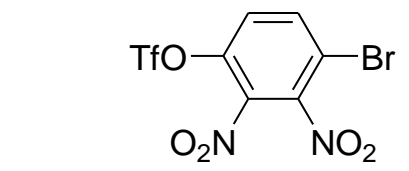

258

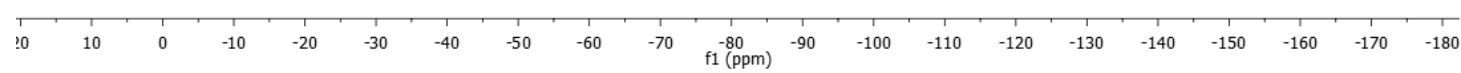

Figure 7.4 ${ }^{13} \mathrm{C}$ and ${ }^{19} \mathrm{~F}$ NMR of compound 258 

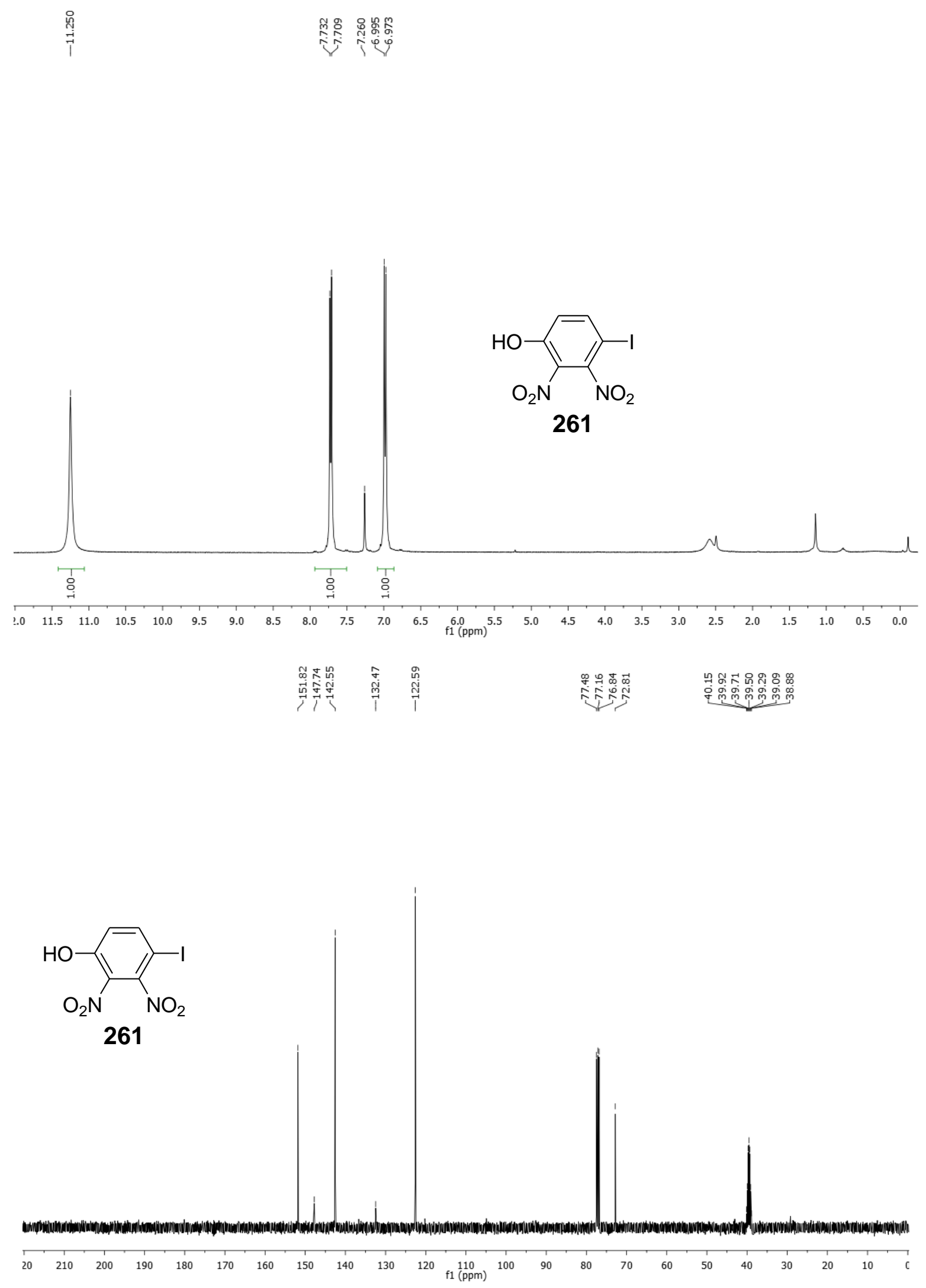

Figure 7.5 ${ }^{1} \mathrm{H}$ and ${ }^{13} \mathrm{C}$ NMR of compound 261 


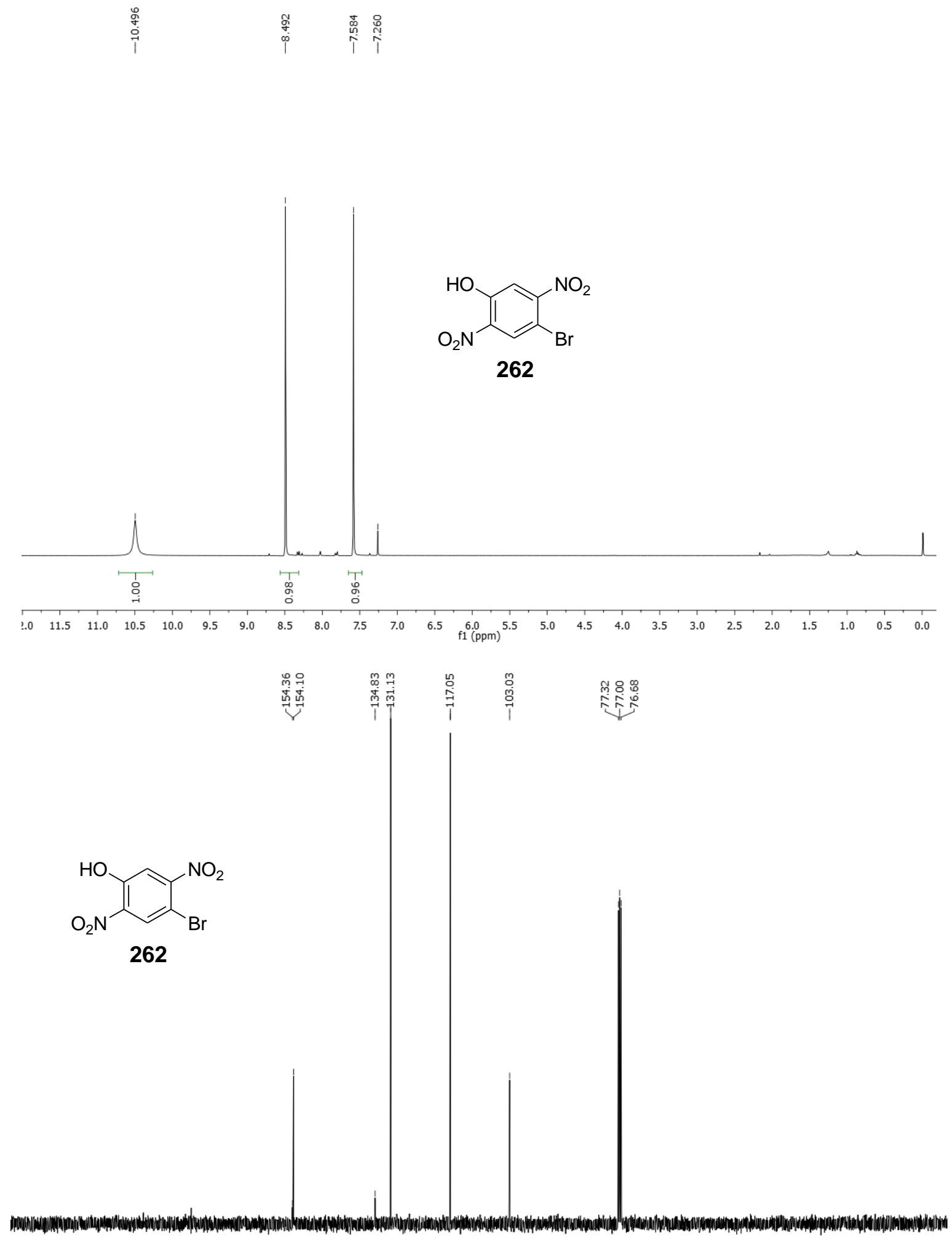

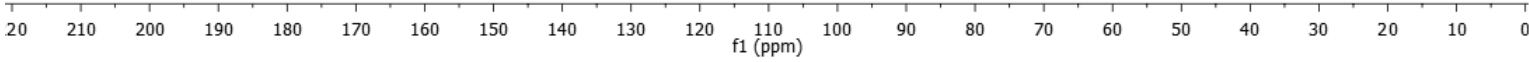

Figure 7.6 ${ }^{1} \mathrm{H}$ and ${ }^{13} \mathrm{C}$ NMR of compound 262 


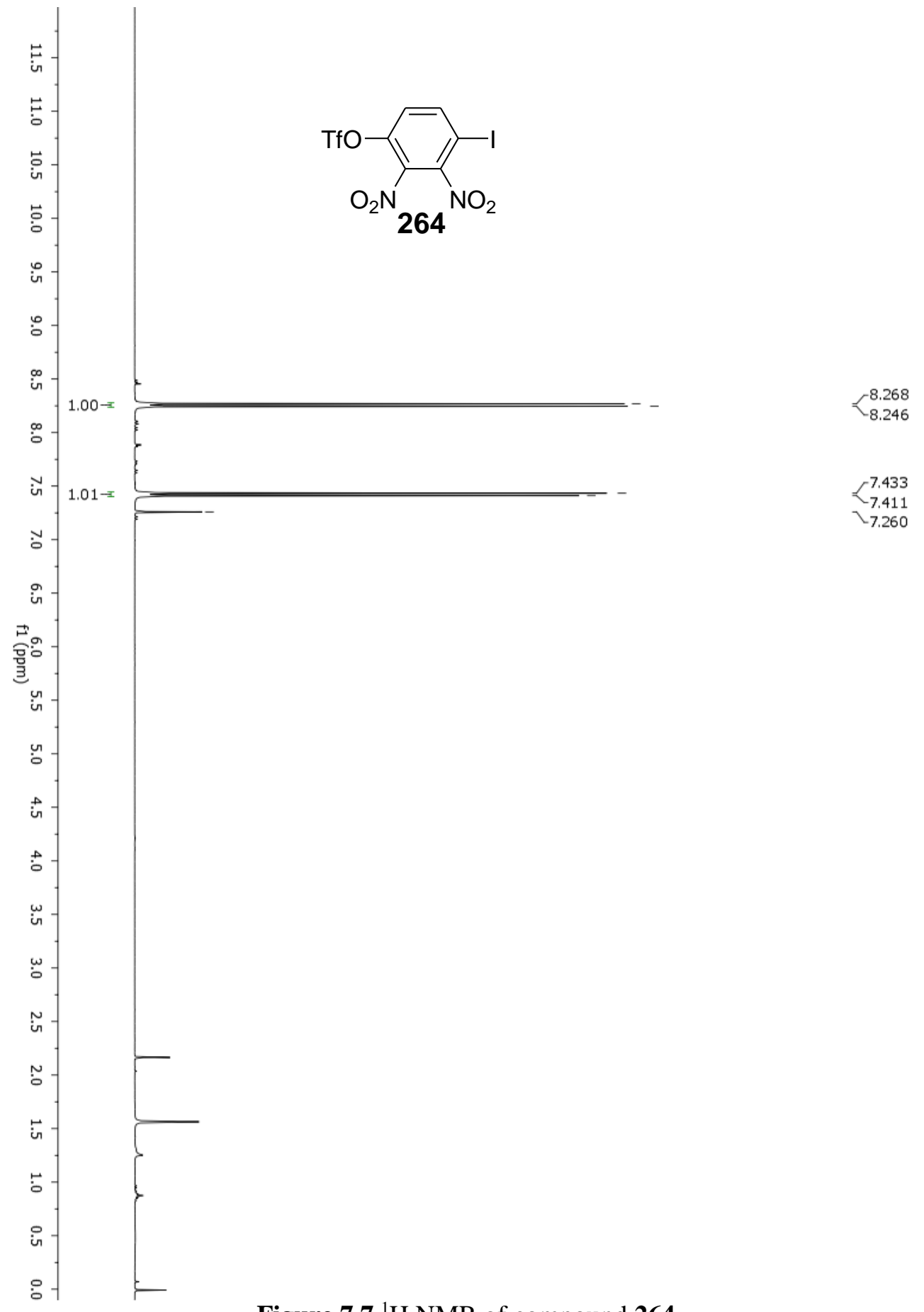

Figure $7.7{ }^{1} \mathrm{H}$ NMR of compound 264 


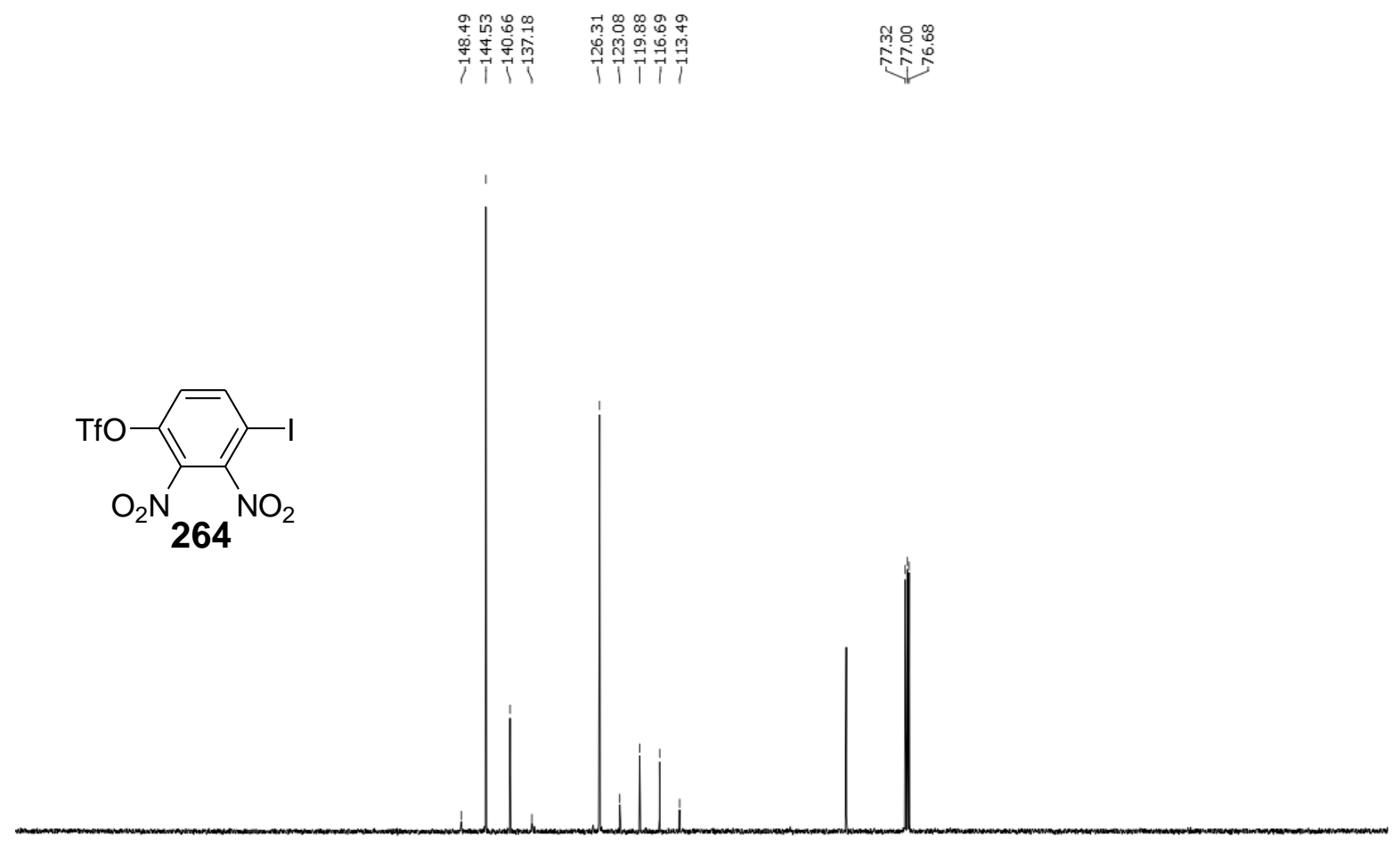

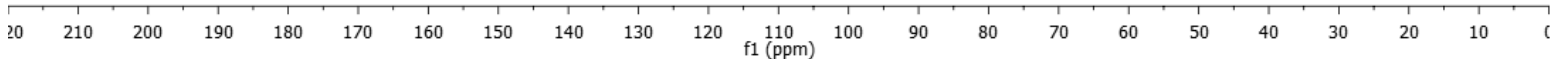
$\stackrel{\substack{+\mathfrak{i}}}{\mathfrak{i}}$<smiles>O=[N+]([O-])c1c(O)ccc(I)c1[N+](=O)[O-]</smiles>

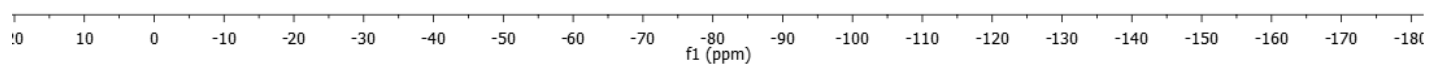

Figure 7.8 ${ }^{13} \mathrm{C}$ and ${ }^{19} \mathrm{~F}$ NMR of compound 264 


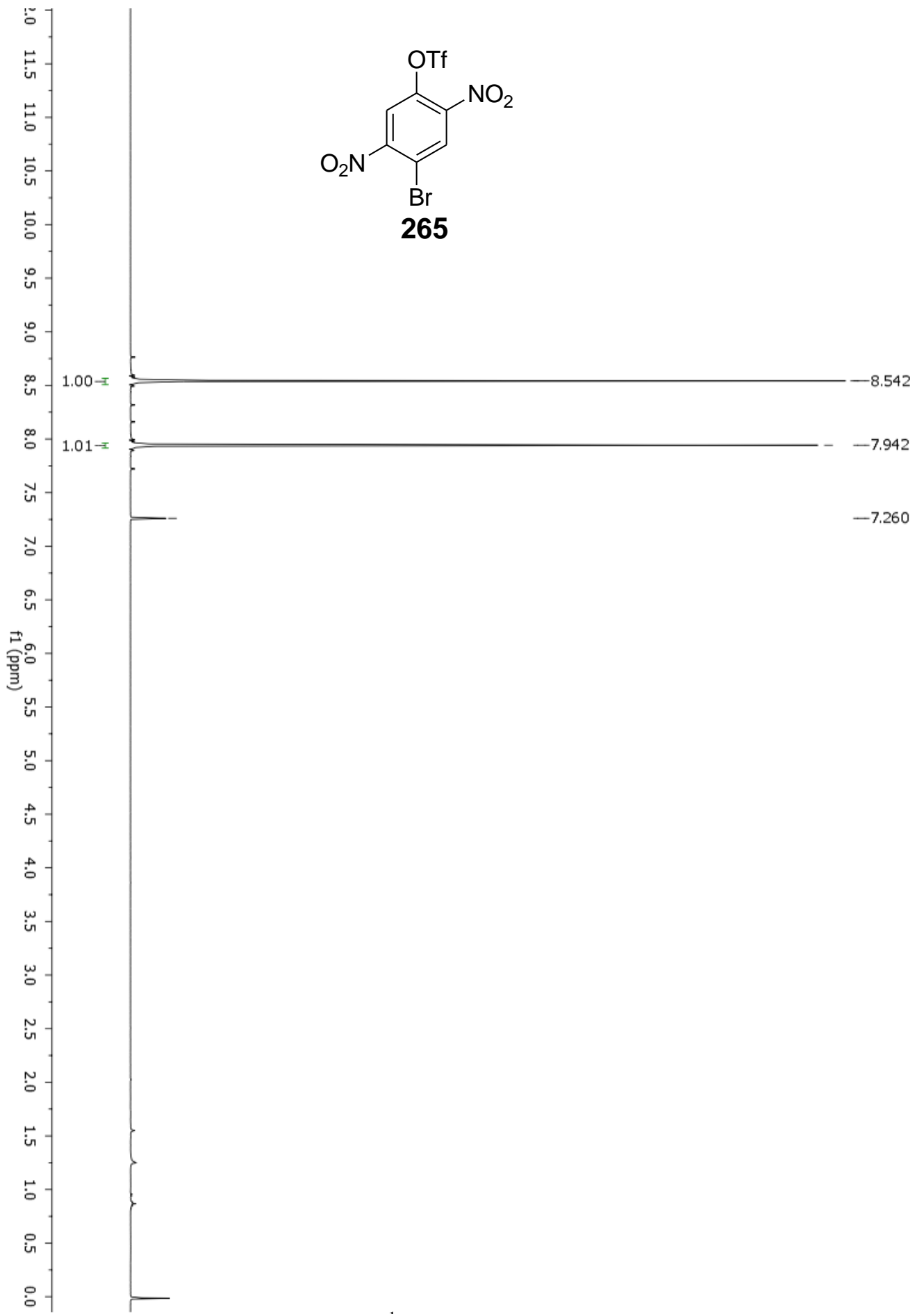

Figure 7.9 ${ }^{1} \mathrm{H}$ NMR of compound 265 

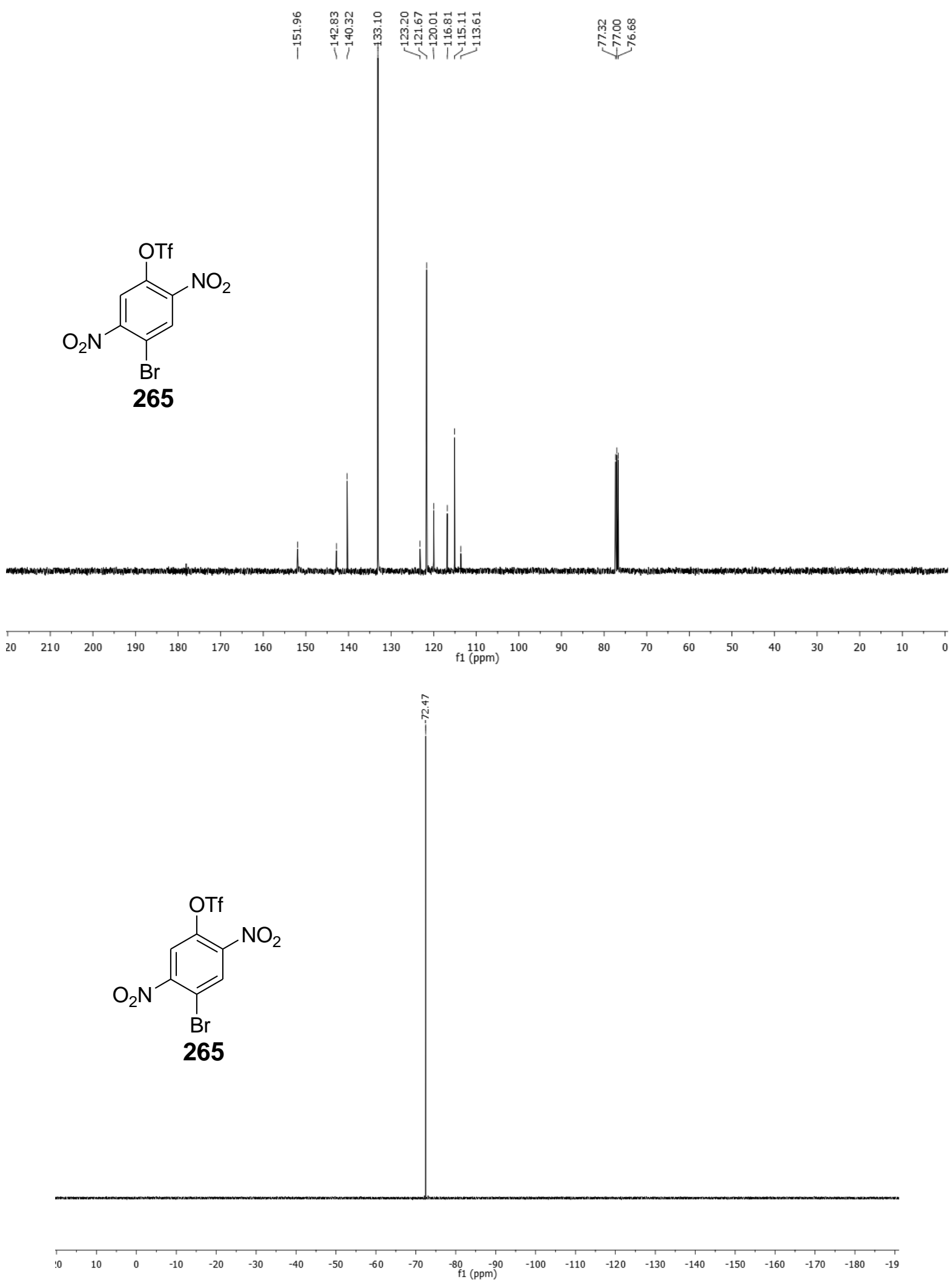

Figure 7.10 ${ }^{13} \mathrm{C}$ and ${ }^{19} \mathrm{~F}$ NMR of compound 265 


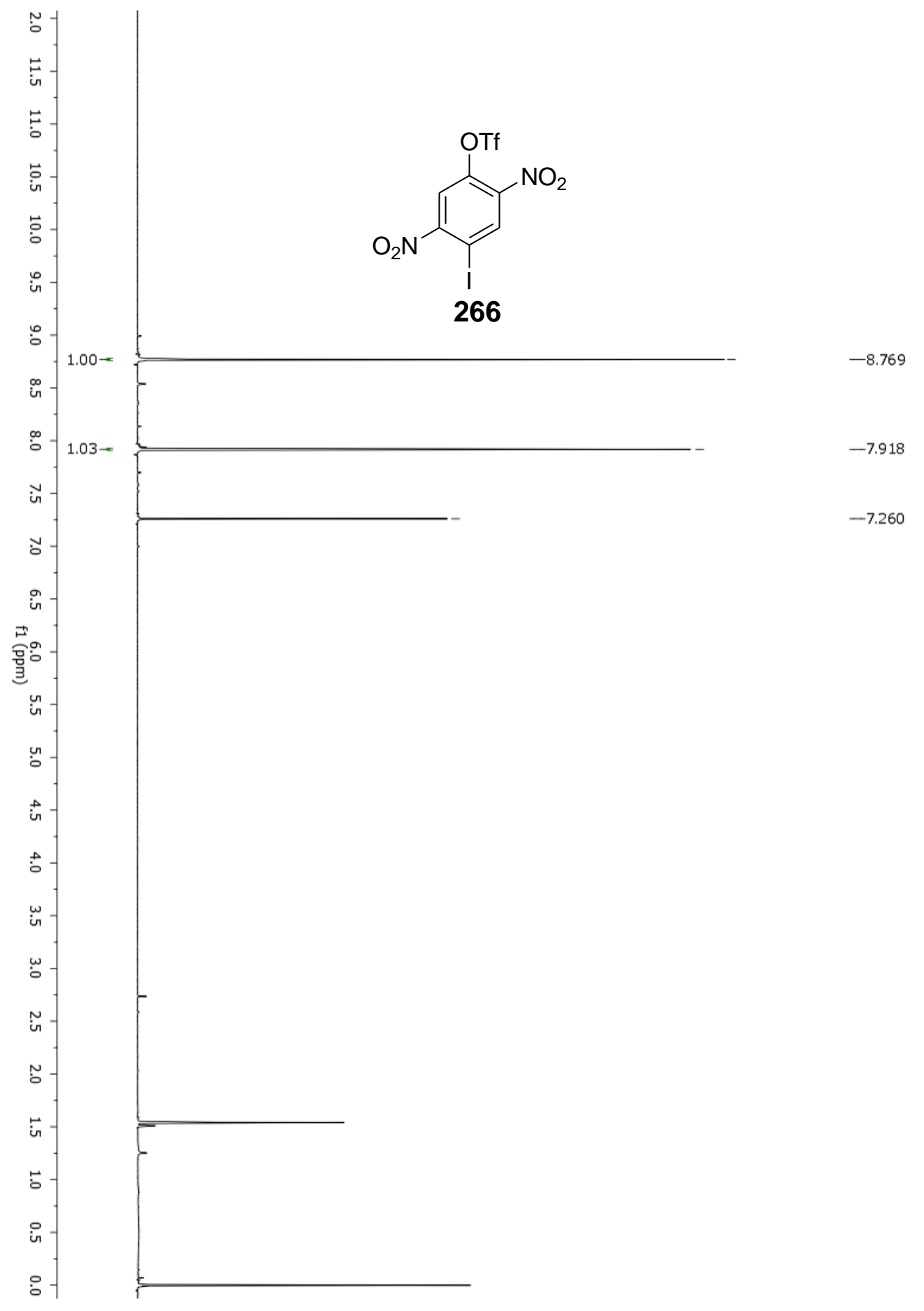

Figure 7.11 ${ }^{1} \mathrm{H}$ NMR of compound 266 

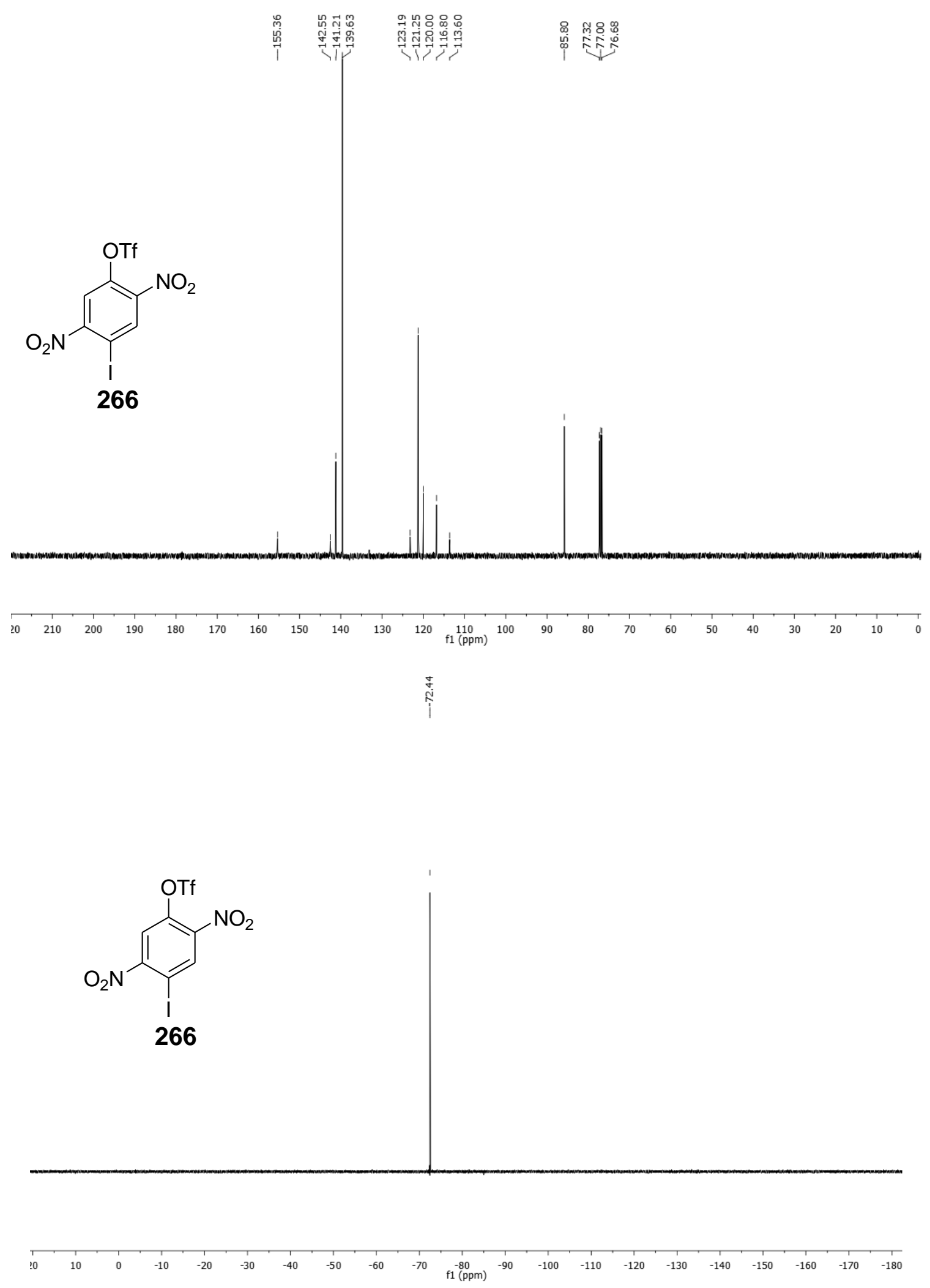

Figure 7.12 ${ }^{13} \mathrm{C}$ and ${ }^{19} \mathrm{~F}$ NMR of compound 266 


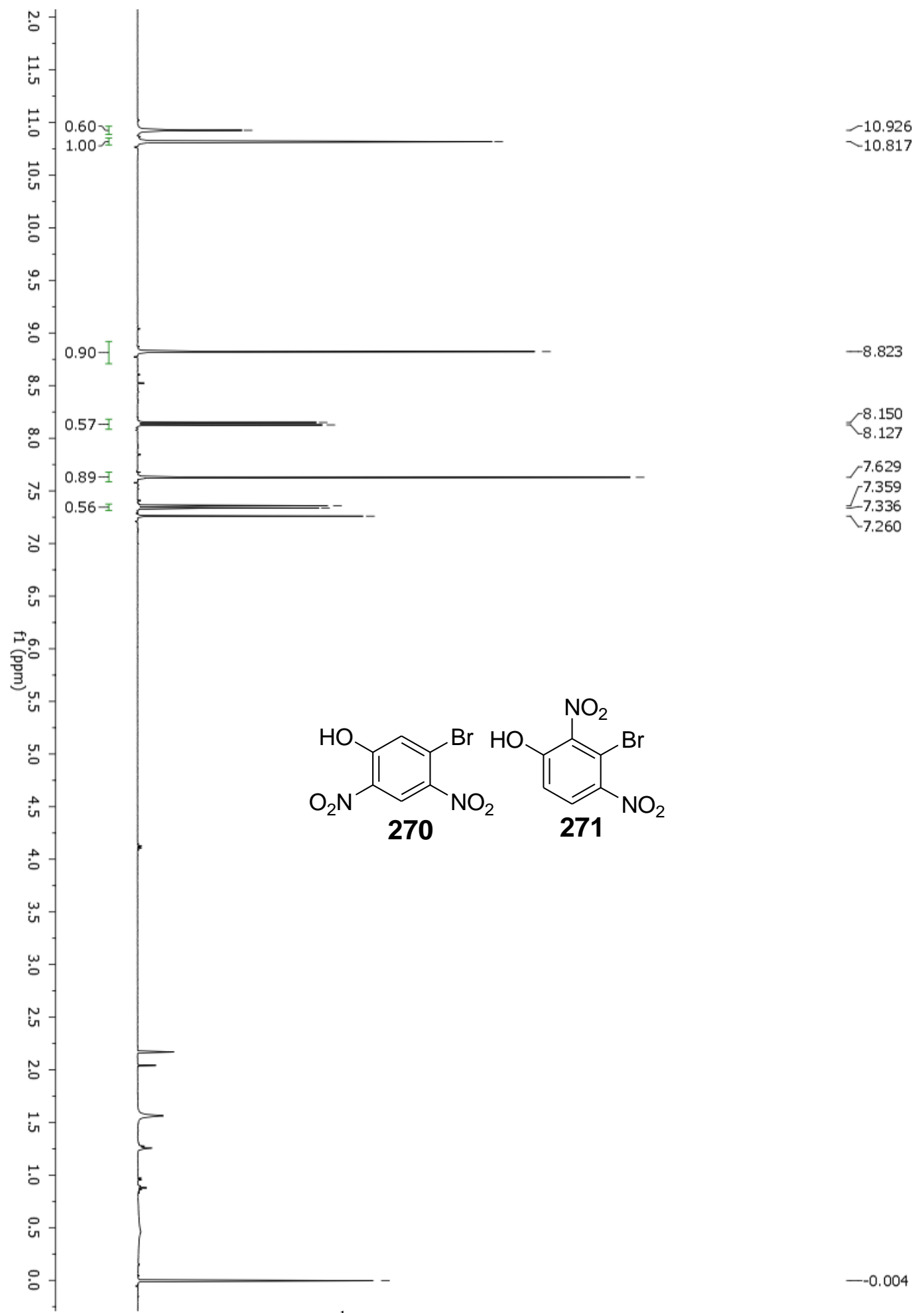

Figure 7.13 ${ }^{1} \mathrm{H}$ NMR of a mixture of 270 and 271 


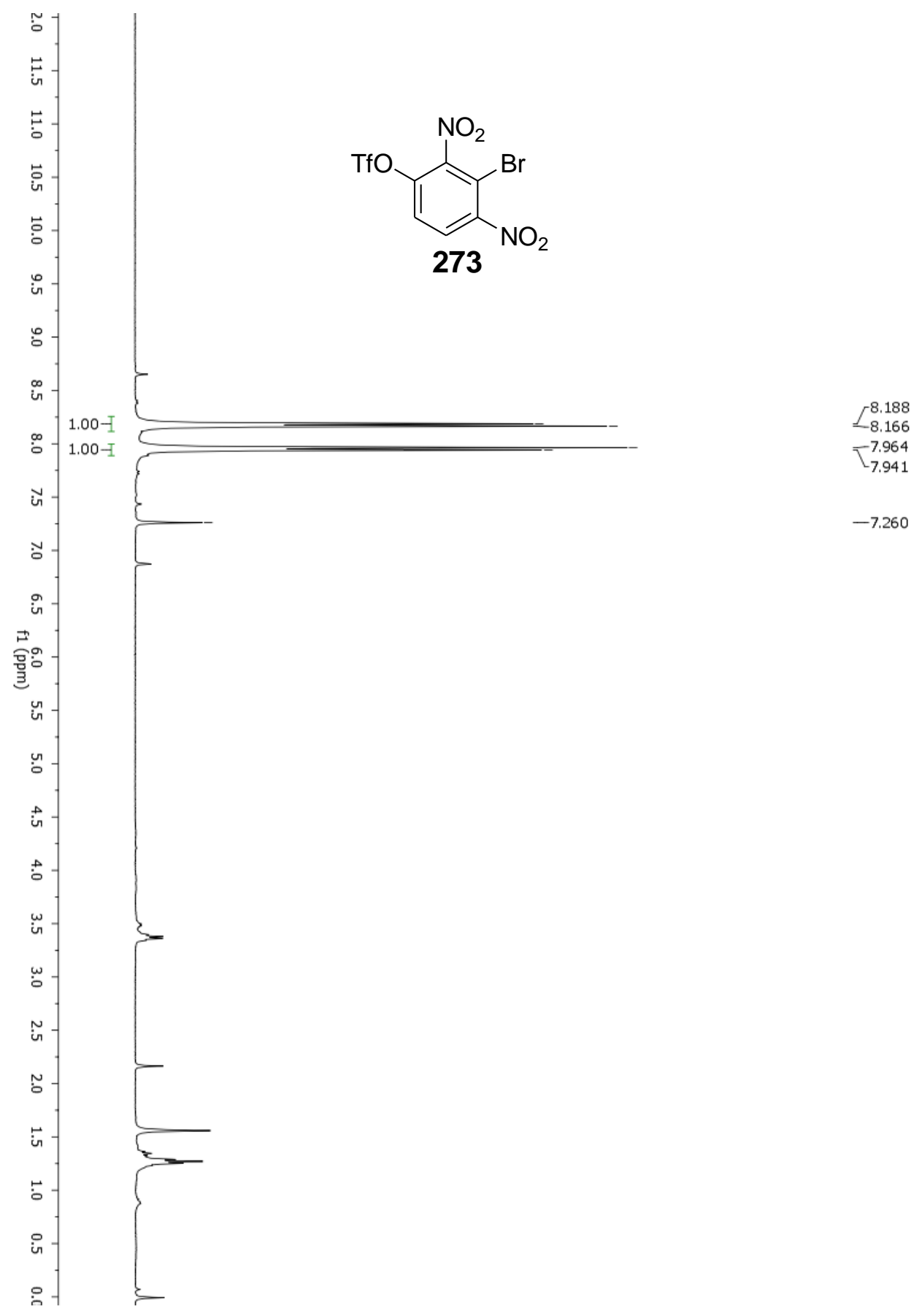

Figure $7.14{ }^{1} \mathrm{H}$ NMR of compound 273 

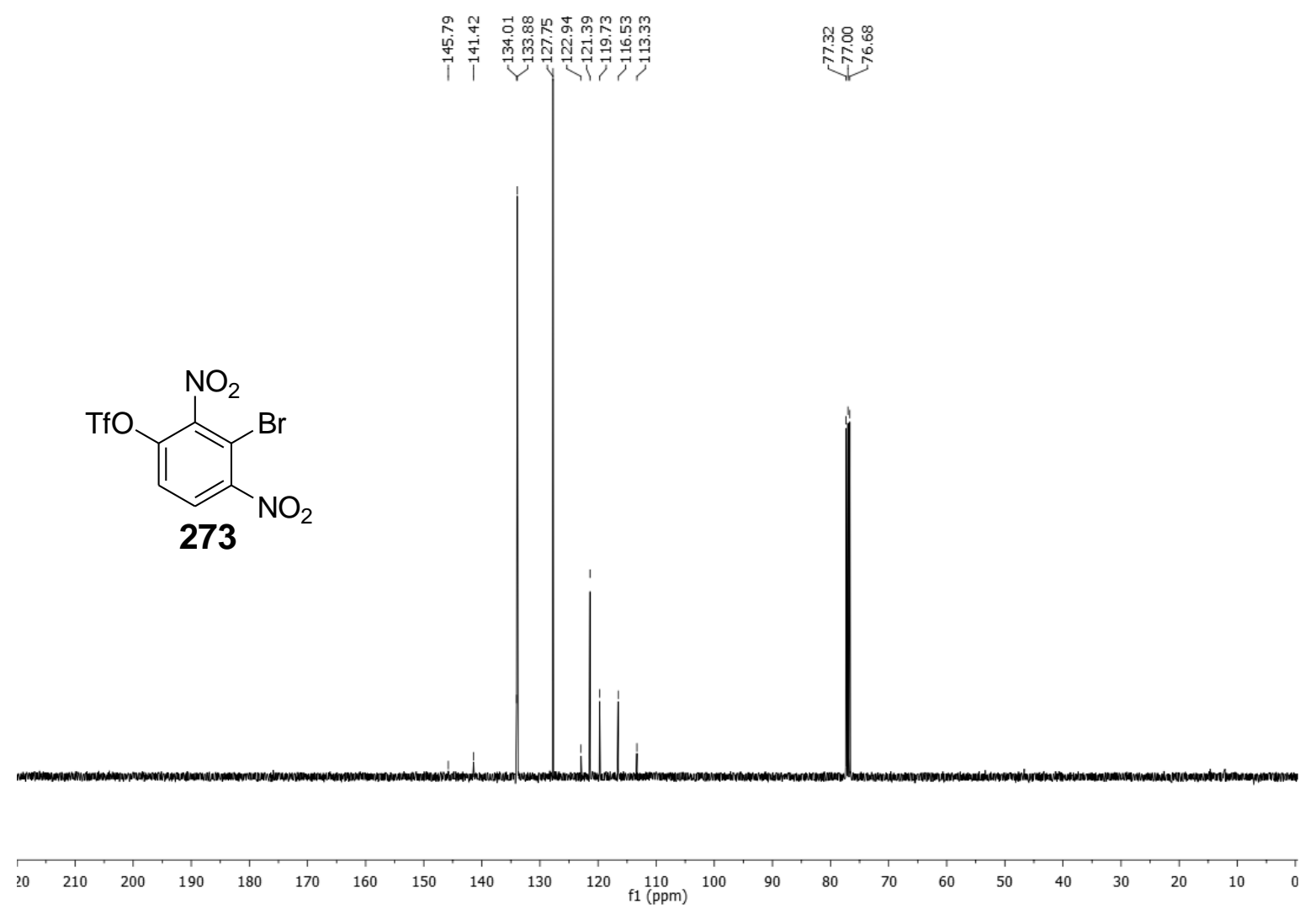

$\stackrel{\substack{\infty \\ i}}{i}$

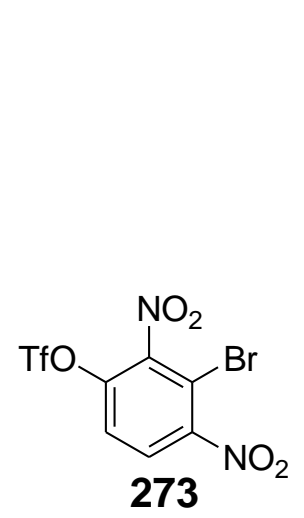

\section{$\stackrel{\substack{n \\ i}}{i}$}

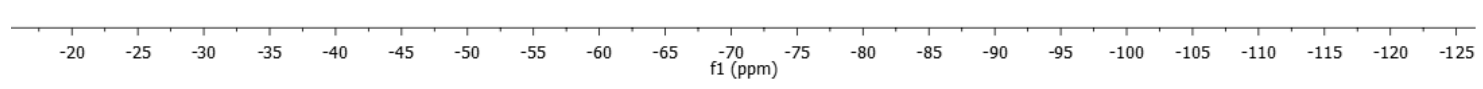

Figure $7.15{ }^{13} \mathrm{C}$ and ${ }^{19} \mathrm{~F}$ NMR of compound 273 

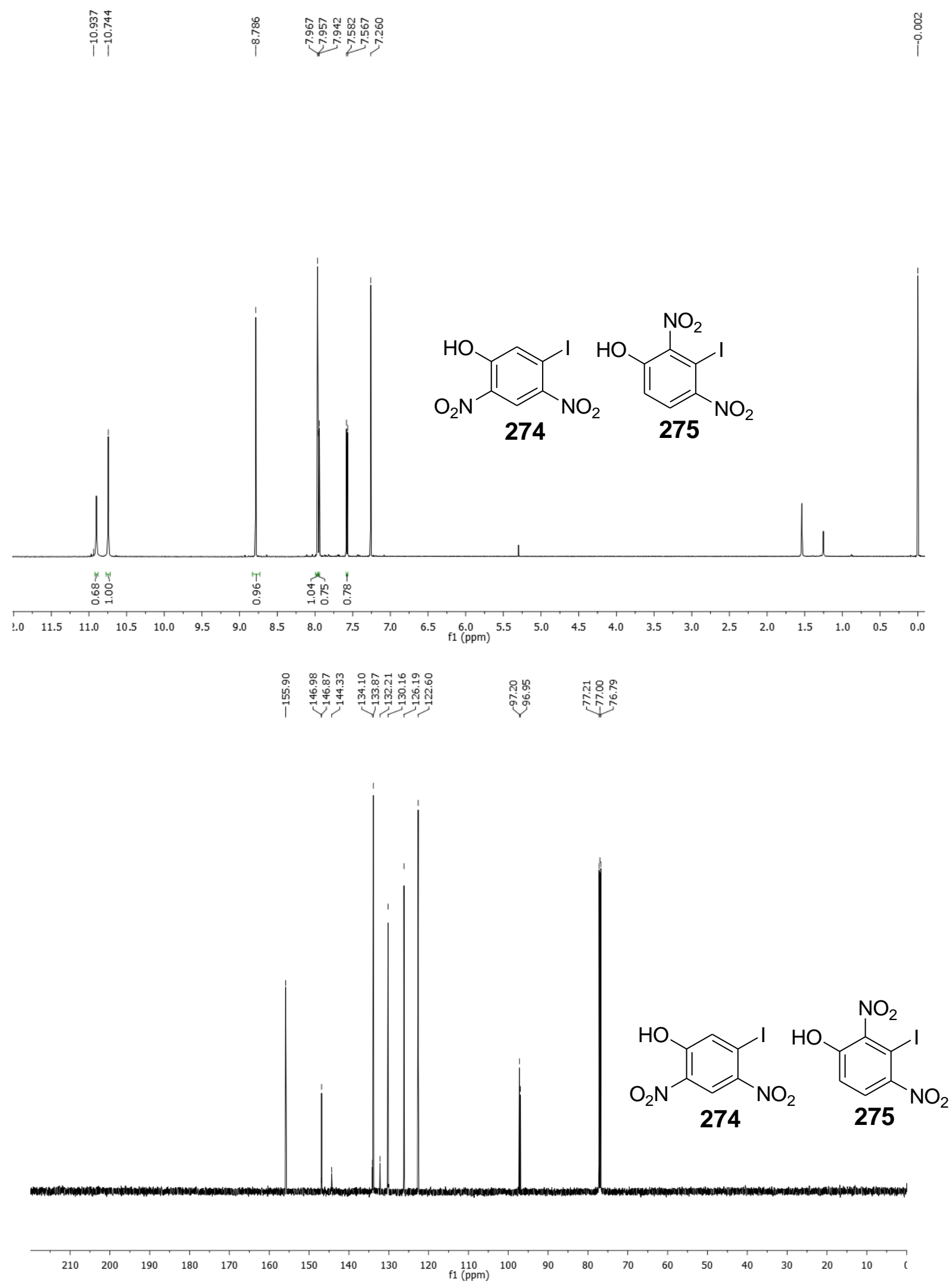

Figure $7.16{ }^{1} \mathrm{H}$ and ${ }^{13} \mathrm{C}$ NMR of a mixture of 274 and 275 


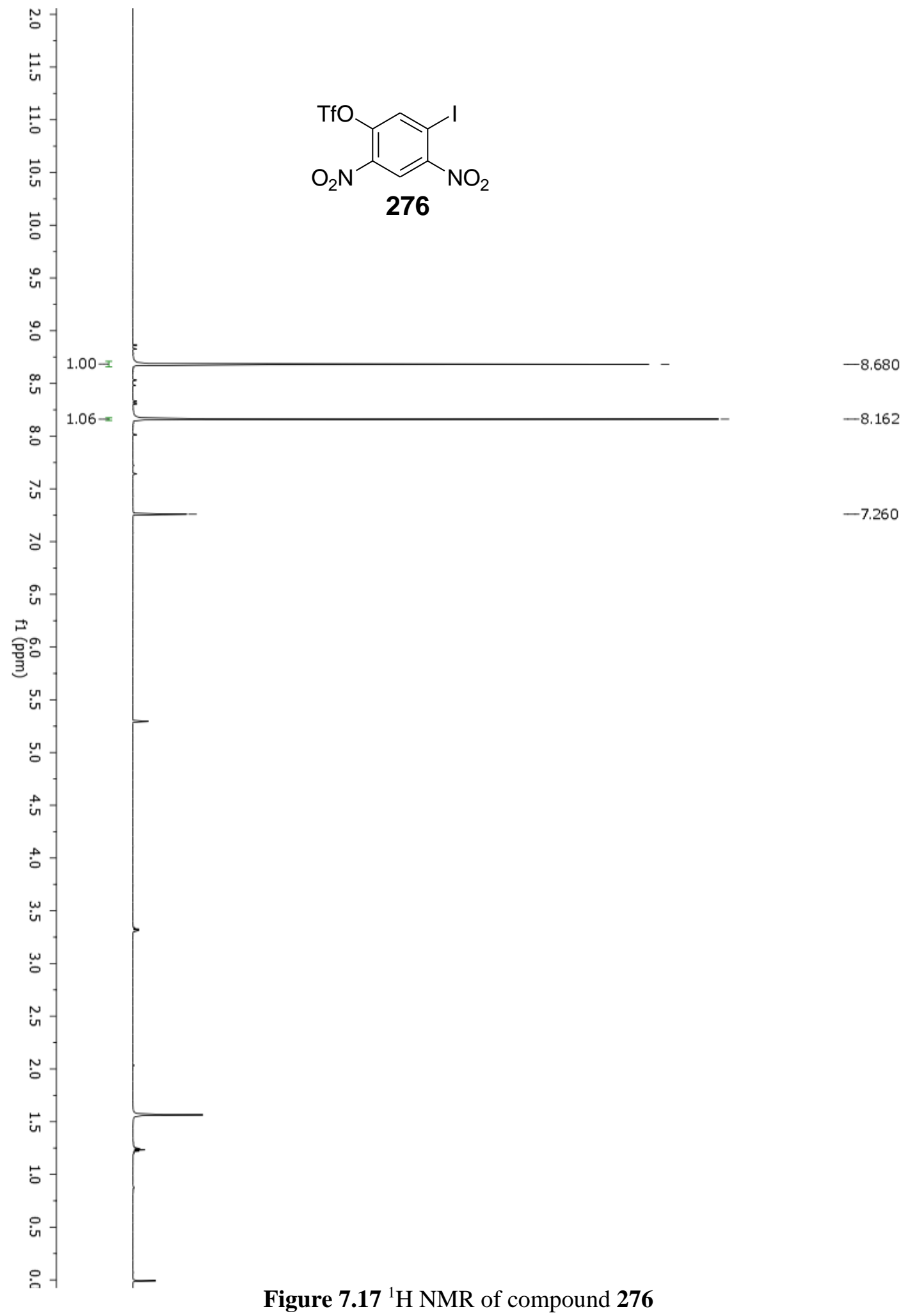




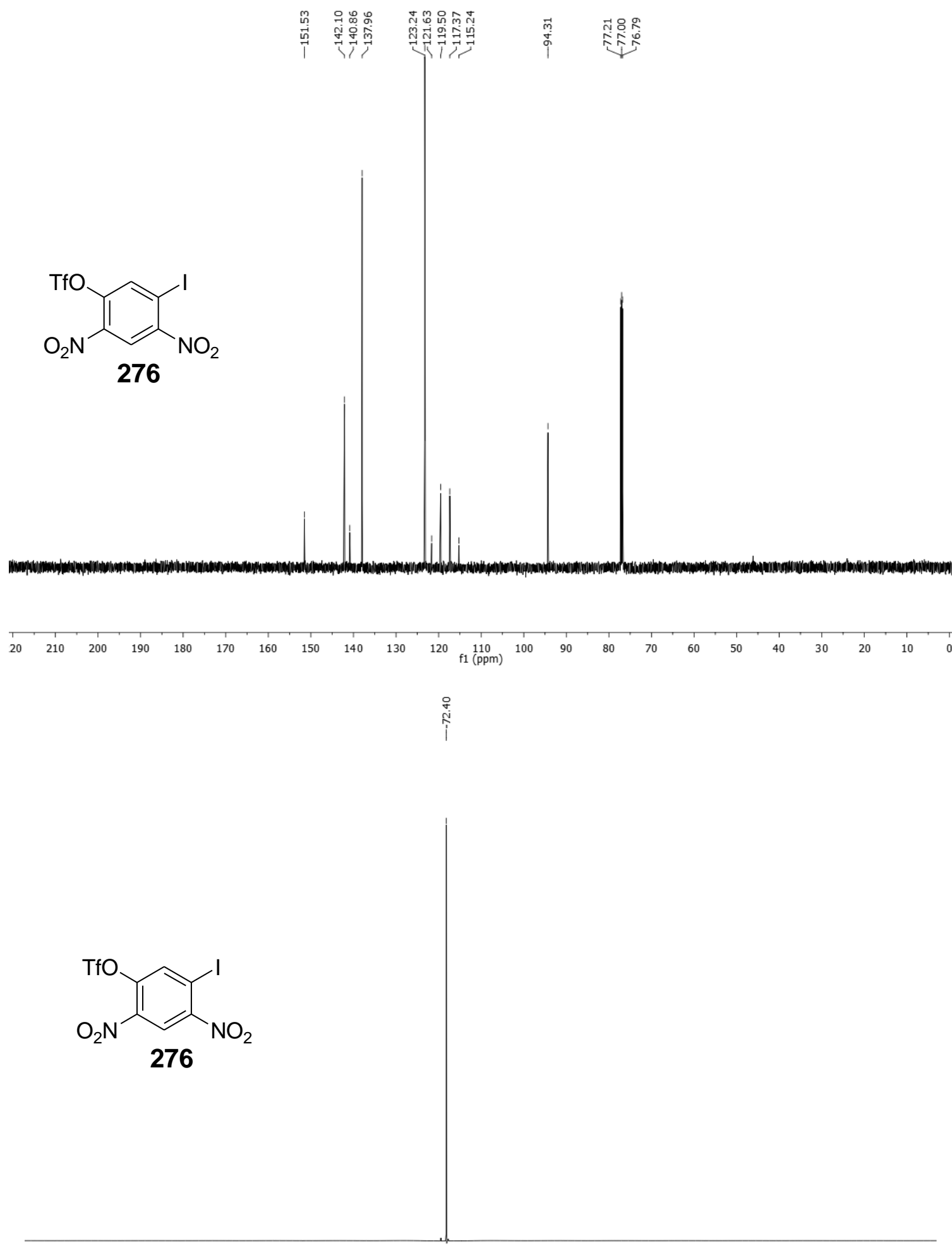

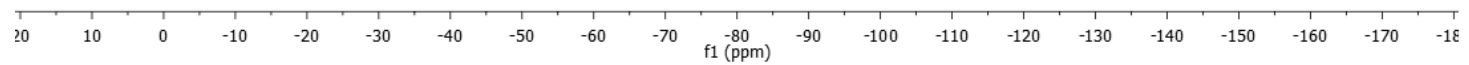

Figure 7.18 ${ }^{13} \mathrm{C}$ and ${ }^{19} \mathrm{~F}$ NMR of compound 276 


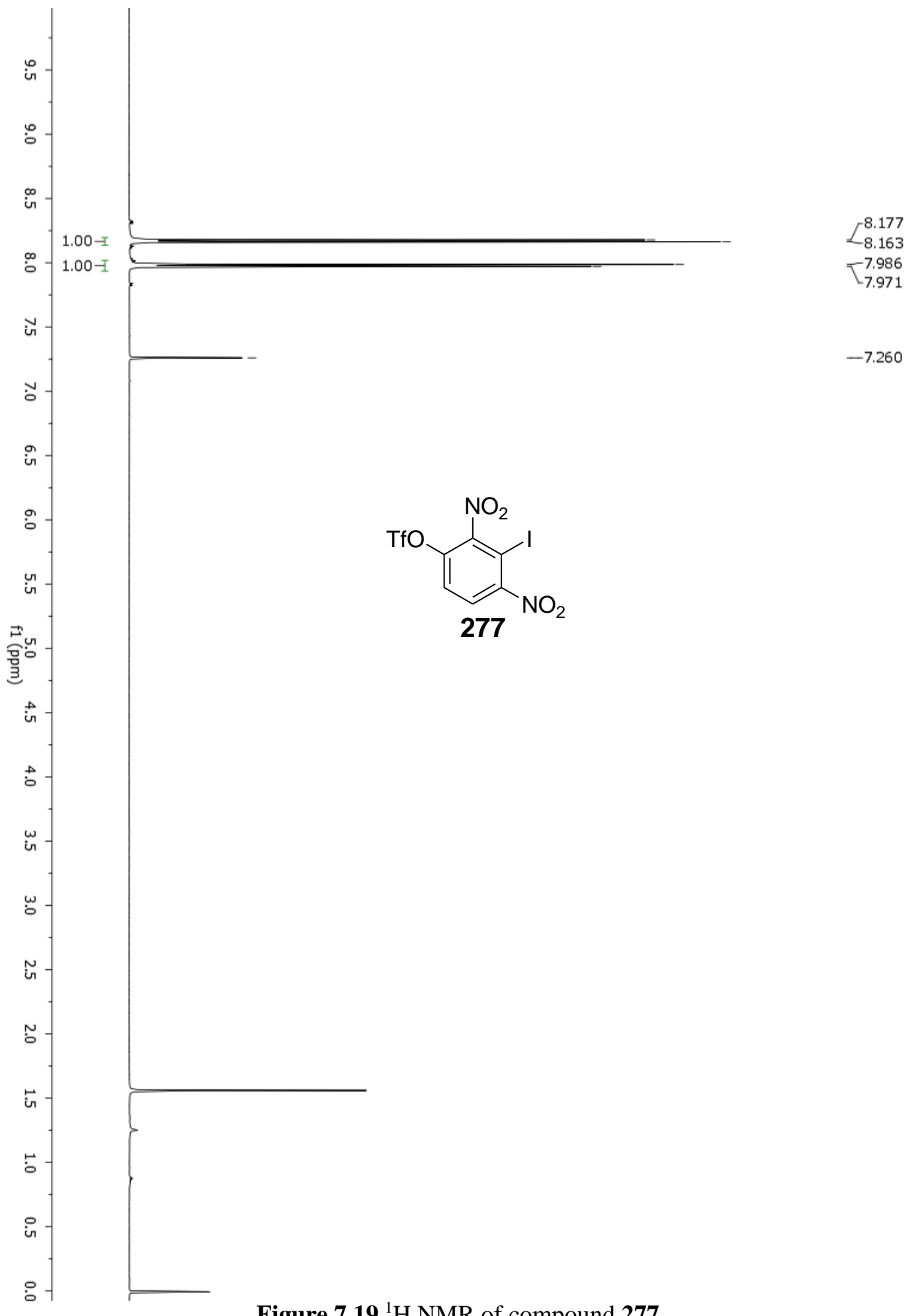

Figure $7.19{ }^{1} \mathrm{H}$ NMR of compound 277 

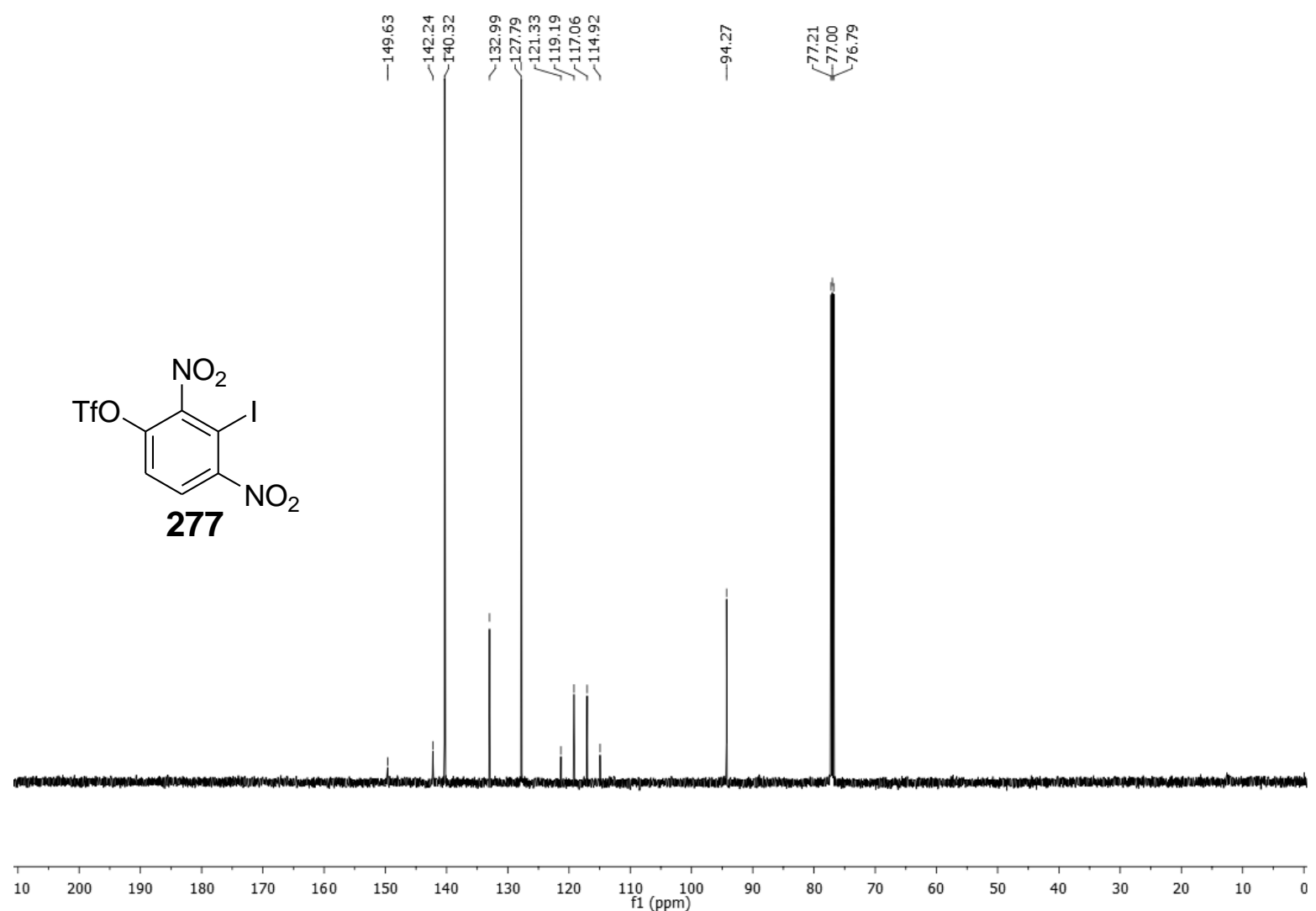

$\stackrel{\substack{i \\ i}}{i}$

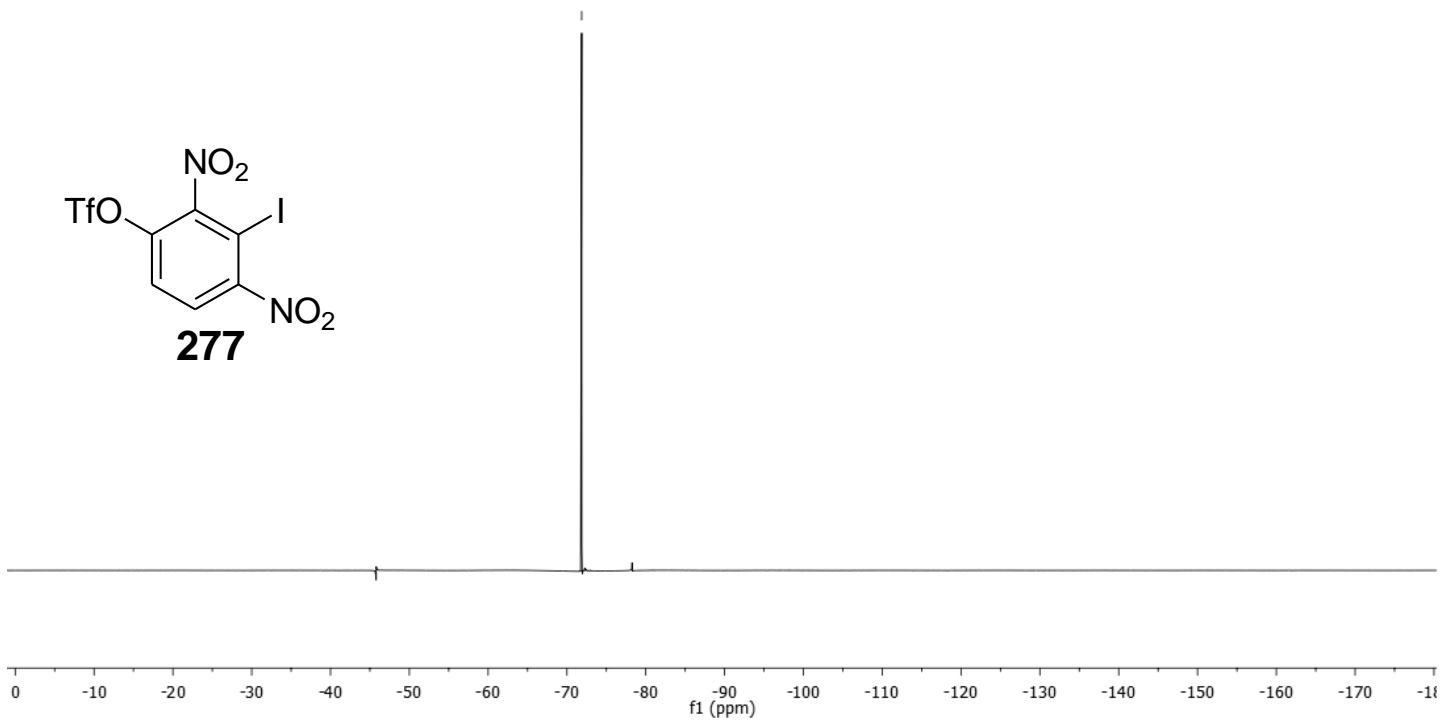

Figure 7.20 ${ }^{13} \mathrm{C}$ and ${ }^{19} \mathrm{~F}$ NMR of compound 277 

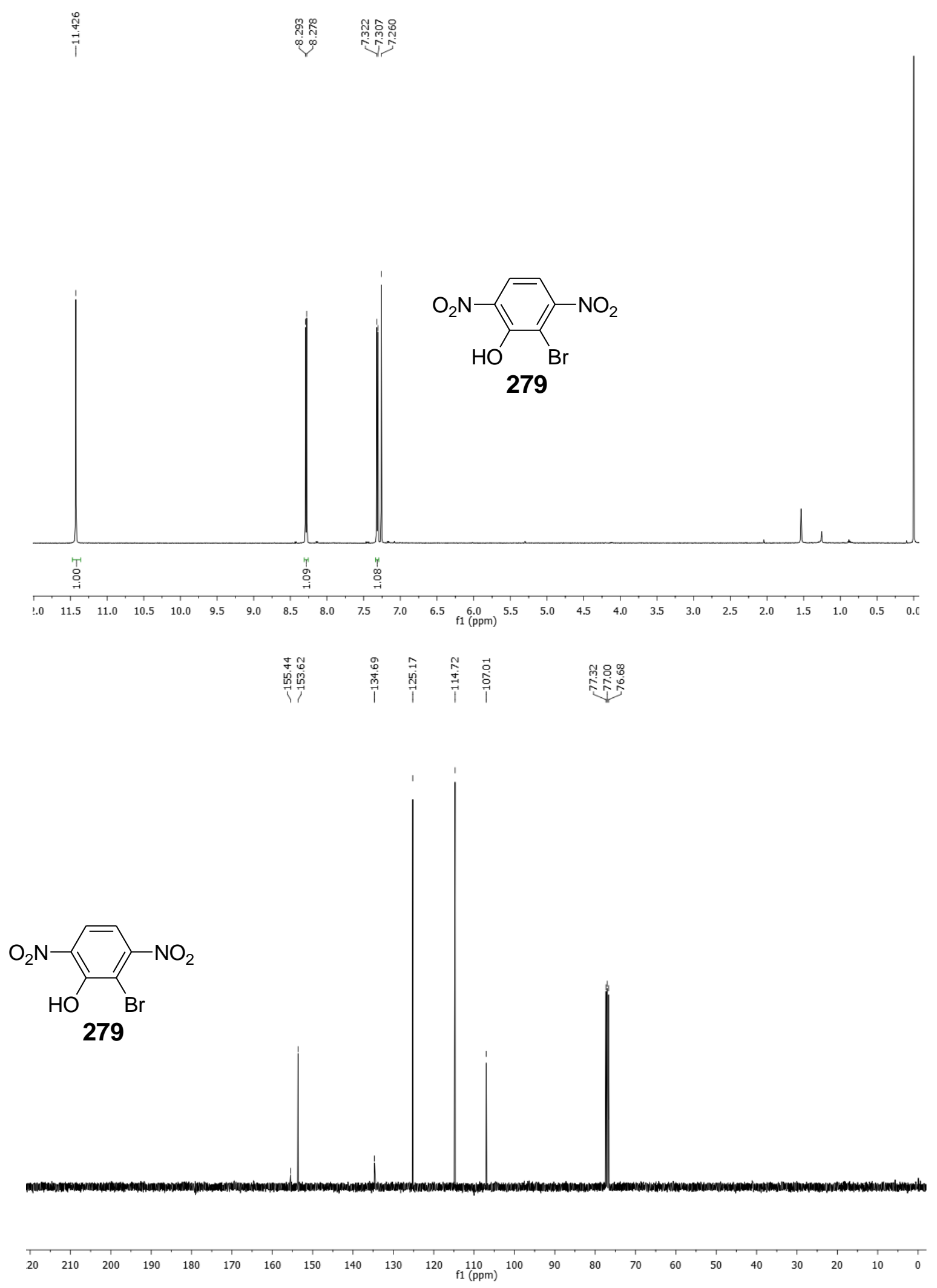

Figure 7.21 ${ }^{1} \mathrm{H}$ and ${ }^{13} \mathrm{C}$ NMR of compound 279 


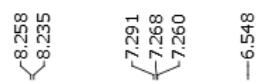

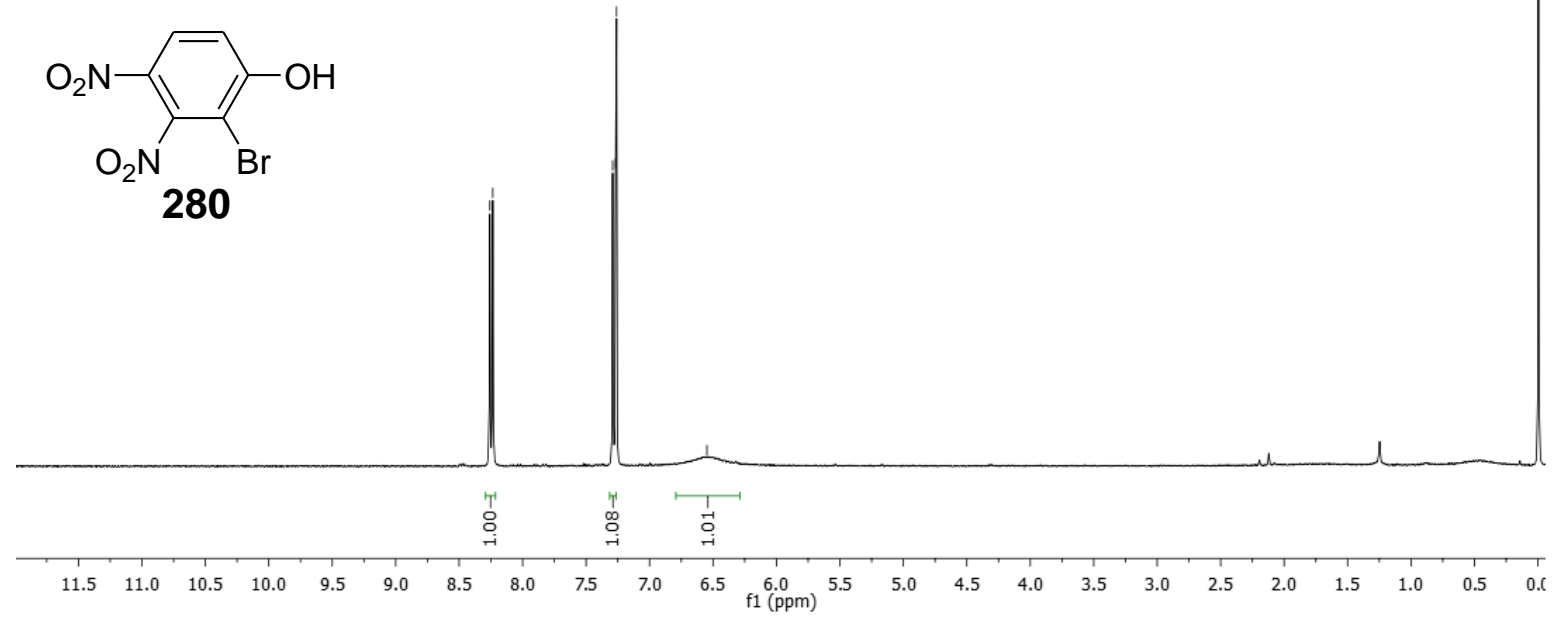

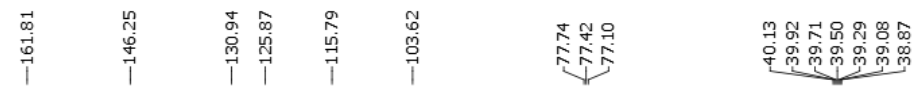
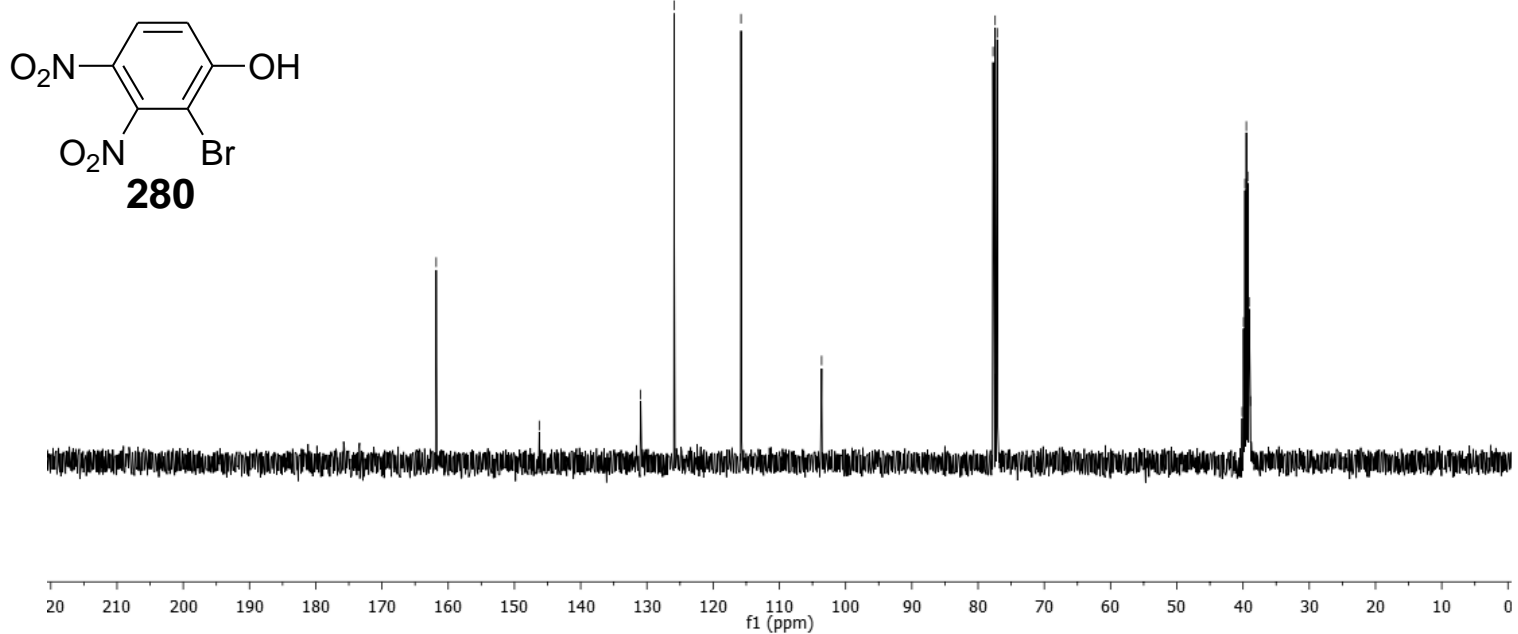

Figure 7.22 ${ }^{1} \mathrm{H}$ and ${ }^{13} \mathrm{C}$ NMR of compound 280 


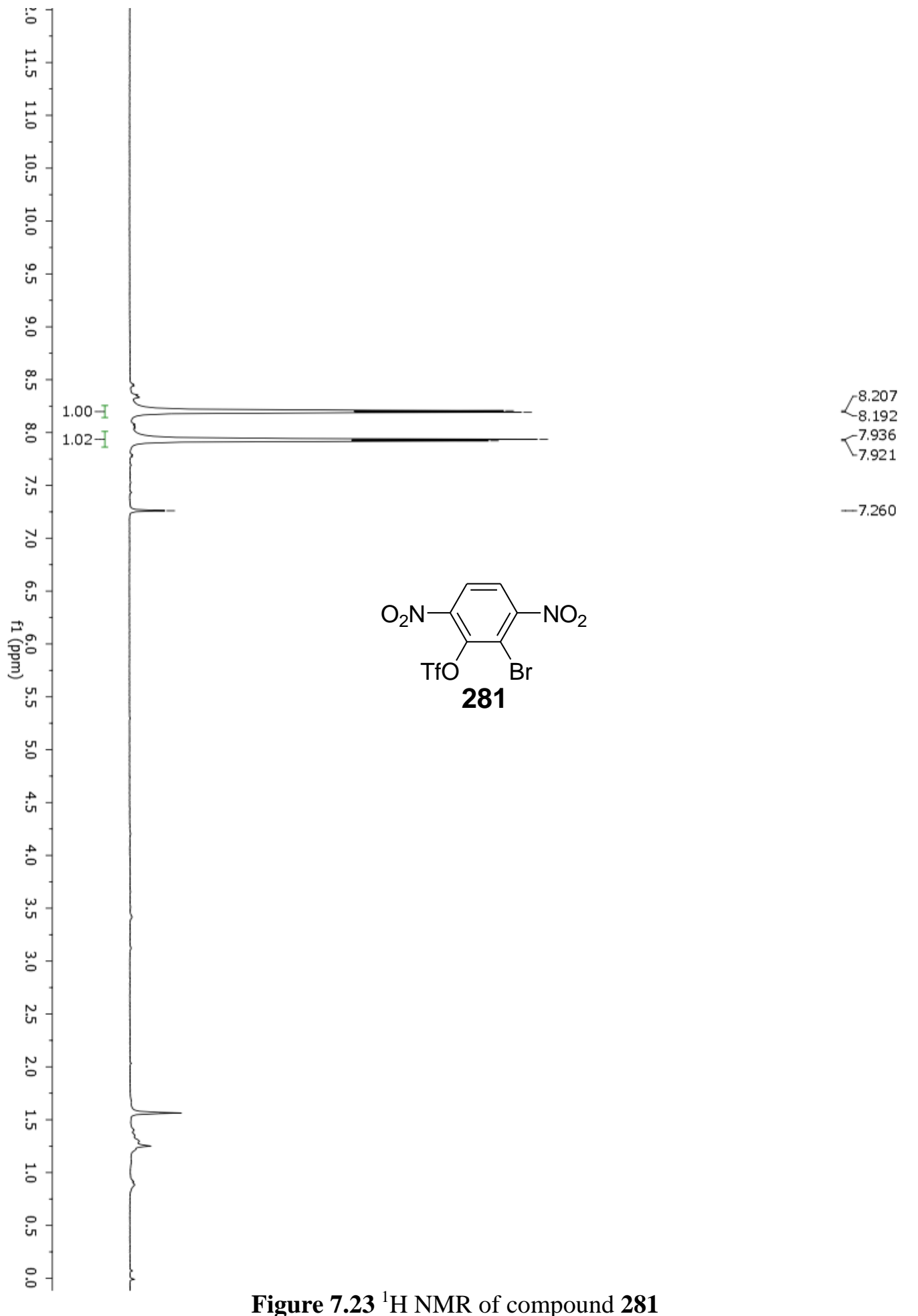

Figure $7.23{ }^{1} \mathrm{H}$ NMR of compound 281 

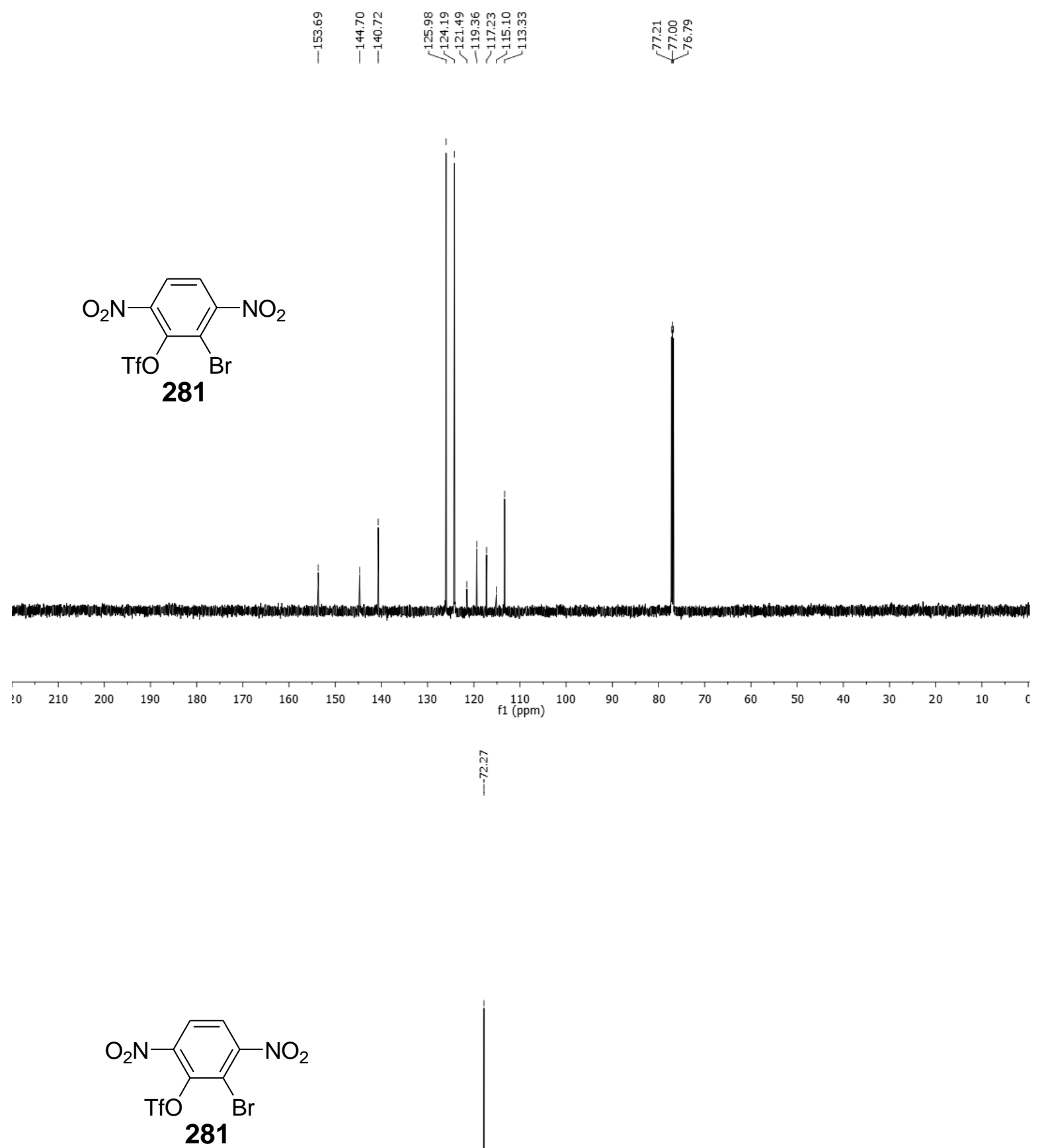

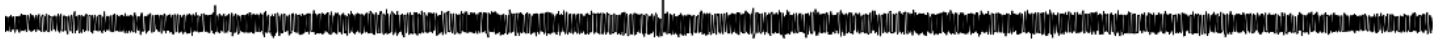

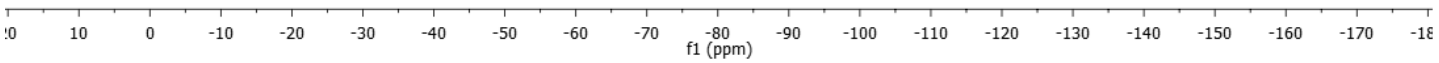

Figure 7.24 ${ }^{13} \mathrm{C}$ and ${ }^{19} \mathrm{~F}$ NMR of compound 281 

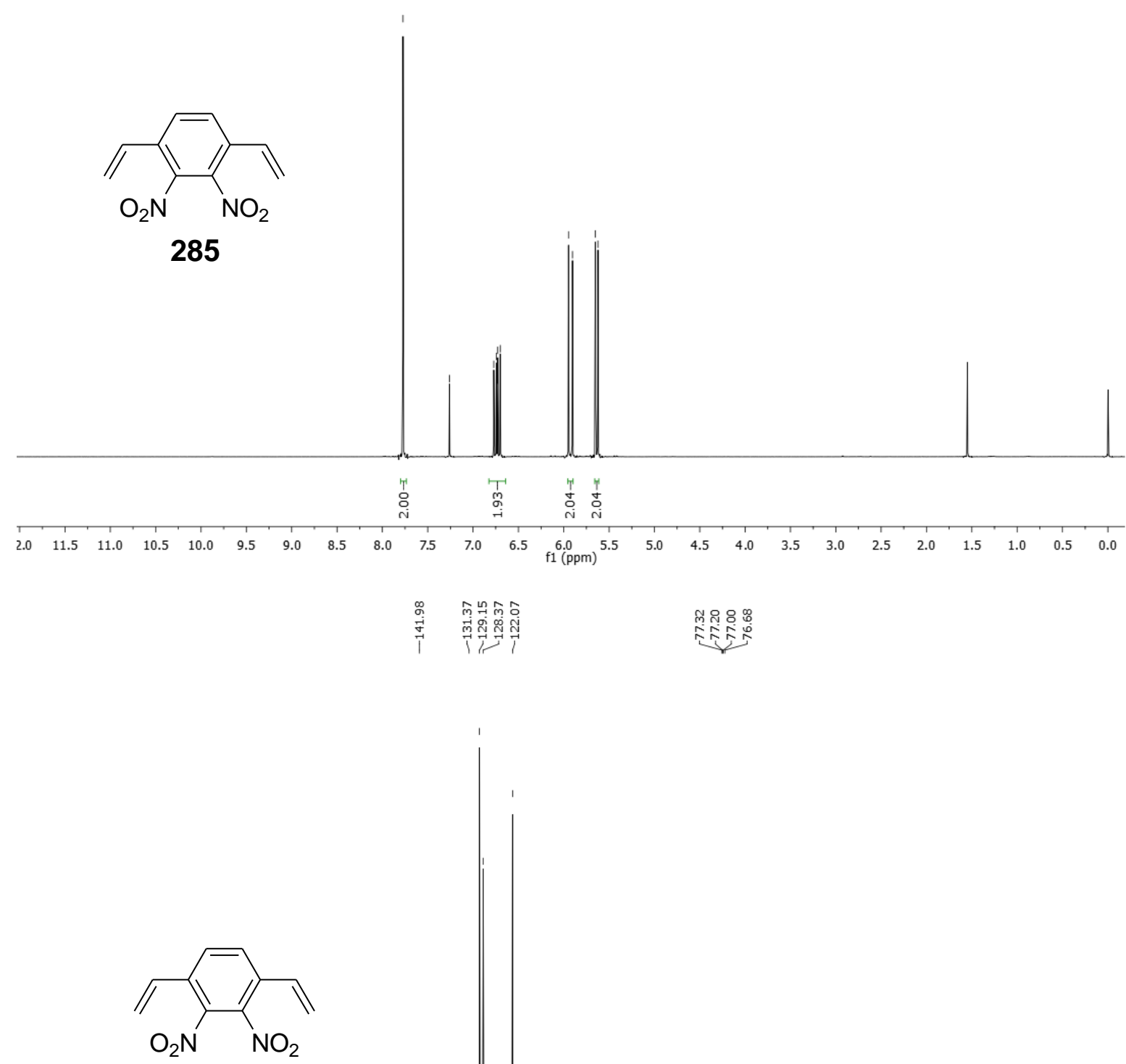

285

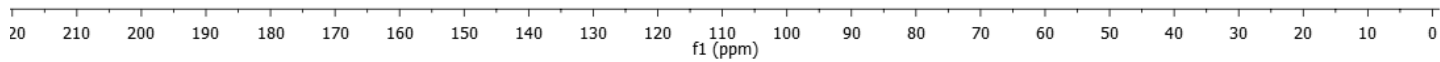

Figure 7.25 ${ }^{1} \mathrm{H}$ and ${ }^{13} \mathrm{C}$ NMR of compound 285 


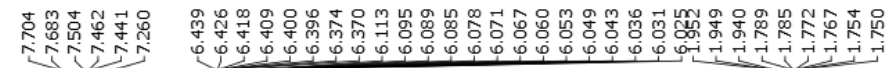
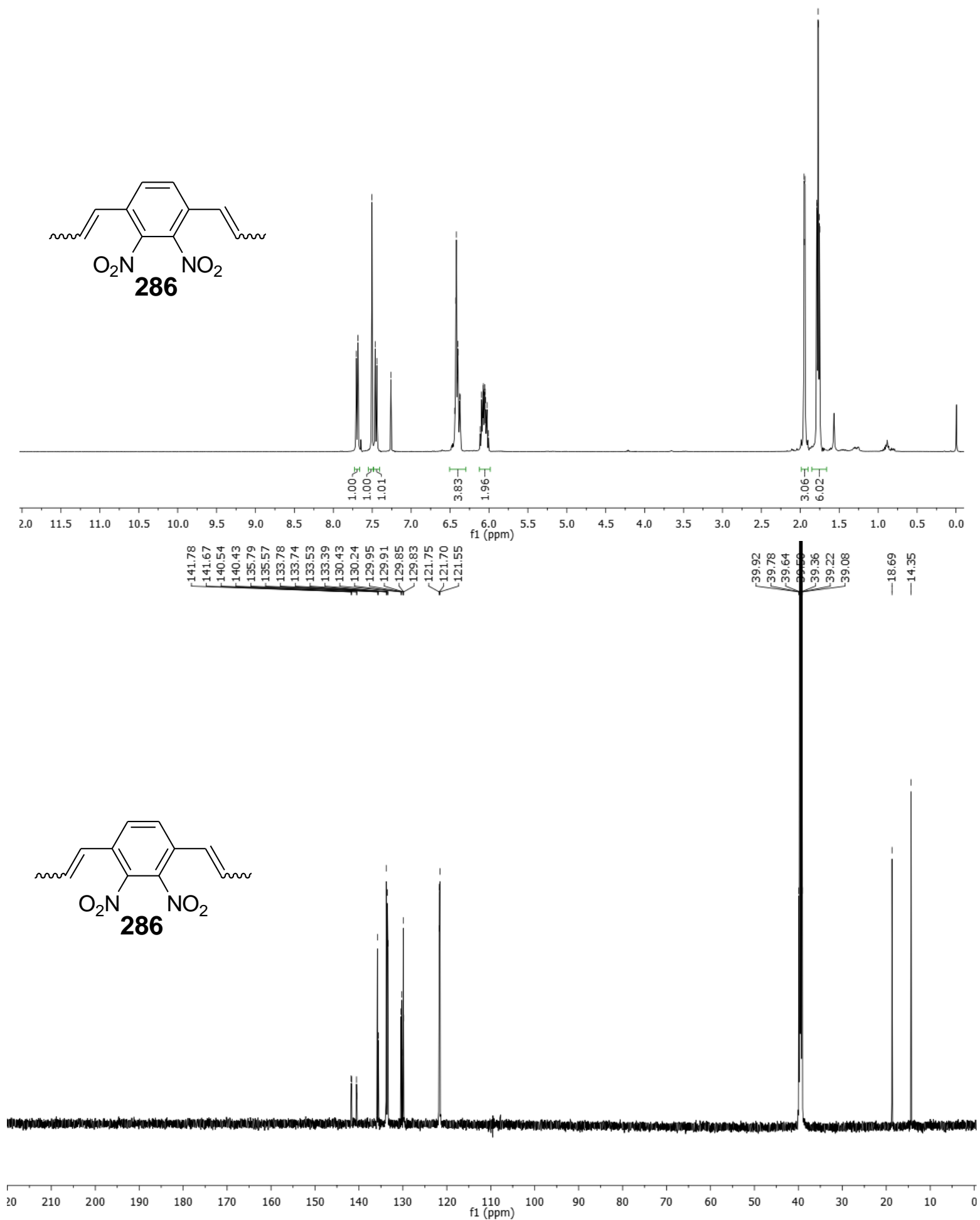

Figure 7.26 ${ }^{1} \mathrm{H}$ and ${ }^{13} \mathrm{C}$ NMR of compound 286 


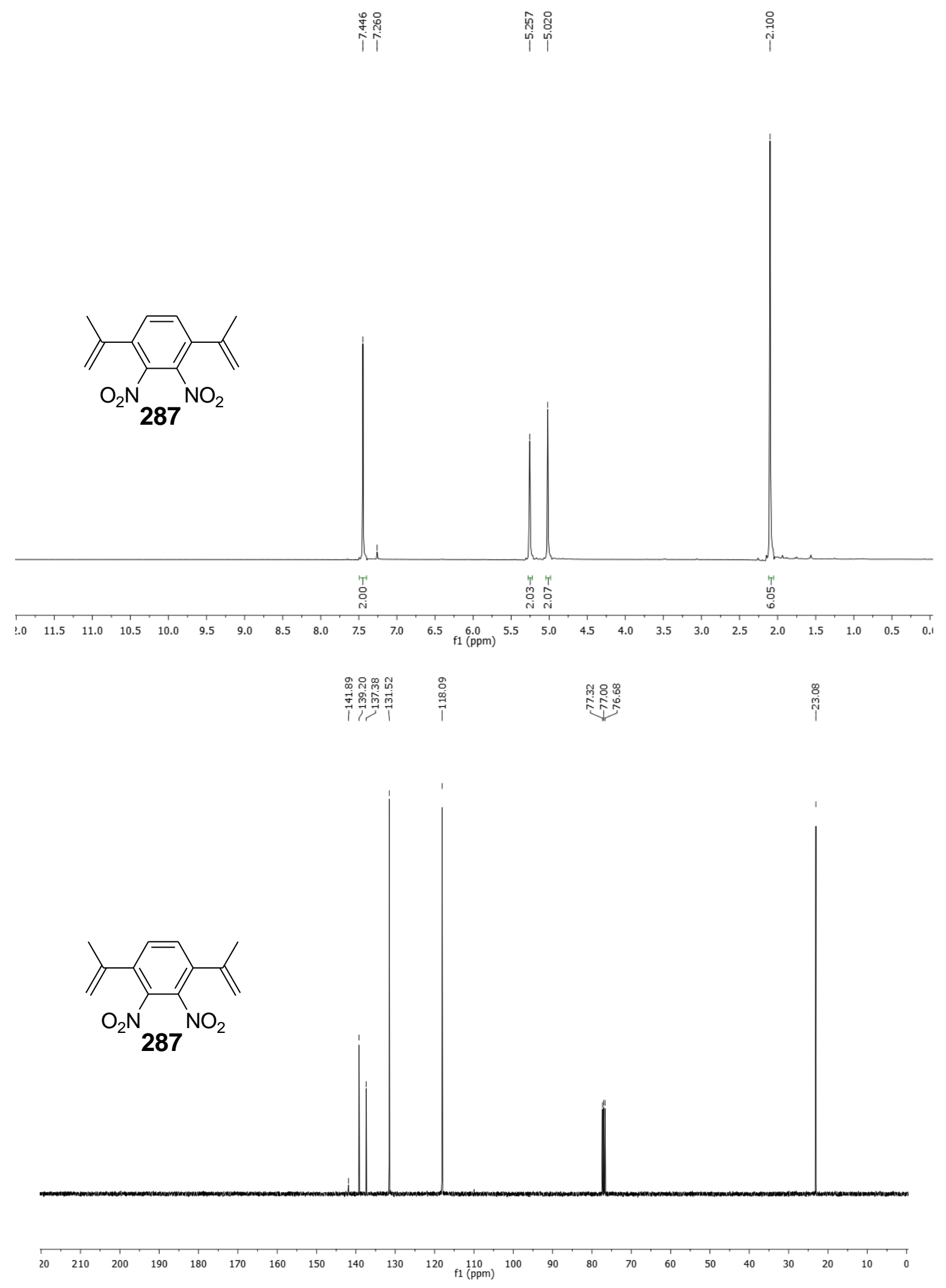

Figure 7.27 ${ }^{1} \mathrm{H}$ and ${ }^{13} \mathrm{C}$ NMR of compound 287 

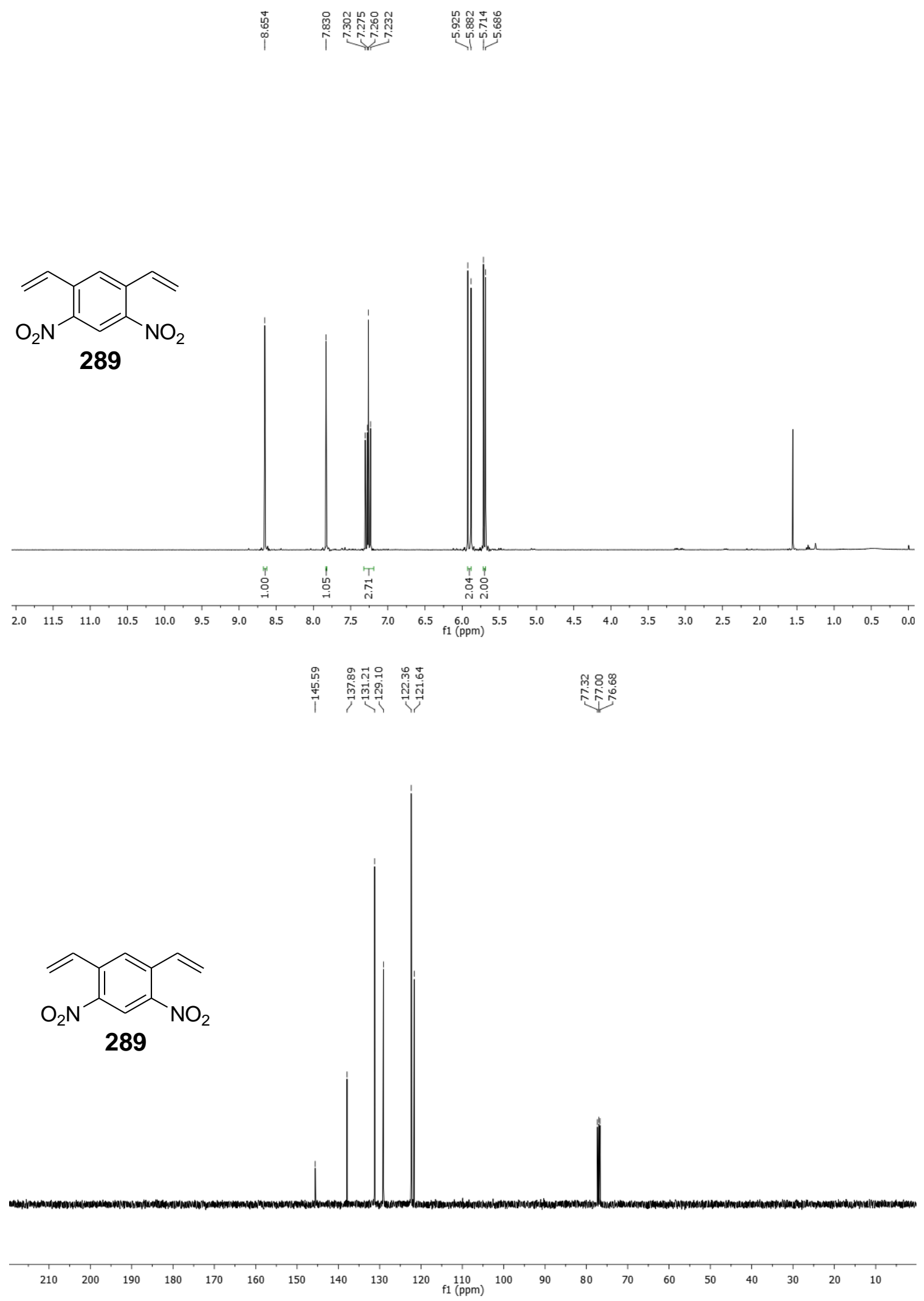

Figure 7.28 ${ }^{1} \mathrm{H}$ and ${ }^{13} \mathrm{C}$ NMR of compound 289 


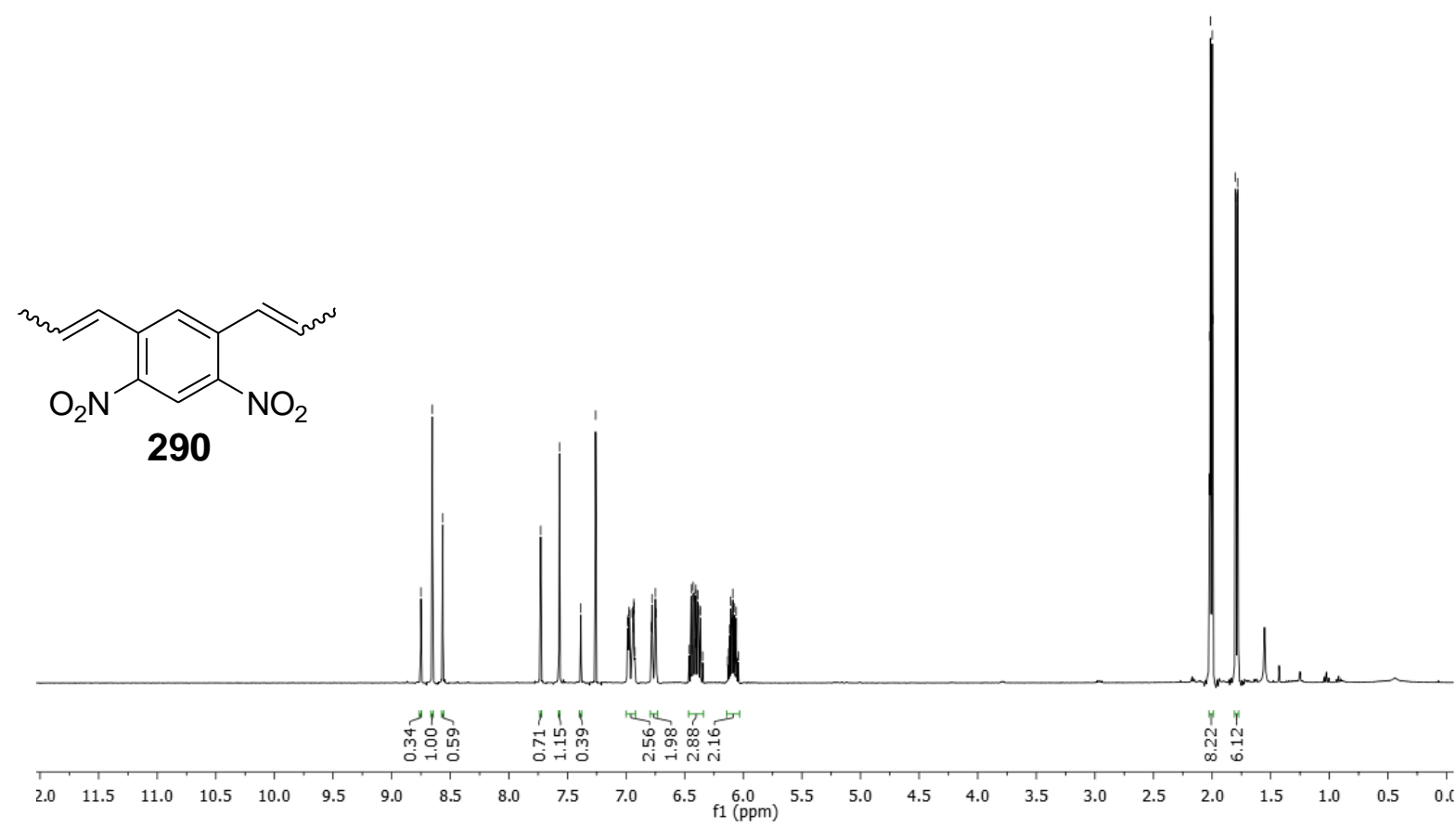

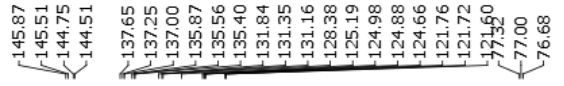
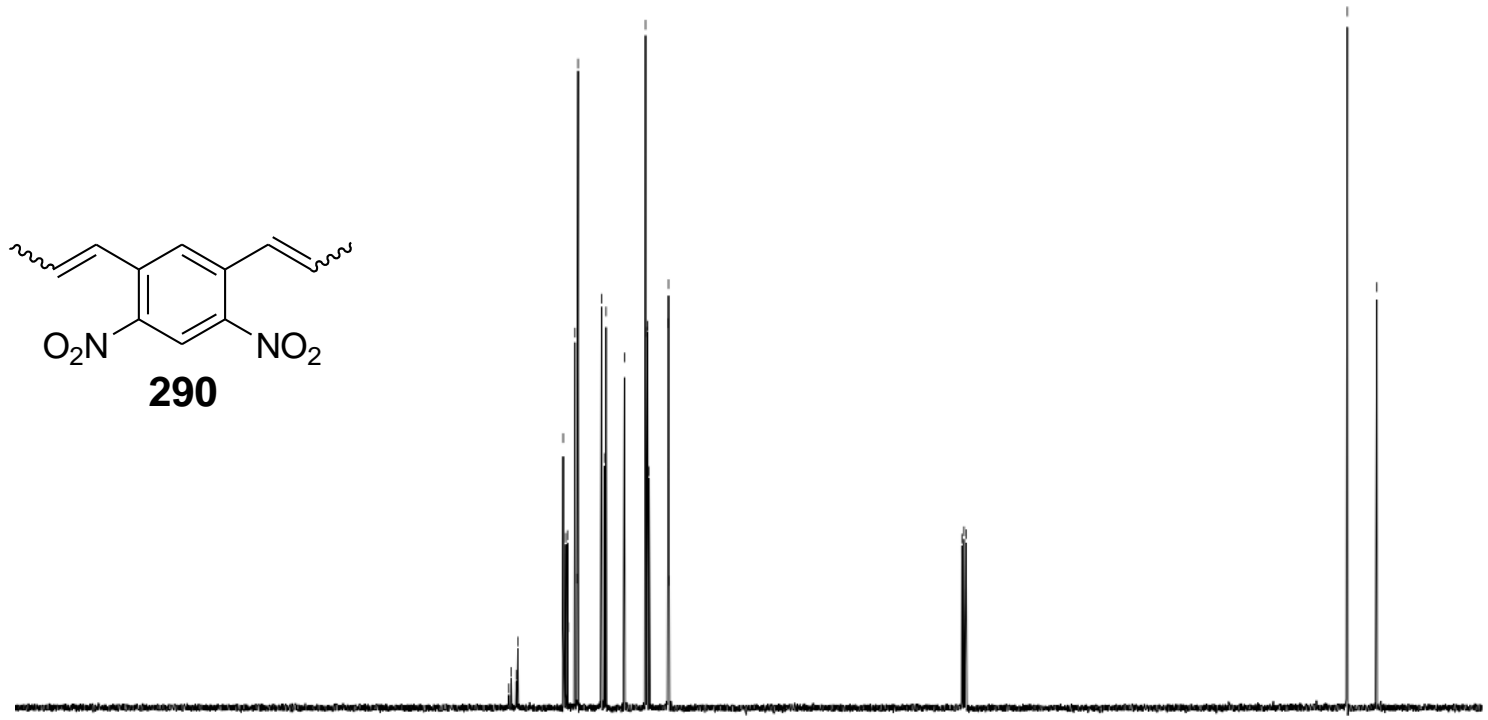

$20 \quad 210 \quad 200 \quad 190 \quad 180$
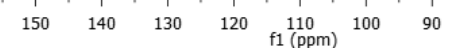

Figure 7.29 ${ }^{1} \mathrm{H}$ and ${ }^{13} \mathrm{C}$ NMR of compound 290 

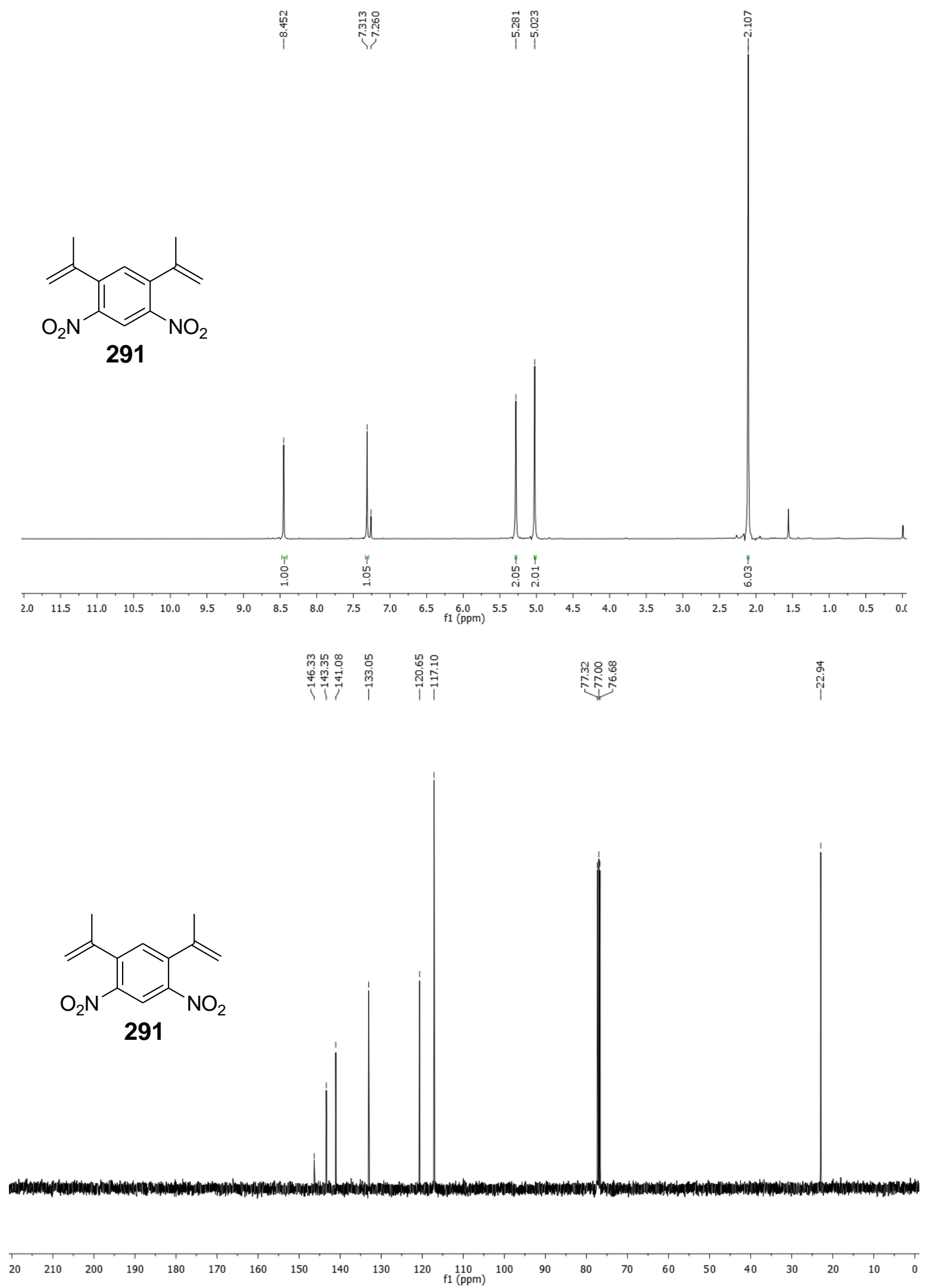

Figure 7.30 ${ }^{1} \mathrm{H}$ and ${ }^{13} \mathrm{C}$ NMR of compound 291 

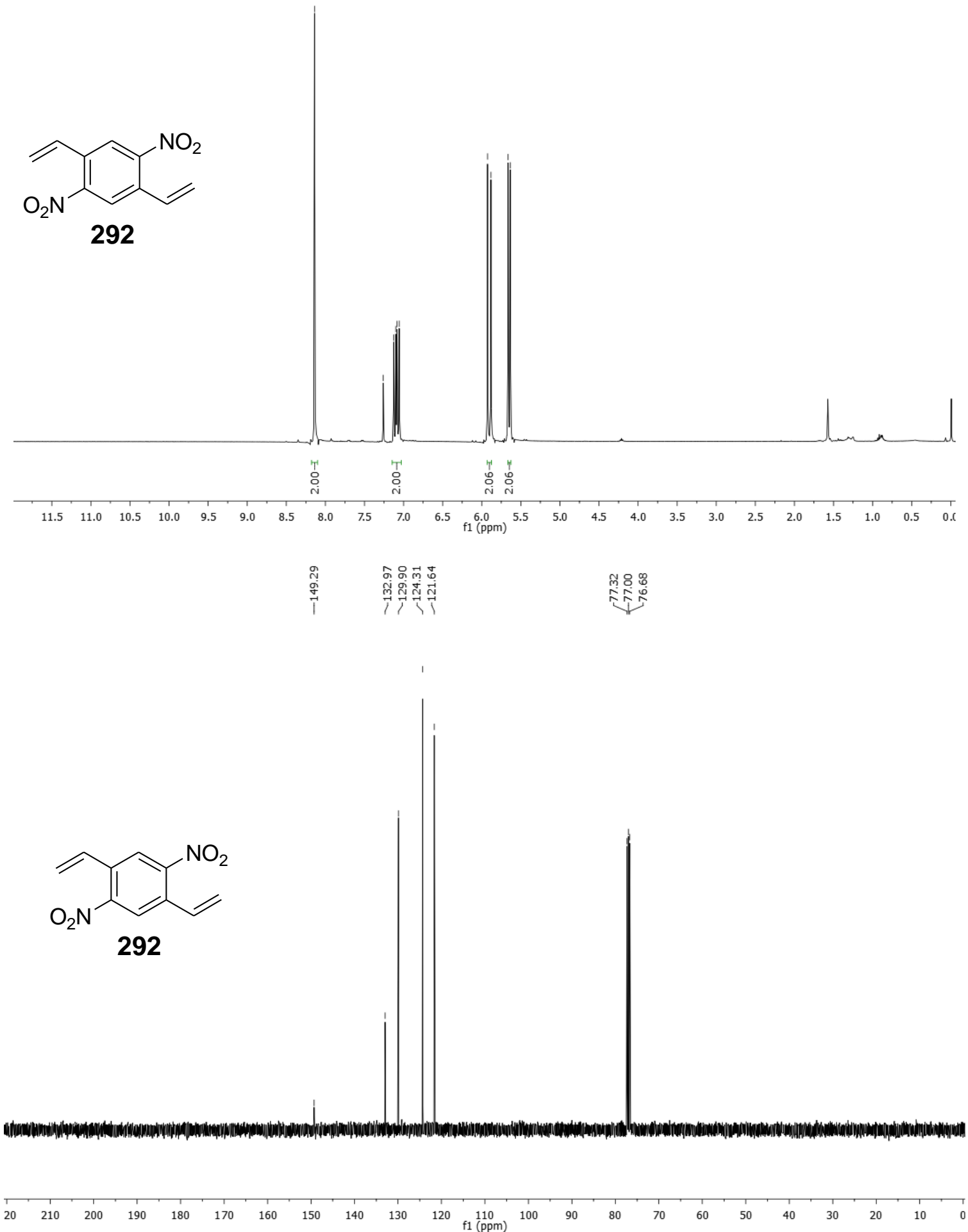

Figure 7.31 ${ }^{1} \mathrm{H}$ and ${ }^{13} \mathrm{C}$ NMR of compound 292 


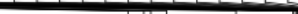
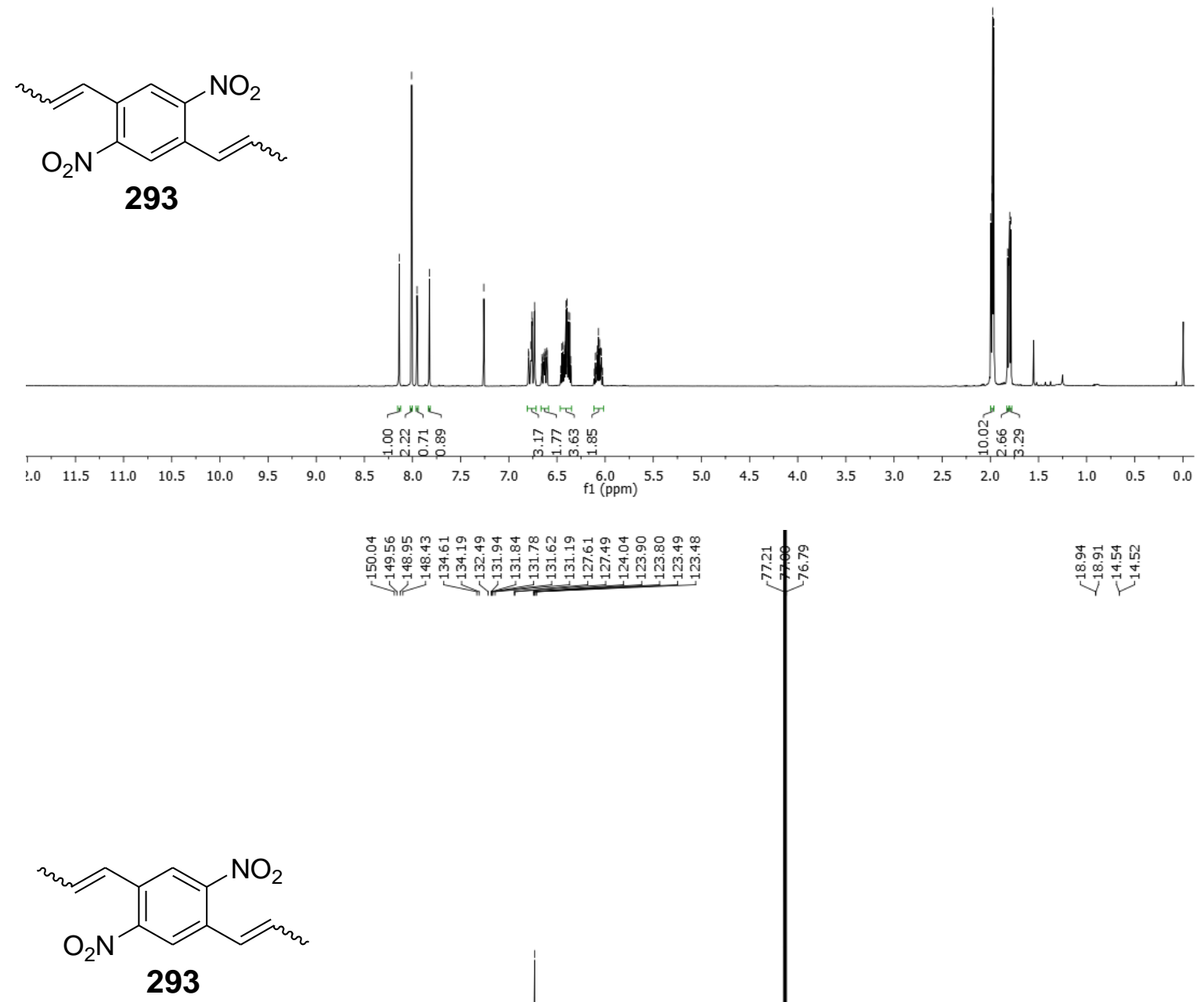

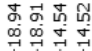

$\underbrace{\infty}_{\substack{\infty \\ \rightarrow}}$

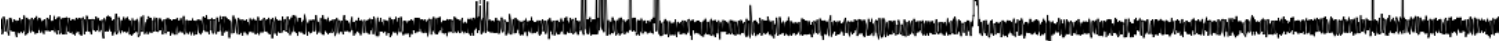

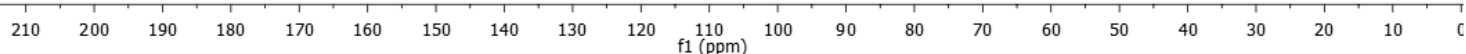

Figure 7.32 ${ }^{1} \mathrm{H}$ and ${ }^{13} \mathrm{C}$ NMR of compound 293 

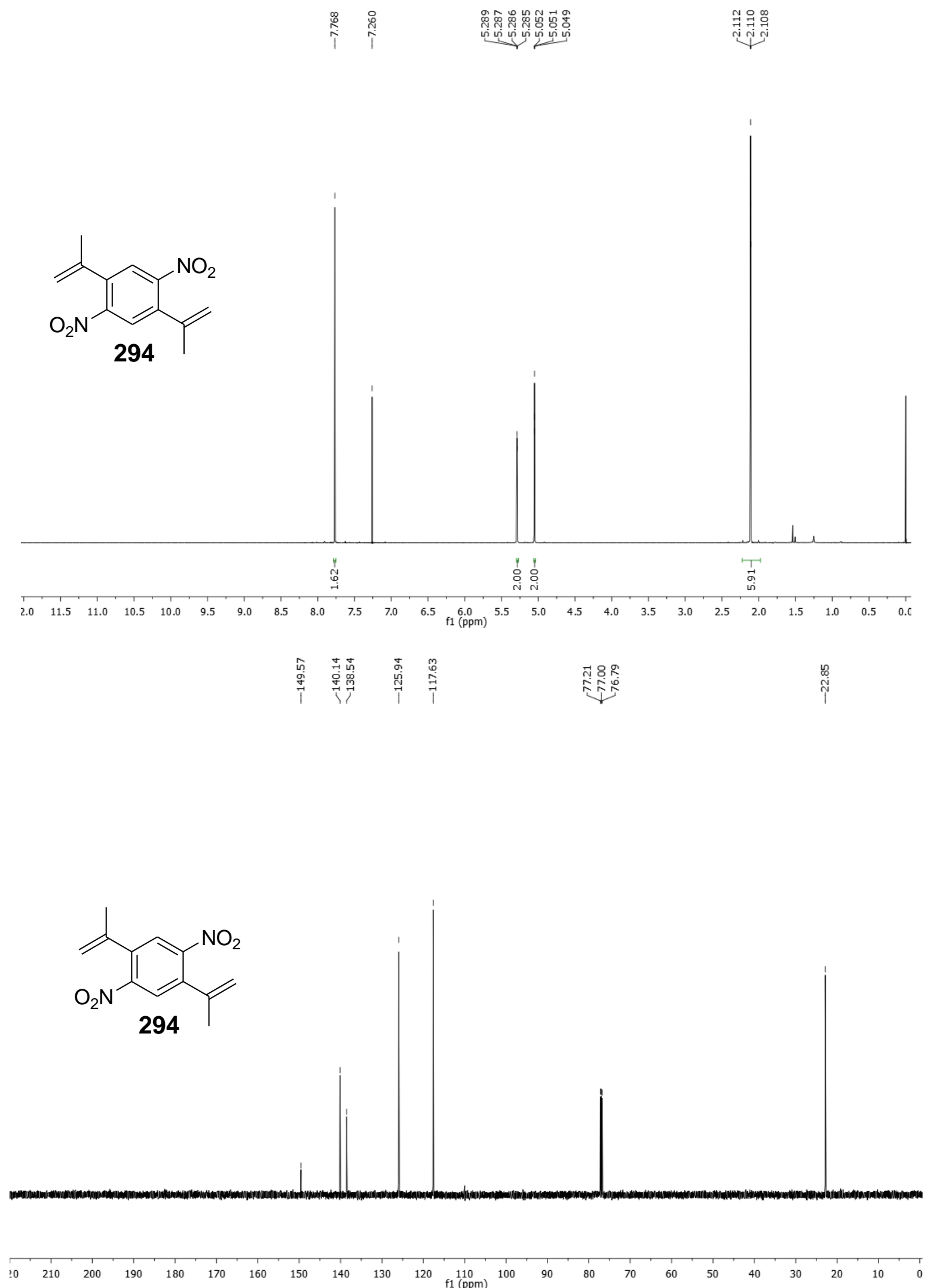

Figure 7.33 ${ }^{1} \mathrm{H}$ and ${ }^{13} \mathrm{C}$ NMR of compound 294 

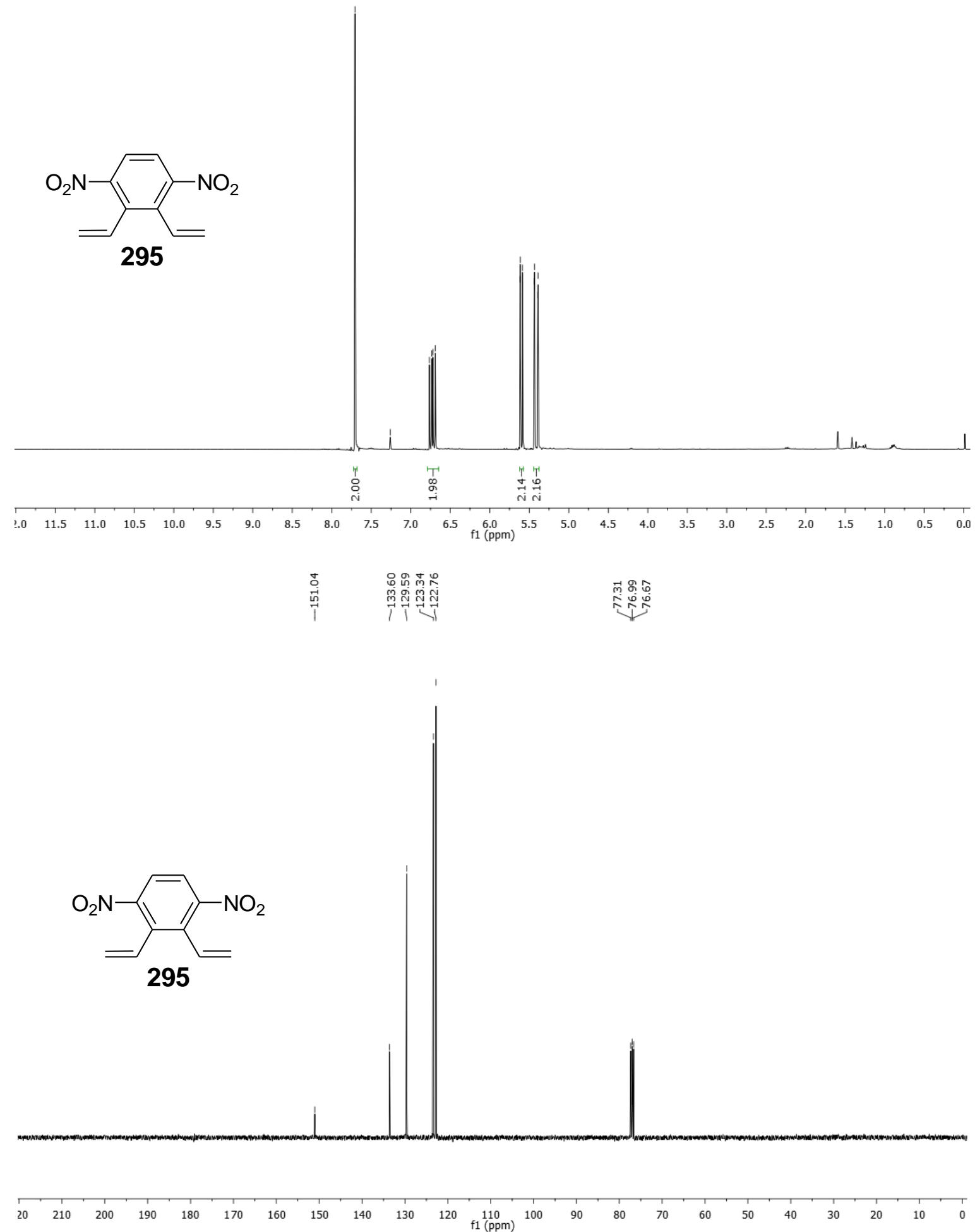

Figure 7.34 ${ }^{1} \mathrm{H}$ and ${ }^{13} \mathrm{C}$ NMR of compound 295 


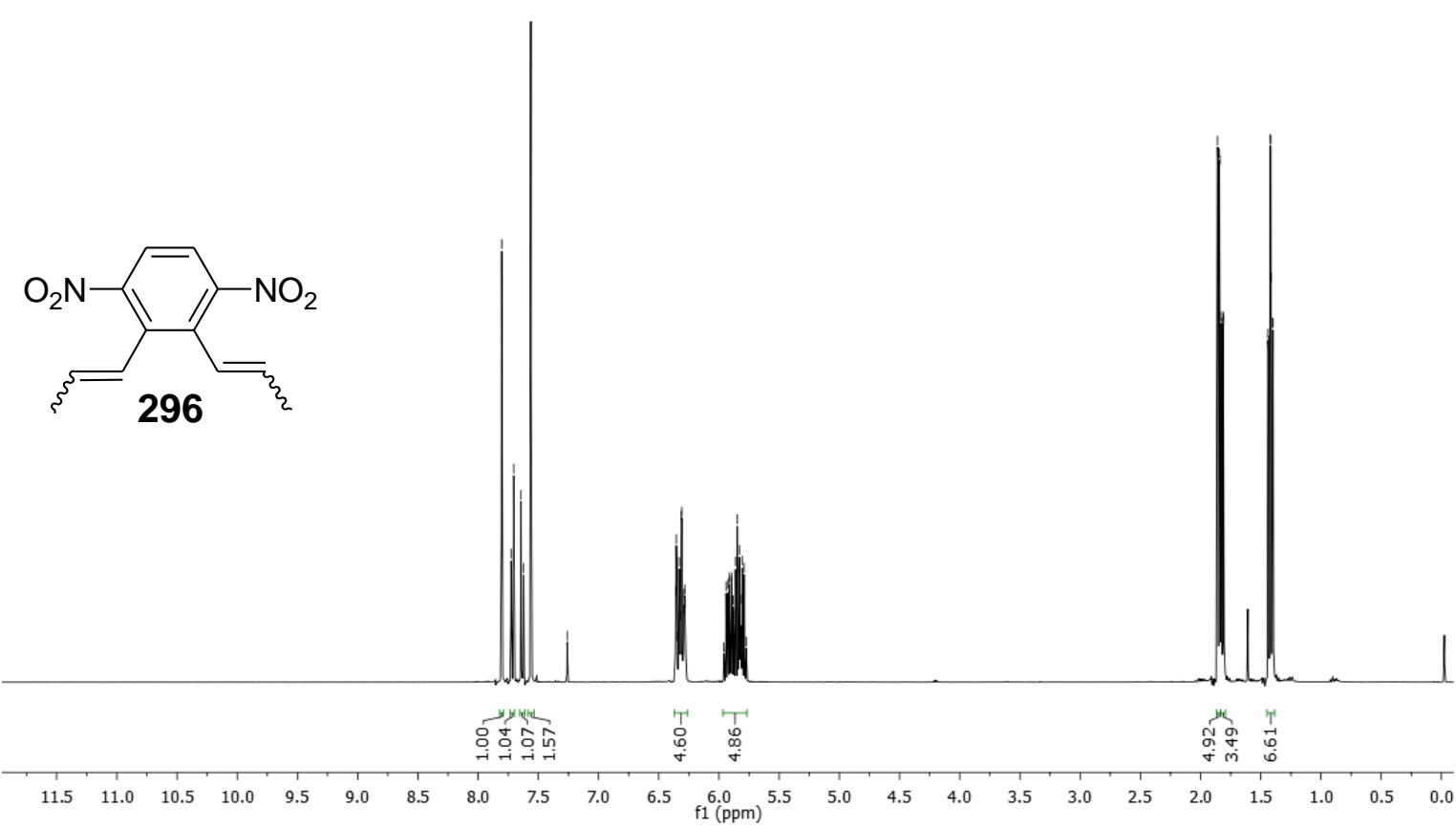

\section{뜽ํำ}

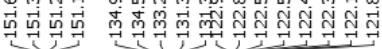
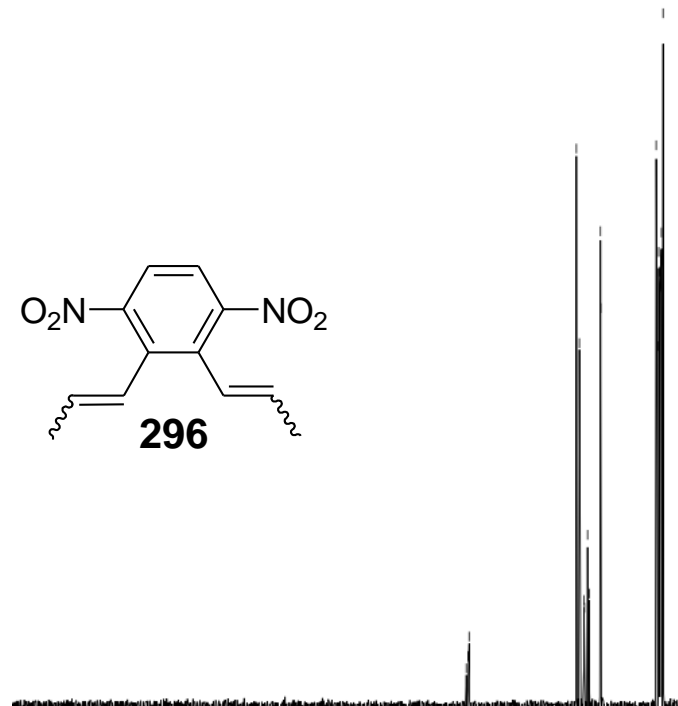

20

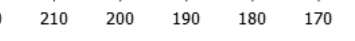

Figure 7.35 ${ }^{1} \mathrm{H}$ and ${ }^{13} \mathrm{C}$ NMR of compound 296 

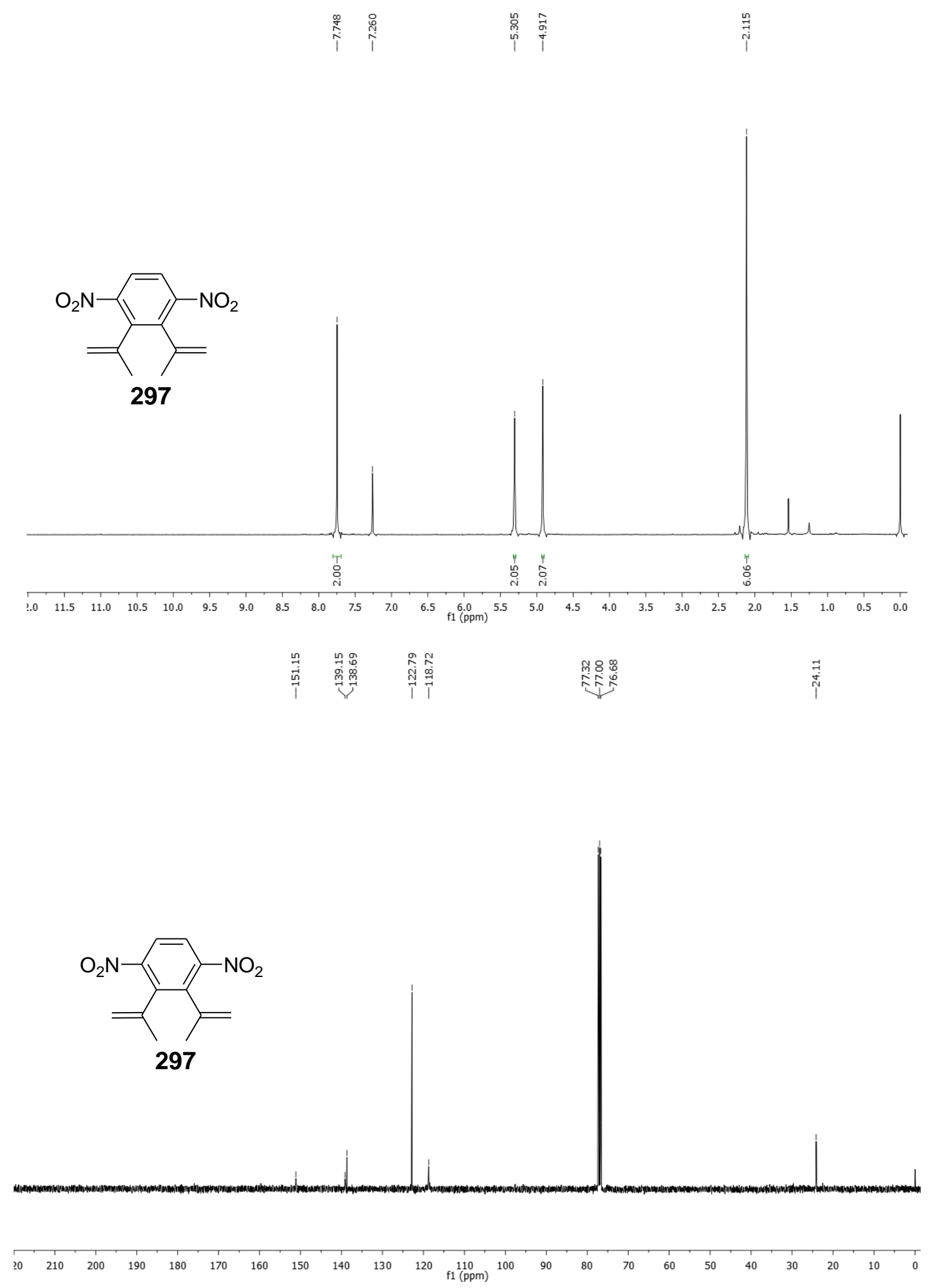

Figure 7.36 ${ }^{1} \mathrm{H}$ and ${ }^{13} \mathrm{C}$ NMR of compound 297 

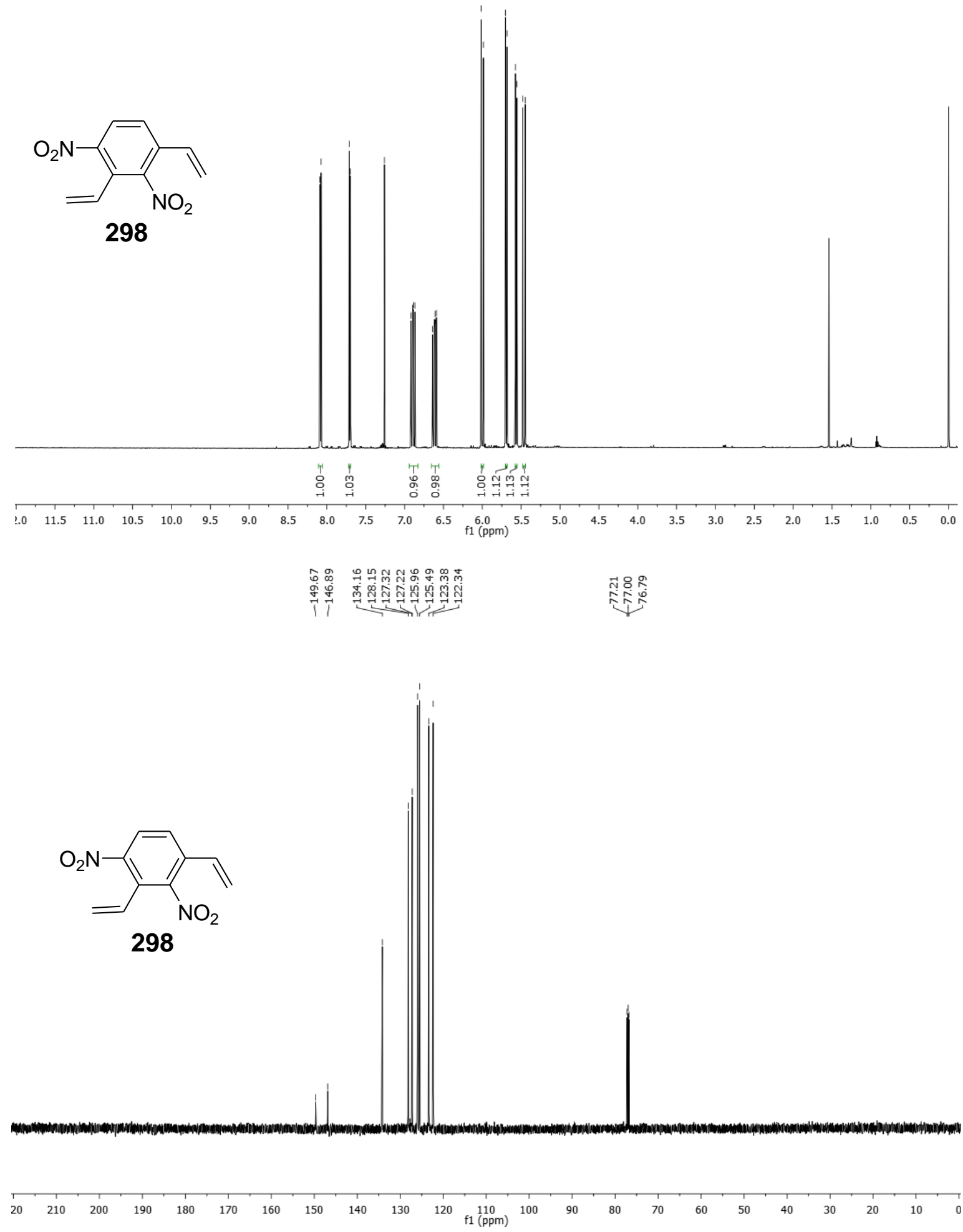

Figure 7.37 ${ }^{1} \mathrm{H}$ and ${ }^{13} \mathrm{C}$ NMR of compound 298 


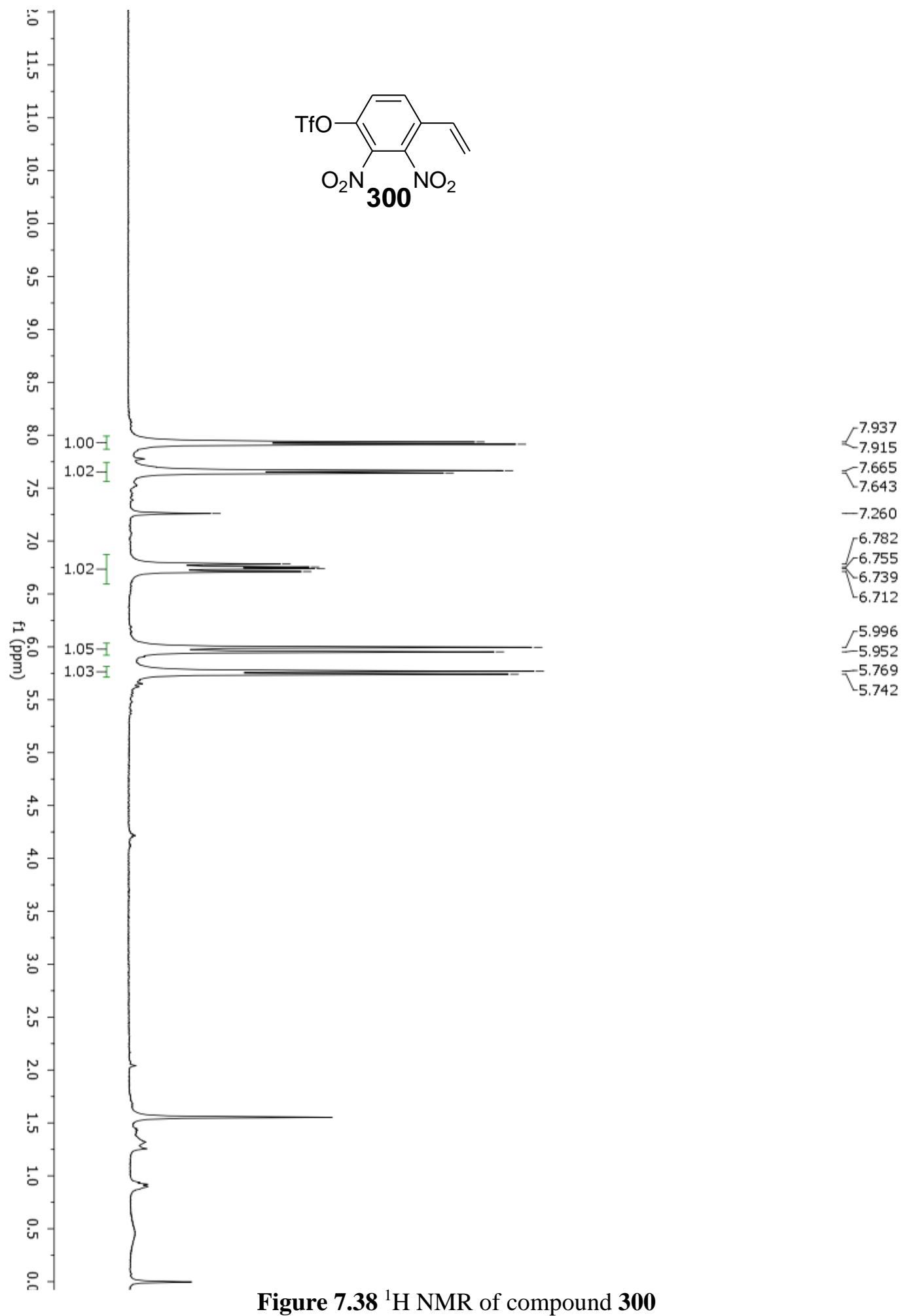



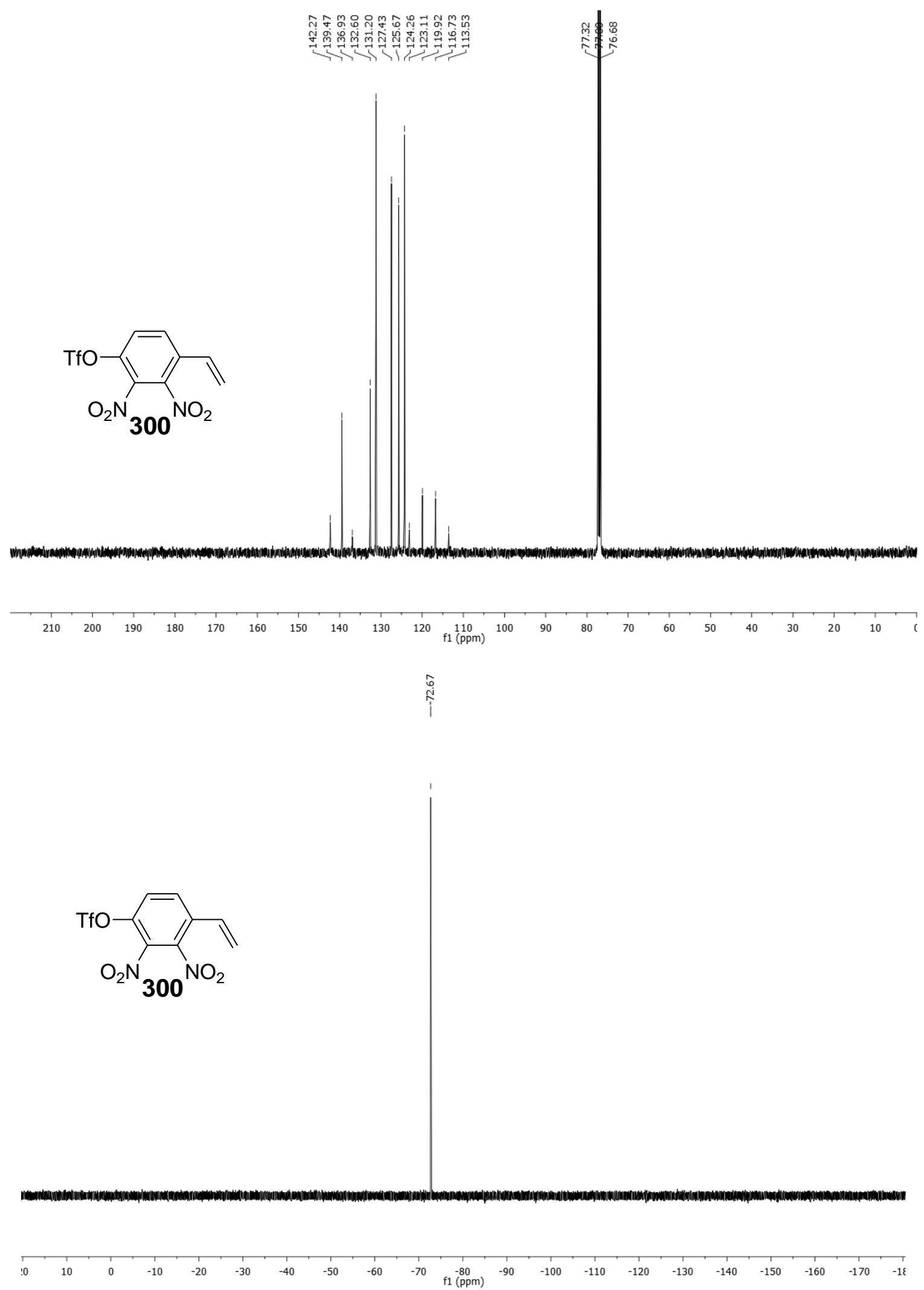

Figure 7.39 ${ }^{13} \mathrm{C}$ and ${ }^{19} \mathrm{~F}$ NMR of compound $\mathbf{3 0 0}$ 


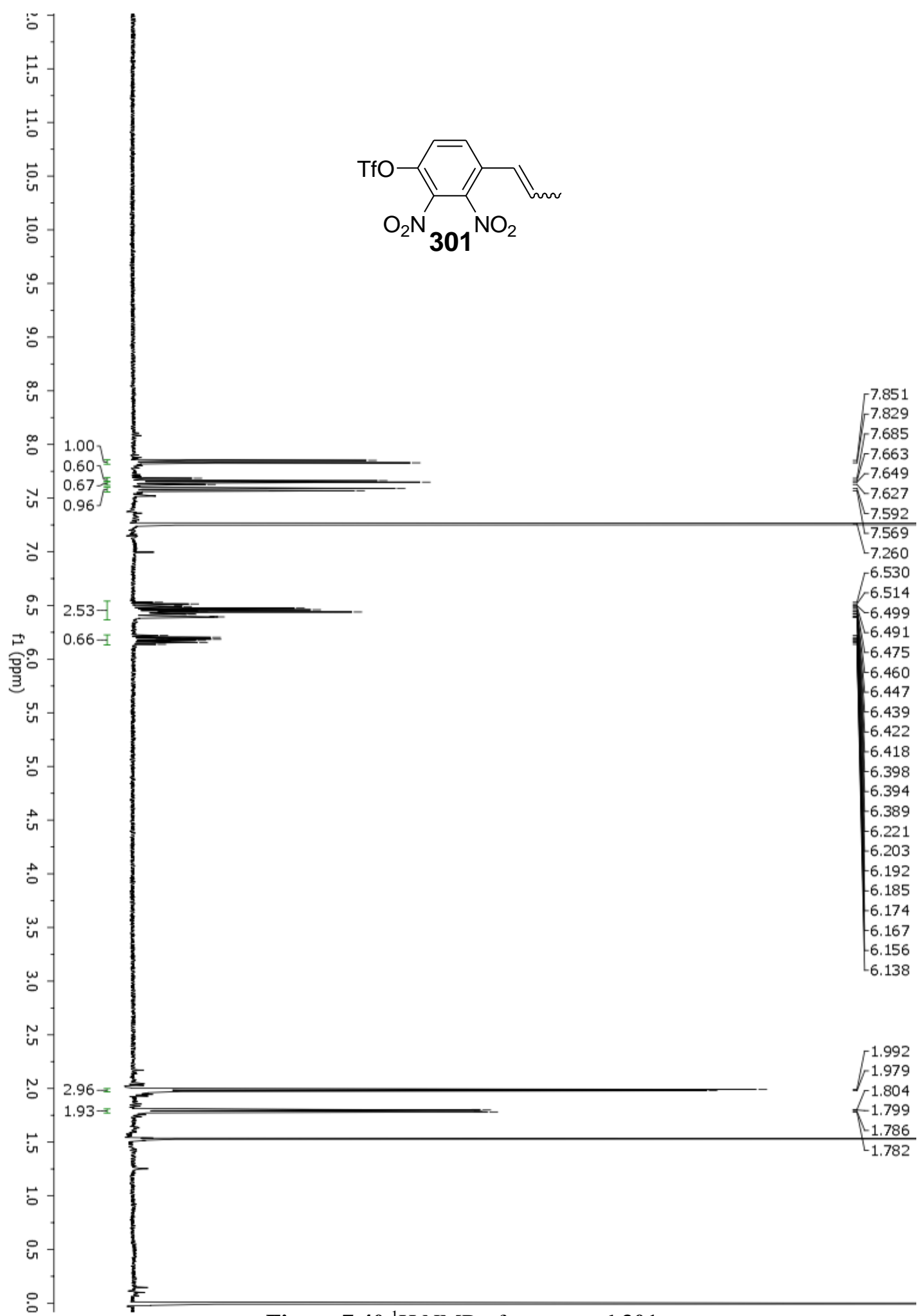

Figure $7.40{ }^{1} \mathrm{H}$ NMR of compound 301 

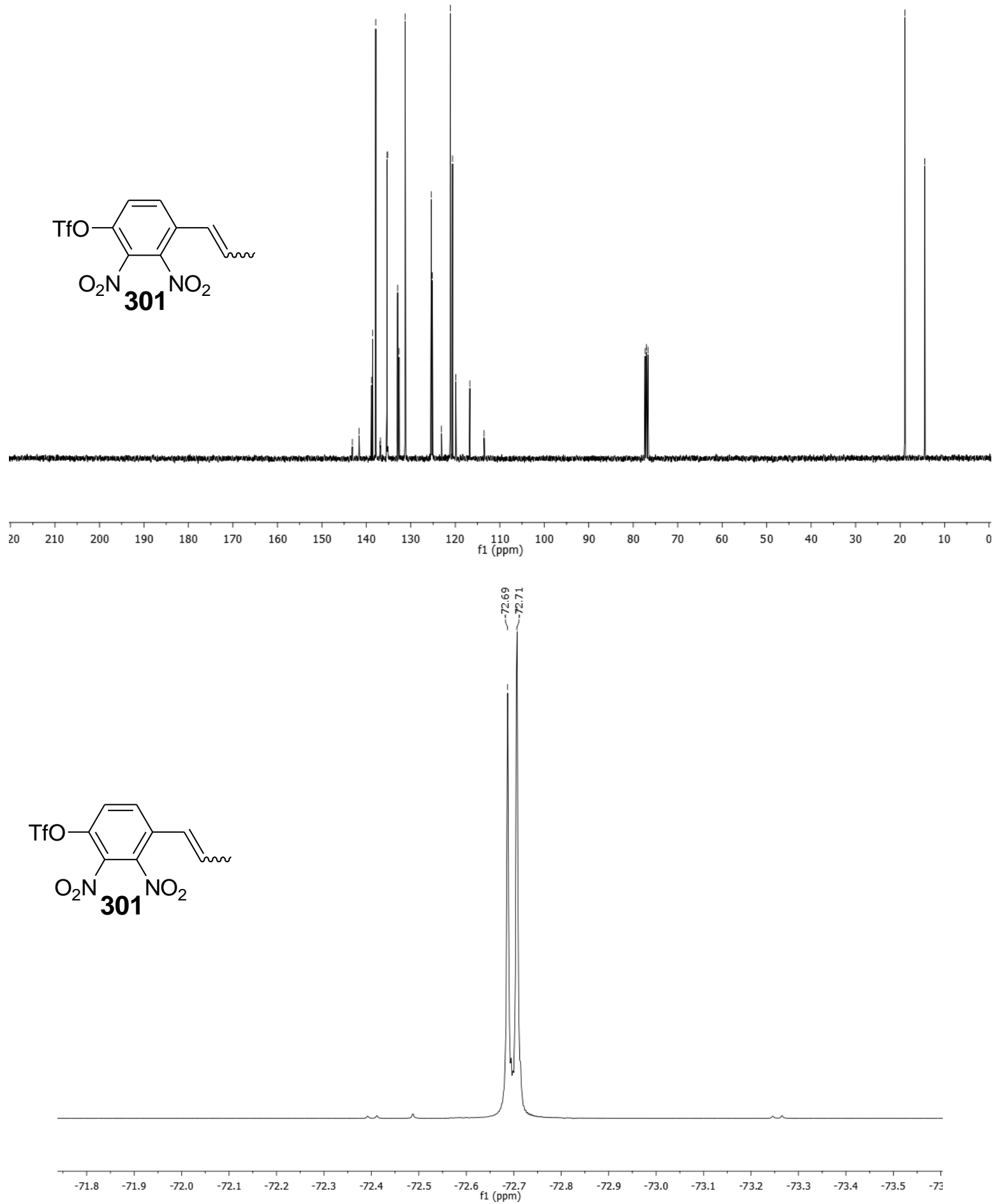

Figure 7.41 ${ }^{13} \mathrm{C}$ and ${ }^{19} \mathrm{~F}$ NMR of compound $\mathbf{3 0 0}$ 

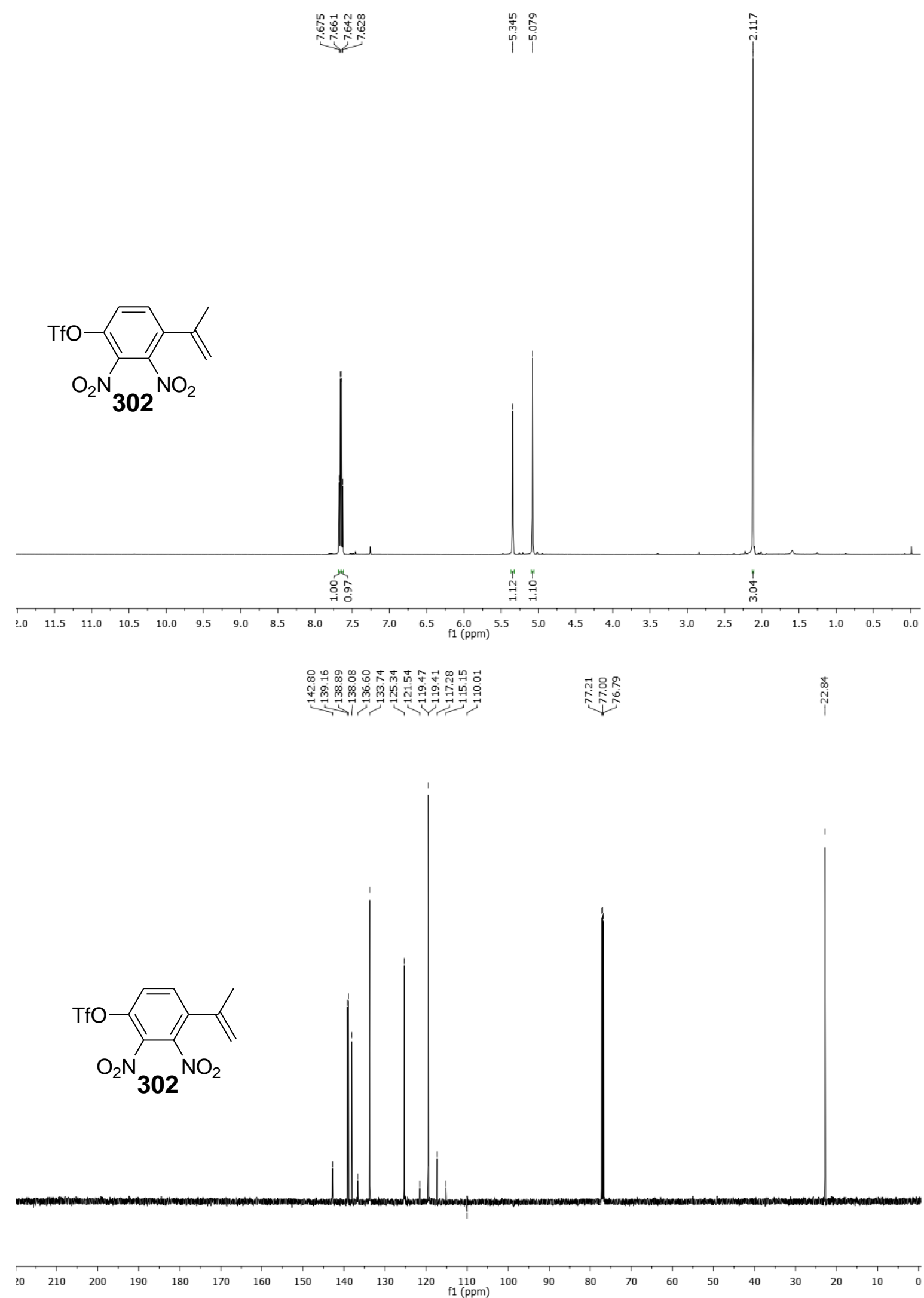

Figure 7.42 ${ }^{1} \mathrm{H}$ and ${ }^{13} \mathrm{C}$ NMR of compound $\mathbf{3 0 2}$ 


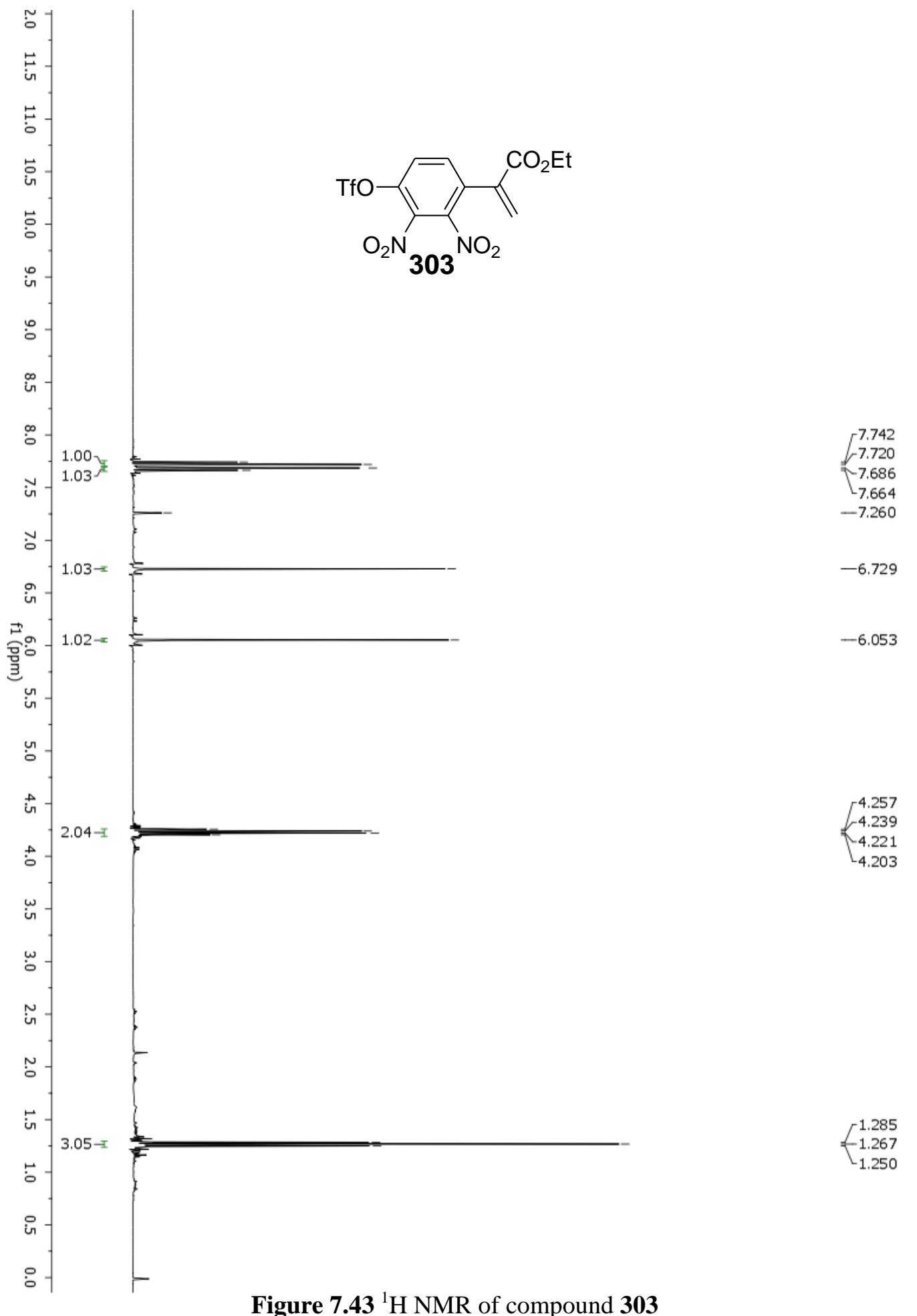

Figure $7.43{ }^{1} \mathrm{H}$ NMR of compound 303 

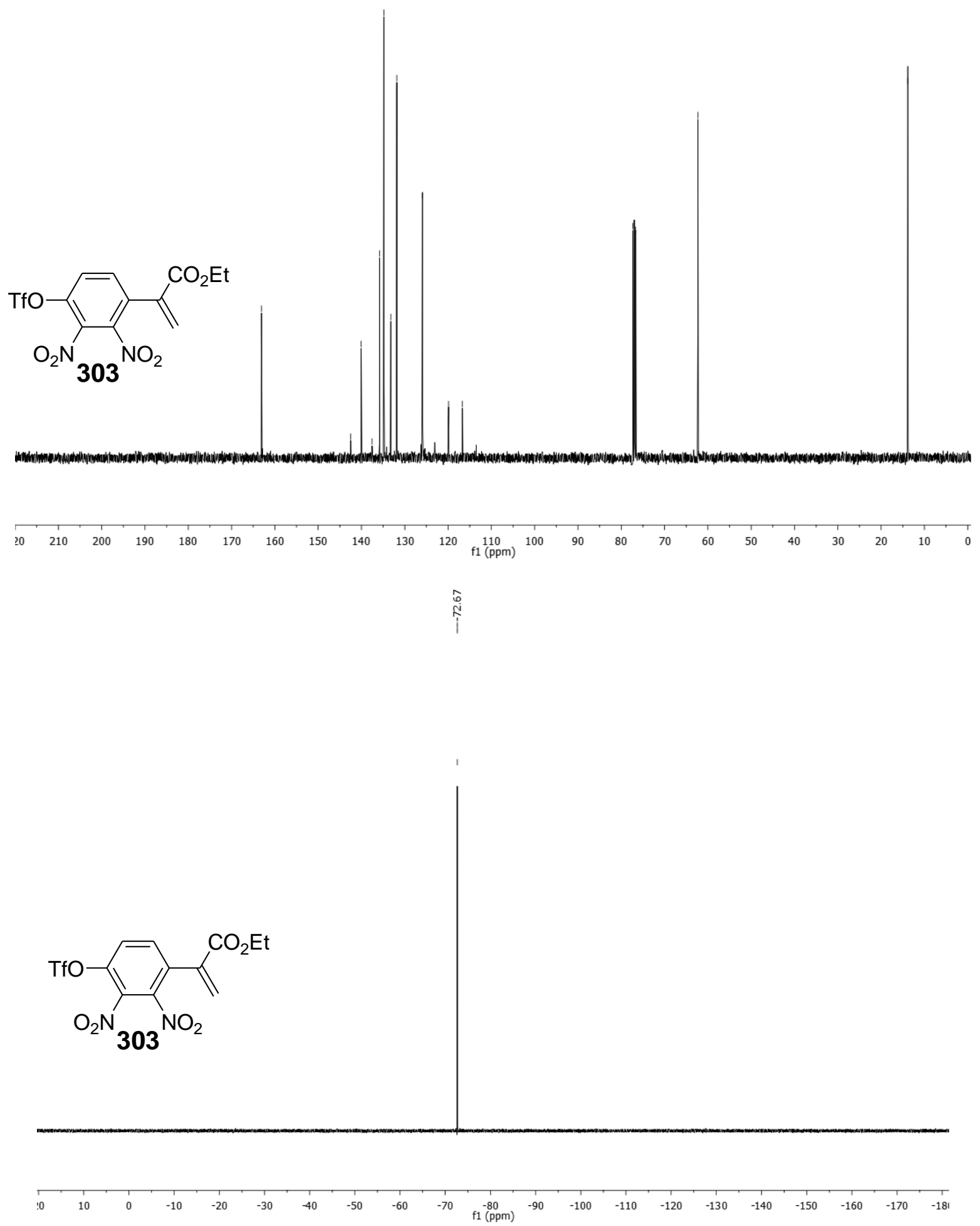

Figure 7.44 ${ }^{13} \mathrm{C}$ and ${ }^{19} \mathrm{~F}$ NMR of compound $\mathbf{3 0 3}$ 


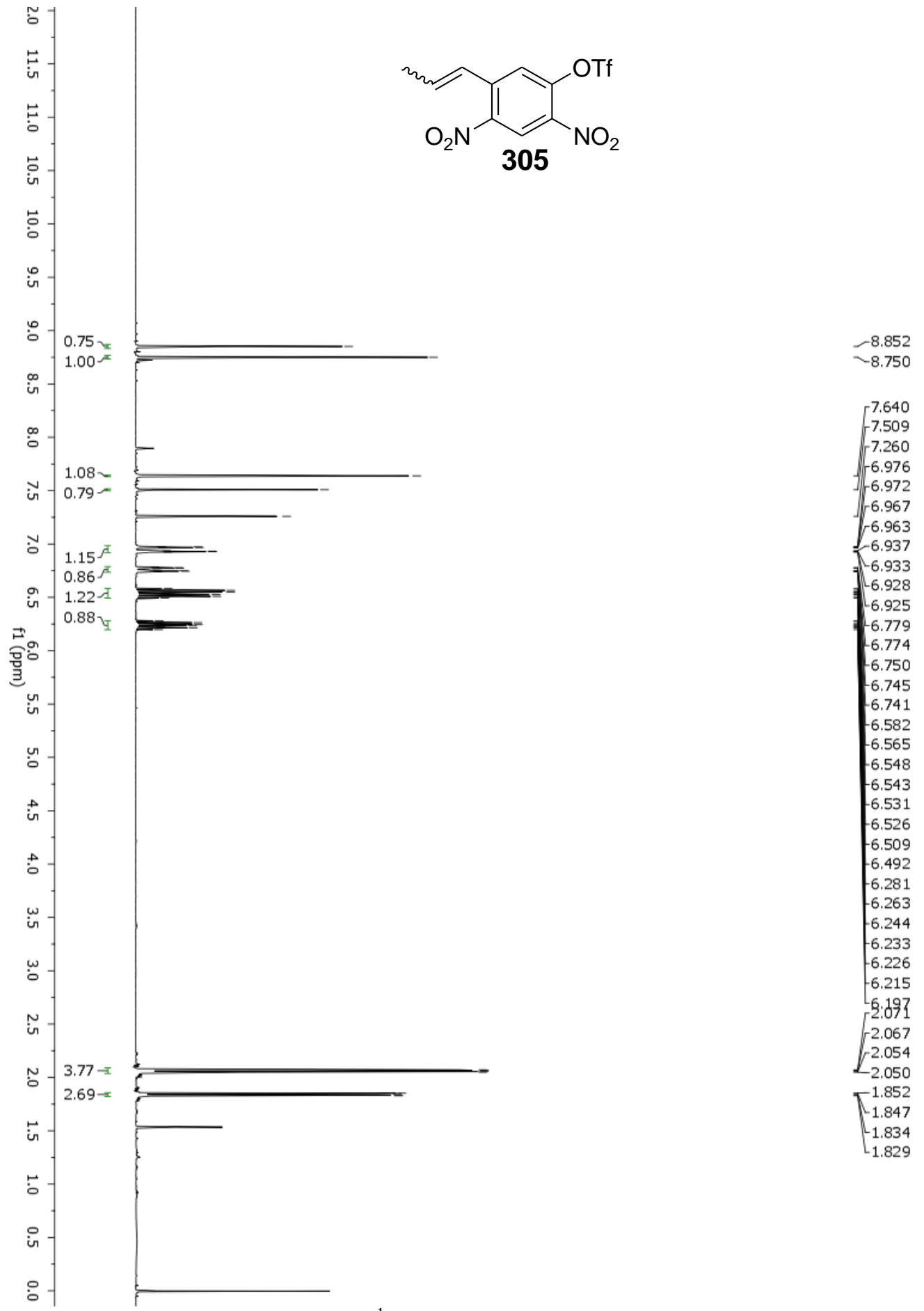

Figure 7.45 ${ }^{1} \mathrm{H}$ NMR of compound $\mathbf{3 0 5}$ 

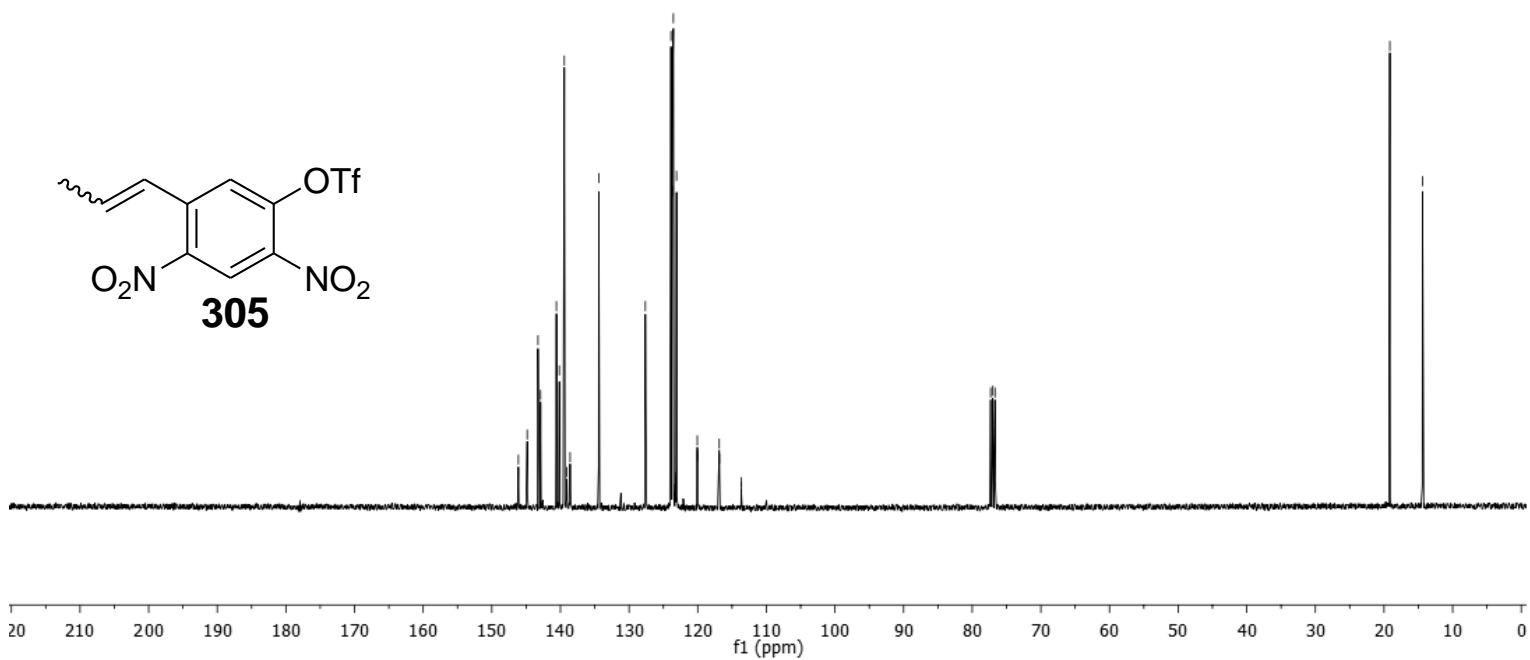

ヘָ
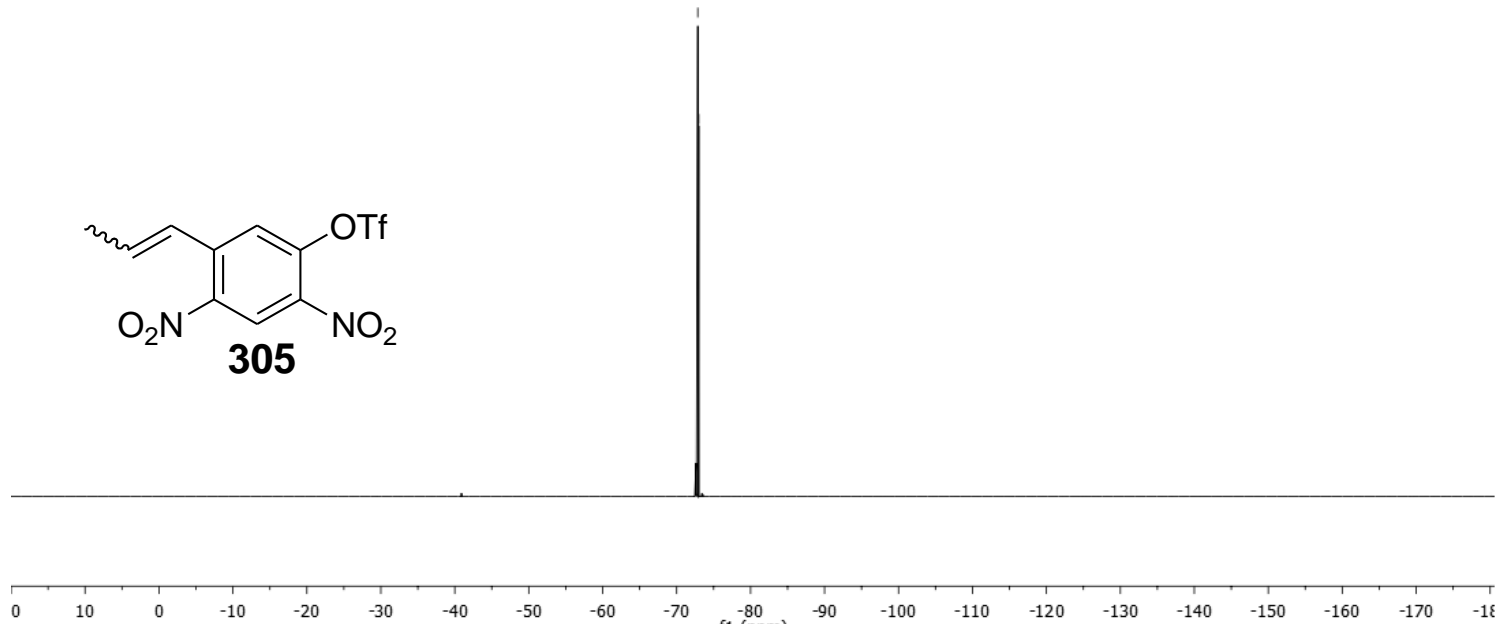

Figure 7.46 ${ }^{13} \mathrm{C}$ and ${ }^{19} \mathrm{~F}$ NMR of compound $\mathbf{3 0 5}$ 


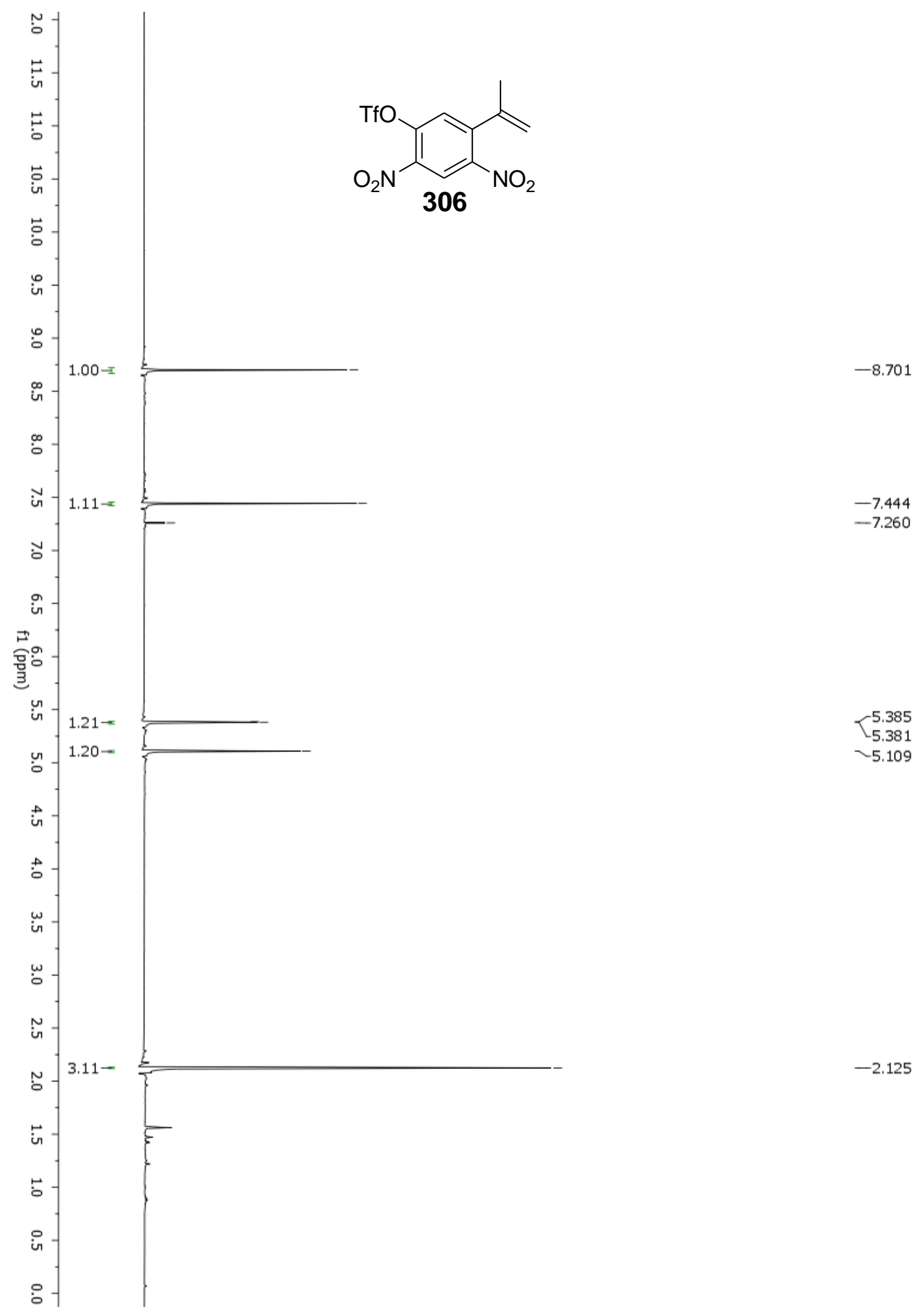

Figure $7.47{ }^{1} \mathrm{H}$ NMR of compound 306 

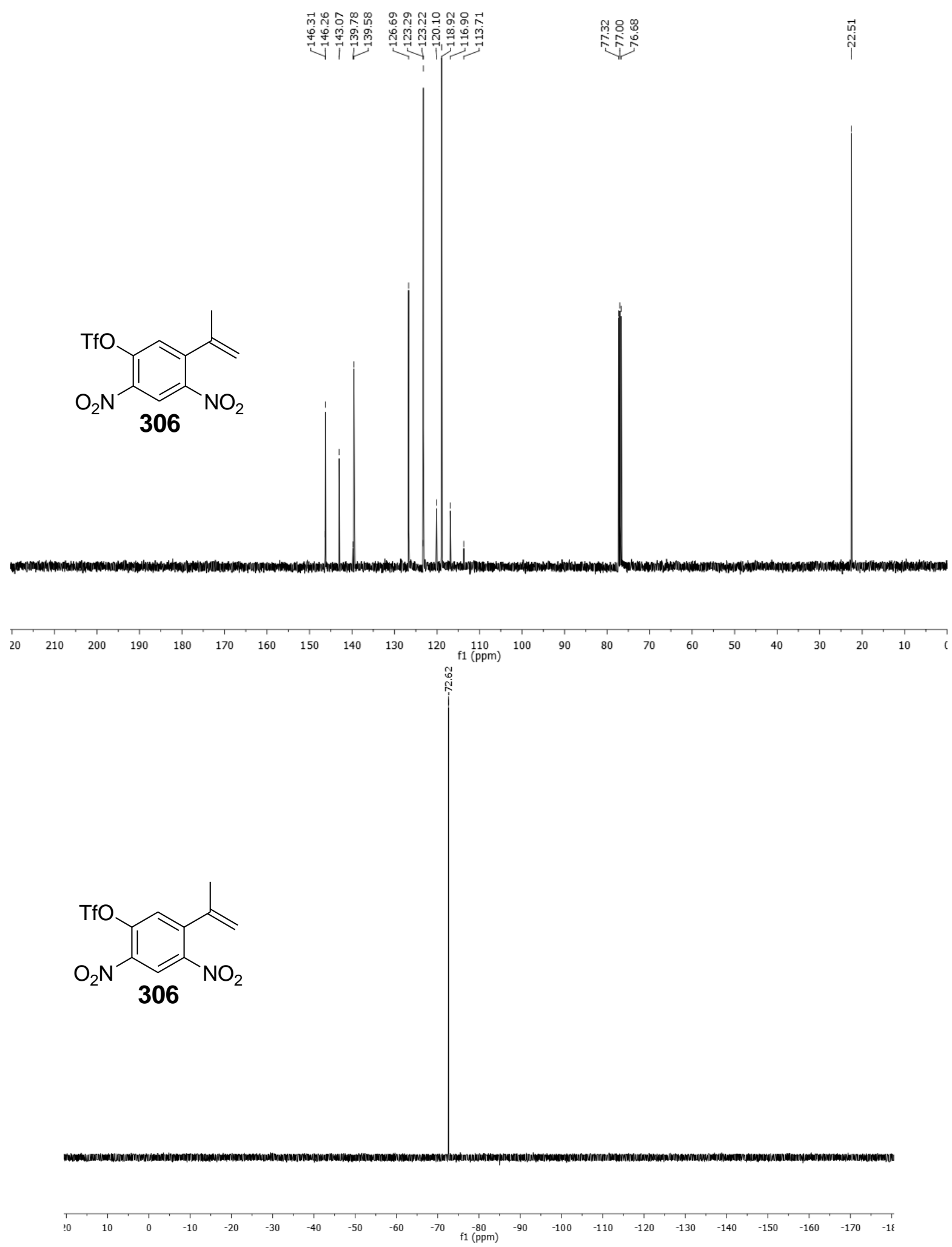

Figure $7.48{ }^{13} \mathrm{C}$ and ${ }^{19} \mathrm{~F}$ NMR of compound 306 


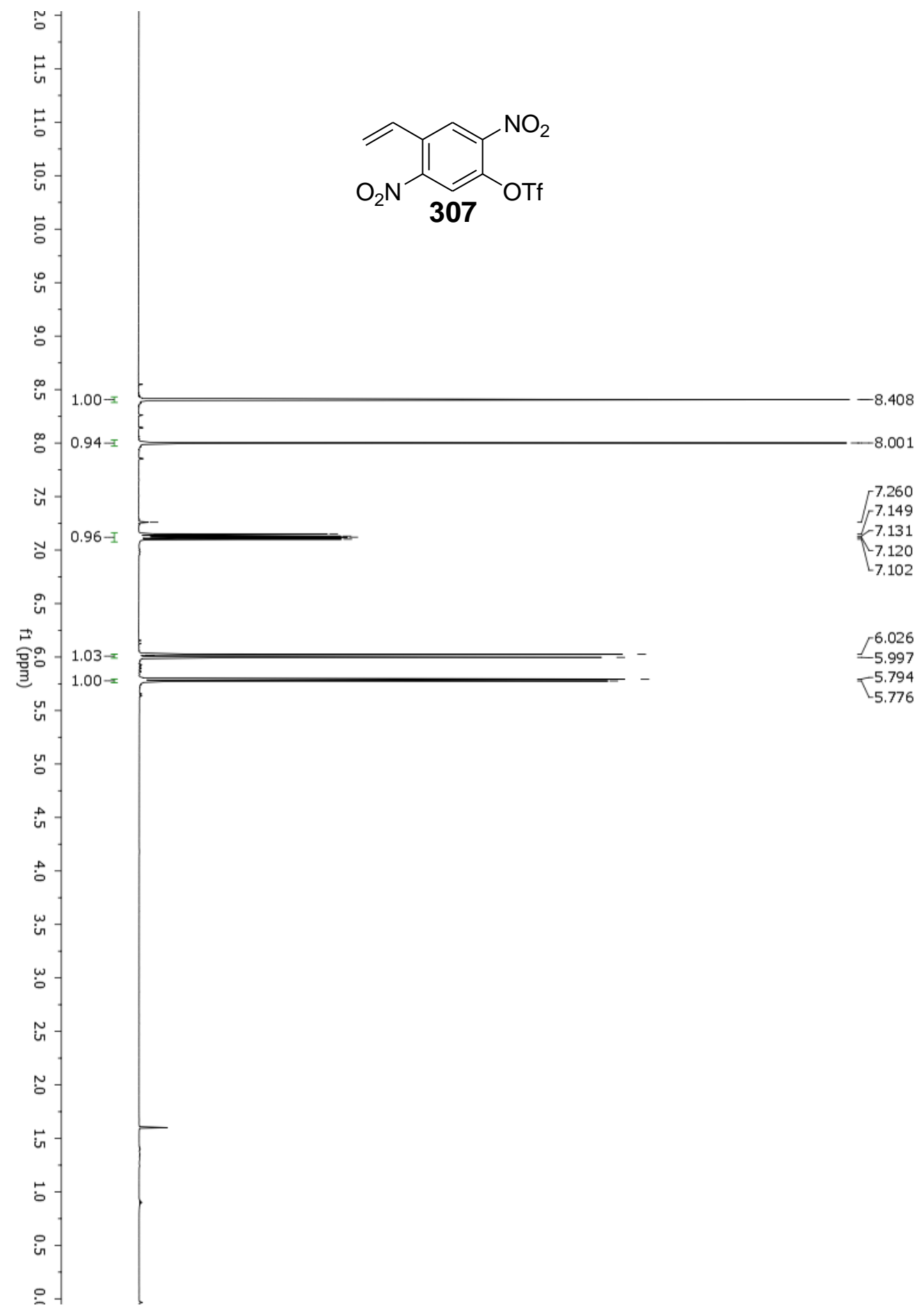

Figure 7.49 ${ }^{1} \mathrm{H}$ NMR of compound 307 

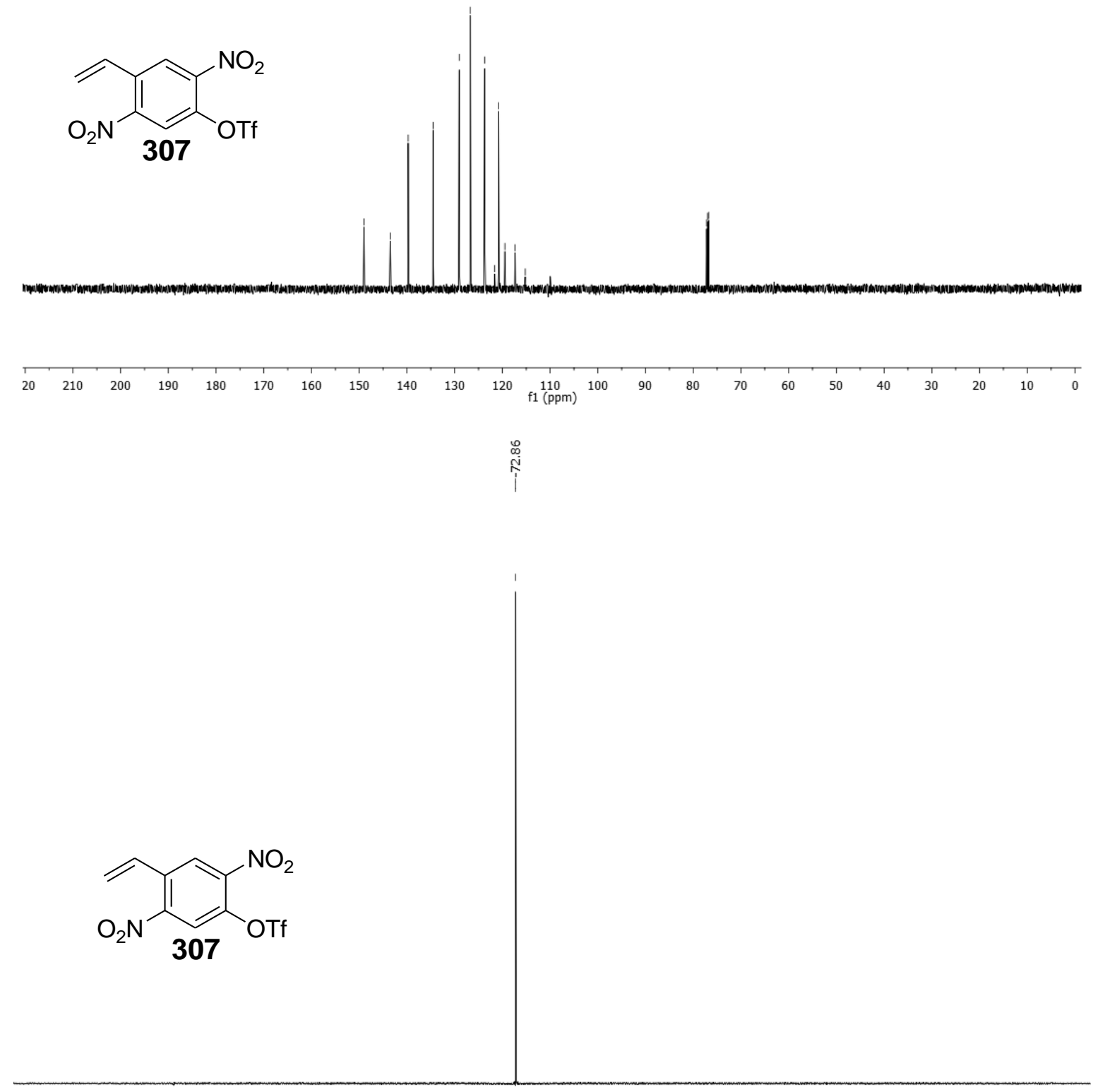

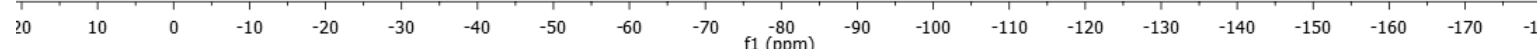

Figure 7.50 ${ }^{13} \mathrm{C}$ and ${ }^{19} \mathrm{~F}$ NMR of compound $\mathbf{3 0 7}$ 


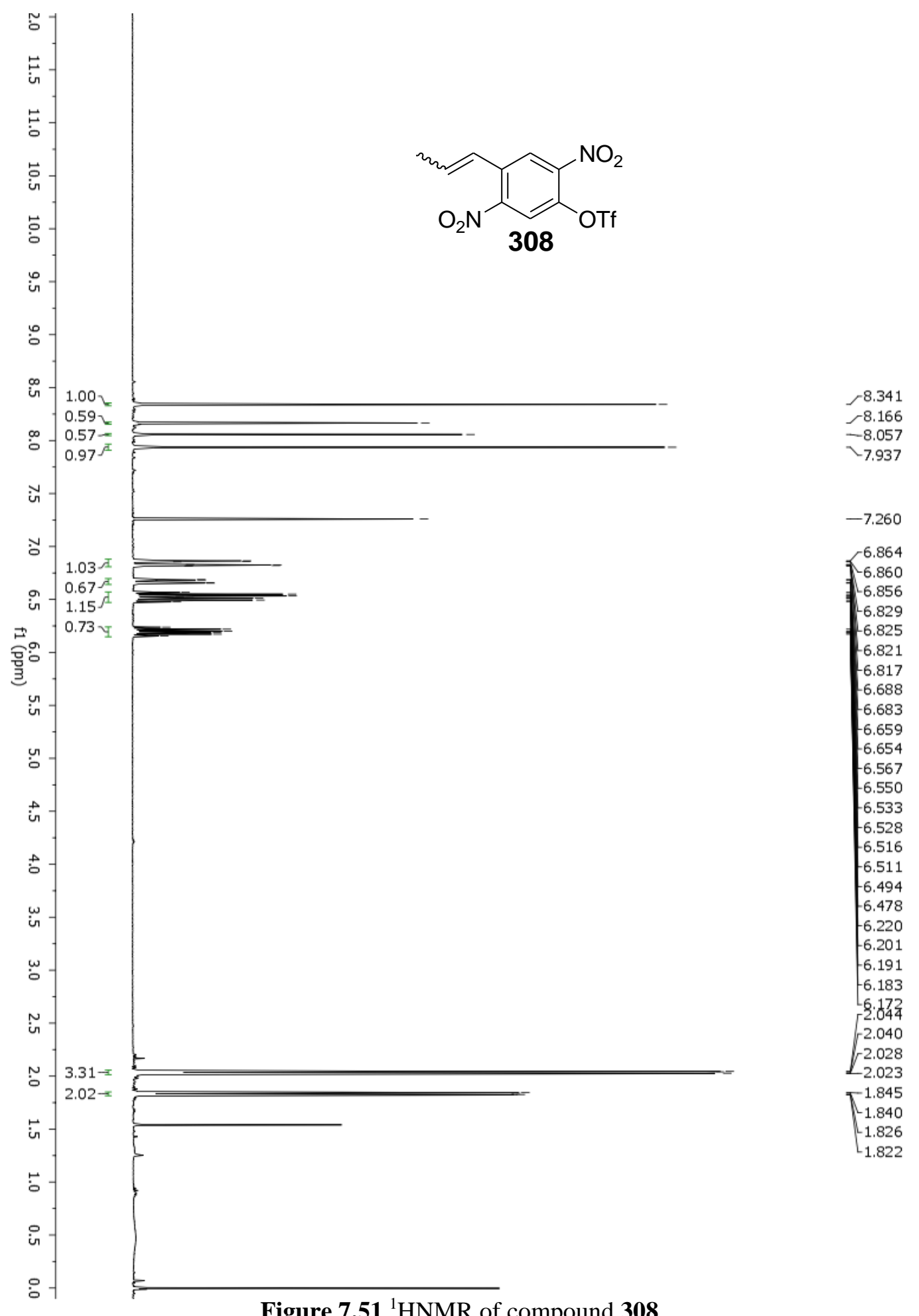

Figure $7.51{ }^{1} \mathrm{HNMR}$ of compound $\mathbf{3 0 8}$ 

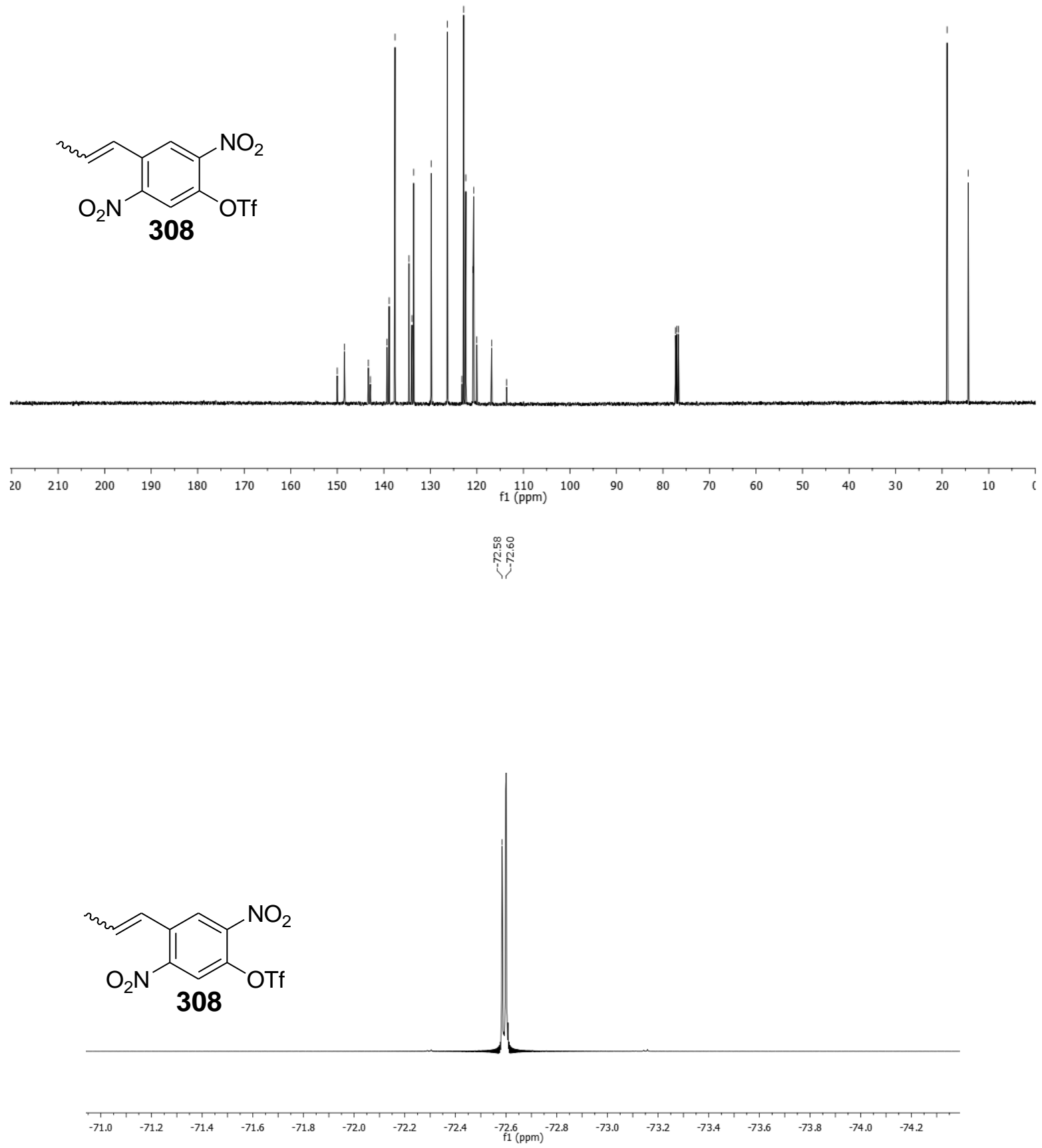

Figure 7.52 ${ }^{13} \mathrm{C}$ and ${ }^{19} \mathrm{~F}$ NMR of compound $\mathbf{3 0 8}$ 


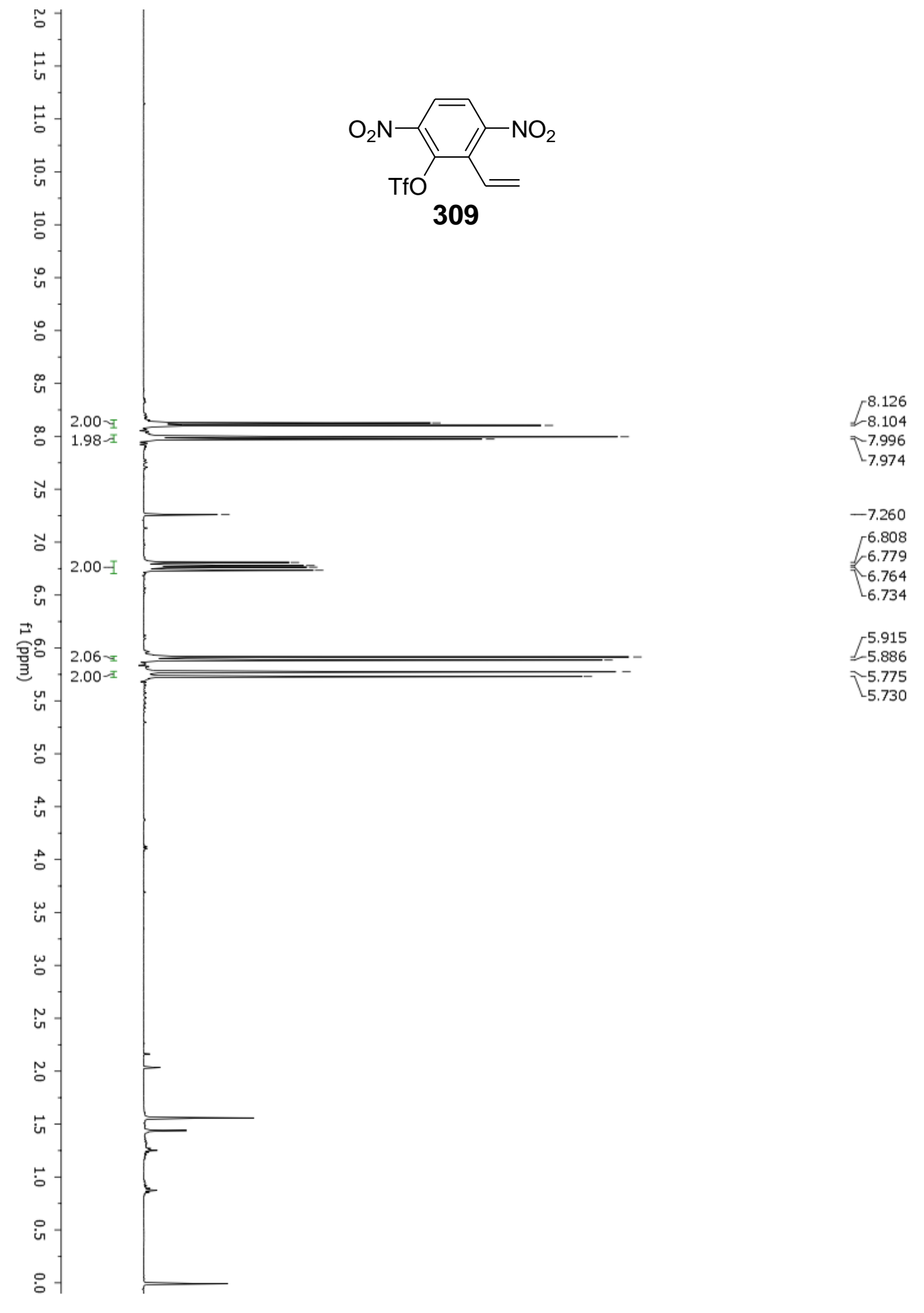

Figure $7.53{ }^{1} \mathrm{H}$ NMR of compound 309 

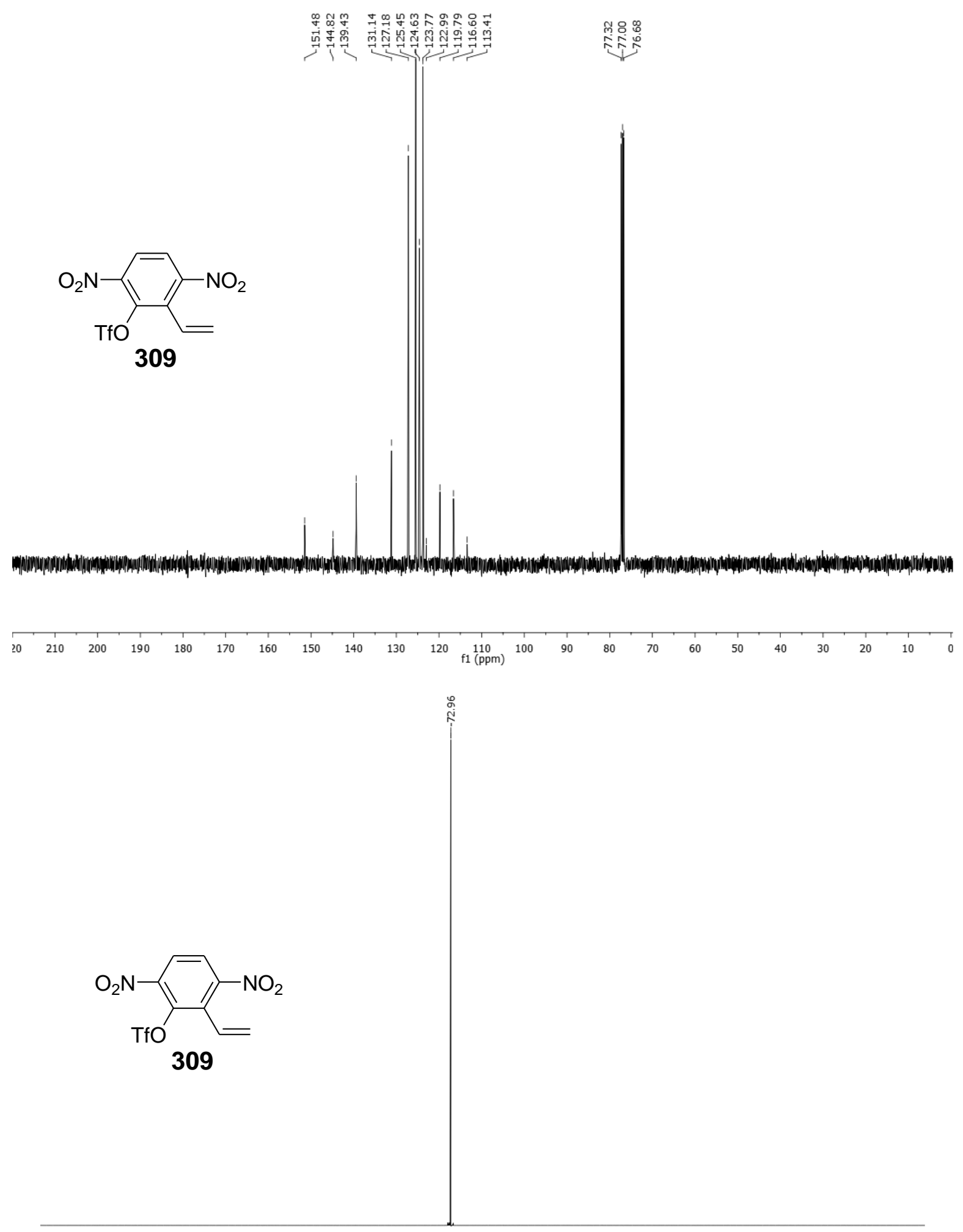

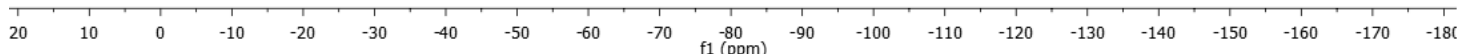

Figure 7.54 ${ }^{13} \mathrm{C}$ and ${ }^{19} \mathrm{~F}$ NMR of compound 309 


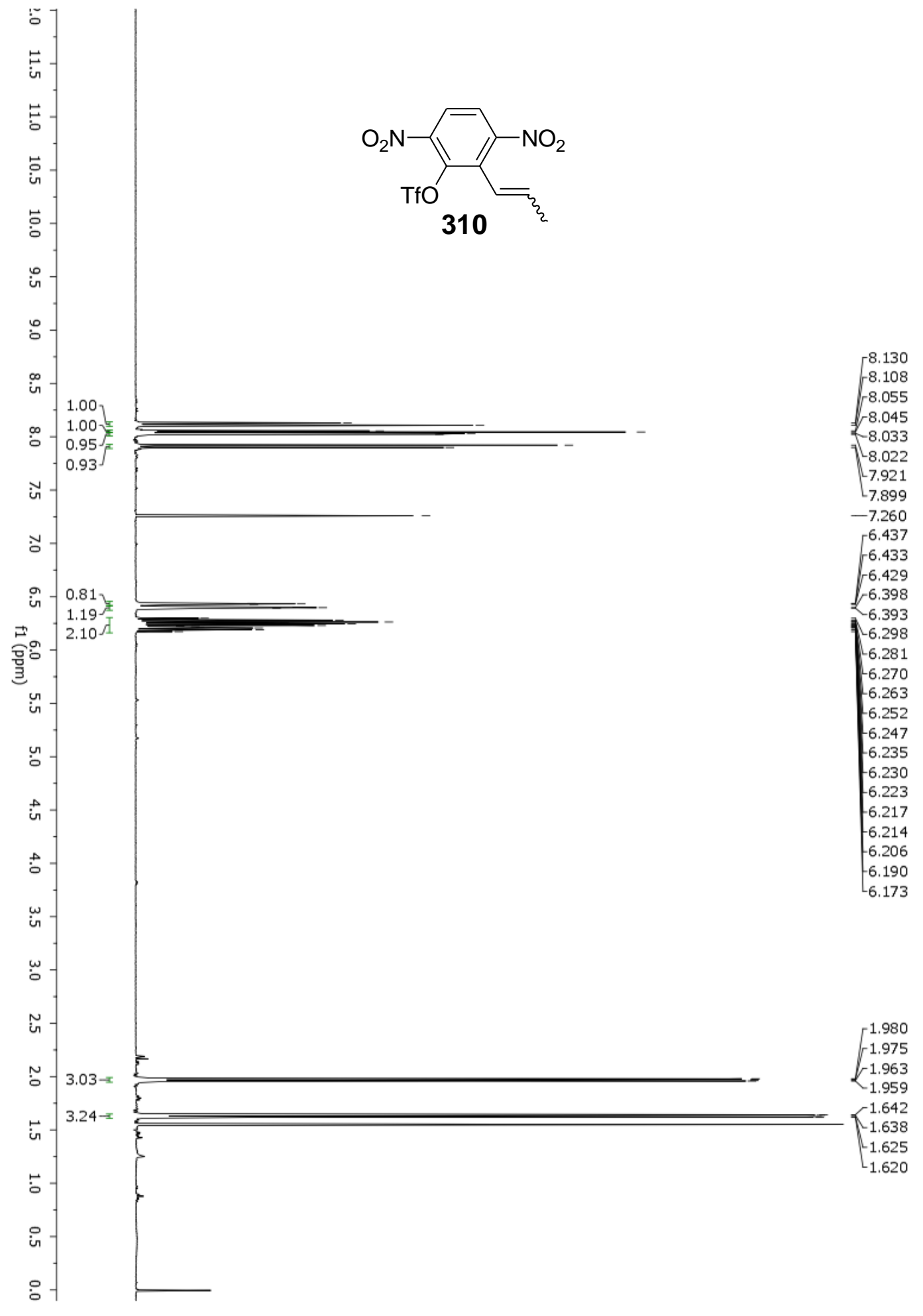

Figure 7.55 ${ }^{1} \mathrm{H}$ NMR of compound 310 

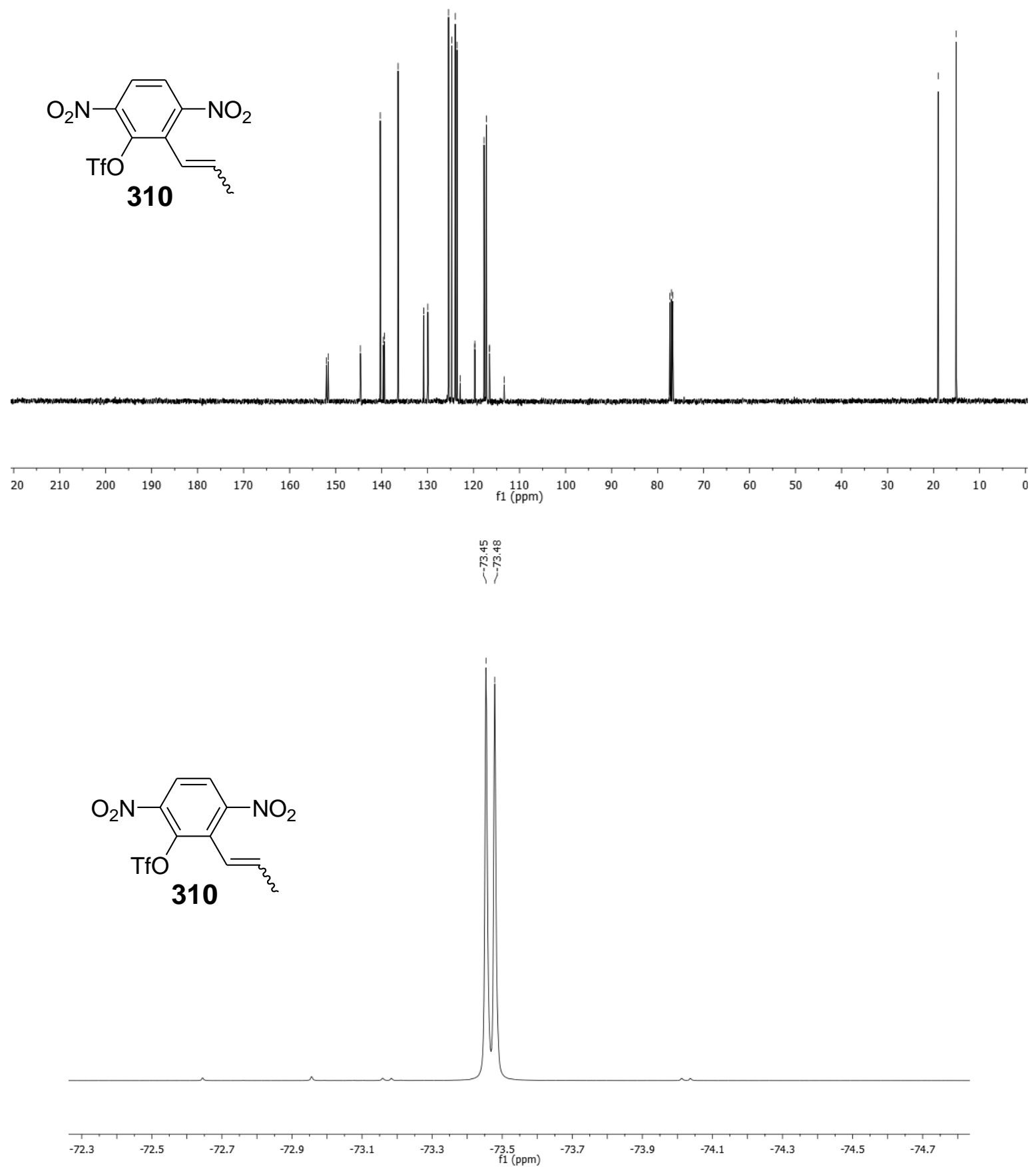

Figure 7.56 ${ }^{13} \mathrm{C}$ and ${ }^{19} \mathrm{~F}$ NMR of compound 310 


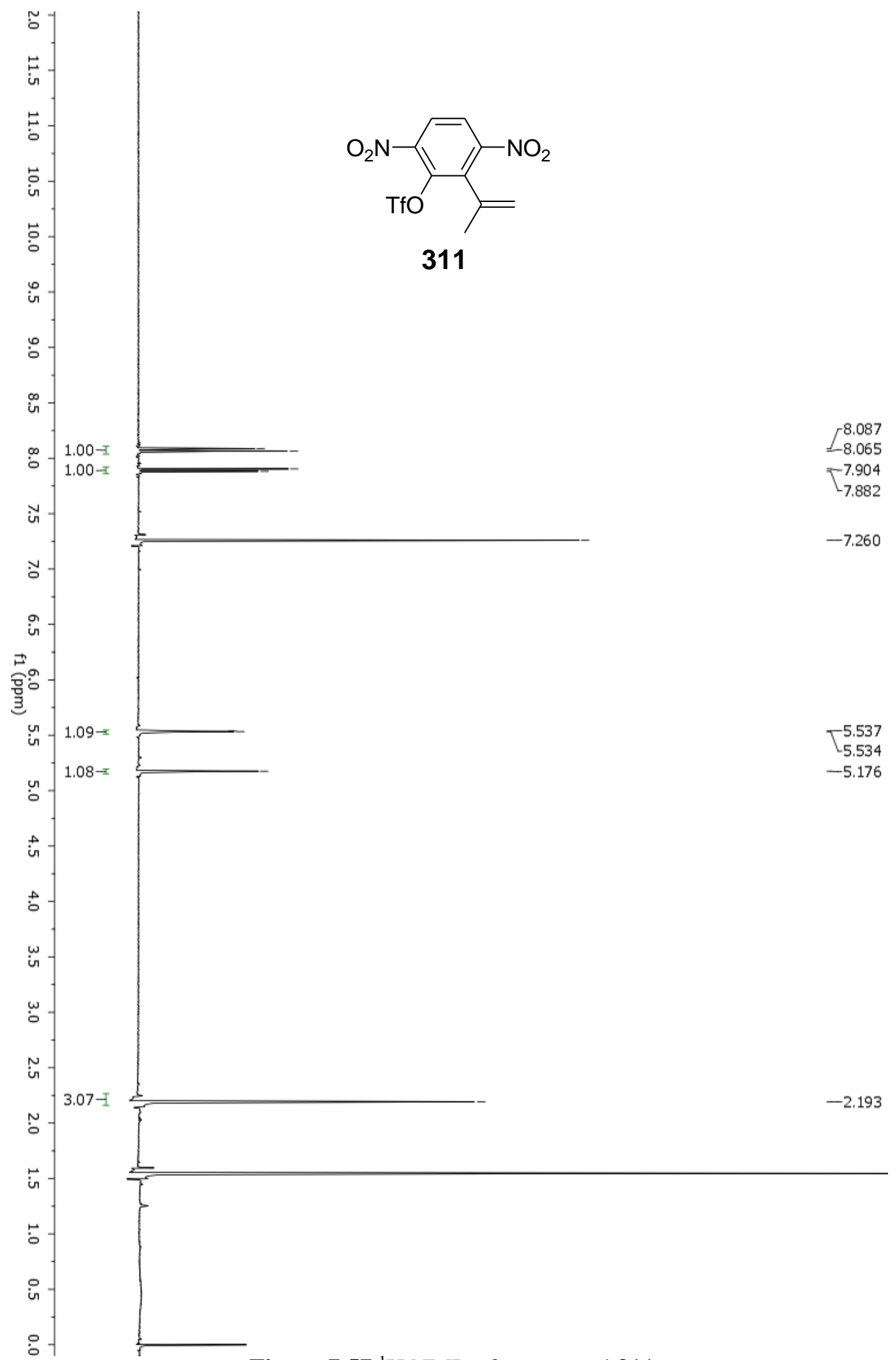

Figure $7.57{ }^{1} \mathrm{H}$ NMR of compound 311 

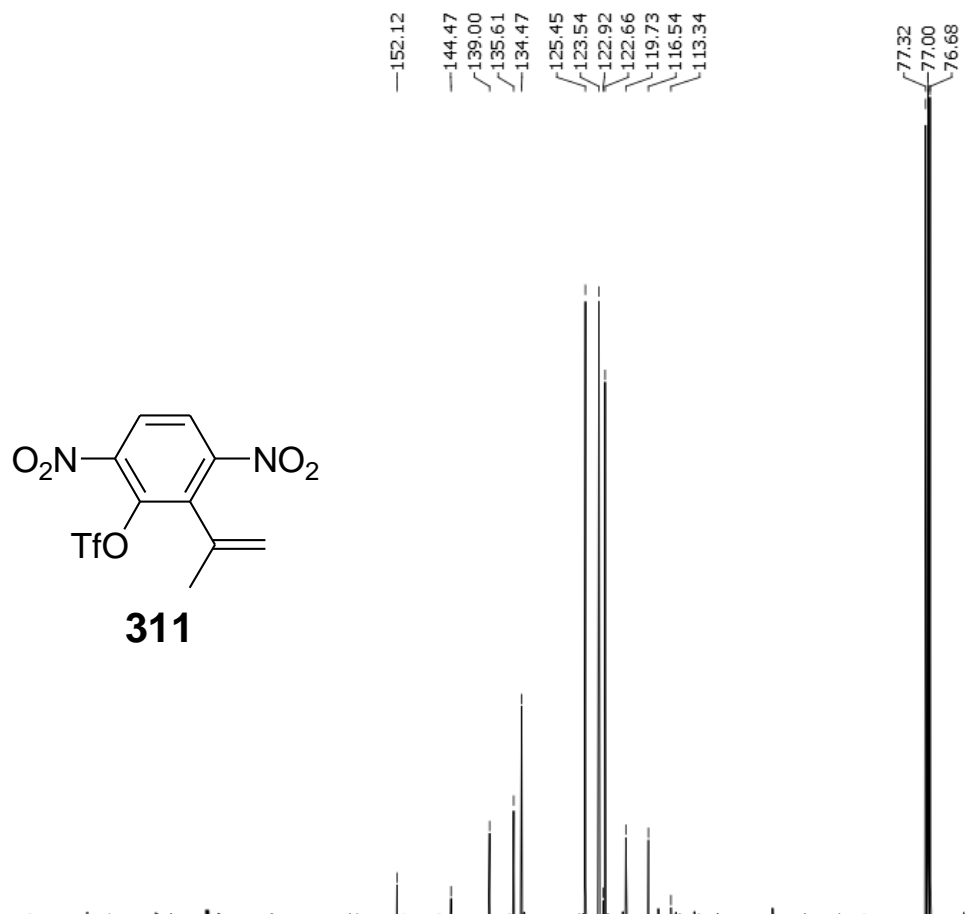

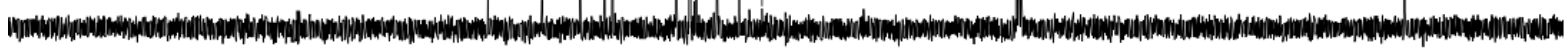
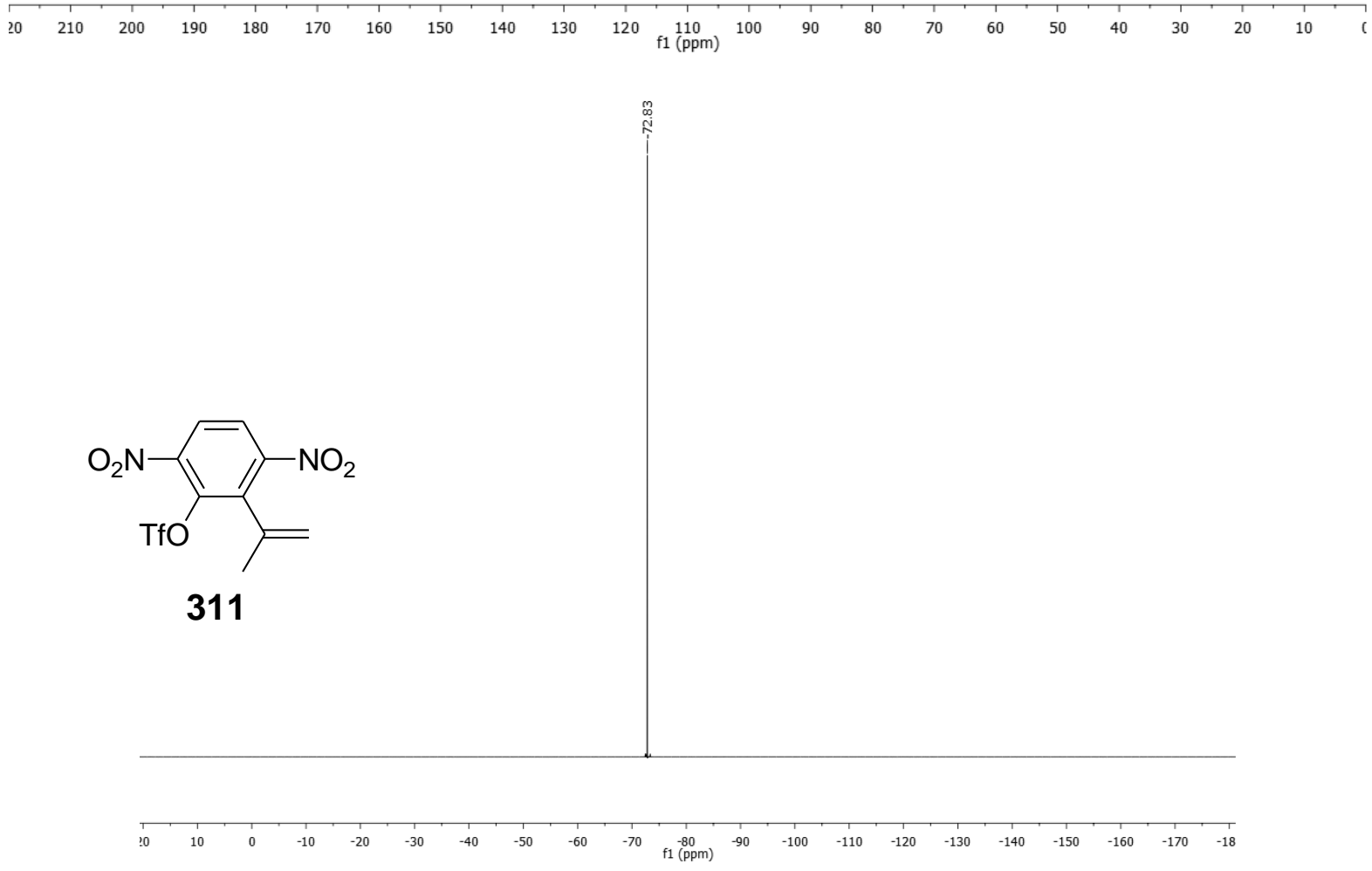

Figure 7.58 ${ }^{13} \mathrm{C}$ and ${ }^{19} \mathrm{~F}$ NMR of compound 311 


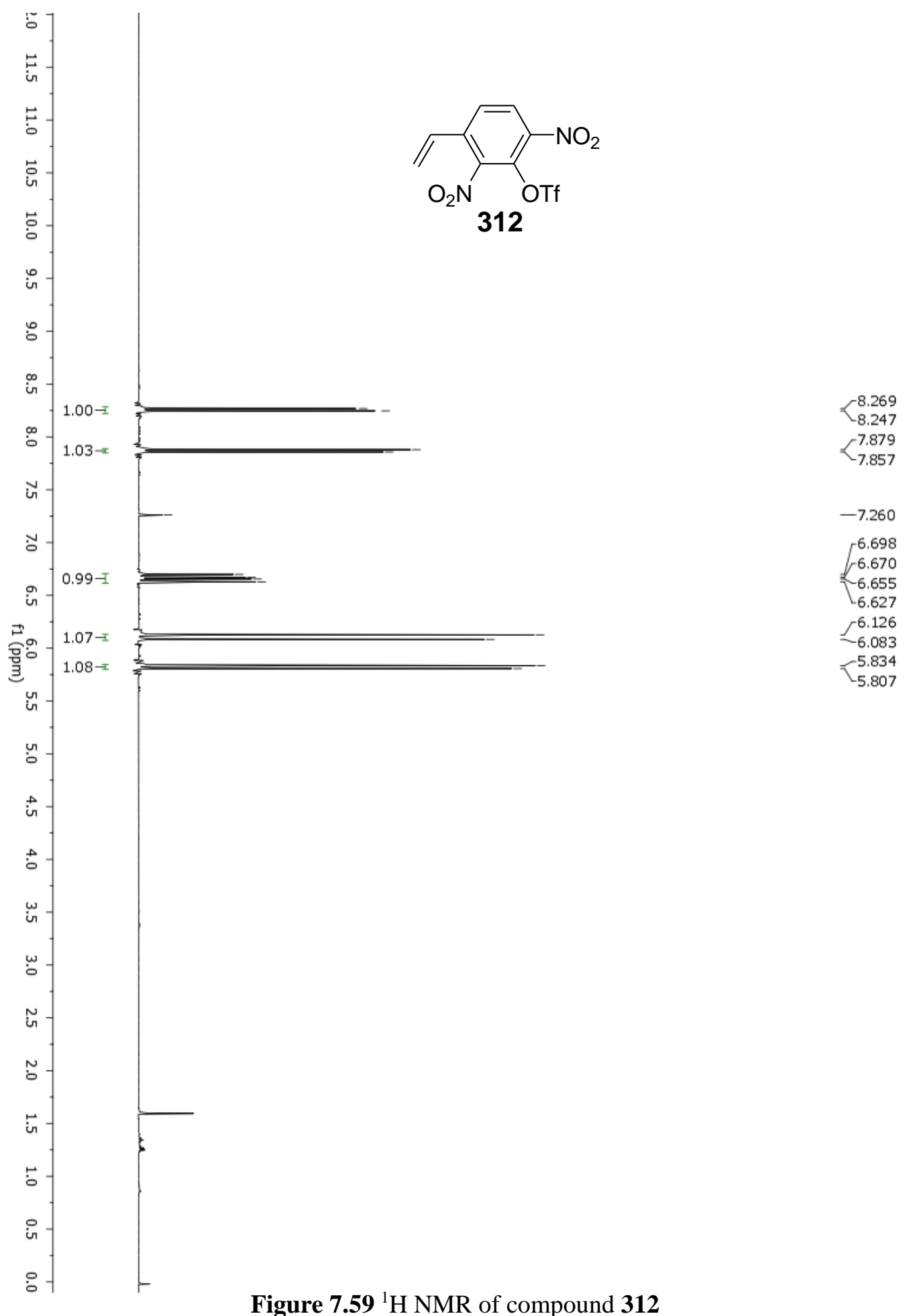

Figure $7.59{ }^{1} \mathrm{H}$ NMR of compound 312 

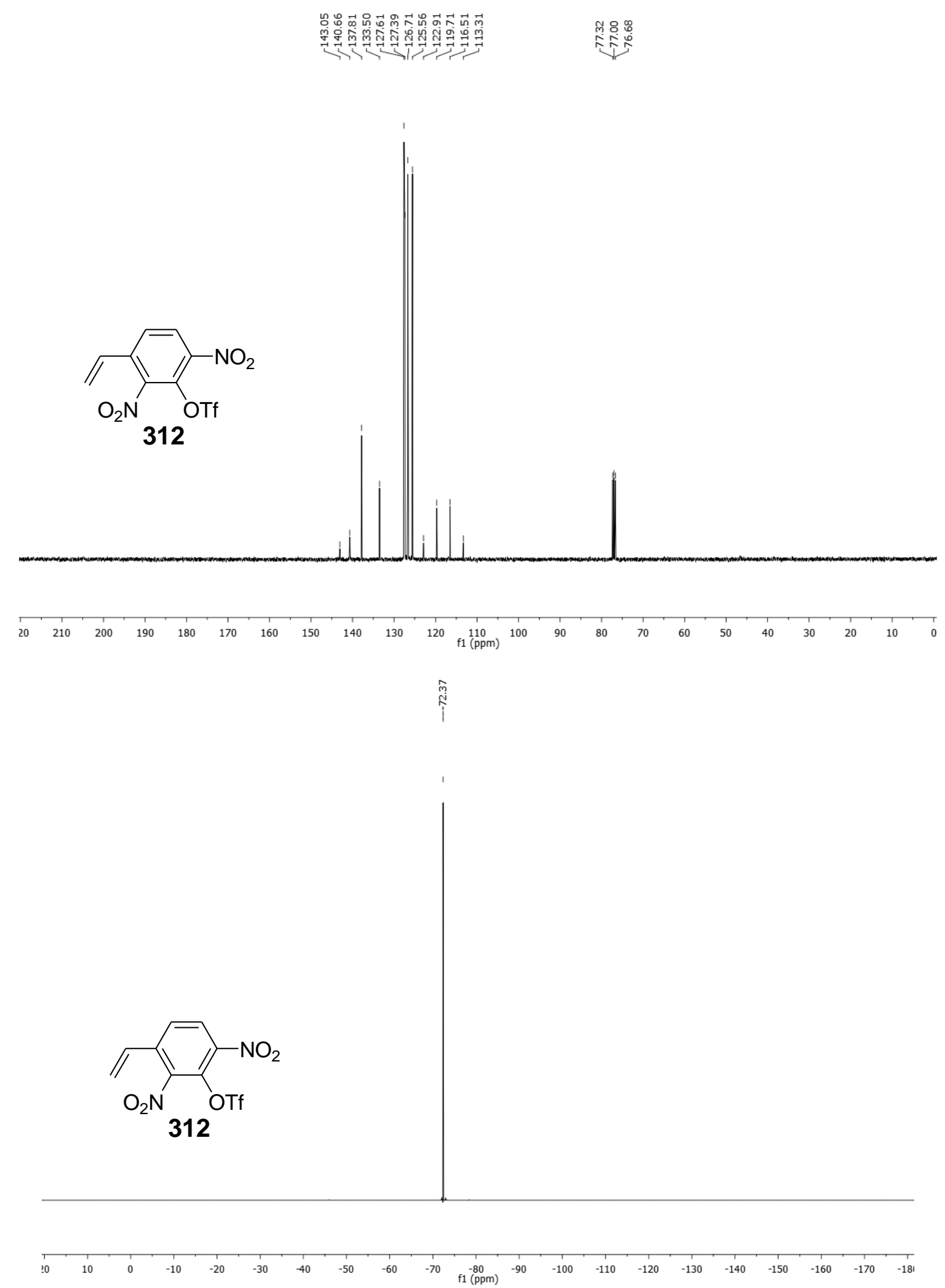

Figure 7.60 ${ }^{13} \mathrm{C}$ and ${ }^{19} \mathrm{~F}$ NMR of compound 312 


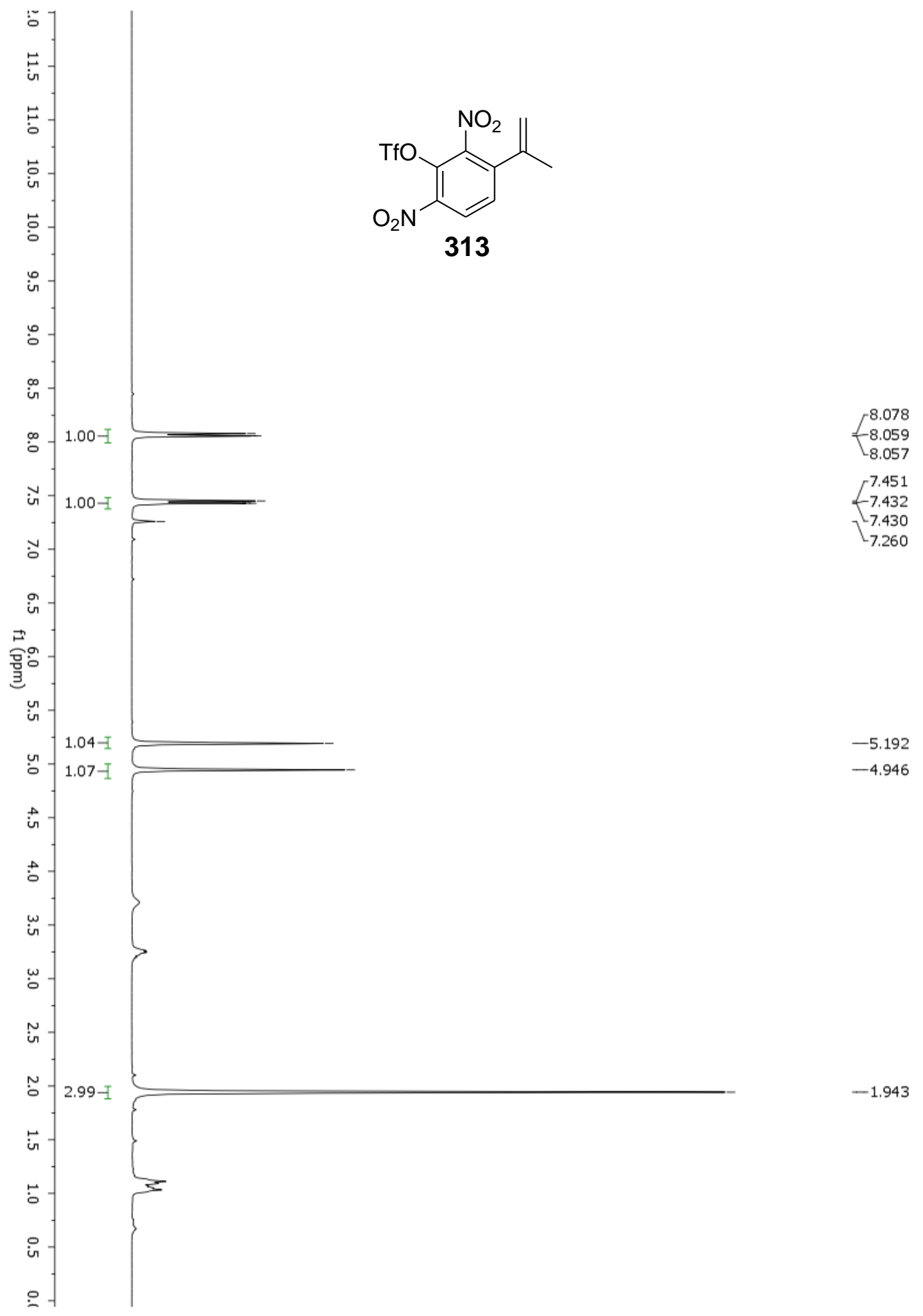

Figure 7.61 ${ }^{1} \mathrm{H}$ NMR of compound 313 

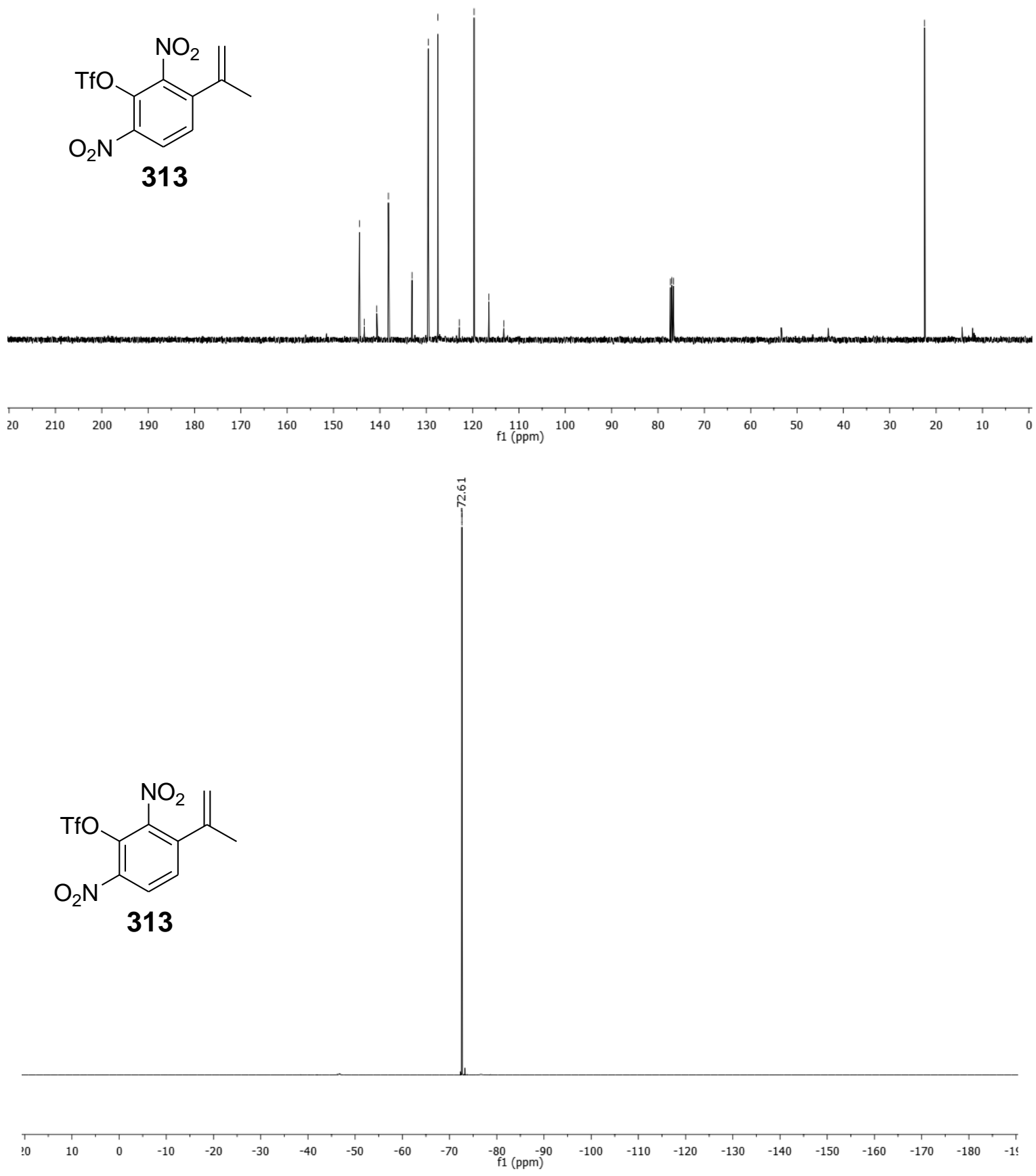

Figure 7.62 ${ }^{13} \mathrm{C}$ and ${ }^{19} \mathrm{~F}$ NMR of compound 313 

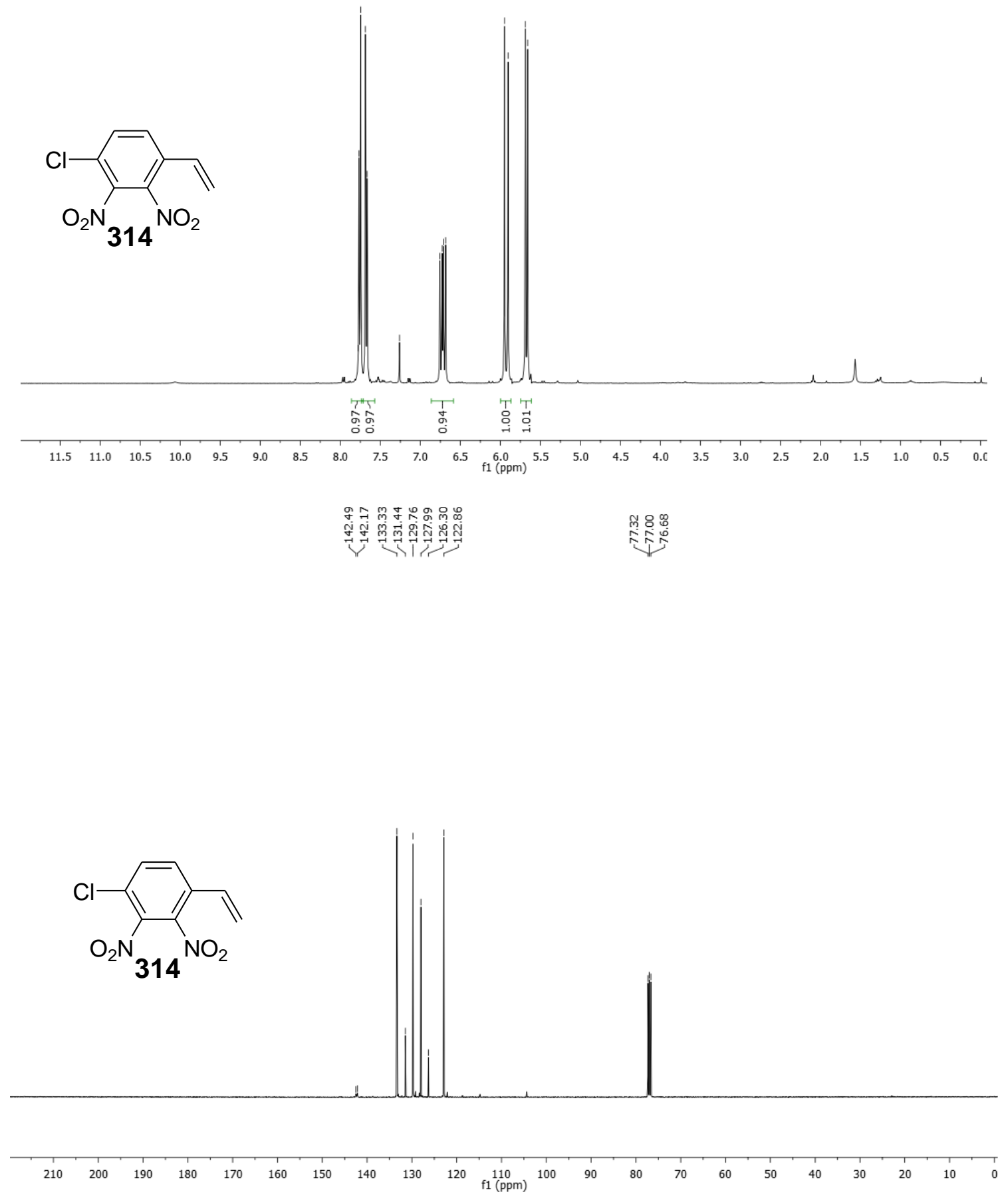

Figure 7.63 ${ }^{1} \mathrm{H}$ and ${ }^{13} \mathrm{C}$ NMR of compound 314 

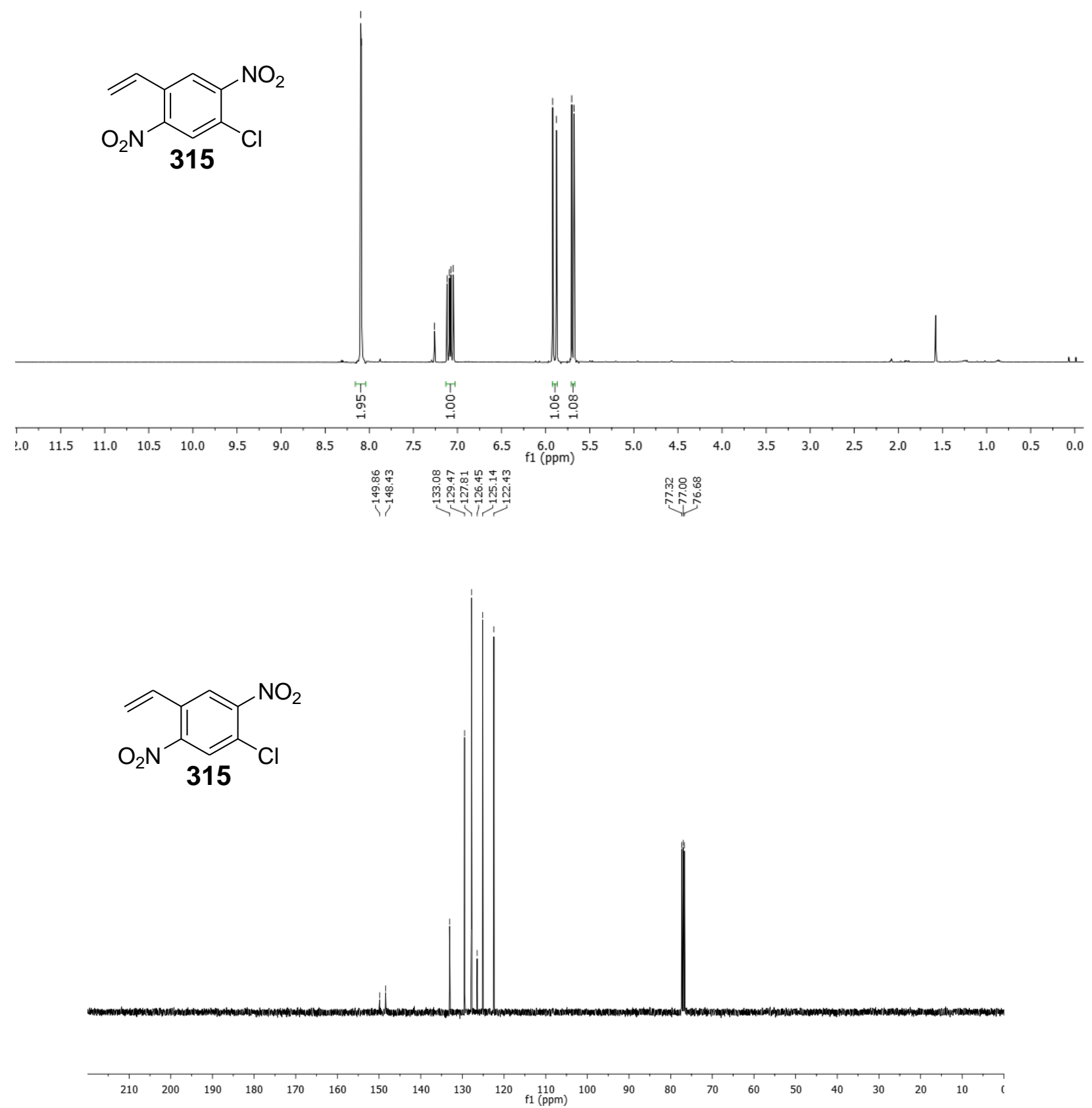

Figure 7.64 ${ }^{1} \mathrm{H}$ and ${ }^{13} \mathrm{C}$ NMR of compound 315 

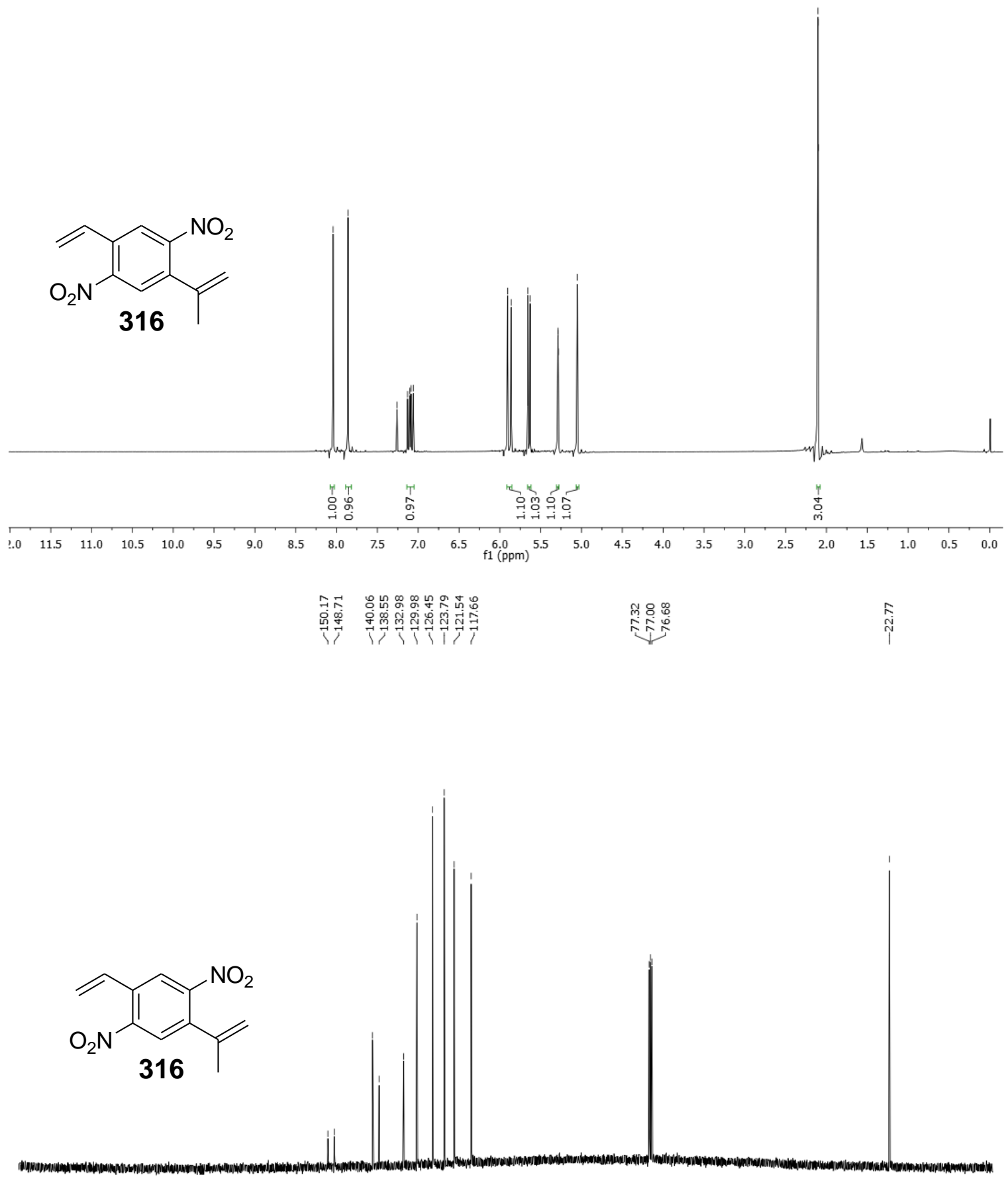

$20 \quad 210 \quad 200 \quad 190-180 \quad 170$

Figure 7.65 ${ }^{1} \mathrm{H}$ and ${ }^{13} \mathrm{C}$ NMR of compound 316 

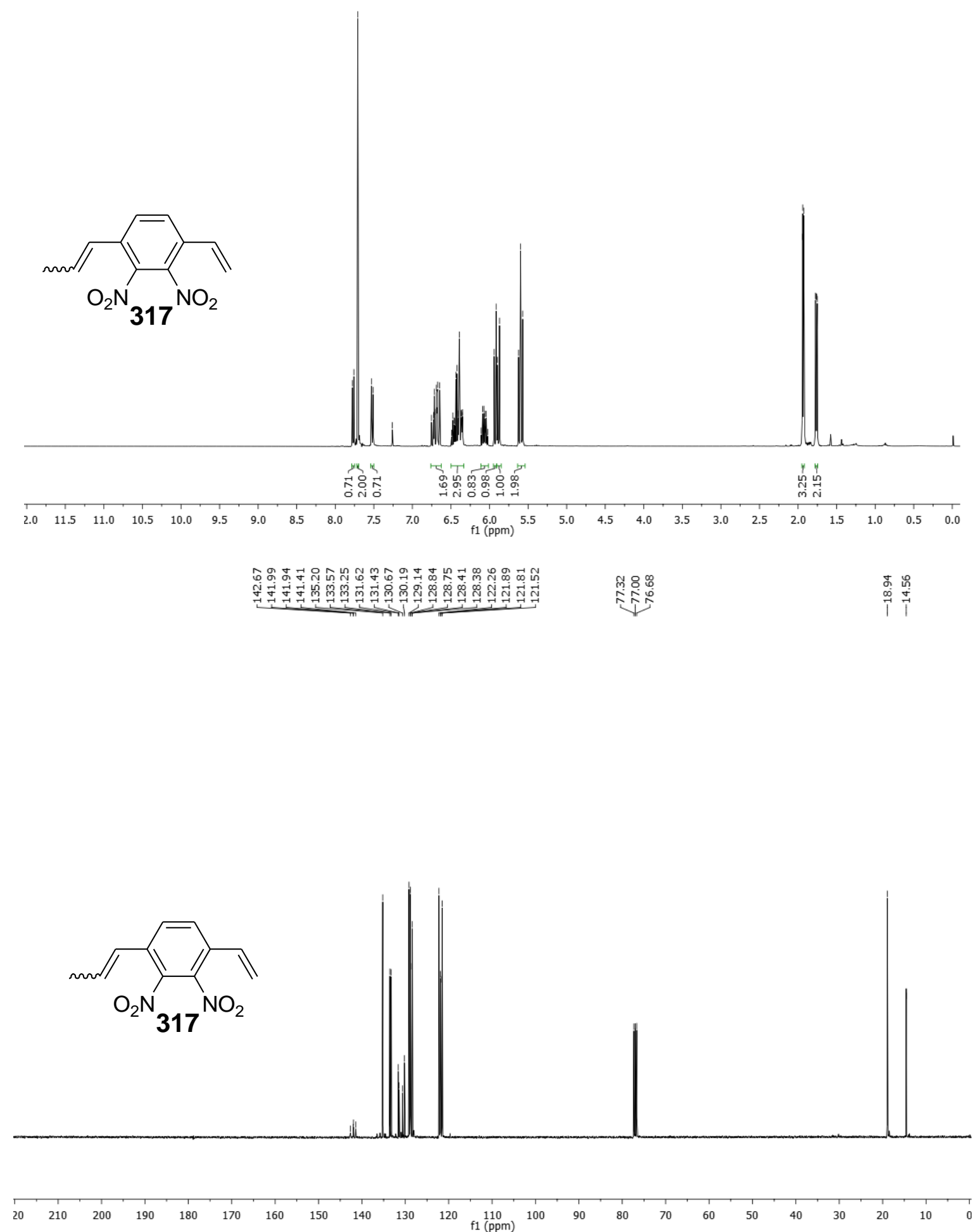

Figure 7.66 ${ }^{1} \mathrm{H}$ and ${ }^{13} \mathrm{C}$ NMR of compound 317 

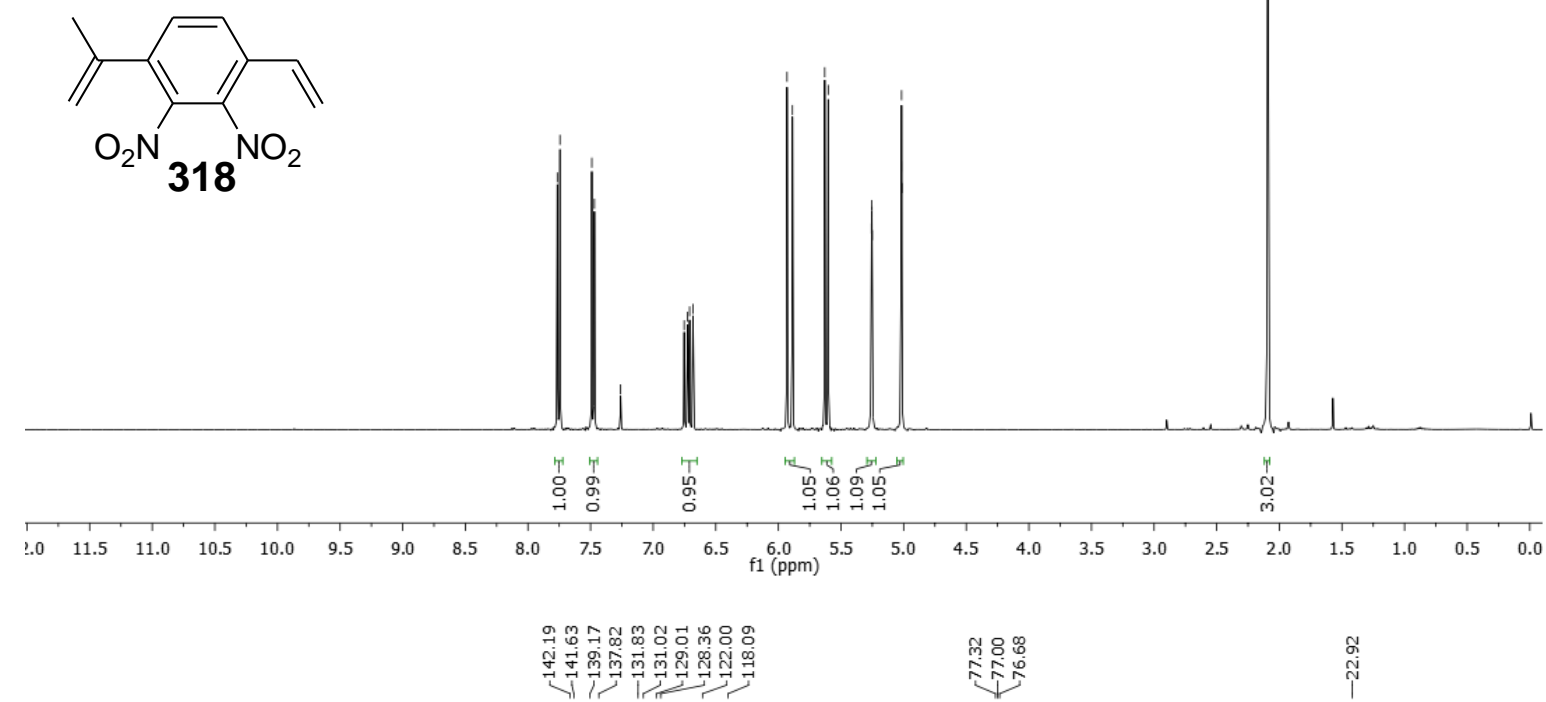

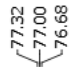
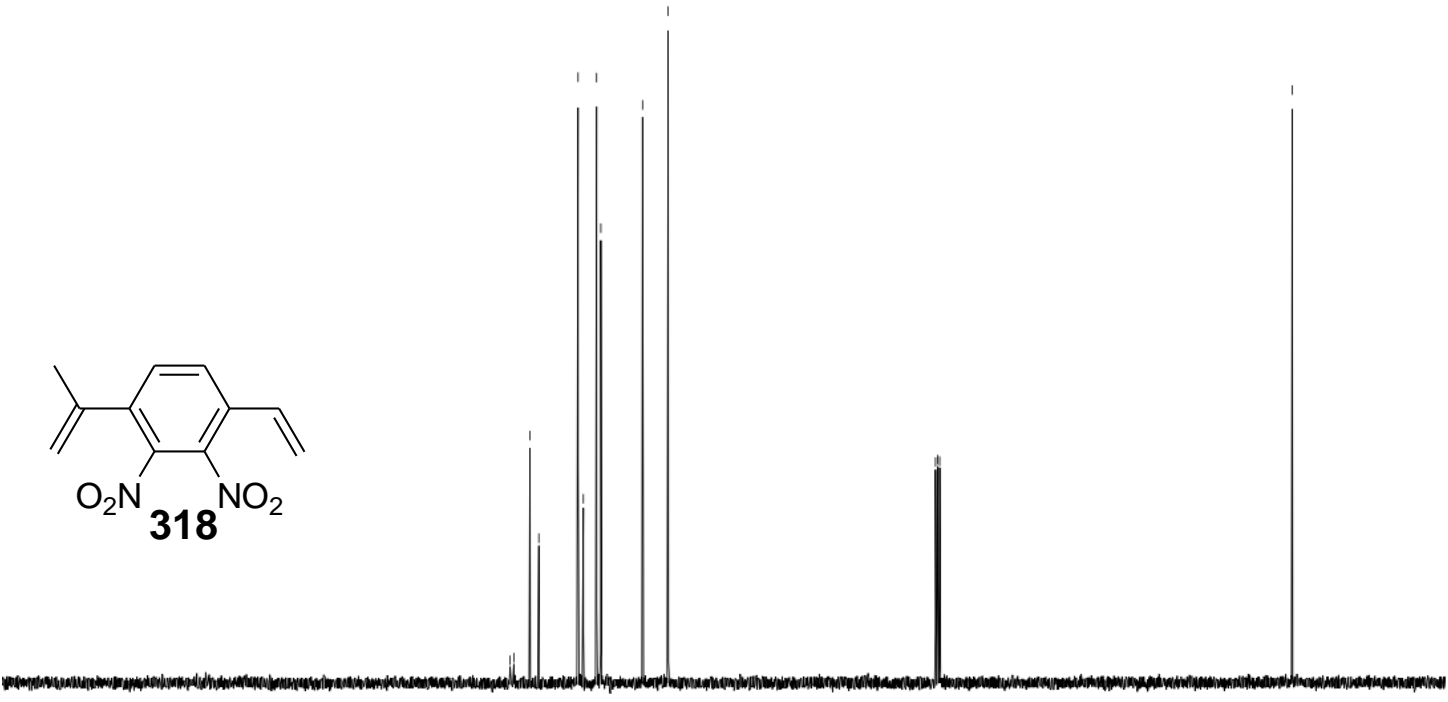

210

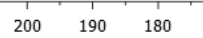

Figure 7.67 ${ }^{1} \mathrm{H}$ and ${ }^{13} \mathrm{C}$ NMR of compound 318 


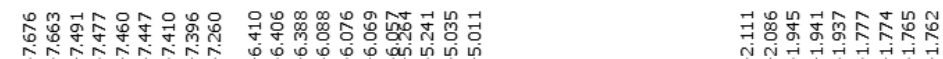

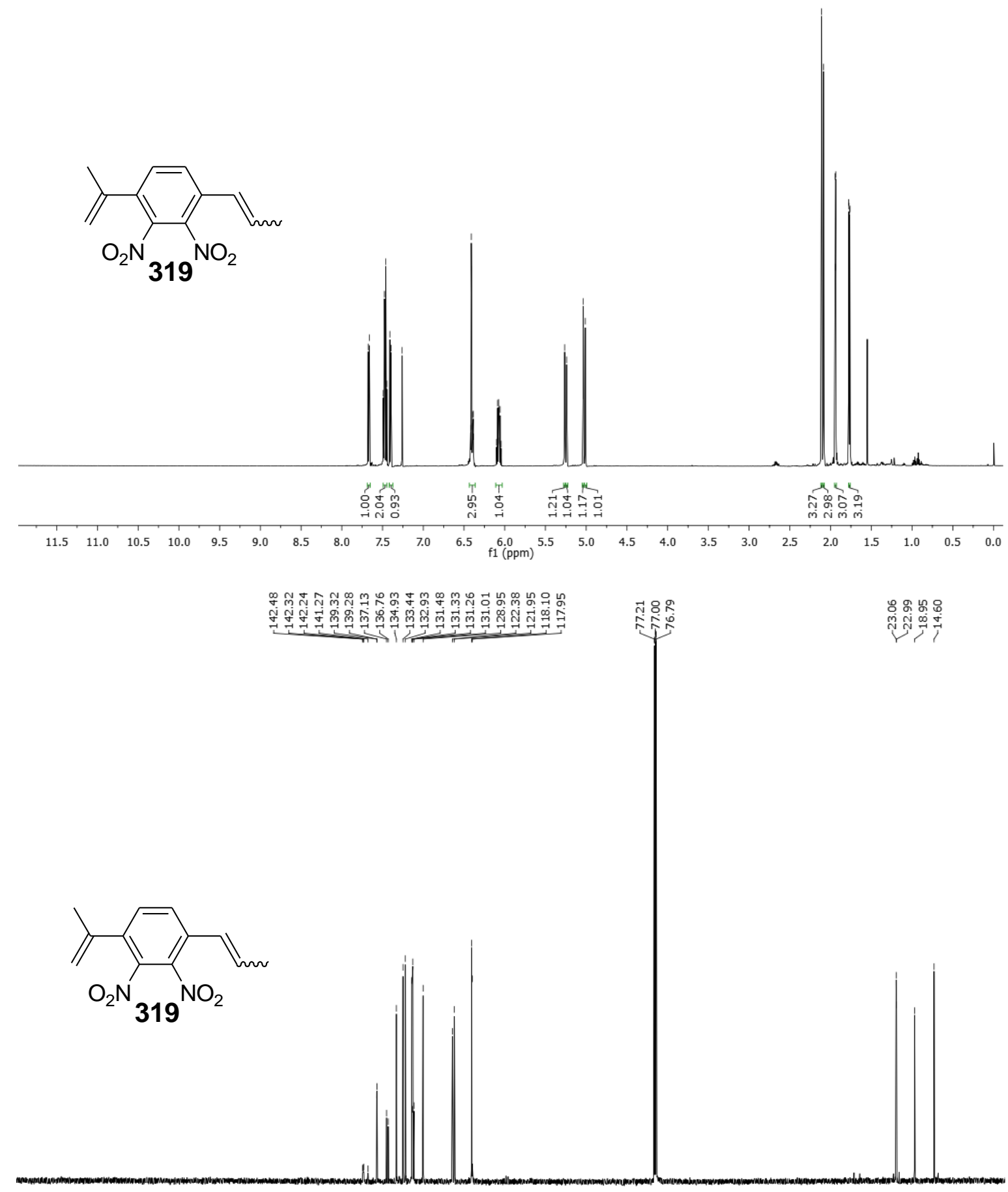

210
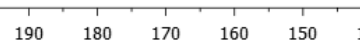

$120 \underset{f 1(\mathrm{ppm})}{110} 100$

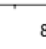

Figure 7.68 ${ }^{1} \mathrm{H}$ and ${ }^{13} \mathrm{C}$ NMR of compound 319 


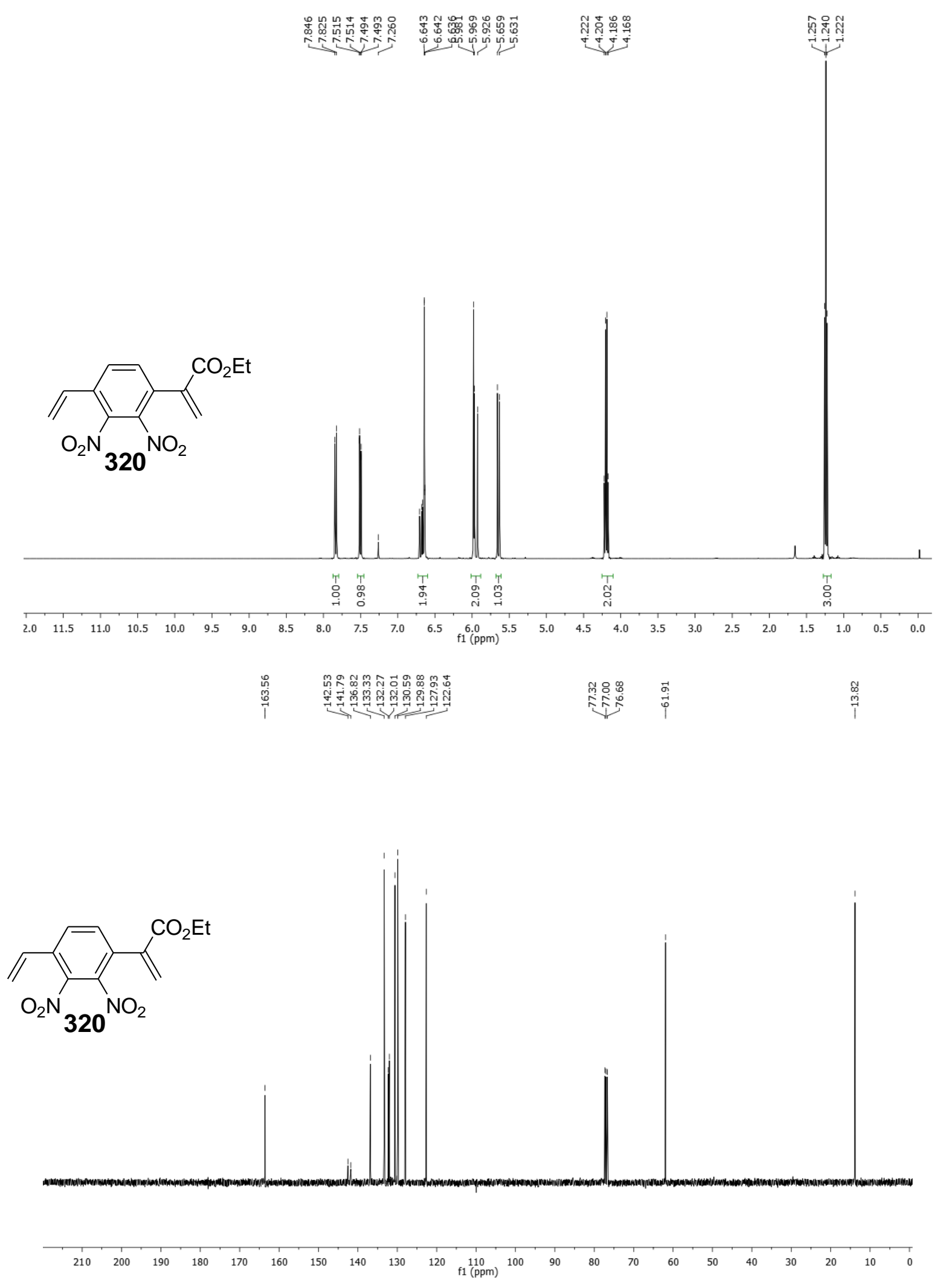

Figure 7.69 ${ }^{1} \mathrm{H}$ and ${ }^{13} \mathrm{C}$ NMR of compound 320 

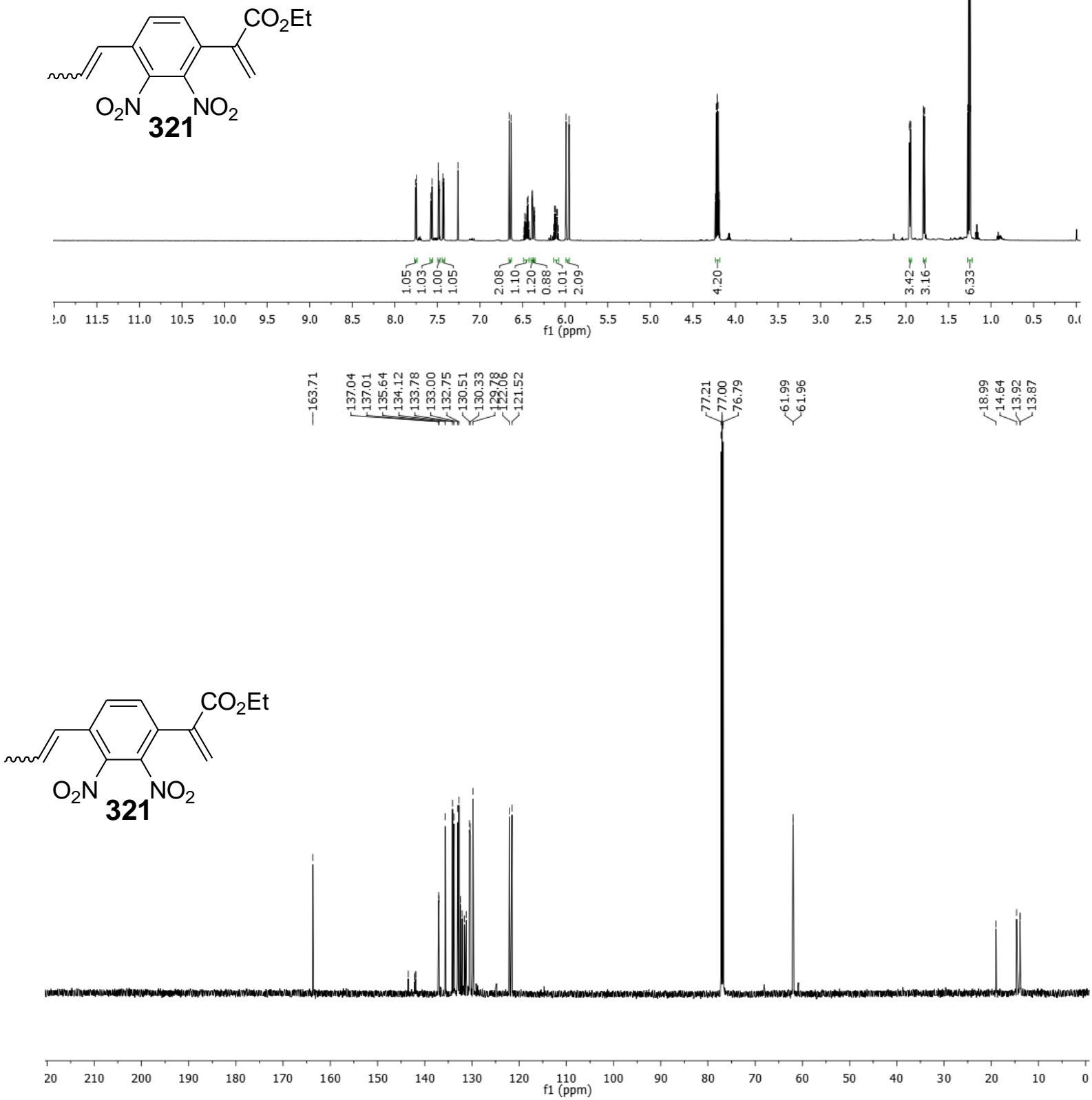

Figure 7.70 ${ }^{1} \mathrm{H}$ and ${ }^{13} \mathrm{C}$ NMR of compound 321 


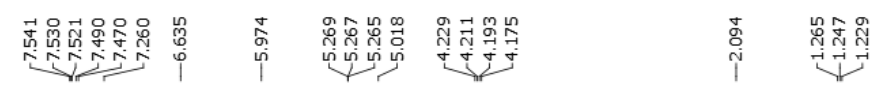
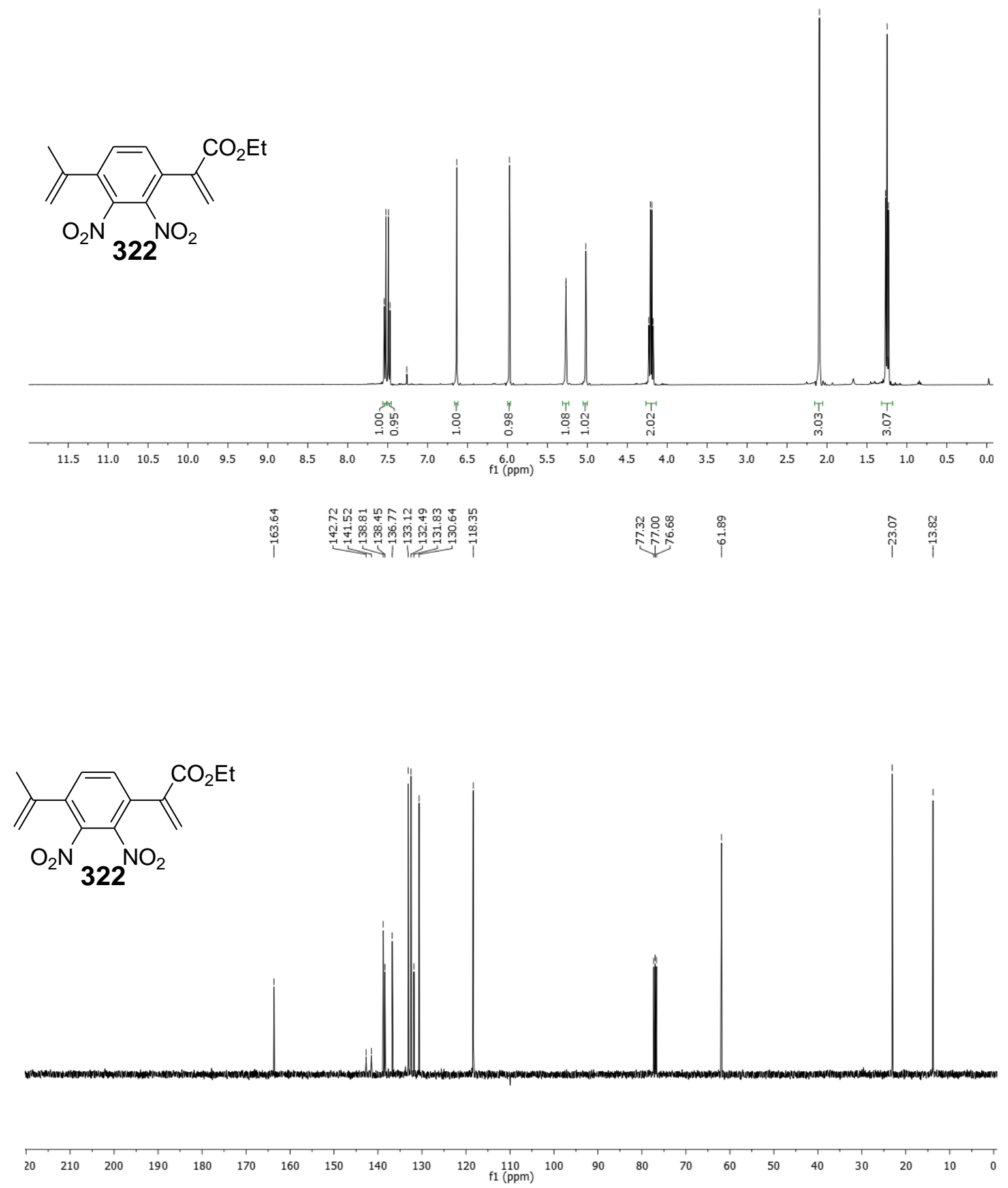

Figure $7.71{ }^{1} \mathrm{H}$ and ${ }^{13} \mathrm{C}$ NMR of compound 322 
<smiles>C=Cc1cc(C(=C)C)c([N+](=O)[O-])cc1[N+](=O)[O-]</smiles>

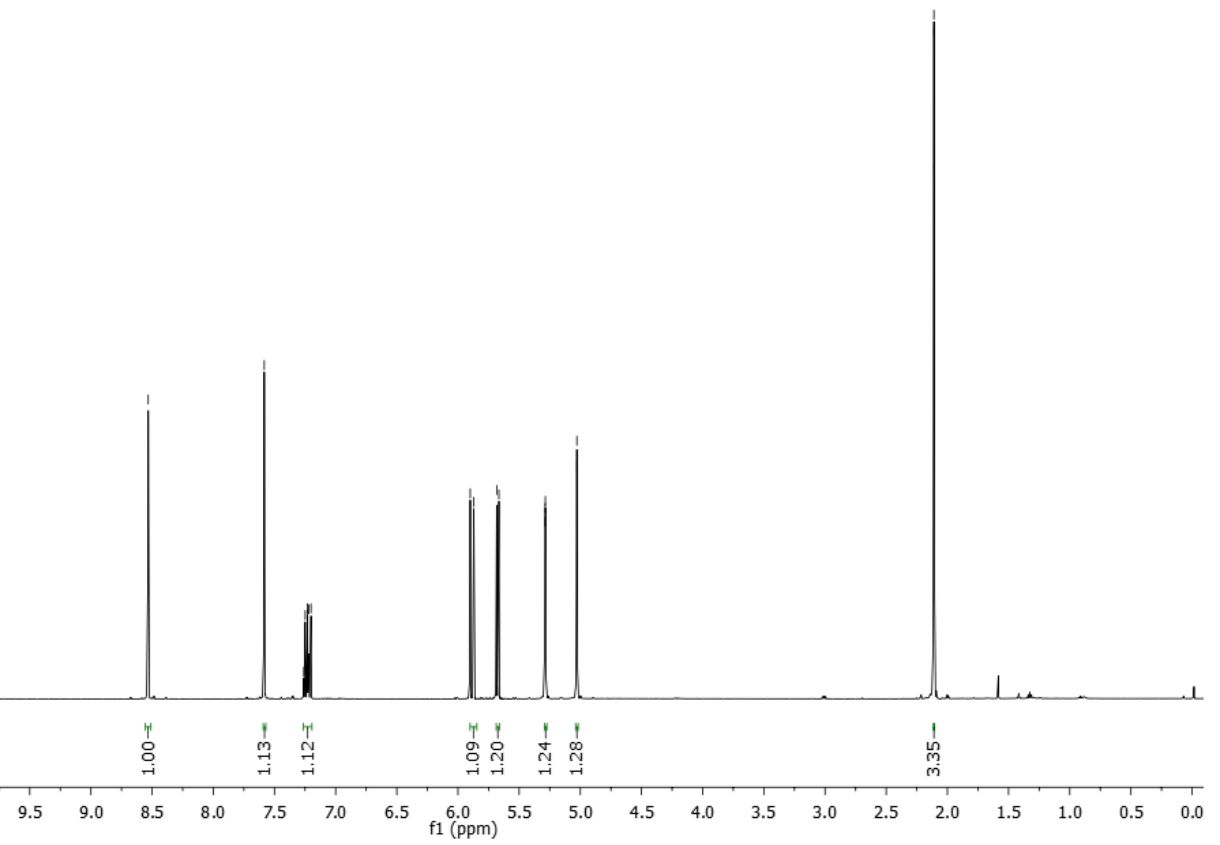

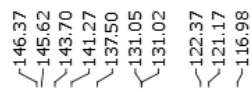
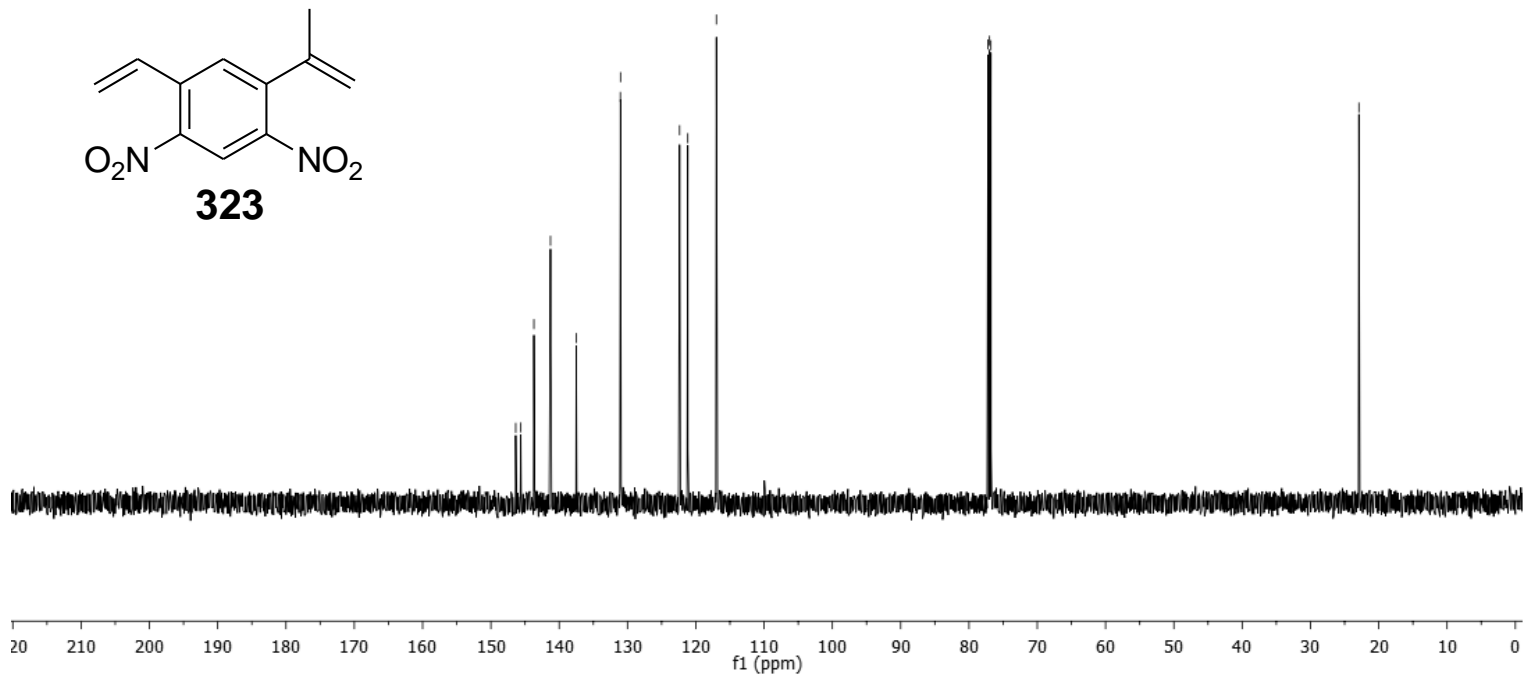

Figure 7.72 ${ }^{1} \mathrm{H}$ and ${ }^{13} \mathrm{C}$ NMR of compound 323 

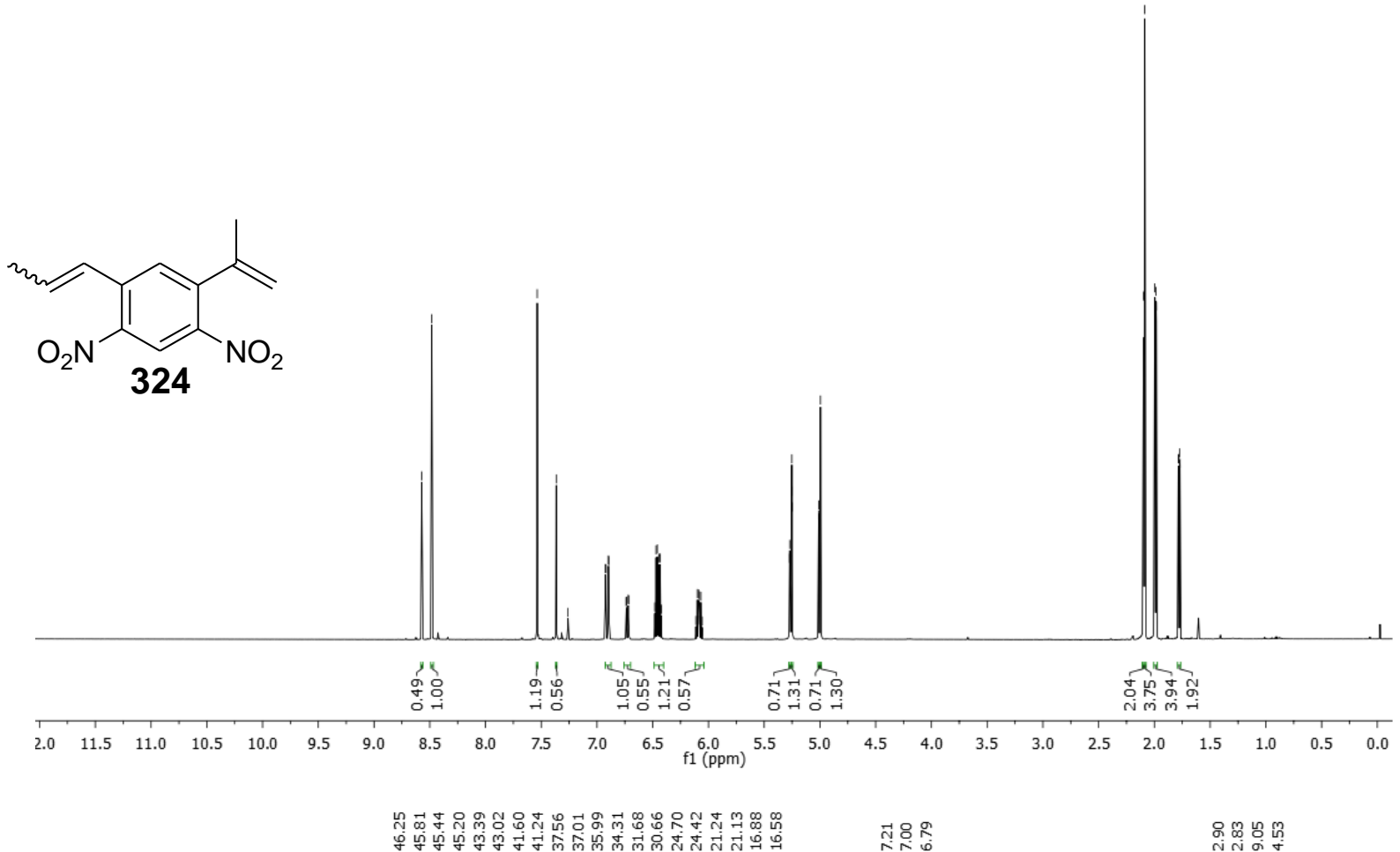

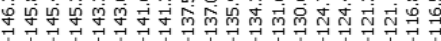

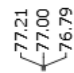

용ํำ

nูก
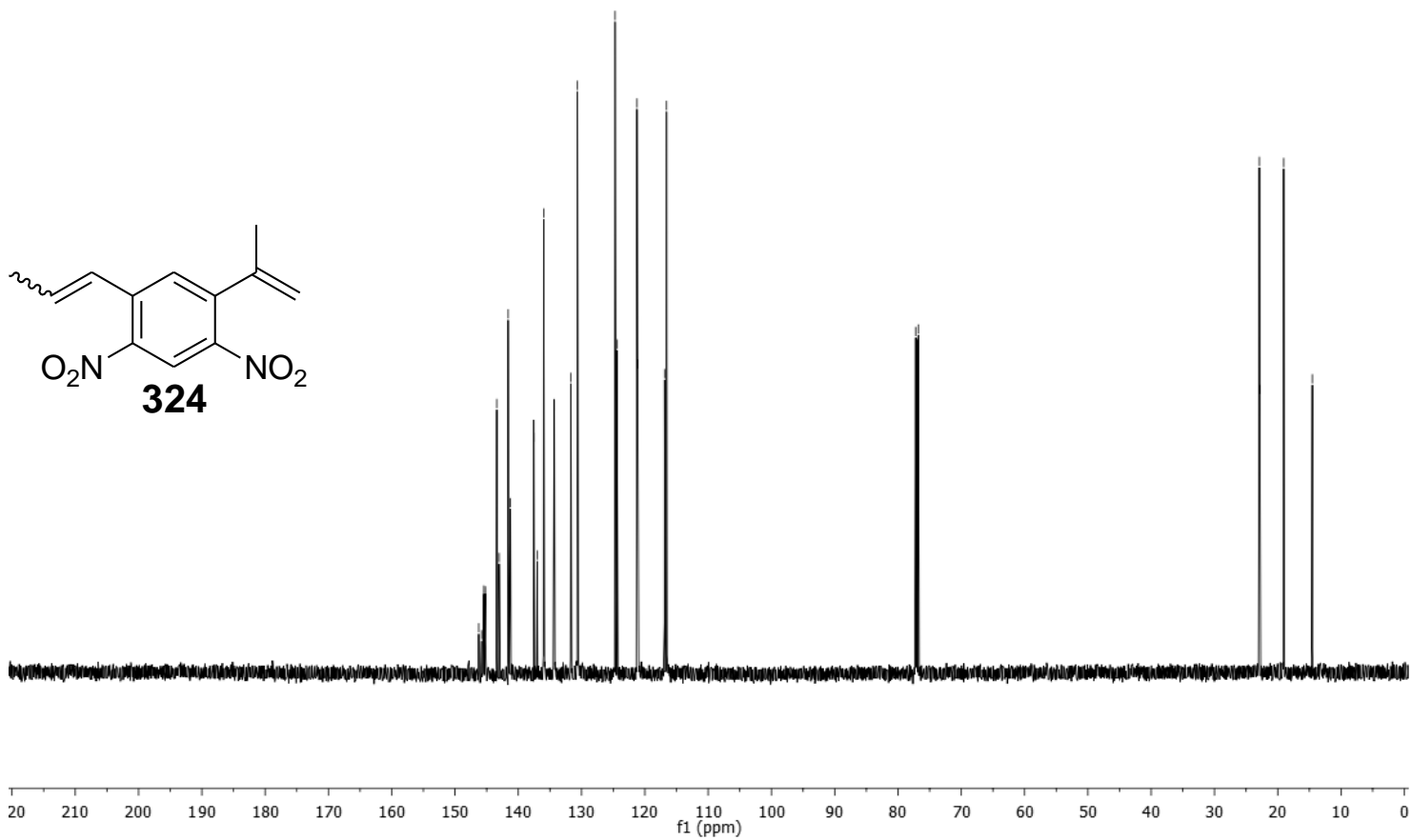

Figure 7.73 ${ }^{1} \mathrm{H}$ and ${ }^{13} \mathrm{C}$ NMR of compound 324 

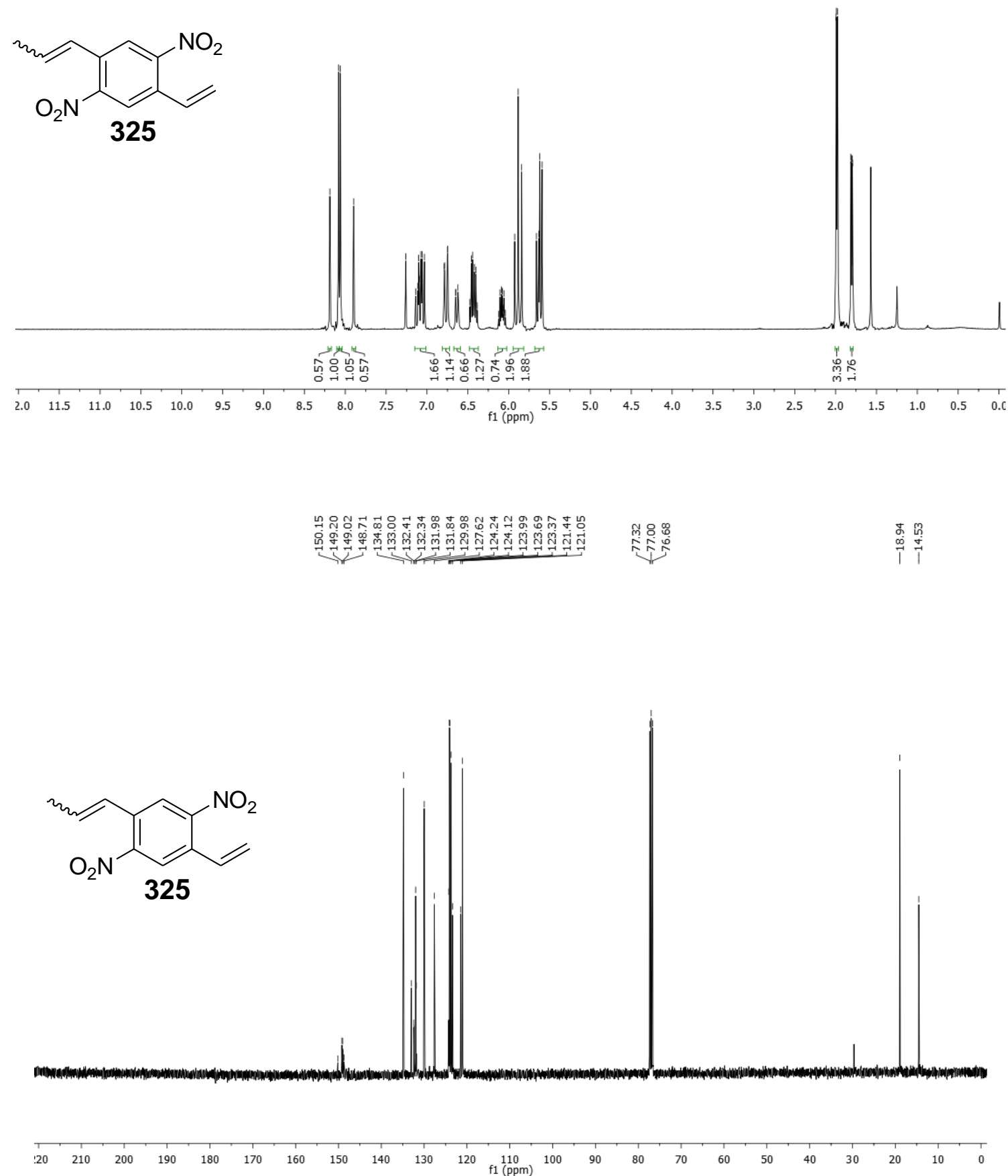

Figure 7.74 ${ }^{1} \mathrm{H}$ and ${ }^{13} \mathrm{C}$ NMR of compound 325 

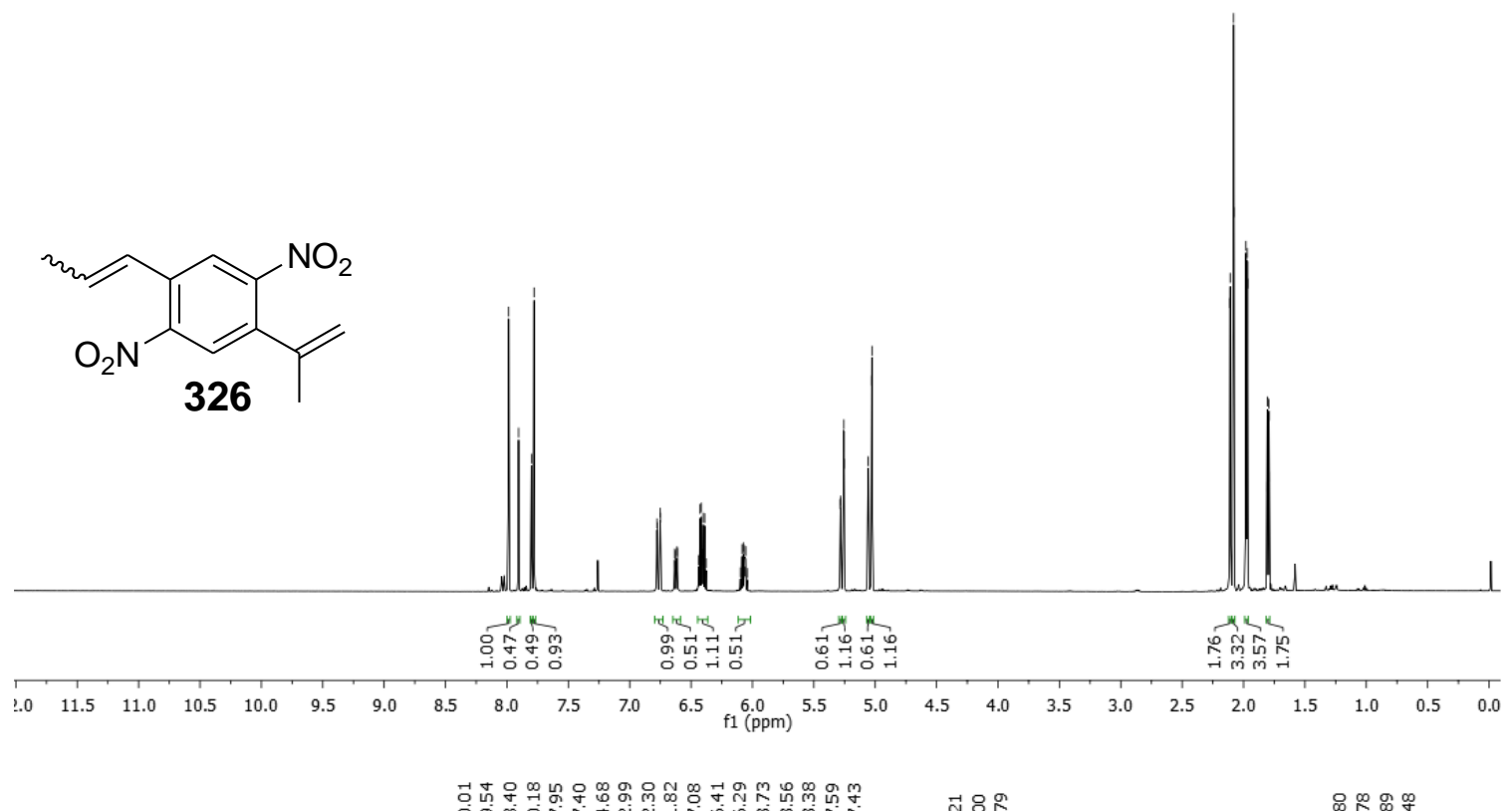

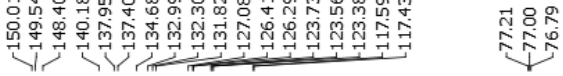

$\circ \stackrel{\infty}{\infty} \stackrel{\infty}{\square}$
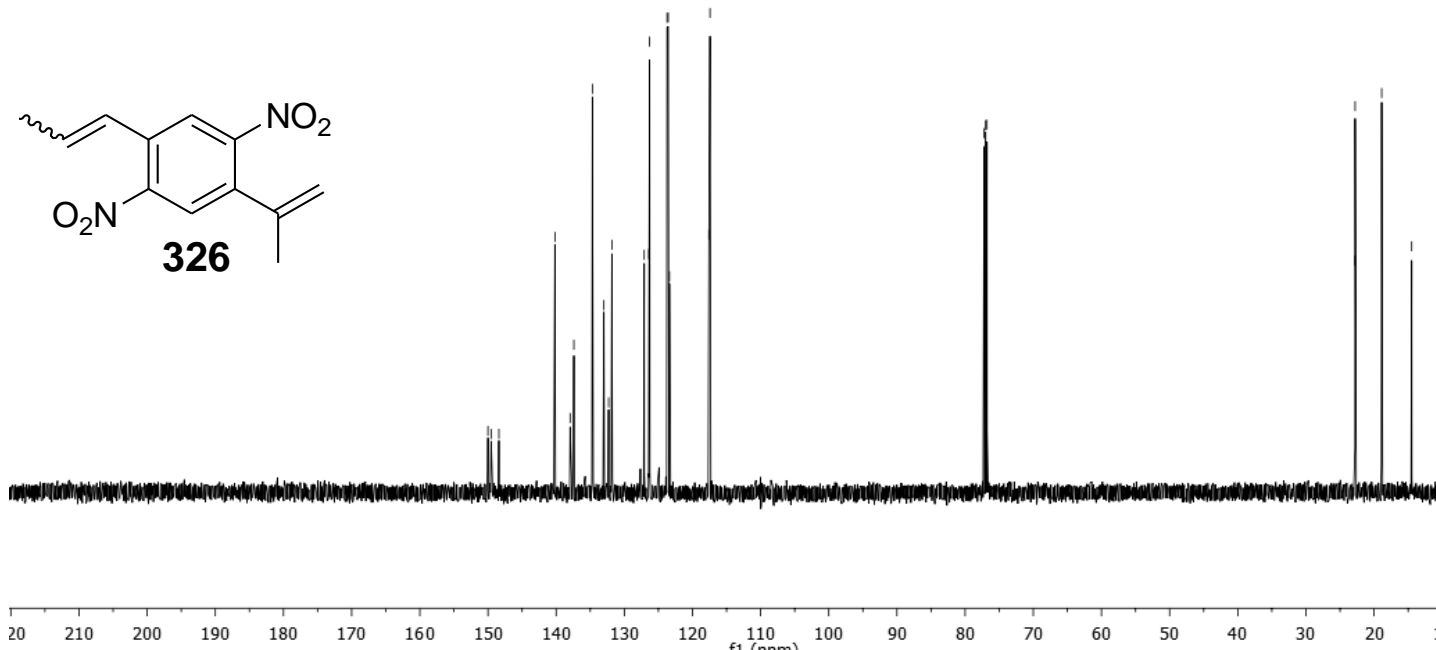

Figure 7.75 ${ }^{1} \mathrm{H}$ and ${ }^{13} \mathrm{C}$ NMR of compound 326 

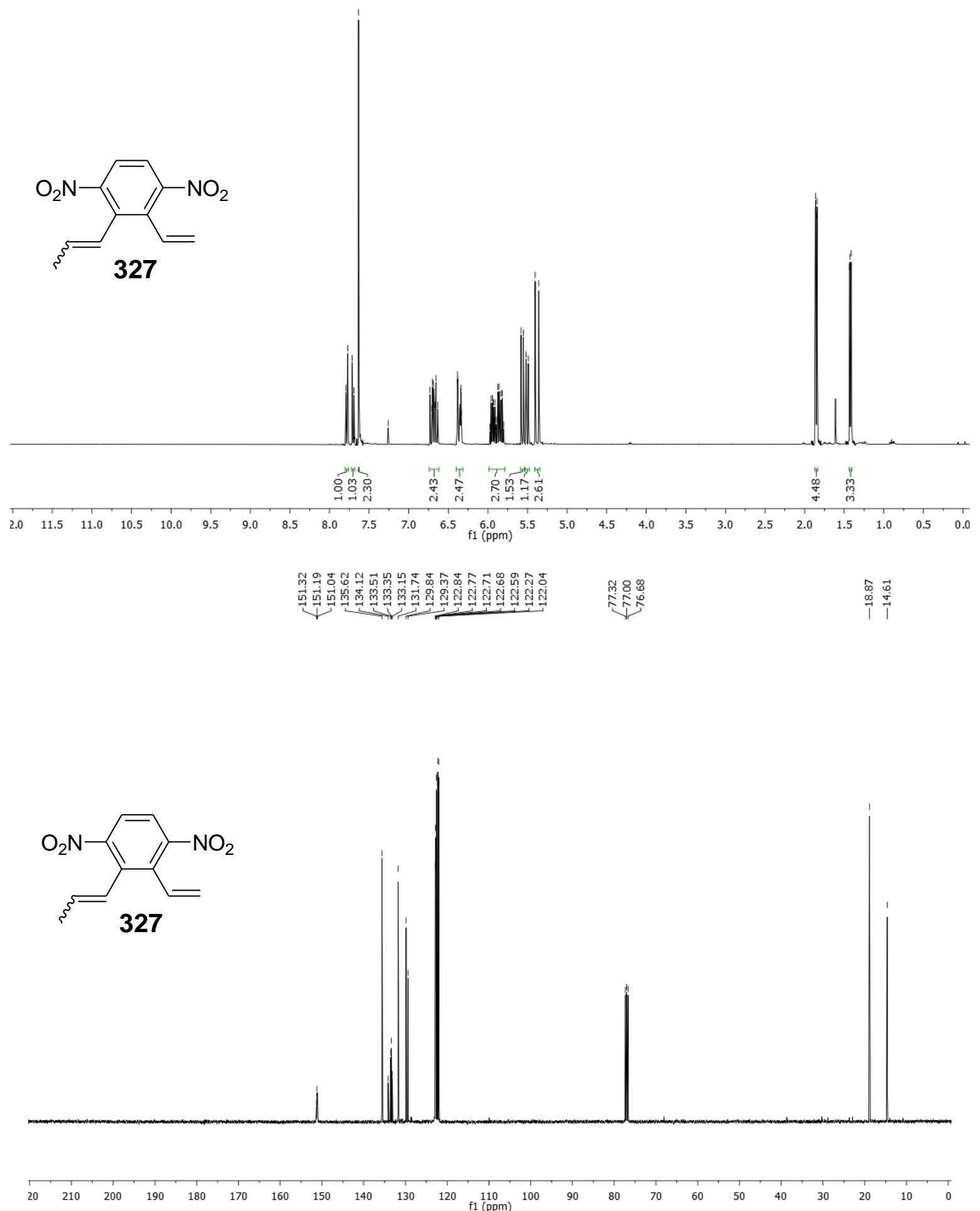

Figure 7.76 ${ }^{1} \mathrm{H}$ and ${ }^{13} \mathrm{C}$ NMR of compound 327 


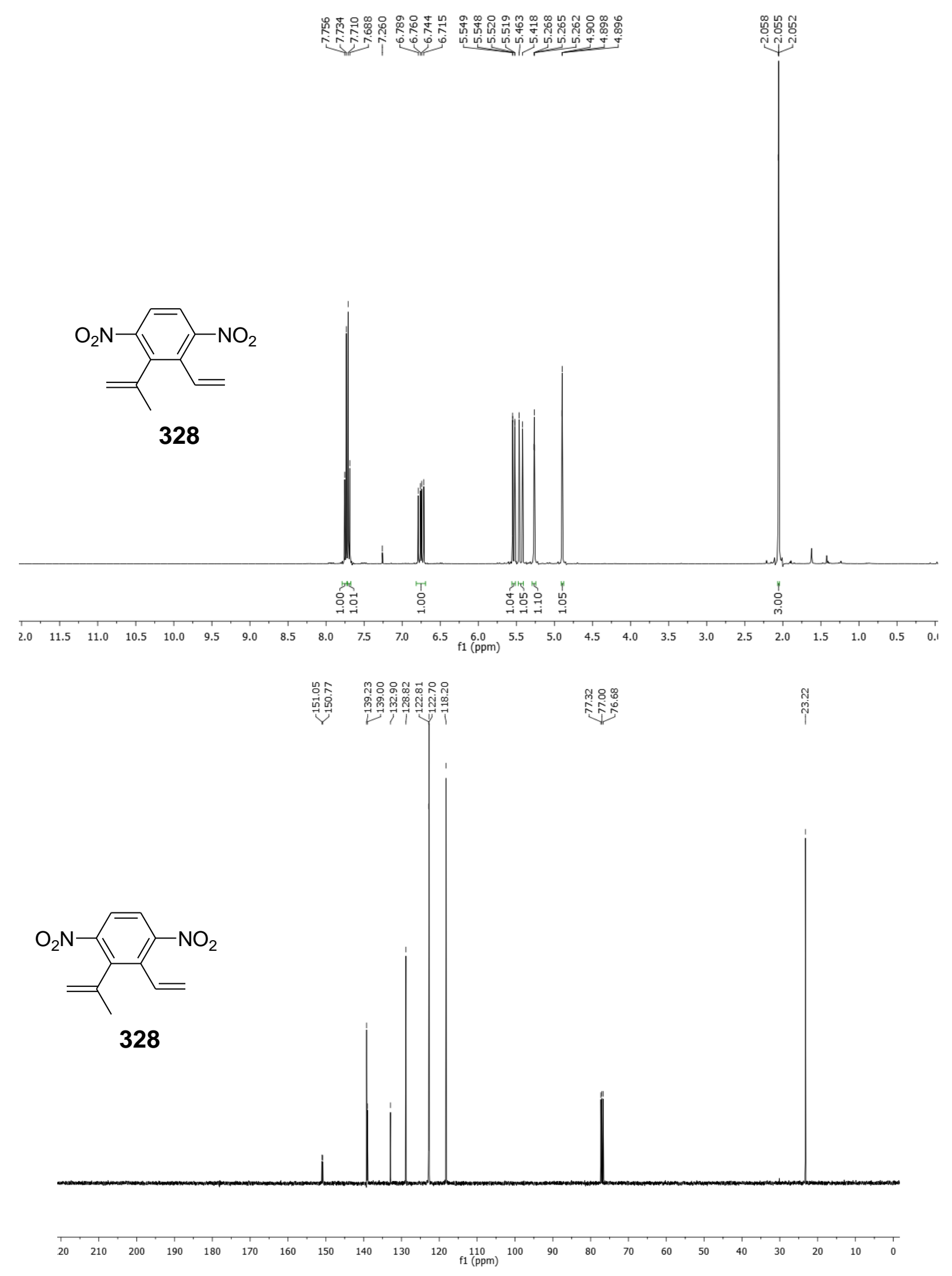

Figure 7.77 ${ }^{1} \mathrm{H}$ and ${ }^{13} \mathrm{C}$ NMR of compound $\mathbf{3 2 8}$ 

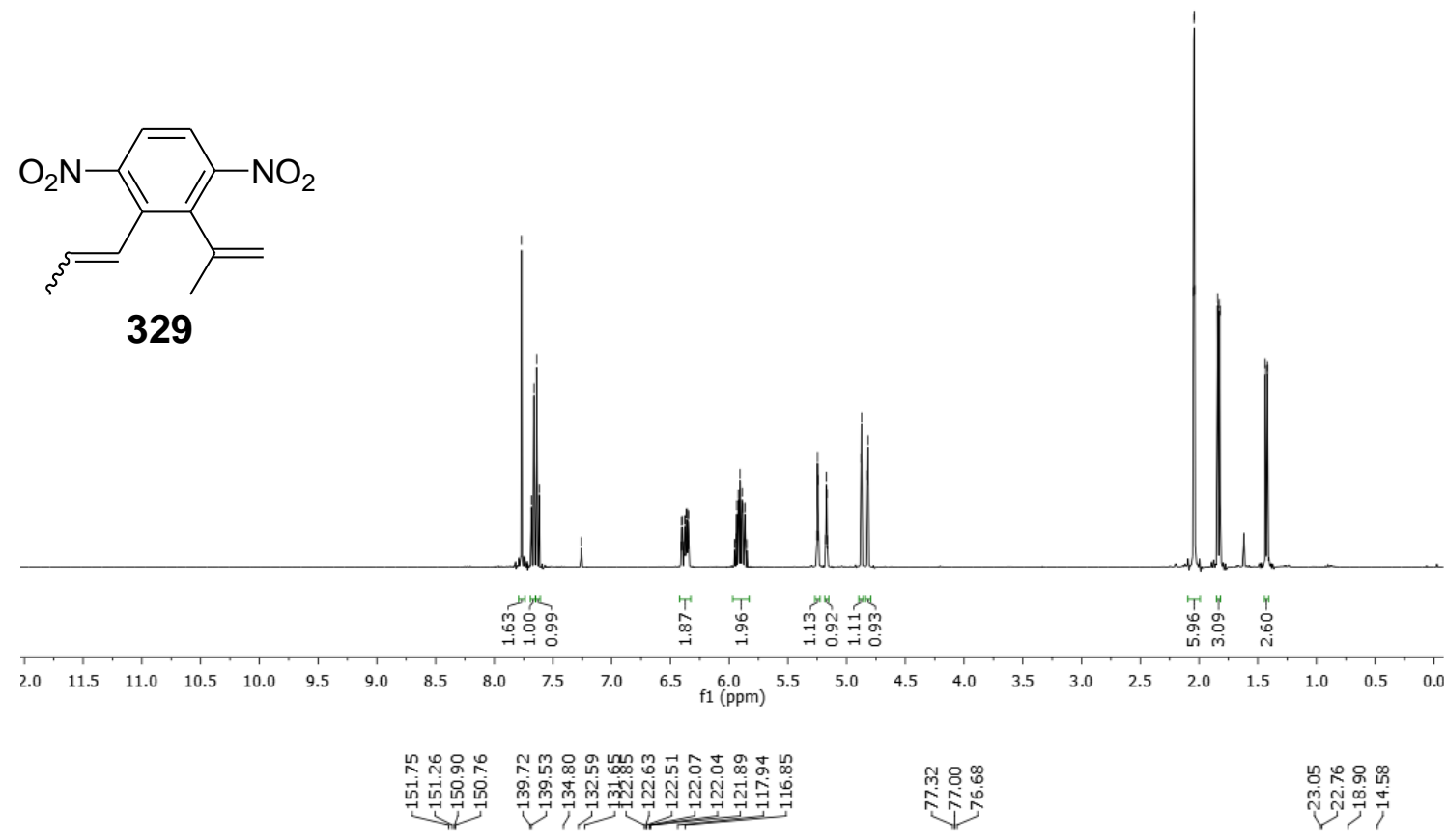

눙요욨

$\min _{\substack{i \\ N}}^{\infty}$

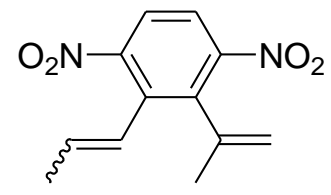

329

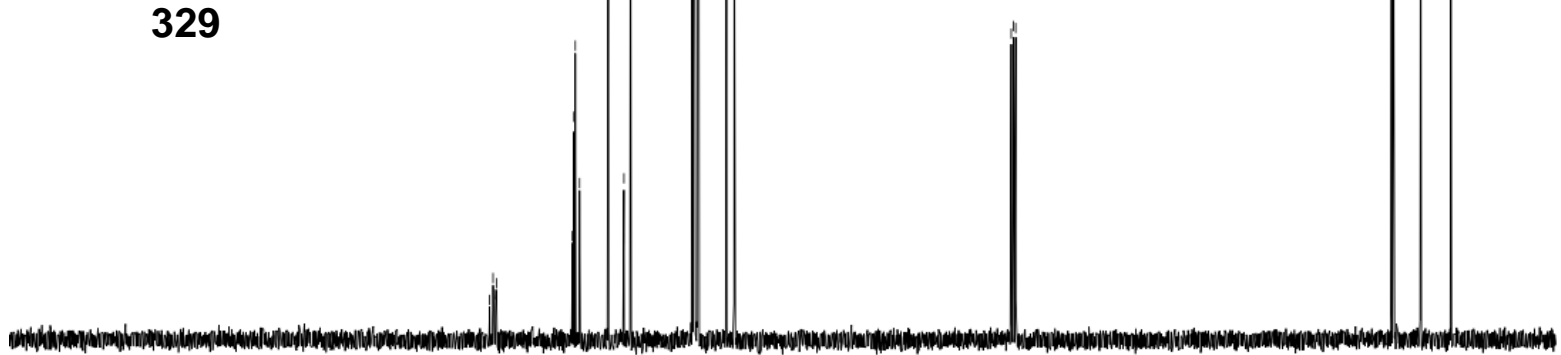

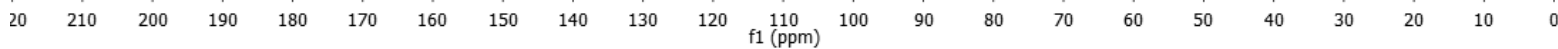

Figure 7.78 ${ }^{1} \mathrm{H}$ and ${ }^{13} \mathrm{C}$ NMR of compound 329 

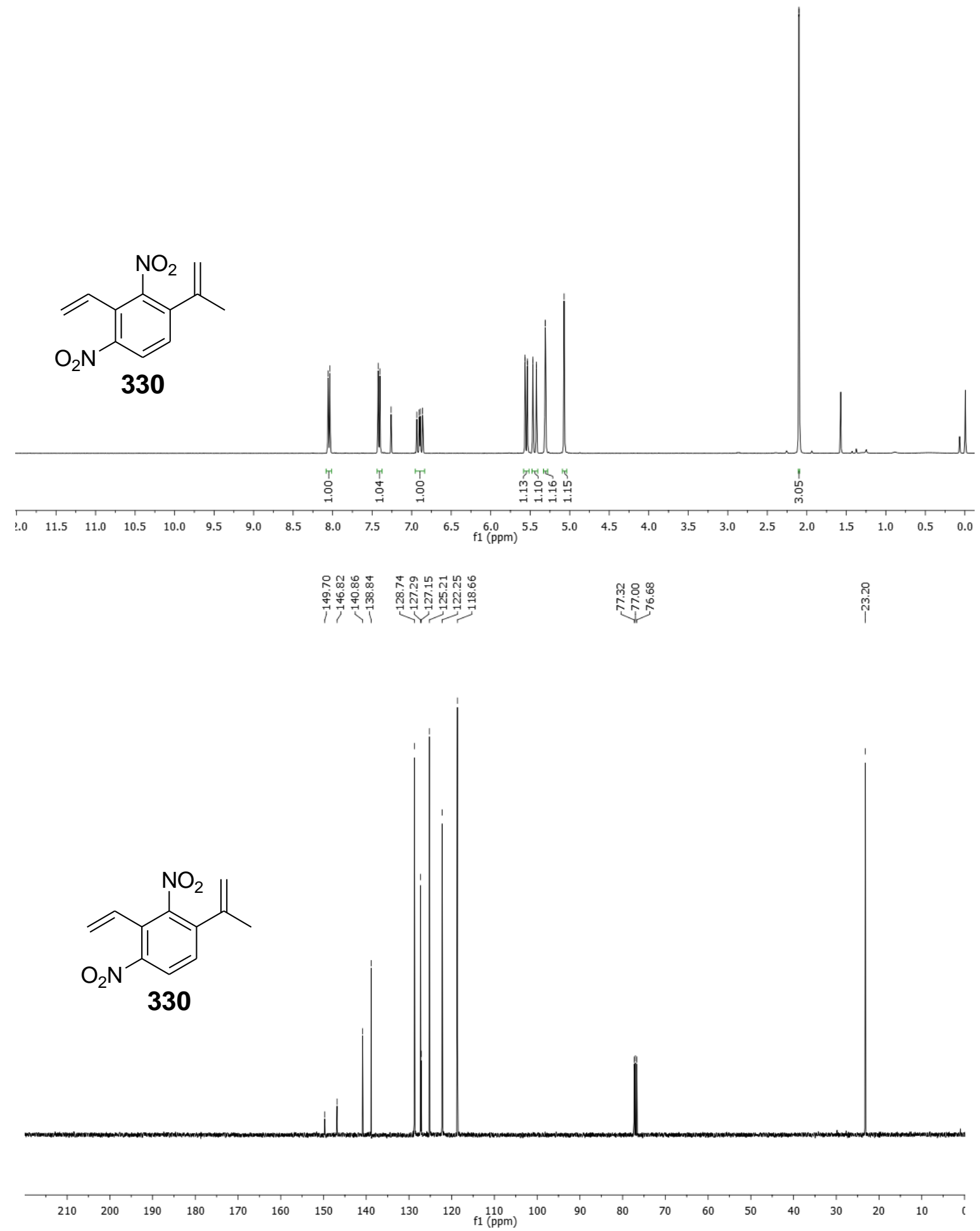

Figure 7.79 ${ }^{1} \mathrm{H}$ and ${ }^{13} \mathrm{C}$ NMR of compound 330 

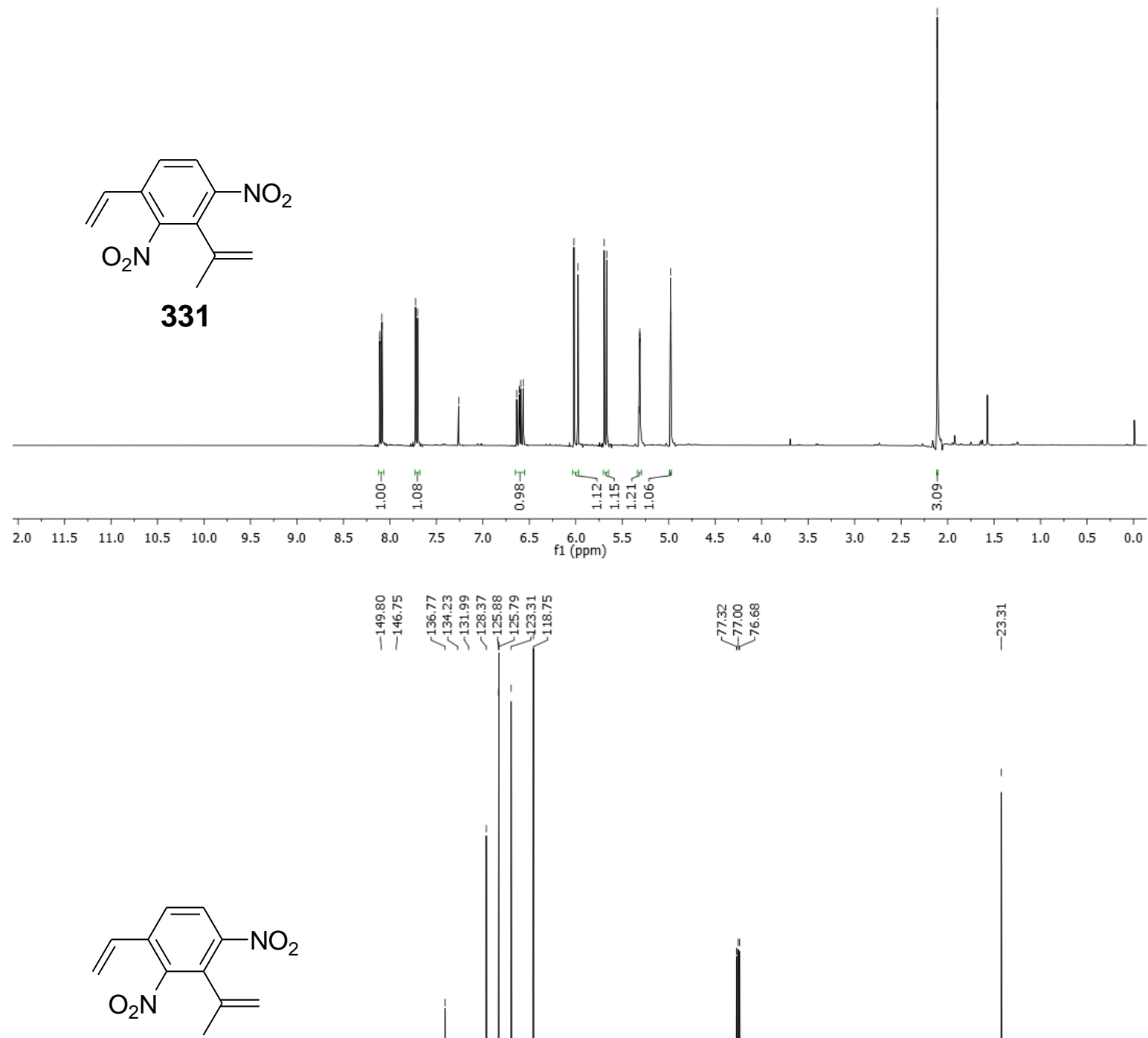

331
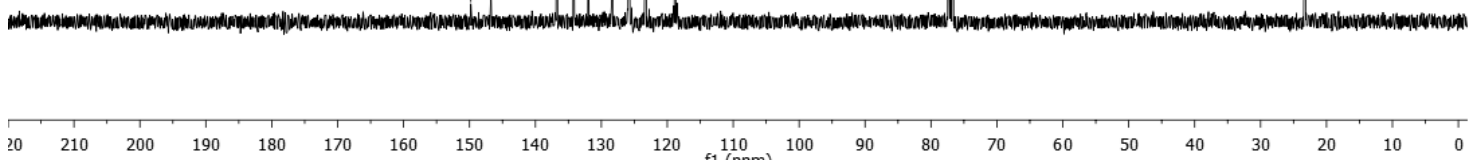

Figure 7.80 ${ }^{1} \mathrm{H}$ and ${ }^{13} \mathrm{C}$ NMR of compound 331 


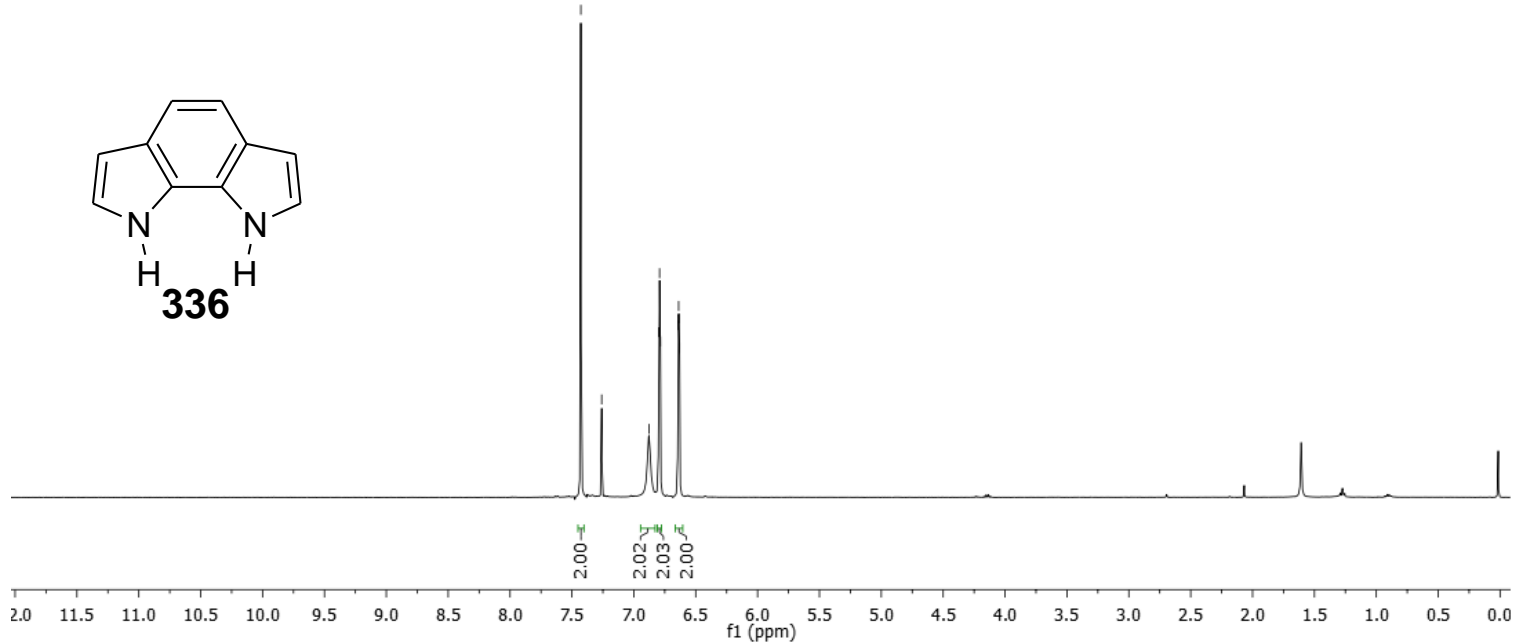

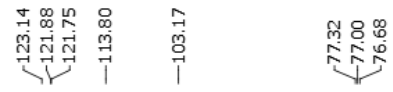
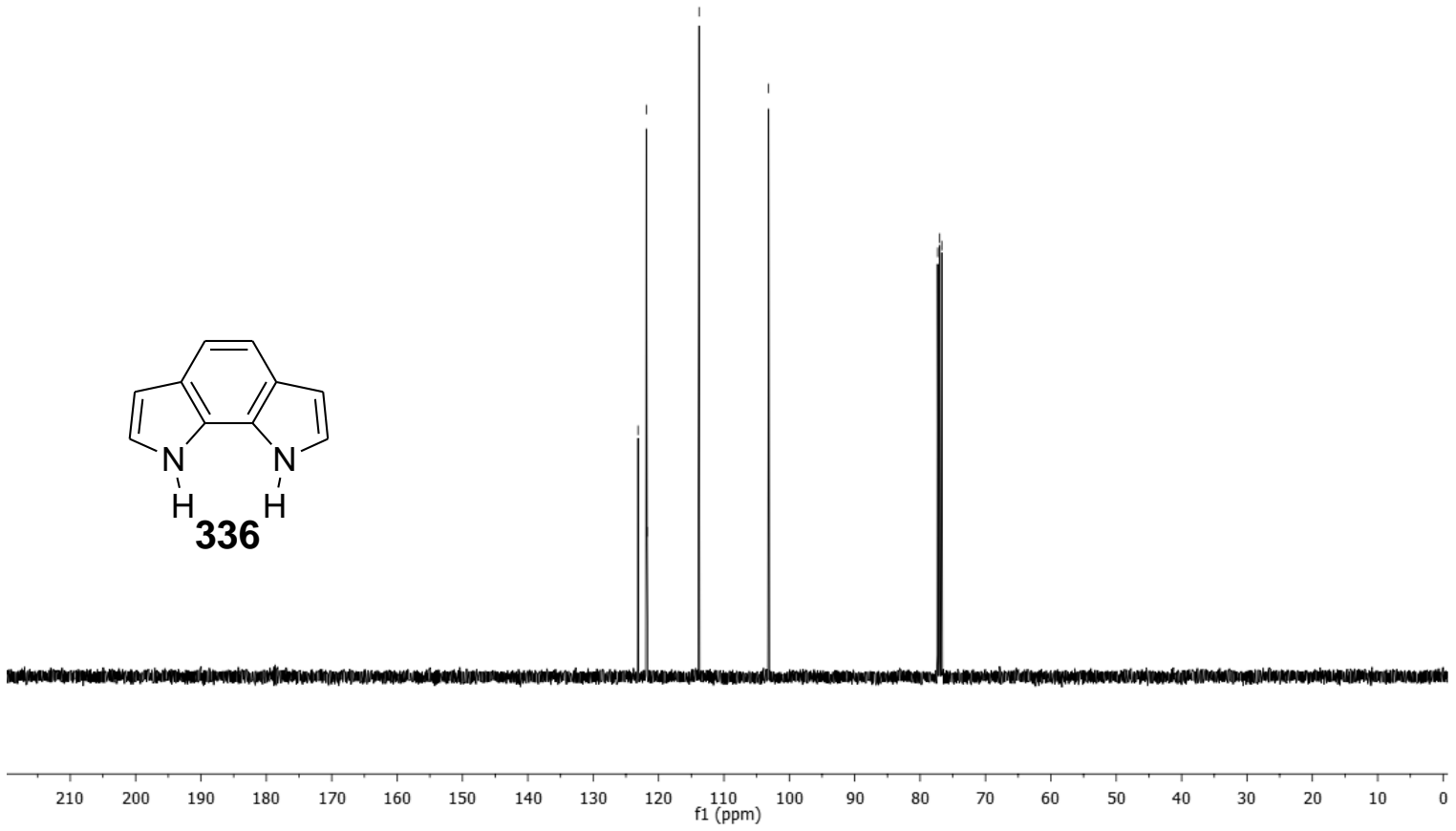

Figure 7.81 ${ }^{1} \mathrm{H}$ and ${ }^{13} \mathrm{C}$ NMR of compound 336 

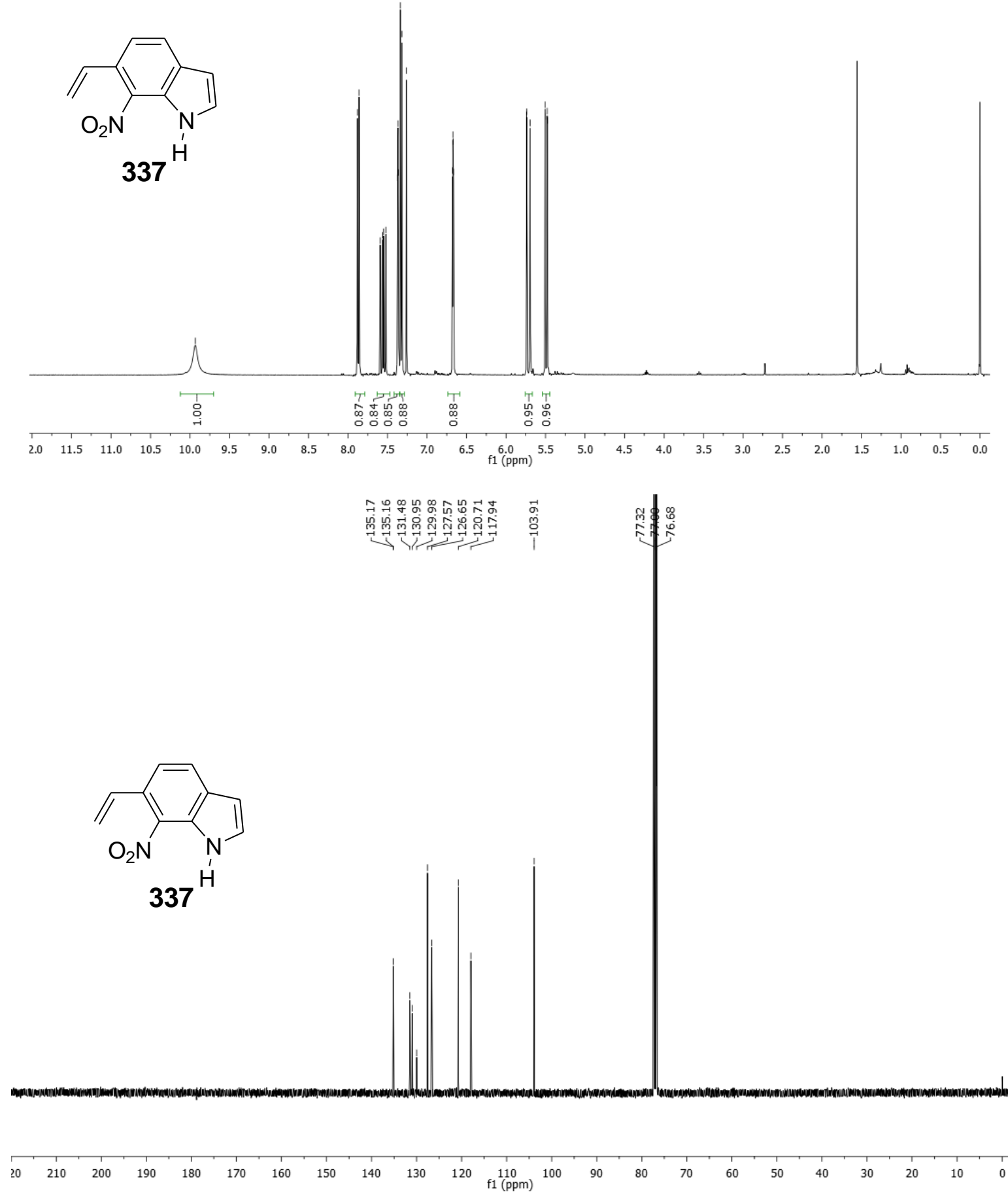

Figure 7.82 ${ }^{1} \mathrm{H}$ and ${ }^{13} \mathrm{C}$ NMR of compound 337 

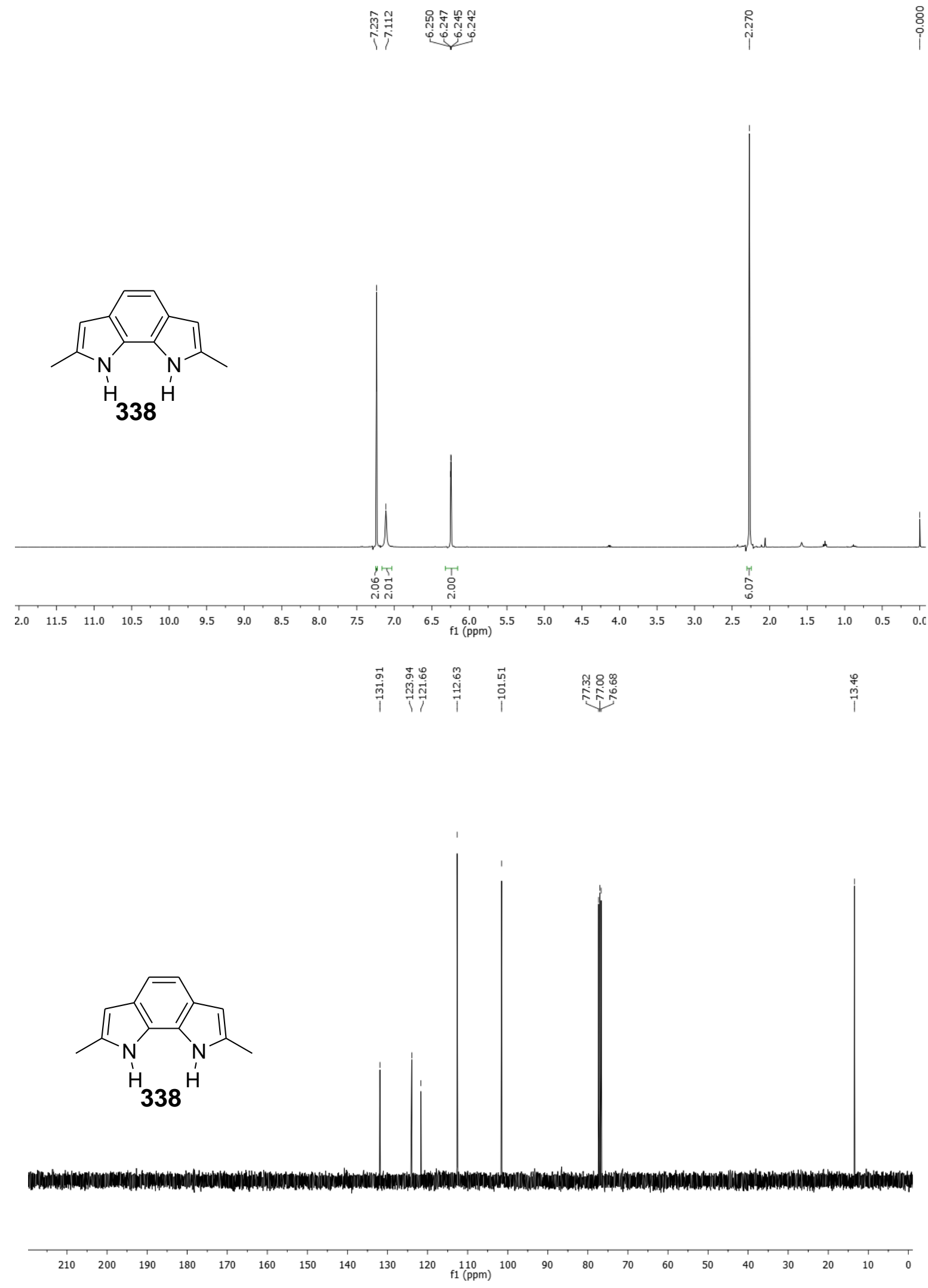

Figure 7.83 ${ }^{1} \mathrm{H}$ and ${ }^{13} \mathrm{C}$ NMR of compound 338 

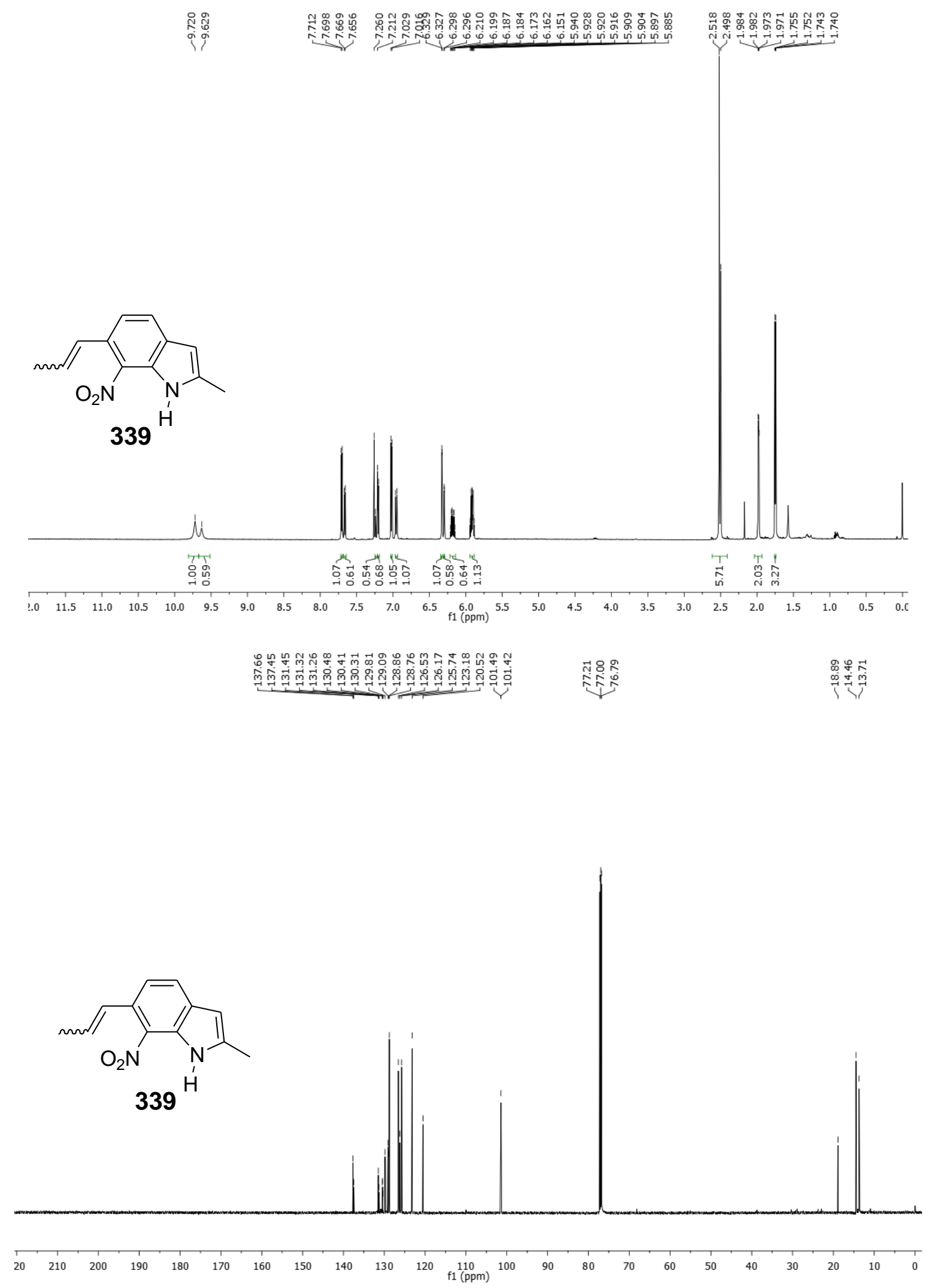

Figure 7.84 ${ }^{1} \mathrm{H}$ and ${ }^{13} \mathrm{C}$ NMR of compound 339 


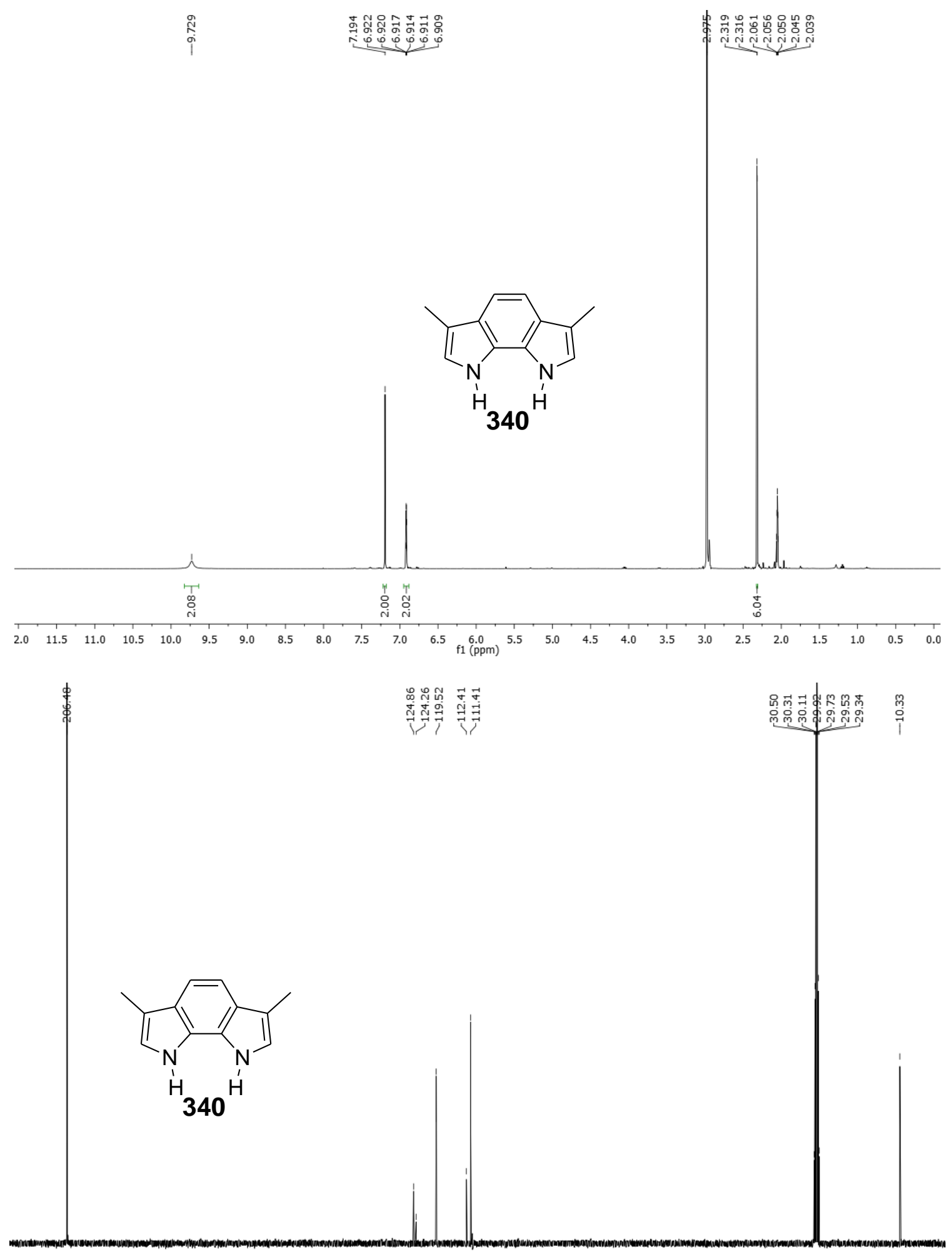

\begin{tabular}{llllllllllllllllllllllll}
\hline & 210 & 200 & 190 & 180 & 170 & 160 & 150 & 140 & 130 & 120 & 110 & 100 & 90 & 80 & 70 & 60 & 50 & 40 & 30 & 20 & 10 & 0
\end{tabular}

Figure 7.85 ${ }^{1} \mathrm{H}$ and ${ }^{13} \mathrm{C}$ NMR of compound 340 

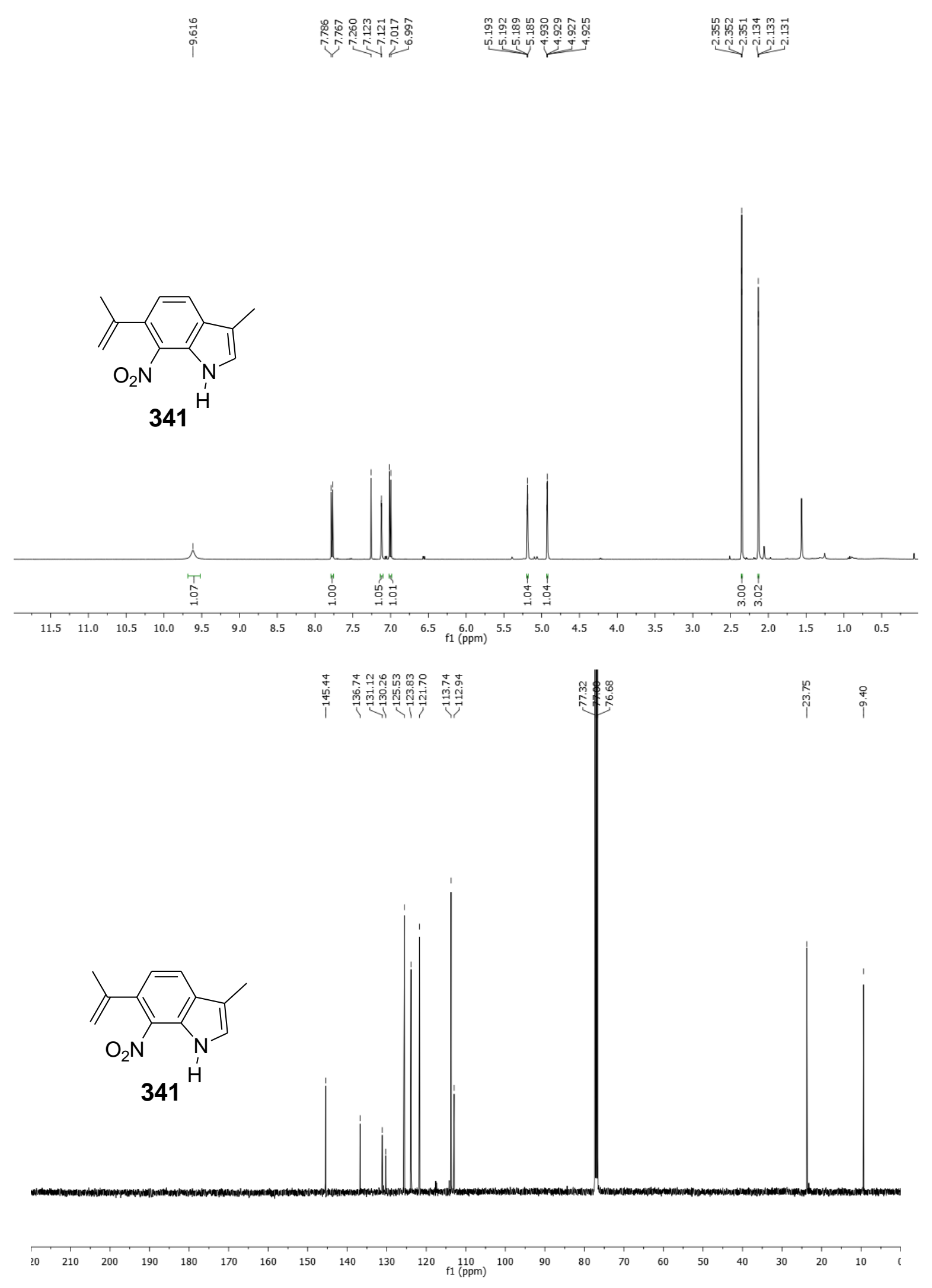

Figure 7.86 ${ }^{1} \mathrm{H}$ and ${ }^{13} \mathrm{C}$ NMR of compound 341 

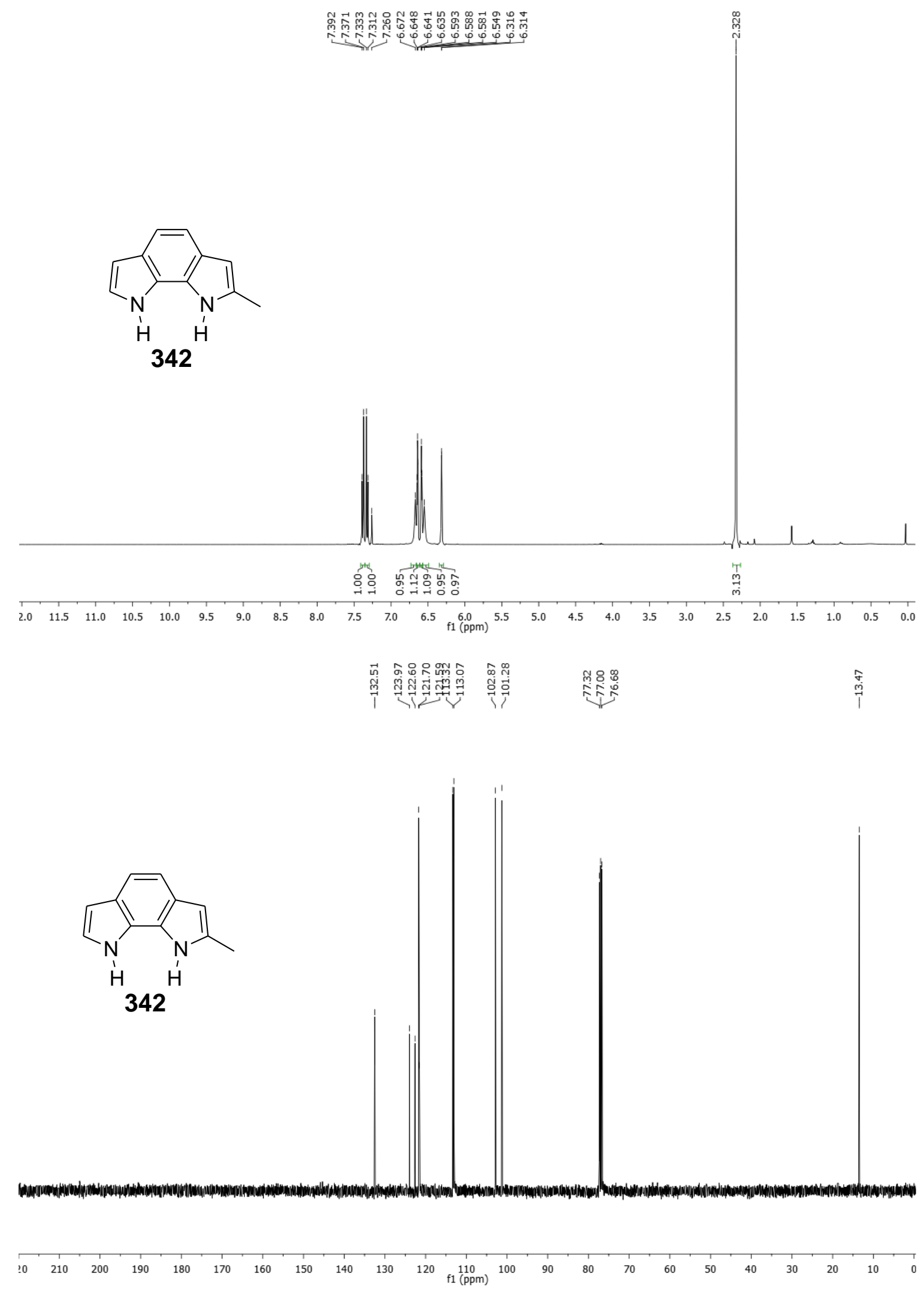

Figure 7.87 ${ }^{1} \mathrm{H}$ and ${ }^{13} \mathrm{C}$ NMR of compound 342 


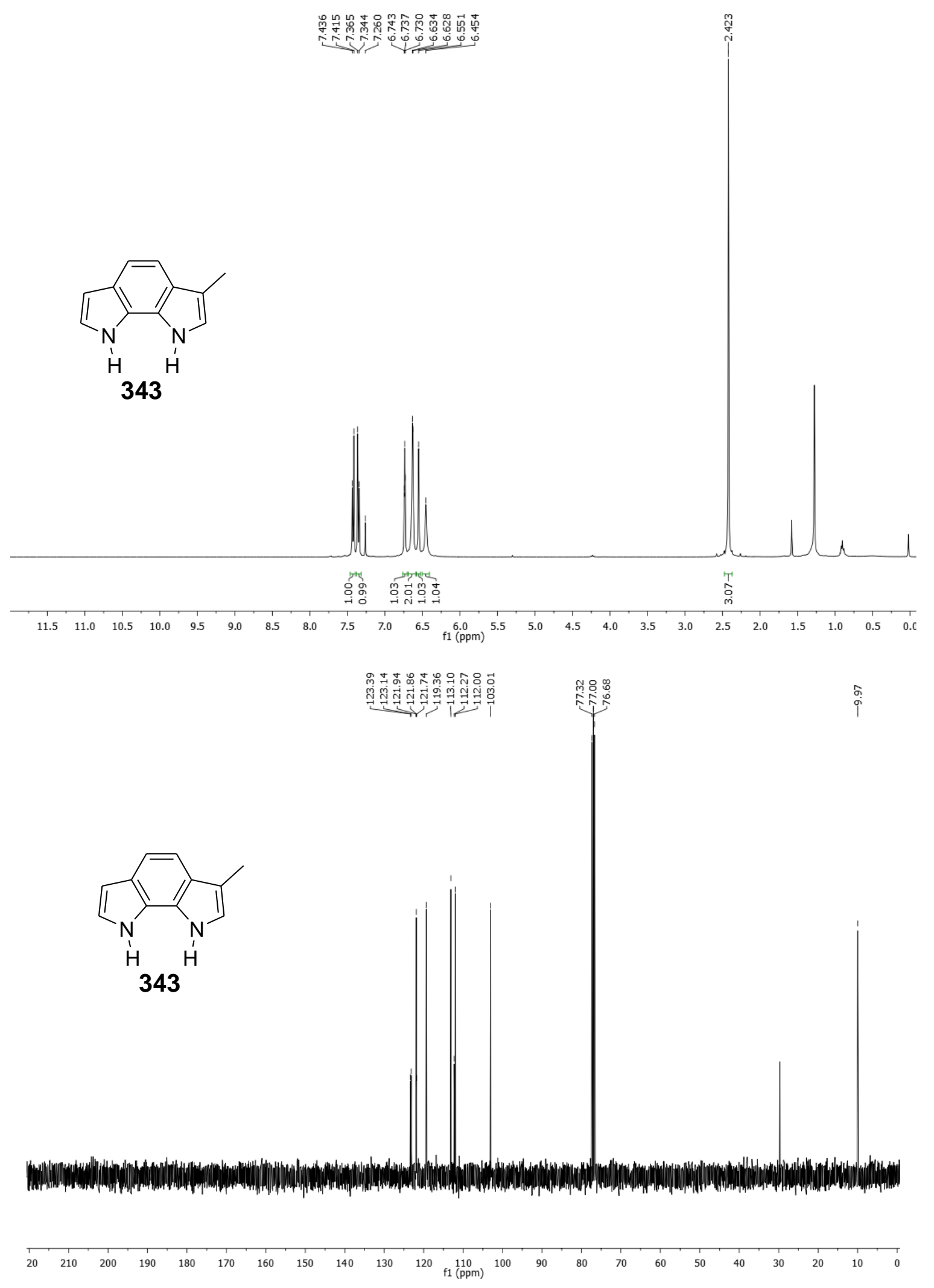

Figure 7.88 ${ }^{1} \mathrm{H}$ and ${ }^{13} \mathrm{C}$ NMR of compound 343 

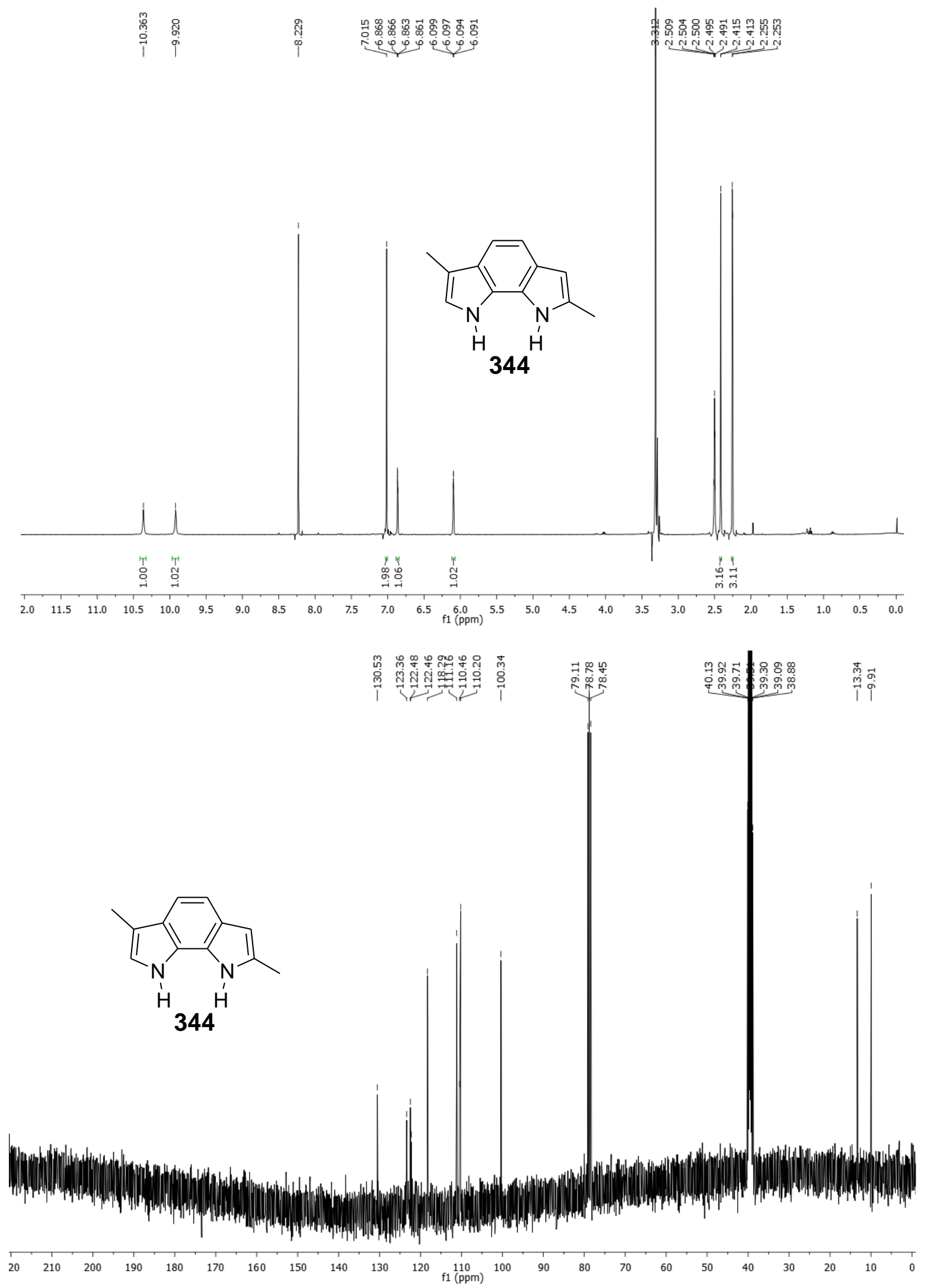

Figure 7.89 ${ }^{1} \mathrm{H}$ and ${ }^{13} \mathrm{C}$ NMR of compound 344 

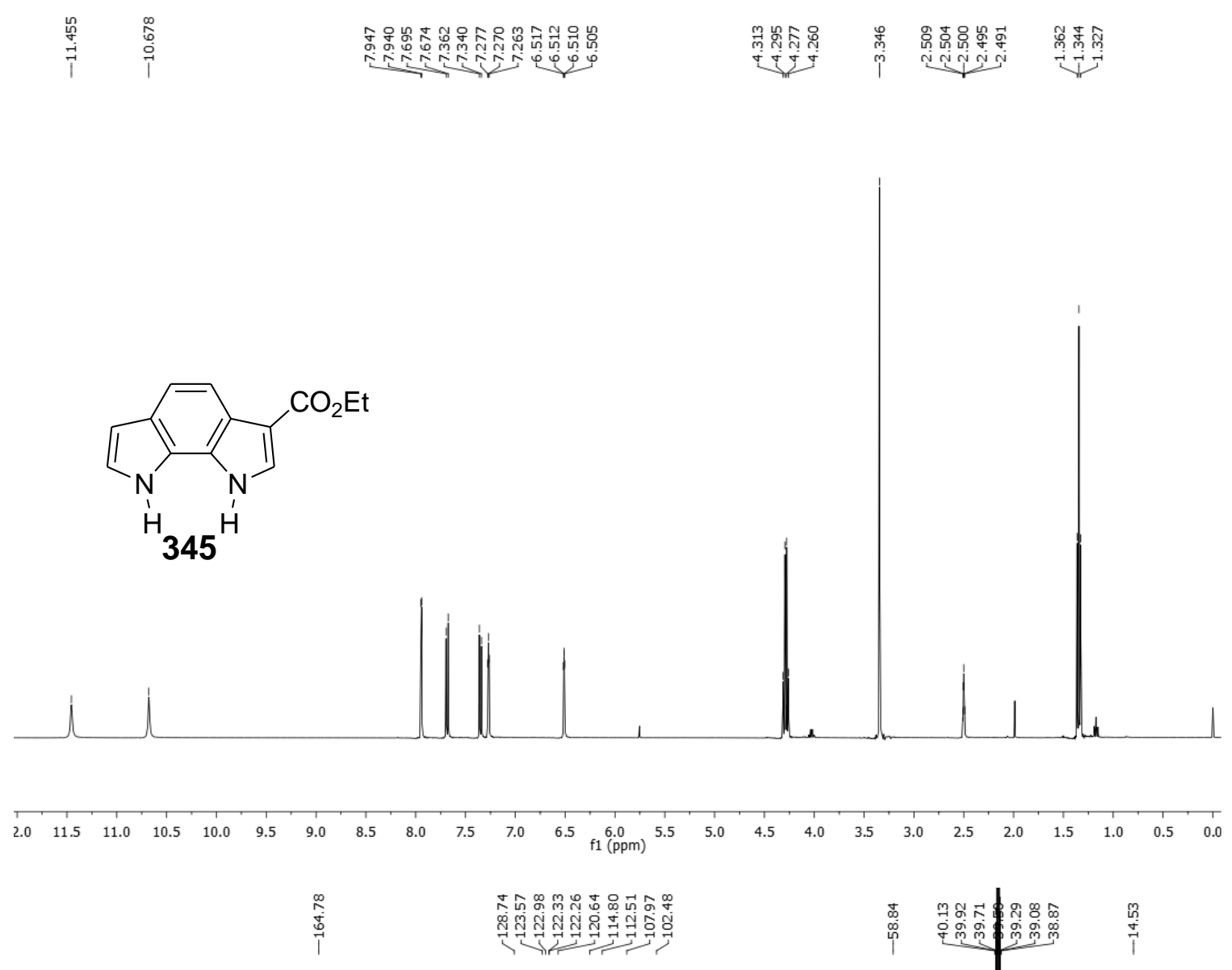

年
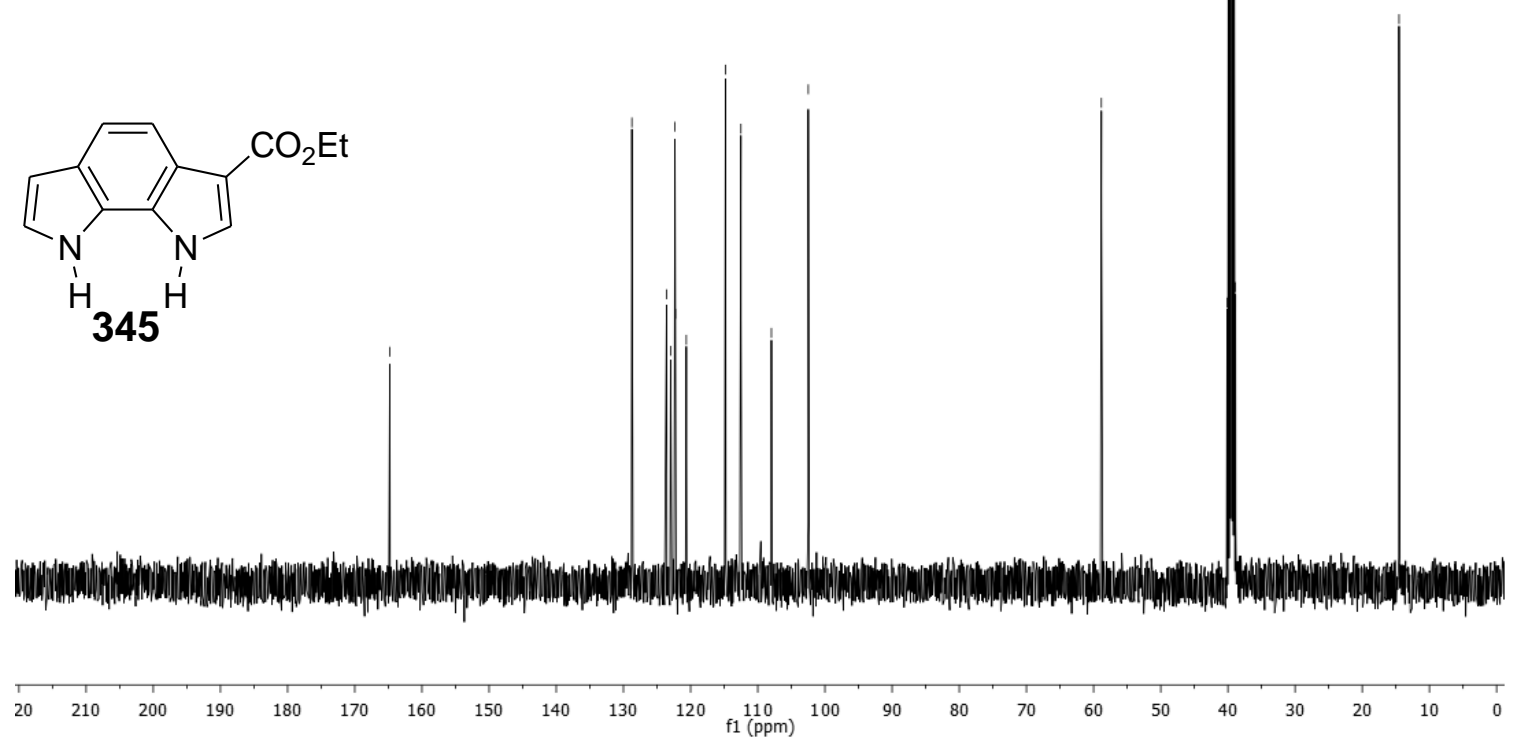

Figure 7.90 ${ }^{1} \mathrm{H}$ and ${ }^{13} \mathrm{C}$ NMR of compound 345 

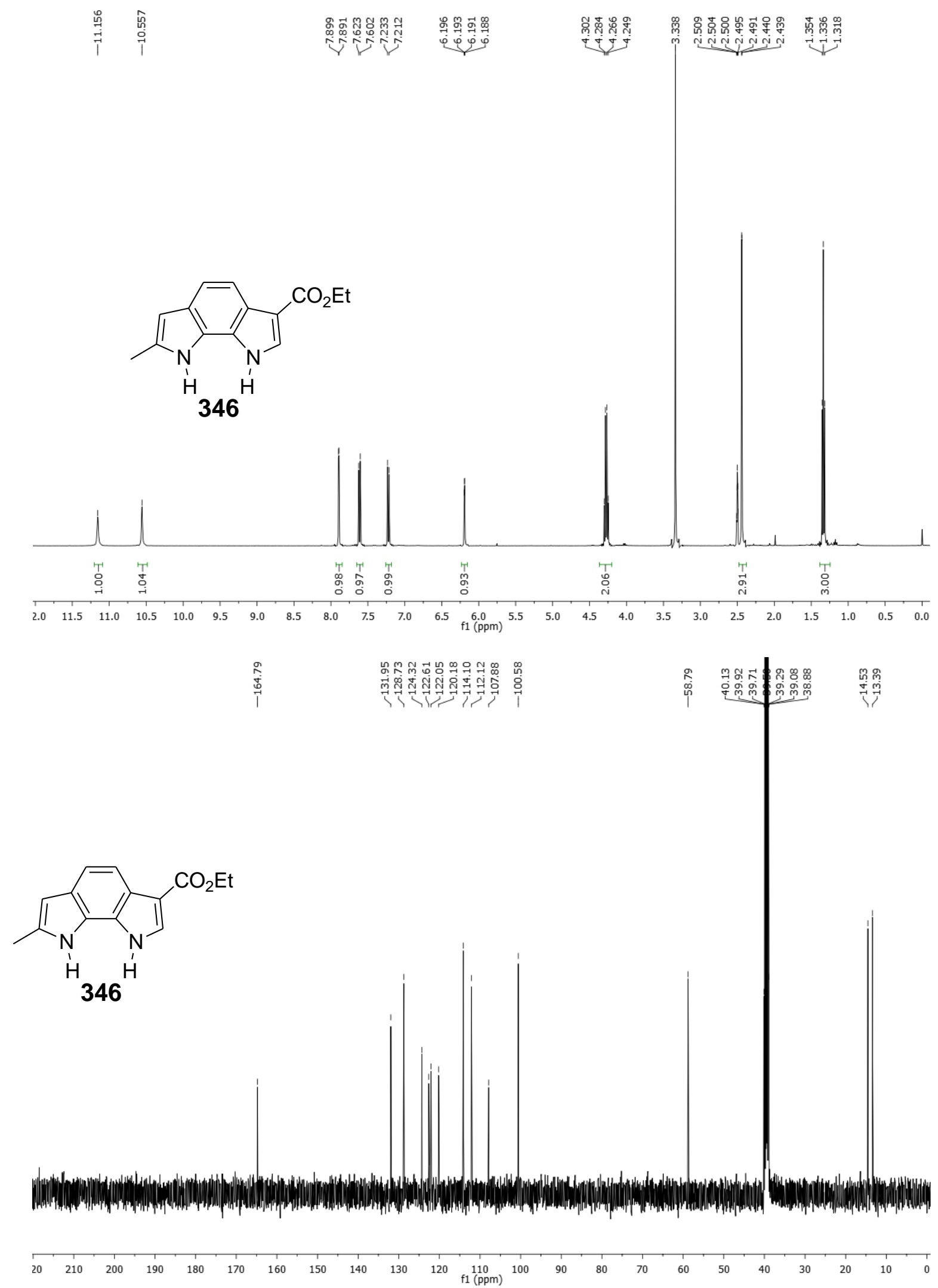

Figure 7.91 ${ }^{1} \mathrm{H}$ and ${ }^{13} \mathrm{C}$ NMR of compound 346 

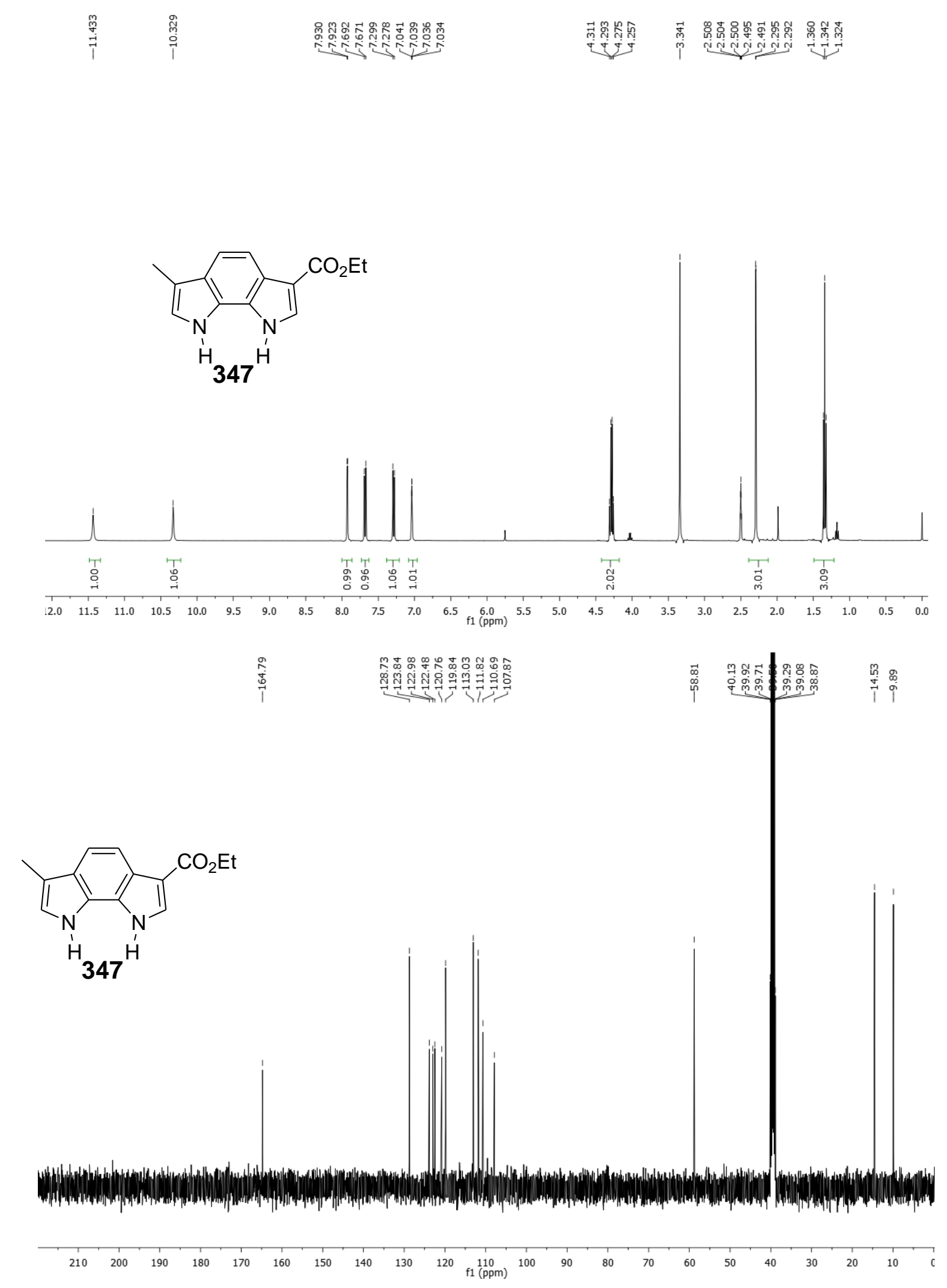

Figure 7.92 ${ }^{1} \mathrm{H}$ and ${ }^{13} \mathrm{C}$ NMR of compound 347 

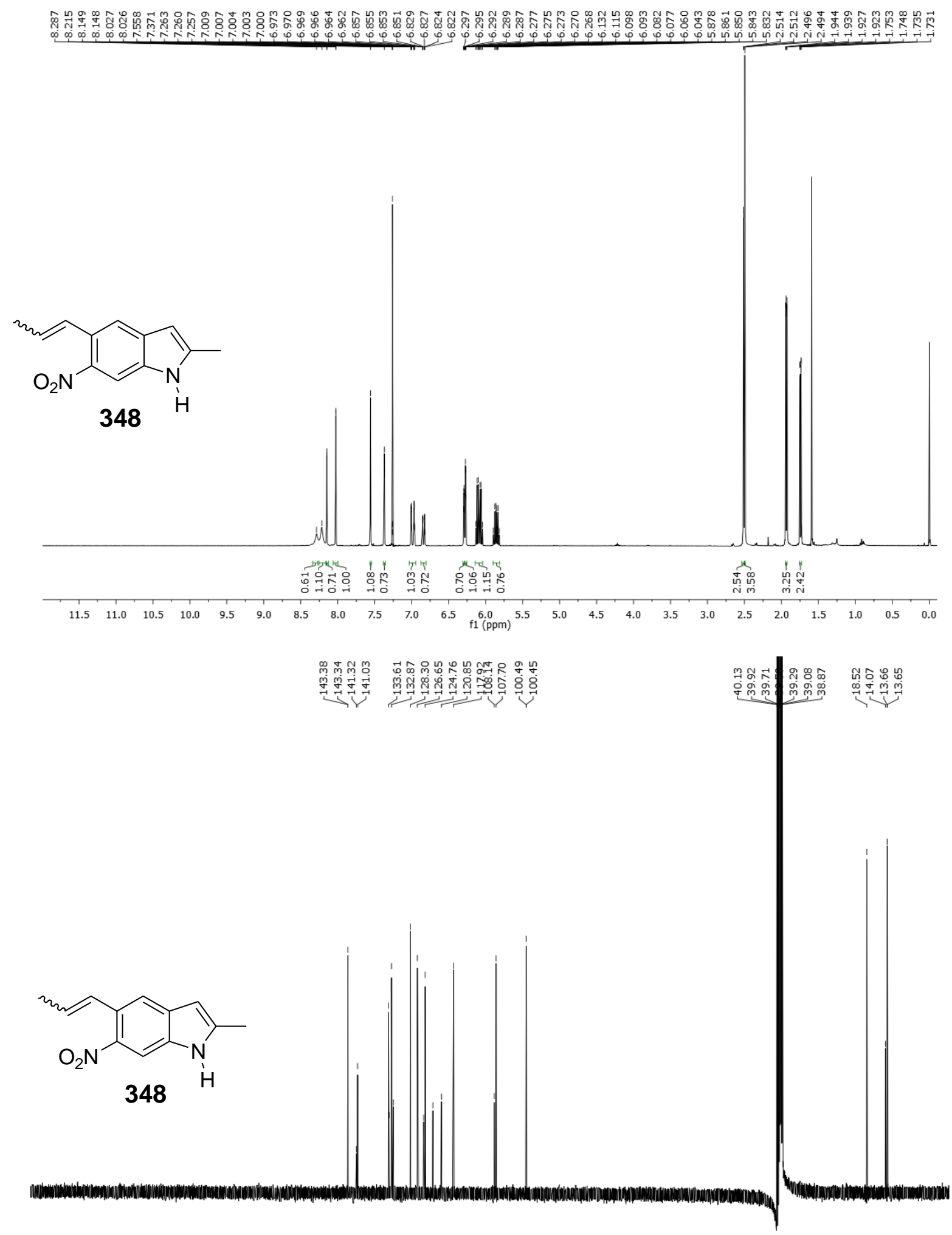

$\begin{array}{llllllllllllllllllllllll}210 & 200 & 190 & 180 & 170 & 160 & 150 & 140 & 130 & 120 & \begin{array}{c}110 \\ \mathrm{f} 1(\mathrm{ppm})\end{array} & 100 & 90 & 80 & 70 & 60 & 50 & 40 & 30 & 20 & 10 & 0\end{array}$

Figure 7.93 ${ }^{1} \mathrm{H}$ and ${ }^{13} \mathrm{C}$ NMR of compound 348 

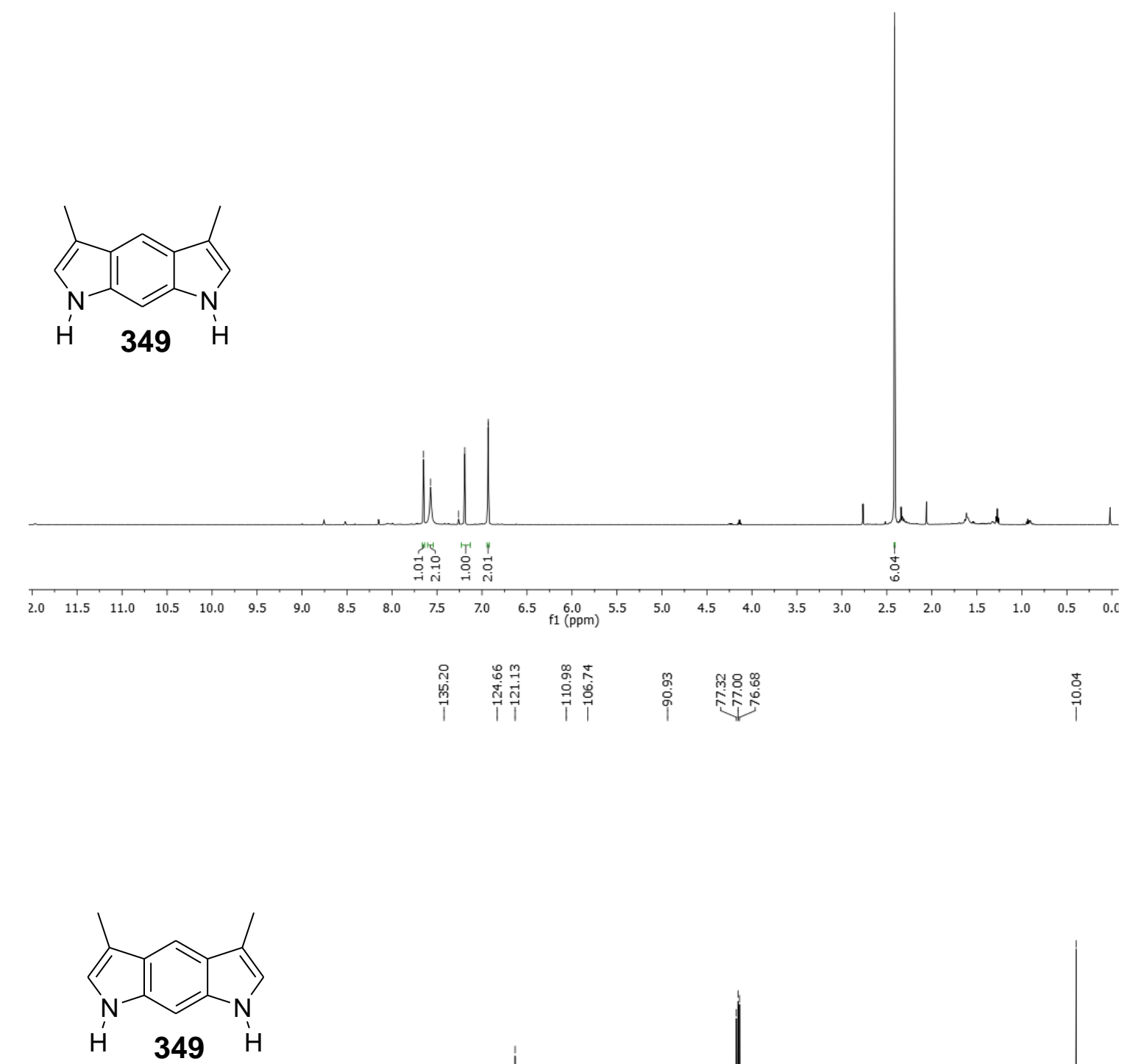

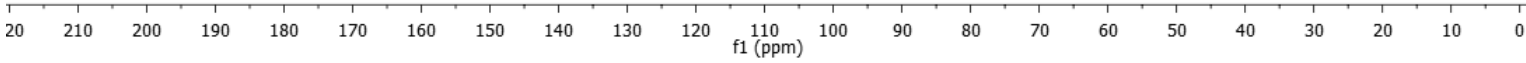

Figure 7.94 ${ }^{1} \mathrm{H}$ and ${ }^{13} \mathrm{C}$ NMR of compound 349 

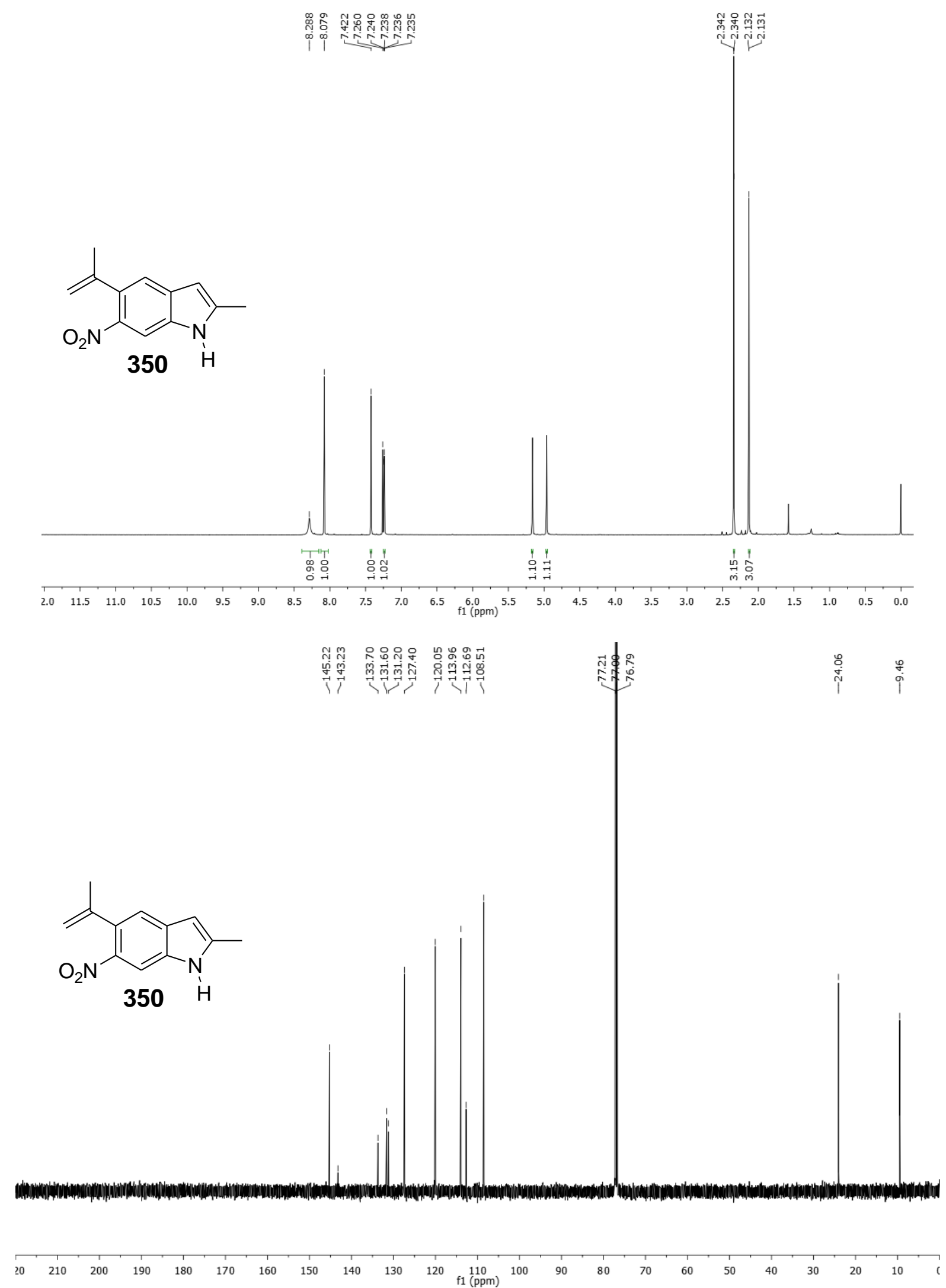

Figure 7.95 ${ }^{1} \mathrm{H}$ and ${ }^{13} \mathrm{C}$ NMR of compound 350 

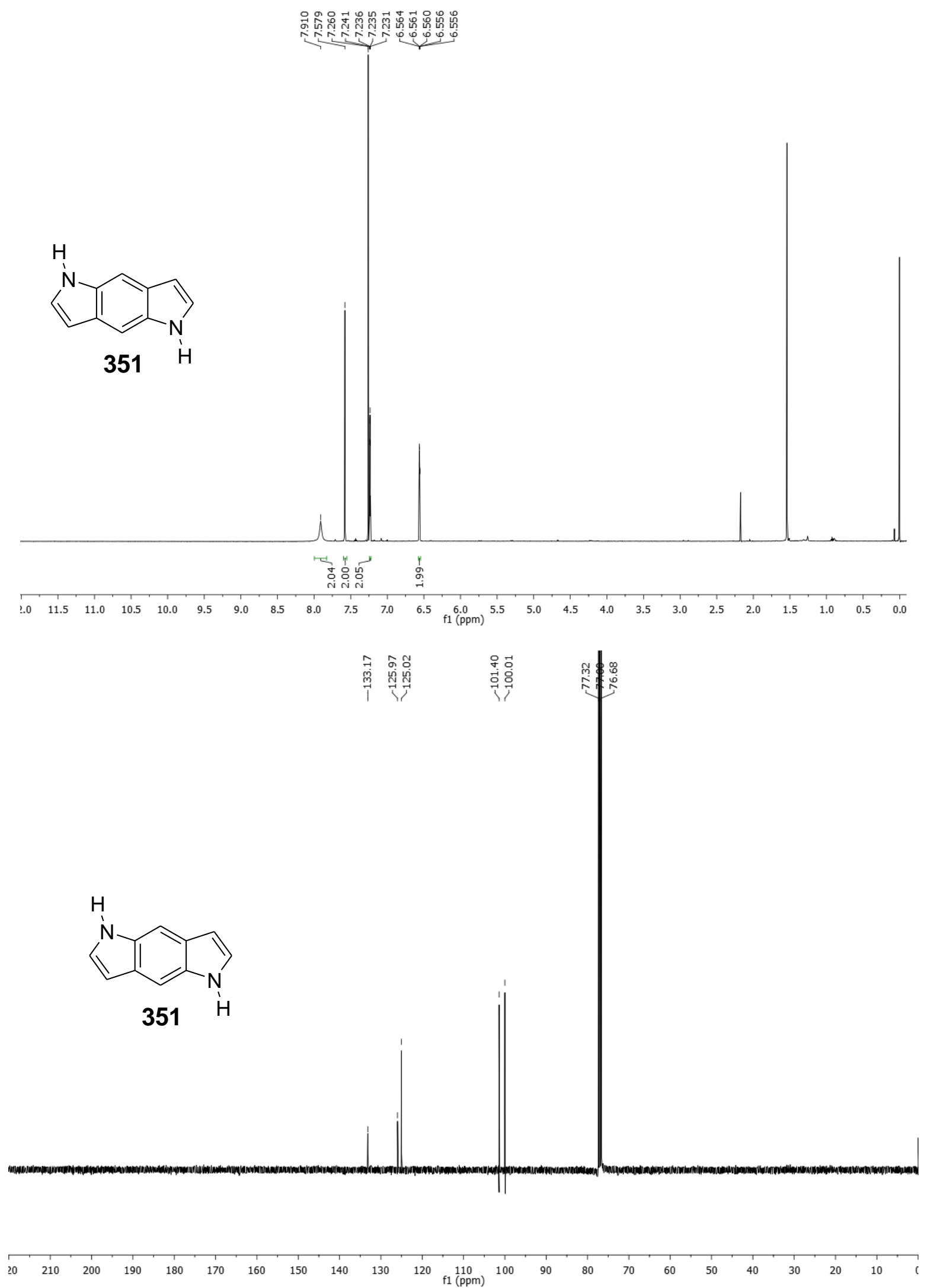

Figure 7.96 ${ }^{1} \mathrm{H}$ and ${ }^{13} \mathrm{C}$ NMR of compound 351 

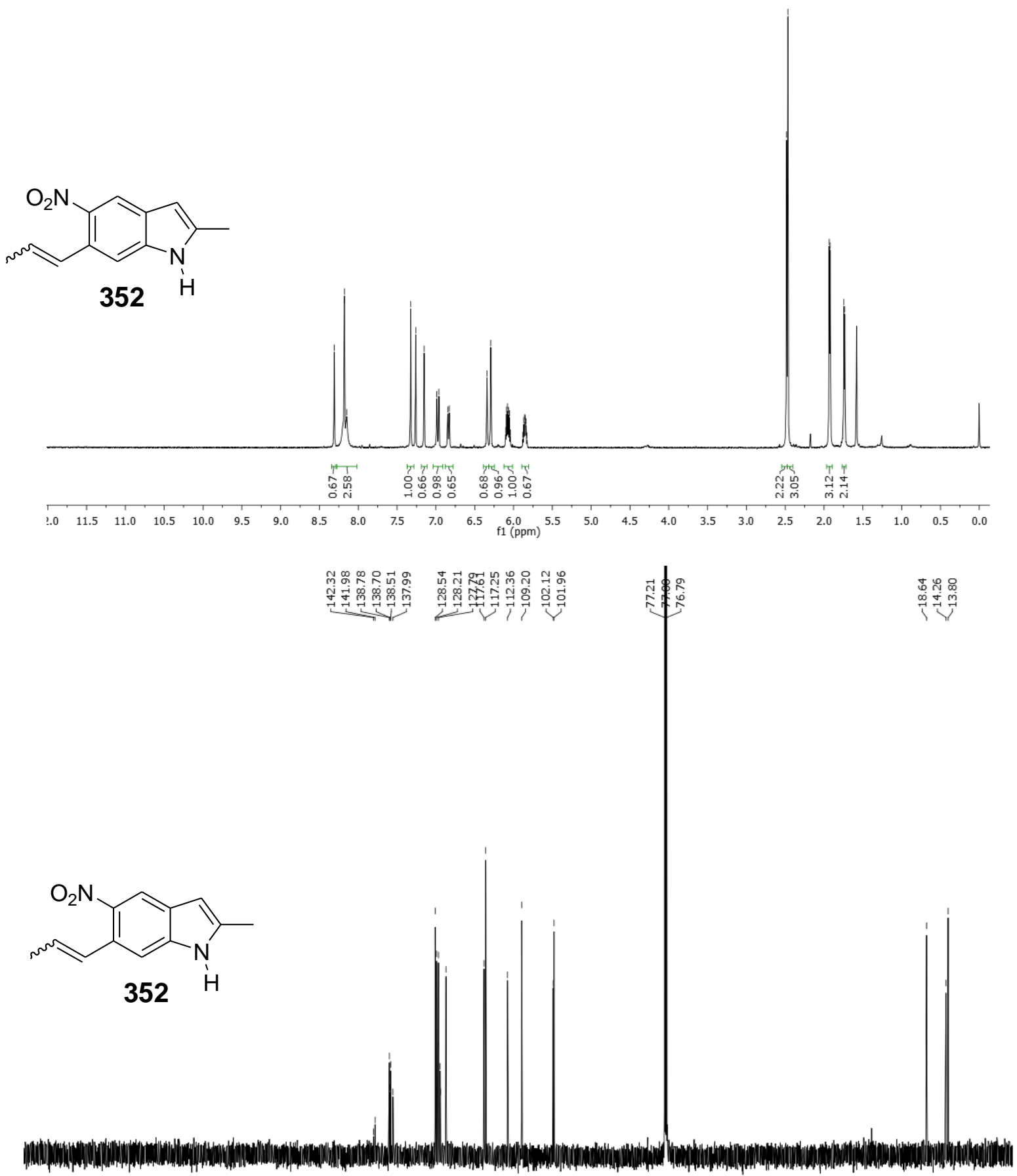

Figure 7.97 ${ }^{1} \mathrm{H}$ and ${ }^{13} \mathrm{C}$ NMR of compound $\mathbf{3 5 2}$ 

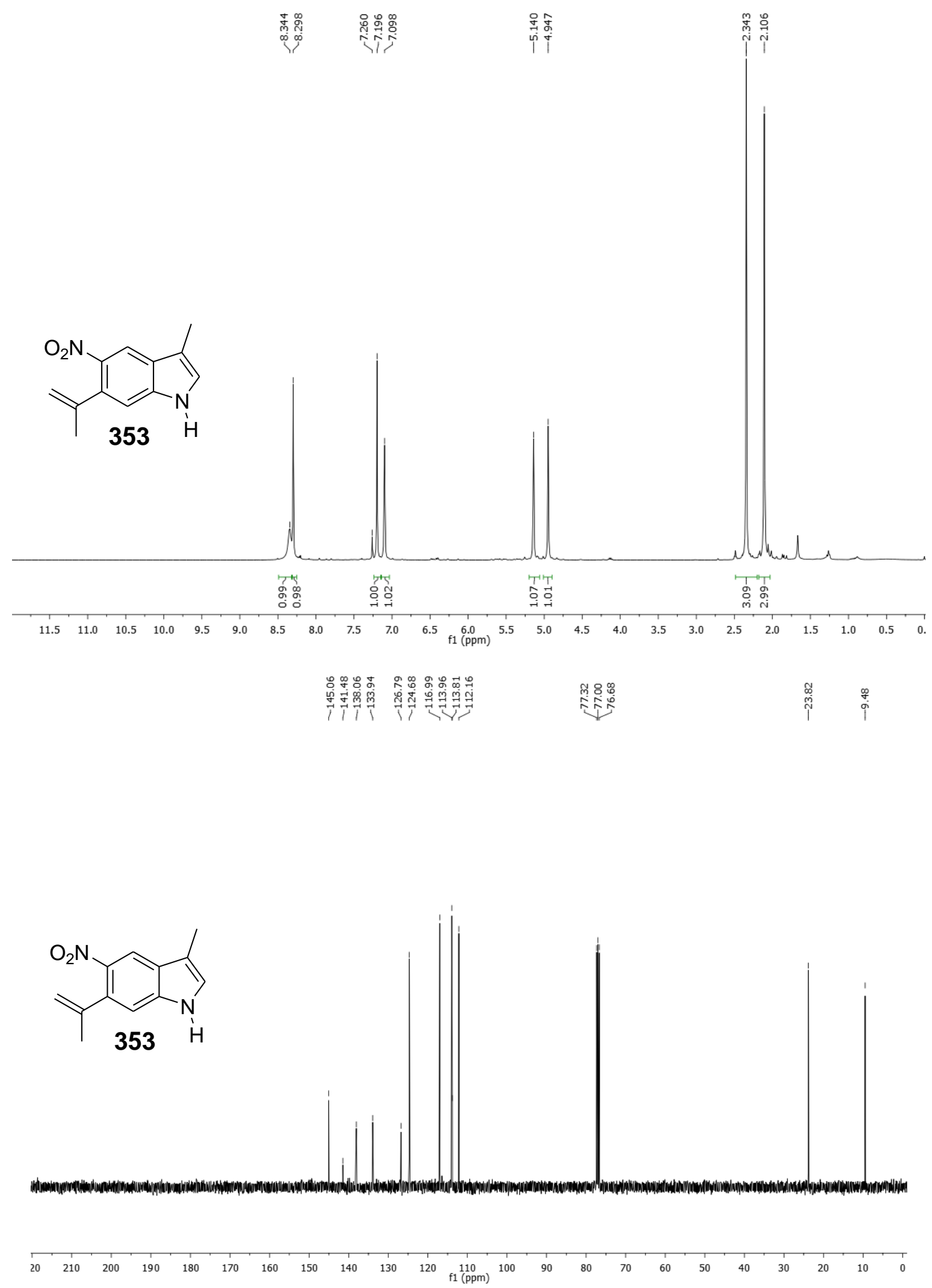

Figure 7.98 ${ }^{1} \mathrm{H}$ and ${ }^{13} \mathrm{C}$ NMR of compound 353 


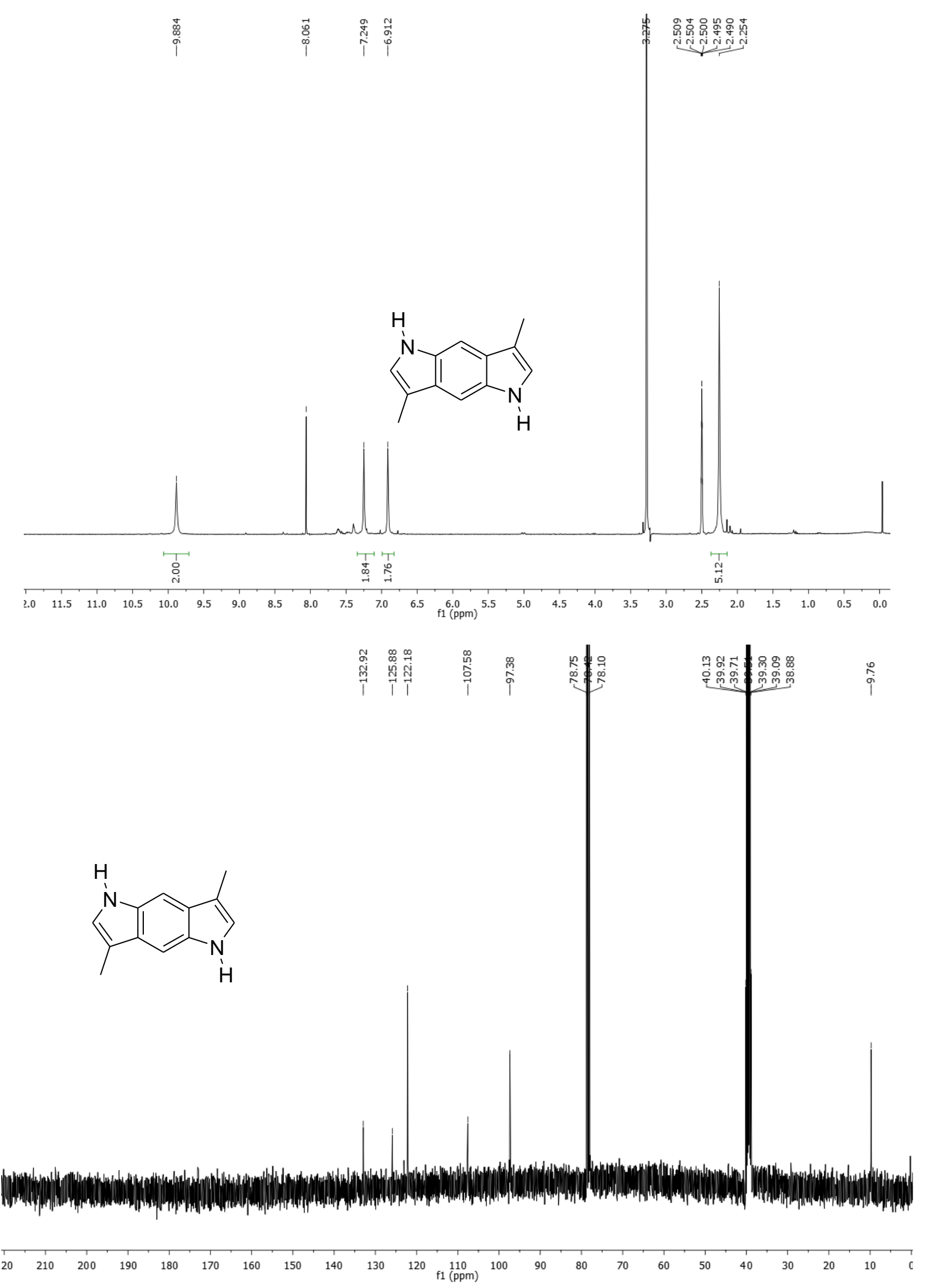

Figure 7.99 ${ }^{1} \mathrm{H}$ and ${ }^{13} \mathrm{C} \mathrm{NMR}$ 


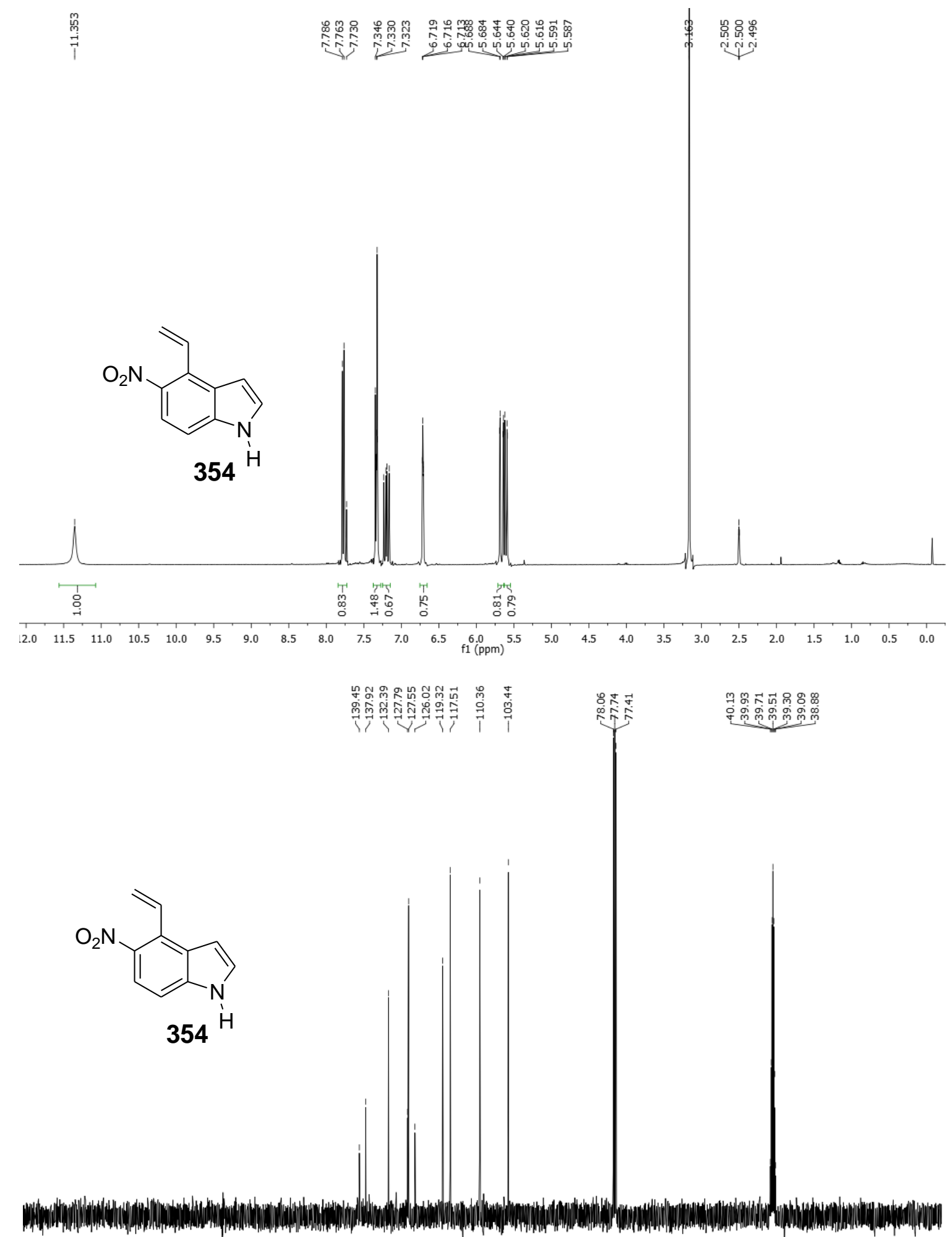

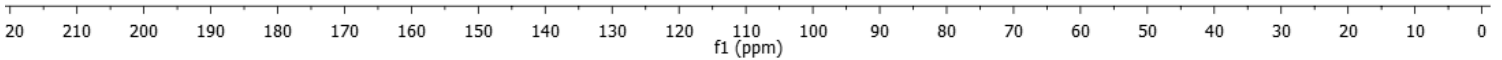

Figure 7.100 ${ }^{1} \mathrm{H}$ and ${ }^{13} \mathrm{C}$ NMR of compound 354 

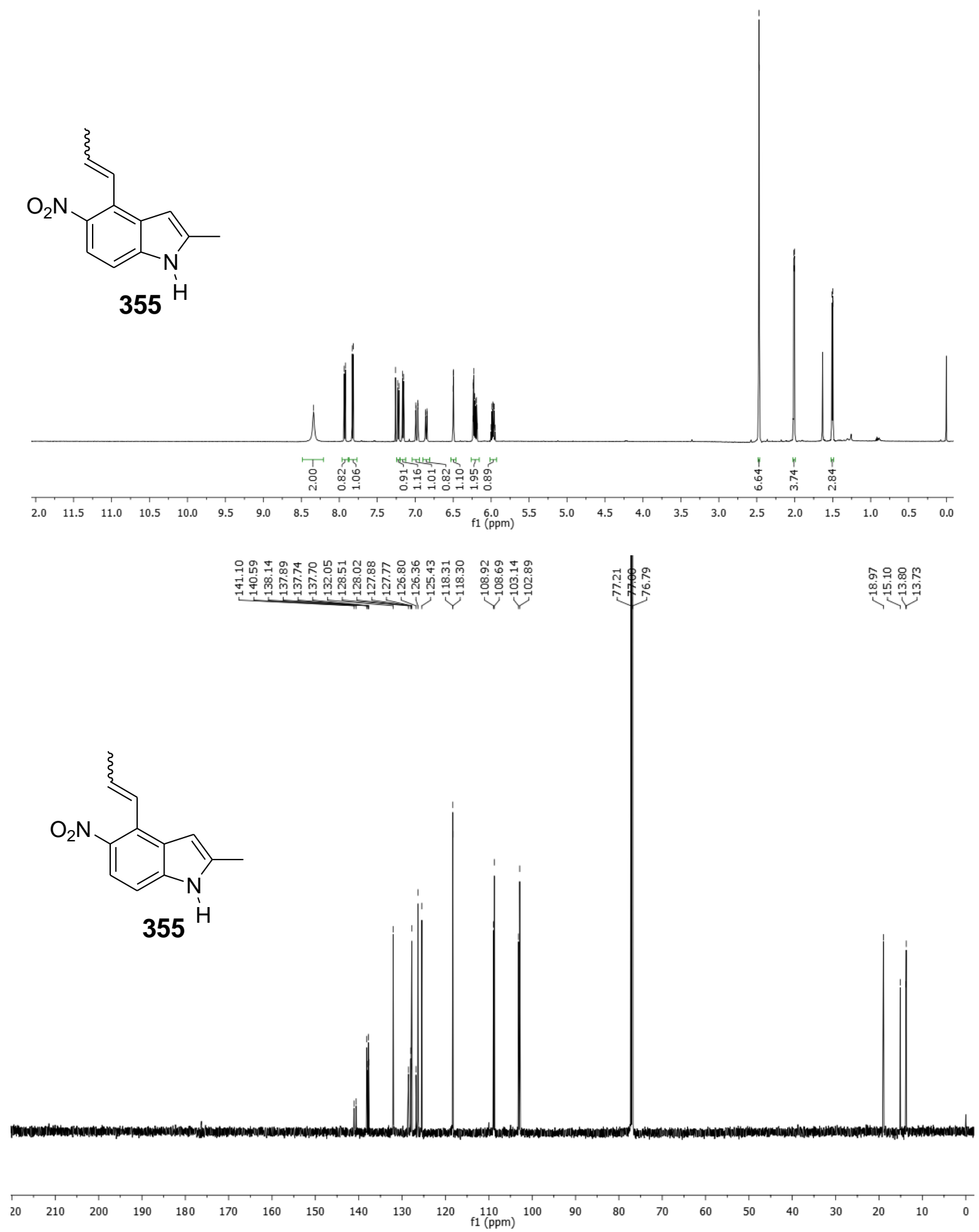

Figure 7.101 ${ }^{1} \mathrm{H}$ and ${ }^{13} \mathrm{C}$ NMR of compound 355 

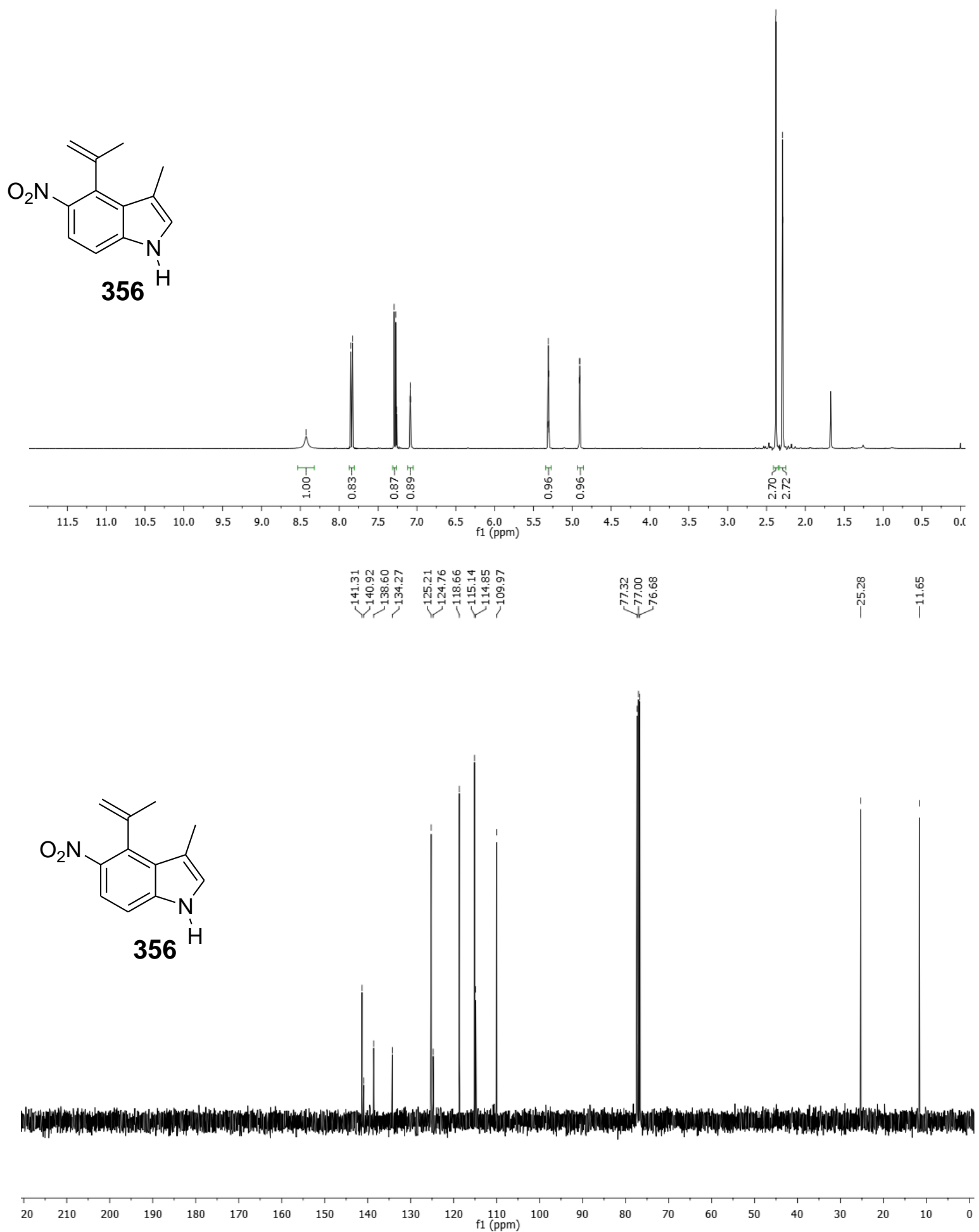

Figure 7.102 ${ }^{1} \mathrm{H}$ and ${ }^{13} \mathrm{C}$ NMR of compound 356 


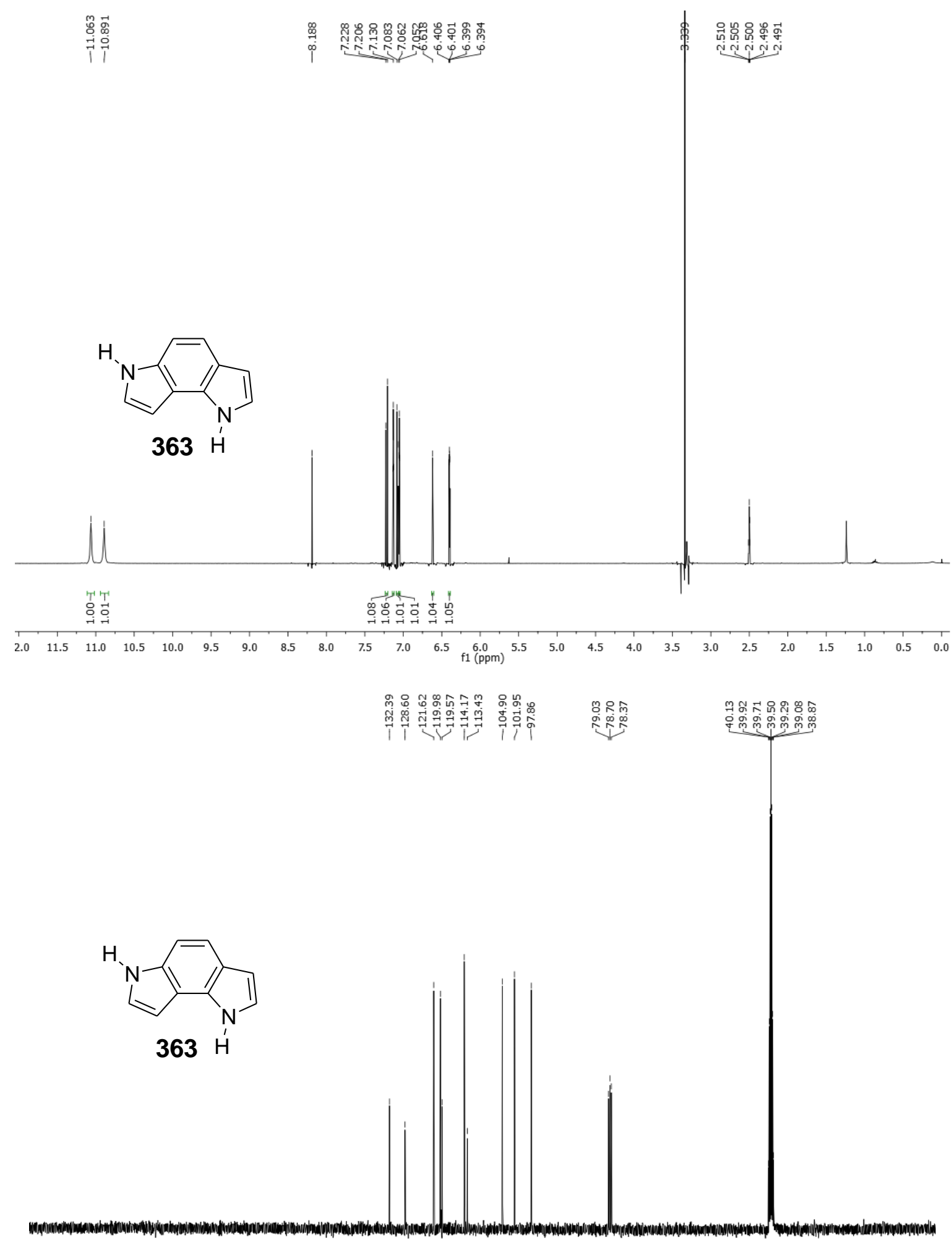

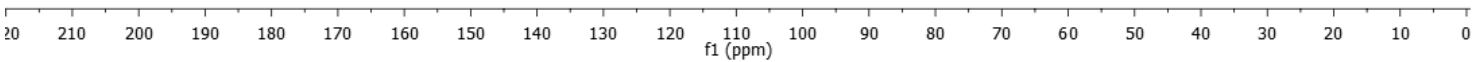

Figure $7.103{ }^{1} \mathrm{H}$ and ${ }^{13} \mathrm{C} \mathrm{NMR}$ of compound 363 

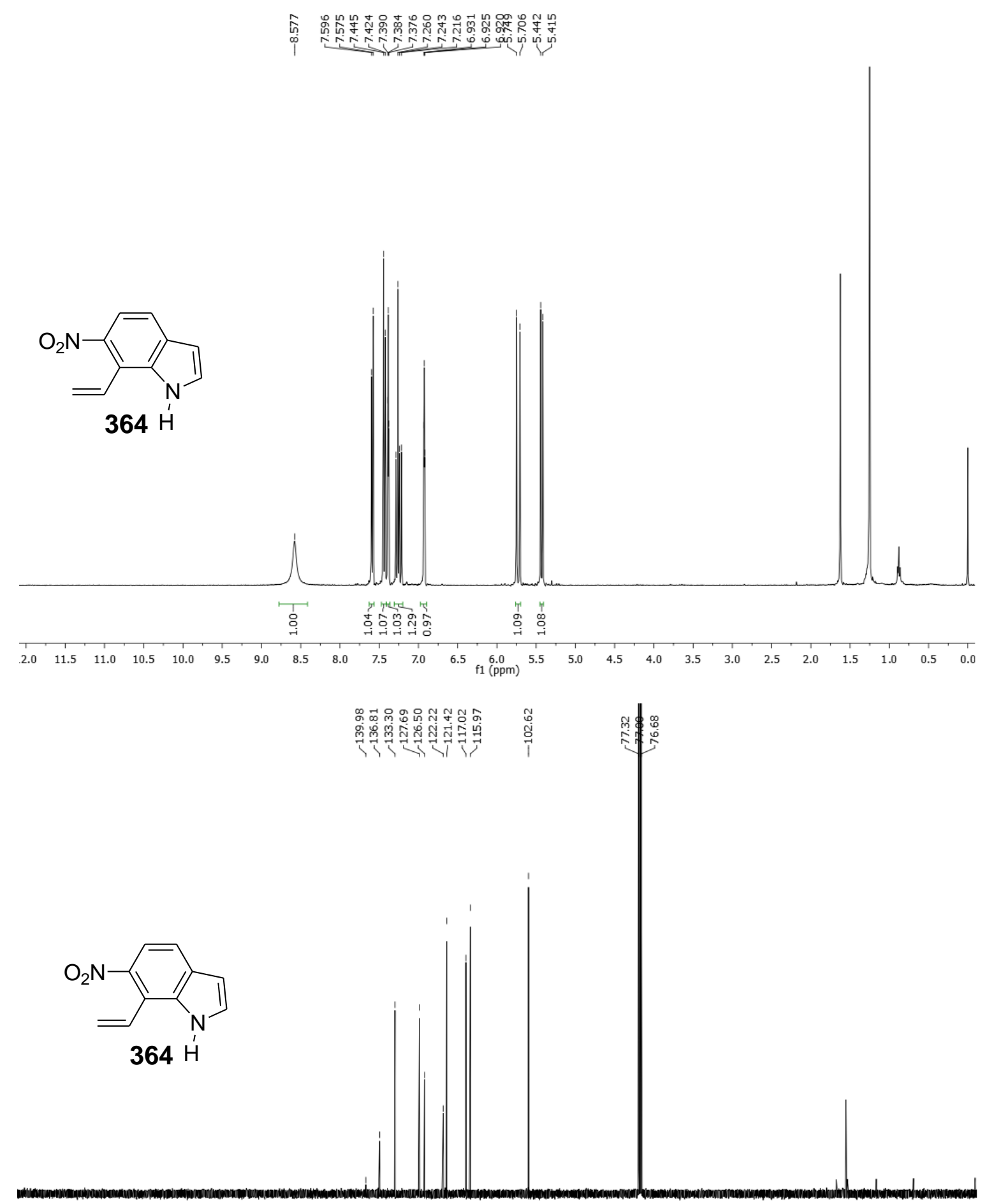

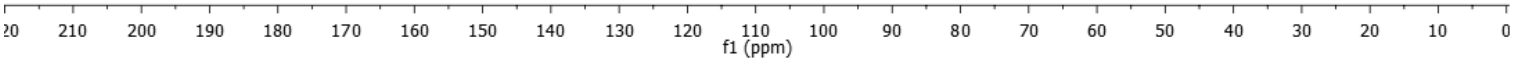

Figure 7.104 ${ }^{1} \mathrm{H}$ and ${ }^{13} \mathrm{C} \mathrm{NMR}$ of compound 364 

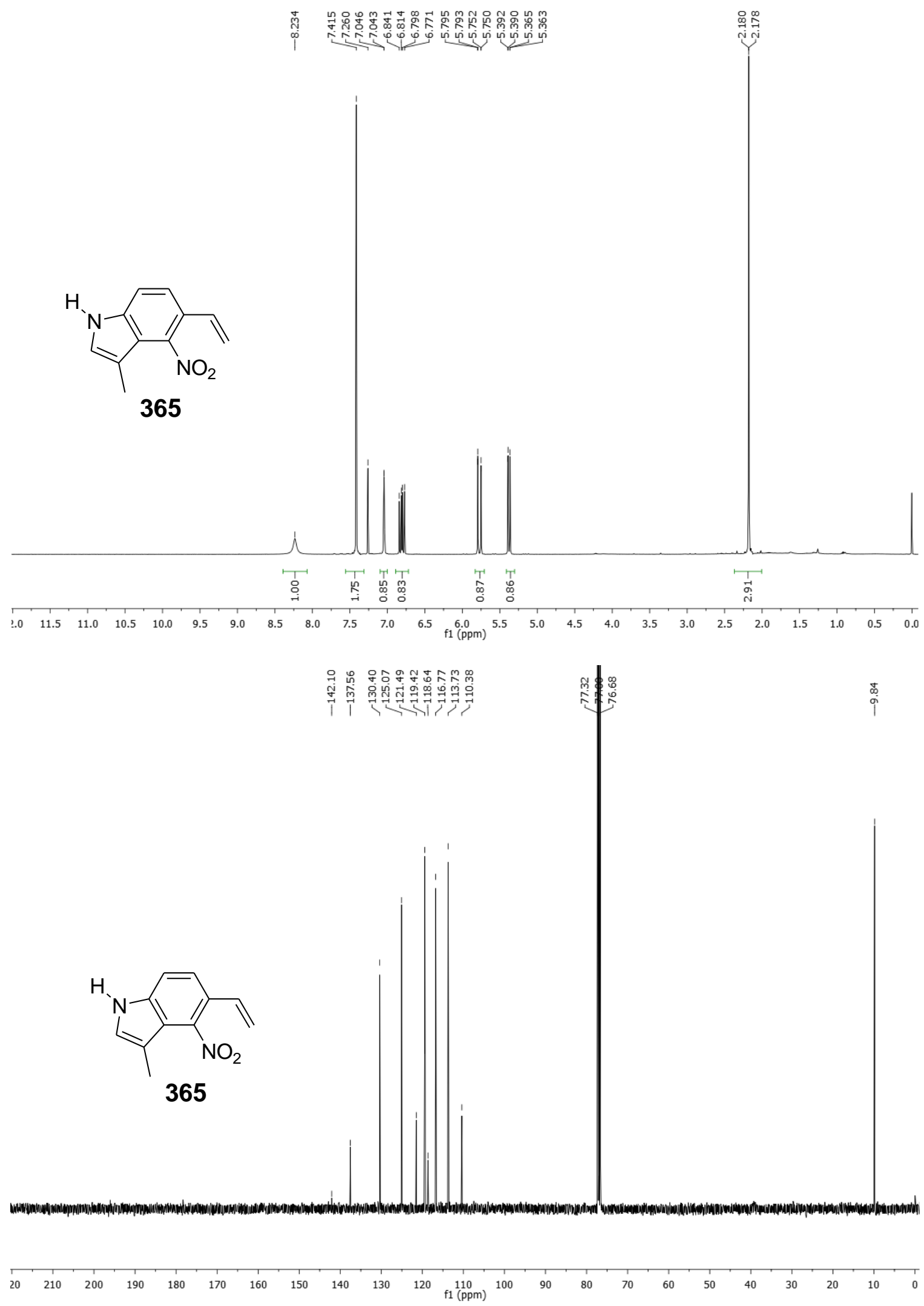

Figure $7.105{ }^{1} \mathrm{H}$ and ${ }^{13} \mathrm{C}$ NMR of compound 365 


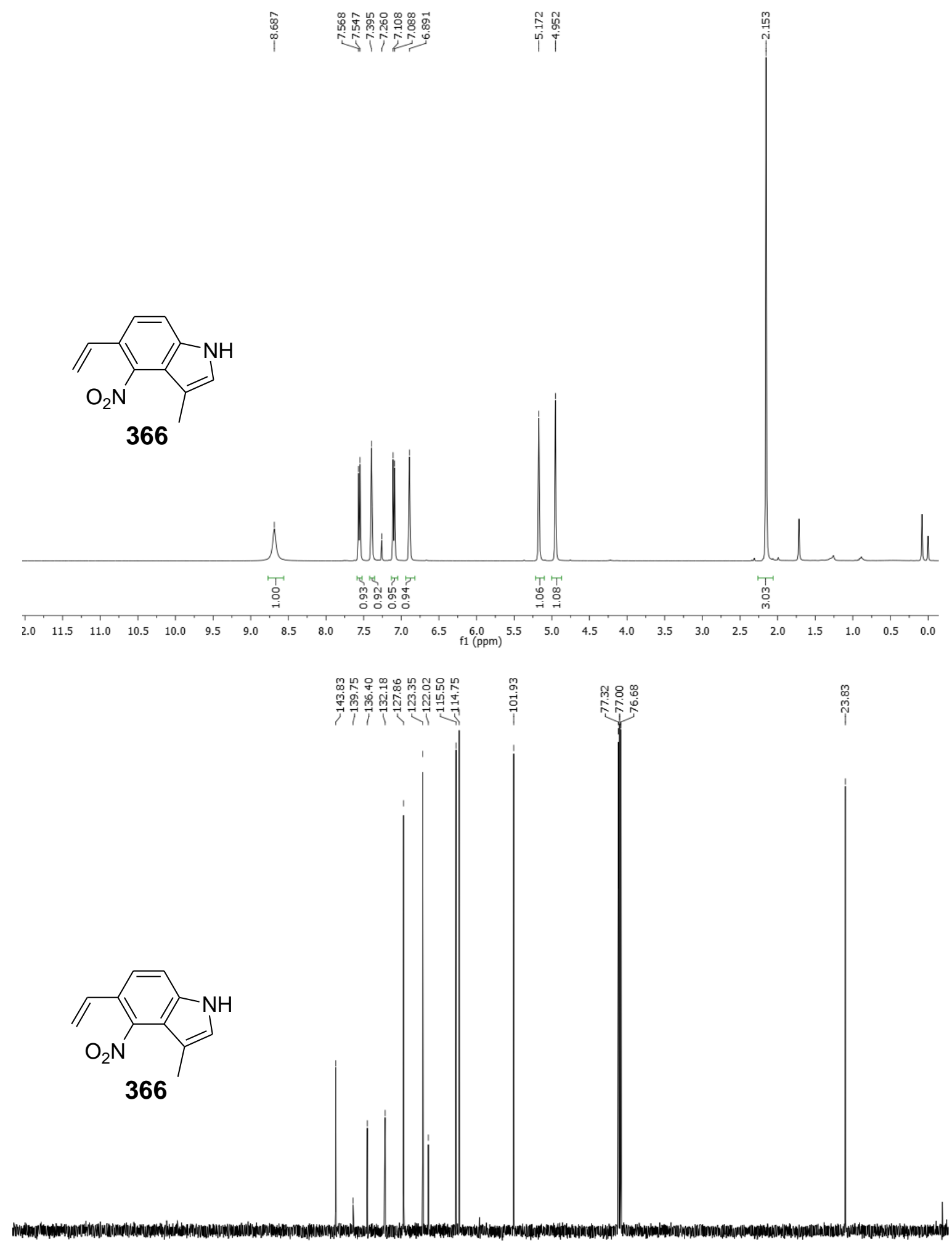

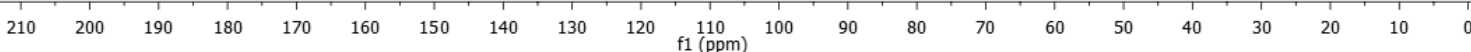

Figure $7.106{ }^{1} \mathrm{H}$ and ${ }^{13} \mathrm{C} \mathrm{NMR}$ of compound 366 

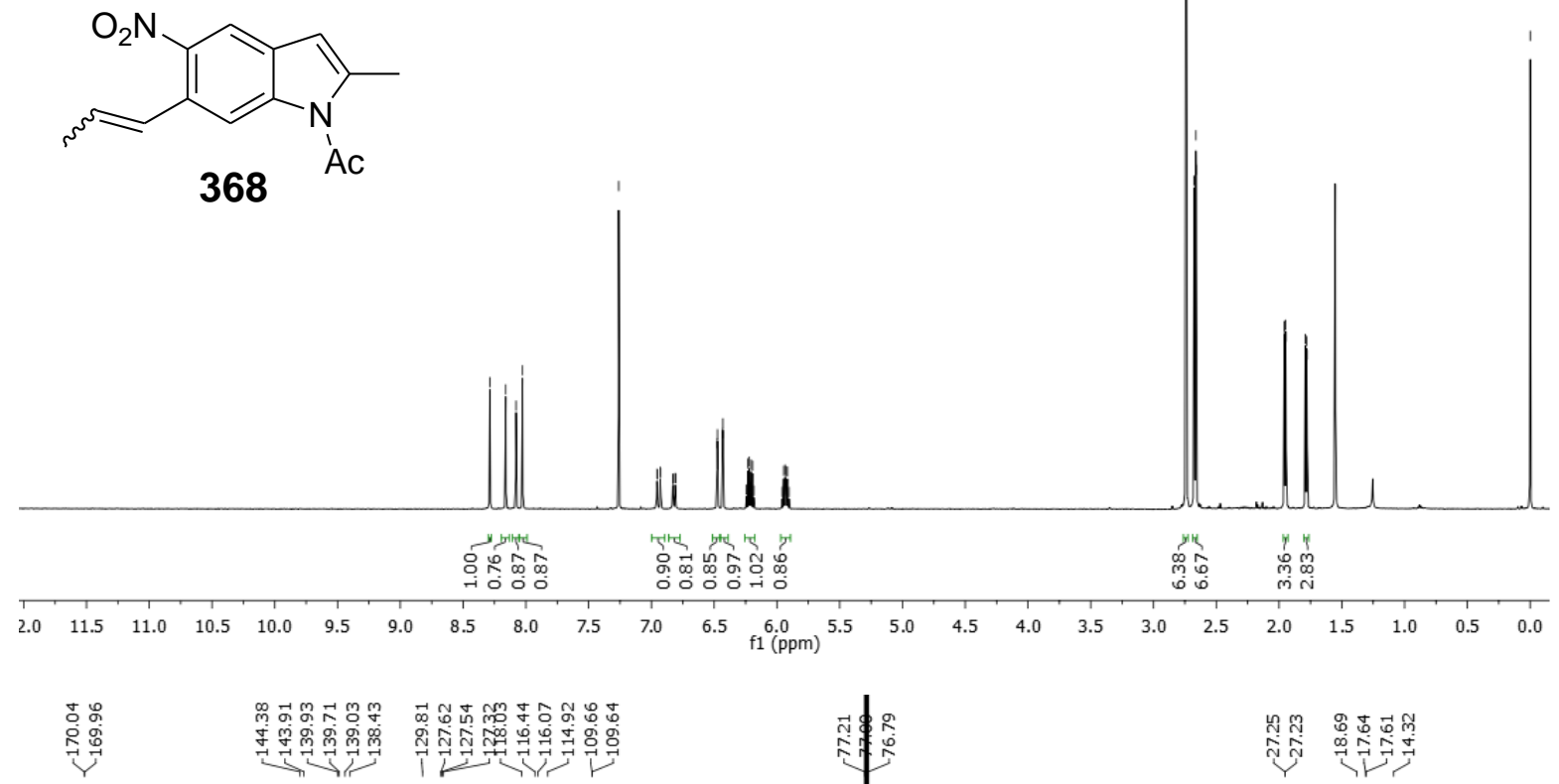

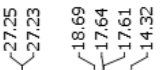
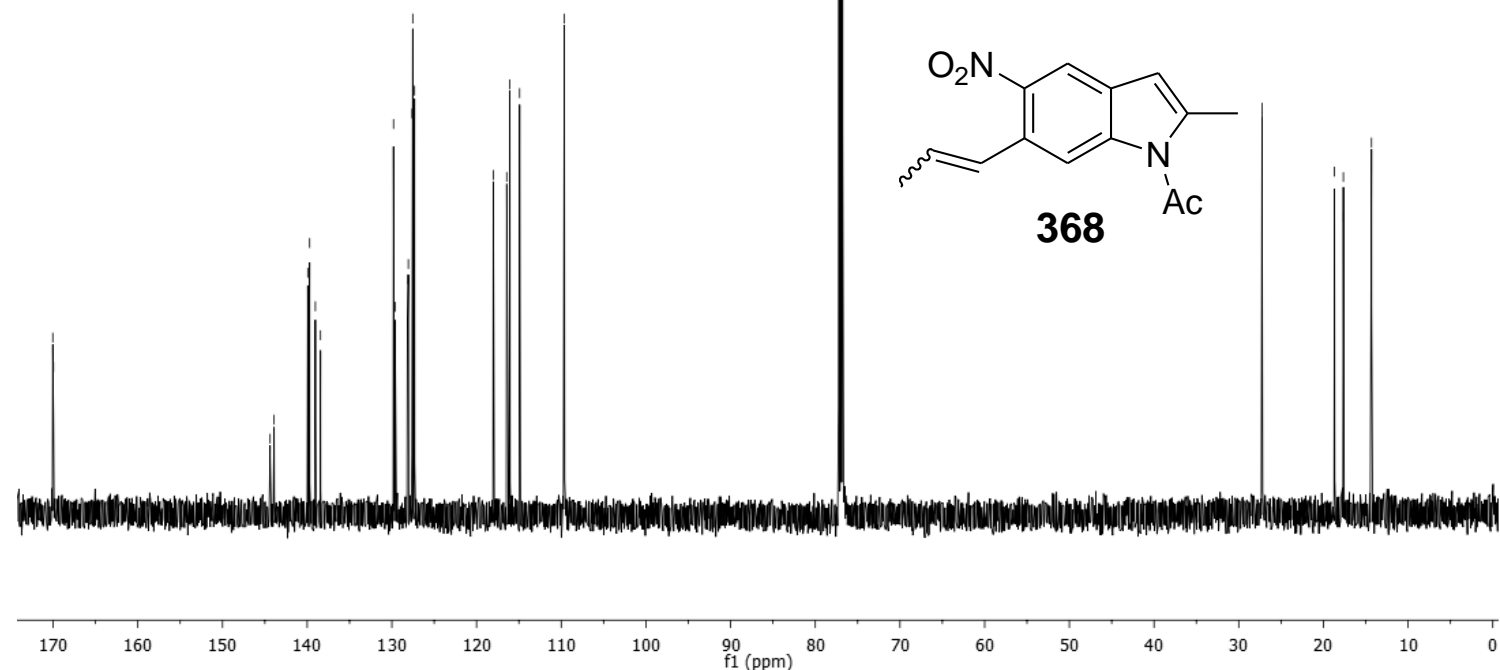

Figure $7.107{ }^{1} \mathrm{H}$ and ${ }^{13} \mathrm{C}$ NMR of compound 368 

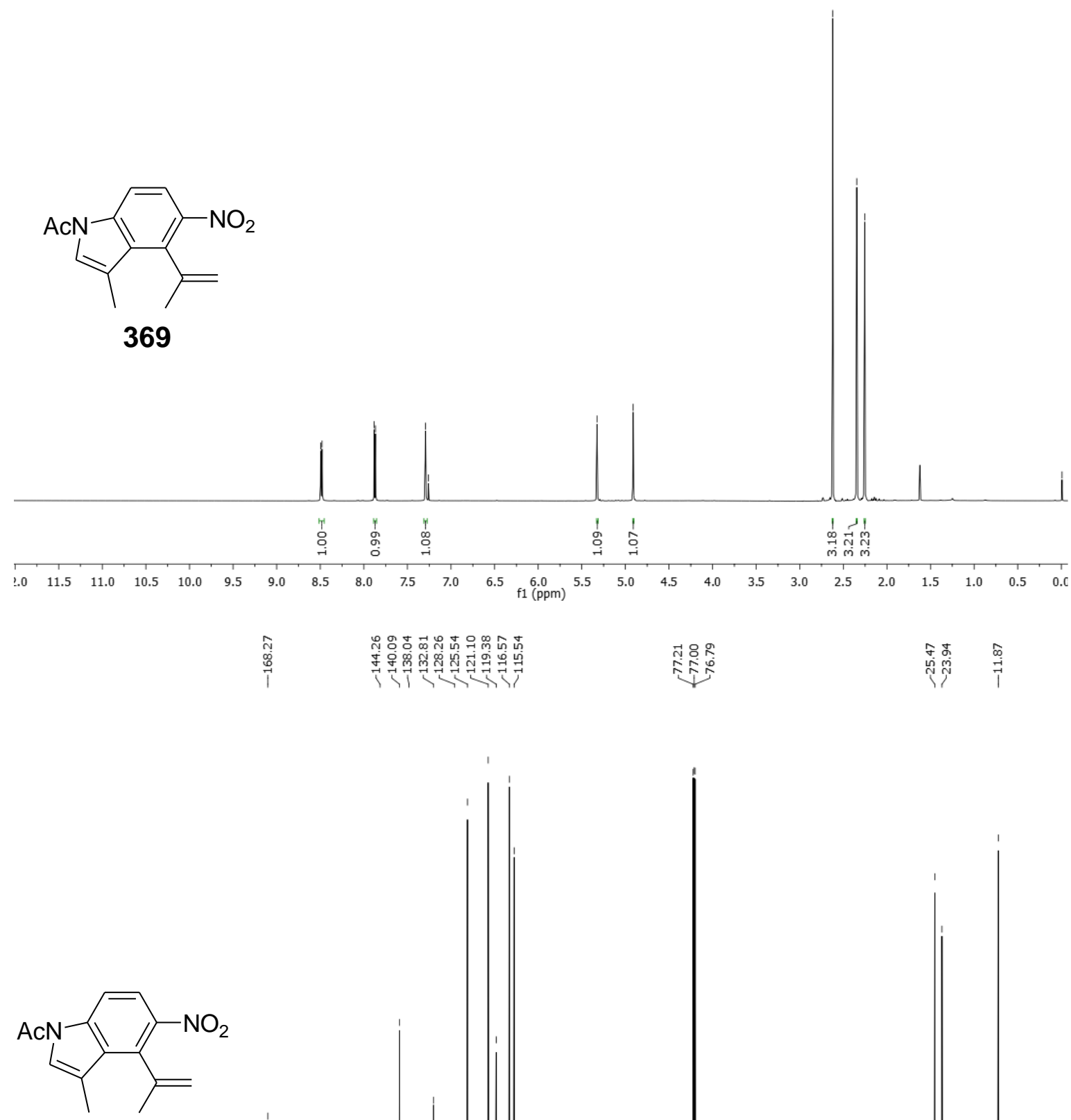

369

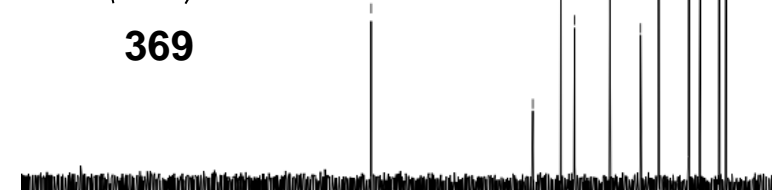

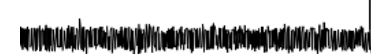
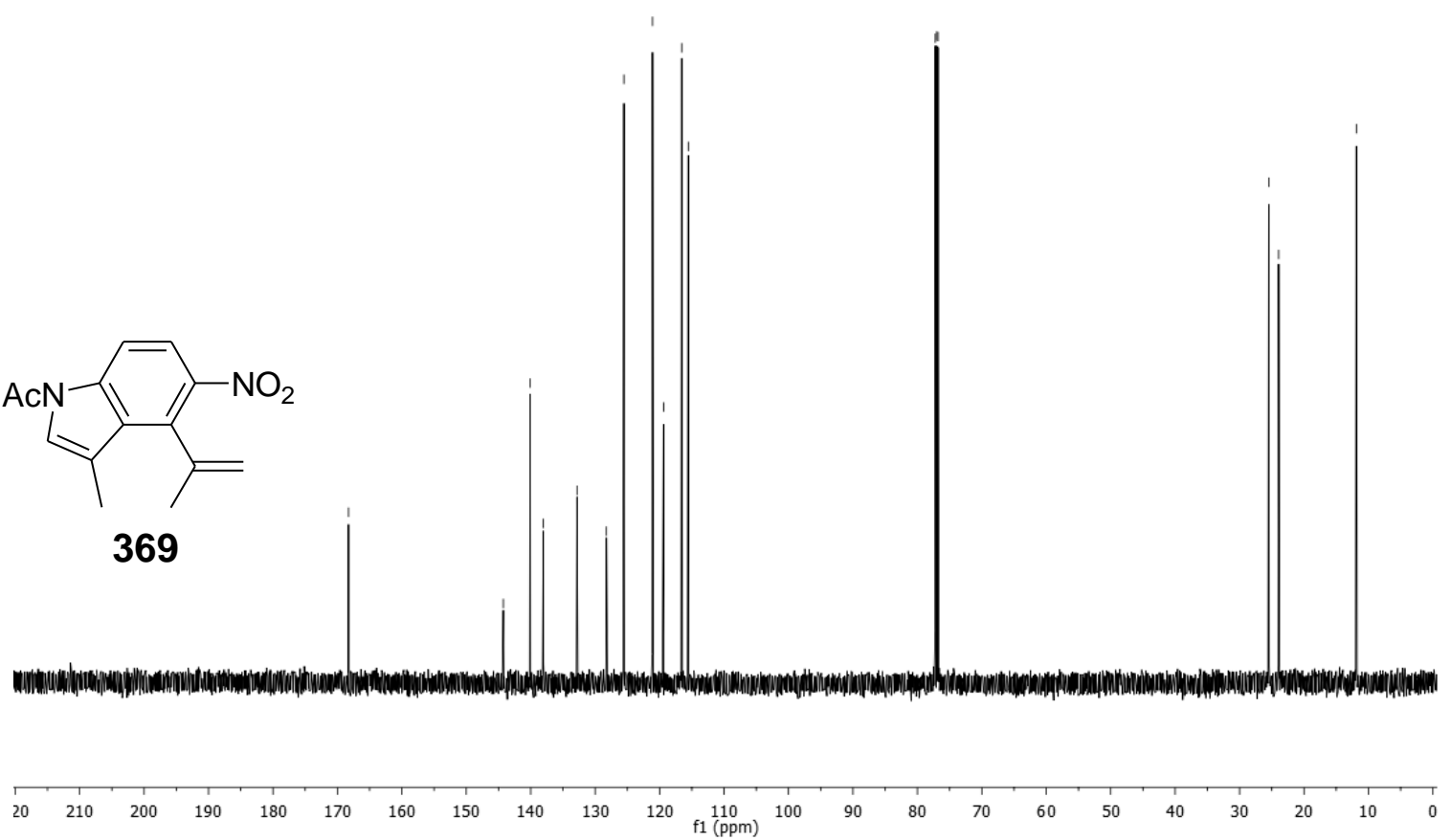

Figure 7.108 ${ }^{1} \mathrm{H}$ and ${ }^{13} \mathrm{C}$ NMR of compound 369 


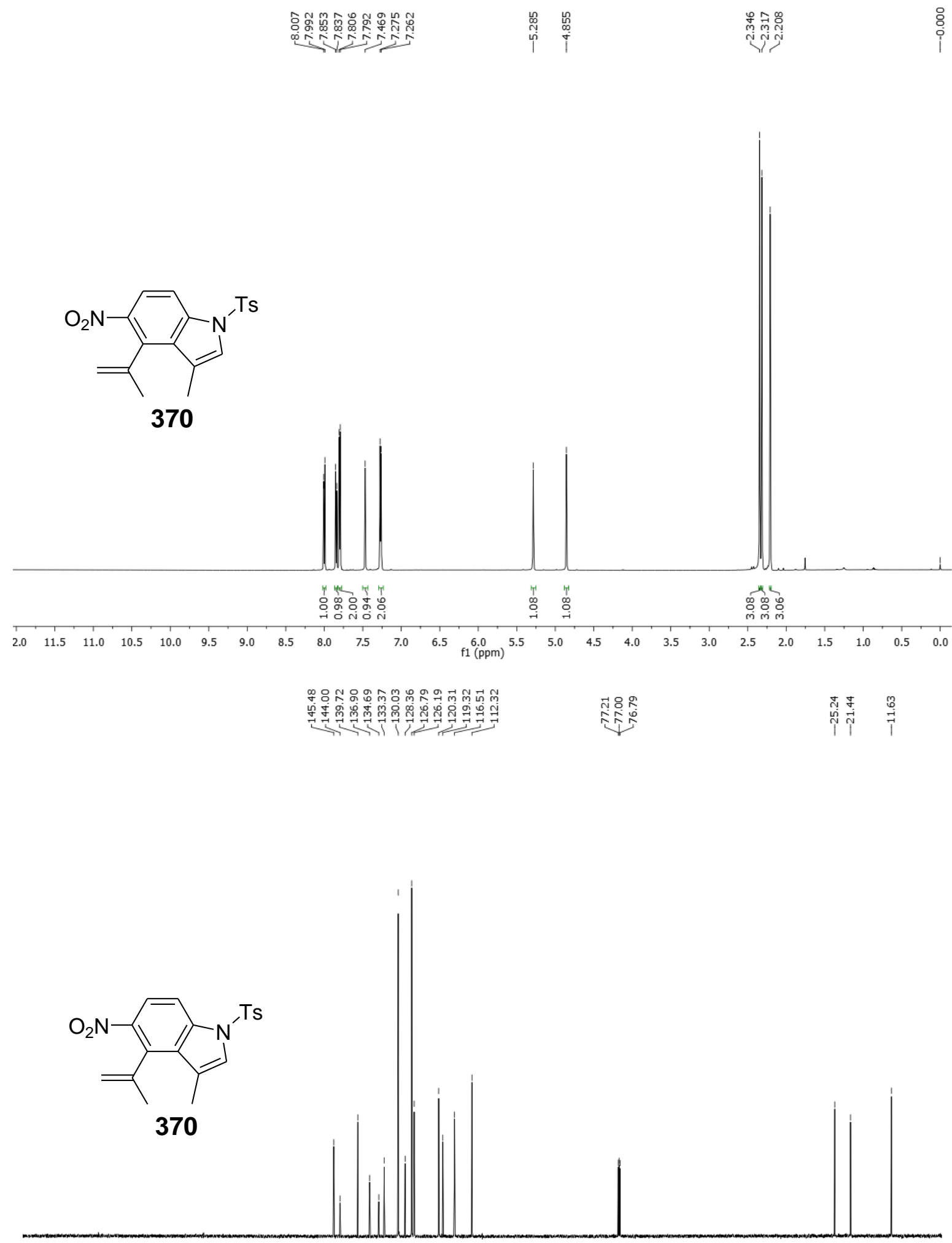

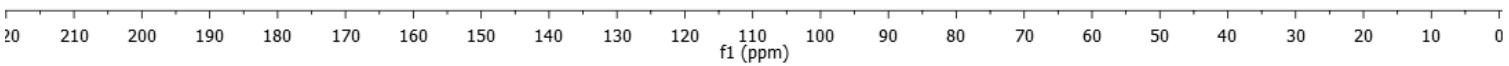

Figure 7.109 ${ }^{1} \mathrm{H}$ and ${ }^{13} \mathrm{C} \mathrm{NMR}$ of compound 370 

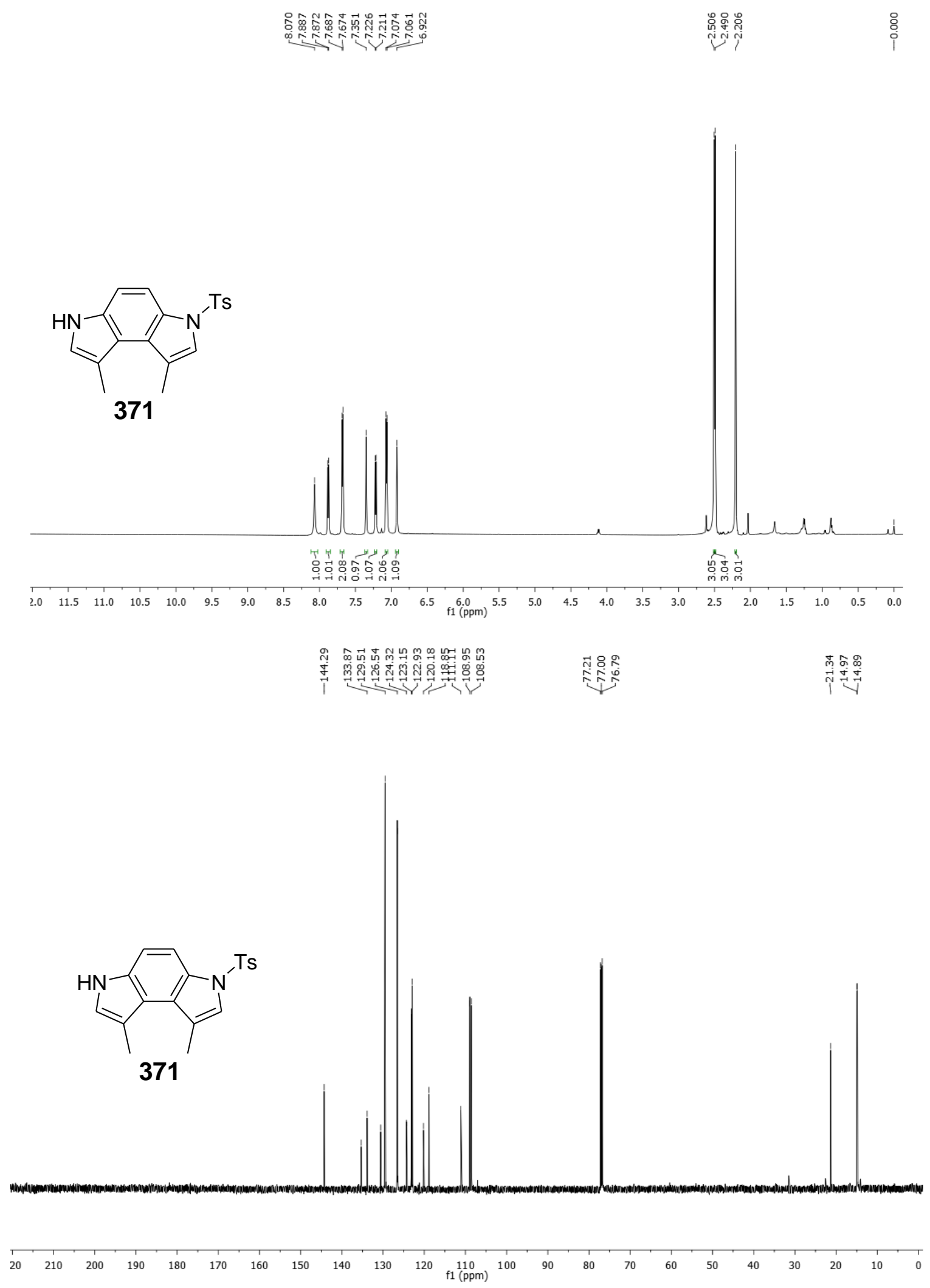

Figure 7.110 ${ }^{1} \mathrm{H}$ and ${ }^{13} \mathrm{C}$ NMR of compound 371 


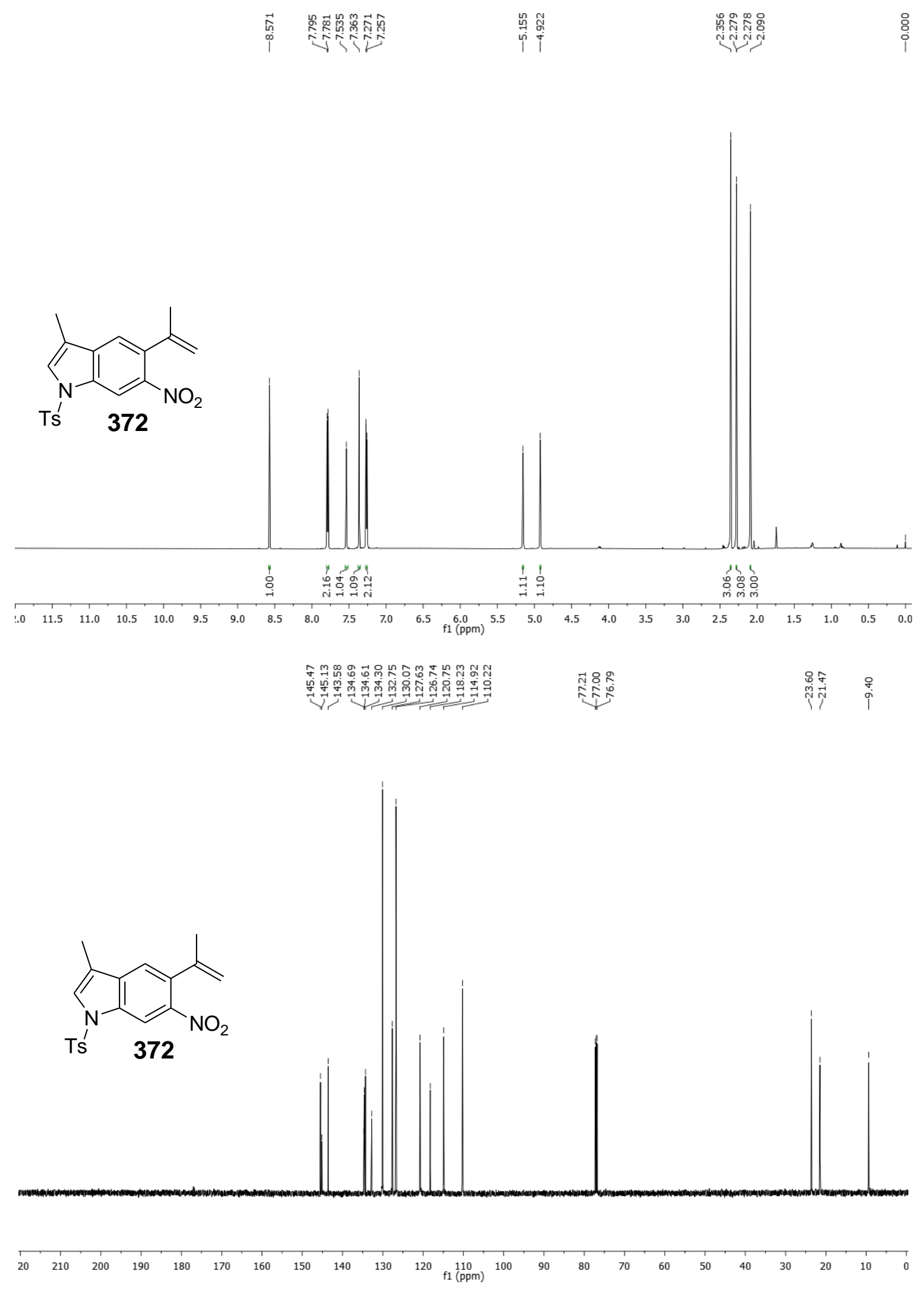

Figure $7.111{ }^{1} \mathrm{H}$ and ${ }^{13} \mathrm{C}$ NMR of compound 372 


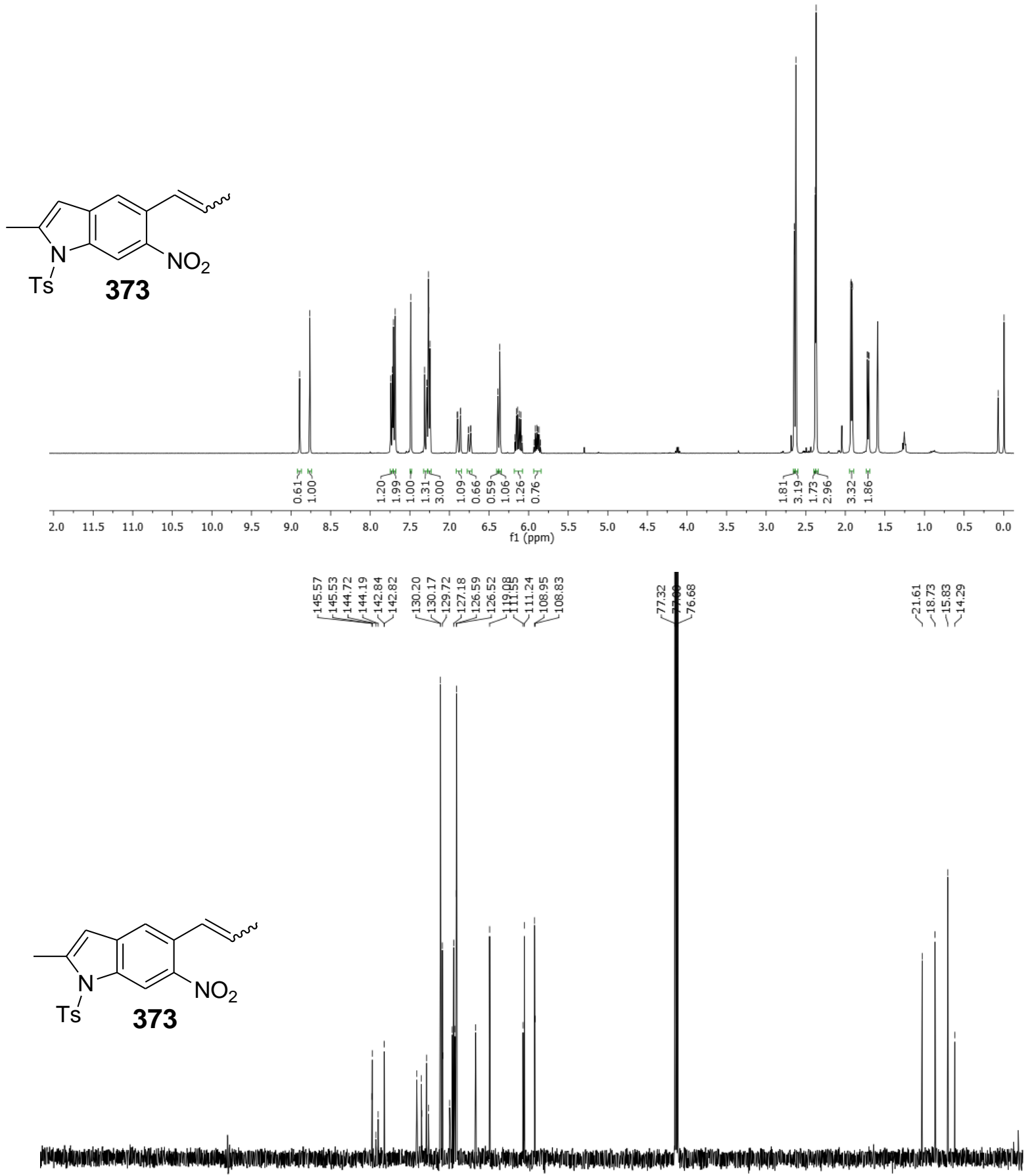

$\begin{array}{llllllllllllllllllllllll}20 & 210 & 200 & 190 & 180 & 170 & 160 & 150 & 140 & 130 & 120 & 110 & 100 & 90 & 80 & 70 & 60 & 50 & 40 & 30 & 20 & 10 & 0\end{array}$

Figure 7.112 ${ }^{1} \mathrm{H}$ and ${ }^{13} \mathrm{C}$ NMR of compound 373 


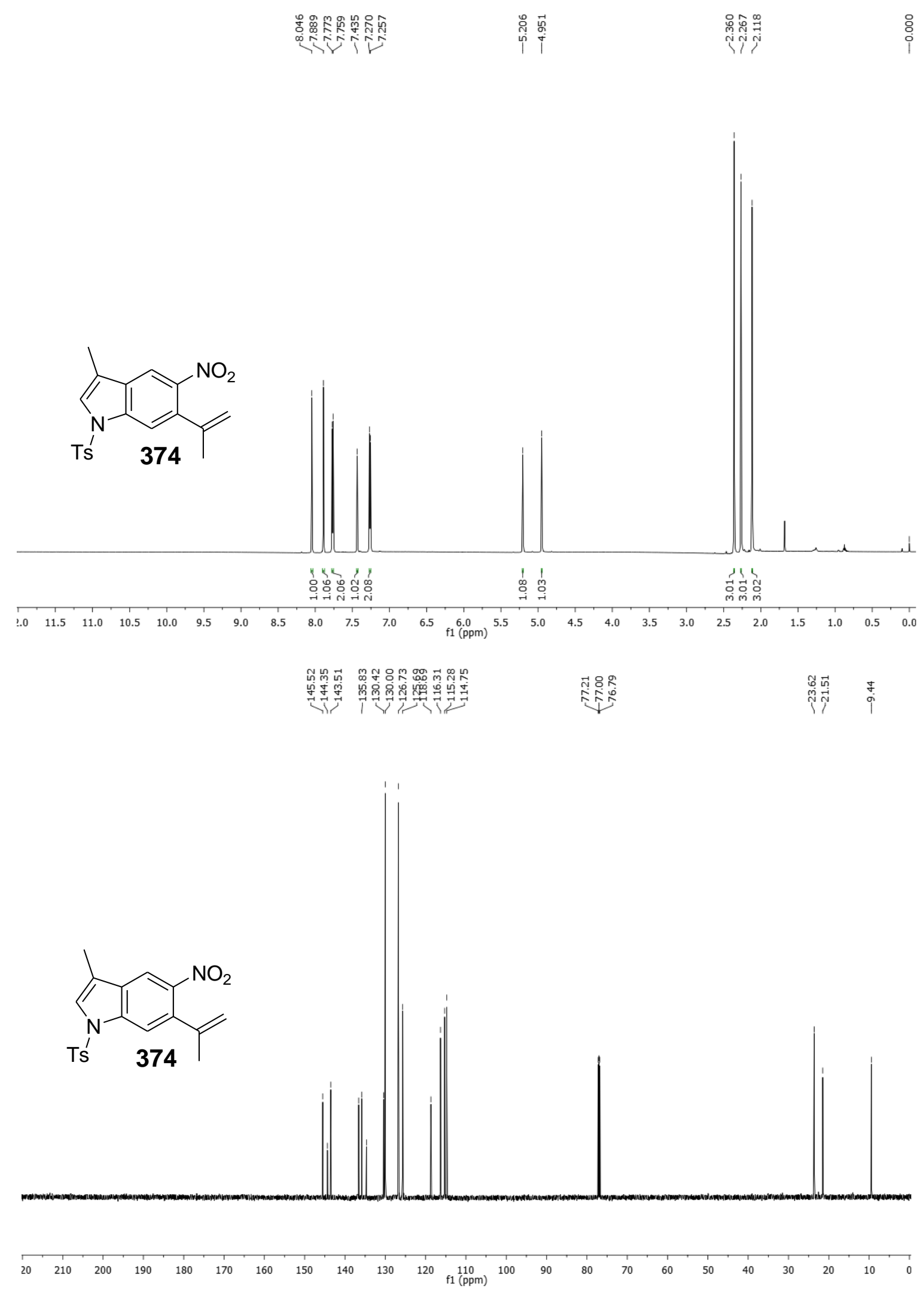

Figure $7.113{ }^{1} \mathrm{H}$ and ${ }^{13} \mathrm{C}$ NMR of compound 374 

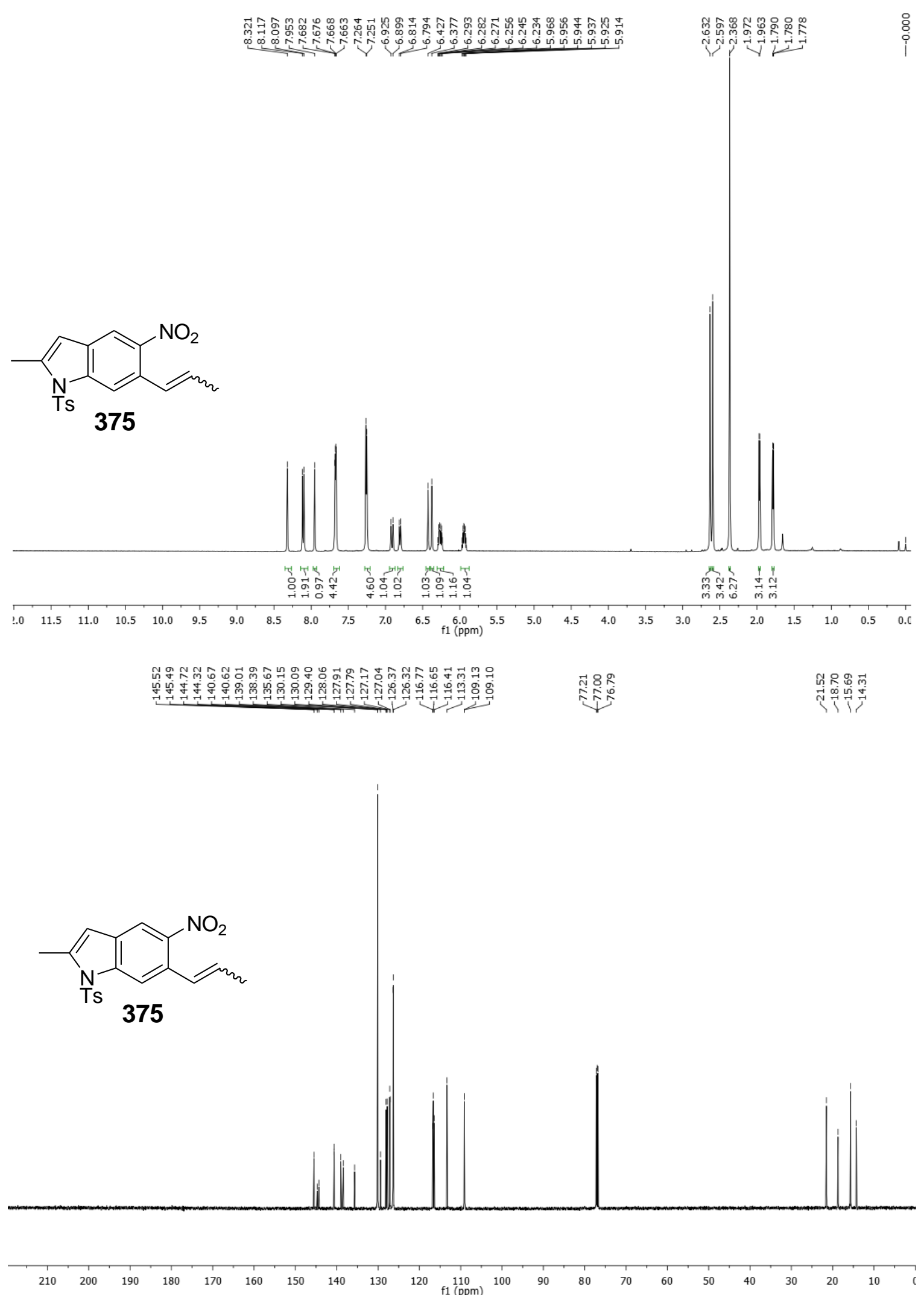

Figure $7.114{ }^{1} \mathrm{H}$ and ${ }^{13} \mathrm{C}$ NMR of compound 375 

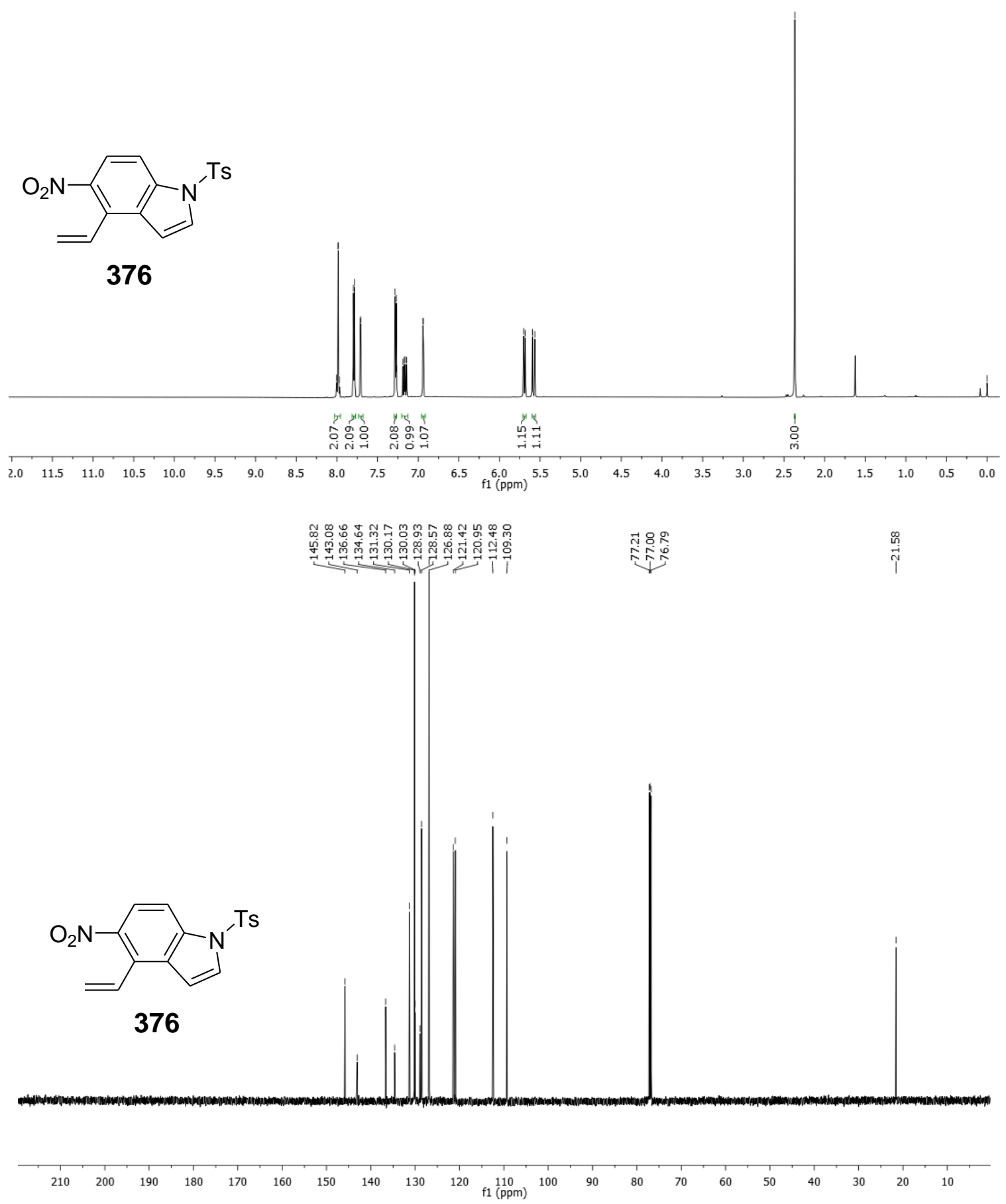

Figure 7.115 ${ }^{1} \mathrm{H}$ and ${ }^{13} \mathrm{C}$ NMR of compound 376 

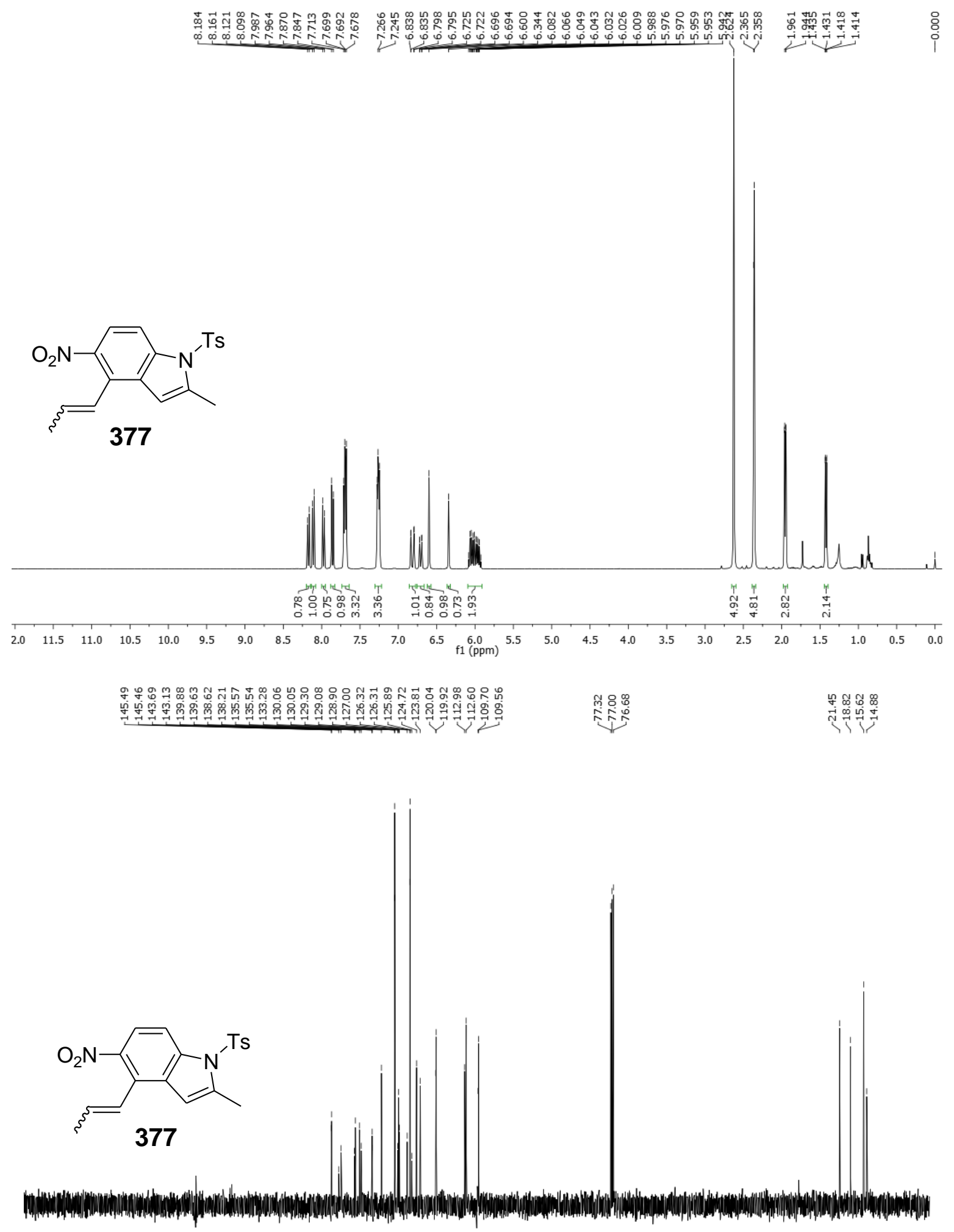

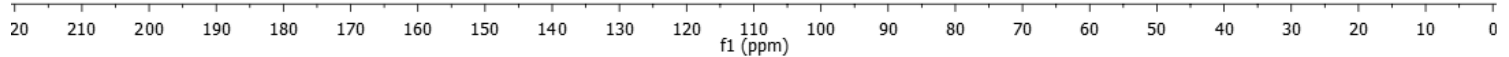

Figure $7.116{ }^{1} \mathrm{H}$ and ${ }^{13} \mathrm{C} \mathrm{NMR}$ of compound 377 

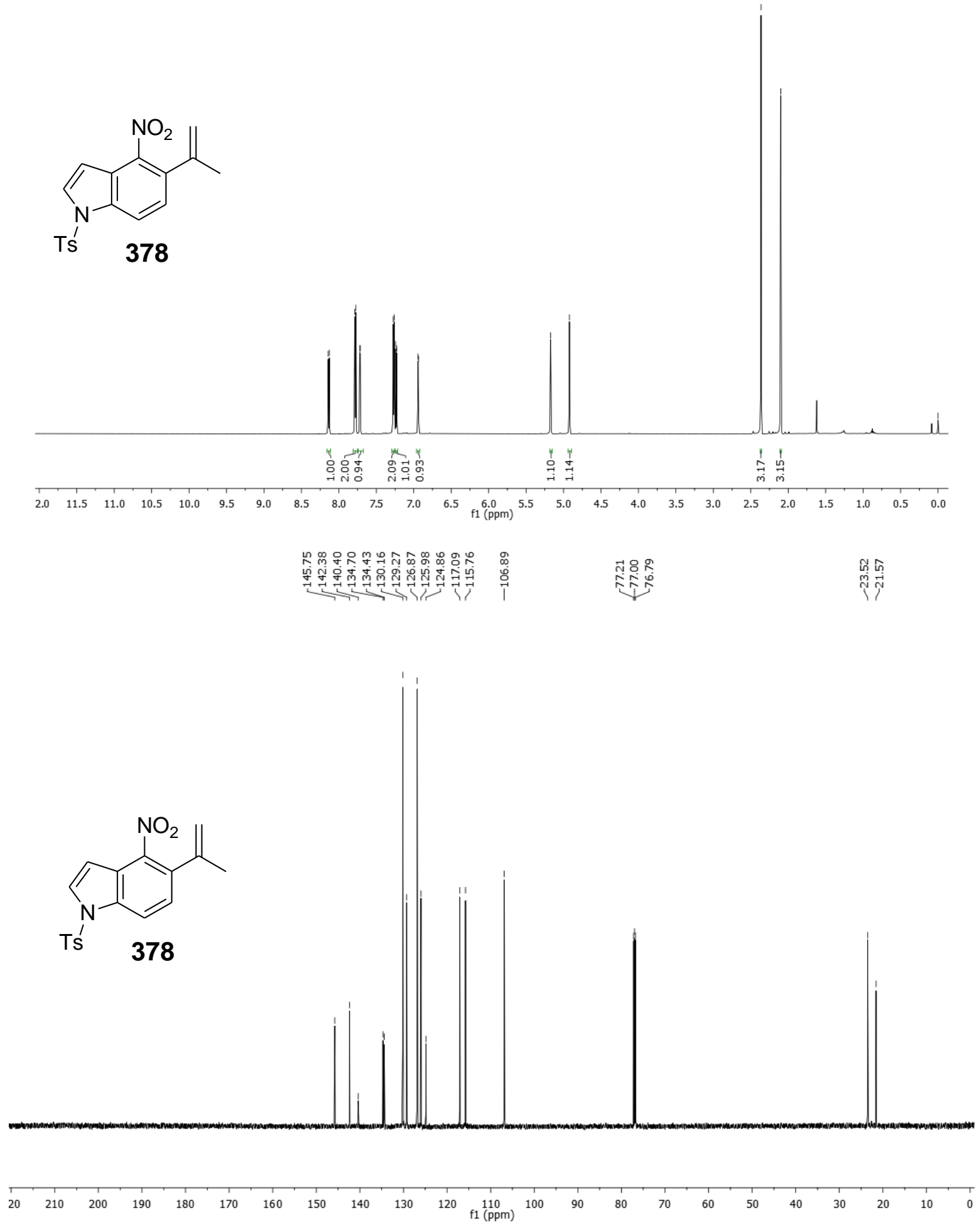

Figure 7.117 ${ }^{1} \mathrm{H}$ and ${ }^{13} \mathrm{C}$ NMR of compound 378 


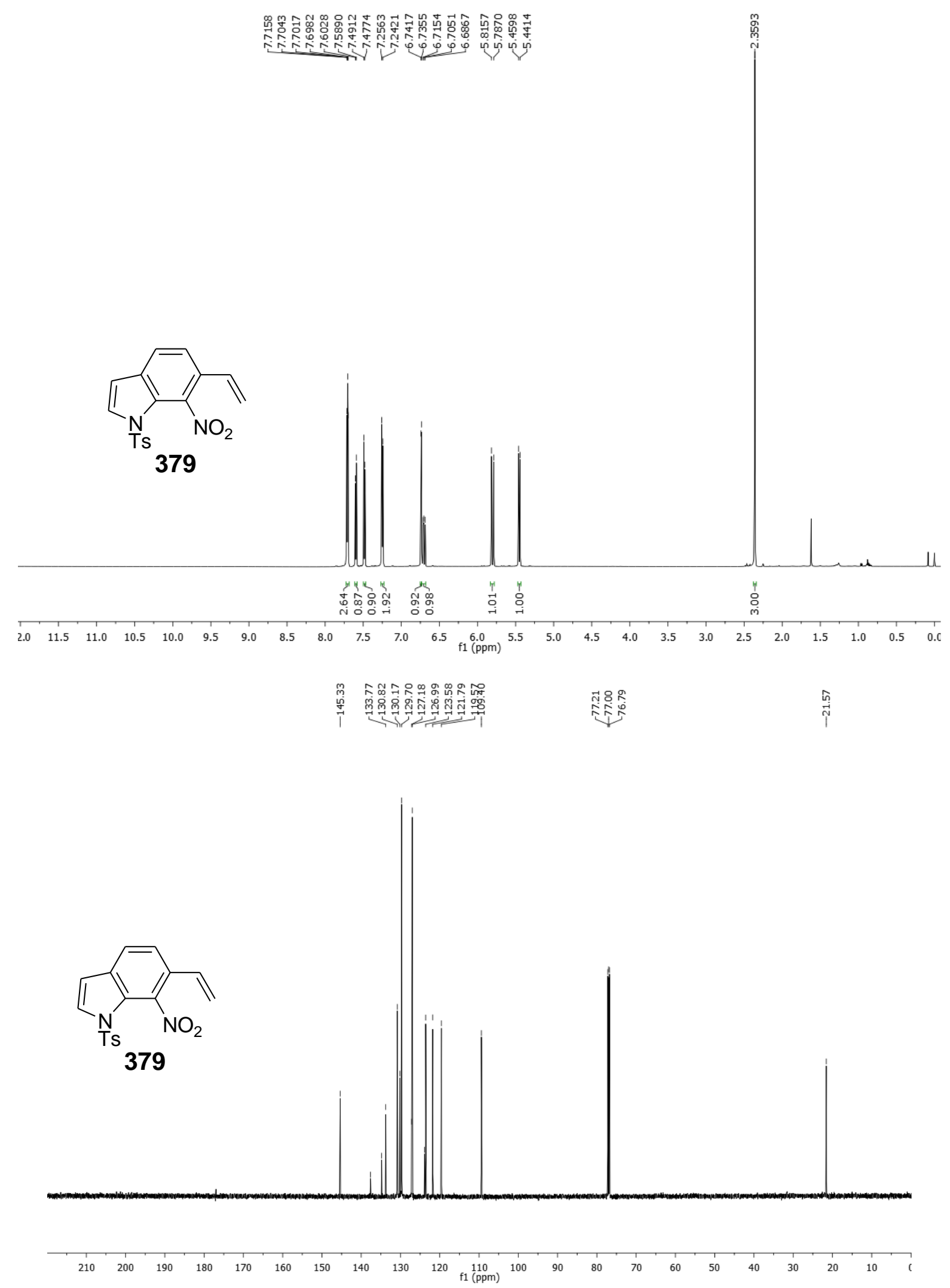

Figure 7.118 ${ }^{1} \mathrm{H}$ and ${ }^{13} \mathrm{C}$ NMR of compound 379 

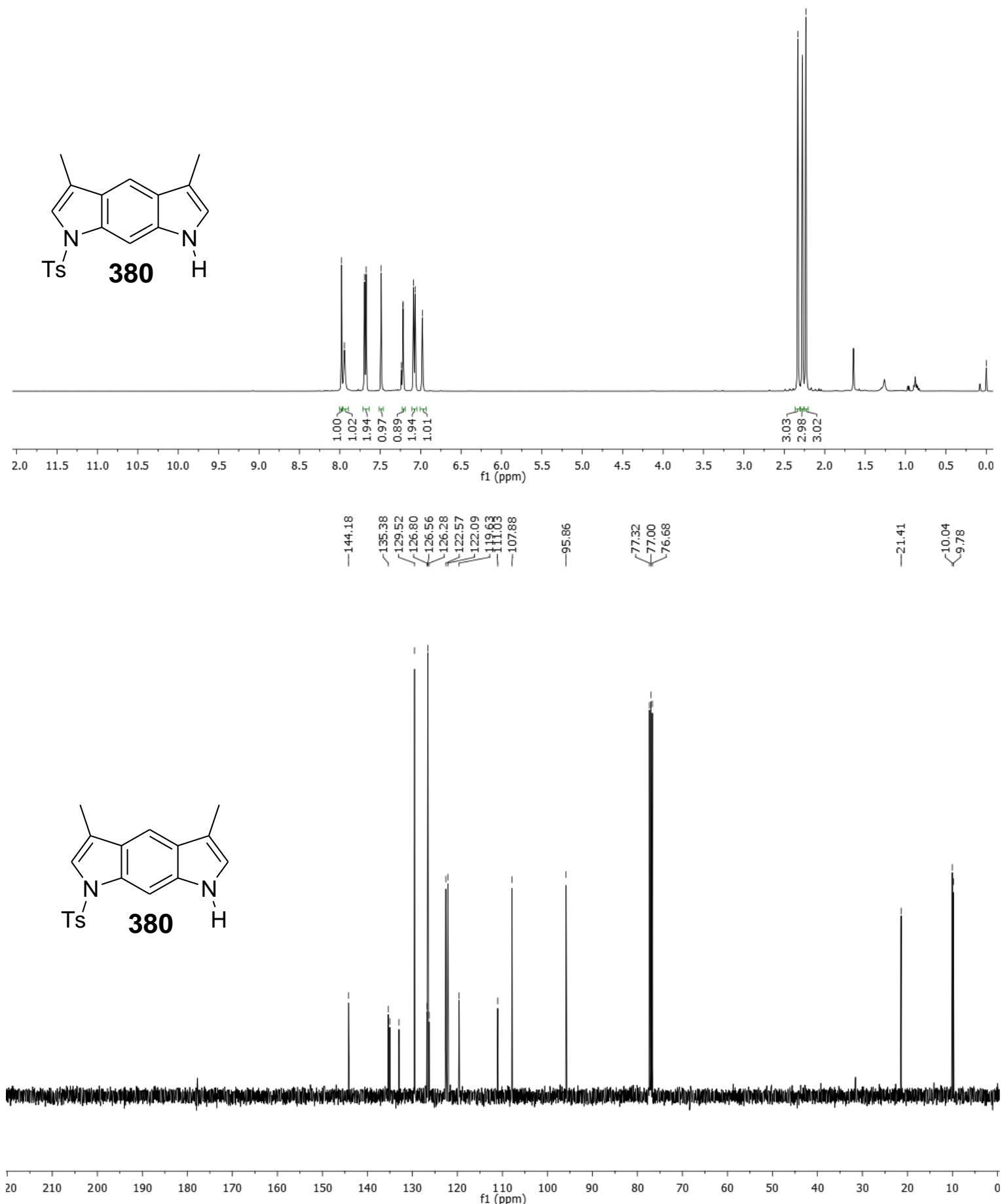

Figure 7.119 ${ }^{1} \mathrm{H}$ and ${ }^{13} \mathrm{C}$ NMR of compound 380 

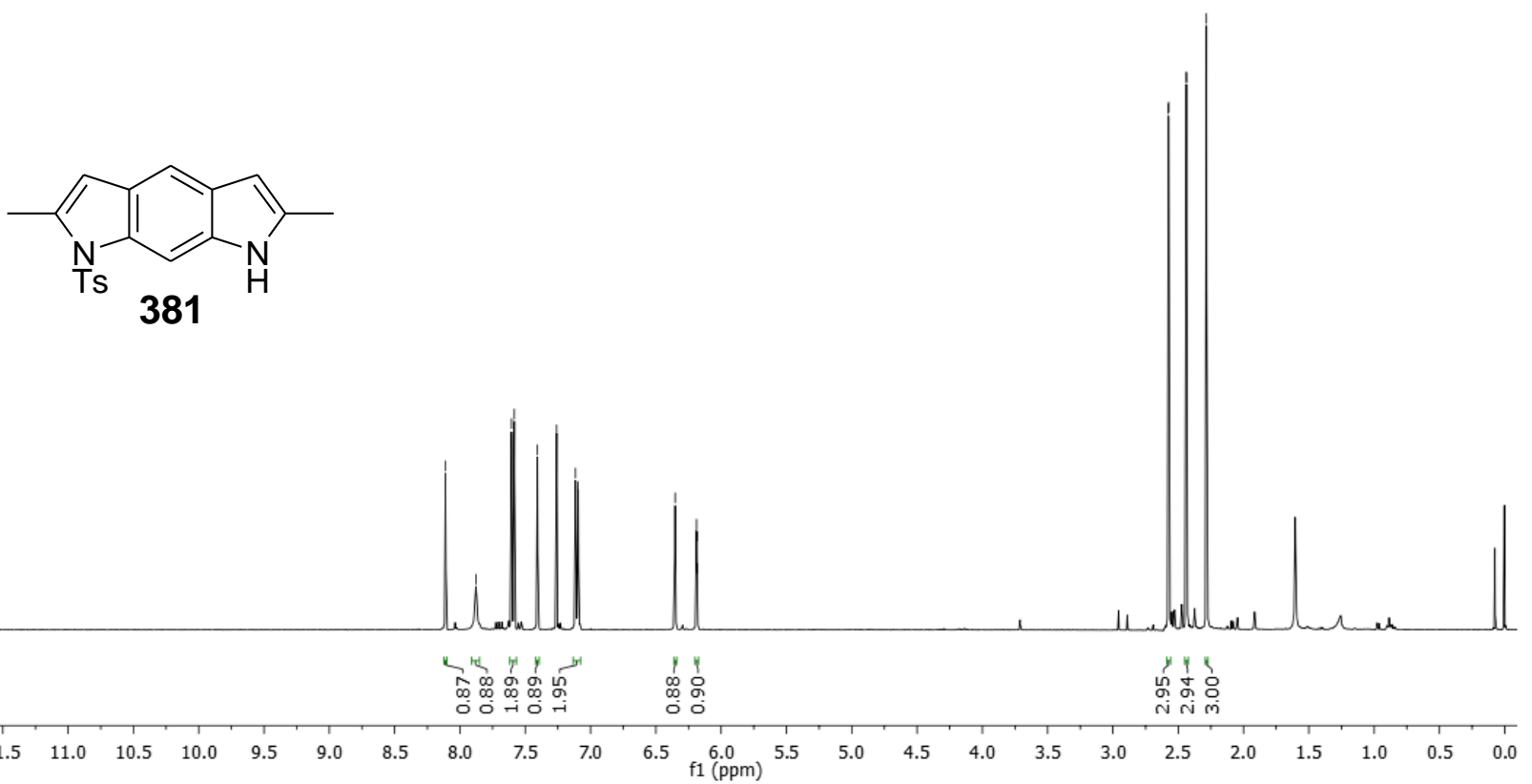

Figure $7.120{ }^{1} \mathrm{H}$ and ${ }^{13} \mathrm{C}$ NMR of compound 381 


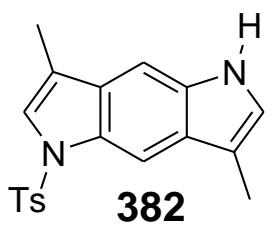

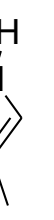
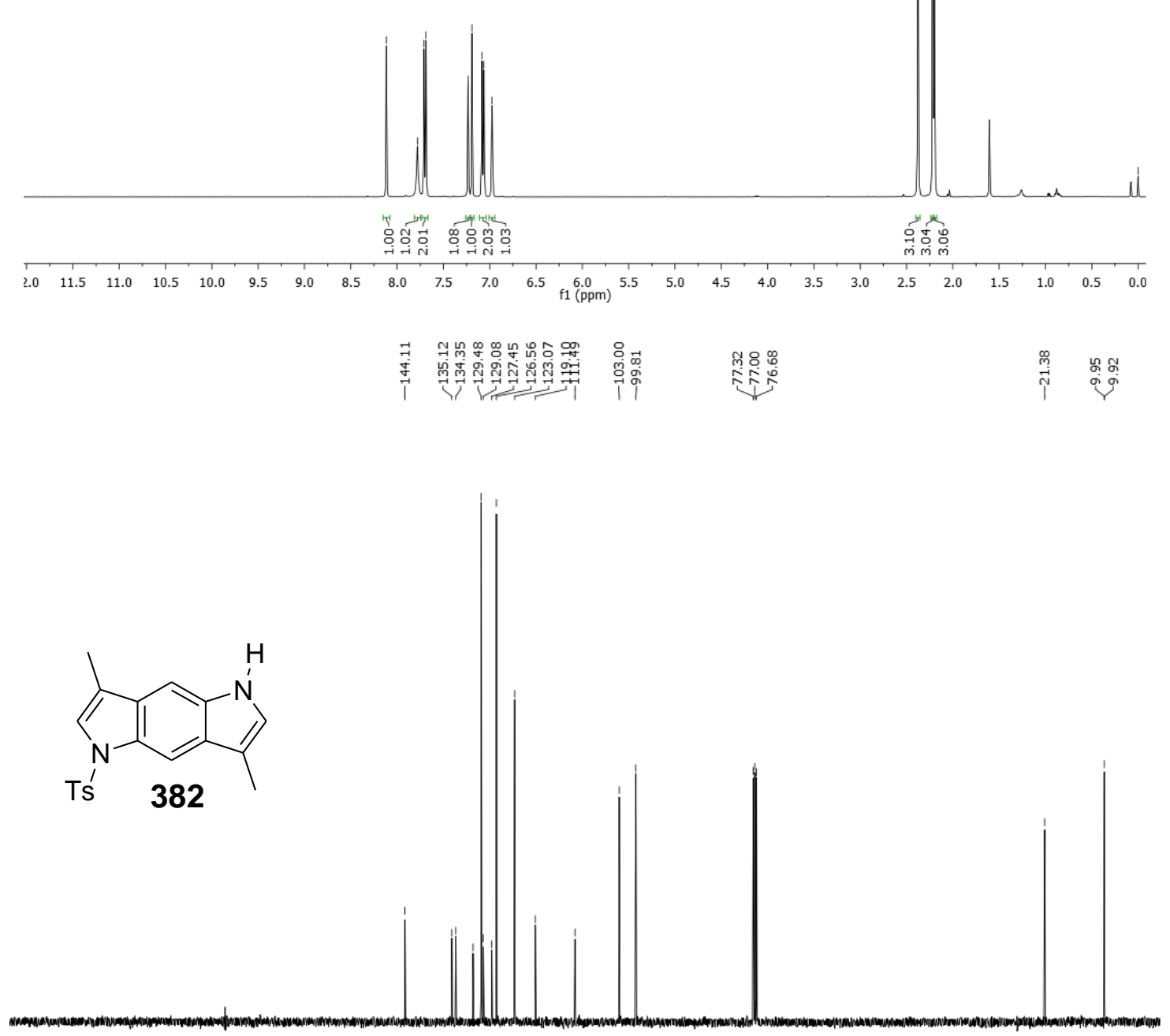

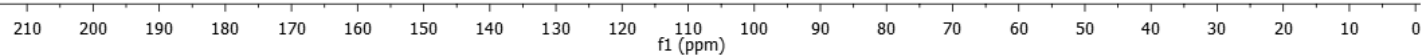

Figure 7.121 ${ }^{1} \mathrm{H}$ and ${ }^{13} \mathrm{C}$ NMR of compound 382 

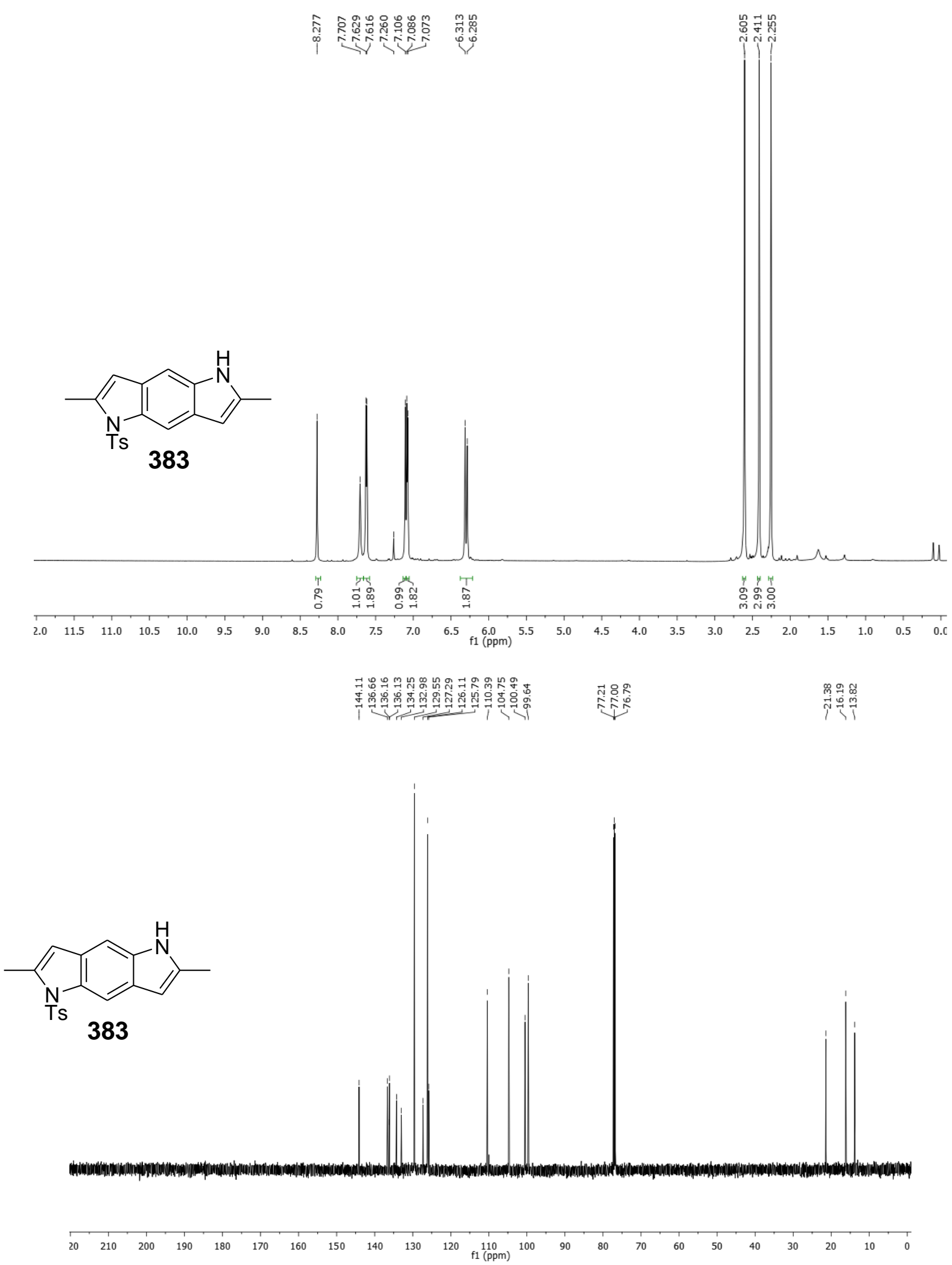

Figure 7.122 ${ }^{1} \mathrm{H}$ and ${ }^{13} \mathrm{C}$ NMR of compound 383 


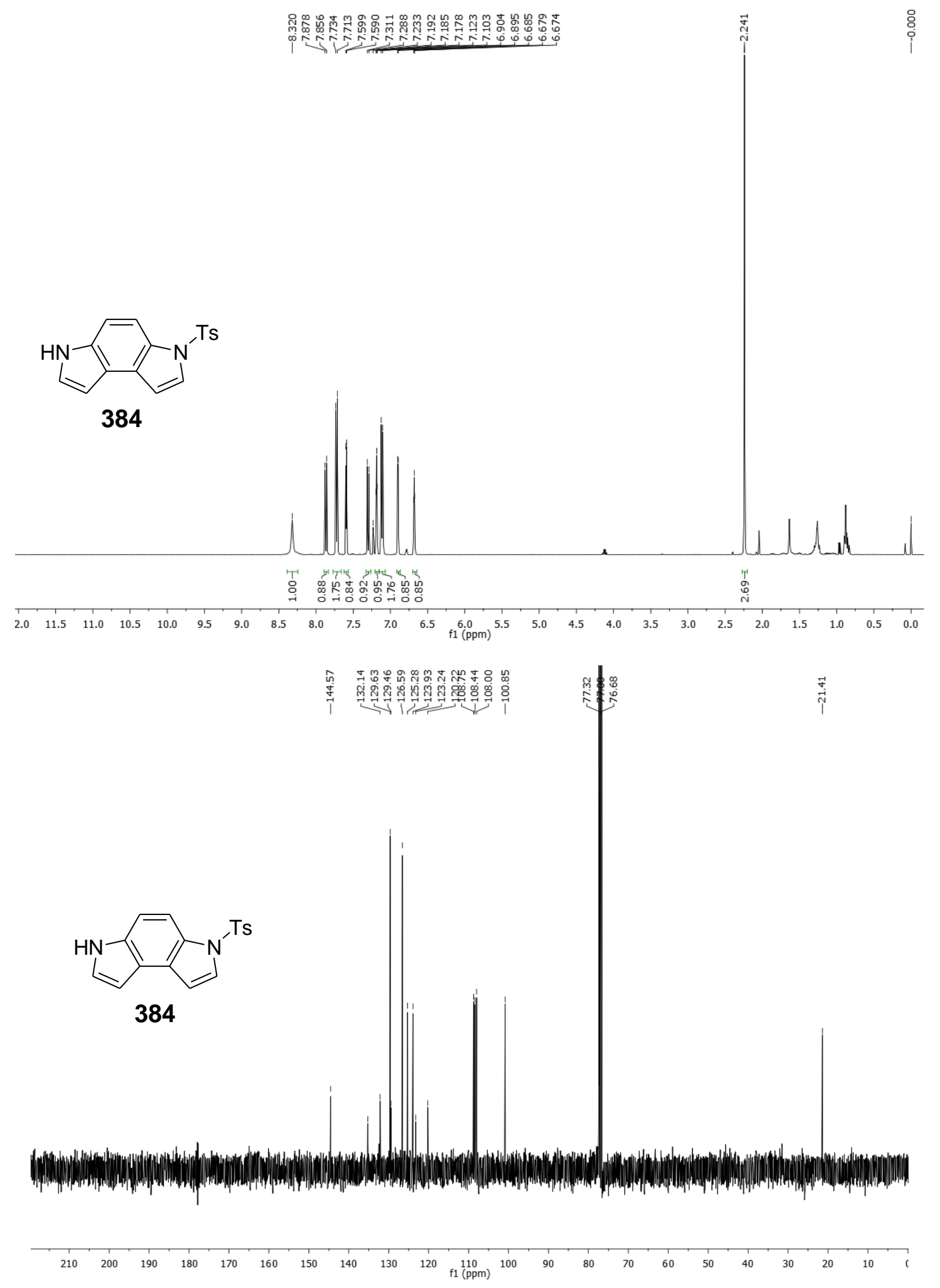

Figure $7.123{ }^{1} \mathrm{H}$ and ${ }^{13} \mathrm{C}$ NMR of compound 384 


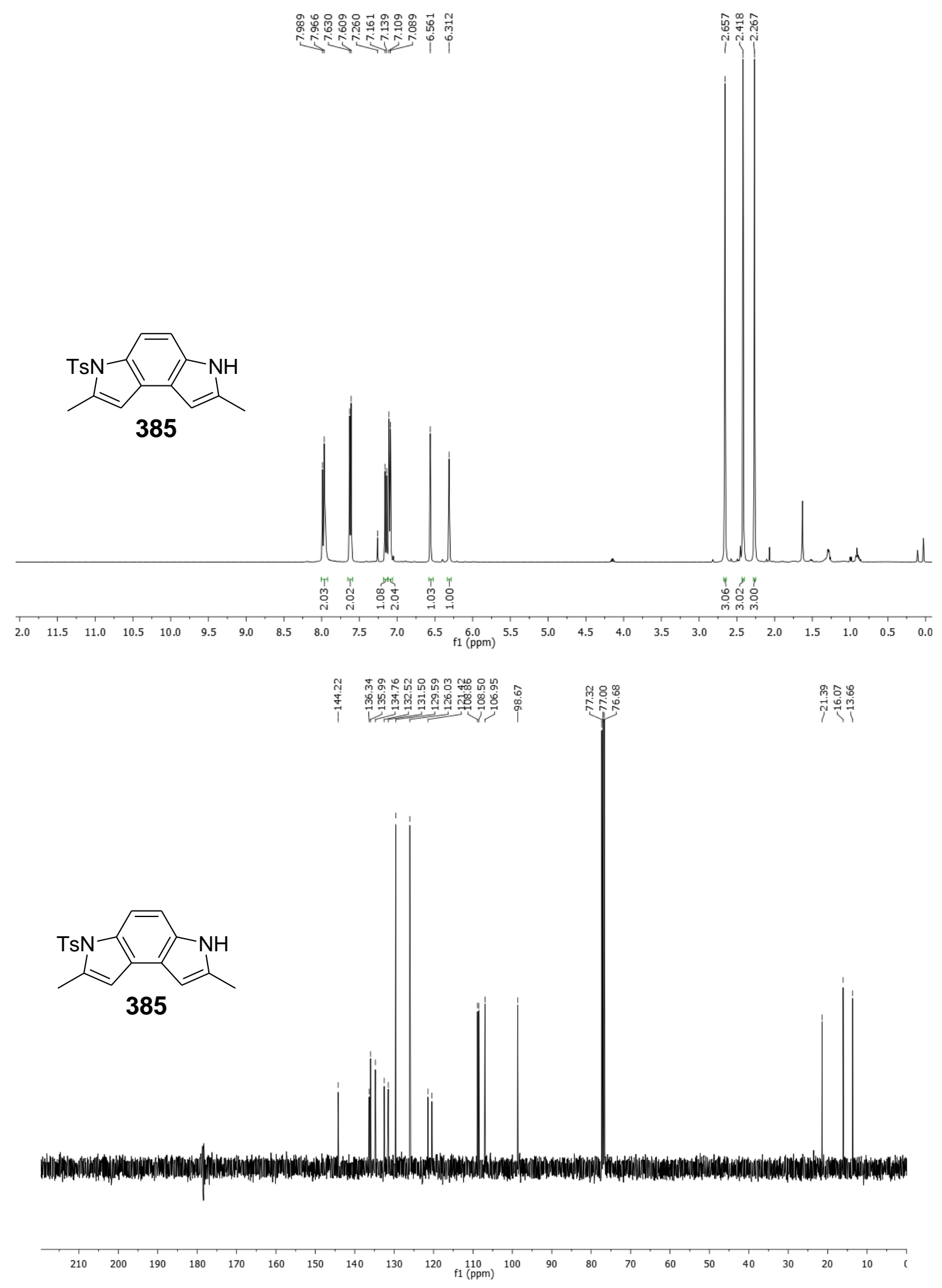

Figure 7.124 ${ }^{1} \mathrm{H}$ and ${ }^{13} \mathrm{C}$ NMR of compound 385 

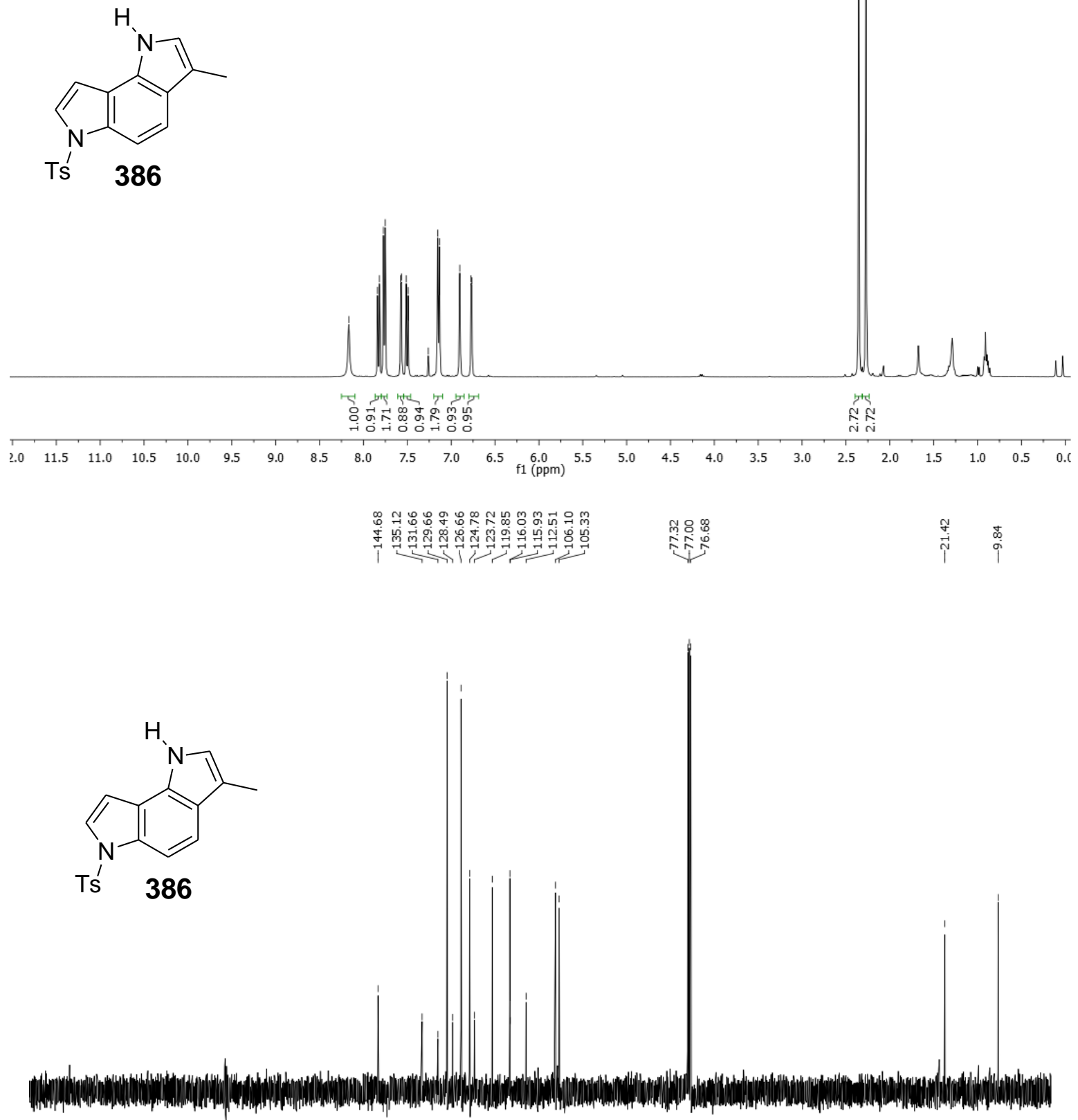

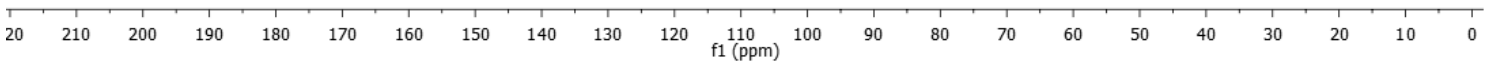

Figure 7.125 ${ }^{1} \mathrm{H}$ and ${ }^{13} \mathrm{C}$ NMR of compound 386 
Synthesis of indoles, biindoles, indole alkaloids, pyrroloindoles, and benzimidazoles from aromatic nitro compounds and a study of the chemoselectivity in the Kosugi-Migita-Stille coupling

Nurul Hoda Ansari

Dissertation submitted to the Eberly College of Arts and Sciences at West Virginia University in partial fulfillment of the requirements for the degree of

Doctor of Philosophy in Chemistry

C. Eugene Bennett Department of Chemistry

APPROVAL OF THE EXAMINING COMMITTEE

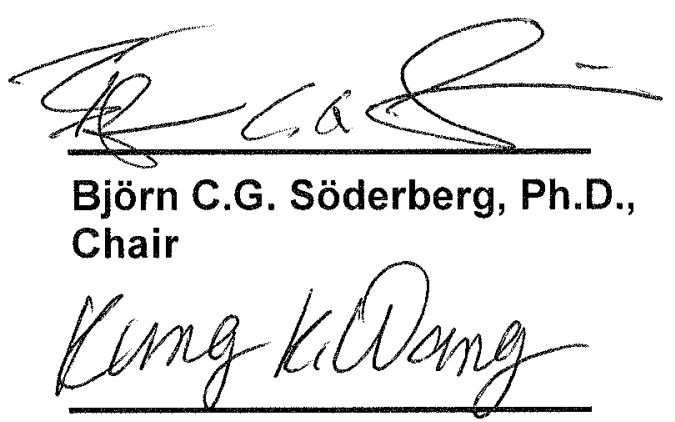

Rung K. Wang, Ph.D.

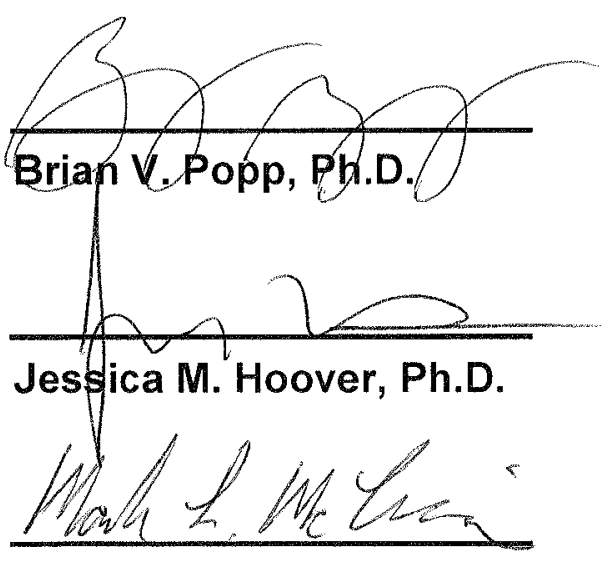

Mark L. McLaughlin, Ph.D. 